\title{
MYOKINES, ADIPOKINES, CYTOKINES IN MUSCLE PATHOPHYSIOLOGY
}

EDITED BY: Valentina Di Felice, Dario Coletti and Marilia Seelaender PUBLISHED IN: Frontiers in Physiology

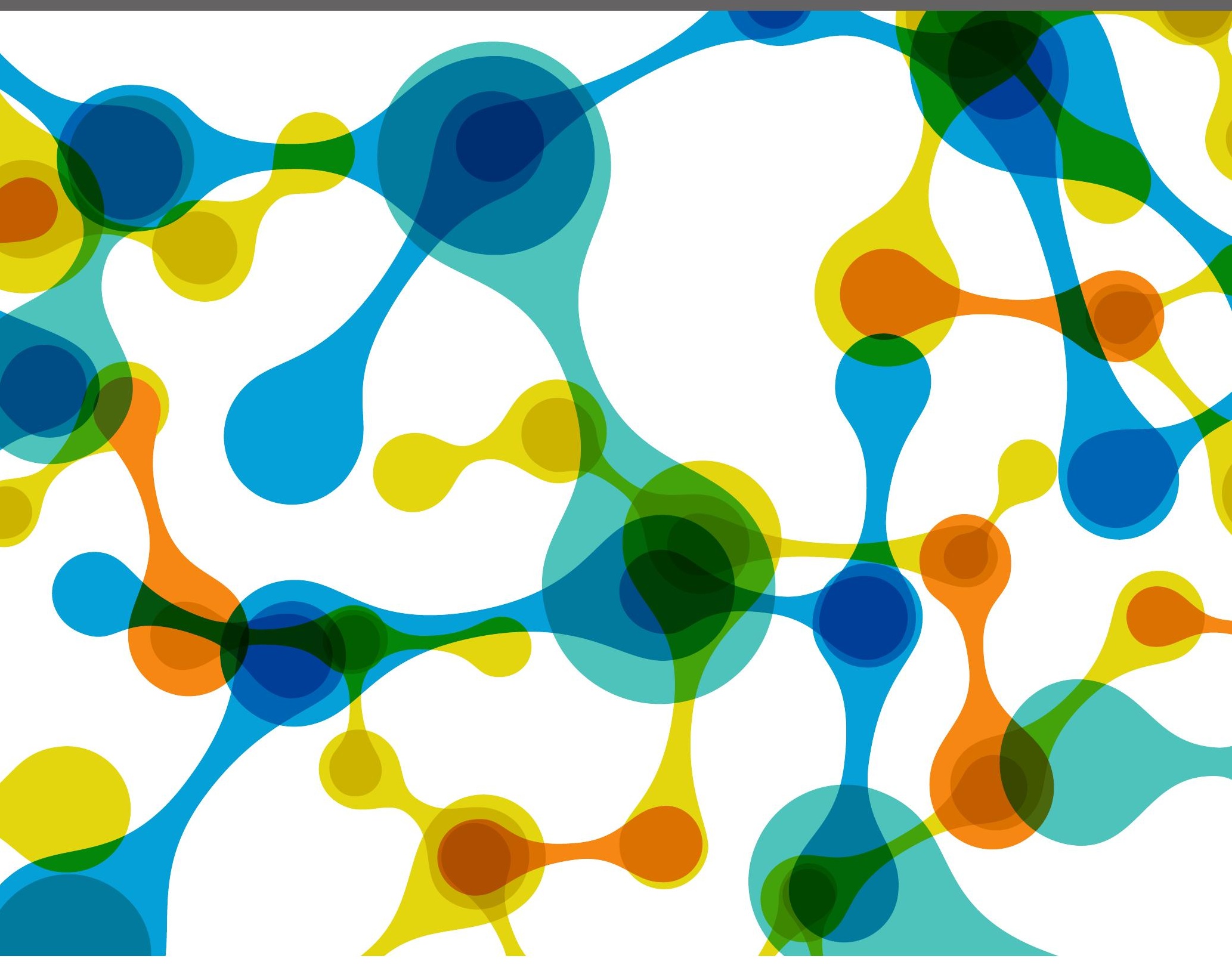

frontiers Research Topics 


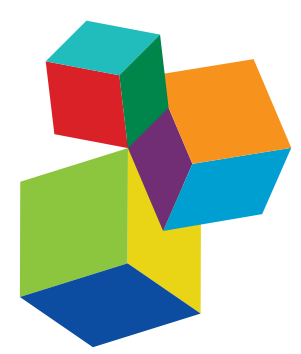

Frontiers eBook Copyright Statement

The copyright in the text of individual articles in this eBook is the property of their respective authors or their respective institutions or funders. The copyright in graphics and images within each article may be subject to copyright of other parties. In both cases this is subject to a license granted to Frontiers.

The compilation of articles constituting this eBook is the property of Frontiers.

Each article within this eBook, and the eBook itself, are published under the most recent version of the Creative Commons CC-BY licence.

The version current at the date of publication of this eBook is CC-BY 4.0. If the CC-BY licence is updated, the licence granted by Frontiers is automatically updated to the new version.

When exercising any right under the CC-BY licence, Frontiers must be attributed as the original publisher of the article or eBook, as applicable.

Authors have the responsibility of ensuring that any graphics or other materials which are the property of others may be included in the

CC-BY licence, but this should be checked before relying on the

CC-BY licence to reproduce those materials. Any copyright notices relating to those materials must be complied with.

Copyright and source acknowledgement notices may not be removed and must be displayed in any copy, derivative work or partial copy which includes the elements in question.

All copyright, and all rights therein, are protected by national and international copyright laws. The above represents a summary only.

For further information please read Frontiers' Conditions for Website

Use and Copyright Statement, and the applicable CC-BY licence.

ISSN 1664-8714

ISBN 978-2-88966-272-2

DOI 10.3389/978-2-88966-272-2

\section{About Frontiers}

Frontiers is more than just an open-access publisher of scholarly articles: it is a pioneering approach to the world of academia, radically improving the way scholarly research is managed. The grand vision of Frontiers is a world where all people have an equal opportunity to seek, share and generate knowledge. Frontiers provides immediate and permanent online open access to all its publications, but this alone is not enough to realize our grand goals.

\section{Frontiers Journal Series}

The Frontiers Journal Series is a multi-tier and interdisciplinary set of open-access, online journals, promising a paradigm shift from the current review, selection and dissemination processes in academic publishing. All Frontiers journals are driven by researchers for researchers; therefore, they constitute a service to the scholarly community. At the same time, the Frontiers Journal Series operates on a revolutionary invention, the tiered publishing system, initially addressing specific communities of scholars, and gradually climbing up to broader public understanding, thus serving the interests of the lay society, too.

\section{Dedication to Quality}

Each Frontiers article is a landmark of the highest quality, thanks to genuinely collaborative interactions between authors and review editors, who include some of the world's best academicians. Research must be certified by peers before entering a stream of knowledge that may eventually reach the public - and shape society; therefore, Frontiers only applies the most rigorous and unbiased reviews.

Frontiers revolutionizes research publishing by freely delivering the most outstanding research, evaluated with no bias from both the academic and social point of view. By applying the most advanced information technologies, Frontiers is catapulting scholarly publishing into a new generation.

\section{What are Frontiers Research Topics?}

Frontiers Research Topics are very popular trademarks of the Frontiers Journals Series: they are collections of at least ten articles, all centered on a particular subject. With their unique mix of varied contributions from Original Research to Review Articles, Frontiers Research Topics unify the most influential researchers, the latest key findings and historical advances in a hot research area! Find out more on how to host your own Frontiers Research Topic or contribute to one as an author by contacting the Frontiers Editorial Office: researchtopics@frontiersin.org 


\section{MYOKINES, ADIPOKINES, CYTOKINES IN MUSCLE PATHOPHYSIOLOGY}

Topic Editors:

Valentina Di Felice, University of Palermo, Italy Dario Coletti, Sapienza University of Rome, Italy Marilia Seelaender, University of São Paulo, Brazil

Citation: Felice, V. D., Coletti, D., Seelaender, M., eds. (2020). Myokines, Adipokines, Cytokines in Muscle Pathophysiology. Lausanne: Frontiers Media SA. doi: 10.3389/978-2-88966-272-2 


\section{Table of Contents}

05 Editorial: Myokines, Adipokines, Cytokines in Muscle Pathophysiology Valentina Di Felice, Dario Coletti and Marilia Seelaender

09 Localization and Expression of Nuclear Factor of Activated T-Cells 5 in Myoblasts Exposed to Pro-inflammatory Cytokines or Hyperosmolar Stress and in Biopsies from Myositis Patients

Sandrine Herbelet, Elly De Vlieghere, Amanda Gonçalves, Boel De Paepe, Karsten Schmidt, Eline Nys, Laurens Weynants, Joachim Weis, Gert Van Peer, Jo Vandesompele, Jens Schmidt, Olivier De Wever and Jan L. De Bleecker

Inhibition of the Activin Receptor Type-2B Pathway Restores Regenerative Capacity in Satellite Cell-Depleted Skeletal Muscle Luigi Formicola, Alice Pannérec, Rosa Maria Correra, Barbara Gayraud-Morel, David Ollitrault, Vanessa Besson, Shahragim Tajbakhsh, Jennifer Lachey, Jasbir S. Seehra, Giovanna Marazzi and David A. Sassoon

37 Aged Nicotinamide Riboside Kinase 2 Deficient Mice Present an Altered Response to Endurance Exercise Training

Robin Deloux, Cynthia Tannous, Arnaud Ferry, Zhenlin Li and Mathias Mericskay

46 Physical Exercise-Induced Myokines and Muscle-Adipose Tissue Crosstalk: A Review of Current Knowledge and the Implications for Health and Metabolic Diseases

Luana G. Leal, Magno A. Lopes and Miguel L. Batista Jr.

63 HDAC4 Regulates Skeletal Muscle Regeneration via Soluble Factors Alessandra Renzini, Nicoletta Marroncelli, Chiara Noviello, Viviana Moresi and Sergio Adamo

74 The Role of Inflammation and Immune Cells in Blood Flow Restriction Training Adaptation: A Review

Fabrício Eduardo Rossi, Marcelo Conrado de Freitas, Nelo Eidy Zanchi, Fábio Santos Lira and Jason M. Cholewa

83 Skeletal Muscle: A Significant Novel Neurohypophyseal Hormone-Secreting Organ

Sergio Adamo, Eva Pigna, Rosamaria Lugarà, Viviana Moresi, Dario Coletti and Marina Bouché

87 Skeletal Muscle Atrophy in Simulated Microgravity Might Be Triggered by Immune-Related microRNAs

Laura Teodori, Alessandra Costa, Luigi Campanella and Maria C. Albertini

93 Effect of Exercise on Fatty Acid Metabolism and Adipokine Secretion in Adipose Tissue

Adriana Mika, Filippo Macaluso, Rosario Barone, Valentina Di Felice and

Tomasz Sledzinski

100 Role of Myokines in Regulating Skeletal Muscle Mass and Function Jong Han Lee and Hee-Sook Jun

109 C-Reactive Protein is Elevated Only in High Creatine Kinase Responders to Muscle Damaging Exercise

Ashwin W. Isaacs, Filippo Macaluso, Carine Smith and Kathryn H. Myburgh 
118 The Skeletal Muscle as an Active Player Against Cancer Cachexia

Fabio Penna, Riccardo Ballarò, Marc Beltrà, Serena De Lucia,

Lorena García Castillo and Paola Costelli

133 Expression Pattern of Angiogenic Factors in Healthy Heart in Response to Physical Exercise Intensity

Marianna Bellafiore, Giuseppe Battaglia, Antonino Bianco and Antonio Palma

141 Exercise-Induced Myokines With Therapeutic Potential for Muscle Wasting

Rosanna Piccirillo

150 A Short-Term High-Fat Diet Alters Glutathione Levels and IL-6 Gene Expression in Oxidative Skeletal Muscles of Young Rats

David E. Andrich, Lilya Melbouci, Ya Ou, Nickolas Auclair, Jocelyne Mercier, Jean-Christophe Grenier, Fábio Santos Lira, Luis B. Barreiro,

Gawiyou Danialou, Alain-Steve Comtois, Jean-Claude Lavoie and

David H. St-Pierre

160 FGF21 as Modulator of Metabolism in Health and Disease

Caterina Tezze, Vanina Romanello and Marco Sandri

169 The Mechanical Stimulation of Myotubes Counteracts the Effects of Tumor-Derived Factors Through the Modulation of the Activin/Follistatin Ratio

Alexandra Baccam, Alexandra Benoni-Sviercovich, Marco Rocchi,

Viviana Moresi, Marilia Seelaender, Zhenlin Li, Sergio Adamo, Zhigang Xue

and Dario Coletti

178 The JAK/STAT Pathway in Skeletal Muscle Pathophysiology

Viviana Moresi, Sergio Adamo and Libera Berghella

188 Eccentric Muscle Contractions: Risks and Benefits

Stéphanie Hody, Jean-Louis Croisier, Thierry Bury, Bernard Rogister and

Pierre Leprince

206 Extracellular Vesicles: Delivery Vehicles of Myokines

Eleonora Trovato, Valentina Di Felice and Rosario Barone

219 Exercise and Conjugated Linoleic Acid Supplementation Induce Changes in the Composition of Liver Fatty Acids

Adriana Mika, Aleksandra Czumaj, Piotr Stepnowski, Filippo Macaluso,

Giulio Spinoso, Rosario Barone, Valentina Di Felice and Tomasz Sledzinski

226 Longitudinal Physical Activity Change During Hemodialysis and Its Association With Body Composition and Plasma BAIBA Levels

Alessio Molfino, Maria Ida Amabile, Thomas Ammann, Silvia Lai,

Alessandra Grosso, Luana Lionetto, Alessandra Spagnoli, Maurizio Simmaco,

Massimo Monti, Alessandro Laviano, Maria Grazia Chiappini and

Maurizio Muscaritoli

234 Hepatocyte Growth Factor Regulates Macrophage Transition to the M2 Phenotype and Promotes Murine Skeletal Muscle Regeneration

Wooshik Choi, Jaeman Lee, Junghun Lee, Sang Hwan Lee and Sunyoung Kim

245 Fibro-Adipogenic Progenitors Cross-Talk in Skeletal Muscle: The Social Network

Beatrice Biferali, Daisy Proietti, Chiara Mozzetta and Luca Madaro 


\title{
Editorial: Myokines, Adipokines, Cytokines in Muscle Pathophysiology
}

\author{
Valentina Di Felice ${ }^{1}$, Dario Coletti ${ }^{2,3,4 *}$ and Marilia Seelaender ${ }^{5}$ \\ ${ }^{1}$ Department of Biomedicine, Neurosciences and Advanced Diagnostics, University of Palermo, Palermo, Italy, ${ }^{2}$ Sorbonne \\ Université, CNRS, INSERM, Institut de Biologie Paris-Seine, IBPS, UMR 8256 Biological Adaptation and Ageing, Paris, \\ France, ${ }^{3}$ Department of Anatomical, Histological, Forensic Sciences and Orthopedics, Sapienza University of Rome, Rome, \\ Italy, ${ }^{4}$ Department of Anatomical, Histological, Forensic Sciences and Orthopedics, Interuniversity Institute of Myology, \\ Rome, Italy, ${ }^{5}$ Department of Surgery, LIM26 HC, Medical School of the University of São Paulo, São Paulo, Brazil
}

Keywords: inflammation, myokines, skeletal muscle disease, organ cross talk, physical exercise

\section{Editorial on the Research Topic}

Myokines, Adipokines, Cytokines in Muscle Pathophysiology

\section{INTRODUCTION}

Individual striated muscle fibers communicate in both a paracrine and endocrine fashion and are also involved in the crosstalk with other tissues and organs such as the adipose tissue, immune system, liver, pancreas, bones, and brain (Delezie and Handschin, 2018). The striated muscle, which accounts for $\sim 40 \%$ of body mass, presents high biosynthetic activity, and extensive vascularization, features that endorse current thinking that muscle is the largest endocrine system of the body (Benatti and Pedersen, 2015). There are hundreds of muscle secretory products, collectively known as myokines, including proteins, miRNA, and exosomes (Barone et al., 2016). Muscle secretion is significantly affected by muscle contraction (Son et al., 2018) due to the activation of mechanotransduction pathways (Coletti et al., 2016a). It has been suggested that the adipose tissue is also an endocrine organ, producing adipokines- leptin, and other hormones, in addition to cytokines (Galic et al., 2010). The inflammatory infiltrate in fat depots affects the course of several diseases, including cancer (Batista et al., 2012; Sawicka and Krasowska, 2016; Neto et al., 2018; Opatrilova et al., 2018), and an extensive review on the role of adipokines in disease has been published elsewhere (Orzechowski et al., 2014).

Myokines, adipokines, and cytokines are major therapeutic targets in both muscular and non-muscular diseases (Lindegaard et al., 2013; Manole et al., 2018), and understanding of their role in tissue crosstalk represents a subject of great interest in current biology. We have therefore chosen to address this paradigm within this Frontiers special issue on "Myokines, Adipokines, Cytokines in Muscle Pathophysiology.”

\section{PROMOTING MUSCLE REGENERATION IN MUSCLE DISEASES}

Received: 08 August 2020 Accepted: 24 August 2020 Published: 23 October 2020

Citation:

Di Felice V, Coletti $D$ and Seelaender M (2020) Editorial: Myokines, Adipokines, Cytokines in

Muscle Pathophysiology.

Front. Physiol. 11:592856.

doi: 10.3389/fphys.2020.592856

Inflammation impairs muscle regeneration, by affecting pro-myogenic genes, including NFAT5. In a cellular model of myositis, Herbelet et al. showed that impaired NFAT5 expression and nuclear translocation induced by the inflammatory cytokines IL-1 and INF-gamma. In patients with polymyositis and dermatomyositis, NFAT5 was unaffected, whereas in inclusion body myositis, NFAT5 was not expressed at all. This state is characterized by sub-clinical inflammation and oxidative stress is found in individuals consuming High Fat Diets (HFD) and contributes to the pathogenesis of the metabolic dysfunction observed in obesity. This has been demonstrated by Andrich et al. in rats consuming HFD for 14 days. HFD reduced GSH levels in the musculature, an 
indicator of altered antioxidant defense, but also, increased IL-6 gene expression. Rossi et al. reviewed the role of inflammation in skeletal muscle remodelling, highlighting that hypoxia and IL-6, upon strength training, augment macrophage and neutrophil recruitment.

HGF/c-met plays an important role in infiltrating macrophages during muscle regeneration (Choi et al.). HGF regulates macrophage transition from the inflammatory to the anti-inflammatory phenotype and acts on various cell types, including muscle progenitors, coordinating muscle regeneration.

It is noteworthy that severe conditions in which the muscle wastes away, such as cachexia, occur in the absence of overt local inflammation, and the atrophic muscle is not enriched in inflammatory cells (Berardi et al., 2008). Therefore, inflammatory cytokines in cachexia lead to protein breakdown and apoptosis, without establishing a chronic degeneration-inflammation cycle related to the detrimental action of local inflammatory cells (de Castro et al., 2019).

\section{STEM CELLS TO THE RESCUE}

Stem cell-mediated muscle regeneration is regulated by circulating hormones and growth factors, by signals released by damaged muscle fibers, and by the extracellular matrix (Musarò et al., 2007). Myostatin (MST) and activin bind the activin receptor-2B (AcvR2B), with the effect of negatively regulating muscle growth and myogenesis (Lee and McPherron, 2001). Formicola et al. demonstrated that inhibition of the AcvR2B receptor rescues muscle regenerative potential, in parallel with the downregulation of ectopic fat deposition and fibrosis.

Muscle SC proliferation is affected in vivo by the expression of the histone deacetylase HDAC4, which acts as a transcriptional activator or repressor (Marroncelli et al., 2018). The paper by Renzini et al. demonstrated that the deletion of HDAC4 affects muscle regeneration and suggested that HDAC4 controls muscle regeneration in vivo via soluble factors.

Satellite cells are important, but not unique players in muscle regeneration. In another contribution, Biferali et al. review the role of fibro-adipogenic progenitors (FAPs) in muscle regeneration. After muscle injury, FAPs undergo massive expansion, followed by subsequent macrophage-mediated clearance. During this critical time window, FAPs establish a dynamic network of interactions supporting SC differentiation.

\section{PARACRINE AND ENDOCRINE MEDIATORS IN MUSCLE PATHOLOGIES}

A comprehensive review written by Penna et al. discusses the role of several pro-inflammatory cytokines produced by tumors and how they contribute to cachexia in cancer. Pro-cachectic factors also exert direct effects on muscle cells in vitro, as shown by Baccam et al., who demonstrated that tumor-derived-factors induce the activin-mediated atrophy of myotubes. A high level of circulating activin is an adverse prognostic factor in cancer patients (Loumaye et al., 2017), yet, the direct role of activin, beyond that of a marker of cachexia, has not been demonstrated.
Follistatin, the physiological activin inhibitor produced by the myotubes upon mechanical stimulation, protects these cells from atrophy. These findings are in agreement with those reported in this same Frontiers special issue by Formicola et al..

The clinical relevance of muscle activity-dependent protective factors is highlighted in inactive patients, submitted to hemodialysis (HD), who show reduced levels of the betaaminoisobutyric acid (BAIBA), a factor that has beneficial effects on muscle metabolism in an autocrine/paracrine manner. In HD patients, BAIBA levels were reduced in inactive individuals (Molfino et al.), confirming the importance of supplying nutritional support (Garcia et al., 2019) and/or exercise intervention in patients who have had long periods of physical inactivity.

Microgravity induces muscle atrophy in astronauts and Teodori et al. have investigated the role of miRNAs in the immune response to simulated microgravity in silico, revealing that microgravity induced conflicting signals that were responsible for muscle atrophy.

\section{MUSCLE AS AN ENDOCRINE ORGAN}

Over 600 myokines are released by the skeletal muscle (Görgens et al., 2015). The already long list of myokines keeps evolving, as new candidates are proposed to be classified into this category. This Frontiers special issue also includes several contributions on the subject, including a comprehensive review of the myokines released as a response to muscle contraction, by Lee and Jun. The potential of myokine to counteract muscle wasting under various conditions is further discussed by Piccirillo et al..

Adamo et al. have suggested that the skeletal muscle produces two hormones classically known as neurohypophyseal factors, i.e., vasopressin and oxytocin. The skeletal muscle may be both a source, as well as a target of these hormones. In a very provocative way, the findings reviewed by Adamo et al. challenge the classical view of endocrine glands as unique, anatomically defined sources of a given factor.

The skeletal muscle is important source of Fibroblast growth factor 21 (FGF21). Whether the action of one such factor is beneficial or detrimental for the muscle is still unclear, especially in humans. By examining the circulating levels of FGF21 in several physiological or pathological conditions we may gather further insight on its relevance and therapeutic value for treating metabolic diseases (Tezze et al.).

\section{HUMORAL MEDIATORS OF THE ORGAN CROSS-TALK}

The perspective of white adipose tissue (WAT) has evolved from one in which it is a mere site for fatty acid storage and metabolism, to the current concept that WAT is a major endocrine organ with a specific role in the control of inflammation. Taking this into account, more recent studies have explored how adipocytes and WAT stromavascular components affect the striated muscle and a plethora of other tissues, especially in pathological conditions (Riccardi et al., 2020). 
Two articles in this issue address and discuss the crosstalk between muscle and adipose tissue: first a review on the myokine regulating both brown and white adipose tissue biology: myostatin, IL-6, IL-15, Irisin, FGF21, Angiopoietin-like protein (ANGPTL) and BAIBA (Leal et al.); second, a report on exerciseinduced myokines release, affecting, adipokine release by WAT (Mika et al.).

It is well-established that exercise has beneficial effects on the heart. To highlight novel mechanisms within this context, Bellafiore et al. investigated the signaling molecules that regulate capillary growth in the healthy myocardium as a result of exercise. VEGFR-1/Flt-1, VEGFR-2/Flk-1, HIF-1 $\alpha$, and iNOS, act as myokines regulating angiogenesis in response to endurance training, consistent with the observed stimulation of capillary network development in healthy hearts, following physical exercise (Brown and Hudlicka, 2003).

The existence of organ crosstalk, involving multiple sources of the same molecule, such as of a specific cytokine, and the pleiotropic effects of many of these mediators, generate paradoxical effects and make it difficult to have a holistic view of the physiopathology of muscle, fat, and the immune system. An example of this complexity is IL-6, which is secreted by the muscle upon exercise but also by the immune cells and WAT during inflammation, inducing both hypertrophy and atrophy of the skeletal muscle while, at the same time, being responsive to exercise in the muscle and fat (Rosa Neto et al., 2009). An excellent review by Pedersen and Febbraio (2008) both shedslight and reconciles different views regarding IL- 6 and its role in the metabolism in health and disease. The full characterization of the specific signaling pathways activated in different organs and conditions is of pivotal importance for understanding the effects of cytokine in different tissues. The Janus kinase (JAK) or signal transducer and activator of transcription (STAT) pathway, is a key intracellular mediator of a variety of factors, including the IL-6 family (Moresi et al.). IL-6-mediated activation of the JAK/STAT pathway may have opposing effects, promoting muscle hypertrophy and satellite proliferation, on one hand, and favoring muscle wasting, on the other hand.

\section{EXERCISE ENDOCRINOLOGICAL EFFECTS}

It has been established that exercise plays a major role in the prevention of human diseases through endocrinological, metabolic, genetic, and even epigenetic mechanisms (Coletti et al., 2016b; Grazioli et al., 2017). However, not all types of exercise are equal. Hody et al. reviewed the benefits and the risks of eccentric contractions, such as downhill running. For its unique features, eccentric exercise has been proposed for innovative rehabilitative protocols, despite the fact-and actually for the very reason that-it is a muscle-damaging exercise protocol. Isaacs et al. have reported that the exertion caused by rhabdomyolysis induced plyometric exercise is associated with different extents of muscle damage and that it is related to pain in healthy subjects. This heterogeneity correlates with a selective increase of C-reactive protein, which should be considered when prescribing exercise for pathological conditions.

In addition to damage and inflammatory markers, exercise induces the release of extracellular vesicles from the muscle. Trovato et al. unveiled and extensively examined the role of vesicles in myokine delivery. The ways in which exerciserelated myokines (exerkines) are involved in tissue crosstalk during physical exercise is a topic of interest and is likely to attract further research attention in the future. Exercise affects vasopressin and oxytocin expression in the neurons of the paraventricular nucleus (Farina et al., 2014) and induces a fivefold increase in the circulating levels of the neurohypophyseal hormone vasopressin (Melin et al., 1980). This increase is associated with the beneficial effects of exercise on muscle homeostasis and suggests a model whereby physical activity stimulates muscle secretion of the neurohypophyseal hormones, which increases muscle responsiveness to the same hormones through the up-regulation of their receptors (Adamo et al.). Taken together, this evidence reveals the molecular bases of the humoral crosstalk between muscles and the brain during exercise, reflecting the Latin motto mens sana in corpore sano, i.e., "a sound mind in a sound body."

There are age-dependent differences in adaptation to exercise (Harber et al., 2012). Aged mice that are deficient in Nicotinamide Riboside Kinase 2 (NMRK2) have shown a maladaptive metabolic response to exercise in both types of striated muscle (Deloux et al.). These are not the first findings to indicate the age-dependent effects of exercise and training adaptation on muscle metabolism. For instance, young Serum Response Factor KO mice do adapt normally to endurance exercise (Djemai et al., 2019) despite the fact that these mice show muscle functional deficits with aging (Lahoute et al., 2008).

Mika, Macaluso et al. showed that exercise and linoleic acid change hepatic fatty acid composition by increasing n-3 polyunsaturated and branched chain fatty acid incorporation. These findings are particularly interesting in light of the beneficial effects of linoleic supplements in muscle pathology (Macaluso et al., 2012; Carotenuto et al., 2016a,b,c).

Taken together, the articles in this special issue demonstrate that myokines, adipokines, and cytokines are central players in muscle physiopathology. The striated muscle must, therefore, be studied and managed using multidisciplinary approaches to consider organ crosstalk and in order to propose state-to-theart, tailored therapeutic interventions that encompass the full complexity and unique characteristics of each patient.

\section{AUTHOR CONTRIBUTIONS}

VDF, DC, and MS contributed equally to the writing of the paper and management of the special issue. All authors contributed to the article and approved the submitted version. 


\section{REFERENCES}

Barone, R., Macaluso, F., Sangiorgi, C., Campanella, C., Marino Gammazza, A., Moresi, V., et al. (2016). Skeletal muscle Heat shock protein 60 increases after endurance training and induces peroxisome proliferator-activated receptor gamma coactivator $1 \alpha 1$ expression. Sci. Rep. 6:19781. doi: 10.1038/srep19781

Batista, M. L., Peres, S. B., McDonald, M. E., Alcantara, P. S., Olivan, M., Otoch, J. P., et al. (2012). Adipose tissue inflammation and cancer cachexia: possible role of nuclear transcription factors. Cytokine 57, 9-16. doi: $10.1016 /$ j.cyto.2011.10.008

Benatti, F. B., and Pedersen, B. K. (2015). Exercise as an anti-inflammatory therapy for rheumatic diseases-myokine regulation. Nat. Rev. Rheumatol. 11, 86-97. doi: 10.1038/nrrheum.2014.193

Berardi, E., Aulino, P., Murfuni, I., Toschi, A., Padula, F., Scicchitano, B. M., et al. (2008). Skeletal muscle is enriched in hematopoietic stem cells and not inflammatory cells in cachectic mice. Neurol. Res. 30, 160-169. doi: $10.1179 / 174313208 \times 281046$

Brown, M. D., and Hudlicka, O. (2003). Modulation of physiological angiogenesis in skeletal muscle by mechanical forces: involvement of VEGF and metalloproteinases. Angiogenesis 6, 1-14. doi: 10.1023/A:1025809808697

Carotenuto, F., Albertini, M. C., Coletti, D., Vilmercati, A., Campanella, L., Darzynkiewicz, Z., et al. (2016a). How diet intervention via modulation of DNA damage response through MicroRNAs may have an effect on cancer prevention and aging, an in silico study. Int. J. Mol. Sci. 17:752. doi: 10.3390/ijms17050752

Carotenuto, F., Coletti, D., Di Nardo, P., and Teodori, L. (2016b). $\alpha$-linolenic acid reduces TNF-induced apoptosis in $\mathrm{C} 2 \mathrm{C} 12$ myoblasts by regulating expression of apoptotic proteins. Eur. J. Transl. Myol. 26:6033. doi: 10.4081/ejtm.2016.6033

Carotenuto, F., Costa, A., Albertini, M. C., Rocchi, M. B., Rudov, A., Coletti, D., et al. (2016c). Dietary flaxseed mitigates impaired skeletal muscle regeneration: in vivo, in vitro and in silico studies. Int. J. Med. Sci. 13, 206-219. doi: $10.7150 /$ ijms. 13268

Coletti, D., Aulino, P., Pigna, E., Barteri, F., Moresi, V., Annibali, D., et al. (2016a). Spontaneous physical activity downregulates Pax7 in cancer cachexia. Stem Cells Int. 2016:6729268. doi: 10.1155/2016/6729268

Coletti, D., Daou, N., Hassani, M., Li, Z., and Parlakian, A. (2016b). Serum response factor in muscle tissues: from development to ageing. Eur. J. Transl. Myol. 26:6008. doi: 10.4081/ejtm.2016.6008

de Castro, G. S., Simoes, E., Lima, J. D. C. C., Ortiz-Silva, M., Festuccia, W. T., Tokeshi, F., et al. (2019). Human cachexia induces changes in mitochondria, autophagy and apoptosis in the skeletal muscle. Cancers 11:1264. doi: $10.3390 /$ cancers 11091264

Delezie, J., and Handschin, C. (2018). Endocrine crosstalk between skeletal muscle and the brain. Front. Neurol. 9:698. doi: 10.3389/fneur.2018.00698

Djemai, H., Hassani, M., Daou, N., Li, Z., Sotiropoulos, A., Noirez, P., et al. (2019). Srf KO and wild-type mice similarly adapt to endurance exercise. Eur. J. Transl. Myol. 29:8205. doi: 10.4081/ejtm.2019.8205

Farina, E. V., Cappello, F., Lipari, L., Valentino, A., Di Felice, V., and Valentino, B. (2014). Presence of oxytocin, vasopressin and atrial natriuretic peptide and their modification in rat hypothalamic paraventricular nucleus during resistance training. Anat. Histol. Embryol. 43, 159-163. doi: 10.1111/ahe.12051

Galic, S., Oakhill, J. S., and Steinberg, G. R. (2010). Adipose tissue as an endocrine organ. Mol. Cell Endocrinol. 316, 129-139. doi: 10.1016/j.mce.2009.08.018

Garcia, M., Seelaender, M., Sotiropoulos, A., Coletti, D., and Lancha, A. H. (2019). Vitamin D, muscle recovery, sarcopenia, cachexia, and muscle atrophy. Nutrition 60, 66-69. doi: 10.1016/j.nut.2018.09.031

Görgens, S. W., Eckardt, K., Jensen, J., Drevon, C. A., and Eckel, J. (2015). Exercise and regulation of adipokine and myokine production. Prog. Mol. Biol. Transl. Sci. 135, 313-336. doi: 10.1016/bs.pmbts.2015.07.002

Grazioli, E., Dimauro, I., Mercatelli, N., Wang, G., Pitsiladis, Y., Di Luigi, L., et al. (2017). Physical activity in the prevention of human diseases: role of epigenetic modifications. BMC Genomics 18(Suppl. 8):802. doi: 10.1186/s12864-017-4193-5

Harber, M. P., Konopka, A. R., Undem, M. K., Hinkley, J. M., Minchev, K., Kaminsky, L. A., et al. (2012). Aerobic exercise training induces skeletal muscle hypertrophy and age-dependent adaptations in myofiber function in young and older men. J. Appl. Physiol. 113, 1495-1504. doi: 10.1152/japplphysiol.00786.2012
Lahoute, C., Sotiropoulos, A., Favier, M., Guillet-Deniau, I., Charvet, C., Ferry, A., et al. (2008). Premature aging in skeletal muscle lacking serum response factor. PLoS ONE 3:e3910. doi: 10.1371/journal.pone.0003910

Lee, S. J., and McPherron, A. C. (2001). Regulation of myostatin activity and muscle growth. Proc. Natl. Acad. Sci. U.S.A. 98, 9306-9311. doi: $10.1073 /$ pnas. 151270098

Lindegaard, B., Hvid, T., Grøndahl, T., Frosig, C., Gerstoft, J., Hojman, P., et al. (2013). Expression of fibroblast growth factor-21 in muscle is associated with lipodystrophy, insulin resistance and lipid disturbances in patients with HIV. PLoS ONE 8:e55632. doi: 10.1371/journal.pone.0055632

Loumaye, A., de Barsy, M., Nachit, M., Lause, P., van Maanen, A., Trefois, P., et al. (2017). Circulating Activin A predicts survival in cancer patients. J. Cachexia Sarcopenia Muscle 8, 768-777. doi: 10.1002/jcsm.12209

Macaluso, F., Morici, G., Catanese, P., Ardizzone, N. M., Gammazza, A. M., Bonsignore, G., et al. (2012). Effect of conjugated linoleic acid on testosterone levels in vitro and in vivo after an acute bout of resistance exercise. J. Strength Condit. Res. 26, 1667-1674. doi: 10.1519/JSC.0b013e318231ab78

Manole, E., Ceafalan, L. C., Popescu, B. O., Dumitru, C., and Bastian, A. E. (2018). Myokines as possible therapeutic targets in cancer cachexia. J. Immunol. Res. 2018:8260742. doi: 10.1155/2018/8260742

Marroncelli, N., Bianchi, M., Bertin, M., Consalvi, S., Saccone, V., De Bardi, M., et al. (2018). HDAC4 regulates satellite cell proliferation and differentiation by targeting P21 and Sharp1 genes. Sci. Rep. 8:3448. doi: 10.1038/s41598-018-21835-7

Melin, B., Eclache, J. P., Geelen, G., Annat, G., Allevard, A. M., Jarsaillon, E., et al. (1980). Plasma AVP, neurophysin, renin activity, and aldosterone during submaximal exercise performed until exhaustion in trained and untrained men. Eur. J. Appl. Physiol. Occup. Physiol. 44, 141-151. doi: 10.1007/BF00421092

Musarò, A., Giacinti, C., Pelosi, L., Dobrowolny, G., Barberi, L., Nardis, C., et al. (2007). Stem cell-mediated muscle regeneration and repair in aging and neuromuscular diseases. Eur. J. Histochem. 51(Suppl. 1), 35-43.

Neto, N. I. P., Murari, A. S. P., Oyama, L. M., Otoch, J. P., Alcântara, P. S., Figuerêdo, M. J., et al. (2018). Peritumoural adipose tissue pro-inflammatory cytokines are associated with tumoural growth factors in cancer cachexia patients. J. Cachexia Sarcopenia Muscle 9, 1101-1108. doi: 10.1002/jcsm.12345

Opatrilova, R., Caprnda, M., Kubatka, P., Valentova, V., Uramova, S., Nosal, V., et al. (2018). Adipokines in neurovascular diseases. Biomed. Pharmacother. 98, 424-432. doi: 10.1016/j.biopha.2017.12.074

Orzechowski, A., Rostagno, A. A., Pucci, S., and Chiocchia, G. (2014). Cytokines and disease. Mediators Inflamm. 2014:209041. doi: 10.1155/2014/209041

Pedersen, B. K., and Febbraio, M. A. (2008). Muscle as an endocrine organ: focus on muscle-derived interleukin-6. Physiol. Rev. 88, 1379-1406. doi: 10.1152/physrev.90100.2007

Riccardi, D. M. D. R., das Neves, R. X. E. M. de Matos-Neto, Camargo, R. G., Lima, J. D. C., Radloff, K., et al. (2020). Plasma lipid profile and systemic inflammation in patients with cancer cachexia. Front. Nutr. 7:4. doi: 10.3389/fnut.2020.00004

Rosa Neto, J. C., Lira, F., S., Oyama, L., M., Zanchi, N., Yamashita, A. S., et al. (2009). Exhaustive exercise causes an anti-inflammatory effect in skeletal muscle and a pro-inflammatory effect in adipose tissue in rats. Eur. J. Appl. Physiol. 106, 697-704. doi: 10.1007/s00421-009-1070-1

Sawicka, K., and Krasowska, D. (2016). Adipokines in connective tissue diseases. Clin. Exp. Rheumatol. 34, 1101-1112.

Son, J. S., Chae, S. A., Testroet, E. D., Du, M., and Jun, H. (2018). Exercise-induced myokines: a brief review of controversial issues of this decade. Expert. Rev. Endocrinol. Metab. 13, 51-58. doi: 10.1080/17446651.2018.1416290

Conflict of Interest: The authors declare that the research was conducted in the absence of any commercial or financial relationships that could be construed as a potential conflict of interest.

Copyright $\odot 2020$ Di Felice, Coletti and Seelaender. This is an open-access article distributed under the terms of the Creative Commons Attribution License (CC BY). The use, distribution or reproduction in other forums is permitted, provided the original author(s) and the copyright owner(s) are credited and that the original publication in this journal is cited, in accordance with accepted academic practice. No use, distribution or reproduction is permitted which does not comply with these terms. 


\section{OPEN ACCESS}

Edited by:

Dario Coletti

Sapienza Università di Roma, Italy

Reviewed by:

Kunihiro Sakuma,

Tokyo Institute of Technology, Japan

Nissrine Daou,

Université Pierre et Marie Curie,

France

*Correspondence:

Jan L. De Bleecker

jan.debleecker@ugent.be

Sandrine Herbelet

sandrine.herbelet@ugent.be

Specialty section:

This article was submitted to

Striated Muscle Physiology,

a section of the journal

Frontiers in Physiology

Received: 12 October 2017 Accepted: 07 February 2018 Published: 21 February 2018

Citation:

Herbelet S, De Vlieghere E, Gonçalves A, De Paepe B, Schmidt K,

Nys E, Weynants L, Weis J, Van Peer G, Vandesompele J, Schmidt J,

De Wever O and De Bleecker JL (2018) Localization and Expression of Nuclear Factor of Activated T-Cells 5

in Myoblasts Exposed to

Pro-inflammatory Cytokines or Hyperosmolar Stress and in Biopsies from Myositis Patients.

Front. Physiol. 9:126.

doi: 10.3389/fphys.2018.00126

\section{Localization and Expression of} Nuclear Factor of Activated T-Cells 5 in Myoblasts Exposed to Pro-inflammatory Cytokines or Hyperosmolar Stress and in Biopsies from Myositis Patients
Sandrine Herbelet ${ }^{1 \star}$, Elly De Vlieghere ${ }^{2}$, Amanda Gonçalves ${ }^{3,4,5}$, Boel De Paepe ${ }^{1}$,
Karsten Schmidt $^{6}$, Eline Nys ${ }^{1}$, Laurens Weynants ${ }^{1}$, Joachim Weis ${ }^{7}$, Gert Van Peer
Jo Vandesompele

${ }^{1}$ Department of Neurology, Ghent University and Ghent University Hospital, Ghent, Belgium, ${ }^{2}$ Cancer Research Institute Ghent and Department of Radiation Oncology and Experimental Cancer Research, Ghent University, Ghent, Belgium, ${ }^{3}$ VIB Inflammation Research Center, Ghent, Belgium, ${ }^{4}$ Department of Biomedical Molecular Biology, Ghent University, Ghent, Belgium, ${ }^{5}$ VIB Bio Imaging Core Gent, Ghent, Belgium, ${ }^{6}$ Department of Neurology and Department of Experimental and Clinical Neuroimmunology, University of Göttingen, Göttingen, Germany, ${ }^{7}$ Institute of Neuropathology, RWTH Aachen Medical School, Aachen, Germany, ${ }^{8}$ Center for Medical Genetics and Cancer Research Institute Ghent, Ghent, Belgium
Aims: Regeneration in skeletal muscle relies on regulated myoblast migration and differentiation, in which the transcription factor nuclear factor of activated T-cells 5 (NFAT5) participates. Impaired muscle regeneration and chronic inflammation are prevalent in myositis. Little is known about the impact of inflammation on NFAT5 localization and expression in this group of diseases. The goal of this study was to investigate NFAT5 physiology in unaffected myoblasts exposed to cytokine or hyperosmolar stress and in myositis.

Methods: NFAT5 intracellular localization and expression were studied in vitro using a cell culture model of myositis. Myoblasts were exposed to DMEM solutions enriched with pro-inflammatory cytokines IFN- $\gamma$ with IL-1 $\beta$ or hyperosmolar DMEM obtained by $\mathrm{NaCl}$ supplementation. NFAT5 localization was visualized using immunohistochemistry $(\mathrm{IHC})$ and Western blotting (WB) in fractionated cell lysates. NFAT5 expression was assessed by WB and RT-qPCR. In vivo localization and expression of NFAT5 were studied in muscle biopsies of patients diagnosed with polymyositis $(n=6)$, dermatomyositis $(n=10)$, inclusion body myositis $(n=11)$ and were compared to NFAT5 localization and expression in non-myopathic controls $(n=13)$. Muscle biopsies were studied by means of quantitative $\mathrm{IHC}$ and WB of total protein extracts.

Results: In unaffected myoblasts, hyperosmolar stress ensues in NFAT5 nuclear translocation and increased NFAT5 mRNA and protein expression. In contrast, pro-inflammatory cytokines did not lead to NFAT5 nuclear translocation nor increased expression. Cytokines IL-1 $\beta$ with IFN- $\gamma$ induced colocalization of NFAT5 with histone deacetylase 6 (HDAC6), involved in cell motility. In muscle biopsies from dermatomyositis 
and polymyositis patients, NFAT5 colocalized with HDAC6, while in IBM, this was often absent.

Conclusions: Our data suggest impaired NFAT5 localization and expression in unaffected myoblasts in response to inflammation. This disturbed myogenic NFAT5 physiology could possibly explain deleterious effects on muscle regeneration in myositis.

Keywords: NFAT5, myoblasts, hyperosmolar stress, pro-inflammatory cytokines, myositis

\section{INTRODUCTION}

Idiopathic inflammatory myopathies (IIM) are autoimmune muscle diseases comprising polymyositis (PM), dermatomyositis (DM), inclusion body myositis (IBM), overlap myositis (OM), and immune mediated necrotizing myopathy (IMNM) (Dalakas, 1991; Dalakas and Hohlfeld, 2003; Hoogendijk et al., 2004; De Bleecker et al., 2013). These diseases are characterized by chronic inflammation and presence of pro-inflammatory cytokines. DM is a complement mediated endotheliopathy whereas IBM and PM are cytotoxic T-cell mediated diseases (De Bleecker and Engel, 1995). In IBM, protein misfolding and autophagosome dysfunction do occur (Benveniste et al., 2015). In IIM, affected muscle fibers suffer necrosis (Loell and Lundberg, 2011). Attempts to regenerate muscle tissue in IIM are compromised. Impaired proliferation and altered fusion capacity has been described in primary cell cultures from patients diagnosed with IIM (Cseri et al., 2015).

Skeletal muscle tissue regeneration occurs in five phases: degeneration by necrosis, transient inflammation, regeneration, remodeling, and functional repair (Musarò, 2014). After activation, muscle stem cells become myoblasts, which will fuse with damaged myofibers or with each other, forming new myofibers (Hawke and Garry, 2001; Musarò, 2014). Transient promyogenic inflammation is tightly regulated and perturbation in the magnitude of the pro-inflammatory cytokines induces chronic inflammation, which is deleterious to muscle repair. One of the possible mechanisms explored in the past to explain this effect is the activation of nuclear factor- $\mathrm{KB}$ (NF-kB) by pro-inflammatory cytokines, interfering with the expression of muscle proteins in differentiating myoblasts (Langen et al., 2001).

Nuclear factor of activated T-cells 5 (NFAT5) belongs to the Rel family of transcription factors and is closely related to NF-kB (López-Rodríguez et al., 2001). It is expressed in skeletal muscle, kidney cells, lymphocytes, thymus and testes (Miyakawa

Abbreviations: BLAST, Basic local alignment search tool; DM, dermatomyositis; DMEMCyto, DMEM supplemented with pro-inflammatory cytokines IFN $-\gamma+$ IL-1 $\beta$; DMEM18, DMEM enriched with $\mathrm{NaCl}$, increasing osmolarity by $18 \mathrm{mM}$; DMEM60, DMEM enriched with $\mathrm{NaCl}$, increasing osmolarity by $60 \mathrm{mM}$; FITC, fluorescein isothiocyanate; GAPDH, glyceraldehyde 3-phosphate dehydrogenase; HDAC6, histone deacetylase 6; HSP70, heat shock protein 70; HSP90, heat shock protein 90; IBM, inclusion body myositis; IF, immunofluorescence microscopy; IFN- $\gamma$, interferon gamma; IIM, idiopathic inflammatory myopathies; IL-1 $\beta$, interleukin 1 beta; LT $\beta$, lymphotoxin beta; MIQE, minimum information for publication of quantitative real-time PCR experiments; $\mathrm{NaCl}$, sodium chloride; NCAM, neural cell adhesion molecule; NFAT5, nuclear factor of activated T-cells 5; PARP, poly (ADP-ribose) polymerase; PM, polymyositis; RT-qPCR, quantitative real-time PCR; siRNA, silencing RNA; UMyo, unaffected myoblasts; WB, Western blotting. et al., 1998; López-Rodríguez et al., 1999; Trama et al., 2000). Five different isotypes have been described for NFAT5: NFAT5a, NFAT5b, NFAT5c, NFAT5d1, and NFAT5d2 (Eisenhaber et al., 2011). Approximately half of endogenous NFAT5 under isotonic conditions is isoform NFAT5a, attached to the plasma membrane by palmitoylation and myristoylation. NFAT $5 \mathrm{~b}$ and NFAT5c are diffusely present in the cytoplasm. In NFAT5a, only the plasmamembrane bound fraction is sensitive to osmolar changes, diffusing to the nucleus upon stimulation (Eisenhaber et al., 2011). NFAT5 in general is tonicity sensitive in skeletal muscle cells and is involved in myoblast migration and differentiation (O'Connor et al., 2007). Cell motility and migration is regulated by histone deacetylase 6 (HDAC6). Besides, NFAT5 regulates heat-shock protein 90 (Hsp90), involved in IIM (Boyault et al., 2007; Zhang et al., 2007; De Paepe et al., 2012).

In virtually all cells, maintaining cellular homeostasis is met with nuclear translocation of NFAT5 and production of organic osmolytes (Evans et al., 2009), restoring homeostasis without disturbing cell function. The NFAT5 pathway can be activated by osmotic shock or receptor activation, yielding a different gene activation program (Kim N. H. et al., 2014). Extracellular hypertonicity drains intracellular water to the extracellular medium causing cell shrinkage (Lang et al., 1998) and DNA damage. This is counteracted by electrolyte uptake (i.e., regulatory volume increase) leading to high intracellular ionic strength (Lang et al., 1998; Alfieri and Petronini, 2007). The latter is neutralized by uptake and production of osmolytes such as sorbitol, betaine and taurine (Brown, 1976, 1978; Lang et al., 1998) after NFAT5 translocation to the nucleus (Ko et al., 2000; Dahl et al., 2001). NFAT5 attempts to rescue osmolarity by restoring cell volume and protecting DNA of incoming $\mathrm{K}^{+}$and $\mathrm{Na}^{+}$ions (Ferraris et al., 1996; Miyakawa et al., 1998). NFAT5 is an activator of tumor necrosis factor (TNF), lymphotoxin $\beta$ (LT $\beta$ ) and heat shock protein 70 (HSP70) transcription (Woo et al., 2002; Esensten et al., 2005; Creus et al., 2012). Both TNF- $\alpha$, LT $\beta$ and HSP70 are involved in IIM (De Bleecker et al., 1999; Creus et al., 2012; De Paepe et al., 2012).

In this study we have hypothesized that there is impaired NFAT5 localization and expression in myoblasts exposed to pro-inflammatory cytokines IFN- $\gamma$ with IL- $1 \beta$ since IIM are characterized by chronic inflammation, deleterious to muscle regeneration. In myoblasts exposed to hyperosmolar conditions, we expect NFAT5 translocation to the nucleus and increased NFAT5 protein expression. According to our hypothesis, we expect impaired NFAT5 localization and expression in muscle biopsies from patients diagnosed with IIM. Exploring the putative colocalization of NFAT5 with HDAC6, involved in cell 
motility, in both myoblasts and muscle biopsies may further our understanding of NFAT5's cellular interactions in skeletal muscle tissue under pro-inflammatory or hyperosmolar conditions.

\section{MATERIALS AND METHODS}

\section{In Vitro Culture of Myoblasts from Non-myopathic Individuals}

Two primary myoblast cell cultures obtained from the Myobank Banque D'ADN, France, were used for in vitro experimentation and were kept under passage 12, to avoid cellular senescence. Myoblasts obtained from unaffected individuals were labeled "UMyo." Hence, cell cultures UMyo1 and UMyo2 originated from unaffected individuals (Supplementary Table 1). Consent was obtained from all subjects and the study was approved by local Ethic Committees. All cultures were grown in DMEM containing glucose and 1\% L-glutamine (Life technologies, Carlsbad, USA), supplemented with 10\% FCS (Cambrex, Bioscience, Walkersville, USA), penicillin (50 $\mathrm{IU} / \mathrm{ml})$ + streptomycin $(50 \mathrm{mg} / \mathrm{ml})$ (Gibco, Invitrogen, Carlsbad, USA) ("DMEM"). Myogenicity was assessed in UMyo by IHC using an antibody against CD56 (neural cell adhesion molecule, NCAM) (Supplementary Table 2 and Supplementary Figure 1A, UMyo1).

UMyo7 and UMyo8 were obtained from the Laboratory of Experimental and Clinical Neuroimmunology in Göttingen, Germany. These cultures were grown in Skeletal Muscle Cell Growth Medium + Supplement Mix (Promocell, Heidelberg, Germany) at $37^{\circ} \mathrm{C}$ and $5 \% \mathrm{CO}_{2}$.

\section{Exposure of Unaffected Myoblasts to Pro-inflammatory Cytokines IFN- $\gamma$ with IL-1 $\beta$ or Hyperosmolar $\mathrm{NaCl}$ Concentrations}

UMyo1 and UMyo2 were transferred at $80 \%$ confluence to 8chamber slides for IHC studies and to $175-\mathrm{cm}^{2}$ flasks with DMEM for WB and RT-qPCR. Two $175-\mathrm{cm}^{2}$ flasks were used per condition to obtain sufficient amounts of cells for protein and mRNA extraction. To mimic inflammatory conditions seen in myositis, myoblasts were exposed to a mixture of proinflammatory cytokines IFN- $\gamma$ with IL- $1 \beta$ diluted in DMEM to respective concentrations of $300 \mathrm{U} / \mathrm{ml}$ and $20 \mathrm{ng} / \mathrm{ml}$ (R\&D Systems, Minneapolis, USA) for 7 or $24 \mathrm{~h}$ (Schmidt et al., 2008). Unstimulated myoblasts served as controls.

To examine the impact of differentiation on NFAT5 physiology in myoblasts, UMyo7 and UMyo8 were transferred to 8-chamber slides (LabTek II, Nunc, Penfield, USA) with DMEM supplemented with $0.5 \%$ chick embryo extract (CEE, Accurate, Westbury, USA). At $80 \%$ confluence, fusion was induced by adding heat-treated horse serum for $48 \mathrm{~h}$.

In DMEM, osmolarity was $110 \mathrm{mM}(280 \mathrm{mOsm} / \mathrm{L})$ with a $\mathrm{NaCl}$ concentration of $6.40 \mathrm{mg} / \mathrm{ml}$. Preparing hyperosmolar DMEM solutions was performed by adding $1.10 \mathrm{mg} / \mathrm{ml} \mathrm{NaCl}$, to obtain an increase of $18 \mathrm{mM}$, raising the concentration of the solution to $128 \mathrm{mM}$ ( $333 \mathrm{mOsm} / \mathrm{L})$. This solution is referred to as "DMEM18" and corresponds to the change in osmolarity after addition of cytokines to DMEM. Addition of $3.50 \mathrm{mg} / \mathrm{ml}$
$\mathrm{NaCl}$ to DMEM to obtain an increase of $60 \mathrm{mM}$ of the solution, resulted in a final osmolarity of $443 \mathrm{mOsm} / \mathrm{L}$. The increase in $60 \mathrm{mM}$ was based on $\mathrm{Na}^{+}$concentrations in blood in the range of 135-145 mM combined with the recent insight pointing to excess salt intake as a worsening factor in autoimmune diseases (Sigaux et al., 2017). This solution is designated as "DMEM60." DMEM supplemented with cytokine mixture IFN- $\gamma$ with IL- $1 \beta$ displayed an osmolarity of $339 \mathrm{mOsm} / \mathrm{L}$ and was labeled as "DMEMCyto." To study the impact of cytokines only, addition of DMEM18 (333 mOsm/L) to untreated cells yielded hyperosmolar controls. This hyperosmolar condition was used as control to compensate for the osmolar change induced in DMEMCyto. Osmolarity of the media supplemented with $\mathrm{NaCl}$ or cytokines was measured using an osmometer (Osmometer Automatic, Knauer, RS451050, Berlin, Germany).

\section{Patients}

Diagnostic limb muscle biopsies $(n=27)$ were performed after written informed consent had been obtained from patients or their legal representatives with PM $(n=6)$, DM $(n=10)$, IBM $(n=11)$ from the University Hospitals of Ghent and Antwerp (Belgium) and the Institute of Neuropathology, RWTH Aachen Medical School (Germany). All patients fulfilled the conventional criteria for diagnosis (Dalakas and Hohlfeld, 2003; De Bleecker et al., 2013). From the cryoblocks remaining after diagnostic work-up, $6 \mu \mathrm{m}$ transversal sections were obtained for IHC and protein extraction. PM is only diagnosed when non-necrotic invaded muscle fibers are present in the diagnostic biopsy and patients have reacted to immunotherapy. At the time of biopsy, all patients were free of immunotherapy and all of them had a progressive disease. The patients' medical files were searched for concomitant diseases (Supplementary Table 3). As NFAT5 is elevated in diabetic patients (Yang et al., 2006), this has to be taken into account during the analysis of the results. The same cautious approach is required if other autoimmune diseases are present. Muscle biopsies from healthy individuals with no clinical, electromyographic, or histologic evidence of myopathy and free from any known other disease served as controls $(n=$ 13). The study was approved by the Ghent University Hospital Medical Ethics Committee and the RWTH Aachen Medical School Ethics Committee.

\section{Muscle Biopsies, Quantitative Immunofluorescent Double and Triple Staining, Confocal Microscopy, and Image Analysis}

Frozen $6 \mu \mathrm{m}$ transversal muscle tissue sections were used for double immunofluorescence (IF) performed as described previously (De Bleecker et al., 2002). Supplementary Table 2 summarizes antibodies and concentrations used during staining for $1 \mathrm{~h}$. Secondary staining was performed for $1 \mathrm{~h}$ with secondary antibodies linked to the fluorescence markers AlexaFluor488 (green), FITC (green), AlexaFluor-555 (red) (Invitrogen, Waltham, USA) or Cy3 (red) (Jackson ImmunoResearch Laboratories West Grove, USA). Sections were imaged with a Leica SP8 AOBS confocal microscope (Leica, Mannheim, 
Germany). Images were taken using a 63X HCX PL Apo 1.4 NA oil objective. Z-sections were made at the resolution limit, in this case $126 \mathrm{~nm}$, to produce a high-resolution stack suitable for colocalization measurements. Images were acquired in a sequential mode, scanned as 246 X $246 \mathrm{~nm}$ per pixel. To test for antibody specificity, NFAT5 siRNA studies were completed. As NFAT5 Rabbit yielded unspecific binding in myonuclei, NFAT5 Goat was selected as the antibody of choice (Supplementary Figure 1B). NFAT5 Goat binds to all five NFAT5 isoforms. Amino acid sequence analysis by BLAST (Basic Local Alignment Search Tool, NIH, Bethesda, USA) of the NFAT5 Rabbit antibody yielded recognition of two proteins, rendering the antibody aspecific to NFAT5 (LLVSLQNQGN NLTGSF). The NFAT5 Goat was specific to NFAT5 (MPSDFISLLS ADLDLESPKS LYSRESVYDL LPKELQLPPS RETSVASMSQ).

\section{Quantitative Western Blotting (WB)}

Total protein extracts were prepared from $175-\mathrm{cm}^{2}$ flasks using appropriate lysis buffer (Laemmli 1x; 2-Mercaptoethanol $0.1 \%$, Bromophenol Blue 0.01\%, Glycerol 10\%, SDS 2\%, Tris$\mathrm{HCl} 60 \mathrm{mM} \mathrm{pH}$ 6.8) followed by sonification. For patients' biopsies, protein extracts were prepared by homogenizing frozen muscle samples in 2 volumes of extraction buffer $(50 \mathrm{mM}$ TrisHCl, 2 mM EDTA pH 7.4) supplemented with protease inhibitor (TM mini protease inhibitor cocktail; Roche, Bazel, Switzerland). To pellet debris, samples were centrifuged at $2,000 \mathrm{~g}$ for $10 \mathrm{~min}$. Proteins were transferred to nitrocellulose membranes by electroblotting and incubated with primary antibodies (Hendrix et al., 2010) (Supplementary Table 4) and anti-GADPH (Abcam, Cambridge, Massachusetts, USA) to correct for protein concentration between samples. PARP was used as a nuclear control in WB of fractionated cell lysates (BD Biosciences, Franklin Lakes, New Jersey, USA) and GAPDH as a cytosolic marker. Immunoreaction was visualized using chemiluminescence (WesternBright ${ }^{\mathrm{TM}}$ Sirius, Advansta, Menlo Park, California, USA) and Proxima 2650 (Isogen Life Science, De Meern, The Netherlands).

In cell cultures, cell fractionation into nuclear and cytosolic parts was performed by adding a hypotonic buffer $(20 \mathrm{mM}$ HEPES, $20 \%$ glycerol, $10 \mathrm{mM} \mathrm{NaCl}, 1.5 \mathrm{mM} \mathrm{MgCl}_{2}, 0.2 \mathrm{mM}$ EDTA, $0.1 \%$ Triton $\mathrm{x}-100$ and MQ) for cell lysis. Nuclei were separated into a pellet after gentle centrifugation at $800 \mathrm{rpm}$ for $10^{\prime}$. After resuspending the pellet in a hypertonic buffer (cf. hypotonic buffer with $500 \mathrm{mM} \mathrm{NaCl}$ instead of $10 \mathrm{mM} \mathrm{NaCl}$ ), rotating it and spinning down, a supernatant containing the nuclear fraction was obtained. NFAT5 antibody selectivity for WB was assessed using siRNA NFAT5 (h): sc-43968 (Santa Cruz Biotechnology, Santa Cruz, California, USA) in one cell culture at $20 \mathrm{nM}$ starting from a stock solution at $10 \mu \mathrm{M}$. Scrambled RNA (scRNA) was used as internal control for siRNA specificity. ScRNA was prepared by diluting $2 \mu \mathrm{L}$ in $2 \mathrm{~mL}$ serum free DMEM following the supplier's protocol. Lipofectamine 2000 (Invitrogen) was used for transfection by diluting $75 \mathrm{~mL}$ in $2 \mathrm{~mL}$ serum free DMEM followed by incubation for $5^{\prime}$ at RT. As lipofectamine was produced in duplicate, each vial was added to the respective siRNA and scRNA solutions and gently mixed for $20^{\prime}$ at RT. In one $75 \mathrm{~cm}^{2}$ flask with confluent layer, the siRNA with lipofectamine was added. In the second $75 \mathrm{~cm}^{2}$ flask with confluent layer, scRNA with lipofectamine was added. Both flasks were incubated for $24 \mathrm{~h}$ at $37^{\circ} \mathrm{C}$ with $5 \% \mathrm{CO}_{2}$. We used a DMEM solution with an osmolarity increased to $135 \mathrm{mM}$ and results were obtained by WB of total cell extractions.

\section{RT-qPCR}

One $175-\mathrm{cm}^{2}$ flask per treatment condition was grown to full confluency and cell cultures were exposed to cytokines or hyperosmolar stress for 7 or $24 \mathrm{~h}$. Total lysis of cell cultures was obtained by following a well-described protocol (Vandesompele et al., 2002) with a minimum purity of $1.9 / 2$ measured by the A260/A280 ratio on BioDrop ${ }^{\text {TM }}$ Touch Duo PC (Harvard Bioscience Inc., Holliston, Massachusetts, USA). Results in line with the MIQE guidelines (Bustin et al., 2009) were considered relevant after calculation with qBase+ Software version 2.6 (www.qbaseplus.com) (Biogazelle, Zwijnaarde, Belgium) (Hellemans et al., 2007). geNorm was used to determine the most stable reference genes from a set of tested candidate reference genes (Supplementary Table 5).

\section{Statistical Analysis}

For statistical analysis of quantitative confocal microscopy images, the Mann Whitney U test was performed in SPSS 23.0 (IBM, Armonk, New York, USA), $p<0.05$ were considered statistically significant. The statistical analysis of colocalization in confocal images was performed with Volocity 6.1.3 (Perkin Elmer, Coventry, UK). Background levels were obtained by measuring the voxel count of the signal outside the cells and subtracted. Colocalization indexes M1 (NFAT5) and M2 (HDAC6) (which indicate the quantity of colocalization in each individual channel), Mander's Overlap coefficient (R) and Pearson's correlation coefficient $(r)$ were calculated using the appropriate algorithm in the Volocity software package. Pearson's correlation was solely chosen as a thresholding method because measurements above background in both channels are included in the calculations (Costes et al., 2004). M1 stands for the quantity of red signal in the green channel, and M2 for the quantity of green signal in the red channel. One-way ANOVA with Tukey's multiple comparison test was applied to the values obtained from RT-qPCR analyzed by qBase+.

\section{RESULTS}

In UMyo, myoblast myogenicity was investigated by IHC. Double IF staining with NFAT5 and NCAM yielded superposition of both dyes after merging the immunofluorescent detection channels (Supplementary Figure 1A).

All results described below were obtained in cell cultures UMyo1 and UMyo2 where differentiation occurred by spontaneous fusion, without any differentiation medium (Supplementary Figure 2). In both cell cultures, myoblasts with single myonuclei could be observed as well as nascent myotubes harboring different myonuclei per cell, albeit to a lesser extent. 


\section{NFAT5 Localization in Unaffected Hyperosmolar Stressed Myoblasts}

The impact of osmolar stress on NFAT5 localization was studied in cultured myoblasts. Two primary myoblast cultures from non-myopathic individuals (UMyo) were exposed to hyperosmolar DMEM. NFAT5 localization was studied by IHC and WB of fractionated cell lysates. In WB, PARP was used as a marker for the nuclear fraction, GAPDH as a cytosolic marker. Results are displayed in Figure 1. Variation in results amongst different cell cultures are displayed in Supplementary Figure 3.

By IHC and WB in UMyo1, NFAT5 was merely localized in the cytoplasm of untreated myoblasts $(n=3)$. Exposure to increasing hyperosmolar conditions showed a decrease in NFAT5 cytoplasmic staining $(n=3)$ and NFAT5 immunoreactivity in the cytosolic fraction $(n=1)$. NFAT5 nuclear staining increased $(n=3)$ along with NFAT5 immunoreactivity in the nuclear fraction $(n=1)$. In WB specifically, PARP was merely expressed in the nuclear fraction and GAPDH immunoreactivity was mainly visible in the cytosolic compartment (Figure 1).

Variation in NFAT5 localization in UMyo was explored by IHC after exposure to DMEM60. In all UMyo cultures, NFAT5 cytoplasmic staining appeared in a punctate pattern in untreated conditions. NFAT5 nuclear staining was observed in all UMyo after hyperosmolar stress (Supplementary Figure 3A). In UMyo7 and UMyo8, where DMEM was supplemented with heat-treated horse serum, addition of heat-treated horse serum did not influence NFAT5 localization as visualized in UMyo8 exposed to DMEM18 or DMEM60 (Supplementary Figure 1C).

\section{Impaired NFAT5 Nuclear Localization in Unaffected Myoblasts Exposed to Pro-inflammatory Cytokines IFN- $\gamma$ with IL-1及}

The influence of cytokine stress on NFAT5 localization was studied in cultured myoblasts. Two primary myoblast cultures from non-myopathic individuals were exposed to DMEM enriched with pro-inflammatory cytokine mixture IFN$\gamma(300 \mathrm{U} / \mathrm{ml})$ with IL-1 $\beta(20 \mathrm{ng} / \mathrm{ml})$ (DMEMCyto). NFAT5 localization was studied by IHC and WB of fractionated cell lysates. In WB, PARP was used as a marker for the nuclear fraction, GAPDH as a cytosolic marker. To study the impact of cytokines alone, addition of DMEM18 to untreated cells yielded hyperosmolar controls. This hyperosmolar condition was used as control to compensate for the osmolar change induced in DMEMCyto. Results are displayed in Figure 2.

By IHC and WB in UMyo1, control myoblasts showed NFAT5 cytoplasmic and nuclear staining and NFAT5 immunoreactivity in the cytosolic and nuclear compartments (cf. 3.1). In UMyo1 exposed to pro-inflammatory cytokines IFN- $\gamma$ with IL-1 $\beta$, NFAT5 nuclear staining $(n=3)$ and immunoreactivity $(n=1)$ were strongly decreased in almost all cells (Figure 2).

\section{NFAT5 Expression in Unaffected Myoblasts Exposed to Hyperosmolar or Cytokine Stress}

NFAT5 expression was studied by means of WB and RTqPCR. Firstly, RT-qPCR explored the presence of NFAT5 mRNA

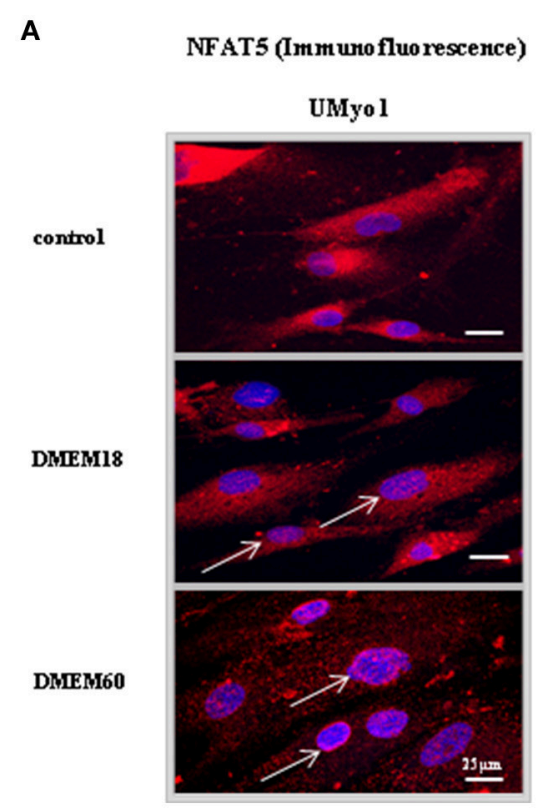

B
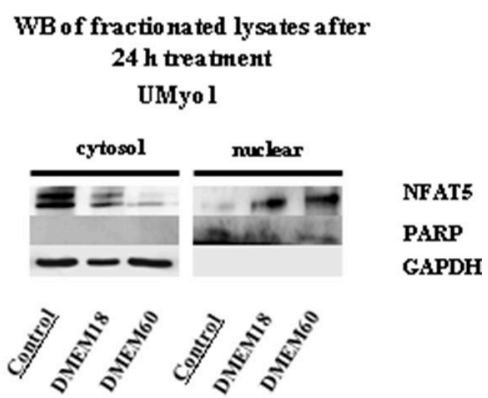

FIGURE 1 | NFAT5 localization in unaffected myoblasts after $24 \mathrm{~h}$ exposure to hyperosmolar conditions. (A) Immunofluorescent staining was performed in UMyo1 (unaffected myoblasts) with NFAT5 Goat antibody, followed by a Cy3 (red)-labeled secondary antibody. Myonuclei were counterstained with DAPI staining (blue). White arrows point at increased NFAT5 nuclear staining in UMyo1 exposed to DMEM18 or DMEM60 (DMEM with a total increase in osmolarity of 18 or $60 \mathrm{mM}$ ) $(n=3)$. (B) By WB, NFAT5 immunoreactivity increased in the nuclear fraction of UMy01 after hyperosmolar stress. Cytosolic NFAT5 immunoreactivity decreased with increasing hyperosmolar stress $(n=1)$. 
A

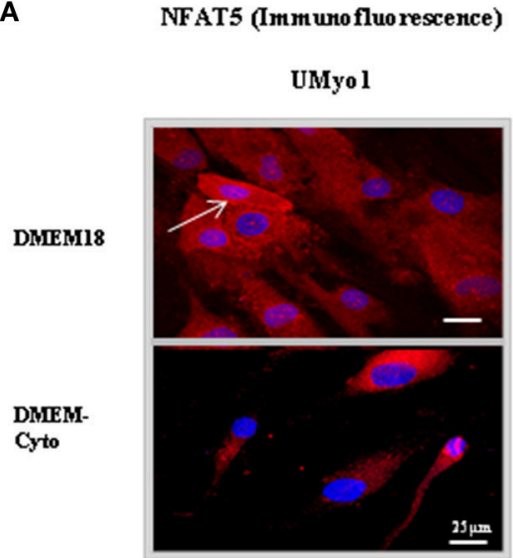

B

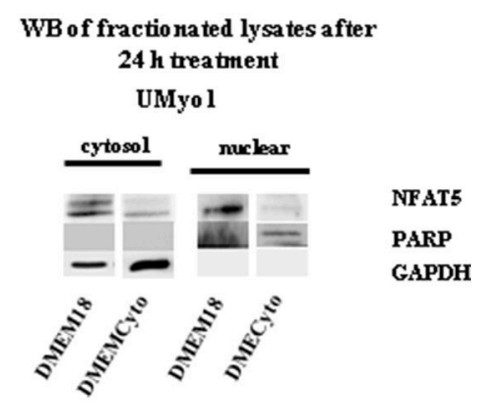

FIGURE 2 | NFAT5 localization in unaffected myoblasts after $24 \mathrm{~h}$ exposure to DMEM supplemented with pro-inflammatory cytokines IFN- $\gamma$ with IL-1 $\beta$.

(A) Immunofluorescent staining was performed in UMy01 (unaffected myoblasts) with NFAT5 Goat antibody, followed by a Cy3 (red)-labeled secondary antibody. Myonuclei were counterstained with DAPI staining (blue). NFAT5 nuclear staining was often absent in UMyo1 exposed to DMEMCyto (DMEM enriched with IFN- $\gamma$ with IL-1 13 ). The white arrow points at increased NFAT5 nuclear staining in UMyo1 exposed to DMEM18 (DMEM with a total increase in osmolarity of $18 \mathrm{mM})(n=3)$. (B) By WB, NFAT5 immunoreactivity decreased in the cytosolic and nuclear fraction of UMyo1 $(n=1)$.

after 7 and $24 \mathrm{~h}$ of exposure to cytokine or hyperosmolar stress. Secondly, WB was performed on total cell lysates exposed to hyperosmolar or cytokine stress for $24 \mathrm{~h}$, to study protein expression. The results are presented in Figure 3 and Supplementary Figure 3B.

In UMyo1, a fold change in NFAT5 mRNA expression of $7.04( \pm 1.53)(p<0.001)$ was measured for NFAT5 after $7 \mathrm{~h}$ of exposure to DMEM60, with subsequent significant increase in NFAT5 protein level $(n=3)$. Following conditions yielded no significant increase in NFAT5 mRNA expression: exposure for $24 \mathrm{~h}$ to DMEM60, 7 or $24 \mathrm{~h}$ exposure to DMEMCyto. Exposure for $24 \mathrm{~h}$ to DMEMCyto had a minor influence on NFAT5 protein expression $(n=3)$ (Figure 3).

Variation in NFAT5 protein expression amongst cell cultures was studied by WB of total cell lysates of UMyo1 and UMyo2. DMEM60 increased significantly NFAT5 protein expression $(n=3)$ (Supplementary Figure 3B).

\section{NFAT5 Colocalization with HDAC6 in Unaffected Myoblasts Exposed to Pro-inflammatory Cytokines IFN $-\gamma$ with \\ IL-1 $\beta$}

By confocal microscopy, NFAT5 colocalization with HDAC6 was studied in UMyol exposed to hyperosmolar or cytokine stress. Colocalization of red and green dyes yielded a yellow to orange signal. Colocalization was measured across the whole image by Volocity and depicted in a diagram. Results are displayed in Figure 4.

In UMyo1 exposed to DMEM18 or DMEM60, both red (NFAT5) and green (HDAC6) signals were distinctly present after superposition. Colocalization with HDAC6 was absent
(Figures 4A,C). Exposure to DMEMCyto induced NFAT5 colocalization with HDAC6, visible as a yellowish coloration in the cytoplasm of the cells, after merging the red and green detection channels. Displaying the colocalization indexes of NFAT5 and HDAC6 in a graph after exposure to DMEM18, DMEM60 or DMEMCyto yielded the same results (arrows, Figures 4B,C).

\section{NFAT5 Colocalizes with HDAC6 in PM and DM, but Not in IBM}

As visualized by IHC, 2/6 patients diagnosed with PM had a very discrete, diffuse NFAT5 surface staining. In DM, similar features were observed as in PM. In IBM, 4/8 patients expressed a positive muscle fiber surface NFAT5 staining. In IIM, NCAM (CD56) positive fibers (regenerating fibers) showed an absence of NFAT5 muscle fiber surface staining. The vast majority of inflammatory cells were negative for NFAT5 in IIM biopsies (Figure 5A and Supplementary Table 6).

By quantitative analysis of confocal microscopy images, NFAT5 and HDAC6 colocalized in PM $(p<0.01)$ and DM $(p<0.001$ ), but not in IBM (Figures 5A,B). By WB of total lysates of patients' biopsies, NFAT5 was expressed in unaffected individuals free of any disease, where NFAT5 expression ranged from once to twice amount of NFAT5 protein. One individual was randomly selected as the standard individual (black arrow) and used in WB of PM, DM and IBM patients' biopsies, allowing for a comparison between blots. In PM, two out of three patients had a NFAT5 protein expression similar to controls. One patient had a decreased NFAT5 protein expression. In DM, NFAT5 protein expression was in the same range as controls and only one individual had no detectable levels of NFAT5 protein. In IBM, two out of three patients had no detectable levels of NFAT5 protein (Figure 5C). 
A Relative NFAT5 mRNA expession after Thtreatment

UMyol

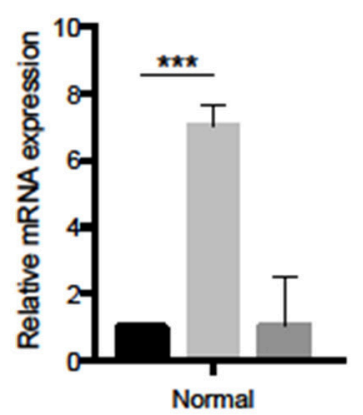

B

Westernblotting of total proteinlysates after 24 h treatment
Relative NFAT5 mRNA expression

after 24 h treatment

UMyol

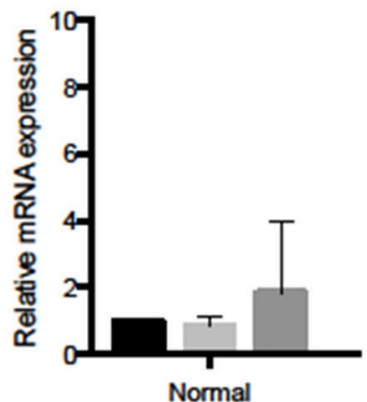

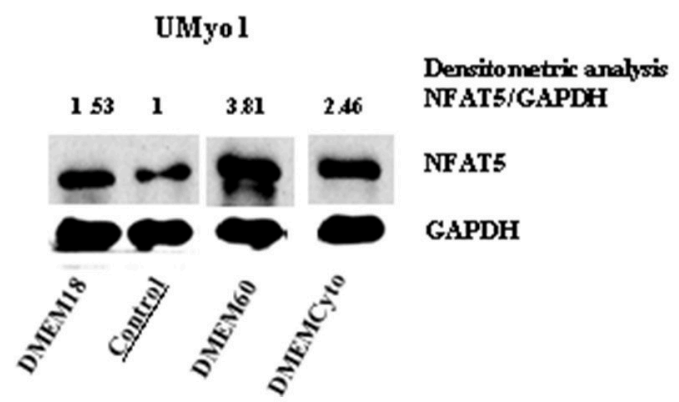

FIGURE 3 | NFAT5 mRNA and protein expression in unaffected myoblasts exposed to hyperosmolar or pro-inflammatory conditions. (A) In UMyo1 (unaffected myoblasts), exposure to DMEM60 for $7 \mathrm{~h}$ significantly increased NFAT5 mRNA expression $(p<0.001)$. In contrast, NFAT5 mRNA was not increased in UMyo1 after $24 \mathrm{~h}$ hyperosmolar treatment and after 7 or $24 \mathrm{~h}$ exposure to DMEMCyto $(n=3)$. Untreated UMyo1 were used as control. (B) By WB of total cell lysates, an increase in NFAT5 protein levels was measured in UMyo1 after $24 \mathrm{~h}$ hyperosmolar challenge $(n=3)$.

Figure 6 presents a schematic overview of the obtained results in NFAT5 physiology in unaffected myoblasts exposed to hyperosmolar or cytokine enriched DMEM solutions.

\section{DISCUSSION}

This study describes two main findings in NFAT5 physiology in unaffected muscle stem cells. Firstly, NFAT5 is translocated to the myonuclei and upregulated in myoblast cell cultures from unaffected individuals exposed to hyperosmolar DMEM solutions. Specifically in DMEM with an osmolarity increased by $60 \mathrm{mM}$, myoblasts display increased expression of NFAT5 mRNA and protein (IHC, WB, RT-qPCR). Secondly, after exposure of unaffected myoblasts to DMEM supplemented with cytokines IFN- $\gamma$ with IL-1 $\beta$, NFAT5 translocation to the myonuclei is not detectable. Instead, NFAT5 colocalizes with HDAC6 in the cytoplasm of myoblasts. NFAT5 mRNA and protein levels are not increased.

NFAT5 localization has been described in the past as being merely nuclear in myoblasts, after staining with NFAT5 Rabbit antibody for IHC (O'Connor et al., 2007). In the current study, NFAT5 is merely localized in the cytoplasm of myoblasts after staining with NFAT5 Goat for IHC. This observation may be explained by NFAT5 antibody specificity. NFAT5 Goat displays specific binding. NFAT5 staining is absent from both the cytoplasm and the nucleus after siRNA NFAT5 studies. NFAT5 Rabbit seems to display aspecific binding in the nucleus and to a lesser extend in the cytoplasm of myoblasts after siRNA NFAT5.

Our first observation corroborates with exposure of myoblasts to mannitol, a membrane-impermeant osmotic protein, where the NFAT5 reporter increases with increasing hyperosmolar mannitol concentrations (O'Connor et al., 2007). In the present study, adding hyperosmolar $\mathrm{NaCl}$ changes the cell environment to a hyperosmolar stressful setting. This leads to water drainage out of the cell by osmosis (Lang et al., 1998). To counteract this deleterious effect, NFAT5 is activated and translocates to the myonucleus where it binds with DNA (Ko et al., 2000; Dahl et al., 2001), with subsequent upregulation of NFAT5 mRNA and NFAT5 protein. NFAT5 is not colocalizing with HDAC6 in this study. This may point to a normal NFAT5 physiology in unaffected myoblasts after exposure to hyperosmolar conditions.

In the second observation, NFAT5 translocation could not be detected in unaffected myoblasts triggered by DMEM enriched with cytokines IFN- $\gamma$ with IL- $1 \beta$ (IHC and WB). IFN- $\gamma$ has 


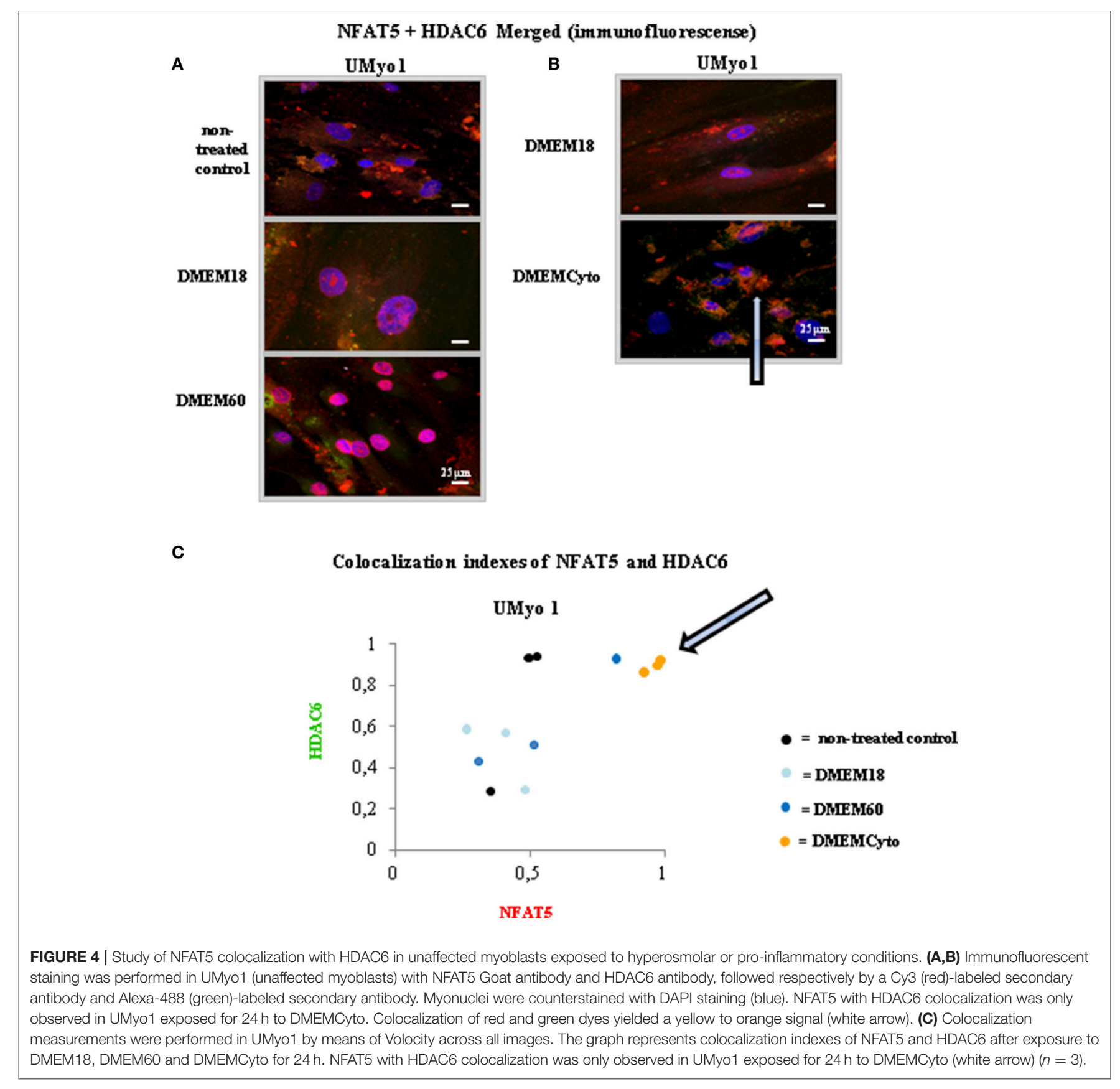

been detected in muscle tissue of all the major subtypes of IIM (Figarella-Branger et al., 2003; Preuße et al., 2012). IFN- $\gamma$, IL$1 \beta$, and TNF- $\alpha$ were found to be markedly increased in IBM muscle compared to PM and DM tissue (Schmidt et al., 2008). Cytoplasmic NFAT5 was detected by IHC and WB, whereas NFAT5 was absent from the nucleus both in IHC and WB. By means of quantitative IHC, cytoplasmic NFAT5 colocalized with HDAC6. In NFAT5 physiology, entry into the nucleus depends of the nuclear localization system (NLS) localized in an amino acid cluster with residues 202 to 204. Mutations in this cluster abolish nuclear import of NFAT5 (Tong et al., 2006). NFAT5 amino acid modifications have been described for NFAT5, such as increased phosphorylation at threonine 135 (Gallazzini et al., 2011) or sumoylation after hyperosmolar stress (Kim J. A. et al., 2014). In the current study, NFAT5 may not be able to translocate to the nucleus after cytokine stimulation by alteration of the NLS or by conformational change of NFAT5. In unaffected myoblasts, this might be a mechanism to orchestrate the cell's appropriate response to hyperosmotic vs. pro-inflammatory conditions leading to a different downstream factor activation scheme (De Paepe et al., 2016). NF- $\mathrm{KB}$ is upregulated in myoblasts after exposure to pro-inflammatory cytokines. Under these conditions, it interferes with proteins involved in myogenesis (Langen et al., 2001). In this study, 

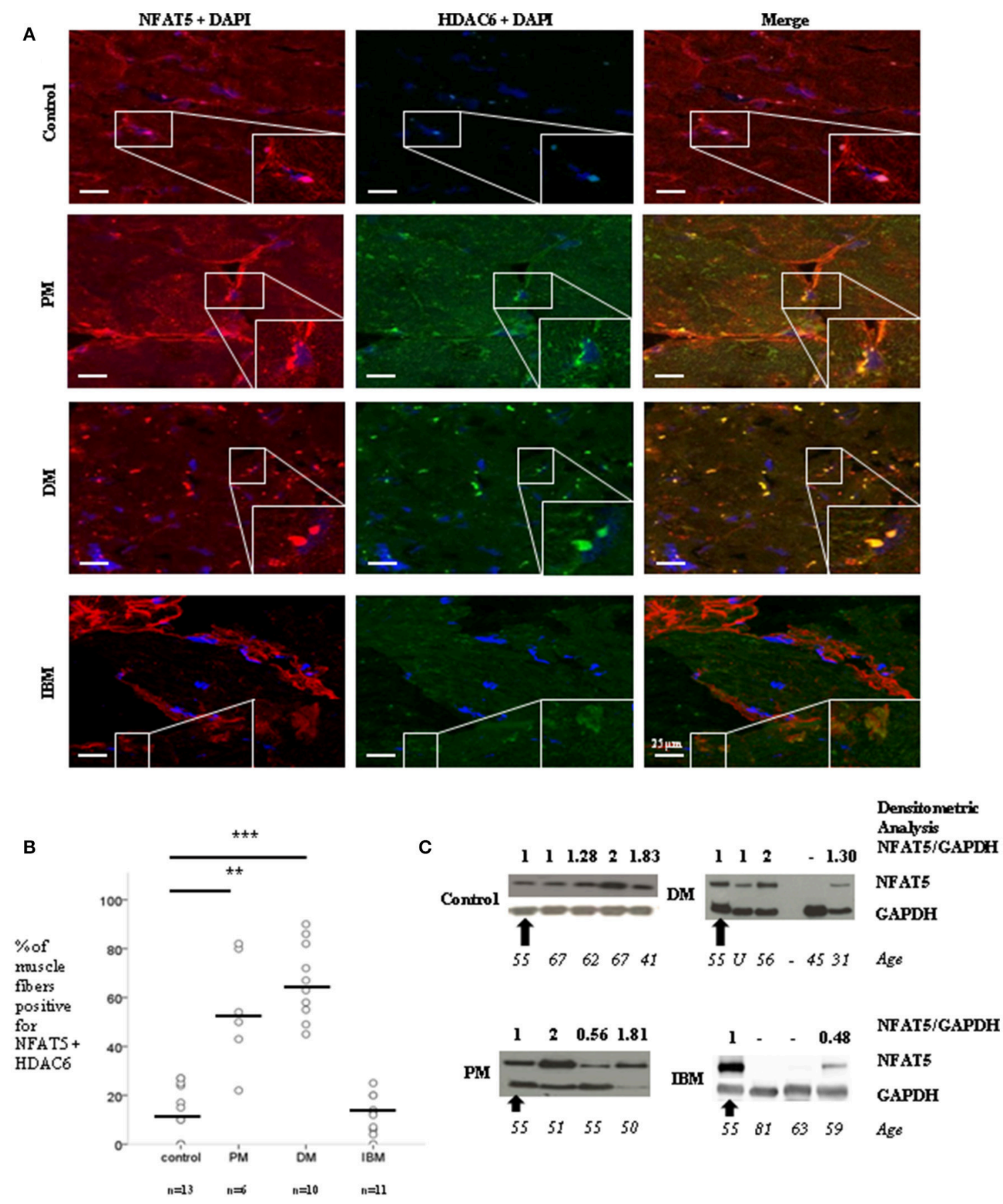

$U=u n k n o w n$

$* t=p<0.01$

FIGURE 5 | Study of NFAT5 localization and expression in control, PM, DM, and IBM biopsies (A,B) Immunofluorescent staining was performed in control, PM, DM, and IBM biopsies with NFAT5 Goat antibody and HDAC6 antibody, followed respectively by a Cy3 (red)-labeled secondary antibody and Alexa-488 (green)-labeled secondary antibody. Myonuclei were counterstained with DAPI staining (blue). In PM, and DM, NFAT5 colocalized with HDAC6, as displayed in the rectangular subsets. This is visible as yellow structures next to the nucleus. Absence of NFAT5 colocalization with HDAC6 was noticed in IBM. By quantitative analysis of confocal microscopy images, NFAT5 and HDAC6 colocalized in PM $(p<0.01)$ and DM $(p<0.001)$, but not in IBM. (C) By WB of total protein extraction of biopsies from control individuals and patients diagnosed with PM, DM, and IBM, different NFAT5 protein levels were measured. PM merely displayed NFAT5 protein levels in the same range as controls. In DM, 1/3 patients had no detectable NFAT5 protein levels. In IBM, 2/3 patients had no detectable levels of NFAT5 protein. 


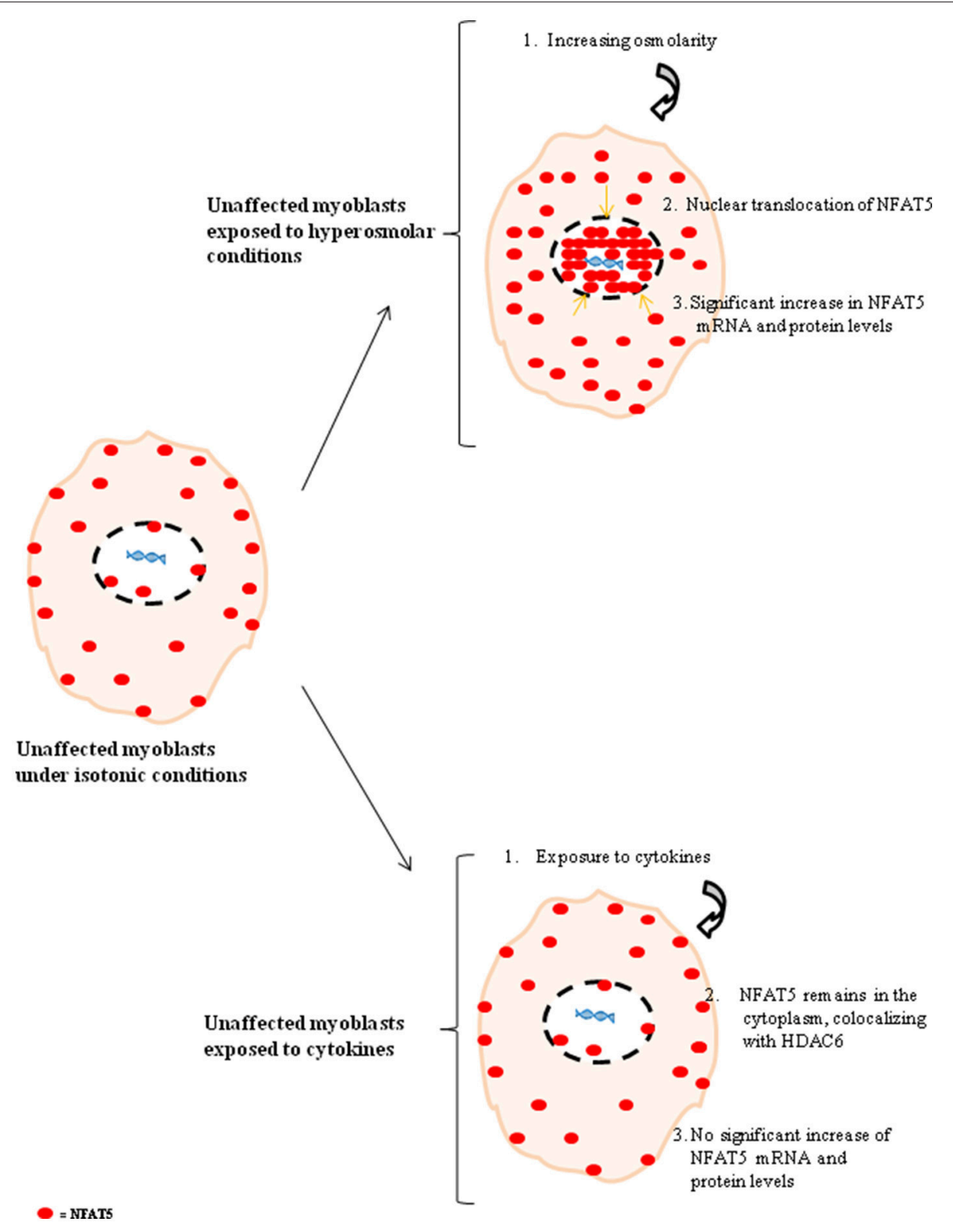

FIGURE 6 | Schematic overview of NFAT5 localization and expression in unaffected myoblasts exposed to hyperosmolar or pro-inflammatory conditions. In unaffected myoblasts under isotonic conditions, NFAT5 is located in the cytoplasm were it is attached to the plasma membrane and resides in the cytoplasm. Increased osmolarity in these myoblasts induced NFAT5 translocation to the nucleus, with subsequent NFAT5 mRNA and protein level increase (upper myoblast). In unaffected myoblasts exposed to pro-inflammatory cytokines IFN- $\gamma$ with IL-1 $\beta$, NFAT5 was not translocating to the nucleus and NFAT5 mRNA and protein level were not increased (lower myoblast).

exposure of unaffected myoblasts to DMEM garnished with IFN- $\gamma+$ IL- $1 \beta$ results in NFAT5 being localized in the cytoplasm and colocalizing with HDAC6, possibly pointing to NFAT5 interacting with HDAC6. HDAC6 is involved in cell motility and regulates heat shock protein 90 (Boyault et al., 2007; Zhang et al., 2007). Besides, HDAC6 is downregulated in embryonic stem cells during differentiation toward the myogenic cell lineage (Lee et al., 2015).

By WB of fractionated cell extracts in UMyo, three bands for NFAT5 are noticed, located around $170 \mathrm{kDa}$. In UMyo control cells, two bands are located in the cytoplasmic compartment and one band in the myonucleus. After hyperosmolar treatment, the two bands located in the cytoplasmic fraction disappear. The five following isotypes have been described for NFAT5: NFAT5a, NFAT5b, NFAT5c, NFAT5d1, and NFAT5d2 obtained by alternative splicing. Eisenhaber et al. (2011) describe diffusion to the nucleus of the plasma-membrane bound fraction of NFAT5a after exposure to salt stress of $350 \mathrm{mosm} / \mathrm{L}$. This condition induces nuclear translocation of all isoforms following a linear curve with time, with complete migration to the nucleus 
of NFAT5b and NFAT5c (Eisenhaber et al., 2011). By consensus, isoform NFAT5 $\mathrm{c}$ has been chosen as the canonical sequence and has a molecular weight of $166 \mathrm{kDa}$. Isoform NFAT5a weights $158 \mathrm{kDa}$ (Germann et al., 2012). Isoform NFAT5b displays a molecular weight of $11 \mathrm{kDa}$ (Germann et al., 2012). NFAT5 isoforms $\mathrm{d} 1$ and $\mathrm{d} 2$, also called NFAT5z1 and NFAT5z2 or NFAT5d and NFAT5e, have very similar molecular weights of respectively $167.7 \mathrm{kDa}$ and $167.8 \mathrm{kDa}$ (Xiao et al., 2015).

NFAT5 is a key regulator of both myoblast migration and differentiation during skeletal muscle myogenesis. Migratory defects in NFAT5+/- myoblasts are described alongside a decreased number of differentiated myoblasts during early differentiation in dominant-negative NFAT5 cells (O'Connor et al., 2007). Myofiber regeneration is a hallmark of DM and PM and regeneration is known to be impaired in IBM (Morosseti et al., 2006). Undetectable NFAT5 protein levels in almost all IBM patients is noticed in this current study, when studying NFAT5 expression by WB.

Combination of cytokine with hyperosmolar $\mathrm{NaCl}$, possibly explaining the interplay between both conditions, led to complete cell death and was not pursued.

In summary, we describe normal NFAT5 localization and expression in unaffected myoblasts exposed to hyperosmolar stress with NFAT5 translocation to the nucleus, increased NFAT5 mRNA and protein levels. In unaffected myoblasts exposed to cytokine stress, NFAT5 localization and expression are impaired, with NFAT5 mainly located in the cytoplasm. In PM and DM patients' biopsies, NFAT5 remains within the normal range, whereas in IBM, an absence of NFAT5 expression can be seen. As NFAT5 is a core protein to myogenesis, our findings may help further our understanding of impaired muscle regeneration in myositis.

\section{ETHICS STATEMENT}

This study was carried out in accordance with the recommendations of Medical Ethics Committees at Ghent University Hospital and Antwerp University Hospital, as well as

\section{REFERENCES}

Alfieri, R. R., and Petronini, P. G. (2007). Hyperosmotic stress response: comparison with other cellular stresses. Pflugers Arch. 454, 173-185. doi: 10.1007/s00424-006-0195-x

Benveniste, O., Stenzel, W., Hilton-Jones, D., Sandri, M., Boyer, O., and van Engelen, B. G. (2015). Amyloid deposits and inflammatory infiltrates in sporadic inclusion body myositis: the inflammatory egg comes before the degenerative chicken. Acta Neuropathol. 129, 611-624. doi: 10.1007/s00401-015-1384-5

Boyault, C., Zhang, Y., Fritah, S., Caron, C., Gilquin, B., Kwon, S. H., et al. (2007). HDAC6 controls major cell response pathways to cytotoxic accumulation of protein aggregates. Genes Dev. 21, 2172-2181. doi: 10.1101/gad. 436407

Brown, A. D. (1976). Microbial water stress. Bacteriol. Rev. 40, 803-846.

Brown, A. D. (1978). Compatible solutes and extreme water stress in eukaryotic micro-organisms. Adv. Microb. Physiol. 17, 181-242. doi: 10.1016/S0065-2911(08)60058-2 the RWTH Aachen Medical School Ethics Committee. Written informed consent was obtained from all subjects, in accordance with the Declaration of Helsinki. The protocol was approved by the Medical Ethics Committees at Ghent University Hospital and Antwerp University Hospital and the RWTH Aachen Medical School Ethics Committee.

\section{AUTHOR CONTRIBUTIONS}

SH: Carried out the literature search and was responsible for the study design, data collection, data analysis, and interpretation, generating figures and writing the manuscript; ED, JV, OD and JD: Directed the study design, performed supervision, data analysis and interpretation, and assisted in the writing process; GV: Analyzed the data, while AG, BD, EN, LW, JW, JS both collected and analyzed the data. All authors contributed to the revision of the manuscript in terms of content.

\section{ACKNOWLEDGMENTS}

The authors express their gratitude to Ms. Sophie D'hose, Ms. Marit Strotbek, Ms. Katrien Vanderheyden, and Ms. Nurten Yigit for their excellent laboratory assistance. SH sincerely wishes to thank all her co-workers at the LECR laboratory for their dedication, assistance and expertise. This work was supported by the Association Française contre les Myopathies (AFM) and the Association Belge contre les Maladies neuroMusculaires (ABMM). Primary myotube cultures were provided by the MYOBANK-AFM de l'Institut de Myologie code BB-003300012 and the Banque d'ADN et de Cellules du Généthon BB-003300010 .

\section{SUPPLEMENTARY MATERIAL}

The Supplementary Material for this article can be found online at: https://www.frontiersin.org/articles/10.3389/fphys. 2018.00126/full\#supplementary-material of quantitative real-time PCR experiments. Clin. Chem. 55, 611-622. doi: 10.1373/clinchem.2008.112797

Costes, S. V., Daelemans, D., Cho, E. H., Dobbin, Z., Pavlakis, G., and Lockett, S. (2004). Automatic and quantitative measurement of protein-protein colocalization in live cells. Biophys. J. 86, 3993-4003. doi: 10.1529/biophysj.103.038422

Creus, K. K., De Paepe, B., Weis, S., and De Bleecker, J. (2012). The multifaceted character of lymphotoxin beta in inflammatory myopathies and muscular dystrophies. Neuromuscul. Disord. 22, 712-719. doi: 10.1016/j.nmd.2012.04.012

Cseri, K., Vincze, J., Cseri, J., Fodor, J., Csernátony, Z., Csernoch, L., et al. (2015). HMGB1 expression and muscle regeneration in idiopathic inflammatory myopathies and degenerative joint diseases. J. Muscle Res. Cell Motil. 36, 255-262. doi: 10.1007/s10974-015-9411-7

Dahl, S. C., Handler, J. S., and Kwon, H. M. (2001). Hypertonicityinduced phosphorylation and nuclear localization of the transcription 
factor TonEBP. Am. J. Physiol. Cell Physiol. 280, C248-C253. doi: 10.1152/ajpcell.2001.280.2.C248

Dalakas, M. C. (1991). Polymyositis, dermatomyositis and inclusion-body myositis. N. Engl. J. Med. 326, 1487-1498. doi: 10.1056/NEJM199111213252107

Dalakas, M. C., and Hohlfeld, R. (2003). Polymyositis and dermatomyositis. Lancet 362, 971-982. doi: 10.1016/S0140-6736(03)14 368-1

De Bleecker, J. L., De Paepe, B., Vanwalleghem, I. E., and Schröder, J. M. (2002). Differential expression of chemokines in inflammatory myopathies. Neurology. 58, 1779-1785. doi: 10.1212/WNL.58.12.1779

De Bleecker, J. L., and Engel, A. G. (1995). Immunocytochemical study of CD45 T cell isoforms in inflammatory myopathies. Am. J. Pathol. 146, 1178-1187.

De Bleecker, J. L., Lundberg, I. E., de Visser, M., the, E. N. M. C., and Myositis Muscle Biopsy Study Group, De Paepe, B. (2013). 193rd ENMC international workshop: pathology diagnosis of idiopathic inflammatory myopathies, 30 November- 2 December 2012, Naarden, The Netherlands. Neuromuscul. Disord. 23, 945-951. doi: 10.1016/j.nmd.2013.0 7.007

De Bleecker, J. L., Meire, V. I., Declercq, W., and Van Aken, E. H. (1999). Immunolocalization of tumor necrosis factor-alpha and its receptors in inflammatory myopathies. Neuromuscul. Disord. 9, 239-246. doi: 10.1016/S0960-8966(98)00126-6

De Paepe, B. Creus, K. K., Weis, J., and De Bleecker, J. L. (2012). Heat shock protein families 70 and 90 in Duchenne muscular dystrophy and inflammatory myopathy: balancing muscle protection and destruction. Neuromuscul. Disord. 22, 26-33. doi: 10.1016/j.nmd.2011.07.007

De Paepe, B., Martin, J.-J., Herbelet, S., Jimenez-Mallebrera, C., Iglesias, E., Jou, C., et al. (2016). Activation of osmolyte pathways in inflammatory myopathy and Duchenne muscular dystrophy points to osmoregulation as a contributing pathogenic mechanism. Lab. Invest. 96, 872-884. doi: 10.1038/labinvest.2016.68

Eisenhaber, B., Sammer, M., Lua, W. H., Benetka, W., Liew, L. L., Yu, W., et al. (2011). Nuclear import of a lipid-modified transcription factor: mobilization of NFAT5 isoform a by osmotic stress. Cell Cycle 10, 3897-3911. doi: $10.4161 /$ cc.10.22.18043

Evans, N. P., Misyak, S. A., Robertson, J. L., Bassaganya-Riera, J., and Grange, R. W. (2009). Dysregulated intracellular signaling and inflammatory gene expression during initial disease onset in Duchenne muscular dystrophy. Am. J. Phys. Med. Rehabil. 88, 502-522. doi: 10.1097/PHM.0b013e3181a5a24f

Ferraris, J. D., Williams, C. K., Jung, K. Y., Bedford, J. J., Burg, M. B., and García-Pérez, A. (1996). ORE, a eukaryotic minimal essential osmotic response element. The aldose reductase gene in hyperosmotic stress. J. Biol. Chem. 271, 18318-13821. doi: 10.1074/jbc.271.31.18318

Figarella-Branger, D., Civatte, M., Bartoli, C., and Pellissier, J. F. (2003). Cytokines, chemokines, and cell adhesion molecules in inflammatory myopathies. Muscle Nerve 28, 659-682. doi: 10.1002/mus.10462

Gallazzini, M., Heussler, G. E., Kunin, M., Izumi, Y., Burg, M. B., and Ferraris, J. D. (2011). High NaCl-induced activation of CDK5 increases phosphorylation of the osmoprotective transcription factor TonEBP/OREBP at threonine 135, which contributes to its rapid nuclear localization. Mol. Biol. Cell. 22, 703-714. doi: 10.1091/mbc.E10-08-0681

Germann, S., Gratadou, L., Zonta, E., Dardenne, E., Gaudineau, B., Fougère, M., et al. (2012). Dual role of the ddx5/ddx17 RNA helicases in the control of the pro-migratory NFAT5 transcription factor. Oncogene 31, 4536-4549. doi: $10.1038 /$ onc. 2011.618

Hawke, T. J., and Garry, D. J. (2001). Myogenic satellite cells: physiology to molecular biology. J. Appl. Physiol. 91, 534-551. doi: 10.1152/jappl.2001.91.2.534

Hellemans, J., Mortier, G., De Paepe, A., Speleman, F., and Vandesompele, J. (2007). qBase relative quantification framework and software for management and automated analysis of real-time quantitative PCR data. Genome Biol. 8:R19. doi: 10.1186/gb-2007-8-2-r19

Hendrix, A., Braems, G., Bracke, M., Seabra, M., Gahl, W., De Wever, O., et al. (2010). The secretory small GTPase Rab27B as a marker for breast cancer progression. Oncotarget 1, 304-308. doi: 10.18632/oncotarget.100809

Hoogendijk, J. E., Amato, A. A., Lecky, B. R., Choy, E. H., Lundberg, I. E., Rose, M. R., et al. (2004). 119th ENMC international workshop: trial design in adult idiopathic inflammatory myopathies, with the exception of inclusion body myositis, 10-12 October 2003, Naarden, The Netherlands. Neuromuscul. Disord. 14, 337-345. doi: 10.1016/j.nmd.2004.0 2.006

Esensten, J. H., Tsytsykova, A. V., Lopez-Rodriguez, C., Ligeiro, F. A.,Rao, A., and Goldfeld, A. (2005). NFAT5 binds to the TNF promoter distinctly from NFATp, C., 3 and 4, and activates TNF transcription during hypertonic stress alone. Nucleic Acids Res. 33, 3845-3854. doi: 10.1093/nar/gki701

Kim, J. A., Kwon, M. J., Lee-Kwon, W., Choi, S. Y., Sanada, S., and Kwon, H. M. (2014). Modulation of TonEBP activity by SUMO modification in response to hypertonicity. Front. Physiol. 5:200. doi: 10.3389/fphys.2014.00200

Kim, N. H., Choi, S., Han, E. J., Hong, B. K., Choi, S. Y., Kwon, H. M., et al. (2014). The xanthine oxidase -NFAT5 pathway regulates macrophage activation and TLR-induced inflammatory arthritis. Eur. J. Immunol. 44, 2721-2736. doi: 10.1002/eji.201343669

Ko, B. C. B., Turck, C. W., Lee, K. W. Y., Yang, Y., and Chung, S. S. M. (2000). Purification, identification, and characterization of an osmotic response binding protein. Biochem. Biophys. Res. Commun. 270, 52-61. doi: 10.1006/bbrc. 2000.2376

Lang, F., Busch, G. L., Ritter, M., Volkl, H., Waldegger, S., Gulbins, E., et al. (1998). Functional significance of cell volume regulatory mechanisms. Physiol. Rev. 78, 247-306. doi: 10.1152/physrev.1998.78.1.247

Langen, R. C., Schols, A. M., Kelder, M. C., Wouters, E. F., and Janssen-Heininger, Y. M. (2001). Inflammatory cytokines inhibit myogenic differentiation through activation of nuclear factor-kappaB. FASEB J. 15, 1169-1180. doi: $10.1096 /$ fj. $00-0463$

Lee, S.-W., Yang, J., Kim, S.-Y., Jeong, H.-K., Lee, J., Kim, W. J., et al. (2015). Micro-RNA-26a induced by hypoxia targets HDAC6 in myogenic differentiation of embryonic stem cells. Nucleic Acids Res. 43, 2057-2073. doi: 10.1093/nar/gkv088

Loell, I., and Lundberg, I. E. (2011). Can muscle regeneration fail in chronic inflammation: a weakness in inflammatory myopathies? J. Intern. Med. 269, 243-257. doi: 10.1111/j.1365-2796.2010.02334.x

López-Rodríguez, C., Aramburu, J., Jin, L., Rakeman, A. S., Michino, M., and Rao, A. (2001). Bridging the NFAT and NF-кB families: NFAT5 dimerization regulates cytokine gene transcription in response to osmotic stress. Immunity 15, 47-58. doi: 10.1016/S1074-7613(01)00165-0

López-Rodríguez, C., Aramburu, J., Rakeman, A. S., and Rao, A. (1999). NFAT5, a constitutively nuclear NFAT protein that does not cooperate with Fos and Jun. Proc Natl Acad Sci U.S.A. 96, 7214-7219. doi: 10.1073/pnas.96.13.7214

Miyakawa, H., Woo, S. K., Chen, C. P., Dahl, S. C., Handler, J. S., Kwon, H. M., et al. (1998). Cis- and trans-acting factors regulating transcription of the BGT1 gene in response to hypertonicity. Am. J. Physiol. Renal Physiol. 274, F753-F761. doi: 10.1152/ajprenal.1998.274.4.F753

Morosseti, R., Mirabella, M., Gliubizzi, C., Broccolini, A., De Angelis, L., Tagliafico, E., et al. (2006). MyoD expression restores defective myogenic differentiation of human mesoangioblasts from inclusion-body myositis muscle. Proc Natl Acad Sci U.S.A. 103, 16995-17000. doi: 10.1073/pnas.06033 86103

Musarò, A. (2014). The basis of muscle regeneration. Adv. Biol. 2014:612471. doi: $10.1155 / 2014 / 612471$

O'Connor, R. S., Mills, S. T., Jones, K. A., Ho, S. N., and Pavlath, G. K. (2007). A combinatorial role for NFAT5 in both myoblast migration and differentiation during skeletal muscle myogenesis. J. Cell. Sci. 120, 149-159. doi: $10.1242 /$ jcs. 03307

Preuße, C., Goebel, H. H., Held, J., Wengert, O., Scheibe, F., Irlbacher, K., et al. (2012). Immune-mediated necrotizing myopathy is characterized by a specific Th1-M1 polarized immune profile. Am. J. Pathol. 181, 2161-2171. doi: 10.1016/j.ajpath.2012.08.033

Schmidt, J., Barthel, K., Wrede, A., Salajegheh, M., and Bähr, M. (2008). Interrelation of inflammation and APP in SIBM: IL- $1 \beta$ induces accumulation of $\beta$-amyloid in skeletal muscle. Brain. 131, 122-1240. doi: 10.1093/brain/awn053

Sigaux, J., Semerano, L., Favre, G., Bessis, N., and Boissier, M. C. (2017). Salt, inflammatory joint disease, and autoimmunity. Joint Bone Spine. doi: 10.1016/j.jbspin.2017.06.003. [Epub ahead of print].

Tong, E. H., Guo, J. J., Huang, A. L., Liu, H., Hu, C. D., Chung, S. S., et al. (2006). Regulation of nucleocytoplasmic trafficking of transcription factor OREBP/TonEBP/NFAT5. J. Biol. Chem. 281, 23870-23879. doi: $10.1074 / j b c . M 602556200$ 
Trama, J., Lu, Q. J., Howley, R. G., and Ho, S. N. (2000). The NFAT-related protein NFATL1 (TonEBP/NFAT5) is induced upon $\mathrm{T}$ cell activation in a calcineurin-dependent manner. J. Immunol. 165, 4884-4894. doi: 10.4049/jimmunol.165.9.4884

Vandesompele, J., De Preter, K., Pattyn, F., Poppe, B., Van Roy, N., De Paepe, A., et al. (2002). Accurate normalization of real-time quantitative RT-PCR data by geometric averaging of multiple internal control genes. Genome Biol. 3:RESEARCH0034. doi: 10.1186/gb-2002-3-7-research0034

Woo, S. K., Lee, S. D., Na, K. Y., Park, W. K., and Kwon, H. M. (2002). Ton EBP/NFAT5 stimulates transcription of HSP70 in response to hypertonicity. Mol. Cell Biol. 22, 5753-5760. doi: 10.1128/MCB.22.16.5753-5760.2002

Xiao, Z., Chang, J. G., Hendriks, I. A., Sigurðsson, J. O., Olsen, J. V., and Vertegaal, A. C. (2015). System-wide analysis of SUMOylation dynamics in response to replication stress reveals novel small ubiquitin-like modified target proteins and acceptor lysines relevant for genome stability. Mol. Cell. Proteomics. 14, 1419-1434. doi: 10.1074/mcp.O114.044792

Yang, B., Hodgkinson, A. D., Oates, P. J., Kwon, H. M., Millward, B. A., and Demaine, A. G. (2006). Elevated activity of transcription factor nuclear factor of activated T-cells 5 (NFAT5) and diabetic nephropathy. Diabetes 55, 1450-1455. doi: $10.2337 / \mathrm{db} 05-1260$
Zhang, X., Yuan, Z., Zhang, Y., Yong, S., Salas-Burgos, A., Koomen., J., et al. (2007) HDAC6 modulates cell motility by altering the acetylation level of cortactin. Mol. Cell. 27, 197-213. doi: 10.1016/j.molcel.2007.05.033

Conflict of Interest Statement: JS has received personal compensation for activities with Bayer, Biotest, CSL Behring, Novartis, and Octapharma as a consultant, both honoraria, and research grants.

The other authors declare that the research was conducted in the absence of any commercial or financial relationships that could be construed as a potential conflict of interest.

Copyright (c) 2018 Herbelet, De Vlieghere, Gonçalves, De Paepe, Schmidt, Nys, Weynants, Weis, Van Peer, Vandesompele, Schmidt, De Wever and De Bleecker. This is an open-access article distributed under the terms of the Creative Commons Attribution License (CC BY). The use, distribution or reproduction in other forums is permitted, provided the original author(s) and the copyright owner are credited and that the original publication in this journal is cited, in accordance with accepted academic practice. No use, distribution or reproduction is permitted which does not comply with these terms. 
OPEN ACCESS

Edited by:

Dario Coletti,

Sapienza Università di Roma, Italy

Reviewed by:

Pier Lorenzo Puri,

Sanford-Burnham Institute for Medical

Research, United States

John Joseph McCarthy,

University of Kentucky, United States

${ }^{*}$ Correspondence:

Giovanna Marazzi

giovanna.em.marazzi@gmail.com

David A. Sassoon

david.a.sassoon@gmail.com

tThese authors have contributed equally to this work.

¥Present Address:

Giovanna Marazzi and David A.

Sassoon,

Institut National de la Santé et de la Recherche Médicale (INSERM),

UMR970, Paris Cardiovascular

Research Center (PARCC), Paris,

France

Specialty section

This article was submitted to Striated Muscle Physiology,

a section of the journal

Frontiers in Physiology

Received: 02 February 2018 Accepted: 20 April 2018 Published: 24 May 2018

Citation:

Formicola L, Pannérec A, Correra RM

Gayraud-Morel B, Olitrault D, Besson V, Tajbakhsh S, Lachey J,

Seehra JS, Marazzi G and Sassoon DA (2018) Inhibition of the Activin Receptor Type-2B Pathway

Restores Regenerative Capacity in

Satellite Cell-Depleted Skeletal

Muscle. Front. Physiol. 9:515.

doi: 10.3389/fphys.2018.00515

\section{Inhibition of the Activin Receptor} Type-2B Pathway Restores Regenerative Capacity in Satellite Cell-Depleted Skeletal Muscle

\author{
Luigi Formicola $^{1 \dagger}$, Alice Pannérec ${ }^{1 \dagger}$, Rosa Maria Correra ${ }^{1}$, Barbara Gayraud-Morel ${ }^{2}$, \\ David Ollitrault ${ }^{1}$, Vanessa Besson ${ }^{1}$, Shahragim Tajbakhsh ${ }^{2}$, Jennifer Lachey ${ }^{3,4}$, \\ Jasbir S. Seehra ${ }^{3,4}$, Giovanna Marazzi ${ }^{1 * \neq}$ and David A. Sassoon ${ }^{\text {*f }}$ \\ ${ }^{1}$ UMR S 1166 French National Institute of Health and Medical Research, France and the Institute of Cardiometabolism and \\ Nutrition, Stem Cells and Regenerative Medicine, University of Pierre and Marie Curie Paris VI, Paris, France, ${ }^{2}$ Centre \\ National de la Recherche Scientifique URA 2578, Institut Pasteur, Stem Cells and Development, Paris, France, ${ }^{3}$ Acceleron \\ Pharma, Cambridge, MA, United States, ${ }^{4}$ Ember Therapeutics, Watertown, MA, United States
}

Degenerative myopathies typically display a decline in satellite cells coupled with a replacement of muscle fibers by fat and fibrosis. During this pathological remodeling, satellite cells are present at lower numbers and do not display a proper regenerative function. Whether a decline in satellite cells directly contributes to disease progression or is a secondary result is unknown. In order to dissect these processes, we used a genetic model to reduce the satellite cell population by $\sim 70-80 \%$ which leads to a nearly complete loss of regenerative potential. We observe that while no overt tissue damage is observed following satellite cell depletion, muscle fibers atrophy accompanied by changes in the stem cell niche cellular composition. Treatment of these mice with an Activin receptor type-2B (AcvR2B) pathway blocker reverses muscle fiber atrophy as expected, but also restores regenerative potential of the remaining satellite cells. These findings demonstrate that in addition to controlling fiber size, the AcvR2B pathway acts to regulate the muscle stem cell niche providing a more favorable environment for muscle regeneration.

Keywords: muscle atrophy, muscle regeneration, muscle stem cell niche, satellite cells, TGF $\beta$ signaling

\section{INTRODUCTION}

Chronic degenerative muscle diseases eventually lead to a collapse in the ability of muscle to regenerate. In the case of Duchenne muscular dystrophy (DMD), afflicted boys show subtle motor defects during early postnatal life that rapidly increase with age leading to paralysis and premature death (Parker et al., 2005; Tabebordbar et al., 2013). It has been proposed that the loss of regenerative capacity in DMD results from an exhaustion of the muscle progenitor satellite cell pool during disease progression (Blau et al., 1983, 1985; Heslop et al., 2000; Jiang et al., 2014; Lu et al., 2014). During the early phase of DMD, muscle undergoes continuous rounds of degeneration/regeneration but eventually regenerative competence declines accompanied by a decrease in satellite cell number (Wallace and McNally, 2009; Mann et al., 2011; Tabebordbar et al., 2013). Following a genetically induced depletion of satellite cells, muscle damage leads to a 
replacement of myofibers by fibrosis and fat deposition (Lepper et al., 2011; Murphy et al., 2011; Sambasivan et al., 2011). Whether the decline in satellite cells contributes to disease progression is unclear, in part since dissecting the relative contribution of a decreased satellite cell number in mouse DMD models is hampered by the fact that these models do not reflect the severity of disease progression in boys (Hoffman et al., 1987; Coulton et al., 1988; Tabebordbar et al., 2013).

We wished to address whether a reduction in the number of satellite cells has a direct effect upon the muscle tissue in the absence of injury and in turn, whether changes in the muscle tissue have an adverse effect upon satellite cell function. This question is particularly relevant as it has been demonstrated that small numbers of satellite cells engraft with high efficiency into healthy skeletal muscle (Collins et al., 2005; Sacco et al., 2008), thus a decline but not complete elimination of the satellite cell pool should be sustainable. Furthermore, satellite cells obtained from mdx mice, a model for Duchenne, contribute robustly when engrafted into healthy muscle (Boldrin et al., 2015). These observations raise the question as to why a reduced satellite cell population cannot replace damaged muscle fibers in diseased muscle?

A number of secreted growth factors have been shown to regulate satellite cell function. Different combinations of TGF $\beta$ superfamily receptors and ligands control muscle growth, progenitor activation, fibrosis and ectopic bone and fat formation (Yamaguchi, 1995; Glass, 2010; Sako et al., 2010; Serrano et al., 2011; Bonaldo and Sandri, 2013; Sartori et al., 2013). Myostatin (MST) is a high affinity ligand for the activin receptor-2B (AcvR2B) and a potent negative regulator of muscle growth that blocks myogenic progression (McPherron et al., 1997; Thomas et al., 2000; Lee and McPherron, 2001). Myostatin-null mice display a pronounced increase in muscle mass coupled with reduced fibrosis following injury (McPherron and Lee, 2002; McCroskery et al., 2005). MST has been shown to stimulate fibrosis (McCroskery et al., 2005; Artaza et al., 2008; Z Hosaka et al., 2012) whereas suppression of MST, using a soluble form of the AcvR2B, has been used to treat congenital myopathies, sarcopenia and cachexia resulting in reduced fibrosis (Wagner et al., 2002; Siriett et al., 2006; Morrison et al., 2009; Cadena et al., 2010; Zhou et al., 2010; George Carlson et al., 2011; Lawlor et al., 2011; Pistilli et al., 2011; Chiu et al., 2013; LachTrifilieff et al., 2014). Follistatin (FST) binds to and blocks MST activity results in muscle hypertrophy (Lee and McPherron, 2001; Amthor et al., 2004; Lee et al., 2010). Other growth factors such as insulin-like growth factor 1 (IGF-1) are potent activators of muscle growth that act in part by blocking the downstream MST-induced pathway (Bodine et al., 2001; Rommel et al., 2001; Trendelenburg et al., 2009; Oberbauer, 2013). While manipulation of the AcvR2B pathway provides a basis for development of therapeutic approaches for degenerative myopathies (Glass, 2010; Zhou and Lu, 2010; Ceco and McNally, 2013; Tabebordbar et al., 2013), it remains unaddressed whether these therapies act via a primary effect upon myofibers or target additional cell types including satellite cells and/or the stem cell niche. Satellite cells are highly responsive to neighboring cell populations in vivo (Ten Broek et al., 2010; Yin et al.,
2013 ) including myofibers, vessels, pericytes, and fibroadipogenic progenitors (FAPs) (Joe et al., 2010; Uezumi et al., 2010; Dellavalle et al., 2011; Pannérec et al., 2012, 2013) as well as invading macrophages following injury (Kharraz et al., 2013; Tabebordbar et al., 2013; Yin et al., 2013; Farup et al., 2015). While FAPs generate fat in diseased muscle, a depletion of interstitial cells including FAPs results in poor regeneration (Murphy et al., 2011), indicating that interstitial cells play a critical role in proper muscle regeneration (Murphy et al., 2011).

In this study, we used a genetic mouse model for depleting satellite cells that relies upon diphtheria toxin targeted cell death to satellite cells (Sambasivan et al., 2011). In this model, injured muscle undergoes pronounced fibrosis and fat formation coupled with a near complete loss of fiber regeneration (Lepper et al., 2011; Murphy et al., 2011; Sambasivan et al., 2011). We found that diphtheria toxin injection resulted consistently in $~ 70-80 \%$ satellite cell reduction, thereby providing a baseline by which we could directly assess the effects of satellite cell reduction in the absence of injury and/or underlying disease state. In addition, this model allows for a reduction of satellite cells in a single muscle group as opposed muscles that provide essential functions such as the diaphragm. We found that satellite cell depletion resulted in muscle fiber atrophy in the absence of injury. Whereas inhibition of the AcvR2B results in muscle fiber hypertrophy in muscle containing an intact satellite cell population (Morrison et al., 2009; Pistilli et al., 2011), we observed that satellite cell depleted muscles undergo a limited hypertrophic response in which muscle fiber size is only restored to levels found in intact untreated muscles. In addition to changes in the muscle fibers, we observed that PW1+ interstitial cells undergo a marked increase in cell number following AcvR2B pathway inhibition suggesting that the stem cell niche is responsive. This prompted us to test whether this change in the stem cell niche would have an impact upon the regenerative potential in muscle containing fewer satellite cells. We report here that treatment of satellite cell depleted muscle with an AcvR2B inhibitor prior to injury rescues regenerative potential concomitant with a near complete inhibition of ectopic fat formation and fibrosis. Using genetic lineage tracing, we found that the regenerated fibers originated from the residual pool of satellite cells in the satellite cell-depleted mice. These data reveal that a decline in satellite cells has a profound effect on the tissue environment compromising myogenic competence and that inhibition of the AcvR2B pathway restores the regenerative potential of a reduced satellite cell population.

\section{MATERIALS AND METHODS}

\section{Mice Models}

Animal models used were C57Bl6J mice (Elevage Janvier); $P W 1^{I R E S n L a c Z}$ transgenic reporter mice $\left(\mathrm{PW}^{\text {nlacZ }}\right)$ (Besson et al., 2011); knock-in heterozygous $\operatorname{Pax} 7^{D T R /+}$ mice, in which the diphtheria toxin receptor (DTR) is expressed under the control of the Pax7 promoter (Sambasivan et al., 2011); Tg:Pax7CreER ${ }^{T 2}$ mice, carrying a BAC where a tamoxifen-inducible Cre recombinase/estrogen receptor fusion protein, $\mathrm{CreER}^{\mathrm{T} 2}$ 
(Metzger and Chambon, 2001), is expressed under the control of Pax7 (Mourikis et al., 2012); ROSA26 ${ }^{\text {mTomato/mGFP }}$ (ROSA ${ }^{\mathrm{mTmG}}$ ) mice (Muzumdar et al., 2007) (Jackson Laboratories). Pax $7^{D T R /+}$ mice were crossed with Tg:Pax7CreER $R^{T 2}$ and ROSA ${ }^{\mathrm{mTmG}}$ to obtain Pax $7^{\text {DTR/+ }}:$ Pax7CreER $^{\mathrm{T} 2}$ :ROSA ${ }^{\mathrm{mTmG}}$ animals.

\section{Ethics Statement}

Approval for the animal (mouse) work performed in this study was obtained through review by the French Ministry of Education, Agreement \#A751320.

\section{RAP-031 Treatment}

Five-weeks old C57Bl6 or PW1 $1^{\text {nLacZ }}$ males and 10 -weeks old Pax7 ${ }^{\text {DTR } /+}$ or Pax $7^{\text {DTR/+ }}$ :Pax7CreER ${ }^{\mathrm{T} 2}:$ ROSA $^{\text {mTmG }}$ mice were injected intra-peritoneally with RAP-031 or vehicle (TBS) $10 \mathrm{mg}$ per $\mathrm{kg}^{-1}$.

\section{Tamoxifen Treatment}

Six weeks-old $\mathrm{Pax} 7^{\mathrm{DTR} /+}: \mathrm{Pax}_{7 \mathrm{CreER}}{ }^{\mathrm{T} 2}$ ROSA $^{\mathrm{mTmG}}$ mice were injected intra-peritoneally every day for 4 days with tamoxifen (250-300 ml, $20 \mathrm{mg} / \mathrm{ml}$; Sigma Aldrich) diluted in sunflower seed oil/5\% ethanol.

\section{Toxins Injections}

Mice were anesthetized by intra-peritoneal injection of ketamine $\left(100 \mathrm{mg} \cdot \mathrm{kg}^{-1}\right)$ and xylazine $\left(10 \mathrm{mg} \cdot \mathrm{kg}^{-1}\right)$ in sterile saline solution. A total volume of 15-30 $\mu$ l was used for one single intramuscular injection of diphtheria toxin (DT) from Corynebacterium diphtheria (Sigma Aldrich) at $1 \mathrm{ng} \cdot \mathrm{g}^{-1}$ of body weight or PBS into the TA muscle using $30 \mathrm{G}$ Hamilton syringe. Muscle injury was induced by intramuscular injection of $40 \mu \mathrm{l}$ of cardiotoxin from Naja mossambica (Latoxan) at a concentration of $10 \mu \mathrm{M}$.

\section{Western Blot Analysis}

TA muscles were homogenized in a lysis buffer $(150 \mathrm{mM} \mathrm{NaCl}$, $50 \mathrm{mM}$ Hepes pH7.6, 1\% NP-40, 0.5\% Sodium deoxycholate, 5 mM EDTA) supplemented with $1 \mathrm{mM}$ PMSF, protease inhibitor cocktail (Roche, Cat. No. 04693124001), $20 \mathrm{mM} \mathrm{NaF,} 10 \mathrm{mM}$ b-glycerophosphate, $5 \mathrm{mM}$ Na-pyrophosphate, and $1 \mathrm{mM} \mathrm{Na-}$ orthovanadate. Equal amount of protein were separated by electrophoresis (Novex NuPAGE Bis-Tris protein gel 12\%) and transferred to a PVDF membrane in $20 \%$ methanol transfer buffer. Membranes were probed with p-SMAD2 (S465/467)/ Smad3 (S423/425), SMAD2/3 total (Cell signaling) and GAPDH (Abcam). Anti-body binding was visualized using horseradish peroxidase (HRP)-conjugated species-specific secondary antibodies (Jackson ImmunoResearch) followed by enhanced chemiluminescence (Pierce).

\section{FACS Analysis}

For fluorescence activated cell sorting (FACS), hindlimb muscles from 7 weeks old PW $1^{\text {nLacZ }}$ mice were digested (Mitchell et al., 2010) and FACS sorted as described previously (Pannérec et al., 2013): briefly, muscles were minced and incubated with a solution of collagenase/dispase for $90 \mathrm{~min}$ at $37^{\circ} \mathrm{C}$, then cells were washed three times in BSA $0.2 \%$ (Jackson Immunoresearch) diluted in Hank's Balanced Salt Solution
(HBSS) (Life Technologies) and then incubated for $1 \mathrm{~h}$ on ice with the following primary antibodies at a concentration of 10 ng. $\mathrm{ml}^{-1}$ : rat anti-mouse CD45-APC (BD Biosciences), rat antimouse Ter119-APC (BD Biosciences), rat anti-mouse CD34E450 (eBiosciences), rat anti-mouse Sca1-A700 (eBiosciences), and rat anti-mouse PDGFR $\alpha$-PE (CD104a, eBiosciences). Cells were washed and incubated with $\mathrm{C}_{12} \mathrm{FDG}, 60 \mu \mathrm{M}$, (Life Technologies) $1 \mathrm{~h}$ at $37^{\circ} \mathrm{C}$ and analyzed and sorted on a FACSAria (Becton Dickinson) with appropriate isotype matching controls as previously reported (Pannérec et al., 2013). Ter119 ${ }^{\text {pos }}$ and CD45 ${ }^{\text {pos }}$ cells were negatively selected and the remaining cells were gated based on the other markers: satellite cells were sorted in the $\mathrm{CD} 34^{\text {pos }} \mathrm{Sca} 1^{\text {neg }}$ fraction, while PDGFR $\alpha^{\text {pos }}$ and PDGFR $\alpha^{\text {neg }}$ PICs were sorted in the $\mathrm{CD}_{34}{ }^{\text {pos }} \mathrm{Sca}_{1}{ }^{\text {pos }} \mathrm{C}_{12} \mathrm{FDG}^{\text {pos }}\left(\mathrm{PW} 1^{\text {pos }}\right)$ fraction (Pannérec et al., 2013). At least 3 independent experiments were performed for CTL and RAP-031-injected mice groups.

\section{Cell Culture}

For transwell co-culture experiments, FACS-sorted cell populations were maintained in high serum medium (GM) as described previously (Pannérec et al., 2013). Satellite cells were plated in the lower chamber at a density of $100 \mathrm{cells} / \mathrm{cm}^{2}$. PICs were plated on the membrane of the insert well $(1 \mu \mathrm{m}$ pore size, BD Biosciences) at 3,000 cells per $\mathrm{cm}^{2}$. Cells were grown for 1 day in GM before adding the following factors: recombinant myostatin ( $R \& D$ Systems) was added at 0, 20, 200, or 2,000 ng. $\mathrm{mL}^{-1}$; human follistatin blocking antibody $(\alpha \mathrm{FST})$ (R\&D Systems), mouse IGF-1 blocking antibody ( $\alpha \mathrm{IGF1)}$ (R\&D Systems) and isotype matched IgG (R\&D Systems) were used at $4 \mu \mathrm{g} \cdot \mathrm{mL}^{-1}$ (R\&D Systems). After 2 days, cells were fixed with $4 \%(\mathrm{w} / \mathrm{v})$ paraformaldehyde (Sigma Aldrich) and the number of cells per colony was counted. At least 3 independent experiments were performed for each condition.

\section{Gene Expression Analysis}

RNA extracts were prepared from freshly FACS sorted cells using RNeasy micro-kit (Qiagen). cDNA was generated by random-primed reverse transcription using the SuperScript First Strand kit (Life Technologies). cDNAs were analyzed by semiquantitative PCR using the ReddyMix PCR Master Mix (Thermo Scientific) under the following cycling conditions: $94^{\circ} \mathrm{C}$ for $5 \mathrm{~min}$ followed by 32 cycles of amplification $\left(94^{\circ} \mathrm{C}\right.$ for $30 \mathrm{~s}, 60^{\circ} \mathrm{C}$ for $30 \mathrm{~s}$ and $72^{\circ} \mathrm{C}$ for $1 \mathrm{~min}$ ) and a final incubation at $72^{\circ} \mathrm{C}$ for $10 \mathrm{~min}$. Primers are listed in Supplementary Table 1.

\section{Histological Analyses}

Tibialis anterior (TA), soleus, plantaris, and gastrocnemius muscles were removed, weighed and snap frozen in liquid nitrogen-cooled isopentane (Sigma Aldrich) as previously described (Mitchell et al., 2010; Pannérec et al., 2013). In the case of $\mathrm{Pax}^{\mathrm{DTR} /+}: \mathrm{Pax}^{\mathrm{C} C \mathrm{CER}^{\mathrm{T} 2}} \mathrm{ROSA}^{\mathrm{mTmG}}$ mice, TA muscles were fixed $2 \mathrm{~h}$ in $4 \%(\mathrm{w} / \mathrm{v}$ ) paraformaldehyde (Sigma Aldrich), incubated overnight in $20 \%(\mathrm{w} / \mathrm{v})$ sucrose (Sigma Aldrich) and frozen in liquid nitrogen-cooled isopentane (Sigma Aldrich). Muscles were cryosectioned $(7 \mu \mathrm{m})$ before processing. Cryosections were stained with haematoxilin and eosin (H\&E) 
(Sigma Aldrich). Fat tissue was detected by Oil-Red O staining (Sigma Aldrich): cryosections were fixed in 10\% formalin (Sigma Aldrich) for $5 \mathrm{~min}$ at $4^{\circ} \mathrm{C}$, rinsed in water and then $100 \%$ propylene glycol (Sigma Aldrich) for $10 \mathrm{~min}$, stained with Oil red O (Sigma Aldrich) for $10 \mathrm{~min}$ at $60^{\circ} \mathrm{C}$, placed in $85 \%$ propylene glycol for $2 \mathrm{~min}$ and rinsed in water. Nuclei were counterstained with Mayer's Hematoxylin Solution (Sigma). Collagen deposition was detected by Sirius Red staining (Sigma Aldrich): cryosections were stained with Mayer's haematoxylin (Sigma Aldrich) for $10 \mathrm{~min}$, rinsed in running tap water for $20 \mathrm{~min}$, then stained with $1.3 \%$ (w/v) Picro-sirius Red solution (Sigma Aldrich) for $45 \mathrm{~min}$ and washed twice in acidified water. The fat and fibrotic index was calculated as percent area of fat (red-colored Oil-Red O-stained areas) and collagen (pink-colored areas) of the total tissue area using NIH Image J Software (http://rsbweb.nih.gov/ $\mathrm{ij} /$ ) as reported previously (Tanano et al., 2003; Mozzetta et al., 2013).

For immunofluorescence, TA cryosections were fixed in $4 \%$ $(\mathrm{w} / \mathrm{v})$ paraformaldehyde and processed for immunostaining as described previously (Mitchell et al., 2010; Pannérec et al., 2013). Primary antibodies were: PW1 (Relaix et al., 1996) (rabbit, 1:3,000), Pax7 (mouse, Developmental Studies Hybridoma Bank, 1:15), M-Cadherin (mouse, Nanotools, 1:100), Ki67 (mouse, BD Biosciences, 1:100), Ki67 (rabbit, Abcam, 1:100, GFP (chicken, Abcam, 1:500), laminin (rabbit, Sigma, 1:100). Antibody binding was revealed using species-specific secondary antibodies coupled to Alexa Fluor 488 (Molecular Probes), Cy3 or Cy5 (Jackson Immunoresearch). Nuclei were counterstained with DAPI (Sigma Aldrich). For quantitative analyses of immunostained tissues, positive cells for at least 300 myofibers were counted in at least 5 randomly chosen fields per muscle section, $n=3$ animals for each group. Fiber size distribution was measured from cryosections obtained from the mid-belly of TA stained with laminin, images were captured on a Zeiss AxioImagerZ1 microscope, and morphometric analysis was performed using MetaMorph7.5 (Molecular Devices). For uninjured muscles, at least 400 fibers from randomly chosen fields were analyzed per muscle section, $n=3$ animals for each group. For injured muscles, between 200 and 575 fibers from randomly chosen fields were analyzed per muscle section, $n=3$ animals for each group.

\section{Statistical Analysis}

All statistics were performed with an unpaired Student's $t$-test using the GraphPad software. Values represent the mean \pm s.e.m. ${ }^{*} p<0.05^{* *} p<0.011^{* * *} p<0.001$ and ${ }^{* * * *} p<0.0001$.

\section{RESULTS}

\section{Satellite Cell Depletion Results in Muscle Fiber Atrophy and Reduces Hypertrophy in Response to AcvR2B Pathway Inhibition}

We used the $\operatorname{Pax} 7^{D T R /+}$ mouse model in which the DT receptor is expressed under the control of $P a x 7$ such that injection of DT results in a substantial depletion of the satellite cell population (Sambasivan et al., 2011). Ten week-old $\operatorname{Pax} 7^{D T R /+}$ mice were injected with DT in the Tibialis Anterior (TA) (CTL DT mice) and PBS in the contralateral muscle as a control (CTL PBS mice) and allowed to recover for 4 weeks (Figure 1A). We observed that DT-induced satellite cell depletion resulted in a marked reduction $(20 \%)$ in fiber size indicating that satellite cells are required to maintain proper fiber size in resting muscle (median value of fiber area distribution: CTL PBS $=1058.67 \pm 141.49$ $\mu \mathrm{m}^{2}$; CTL DT $\left.=831.33 \pm 85.38 \mu \mathrm{m}^{2}\right)$ (Figures 1C,D). It has been shown previously that a soluble form of the AcvR2B (RAP031) containing the ligand-binding site inhibits AcvR2B signaling by functioning as a decoy receptor in vivo and that this inhibition results in marked hypertrophy due to inhibition of MST and activin activity (Akpan et al., 2009; Koncarevic et al., 2010; George Carlson et al., 2011; Pistilli et al., 2011). We therefore tested RAP-031 in muscle containing an intact population of satellite cells (PBS-injected control referred to here as "intact") and DT-induced satellite cell depleted mice. Mice were injected intraperitoneally with RAP-031 or vehicle (CTL) twice a week for 2 weeks (Figure 1A). In order to confirm AcvR2B inhibition following RAP-031 administration, we performed Western Blot analyses on TA muscle extracts, which revealed a $50 \%$ reduction of the ratio between the phosphorylated form of Smad2 (pSmad2) and the total amount of Smad2 in RAP-031 treated animals, regardless of the status of the satellite cell pool (both in PBS and DT muscles), thus confirming inhibition of the AcvR2B signaling following RAP-031 administration (Figure 1B). Moreover, as reported by others (Cadena et al., 2010; George Carlson et al., 2011; Pistilli et al., 2011), intact muscle exposed to RAP-031 displayed a pronounced increase in fiber size (median value of fiber area distribution: CTL PBS $=1058.67 \pm 141.49 \mu \mathrm{m}^{2}$; RAP PBS $=1435.00 \pm 105.67 \mu \mathrm{m}^{2}$ ) (Figures 1C,D). However, in satellite cell-depleted muscle, RAP-031 treatment rescued the reduction of muscle fiber size previously induced by DT injection, but failed to generate a net fiber hypertrophic effect (median value of fiber area distribution: RAP DT $=969.00 \pm$ $61.25 \mu \mathrm{m}^{2}$ ) (Figures 1C,D). Taken together, these data indicate that a reduced satellite cell compartment limits muscle fiber hypertrophy in response to AcvR2B inhibition.

\section{Depletion of the Satellite Cell Compartment Alters the Stem Cell Niche}

Fiber hypertrophy is typically accompanied by an increase in myonuclear content in intact muscle, a process referred to as nuclear accretion (Smith et al., 2001; Pallafacchina et al., 2013). We observed that RAP-031 treatment of intact muscles resulted in a marked increase in sublaminal nuclear content as compared to vehicle-injected mice (Figure 1E) consistent with previous studies (Smith et al., 2001; McCroskery et al., 2003; Zhou et al., 2010; Wang and McPherron, 2012). In contrast, we did not observe a change in sublaminal nuclei content in DT RAP031 mice as compared to controls (Figure 1E) indicating that in presence of a reduced satellite cell pool, other progenitors with myogenic capacities (pericytes, myoPICs) are not recruited into the myofibers. We do note a minor increase in sublaminal nuclei following satellite cell depletion in the absence of RAP031 exposure (Figure 1E), however this is likely due to a reduced fiber volume, nonetheless, we cannot rule out that a portion of the surviving satellite cells or other resident progenitor cells fuse with the muscle fibers in response to satellite cell depletion or DT-treatment. 
A

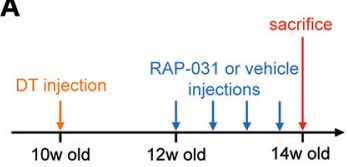

C

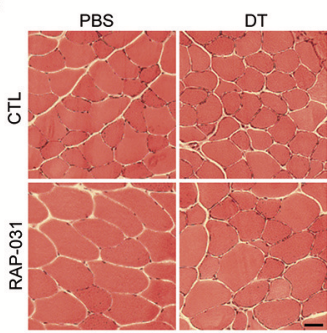

D
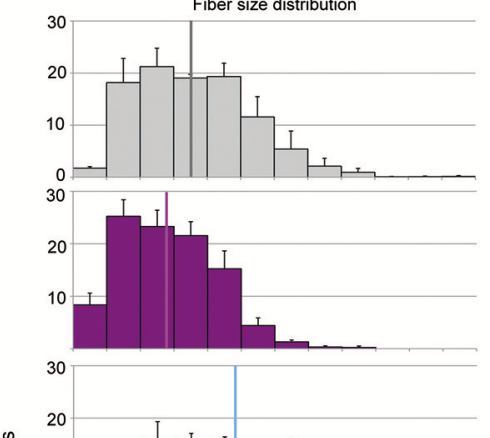

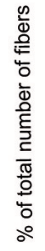

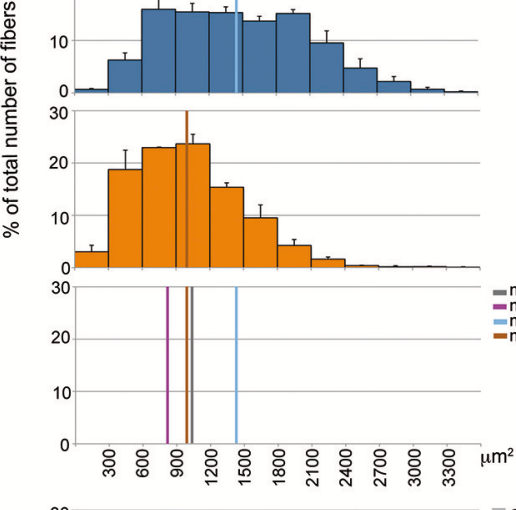

B

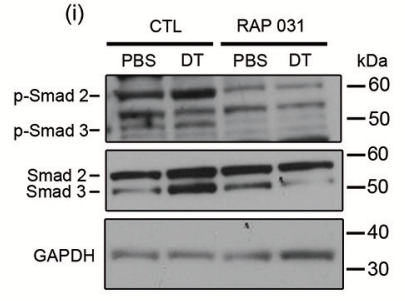

E

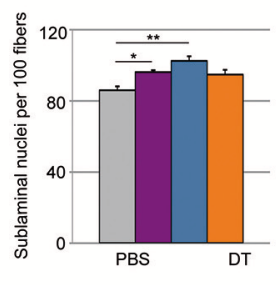

CTLPBS RAP-031 PBS RAP-031 DT

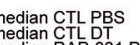
$=$ median CTL DT
$=$ median RAP-O31 PBS
-median RAP-031 DT

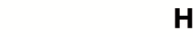

H CTLPBS CTLDT

(ii)

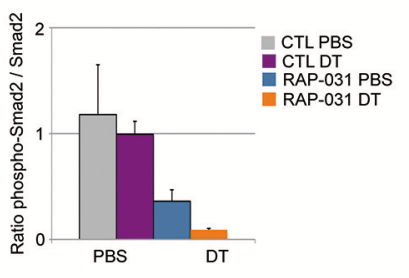

F

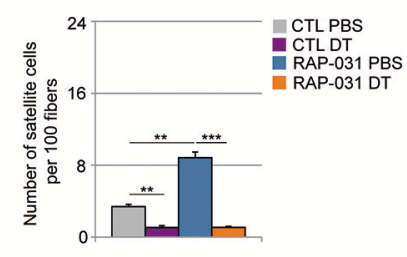

G
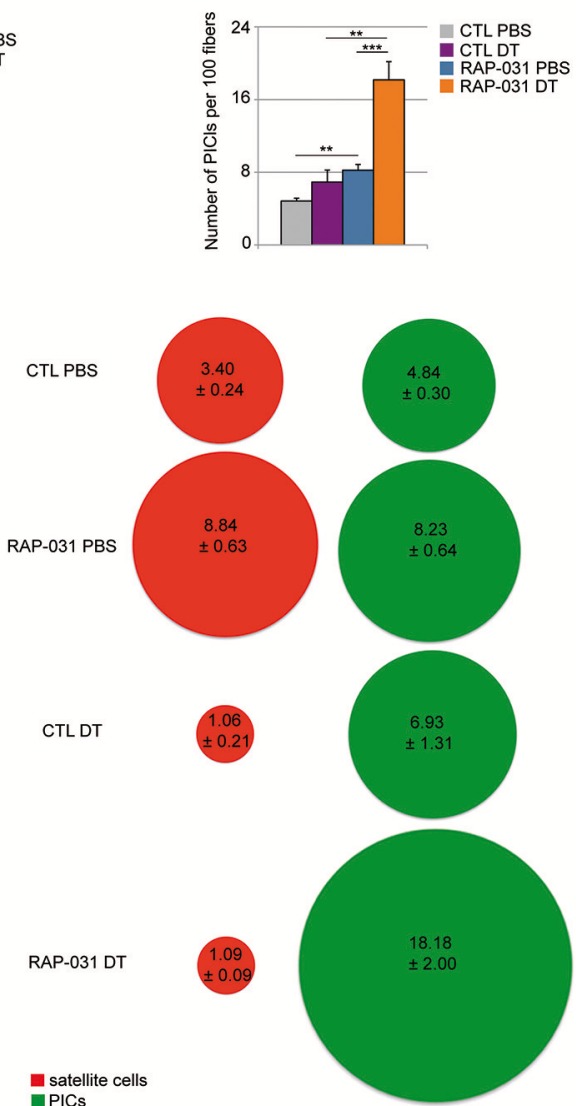

FIGURE 1 | RAP-031 treatment rescues the reduction of muscle fiber size induced by satellite cell depletion and increases PICs in satellite cell-depleted muscle. (A) Strategy: the right TA of 10 week-old Pax $7^{D T R /+}$ males was injected with diphteria toxin (DT) to deplete satellite cells while the contralateral muscle was injected with PBS 2 weeks before RAP-031 treatment. Mice were intraperitoneally injected with RAP-031 or vehicle (CTL) twice a week for 2 weeks and sacrificed 14 days after the first injection. (B) Western Blot of TA protein extracts with antibodies against phospho-Smad2/3, total Smad2/3 and GAPDH. (i) Representative image of the blots. Molecular size in $\mathrm{kDa}$ is shown on the right side. (ii) Ratio between levels of phospho-Smad2 and levels of total Smad2. The graph shows a marked reduction in the ratio pSmad2/Smad2 in RAP-031 muscles, indicating an inhibition of the AcvR2B pathway following systemic RAP-031 treatment. $N=2$ muscles per group. (C) Cross-sections images of TA muscles from CTL (Upper) or RAP-031 (Lower) mice injected with PBS (Left) or DT (Right) stained with hematoxylin and eosin. Scale bar, $60 \mu \mathrm{m}$. (D) Fiber size distribution in CTL PBS (gray), CTL DT (purple), RAP-031 PBS (blue), and RAP-031 DT (orange) TAs. RAP-031 treatment rescued the reduction of muscle fiber size induced by satellite cell depletion. Values represent the mean number \pm s.e.m. per 100 fibers for each size. $N=3$ animals for each group. Median of fiber area distributions are shown in the graphs. (E) Quantification of the number of sublaminal nuclei per 100 fibers in TA from CTL and RAP-031 mice injected with PBS or DT. RAP-031 treatment did not result in myonuclei addition when satellite cells are depleted. Values represent the mean number \pm s.e.m. per 100 fibers. At

(Continued) 
FIGURE 1 | least 300 fibers from randomly chosen fields were counted for each animal, $n=3$ animals were considered for each group. (F,G) Quantification of satellite cells (E) and PICs (F) per 100 fibers in TA from CTL and RAP-031 mice injected with PBS or DT. (H) Ratio between PICs (green) and satellite cells (red) per 100 fibers in TA from CTL and RAP-031 mice injected with PBS or DT was profoundly altered in DT-injected mice after RAP-031 treatment. In (E-G), satellite cells were determined as M-Cadherin ${ }^{p o s}$ cells underneath the basal lamina, PICs were determined as interstitial PW1 ${ }^{\text {pos }}$ M-Cadherin ${ }^{\text {neg }}$ cells. In (E-G), values represent the mean number of positive cells \pm s.e.m. per 100 fibers. At least 300 fibers from randomly chosen fields were counted for each animal, $n=3$ animals were considered for each group. For all values, ${ }^{*} p<0.05,{ }^{* *} p<0.01$, and ${ }^{* * *} p<0.001$.

We next sought to determine the status of satellite cells and other resident progenitor cell populations in response to satellite cell depletion in the presence or absence of RAP031 exposure. Muscle tissue sections from PBS or DT-injected muscles from vehicle and RAP-031 treated mice were stained for PW1/Peg3 (to label PICs) and M-Cadherin (to label satellite cells) (Supplementary Figure 1). As expected (Sambasivan et al., 2011), satellite cell numbers were markedly decreased following DT injection to levels of $\sim 25-30 \%$ as compared to levels in intact muscles (Figure 1F). Furthermore, whereas RAP-031 treatment induced a 2 -fold increase in satellite cell number in intact muscles (Figure 1F), we did not observe an increase in satellite cell number in satellite cell depleted muscles in response to RAP-031 treatment (Figure 1F). These results suggest that once the satellite cell number is below a specific or threshold level, their response to AcvR2B inhibition is abrogated. As shown previously (Sambasivan et al., 2011) and observed in this study, PICs are clearly detectable following satellite cell depletion and we observed a modest increase in PICs number following satellite cell depletion as compared to intact muscles (Figure 1G). Furthermore, we observed that PICs undergo a 2fold increase in intact muscles and a 4-fold increase in satellite cell depleted muscles following RAP-031 treatment (Figure 1G), indicating that PICs increase in number in response to AcvR2B inhibition in all conditions and that this response is increased when satellite cell numbers are reduced. Whether the increase in PICs is due to a proliferation of previously existing PICs or de novo PW1 expression in a subset of interstitial cells is not known and requires the generation of novel transgenic mouse models to specifically track PICs. Since we observed a deregulation of the number and proportion of muscle cell populations in our experimental conditions, we wished to better characterize the status of the muscle stem cell niche. We therefore checked for the expression of a marker of myogenic cell activation (MyoD) and a marker of cell proliferation (Ki67) in combination with markers for satellite cells (PW1, Pax7) and PICs (PW1) via immunostaining in TA cryosections. We did not detect MyoD expression in TA from any of the conditions tested (data not shown), indicating that neither DT-induced satellite cell pool depletion nor AcvR2B inhibition induced myogenic activation of satellite cells or any other muscle progenitors. In addition, we detected only rare Ki67 ${ }^{\text {pos }}$ cells in all the conditions examined, among which few Ki67 ${ }^{\text {pos }}$ PICs and no Ki67 ${ }^{\text {pos }}$ satellite cell in DT RAP-031 mice (data not shown), indicating that satellite cells and a majority of PICs were in quiescent cell cycle state (G0 resting phase) at the end of the observation period, both in the PBS and DT-injected mice, and regardless of AcvR2B inhibition treatment.

We had shown previously that PICs and satellite cells are present in a $\sim 1: 1$ ratio in resting muscle throughout postnatal life, whereas the constitutive loss of Pax7, which leads to a concomitant decrease of satellite cells and an increase in PICs such that the ratio of PICs and satellite cells is altered (Mitchell et al., 2010). We observed that the increase in PICs is present at birth prior to any detectable loss of satellite cells indicating that a change in the PIC population is an early event following compromised satellite cell function in the Pax7 mutant mouse (Mitchell et al., 2010). Furthermore, we have shown that PICs encompass the fibroadipogenic progenitors (FAPs) as well as a population of myogenically competent interstitial cells (myoPICs) (Pannérec et al., 2013). Since FAPs have already been shown to exert a promyogenic effect upon activated satellite cells but also underlie the fibrotic and adipogenic response in muscle tissue in pathological settings (Joe et al., 2010; Uezumi et al., 2010, 2011), we set out to determine whether these populations were altered in response to satellite cell depletion. Consistent with our previous observations, we observed that PICs and satellite cells are present in a $\sim 1: 1$ ratio in normal resting muscle (Figure 1H). Following AcvR2B inhibition, this ratio does not display a significant change despite the increase in cell number suggesting a tight regulation of the stem cell niche in both normal and hypertrophic muscle. In contrast, depletion of the satellite cell compartment resulted in an alteration of the PIC/satellite cells ratio in favor of PICs $(\sim 7: 1)$ however, we observed that RAP-031 treatment resulted in a PIC/satellite cells ratio of $\sim 17: 1$ in satellite cell-depleted muscle (Figure $\mathbf{1 H}$ ). Analyses performed on younger mice (5 week-old) (Supplementary Figure 2A) also showed an increase in both satellite cells and PICs following RAP-031 treatment (Supplementary Figure 2B), however PICs displayed a more pronounced increase as compared to satellite cells, leading to an altered ratio in favor of PICs (Supplementary Figure 2C). Moreover, we observed that the proportions of FAPs (PW $1^{\text {pos }} \mathrm{PDGFR} \alpha^{\text {pos }}$ ) and myoPICs $\left(\mathrm{PW} 1^{\text {pos}} \mathrm{PDGFR} \alpha^{\text {neg }}\right)$ were unchanged in RAP-031 as compared to vehicle-injected mice (Supplementary Figures 2D-F), indicating that both PW1 expressing interstitial populations were equally increased after AcvR2B blockade. Taken together, these data indicate that AcvR2B pathway inhibition has a profound impact on the muscle stem cell niche in intact as well as in satellite cell-depleted muscles.

\section{AcvR2B Inhibition Restores the Regenerative Capacity and Inhibits Fibrosis/Adipogenesis in Muscle Containing Few Satellite Cells}

Satellite cell depletion results in a severely compromised regenerative response coupled with pronounced fibrosis and fat deposition (Lepper et al., 2011; Sambasivan et al., 2011) 
and this outcome is strikingly similar to late stage degenerative myopathies (Maier and Bornemann, 1999; Shefer et al., 2006; Tabebordbar et al., 2013). FAPs have been proposed to exert a promyogenic role on satellite cells during regeneration but also to directly contribute to fat and fibrosis in diseased muscle (Joe et al., 2010; Uezumi et al., 2010; Murphy et al., 2011), therefore we sought to determine whether the changes observed in the PICs population, which includes the FAPs, in both intact and satellite cell depleted muscles following RAP-031 treatment reflected a general change in the stem cell niche that in turn may provide a more favorable regenerative environment. Previously we showed that PICs and satellite cells have distinct transcriptomes (Pannérec et al., 2013). Closer examination of these previous data revealed a striking reciprocal expression of TGF $\beta$ ligands and their receptors in PICs and satellite cells although these earlier studies were performed on cells isolated from juvenile muscles and without separating the population based upon PDGFR $\alpha$ expression (Pannérec et al., 2013). Nonetheless, the close overlap between juvenile and adult satellite cell transcriptomes (Pannérec et al., 2013) prompted us to examine the expression of specific TGF $\beta$ family members and receptors in adult satellite cells, FAPs and myoPICs. Semi-quantitative PCR analyses revealed that adult satellite cells express activinA and MST (Supplementary Figure 3A), which are factors known to inhibit myogenesis and induce muscle atrophy (McPherron et al., 1997; Lee et al., 2010). In contrast, FAPs expressed FST and IGF-1 (Supplementary Figure 3A), which have been demonstrated to exert a promyogenic effect on muscle fibers and activated satellite cells (Glass, 2010; Lee et al., 2010) (Supplementary Figure 3A). We note that the myoPICs do not show this reciprocal expression of TGF $\beta$ family members, although they express different extracellular and intracellular players of the TGF $\beta$ pathway (Supplementary Figure 3A), suggesting a response to TGF $\beta$ pathway manipulation. Furthermore, we observed that satellite cell capacity to form clones in vitro is dramatically reduced when these cells are cultured in presence of increasing doses of exogenous MST (Supplementary Figures 3B,C), consistent with previous observations (McCroskery et al., 2003). We found that the decline of satellite cell clone-forming capacity in presence of MST can be reversed by the concomitant co-culture with PICs (Supplementary Figures 3B,C) and that this rescue can be abrogated by the addition of blocking antibodies against FST and IGF-1 to the medium (Supplementary Figure 3C). Taken together, these data are consistent with a model in which the PICs are a key cellular constituent of the satellite stem cell niche and serve to provide a promyogenic effect in healthy muscle in response to injury (Joe et al., 2010; Uezumi et al., 2010; Murphy et al., 2011; Mozzetta et al., 2013). We found here that PICs are strongly increased in number in satellite celldepleted muscle following RAP-031 treatment as compared to CTL DT-injected mice (Figure 1G), while satellite cell number did not change and remained at a level of $30 \%$ of the intact muscle population (Figure 1F). Satellite cell-depleted TA muscles and control (PBS-injected) contralateral muscles were therefore injured by cardiotoxin (Ctx) 1 day before the last RAP-031 or vehicle injection and the level of regeneration was assessed at 2 weeks following injury. As expected, all RAP-031-treated ( $n=$
9) and CTL $(n=9)$ TA muscles injected with PBS displayed robust myofiber regeneration while CTL satellite cell-depleted muscles displayed severely compromised regeneration coupled with fat deposition and fibrosis (Figure 2A). We observed rare and highly restricted regions of newly regenerated fibers in only 2 of the 5 CTL satellite cell depleted muscles (CTL DT) suggesting that restricted surviving satellite cells can be mobilized locally but fail to contribute significantly toward replacing muscle tissue. In contrast, RAP-031 treatment of satellite cell-depleted muscles resulted in a marked rescue in overall regeneration coupled with a near complete inhibition of fat formation and fibrosis in 7 out of 9 samples (Figure 2A). We note that depletion of satellite cells in vehicle-injected mice resulted in a strong decrease in the number of centrally nucleated fibers after injury compared to muscles injected with PBS (8.8-fold), whereas this decrease was less than 1.5-fold in RAP-031 DT mice as compared to CTL PBS muscles (Figure 2B). Despite the rescue in regenerative capacity following RAP-031 treatment, satellite cell-depleted muscles exhibited a 30\% reduced fiber size as compared to normal intact muscles (median value of fiber area distribution: CTL PBS $=1,455.13 \pm 175.68 \mu \mathrm{m}^{2}$; RAP DT $=1003.80 \pm 136.18 \mu \mathrm{m}^{2}$ ) (Figure 2C), indicating that AcvR2B pathway inhibition via RAP-031 administration before injury is able to rescue overall regeneration capacity in satellite cell-depleted muscles, but does not rescue satellite cell depletion-induced muscle mass loss after injury. We note that while RAP-031 treatment is able to increase fiber size in homeostatic intact muscles (Figure 1D), it does not induce muscle fiber hypertrophy after injury (median value of fiber area distribution: CTL PBS $=1,455.13 \pm 175.68 \mu \mathrm{m}^{2}$; RAP $\mathrm{PBS}=1,208.55 \pm 190.74 \mu \mathrm{m}^{2}$ ) (Figure 2C) suggesting that the AcvR2B signaling pathway in resting and regenerating muscle acts differently. Moreover, in RAP-031 satellite cell-depleted muscle, regeneration was coupled with a marked decrease of fat as well as fibrotic zones to levels comparable to CTL intact muscle (Figure 2D).

We next compared the number of satellite cells in injured and uninjured muscles. As expected (Wang and Rudnicki, 2012; Yin et al., 2013), satellite cells were increased in CTL PBS-injected muscle after injury (Figure 2E). In contrast, satellite cell numbers were below the level of detection in CTL DT-injected contralateral muscles after injury (Figure 2E), indicating that the failure of regeneration was accompanied by a complete loss of satellite cell self-renewal after Ctx injection. We found that RAP-031 treatment did not significantly change the amount of satellite cells present in the regenerated intact muscle (Figure 2E) indicating that regeneration of RAP-031 treated muscle is accompanied by the re-establishment of the normal intact satellite cell population number. Similarly, satellite cell-depleted muscles pretreated with RAP-031 gave rise to new muscle with a satellite cell population that was restored to $\sim 30 \%$ of the intact muscle population (Figure 2E). These data demonstrate that inhibition of the AcvR2B pathway in muscle tissue containing a reduced population of satellite cells is able to rescue regeneration but that the satellite cell population is only restored to pre-injured levels. 


\section{A}
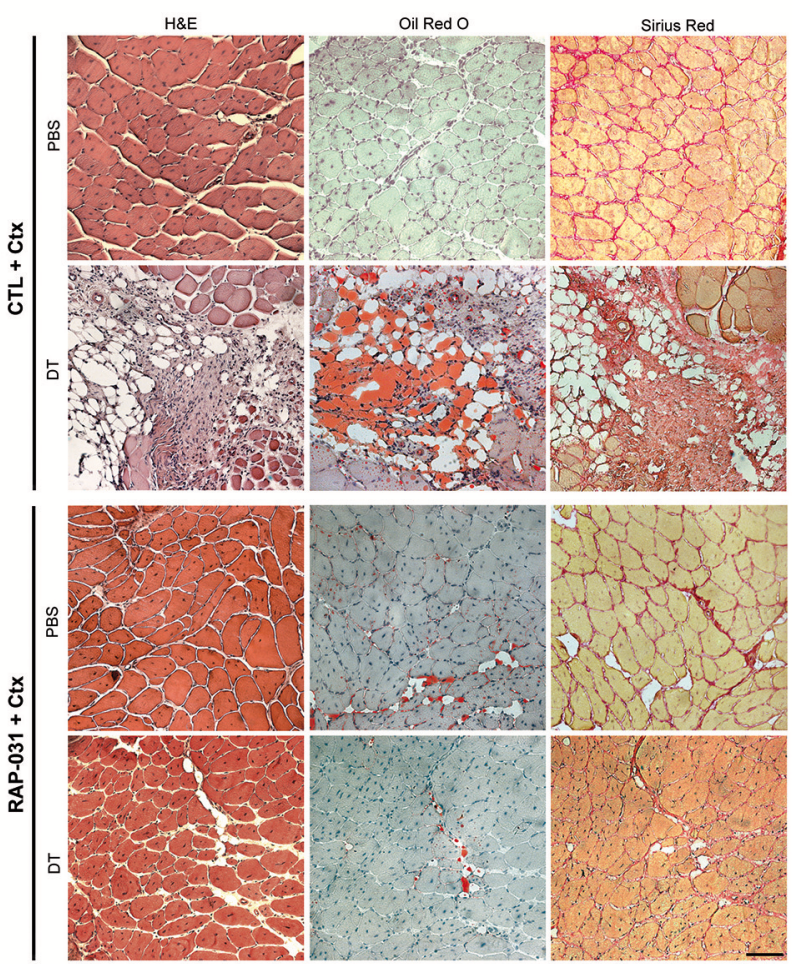

B

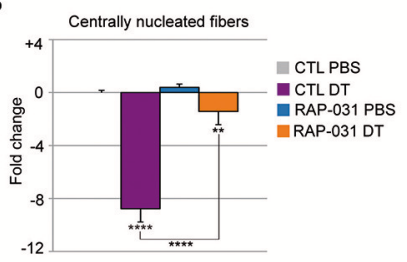

C

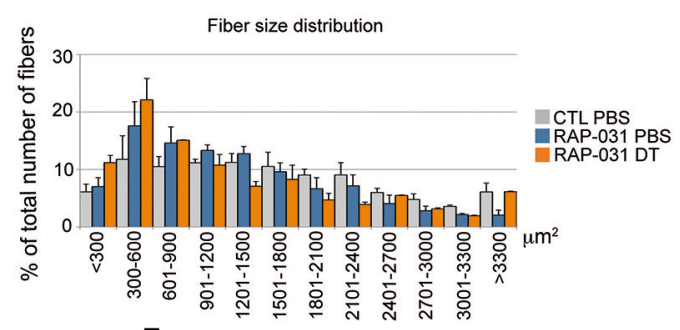

D
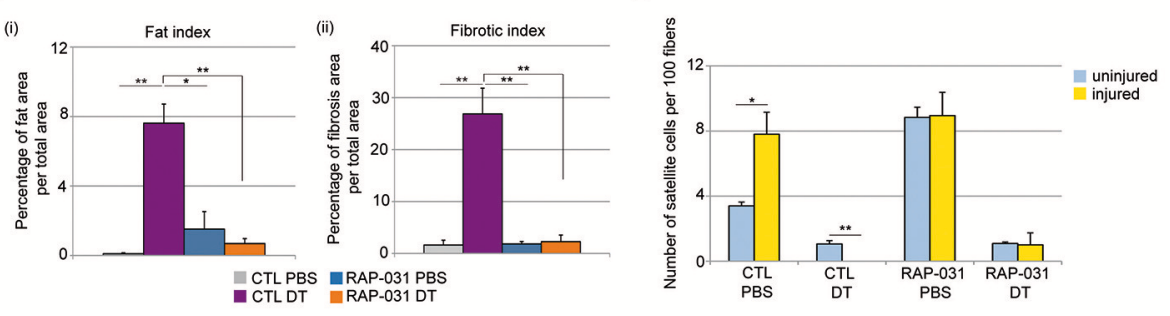

FIGURE 2 | AcvR2B pathway inhibition improves overall fiber regeneration and ameliorates tissue homeostasis in injured satellite cell-depleted muscles.

(A) Cross-sections of TA muscle from CTL (Upper) and RAP-031 (Lower) Pax7DTR/+ mice injected with DT or PBS stained with haematoxylin and eosin (Left), Oil-Red $\mathrm{O}$ (Middle) and Sirius Red (Right). Regeneration is more efficient in RAP-031 DT mice as compared to CTL DT mice, with a marked reduction in fat and fibrotic tissue deposition. Scale bar, $85 \mu \mathrm{m}$. (B) Quantitative analysis of regenerative capacity of TA muscles shown in (A). The number of centrally nucleated fibers per field in TAs from CTL mice injected with PBS was considered as the baseline (zero) and values represent the fold change \pm s.e.m. normalized accordingly. Muscle fiber regeneration is significantly rescued in RAP-031 DT mice as compared to CTL DT mice. Five randomly chosen fields from one mid-belly TA section were analyzed for each animal, $n=3$ animals were analyzed for each group. (C) Fiber size distribution in CTL PBS (gray), RAP-031 PBS (blue), and RAP-031 DT (orange) TAs. Satellite cell-depleted muscles exposed to RAP-031 before injury exhibited regenerated fibers with a reduced size as compared to intact muscles. Values represent the mean number \pm s.e.m. per 100 fibers for each size. $N=3$ animals for each group. (D) Quantitative analysis of fat (i) and fibrotic (ii) areas of muscles showed in (A). One mid-belly TA section was analyzed with ImageJ software for each animal and $n=3$ animals were analyzed for each group. Fat areas were determined by Oil-Red $O$ staining and fibrotic areas were determined by Sirius-Red staining. Values represent the percentage of fat (i) or fibrotic (ii) area on whole muscle area.

(E) Quantification of satellite cells per 100 fibers in TA from uninjured (blue) and injured (yellow) Pax7DTR/+ CTL and RAP-031 mice injected with PBS or DT. Satellite cells were undetectable after injury in CTL DT muscle, however RAP-031 treatment was able to prevent satellite cell loss. Satellite cells were determined as Pax7 pos cells underneath the basal lamina. At least 5 randomly chosen fields, corresponding to at least 300 fibers, were counted for each animal, $n=3$ animals were considered for each group. ${ }^{\star} p<0.05,{ }^{* *} p<0.01,{ }^{* * * *} p<0.001$. 


\section{AcvR2B Inhibition Rescues Residual Satellite Cell Regenerative Capacity}

Our results raised the question as to whether the regeneration we observed in RAP-031 treated satellite cell-depleted muscle was primarily due to the activation of the residual satellite cells or due to the recruitment of non-satellite cell myogenic populations. We demonstrated previously that PICs contain a population of myogenic cells and several studies have shown the presence of myogenic cells other than satellite cells in normal muscle tissue however their participation in regeneration is unproven (Mitchell et al., 2010; Pannérec et al., 2013). To determine the origin of regenerating myofibers, we crossed $\mathrm{Pax} 7^{\mathrm{DTR} /+}$ mice with Tg:Pax7CreER ${ }^{T 2}$ mice (Mourikis et al., 2012) and with reporter line ROSA26 $6^{\text {mTomato } / m G F P}$ (ROSA ${ }^{\mathrm{mTmG}}$ ) (Muzumdar et al., 2007). The resulting Pax7 $^{\text {DTR/+ }}:$ Pax7CreER $^{\mathrm{T} 2}$ :ROSA ${ }^{\mathrm{mTmG}}$ mice express a tamoxifeninducible Cre (Metzger and Chambon, 2001) such that Pax7-expressing satellite cells and their progeny are specifically marked by membranous GFP (mGFP), while all the other cells are marked by membranous Tomato (mTomato) (Mourikis et al., 2012). To verify the efficiency of Cre-mediated labeling of the satellite cell population, 6 week-old Pax7CreER ${ }^{\mathrm{T} 2}: \mathrm{ROSA}^{\mathrm{mTmG}}$ mice were injected with tamoxifen and sacrificed 6 weeks later (Supplementary Figure 4A). We stained TA muscles for mGFP and Pax7 and observed that all Pax7-positive cells were positive for mGFP (Supplementary Figure 4B) indicating that the expression of mGFP labeled the entirety of the Pax $7^{\text {pos }}$ satellite cell population. In addition, we observed mGFPpos sublaminal monuclear cells that did not express Pax7 at detectable levels indicating that mGFP labeling identified a larger set of satellite cells, which displayed low or undetectable levels of Pax7 at the time of the analysis (Supplementary Figure 4B). Taken together, we conclude that nearly $100 \%$ of the satellite cell population was marked by mGFP expression such that the Pax7 $7^{\mathrm{DTR} /+}:$ Pax7CreER $^{\mathrm{T} 2}: \mathrm{ROSA}^{\mathrm{mTmG}}$ mice provides a genetic tool for determining whether newly regenerated fibers are derived from satellite cells in which case fibers are predicted to express mGFP whereas the presence of $\mathrm{mGFP}^{\text {neg }} \mathrm{mTomato}^{\text {pos }}$ regenerating fibers would indicate a contribution of non-satellite progenitor cells. We then used 6 week-old $\operatorname{Pax}^{\mathrm{DTR} /+}: \mathrm{Pax}_{\mathrm{C}} \mathrm{CreER}^{\mathrm{T} 2}: \mathrm{ROSA}^{\mathrm{mTmG}}$ mice injected with tamoxifen to activate mGFP expression in satellite cells followed by a single injection of DT in the right TA and PBS in the contralateral muscle 4 weeks after the last tamoxifen injection. Muscles were allowed to recover for 2 weeks and then treated with RAP-031. Muscles were injured by cardiotoxin 1 day before the last RAP-031 injection and the regeneration was assessed 2 weeks later (Figure 3A). In satellite cell-depleted muscles from RAP-031 mice, we observed that the majority of centrally nucleated fibers $(>80 \%)$ were $\mathrm{mGFP}^{\text {pos }}$ (Figures 3B,C), comparable to vehicle (CTL) PBS and RAP-031 PBS conditions (Figures 3B,C), indicating that the reduced pool of satellite cells can be mobilized by RAP-031 treatment in satellite cell depleted muscles after injury. While these data confirm previous studies demonstrating that the satellite cells are the major muscle progenitor (Relaix and Zammit, 2012) we note that $\sim 15 \%$ of the centrally-nucleated fibers in intact muscle are exclusively marked by mTomato (Figure 3D), strongly suggesting a contribution of non-satellite cells. Interestingly, we observed a $10 \%$ increase in the number of $\mathrm{mGFP}^{\text {neg }} \mathrm{mTomato}^{\text {pos }}$ centrally nucleated fibers and a concomitant $10 \%$ decrease of mGFPpos centrally nucleated fibers in intact muscles from RAP-031 treated mice as compared to CTL mice (Figures 3C,D), suggesting that AcvR2B pathway inhibition stimulates non-satellite cell recruitment. Alternatively, there may be a small population of satellite cells that are resistant to Cre labeling that accounts for the generation of tomato positive fibers.

\section{DISCUSSION}

In addition to playing a key role during myofiber regeneration, satellite cells can fuse to existing myofibers during muscle hypertrophy leading to a stabilization of the larger myofiber mass (Amthor et al., 2009; McCarthy et al., 2011; Lee et al., 2012; Wang and McPherron, 2012). In this study, we found that a $\sim 70-80 \%$ reduction in the number of satellite cells triggers a decrease in myofiber size suggesting a role for satellite cells in maintaining fiber size. Interestingly, previous studies showed that depletion of satellite cells in adult mice (4-month old) did not reduce muscle fiber size nor exacerbate age-related sarcopenia (Fry et al., 2015; Murach et al., 2017), however depletion performed at 2.5 months of age (this study) resulted in fiber size reduction. Moreover, we found that myofiber hypertrophy is limited in muscles containing fewer satellite cells following AcvR2B pathway inhibition. Our observations are therefore consistent with a previous study in which satellite cell depletion in young mice ( 2.5 months old) abrogates subsequent mechanical overload-induced fiber hypertrophy (Murach et al., 2017), while satellite cells are usually reported to be dispensable in hypertrophy processes at later ages ( $>4$ months) (Murach et al., 2017, 2018). Taken together, these data suggest an active, agedependent role for satellite cells in normal late postnatal muscle growth.

The central question of this study was why a reduced satellite cell population cannot properly regenerate and our observations here suggest that the microenvironment is altered in response to a decline in satellite cells. Previous reports demonstrated that as few as 10 satellite cells can generate hundreds of myofibers when engrafted in healthy muscle. It has also been proposed that the diseased muscle environment does not favor optimal satellite cell function (Mann et al., 2011; Serrano et al., 2011; Pannérec et al., 2012; Tabebordbar et al., 2013; Yin et al., 2013). Consistent with this proposal, it has been shown that regenerative capacity declines sharply when the satellite cell pool declines to $50 \%$ of initial satellite cell complement in the double mouse mutant for utrophin and dystrophin (Lu et al., 2014). Our results confirm satellite cells contribute to muscle homeostasis.

The importance of niche cells has been demonstrated in multiple tissues which serve as cellular partners to govern progenitor activation, self-renewal and survival (Leatherman, 2013). We demonstrated previously that PW1/Peg3 expression can be used to identify a large array of adult progenitor/stem cells including associated niche cells (Besson et al., 2011). As one 


\section{A}

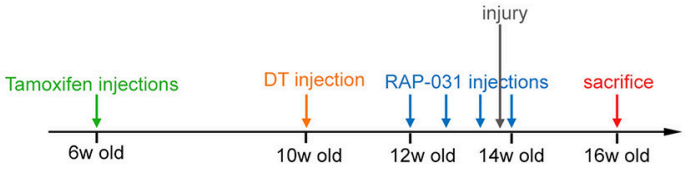

B
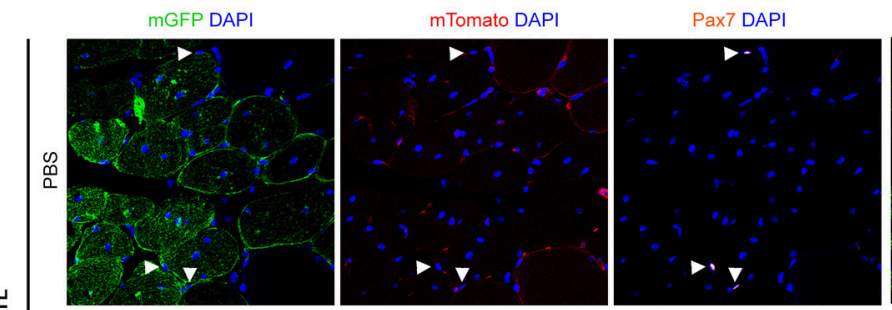

mGFP mTomato Pax7 DAPI

ப̌
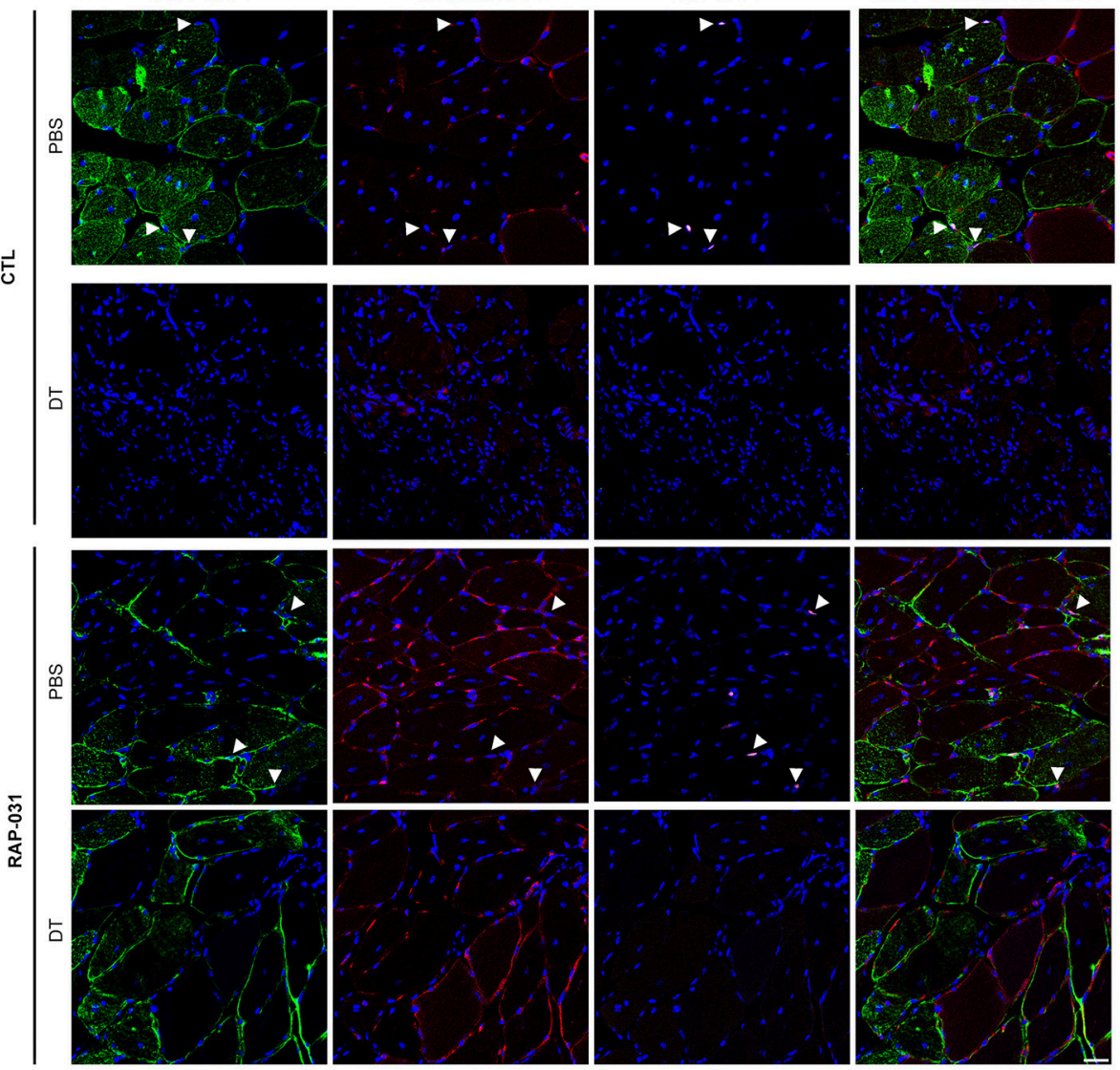

C
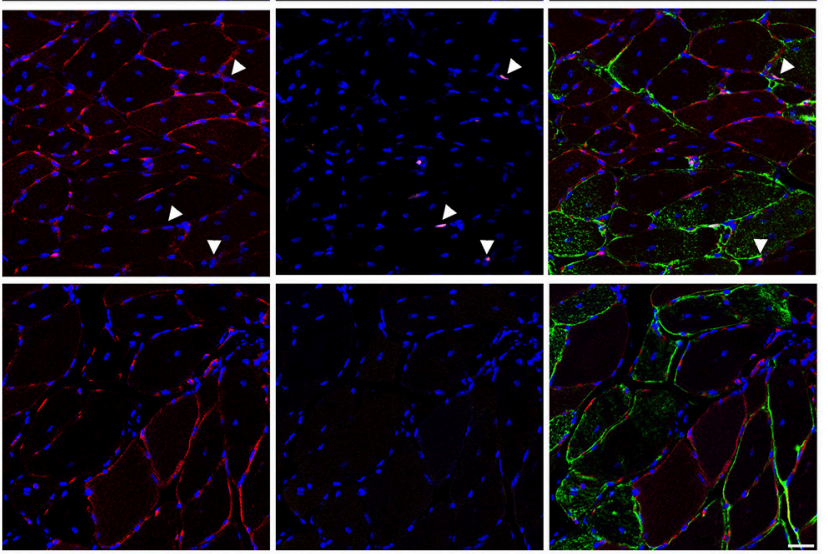

D
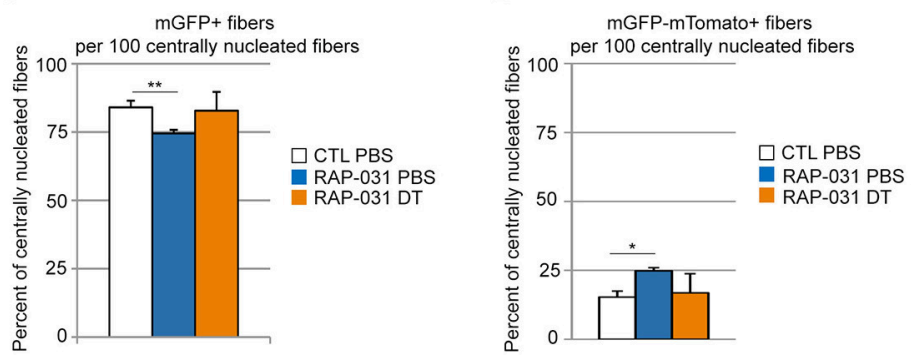

FIGURE 3 | Regeneration in satellite cell-depleted muscles following AcvR2B pathway inhibition is mainly executed by the residual satellite cells. (A) Strategy: 6 week-old Pax $7^{\mathrm{DTR} /+}: \mathrm{Pax}_{\mathrm{C} C r e E R^{\mathrm{T}} 2}: \mathrm{ROSA}^{\mathrm{mTmG}}$ mice were injected with tamoxifen to label satellite cells. 4 weeks later, the right TA was injected with DT to deplete the satellite cell pool. The contralateral muscle was injected with PBS. After 2 weeks, mice were injected with either RAP-031 or vehicle (CTL) twice a week for 2 weeks. The day before the last injection of RAP-031 or vehicle both TAs were injured by cardiotoxin injection. Mice were sacrificed 2 weeks after injury. (B) Representative images of cross-sections from injured TA muscles of CTL (Upper) and RAP-031 (Lower) mice injected with DT or PBS as described in (A), immunostained for mGFP (green) to identify satellite cell-derived fibers and Pax7 (orange) to identify satellite cells. mTomato fluorescence is shown in red, DAPI staining (blue) identifies nuclei. The majority of regenerating fibers are satellite cell-derived (green) regardless of treatment. White arrowheads: satellite cells. Scale bar, $30 \mu \mathrm{m}$. (C,D) Quantification of the number of mGFPpos $\mathbf{( C )}$ and mGFPneg mTomatopos (D) fibers per 100 centrally nucleated fibers for CTL and RAP-03 mice injected with PBS or DT, as described in (A). Values represent the mean number of positive fibers \pm s.e.m. per 100 centrally nucleated fibers. Random images were captured at 20X magnification and at least 5 different fields for each section were counted, $n=3$ animals were considered for each group. ${ }^{*} p<0.05,{ }^{* *} p<0.01$. 


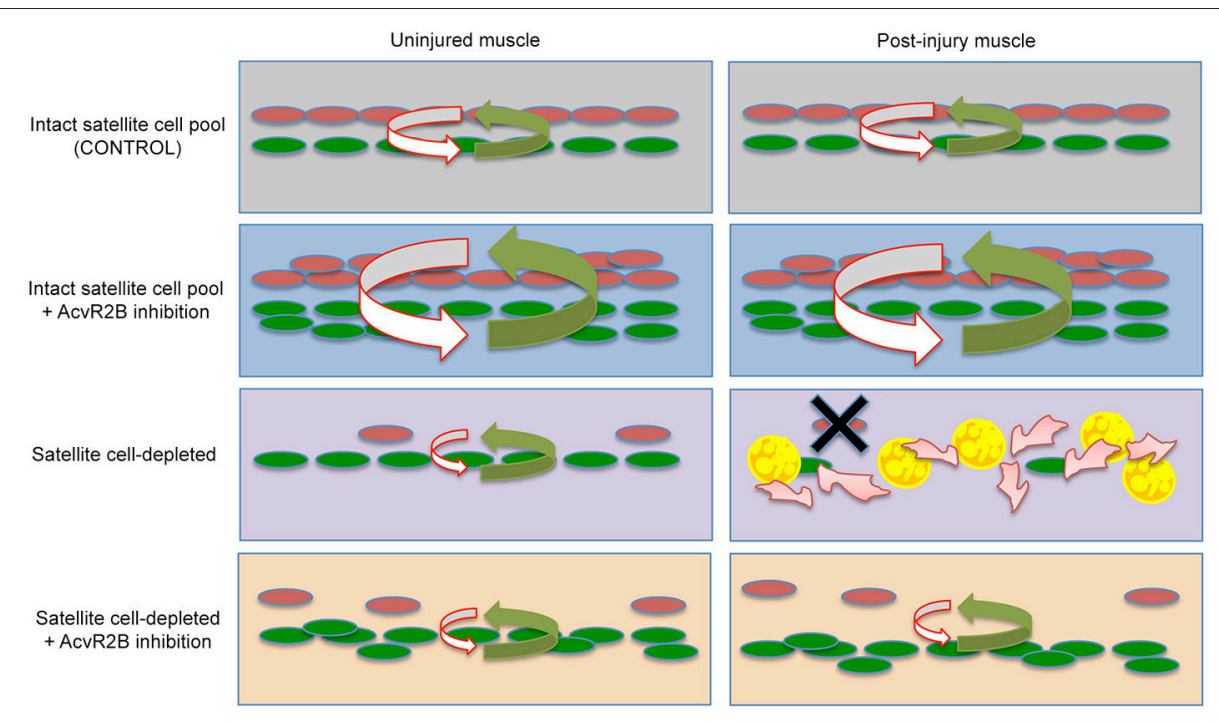

FIGURE 4 | Proposed cellular model of satellite cell and stem cell niche interactions. Top Row- In resting muscle containing an intact population of satellite cells (Left), pro-myogenic signals (green arrow) from PICs/FAPs (green), and anti-adipogenic signals (red arrow) from satellite cells (red) are kept in a tight balance, which is maintained after injury (Right). Second Row- Following AcvR2B inhibition, PICs and satellite cell populations expand, however pro- and anti-myogenic balance in maintained before and after injury. Third Row- Satellite cell depletion disrupts the balance and overall levels of factors regulating the niche (Left) and signaling within the niche is completely deregulated following injury (Right). This results in a complete loss of satellite cells and consequently a loss of satellite cell derived anti-adipogenic signals allowing FAP progression into fat and fibrosis (Right). Fourth Row- AcvR2B inhibitor treatment of satellite cell depleted muscle reinforces the niche (Left) and allows for satellite cell myogenic progression/self-renewal and consequently the maintenance of a minimal balance of factors required to restore the muscle tissue and stem cell niche (Right).

example, PW1/Peg3 is expressed in the hair follicle bulge cells that are a major reservoir of skin progenitors as well as in closely associated dermal papilla cells (Besson et al., 2011, 2017) which have a dual role as mesenchymal stem cells and as critical niche support cells (Driskell et al., 2011; Wong et al., 2012). We suggest that the expression of PW1/Peg3 in the satellite cells and a subset of interstitial cells including most notably the FAPs, defines a similar niche in skeletal muscle.

While our lineage tracing analyses confirms that satellite cells are the major source of regenerated muscle in all cases, several non-satellite cell populations have been shown to have pronounced myogenic activity (Pannérec et al., 2012). In intact skeletal muscle, $\sim 15 \%$ of the regenerated muscle fibers do not express the satellite cell lineage marker following damage and increase to $\sim 25 \%$ following AcvR2B pathway inhibition. Since all Pax7-positive cells express GFP following tamoxifen injection, our data suggest that non-satellite cell progenitors participate directly in muscle regeneration but require an intact satellite cell compartment although lineage-specific markers for the myoPIC population is required to assess the origin of these cells.

It has been described previously that satellite cells inhibit adipogenic differentiation of FAPs and that FAPs are promyogenic for satellite cells (Joe et al., 2010; Uezumi et al., 2010, 2011). We report here that in normal resting muscle, satellite cells express myogenic inhibitory factors such as MST and activinA, whereas FAPs express promyogenic factors including FST and IGF-1. These observations provide a framework (Figure 4) in which satellite cells are present in a niche which includes the FAPs and that the balance of pro- and anti-myogenic signals keeps myogenesis and fibro/adipogenesis tightly regulated.

The AcvR2B pathway has been a focus for the development of therapeutics for degenerative muscle diseases due to the recognition that these molecules antagonize myostatin and activin activity (Glass, 2010; Zhou and Lu, 2010; Ceco and McNally, 2013). A recent study has revealed that histone deacetylase (HDAC) inhibitors provide a promising route to treat muscular dystrophy in murine models via the induction of follistatin in interstitial muscle cells consistent with our results here showing that muscle mass is regulated by complex interactions between multiple cells types (Mozzetta et al., 2013). It is of interest that the beneficial effects of HDAC inhibitors decrease efficacy with age (Mozzetta et al., 2013). Similarly, we report here that AcvR2B inhibition in young mice (5 weeks) causes an increase in the PIC/satellite cell ratio disrupting the typical 1:1 ratio, while a 1:1 ratio is maintained in 10-weekold mice. Taken together, these data indicate that changes in regenerative capacity and stem cell competence during postnatal life reflect a loss of plasticity in the stem cell niche.

\section{CONCLUSIONS}

Our results point to a mechanistic convergence for satellite cells governing both regeneration and the maintenance of muscle fiber size. Whereas $20-30 \%$ of the normal population of satellite cells is insufficient to give rise to new muscle following damage, this reduced population can execute the regeneration program 
if the AcvR2B pathway is pharmacologically targeted. We propose that pharmacological targeting of the AcvR2B pathway restores a balance of regulatory factors within the muscle stem cell niche, in part through the regulation of cells that exert a promyogenic effect as well as cells capable of generating fibrotic and fat tissue, such as PICs/FAPs, that can interfere with the proper reassembly of muscle tissue (Figure 4), rather than directly promoting regeneration through the activation of satellite cells. This idea is also supported by our observation that AcvR2B inhibition treatment before injury does not alter the activation status of either the satellite cell population nor other cells types (such as PICs/FAPs) suggesting that all the cellular components of the muscle stem cell niche are important for proper regeneration. The capacity to restore the regenerative potential of muscle containing a reduced progenitor population through pharmacological intervention will impact the design of future therapeutic approaches for degenerative myopathies.

\section{AUTHOR CONTRIBUTIONS}

LF, AP, RC, DO, and BG-M performed experiments with reagents provided by JL and JS and expertise in using the mouse models was provided by VB, BG-M, and ST. All authors designed experiments, analyzed and interpreted data. LF, AP, RC, GM, and DS prepared the manuscript.

\section{ACKNOWLEDGMENTS}

We thank Catherine Blanc and Benedicte Hoareau (Flow Cytometry Core CyPS, Pierre \& Marie Curie University, PitieSalpetriere Hospital, Paris, France) for their assistance and help in sorting strategies; Antonio Musarò (DAHFMO-Unit of Histology and Medical Embryology, Sapienza University of Rome, Rome, Italy) for providing PCR primers for IGF-1 and for helpful discussions. This work was supported by the French Ministry of Research Chaire d'Excellence and the Muscular Dystrophy Association of America to DS and the European Community Seventh Framework Program projects OPTISTEM (Optimization of stem cell therapy for degenerative epithelial and muscle diseases contract number Health-F5-2009-223098) and ENDOSTEM (Activation of vasculature associated stem cells and muscle stem cells for the repair and maintenance of muscle tissue-agreement number 241440). ST acknowledges support from the Institut Pasteur and the Agence Nationale de la Recherche (Laboratoire d'Excellence Revive, Investissement d'Avenir; ANR-10-LABX-73). The Myology Group was a beneficiary of a Strategic Plan Support from the Association Française contre les Myopathies (AFM) and is affiliated the ANR Laboratoire d'Excellence program REVIVE and the IHU-ICAN projects. AP was supported by the French Ministry of Research and the Fondation pour la Recherche Médicale (Programme Espoirs de la Recherche, number FDT20110922527). RC was supported by a grant from the Marie Curie Training Programme (INGENIUM-ITN FP7). DO was supported by funding from REVIVE. In addition, we thank members of the Brack laboratory (UCSF) for a careful read and critique of this study prior to submission.

\section{SUPPLEMENTARY MATERIAL}

The Supplementary Material for this article can be found online at: https://www.frontiersin.org/articles/10.3389/fphys. 2018.00515/full\#supplementary-material

\section{Supplementary Figure 1 | Representative pictures of immuno-stainings in} uninjured TA muscles. Representative images of cross-sections from uninjured TA muscles of CTL (Upper) and RAP-031 (Lower) mice injected with DT or PBS, immunostained for PW1 (green), M-cadherin (red), Laminin (orange). DAPI staining (blue) identifies nuclei. Satellite cells (white arrowheads) are identified as M-cadherin pos cells. PICs (yellow arrowheads) are identified as PW1pos interstitial cells. Scale bar, $20 \mu \mathrm{m}$.

Supplementary Figure 2 | RAP-031 treatment of intact muscle from young mice alters the muscle stem cell niche. (A) Strategy: 5 week-old male wild-type mice were injected intraperitoneally with RAP-031 or vehicle (CTL) every 3 days and sacrificed 2 weeks after the first injection. (B) Quantification of satellite cells and PICs per 100 fibers in CTL and RAP-031 TA muscles showed an increase of both cells types after RAP-031 treatment. (C) Quantification of progenitor cells shown in (B) for CTL and RAP-031 Tibialis Anterior revealed an altered ratio between PICs (green) and satellite cells (red) in RAP-031 vs. CTL. For (B,C) PICs were determined as interstitial PW1 ${ }^{\text {pos } P a x 7^{\text {neg }}}$ cells, satellite cells as Pax ${ }^{\text {pos }}$ cells underneath the basal lamina. (D,E) Flow cytometric analyses of single cells from 7-week old limb muscles from CTL (D) or RAP-031-injected (E) PW1 ${ }^{\text {nLacZ }}$ mice following the protocol described in (A). Cells were stained for CD45, Ter119, CD34, Sca1, PDGFR $\alpha$, and $\mathrm{C}_{12}$ FDG (to reveal PW1 expression via $\beta$-galactosidase activity), as reported in Pannérec et al. (2013). CD45neg Ter119neg were selected. The gates used to isolate FAPs (CD34 ${ }^{\text {pos }}$ Sca $1^{\text {pos }}$ PW1 $\left.{ }^{\text {pos } P D G F R ~}{ }^{\text {pos }}\right)$ and myoPICs $\left(C D 34^{\text {pos }}\right.$ Sca $^{\text {pos }}{ }^{\text {PW }} 1^{\text {pos } P D G F R ~} \alpha^{\text {neg }}$ ) are shown. Satellite cells (SAT) are also shown in red (CD34 ${ }^{\text {pos }}$ Sca $\left.{ }^{\text {neg }}\right)$. (F) Number of FAPs (PW1 $\left.{ }^{\text {pos } P D G F R} \alpha^{\text {pos }}\right)$ and myoPICs (PW1 ${ }^{\text {pos PDGFR }}{ }^{\text {pos }}$ ) from CTL and RAP-031 mice as presented as the

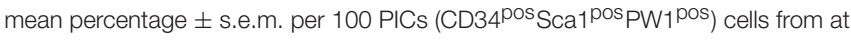
least 3 independent experiments. ${ }^{*} p<0.05,{ }^{* *} p<0.01$, and ${ }^{* * *} p<0.001$.

Supplementary Figure $\mathbf{3}$ | Adult PICs and satellite cells express TGF $\beta$ pathway related genes. (A) Semi-quantitative PCR of selected genes involved in the TGF $\beta$ and IGF-1 pathway in freshly sorted adult satellite cells (SAT), FAPs (PW1 ${ }^{\text {pos }}$ PDGFR $\alpha^{\text {pos }}$ ), and myoPICs (PW $\left.{ }^{\text {pos } P D G F R ~} \alpha^{\text {neg }}\right)$ sorted as shown in (Supplementary Figure 1D). PC, Positive Control: whole muscle extract from 7 week-old PW1nLacZ mice, except for activin a subunit, IGF-1 IEa and IEb (whole liver extract from 7 week-old PW1nLacZ mice) and for TGF $\beta$ R2 (whole brain extract from 7 week-old PW1nLacZ mice). (B) Schematic transwell membrane system used: PICs (green) were plated in the upper well on a semipermeable membrane (insert) and satellite cells (red) were plated in the lower chamber. (C) Quantitative analyses of satellite cell proliferation in growth medium containing $0,20,200 \mathrm{ng} / \mathrm{ml}$, or $2 \mu \mathrm{g} / \mathrm{ml}$ of recombinant myostatin. Satellite cells were cultured alone (red bars) or in the presence of PICs and isotype-matched IgG (gray bars). Satellite cells and PICs were co-cultured in presence of a blocking antibody to follistatin ( $\alpha F S T$, blue bars) or to IGF-1 ( $\alpha$ IGF-1, black bars) or blocking antibodies to IGF-1 and FST together ( $\alpha$ FST + alGF1, white bars). Large colonies (>12 cells) were counted and shown as a percentage of the number of total colonies.

Supplementary Figure 4 | Efficiency of inducible Pax7-driven Cre mediated recombination of RosamTomatomGFP locus. (A) 6 week-old

Pax7CreER ${ }^{T 2}:$ ROSA $^{\text {mTmG }}$ mice were injected with tamoxifen every day for 4 days and sacrificed 6 weeks later. (B) Quantification analysis of satellite cells in mice treated as in (A). (i) $100 \%$ of Pax $7^{\text {pos }}$ satellite cells express mGFP. Values represent the mean number of GFPpos and GFPneg cells \pm s.e.m. per 100 Pax7 ${ }^{\text {pos }}$ cells. (ii) The majority (>80\%) of GFPpos mononuclear cells co-express Pax7, however $<20 \%$ of the GFPpos mononuclear cells are not co-labeling with Pax7. Values represent the mean number of $\mathrm{Pax} 7^{\text {Pos }}$ and Pax $7^{\text {neg }}$ cells \pm s.e.m. per 100 GFPpos mononuclear cells. For quantification, immunostaining for Pax 7 and GFP was performed on TA muscle section from $n=4$ mice and at least 5 different randomly chosen fields were counted for each section, corresponding to an average number of fibers of $>120$ per section.

Supplementary Table 1 | List of primers used for semi-qPCR. 


\section{REFERENCES}

Akpan, I., Goncalves, M. D., Dhir, R., Yin, X., Pistilli, E. E., Bogdanovich, S., et al. (2009). The effects of a soluble activin type IIB receptor on obesity and insulin sensitivity. Int. J. Obes. (Lond). 33, 1265-1273. doi: 10.1038/ijo.2009.162

Amthor, H., Nicholas, G., McKinnell, I., Kemp, C. F., Sharma, M., Kambadur, R., et al. (2004). Follistatin complexes Myostatin and antagonises Myostatin-mediated inhibition of myogenesis. Dev. Biol. 270, 19-30. doi: 10.1016/j.ydbio.2004.01.046

Amthor, H., Otto, A., Vulin, A., Rochat, A., Dumonceaux, J., Garcia, L., et al. (2009). Muscle hypertrophy driven by myostatin blockade does not require stem/precursor-cell activity. Proc. Natl. Acad. Sci. U.S.A. 106, 7479-7484. doi: $10.1073 /$ pnas.0811129106

Artaza, J. N., Singh, R., Ferrini, M. G., Braga, M., Tsao, J., and Gonzalez-Cadavid, N. F. (2008). Myostatin promotes a fibrotic phenotypic switch in multipotent $\mathrm{C} 3 \mathrm{H} 10 \mathrm{~T} 1 / 2$ cells without affecting their differentiation into myofibroblasts. J. Endocrinol. 196, 235-249. doi: 10.1677/JOE-07-0408

Besson, V., Kyryachenko, S., Janich, P., Benitah, S. A., Marazzi, G., and Sassoon, D. (2017). Expression analysis of the stem cell marker Pw1/Peg3 Reveals a CD34 Negative Progenitor Population in the Hair Follicle. Stem Cells. 35, 1015-1027. doi: $10.1002 /$ stem. 2540

Besson, V., Smeriglio, P., Wegener, A., Relaix, F., Nait Oumesmar, B., Sassoon, D. A., et al. (2011). PW1 gene/paternally expressed gene 3 (PW1/Peg3) identifies multiple adult stem and progenitor cell populations. Proc. Natl. Acad. Sci. U.S.A. 108, 11470-11475. doi: 10.1073/pnas.1103873108

Blau, H. M., Webster, C., and Pavlath, G. K. (1983). Defective myoblasts identified in Duchenne muscular dystrophy. Proc. Natl. Acad. Sci. U.S.A. 80, 4856-4860.

Blau, H. M., Webster, C., Pavlath, G. K., and Chiu, C. P. (1985). Evidence for defective myoblasts in Duchenne muscular dystrophy. Adv. Exp. Med. Biol. 182, 85-110.

Bodine, S. C., Stitt, T. N., Gonzalez, M., Kline, W. O., Stover, G. L., Bauerlein, R., et al. (2001). Akt/mTOR pathway is a crucial regulator of skeletal muscle hypertrophy and can prevent muscle atrophy in vivo. Nat. Cell Biol. 3, 1014-1019. doi: 10.1038/ncb1101-1014

Boldrin, L., Zammit, P. S., and Morgan, J. E. (2015). Satellite cells from dystrophic muscle retain regenerative capacity. Stem Cell Res. 14, 20-29. doi: 10.1016/j.scr.2014.10.007

Bonaldo, P., and Sandri, M. (2013). Cellular and molecular mechanisms of muscle atrophy. Dis. Model. Mech. 6, 25-39. doi: 10.1242/dmm.010389

Cadena, S. M., Tomkinson, K. N., Monnell, T. E., Spaits, M. S., Kumar, R., Underwood, K. W., et al. (2010). Administration of a soluble activin type IIB receptor promotes skeletal muscle growth independent of fiber type. J. Appl. Physiol. 109, 635-642. doi: 10.1152/japplphysiol.008 66.2009

Ceco, E., and McNally, E. M. (2013). Modifying muscular dystrophy through transforming growth factor-beta. FEBS J. 280, 4198-4209. doi: $10.1111 /$ febs. 12266

Chiu, C. S., Peekhaus, N., Weber, H., Adamski, S., Murray, E. M., Zhang, H. Z., et al. (2013). Increased muscle force production and bone mineral density in ActRIIB-Fc-treated mature rodents. J. Gerontol. A Biol. Sci. Med. Sci. 68, 1181-1192. doi: 10.1093/gerona/glt030

Collins, C. A., Olsen, I., Zammit, P. S., Heslop, L., Petrie, A., Partridge, T. A., et al. (2005). Stem cell function, self-renewal, and behavioral heterogeneity of cells from the adult muscle satellite cell niche. Cell 122, 289-301. doi: 10.1016/j.cell.2005.05.010

Coulton, G. R., Morgan, J. E., Partridge, T. A., and Sloper, J. C. (1988). The mdx mouse skeletal muscle myopathy: I. A histological, morphometric and biochemical investigation. Neuropathol. Appl. Neurobiol. 14, 53-70.

Dellavalle, A., Maroli, G., Covarello, D., Azzoni, E., Innocenzi, A., Perani, L., et al. (2011). Pericytes resident in postnatal skeletal muscle differentiate into muscle fibres and generate satellite cells. Nat. Commun. 2:499. doi: $10.1038 /$ ncomms 1508

Driskell, R. R., Clavel, C., Rendl, M., and Watt, F. M. (2011). Hair follicle dermal papilla cells at a glance. J. Cell Sci. 124(Pt 8), 1179-1182. doi: 10.1242/jcs.082446

Farup, J., Madaro, L., Puri, P. L., and Mikkelsen, U. R. (2015). Interactions between muscle stem cells, mesenchymal-derived cells and immune cells in muscle homeostasis, regeneration and disease. Cell Death Dis. 6:e1830. doi: $10.1038 /$ cddis. 2015.198
Fry, C. S., Lee, J. D., Mula, J., Kirby, T. J., Jackson, J. R., Liu, F., et al. (2015). Inducible depletion of satellite cells in adult, sedentary mice impairs muscle regenerative capacity without affecting sarcopenia. Nat. Med. 21, 76-80. doi: $10.1038 / \mathrm{nm} .3710$

George Carlson, C., Bruemmer, K., Sesti, J., Stefanski, C., Curtis, H., Ucran, J., et al. (2011). Soluble activin receptor type IIB increases forward pulling tension in the mdx mouse. Muscle Nerve 43, 694-699. doi: 10.1002/mus.21944

Glass, D. J. (2010). Signaling pathways perturbing muscle mass. Curr. Opin. Clin. Nutr. Metab. Care 13, 225-229. doi: 10.1097/MCO.0b013e32833862df

Heslop, L., Morgan, J. E., and Partridge, T. A. (2000). Evidence for a myogenic stem cell that is exhausted in dystrophic muscle. J. Cell Sci. 113(Pt 12), 2299-2308.

Hoffman, E. P., Brown, R. H. Jr., and Kunkel, L. M. (1987). Dystrophin: the protein product of the Duchenne muscular dystrophy locus. Cell 51, 919-928.

Jiang, C., Wen, Y., Kuroda, K., Hannon, K., Rudnicki, M. A., and Kuang, S. (2014). Notch signaling deficiency underlies age-dependent depletion of satellite cells in muscular dystrophy. Dis. Model. Mech. 7, 997-1004. doi: $10.1242 / \mathrm{dmm} .015917$

Joe, A. W., Yi, L., Natarajan, A., Le Grand, F., So, L., Wang, J., et al. (2010). Muscle injury activates resident fibro/adipogenic progenitors that facilitate myogenesis. Nat. Cell Biol. 12, 153-163. doi: 10.1038/ncb2015

Kharraz, Y., Guerra, J., Mann, C. J., Serrano, A. L., and Muñoz-Cánoves, P. (2013). Macrophage plasticity and the role of inflammation in skeletal muscle repair. Mediat. Inflamm. 2013:491497. doi: 10.1155/2013/491497

Koncarevic, A., Cornwall-Brady, M., Pullen, A., Davies, M., Sako, D., Liu, J., et al. (2010). A soluble activin receptor type IIb prevents the effects of androgen deprivation on body composition and bone health. Endocrinology 151, 4289-4300. doi: 10.1210/en.2010-0134

Lach-Trifilieff, E., Minetti, G. C., Sheppard, K., Ibebunjo, C., Feige, J. N., Hartmann, S., et al. (2014). An antibody blocking activin type II receptors induces strong skeletal muscle hypertrophy and protects from atrophy. Mol. Cell. Biol. 34, 606-618. doi: 10.1128/MCB.01307-13

Lawlor, M. W., Read, B. P., Edelstein, R., Yang, N., Pierson, C. R., Stein, M. J., et al. (2011). Inhibition of activin receptor type IIB increases strength and lifespan in myotubularin-deficient mice. Am. J. Pathol. 178, 784-793. doi: 10.1016/j.ajpath.2010.10.035

Leatherman, J. (2013). Stem cells supporting other stem cells. Front. Genet. 4:257. doi: 10.3389/fgene.2013.00257

Lee, S. J., and McPherron, A. C. (2001). Regulation of myostatin activity and muscle growth. Proc. Natl. Acad. Sci. U.S.A. 98, 9306-9311. doi: 10.1073/pnas.151270098

Lee, S. J., Huynh, T. V., Lee, Y. S., Sebald, S. M., Wilcox-Adelman, S. A., Iwamori, N., et al. (2012). Role of satellite cells versus myofibers in muscle hypertrophy induced by inhibition of the myostatin/activin signaling pathway. Proc. Natl. Acad. Sci. U.S.A. 109, E2353-E2360. doi: 10.1073/pnas.1206410109

Lee, S. J., Lee, Y. S., Zimmers, T. A., Soleimani, A., Matzuk, M. M., Tsuchida, K., et al. (2010). Regulation of muscle mass by follistatin and activins. Mol. Endocrinol. 24, 1998-2008. doi: 10.1210/me.2010-0127

Lepper, C., Partridge, T. A., and Fan, C. M. (2011). An absolute requirement for Pax7-positive satellite cells in acute injury-induced skeletal muscle regeneration. Development 138, 3639-3646. doi: 10.1242/dev.067595

Lu, A., Poddar, M., Tang, Y., Proto, J. D., Sohn, J., Mu, X., et al. (2014). Rapid depletion of muscle progenitor cells in dystrophic mdx/utrophin-/- mice. Hum. Mol. Genet. 23, 4786-4800. doi: 10.1093/hmg/ddu194

Maier, F., and Bornemann, A. (1999). Comparison of the muscle fiber diameter and satellite cell frequency in human muscle biopsies. Muscle Nerve 22, 578-583.

Mann, C. J., Perdiguero, E., Kharraz, Y., Aguilar, S., Pessina, P., Serrano, A. L., et al. (2011). Aberrant repair and fibrosis development in skeletal muscle. Skelet. Muscle 1:21. doi: 10.1186/2044-5040-1-21

McCarthy, J. J., Mula, J., Miyazaki, M., Erfani, R., Garrison, K., Farooqui, A. B., et al. (2011). Effective fiber hypertrophy in satellite cell-depleted skeletal muscle. Development 138, 3657-3666. doi: 10.1242/dev.068858

McCroskery, S., Thomas, M., Maxwell, L., Sharma, M., and Kambadur, R. (2003). Myostatin negatively regulates satellite cell activation and self-renewal. J. Cell Biol. 162, 1135-1147. doi: 10.1083/jcb.200207056

McCroskery, S., Thomas, M., Platt, L., Hennebry, A., Nishimura, T., McLeay, L., et al. (2005). Improved muscle healing through enhanced regeneration and reduced fibrosis in myostatin-null mice. J. Cell Sci. 118(Pt 15), 3531-3541. doi: $10.1242 /$ jcs. 02482 
McPherron, A. C., and Lee, S. J. (2002). Suppression of body fat accumulation in myostatin-deficient mice. J. Clin. Invest. 109, 595-601. doi: 10.1172/JCI13562

McPherron, A. C., Lawler, A. M., and Lee, S. J. (1997). Regulation of skeletal muscle mass in mice by a new TGF-beta superfamily member. Nature $387,83-90$. doi: $10.1038 / 387083 \mathrm{a} 0$

Metzger, D., and Chambon, P. (2001). Site- and time-specific gene targeting in the mouse. Methods 24, 71-80. doi: 10.1006/meth.2001.1159

Mitchell, K. J., Pannérec, A., Cadot, B., Parlakian, A., Besson, V., Gomes, E. R., et al. (2010). Identification and characterization of a non-satellite cell muscle resident progenitor during postnatal development. Nat. Cell Biol. 12, 257-266. doi: $10.1038 /$ ncb2025

Morrison, B. M., Lachey, J. L., Warsing, L. C., Ting, B. L., Pullen, A. E., Underwood, K. W., et al. (2009). A soluble activin type IIB receptor improves function in a mouse model of amyotrophic lateral sclerosis. Exp. Neurol. 217, 258-268. doi: 10.1016/j.expneurol.2009.02.017

Mourikis, P., Sambasivan, R., Castel, D., Rocheteau, P., Bizzarro, V., and Tajbakhsh, S. (2012). A critical requirement for notch signaling in maintenance of the quiescent skeletal muscle stem cell state. Stem Cells 30, 243-252. doi: 10.1002/stem.775

Mozzetta, C., Consalvi, S., Saccone, V., Tierney, M., Diamantini, A., Mitchell, K. J., et al. (2013). Fibroadipogenic progenitors mediate the ability of HDAC inhibitors to promote regeneration in dystrophic muscles of young, but not old Mdx mice. EMBO Mol. Med. 5, 626-639. doi: 10.1002/emmm.201202096

Murach, K. A., Fry, C. S., Kirby, T. J., Jackson, J. R., Lee, J. D., White, S. H., et al. (2018). Starring or Supporting Role? Satellite Cells and Skeletal Muscle Fiber Size Regulation. Physiology (Bethesda) 33, 26-38. doi: 10.1152/physiol.00019.2017

Murach, K. A., White, S. H., Wen, Y., Ho, A., Dupont-Versteegden, E. E., McCarthy, J. J., et al. (2017). Differential requirement for satellite cells during overload-induced muscle hypertrophy in growing versus mature mice. Skelet. Muscle 7:14. doi: 10.1186/s13395-017-0132-z

Murphy, M. M., Lawson, J. A., Mathew, S. J., Hutcheson, D. A., and Kardon, G. (2011). Satellite cells, connective tissue fibroblasts and their interactions are crucial for muscle regeneration. Development 138, 3625-3637. doi: $10.1242 /$ dev. 064162

Muzumdar, M. D., Tasic, B., Miyamichi, K., Li, L., and Luo, L. (2007). A global double-fluorescent Cre reporter mouse. Genesis 45, 593-605. doi: $10.1002 /$ dvg. 20335

Oberbauer, A. M. (2013). The Regulation of IGF-1 gene transcription and splicing during development and aging. Front. Endocrinol. (Lausanne) 4:39. doi: 10.3389/fendo.2013.00039

Pallafacchina, G., Blaauw, B., and Schiaffino, S. (2013). Role of satellite cells in muscle growth and maintenance of muscle mass. Nutr. Metab. Cardiovasc. Dis. 23 (Suppl. 1), S12-S18. doi: 10.1016/j.numecd.2012.02.002

Pannérec, A., Formicola, L., Besson, V., Marazzi, G., and Sassoon, D. A. (2013). Defining skeletal muscle resident progenitors and their cell fate potentials. Development. 140, 2879-2891. doi: 10.1242/dev.089326

Pannérec, A., Marazzi, G., and Sassoon, D. (2012). Stem cells in the hood: the skeletal muscle niche. Trends Mol. Med. 18, 599-606. doi: 10.1016/j.molmed.2012.07.004

Parker, A. E., Robb, S. A., Chambers, J., Davidson, A. C., Evans, K., O’Dowd, J., et al. (2005). Analysis of an adult Duchenne muscular dystrophy population. QJM 98, 729-736. doi: 10.1093/qjmed/hci113

Pistilli, E. E., Bogdanovich, S., Goncalves, M. D., Ahima, R. S., Lachey, J., Seehra, J., et al. (2011). Targeting the activin type IIB receptor to improve muscle mass and function in the mdx mouse model of Duchenne muscular dystrophy. Am. J. Pathol. 178, 1287-1297. doi: 10.1016/j.ajpath.2010.11.071

Relaix, F., and Zammit, P. S. (2012). Satellite cells are essential for skeletal muscle regeneration: the cell on the edge returns centre stage. Development 139, 2845-2856. doi: 10.1242/dev.069088

Relaix, F., Weng, X., Marazzi, G., Yang, E., Copeland, N., Jenkins, N., et al. (1996). Pwl, a novel zinc finger gene implicated in the myogenic and neuronal lineages. Dev. Biol. 177, 383-396. doi: 10.1006/dbio.1996.0172

Rommel, C., Bodine, S. C., Clarke, B. A., Rossman, R., Nunez, L., Stitt, T. N., et al. (2001). Mediation of IGF-1-induced skeletal myotube hypertrophy by PI(3)K/Akt/mTOR and PI(3)K/Akt/GSK3 pathways. Nat. Cell Biol. 3, 1009-1013. doi: 10.1038/ncb1101-1009
Sacco, A., Doyonnas, R., Kraft, P., Vitorovic, S., and Blau, H. M. (2008). Selfrenewal and expansion of single transplanted muscle stem cells. Nature 456, 502-506. doi: 10.1038/nature07384

Sako, D., Grinberg, A. V., Liu, J., Davies, M. V., Castonguay, R., Maniatis, S., et al. (2010). Characterization of the ligand binding functionality of the extracellular domain of activin receptor type IIb. J. Biol. Chem. 285, 21037-21048. doi: 10.1074/jbc.M110.114959

Sambasivan, R., Yao, R., Kissenpfennig, A., Van Wittenberghe, L., Paldi, A., Gayraud-Morel, B., et al. (2011). Pax7-expressing satellite cells are indispensable for adult skeletal muscle regeneration. Development 138, 3647-3656. doi: 10.1242/dev.067587

Sartori, R., Schirwis, E., Blaauw, B., Bortolanza, S., Zhao, J., Enzo, E., et al. (2013). BMP signaling controls muscle mass. Nat. Genet. 45, 1309-1318. doi: $10.1038 /$ ng. 2772

Serrano, A. L., Mann, C. J., Vidal, B., Ardite, E., Perdiguero, E., and MuñozCánoves, P. (2011). Cellular and molecular mechanisms regulating fibrosis in skeletal muscle repair and disease. Curr. Top. Dev. Biol. 96, 167-201. doi: 10.1016/B978-0-12-385940-2.00007-3

Shefer, G., Van de Mark, D. P., Richardson, J. B., and Yablonka-Reuveni, Z. (2006). Satellite-cell pool size does matter: defining the myogenic potency of aging skeletal muscle. Dev. Biol. 294, 50-66. doi: 10.1016/j.ydbio.2006. 02.022

Siriett, V., Platt, L., Salerno, M. S., Ling, N., Kambadur, R., and Sharma, M. (2006). Prolonged absence of myostatin reduces sarcopenia. J. Cell. Physiol. 209, 866-873. doi: $10.1002 / j \mathrm{cp} .20778$

Smith, H. K., Maxwell, L., Rodgers, C. D., McKee, N. H., and Plyley, M. J. (2001). Exercise-enhanced satellite cell proliferation and new myonuclear accretion in rat skeletal muscle. J. Appl. Physiol. (1985) 90, 1407-1414. doi: 10.1152/jappl.2001.90.4.1407

Tabebordbar, M., Wang, E. T., and Wagers, A. J. (2013). Skeletal muscle degenerative diseases and strategies for therapeutic muscle repair. Annu. Rev. Pathol. 8, 441-475. doi: 10.1146/annurev-pathol-011811-132450

Tanano, H., Hasegawa, T., Kimura, T., Sasaki, T., Kawahara, H., Kubota, A., et al. (2003). Proposal of fibrosis index using image analyzer as a quantitative histological evaluation of liver fibrosis in biliary atresia. Pediatr. Surg. Int. 19, 52-56. doi: 10.1007/s00383-002-0883-3

Ten Broek, R. W., Grefte, S., and Von den Hoff, J. W. (2010). Regulatory factors and cell populations involved in skeletal muscle regeneration. J. Cell. Physiol. 224, 7-16. doi: 10.1002/jcp.22127

Thomas, M., Langley, B., Berry, C., Sharma, M., Kirk, S., Bass, J., et al. (2000). Myostatin, a negative regulator of muscle growth, functions by inhibiting myoblast proliferation. J. Biol. Chem. 275, 40235-40243. doi: 10.1074/jbc.M004356200

Trendelenburg, A. U., Meyer, A., Rohner, D., Boyle, J., Hatakeyama, S., and Glass, D. J. (2009). Myostatin reduces Akt/TORC1/p70S6K signaling, inhibiting myoblast differentiation and myotube size. Am. J. Physiol,. Cell Physiol. 296, C1258-C1270. doi: 10.1152/ajpcell.00105.2009

Uezumi, A., Fukada, S., Yamamoto, N., Takeda, S., and Tsuchida, K. (2010). Mesenchymal progenitors distinct from satellite cells contribute to ectopic fat cell formation in skeletal muscle. Nat. Cell Biol. 12, 143-152. doi: $10.1038 / \mathrm{ncb} 2014$

Uezumi, A., Ito, T., Morikawa, D., Shimizu, N., Yoneda, T., Segawa, M., et al. (2011). Fibrosis and adipogenesis originate from a common mesenchymal progenitor in skeletal muscle. J. Cell Sci. 124(Pt 21), 3654-3664. doi: $10.1242 /$ jcs. 086629

Wagner, K. R., McPherron, A. C., Winik, N., and Lee, S. J. (2002). Loss of myostatin attenuates severity of muscular dystrophy in $\mathrm{mdx}$ mice. Ann. Neurol. 52, 832-836. doi: 10.1002/ana.10385

Wallace, G. Q., and McNally, E. M. (2009). Mechanisms of muscle degeneration, regeneration, and repair in the muscular dystrophies. Annu. Rev. Physiol. 71, 37-57. doi: 10.1146/annurev.physiol.010908. 163216

Wang, Q., and McPherron, A. C. (2012). Myostatin inhibition induces muscle fibre hypertrophy prior to satellite cell activation. J. Physiol. 590(Pt 9), 2151-2165. doi: 10.1113/jphysiol.2011.226001

Wang, Y. X., and Rudnicki, M. A. (2012). Satellite cells, the engines of muscle repair. Nat. Rev. Mol. Cell Biol. 13, 127-133. doi: 10.1038/nrm3265 
Wong, V. W., Levi, B., Rajadas, J., Longaker, M. T., and Gurtner, G. C. (2012). Stem cell niches for skin regeneration. Int. J. Biomater. 2012:926059. doi: $10.1155 / 2012 / 926059$

Yamaguchi, A. (1995). Regulation of differentiation pathway of skeletal mesenchymal cells in cell lines by transforming growth factor-beta superfamily. Semin. Cell Biol. 6, 165-173.

Yin, H., Price, F., and Rudnicki, M. A. (2013). Satellite cells and the muscle stem cell niche. Physiol. Rev. 93, 23-67. doi: 10.1152/physrev.00043. 2011

Z Hosaka, Y., Ishibashi, M., Wakamatsu, J., Uehara, M., and Nishimura, T. (2012). Myostatin regulates proliferation and extracellular matrix mRNA expression in NIH3T3 fibroblasts. Biomed. Res. 33, 355-361. doi: 10.2220/biomedres. 33.355

Zhou, L., and Lu, H. (2010). Targeting fibrosis in Duchenne muscular dystrophy. J. Neuropathol. Exp. Neurol. 69, 771-776. doi: 10.1097/NEN.0b013e3181e $9 \mathrm{a} 34 \mathrm{~b}$
Zhou, X., Wang, J. L., Lu, J., Song, Y., Kwak, K. S., Jiao, Q., et al. (2010). Reversal of cancer cachexia and muscle wasting by ActRIIB antagonism leads to prolonged survival. Cell 142, 531-543. doi: 10.1016/j.cell.2010.07.011

Conflict of Interest Statement: The authors declare that the research was conducted in the absence of any commercial or financial relationships that could be construed as a potential conflict of interest.

Copyright (๑) 2018 Formicola, Pannérec, Correra, Gayraud-Morel, Ollitrault, Besson, Tajbakhsh, Lachey, Seehra, Marazzi and Sassoon. This is an open-access article distributed under the terms of the Creative Commons Attribution License (CC $B Y)$. The use, distribution or reproduction in other forums is permitted, provided the original author(s) and the copyright owner are credited and that the original publication in this journal is cited, in accordance with accepted academic practice. No use, distribution or reproduction is permitted which does not comply with these terms. 


\section{OPEN ACCESS}

Edited by:

Valentina Di Felice,

Università degli Studi di Palermo, Italy

Reviewed by:

Marina Bouche,

Università degli Studi di Roma La

Sapienza, Italy

Antonio Paolo Beltrami,

Università degli Studi di Udine, Italy

*Correspondence:

Mathias Mericskay mathias.mericskay@inserm.fr

Specialty section:

This article was submitted to

Striated Muscle Physiology,

a section of the journal

Frontiers in Physiology

Received: 05 July 2018

Accepted: 27 August 2018 Published: 19 September 2018

Citation:

Deloux R, Tannous C, Ferry A, Li Z and Mericskay M (2018) Aged Nicotinamide Riboside Kinase 2 Deficient Mice Present an Altered Response to Endurance Exercise Training. Front. Physiol. 9:1290. doi: 10.3389/fphys.2018.01290

\section{Aged Nicotinamide Riboside Kinase 2 Deficient Mice Present an Altered Response to Endurance Exercise Training}

\author{
Robin Deloux ${ }^{1,2}$, Cynthia Tannous ${ }^{1,2}$, Arnaud Ferry ${ }^{3,4}$, Zhenlin $L^{2}{ }^{2}$ and Mathias Mericskay ${ }^{1 *}$ \\ 'Signalling and Cardiovascular Pathophysiology-UMR-S 1180, Univ. Paris-Sud, INSERM, Université Paris-Saclay, \\ Châtenay-Malabry, France, ${ }^{2}$ Department of Biology of Adaptation and Ageing, CNRS UMR8256, INSERM U1164, Institute of \\ Biology Paris-Seine, DHU FAST, Sorbonne Universités, Paris, France, ${ }^{3}$ Sorbonne Paris Cité, Université Paris Descartes, \\ Paris, France, ${ }^{4}$ Institut de Myologie, UMR-S 794, INSERM, CNRS, Sorbonne Universités, Paris, France
}

Background: Skeletal muscle aging is marked by the development of a sarcopenic phenotype, a global decline of muscle energetic capacities, and an intolerance to exercise. Among the metabolic disorders involved in this syndrome, NAD metabolism was shown to be altered in skeletal muscle, with an important role for the NAMPT enzyme recycling the nicotinamide precursor. An alternative pathway for NAD biosynthesis has been described for the nicotinamide riboside vitamin B3 precursor used by the NMRK kinases, including the striated muscle-specific NMRK2.

Aim: With this study, our goal is to explore the ability of 16-month-old $\mathrm{Nmrk}^{-1-}$ mice to perform endurance exercise and study the consequences on muscle adaptation to exercise.

Methods: 10 control and $6 \mathrm{Nmrk} 2^{-/-}$mice were used and randomly assigned to sedentary and treadmill endurance training groups. After 9 weeks of training, heart and skeletal muscle samples were harvested and used for gene expression analysis, NAD levels measurements and immunohistochemistry staining.

Results: Endurance training triggered a reduction in the expression of Cpt1b and AcadL genes involved in fatty acid catabolism in the heart of $\mathrm{Nmrk2}^{-/-}$mice, not in control mice. NAD levels were not altered in heart or skeletal muscle, nor at baseline neither after exercise training in any group. Myh7 gene encoding for the slow MHC-I was more strongly induced by exercise in $\mathrm{Nmrk}^{-1-}$ mice than in controls. Moreover, $I L-15$ expression levels is higher in $\mathrm{Nmrk2}^{-/-}$mice skeletal muscle at baseline compared to controls. No fiber type switch was observed in plantaris after exercise, but fast fibers diameter was reduced in aged control mice, not in $\mathrm{Nmrk2}^{-/-}$mice. No fiber type switch or diameter modification was observed in soleus muscle.

Conclusion: In this study, we demonstrated for the first time a phenotype in old $\mathrm{Nmrk2}^{-1-}$ mice in response to endurance exercise training. Although NMRK2 seems to 
be predominantly dispensable to maintain global NAD levels in heart and skeletal muscle, we demonstrated a maladaptive metabolic response to exercise in cardiac and skeletal muscle, showing that NMRK2 has a specific and restricted role in NAD signaling compared to the NAMPT pathway.

Keywords: heart, skeletal muscle, aging, NAD, NMRK2, exercise, endurance training

\section{INTRODUCTION}

Skeletal muscles are highly plastic organs that adapt their shape and performance to the physiological demand. In a simplified way, resistance training leads to hypertrophy of fibers while endurance training stimulates oxidative metabolism in fibers. This plasticity however is progressively lost with aging, which is associated both with a reduction in muscle mass and strength at baseline (sarcopenia) and a reduced gain in performance and muscle remodeling in response to resistance exercise (Kumar et al., 2009). Muscle aging is also associated with reduced endurance exercise capacity that is attributed not only to decreased cardiovascular performance but also to an intrinsic decrease in the quality and quantity of muscle cell mitochondria (Lanza and Nair, 2010; Johnson et al., 2013). Hence, sarcopenic muscles are supposed to differ in the various biochemical pathways involved in exercise response (Ziaaldini et al., 2017). Yet, despite these limitations, regular exercise remains one of the most effective strategy to counteract sarcopenia and lower chronic inflammatory cytokines overload although optimization of the protocols and a better understanding of the mechanisms involved in the lower response of sarcopenic muscles resistance training are still required (Cruz-Jentoft et al., 2014; Denison et al., 2015; Ziaaldini et al., 2017; Monteiro-Junior et al., 2018).

Among the molecular mechanisms involved in the aging process, alterations in the metabolism of the Nicotinamide Adenine Dinucleotide $\left(\mathrm{NAD}^{+}\right)$has been shown to play a major role in most tissues, including heart and skeletal muscles (Mericskay, 2016; Yoshino et al., 2018).

NAD has the particularity of being both a coenzyme and a signaling molecule. As a coenzyme, NAD is recycled from oxidized $\mathrm{NAD}^{+}$to reduced NADH in the oxido-reduction reactions of the energy metabolism, without net consumption of the total NAD pool in these processes. As a signaling molecule, NAD is a co-substrate molecule hydrolyzed by several cellular enzymes, notably the sirtuins (SIRT) deacetylases, the polyADPribose polymerases (PARP) and the $\mathrm{CD} 38$ and $\mathrm{CD} 157$ ADPribose cyclases (Mericskay, 2016; Yoshino et al., 2018). These pathways act as sensors of energetic and redox state to regulate energy metabolism (SIRT1, SIRT3), oxidative stress response and cell survival (PARP1) and $\mathrm{Ca}^{2+}$ signaling (CD38) in all cell types. In aged mouse skeletal muscle, there is more than $50 \%$ decline in $\mathrm{NAD}^{+}$levels that alters SIRT1 activity and reduced the level of nuclear and mitochondrial encoded mitochondrial proteins (Gomes et al., 2013).

The sirtuins, PARP1 and CD38/CD157 proteins all cleave the $\mathrm{NAD}^{+}$into nicotinamide (NAM) and adenine diphosphate-ribose (ADPR) for their enzymatic activities.
Different salvage pathway exist that compensate for this cellular consumption of $\mathrm{NAD}^{+}$(Mericskay, 2016; Yoshino et al., 2018). A major pathway in muscle is initiated by the nicotinamide phosphoribosyl transferase (NAMPT). NAMPT uses the NAM derived from the activity of $\mathrm{NAD}^{+}$consuming enzymes but also nutritional NAM (vitamin B3), to generate nicotinamide mononucleotide (NMN) that is then fused to the ADP moiety of ATP by the nicotinamide adenilyl transferases (NMNAT, 1 to 3) to form the dinucleotide. Genetic depletion of the NAMPT enzyme in adult skeletal muscles leads to fiber degeneration and progressive loss of both muscle strength and treadmill endurance (Frederick et al., 2016). The contribution of the deamidated precursors, nicotinic acid and tryptophan to $\mathrm{NAD}^{+}$synthesis is minimal in skeletal muscles (Mori et al., 2014).

More recently, an alternative pathway for the synthesis of NMN was discovered based on the phosphorylation of the nucleoside form of the NAM base, the nicotinamide riboside (NR), by the NR kinases NRK1 and NRK2 (official symbol NMRK1 and NMRK2; Bieganowski and Brenner, 2004; Belenky et al., 2007). NMRK2 is expressed predominantly in skeletal muscles whereas NMRK1 seems to be ubiquitously expressed but more abundant in liver and kidneys (Ratajczak et al., 2016; Fletcher et al., 2017). Nmrk2-/- mice (12-14 weeks) showed no alteration in total NAD levels in skeletal muscles and no phenotype at baseline or after 6 weeks of endurance exercise protocol as regard muscle fiber cross-sectional area and muscle fiber type distribution (Fletcher et al., 2017).

Considering that NAD metabolism becomes crucial during aging, we explored in the present study the ability of middleage Nmrk2 $2^{-I-}$ mice (16 month) to perform endurance exercise training and the impact of this training on muscle fiber crosssectional area, muscle fiber type distribution and myokines expression in different muscles.

\section{MATERIALS AND METHODS}

\section{Animals and Endurance Training}

All experiments with animals conformed to the Directive 2010/63/EU of the European Parliament and were approved by the ethics committee Charles Darvin \#5 and authorized by the Ministry of Research in application of the French Rural Code, notably the articles R. 214-87 to R. 214-126 (agreement 00369.03: Role of the kinase NMRK2 in skeletal muscles). The animal experiments were performed in the UPMC university Center for Experimental Exploration, UMS 028, 105 Boulevard de l'Hôpital, Paris 75013, holding the agreement \# A751315.

Constitutive Nmrk2 knocked-out mice were obtained and genotyped in a C57BL/6NTac genetic background as previously 
described (Vaur et al., 2017). Homozygous Nmrk2-/- mice were viable and fertile. For this study, 16 sixteen-month old female mice were used $(n=10 \mathrm{C} 57 \mathrm{BL} / 6 \mathrm{~N}$ wild-type mice and $n=6 \mathrm{Nmrk2} 2^{-1-}$ mice). Half of the animals were then randomly assigned to sedentary or endurance training groups ( $n=5 \mathrm{C} 57 \mathrm{BL} / 6 \mathrm{~N}$ wild-type mice and $n=3 \mathrm{Nrmk} 2^{-/-}$mice for each condition). Mice were acclimated to the treadmill for 3 consecutive days, and first submitted to an exercise performance test involving a warm up phase at 5 to $15 \mathrm{~cm} / \mathrm{s}$ for $15 \mathrm{~min}$, followed by an acute exercise phase where the speed of the treadmill was progressively increased until reaching signs of exhaustion (more than 5 shocks within $60 \mathrm{~s}$, or more than 5 consecutive seconds on the shock grid with attempting to reengage on the treadmill). Endurance training protocol was carried out for 9 weeks and was composed of a warm up phase at 5 to $15 \mathrm{~cm} / \mathrm{s}$ for $15 \mathrm{~min}$ followed by 0 to $45 \mathrm{~min}$ at $15 \mathrm{~cm} / \mathrm{s}$ and after that $45 \mathrm{~min}$ in which speed was progressively increased from 15 to $30 \mathrm{~cm} / \mathrm{s}$. Protocol was strictly identical for every animal used in this study.

\section{RT-qPCR Analysis}

Total RNA were extracted from tissue samples using TRI Reagent ${ }^{\circledR}$ (Molecular Research Center) and a tissue homogenizer (Precellys ${ }^{\circledR}$, Bertin Instruments) following the manufacturer instructions. RNA concentrations were quantified by spectrophotometry using NanoDrop 2000 (Thermo Fisher Scientific). cDNAs were reversed transcripted from $2 \mu \mathrm{g}$ of RNA using the iScript $^{\mathrm{TM}}$ Reverse Transcription kit (Biorad). Quantitative PCR reactions were carried out on $\mathrm{CFX}^{\mathrm{TM}}$ Real-Time PCR Detection System (Biorad), in triplicates for each sample in a $7.5 \mu \mathrm{L}$ volume containing $3.75 \mu \mathrm{L}$ of SsoAdvanced ${ }^{\mathrm{TM}}$ Universal SYBR ${ }^{\circledR}$ Green Supermix, $0.5 \mu \mathrm{M}$ of each forward and reverse primers and $2.5 \mu \mathrm{L}$ of $1: 20$ diluted cDNA. Primers sequences used in this study are available on request. The expression of Hprt (Hypoxanthine-guanine phosphoribosyltransferase) gene or the mean of the expression of RplpO (Ribosomal Protein, Large, P0) and Ywhaz (Tyrosine 3-Monooxygenase/Tryptophan 5-Monooxygenase Activation Protein Zeta) genes were used as a reference for normalization in heart and gastrocnemius muscle, and plantaris and soleus muscles, respectively.

\section{NAD Extraction and Quantification}

Metabolites were extracted by homogenizing tissue samples in a buffered ethanol solution (75\% ethanol/25\% HEPES $10 \mathrm{mM}$ $\mathrm{pH} 7.1,10 \mu \mathrm{L} / \mathrm{mg}$ of tissue) with a tissue homogenizer (Precellys ${ }^{\circledR}$, Bertin Instruments). Extracts were heated at $80^{\circ} \mathrm{C}$ for $5 \mathrm{~min}$, chilled on ice and centrifuged for at $15000 \mathrm{~g}$ for $15 \mathrm{~min}$ at $4^{\circ} \mathrm{C}$. NAD levels were quantified using an MTT-formazan recycling assay. Samples extracts were diluted in water to a final volume of $25 \mu \mathrm{L}$. After adding 100 $\mu \mathrm{L}$ of reaction buffer $(600 \mathrm{mM}$ ethanol, $0.5 \mathrm{mM}$ 3-(4.5dimethylthiazol-2-yl)-2.5-diphenyltetrazolium bromide (MTT), $2 \mathrm{mM}$ phenazine ethosulfate (PES), $120 \mathrm{mM}$ Bicine (pH7.8), yeast alcohol dehydrogenase $0.05 \mathrm{mg} / \mathrm{mL}$ (SIGMA A3263), kinetics of the reaction was assessed by measuring OD at $550 \mathrm{~nm}$ every $30 \mathrm{~s}$ for 40 min using a TECAN Infinite F500 microplate reader.
Samples NAD concentrations were determined by comparing the slope of the reaction $(\mathrm{OD} / \mathrm{s})$ to a range of standard $\mathrm{NAD}^{+}$ concentrations.

\section{Immunofluorescent Staining}

Immediately after sacrifice, fast (plantaris) and slow-twitch (soleus) muscles where embed in Tissue-Tek (Sakura, USA) and frozen in liquid nitrogen-cooled isopentane at $-150^{\circ} \mathrm{C}$. They when then stored at $-80^{\circ} \mathrm{C}$ and sliced into $8 \mu \mathrm{m}$ cryosections with CM1860 Cryostat, (Leica). Immunohistochemical staining of Myosin Heavy Chain (MHC) isoforms was performed using mouse monoclonal antibodies BAD5 (MHC-I specific, alternate name $\beta$-MHC, IgG2b, 1:100 dilution), SC-71 (MHC-IIA specific, IgG1, 1:100 dilution) and BF-F3 (MHC-IIB specific, IgM, 1:100 dilution) respectively, obtained from Developmental Studies Hybridoma Bank (DSHB, University of Iowa). Laminin staining was also performed for labeling basement membranes using a rabbit polyclonal antibody (L9393, Sigma). Briefly, muscle sections were fixed with 4\% PFA for $5 \mathrm{~min}$, washed twice ( $5 \mathrm{~min}$ each) in PBS, permeabilized in $0.1 \%$ Triton for $10 \mathrm{~min}$, washed twice in PBS, saturated in 5\% IgG-free BSA for $45 \mathrm{~min}$, washed once in PBS, incubated with 1:100 mouse FAB for $20 \mathrm{~min}$, washed once in PBS. Primary antibodies were then incubated overnight at $4{ }^{\circ} \mathrm{C}$. After washing 3 times in $0.1 \%$ Tween 20 in PBS, secondary antibodies were used to selectively bind to each primary antibody: goat anti-mouse IgG2b conjugated with Alexa Fluor ${ }^{\circledR} 350$, goat anti-mouse IgG1 conjugated with Alexa Fluor ${ }^{\circledR}$ 555 , goat anti-mouse IgM conjugated with Alexa Fluor ${ }^{\circledR} 488$, and goat anti-rabbit conjugated with Alexa Fluor ${ }^{\circledR}$ 633, all diluted at $1: 400$ in 5\% IgG-free BSA. After 3 washes in $0.1 \%$ Tween 20 in PBS, muscle section were mounted using 70\% glycerol. Pictures were taken using and inverted fluorescence microscope (DMi8, Leica). Pure MHC-IIX fibers were not stained by these antibodies and appeared black. Fiber type distribution and MinFeret were assessed using ImageJ software.

\section{Statistical Analysis}

Animals were assigned to sedentary or endurance training groups by randomization. Shapiro-Wilk test were applied to test for normality of distribution before to use parametric test. To assess statistical significance between control and $N m r k 2^{-/-}$ mice at exhaustion test, $t$-tests for independent samples were performed. For RT-qPCR analysis, NAD levels and fiber type and MinFeret comparisons, two-way ANOVA for independent samples were performed, followed by post-hoc Tukey tests for multiple comparison when an interaction between the genotype and the endurance training factors was established. Global MinFeret distribution statistical differences were assessed using Chi-square test. Values are expressed as mean \pm SEM.

\section{RESULTS}

\section{Endurance Training and Muscle Performance in Nmrk2-/- Mice}

Considering the old age of the mice, 16 months at the beginning of the endurance training protocol, and the potential effect of Nmrk2 deficiency on exercise capacities, training protocol was 
adapted by starting at a very low speed $(15 \mathrm{~cm} / \mathrm{s}, 100 \mathrm{~m}$ run per session) that was progressively increased to reach $30 \mathrm{~cm} / \mathrm{s}$ ( $890 \mathrm{~m}$ per session) at the end of the protocol. All animals were able to follow the complete training (Figure 1A). To assess if there was any difference in term of exercise capacity and endurance between control and $N m r k 2^{-/-}$mice, animals were submitted to a treadmill exhaustion test 1 week after the beginning of the protocol. Nmrk2 ${ }^{-/-}$mice reached exhaustion after $24.0 \pm 2.3 \mathrm{~min}$ vs. $22.6 \pm 1.8 \mathrm{~min}$ for control mice (Figure 1B), at a speed of $39.0 \pm 2.3 \mathrm{~cm} / \mathrm{s} v s .36 .7 \pm 1.8 \mathrm{~cm} / \mathrm{s}$ for control mice (Figure 1C), with no statistical difference between the groups. After 9 weeks of training, there was no significant body weight change in trained control and Nmrk2 ${ }^{-/-}$mice (Figure 1D).

\section{Effects of Endurance Training on Cardiac Gene Expression and NAD Levels}

To gain insight on NAD metabolism modulation in heart by exercise in old mice, we first analyzed the gene expression profile of several main enzymes responsible for its biosynthesis and consumption. Nmrk2 gene expression was absent as expected in $N m r k 2^{-/}$mice, but not modified by training in control mice. Nmrk1 and Nampt expressions were not modulated by endurance training in control and in $N m r k 2^{-/-}$mice, neither was the expression of the sirtuins Sirt1 and Sirt3 (Figure 2A).
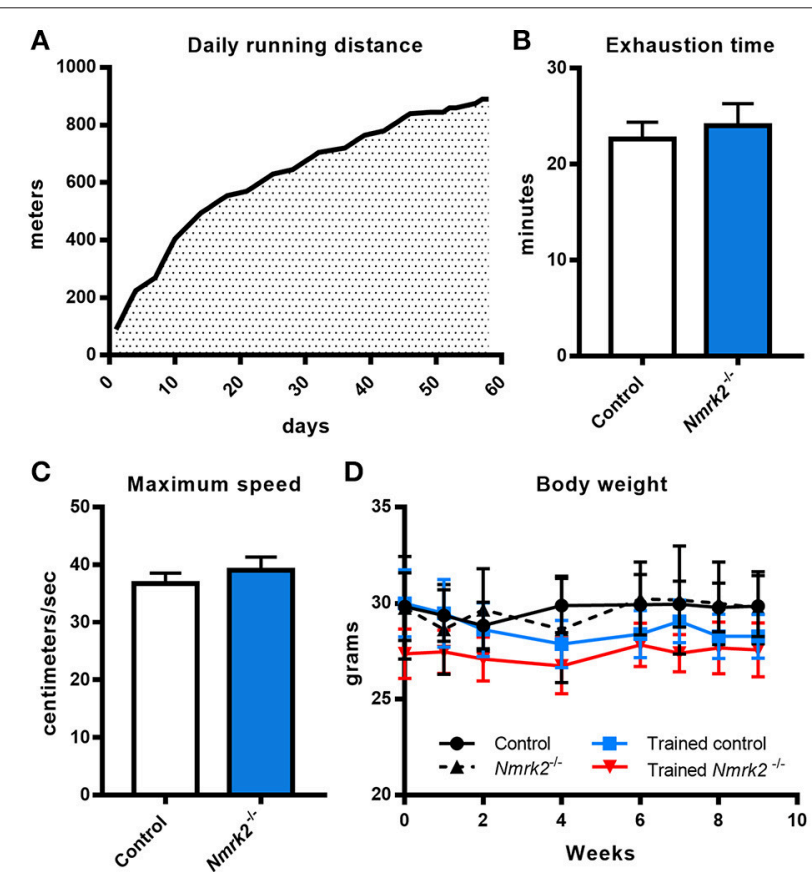

FIGURE 1 | Daily running distance during endurance training, endurance test and body weight evolution. (A) Evolution of the daily running distance imposed to control and $\mathrm{Nmrk2}^{-/-}$mice during the 9 weeks of endurance training. (B, C) Maximum speed (B) and time (C) reached at exhaustion during endurance test. (D) Evolution of body weight along the training protocol. $N=5$ in each sedentary and trained control group, and $N=3$ in each sedentary and trained Nmrk2 ${ }^{-/-}$group. Statistical analysis: (B,C), $t$-test for independant samples. (D) two-way ANOVA for independent samples.
In line with those observations, we did not observe a difference regarding NAD levels in cardiac muscle (Figure 2B). Pgc1a, known to be activated in the heart by exercise, was slightly increased without reaching statistical significance, when there was no induction in Nmrk2 $2^{-/-}$mice (Figure 2C). Cardiac stress marker Bnf was not clearly modulated by exercise or lack of NMRK2 (Figure 2D). The Myh7 gene encoding the slow $\beta$-MHC was increased in only one sedentary $N m r k 2^{-/-}$mouse that did not show any other major diffference in other tested genes in the heart and skeletal muscle compared to the rest of the group. Interestingly, Cpt $1 b$ and $A c a d L$ genes, involved in fatty acids catabolism, were significantly lowered by exercise in $\mathrm{Nmrk} 2^{-/-}$ mice, but not control mice ( $p<0.05$, Figure 2E). No clear effects could be identified on $P d k 2$ and $P d k 4$ expression, two isoforms of PDK, which negatively regulates glycolysis, neither on glucose transporters Glut1 and Glut4 (Figure 2F).

\section{Effects of Endurance Training on Skeletal Muscle Gene Expression and NAD Levels}

Nmrk2 being highly expressed in skeletal muscle, we also investigated NAD metabolism modulation enzymes in gastrocnemius muscle. No effect was observed regarding Nampt or Sirt1 and Sirt3 genes expression, but Nmrk1 was reduced in $N m r k 2^{-/-}$mice in comparison to controls (genotype effect, $p<0.01$, Figure 3A). In parallel with these observations, no major modulation of total NAD levels was observed (Figure 3C). We also studied the expression levels of myosin heavy-chain isoforms, known to be modulated by endurance training in young mice. Interestingly, Myh7 gene coding for the slow MHC-I isoform was strongly induced by exercise in $\mathrm{Nrmk} 2^{-/-}$ mice $(p<0.01$, Figure 3B), to a higher level compared to trained control mice $(p<0.05)$ when no statistically significant effect of exercise was found in control mice. No modulation of other $\mathrm{MHC}$ isoforms gene expression was observed ( $M y h 2$ for MHC-II1, Myh1 for MHC-IIX or Myh4 for MHC-IIB).

Considering the emerging role of skeletal muscle in autocrine and paracrine signaling, we also analyzed the expression of several myokines in slow (soleus) and fast-twitch (plantaris) muscles. In soleus muscle, an effect of training and genotype was observed for $I L-15$ expression, a myokine that mediates muscle-fat crosstalk, seems to be higher in trained Nmrk2-/mice but no interaction could be identified (Figure 3D). Sparc, a myokine linked to muscle atrophy, and Fndc5, a precursor of irisin correlated to browning of adipose tissue, were not modified by exercise, even in control animals. No modifications of the expression of these 3 genes was observed in plantaris muscle although the global profile was strikingly similar to the one observed in the soleus muscle (Figure 3E).

\section{Modulation of MHC Isoforms Distribution and Minferet Diameter in Fast and Mixed Muscles}

To go further in the understanding of the effects of NMRK2 deficiency on skeletal muscle adaptation to exercise, we analyzed the distribution of MHC isoforms and minimal Feret diameter of fibers in fast plantaris muscle and the mixed more oxidative 

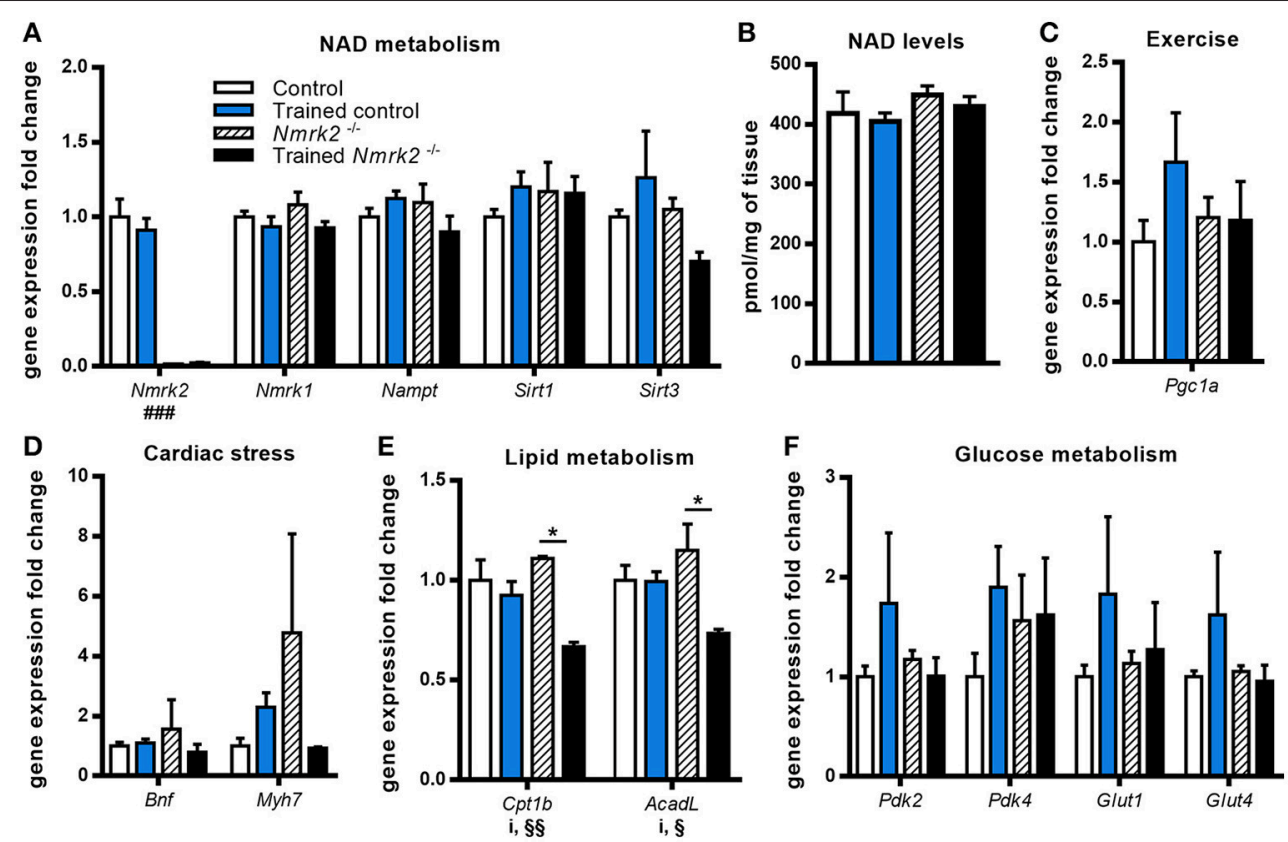

FIGURE 2 | Gene expression analysis and global NAD levels in left ventricle. (A) Relative quantification by RT-qPCR of Nmrk1, Nmrk2, Nampt, Sirt1, and Sirt3 mRNA levels in left ventricule. (B) Myocardial NAD levels. (C-F) Relative quantification by RT-qPCR of Pgc1 $\alpha$ (C), Bnf and Myh7 (D), Cpt1b and AcadL (E), Pdk2, Pdk4, Glut1, and Glut4 (F) mRNA levels in left ventricule. $n=5$ in control and trained control groups, $n=3$ in Nmrk2 ${ }^{-/-}$and trained Nmrk2 ${ }^{-/-}$groups. Results are expressed as mean values \pm SEM. Statistical analysis: two-way ANOVA for independent samples. ${ }^{i} p<0.05$ for interaction, $\# \# \# p<0.001$ for genotype effect, $\S_{p}<0.05$ and $\S \S<0.01$ for endurance training effect. ${ }^{*} p<0.05$ from Tukey's multiple comparisons test when applicable.
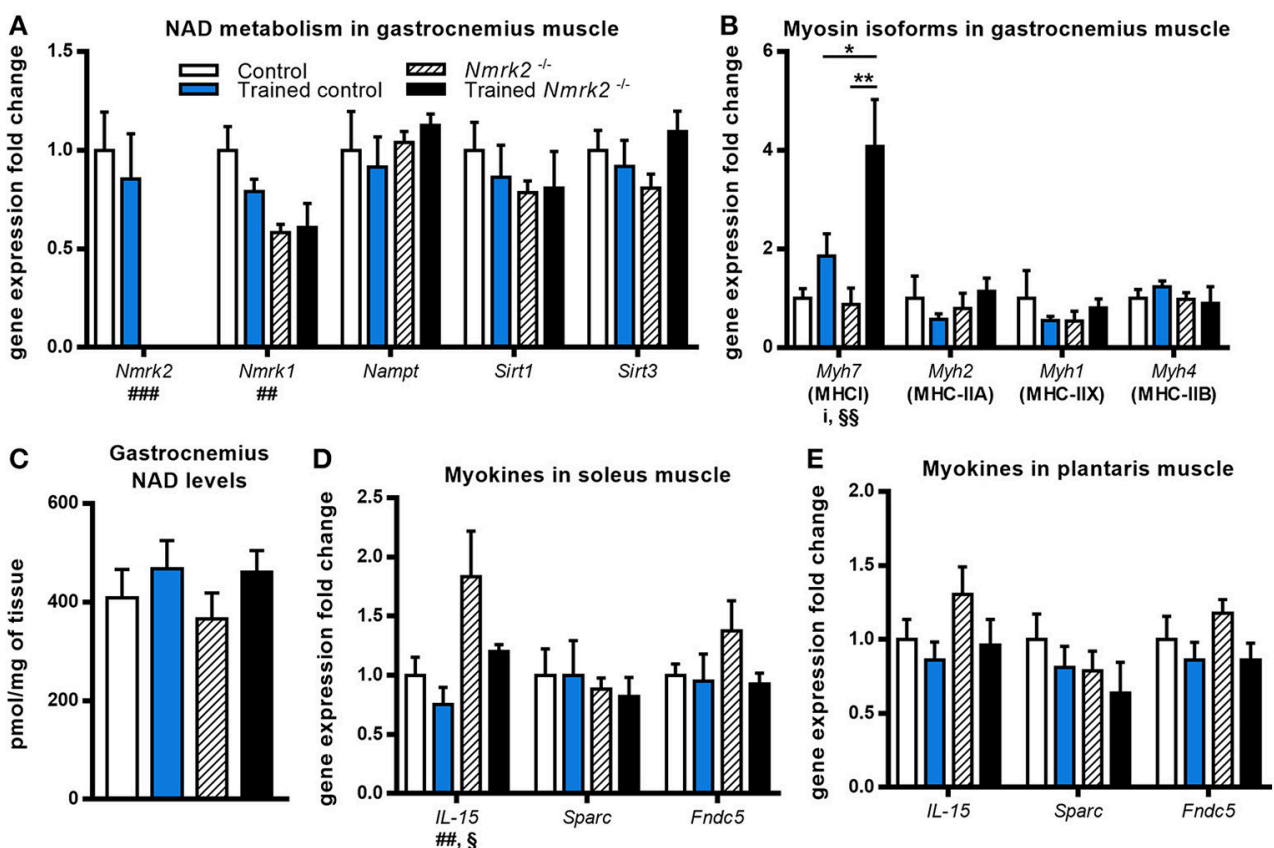

FIGURE 3 | Gene expression analysis and global NAD levels in skeletal muscle. (A,B) Relative quantification by RT-qPCR of Nmrk1, Nmrk2, Nampt, Sirt1, Sirt3 (A), and Myh7, Myh2, Myh1, and Myh4 myosin isoforms (B) mRNA levels in gastrocnemius muscle. (C) Gastrocnemius NAD levels. (D,E) Relative quantification by RT-qPCR of IL-15, Sparc, and Fndc5 myokines mRNA levels in soleus (D) and plantaris muscle (E) respectively. Results are expressed as mean values \pm SEM Statistical analysis: two-way ANOVA for independent samples. ${ }^{i} p<0.05$ for interaction, $\# \# p<0.01$ and $\# \# \# p<0.001$ for genotype effect, ${ }^{\S} p<0.05$ and $\S_{\S} p<0.01$ for endurance training effect. ${ }^{*} p<0.05$ and ${ }^{* \star} p<0.01$ from Tukey's multiple comparisons test when applicable. 
soleus muscles by immuno-histochemical staining. In plantaris muscle, no fiber type switch with exercise could be detected (Figures 4A-E). As expected, no type I fibers were detected in this fast muscle. MinFeret diameter trend to be reduced with exercise in control mice, reaching statistical significance for MHC-IIX fibers, when no changes were observed in Nmrk2-/mice (Figure 4F). Analysis of MinFeret diameter distributions of all muscle fibers showed a statistically significant shift of distribution toward thinner muscle fibers with training in control mice ( $p<0.0001$, Figure 4G), when no effect of training was observed with $\mathrm{Nmrk}^{-/-}$mice (Figure $4 \mathbf{H}$ ).

In the soleus muscle, there was no difference in term of fiber type repartition between control and Nmrk2 $2^{-/}$mice, and no fiber type switching was observed with exercise in any group (Figures 5A-E). Note that no Type IIb fast twitch glycolytic fibers were detected in this muscle as expected. No difference either was found regarding all muscle fibers MinFeret diameter distributions, in term of genotype or exercise effect (Figures F-H).

\section{DISCUSSION}

The stimulation of $\mathrm{NAD}^{+}$synthesis by the NR vitamin $\mathrm{B} 3$, the substrate of NMRK enzymes, has been shown to be beneficial for muscle physiology and mitochondrial function in the context of aging or genetic myopathies in many studies (Mouchiroud et al., 2013; Cerutti et al., 2014; Khan et al., 2014; Frederick et al., 2016; Ryu et al., 2016; Zhang et al., 2016). One exception being a short report that NR mildly decreases exercise performance in rats in a swimming exhaustion test although no muscle phenotyping or molecular analyses were performed to understand this lack of response (Kourtzidis et al., 2016).

We have shown recently that Nmrk2 levels are strongly induced in the context dilated cardiomyopathy triggered by heart-specific deletion of the SRF transcription factor and that NR-supplemented diet or voluntary wheel running delays the onset of heart failure in this model (Deloux et al., 2017; Diguet et al., 2018). NMRK2, a striated muscle specific kinase has recently been shown to be required in muscle cells, in redundancy with the more ubiquitous NMRK1, to respond to the boosting effect of NR on NAD level (Fletcher et al., 2017). Here, we showed that aged NMRK2 deficient mice present an altered response to exercise training. In the heart, $C p t 1 b$ and $A c a d L$ genes, involved in $\beta$-oxidation, were reduced with exercise in Nmrk2 $2^{-/-}$mice. In gastrocnemius muscle, Nmrk1 levels were reduced in NMRK2 deficient mice, and slow MHC isoform Myh7 was more strongly induced by exercise in $N m r k 2^{-/-}$mice than in controls. Endurance training reduced the diameter of fibers in control mice but not in $N m r k 2^{-/-}$mice.

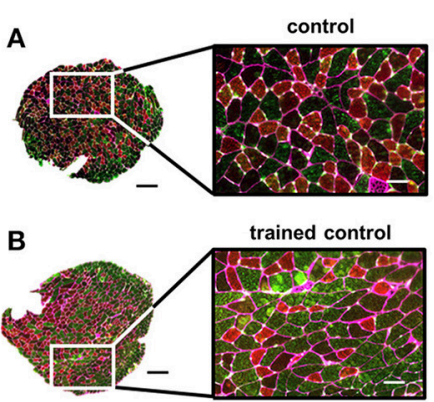

C

Nmrk2 $\%$
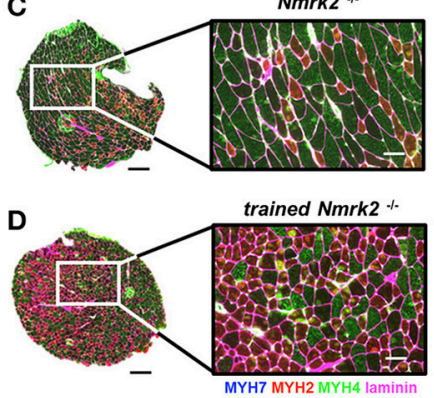

E Fiber type repartition in plantaris muscle

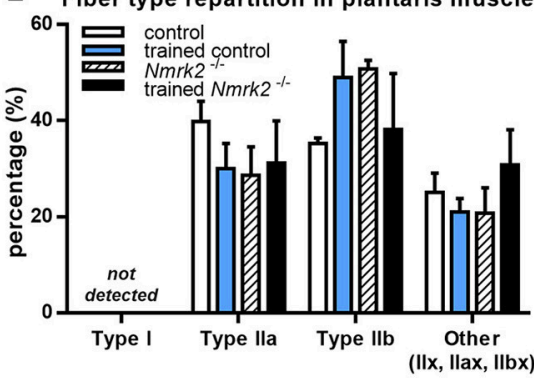

$\mathbf{F}$

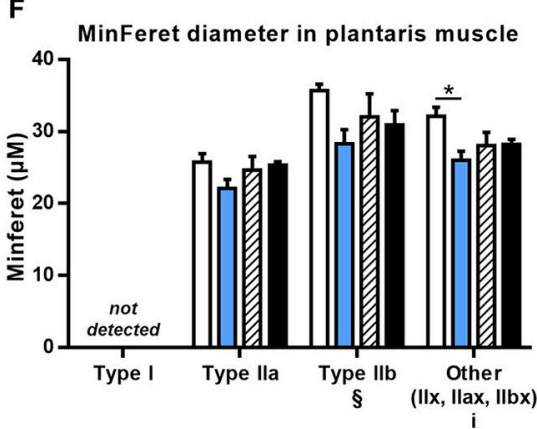

G

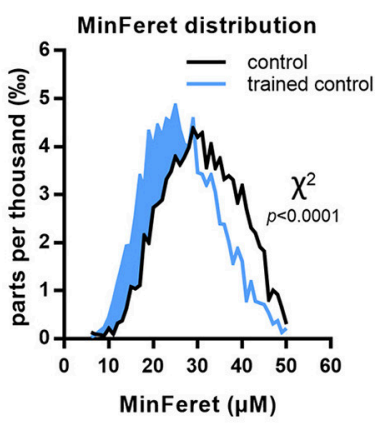

H

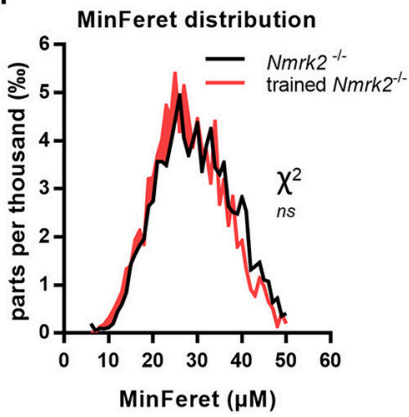

FIGURE 4 | MHC isoforms distribution and MinFeret diameter in plantaris muscle. (A-D) Myosin isoforms and laminin immunofluorescence histochemistry (MHC-I (MYH7) in blue, MHC-IIA (MYH2) in red, MHC-IIB (MYH4) in green, laminin in magenta) on 8- $\mu \mathrm{m}$ transversal cryosections of plantaris muscle from 18 weeks old mice, WT or Nmrk2 $2^{-1-}$, sedentary or trained. Scale bars $=200 \mu \mathrm{m}$ for whole muscle images, $60 \mu \mathrm{M}$ for magnified images. (E) Fiber type proportions calculated from whole muscle sections. Fibers negative or mixed for Type I, IIA, or IIB MHC isoforms where assigned to a separated category, in comparaison to pure MHC isoforms positive fibers. (F) Fibers MinFeret diameter $(\mu \mathrm{M})$. (G,H) Global MinFeret distributions whithin control and trained control mice (G), and $\mathrm{Nmrk2}^{-/-}$and trained $\mathrm{Nmrk2}^{-/-}$mice (H). $n=5$ in control and trained control groups, $n=3$ in Nmrk2 ${ }^{-/-}$and trained Nmrk2 ${ }^{-/-}$groups. Results are expressed as mean values \pm SEM. Statistical analysis (E, F), two-way ANOVA for independent samples. ${ }^{i} p<0.05$ for interaction, ${ }^{\S} p<0.05$ for endurance training effect. ${ }^{*} p<0.05$ from Tukey's multiple comparisons test when applicable. (G,H), Chi-square test for MinFeret distribution comparisons. 

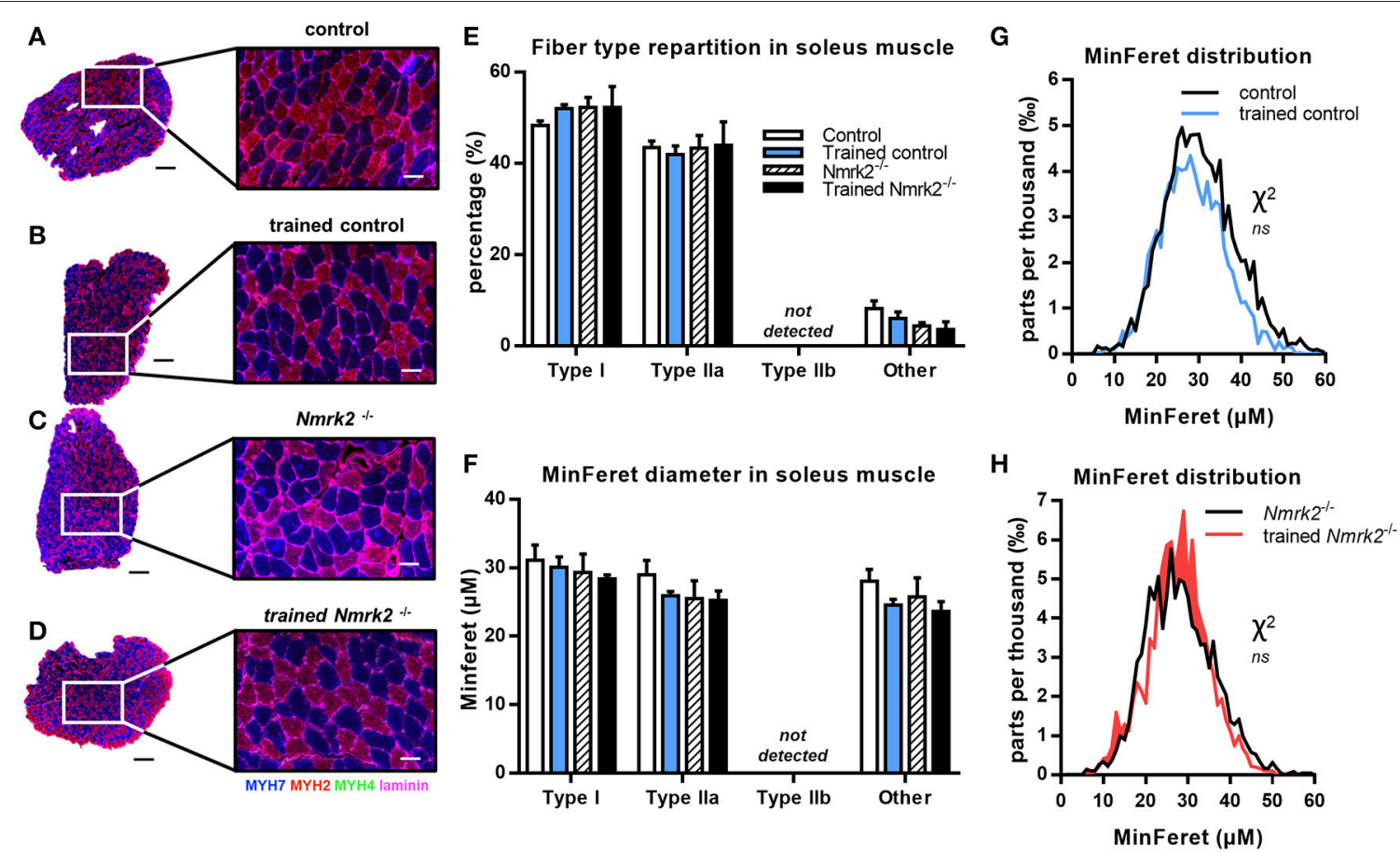

FIGURE 5 | MHC isoforms distribution and MinFeret diameter in soleus muscle. (A-D) Myosin isoforms and laminin immunofluorescence histochemistry [MHC-I (MYH7) in blue, MHC-IIA (MYH2) in red, MHC-IIB (MYH4) in green, laminin in magenta] on 8- $4 \mathrm{~m}$ transversal cryosections of soleus muscle from 18 weeks old mice, WT or $\mathrm{Nmrk}^{-/-}$, sedentary or trained. Scale bars $=200 \mu \mathrm{m}$ for whole muscle images, $60 \mu \mathrm{M}$ for magnified images. (E) Fiber type proportions calculated from whole muscle sections. Fibers negative or mixed for Type I, IIA, or IIB MHC isoforms where assigned to a separated category, in comparaison to pure MHC isoforms positive fibers. (F) Fibers MinFeret diameter $(\mu \mathrm{M})$. (G,H) Global MinFeret distributions whithin control and trained control mice (G), and $\mathrm{Nmrk}^{-/-}$and trained $\mathrm{Nmrk2}{ }^{-/-}$mice (H). $n=5$ in control and $n=4$ in trained control groups, $n=3$ in Nmrk2 $-/-$ and trained Nmrk2 ${ }^{-/-}$groups. Results are expressed as mean values \pm SEM. Statistical analysis (E,F), two-way ANOVA for independent samples. (G,H), Chi-square test for MinFeret distribution comparisons.

In cardiac and skeletal muscle, NAD levels has been described to be highly regulated by NAMPT, that acts as a rate limiting enzyme recycling NAM into $\mathrm{NMN}$ to form $\mathrm{NAD}^{+}$(Mori et al., 2014). NMRK1 and NMRK2 enzymes were identified more recently as alternative enzyme using NR as an alternative precursor to salvage $\mathrm{NAD}^{+}$levels (Bieganowski and Brenner, 2004). Here we show that global steady tissue levels of NAD are not altered in the heart and skeletal muscle despite the absence of NMRK2. This result is in agreement with a recently published study in young Nmrk2-/- mice that also reported there was no alteration of global NAD levels in quadriceps muscle in the KO mice in comparison to controls, with concentrations around $400 \mathrm{pmol} / \mathrm{mg}$ of tissue, which are identical to our findings in 18 month-old mice (Fletcher et al., 2017). In contrast, however, the authors did not observe a difference in term of Nmrk1 expression in young Nmrk2 $2^{-/}$mice, when we found a clear reduction at 18-month of age. However Nampt expression is maintained at this age in Nmrk2 $2^{-/-}$mice, which is apparently sufficient to sustain global levels of NAD. Interestingly, while NAD levels were strongly reduced in the skeletal muscle-specific Nampt $t^{-/-}$ mice that were intolerant to exercise, NR had a potent rescuing effect on exercise intolerance, fiber size and ATP production despite having no impact on total NAD tissue levels and very minor effect on mitochondrial NAD pool (Frederick et al., 2016).
These finding show that the NMN produced from NR by NMRK enzymes is not equivalent to the NMN produced by NAMPT to synthesize NAD. So, while it is clear now that NMRK2 is dispensable for the maintenance of global tissue NAD levels, it suggests that NMRK2 is involved in the synthesis of a subfraction of the NAD pool that is efficiently used by the muscle cells.

Skeletal muscle adaptations to exercise varies depending on the type of exercise. To focus on endurance exercise, different studies have clearly demonstrated in human that endurance training induces a switch from glycolytic to a more oxidative phenotype (Andersen and Henriksson, 1977). In young rats, 10 weeks running program triggers a similar shift toward slower oxidative isoforms of myosin in plantaris muscle, when no shift was reported in soleus muscle (Fitzsimons et al., 1990). Here, we show that in aged mice, treadmill running exercise increases the expression of $M y h 7$ gene, which encodes a slow contractile isoform of myosin heavy chain (MHCI) that uses less ATP per unit of work because of its lower myosin ATPase activity, relative to fast myosin isoforms, and is thus more metabolically efficient in the context of endurance training. Interestingly, the increase in $M y h 7$ expression was significantly stronger in $N m r k 2^{-/-}$ mice compared to controls, suggesting that NMRK2 signaling is normally blunting the expression of slow myosin in muscle or reciprocally that the lack of NMRK2 reults in a higher need 
for the muscle to reduce the energy required for contraction. We also found that endurance exercise led to a reduction of the mean diameter of muscle fibers in the plantaris but not the soleus of control mice whereas this phenomenon did not occur in $N m r k 2^{-/-}$mice. It is important to notice that Nmrk2 expression is 2 times higher in plantaris muscle compared to soleus (Fletcher et al., 2017), which may explain while it function is more easily put in evidence in this muscle.

Considering the emerging role of skeletal muscle in autocrine and paracrine signaling, we also analyzed the expression of several myokines known to be activated by exercise. Among them, IL-15 is a cytokine secreted by skeletal muscle that promotes endurance adaptations via a stimulation of the oxidative energy metabolism and the Sirt1/PGC1a axis in young mice (Quinn et al., 2013). IL-15 levels are increased by exercise in humans (Rinnov et al., 2014). On the other skeletal muscle aging and sarcopenia are associated with low levels of IL-15 (Yalcin et al., 2018). Here, we show that exercise reduced IL-15 mRNA level in aged control and Nmrk2 $2^{-/-}$mice. On the other hand, we showed that the $I L-15$ gene expression is upregulated at baseline in the soleus of Nmrk2 $2^{-/-}$mice. Since we observed a trend to have thinner muscle fibers in plantaris but not soleus muscle of Nmrk2 $2^{-/-}$compared to controls, it suggests that the local increase in IL-15 production in the soleus may contribute to preserve muscle mass. The link between NMRK2-dependent NAD metabolism and IL-15 signaling in oxidative metabolism will deserve to be further explored in the future. In the same line,

\section{REFERENCES}

Andersen, P., and Henriksson, J. (1977). Training induced changes in the subgroups of human type II skeletal muscle fibres. Acta Physiol. Scand. 99, 123-125. doi: 10.1111/j.1748-1716.1977.tb10361.x

Belenky, P., Racette, F. G., Bogan, K. L., McClure, J. M., Smith, J. S., and Brenner, C. (2007). Nicotinamide riboside promotes Sir2 silencing and extends lifespan via Nrk and Urh1/Pnp1/Meul pathways to NAD+. Cell 129, 473-484. doi: 10.1016/j.cell.2007.03.024

Bieganowski, P., and Brenner, C. (2004). Discoveries of nicotinamide riboside as a nutrient and conserved NRK genes establish a Preiss-Handler independent route to NAD+ in fungi and humans. Cell 117, 495-502. doi: 10.1016/S0092-8674(04)00416-7

Cerutti, R., Pirinen, E., Lamperti, C., Marchet, S., Sauve, A. A., Li, W., et al. (2014). NAD(+)-dependent activation of Sirtl corrects the phenotype in a mouse model of mitochondrial disease. Cell Metab. 19, 1042-1049. doi: 10.1016/j.cmet.2014.04.001

Cruz-Jentoft, A. J., Landi, F., Schneider, S. M., Zúñiga, C., Arai, H., Boirie, Y., et al. (2014). Prevalence of and interventions for sarcopenia in ageing adults: a systematic review. report of the international sarcopenia initiative (EWGSOP and IWGS). Age Ageing 43, 748-759. doi: 10.1093/ageing/afu115

Deloux, R., Vitiello, D., Mougenot, N., Noirez, P., Li, Z., Mericskay, M., et al. (2017). Voluntary exercise improves cardiac function and prevents cardiac remodeling in a mouse model of dilated cardiomyopathy. Front. Physiol. 8:899. doi: 10.3389/fphys.2017.00899

Denison, H. J., Cooper, C., Sayer, A. A., and Robinson, S. M. (2015). Prevention and optimal management of sarcopenia: a review of combined exercise and nutrition interventions to improve muscle outcomes in older people. Clin. Int. Aging 10, 859-869. doi: 10.2147/CIA.S55842

Diguet, N., Trammell, S. A. J., Tannous, C., Deloux, R., Piquereau, J., Mougenot, N., et al. (2018). nicotinamide riboside preserves cardiac function our observation that genes involved in fatty acids $\beta$-oxidation (FAO) are abnormally repressed in the heart of $\mathrm{Nmrk2}^{-/-}$mice upon training while it is well known that FAO is increased during exercise to respond to the higher energy demand (Jeppesen and Kiens, 2012) suggest a maladaptative metabolic response in absence of NMRK2.

In conclusion, we demonstrated for the first time a phenotype in old $N m r k 2^{-/-}$mice in response to endurance training suggesting that NMRK2 deficient mice are prone to develop muscle dysfunction with aging.

\section{AUTHOR CONTRIBUTIONS}

$\mathrm{MM}$ and ZL conceived the study. RD and CT performed the laboratory experiments. AF performed and supervised RD for the endurance training protocol. $\mathrm{RD}$ and $\mathrm{MM}$ wrote the draft of the manuscript. AF corrected the draft of the manuscript. All authors have read and approved the final manuscript.

\section{ACKNOWLEDGMENTS}

This work was supported by the Association Française contre les Myopathies (AFM grant \#16282, ZL, MM). RD and CT were supported by a $\mathrm{PhD}$ fellowship of French Ministère de la Recherche et de l'Enseignement Supérieur. We thank the UPMC university Center for Experimental Exploration, UMS 028 for taking care of the mice. in a mouse model of dilated cardiomyopathy. Circulation 137, 2256-2273. doi: 10.1161/CIRCULATIONAHA.116.026099

Fitzsimons, D. P., Diffee, G. M., Herrick, R. E, and Baldwin, K. M. (1990). Effects of endurance exercise on isomyosin patterns in fast- and slow-twitch skeletal muscles. J. App. Physiol. 68, 1950-1955.

Fletcher, R. S., Ratajczak, J., Doig, C. L., Oakey, L. A., Callingham, R., and Da Silva, Xavier, G., et al. (2017). Nicotinamide riboside kinases display redundancy in mediating nicotinamide mononucleotide and nicotinamide riboside metabolism in skeletal muscle cells. Mol. Metab. 6, 819-832. doi: 10.1016/j.molmet.2017.05.011

Frederick, D. W., Loro, E., Liu, L., Davila, A. Jr., Chellappa, K., Silverman, I., et al. (2016). Loss of NAD homeostasis leads to progressive and reversible degeneration of skeletal muscle. Cell Metab. 24, 269-282. doi: 10.1016/j.cmet.2016.07.005

Gomes, A. P., Price, N. L., Ling, A. J., Moslehi, J. J., Montgomery, M. K., Rajman, L., White, J. P., et al. (2013). Declining NAD(+) induces a pseudohypoxic state disrupting nuclear-mitochondrial communication during aging. Cell 155, 1624-1638. doi: 10.1016/j.cell.2013.11.037

Jeppesen, J., and Kiens, B. (2012). Regulation and limitations to fatty acid oxidation during exercise. J. Physiol. 590, 1059-1068. doi: 10.1113/jphysiol.2011.225011

Johnson, M. L., Robinson, M. M., and Nair, K. S. (2013). Skeletal muscle aging and the mitochondria. Trends Endocrinol. Metabol. 24, 247-256. doi: 10.1016/j.tem.2012.12.003

Khan, N. A., Auranen, M., Paetau, I., Pirinen, E., Euro, L., Forsstrom, S., et al. (2014). Effective treatment of mitochondrial myopathy by nicotinamide riboside, a vitamin B3. EMBO Mol. Med. 6, 721-731. doi: 10.1002/emmm.201403943

Kourtzidis, I. A., Stoupas, A. T., Gioris, I. S., Veskoukis, A. S., Margaritelis, N. V., Taitzoglou, M., et al. (2016). The NAD $(+)$ precursor nicotinamide riboside decreases exercise performance in rats. J. Int. Soc. Sports Nutr. 13, 32. doi: 10.1186/s12970-016-0143-x 
Kumar, V., Selby, A., Rankin, D., Patel, R., Atherton, P., Hildebrandt, W., et al. (2009). Age-related differences in the dose-response relationship of muscle protein synthesis to resistance exercise in young and old men. J. Physiol. 587, 211-217. doi: 10.1113/jphysiol.2008.164483

Lanza, I. R., and Nair, K. S. (2010). Mitochondrial Function as a Determinant of Life Span. Pflugers Archiv. 459, 277-289. doi: 10.1007/s00424-009-0724-5

Mericskay, M. (2016). Nicotinamide adenine dinucleotide homeostasis and signalling in heart disease: Pathophysiological implications and therapeutic potential. Arch. Cardiovasc. Dis. 109, 207-215. doi: 10.1016/j.acvd.2015. 10.004

Monteiro-Junior, R. S, de Tarso Maciel-Pinheiro, P., da Matta Mello Portugal, E., da Silva Figueiredo, L. F., Terra, R., Carneiro, L. S. F, et al. (2018). Effect of exercise on inflammatory profile of older persons: systematic review and meta-analyses. J. Phys. Act. Health 15, 64-71. doi: 10.1123/jpah.2016-0735

Mori, V., Amici, A., Mazzola, F., Di Stefano, M., Conforti, L., Magni, G., et al. (2014). Metabolic profiling of alternative NAD biosynthetic routes in mouse tissues. PloS ONE 9:e113939. doi: 10.1371/journal.pone.0113939

Mouchiroud, L., Houtkooper, R. H., Moullan, N., Katsyuba, E., Ryu, D., Canto, C., et al. (2013). The NAD(+)/sirtuin pathway modulates longevity through activation of mitochondrial UPR and FOXO signaling. Cell 154, 430-441. doi: 10.1016/j.cell.2013.06.016

Quinn, L. S., Anderson, B. G., Conner, J. D., and Wolden-Hanson, T. (2013). IL-15 Overexpression promotes endurance, oxidative energy metabolism, and muscle PPAR $\delta$, SIRT1, PGC- $1 \alpha$, and PGC- $1 \beta$ expression in male mice. Endocrinology 154, 232-245. doi: 10.1210/en.2012-1773

Ratajczak, J., Joffraud, M., Trammell, S. A. J., Ras, R., Canela, N., Boutant, M., et al. (2016). NRK1 controls nicotinamide mononucleotide and nicotinamide riboside metabolism in mammalian cells. Nat. Commun. 7:13103. doi: $10.1038 /$ ncomms 13103

Rinnov, A., Yfanti, C., Nielsen, S., Akerström, T. C., Peijs, L., Zankari, A., et al. (2014). Endurance training enhances skeletal muscle interleukin-15 in human male subjects. Endocrine 45, 271-278. doi: 10.1007/s12020-013-9969-z
Ryu, D., Zhang, H., Ropelle, E. R., Sorrentino, V., Mázala, D. A. G., Mouchiroud, L., et al. (2016). NAD+ repletion improves muscle function in muscular dystrophy and counters global PARylation. Sci. Trans. Med. 8:361ra139. doi: 10.1126/scitranslmed.aaf5504

Vaur, P., Brugg, B., Mericskay, M., Li, Z., Schmidt, M. S., Vivien, D., et al. (2017). Nicotinamide riboside, a form of vitamin B3, protects against excitotoxicity-induced axonal degeneration. FASEB J. 31, 5440-5452. doi: 10.1096/fj.201700221RR

Yalcin, A., Silay, K., Balik, A. R., Avcioglu, G., and Aydin, A. S. (2018). The relationship between plasma interleukin-15 levels and sarcopenia in outpatient older people. Aging Clin. Exp. Res. 30, 783-790. doi: 10.1007/s40520-017-0848-y

Yoshino, J., Baur, J. A., and Imai, S. I. (2018). NAD+ intermediates: the biology and therapeutic potential of NMN and NR. Cell Metabol. 27, 513-528. doi: 10.1016/j.cmet.2017.11.002

Zhang, H., Ryu, D., Wu, Y., Gariani, K., Wang, X., Luan, P., et al. (2016). NAD(+) repletion improves mitochondrial and stem cell function and enhances life span in mice. Science 352, 1436-1443. doi: 10.1126/science.aaf2693

Ziaaldini, M. M., Marzetti, E., Picca, A., and Murlasits, Z. (2017). biochemical pathways of sarcopenia and their modulation by physical exercise: a narrative review. Front. Med. 4:167. doi: 10.3389/fmed.2017.00167

Conflict of Interest Statement: The authors declare that the research was conducted in the absence of any commercial or financial relationships that could be construed as a potential conflict of interest.

Copyright (c) 2018 Deloux, Tannous, Ferry, Li and Mericskay. This is an open-access article distributed under the terms of the Creative Commons Attribution License (CC $B Y)$. The use, distribution or reproduction in other forums is permitted, provided the original author(s) and the copyright owner(s) are credited and that the original publication in this journal is cited, in accordance with accepted academic practice. No use, distribution or reproduction is permitted which does not comply with these terms. 
OPEN ACCESS

Edited by:

Dario Coletti,

Università degli Studi di Roma La

Sapienza, Italy

Reviewed by:

Libera Berghella,

California Institute of Technology,

United States

Ara Parlakian,

Université Pierre et Marie Curie,

France

*Correspondence:

Miguel L. Batista Jr.

migueljir@me.com

Specialty section:

This article was submitted to

Striated Muscle Physiology,

a section of the journal

Frontiers in Physiology

Received: 20 June 2018

Accepted: 29 August 2018

Published: 24 September 2018

Citation:

Leal LG, Lopes MA and Batista ML Jr

(2018) Physical Exercise-Induced

Myokines and Muscle-Adipose Tissue

Crosstalk: A Review of Current

Knowledge and the Implications for Health and Metabolic Diseases.

Front. Physiol. 9:1307.

doi: 10.3389/fphys.2018.01307

\section{Physical Exercise-Induced Myokines and Muscle-Adipose Tissue Crosstalk: A Review of Current Knowledge and the Implications for Health and Metabolic Diseases}

\author{
Luana G. Leal ${ }^{1,2}$, Magno A. Lopes ${ }^{1}$ and Miguel L. Batista Jr. ${ }^{1,2 *}$ \\ ${ }^{1}$ Integrated Group of Biotechnology, Laboratory of Adipose Tissue Biology, University of Mogi das Cruzes, São Paulo, Brazil, \\ ${ }^{2}$ Technological Research Group, University of Mogi das Cruzes, São Paulo, Brazil
}

Physical exercise has beneficial effects on metabolic diseases, and a combined therapeutic regimen of regular exercise and pharmaceutical treatment is often recommended for their clinical management. However, the mechanisms by which exercise produces these beneficial effects are not fully understood. Myokines, a group of skeletal muscle (SkM) derived peptides may play an important part in this process. Myokines are produced, expressed and released by muscle fibers under contraction and exert both local and pleiotropic effects. Myokines such as IL-6, IL-10, and IL-1ra released during physical exercise mediate its health benefits. Just as exercise seems to promote the myokine response, physical inactivity seems to impair it, and could be a mechanism to explain the association between sedentary behavior and many chronic diseases. Myokines help configure the immune-metabolic factor interface and the health promoting effects of physical exercise through the release of humoral factors capable of interacting with other tissues, mainly adipose tissue (AT). AT itself secretes proinflammatory cytokines (adipokines) as a result of physical inactivity and it is well recognized that AT inflammation can lead to the development of metabolic diseases, such as type 2 diabetes mellitus (T2DM) and atherosclerosis. On the other hand, the browning phenotype of AT has been suggested to be one of the mechanisms through which physical exercise improves body composition in overweight/obese individuals. Although, many cytokines are involved in the crosstalk between SkM and AT, in respect of these effects, it is IL-6, IL-15, irisin, and myostatin which seem to have the decisive role in this "conversation" between AT and SkM. This review article proposes to bring together the latest "state of the art" knowledge regarding Myokines and muscle-adipose tissue crosstalk. Furthermore, it is intended to particularly focus on the immune-metabolic changes from AT directly mediated by myokines.

Keywords: IL-6, irisin, browning, inflammation, immunometabolism, exercise-factor 


\section{INTRODUCTION}

\section{General Characterization}

Physical inactivity is a global health problem, and recent data indicate that approximately one-third of the world's adult population is physically inactive. This means that these individuals do not perform the minimum $150 \mathrm{~min}$ a week of moderate to vigorous aerobic physical activity recommended by the World Health Organization (Ruiz-Casado et al., 2017). Physical inactivity is directly related to higher risk rates for major non-communicable diseases (NCDs). NCDs are a set of diseases of long duration and generally slow progression. The four main types of noncommunicable diseases are cardiovascular diseases (e.g., heart attacks and stroke), cancer, chronic respiratory diseases (e.g., chronic obstructive pulmonary disease and asthma) and type 2 diabetes mellitus (T2DM; WHO, 2014). In 2008, NCDs, such as coronary heart disease, T2DM and colon and breast cancers were responsible for about five million deaths or about 9\% of total global premature mortality (Figure 1; Lee et al., 2012; Lobelo et al., 2014).

The benefits of physical exercise as a protective factor against NCDs have been documented since 450 BC, with Hippocrates, the "father of Western medicine," stating that "Walking is man's best medicine" and "If there is a deficiency in food and exercise the body will fall sick" (Febbraio, 2017). Several biological mechanisms may be responsible for the reduction of risk factors for chronic diseases and premature death associated with physical exercise (Warburton et al., 2006) and there is currently a consensus in the literature that regular physical exercise is directly related to: (1) improvements in body composition, including reduced abdominal adiposity and body weight (Thomas et al., 2013; Smith, 2018); (2) improved lipoprotein protein profiles, through reduced triglyceride and low-density lipoproteins [LDL] levels, and increased high density lipoprotein [HDL] levels (Warburton et al., 2006; Mitsui et al., 2012); (3) greater efficiency in glucose homeostasis and insulin sensitivity (Tan et al., 2018); (4) reduced blood pressure (Sharman et al., 2015; Imazu et al., 2017); (5) reduced systemic inflammation (Lira et al., 2009; Rosa Neto et al., 2009; Batista et al., 2010) and (6) increased cardiac function (Barauna et al., 2005; Laterza et al., 2007; Batista et al., 2008).

Unhealthy lifestyles comprising unhealthy diet combined with lack of physical activity may result in increased blood pressure, increased blood glucose, elevated blood lipids and obesity. These are called metabolic risk factors that lead to cardiovascular disease, the leading NCDs in terms of premature deaths (GBD 2015 Risk Factors Collaborators, 2016). There are four metabolic markers referred to as risk factors for NCDs: (1) raised blood pressure; (2) overweight/obesity; (3) hyperglycemia and (4) hyperglicidemia. High blood pressure (19\% of overall deaths) followed by overweight and obesity and increased blood glucose are related to the higher metabolic risk factors that culminate in death (GBD 2015 Risk Factors Collaborators, 2016). Obesity is considered a global epidemiological health problem and is associated with the development of numerous metabolic diseases, such as: insulin resistance (Martin et al., 2015), Type II Diabetes (Pulgaron and Delamater, 2014), atherosclerosis
(Lovren et al., 2015), non-alcoholic hepatic steatosis (Li et al., 2014; Negrin et al., 2014), hypertension (Paley and Johnson, 2018) and coronary heart disease (Warburton et al., 2006; Figure 1).

Physical exercise is an important non-pharmacological treatment option not only as a preventive agent, but also in the treatment of numerous metabolic diseases, such as type II diabetes, hypertension, and cardiovascular disease (Nunan et al., 2013; Mahtani et al., 2015). The mechanisms involved in the benefits generated by physical exercise against metabolic diseases are related to the increase in total energy expenditure and consequent reduction in the accumulation of fat mass, including a reduction in total body mass (Lancaster and Febbraio, 2014). For decades, it has been known that there is a direct relationship between obesity and inflammation and the development of NCDs (Cani and Jordan, 2018). Numerous studies have sought to elucidate which components of the immune system, as well as which molecules from local inflammation, could be related to the development of NCDs (Lancaster and Febbraio, 2014). In obesity, the immune cell profile in AT is substantially altered, for example, there is an accumulation of proinflammatory macrophages, neutrophils, and CD8 $+\mathrm{T}$ lymphocytes, while regulatory and eosinophilic $\mathrm{T}$ cells are substantially reduced. This process contributes to inflammation that culminates in the development of systemic insulin resistance (Osborn and Olefsky, 2012; Mraz and Haluzik, 2014).

Considering the role of physical exercise as a direct modulating agent of the benefits related to the general improvement of physiological variables involved in NCDs in recent years, several studies have characterized the role of skeletal striated muscle in this context (Pedersen and Febbraio, 2012; Pedersen, 2013). It is well established that SkM constitutes a key organ for the oxidation of lipid, acting as an elaborate energy production and consumption system that influences the whole body's energy metabolism (Iizuka et al., 2014). SkM tissue is composed of several cell types including muscle fibers, stem cells, fibroblasts, pericytes, adipocytes, motor neurons, and connective tissue. In addition, the muscular satellite cells play an important role in the cellular signaling process with neighboring cells, thus being a bioactive secretory factor (Sheehan et al., 2000). For almost half a century, researchers have hypothesized that musculoskeletal cells have a humoral factor, which is activated by increased demand for glucose during muscle contractions (Nielsen and Pedersen, 2008).

The concept of SkM as an immunogenic producer/secretor of cytokines, which exert an endocrine function, was proposed by Pedersen (2013), who proposed that such cytokines and other peptides should be classified as myokines (Pedersen, 2013). The discovery of the role of SkM as an endocrine organ expanded the knowledge of how the nervous, endocrine and immune systems contribute synergistically to the maintenance of body homeostasis (Febbraio and Pedersen, 2005; Karstoft and Pedersen, 2016). Myokines are an important local agent, having effects on metabolism, angiogenesis and muscle growth, as well as being capable of systemically acting on other organs and systems (Febbraio and Pedersen, 2005). A tissue of great metabolic importance that has been the subject of studies on crosstalk with 

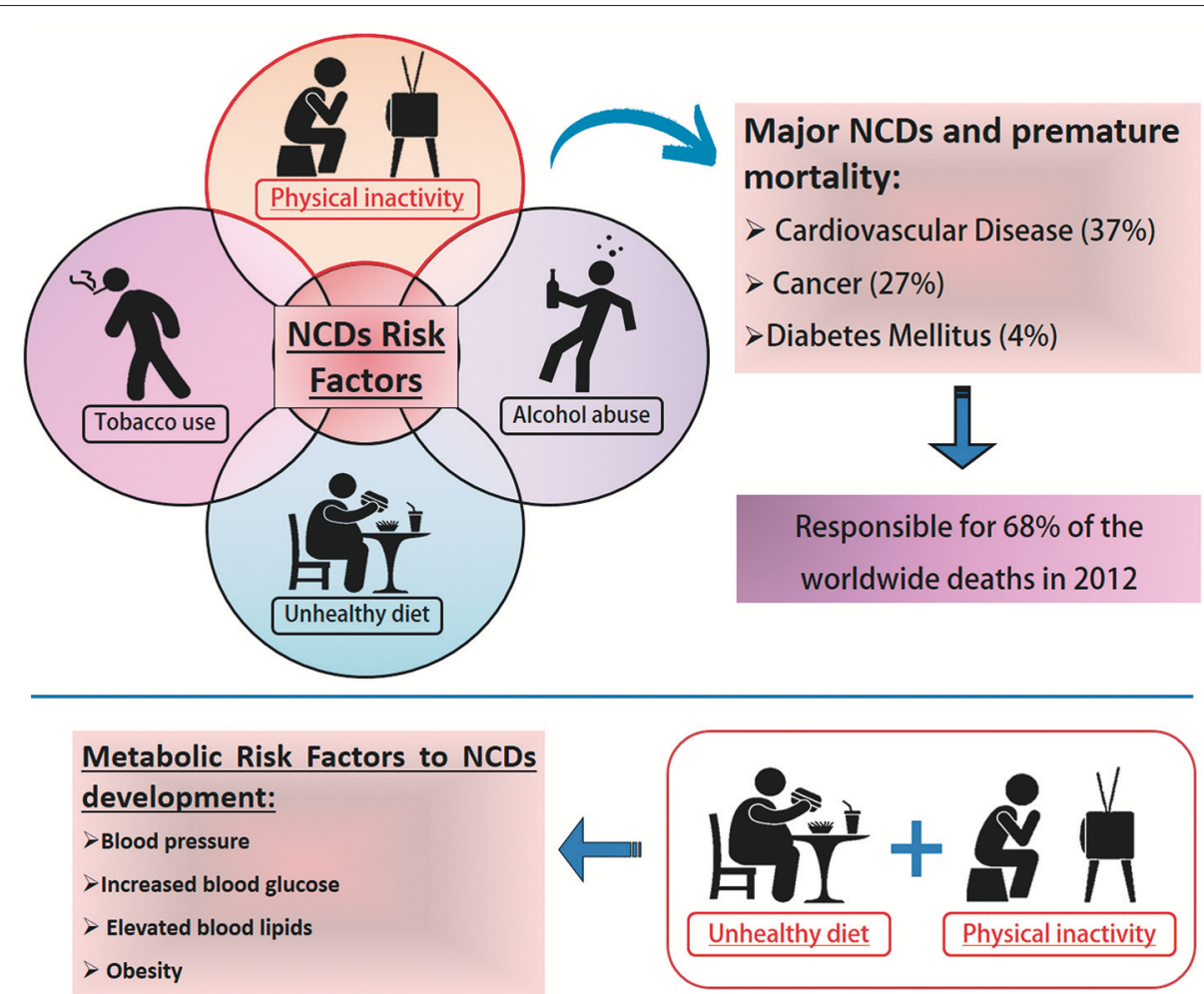

FIGURE 1 | Noncommunicable Diseases (NCDs) Risk Factors. NCDs have become a major global public health problem. Tobacco and alcohol (misuse) use, unhealthy diet and physical inactivity represent important health risks. Physical inactivity is a leading cause of death worldwide. It increases the risk of major noncommunicable diseases (NCDs) such as coronary heart disease, type 2 diabetes and breast, and colon cancers, responsible in 2008 for an estimated five million deaths or about $9 \%$ of the total global premature mortality. According to the World Health Organization, poor life habits such as physical inactivity and unhealthy diets are the main indications for the development of metabolic disorders that increase the risk factors for the development of NCDs. Data available at: http://www.who.int/ news-room/fact-sheets/detail/noncommunicable-diseases.

SkM is adipose tissue (AT). AT is widely known, not only for its ability to store energy, but also for its important endocrine component. AT in mammals consists of at least two different functional types: white and brown (Rosen and Spiegelman, 2006). White adipose tissue (WAT) is the main site of energy storage and release of hormones and cytokines that modulate whole body metabolism and insulin resistance, whereas brown adipose tissue (BAT) is important both for energy expenditure and the release of energy as heat. This thermogenic effect is mediated by the expression of decoupling protein-1 (UCP1; Cypess et al., 2009).

Both AT and SkM are significantly affected by exercise. One of the adaptations of these tissues to exercise is the secretion of molecules capable of modulating not only local metabolism but also systemic metabolism (pleiotropic). These secreted molecules can act in an endocrine manner to facilitate crosstalk between these tissues (tissue-to-tissue) and thus work together to improve overall metabolic health. In this review, we sought to produce an update on the state of knowledge in relation to the potential of physical exercise as inducer of myokines capable of modulating AT, as well as the impact of this crosstalk on health maintenance and promotion.

\section{Physical Exercise as an Inducer of an Anti-inflammatory Response}

In recent years, several well-designed studies have demonstrated that performing acute aerobic exercise is an important mediator of systemic anti-inflammatory response (Petersen and Pedersen, 2005; Pedersen and Fischer, 2007; Pedersen et al., 2007). Muscle contraction induced by physical exercise results in increased gene expression and secretion of interleukin-6 (IL-6) by skeletal myocyte. Consequently, depending on the exercise variables (volume, intensity, density), there is an increase in IL-6 plasma levels (Steensberg et al., 2003). Following acute aerobic exercise, there is an increase in the cytokines interleukin-10 (IL-10), interleukin-1 receptor antagonist (IL1-ra), and the soluble receptors of the tumor necrosis factor I and II (TNF I and II). This set of changes is characterized as an "anti-inflammatory effect" (Petersen and Pedersen, 2005).

Given this condition, the hypothesis that has been proposed is that the regular practice of physical exercise, organized in a exercise training program (chronic effect), exerts an antiinflammatory effect induced by the sessions (acute effect), which leads to improved protection against chronic inflammatory situations, levels of proinflammatory cytokines and C-reactive 
protein (Petersen and Pedersen, 2005; Fischer, 2006; Lira et al., 2009; Batista et al., 2010). However, the possible mechanisms modulating this "beneficial" effect are not well established.

In general, this anti-inflammatory effect has been shown to be more evident in some pathological conditions, such as atherosclerosis, type II diabetes, obesity and heart failure (HF), especially those presenting a two- to three-fold systemic increase in the levels of proinflammatory cytokines and C-reactive protein (Fischer et al., 2007). In addition, this cytokine production profile that occurs during and immediately after physical exercise is dependent on several factors, such as; population (sedentary, presence of diseases, etc.), the intensity or duration of the physical exercise, glucose availability and sample collection time (Flynn et al., 2007).

In addition to this, several cross-sectional studies have shown that there is a slight increase in the plasma levels of proinflammatory molecules under various conditions, such as physical inactivity (sedentary; Petersen and Pedersen, 2005; Flynn et al., 2007; Pedersen, 2007; Pedersen et al., 2007) in elderly people (Bruunsgaard et al., 2003), as well as in patients with diseases such as intermittent claudication (Tisi et al., 1997), T2DM (Boule et al., 2001), and atherosclerosis, among others. Under these conditions, the term "chronic low-grade systemic inflammation" has been used to characterize a 2- to 3-fold increase in plasma levels of TNF- $\alpha$, IL- $1 \alpha$, IL-6, IL-1ra, sTNFR1 and 2, and C-reactive protein (CRP), inflammatory markers noted as important both in the development and the progression of these inflammatory processes. Despite the evident correlation between exercise and anti-inflammatory effects, little information has been produced so far to explain the possible mechanisms responsible for the relationship between physical training and the reduction in these markers (Pedersen and Fischer, 2007). In these disease conditions, the origin of this systemic alteration is not well characterized, however, it has been proposed that WAT and peripheral blood mononuclear cells (especially lymphocytes) may be the main source of these cytokines (Steensberg et al., 2003; Fischer, 2006; Pedersen and Fischer, 2007).

\section{Chronic Effect of Exercise: Physical Training and Neuroimmune-Metabolic Response}

In general, exercise training programs are usually characterized by repetitive phases of overload, overreaching, maintenance of overtraining and recovery (Steinacker et al., 2004). The overload phases are characterized by a difference between the total amount of the overload (volume $\mathrm{x}$ intensity $\mathrm{x}$ density) and the recovery time between the exercise training sessions. Recovery between exercise sessions is necessary to allow recuperation, and over time, an improvement in exercise performance, metabolism and homeostasis.

On the other hand, if the recovery time is insufficient a chronic state of changes (molecular, biochemical and regulatory) can be produced that compromise well-being, increase the incidence of illness and decrease physical performance. The balance between exercise and recovery determines the positive or "beneficial" outcomes or adaptations of a given period of exercise training (Lehmann et al., 1993b; Steinacker et al., 2004).
Lehmann et al. proposed a biphasic model of response to training overload (Lehmann et al., 1993a) involving predominantly: 1-peripheral mechanisms in the early stages of overload in the organism, and 2-central mechanisms in the more pronounced and lasting phases of the overload period, with the hypothalamus as a central integrator of all afferent signaling to the brain and having an important role in regulating central responses to stress and physical training (Steinacker et al., 2004). These interactions involve afferent information from the autonomic nervous system, direct metabolic effects, hormones, cytokines and also information from superior brain centers, demonstrating a complex interaction involving bi-directional communication between the neuroendocrine and immune systems (Spinedi and Gaillard, 1998).

Researchers have examined the interaction of various systems in relation to the stimulus provided by physical exercise (Pedersen et al., 2007; Ruiz-Casado et al., 2017; Ishiuchi et al., 2018). Many researchers have sought to examine the role of the SkM themselves, particularly peripheral factors probably originating from successive muscle contractions during exercise that mediate changes in the tissue itself as well as having a systemic effect on other organs such as the liver and AT.

\section{Adipose Tissue}

Currently, AT is characterized as an important endocrine organ present in the body, related both to the expression of various cytokines and to the regulation and coordination of energy homeostasis, blood pressure, immune function, angiogenesis, mechanical shock protection, and thermogenic function (Prins, 2002; Trayhurn and Wood, 2004). The cytokines that are produced in the AT are called adipokines, molecules which have properties that allow them to generate autocrine and paracrine effects, or even a systemic effect, thanks to their endocrine characteristic, being able to reach distant tissues and have metabolic consequences in the whole organism (Kershaw and Flier, 2004).

Histologically, AT is characterized as connective tissue with special properties (Sheehan et al., 2000). In anatomical terms, two different specialized types of AT with different origins and functions, known as WAT and BAT, are described (Tsoli et al., 2012). WAT can be found in different regions in the body, being responsible mainly for the storing excess energy inside its cells in the form of triacylglycerol (TG). This stored energy is used when there is a caloric deficit (Kajimura et al., 2015). The anatomic location of AT determines its metabolic identity and central functions. In humans, WAT is located in many different deposits, such as the intra-abdominal visceral deposit, whose deregulation is associated with greater risks for metabolic diseases; and the subcutaneous deposit that can transmit protective effects on energy homeostasis (Kusminski et al., 2016). On the other hand, BAT has a more limited distribution. In mice, large BAT deposits, including interscapular, axillary and cervical BAT, develop in the prenatal period and provide a source of heat to protect newborns against exposure to cold (Kajimura et al., 2015). In adult men, the highest concentration of BAT occurs in the interscapular region (Kajimura et al., 2015). Its cellular structure presents a great concentration of mitochondria, having as one 
of its main function the thermogenic control of the organism (Sidossis and Kajimura, 2015). This process occurs through a mitochondrial protein called uncoupling protein 1 (UCP-1), which has the property of dissipating energy in the form of heat, a process described as non-shivering thermogenesis. Accordingly, this process results in the maintenance of body temperature when exposed to extreme situations (e.g., low temperatures; Karamanlidis et al., 2007; Wu et al., 2013).

As regards cellularity, both WAT and BAT are composed of several cell types, such as fibroblasts, endothelial cells, immune cells, nerves, preadipocytes and adipocytes, where the latter changes shape and function between WAT and BAT. While WAT adipocytes are characterized by the presence of a single large lipid (unilocular) droplet, BAT maintains multiple small droplets (multilocular) within its adipocytes (Sidossis and Kajimura, 2015). Although WAT is morphologically and metabolically different from BAT, about 30 years ago it was seen that there were adipocytes in the WAT that had morphological similarities with the BAT adipocytes, which until then were believed to be the only ones that had the thermogenic property (Harms and Seale, 2013; $\mathrm{Wu}$ et al., 2013). These adipocytes, as in the BAT adipocytes, were multilocular, had a larger number of mitochondria and when stimulated by low temperatures received $\beta 3$-adrenergic stimulation, expressed UCP-1, elevated cellular metabolism and finally dissipated energy in the form of heat (Wu et al., 2013). These white cells with characteristics of brown cells were named as beige cells or "brite-brown in white," and the name given to this phenomenon of cellular differentiation was browning of WAT (Wu et al., 2012, 2013).

\section{Metabolic Diseases and Systemic Repercussions of AT}

As already mentioned, both physical inactivity and NCDs, particularly those diseases characterized by metabolic diseases, present a clear morpho-functional dysfunction of AT. Taken together, these stressors, depending on their intensity and duration, result in the remodeling of the AT. This process is characterized by a set of changes, such as: modification of the adipocyte area (hypertrophy, hyperplasia or atrophy; Arner, 2000); disturbance in fatty acid turnover (lipolysis and lipogenesis; Arner et al., 2010); rearrangement of extra-cellular matrix components (Scherer, 2016); inflammation (Batista et al., 2012); modulation of the browning phenotype related to the thermogenic effect (Kir et al., 2014); among others. The end results of this process are both local (i.e., in tissue) and systemic (ectopic fat, insulin resistance, etc.) impairment of the normal physiological functions of this tissue.

Physical inactivity contributes to being overweight or obese and the development of chronic conditions, including cardiovascular diseases, T2DM, gallbladder disease and osteoarthritis (Greenwood et al., 2010). Obesity, the excessive accumulation of body fat (mainly due to an imbalance between energy intake and expenditure) is at the heart of all these problems. Excessive fat accumulation in the adipocytes can result in an imbalance in TG turnover, inflammation in the AT and a consequent increase in the secretion of a large number of proinflammatory factors, such as TNF- $\alpha$, and others (Mraz and
Haluzik, 2014). Excess AT and consequent dysfunction plays an important role in the pathophysiology of other metabolic diseases such as metabolic syndrome (Grundy, 2015).

On the other hand, people with a deficiency of AT (for example, lipodystrophy) can also be diagnosed with metabolic syndrome. This happens because in this case redistribution of fat is almost always directed to SkM which directly leads to insulin resistance (Grundy, 2015). In cancer cachexia, AT is affected early, prior to the establishment of the main signs and symptoms of the syndrome (Fouladiun et al., 2005). The breakdown in fatty acid turnover is the most well characterized physiological event, evidenced by the increase of lipolysis and changes in of lipogenesis (Henriques et al., 2017). Increases of cellular infiltrate, in particular macrophages (Batista et al., 2013), increased collagen and impairment of adipocyte turnover (Franco et al., 2017), has also been described. As a result, AT loses its TG storage capacity and, consequently, presents a reduction in the mass of this tissue. This scenario (AT mass loss) presents a negative correlation with cancer patient survival (Ebadi et al., 2017).

\section{Skeletal Muscle as an Immunogenic Organ The Concept of Myokines}

The SkM is a highly organized tissue at the micro and macroscopic level and is the body's main protein reserve (Hornberger, 2011; Bentzinger et al., 2013). SkM tissue is an important component related to quality of life, health, survival, and metabolic balance (Rodriguez et al., 2014; Salanova et al., 2014). It is a plastic tissue and continuously adaptable to various situations such as mechanical stimulation or disuse, leading to the condition of maintenance and/or muscular hypertrophy (increase of muscle mass) and atrophy (reduction of muscle mass), respectively. In addition, such tissue has a high capacity to alter its phenotype, depending on the mechanical load applied to it (Williamson et al., 2001). SkM tissue is also identified as an organ that synthesizes and secretes cytokines and other peptides, and these molecules have been given the name myokines (Pedersen et al., 2007). $\mathrm{SkM}$ is one of the largest organs of the human body, and in addition to its important functions such as providing locomotion, maintaining body temperature and metabolic homeostasis (Henningsen et al., 2010), the discovery that muscle contraction secretes proteins defined a new paradigm; SkM is a secretory organ, synthesizing and secreting myokines in response to muscle contraction. The products secreted by this tissue can influence the metabolism and function of SkM, in addition to other tissues and organs (Pedersen, 2013). Studies on the humoral component of SkM date back to the middle of the twentieth century, with a focus on the role of physical exercise as a modulator of glucose metabolism, with no conclusion about the mechanisms involved in this regulatory process (Goldstein, 1961). The ability of SkM to secrete myokines from muscle contraction was termed as "work stimulus," "work factor," or "exercise factor" (Figure 2; Pedersen, 2011). The concept of "exercise factor" was based on the fact that muscle contractions engage metabolic and physiological responses in other organs, and that these are not mediated by 


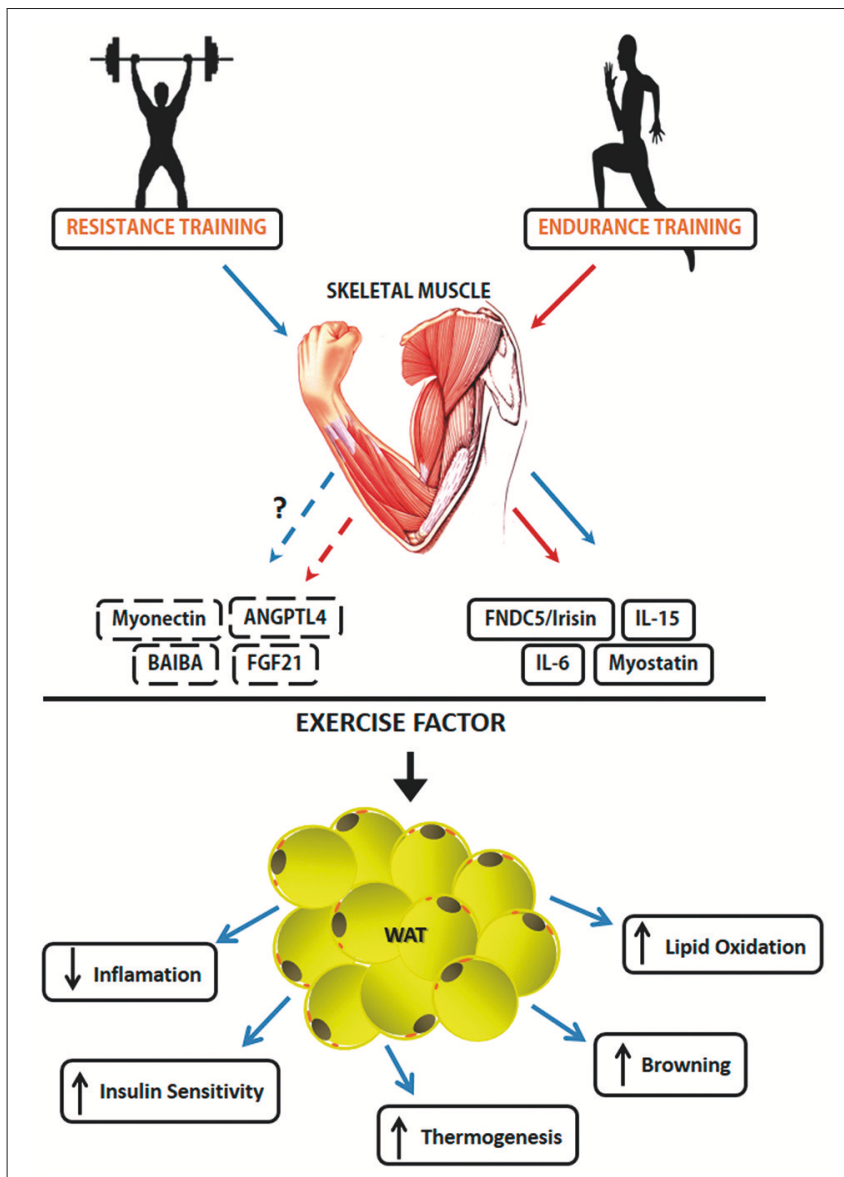

FIGURE 2 | Myokines involved in induced-exercise muscle-adipose tissue crosstalk. The secretion and action of different types of Myokines is exercise type-dependent. Some Myokines like Irisin, Myostatin, IL-6 e IL-15 have been the subject of a number of published studies about their mechanisms. However, FGF21, Myonectin, ANGPTL4, and BAIBA need more studies about their role as exercise factor. Solid lines represent already known mechanisms, while broken lines represent the mechanisms that still have to be unraveled. The red line represents factors released during endurance training practice while the blue line represents factors released during resistance training practice. The Myokines released by exercise and their effects on White Adipose Tissue. Muscle contraction-induces a set of molecules that have an endocrine function. Muscle-adipose tissue crosstalk exerts functions such as reduction of adiposity, increase in thermogenesis due to increased adrenergic activity, increased markers of the browning phenomenon and increased lipolytic activity.

the nervous system (Pedersen et al., 2003). This concept was established after a study with electrical stimulation in paralyzed muscles, in which it was verified that the capacity of muscle contractions in modulating other tissues occurs through an independent pathway of activation of the nervous system (Kjaer et al., 1996). Thus, myokines began to be understood as a protective factor against disease and the effects of physical inactivity.

Table 1 provides a list of original articles that investigated the potential of exercise/physical training as an inducer of myokines and evidence of mechanisms or outcomes relating to the muscleadipose tissue crosstalk.

\section{Myokines That Act on Crosstalk Between SkM and AT}

As described above, myokines are released from SkM in response to exercise. Thus, following the characterization of irisin as a myokine capable of activating adipose tissue browning in rodents in response to exercise (Bostrom et al., 2012) the concept that factors secreted by SkM could signal to other organs has grown and developed. More recently, Lee et al. (2014) shown that irisin and FGF21, both induced by submaximal exercise, have a function in promoting the browning of adipose tissue to meet the increased demand for fat oxidation. Moreover, the characterization of these two molecules opened an avenue of possibilities in the search for new factors that will add to our understanding of muscle-adipose tissues crosstalk and the options for treatment (Figure 2). In this review article, the main proteins identified in the literature that are expressed/secreted by SkM and have a capacity to act on the crosstalk between muscle-adipose tissues will be explored.

\section{Myostatin}

Transforming growth factor beta superfamily (TGF- $\beta$ ) and related factors such as myokines are first candidate molecules identified in SkM, and reported to induce biological signals that regulate cell growth, regeneration, differentiation, transformation and death of SkM (Iizuka et al., 2014). Among them are: myostatin, activins or inhibins, follistatin and bone morphogenic proteins (BMP). Myostatin is a protein related to the control of muscle growth and body metabolism (Feng and Derynck, 2005).

Among the different proteins of the TGF- $\beta$ family, myostatin (also known as GDF8) is a protein secreted during embryonic development and its function is to limit muscle growth in the physiological situation during development. However, it is known today that myostatin is also expressed and secreted even in adulthood (Argiles et al., 2012). Myostatin circulates in the blood in a latent form and when cleaved, it presents itself actively. Once activated, it has high affinity to ACTRIIB Activins receptors (de Caestecker, 2004). Once attached to its receptor, it triggers the activation of the Smad family transcription factors (Smad2 and Smad3). This activation in turn culminates in muscle atrophy, through the subsequent activation of the Forkhead Box family transcription factors, FOXO $(1,2$, and 3$)$ together with inhibition of the AKT/mTOR pathway (Braun and Gautel, 2011). The importance of myostatin as an atrophic component is highlighted in patients with HF and cancer, who present increased serum levels of this protein, which can result in cachexia, which can only be reversed by the deletion of the gene, as can be observed in in vitro assays and transgenic animal models (George et al., 2010; Heineke et al., 2010).

In addition to the ability to modulate the Smad, FOXO and AKT pathways, the interaction between myostatin and physical exercise also appears to occur through the transcription factor peroxisome proliferator-activated receptor gamma co-activator 1-alpha (PGC-1 $\alpha$ ) in muscle, which in turn is stimulated by exercise (Han et al., 2013). Studies have shown that inhibition of myostatin results in up-regulation of PGC-1 $\alpha$ activity in the SkM, thus stimulating mitochondrial biogenesis. PGC- $1 \alpha$ in turn binds 
TABLE 1 | List of original papers that investigate proteins expressed and/or secreted by skeletal muscle which act on muscle-adipose tissue crosstalk.

\begin{tabular}{|c|c|c|c|c|c|}
\hline References & Experimental model & Training protocol & $\begin{array}{l}\text { Myokines } \\
\text { analyzed }\end{array}$ & Main results & $\begin{array}{l}\text { Evidence of muscle-adipose } \\
\text { tissue crosstalk }\end{array}$ \\
\hline $\begin{array}{l}\text { Norheim } \\
\text { et al., } 2014\end{array}$ & $\begin{array}{l}\text { Healthy and physically } \\
\text { inactive men }\end{array}$ & $\begin{array}{l}\text { Combined strength and endurance } \\
\text { training for } 12 \text { weeks, including two } \\
\text { endurance bicycle sessions ( } 60 \\
\text { min-70\% } \mathrm{VO}_{2} \text { máx) and two whole } \\
\text { body strength training sessions } \\
\text { ( } 60 \text { min) per week. }\end{array}$ & FNDC5/Irisin & $\begin{array}{l}\uparrow \text { FNDC5 mRNA expression in } \\
\text { skeletal muscle. } \\
\uparrow \text { Plasma concentration of irisin } \\
\text { after } 45 \text { min ergometer cycling } \\
(\sim 1.2 \text {-fold) and } \downarrow \text { after } 2 \mathrm{~h} \text { rest. }\end{array}$ & $\begin{array}{l}\text { There was little or no effect of } \\
\text { long-term training on selected } \\
\text { browning genes and no correlation } \\
\text { between circulating irisin with UCP1 } \\
\text { mRNA in subcutaneous adipose } \\
\text { tissue. }\end{array}$ \\
\hline $\begin{array}{l}\text { Lee et al., } \\
2014\end{array}$ & $\begin{array}{l}\text { Healthy adults } \\
\text { and volunteers wearing } \\
\text { hospital scrubs rested } \\
\text { in beds }\end{array}$ & $\begin{array}{l}\text { Acute session of exercise on } \\
\text { cycloergometer following graded, } \\
\text { stepwise cold exposure to maximal } \\
\text { capacity (VO2max) and sub-maximal } \\
\text { exercise test at } 40 \% \text { VO2max for } 1 \mathrm{~h} \text {. }\end{array}$ & Irisin & $\begin{array}{l}\uparrow \text { Irisin in maximal exercise group } \\
\text { compared to cold exposure. }\end{array}$ & $\begin{array}{l}\text { In vitro experiments (BAT) provide } \\
\text { mechanistic insight into in vivo } \\
\text { observations. The results indicate that } \\
\text { shivering-stimulated irisin, in concert } \\
\text { with FGF21, phenotypically } \\
\text { transforms white adipocytes to } \\
\text { BAT-like cells to expand overall } \\
\text { thermogenic capacity. }\end{array}$ \\
\hline $\begin{array}{l}\text { Miyamoto- } \\
\text { Mikami et al., } \\
2015\end{array}$ & $\begin{array}{l}\text { Healthy young subjects } \\
\text { and healthy middle } \\
\text {-aged/older adults }\end{array}$ & $\begin{array}{l}\text { Cycling on a leg ergometer for } \\
55 \text { min, } 3 \text { days/week, for } 8 \text { weeks. }\end{array}$ & Irisin & $\begin{array}{l}\uparrow \text { Serum irisin level in the } \\
\text { middle-aged/older training group } \\
\leftrightarrow \text { Serum irisin level in the young } \\
\text { training group }\end{array}$ & $\begin{array}{l}\text { In the middle-aged/older training } \\
\text { group, the endurance } \\
\text { training-induced reduction in visceral } \\
\text { adipose tissue area was negatively } \\
\text { correlated with the change in serum } \\
\text { irisin level }\end{array}$ \\
\hline $\begin{array}{l}\text { Kurdiova } \\
\text { et al., } 2014\end{array}$ & $\begin{array}{l}\text { Middle-aged sedentary } \\
\text { men }\end{array}$ & $\begin{array}{l}\text { Combined training performed } 3 \text { times } \\
\text { per week during } 3 \text { months. Intensity } \\
\text { was maintained at } 70-85 \% \text { of }\end{array}$ & FNDC5/Irisin & $\begin{array}{l}\text { Exercise did not affect } \\
\text { Fndc5/irisin }\end{array}$ & $\begin{array}{l}\text { Was observed a down regulation of } \\
\text { Fndc5/irisin in adipose tissue and } \\
\text { circulation in T2D group. }\end{array}$ \\
\hline
\end{tabular}

Roca-Rivada Lean and obese rats et al., 2013 and obese men

Pekkala et al., Untrained and trained 2013 healthy men

Bueno et al., Wistar rats 2011

Hjorth et al., Sedentary men 2016

Macpherson C57BL/6 mice et al., 2015 at $70-85 \%$ of maximal heart rate and strength training Intensity was maintained at $70-85 \%$ of maximal heart rate.

Free access to the activity wheel for 1 FNDC5/Irisin or 3 weeks

Acute low-intensity aerobic exercise was performed with bicycle ergometer for $1 \mathrm{~h}$ at a low intensity of $50 \%$ VO2max

Single resistance exercise of 5 sets of 10 repetitions in leg press until failure. Heavy-intensity endurance exercise, twice a week and combined EE and RE training

$1.5 \mathrm{~h} /$ day, $45 \mathrm{~min}$ at 9:00 AM and $45 \mathrm{~min}$ at 05:00 h PM, for 4 weeks.

12 weeks of two interval bicycle sessions (60 min, 45-minbicycle test at $70 \%$ of VO2max) and two full-body strength training sessions (60 min) per week

Mice ran for $120 \mathrm{~min}$ at $15 \mathrm{~m} / \mathrm{min}, \quad$ IL-6 with an incline of $5 \%$.
Myostatin

$\leftrightarrow$ In mRNA myostatin between exercised and sedentary normal diet and HFD rats

FNDC5/irisin

$\uparrow$ FNDC5/irisin in muscle after 3 weeks of exercise $\uparrow$ FNDC5/irisin in adipose tissue after 1 week of exercise and $\downarrow$ after 3 weeks exercise

$\uparrow$ FNDC5 mRNA only in young men post-RE

Myostatin

$\downarrow$ mRNA myostatin in skeletal muscle after acute and long-term exercise

In cultured muscle cells myostatin promoted an insulin-independent increase in glucose uptake.

$\uparrow$ Enhanced rate of glucose oxidation and lactate production in muscle cells incubated with myostatin

$\uparrow$ Expression and secretion of IL-6 and IL-10 in skeletal muscle in exercise HFD group
$75 \%$ of irisin expression by skeletal muscle and $28 \%$ by AT. No correlation between expression of UCP1 in subcutaneous fat and circulating irisin

No change in plasma level of irisin on adipose tissue.

Irisin and FNDC5were not associated with glucose tolerance and being overweight, or with metabolic disturbances, respectively

$\uparrow$ In mRNA myostatin in BAT of high-fat rats after swimming $\downarrow$ In mRNA myostatin in mesenteric AT of high-fat trained rats versus sedentary high fat diet rats

The expression of myostatin was correlated negatively with insulin sensitivity $\uparrow$ Myostatin mRNA in AT after 12 weeks of training and correlated positively with insulin sensitivity markers.

$\uparrow$ On infiltrates cells in AT of HFD group $\downarrow$ F480 and CD11 in AT in exercise HFD group 


\begin{tabular}{|c|c|c|c|c|c|}
\hline References & Experimental model & Training protocol & $\begin{array}{l}\text { Myokines } \\
\text { analyzed }\end{array}$ & Main results & $\begin{array}{l}\text { Evidence of muscle-adipose } \\
\text { tissue crosstalk }\end{array}$ \\
\hline $\begin{array}{l}\text { Castellani } \\
\text { et al., } 2015\end{array}$ & C57BL/6 mice & $\begin{array}{l}\text { Mice ran for } 2 \mathrm{~h} \text { at } 15 \mathrm{~m} / \mathrm{min} \text { at a } 5 \% \\
\text { incline, during } 4 \text { weeks }\end{array}$ & IL-6 & $\begin{array}{l}\leftrightarrow \text { No differences in plasma IL- } 6 \\
\text { between sedentary and trained } \\
\text { mice }\end{array}$ & $\begin{array}{l}\uparrow \text { In levels of il- } 6 \text { mRNA epididymal AT } \\
\text { with no increase in IL- } 1 \beta \text { and TNF- } \alpha \\
\uparrow \text { In protein and mRNA levels of IL- } 6 \\
\text { receptor in epididymal AT }\end{array}$ \\
\hline
\end{tabular}

BAT, Brown Adipose Tissue; AT, Adipose Tissue; EE, Endurance Exercise; RE, Resistance Exercise; T2D, Type 2 Diabetes; $\uparrow$, increase, $\downarrow$, decrease and $\leftrightarrow$, no alterations.

to FOXO and inhibits its transcriptional activity (Lebrasseur et al., 2009). Another effect is an increase in AMPK activity in the muscle, which increases insulin sensitivity and responsiveness (Zhang et al., 2011).

However, myostatin is not exclusively secreted by SkM tissue, with studies indicating that AT also secretes this protein. Its importance in this tissue is related to the maintenance of adipose mass and greater sensitivity to insulin (Hamrick et al., 2006; Guo et al., 2009). Myostatin mRNA levels increase substantially in genetically-modified obese mice (leptin-deficient $o b / o b$ mice) and also in those receiving a high fat diet (HFD), so both circulating and SkM myostatin levels are present in obese subjects when compared to non-obese subjects (Allen et al., 2008; Hittel et al., 2009). Transgenic mice that expressed non-functional myostatin (conditioned to SkM), presented not only increased muscle mass but also resistance to weight gain (fat mass) and insulin resistance even when receiving a high-fat diet (Zhao et al., 2005). On the other hand, knockout animals for myostatin presented not only an increase in muscle mass but also an improvement in composition due to a reduction of fat mass, which in turn was due to the presence of the browning phenotype in the WAT, mainly because of an increase in the expression of irisin (Shan et al., 2013).

However, there are few studies in the literature that investigate the direct role of myostatin as an "exercise factor" and its ability to modulate AT. Bueno et al. (2011), evaluated the expression of myostatin and its ACTRIIb receptor in both SkM and AT from obese and insulin resistant rats who practiced swimming training. No change was found in myostatin expression in the SkM of trained animals compared to the control group. However, in the BAT the expression of myostatin and ACTRIIb was increased in the trained obese animals compared to the sedentary obese, while in the mesenteric adipose tissue (meAT) they were reduced (Table 1). The authors suggest that these changes are due to the ability of myostatin to modulate energetic homeostasis in exercise and obesity, since no change was observed in non-obese animals (Bueno et al., 2011). A recent study investigated the relationship between myostatin, physical activity and dysglycaemia in men with or without dysglycaemia who underwent a 30-45 min cycle test before and after 12 weeks of combined training (Hjorth et al., 2016). They found that myostatin mRNA expression was reduced in SkM after acute exercise and was further reduced over 12 weeks of training, while myostatin expression in AT increased after 12 weeks of training and correlated positively with insulin sensitivity markers (Table 1). Analysis at cellular levels with recombinant myostatin showed increased glucose uptake in human SkM cells, suggesting a complex regulatory role of myostatin in SkM homeostasis.

However, these last two studies have not yet elucidated direct evidence on muscle-adipose tissue crosstalk, since modulation in myostatin levels from exercise was due to qPCR analysis from AT. It is clear that myostatin is modulated by exercise, but more work on the modulation of myostatin muscle levels and its direct action in the exercise state are still necessary.

\section{Interleukins}

Interleukins (IL) are a group of cytokines bound to the immune system that have the ability to develop several different cellular responses when bound to surface receptors. These molecules have around 30 different isoforms that act in a paracrine and autocrine way, being strongly linked to inflammatory pathologies (Brocker et al., 2010). They play an important role in inflammatory processes and can exert a pro or anti-inflammatory function (Batista et al., 2010). The main proinflammatory cytokines are IL- 6 and IL-1 $\beta$, while the main anti-inflammatory ones are IL-4, IL-10, and IL-13, which act to inhibit the expression of other proinflammatory proteins (Lira et al., 2009). Other ILs such as IL-4, IL-8, IL-7, and IL-15 are also released by muscle tissue (Pedersen, 2011).

\section{Interleukin-6 (IL-6)}

Interleukin-6 (IL-6) is an important IL that is at high levels after exercise and plays an important role in systemic inflammation (Pratesi et al., 2013). High levels of IL-6 are observed in situations where glycogen levels are low, as a response to metabolic demand, since higher levels of IL- 6 are related to a higher lipolytic rate by activation of the AMPK pathway and/or PI3-kinase activating the oxidation of the fatty acids which leads to greater availability of energy supply from this energy source (Keller et al., 2001).

Some studies suggest that increased IL- 6 during and after an acute exercise session might be related to the type of exercise performed. Higher plasma levels of IL- 6 have been reported in some studies that use running as a model of physical exercise compared to those which use cycling (Nieman et al., 1998; Starkie et al., 2001). According to these studies, the running model leads to a greater release of IL- 6 because of the greater muscle damage which occurs due to its eccentric component being larger compared to that of cycling. Other studies comparing the IL- 6 response in these two exercise models did not show significant differences (Starkie et al., 2001).

Increased levels of IL- 6 after exercise are followed by increased expression of the IL-1 receptor antagonist (IL-1ra) and IL-10, 
such adjustment chronically constitutes an anti-inflammatory component and a response to the increase in circulating IL6 induced by exercise (Febbraio and Pedersen, 2002). Plasma concentrations of IL-6 may increase up to 100-fold after exercise. However, the increase in circulating IL-6 after exercise does not appear linearly over time, with a study finding an exponential acceleration in IL-6 secretion soon after exercise (Macdonald et al., 2003). In a recent study, in which the effects of voluntary running in tumor-bearing mice were evaluated, running animals showed a 29-fold increase in IL-6 levels in SkM, increased secretion of NK cells and a reduction in tumor volume, whereas trained animals receiving an IL- 6 antagonist did not exhibit the same rate of tumor reduction as well as less infiltration of NK cells into the tumor (Pedersen et al., 2016). Such a finding confirms the importance of IL- 6 as an "exercise factor" with the ability to modulate the immune system during tumor progression.

However, studies have shown that IL-6 can induce an anti-inflammatory environment, not only by inducing the production of anti-inflammatory cytokines, but also, under specific conditions, by inhibiting the production of TNF- $\alpha$, as demonstrated in an in vitro study (Beyaert and Fiers, 1999) and in mice (Petersen and Pedersen, 2005). In humans, infusion of rhIL-6, an experimental procedure that mimics the increase in IL- 6 levels induced by exercise, was able to inhibit the increase in endotoxin-induced TNF- $\alpha$ plasma levels (Starkie et al., 2003). In addition, evidence of an anti-inflammatory effect of IL-6 has shown to be related to a direct relation between muscle tissue and AT. This kind of relationship is characterized as crosstalk (Macpherson et al., 2015).

This study was conducted in C57bl/6 mice that received a high fat diet (HFD) and were exposed to running training (Table 1). At the end of the study, it was possible to observe that even without changes in adipose mass, higher levels of IL- 6 expression in the SkM were responsible for the increase in IL-10 expression, together with a significant reduction in inflammatory infiltrates in AT (Macpherson et al., 2015).

As described previously, IL-10 can act on different cell types to induce suppression of the inflammatory response and it has been postulated that it is the main molecule responsible for the "orchestration" of inflammatory reactions, in particular those involving the activation of monocyte/macrophage cells. Therefore, in humans, when IL-10 is added to the culture medium of mononuclear cells and circulating neutrophils stimulated with lipopolysaccharide(LPS), the synthesis of proinflammatory cytokines (TNF- $\alpha$, IL- $1 \beta$, IL-6) is inhibited through post-transcriptional mechanisms, a direct consequence of a higher mRNA degradation rate of the corresponding genes (Christiansen et al., 2010).

\section{Interleukin-15 (IL-15)}

Another IL expressed by SkM with an important metabolic role is IL-15. It has been suggested to be an important modulator of body fat mass, which also actively participates in the innate immune response, playing an important role in the development and function of natural killer (NK) cells and lymphocytes (Quinn et al., 2013).
In a study by Nielsen et al. which analyzed the rate of secretion of IL-15 in SkM of individuals who practiced Resistance Training (RT), it was possible to confirm that $24 \mathrm{~h}$ after the last RT session a two-fold increase in IL-15 mRNA levels was observed, and that this response was also dependent on the type of muscle fiber (Nielsen et al., 2007). Studies indicate that this cytokine is the main agent in crosstalk between SkM tissue and AT (Pedersen et al., 2007). The relationship of IL-15 and AT can be observed in a translational study (Nielsen et al., 2008), which verified in humans an inverse relationship between IL-15 (mRNA) and AT mass indexes. In mice transfected with IL-15 overexpressing plasmid, they showed reductions in AT mass compared to the control group.

The concentration of circulating IL-15 was shown to be closely related to physical exercise, in addition to increasing lipid oxidation and gene expression of peroxisome proliferatoractivated receptor delta (PPAR $\delta$ ) in rodents when they were treated with the cytokine (Quinn et al., 2013). In addition, IL15 is directly related to muscle metabolism, or even diet-induced obesity and insulin sensitivity (Quinn et al., 2011, 2013).

Because it has an anti-inflammatory function, IL-15 inhibits the action of $\mathrm{TNF} \alpha$ in the muscle during cachexia (Pajak et al., 2008). In addition, any deregulation of this cytokine can lead to several pathologies linked to autoimmune diseases such as rheumatoid arthritis and leukemia (Fehniger and Caligiuri, 2001). However, further studies are required to establish its full clinical importance.

Some previous studies demonstrated the link between IL15 release, SkM, and NCDs. In an in vitro study, cells treated with IL-15 had increased expression of heavy chain myosin and induced anabolism in SkM cells without stimulation of precursor myogenic cells (Furmanczyk and Quinn, 2003). Also, the ability of IL-15 to induce hypertrophy in SkM cells has been demonstrated through the reduction of the rate of protein degradation during cachexia and sarcopenia, highlighting the therapeutic power of this cytokine (Quinn et al., 2002). The ability of IL-15 to act on muscle- adipose tissue crosstalk was investigated by the same group, showing IL-15 overexpression in relation to reduced adiposity. To test the hypothesis, transgenic animals that overexpress IL-15 (mRNA and protein) in SkM were used in two experimental conditions: overexpression of musclefree IL-15 in the circulation and overexpression of muscle IL15 without secretion into the circulation. Only the group that had the increased levels of IL-15 from the muscle in circulation had a reduction in adiposity due to the greater targeting of this substrate for energy generation (Quinn et al., 2009). However, no study has been able to provide evidence of the direct mechanism of IL-15 as an "exercise factor" in muscle-adipose tissue crosstalk.

\section{FGF-21}

Fibroblast growth factor 21 (FGF21) is an endocrine-member of the FGF family. The expression and circulating levels of FGF21 are dependent on factors such as nutritional status, diet, hormone levels, and activities of various transcriptional factors. FGF21 acts in the control of glucose and lipid homeostasis (Kharitonenkov et al., 2005). In general, FGF21 stimulates the uptake of glucose by adipocytes and inhibits the production of glucose in the 
liver. In addition, FGF21 appears to protect pancreatic $\beta$ cells from cell death induced by glycotoxicity (Kharitonenkov et al., 2005, 2007). Hojman et al. found that FGF21 is expressed in both plasma and SkM after insulin stimulation in humans, and thus classified FGF21 as an insulin regulatory myokine (Hojman et al., 2009). However, studies that aimed to correlate FGF21 and its importance as "exercise factor" are still inconclusive. A study by Cuevas-Ramos et al. found an increase in serum FGF21 levels in runners after 2 weeks of training, but found no change in their gene expression in SkM (Cuevas-Ramos et al., 2012). However, another study analyzed the expression of FGF21 after an acute session of running exercise in both an experimental mice model and in humans and showed an increase in serum FGF21 levels (Kim et al., 2013b). However, this increase was not in SkM or AT but in the liver (Kim et al., 2013b). The authors, observed that this increase is in turn accompanied by increased hepatic peroxisome proliferatoractivated receptor alpha (PPAR $\alpha)$ expression, required for the activation of lipolysis and also of ATF4 (Kim et al., 2013b), a transcription factor identified in several stress response situations, including autophagy, mitochondrial stress, and amino acid deprivation (Kim et al., 2013a). In this respect, more studies are needed to confirm the importance of FGF21 as "exercise factor," since studies on the systemic effects of this molecule focus on quantifying their plasma levels (Cuevas-Ramos et al., 2012; Lee et al., 2014), rather than on verifying their expression in the SkM during contraction.

\section{Irisin}

Irisin is considered the most promising myokine in the context of metabolism maintenance because it is secreted by SkM and has been suggested to mediate the effect of exercise on WAT metabolism (Aydin et al., 2014). Irisin, formerly called Iris, is a recently identified myokine, released into the circulation by SkM in an exercise-dependent manner. It is reported to have the ability to convert WAT to BAT, a phenomenon known as browning; a phenotype set of adaptations that results in increasing total energy expenditure (Bostrom et al., 2012).

Irisin was discovered and characterized by Bostrom et al. (2012), in a study that sought to elucidate the relevance of the transcriptional coactivator PGC-1 $\alpha$ in the control of obesity (Bostrom et al., 2012). First, an algorithm was used to predict candidate proteins produced by SkM that may mediate the browning process in AT, excluding mitochondrial target proteins (Figure 3). Five candidate proteins were found to be mediated by PGC- $1 \alpha$ in the muscle: IL-15, Fndc5, VEGF $\beta$, Lrg1, and TIMP4. However, only the irisin precursor FNDC5 was able to promote the differentiation of stromal vascular cells isolated from WAT from beige mice (browning phenomenon; Bostrom et al., 2012). Consequently, there was an emerging search for a deeper understanding of the mechanisms of action of irisin, whether they were local or systemic as well as its role as an "exercise factor" (Huh et al., 2012, 2014, 2015; Hecksteden et al., 2013; Moraes et al., 2013; Pekkala et al., 2013; Roca-Rivada et al., 2013; Aydin et al., 2014; Daskalopoulou et al., 2014; Pardo et al., 2014; Tsuchiya et al., 2014; Kim et al., 2015; Miyamoto-Mikami et al., 2015; Fagundo et al., 2016; Duft et al., 2017). However, most of the studies only analyzed plasma levels of FNDC5/irisin and did not assess its expression by SkM. A study aimed to verify the physiological variables effect of irisin in healthy groups of swimmers of different ages and explore the direct effects on muscle metabolism, and found: (1) higher irisin serum levels in the younger group compared to the older group (age effect); (2) serum irisin levels were elevated in the swimmer groups (training effect); (3) rates of plasma irisin were related to the intensity of exercise training, with the group that performed intermittent high-intensity exercise having higher levels; (4) Higher rates of irisin were related to the metabolism of glucose and lipids in SkM through AMPK phosphorylation; despite the differences in basal irisin levels, exercise-induced irisin secretion is independent of age or fitness level. Increased irisin can directly modulate muscle metabolism through 5' AMP-activated protein kinase (AMPK) activation (Huh et al., 2014).

Another study aimed to elucidate the association between endurance exercise and the impact of irisin induction on body fat in 25 healthy ( \pm 21 years old) and elderly ( \pm 68 years) individuals who practiced 8 weeks of cycloergometer training at $60-70 \%$ of VO2peak for $45 \mathrm{~min}, 3 \mathrm{x} /$ week. The main results indicated an increase in serum irisin levels in the elderly group compared to the young group (age $21 \pm 1$ years) accompanied by a reduction in visceral adiposity which was negatively correlated with irisin levels $(r=-0.54, P<0.05$; Miyamoto-Mikami et al., 2015). However, the irisin modulation seemed to be dependent on the modality and intensity of the exercise. In this respect, irisin secretion was observed in volunteers of different ages submitted to a protocol of aerobic exercise of moderate to maximum intensity (70-75\% VO2max until exhaustion; Huh et al., 2014). In this study, it was possible to verify that intense exercise, until exhaustion, promoted higher irisin serum levels after exercise in the young group compared to the old group (age 67.9 \pm 5.0 ). The same study also analyzed the secretion of irisin in swimming adolescents in groups that either practiced high or moderate intensity swimming. It was observed that there was a significant increase in serum irisin levels immediately and up to $1 \mathrm{~h}$ after an exercise session in the group that practiced high-intensity swimming compared to the moderate intensity group.

Norhein et al. argue that the regulatory effect of physical training on the expression of muscular FNDC5 is not clear. In this study, 26 physically inactive men (normoglycemic and diabetic) were submitted to a 12 -week physical training program combining strength and endurance exercises (Table 1). Muscle biopsies were performed before and after the intervention and it was observed that muscle mRNA levels for both PGC- $1 \alpha$ and FNDC5 were increased after exercise. However, circulating levels of irisin were only increased acutely and shortly after the exercise session (1.2-fold; Table 1). As far as the analysis of muscle-adipose tissue crosstalk was concerned, few effects were seen in AT browning: AT UCP1 mRNA levels were not directly correlated with FNDC5 levels in both AT and in SkM (Norheim et al., 2014). Pekkala et al. analyzed the effects of different short-term and long-term exercise protocols on the FNDC5 and PGC- $1 \alpha$ of healthy trained and untrained men, who either did low intensity aerobic exercise, resistance training, high intensity exercise or combined endurance training, and assessed levels of muscle and irisin serum, as well as studying the associations of irisin and FNDC5 with health parameters (Pekkala et al., 


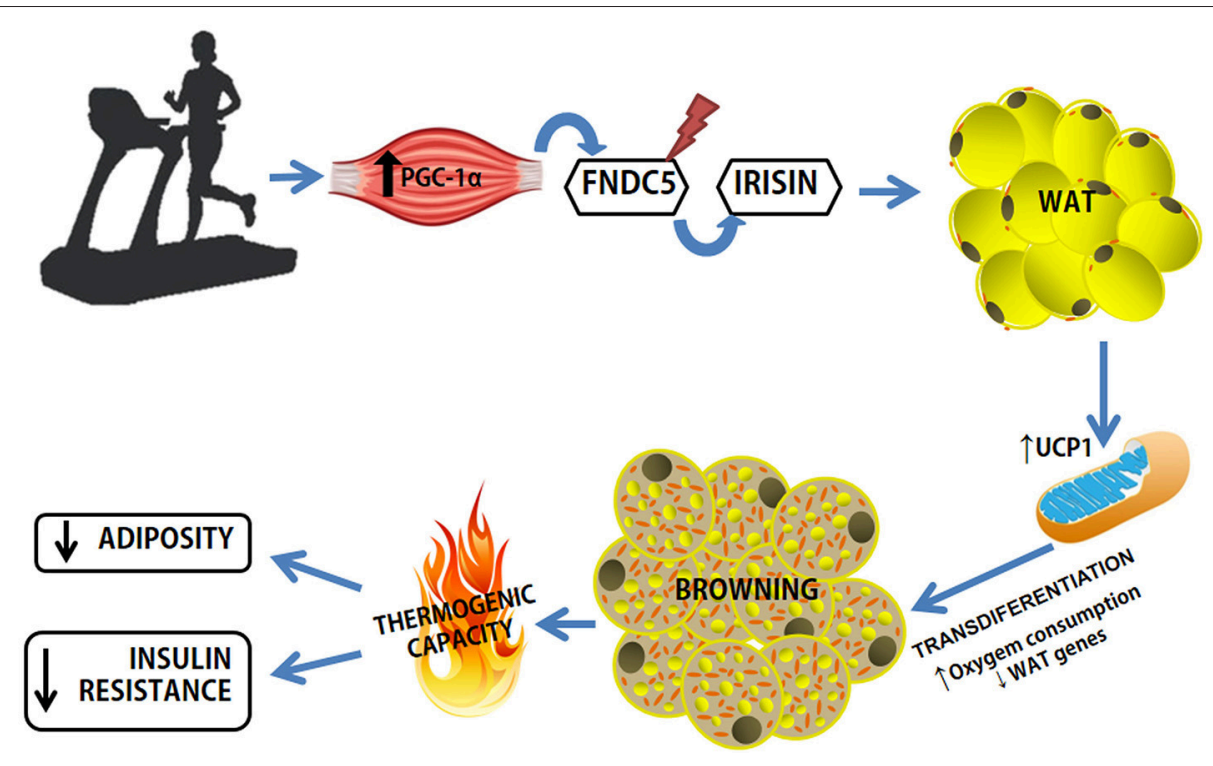

FIGURE 3 | Exercise-induced browning in WAT. Increased expression of PGC-1a in muscle as a result of exercise causes increased expression of FNDC5, a type I membrane protein, which C-terminally cleaved and secreted as irisin into the circulation. Irisin binds to a receptor yet unknown in WAT adipocytes and leads to phenotypic modification. WAT, white adipose tissue; FNDC5, Fibronectin Type III Domain Containing 5; UCP1, Uncoupling Protein Type 1.

2013). In this study, it was observed that muscle irisin levels were only increased in the young group that performed a single resistance training session, without any changes in irisin serum levels regardless of training. No association was found between levels of FNDC5 and health parameters (Table 1). The group also concluded that data on exercise and irisin modulation are questionable.

However, it is important to note that irisin is not a protein exclusively secreted by muscle tissue. The study presented by Arturo Roca-Rivada et al. was a pioneer in showing that WAT also secretes FNDC5, the precursor of irisin, indicating that it is not only a myokine but also an adipokine (Roca-Rivada et al., 2013). The study was based on gene and protein expression analyses by explant assay of subcutaneous and visceral AT from rats. He also found that both tissues secreted FNDC5/irisin under the stimulus of endurance training.

\section{ANGPTL4}

Angiopoietin-like protein (ANGPTL) is a group of proteins that can be secreted by the AT, SkM, intestinal, and liver cells and is responsible for several processes, among them energy metabolism, and TG turnover modulation in AT and the regulation of blood glucose levels (Ingerslev et al., 2017; Popova et al., 2018). Despite the name, ANGPTLs are not specific ligands for angiopoietin, but are named after the structural similarity with this protein (Cinkajzlova et al., 2018).

ANGPTL is regulated through peroxisome proliferatoractivated receptor (PPAR) and has the ability to regulate serum triglyceride levels. This ability is related to increased lipoprotein lipase (LPL) activity, promoting lipolysis in AT, and improving glucose tolerance (Ingerslev et al., 2017). Specifically, plasma ANGPTL4 acts on myotubes, and is well-established as a myokine derived from physical exercise (exercise factor; Raschke and Eckel, 2013; Ingerslev et al., 2017). The performance of this protein is related to several physiological processes, among them are insulin sensitivity, lipid metabolism, adipogenesis (Cinkajzlova et al., 2018), and the increase of circulating fatty acids, which are generated mainly by chronic caloric restrictions, fasting, and aerobic physical training (Raschke and Eckel, 2013).

It was seen that when muscle contraction was stimulated in myocytes during exercise using electrical pulse stimulus, there was an increase in the gene expression of ANGPTL 4 after $4 \mathrm{~h}$, and an increase of the supernatant proteins after $8 \mathrm{~h}$ of stimulation, corroborating the link between exercise and the expression of ANGPTL4 (Scheler et al., 2013). However, there are contrary findings, as in the study by Catoire et al. using acute endurance exercise in a single leg, ANGPTL4 was shown to be more highly expressed in unexercised legs than in exercised legs (Catoire et al., 2014).

The practice of fasting combined with physical exercise is indicated as an important inducer of ANGPTL4 expression and may be beneficial to those with high serum levels of long chain fatty acids (Ingerslev et al., 2017). In addition, an increase in serum ANGPTL4 in obese patients (with or without T2DM) was observed, and the same was observed in patients who underwent bariatric surgery and in fasting patients, while it was reduced in patients with anorexia (Cinkajzlova et al., 2018). However, even with published data indicating a role for ANGPTL4 in obesity, and glucose intolerance (Morris, 2018), this protein is still poorly studied and there is much about its therapeutic role that needs to be further investigated.

\section{BAIBA}

$\beta$-aminoisobutyric acid (BAIBA) are natural metabolites of thymine and valine secreted primarily by myocytes during 
the practice of physical exercise (Roberts et al., 2014). The increase of PGC1 $\alpha$ observed during physical exercise results in a concomitant increase in BAIBA serum levels. In the same study, in vitro, when BAIBA was cultivated with primary cells from subcutaneous adipose tissue (scAT), there was an up regulation of brown and beige adipocytes marker, such as UCP1 and CIDEA. In this way, it was shown that BAIBA has a potential to induce browning in white adipocytes, in addition to demonstrating that physical exercise increases its production by the body. Moreover, BAIBA has been proposed as a promising molecule capable of inducing the thermogenic framework by an independent pathway of adrenergic activation (Jeremic et al., 2017).

BAIBA also has an important therapeutic role against obesity (Ginter and Simko, 2014), mainly by stimulating the oxidation of fatty acids, reducing the process of lipogenesis in WAT, and attenuating inflammation and insulin resistance (Jung et al., 2018). The regulation of BAIBA is mediated through AMPK signaling, and plays an important role in several pathologies. In addition to obesity, BAIBA is reported to attenuate hepatic apoptosis by reducing ER stress on hyperlipidemia and improving renal fibrosis (Jung et al., 2018). Furthermore, it has been shown that circulating levels of BAIBA have an important role against cardiometabolic risks (Kammoun and Febbraio, 2014). Thus, with these data, it is possible to determine that the crosstalk between SkM, cardiac muscle, liver and WAT and BAT has a strong correlation with the practice of physical exercise (Kammoun and Febbraio, 2014).

\section{Myonectin}

Myonectin, also known as C1q/TNF-related protein 15 (CTRP15), is a new myokine recently found as a protein typical of fatty acid metabolism in response to exercise (Seldin et al., 2012; Toloza et al., 2018), although in other studies the relationship between the expression of myonectin and physical exercise is contradictory (Peterson et al., 2014).

The study by Seldin et al., which made a general characterization of this myokine, showed that it is widely expressed in SkM, more precisely in myotubes (Seldin et al., 2012). In addition, it is described as a potent inducer of cell differentiation in $\mathrm{C} 2 \mathrm{C} 12$ cells, which, according to the authors, indicates that this protein is produced in muscle fibers, not in satellite cells (Seldin et al., 2012). A test performed in vivo using the soleus and plantaris muscles showed increased gene expression and circulating levels of myonectin in animals that had been fed (compared to animals that had not). It was also shown that in the presence of carbohydrates or lipids in the gastrointestinal tract there was potent stimulation of myonectin expression in the assessed muscles (Seldin et al., 2012), suggesting a strong correlation between myonectin and nutrient uptake (Toloza et al., 2018).

High levels of myonectin were observed in individuals with T2DM, glucose intolerance, and obesity (Li et al., 2018). Other members of the CTRP family are expressed by AT, and they are described as adiponectin paralogue genes (Wong et al., 2008).This gene family is up-regulated in ob/ob mice and are highly expressed when stimulated with peroxisome proliferatoractivated receptor gamma (PPAR $\gamma$ ) agonists (Wong et al., 2008), however, because it is newly discovered, there is as yet little in the literature regarding the direct crosstalk between myonectin and AT.

In summary, myonectin, as well as irisin, promote glucose and fatty acid uptake and oxidation in both the liver and AT, acting more specifically on lipid metabolism mediated by CD36, fatty acid transporter protein (FATP) and fatty acid binding protein (FABP4), but not participating in lipolysis and glucose homeostasis (Gamas et al., 2015). Myonectin, is, therefore, a potent target for studies that focus on identifying future therapies, mainly related to insulin resistance. It is fundamental to characterize molecules that may present a function mimicking the effects of physical exercise and consequently positively modulating lipid homeostasis and lipogenesis in AT (Gamas et al., 2015).

\section{CONCLUSION}

Over the last decades, exercise training has been suggested as a preventive and therapeutic strategy for managing and treating several NCDs, including T2DM, hypertension, heart disease, obesity, and sarcopenia. Physical exercise is known to improve metabolic health through adaptations to several tissues, including SkM and AT. Although metabolic improvements from exercise training in SkM and AT are important in themselves, an important concept that has been evidenced in exercise physiology is the concept of tissue communication, or "crosstalk." In addition, many studies have proposed that in NCDs, mainly in those related to metabolic diseases such as obesity, a low-grade chronic inflammatory profile is a wellcharacterized scenario. Interestingly, several recent studies have characterized and identified various myokines released from SkM during and after a single bout of exercise. In particular, myokines may act pleiotropically, mediating many aspects related to AT metabolism. Myokines have also provided a new basis to understand the molecular mechanisms underlying the beneficial effects of exercise training on the reduction of morbidity and mortality rates. Although the identified myokines share a common role in regulating metabolism, how each myokine works and how these myokines work together still remains to be elucidated. In addition, given the role of myokines in fine-tuning the metabolic process associated with exercise, the development of specific exercise regimens or compounds derived from myokines that mimic the effects of exercise are promising areas to explore in the treatment of metabolic diseases through exercise.

\section{AUTHOR CONTRIBUTIONS}

MB, LL, and ML conceived the review. LL and ML analyzed the data and MB, LL, and ML wrote the paper.

\section{FUNDING}

This work was supported by São Paulo Research Foundation (FAPESP) Grants: 2010/51078-1, 2015/19259-0 
and $\mathrm{CNPq} 311966 / 2015-2$ to $\mathrm{MB}$. The contents of this article are solely the responsibility of the authors and do not necessarily represent the official views of FAPESP.

\section{REFERENCES}

Allen, D. L., Cleary, A. S., Speaker, K. J., Lindsay, S. F., Uyenishi, J., Reed, J. M., et al. (2008). Myostatin, activin receptor IIb, and follistatin-like-3 gene expression are altered in adipose tissue and skeletal muscle of obese mice. Am. J. Physiol. Endocrinol. Metab. 294, E918-E927. doi: 10.1152/ajpendo.00798.2007

Argiles, J. M., Orpi, M., Busquets, S., and Lopez-Soriano, F. J. (2012). Myostatin: more than just a regulator of muscle mass. Drug Discov. Today 17, 702-709. doi: 10.1016/j.drudis.2012.02.001

Arner, E., Westermark, P. O., Spalding, K. L., Britton, T., Ryden, M., Frisen, J., et al. (2010). Adipocyte turnover: relevance to human adipose tissue morphology. Diabetes 59, 105-109. doi: 10.2337/db09-0942

Arner, P. (2000). Obesity-a genetic disease of adipose tissue? Br. J. Nutr. 83(Suppl. 1), S9-S16. doi: 10.1017/S0007114500000891

Aydin, S., Kuloglu, T., Aydin, S., Kalayci, M., Yilmaz, M., Cakmak, T., et al. (2014). A comprehensive immunohistochemical examination of the distribution of the fat-burning protein irisin in biological tissues. Peptides 61, 130-136. doi: 10.1016/j.peptides.2014.09.014

Barauna, V. G., Batista, M. L. Jr., Costa Rosa, L. F., Casarini, D. E., Krieger, J. E., and Oliveira, E. M. (2005). Cardiovascular adaptations in rats submitted to a resistance-training model. Clin. Exp. Pharmacol. Physiol. 32, 249-254. doi: 10.1111/j.1440-1681.2005.04180.x

Batista, M. L. Jr., Neves, R. X., Peres, S. B., Yamashita, A. S., Shida, C. S., Farmer, S. R., et al. (2012). Heterogeneous time-dependent response of adipose tissue during the development of cancer cachexia. J. Endocrinol. 215, 363-373. doi: 10.1530/JOE-12-0307

Batista, M. L. Jr., Olivan, M., Alcantara, P. S., Sandoval, R., Peres, S. B., Neves, R. X., et al. (2013). Adipose tissue-derived factors as potential biomarkers in cachectic cancer patients. Cytokine 61, 532-539. doi: 10.1016/j.cyto.2012.10.023

Batista, M. L. Jr., Rosa, J. C., Lopes, R. D., Lira, F. S., Martins, E. Jr., Yamashita, A. S., et al. (2010). Exercise training changes IL-10/TNF-alpha ratio in the skeletal muscle of post-MI rats. Cytokine 49, 102-108. doi: 10.1016/j.cyto.2009.10.007

Batista, M. L. Jr., Santos, R. V., Lopes, R. D., Lopes, A. C., Costa Rosa, L. F., and Seelaender, M. C. (2008). Endurance training modulates lymphocyte function in rats with post-MI CHF. Med. Sci. Sports Exerc. 40, 549-556. doi: 10.1249/MSS.0b013e31815ed6d2

Bentzinger, C. F., Lin, S., Romanino, K., Castets, P., Guridi, M., Summermatter, S., et al. (2013). Differential response of skeletal muscles to mTORC1 signaling during atrophy and hypertrophy. Skelet. Muscle 3:6. doi: 10.1186/2044-5040-3-6

Beyaert, R., and Fiers, W. (1999). "Tumor necrosis factor and lymphotoxin," in Cytokines 2 Edn, eds A. Mire-Sluis and R. Thorpe (Blanche Lane: Academic Press), 335-345.

Bostrom, P., Wu, J., Jedrychowski, M. P., Korde, A., Ye, L., Lo, J. C., et al. (2012). A PGC1-alpha-dependent myokine that drives brown-fat-like development of white fat and thermogenesis. Nature 481, 463-468. doi: 10.1038/nature10777

Boule, N., Haddad, E., Kenny, G., Wells, G., and Sigal, R. (2001). Effects ofexercise on glycemic control and body mass in type 2 diabetes mellitus: a meta-analysis of controlled clinical trials. JAMA 286, 1218-1227. doi: $10.1001 /$ jama.286.10.1218

Braun, T., and Gautel, M. (2011). Transcriptional mechanisms regulating skeletal muscle differentiation, growth and homeostasis. Nat. Rev. Mol. Cell Biol. 12, 349-361. doi: 10.1038/nrm3118

Brocker, C., Thompson, D., Matsumoto, A., Nebert, D. W., and Vasiliou, V. (2010). Evolutionary divergence and functions of the human interleukin (IL) gene family. Hum. Genomics 5, 30-55. doi: 10.1186/1479-7364-5-1-30

Bruunsgaard, H., Ladelund, S., Pedersen, A., Schroll, M., Jorgensen, T., and Pedersen, B. (2003). Predicting death from tumour necrosis factor-alpha and interleukin-6 in 80-year-old people. Clin. Exp. Immunol. 132, 24-31. doi: 10.1046/j.1365-2249.2003.02137.x

\section{ACKNOWLEDGMENTS}

We thank all members of Laboratory of Adipose Tissue Biology for helpful discussions and critical reading of the manuscript.

Bueno, P. G., Bassi, D., Contrera, D. G., Carnielli, H. M., Silva, R. N., Nonaka, K. O., et al. (2011). Post-exercise changes in myostatin and actRIIB expression in obese insulin-resistant rats. Mol. Cell. Endocrinol. 339, 159-164. doi: 10.1016/j.mce.2011.04.006

Cani, P. D., and Jordan, B. F. (2018). Gut microbiota-mediated inflammation in obesity: a link with gastrointestinal cancer. Nat. Rev. Gastroenterol. Hepatol. 15. doi: 10.1038/s41575-018-0025-6

Castellani, L., Perry, C. G., Macpherson, R. E., Root-McCaig, J., Huber, J. S., Arkell, A. M., et al. (2015). Exercise-mediated IL-6 signaling occurs independent of inflammation and is amplified by training in mouse adipose tissue. J. Appl. Physiol. 119, 1347-1354. doi: 10.1152/japplphysiol.00551.2015

Catoire, M., Mensink, M., Kalkhoven, E., Schrauwen, P., and Kersten, S. (2014). Identification of human exercise-induced myokines using secretome analysis. Physiol. Genomics 46, 256-267. doi: 10.1152/physiolgenomics.00174. 2013

Christiansen, T., Paulsen, S. K., Bruun, J. M., Pedersen, S. B., and Richelsen, B. (2010). Exercise training versus diet-induced weight-loss on metabolic risk factors and inflammatory markers in obese subjects: a 12-week randomized intervention study. Am. J. Physiol. Endocrinol. Metab. 298, E824-E831. doi: 10.1152/ajpendo.00574.2009

Cinkajzlova, A., Mraz, M., Lacinova, Z., Klouckova, J., Kavalkova, P., Kratochvilova, H., et al. (2018). Angiopoietin-like protein 3 and 4 in obesity, type 2 diabetes mellitus, and malnutrition: the effect of weight reduction and realimentation. Nutr. Diabetes 8:21. doi: 10.1038/s41387-018-0032-2

Cuevas-Ramos, D., Almeda-Valdes, P., Meza-Arana, C. E., Brito-Cordova, G., Gomez-Perez, F. J., Mehta, R., et al. (2012). Exercise increases serum fibroblast growth factor 21 (FGF21) levels. PLoS ONE 7:e38022. doi: 10.1371/journal.pone.0038022

Cypess, A. M., Lehman, S., Williams, G., Tal, I., Rodman, D., Goldfine, A. B., et al. (2009). Identification and importance of brown adipose tissue in adult humans. N. Engl. J. Med. 360, 1509-1517. doi: 10.1056/NEJMoa0810780

Daskalopoulou, S. S., Cooke, A. B., Gomez, Y. H., Mutter, A. F., Filippaios, A., Mesfum, E. T., et al. (2014). Plasma irisin levels progressively increase in response to increasing exercise workloads in young, healthy, active subjects. Eur. J. Endocrinol. 171, 343-352. doi: 10.1530/EJE-14-0204

de Caestecker, M. (2004). The transforming growth factor-beta superfamily of receptors. Cytokine Growth Factor Rev. 15, 1-11. doi: 10.1016/j.cytogfr.2003.10.004

Duft, R. G., Castro, A., Bonfante, I. L. P., Brunelli, D. T., Chacon-Mikahil, M. P. T., and Cavaglieri, C. R. (2017). Metabolomics approach in the investigation of metabolic changes in obese men after 24 weeks of combined training. J. Proteome Res. 16, 2151-2159. doi: 10.1021/acs.jproteome.6b00967

Ebadi, M., Martin, L., Ghosh, S., Field, C. J., Lehner, R., Baracos, V. E., et al. (2017). Subcutaneous adiposity is an independent predictor of mortality in cancer patients. Br. J. Cancer 117, 148-155. doi: 10.1038/bjc.2017.149

Fagundo, A. B., Jimenez-Murcia, S., Giner-Bartolome, C., Aguera, Z., Sauchelli, S., Pardo, M., et al. (2016). Modulation of irisin and physical activity on executive functions in obesity and morbid obesity. Sci. Rep. 6:30820. doi: $10.1038 /$ srep30820

Febbraio, M. A. (2017). Exercise metabolism in 2016: Health benefits of exercise - more than meets the eye! Nat. Rev. Endocrinol. 13, 72-74. doi: $10.1038 /$ nrendo.2016.218

Febbraio, M. A., and Pedersen, B. K. (2002). Muscle-derived interleukin6: mechanisms for activation and possible biological roles. FASEB J. 16, 1335-1347. doi: 10.1096/fj.01-0876rev

Febbraio, M. A., and Pedersen, B. K. (2005). Contraction-induced myokine production and release: is skeletal muscle an endocrine organ? Exerc. Sport Sci. Rev. 33, 114-119. doi: 10.1097/00003677-200507000-00003

Fehniger, T. A., and Caligiuri, M. A. (2001). Interleukin 15: biology and relevance to human disease. Blood 97, 14-32. doi: 10.1182/blood.V97.1.14 
Feng, X. H., and Derynck, R. (2005). Specificity and versatility in tgfbeta signaling through Smads. Annu. Rev. Cell Dev. Biol. 21, 659-693. doi: 10.1146/annurev.cellbio.21.022404.142018

Fischer, C. (2006). Interleukin-6 in acute exercise and training: what is the biological relevance? Exerc. Immunol. Rev. 12, 6-33.

Fischer, C. P., Berntsen, A., Perstrup, L. B., Eskildsen, P., and Pedersen, B. K. (2007). Plasma levels of interleukin-6 and C-reactive protein are associated with physical inactivity independent of obesity. Scand. J. Med. Sci. Sports 17, 580-587. doi: 10.1111/j.1600-0838.2006.00602.x

Flynn, M. G., Mcfarlin, B. K., and Markofski, M. M. (2007). The antiinflammatory actions of exercise training. Am. J. Lifestyle Med. 1, 220-235. doi: $10.1177 / 1559827607300283$

Fouladiun, M., Korner, U., Bosaeus, I., Daneryd, P., Hyltander, A., and Lundholm, K. G. (2005). Body composition and time course changes in regional distribution of fat and lean tissue in unselected cancer patients on palliative care-correlations with food intake, metabolism, exercise capacity, and hormones. Cancer 103, 2189-2198. doi: 10.1002/cncr.21013

Franco, F. O., Lopes, M. A., Henriques, F. S., Neves, R. X., Bianchi Filho, C., and Batista, M. L. Jr. (2017). Cancer cachexia differentially regulates visceral adipose tissue turnover. J. Endocrinol. 232, 493-500. doi: 10.1530/JOE-16-0305

Furmanczyk, P. S., and Quinn, L. S. (2003). Interleukin-15 increases myosin accretion in human skeletal myogenic cultures. Cell Biol. Int. 27, 845-851. doi: 10.1016/S1065-6995(03)00172-0

Gamas, L., Matafome, P., and Seica, R. (2015). Irisin and myonectin regulation in the insulin resistant muscle: implications to adipose tissue: muscle crosstalk. J. Diabetes Res. 2015:359159. doi: 10.1155/2015/359159

GBD 2015 Risk Factors Collaborators (2016). Global, regional, and national comparative risk assessment of 79 behavioural, environmental and occupational, and metabolic risks or clusters of risks, 1990-2015: a systematic analysis for the Global Burden of Disease Study 2015. Lancet 388, 1659-1724. doi: 10.1016/S0140-6736(16)31679-8

George, I., Bish, L. T., Kamalakkannan, G., Petrilli, C. M., Oz, M. C., Naka, Y., et al. (2010). Myostatin activation in patients with advanced heart failure and after mechanical unloading. Eur. J. Heart Fail. 12, 444-453. doi: 10.1093/eurjhf/hfq039

Ginter, E., and Simko, V. (2014). Recent data on obesity research: beta-aminoisobutyric acid. Bratisl. Lek. Listy 115, 492-493. doi: 10.4149/BLL_2014_095

Goldstein, M. S. (1961). Humoral nature of the hypoglycemic factor of muscular work. Diabetes 10, 232-234. doi: 10.2337/diab.10.3.232

Greenwood, J. L., Joy, E. A., and Stanford, J. B. (2010). The physical activity vital sign: a primary care tool to guide counseling for obesity. J. Phys. Act. Health 7, 571-576. doi: 10.1123/jpah.7.5.571

Grundy, S. M. (2015). Adipose tissue and metabolic syndrome: too much, too little or neither. Eur. J. Clin. Invest. 45, 1209-1217. doi: 10.1111/eci. 12519

Guo, T., Jou, W., Chanturiya, T., Portas, J., Gavrilova, O., and Mcpherron, A. C. (2009). Myostatin inhibition in muscle, but not adipose tissue, decreases fat mass and improves insulin sensitivity. PLoS ONE 4:e4937. doi: 10.1371/journal.pone.0004937

Hamrick, M. W., Pennington, C., Webb, C. N., and Isales, C. M. (2006). Resistance to body fat gain in 'double-muscled' mice fed a high-fat diet. Int. J. Obes. 30, 868-870. doi: $10.1038 /$ sj.ijo.0803200

Han, H. Q., Zhou, X., Mitch, W. E., and Goldberg, A. L. (2013). Myostatin/activin pathway antagonism: molecular basis and therapeutic potential. Int. J. Biochem. Cell Biol. 45, 2333-2347. doi: 10.1016/j.biocel.2013.05.019

Harms, M., and Seale, P. (2013). Brown and beige fat: development, function and therapeutic potential. Nat. Med. 19, 1252-1263. doi: 10.1038/nm.3361

Hecksteden, A., Wegmann, M., Steffen, A., Kraushaar, J., Morsch, A., Ruppenthal, S., et al. (2013). Irisin and exercise training in humans results from a randomized controlled training trial. BMC Med. 11:235. doi: 10.1186/1741-7015-11-235

Heineke, J., Auger-Messier, M., Xu, J., Sargent, M., York, A., Welle, S., et al. (2010). Genetic deletion of myostatin from the heart prevents skeletal muscle atrophy in heart failure. Circulation 121, 419-425. doi: 10.1161/CIRCULATIONAHA.109.882068

Henningsen, J., Rigbolt, K. T., Blagoev, B., Pedersen, B. K., and Kratchmarova, I. (2010). Dynamics of the skeletal muscle secretome during myoblast differentiation. Mol. Cell. Proteomics 9, 2482-2496. doi: 10.1074/mcp.M110.002113

Henriques, F. S., Sertie, R. A. L., Franco, F. O., Knobl, P., Neves, R. X., et al. Jr (2017). Early suppression of adipocyte lipid turnover induces immunometabolic modulation in cancer cachexia syndrome. FASEB J. 31, 1976-1986. doi: 10.1096/fj.201601151R

Hittel, D. S., Berggren, J. R., Shearer, J., Boyle, K., and Houmard, J. A. (2009). Increased secretion and expression of myostatin in skeletal muscle from extremely obese women. Diabetes 58, 30-38. doi: 10.2337/db08-0943

Hjorth, M., Pourteymour, S., Gorgens, S. W., Langleite, T. M., Lee, S., Holen, T., et al. (2016). Myostatin in relation to physical activity and dysglycaemia and its effect on energy metabolism in human skeletal muscle cells. Acta Physiol. 217, 45-60. doi: 10.1111/apha.12631

Hojman, P., Pedersen, M., Nielsen, A. R., Krogh-Madsen, R., Yfanti, C., Akerstrom, T., et al. (2009). Fibroblast growth factor-21 is induced in human skeletal muscles by hyperinsulinemia. Diabetes 58, 2797-2801. doi: 10.2337/db09-0713

Hornberger, T. A. (2011). Mechanotransduction and the regulation of mTORC1 signaling in skeletal muscle. Int. J. Biochem. Cell Biol. 43, 1267-1276. doi: 10.1016/j.biocel.2011.05.007

Huh, J. Y., Mougios, V., Kabasakalis, A., Fatouros, I., Siopi, A., Douroudos, I.i, et al. (2014). Exercise-induced irisin secretion is independent of age or fitness level and increased irisin may directly modulate muscle metabolism through AMPK activation. J. Clin. Endocrinol. Metab. 99, E2154-E2161. doi: 10.1210/jc.2014-1437

Huh, J. Y., Panagiotou, G., Mougios, V., Brinkoetter, M., Vamvini, M. T., Schneider, B. E., et al. (2012). FNDC5 and irisin in humans: I. Predictors of circulating concentrations in serum and plasma and II. mRNA expression and circulating concentrations in response to weight loss and exercise. Metabolism 61, 1725-1738. doi: 10.1016/j.metabol.2012.09.002

Huh, J. Y., Siopi, A., Mougios, V., Park, K. H., and Mantzoros, C. S. (2015). Irisin in response to exercise in humans with and without metabolic syndrome. J. Clin. Endocrinol. Metab. 100, E453-E457. doi: 10.1210/jc.2014-2416

Iizuka, K., Machida, T., and Hirafuji, M. (2014). Skeletal muscle is an endocrine organ. J. Pharmacol. Sci. 125, 125-131. doi: 10.1254/jphs.14R02CP

Imazu, A. A., Goessler, K. F., Casonatto, J., and Polito, M. D. (2017). The influence of physical training status on postexercise hypotension in patients with hypertension: a cross-sectional study. Blood Press. Monit. 22, 196-201. doi: 10.1097/MBP.0000000000000255

Ingerslev, B., Hansen, J. S., Hoffmann, C., Clemmesen, J. O., Secher, N. H., Scheler, M., et al. (2017). Angiopoietin-like protein 4 is an exercise-induced hepatokine in humans, regulated by glucagon and cAMP. Mol. Metab. 6, 1286-1295. doi: 10.1016/j.molmet.2017.06.018

Ishiuchi, Y., Sato, H., Tsujimura, K., Kawaguchi, H., Matsuwaki, T., Yamanouchi, K., et al. (2018). Skeletal muscle cell contraction reduces a novel myokine, chemokine (C-X-C motif) ligand 10 (CXCL10): potential roles in exercise-regulated angiogenesis. Biosci. Biotechnol. Biochem. 82, 97-105. doi: 10.1080/09168451.2017.1411778

Jeremic, N., Chaturvedi, P., and Tyagi, S. C. (2017). Browning of white fat: novel insight into factors, mechanisms, and therapeutics. J. Cell. Physiol. 232, 61-68. doi: $10.1002 /$ jcp. 25450

Jung, T. W., Park, H. S., Choi, G. H., Kim, D., and Lee, T. (2018). $\beta$ aminoisobutyric acid attenuates LPS-induced inflammation and insulin resistance in adipocytes through AMPK-mediated pathway. J. Biomed. Sci. 25:27. doi: $10.1186 / \mathrm{s} 12929-018-0431-7$

Kajimura, S., Spiegelman, B. M., and Seale, P. (2015). Brown and beige fat: physiological roles beyond heat generation. Cell Metab. 22, 546-559. doi: 10.1016/j.cmet.2015.09.007

Kammoun, H. L., and Febbraio, M. A. (2014). Come on BAIBA light my fire. Cell Metab. 19, 1-2. doi: 10.1016/j.cmet.2013.12.007

Karamanlidis, G., Karamitri, A., Docherty, K., Hazlerigg, D. G., and Lomax, M. A. (2007). C/EBPbeta reprograms white 3T3-L1 preadipocytes to a Brown adipocyte pattern of gene expression. J. Biol. Chem. 282, 24660-24669. doi: $10.1074 /$ jbc.M703101200

Karstoft, K., and Pedersen, B. K. (2016). Skeletal muscle as a gene regulatory endocrine organ. Curr. Opin. Clin. Nutr. Metab. Care 19, 270-275. doi: 10.1097/MCO.0000000000000283

Keller, C., Steensberg, A., Pilegaard, H., Osada, T., Saltin, B., Pedersen, B. K., et al. (2001). Transcriptional activation of the IL-6 gene in human contracting 
skeletal muscle: influence of muscle glycogen content. FASEB J. 15, 2748-2750. doi: 10.1096/fj.01-0507fje

Kershaw, E. E., and Flier, J. S. (2004). Adipose tissue as an endocrine organ. J. Clin. Endocrinol. Metab. 89, 2548-2556. doi: 10.1210/jc.2004-0395

Kharitonenkov, A., Shiyanova, T. L., Koester, A., Ford, A. M., Micanovic, R., Galbreath, E. J., et al. (2005). FGF-21 as a novel metabolic regulator. J. Clin. Invest. 115, 1627-1635. doi: 10.1172/JCI23606

Kharitonenkov, A., Wroblewski, V. J., Koester, A., Chen, Y. F., Clutinger, C. K., Tigno, X. T., et al. (2007). The metabolic state of diabetic monkeys is regulated by fibroblast growth factor-21. Endocrinology 148, 774-781. doi: 10.1210/en.2006-1168

Kim, H. J., So, B., Choi, M., Kang, D., and Song, W. (2015). Resistance exercise training increases the expression of irisin concomitant with improvement of muscle function in aging mice and humans. Exp. Gerontol. 70, 11-17. doi: 10.1016/j.exger.2015.07.006

Kim, K. H., Jeong, Y. T., Oh, H., Kim, S. H., Cho, J. M., Kim, Y. N., et al. (2013a). Autophagy deficiency leads to protection from obesity and insulin resistance by inducing Fgf21 as a mitokine. Nat. Med. 19, 83-92. doi: 10.1038/nm.3014

Kim, K. H., Kim, S. H., Min, Y. K., Yang, H. M., Lee, J. B., and Lee, M. S. (2013b). Acute exercise induces FGF21 expression in mice and in healthy humans. PLoS ONE 8:e63517. doi: 10.1371/journal.pone.0063517

Kir, S., White, J. P., Kleiner, S., Kazak, L., Cohen, P., Baracos, V. E., et al. (2014). Tumour-derived PTH-related protein triggers adipose tissue browning and cancer cachexia. Nature 513, 100-104. doi: 10.1038/nature13528

Kjaer, M., Pollack, S. F., Mohr, T., Weiss, H., Gleim, G. W., Bach, F. W., et al. (1996). Regulation of glucose turnover and hormonal responses during electrical cycling in tetraplegic humans. Am. J. Physiol. 271, R191-R199. doi: 10.1152/ajpregu.1996.271.1.R191

Kurdiova, T., Balaz, M., Vician, M., Maderova, D., Vlcek, M., Valkovic, L., et al. (2014). Effects of obesity, diabetes and exercise on Fndc5 gene expression and irisin release in human skeletal muscle and adipose tissue: in vivo and in vitro studies. J. Physiol. 592, 1091-1107. doi: 10.1113/jphysiol.2013.264655

Kusminski, C. M., Bickel, P. E., and Scherer, P. E. (2016). Targeting adipose tissue in the treatment of obesity-associated diabetes. Nat. Rev. Drug Discov. 15, 639-660. doi: 10.1038/nrd.2016.75

Lancaster, G. I., and Febbraio, M. A. (2014). The immunomodulating role of exercise in metabolic disease. Trends Immunol. 35, 262-269. doi: 10.1016/j.it.2014.02.008

Laterza, M. C., De Matos, L. D., Trombetta, I. C., Braga, A. M., Roveda, F., Alves, M. J., et al. (2007). Exercise training restores baroreflex sensitivity in never-treated hypertensive patients. Hypertension 49, 1298-1306. doi: 10.1161/HYPERTENSIONAHA.106.085548

Lebrasseur, N. K., Schelhorn, T. M., Bernardo, B. L., Cosgrove, P. G., Loria, P. M., and Brown, T. A. (2009). Myostatin inhibition enhances the effects of exercise on performance and metabolic outcomes in aged mice. J. Gerontol. A Biol. Sci. Med. Sci. 64, 940-948. doi: 10.1093/gerona/glp068

Lee, I. M., Shiroma, E. J., Lobelo, F., Puska, P., Blair, S. N., Katzmarzyk, P. T., et al. (2012). Effect of physical inactivity on major non-communicable diseases worldwide: an analysis of burden of disease and life expectancy. Lancet 380, 219-229. doi: 10.1016/S0140-6736(12)61031-9

Lee, P., Linderman, J. D., Smith, S., Brychta, R. J., Wang, J., Idelson, C., et al. (2014). Irisin and FGF21 are cold-induced endocrine activators of brown fat function in humans. Cell Metab. 19, 302-309. doi: 10.1016/j.cmet.2013.12.017

Lehmann, M., Foster, C., and Keul, J. (1993a). Overtraining in endurance athletes: a brief review. Med. Sci. Sports Exerc. 25, 854-862. doi: 10.1249/00005768-199307000-00015

Lehmann, M., Knizia, K., Gastmann, U., Petersen, K., Khalaf, A., Bauer, S., et al. (1993b). Influence of 6-week, 6 days per week, training on pituitary function in recreational athletes. Br. J. Sports Med. 27, 186-192. doi: 10.1136/bjsm.27.3.186

Li, K., Liao, X., Wang, K., Mi, Q., Zhang, T., Jia, Y., et al. (2018). Myonectin predicts the development of type 2 diabetes. J. Clin. Endocrinol. Metab. 103, 139-147. doi: 10.1210/jc.2017-01604

Li, Y., Wong, K., Giles, A., Jiang, J., Lee, J. W., Adams, A. C., et al. (2014). Hepatic SIRT1 attenuates hepatic steatosis and controls energy balance in mice by inducing fibroblast growth factor 21. Gastroenterology 146, 539-549 e537. doi: 10.1053/j.gastro.2013.10.059

Lira, F. S., Koyama, C. H., Yamashita, A. S., Rosa, J. C., Zanchi, N. E., Batista, M. L. Jr., et al. (2009). Chronic exercise decreases cytokine production in healthy rat skeletal muscle. Cell Biochem. Funct. 27, 458-461. doi: 10.1002/ cbf.1594

Lobelo, F., Stoutenberg, M., and Hutber, A. (2014). The exercise is medicine global health initiative: a 2014 update. Br. J. Sports Med. 48, 1627-1633. doi: 10.1136/bjsports-2013-093080

Lovren, F., Teoh, H., and Verma, S. (2015). Obesity and atherosclerosis: mechanistic insights. Can. J. Cardiol. 31, 177-183. doi: 10.1016/j.cjca.2014.11.031

Macdonald, C., Wojtaszewski, J. F., Pedersen, B. K., Kiens, B., and Richter, E. A. (2003). Interleukin-6 release from human skeletal muscle during exercise: relation to AMPK activity. J. Appl. Physiol. 95, 2273-2277. doi: 10.1152 /japplphysiol.00242.2003

Macpherson, R. E., Huber, J. S., Frendo-Cumbo, S., Simpson, J. A., and Wright, D. C. (2015). Adipose tissue insulin action and IL-6 signaling after exercise in obese mice. Med. Sci. Sports Exerc. 47, 2034-2042. doi: 10.1249/MSS.0000000000000660

Mahtani, K. R., Mcmanus, J., and Nunan, D. (2015). Physical activity and obesity editorial: is exercise pointless or was it a pointless exercise? Br. J. Sports Med. 49, 969-970. doi: 10.1136/bjsports-2015-095005

Martin, K. A., Mani, M. V., and Mani, A. (2015). New targets to treat obesity and the metabolic syndrome. Eur. J. Pharmacol. 763, 64-74. doi: 10.1016/j.ejphar.2015.03.093

Mitsui, T., Nakamura, T., Ito, T., Umemoto, Y., Sakamoto, K., Kinoshita, T., et al. (2012). Exercise significantly increases plasma adrenaline and oxidized low-density lipoprotein in normal healthy subjects but not in persons with spinal cord injury. Arch. Phys. Med. Rehabil. 93, 725-727. doi: 10.1016/j.apmr.2011.08.046

Miyamoto-Mikami, E., Sato, K., Kurihara, T., Hasegawa, N., Fujie, S., Fujita, S., et al. (2015). Endurance training-induced increase in circulating irisin levels is associated with reduction of abdominal visceral fat in middleaged and older adults. PLoS ONE 10:e0120354. doi: 10.1371/journal.pone. 0120354

Moraes, C., Leal, V. O., Marinho, S. M., Barroso, S. G., Rocha, G. S., Boaventura, G. T., et al. (2013). Resistance exercise training does not affect plasma irisin levels of hemodialysis patients. Horm. Metab. Res. 45, 900-904. doi: $10.1055 / \mathrm{s}-0033-1354402$

Morris, A. (2018). Obesity: ANGPTL4 - the link binding obesity and glucose intolerance. Nat. Rev. Endocrinol. 14:251. doi: 10.1038/nrendo.2018.35

Mraz, M., and Haluzik, M. (2014). The role of adipose tissue immune cells in obesity and low-grade inflammation. J. Endocrinol. 222, R113-R127. doi: 10.1530/JOE-14-0283

Negrin, K. A., Roth Flach, R. J., Distefano, M. T., Matevossian, A., Friedline, R. H., Jung, D., et al. (2014). IL-1 signaling in obesity-induced hepatic lipogenesis and steatosis. PLoS ONE 9:e107265. doi: 10.1371/journal.pone.0107265

Nielsen, A. R., Hojman, P., Erikstrup, C., Fischer, C. P., Plomgaard, P., Mounier, R., et al. (2008). Association between interleukin-15 and obesity: interleukin-15 as a potential regulator of fat mass. J. Clin. Endocrinol. Metab. 93, 4486-4493. doi: 10.1210/jc.2007-2561

Nielsen, A. R., Mounier, R., Plomgaard, P., Mortensen, O. H., Penkowa, M., Speerschneider, T., et al. (2007). Expression of interleukin-15 in human skeletal muscle effect of exercise and muscle fibre type composition. J. Physiol. 584, 305-312. doi: 10.1113/jphysiol.2007.139618

Nielsen, S., and Pedersen, B. K. (2008). Skeletal muscle as an immunogenic organ. Curr. Opin. Pharmacol. 8, 346-351. doi: 10.1016/j.coph.2008.02.005

Nieman, D. C., Nehlsen-Cannarella, S. L., Fagoaga, O. R., Henson, D. A., Utter, A., Davis, J. M., et al. (1998). Influence of mode and carbohydrate on the cytokine response to heavy exertion. Med. Sci. Sports Exerc. 30, 671-678. doi: 10.1097/00005768-199805000-00005

Norheim, F., Langleite, T. M., Hjorth, M., Holen, T., Kielland, A., Stadheim, H. K., et al. (2014). The effects of acute and chronic exercise on PGC-1alpha, irisin and browning of subcutaneous adipose tissue in humans. FEBS J. 281, 739-749. doi: $10.1111 /$ febs.12619

Nunan, D., Mahtani, K. R., Roberts, N., and Heneghan, C. (2013). Physical activity for the prevention and treatment of major chronic disease: an overview of systematic reviews. Syst. Rev. 2:56. doi: 10.1186/2046-4053-2-56

Osborn, O., and Olefsky, J. M. (2012). The cellular and signaling networks linking the immune system and metabolism in disease. Nat. Med. 18, 363-374. doi: $10.1038 / \mathrm{nm} .2627$ 
Pajak, B., Orzechowska, S., Pijet, B., Pijet, M., Pogorzelska, A., Gajkowska, B., et al. (2008). Crossroads of cytokine signaling-the chase to stop muscle cachexia. J. Physiol. Pharmacol. 59(Suppl. 9), 251-264.

Paley, C. A., and Johnson, M. I. (2018). Abdominal obesity and metabolic syndrome: exercise as medicine? BMC Sports Sci. Med. Rehabil. 10:7. doi: 10.1186/s13102-018-0097-1

Pardo, M., Crujeiras, A. B., Amil, M., Aguera, Z., Jimenez-Murcia, S., Banos, R., et al. (2014). Association of irisin with fat mass, resting energy expenditure, and daily activity in conditions of extreme body mass index. Int. J. Endocrinol. 2014:857270. doi: 10.1155/2014/857270

Pedersen, B. K. (2007). State of the art reviews: health benefits related to exercise in patients with chronic low-grade systemic inflammation. Am. J. Lifestyle Med. 1, 289-298. doi: 10.1177/1559827607301410

Pedersen, B. K. (2011). Muscles and their myokines. J. Exp. Biol. 214, 337-346. doi: $10.1242 /$ jeb.048074

Pedersen, B. K. (2013). Muscle as a secretory organ. Compr. Physiol. 3, 1337-1362. doi: $10.1002 /$ cphy.c120033

Pedersen, B. K., Akerstrom, T. C. A., Nielsen, A. R., and Fischer, C. P. (2007). Role of myokines in exercise and metabolism. J. Appl. Physiol. 103, 1093-1098. doi: 10.1152/japplphysiol.00080.2007

Pedersen, B. K., and Febbraio, M. A. (2012). Muscles, exercise and obesity: skeletal muscle as a secretory organ. Nat. Rev. Endocrinol. 8, 457-465. doi: $10.1038 /$ nrendo.2012.49

Pedersen, B. K., and Fischer, C. P. (2007). Beneficial health effects of exercise - the role of IL-6 as a myokine. Trends Pharmacol. Sci. 28, 152-156. doi: 10.1016/j.tips.2007.02.002

Pedersen, B. K., Steensberg, A., Fischer, C., Keller, C., Keller, P., Plomgaard, P., et al. (2003). Searching for the exercise factor: is IL-6 a candidate? J. Muscle Res. Cell Motil. 24, 113-119. doi: 10.1023/A:1026070911202

Pedersen, L., Idorn, M., Olofsson, G. H., Lauenborg, B., Nookaew, I., Hansen, R. H., et al. (2016). Voluntary running suppresses tumor growth through epinephrine- and IL-6-Dependent NK cell mobilization and redistribution. Cell Metab. 23, 554-562. doi: 10.1016/j.cmet.2016.01.011

Pekkala, S., Wiklund, P. K., Hulmi, J. J., Ahtiainen, J. P., Horttanainen, M., Pollanen, E., et al. (2013). Are skeletal muscle FNDC5 gene expression and irisin release regulated by exercise and related to health? J. Physiol. 591, 5393-5400. doi: 10.1113/jphysiol.2013.263707

Petersen, A. M. W., and Pedersen, B. K. (2005). The anti-inflammatory effect of exercise. J. Appl. Physiol. 98, 1154-1162. doi: 10.1152/japplphysiol.00164.2004

Peterson, J. M., Mart, R., and Bond, C. E. (2014). Effect of obesity and exercise on the expression of the novel myokines, Myonectin and Fibronectin type III domain containing 5. Peer J. 2:e605. doi: 10.7717/peerj.605

Popova, P., Vasilyeva, L., Tkachuck, A., Puzanov, M., Golovkin, A., Bolotko, Y., et al. (2018). A randomised, controlled study of different glycaemic targets during gestational diabetes treatment: effect on the level of adipokines in cord blood and ANGPTL4 expression in human umbilical vein endothelial cells. Int. J. Endocrinol. 2018:6481658. doi: 10.1155/2018/64 81658

Pratesi, A., Tarantini, F., and Di Bari, M. (2013). Skeletal muscle: an endocrine organ. Clin. Cases Miner. Bone Metab. 10, 11-14. doi: $10.11138 / \mathrm{ccmbm} / 2013.10 .1 .011$

Prins, J. B. (2002). Adipose tissue as an endocrine organ. Best Pract. Res. Clin. Endocrinol. Metab. 16, 639-651. doi: 10.1053/beem.2002.0222

Pulgaron, E. R., and Delamater, A. M. (2014). Obesity and type 2 diabetes in children: epidemiology and treatment. Curr. Diab. Rep. 14:508. doi: 10.1007/s11892-014-0508-y

Quinn, L. S., Anderson, B. G., Conner, J. D., Pistilli, E. E., and Wolden-Hanson, T. (2011). Overexpression of interleukin-15 in mice promotes resistance to diet-induced obesity, increased insulin sensitivity, and markers of oxidative skeletal muscle metabolism. Int. J. Interferon. Cytokine Mediat. Res. 3, 29-42. doi: 10.2147/IJICMR.S19007

Quinn, L. S., Anderson, B. G., Conner, J. D., and Wolden-Hanson, T. (2013). IL-15 overexpression promotes endurance, oxidative energy metabolism, and muscle PPARdelta, SIRT1, PGC-1alpha, and PGC-1beta expression in male mice. Endocrinology 154, 232-245. doi: 10.1210/en.2012-1773

Quinn, L. S., Anderson, B. G., Drivdahl, R. H., Alvarez, B., and Argiles, J. M. (2002). Overexpression of interleukin-15 induces skeletal muscle hypertrophy in vitro: implications for treatment of muscle wasting disorders. Exp. Cell Res. 280, 55-63. doi: 10.1006/excr.2002.5624

Quinn, L. S., Anderson, B. G., Strait-Bodey, L., Stroud, A. M., and Argiles, J. M. (2009). Oversecretion of interleukin-15 from skeletal muscle reduces adiposity. Am. J. Physiol. Endocrinol. Metab. 296, E191-E202. doi: 10.1152/ajpendo.90506.2008

Raschke, S., and Eckel, J. (2013). Adipo-myokines: two sides of the same coinmediators of inflammation and mediators of exercise. Mediators Inflamm. 2013:320724. doi: 10.1155/2013/320724

Roberts, L. D., Bostrom, P., O’sullivan, J. F., Schinzel, R. T., Lewis, G. D., Dejam, A., et al. (2014). $\beta$-Aminoisobutyric acid induces browning of white fat and hepatic beta-oxidation and is inversely correlated with cardiometabolic risk factors. Cell Metab. 19, 96-108. doi: 10.1016/j.cmet.2013.12.003

Roca-Rivada, A., Castelao, C., Senin, L. L., Landrove, M. O., Baltar, J., Belen Crujeiras, A., et al. (2013). FNDC5/irisin is not only a myokine but also an adipokine. PLoS ONE 8:e60563. doi: 10.1371/journal.pone.0060563

Rodriguez, J., Vernus, B., Chelh, I., Cassar-Malek, I., Gabillard, J. C., Hadj Sassi, A., et al. (2014). Myostatin and the skeletal muscle atrophy and hypertrophy signaling pathways. Cell. Mol. Life Sci. 71, 4361-4371doi: 10.1007/s00018-0141689-x

Rosa Neto, J. C., Lira, F. S., Oyama, L. M., Zanchi, N. E., Yamashita, A. S., Batista, M. L. Jr., et al. (2009). Exhaustive exercise causes an anti-inflammatory effect in skeletal muscle and a pro-inflammatory effect in adipose tissue in rats. Eur. J. Appl. Physiol. 106, 697-704. doi: 10.1007/s00421-009-1070-1

Rosen, E. D., and Spiegelman, B. M. (2006). Adipocytes as regulators of energy balance and glucose homeostasis. Nature 444, 847-853. doi: 10.1038/nature05483

Ruiz-Casado, A., Martin-Ruiz, A., Perez, L. M., Provencio, M., Fiuza-Luces, C., and Lucia, A. (2017). Exercise and the Hallmarks of Cancer. Trends Cancer 3, 423-441. doi: 10.1016/j.trecan.2017.04.007

Salanova, M., Gelfi, C., Moriggi, M., Vasso, M., Vigano, A., Minafra, L., et al. (2014). Disuse deterioration of human skeletal muscle challenged by resistive exercise superimposed with vibration: evidence from structural and proteomic analysis. FASEB J. 28, 4748-4763. doi: 10.1096/fj.14-252825

Scheler, M., Irmler, M., Lehr, S., Hartwig, S., Staiger, H., Al-Hasani, H., et al. (2013). Cytokine response of primary human myotubes in an in vitro exercise model. Am. J. Physiol. Cell Physiol. 305, C877-C886. doi: 10.1152/ajpcell.000 43.2013

Scherer, P. E. (2016). The multifaceted roles of adipose tissue-therapeutic targets for diabetes and beyond: the 2015 banting lecture. Diabetes 65, 1452-1461. doi: $10.2337 / \mathrm{db} 16-0339$

Seldin, M. M., Peterson, J. M., Byerly, M. S., Wei, Z., and Wong, G. W. (2012). Myonectin (CTRP15), a novel myokine that links skeletal muscle to systemic lipid homeostasis. J. Biol. Chem. 287, 11968-11980. doi: 10.1074/jbc.M111.336834

Shan, T., Liang, X., Bi, P., and Kuang, S. (2013). Myostatin knockout drives browning of white adipose tissue through activating the AMPK-PGC1alphaFndc5 pathway in muscle. FASEB J. 27, 1981-1989. doi: 10.1096/fj.12-225755

Sharman, J. E., La Gerche, A., and Coombes, J. S. (2015). Exercise and cardiovascular risk in patients with hypertension. Am. J. Hypertens. 28, 147-158. doi: 10.1093/ajh/hpu191

Sheehan, S. M., Tatsumi, R., Temm-Grove, C. J., and Allen, R. E. (2000). HGF is an autocrine growth factor for skeletal muscle satellite cells in vitro. Muscle Nerve 23, 239-245. doi: 10.1002/(SICI)1097-4598(200002)23:2<239::AID-MUS15>3. $0 . \mathrm{CO} ; 2-\mathrm{U}$

Sidossis, L., and Kajimura, S. (2015). Brown and beige fat in humans: thermogenic adipocytes that control energy and glucose homeostasis. J. Clin. Invest. 125, 478-486. doi: 10.1172/JCI78362

Smith, J. K. (2018). Exercise, obesity and CNS control of metabolic homeostasis: a review. Front. Physiol. 9:574. doi: 10.3389/fphys.2018.00574

Spinedi, E., and Gaillard, R. C. (1998). A regulatory loop between the hypothalamo-pituitary-adrenal (HPA) axis and circulating leptin: a physiological role of ACTH. Endocrinology 139, 4016-4020. doi: 10.1210/endo.139.9.6291

Starkie, R., Ostrowski, S. R., Jauffred, S., Febbraio, M., and Pedersen, B. K. (2003). Exercise and IL-6 infusion inhibit endotoxin-induced TNF-\{alpha\} production in humans. FASEB J. 17, 884-886. doi: 10.1096/fj.02-0670fje 
Starkie, R. L., Arkinstall, M. J., Koukoulas, I., Hawley, J. A., and Febbraio, M. A. (2001). Carbohydrate ingestion attenuates the increase in plasma interleukin6, but not skeletal muscle interleukin-6 mRNA, during exercise in humans. J. Physiol. 533, 585-591. doi: 10.1111/j.1469-7793.2001.0585a.x

Steensberg, A., Fischer, C. P., Keller, C., Moller, K., and Pedersen, B. K. (2003). IL-6 enhances plasma IL-1ra, IL-10, and cortisol in humans. Am. J. Physiol. Endocrinol. Metab. 285, E433-E437. doi: 10.1152/ajpendo.00074.2003

Steinacker, J., Lormes, W., Reissnecker, S., and Liu, Y. (2004). New aspects of the hormone and cytokine response to training. Eur. J. Appl. Physiol. 91, 382-391. doi: 10.1007/s00421-003-0960-x

Tan, S., Du, P., Zhao, W., Pang, J., and Wang, J. (2018). Exercise training at maximal fat oxidation intensity for older women with type 2 diabetes. Int. J. Sports Med. 39, 374-381. doi: 10.1055/a-0573-1509

Thomas, G. A., Kraemer, W. J., Comstock, B. A., Dunn-Lewis, C., Maresh, C. M., and Volek, J. S. (2013). Obesity, growth hormone and exercise. Sports Med. 43, 839-849. doi: 10.1007/s40279-013-0064-7

Tisi, P., Hulse, M., Chulakadabba, A., Gosling, P., and Shearman, C. (1997). Exercise training for intermittent claudication: does it adversely affect biochemical markers of the exercise-induced inflammatory response? Eur. J. Vasc. Endovasc. Surg. 14, 344-350. doi: 10.1016/S1078-5884(97)8 0283-3

Toloza, F. J. K., Mantilla-Rivas, J. O., Perez-Matos, M. C., Ricardo-Silgado, M. L., Morales-Alvarez, M. C., Pinzon-Cortes, J. A., et al. (2018). Plasma levels of myonectin but not myostatin or fibroblast-derived growth factor 21 are associated with insulin resistance in adult humans without diabetes mellitus. Front. Endocrinol. 9:5. doi: 10.3389/fendo.2018.00005

Trayhurn, P., and Wood, I. S. (2004). Adipokines: inflammation and the pleiotropic role of white adipose tissue. Br. J. Nutr. 92, 347-355. doi: 10.1079/BJN20041213

Tsoli, M., Moore, M., Burg, D., Painter, A., Taylor, R., Lockie, S. H., et al. (2012). Activation of thermogenesis in brown adipose tissue and dysregulated lipid metabolism associated with cancer cachexia in mice. Cancer Res. 72, 4372-4382. doi: 10.1158/0008-5472.CAN-11-3536

Tsuchiya, Y., Ando, D., Goto, K., Kiuchi, M., Yamakita, M., and Koyama, K. (2014). High-intensity exercise causes greater irisin response compared with low-intensity exercise under similar energy consumption. Tohoku J. Exp. Med. 233, 135-140. doi: 10.1620/tjem.233.135
Warburton, D. E., Nicol, C. W., and Bredin, S. S. (2006). Health benefits of physical activity: the evidence. CMAJ 174, 801-809. doi: 10.1503/cmaj.051351

WHO (2014). Global Status Report on Noncommunicable Diseases 2014.

Williamson, D. L., Gallagher, P. M., Carroll, C. C., Raue, U., and Trappe, S. W. (2001). Reduction in hybrid single muscle fiber proportions with resistance training in humans. J. Appl. Physiol. 91, 1955-1961. doi: 10.1152/jappl.2001.91.5.1955

Wong, G. W., Krawczyk, S. A., Kitidis-Mitrokostas, C., Revett, T., Gimeno, R., and Lodish, H. F. (2008). Molecular, biochemical and functional characterizations of C1q/TNF family members: adipose-tissue-selective expression patterns, regulation by PPAR-gamma agonist, cysteine-mediated oligomerizations, combinatorial associations and metabolic functions. Biochem. J. 416, 161-177. doi: 10.1042/BJ20081240

Wu, J., Bostrom, P., Sparks, L. M., Ye, L., Choi, J. H., Giang, A. H., et al. (2012). Beige adipocytes are a distinct type of thermogenic fat cell in mouse and human. Cell 150, 366-376. doi: 10.1016/j.cell.2012.05.016

Wu, J., Cohen, P., and Spiegelman, B. M. (2013). Adaptive thermogenesis in adipocytes: is beige the new brown? Genes Dev. 27, 234-250. doi: $10.1101 /$ gad.211649.112

Zhang, C., Mcfarlane, C., Lokireddy, S., Bonala, S., Ge, X., Masuda, S., et al. (2011). Myostatin-deficient mice exhibit reduced insulin resistance through activating the AMP-activated protein kinase signalling pathway. Diabetologia 54, 1491-1501. doi: 10.1007/s00125-011-2079-7

Zhao, B., Wall, R. J., and Yang, J. (2005). Transgenic expression of myostatin propeptide prevents diet-induced obesity and insulin resistance. Biochem. Biophys. Res. Commun. 337, 248-255. doi: 10.1016/j.bbrc.2005.09.044

Conflict of Interest Statement: The authors declare that the research was conducted in the absence of any commercial or financial relationships that could be construed as a potential conflict of interest.

Copyright (c) 2018 Leal, Lopes and Batista. This is an open-access article distributed under the terms of the Creative Commons Attribution License (CC BY). The use, distribution or reproduction in other forums is permitted, provided the original author(s) and the copyright owner(s) are credited and that the original publication in this journal is cited, in accordance with accepted academic practice. No use, distribution or reproduction is permitted which does not comply with these terms. 


\title{
HDAC4 Regulates Skeletal Muscle Regeneration via Soluble Factors
}

\author{
Alessandra Renzini ${ }^{1+}$, Nicoletta Marroncelli ${ }^{1 \dagger}$, Chiara Noviello ${ }^{1}$, Viviana Moresi ${ }^{1,2 *}$ and \\ Sergio Adamo' \\ ${ }^{1}$ DAHFMO Unit of Histology and Medical Embryology, Interuniversity Institute of Myology, Sapienza University of Rome, \\ Rome, Italy, ${ }^{2}$ Laboratory of Cardiovascular Endocrinology, IRCCS San Raffaele Pisana, Rome, Italy
}

Skeletal muscle possesses a high ability to regenerate after an insult or in pathological conditions, relying on satellite cells, the skeletal muscle stem cells. Satellite cell behavior is tightly regulated by the surrounding microenvironment, which provides multiple signals derived from local cells and systemic factors. Among epigenetic mechanisms, histone deacetylation has been proved to affect muscle regeneration. Indeed, panhistone deacetylase inhibitors were found to improve muscle regeneration, while deletion of histone deacetylase 4 (HDAC4) in satellite cells inhibits their proliferation and differentiation, leading to compromised muscle regeneration. In this study, we delineated the HDAC4 function in adult skeletal muscle, following injury, by using a tissue-specific null mouse line. We showed that HDAC4 is crucial for skeletal muscle regeneration by mediating soluble factors that influence muscle-derived cell proliferation and differentiation. These findings add new biological functions to HDAC4 in skeletal muscle that need considering when administering histone deacetylase inhibitors.

Keywords: HDAC inhibitors, satellite cells, muscle regeneration, soluble factors, muscular dystrophies

\section{INTRODUCTION}

Skeletal muscle possesses a high capacity to regenerate and muscle regeneration has been extensively studied since the $50^{\prime}$ s. In addition to physiological demand, such as during muscle growth or upon exercise, new muscle fibers are generated in response to muscle damage following injury or in degenerative diseases. Although several types of cells, including muscle-derived cells (MDCs), contribute to skeletal muscle regeneration, a crucial role of adult muscle stem cells, i.e., satellite cells (SCs), in this process is now well established (Torrente et al., 2001; Jankowski et al., 2002).

Usually quiescent, upon proper stimulation SCs become activated, proliferate, differentiate, and fuse to repair damaged myofibers or to create newly formed ones (Dumont et al., 2015). SC expansion, commitment, differentiation and fusion are sequential phases, strictly controlled by numerous transcription factors, also considered as specific stage-markers of SCs. Quiescent and activated SCs do express Pax7, a paired box transcription factor (Le Grand and Rudnicki, 2007; Wang and Rudnicki, 2012). Upon activation, SCs proliferate and can either generate selfrenewing stem cells or differentiated ones, depending on symmetric or asymmetric division (Kuang et al., 2007). Once SCs are committed to myogenesis, they start expressing other transcription factors, such as Myf5 or MyoD (Dumont et al., 2015). The transition from myoblast to myotube is mainly regulated by $\mathrm{MyoD}$, which directly regulates the transcription of the other myogenic regulatory factor (MRF) family members, myogenin and MRF4. In addition, myocyte enhancer factor-2 (Mef2) proteins co-operate with MRFs to activate the expression of skeletal muscle 
terminal differentiation genes, such as myosin heavy chains (MHCs) or creatine kinase (Lilly et al., 1994; Black and Olson, 1998) Myocytes fusion requires the expression of specific genes, including myomaker and myomerger (Millay et al., 2013; Quinn et al., 2017).

In adult skeletal myofibers, SCs reside between the basal lamina and the sarcolemma, in a surrounding microenvironment referred to as "niche." SC niche consists of the basement membrane, extracellular matrix, vascular and neural networks, several types of surrounding and interstitial cells and several diffusible molecules (Yin et al., 2013; Furuichi and Fujii, 2017). Structural and biochemical cues from the niche act on SC behavior to regulate cell quiescence, self-renewal, proliferation or differentiation, through cell-cell interactions or paracrine signals. Soluble factors play a critical role in the maintenance of muscle homeostasis in physiological conditions and may be involved in the progression of skeletal muscle diseases (De Pasquale et al., 2012; De Paepe and De Bleecker, 2013; Furuichi and Fujii, 2017). An increasing number of diffusible molecules secreted by skeletal muscle have been discovered playing a role in SC behavior. Among them, fibroblast growth factor (FGF), transforming growth factor-beta (TGF- $\beta$ ), tumor necrosis factoralpha (TNF- $\alpha$ ) or obestatin modulate different phases of muscle regeneration, in both physiological and pathological conditions (Floss et al., 1997; Coletti et al., 2005; Gurriarán-Rodríguez et al., 2015; Delaney et al., 2017; Pawlikowski et al., 2017). Also, circulating hormones regulate SC pool and skeletal muscle regeneration (Moresi et al., 2009; Costa et al., 2014; Kim et al., 2016).

Several epigenetic mechanisms are active at multiple steps of muscle regeneration, fine-tuning SC gene expression (Giordani and Puri, 2013; Moresi et al., 2015). Proper chromatin remodeling accompanies SC activation and differentiation. A permissive chromatin state that characterizes and distinguishes the pluripotency of stem cells is established by the general lack of repressive mark trimethylation of lysine 27 on histone H3 (H3K27me3) and the concomitant presence of the permissive mark trimethylation of lysine 4 on histone $\mathrm{H} 3$ (H3K4me3) at the transcription start sites (Dilworth and Blais, 2011; Moresi et al., 2015). For instance, Pax7 expression is progressively silenced during SC differentiation, changing its chromatin status from a transcriptionally permissive state, characterized by elevated levels of $\mathrm{H} 3 \mathrm{~K} 4 \mathrm{me} 3$, to a repressive one, enriched in $\mathrm{H} 3 \mathrm{~K} 27 \mathrm{me} 3$. In addition to histone methylation, histone acetyltransferases (HATs) and histone deacetylases (HDACs) modify the acetylation status of histones or transcription factors, thereby acting as transcriptional activators or repressors, respectively (Peserico and Simone, 2011). Among HDAC family members, class I HDACs inhibit MyoD gene transcription and activity. During SC differentiation, the sequential interaction of class I HDACs with MyoD and the hypo-phosphorylated pRb complex allows the transcriptional activation of the differentiation genes, such as myogenin or muscle creatine kinase (Puri et al., 2001). Instead, the class II HDAC member HDAC4 promotes SC proliferation, by repressing the transcription of the cell cycle inhibitor Cdkn1a, and differentiation, by inhibiting the expression of Sharp1 gene (Marroncelli et al., 2018). Moreover, the class II HDACs translocate from the nucleus into the cytoplasm, thereby releasing the inhibition on Mef2 and their target genes ( $\mathrm{Lu}$ et al., 2000).

Muscle regeneration: (i) relies on SCs, which are influenced by their "niche"; (ii) takes place in sequential stages, each one strictly regulated by cell-autonomous and non-autonomous cues; (iii) HDAC4 function in SCs has been partially elucidated. Therefore, in this study, we aimed to define the HDAC4 functions in adult skeletal muscle, by analyzing muscle regeneration in vivo in mice carrying a tissue-specific deletion of HDAC4.

With this purpose, we studied muscle regeneration in a mouse model in which HDAC4 was deleted specifically in skeletal muscle (HDAC4 ${ }^{\mathrm{fl} / \mathrm{fl}}$ myogenin;Cre mice, thereafter named HDAC4 mKO mice). Here we report that HDAC4 in skeletal muscle is required for proper timing and efficiency of muscle regeneration, besides HDAC4 functions in SCs. Indeed, deletion of HDAC4 compromises muscle regeneration process in vivo. MDCs from HDAC4mKO mice efficiently proliferate and differentiate in vitro, suggesting that HDAC4 mediates muscle regeneration in vivo, via soluble factors. Indeed, sera from injured HDAC4 mKO mice inhibit proliferation and differentiation of HDAC4 ${ }^{\mathrm{fl} / \mathrm{fl}} \mathrm{MDCs}$, highlighting the multiple HDAC4 functions in muscle regeneration.

\section{MATERIALS AND METHODS}

\section{Animals}

Mice were treated in strict accordance with the guidelines of the Institutional Animal Care and Use Committee, and to relevant national and European legislation, throughout the experiments. Animal protocols were approved by the Italian Ministry of Health (authorization \# 244/2013-B). HDAC4 $4^{\mathrm{fl} / \mathrm{fl}}$ myogenin-Cre (HDAC4 mKO) mice were generated by crossing HDAC4 ${ }^{\mathrm{fl} / \mathrm{fl}}$ with a mouse line expressing the Cre recombinase under the control of myogenin promoter, i.e., myogenin-Cre mice. $\mathrm{HDAC}^{\mathrm{fl} / \mathrm{fl}}$ mice were used as controls.

\section{Freeze Injury}

Freeze injury was performed in 8-week-old $\mathrm{HDAC}^{\mathrm{fl} / \mathrm{fl}}$ and HDAC4 mKO male mice. Mice were anesthetized with intraperitoneal (IP) injections of $50 \mathrm{mg} / \mathrm{kg}$ Zoletyl, $10 \mathrm{mg} / \mathrm{kg}$ Xylazine solution. To induce freeze injury, the tip of a steel probe precooled in dry ice was applied, for 10 seconds, to posterior muscles of anesthetized mice. This procedure induces a focal, reproducible, injury (Moresi et al., 2008; Marroncelli et al., 2018).

\section{Serum Collection}

The sera were collected by cardiac puncture in the right atrium of anesthetized male mice, 4 days after injury. About $1 \mathrm{ml}$ of blood was transferred to an Eppendorf tube and allowed to stand at room temperature for $30 \mathrm{~min}$. Blood was centrifuged at $3000 \mathrm{rpm}$ for $10 \mathrm{~min}$ and the supernatant serum was recovered. Samples were stored at $4^{\circ} \mathrm{C}$ for up to 6 months. 


\section{Histological Analyses}

Tibialis anterior muscles were dissected and embedded in Jung tissue freezing medium (Leica, Wetzlar, Germany) and frozen in liquid nitrogen precooled isopentane. Cryosections of $8 \mu \mathrm{m}$ were obtained by using a Leica cryostat. Muscles were sectioned throughout the entire length, and the injury/regeneration site was identified by extemporary toluidine blue staining. Histological sections were collected at the level of the injury/regeneration site. The section containing the maximal area of injury/regeneration was identified and further analyzed. For histological analyses, cryosections were fixed in $4 \%$ paraformaldehyde (PFA) buffered solution and stained with hematoxylin/eosin using standard method. The sections were examined with an Axioskop 2 plus system (Zeiss) microscope with relative camera AxioCamHRc and software.

\section{Morphometric Analyses}

Photomicrographs of regenerating muscles were taken at standard resolution $(1300 \times 1030$ pixel $)$ and the cross-sectional area of regenerating fibers, identified by morphological criteria (presence of centrally located nuclei) was measured by using Image J, Scion Image software. Due to the well-known wide distribution of CSAs of fibers in a regenerating muscle, we used the median of the CSA measurements to characterize our population. In addition, regenerating fiber distribution was generated by clustering the regenerating fibers into classes and expressing the value as percentage, over the regenerating fiber number.

\section{Muscle Derived Cell Isolation}

Muscle-derived cells were isolated from hind-limb muscles of 3-week-old male mice by sequential enzymatic digestions: firstly, for $30 \mathrm{~min}$ at $37^{\circ} \mathrm{C}$, with freshly prepared $1 \mathrm{mg} / \mathrm{ml}$ collagenase/dispase (Roche Diagnostics, Mannheim, Germany) in phosphate-buffered saline (PBS), followed by a second one, for $15 \mathrm{~min}$ at $37^{\circ} \mathrm{C}$, with $0.1 \mathrm{mg} / \mathrm{ml}$ type II collagenase (Sigma-Aldrich) in PBS. The enzymatic reaction was blocked by adding cell growth medium, then the cell suspension was filtered through 40-micron cell strainer filter (Falcon) and mildly centrifuged. Cells were re-suspended in growth medium and, after two preplatings of $1 \mathrm{~h}$ each to deplete fibroblasts, MDCs were plated on $0.01 \%$ collagen (Sigma-Aldrich)-coated dishes.

\section{Culture Conditions and Treatments}

Cells were cultured with Dulbecco's modified Eagle medium supplemented with $20 \%$ horse serum (Sigma-Aldrich), $100 \mathrm{U} / \mathrm{ml}$ penicillin (Sigma-Aldrich), $100 \mu \mathrm{g} / \mathrm{ml}$ streptomycin (SigmaAldrich), $50 \mu \mathrm{g} / \mathrm{ml}$ gentamicin (Sigma-Aldrich), 3\% of chicken embryo extract as growing medium (GM). After 3 days, or when cells reached $50 \%$ of confluence, GM was replaced with a differentiation medium (DM), consisting of a 1:10 dilution of GM. For conditioned cultures, horse serum was replaced with murine serum, derived from HDAC4 mKO or HDAC4 $4^{\mathrm{ll} / \mathrm{f}}$ injured mice, 4 days after the surgical procedure.

\section{Immunostaining Analyses}

For MHC immunofluorescence, differentiated cells were fixed in 4\% PFA buffered solution for $10 \mathrm{~min}$ and then blocked in 10\% goat serum in PBS for $1 \mathrm{~h}$. Cells were incubated overnight with sarcomeric MHC antibody (clone MF 20, Developmental Studies Hybridoma Bank), diluted 1:10 in 1\% BSA PBS. Fluorescent conjugated secondary anti-mouse IgG1 antibody (Alexa488, Invitrogen) diluted 1:500 in 1\% BSA PBS was used to detect the primary antibody. For Ki-67 immunofluorescence, growing cells at $24 \mathrm{~h}$ were fixed in 4\% PFA buffered solution for $10 \mathrm{~min}$, permeabilized in $0.2 \%$ Triton in PBS for $30 \mathrm{~min}$, then blocked in 3\% BSA in PBS for $20 \mathrm{~min}$. Cells were incubated overnight with 1:100 Ki-67 antibody (Santa Cruz Biotechnology) in $0.5 \%$ BSA in PBS. Fluorescent conjugated secondary anti-goat antibody (Alexa 555, Invitrogen) diluted $1: 200$ in $0.5 \%$ BSA in PBS was used to detect the primary antibody. Nuclei were counterstained with $[0.5 \mu \mathrm{g} / \mathrm{ml}]$ Hoechst and samples were mounted with $60 \%$ glycerol in Tris $\mathrm{HCl} 0.2 \mathrm{M}$ pH 9.3.

\section{RT-PCR and Real-Time PCR}

Total RNA from Tibialis Anterior muscle was isolated using TRIzoL reagent (Thermo Fisher), according to manufacturer's instructions, 4 days following injury. cDNA synthesis was performed from 0.5 to $1 \mu \mathrm{g}$ of RNA using Reverse Transcription Kit (Takara), following the manufacturer's instructions. Quantitative PCR was performed using the ABI PRISM 700 SDS (Applied Biosystems) with SYBR Green reagent (Applied Biosystems) and primer listed in Table $\mathbf{1 .}$

\section{Statistics}

Statistical significance was determined using two-tailed Student's $t$-test with a significance level $<0.05$, or by using one-way analysis of variance (ANOVA), followed by Tukey's HSD as a post hoc test, when more than two conditions needed to be compared. All values are expressed as mean \pm standard error of the mean (SEM). VassarStats, a statistical computation website

TABLE 1 | Primers used for real-time PCR.

\begin{tabular}{llll}
\hline Gene & Reference number & \multicolumn{1}{c}{ Forward primer } & Reverse primer \\
\hline HDAC4 & NM_207225.2 & GTCTTGGGAATGTACGACGC & GTTGCCAGAGCTGCTATTG \\
Pax7 & NM_011039.2 & TCCCCCTGGAAGTGTCCA & TGGGAACACGGAGCTGA \\
MyoD & M84918.1 & ACCCAGGAACTGGGTGGA & AAGTCGTCTGCTGTCTCAAA \\
Myogenin & NM_031189.2 & GCACTGGAGTCGGTCCCAA & TATCCTCCACCGTGATGCTG \\
e-MHC & M11154.1 & TGGTCGTAATCAGCAGCA & TCGTCTCGCTTGGCAA
\end{tabular}


available at http://vassarstats.net/, was used for the statistical analyses.

\section{RESULTS}

\section{HDAC4 Expression Is Modulated in Skeletal Muscle Upon Injury}

Aiming to investigate the role of HDAC4 in skeletal muscle regeneration, we evaluated its expression levels in regenerating skeletal muscle over time. The tibialis anterior muscle of adult $\mathrm{HDAC} 4^{\mathrm{fl} / \mathrm{fl}}$ male mice was subjected to a localized, reproducible, freeze injury to induce regeneration, (Moresi et al., 2008) and HDAC4 expression was evaluated over time, by real-time PCR (Figure 1). HDAC4 expression in skeletal muscle is significantly induced upon injury, compared to un-injured muscles, and reached a peak of expression at day 4 , suggesting a role for this epigenetic factor in the early phases of muscle regeneration.

\section{Deletion of HDAC4 in Skeletal Muscle Compromised Muscle Regeneration}

The expression of HDAC4 is significantly up-regulated in skeletal muscle in response to injury. Therefore, to define HDAC4 functions in adult skeletal muscle, we analyzed muscle regeneration in a mouse line in which HDAC4 deletion is mediated by a Cre-recombinase under the control of the myogenin promoter, i.e., since the embryonic stage E8.5 (HDAC4 mKO mice) (Cheng et al., 1992). This mouse line does not show overt abnormalities in skeletal muscle under physiological conditions (Moresi et al., 2010; Pigna et al., 2018). However, 1 week after injury, HDAC4 mKO mice showed smaller regenerating fibers than $\mathrm{HDAC}_{4}^{\mathrm{fl} / \mathrm{fl}}$ mice, used as controls, by histological analyses (Figure 2A). Morphometric analyses of regenerating myofiber cross-sectional area (CSA)

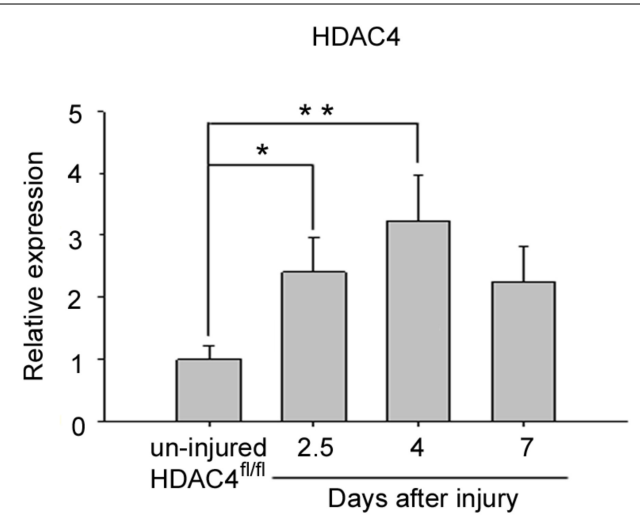

FIGURE 1 | HDAC4 expression is up-regulated in regenerating HDAC4 4 fl/fl muscles. Real-time PCR for HDAC4 in regenerating muscles, at the indicated time points following injury, over un-injured muscles. Data are presented as mean + /- SEM. $n=6$ mice for each condition. One-way ANOVA showed a significant effect of the treatment between groups $(F=5.16 ; d f=3 ; p=0.01$ ) and a significant interaction between un-injured $\mathrm{HDAC}^{\mathrm{fl} / \mathrm{fl}}$ mice and 2.5 days $\left({ }^{*} p<0.05\right)$ or 4 days $\left({ }^{* *} p<0.01\right)$ after injury by Tukey's HSD test. confirmed that HDAC4 mKO mice exhibited smaller, centrally nucleated myofibers with respect to $\mathrm{HDAC}^{\mathrm{fl} / \mathrm{fl}}$ mice (Figure $2 \mathbf{B}$ ). Regenerating fiber distribution confirmed that HDAC4 KO mice displayed a significantly higher number of smaller regenerating myofibers $\left(400-599 \mu \mathrm{m}^{2}\right)$ than $\mathrm{HDAC}^{\mathrm{fl} / \mathrm{fl}}$ mice, at the expenses of the larger ones (1000-1199 $\mu \mathrm{m}^{2}$ ) (Figure 2C). Molecular analyses performed at the time of maximal expression of HDAC4 in regenerating muscles, i.e., 4 days following injury, corroborated a significant reduction of the expression of myogenic markers of early, intermediate and terminal differentiation, i.e., Pax7, MyoD, myogenin and embryonic $\mathrm{MHC}$, in HDAC4 $\mathrm{mKO}$ mice, compared to $\mathrm{HDAC} 4^{\mathrm{fl} / \mathrm{fl}}$ mice (Figure 2D).

To discriminate whether the HDAC4 deletion in skeletal muscle resulted in delayed or compromised muscle regeneration, we analyzed regenerating muscles at later time points, 2 weeks and 1 month after injury. Differences in muscle regeneration ability persisted between genotypes after 2 weeks and after 1 month following injury, as shown by histological and morphometrical analyses of regenerating fiber CSA (Figure 3), indicating that deletion of HDAC4 in skeletal muscle is sufficient to hamper muscle regeneration.

\section{HDAC4 mKO Muscle Derived Cells Efficiently Proliferate and Differentiate in vitro}

Because HDAC4 is crucial for muscle stem cell proliferation and differentiation (Marroncelli et al., 2018), we wondered whether MDC proliferation and/or differentiation was affected in HDAC4 mKO mice. MDCs were isolated, and cell proliferation was assessed by immunofluorescence for Ki-67, a protein associated with cellular proliferation, after $24 \mathrm{~h}$ in growing condition. HDAC4 mKO MDCs showed a similar amount of Ki-67 positive cells, and of total MDCs, respect to $\mathrm{HDAC} 4^{\mathrm{fl} / \mathrm{fl}}$ mice (Figures 4A-C). Terminal differentiation was induced and assessed by immunofluorescence for MHC (Figure 4D). No significant differences in terminal differentiation were detected between HDAC4 $\mathrm{mKO}$ and HDAC4 ${ }^{\mathrm{fl} / \mathrm{fl}} \mathrm{MDCs}$, as also quantified by the differentiation (i.e., the number MHC positive cells, over total nuclei) or the fusion (the number of myonuclei in myotubes, over total nuclei) indexes (Figure 4E).

These data indicate that deletion of HDAC4, upon myogenin expression, does not affect MDC proliferation or differentiation, implying that $\mathrm{HDAC} 4$ controls muscle regeneration in vivo via soluble factors.

\section{HDAC4 mKO Serum Negatively Affects MDC Proliferation and Differentiation}

To prove that HDAC4 affects MDC biology in a cell nonautonomous manner, HDAC4 $\mathrm{mKO}$ and $\mathrm{HDAC}^{\mathrm{fl} / \mathrm{fl}}$ sera were withdrawn 4 days following injury, at the time of the HDAC4 maximal expression in skeletal muscle. Control MDCs were cultured with conditioned media by using HDAC4 $\mathrm{mKO}$ or $\mathrm{HDAC}^{\mathrm{fl} / \mathrm{fl}}$ sera, and MDC proliferation was evaluated after $24 \mathrm{~h}$ in growing condition, by $\mathrm{Ki}-67$ immunofluorescence (Figure 5A). Quantification of Ki-67-positive cells revealed that 
A

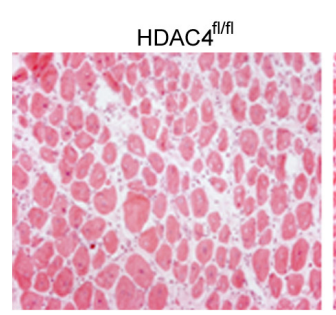

C

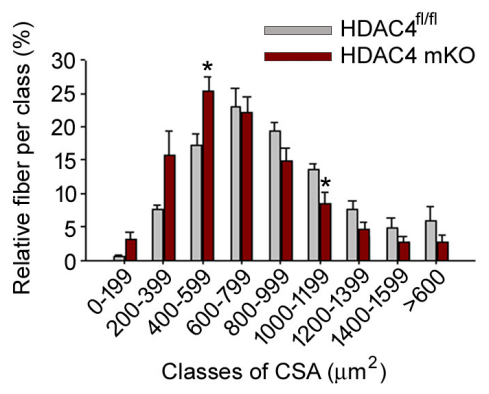

D

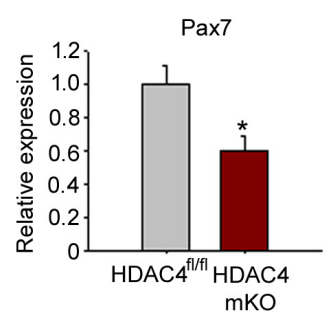

HDAC4 mKO

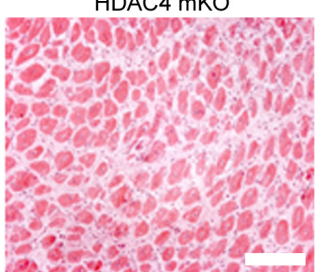

B

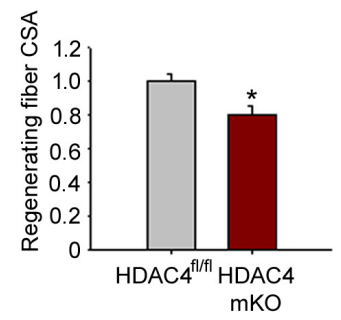

FIGURE 2 | HDAC4 mKO mice exhibit delayed muscle regeneration. (A) Representative images of HDAC4 mKO and HDAC4 ${ }^{f / f l}$ tibialis anterior regenerating muscles, 1 week after injury. Scale bar $=50$ micron. (B) Regenerating fiber CSA of HDAC4 mKO and HDAC4 ${ }^{\mathrm{fl} / \mathrm{fl}}$ mice, 1 week after injury. Data are presented as median $+/$ - SEM. $n=8$ mice for genotype. ${ }^{*} p<0.05$ by Student's $t$-test. (C) Morphometric analysis of the distribution of regenerating fiber cross-sectional area, 1 week after injury. $n=8$ mice for genotype. Data are presented as average +/- SEM. ${ }^{*} p<0.05$ by Student's $t$-test. (D) Gene expression of indicated myogenic markers in HDAC4 mKO and HDAC4 ${ }^{\mathrm{fl} / \mathrm{fl}}$ mice, by real-time PCR, 4 days following injury. Data are presented as mean $+/-\mathrm{SEM} . n=8$ mice for genotype. ${ }^{*} p<0.05$; ${ }^{* *} p<0.005$ by Student's $t$-test.

HDAC4 $\mathrm{mKO}$ sera reduced the MDC proliferating cell number, compared to HDAC4 $4^{\mathrm{fl} / \mathrm{fl}}$ sera (Figure 5B), data also confirmed by the quantification of the MDCs (Figure 5C).

To investigate if the reduction in MDC proliferation influences their terminal differentiation, control MDC were induced to differentiate, by mean of conditioned media with HDAC4 mKO or HDAC4 $4^{\mathrm{f} / \mathrm{fl}}$ mouse sera, as control. HDAC4 mKO sera significantly reduced also MDC terminal differentiation and fusion, as assessed by immunofluorescence for MHC and quantification of the differentiation and fusion indexes (Figures 5D,E). Quantification of MDCs, after 3 days of differentiation, confirmed a significant reduction in the MDC number upon HDAC4 mKO serum treatment with respect to controls (Figure 5F). These data demonstrate that HDAC4 mediates the production and release of circulating factors able to negatively affect MDC proliferation and differentiation.

\section{DISCUSSION}

Numerous studies clarified that MDC biology is controlled by cell-autonomous and non-autonomous cues, as well as by epigenetic mechanisms (Boonen and Post, 2008; Moresi et al., 2015). In this study, we investigated which functions HDAC4 plays during muscle regeneration. HDAC4 is a stress-responsive epigenetic factor known to regulate multiple responses in skeletal muscle, including satellite cells biology upon injury (Moresi et al., 2010; Choi et al., 2014; Marroncelli et al., 2018). We found HDAC4 to be induced to a significant extent in skeletal muscle upon injury, in line with previous findings (Choi et al., 2014), with the expression peaking in the early phases of regeneration, suggesting that HDAC4 mediates skeletal muscle response to injury. To dissect the HDAC4 biological functions in skeletal muscle, excluding SCs in the early phases of differentiation, we generated HDAC4 mKO mice, in which HDAC4 deletion occurs upon myogenin expression. HDAC4 mKO mice do not show skeletal muscle abnormalities at baseline (Moresi et al., 2010; Pigna et al., 2018). However, following injury, deletion of HDAC4 in skeletal muscle significantly hampered muscle regeneration. When HDAC4 deletion occurs in myogenin-positive cells, MDCs efficiently proliferate and differentiate in vitro, differently from HDAC4 KO SCs, having compromised expansion and differentiation in a cell-autonomous manner (Choi et al., 2014; Marroncelli 
A

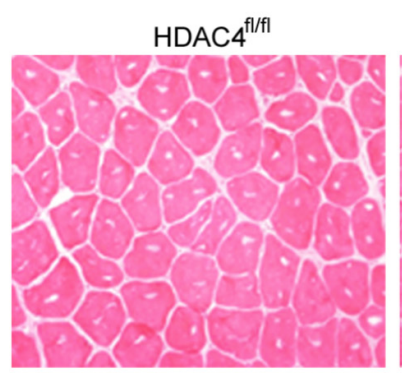

C

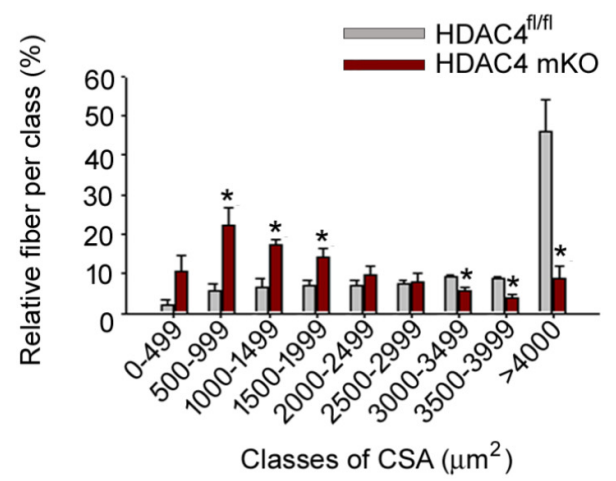

D
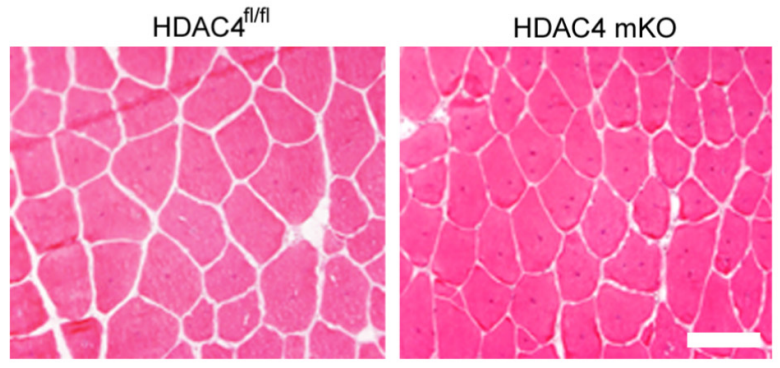

$\mathbf{F}$

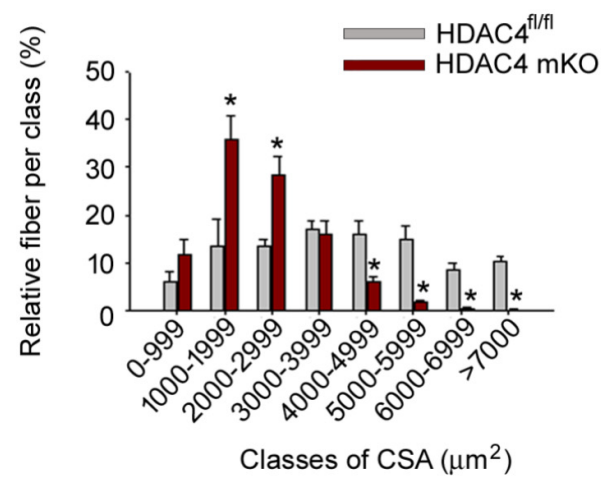

B

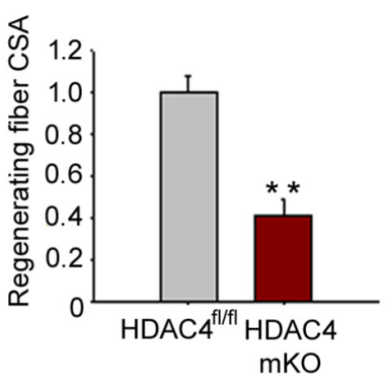

E

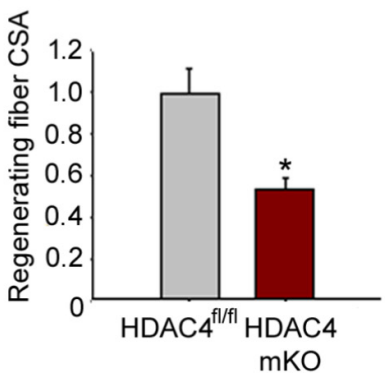

FIGURE 3 | HDAC4 mKO mice show impaired muscle regeneration. (A) Representative images of HDAC4 mKO and HDAC4 ${ }^{\mathrm{fl} / \mathrm{fl}}$ tibialis anterior regenerating muscles, 2 weeks after injury. Scale bar $=50$ micron. (B) Regenerating fiber CSA of HDAC4 mKO and HDAC4 $4^{\mathrm{f} / \mathrm{fl}}$ mice, 2 weeks after injury. Data are presented as median $+/-$ SEM. $n=8$ mice for genotype. ${ }^{* *} p<0.02$ by Student's $t$-test. (C) Morphometric analysis of the distribution of regenerating fiber cross-sectional area, 2 weeks after injury. $n=8$ mice for genotype. Data are presented as average + -- SEM. ${ }^{*} p<0.05$ by Student's $t$-test. (D) Representative images of HDAC4 mKO and $\mathrm{HDAC}^{\mathrm{fl} / \mathrm{fl}}$ tibialis anterior regenerating muscles, 1 month after injury. Scale bar $=50$ micron. (E) Regenerating fiber CSA of HDAC4 mKO and HDAC4 ${ }^{f / / f l}$ mice, 1 month after injury. $n=8$ mice for genotype. Data are presented as median $+/-$ SEM. ${ }^{*} p<0.05$ by Student's $t$-test. (F) Morphometric analysis of the distribution of regenerating fiber cross-sectional area, 1 month after injury. $n=5$ mice for genotype. Data are presented as average $+/-$ SEM. * $p<0.05$ by Student's $t$-test.

et al., 2018). Despite MDC ability to differentiate in vitro, compromised muscle regeneration in HDAC4 mKO mice indicates that, in the injured muscle, HDAC4 influences MDCs behavior, evidencing novel HDAC4 cellular functions depending on the timing and/or the cell sub-type where this enzyme is expressed. 


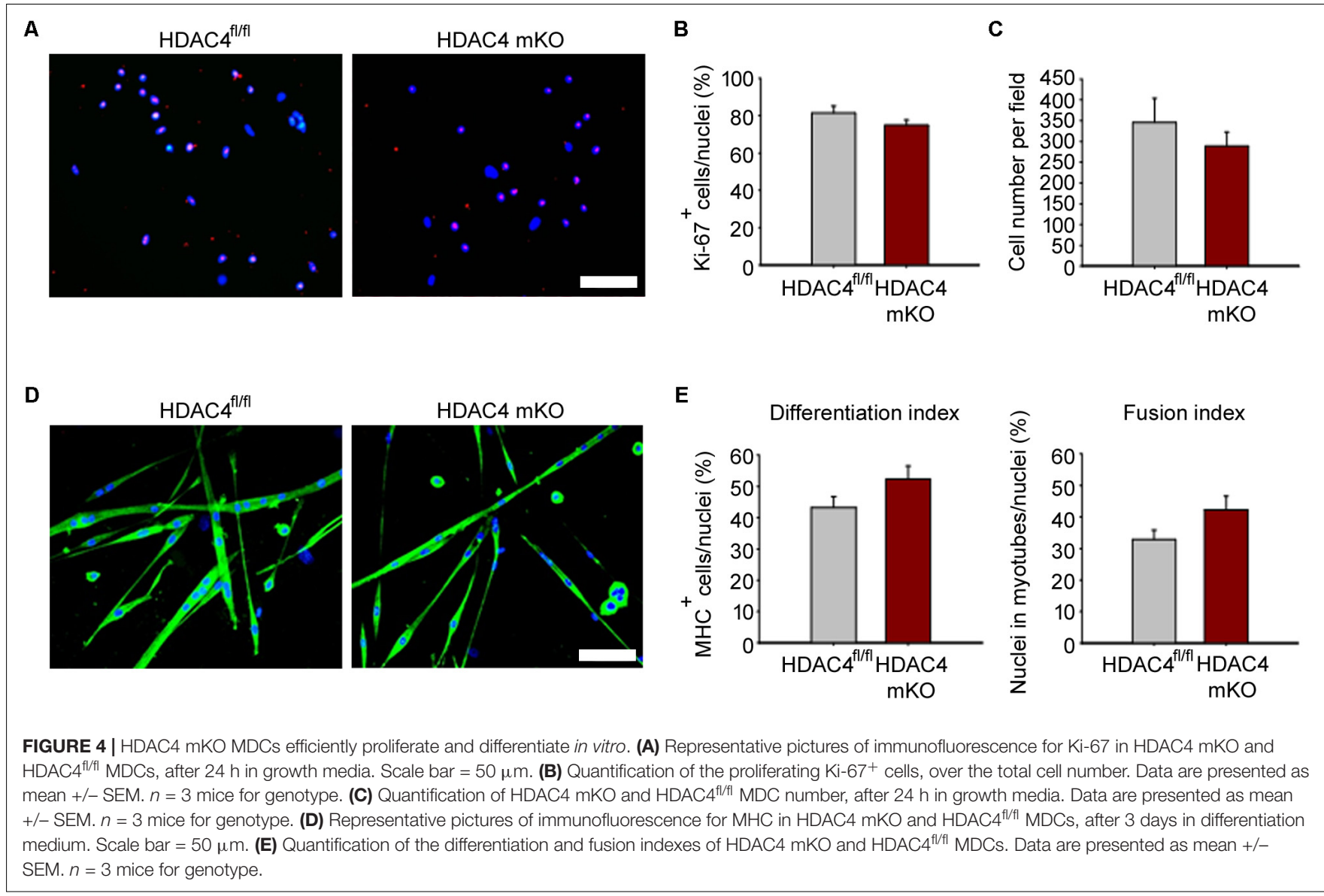

Interestingly, the decrease of the expression level of the SCs marker Pax7 4 days after injury indicates a deficit in either SC number or proliferation. The paired box transcription factor Pax7 is the master regulator of SC (Seale et al., 2000), required for SC function in adult skeletal muscle. Indeed, upon Pax7 deletion, SCs exhibit cell-cycle arrest and dysregulation of MRFs, muscles resulted smaller and muscle regeneration was severely impaired (Oustanina et al., 2004; Kuang et al., 2006; Relaix et al., 2006). Since HDAC4 mKO MDCs were able to proliferate or differentiate efficiently in vitro, soluble factors likely mediate the reduction of Pax7 expression in regenerating HDAC4 mKO muscles.

By using culture media conditioned with sera from injured HDAC4 $\mathrm{mKO}$ or $\mathrm{HDAC}_{4}^{\mathrm{fl} / \mathrm{fl}}$ mice, we proved that HDAC4 does mediate the secretion of circulating factors able to influence MDC proliferation and differentiation. Numerous environmental factors regulate adult myogenesis during regeneration. Growth factors released from injured myofibers strictly regulate the activation of quiescent SC (Furuichi and Fujii, 2017). SC proliferation is supported by mitogens such as FGF and insulinlike growth factor (IGF), which get up-regulated in skeletal muscle after injury (Guthridge et al., 1992; Bischoff and Heintz, 1994). Furthermore, activated SCs secrete miRNAs - containing exosomes, which in turn modulate SC proliferation and differentiation (Harding and Velleman, 2016). SC differentiation is strictly influenced by secreted factors as well. For instance, insulin-like growth factor 1 (IGF1) and interleukin 15 (IL-15) secretion is induced after membrane damage or during exercise, these myokines promote SC differentiation and contributes to muscle hypertrophy by enhancing protein synthesis (DeVol et al., 1990; Adams, 2002; Quinn et al., 2002; Furmanczyk and Quinn, 2003). Among soluble factors acting on SC differentiation, several inflammatory cytokines are known to inhibit this process. TNF- $\alpha$ and interleukin 1 (IL-1) are inflammatory mediators of muscle wasting and interfere with the expression of myogenic factors in differentiating myoblasts, by activating the nuclear factor - kappa beta $(\mathrm{NFk} \beta)$ and caspases (Langen et al., 2001; Moresi et al., 2008, 2009). In addition to inhibit SC activation and self-renewal, numerous secreted factors negatively affect also SC differentiation and fusion, among them myostatin and growth differentiation factor11 (GDF11) (TGFß superfamily's members) are known to hamper muscle regeneration by inhibiting SC activity on distinct phases during myogenesis (Reisz-Porszasz et al., 2003; Egerman et al., 2015).

HDACs have been shown to regulate soluble factors in different cell types. In neuronal and glial cells, the release of brain-derived neurotrophic factor (BDNF) and fibroblast growth factor 1 (FGF1) is mediated by HDACs (Hossain 
A

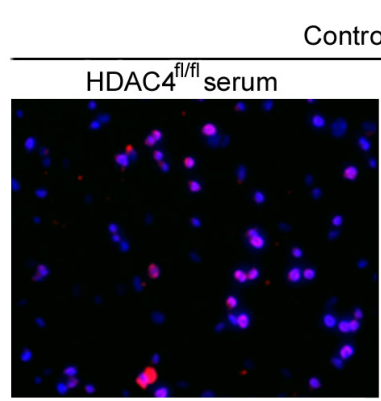

D

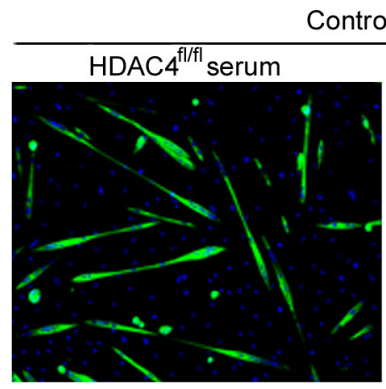

E

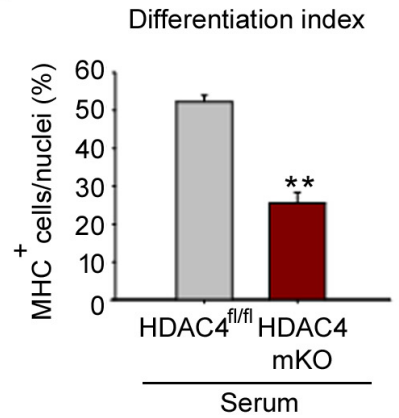

B

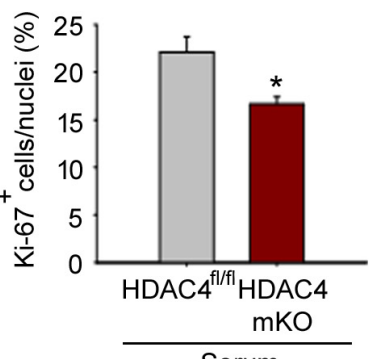

Serum
C

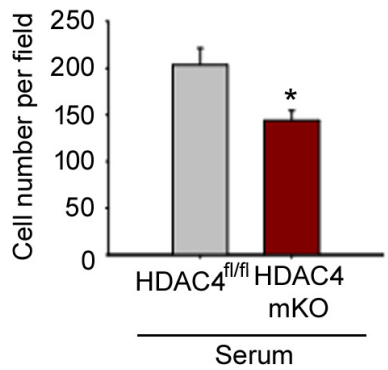

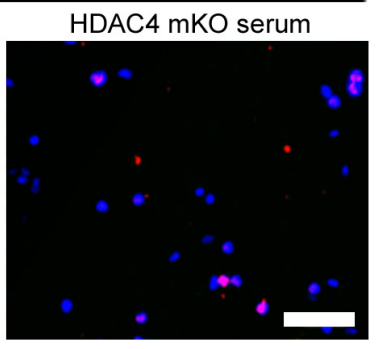

ontrol MDC

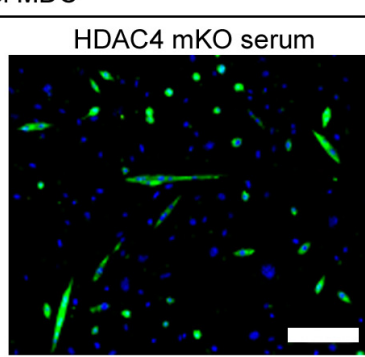

FIGURE 5 | HDAC4 mKO serum negatively affects control MDC proliferation and differentiation. (A) Representative pictures of immunofluorescence for Ki-67 in control MDCs, after $24 \mathrm{~h}$ in conditioned media with sera from either injured HDAC4 mKO or HDAC4l/fl mice. Scale bar $=50 \mu \mathrm{m}$. (B) Quantification of the proliferating $\mathrm{Ki}-67^{+}$cells, over the total cell number. Data are presented as mean $+/-$SEM. $n=6$ mice for genotype. * $p<0.05$ by Student's $t$-test. (C) Quantification of MDC number cultured with sera from either injured HDAC4 mKO or HDAC4 ${ }^{\mathrm{fl} / \mathrm{fl}}$ mice. Data are presented as mean $+/-\mathrm{SEM}$. $n=6$ mice for genotype. * $p<0.05$ by Student's t-test. (D) Representative pictures of immunofluorescence for MHC in control MDCs cultured with conditioned media with sera from either HDAC4 mKO or HDAC4 $^{\mathrm{fl} / \mathrm{fl}}$ injured mice, after 3 days in differentiation medium. Scale bar $=100 \mu \mathrm{m}$. (E) Quantification of the differentiation and fusion indexes of control MDCs cultured with sera from either injured HDAC4 mKO or HDAC4 $4^{\mathrm{fl} / \mathrm{fl}}$ mice, after 3 days in differentiation medium. Data are presented as mean $+/-$ SEM. $n=6$ mice for genotype. ${ }^{* *} p<0.005$ by Student's $t$-test. (F) Number of control MDCs cultured with sera from either injured HDAC4 mKO or HDAC4 ${ }^{\text {fl/fl }}$ mice, after 3 days in differentiation medium. Data are presented as mean +/- SEM. $n=6$ mice for genotype. ** $p<0.005$ by Student's $t$-test.

et al., 2018), as well as in fibroblasts the production of several proinflammatory cytokines/chemokines (Li et al., 2011), thus contributing to chronic inflammatory processes. Moreover, HDACs modulate IL-4 expression and secretion in mast cells and monocyte-derived DCs (moDCs) (López-Bravo et al., 2013; Nakamaru et al., 2015). In addition to its classical roles, new evidence links HDACs and soluble factors. For instance, cytoplasmic protein acetylation/deacetylation balance has been involved in extracellular vesicles content and release in colon cancer cells (Li et al., 2016; Chao et al., 2017). In skeletal muscle, the expression of several soluble factors, such as TGF- $\beta$ and follistatin, are modulated by HDAC4 during myogenesis (Sun et al., 2010; Winbanks et al., 2011). Moreover, HDAC4 promotes skeletal muscle reinnervation via the release of FGF binding protein 1 (Williams et al., 2009).

A preclinical study in a murine Duchenne Muscular Dystrophy model demonstrated that Givinostat, a class I and 
II HDAC inhibitor, improves skeletal muscle regeneration by reducing fibrotic and adipose tissues (Mozzetta et al., 2013). Similarly, trichostatin A (TSA), another class I-II HDAC inhibitor, promotes skeletal muscle regeneration by targeting fibro-adipogenic cells and inducing the expression and the release of follistatin, a pro-myogenic soluble factor identified as a central mediator in myoblast recruitment and fusion (Iezzi et al., 2004; Mozzetta et al., 2013). Our results are in apparent contrast with the findings of previous studies obtained by administering HDAC inhibitors during muscle regeneration. However, different experimental conditions could reconcile such discrepancy. In those studies, muscle regeneration was improved by daily systemic administration of class I and II HDAC inhibitors after injury, which is different from our study exploiting HDAC4 mKO mice, a tissue-specific KO mouse bringing the genetic deletion at embryonic stage E8.5 of one member of the class IIa HDACs.

\section{CONCLUSION}

The present study sheds further light on multiple HDAC4 functions in skeletal muscle following injury stress. HDAC4 not only promotes SC replenishment and differentiation in a cell-autonomous manner via the epigenetic regulation of gene transcription but also influences MDC proliferation and differentiation via muscle derived-soluble factors. These findings should be considered when administering class I-II HDAC inhibitors to treat muscular diseases.

\section{REFERENCES}

Adams, G. R. (2002). Invited review: autocrine/paracrine IGF-I and skeletal muscle adaptation. J. Appl. Physiol. 93, 1159-1167. doi: 10.1152/japplphysiol.01264. 2001

Bischoff, R., and Heintz, C. (1994). Enhancement of skeletal muscle regeneration. Dev. Dyn. 201, 41-54. doi: 10.1002/aja.1002010105

Black, B. L., and Olson, E. N. (1998). Transcriptional control of muscle development by myocyte enhancer factor-2 (mef2) proteins. Annu. Rev. Cell Dev. Biol. 14, 167-196. doi: 10.1146/annurev.cellbio.14.1.167

Boonen, K. J. M., and Post, M. J. (2008). The muscle stem cell niche: regulation of satellite cells during regeneration. Tissue Eng. B Rev. 14, 419-431. doi: 10.1089/ ten.teb.2008.0045

Chao, O. S., Chang, T. C., Di Bella, M. A., Alessandro, R., Anzanello, F., Rappa, G., et al. (2017). The HDAC6 inhibitor tubacin induces release of CD133+extracellular vesicles from cancer cells. J. Cell. Biochem. 118, 44144424. doi: $10.1002 /$ jcb. 26095

Cheng, T.-C., Hanley, T. A., Mudd, J., Merlie, J. P., and Olson, E. N. (1992). Mapping of myogenin transcription during embryogenesis using transgenes linked to the myogenin control region. J. Cell Biol. 119, 1649-1656. doi: 10.1083/ jcb.119.6.1649

Choi, M.-C., Ryu, S., Hao, R., Wang, B., Kapur, M., Fan, C.-M., et al. (2014). HDAC4 promotes Pax7-dependent satellite cell activation and muscle regeneration. EMBO Rep. 15, 1175-1183. doi: 10.15252/embr. 201439195

Coletti, D., Moresi, V., Adamo, S., Molinaro, M., and Sassoon, D. (2005). Tumor necrosis factor- $\alpha$ gene transfer induces cachexia and inhibits muscle regeneration. Genesis 43, 120-128. doi: 10.1002/gene.20160

Costa, A., Rossi, E., Scicchitano, B. M., Coletti, D., Moresi, V., and Adamo, S. (2014). Neurohypophyseal hormones: novel actors of striated muscle development and homeostasis. Eur. J. Transl. Myol. 24:3790. doi: 10.4081/ejtm. 2014.3790

\section{AUTHOR CONTRIBUTIONS}

$\mathrm{AR}, \mathrm{NM}$, and $\mathrm{CN}$ performed the experiments. $\mathrm{VM}$ and SA conceived the project and analyzed the data. All authors contributed to critical analysis. AR, VM, and SA wrote the manuscript and all authors approved the final manuscript for publication.

\section{FUNDING}

This study has been funded by Ricerca finalizzata (GR-20102311055) from the Italian Ministry of Health and partially supported by Sapienza research projects 2016 (SapMedi2016) and 2017 (RM11715C78539BD8).

\section{ACKNOWLEDGMENTS}

We are grateful to Carla Ramina for her technical assistance. We are grateful to Prof. Eric N Olson and Rhonda Bassel-Duby (University of Texas Southwestern Medical Center, Dallas, TX, United States) for the mouse line and the scientific advice. The MF 20 monoclonal antibody was developed by D. A. Fishman and obtained from the Developmental Studies Hybridoma Bank, created by the NICHD of the NIH and maintained at the University of Iowa, Department of Biology, Iowa City, IA, United States, 52242.

De Paepe, B., and De Bleecker, J. L. (2013). Cytokines and chemokines as regulators of skeletal muscle inflammation: presenting the case of Duchenne muscular dystrophy. Mediators Inflamm. 2013:540370. doi: 10.1155/2013/ 540370

De Pasquale, L., D’Amico, A., Verardo, M., Petrini, S., Bertini, E., and De Benedetti, F. (2012). Increased muscle expression of interleukin-17 in Duchenne muscular dystrophy. Neurology 78, 1309-1314. doi: 10.1212/WNL. 0b013e3182518302

Delaney, K., Kasprzycka, P., Ciemerych, M. A., and Zimowska, M. (2017). The role of TGF- $\beta 1$ during skeletal muscle regeneration. Cell Biol. Int. 41, 706-715. doi: 10.1002/cbin.10725

DeVol, D. L., Rotwein, P., Sadow, J. L., Novakofski, J., and Bechtel, P. J. (1990). Activation of insulin-like growth factor gene expression during work-induced skeletal muscle growth. Am. J. Physiol. Metab. 259, E89-E95. doi: 10.1152/ ajpendo.1990.259.1.E89

Dilworth, F. J., and Blais, A. (2011). Epigenetic regulation of satellite cell activation during muscle regeneration. Stem Cell Res. Ther. 2:18. doi: 10.1186/ scrt59

Dumont, N. A., Bentzinger, C. F., Sincennes, M.-C., and Rudnicki, M. A. (2015). Satellite cells and skeletal muscle regeneration. Compr. Physiol. 5, 1027-1059. doi: 10.1002/cphy.c140068

Egerman, M. A., Cadena, S. M., Gilbert, J. A., Meyer, A., Nelson, H. N., Swalley, S. E., et al. (2015). GDF11 increases with age and inhibits skeletal muscle regeneration. Cell Metab. 22, 164-174. doi: 10.1016/j.cmet.2015. 05.010

Floss, T., Arnold, H. H., and Braun, T. (1997). A role for FGF-6 in skeletal muscle regeneration. Genes Dev. 11, 2040-2051. doi: 10.1101/gad.11. 16.2040

Furmanczyk, P. S., and Quinn, L. S. (2003). Interleukin15 increases myosin accretion in human skeletal myogenic cultures. Cell Biol. Int. 27, 845-851. doi: 10.1016/S1065-6995(03) 00172-0 
Furuichi, Y., and Fujii, N. L. (2017). Mechanism of satellite cell regulation by myokines. J. Phys. Fit. Sport Med. 6, 311-316. doi: 10.7600/jp fsm.6.311

Giordani, L., and Puri, P. L. (2013). Epigenetic control of skeletal muscle regeneration. FEBS J. 280, 4014-4025. doi: 10.1111/febs.12383

Gurriarán-Rodríguez, U., Santos-Zas, I., González-Sánchez, J., Beiroa, D., Moresi, V., Mosteiro, C. S., et al. (2015). Action of obestatin in skeletal muscle repair: stem cell expansion, muscle growth, and microenvironment remodeling. Mol. Ther. 23, 1003-1021. doi: 10.1038/mt.2015.40

Guthridge, M., Wilson, M., Cowling, J., Bertolini, J., and Hearn, M. T. W. (1992). The role of basic fibroblast growth factor in skeletal muscle regeneration. Growth Factors 6, 53-63. doi: 10.3109/08977199209 008871

Harding, R. L., and Velleman, S. G. (2016). MicroRNA regulation of myogenic satellite cell proliferation and differentiation. Mol. Cell. Biochem. 412, 181-195. doi: 10.1007/s11010-015-2625-6

Hossain, M. S., Oomura, Y., and Katafuchi, T. (2018). Glucose can epigenetically alter the gene expression of neurotrophic factors in the murine brain cells. Mol. Neurobiol. 55, 3408-3425. doi: 10.1007/s12035-017-0578-3

Iezzi, S., Di Padova, M., Serra, C., Caretti, G., Simone, C., Maklan, E., et al. (2004). Deacetylase inhibitors increase muscle cell size by promoting myoblast recruitment and fusion through induction of follistatin. Dev. Cell 6, 673-684. doi: 10.1016/S1534-5807(04)00107-8

Jankowski, R. J., Deasy, B. M., and Huard, J. (2002). Muscle-derived stem cells. Gene Ther. 9, 642-647. doi: 10.1038/sj.gt.3301719

Kim, J.-H., Han, G.-C., Seo, J.-Y., Park, I., Park, W., Jeong, H.-W., et al. (2016). Sex hormones establish a reserve pool of adult muscle stem cells. Nat. Cell Biol. 18, 930-940. doi: 10.1038/ncb3401

Kuang, S., Chargé, S. B., Seale, P., Huh, M., and Rudnicki, M. A. (2006). Distinct roles for Pax7 and Pax3 in adult regenerative myogenesis. J. Cell Biol. 172, 103-113. doi: 10.1083/jcb.200508001

Kuang, S., Kuroda, K., Le Grand, F., and Rudnicki, M. A. (2007). Asymmetric selfrenewal and commitment of satellite stem cells in muscle. Cell 129, 999-1010. doi: 10.1016/j.cell.2007.03.044

Langen, R. C., Schols, A. M., Kelders, M. C., Wouters, E. F., and Janssen-Heininger, Y. M. (2001). Inflammatory cytokines inhibit myogenic differentiation through activation of nuclear factor-kappaB. FASEB J. 15, 1169-1180. doi: 10.1016/j.cell. 2007.03.044

Le Grand, F., and Rudnicki, M. A. (2007). Skeletal muscle satellite cells and adult myogenesis. Curr. Opin. Cell Biol. 19, 628-633. doi: 10.1016/J.CEB.2007.09.012

Li, M., Riddle, S. R., Frid, M. G., El Kasmi, K. C., McKinsey, T. A., Sokol, R. J., et al. (2011). Emergence of fibroblasts with a proinflammatory epigenetically altered phenotype in severe hypoxic pulmonary hypertension. J. Immunol. 187, 2711-2722. doi: 10.4049/jimmunol.1100479

Li, Z., Zhuang, M., Zhang, L., Zheng, X., Yang, P., and Li, Z. (2016). Acetylation modification regulates GRP78 secretion in colon cancer cells. Sci. Rep. 6:30406. doi: $10.1038 /$ srep30406

Lilly, B., Galewsky, S., Firulli, A. B., Schulz, R. A., and Olson, E. N. (1994). D-MEF2: a MADS box transcription factor expressed in differentiating mesoderm and muscle cell lineages during Drosophila embryogenesis. Proc. Natl. Acad. Sci. U.S.A. 91, 5662-5666.

López-Bravo, M., Minguito de la Escalera, M., Domínguez, P. M., GonzálezCintado, L., del Fresno, C., Martín, P., et al. (2013). IL-4 blocks TH1polarizing/inflammatory cytokine gene expression during monocyte-derived dendritic cell differentiation through histone hypoacetylation. J. Allergy Clin. Immunol. 132, 1409.e13-1419.e13. doi: 10.1016/j.jaci.2013.08.039

Lu, J., McKinsey, T. A., Nicol, R. L., and Olson, E. N. (2000). Signal-dependent activation of the MEF2 transcription factor by dissociation from histone deacetylases. Proc. Natl. Acad. Sci. U.S.A. 97, 4070-4075. doi: 10.1073/pnas. 080064097

Marroncelli, N., Bianchi, M., Bertin, M., Consalvi, S., Saccone, V., De Bardi, M., et al. (2018). HDAC4 regulates satellite cell proliferation and differentiation by targeting P21 and Sharp1 genes. Sci. Rep. 8:3448. doi: 10.1038/s41598-01821835-7

Millay, D. P., O’Rourke, J. R., Sutherland, L. B., Bezprozvannaya, S., Shelton, J. M., Bassel-Duby, R., et al. (2013). Myomaker is a membrane activator of myoblast fusion and muscle formation. Nature 499, 301-305. doi: 10.1038/nat ure 12343
Moresi, V., Garcia-Alvarez, G., Pristerà, A., Rizzuto, E., Albertini, M. C., Rocchi, M., et al. (2009). Modulation of caspase activity regulates skeletal muscle regeneration and function in response to vasopressin and tumor necrosis factor. PLoS One 4:e5570. doi: 10.1371/journal.pone. 0005570

Moresi, V., Marroncelli, N., and Adamo, S. (2015). New insights into the epigenetic control of satellite cells. World J. Stem Cells 7, 945-955. doi: 10.4252/wjsc.v7. i6.945

Moresi, V., Pristerà, A., Scicchitano, B. M., Molinaro, M., Teodori, L., Sassoon, D., et al. (2008). Tumor necrosis factor- $\alpha$ inhibition of skeletal muscle regeneration is mediated by a caspase-dependent stem cell response. Stem Cells 26, 997-1008. doi: 10.1634/stemcells.2007-0493

Moresi, V., Williams, A. H., Meadows, E., Flynn, J. M., Potthoff, M. J., McAnally, J., et al. (2010). Myogenin and class II HDACs control neurogenic muscle atrophy by inducing E3 ubiquitin ligases. Cell 143, 35-45. doi: 10.1016/j.cell.2010. 09.004

Mozzetta, C., Consalvi, S., Saccone, V., Tierney, M., Diamantini, A., Mitchell, K. J., et al. (2013). Fibroadipogenic progenitors mediate the ability of HDAC inhibitors to promote regeneration in dystrophic muscles of young, but not old Mdx mice. EMBO Mol. Med. 5, 626-639. doi: 10.1002/emmm. 201202096

Nakamaru, Y., Takagi, D., Homma, A., Hatakeyama, S., and Fukuda, S. (2015). Oxidative stress regulates IL-4 gene expression in mast cells through the reduction of histone deacetylase. Otolaryngol. Head Neck Surg. 152, 48-52. doi: $10.1177 / 0194599814559189$

Oustanina, S., Hause, G., and Braun, T. (2004). Pax7 directs postnatal renewal and propagation of myogenic satellite cells but not their specification. EMBO J.23, 3430-3439. doi: 10.1038/sj.emboj.7600346

Pawlikowski, B., Vogler, T. O., Gadek, K., and Olwin, B. B. (2017). Regulation of skeletal muscle stem cells by fibroblast growth factors. Dev. Dyn. 246, 359-367. doi: $10.1002 /$ dvdy.24495

Peserico, A., and Simone, C. (2011). Physical and functional hat/hdac interplay regulates protein acetylation balance. J. Biomed. Biotechnol. 2011:371832. doi: $10.1155 / 2011 / 371832$

Pigna, E., Renzini, A., Greco, E., Simonazzi, E., Fulle, S., Mancinelli, R., et al. (2018). HDAC4 preserves skeletal muscle structure following long-term denervation by mediating distinct cellular responses. Skelet. Muscle 8:6. doi: 10.1186/s13395018-0153-2

Puri, P. L., Iezzi, S., Stiegler, P., Chen, T. T., Schiltz, R. L., Muscat, G. E., et al. (2001). Class I histone deacetylases sequentially interact with $\mathrm{MyoD}$ and $\mathrm{pRb}$ during skeletal myogenesis. Mol. Cell 8, 885-897. doi: 10.1186/s13395-018-0153-2

Quinn, L. S., Anderson, B. G., Drivdahl, R. H., Alvarez, B., and Argilés, J. M. (2002). Overexpression of interleukin-15 induces skeletal muscle hypertrophy in vitro: implications for treatment of muscle wasting disorders. Exp. Cell Res. 280, 55-63. doi: 10.1016/S1097-2765(01)00373-2

Quinn, M. E., Goh, Q., Kurosaka, M., Gamage, D. G, Petrany, M. J, Prasad, V., et al. (2017). Myomerger induces fusion of non-fusogenic cells and is required for skeletal muscle development. Nat. Commun. 8:15665. doi: 10.1038/ ncomms 15665

Reisz-Porszasz, S., Bhasin, S., Artaza, J. N., Shen, R., Sinha-Hikim, I., Hogue, A., et al. (2003). Lower skeletal muscle mass in male transgenic mice with musclespecific overexpression of myostatin. Am. J. Physiol. Metab. 285, E876-E888. doi: 10.1152/ajpendo.00107.2003

Relaix, F., Montarras, D., Zaffran, S., Gayraud-Morel, B., Rocancourt, D., Tajbakhsh, S., et al. (2006). Pax3 and Pax7 have distinct and overlapping functions in adult muscle progenitor cells. J. Cell Biol. 172, 91-102.

Seale, P., Sabourin, L. A., Girgis-Gabardo, A., Mansouri, A., Gruss, P., and Rudnicki, M. A. (2000). Pax7 is required for the specification of myogenic satellite cells. Cell 102, 777-786. doi: 10.1083/jcb.200508044

Sun, Y., Ge, Y., Drnevich, J., Zhao, Y., Band, M., and Chen, J. (2010). Mammalian target of rapamycin regulates miRNA-1 and follistatin in skeletal myogenesis. J. Cell Biol. 189, 1157-1169. doi: 10.1083/jcb.200912093

Torrente, Y., Tremblay, J. P., Pisati, F., Belicchi, M., Rossi, B., Sironi, M., et al. (2001). Intraarterial injection of muscle-derived CD34(+)Sca-1(+) stem cells restores dystrophin in mdx mice. J. Cell Biol. 152, 335-348. doi: 10.1083/jcb. 200912093

Wang, Y. X., and Rudnicki, M. A. (2012). Satellite cells, the engines of muscle repair. Nat. Rev. Mol. Cell Biol. 13, 127-133. doi: 10.1038/nrm3265 
Williams, A. H., Valdez, G., Moresi, V., Qi, X., McAnally, J., Elliott, J. L., et al. (2009). MicroRNA-206 delays ALS progression and promotes regeneration of neuromuscular synapses in mice. Science 326, 1549-1554. doi: 10.1126/science. 1181046

Winbanks, C. E., Wang, B., Beyer, C., Koh, P., White, L., Kantharidis, P., et al. (2011). TGF-beta regulates miR-206 and miR-29 to control myogenic differentiation through regulation of HDAC4. J. Biol. Chem. 286, 13805-13814. doi: $10.1074 /$ jbc.M110.192625

Yin, H., Price, F., and Rudnicki, M. A. (2013). Satellite cells and the muscle stem cell niche. Physiol. Rev. 93, 23-67. doi: 10.1152/physrev. 00043.2011
Conflict of Interest Statement: The authors declare that the research was conducted in the absence of any commercial or financial relationships that could be construed as a potential conflict of interest.

Copyright (c) 2018 Renzini, Marroncelli, Noviello, Moresi and Adamo. This is an open-access article distributed under the terms of the Creative Commons Attribution License (CC BY). The use, distribution or reproduction in other forums is permitted, provided the original author(s) and the copyright owner(s) are credited and that the original publication in this journal is cited, in accordance with accepted academic practice. No use, distribution or reproduction is permitted which does not comply with these terms. 
OPEN ACCESS

Edited by:

Dario Coletti,

Università degli Studi di Roma La

Sapienza, Italy

Reviewed by:

Nissrine Daou,

Université Pierre et Marie Curie,

France

Marina Bouche

Università degli Studi di Roma La

Sapienza, Italy

*Correspondence:

Fábio Santos Lira

Fabio.lira@unesp.br

Specialty section:

This article was submitted to

Striated Muscle Physiology,

a section of the journal

Frontiers in Physiology

Received: 06 June 2018 Accepted: 11 September 2018

Published: 09 October 2018

Citation:

Rossi FE, de Freitas MC, Zanchi NE, Lira FS and Cholewa JM (2018) The

Role of Inflammation and Immune Cells in Blood Flow Restriction Training Adaptation: A Review.

Front. Physiol. 9:1376.

doi: 10.3389/fphys.2018.01376

\section{The Role of Inflammation and Immune Cells in Blood Flow Restriction Training Adaptation: A Review}

\author{
Fabrício Eduardo Rossi ${ }^{1}$, Marcelo Conrado de Freitas ${ }^{2}$, Nelo Eidy Zanchi ${ }^{3}$, \\ Fábio Santos Lira ${ }^{1 *}$ and Jason M. Cholewa ${ }^{4}$ \\ ${ }^{1}$ Exercise and Immunometabolism Research Group, Department of Physical Education, São Paulo State University (UNESP), \\ Presidente Prudente, Brazil, ${ }^{2}$ Skeletal Muscle Assessment Laboratory, Department of Physical Education, School of \\ Technology and Sciences, São Paulo State University, Presidente Prudente, Brazil, ${ }^{3}$ Laboratory of Cellular and Molecular \\ Biology of Skeletal Muscle (LABCEMME), Department of Physical Education, Federal University of Maranhão (UFMA), São \\ Luís, Brazil, ${ }^{4}$ Department of Kinesiology, Coastal Carolina University, Conway, SC, United States
}

Blood flow restriction (BFR) combined with low-intensity strength training has been shown to increase skeletal muscle mass and strength in a variety of populations. BFR results in a robust metabolic stress which is hypothesized to induce muscle growth via increased recruitment of fast-twitch muscle fibers, a greater endocrine response, and/or enhancing the cellular swelling contribution to the hypertrophic process. Following exercise, neutrophils are the first immune cells to initiate the tissue remodeling process via several mechanisms including an increased production of cytokines and recruitment of monocytes/macrophages, which facilitate the phagocytosis of foreign particles, the differentiation of myoblasts, and the formation of new myotubes. Thus, the purpose of this review was to discuss the mechanisms through which metabolic stress and immune cell recruitment may induce skeletal muscle remodeling following BFR strength training.

Keywords: immune cell, occlusion, hypertrophy, resistance exercise, KAATSU training

\section{INTRODUCTION}

Strength training has been the classically employed exercise program to increase skeletal muscle hypertrophy and strength. The American College of Sports Medicine (Evetovich, 2009) recommends strength training with intensities over $70 \%$ of the one repetition maximum (1RM) to increase muscle mass, and several studies have shown that intensities in excess of 70\% 1RM greater stimulate muscle protein synthesis, satellite cell activity, and decrease proteolysis when compared to lowintensity resistance exercise (Harridge, 2007; Louis et al., 2007). There is a growing body of evidence, however, that lower-intensity strength training induces similar degrees of muscle protein synthesis acutely (Burd et al., 2010, 2012) and muscle hypertrophy chronically (Schoenfeld et al., 2016) when sets are carried out to muscular failure. Although heavier loading seems necessary to maximize muscular strength development (Peterson et al., 2005), high-intensity loads performed in traditional strength training programs may not be a viable option for sarcopenic populations, older individuals with chronic health problems, or individuals with injuries who may be unable to tolerate heavy loading. 
Blood flow restriction (BFR), also known as occlusion or KAATSU training (Abe et al., 2005), combined with low-intensity strength training $(20-30 \% 1 \mathrm{RM})$ has been shown to increase muscle size and strength in the elderly (Libardi et al., 2015), athletes (Takarada et al., 2002), untrained (Clark et al., 2011), and people with injuries (Loenneke et al., 2013). This training model requires the use of inflatable cuffs that are placed at the proximal ends of the upper arms or thighs to restrict blood flow, whereby the external pressure applied is sufficient to maintain arterial inflow whilst occluding venous outflow distal to the occlusion site (Kaijser et al., 1990), thus resulting in an ischemic/hypoxic environment that enhances the training effect in exercising muscle (Takada et al., 2012).

Several studies have compared low-intensity strength training with BFR and high-intensity strength training without BFR and demonstrated similar increases in muscle cross-section area between exercise protocols (Ellefsen et al., 2015; Farup et al., 2015; Libardi et al., 2015), however, the mechanisms responsible for such effects are still poorly understood. Mechanical tension and metabolic stress are suggested to be the factors primarily responsible for hypertrophic adaptations following BFR training (Pearson and Hussain, 2015).

The mechanisms by which mechanical tension induces muscle hypertrophy include mechanotransduction, where sarcolemmalbound mechanosensors (i.e., integrins) stimulate intracellular anabolic and catabolic pathways which convert mechanical energy into chemical signals promoting protein synthesis instead of degradation (Zou et al., 2011). Exercise-induced muscle damage can be influenced by an increase in the volume, velocity, initial muscle length, or the intensity of the exercise. These stimuli can result in an overstretching of the sarcomere to such an extent that it becomes disrupted, resulting in z-disk damage and eventually a disruption of the cytoskeletal matrix (Proske and Morgan, 2001). This may promote activation of the stretch-activated calcium channels or transient receptor potential channels (Allen et al., 2005). Consequently, cytokines are released and there is an immune system activation, directing the local satellite cells to the site of muscle damage and initiating the phagocytosis of debris and tissue remodeling (Tidball and Villalta, 2010).

In contrast to the muscle damage that occurs as a result of traditional strength training, a review conducted by Loenneke et al. (2014a) does not support the hypothesis that BFR training increases the incidence of muscle damage. Sudo et al. (2015) verified muscle fiber damage following high-intensity eccentric contractions and BFR plus eccentric contractions in male Wistar rats with different levels of occlusion pressure (140, 160, and 200 Torr). Despite BFR increased Ribosomal S6 kinase 1 (S6K1) phosphorylation, a downstream target of rapamycin (mTOR)phosphorylation kinase, there was no measurable muscle damage for any of the three different pressures $(2.6 \pm 1.2 \%),(3.0 \pm 0.5 \%)$, $(0.2 \pm 0.1 \%)$, respectively, compared to high-intensity (26.4 $\pm 4.0 \%)$ when verified the histochemical muscle fiber damage (area of damaged fibers/total fiber area analyzed). In humans, Neto et al. (2018) compared low-load resistance exercise $(20 \%$ $1 \mathrm{RM}$ ) plus continuous or intermittent BFR and high-intensity resistance exercise $(80 \% 1 \mathrm{RM})$ and observed higher creatine kinase and lactate dehydrogenase after high-intensity compared with both continuous or intermittent exercise with BFR 24 and $48 \mathrm{~h}$ post-exercise. In addition, Wilson et al. (2013) demonstrated significantly increases muscle activation and muscle thickness, but no changes in muscle soreness, power, and muscle swelling after 4 sets of leg press (30-15-15-15-repetition at 30\% 1 RM) in trained men. Collectively, this research refutes the muscle damage hypothesis post-BFR training.

In this sense, the metabolic stress during BFR as a result of the ischemic/hypoxic environment seems to be the prevailing mechanism whereyby BFR could potentiate hypertrophy during low-intensity strength training. Metabolic stress and/or hypoxia may leads to an increased recruitment of fast-twitch muscle fibers (Suga et al., 2009), increased inflammatory and endocrine response (Fujita et al., 2007), cellular swelling (Loenneke et al., 2013), and elevated intramuscular inorganic phosphates (Takada et al., 2012), all of which have been shown mediate muscle protein signaling and satellite cell proliferation (Figure 1).

Hypoxia-inducible factor-1 alpha (HIF-1 $\alpha$ ) activation contributes to an increased expression of angiogenic factors, vascular endothelial growth factor (VEGF), endothelial nitric oxide synthase (eNOS) (Larkin et al., 2012), and monocyte chemotactic protein-1 (MCP-1). Warren et al. (2004) used a freeze injury model to evaluate muscle repair and inflammation in mice and observed a significant importance for MCP-1, macrophage inflammatory protein (MIP)-1alpha, MIP-1beta, and monocyte chemoattractant protein during muscle repair. Mice that were null mutants for MCP-1 were found to have a slowed recovery from muscle injury compared to wild-type animals as assessed by muscle force production. Shill et al. (2017) observed that BFR using unilateral isometric wrist exercise increased concentrations of fibroblast growth factor,

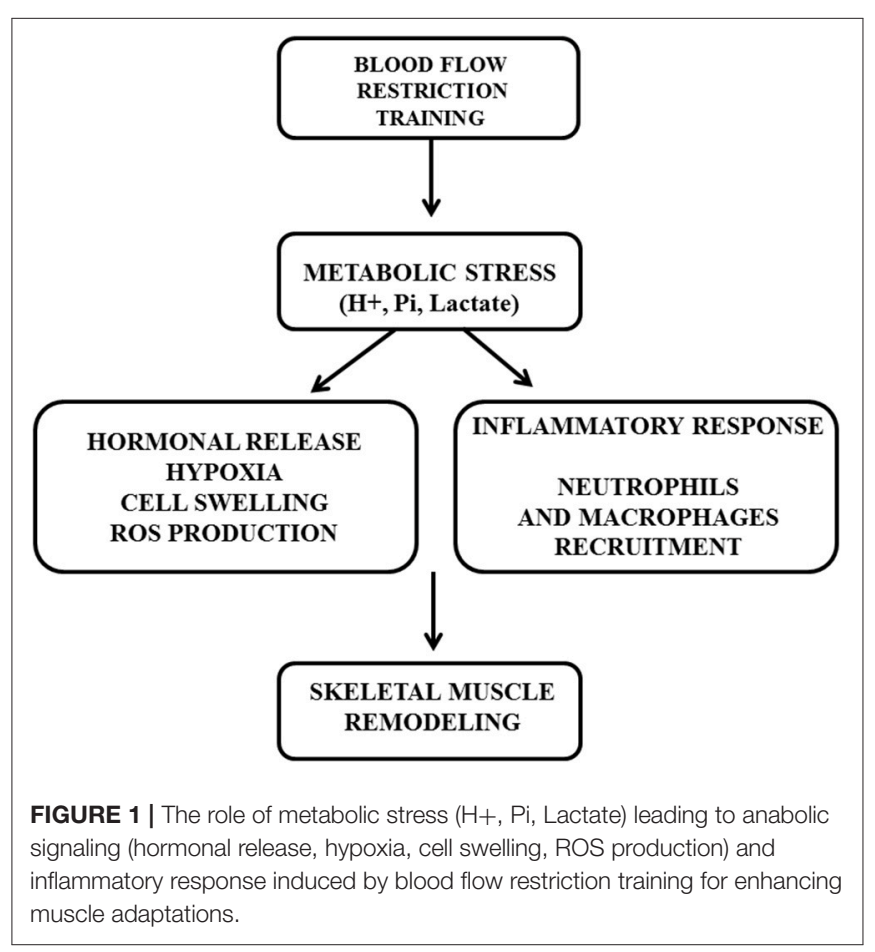


IL-6, IL-10, TNF $\alpha$, and VEGF during exercise and reduced the percentage of oxygen saturation, demonstrating that the hypoxic environment generated during BFR was associated with a heightened inflammatory cytokine production.

Thus, as hypothesized by Pearson and Hussain (2015), both mechanical tension and metabolic stress would synergistically contribute to the hypertrophic adaptations of BFR strength training, with metabolic stress playing the dominant role. Although the efficacy of BFR strength training to induce skeletal muscle hypertrophy has been well demonstrated in the literature (Loenneke and Pujol, 2009; Patterson and Ferguson, 2011; Takada et al., 2012; Loenneke et al., 2013; Pope et al., 2013), the mechanism by which BFR results in an inflammatory response and immune cell recruitment is still poorly understood. Therefore, the purpose of this review is to discuss the relationship between metabolic stress and immune cell recruitment in skeletal muscle remodeling following BFR strength training.

\section{THE INFLAMMATORY RESPONSE DURING BLOOD FLOW RESTRICTION TRAINING: POTENTIAL MECHANISMS}

The stress induced by exercise results in an inflammatory response mediated by an increase in myokine production, which are one of several hundred cytokines and proteoglycan peptides that are produced and released by muscle cells (myocytes) in response to muscular contractions (Giudice and Taylor, 2017). Myokines, specifically IL-6, play a role in skeletal muscle tissue regeneration after damaging exercise, in part by participating in the recruitment of neutrophil, monocytes, and lymphocytes, which phagocytize debris materials (Vannella and Wynn, 2017). In addition, IL- 6 also contributes to the activation, differentiation and proliferation of satellite cells, which migrate and fuse to the spaces where muscle fiber damage occurred and contribute new myonuclei (Serrano et al., 2008). Recently, Gao et al. (2017) investigated the influence of IL-6 on mTORC1 signaling and protein synthesis in cultured myotubes. It was observed that IL-6 can increase protein synthesis by activating the gp130-Akt and mTORC1 pathway in a dose-sensitive manner, suggesting that the IL-6 response to resistance exercise potentiates muscle hypertrophy.

Regarding the influence of BFR on the inflammatory response, Patterson and Ferguson (2011) compared the effects of BFR (five sets of unilateral knee extensions with 20\% 1-RM and 30-s rest between each set) with low-load resistance exercise without BFR older men. The authors observed that both conditions increased serum IL- 6 at 60 and 120 min post-exercise, but GH and VEGF only increased following the BFR condition. Takarada et al. (2000) also compared the IL-6 response after BFR using five sets of 14 repetitions at $20 \%$ of $1-\mathrm{RM}$ vs. the same exercise protocol without occlusion (control). The results showed that plasma IL-6, lactate, GH and norepinephrine was greater in BFR than the control condition without any increase in CK activity $24 \mathrm{~h}$ post-exercise in either condition, suggesting that BFR may induce a higher metabolic stress (accumulation of lactate) and hormonal response without apparent markers of muscle damage.
Supporting this, Nielsen et al. (2017) showed that short-term BFR (unilateral knee extensor exercise to failure at 20\% 1$\mathrm{RM}$ ) generated modest increases in the inflammatory responses (MCP-1, IL-6, and TNF- $\alpha$ ) with lower muscle damage.

According to these findings it appears that the early increase in IL-6 in response to BFR may be in part due to a greater metabolic stress more so than muscle damage. Studies consistently demonstrate that BFR generates a high concentration of lactate accumulation, $\mathrm{H}+$, and a significant decrease in intramuscular $\mathrm{pH}$, suggesting that BFR is highly reliant on rapid glycolytic metabolism (Takarada et al., 2000; Suga et al., 2009, 2012). Previous studies also reported an association between anaerobic metabolism and an increase in the production of inflammatory cytokines (Pedersen and Febbraio, 2005). Additionally, the reduction of muscle glycogen during exercise seems to potentiate the IL-6 response (Pedersen and Febbraio, 2005), and acute ischemic exercise induced by BFR results in a higher degree of glycogen depletion of type II fibers compared with non-ischaemic exercise (Sundberg, 1994). In this context, the increased IL-6 response by BFR may be explained by the predominance of anaerobic glycolytic metabolism and glycogen depletion.

Another possible driver of the IL- 6 response during BFR could be attributed to intramuscular hypoxia. It has been reported that BFR decreases muscle oxygenation, generating a hypoxic environment (Karabulut et al., 2011; Ganesan et al., 2015) leading to a robust activation of HIF- $1 \alpha$. Several studies have demonstrated that HIF- $1 \alpha$ has the potential to increase proinflammatory gene expression, such as IL-6 and TNF- $\alpha$, via increasing nuclear factor kappa $\mathrm{B}(\mathrm{NF}-\mathrm{kB})$ translocation to the cell nucleus (Van Uden et al., 2008; Oliver et al., 2009; Szade et al., 2015). An increased expression of HIF-1 $\alpha$ has also been associated with a high production of inflammatory cytokines (IL6 and TNF- $\alpha$ ). Leire et al. (2013) reported that when cell cultures were treated with AKB-4924, an activator of HIF-1 $\alpha$, IL-6 levels significantly increased, demonstrating the influence of HIF- $1 \alpha$ on IL-6 production. In support of this hypothesis, Drummond et al. (2008) found that bilateral knee extension exercise with BFR (4 sets of $30,15,15,15$ repetition at $20 \%$ of $1 \mathrm{RM}$, with $30 \mathrm{~s}$ rest period) increased HIF- $1 \alpha$ gene expression. Shill et al. (2017) observed that BFR using unilateral isometric wrist exercise increased concentrations of fibroblast growth factor, IL-6, IL$10, \mathrm{TNF} \alpha$, and VEGF and reduced the percentage of oxygen saturation, demonstrating a link between the BFR induced hypoxic environment and cytokine production (Figure 2).

In summary, studies have demonstrated that BFR increases the IL- 6 concentrations absent of significant muscle damage, suggesting that other mechanisms likely regulates the IL-6 response to BFR. It seems that the metabolic stress associated with glycogen depletion and hypoxia could be a potential mechanism by which BFR modulates the acute immune response.

\section{NEUTROPHIL AND MACROPHAGE RESPONSE TO RESISTANCE EXERCISE}

Multiple immune cell types play a critical role in muscle repair, regeneration and remodeling following exercise. Neutrophils, 


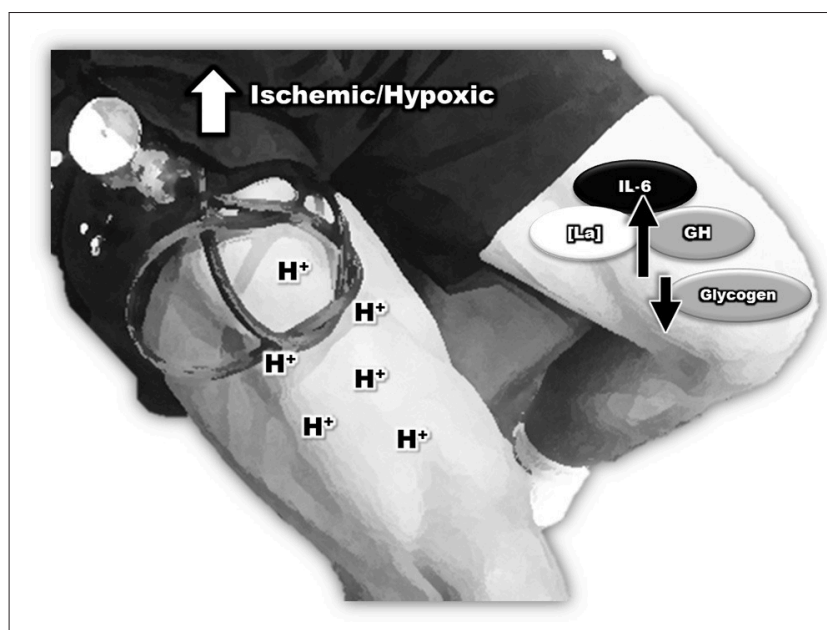

FIGURE 2 | The inflammatory response during blood flow restriction plus low-intensity strength training.

macrophages and $\mathrm{T}$ cells seem to be the first immune cells to initiate the recovery process following exercise-induced muscle damage. Neutrophils constitute $60 \%$ of the circulating leukocytes and are the most abundant cellular component of the human immune system, with a total number of approximately $5 \times 10^{11}$ cells in a $70 \mathrm{~kg}$ individual (Pyne, 1994). It has been suggested (Pyne, 1994) that neutrophil recruitment is an essential role in the initial stages of muscle repair and regeneration after exercise, as neutrophils act to clear cellular debris via the release of proteolytic enzymes for several hours after exercise.

Neutrophils accumulation in skeletal muscle likely starts immediately after exercise, as Frenette et al. (2000) showed a $60 \%$ increase in neutrophil invasion $2 \mathrm{~h}$ after muscle injury. Previous studies demonstrated neutrophil accumulation in the blood vessels of skeletal muscle occurs after 1 and $24 \mathrm{~h}$ after eccentric exercise (Raastad et al., 2003; Paulsen et al., 2010), and histological studies have demonstrated that after $24-48 \mathrm{~h}$ of exercise, there is an increase of leukocytes infiltratation in the extracellular space within the muscle (Hellsten et al., 1997; Paulsen et al., 2010). Animal studies have also demonstrated a clear influence of neutrophils on muscle regeneration after exercise, as depletion of neutrophils in mice before muscle injury impairs skeletal muscle regeneration, likely as a result of a reduced capacity to remove tissue debris by phagocytes that slowed regenerative process (Teixeira et al., 2003).

After the acute neutrophil accumulation, macrophages, a subpopulation of leukocytes, infiltrate the muscle and play an important role in the later stages of muscle repair and regeneration. The monocytes/macrophages are attracted in skeletal muscle tissue by chemotactic factors such as fractalkine (CX3CL1) and monocyte chemotactic protein-1 (MCP-1), contributing to skeletal muscle repair by phagocytosis of foreign particles, secreting pro-inflammatory cytokines (e.g., TNF $\alpha$ and IL-6) and stimulating satellite cell proliferation (Peake and Neubauer, 2017). Previous studies report that the increase in macrophages in human skeletal muscle occurred at later time points in recovery, around at $48 \mathrm{~h}$ to 7 days after exercise (Jones et al., 1986; Marklund et al., 2013). It has been demonstrated that macrophages play a critical role for muscle regeneration, as inhibiting the recruitment of monocytes to injured muscles impairs muscle regeneration ( $\mathrm{Lu}$ et al., 1998). Warren et al. (2004) used a freeze injury model to evaluate muscle repair and inflammation in mice and observed a significant importance for MCP-1 and macrophage recruitment to potentiate skeletal muscle regeneration. Mice that were null mutants for Chemokine Receptor 5 (CCR5) and MCP-1 were found to have slowed recovery from muscle injury compared to wild-type animals as assessed by muscle force production as an index of recovery.

Additionally, both macrophages and neutrophils appear to mediate muscle hypertrophy by the secretion of proinflammatory cytokines (IL-6 and TNF $\alpha$ ), leading to anabolic signaling through a pathway that phosphorylates mTORC1 (Schoenfeld et al., 2016). It has been demonstrated that the production of ROS may stimulate anabolic signaling, as ROS can act with a signaling molecule to activate mTORC1 signaling via the IGF-1 and MAPK pathways (Kefaloyianni et al., 2006; Handayaningsih et al., 2011). In addition, neutrophils and macrophages can release growth factors such as insulin-like growth factor (IGF-1), basic Fibroblast Growth Factor (bFGF), Transforming Growth Factor (TGF), and Mechano Growth Factor (MGF), and these factors contribute to the muscle regeneration and hypertrophic response by activating satellite cells and mTORC1 signaling (Jiang et al., 1992; Cantini et al., 2002; Koh and Pizza, 2009; Zanou and Gailly, 2013; Figure 3).

\section{NEUTROPHIL AND MACROPHAGE RESPONSE TO BLOOD FLOW RESTRICTION EXERCISE}

After traditional resistance exercise there is an increase in neutrophils (Macintyre et al., 2000; Simonson and Jackson, 2004) and macrophages (Malm et al., 1999); however, the influence of BFR on the immune cell response is less discussed in the literature. Nielsen et al. (2017) investigated the effect of 3 weeks of BFR training (20\%-1RM to concentric failure) compared to work-matched traditional resistance training performed at low (20\%-1RM) or high-intensities (HI; 70\%-1RM) and observed that proinflammatory macrophages (M1) increased $108 \%$ with BFR training and 165\% with low intensity strength training 3 days after training cessation. Additionally, anti-inflammatory phenotype (M2) increased $163 \%$ only with BFR training. Membrane and intracellular HSP27 expression increased 60$132 \%$ at 8 days into the intervention with BFR. The authors concluded that signs of myocellular stress were observed in the early phase of the training intervention with inflammation occurring after the training program. Thus, this research furthers the hypothesis that the macrophage response to BFR training occurs due to a heightened inflammation response but is likely not related to muscle damage.

Regarding neutrophils, Behringer et al. (2017) investigated the effects of moderate-intensity (four sets at 75\% of 1RM until volitional failure) eccentric knee extensions with BFR $(20 \mathrm{mmHg})$ 


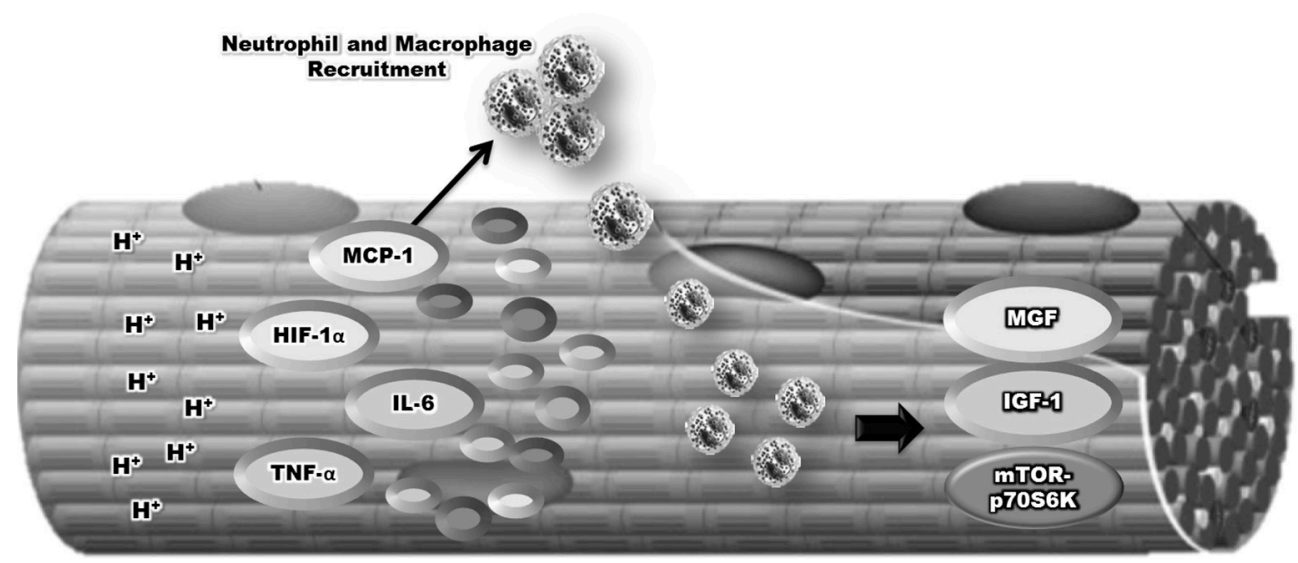

FIGURE 3 | Relationship between inflammatory, neutrophil, and macrophage in the remodeling of skeletal muscle mass induced by blood flow restriction plus strength training.

TABLE 1 | Effect of blood flow restriction plus low-intensity strength training on the inflammatory response.

\begin{tabular}{|c|c|c|}
\hline Author (year) & Aim & Results \\
\hline Takarada et al., 2000 & $\begin{array}{l}\text { Compared the IL- } 6 \text { response after BFR using five sets of } 14 \text { repetitions at } 20 \% \text { of } \\
1-\mathrm{RM} \text { vs. the same exercise protocol without occlusion (control) }\end{array}$ & $\begin{array}{l}\uparrow \mathrm{IL}-6 \text {, lactate, GH, and norepinephrine in BFR condition } \\
\leftrightarrow \text { Creatine kinase in both conditions }\end{array}$ \\
\hline Patterson and & Compared the effects of BFR (five sets of unilateral knee extensions with 20\% & $\uparrow$ IL-6 in both conditions \\
\hline Ferguson, 2011 & $\begin{array}{l}1-\mathrm{RM} \text { and } 30 \text {-s rest between each set) with low-load resistance exercise without } \\
\text { BFR on the inflammatory response }\end{array}$ & $\uparrow \mathrm{GH}$ and VEGF only in BFR condition \\
\hline Nielsen et al., 2017 & $\begin{array}{l}\text { Investigated the effect of } 3 \text { weeks of BFR training ( } 20 \%-1 \mathrm{RM} \text { to concentric } \\
\text { failure) compared to two groups that performed a matched amount of work at } \\
\text { low }(20 \%-1 \mathrm{RM}) \text { or high-intensities (HI; } 70 \%-1 \mathrm{RM})\end{array}$ & $\begin{array}{l}\uparrow \text { macrophages (M1) in BFR and low intensity training } \\
\text { conditions } \\
\uparrow \text { macrophage (M2) only in BFR condition } \\
\uparrow \mathrm{HSP} 27 \text { expression only in BFR condition } \\
\leftrightarrow \mathrm{L}-6 \text {, TNF- } \alpha \text {, and MCP- } 1 \text { in BFR condition } \\
\uparrow \text { CK only in HI condition }\end{array}$ \\
\hline Behringer et al., 2017 & $\begin{array}{l}\text { Investigated the effects of moderate-intensity (four sets at } 75 \% \text { of } 1 \mathrm{RM} \text { until } \\
\text { volitional failure) eccentric knee extensions with BFR }(20 \mathrm{mmHg}) \text { and without } \\
\text { (control) on blood lactate, hormonal response, muscle swelling, biomarkers of } \\
\text { muscle damage and neutrophil counts }\end{array}$ & $\begin{array}{l}\downarrow \text { volume in BFR compared with control condition } \\
\uparrow \text { neutrophil counts, lactate muscle swelling, GH, and } \\
\text { CK in both conditions }\end{array}$ \\
\hline
\end{tabular}

$\uparrow$, significant increased; $\downarrow$, significant decreased; $\leftrightarrow$, no changed.

and without on blood lactate, endocrine response, muscle swelling, biomarkers of muscle damage, and neutrophil counts. The results showed that the total number of repetitions was lower in BFR compared with the control condition, however, despite a lower volume, BFR induced a similar increase in metabolic stress (lactate), muscle swelling, plasma GH, creatine kinase (CK), and neutrophil counts. The authors suggested that the lower oxygen supply during BFR may have led to a greater metabolic stress and membrane damage due reactive oxygen species (ROS) from an ischemia-reperfusion sequence or by invading neutrophils. The higher intensity (75\% 1RM vs. 20-30\% 1RM) and lower occlusion pressure may explain the discrepancies in markers of muscle damage found in this study compared to other BFR studies.

The mechanism by which macrophages and neutrophils are recruited to skeletal muscle after BFR is not fully understood, but can be partially attributed to transcription factors such as HIF- $1 \alpha$ that respond to decreases in available oxygen at the cellular level (Drummond et al., 2008). Leire et al. (2013) investigated the role of AKB-4924, an activator of HIF-1 $\alpha$, on neutrophil recruitment in mice. The results showed that AKB4924 increased the recruitment of neutrophils to the site of inflammation induced by lipopolysaccharide (LPS) injection. When a high dose $(5 \mu \mathrm{g})$ of LPS was injected in HIF-1 $\alpha$ knockout mice, neutrophil recruitment to the injury site was reduced compared with control mice. HIF-1 $\alpha$ activation via hypoxia contributes to increased expression of MCP-1 (also known as CCL2) (Larkin et al., 2012), which is a chemokine with potent macrophage recruitment and activating functions (Lu et al., 1998; Chazaud et al., 2003). Roseguini et al. (2010) demonstrated that intermittent pneumatic compressions, a method conceptually similar to BFR, increases skeletal muscle MCP-1 expression in rats. It is likely the myocellular macrophage infiltration after BFR as demonstrated by Nielsen et al. (2017) may be explained by increased MCP-1 in skeletal muscle via HIF-1 activation. Thus, activation of HIF- $1 \alpha$ may be one potential mechanism that BFR increases neutrophil and macrophage response.

BFR training increases the IL-6 production, which may also induce the recruitment of immune cells (Takarada 
et al., 2000; Patterson and Ferguson, 2011; Nielsen et al., 2017), and in particular, neutrophils. Fielding et al. (2008) showed that mice with alterations in IL-6/gp130 signaling by the knockout of IL-6 receptor (gp130) decreased the rate of neutrophil trafficking during acute inflammation. In addition, the researchers demonstrated that IL- 6 can regulate neutrophil trafficking in a STAT3 dependent-manner. Therefore, increasing IL-6 levels via BFR training could increase neutrophil recruitment for skeletal muscle remodeling.

Another mechanism whereby BFR may increase the recruitment of neutrophils without inducing muscle damage may be attributed to catecholamine release, specifically norepinephrine. Takarada et al. (2000) demonstrated that a BFR protocol using five sets at $20 \%$ of $1-\mathrm{RM}$ and 14 repetitions induced greater norepinephrine production compared with the same exercise protocol without occlusion. Shimizu et al. (2016) compared the influence of BFR (3 sets at 20\% of $1 \mathrm{RM}$ with $30 \mathrm{~s}$ of rest interval) vs. resistance exercise without occlusion (control) on the endocrine response in healthy elderly people. It was observed that BFR generated a higher production of norepinephrine as well as lactate, VEGF and $\mathrm{GH}$ compared with the control condition. Previous studies showed that noradrenaline may act as "danger signals" during fatiguing exercise, thereby increasing neutrophil activation and function. Hinchado et al. (2012) found that after exercise when neutrophils were incubated with norepinephrine there was an increase of chemotaxis, phagocytosis and microbicide capacity, demonstrating an improved neutrophil function. These findings may explain in part the increase of neutrophils counts induced by BRF (Behringer et al., 2017), as norepinephrine seems to play a significant role in neutrophil function and activation.

The immune system appears to act in a straightforward manner to contribute to the plasticity of skeletal muscle tissue via the phagocytosis of foreign particles and increasing inflammation by releasing cytokines that stimulate further infiltration of immune cells in an attempt to repair and regenerate damaged tissues. We believe that hypoxia, metabolic mediators, and IL-6 mediated by BFR during low-intensity strength training could be important factors to potentiate the macrophage and neutrophil recruitment contributing to improvement skeletal muscle remodeling; however, there are few studies in the literature investigating the effects of BFR training on immune cells response according showed at Table 1. Therefore, future studies should be conducted to better understand the immune system response during acute and chronic BFR training, and to further investigate how BFR may be employed as an appropriate treatment in muscle wasting disorders characterized by chronic low-grade inflammation.

\section{FUTURE PERSPECTIVE}

The immune system is virtually involved in every repair and regeneration response in skeletal muscle cells. However, the immune system is not activated only in response to conditions of muscle damage, but also in response to muscle contractions, with muscle metabolites and substrates utilization being major drivers of such response. At the moment, several studies that demonstrate minimal to no muscle damage following BFR contractions refute the muscle damage hypothesis (Wilson et al., 2013; Loenneke et al., 2014b; Sudo et al., 2015). However, a certain degree of caution should be taken when interpreting this data since signs of muscle damage may occur in the absence of either soreness, reduced force production, and/or increased plasma CK. Nevertheless, infiltration of immune cells in the muscle is seen after a BFR exercisewhich poses the question of, mechanistically, who is directing immune cells to the muscle and what tasks they are performing, since there does not appear to be any measurable muscle damage. In regards to "who" is recruiting immune cells to the skeletal muscle, a multitude of mechanisms may exist, from cytokines secreted by the contracting muscle to activation of transcription factors activating chemotactic proteins (Giudice and Taylor, 2017; Vannella and Wynn, 2017), both of which are reinforced by muscle hypoxia. The question of "what task" they are performing may be more related to muscle remodeling, since BFR is a potent method to induce muscle hypertrophy, and, in fact, the studies which show a robust infiltration of immune cells to the muscle are the studies reporting robust muscle hypertrophy in short periods of time (i.e., 19 days) (Nielsen et al., 2012). Although classical hypertrophic pathways, such as mTOR, have been shown to be activated by BFR, non-canonical activations via growth factors secreted by the immune cells may reinforce the intrinsic muscle contraction effects on signaling pathways. Such cell signaling activation can lead to increased protein synthesis and muscle hypertrophy. In this case, multiple pathways and modus operandis may be coordinately working to produce muscle hypertrophy and immune system activation. The question remains: If there is no muscle damage, then what is triggering the immune system to work so hard in the BFR paradigm?

\section{CONCLUSION}

Skeletal muscle hypertrophy is an important goal for individuals pursuing both athletic or rehabilitative gains. With this goal in mind, new strategies have been employed to investigate varying cell signaling pathways, cellular types, and hormonal triggers, and which seem to be activated via tension or metabolic overload. Traditionally, exercises involving muscle damage are well known activators of muscle satellite cells, pro-inflammatory cytokines, and immune cells. However, the recent literature has pointed out that metabolic stimuli (induced by resistance training plus BFR) such as hypoxia and metabolic overload $\left(\mathrm{H}^{+}, \mathrm{P}_{\mathrm{i}}\right.$, lactate accumulation) are also potential activators of IL-6, macrophages and neutrophils. Taken collectively, these anabolic stimuli could help to explain the effectiveness of such methods in the absence of high mechanical forces and muscle damage. Mechanistic studies will help to dissect the importance of each variable in the role of the metabolic response, thus strengthening the concept that BFR exercises, although characterized as low force exercises, are effective not only to increase the MPS response, but also the multitude of immune cell responses that accompanies muscle hypertrophy. 


\section{AUTHOR CONTRIBUTIONS}

FR, MdF, NZ, FL, and JC conceptualized and drafted the paper. All authors revised the work and final approval of the manuscript.

\section{REFERENCES}

Abe, T., Hinata, S., Koizumi, K., and Sato, Y. (2005). Day-to-day change in muscle strength and MRI-measured skeletal muscle size during 7 days KAATSU resistance training: a case study. Int. J. KAATSU Train. Res. 1, 71-76. doi: $10.3806 /$ ijktr.1.71

Allen, D. G., Whitehead, N. P., and Yeung, E. W. (2005). Mechanisms of stretch-induced muscle damage in normal and dystrophic muscle: role of ionic changes. J. Physiol. 567, 723-735. doi: 10.1113/jphysiol.2005. 091694

Behringer, M., Heinke, L., Leyendecker, J., and Mester, J. (2017). Effects of blood flow restriction during moderate-intensity eccentric knee extensions. J. Physiol. Sci. 68, 589-599. doi: 10.1007/s12576-017-0568-2

Burd, N. A., Mitchell, C. J., Churchward-Venne, T. A., and Phillips, S. M. (2012). Bigger weights may not beget bigger muscles: evidence from acute muscle protein synthetic responses after resistance exercise. Appl. Physiol. Nutr. Metab. 37, 551-554. doi: 10.1139/h2012-022

Burd, N. A., West, D. W., Staples, A. W., Atherton, P. J., Baker, J. M., Moore, D. R., et al. (2010). Low-load high volume resistance exercise stimulates muscle protein synthesis more than high-load low volume resistance exercise in young men. PLoS ONE 5:e12033. doi: 10.1371/journal.pone.0012033

Cantini, M., Giurisato, E., Radu, C., Tiozzo, S., Pampinella, F., Senigaglia, D., et al. (2002). Macrophage-secreted myogenic factors: a promising tool for greatly enhancing the proliferative capacity of myoblasts in vitro and in vivo. Neurol. Sci. 23, 189-194. doi: 10.1007/s100720200060

Chazaud, B., Sonnet, C., Lafuste, P., Bassez, G., Rimaniol, A. C., Poron, F., et al. (2003). Satellite cells attract monocytes and use macrophages as a support to escape apoptosis and enhance muscle growth. J. Cell Biol. 163, 1133-1143. doi: $10.1083 /$ jcb. 200212046

Clark, B. C., Manini, T. M., Hoffman, R. L., Williams, P. S., Guiler, M. K., Knutson, M. J., et al. (2011). Relative safety of 4 weeks of blood flow-restricted resistance exercise in young, healthy adults. Scand. J. Med. Sci. Sports 21, 653-662. doi: 10.1111/j.1600-0838.2010.01100.x

Drummond, M. J., Fujita, S., Abe, T., Dreyer, H. C., Volpi, E., and Rasmussen, B. B. (2008). Human muscle gene expression following resistance exercise and blood flow restriction. Med. Sci. Sports Exerc. 40, 691-698. doi: 10.1249/MSS.0b013e318160ff84

Ellefsen, S., Hammarstrom, D., Strand, T. A., Zacharoff, E., Whist, J. E., Rauk, I., et al. (2015). Blood flow-restricted strength training displays high functional and biological efficacy in women: a within-subject comparison with high-load strength training. Am. J. Physiol. Regul. Integr. Comp. Physiol. 309, R767-R779. doi: 10.1152/ajpregu.00497.2014

Evetovich, T. K. (2009). Progression models in resistance training for healthy adults (vol 41, pg 687, 2009). Med. Sci. Sports Exerc. 41, 1351-1351.

Farup, J., De Paoli, F., Bjerg, K., Riis, S., Ringgard, S., and Vissing, K. (2015). Blood flow restricted and traditional resistance training performed to fatigue produce equal muscle hypertrophy. Scand. J. Med. Sci. Sports 25, 754-763. doi: $10.1111 /$ sms.12396

Fielding, C. A., Mcloughlin, R. M., Mcleod, L., Colmont, C. S., Najdovska, M., Grail, D., et al. (2008). IL-6 regulates neutrophil trafficking during acute inflammation via STAT3. J. Immunol. 181, 2189-2195. doi: 10.4049/jimmunol.181. 3.2189

Frenette, J., Cai, B., and Tidball, J. G. (2000). Complement activation promotes muscle inflammation during modified muscle use. Am. J. Pathol. 156, 2103-2110. doi: 10.1016/S0002-9440(10)65081-X

Fujita, S., Abe, T., Drummond, M. J., Cadenas, J. G., Dreyer, H. C., Sato, Y., et al. (2007). Blood flow restriction during low-intensity resistance exercise increases S6K1 phosphorylation and muscle protein synthesis. J. Appl. Physiol. 103, 903-910. doi: 10.1152/japplphysiol.00195.2007

\section{FUNDING}

The authors would like to thank FAPESP for their support (2015/23910-8, 2016/16712-8) and The Coordination for the Improvement of Higher Education Personnel (CAPES).

Ganesan, G., Cotter, J. A., Reuland, W., Cerussi, A. E., Tromberg, B. J., and Galassetti, P. (2015). Effect of blood flow restriction on tissue oxygenation during knee extension. Med. Sci. Sports Exerc. 47, 185-193. doi: 10.1249/MSS.0000000000000393

Gao, S., Durstine, J. L., and Koh, H. J. (2017). Acute myotube protein synthesis regulation by IL-6-related cytokines. Am. J. Physiol. Cell Physiol. 313, C487C500. doi: 10.1152/ajpcell.00112.2017

Giudice, J., and Taylor, J. M. (2017). Muscle as a paracrine and endocrine organ. Curr. Opin. Pharmacol. 34, 49-55. doi: 10.1016/j.coph.2017.05.005

Handayaningsih, A. E., Iguchi, G., Fukuoka, H., Nishizawa, H., Takahashi, M., Yamamoto, M., et al. (2011). Reactive oxygen species play an essential role in IGF-I signaling and IGF-I-induced myocyte hypertrophy in C2C12 myocytes. Endocrinology 152, 912-921. doi: 10.1210/en.2010-0981

Harridge, S. D. (2007). Plasticity of human skeletal muscle: gene expression to in vivo function. Exp. Physiol. 92, 783-797. doi: 10.1113/expphysiol.2006.036525

Hellsten, Y., Frandsen, U., Orthenblad, N., Sjodin, B., and Richter, E. A. (1997) Xanthine oxidase in human skeletal muscle following eccentric exercise: a role in inflammation. J. Physiol. 498(Pt 1), 239-248.

Hinchado, M. D., Giraldo, E., and Ortega, E. (2012). Adrenoreceptors are involved in the stimulation of neutrophils by exercise-induced circulating concentrations of Hsp72: cAMP as a potential "intracellular danger signal." J. Cell. Physiol. 227, 604-608. doi: 10.1002/jcp.22759

Jiang, W., Puntis, M. C., Nakamura, T., and Hallett, M. B. (1992). Neutrophil priming by hepatocyte growth factor, a novel cytokine. Immunology 77, 147-149.

Jones, D. A., Newham, D. J., Round, J. M., and Tolfree, S. E. (1986). Experimental human muscle damage: morphological changes in relation to other indices of damage. J. Physiol. 375, 435-448. doi: 10.1113/jphysiol.1986.sp016126

Kaijser, L., Sundberg, C. J., Eiken, O., Nygren, A., Esbjornsson, M., Sylven, C., et al. (1990). Muscle oxidative capacity and work performance after training under local leg ischemia. J. Appl. Physiol. 69, 785-787. doi: 10.1152/jappl.1990.69.2.785

Karabulut, M., Mccarron, J., Abe, T., Sato, Y., and Bemben, M. (2011). The effects of different initial restrictive pressures used to reduce blood flow and thigh composition on tissue oxygenation of the quadriceps. J. Sports Sci. 29, 951-958. doi: 10.1080/02640414.2011.572992

Kefaloyianni, E., Gaitanaki, C., and Beis, I. (2006). ERK1/2 and p38-MAPK signalling pathways, through MSK1, are involved in NF-kappaB transactivation during oxidative stress in skeletal myoblasts. Cell. Signal. 18, 2238-2251. doi: 10.1016/j.cellsig.2006.05.004

Koh, T. J., and Pizza, F. X. (2009). Do inflammatory cells influence skeletal muscle hypertrophy? Front. Biosci. 1, 60-71.

Larkin, K. A., Macneil, R. G., Dirain, M., Sandesara, B., Manini, T. M., and Buford, T. W. (2012). Blood flow restriction enhances post-resistance exercise angiogenic gene expression. Med. Sci. Sports Exerc. 44, 2077-2083. doi: 10.1249/MSS.0b013e3182625928

Leire, E., Olson, J., Isaacs, H., Nizet, V., and Hollands, A. (2013). Role of hypoxia inducible factor-1 in keratinocyte inflammatory response and neutrophil recruitment. J. Inflamm. 10:28. doi: 10.1186/1476-9255-10-28

Libardi, C. A., Chacon-Mikahil, M. P., Cavaglieri, C. R., Tricoli, V., Roschel, H., Vechin, F. C., et al. (2015). Effect of concurrent training with blood flow restriction in the elderly. Int. J. Sports Med. 36, 395-399. doi: 10.1055/s-0034-1390496

Loenneke, J. P., and Pujol, T. J. (2009). The use of occlusion training to produce muscle hypertrophy. Strength Cond. J. 31, 77-84. doi: 10.1519/SSC.0b013e3181a5a352

Loenneke, J. P., Thiebaud, R. S., and Abe, T. (2014a). Does blood flow restriction result in skeletal muscle damage? A critical review of available evidence. Scand. J. Med. Sci. Sports 24, e415-e422. doi: 10.1111/sms.12210 
Loenneke, J. P., Thiebaud, R. S., Abe, T., and Bemben, M. G. (2014b). Blood flow restriction pressure recommendations: the hormesis hypothesis. Med. Hypotheses 82, 623-626. doi: 10.1016/j.mehy.2014.02.023

Loenneke, J. P., Young, K. C., Wilson, J. M., and Andersen, J. (2013). Rehabilitation of an osteochondral fracture using blood flow restricted exercise: a case review. J. Bodyw. Mov. Ther. 17, 42-45. doi: 10.1016/j.jbmt.2012.04.006

Louis, E., Raue, U., Yang, Y., Jemiolo, B., and Trappe, S. (2007). Time course of proteolytic, cytokine, and myostatin gene expression after acute exercise in human skeletal muscle. J. Appl. Physiol. 103, 1744-1751. doi: 10.1152/japplphysiol.00679.2007

Lu, B., Rutledge, B. J., Gu, L., Fiorillo, J., Lukacs, N. W., Kunkel, S. L., et al. (1998). Abnormalities in monocyte recruitment and cytokine expression in monocyte chemoattractant protein 1-deficient mice. J. Exp. Med. 187, 601-608. doi: $10.1084 /$ jem.187.4.601

Macintyre, D. L., Reid, W. D., Lyster, D. M., and Mckenzie, D. C. (2000). Different effects of strenuous eccentric exercise on the accumulation of neutrophils in muscle in women and men. Eur. J. Appl. Physiol. 81, 47-53. doi: 10.1007/PL00013796

Malm, C., Lenkei, R., and Sjodin, B. (1999). Effects of eccentric exercise on the immune system in men. J. Appl. Physiol. 86, 461-468. doi: 10.1152/jappl.1999.86.2.461

Marklund, P., Mattsson, C. M., Wahlin-Larsson, B., Ponsot, E., Lindvall, B., Lindvall, L., et al. (2013). Extensive inflammatory cell infiltration in human skeletal muscle in response to an ultraendurance exercise bout in experienced athletes. J. Appl. Physiol. 114, 66-72. doi: 10.1152/japplphysiol.01538.2011

Neto, G. R., Novaes, J. S., Salerno, V. P., Gonçalves, M. M., Batista, G. R., and Cirilo-Sousa, M. S.(2018). Does a resistance exercise session with continuous or intermittent blood flow restriction promote muscle damage and increase oxidative stress? J. Sports Sci. 36, 104-110. doi: 10.1080/02640414.2017.12 83430

Nielsen, J. L., Aagaard, P., Bech, R. D., Nygaard, T., Hvid, L. G., Wernbom, M., et al. (2012). Proliferation of myogenic stem cells in human skeletal muscle in response to low-load resistance training with blood flow restriction. J. Physiol. 590, 4351-4361. doi: 10.1113/jphysiol.2012.237008

Nielsen, J. L., Aagaard, P., Prokhorova, T. A., Nygaard, T., Bech, R. D., Suetta, C., et al. (2017). Blood flow restricted training leads to myocellular macrophage infiltration and upregulation of heat shock proteins, but no apparent muscle damage. J. Physiol. 595, 4857-4873. doi: 10.1113/JP273907

Oliver, K. M., Taylor, C. T., and Cummins, E. P. (2009). Hypoxia. Regulation of $\mathrm{NF \kappa B}$ signalling during inflammation: the role of hydroxylases. Arthritis Res. Ther. 11, 215. doi: 10.1186/ar2575

Patterson, S. D., and Ferguson, R. A. (2011). Enhancing strength and postocclusive calf blood flow in older people with training with blood-flow restriction. $J$. Aging Phys. Act. 19, 201-213. doi: 10.1123/japa.19.3.201

Paulsen, G., Egner, I. M., Drange, M., Langberg, H., Benestad, H. B., Fjeld, J. G., et al. (2010). A COX-2 inhibitor reduces muscle soreness, but does not influence recovery and adaptation after eccentric exercise. Scand. J. Med. Sci. Sports 20, e195-e207. doi: 10.1111/j.1600-0838.2009.00947.x

Peake, J. M., and Neubauer, O. (2017). Muscle damage and inflammation during recovery from exercise. J. Appl. Physiol. 122, 559-570. doi: 10.1152/japplphysiol.00971.2016

Pearson, S. J., and Hussain, S. R. (2015). A review on the mechanisms of bloodflow restriction resistance training-induced muscle hypertrophy. Sports Med. 45, 187-200. doi: 10.1007/s40279-014-0264-9

Pedersen, B. K., and Febbraio, M. (2005). Muscle-derived interleukin-6-a possible link between skeletal muscle, adipose tissue, liver, and brain. Brain Behav. Immun. 19, 371-376. doi: 10.1016/j.bbi.2005.04.008

Peterson, M. D., Rhea, M. R., and Alvar, B. A. (2005). Applications of the doseresponse for muscular strength development: a review of meta-analytic efficacy and reliability for designing training prescription. J. Strength Cond. Res. 19, 950-958. doi: 10.1519/R-16874.1

Pope, Z. K., Willardson, J. M., and Schoenfeld, B. J. (2013). Exercise and blood flow restriction. J. Strength Cond. Res. 27, 2914-2926. doi: 10.1519/JSC.0b013e3182874721

Proske, U., and Morgan, D. L. (2001). Muscle damage from eccentric exercise: mechanism, mechanical signs, adaptation and clinical applications. J. Physiol. 537, 333-345. doi: 10.1111/j.1469-7793.2001.00333.x
Pyne, D. B. (1994). Regulation of neutrophil function during exercise. Sports Med. 17, 245-258. doi: 10.2165/00007256-199417040-00005

Raastad, T., Risoy, B. A., Benestad, H. B., Fjeld, J. G., and Hallen, J. (2003). Temporal relation between leukocyte accumulation in muscles and halted recovery $10-20 \mathrm{~h}$ after strength exercise. J. Appl. Physiol. 95, 2503-2509. doi: 10.1152/japplphysiol.01064.2002

Roseguini, B. T., Mehmet Soylu, S., Whyte, J. J., Yang, H. T., Newcomer, S., and Laughlin, M. H. (2010). Intermittent pneumatic leg compressions acutely upregulate VEGF and MCP-1 expression in skeletal muscle. Am. J. Physiol. Heart Circ. Physiol. 298, H1991-H2000. doi: 10.1152/ajpheart.0000 6.2010

Schoenfeld, B. J., Wilson, J. M., Lowery, R. P., and Krieger, J. W. (2016). Muscular adaptations in low- versus high-load resistance training: a meta-analysis. Eur. J. Sport Sci. 16, 1-10. doi: 10.1080/17461391.2014.9 89922

Serrano, A. L., Baeza-Raja, B., Perdiguero, E., Jardi, M., and Munoz-Canoves, P. (2008). Interleukin-6 is an essential regulator of satellite cell-mediated skeletal muscle hypertrophy. Cell Metab. 7, 33-44. doi: 10.1016/j.cmet.2007. 11.011

Shill, D. D., Polley, K. R., Willingham, T. B., Call, J. A., Murrow, J. R., Mccully, K. K., et al. (2017). Experimental intermittent ischemia augments exerciseinduced inflammatory cytokine production. J. Appl. Physiol. 123, 434-441. doi: 10.1152/japplphysiol.01006.2016

Shimizu, R., Hotta, K., Yamamoto, S., Matsumoto, T., Kamiya, K., Kato, M., et al. (2016). Low-intensity resistance training with blood flow restriction improves vascular endothelial function and peripheral blood circulation in healthy elderly people. Eur. J. Appl. Physiol. 116, 749-757. doi: 10.1007/s00421-016-3328-8

Simonson, S. R., and Jackson, C. G. (2004). Leukocytosis occurs in response to resistance exercise in men. J. Strength Cond. Res. 18, 266-271. doi: 10.1519/R-12572.1

Sudo, M., Ando, S., Poole, D. C., and Kano, Y. (2015). Blood flow restriction prevents muscle damage but not protein synthesis signaling following eccentric contractions. Physiol. Rep. 3:e12449. doi: 10.14814/phy2.12449

Suga, T., Okita, K., Morita, N., Yokota, T., Hirabayashi, K., Horiuchi, M., et al. (2009). Intramuscular metabolism during low-intensity resistance exercise with blood flow restriction. J. Appl. Physiol. 106, 1119-1124. doi: 10.1152/japplphysiol.90368.2008

Suga, T., Okita, K., Takada, S., Omokawa, M., Kadoguchi, T., Yokota, T., et al. (2012). Effect of multiple set on intramuscular metabolic stress during lowintensity resistance exercise with blood flow restriction. Eur. J. Appl. Physiol. 112, 3915-3920. doi: 10.1007/s00421-012-2377-x

Sundberg, C. J. (1994). Exercise and training during graded leg ischaemia in healthy man with special reference to effects on skeletal muscle. Acta Physiol. Scand. Suppl. 615, 1-50.

Szade, A., Grochot-Przeczek, A., Florczyk, U., Jozkowicz, A., and Dulak, J. (2015). Cellular and molecular mechanisms of inflammation-induced angiogenesis. IUBMB Life 67, 145-159. doi: 10.1002/iub.1358

Takada, S., Okita, K., Suga, T., Omokawa, M., Kadoguchi, T., Sato, T., et al. (2012). Low-intensity exercise can increase muscle mass and strength proportionally to enhanced metabolic stress under ischemic conditions. J. Appl. Physiol. 113, 199-205. doi: 10.1152/japplphysiol.00149.2012

Takarada, Y., Nakamura, Y., Aruga, S., Onda, T., Miyazaki, S., and Ishii, N. (2000). Rapid increase in plasma growth hormone after low-intensity resistance exercise with vascular occlusion. J. Appl. Physiol. 88, 61-65. doi: 10.1152/jappl.2000.88.1.61

Takarada, Y., Sato, Y., and Ishii, N. (2002). Effects of resistance exercise combined with vascular occlusion on muscle function in athletes. Eur. J. Appl. Physiol. 86, 308-314. doi: 10.1007/s00421-001-0561-5

Teixeira, C. F., Zamuner, S. R., Zuliani, J. P., Fernandes, C. M., Cruz-Hofling, M. A., Fernandes, I., et al. (2003). Neutrophils do not contribute to local tissue damage, but play a key role in skeletal muscle regeneration, in mice injected with Bothrops asper snake venom. Muscle Nerve 28, 449-459. doi: 10.1002/mus. 10453

Tidball, J. G., and Villalta, S. A. (2010). Regulatory interactions between muscle and the immune system during muscle regeneration. Am. J. Physiol. Regul. Integr. Comp. Physiol. 298, R1173-R1187. doi: 10.1152/ajpregu.00735.2009 
Van Uden, P., Kenneth, N. S., and Rocha, S. (2008). Regulation of hypoxia-inducible factor- $1 \alpha$ by NF-кB. Biochem. J. 412, 477-484. doi: 10.1042/BJ20080476

Vannella, K. M., and Wynn, T. A. (2017). Mechanisms of organ injury and repair by macrophages. Annu. Rev. Physiol. 79, 593-617. doi: 10.1146/annurev-physiol-022516-03 4356

Warren, G. L., O'farrell, L., Summan, M., Hulderman, T., Mishra, D., Luster, M. I., et al. (2004). Role of CC chemokines in skeletal muscle functional restoration after injury. Am. J. Physiol. Cell Physiol. 286, C1031-C1036. doi: 10.1152/ajpcell.00467.2003

Wilson, J. M., Lowery, R. P., Joy, J. M., Loenneke, J. P., and Naimo, M. A. (2013). Practical blood flow restriction training increases acute determinants of hypertrophy without increasing indices of muscle damage. J. Strength Cond. Res. 27, 3068-3075. doi: 10.1519/JSC.0b013e31828 alffa

Zanou, N., and Gailly, P. (2013). Skeletal muscle hypertrophy and regeneration: interplay between the myogenic regulatory factors (MRFs) and insulin- like growth factors (IGFs) pathways. Cell. Mol. Life Sci. 70, 4117-4130 doi: 10.1007/s00018-013-1330-4

Zou, K., Meador, B. M., Johnson, B., Huntsman, H. D., Mahmassani, Z., Valero, M. C., et al. (2011). The a7b1-integrin increases muscle hypertrophy following multiple bouts of eccentric exercise. J. Appl. Physiol. 111, 1134-1141. doi: 10.1152/japplphysiol.00081.2011

Conflict of Interest Statement: The authors declare that the research was conducted in the absence of any commercial or financial relationships that could be construed as a potential conflict of interest.

Copyright (C) 2018 Rossi, de Freitas, Zanchi, Lira and Cholewa. This is an open-access article distributed under the terms of the Creative Commons Attribution License (CC $B Y)$. The use, distribution or reproduction in other forums is permitted, provided the original author(s) and the copyright owner(s) are credited and that the original publication in this journal is cited, in accordance with accepted academic practice. No use, distribution or reproduction is permitted which does not comply with these terms. 
OPEN ACCESS

Edited by:

Wataru Aoi,

Kyoto Prefectural University, Japan

Reviewed by:

Antonio Torsello,

Università degli Studi di Milano

Bicocca, Italy

*Correspondence:

Dario Coletti

dario.coletti@uniroma1.it;

dario.coletti@upmc.fr

Specialty section:

This article was submitted to

Striated Muscle Physiology,

a section of the journal

Frontiers in Physiology

Received: 09 September 2018

Accepted: 12 December 2018

Published: 08 January 2019

Citation:

Adamo S, Pigna E, Lugarà $R$,

Moresi V, Coletti $D$ and Bouché $M$

(2019) Skeletal Muscle: A Significant

Novel Neurohypophyseal

Hormone-Secreting Organ.

Front. Physiol. 9:1885.

doi: 10.3389/fphys.2018.01885

\section{Skeletal Muscle: A Significant Novel Neurohypophyseal Hormone-Secreting Organ}

\author{
Sergio Adamo ${ }^{1}$, Eva Pigna ${ }^{1}$, Rosamaria Lugarà ${ }^{1}$, Viviana Moresi ${ }^{1}$, Dario Coletti ${ }^{1,2 *}$ and \\ Marina Bouché ${ }^{1}$ \\ ${ }^{1}$ Section of Histology \& Medical Embryology, Department of Anatomical, Histological, Forensic and Orthopedic Sciences, \\ Interuniversity Institute of Myology, Sapienza University of Rome, Rome, Italy, ${ }^{2}$ Sorbonne Université, CNRS UMR \\ 8256-INSERM ERL U1164, Biological Adaptation and Aging B2A, Paris, France
}

Keywords: vasopressin, oxytocin, myogenesis, muscle regeneration, satellite cell, muscle atrophy and hypertrophy, anabolizing agents

Vasopressin ( $\arg ^{8}$-vasopressin) and oxytocin are closely related nonapeptide hormones, synthesized as pre-hormones in the magnocellular neurons of the paraventricular and supraoptic nuclei of the hypothalamus. Vasopressin and oxytocin are secreted in response to a variety of physiological stimuli, serving such different functions as controlling water balance, milk ejection, uterine contraction, mood, and parental behavior (Lechan and Toni, 2000; Costa et al., 2014a).

\section{MUSCLE AS A TARGET OF NEUROHYPOPHYSEAL HORMONES}

Skeletal muscle or myogenic precursors have never been considered, until recently, as targets of neurohypophyseal hormones. However, very early evidence was provided by Wakelam and collaborators, who showed some mild effects of vasopressin on carbohydrate metabolism in myoblasts (Wakelam and Pette, 1982; Wakelam et al., 1987). These initial observations were corroborated by later studies showing that stimulation of primary chick embryo myoblasts or murine L6 and L5 myogenic cell lines with vasopressin, oxytocin, their analogs and antagonists resulted in the structure- and concentration-dependent activation of phospholipase C (PLC) signaling, the stimulation of myogenic differentiation, and the hypertrophy of newly formed muscle fibers (Teti et al., 1993; Nervi et al., 1995). Worth noting, PLC activates Protein Kinase C family members, among which the theta isoform plays an important role in both muscle differentiation and disease (Marrocco et al., 2014, 2017; Lozanoska-Ochser et al., 2018). The potent pro-myogenic effect of vasopressin was further characterized in myogenic cells cultured in a serum-free medium, a "clean" experimental model that allowed us to establish that L6 myogenic cells express the vasopressin V1a receptor (V1a-R) and that vasopressin elicits a complex signal transduction response in these cells (Minotti et al., 1998; Scicchitano, 2002, 2005; Naro et al., 2003; Toschi et al., 2011; Costa et al., 2014b). Moreover, it was found that V1a-R expression is modulated during the differentiation of L6 cells, probably in a post-translational manner (Alvisi et al., 2008).

Studies from other laboratories highlighted the presence of functional oxytocin receptors (OT-R) in human myoblasts derived from postnatal satellite cells (Breton et al., 2002), and in C2C12 myogenic cells which respond to oxytocin by activating the calcium-CaMKK-AMPK pathway (Lee et al., 2008). A recent in vitro study showed that C2C12 myoblasts express not only OT-R but also oxytocin and that the expression of both products increases upon myogenic differentiation of the cells (Berio et al., 2017). Furthermore, myotubes treated with $17 \beta$-estradiol overexpress oxytocin and OT-R genes by approximately 3 - and 29-fold, respectively (Berio et al., 2017). 
While cell cultures provide useful models to define, under controlled conditions, the effects of vasopressin or oxytocin on myogenic differentiation at the molecular level, in vivo data support the idea that both neurohypophyseal hormones play physiological roles in skeletal muscle. Indeed, a role for neurohypophyseal hormones in prenatal muscle development was first suggested by the presence of immunoreactive vasopressin in human fetal and neonatal skeletal muscle (Smith et al., 1992). Moreover, a >120-fold increase in oxytocin expression was observed in bovine muscle during early to mid-fetal calf development, coincident with active myofiber formation (De Jager et al., 2011), findings in keeping with the above reported in vitro data.

In an in vivo murine experimental model, we reported that V1a-R expression, measurable under basal conditions in muscle, was strongly up-regulated in the early phase of regeneration ( $9 \mathrm{~h}$ after injury) and gradually decreased in the following days, along with the regeneration process. In this model, vasopressin administration promoted muscle regeneration. Furthermore, overexpression of $\mathrm{V} 1 \mathrm{a}-\mathrm{R}$ in muscle sufficed to dramatically enhance post-injury muscle regeneration, without administering exogenous vasopressin (Toschi et al., 2011). In an in vivo mouse model of tumor necrosis factor (TNF)-inhibited muscle regeneration, the administration of vasopressin rescued the inhibitory effect of TNF, likely through a mechanism involving the modulation of HSP70 levels (Moresi et al., 2009). Again, TNFmediated muscle atrophy was rescued by the overexpression of the V1a-R in vivo (Costa et al., 2014b).

Altogether, the in vivo evidence supports the notion that both vasopressin and oxytocin have potent effects, in the development, regeneration and homeostasis of skeletal muscle.

Further insights came from studies conducted in aged mice (Elabd et al., 2014). The authors focused on the reduced muscle regeneration and muscle atrophy (sarcopenia) occurring in aging. While it is known that aging is accompanied by reduced physiological levels of sex steroids, the authors found that circulating oxytocin level is also reduced. Interestingly, satellite cells from aged animals exhibited a significantly lower OT-R expression than those from young animals. Moreover, comparing young and aged mice treated with an oxytocin selective antagonist or with exogenous oxytocin, respectively, the authors demonstrated that oxytocin is required for efficient muscle regeneration (Elabd et al., 2014). The impaired muscle regeneration of aged mice was shown to depend primarily upon reduced proliferation of satellite cells, a phenomenon rescued by exogenous oxytocin administration. The oxytocin effect on satellite cell proliferation was reported to be mediated by the MAPK/ERK pathway (Elabd et al., 2014). In line with the above findings, muscle regeneration in oxytocin $\mathrm{KO}$ mice was severely compromised. Young oxytocin $\mathrm{KO}$ mice displayed a premature decline in muscle regeneration, as well as muscle fibrosis and fat infiltration, showing a muscle phenotype characteristic of sarcopenia (Elabd et al., 2014).

Therefore, both neurohypophyseal hormones appear to regulate positively muscle homeostasis in different models: oxytocin, in the aging and KO mouse models (Elabd et al., 2014); and vasopressin, in injured muscle or TNF-induced muscle wasting (Moresi et al., 2009; Toschi et al., 2011; Costa et al., 2014b).

The apparent overlapping between the observed effects of the two neurohypophyseal hormones in the regulation of muscle differentiation and trophism may depend on the fact that both V1a-R and OT-R cross-bind their ligands, albeit with different affinities (Barberis et al., 1998; Gupta et al., 2008). Furthermore, the results obtained in the myogenic L6 cell line used as an experimental tool to show the effect of neurohypophyseal hormones probably depend on a peculiar expression of the receptors for these hormones, in comparison to other myogenic cell types.

\section{MUSCLE TISSUE AS A SOURCE OF VASOPRESSIN AND OXYTOCIN}

Interestingly, the possibility that myogenic cells express one of the neurohypophyseal hormones, as suggested by the pioneering results of Smith in prenatal human muscle for vasopressin (Smith et al., 1992), was recently proposed again (Berio et al., 2017), with regard to oxytocin and OT-R. Based on these observations, muscle can thus be added to the list of previously unrecognized sites of oxytocin expression, such as testes, ovaries, heart and lungs (Assinder et al., 2000; Jankowski et al., 2004; Kiss and Mikkelsen, 2005; Gutkowska and Jankowski, 2012). Further results supporting this hypothesis, were obtained in studies aimed to investigate the mechanisms triggered by the administration of steroids used to increase muscle mass in livestock farming. Cattle regularly treated with anabolizing agents displayed dramatically enhanced oxytocin mRNA expression in skeletal muscle, accompanied by a $\sim 50$ fold higher level of circulating oxytocin (De Jager et al., 2011). Intriguingly, the authors provided evidence that the hypertrophying effect of anabolic steroids is prevalently mediated by OT-R signaling. Furthermore, in a more recent study, Divari reported that serum levels of oxytocin increased dramatically in cattle regularly treated with $17 \beta$-estradiol, but not with either dexamethasone or placebo. This administration of $17 \beta$ estradiol also resulted in increased (33-fold) skeletal muscle expression of the oxytocin-precursor mRNA (Divari et al., 2013). Increased expression of the oxytocin-precursor mRNA was also found in the muscle of sheep subjected to chronical treatment with a combination of $17 \beta$-estradiol and the synthetic androgen trenbolone acetate (TBA). Also the circulating oxytocin level increased in steroid-treated sheep compared to placebotreated controls (Kongsuwan et al., 2012). Together, the above studies indicate a correlation between the steroid-induced muscle hypertrophy and the increased expression of both oxytocin and OT-R in skeletal muscle.

The mechanisms underlying regulation by steroids of oxytocin expression remain to be fully elucidated. The oxytocin promoter does not possess a classical Estrogen Response Element, whereas it has a high affinity binding-site for nuclear orphan receptors/estrogen related receptor alpha (ERR $\alpha)$. Koohi reported that the estrogen dependent control of the oxytocin 
promoter is independent of classical Estrogen Receptor (ER) binding, but requires a functional $E R R \alpha$ (Koohi et al., 2005). These authors further demonstrated that the estrogenic stimulation of the OT-R occurs through the ERK/MAPKmediated stimulation of the transcriptional activity of ERR $\alpha$. The up-regulation of oxytocin expression by this non-classical mechanism may, in turn, sustain an autocrine feed-forward oxytocin/OT-R loop which amplifies the response to oxytocin, as shown in bone (Colaianni et al., 2012; Berio et al., 2017).

Worth noting, exercise (i.e., muscle contraction) represents a physiological stimulus increasing the levels of circulating neurohypophyseal hormones, as well as their expression (or the expression of their receptors) in several tissues beside skeletal muscle (Martins et al., 2005). Based on several studies in man and other mammals, it is clear that exercise induces a fivefold increase in the circulating levels of vasopressin (Melin et al., 1980; Convertino et al., 1981; Alexander et al., 1991). This increase, associated to the beneficial effects of exercise on muscle homeostasis, suggests a model whereby physical activity stimulates muscle secretion of the neurohypophyseal hormones and induces a generalized sensitization to these factors through the up-regulation of their receptors in various districts. An exercise-mediated increase in vasopressin and/or oxytocin could ultimately contribute to maintaining muscle homeostasis and add to the additional benefits of exercise, including an increased life span and general well-being.

\section{FINAL REMARKS}

The in vitro and in vivo studies discussed above suggest that skeletal muscle is a target of neurohypophyseal hormones, which regulate muscle homeostasis and function in both physiological and pathological conditions. On the other hand, skeletal muscle has been recognized as the source of a wide range of circulating factors, namely myokines, which regulate a number of different functions with paracrine or endocrine mechanisms (Pedersen

\section{REFERENCES}

Alexander, S. L., Irvine, C. H. G., Ellis, M. J., and Donald, R. A. (1991). The effect of acute exercise on the secretion of corticotropin-releasing factor, arginine vasopressin, and adrenocorticotropin as measured in pituitary venous blood from the horse. Endocrinology 128, 65-72. doi: 10.1210/endo128-1-65

Alvisi, M., De Arcangelis, V., Ciccone, L., Palombi, V., Alessandrini, M., Nemoz, G., et al. (2008). Vla vasopressin receptor expression is modulated during myogenic differentiation. Differentiation 76, 371-380. doi: 10.1111/j.1432-0436.2007.00231.x

Assinder, S. J., Carey, M., Parkinson, T., and Nicholson, H. D. (2000). Oxytocin and vasopressin expression in the ovine testis and epididymis: changes with the onset of spermatogenesis. Biol. Reprod. 63, 448-456. doi: 10.1095/biolreprod63.2.448

Barberis, C., Mouillac, B., and Durroux, T. (1998). Structural bases of vasopressin/oxytocin receptor function. J. Endocrinol. 156, 223-229. doi: 10.1677 /joe. 0.1560223

Berio, E., Divari, S., Starvaggi Cucuzza, L., Biolatti, B., and Cannizzo, F. T. (2017). $17 \quad \beta$-estradiol upregulates oxytocin and the oxytocin receptor in C2C12 myotubes. PeerJ 5:e3124. doi: 10.7717/ peerj. 3124 and Febbraio, 2008; Hoffmann and Weigert, 2017). In the light of its abundance in the organism, skeletal muscle may thus be regarded as the largest endocrine gland in the body. In this article, we discuss in vitro and in vivo studies showing that oxytocin is synthesized by muscle and that its secretion significantly contributes to the level of circulating hormones. This activity appears to be central to the mechanisms which regulate muscle homeostasis, to contribute to muscle hypertrophic responses and to be altered in atrophic conditions. As a whole, this evidence suggests that OT and AVP be considered as potential myokines. In addition, these considerations suggest a potential therapeutic use of these molecules, along with more selective and potent analogs, in atrophic and muscle wasting conditions, such as sarcopenia and cachexia, and as a tool in adjuvant therapies against muscular dystrophies and neuromuscular diseases.

\section{AUTHOR CONTRIBUTIONS}

$\mathrm{SA}, \mathrm{MB}$ drafted the manuscript and approved the final version. EP, RL contributed to manuscript writing. DC, VM provided important interpretations, critically revised the manuscript. All authors provided final approval of the opinion content.

\section{ACKNOWLEDGMENTS}

The authors are grateful to Pr. Giulio Cossu, University of Manchester, UK, and to Anna Luisa Mazzotti for critical reading and editing of the manuscript, respectively. SA is founded by Sapienza University research projects 2016 (SapMedi2016) and 2017 (RM11715C78539BD8). DC is funded by AFM (2017-20603), ANR (2013-J13R191), EFEM 2016, IBPS (2014), NIH (2013-1R01CA108857-01subcontractor). MB is founded by Sapienza University of Rome research projects 2017 (RP11715C7D238352), and by Duchenne Parent Project Onlus.

Breton, C., Haenggeli, C., Barberis, C., Heitz, F., Bader, C. R., Bernheim, L., et al. (2002). Presence of functional oxytocin receptors in cultured human myoblasts. J. Clin. Endocrinol. Metab. 87, 1415-1418. doi: 10.1210/jcem.87.3.8537

Colaianni, G., Sun, L., Di Benedetto, A., Tamma, R., Zhu, L. L., Cao, J., et al. (2012). Bone marrow oxytocin mediates the anabolic action of estrogen on the skeleton. J. Biol. Chem. 287, 29159-29167. doi: 10.1074/jbc.M112.365049

Convertino, V. A., Keil, L. C., Bernauer, E. M., and Greenleaf, J. E. (1981). Plasma volume, osmolality, vasopressin, and renin activity during graded exercise in man. J. Appl. Physiol. 50, 123-128. doi: 10.1152/jappl.1981.50.1.123

Costa, A., Rossi, E., Scicchitano, B. M., Coletti, D., Moresi, V., and Adamo, S. (2014a). Neurohypophyseal hormones: novel actors of striated muscle development and homeostasis. Eur. J. Transl. Myol. 24:3790. doi: 10.4081/bam.2014.3.217

Costa, A., Toschi, A., Murfuni, I., Pelosi, L., Sica, G., Adamo, S., et al. (2014b). Local overexpression of V1a-vasopressin receptor enhances regeneration in tumor necrosis factor-induced muscle atrophy. Biomed Res. Int. 2014:235426. doi: $10.1155 / 2014 / 235426$

De Jager, N., Hudson, N. J., Reverter, A., Wang, Y.-H., Nagaraj, S. H., Cafe, L. M., et al. (2011). Chronic exposure to anabolic steroids induces the muscle expression of oxytocin and a more than fiftyfold increase in circulating oxytocin in cattle. Physiol. Genomics. 43, 467-478. doi: 10.1152 /physiolgenomics.00226.2010 
Divari, S., Pregel, P., Cannizzo, F. T., Starvaggi Cucuzza, L., Brina, N., and Biolatti, B. (2013). Oxytocin precursor gene expression in bovine skeletal muscle is regulated by $17 \beta$-oestradiol and dexamethasone. Food Chem. 141, 4358-4366. doi: 10.1016/j.foodchem.2013.07.029

Elabd, C., Cousin, W., Upadhyayula, P., Chen, R. Y., Chooljian, M. S., Li, J., et al. (2014). Oxytocin is an age-specific circulating hormone that is necessary for muscle maintenance and regeneration. Nat. Commun. 5:4082. doi: $10.1038 /$ ncomms5082

Gupta, J., Russell, R. J., Wayman, C. P., Hurley, D., and Jackson, V. M. (2008). Oxytocin-induced contractions within rat and rabbit ejaculatory tissues are mediated by vasopressin V1Areceptors and not oxytocin receptors. Br. J. Pharmacol. 155, 118-126. doi: 10.1038/bjp.2008.226

Gutkowska, J., and Jankowski, M. (2012). Oxytocin revisited: its role in cardiovascular regulation. J. Neuroendocrinol. 24, 599-608. doi: 10.1111/j.1365-2826.2011.02235.x

Hoffmann, C., and Weigert, C. (2017). Skeletal muscle as an endocrine organ: The role of myokines in exercise adaptations. Cold Spring Harb. Perspect. Med. 7: a029793. doi: 10.1101/cshperspect.a029793

Jankowski, M., Danalache, B., Wang, D., Bhat, P., Hajjar, F., Marcinkiewicz, M., et al. (2004). Oxytocin in cardiac ontogeny. Proc. Natl. Acad. Sci.U.S.A. 101, 13074-13079. doi: 10.1073/pnas.0405324101

Kiss, A., and Mikkelsen, J. D. (2005). Oxytocin - Anatomy and functional assignments: a minireview. Endocr. Regul. 39, 97-105.

Kongsuwan, K., Knox, M. R., Allingham, P. G., Pearson, R., and Dalrymple, B. P. (2012). The effect of combination treatment with trenbolone acetate and estradiol-17 $\beta$ on skeletal muscle expression and plasma concentrations of oxytocin in sheep. Domest. Anim. Endocrinol. 43, 67-73. doi: 10.1016/j.domaniend.2012.02.004

Koohi, M. K., Ivell, R., and Walther, N. (2005). Transcriptional activation of the oxytocin promoter by oestrogens uses a novel non-classical mechanism of oestrogen receptor action. J. Neuroendocrinol. 17, 197-207. doi: 10.1111/j.1365-2826.2005.01298.x

Lechan, R. M., and Toni, R. (2000). Functional Anatomy of the Hypothalamus and Pituitary. South Dartmouth, MA: Endotext.

Lee, E. S., Uhm, K. O., Lee, Y. M., Kwon, J., Park, S. H., and Soo, K. H. (2008). Oxytocin stimulates glucose uptake in skeletal muscle cells through the calcium-CaMKK-AMPK pathway. Regul. Pept. 151, 71-74. doi: 10.1016/j.regpep.2008.05.001

Lozanoska-Ochser, B., Benedetti, A., Rizzo, G., Marrocco, V., Di Maggio, R., Fiore, P., et al. (2018). Targeting early PKC $\theta$-dependent T-cell infiltration of dystrophic muscle reduces disease severity in a mouse model of muscular dystrophy. J. Pathol. 244, 323-333. doi: 10.1002/path.5016

Marrocco, V., Fiore, P., Benedetti, A., Pisu, S., Rizzuto, E., Musarò, A., et al. (2017). Pharmacological inhibition of $\operatorname{pkc} \theta$ counteracts muscle disease in a mouse model of duchenne muscular dystrophy. EBioMedicine 16, 150-161. doi: 10.1016/j.ebiom.2017.01.001

Marrocco, V., Fiore, P., Madaro, L., Crupi, A., Lozanoska-Ochser, B., and Bouché, M. (2014). Targeting PKC $\theta$ in skeletal muscle and muscle diseases: good or bad? Biochem. Soc. Trans. 42, 1550-1555. doi: 10.1042/BST20140207

Martins, A. S., Crescenzi, A., Stern, J. E., Bordin, S., and Michelini, L. C. (2005). Hypertension and exercise training differentially affect oxytocin and oxytocin receptor expression in the brain. Hypertension 46, 1004-1009. doi: 10.1161/01.HYP.0000175812.03322.59

Melin, B., Eclache, J. P., Geelen, G., Annat, G., Allevard, A. M., Jarsaillon, E., et al. (1980). Plasma AVP, neurophysin, renin activity, and aldosterone during submaximal exercise performed until exhaustion in trained and untrained men. Eur. J. Appl. Physiol. Occup. Physiol. 44, 141-151. doi: 10.1007/BF00421092

Minotti, S., Scicchitano, B. M., Nervi, C., Scarpa, S., Lucarelli, M., Molinaro, M., et al. (1998). Vasopressin and insulin-like growth factors synergistically induce myogenesis in serum-free medium. Cell Growth Differ. Mol. Biol. J. Am. Assoc. Cancer Res. 9, 155-163.

Moresi, V., Garcia-Alvarez, G., Pristerà, A., Rizzuto, E., Albertini, M. C., Rocchi, M., et al. (2009). Modulation of caspase activity regulates skeletal muscle regeneration and function in response to vasopressin and tumor necrosis factor. PLoS ONE 4:e5570. doi: 10.1371/journal.pone.0005570

Naro, F., De Arcangelis, V., Sette, C., Ambrosio, C., Komati, H., Molinaro, M., et al. (2003). A bimodal modulation of the cAMP pathway is involved in the control of myogenic differentiation in 16 cells. J. Biol. Chem. 278, 49308-49315. doi: 10.1074/jbc.M306941200

Nervi, C., Benedetti, L., Minasia., Molinaro, M., and Adamo, S. (1995). Argininevasopressin induces differentiation of skeletal myogenic cells and up-regulation of myogenin and Myf-5. Cell Growth Differ. 6, 81-89.

Pedersen, B. K., and Febbraio, M. A. (2008). Muscle as an endocrine organ: focus on muscle-derived interleukin-6. Physiol. Rev. 88, 1379-1406. doi: 10.1152/physrev.90100.2007

Scicchitano, B. M. (2002). AVP induces myogenesis through the transcriptional activation of the myocyte enhancer factor 2. Mol. Endocrinol. 16, 1407-1416. doi: $10.1210 /$ me.16.6.1407

Scicchitano, B. M. (2005). Vasopressin-dependent myogenic cell differentiation is mediated by both $\mathrm{Ca}^{2+} /$ calmodulin-dependent kinase and calcineurin pathways. Mol. Biol. Cell. 16, 3632-3641. doi: 10.1091/mbc.E0501-0055

Smith, A., Stephen, R. I., Arkley, M. M., and McIntosh, N. (1992). Immunoreactive arginine vasopressin in human fetal and neonatal skeletal muscle. Early Hum.Dev. 28, 215-222.

Teti, A., Naro, F., Molinaro, M., and Adamo, S. (1993). Transduction of arginine vasopressin signal in skeletal myogenic cells. Am. J. Physiol. 265(1 Pt 1), C113-121.

Toschi, A., Severi, A., Coletti, D., Catizone, A., Musar,ò, A., Molinaro, M. et al. (2011). Skeletal muscle regeneration in mice is stimulated by local overexpression of V1a-vasopressin receptor. Mol. Endocrinol. 25, 1661-1673. doi: 10.1210/me.2011-1049

Wakelam, M. J., Patterson, S., and Hanley, M. R. (1987). L6 skeletal muscle cells have functional V1-vasopressin receptors coupled to stimulated inositol phospholipid metabolism. FEBS Lett. 210, 181-184.

Wakelam, M. J., and Pette, D. (1982). The control of glucose 1,6-bisphosphate by developmental state and hormonal stimulation in cultured muscle tissue. Biochem. J. 204, 765-769. doi: 10.1042/bj2040765

Conflict of Interest Statement: The authors declare that the research was conducted in the absence of any commercial or financial relationships that could be construed as a potential conflict of interest.

Copyright (c) 2019 Adamo, Pigna, Lugarà, Moresi, Coletti and Bouché. This is an open-access article distributed under the terms of the Creative Commons Attribution License (CC BY). The use, distribution or reproduction in other forums is permitted, provided the original author(s) and the copyright owner(s) are credited and that the original publication in this journal is cited, in accordance with accepted academic practice. No use, distribution or reproduction is permitted which does not comply with these terms. 


\section{OPEN ACCESS}

Edited by: Valentina Di Felice, Università degli Studi di Palermo, Italy

Reviewed by: Emidio Marques De Matos Neto, Federal University of Piauí, Brazil

Nissrine Daou,

Université Pierre et Marie Curie,

France

Guglielmo Sorci,

University of Perugia, Italy

*Correspondence:

Laura Teodor

laura.teodori@enea.it

${ }^{\dagger}$ These authors have contributed equally to this work

Specialty section:

This article was submitted to Striated Muscle Physiology, a section of the journal

Frontiers in Physiology

Received: 02 July 2018 Accepted: 21 December 2018 Published: 10 January 2019

Citation:

Teodori L, Costa A, Campanella L and Albertini MC (2019) Skeletal Muscle Atrophy in Simulated Microgravity Might Be Triggered by

Immune-Related microRNAs.

Front. Physiol. 9:1926.

doi: 10.3389/fphys.2018.01926

\section{Skeletal Muscle Atrophy in Simulated Microgravity Might Be Triggered by Immune-Related microRNAs}

\author{
Laura Teodori $^{1 *+}$, Alessandra Costa ${ }^{11}$, Luigi Campanella² and Maria C. Albertini ${ }^{3}$ \\ ${ }^{1}$ Diagnostic and Metrology Laboratory, TECFIS-FSN, ENEA, Frascati, Italy, ${ }^{2}$ Department of Chemistry, Sapienza University \\ of Rome, Rome, Italy, ${ }^{3}$ Department of Biomolecular Sciences, University of Urbino Carlo Bo, Urbino, Italy
}

Exposure to microgravity induces skeletal muscle disorders including atrophy, muscle force decrease, fiber-type shift. Microgravity also contributes to immune-function alterations and modifies microRNAs (miRs) expression. To understand the link between microgravity-induced skeletal muscle atrophy and immune function deregulation, a bioinformatics study was performed. The web platform MiRNet was used for miRstargets interaction analysis from previous proteomic studies on human soleus (SOL) and vastus lateralis $(\mathrm{VL})$ muscles. We predicted miRs targeting deregulated gene expression following bed rest as a model of microgravity exposure; namely, let-7a-5p, miR-125b-5p for over-expressed genes in SOL and VL; miR-1-3p, miR-125b-5p and miR-1-3p, miR95-5p for down-expressed genes in VL and SOL. The predicted miRs have important immune functions, exhibiting a significant role on both inflammation and atrophy. Let-7a down-expression leads to proliferation pathways promotion and differentiation pathway inhibition, whereas miR-1-3p over-expression yields anti-proliferative effect, promoting early differentiation. Such conflicting signals could lead to impairment between proliferation and differentiation in skeletal muscles. Moreover, promotion of an M2-like macrophage phenotype (IL-13, IL-10) by let-7a down-regulation and simultaneous promotion of an M1-like macrophage (IL-6, TNF- $\alpha$ ) phenotype through the over-expression of EEF2 lead to a deregulation between M1/M2 tuning, that is responsible for a first pro-inflammatory/proliferative phase followed by an antiinflammatory pro-myogenic phase during skeletal muscle regeneration after injury. These observations are important to understand the mechanism by which inflammation may play a significant role in skeletal muscle dysfunction in spaceflights, providing new links between immune response and skeletal muscle deregulation, which may be useful to further investigate possible therapeutic intervention.

Keywords: space flight, bioinformatics, miRs prediction, web-based platform, immune function deregulation, skeletal muscle atrophy

\section{INTRODUCTION}

Microgravity poses one of the greatest risks to astronauts during prolonged missions (Williams et al., 2009). Exposure to microgravity results in decreased muscle strength and endurance, raising concerns that muscle atrophy could increase long-term spaceflight risk unless adequate countermeasures were undertaken. Indeed, the employed physical exercise countermeasures are 
still incapable of preventing muscle atrophy, thus increasing needs for development of more focused program. Immune deregulation is also a major issue during spaceflights and was first observed in astronauts following missions, making them most exposed to infections (Johnston et al., 1975). Muscle atrophy is a feature of many diseases affecting skeletal muscle and it also responsible for muscle disease exacerbation (Kovanda et al., 2018). Exposure to microgravity during spaceflights might modify miRs expression as compared to normal gravity. MiRs are short non-coding RNAs able to regulate gene expression. Muscle specific miRs (myomiRs) regulate several processes in skeletal muscle, such as myogenesis, muscle homeostasis and response to external stimuli. Muscle-specific miRs (myomiRs) control various processes in skeletal muscles, from myogenesis and muscle homeostasis to responses to environmental stimuli, such as exercise. MiRs are critical regulators of both adult skeletal muscle differentiation/maintenance and inflammatory cytokine signaling (Bartel, 2004; Baltimore et al., 2008; Sonkoly et al., 2008). We hypothesized that links might exist between skeletal muscle atrophy and inflammation deregulation triggered by microgravity. The aim of the present work is to explore the mechanism by which the immune response to simulated microgravity may play a role in skeletal muscle dysfunction in spaceflights, providing a new mechanism linking the action of inflammatory cytokines to skeletal muscle deregulation. Deregulated proteins associated to bed rest as a microgravity model from a previous study (Moriggi et al., 2010) were employed to build a network identifying new putative miRs involved. MiRNet, an easyto-use web-based tool ${ }^{1}$, helped us to identify new regulatory mechanisms associated to immune microgravity muscle atrophy.

\section{MATERIALS AND METHODS}

The proteome profile previously performed on muscle biopsies (from VL to SOL) from 12 healthy subjects before and after 55 days of bed rest (Moriggi et al., 2010) has been used for this study. The proteins that showed a differential expression in the two conditions in VL and SOL muscles have been divided into 2 different groups: over- and down-expressed. The Swiss-Prot accession number of the deregulated proteins (genes) was used in PubMed ${ }^{2}$ to look for the associated official gene symbol. This latter has been used in miRNet to build a network identifying new putative miRs-targets interactions. The network shows the interactions existing between a miRNA and the genes modulated $^{1}$. We used the mRNA area and we uploaded 4 different lists of gene symbols: VL over-expressed; VL down-expressed; SOL over-expressed; SOL down-expressed. We first performed the analysis inserting $H$. sapiens as organism and the official gene symbol as ID type. We also repeated a second analysis choosing "muscle" as tissue

${ }^{1}$ http://www.mirnet.ca

${ }^{2}$ https://www.ncbi.nlm.nih.gov/pubmed

(human only) option. In this way, a network with nodes and connections between our genes and miRs has been built.

\section{RESULTS}

We used the proteome profile from Moriggi et al. (2010) where 55 days bed rest modulated different muscle genes. A list of modulated SOL and VL miRs was used for miRNet analysis to look for miRs-targets connections. We found putative miRs associated to up- and down-regulated genes (Table 1). Furthermore, when the analysis was performed choosing mRNAs from human muscle, different miRs emerged.

We performed a bibliographic analysis of the muscle miRs and their putative targets emerged from the miRNet analysis. We found that let-7a-5p and miR-1 are evidently involved in a proliferation/differentiation conflicting signaling (Figure 1) and their down- and up-regulation (respectively) play a fundamental role in tissue adaptation under simulated microgravity, resulting

TABLE 1 | Results obtained by miRNet analysis.

\begin{tabular}{ll}
\hline Deregulated proteins & microRNAs (gene symbols; gene IDs) \\
\hline VL Over-expressed & miR-484 \\
& miR-92a-3p \\
& $m i R-16-5 p$ \\
& $m i R-615-3 p$ \\
& $m i R-222-3 p$ \\
& $m i R-320 a$
\end{tabular}

VL Over-expressed in muscle

VL Down-expressed

VL Down-expressed in muscle

SOL Over-expressed in muscle

SOL Down-expressed

SOL Down-expressed in muscle let-7a-5p (EEF2; ID: 1938; eukaryotic translation elongation factor 2) (VCL; ID: 7414; vinculin) miR-125b-5p (VDAC1; ID: 7416; voltage dependent anion channel 1) (VCL; ID: 7414; vinculin) miR-5196-5p miR-4747-5p

miR-1-3p (SUCLA2; ID: 8803; succinate-CoA ligase ADP-forming beta subunit) (PRDX2; ID: 7001; peroxiredoxin 2) miR-125b-5p (PRDX2; ID: 7001; peroxiredoxin 2) miR-222-3p miR-let-7b-5p miR-34a-5p miR-320a

let-7a-5p (VCL; ID: 7414; vinculin) miR-125b-5p (VCL; ID: 7414; vinculin)

miR-30c-5p

$\operatorname{miR}-1-3 p$

miR-30a-5p

miR-1-3p (SUCLA2; ID: 8803; succinate-CoA ligase ADP-forming beta subunit) (ACTC1; ID: 70; actin, alpha, cardiac muscle 1) miR-95-5p (ACTC1; ID: 70; actin, alpha, cardiac muscle 1)

We reported the putative microRNAs that are associated to the over- and downexpressed VL/SOL proteins (genes). The genes associated to muscle tissue are also reported in parenthesis (bold). VL, vastus lateralis human muscle; SOL, human soleus. 


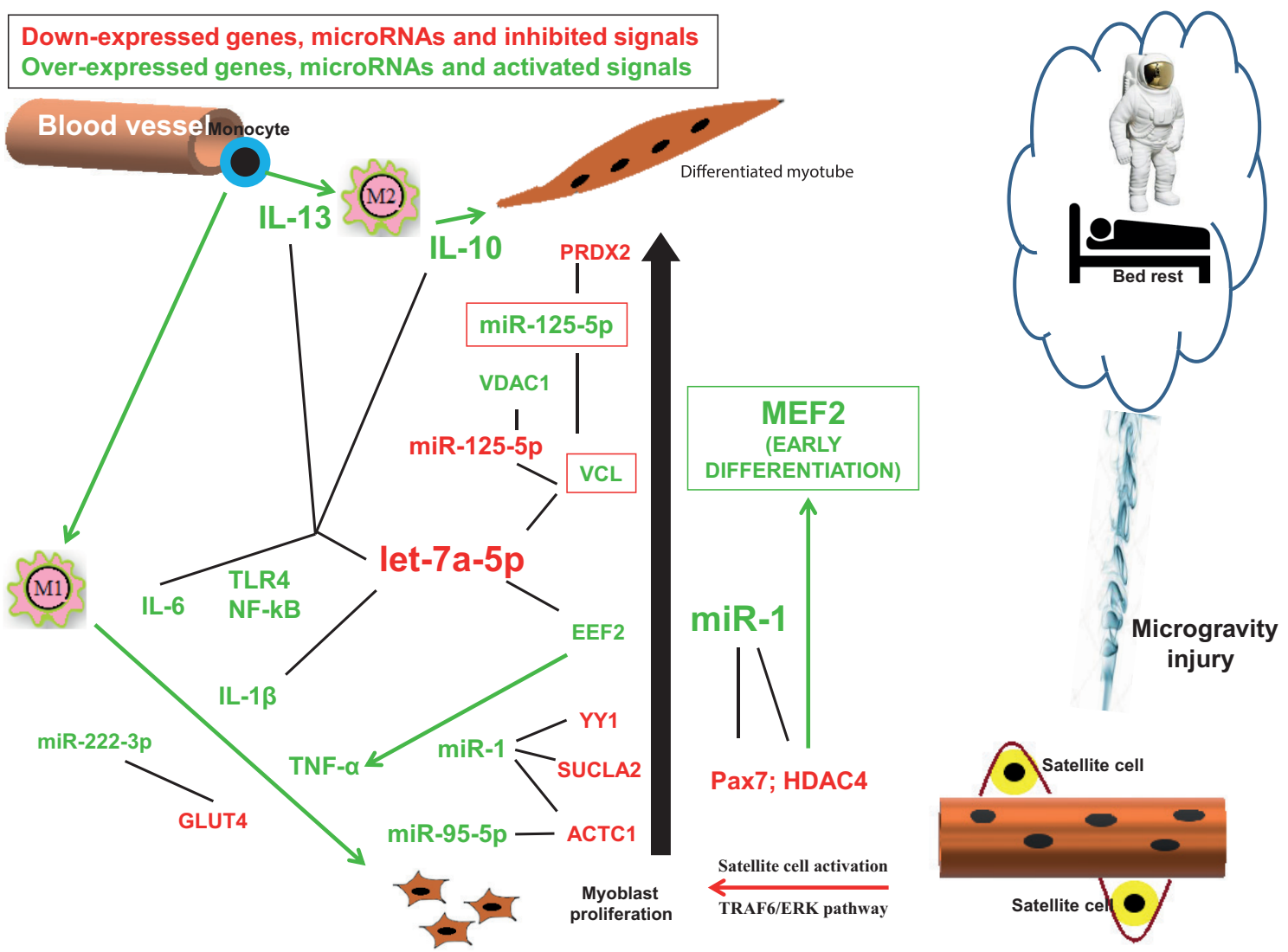

FIGURE 1 | Hypothesis of the conflicting signals responsible for atrophy observed during reduced gravity environment. Down-expressed genes, microRNAs and inhibited signals (arrows) are indicated in red, while over-expressed genes, microRNAs and activated signals (arrows) are indicated in green. Black lines indicate the possible interactions between mRNA and microRNAs evidenced by the miRNet analysis.

in skeletal muscle atrophy, strictly associated to immunefunction dysregulation.

\section{DISCUSSION}

The results of our bioinformatic analysis predicted some miRs dysregulation in healthy subjects undergoing bed rest as microgravity model. Our results evidenced that let-7 downregulation and simultaneous miR-1 up-regulation might play a fundamental role in tissue adaptation under microgravity resulting in skeletal muscle atrophy. Let-7 promotes cell differentiation and cellular proliferation inhibition in several cellular systems (Roush and Slack, 2008). The let-7miRs family plays a key role in modulating inflammatory responses as well (Song and Lee, 2015). Let-7a is involved in inflammation processes by participating to NF- $\kappa \mathrm{B}$ and angiogenesis signaling (Song et al., 2016). When miRs of let-7 family are downregulated, the levels of IL-6 and IL-10 cytokines are downregulated too (Liu et al., 2011; Schulte et al., 2011). Moreover decreased levels of let-7 following microbial infection enhance the expression of TLR4 (Androulidaki et al., 2009; Hu et al., 2009). Let-t is also related to adaptive immune response since it has been show a regulation by let- 7 of the production of IL-13 by T lymphocyte during the inflammatory process due to airway allergic response. Indeed, let-7 can inhibit the anti-inflammatory cytokine IL13 secretion (Fan et al., 2016); on the other hand let-7 miRs expression is dynamically regulated in response to TNF- $\alpha$ (and other factors as serum glucose). Thus let-7 down-expression, as in simulated microgravity, exacerbates inflammation. Finally, our bioinformatics (in silico) analysis of the interactions between miRs and the pathways involved in the inflammatory process, and in skeletal muscle wasting, such as TNF-alpha and NF-kB pathways identified let-7 family as one of the main predicted regulators of the aforementioned pathways. Noteworthy, many transmembrane receptors involved in these pathways (TNFR1, TNFR2, and IL1R) are targets of let-7 miRNA (Brennan et al., 2017).

Our in silico analysis showed that let-7 targets vinculin (VC), a protein connecting integrins to actin filaments, and that is recruited to focal adhesions (FAs) in response to force. VCL is suggested to be linked to FA mechanosensitivity and it is essential for FA stabilization under force (King et al., 2018). Migration of satellite cells (SC), necessary for skeletal muscle development and regeneration, is strictly dependant on the formation of mature FA connecting the cell to the extracellular 
matrix (Goetsch et al., 2014). In particular VCL is critical during the remodeling of the junction, due to the force exerted by the cytoskeleton, to avoid junction opening (Huveneers et al., 2012). TNF- $\alpha$ induces substantial reorganization of actin cytoskeleton and FA (Koukouritaki et al., 1999). In 55 days bed rest, VCL might fail to ensure proper migration and alignment for the cell to differentiate. Cells expressing VCL by $20 \%$ over endogenous level had altered locomotory properties. VCL is a key control protein for FA modeling and it is regulated by tension (Spanjaard and de Rooij, 2013). VCL expression at FA is negatively regulated by TNF-alpha that also indices cytoskeletal actin depolymerization (Scott and Panitch, 2014). Let-7 also targets EEF2 and, in turn, EEF2 suppresses let-7 expression (Ding et al., 2008). EEF2 activation activates subsequent TNF- $\alpha$ elongation (via MKK3/6p38 $\gamma / \delta$ pathway).

The restoration of let-7 levels might represent an interesting strategy for therapeutic approaches to prevent up-regulation of key proteins implicated in microgravity-driven inflammation and other key pathological hallmarks of atrophy processes.

Our in silico research also predicted miR-1-3p deregulation. MiR-1 family (belonging to myomiRs) controls myosin content, fiber type and muscle performance and directly targets HDAC4 (histone deacetylase 4) (Zuo et al., 2015). HDAC4 is directly inhibited by miR-1. This inhibition is responsible for cell-cycle arrest in G1 and G2, an anti-proliferative effect typical of HDAC inhibitors (Sun et al., 2015). HDAC4 has an inhibitory effects upon MEF2 (myocyte enhancer factor) transcription factor, responsible for the activation of early myogenic differentiation (Sun et al., 2015). A mutual regulation also exists between mir-1 and YY1, an inhibitor of myogenic differentiation; in fact miR-1 binds the $3^{\prime}$-UTR of YY1 and YY1 targets miR-1 (Sun et al., 2015).

Expression of miR-1 is regulated by nNOS signaling through HDCA2. HDAC may regulate the expression of iNos and NF$\mathrm{kB}$ dependent genes, which can further induce the expression of cytokines (Kharitonov and Ito, 2009). MiR-1 also controls G6PD, a relevant enzyme in oxidative stress response (Sun et al., 2015).

Glucocorticoids induce skeletal muscle atrophy with miR1 expression through glucocorticoid receptor and myostatin. The results suggest that miR-1 is a catabolic miR and might have a central role in microgravity-induced skeletal muscle atrophy. MiR-1 also blocks Pax7 which has been suggested to have a role in the maintenance of the proliferative phase through the prevention of early differentiation, thus miR1 up-regulation results in a block of proliferation and early differentiation induction. Pax7 is also a regulator of MyoD and may be involved in MyoD up-regulation observed during SC activation. Pax7 has also been demonstrated to be sufficient for the activation of the myogenic program in CD $45+/$ Sca1 + cells isolated from adult skeletal muscle tissue (Zammit et al., 2006). Hosoyama et al. (2017) hypothesize that human skeletal muscle stem/progenitor cells pool may be reduced during microgravity when Pax7 is down-regulated through TRAF6/ERK signaling pathway.

Our results also showed that miR-125b-5p is associated either to over- and down-expressed muscle VL genes and associated to over-expressed muscle SOL VCL gene. MiR-125b-5p targets mitochondrial apoptotic pathways and regulates the phenotype of macrophages targeting B7-H4 (Diao et al., 2017). MiR125b-5p over-expression down-regulates B7-H4 expression in macrophage. B7-H4, an immunoglobulin superfamily molecule that has been shown to inhibit $\mathrm{T}$ cell responses through cell cycle arrest and inhibition of $\mathrm{T}$ cell proliferation and cytokine release. $\mathrm{B} 7-\mathrm{H} 4$ has been also identified in skeletal muscle. MiR$125 \mathrm{~b}$ plays a role in innate immune response. MiR-125-b induces fibrosis by targeting apelin (Chen et al., 2017). MiR-125a and miR-125b indirectly activate NK-kB pathway via suppression of TNF-alpha-induced protein 3 (TNFAIP3, A20) (Chen et al., 2017). Down-regulation of miR-125b-5p increases inflammation (Chen et al., 2017).

Our results also predicted that miR-95-5p is associated with ACTC1 down-expressed SOL gene muscle. MiR-95-5p has been proposed as a new miR biomarker candidate in muscular dystrophy (dystromir). MiR-95 promotes myogenic differentiation by down-regulating the AIMP2 translation. ACTC1 is an AIMP2 interacting protein and both have been found to be implicated in FA.

The results also demonstrated an over-expression of miR-222$3 \mathrm{p}$ and miR-320a in both VL and SL. SLC2A4 gene (GLUT4 protein) (Xu et al., 2015) is a direct target of miR-222-3p. Limb immobilization halved muscle GLUT4 protein concentration in animal models. GLUT4 decrease is also associated with insulin resistance and high levels of inflammatory markers. The oxidative stress-responsive microR-320a is a skeletal muscle mitochondrial metabolism and migration regulator. It regulates glycolysis by directly downregulating phosphofructokinase, in diverse biological systems (Tang et al., 2012). Increased levels of miR-320a may account for an impairment of the glycolytic system by suppressing a key enzyme in the glycolysis process.

Several are the miRs associated to skeletal muscle proliferation and differentiation (myomiRs) e.g., miR-1, 133a/b, 206, 208a/b, $499 \mathrm{a} / \mathrm{b}$ and others are more and more coming to limelight, but except for miR-1, these were not associated to the overand down-expressed VL/SOL proteins (genes) experimentally detected by Moriggi et al. (2010). Moreover, we find some miRs that were not described by others. For examples, in murine gastrocnemius in spaceflight environment the ratio of miR-1/ miR-133a was significantly increased (Allen et al., 2009) but none of ours were found. These differences might be accounted for different models (animals vs. human), different environment (spaceflight vs. bed rest), different target (gastrocnemius vs. VL and SOL).

\section{CONCLUSION}

In conclusion, our bioinformatics analysis revealed that conflicting signals are taking place simultaneously in reduced gravity environment, resulting in impairment between proliferation and differentiation signaling pathways which might be responsible for the observed atrophy (Figure 1). The same predicted dysregulated miRs are, at the same time, also responsible for an inflammation response, which might be 
the reason of atrophy outcome thus suggesting that immune dysregulation is one of the molecular pathways ensuing microgravity-generated muscle atrophy. In this report we highlighted the roles played by the pathways controlled mainly by let-7a and miR-1, miR-125b-5p, miR-95-5p, miR-222-3p. The miRs here discussed are, of course, not exhaustive of the mechanisms involved in skeletal muscle response to microgravity, however, they highlight the important role played

\section{REFERENCES}

Allen, D. L., Bandstra, E. R., Harrison, B. C., Thorng, S., Stodieck, L. S., Kostenuik, P. J., et al. (2009). Effects of spaceflight on murine skeletal muscle gene expression. J. Appl. Physiol. 106, 582-595. doi: 10.1152/japplphysiol.90780. 2008

Androulidaki, A., Iliopoulos, D., Arranz, A., Doxaki, C., Schworer, S., Zacharioudaki, V., et al. (2009). The kinase Akt1 controls macrophage response to lipopolysaccharide by regulating microRNAs. Immunity 31, 220-231. doi: 10.1016/j.immuni.2009.06.024

Baltimore, D., Boldin, M. P., O'Connell, R. M., Rao, D. S., and Taganov, K. D. (2008). MicroRNAs: new regulators of immune cell development and function. Nat. Immunol. 9, 839-845. doi: 10.1038/ni.f.209

Bartel, D. P. (2004). MicroRNAs: genomics, biogenesis, mechanism, and function. Cell 116, 281-297.

Brennan, E., Wang, B., McClelland, A., Mohan, M., Marai, M., Beuscart, O., et al. (2017). Protective effect of let-7 miRNA family in regulating inflammation in diabetes-associated atherosclerosis. Diabetes 66, 2266-2277. doi: 10.2337/db161405

Chen, C., Ponnusamy, M., Liu, C., Gao, J., Wang, K., and Li, P. (2017). MicroRNA as a therapeutic target in cardiac remodeling. Biomed. Res. Int. 2017, 1278436. doi: 10.1155/2017/1278436

Diao, W., Lu, L., Li, S., Chen, J., Zen, K., and Li, L. (2017). MicroRNA125b-5p modulates the inflammatory state of macrophages via targeting B7H4. Biochem. Biophys. Res. Commun. 491, 912-918. doi: 10.1016/j.bbrc.2017. 07.135

Ding, X. C., Slack, F. J., and Grosshans, H. (2008). The let-7 microRNA interfaces extensively with the translation machinery to regulate cell differentiation. Cell Cycle 7, 3083-3090. doi: 10.4161/cc.7.19.6778

Fan, J., Kou, X., Yang, Y., and Chen, N. (2016). MicroRNA-regulated proinflammatory cytokines in sarcopenia. Med. Inflamm. 2016:1438686. doi: $10.1155 / 2016 / 1438686$

Goetsch, K. P., Snyman, C., Myburgh, K. H., and Niesler, C. U. (2014). ROCK-2 is associated with focal adhesion maturation during myoblast migration. J. Cell. Biochem. 115, 1299-1307. doi: 10.1002/jcb. 24784

Hosoyama, T., Ichida, S., Kanno, M., Ishihara, R., Hatashima, T., Ueno, K., et al. (2017). Microgravity influences maintenance of the human muscle stem/progenitor cell pool. Biochem. Biophys. Res. Commun. 493, 998-1003. doi: 10.1016/j.bbrc.2017.09.103

Hu, G., Zhou, R., Liu, J., Gong, A. Y., Eischeid, A. N., Dittman, J. W., et al. (2009). MicroRNA-98 and let-7 confer cholangiocyte expression of cytokine-inducible Src homology 2-containing protein in response to microbial challenge. J. Immunol. 183, 1617-1624. doi: 10.4049/jimmunol.080 4362

Huveneers, S., Oldenburg, J., Spanjaard, E., van der Krogt, G., Grigoriev, I., Akhmanova, A., et al. (2012). Vinculin associates with endothelial VE-cadherin junctions to control force-dependent remodeling. J. Cell Biol. 196, 641-652. doi: $10.1083 /$ jcb. 201108120

Johnston, R. S., Dietlein, L. F. S., Berry, C. A., Parker, J. F., and West, V. (1975). Biomedical Results of Apollo. NASA Technical Report No. 1-581. Arlington, VA: BioTechnology Inc.

Kharitonov, S. A., and Ito, K. (2009). "Chapter 30 - Nitric Oxide," in Asthma and COPD, 2nd Edn, ed. P. J. Barnes (New York, NY: Elsevier Ltd.), 896. by inflammation in muscle atrophy as already demonstrated in other forms of skeletal muscle pathologies.

\section{AUTHOR CONTRIBUTIONS}

LT and MCA made the bioinformatic analysis. LT, MCA, and AC wrote the paper. LC revised the manuscript.

King, G. A., Biebricher, A. S., Heller, I., Peterman, E. J. G., and Wuite, G. J. L. (2018). Quantifying local molecular tension using intercalated DNA fluorescence. Nano Lett. 18, 2274-2281. doi: 10.1021/acs.nanolett.7b04842

Koukouritaki, S. B., Vardaki, E. A., Papakonstanti, E. A., Lianos, E., Stournaras, C., and Emmanouel, D. S. (1999). TNF-alpha induces actin cytoskeleton reorganization in glomerular epithelial cells involving tyrosine phosphorylation of paxillin and focal adhesion kinase. Mol. Med. 5, 382-392.

Kovanda, A., Leonardis, L., Zidar, J., Koritnik, B., Dolenc-Groselj, L., Ristic Kovacic, S., et al. (2018). Differential expression of microRNAs and other small RNAs in muscle tissue of patients with ALS and healthy age-matched controls. Sci. Rep. 8:5609. doi: 10.1038/s41598-018-23 139-2

Liu, Y., Chen, Q., Song, Y., Lai, L., Wang, J., Yu, H., et al. (2011). MicroRNA-98 negatively regulates IL-10 production and endotoxin tolerance in macrophages after LPS stimulation. FEBS Lett. 585, 1963-1968. doi: 10.1016/j.febslet.2011. 05.029

Moriggi, M., Vasso, M., Fania, C., Capitanio, D., Bonifacio, G., Salanova, M., et al. (2010). Long term bed rest with and without vibration exercise countermeasures: effects on human muscle protein dysregulation. Proteomics 10, 3756-3774. doi: 10.1002/pmic.200900817

Roush, S., and Slack, F. J. (2008). The let-7 family of microRNAs. Trends Cell Biol. 18, 505-516. doi: 10.1016/j.tcb.2008.07.007

Schulte, L. N., Eulalio, A., Mollenkopf, H. J., Reinhardt, R., and Vogel, J. (2011). Analysis of the host microRNA response to Salmonella uncovers the control of major cytokines by the let-7 family. EMBO J. 30, 1977-1989. doi: 10.1038/ emboj.2011.94

Scott, R. A., and Panitch, A. (2014). Decorin mimic regulates platelet-derived growth factor and interferon-gamma stimulation of vascular smooth muscle cells. Biomacromolecules 15, 2090-2103. doi: 10.1021/bm500 $224 \mathrm{f}$

Song, J., Jun, M., Ahn, M. R., and Kim, O. Y. (2016). Involvement of miR-Let7A in inflammatory response and cell survival/apoptosis regulated by resveratrol in THP-1 macrophage. Nutr. Res. Pract. 10, 377-384. doi: 10.4162/nrp.2016.10. 4.377

Song, J., and Lee, J. E. (2015). ASK1 modulates the expression of microRNA Let7A in microglia under high glucose in vitro condition. Front. Cell Neurosci. 9:198. doi: $10.3389 /$ fncel.2015.00198

Sonkoly, E., Stahle, M., and Pivarcsi, A. (2008). MicroRNAs and immunity: novel players in the regulation of normal immune function and inflammation. Semin. Cancer Biol. 18, 131-140. doi: 10.1016/j.semcancer.2008. 01.005

Spanjaard, E., and de Rooij, J. (2013). Mechanotransduction: vinculin provides stability when tension rises. Curr. Biol. 23, R159-R161. doi: 10.1016/j.cub.2013. 01.020

Sun, H., Cao, Y., Zhao, Y., Lu, L., Zhou, L., Wang, L., et al. (2015). "MicroRNAs in skeletal muscle differentiation," in MicroRNAs in Regenerative Medicine, ed. C. K. Sen (New York, NY: Elsevier BV), 419-446.

Tang, H., Lee, M., Sharpe, O., Salamone, L., Noonan, E. J., Hoang, C. D., et al. (2012). Oxidative stress-responsive microRNA-320 regulates glycolysis in diverse biological systems. FASEB J. 26, 4710-4721. doi: 10.1096/fj.11-19 7467

Williams, D., Kuipers, A., Mukai, C., and Thirsk, R. (2009). Acclimation during space flight: effects on human physiology. CMAJ 180, 1317-1323. doi: 10.1503/ cmaj.090628 
Xu, P. T., Song, Z., Zhang, W. C., Jiao, B., and Yu, Z. B. (2015). Impaired translocation of GLUT4 results in insulin resistance of atrophic soleus muscle. Biomed. Res. Int. 2015:291987. doi: 10.1155/2015/29 1987

Zammit, P. S., Relaix, F., Nagata, Y., Ruiz, A. P., Collins, C. A., Partridge, T. A., et al. (2006). Pax7 and myogenic progression in skeletal muscle satellite cells. J. Cell Sci. 119(Pt 9), 1824-1832. doi: 10.1242/jcs.02908

Zuo, J., Wu, F., Liu, Y., Xiao, J., Xu, M., Yu, Q., et al. (2015). MicroRNA transcriptome profile analysis in porcine muscle and the effect of miR143 on the MYH7 gene and protein. PLoS One 10:e0124873. doi: 10.1371/journal.pone.0124873
Conflict of Interest Statement: The authors declare that the research was conducted in the absence of any commercial or financial relationships that could be construed as a potential conflict of interest.

Copyright $\odot 2019$ Teodori, Costa, Campanella and Albertini. This is an open-access article distributed under the terms of the Creative Commons Attribution License (CC BY). The use, distribution or reproduction in other forums is permitted, provided the original author(s) and the copyright owner(s) are credited and that the original publication in this journal is cited, in accordance with accepted academic practice. No use, distribution or reproduction is permitted which does not comply with these terms. 


\section{OPEN ACCESS}

Edited by:

Dario Coletti,

Sapienza University of Rome, Italy

Reviewed by:

Nicolas J. Pillon,

Karolinska Institute (KI), Sweden

Kunihiro Sakuma,

Tokyo Institute of Technology, Japan

Christophe Hourdé,

Université Savoie Mont Blanc, France

*Correspondence:

Tomasz Sledzinski

tsledz@gumed.edu.pl

Specialty section:

This article was submitted to

Striated Muscle Physiology,

a section of the journal

Frontiers in Physiology

Received: 16 October 2018

Accepted: 11 January 2019

Published: 28 January 2019

Citation:

Mika A, Macaluso F, Barone R,

Di Felice $V$ and Sledzinski T (2019)

Effect of Exercise on Fatty Acid Metabolism and Adipokine Secretion

in Adipose Tissue.

Front. Physiol. 10:26.

doi: 10.3389/fphys.2019.00026

\section{Effect of Exercise on Fatty Acid Metabolism and Adipokine Secretion in Adipose Tissue}

\author{
Adriana Mika ${ }^{1,2}$, Filippo Macaluso ${ }^{3,4,5}$, Rosario Barone ${ }^{3,4}$, Valentina Di Felice ${ }^{3,4}$ and \\ Tomasz Sledzinski* \\ ${ }^{1}$ Department of Pharmaceutical Biochemistry, Faculty of Pharmacy, Medical University of Gdańsk, Gdańsk, Poland, \\ ${ }^{2}$ Department of Environmental Analysis, Faculty of Chemistry, University of Gdańsk, Gdańsk, Poland, ${ }^{3}$ Department \\ of Biomedicine, Neurosciences, and Advanced Diagnostic (Bi.N.D.), University of Palermo, Palermo, Italy, \\ ${ }^{4}$ Euro-Mediterranean Institute of Science and Technology, Palermo, Italy, ${ }^{5}$ SMART Engineering Solutions \& Technologies \\ (SMARTEST) Research Center, eCampus University, Palermo, Italy
}

Increased physical activity is an optimal way to maintain a good health. During exercise, triacylglycerols, an energy reservoir in adipose tissue, are hydrolyzed to free fatty acids (FAs) which are then released to the circulation, providing a fuel for working muscles. Thus, regular physical activity leads to a reduction of adipose tissue mass and improves metabolism. However, the reduction of lipid reservoir is also associated with many other interesting changes in adipose tissue FA metabolism. For example, a prolonged exercise contributes to a decrease in lipoprotein lipase activity and resultant reduction of FA uptake. This results in the improvement of mitochondrial function and upregulation of enzymes involved in the metabolism of polyunsaturated fatty acids. The exercise-induced changes in adipocyte metabolism are associated with modifications of FA composition. The modifications are adipose tissue depot-specific and follow different patterns in visceral and subcutaneous adipose tissue. Moreover, exercise affects adipokine release from adipose tissue, and thus, may mitigate inflammation and improve insulin sensitivity. Another consequence of exercise is the recently described phenomenon of adipose tissue "beiging," i.e., a switch from energy-storing white adipocyte phenotype to thermogenic FA oxidizing beige adipocytes. This process is regulated by myokines released during the exercise. In this review, we summarize published evidence for the exercise-related changes in FA metabolism and adipokine release in adipose tissue, and their potential contribution to beneficial cardiovascular and metabolic effects of physical activity.

Keywords: exercise, adipose tissue, fatty acid, adipokine, myokine, adipose tissue beiging

\section{INTRODUCTION}

In the 21 st century, when obesity is recognized as a civilization-related, economic and social burden and the numbers of obese and overweight individuals still increase, we need new strategies to prevent and treat those conditions. Since excess body weight results from an imbalance between energy intake and energy expenditure (Jakicic and Otto, 2005), one way to maintain a correct body weight is to stimulate lipid catabolism through increased physical activity. Appropriately designed training simulates lipolysis, i.e., the hydrolysis of triacylglycerols stored in adipose tissue (AT), which results in the release of free fatty acids (FFAs) to circulation and their oxidation in muscles and other tissues. Elevated blood concentration of FFAs, observed in obesity and metabolic 
syndrome, is an adverse condition that may lead to lipotoxicity and ectopic deposition of lipids in other tissues (Mika and Sledzinski, 2017). Thus, efficient uptake and oxidation of FFAs in working muscles are critical for maintaining their normal blood levels. Moreover, exercise contributes to an increase in the number of mitochondria in white AT (WAT) and stimulates the expression of brown adipocyte-specific genes, which leads to "beiging" of WAT and amelioration of glucose intolerance induced by a high-fat diet (Sutherland et al., 2009; Xu et al., 2011; Roberts et al., 2014) (Figure 1). These effects of exercise on AT are associated with significant changes in metabolism and composition of fatty acids (FAs), the main components of adipocytes. Aside from the storage of triacylglycerols, AT acts also as an endocrine organ, releasing many biologically active substances referred to as adipokines. Exercise may also modulate the endocrine function of AT. There are three types of AT: WAT, located subcutaneously and viscerally, brown adipose tissue (BAT), and beige AT formed as a consequence of white adipocyte "beiging," i.e., their phenotypic and metabolic transition to cells similar to brown adipocytes. WAT, abundant in both humans and rodents, is primarily responsible for triacylglycerols storage and release of various adipokines into the blood. While in rodents BAT forms a large interscapular depot, as well as smaller depots in other locations, its existence in humans, around the neck, spine and major blood vessels, has been demonstrated quite recently. BAT, rich in mitochondria, is primarily responsible for thermogenesis (Lehnig and Stanford, 2018). Recent studies in humans and in rodents have identified controversial results in BAT activity in response to regular physical exercise. In trained humans, Vosselman et al. (2015) and Motiani et al. (2017) have observed a decrease in BAT activity (mitochondrial activity, glucose uptake, and thermogenesis, Figure 1). In this review, we discuss the exercise-induced changes in the composition and metabolism of FAs in AT, with particular emphasis on AT depotspecific differences.

\section{EFFECT OF EXERCISE ON FA COMPOSITION IN AT}

The release of FAs from adipocytes to deliver them to working muscles contributes to changes in the amount and composition of AT lipids. However, these effects were shown to depend on the exercise intensity (Nikolaidis and Mougios, 2004). Some studies demonstrated that low-intensity endurance training leads to maximal lipid oxidation, but available evidence in this matter is inconclusive (Romain et al., 2012). Triacylglycerols are the major class of lipids, representing up to $90-99 \%$ of all AT lipids (Nikolaidis and Mougios, 2004). WAT in mammalian body forms a few depots and can generally be classified into visceral and subcutaneous AT differing in terms of the composition of triacylglycerols-forming FA (Garaulet et al., 2006). Published data about the effects of exercise on FA composition in human AT are limited. An early studies revealed a decrease in oleic acid (18:1) and increase in linoleic acid (18:2 n-6) content in subcutaneous AT after chronic training (Allard et al., 1973; Sutherland et al., 1981). The decrease in the level of 18:1, the main monounsaturated FA (MUFA), which was observed in both studies mentioned above, might be associated with reduced activity of stearoyl-CoA desaturase (SCD1) in AT (Nikolaidis and Mougios, 2004). Since metabolic disorders were shown to be associated with enhanced synthesis of 18:1 and other MUFA by SCD1 (Mika et al., 2015), a post-exercise decrease in AT content of 18:1 may be considered a favorable change. Published evidence suggests that physical training may contribute to a preferential mobilization of some FAs from AT. Already after 2 weeks of the training in senior oarsmen, the authors observed a significant decrease in total serum triacylglycerols and cholesterol, along with changes in the FA profile of AT: a decrease in palmitoleic acid (16:1) and an increase in stearic acid (18:0) content, comparing to previously untrained controls (Danner et al., 1984). A more recent study demonstrated that 6 months of increased physical activity contributed to a significant increase in 18:2 n-6 in overweight elderly subjects, while no such effect was observed in untrained controls (Sjögren et al., 2012). Taken altogether, this sparse evidence from human suggests that chronic exercise may contribute to a decrease in 18:1 content, with concomitant increase in $18: 2 \mathrm{n}-6$ and $18: 0$. Since $18: 1$ is the main FA found in triacylglycerols (Ntambi and Miyazaki, 2003; Liu et al., 2011), the decrease in its content may contribute to a relative increase in other FAs. 18:2 n-6 is an essential FA, a substrate for synthesis of other $n-6$ PUFA, that in turn may be than converted into proinflammatory oxylipins, including eicosanoids (Mika and Sledzinski, 2017). However, regular exercise training seems to reduce systemic inflammation (Görgens et al., 2015). More data in this matter originate from rodent models, and based on this evidence we may compare the effect of exercise on FA composition in various WAT depots, as well as in BAT (May et al., 2017). Most of the animal studies demonstrated that chronic exercise contributed to a decrease in MUFA content, which is consistent with the observations made in humans (Nikolaidis and Mougios, 2004). Regarding polyunsaturated FAs (PUFAs), most animal studies showed an increase in their content, especially n-6 PUFAs; however, in some studies, the post-exercise levels of PUFAs were lower than prior to the exercise or remained unchanged. The chronic exercise-induced changes in PUFA content in AT are depot-specific (Bailey et al., 1993; Nikolaidis and Mougios, 2004). Petridou et al. (2005) reported decrease in MUFA levels after chronic exercise and an increase in $n-6$ PUFA content in visceral WAT but not in subcutaneous WAT. Among the MUFAs of visceral fat, chronic exercise contributed to a decrease in 16:1, but not in 18:1 (Petridou et al., 2005); the same phenomenon was also observed by Rocha-Rodrigues et al. (2017b) in a rat model. In recent study conducted by May et al. (2017) the authors performed a comprehensive analysis of FA content in phospholipids and triacylglycerols from subcutaneous WAT and BAT of mice subjected to a 3 -week exercise training. The study demonstrated that while the exercise contributed to a significant increase in MUFA level and a significant decrease in PUFA content in WAT phospholipids, an inverse phenomenon, i.e., a decrease in MUFAs and an increase in PUFAs was observed in BAT phospholipids. Moreover, a significant decrease in triacylglycerol content of SFAs, MUFAs and PUFAs in BAT and triacylglycerol content of PUFAs in WAT was observed 


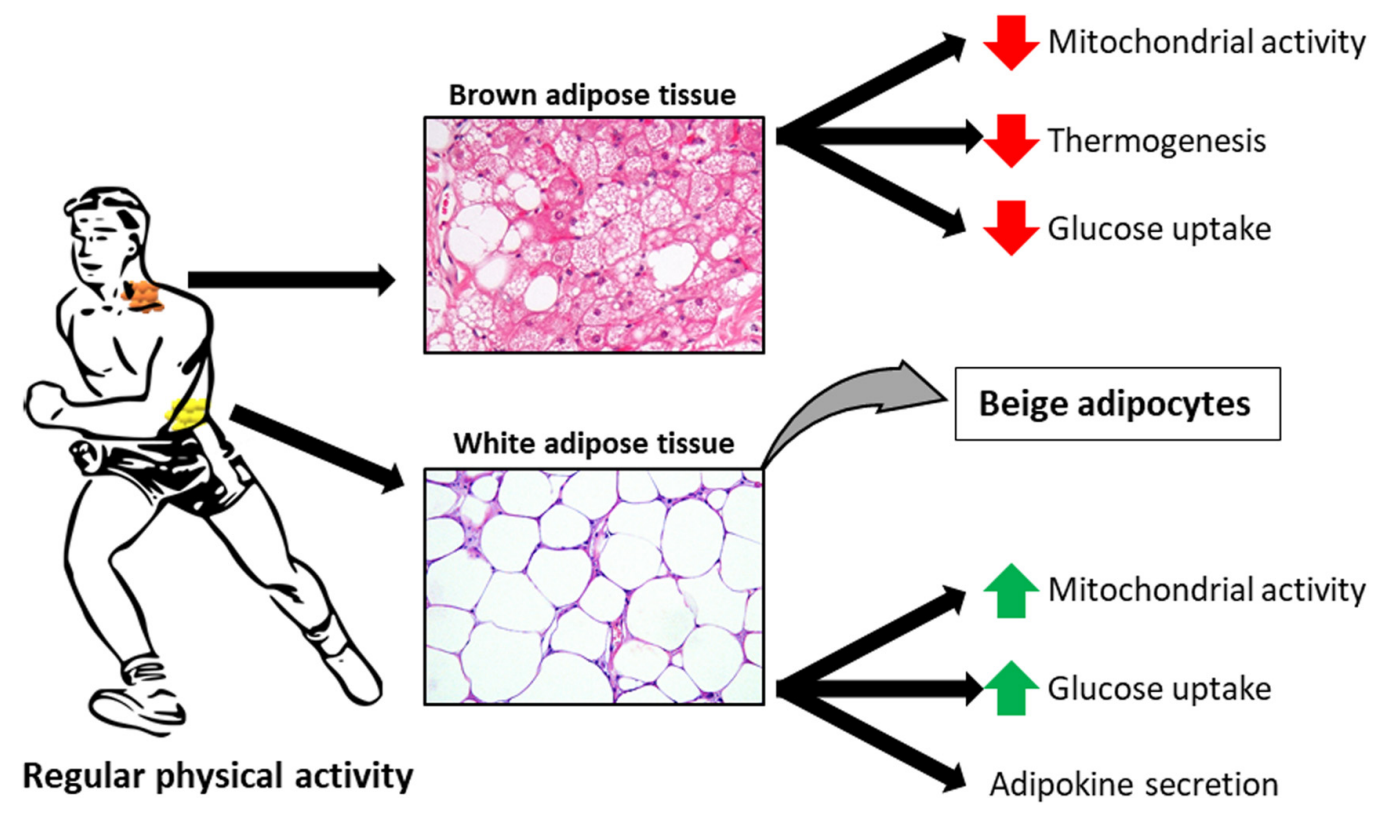

FIGURE 1 | Exercise-induced adaptations to white adipose tissue (WAT), brown adipose tissue (BAT) and beige adipocytes. Histological sections of WAT and BAT are stained with haematoxylin and eosin.

(May et al., 2017). However, it should be stressed that in that study, FA content was expressed in nmol per mg of protein, rather than as a percentage of total FAs as in previously mentioned experiments. These findings suggest that post-exercise changes in FA composition of AT are not only depot- but also lipid moleculespecific. In both WAT and BAT, physical exercise contributed to a significant decrease in the total content of triacylglycerols, but with a concomitant increase in the level of triacylglycerols containing long-chain FAs (58-60 total carbons) (May et al., 2017). The effects of exercise on FA composition in AT and other AT parameters in human and animal studies is summarized in Table 1.

\section{EFFECT OF EXERCISE ON FA METABOLISM IN AT}

The post-exercise decrease in triacylglycerols content in AT is with no doubt a consequence of enhanced lipolysis. The process, initiated by adipose triglyceride lipase (ATGL), is then continued by hormone-sensitive lipase (HSL), upon phosphorylation thereof; eventually, the last FA chain is hydrolyzed by monoacylglycerol lipase (MAGL) (Chen et al., 2015). While the rate of lipolysis is decreased by obesity and highfat diet, chronic exercise was shown to normalize the markers of this process, phosphorylated HSL and ATGL, in mice that have been previously maintained on a high-fat diet (Chen et al., 2015). Surprisingly, however, Holland et al. (2016) demonstrated that voluntary wheel running for 42 days contributed to a decrease in phosphorylated HSL level in rats. In contrast, chronic exercise was shown to stimulate the activity of lipolytic enzymes in the adipocytes of obese mice (Woo and Kang, 2016), and a recent study demonstrated that endurance exercise contributed to an increase in triacylglycerol lipase activity in human AT, especially during the first 10 min of the training (Petridou et al., 2017). An upregulation of HSL after chronic exercise was also mentioned in a review paper published by Steinberg (2009). Moreover, irisin, an adipokine released by working muscles, was shown to induce the expression of ATGL and HSL in 3T3L1 adipocytes (Gao et al., 2016). Thus, the results of most published studies suggest that physical exercise may stimulate lipolytic activity within AT, that may contribute to more efficient reduction of AT mass and/or prevent accumulation thereof.

Physical activity may also modulate FA synthesis, desaturation and elongation. The reduction of MUFA content after chronic exercise reported by many authors might be a consequence of a decrease in FA desaturation by SCD1 (Nikolaidis and Mougios, 2004). However, this conclusion is based on the desaturation indices calculated from SFA and MUFA contents in AT. Thus, it cannot be excluded that those parameters were also influenced by preferential uptake and release of certain FAs in AT during exercise (Halliwell et al., 1996). One study demonstrated that chronic exercise did not affect the expression of SCD1 in mice subcutaneous WAT, but contributed to lesser activity of this enzyme in BAT (May et al., 2017). Also in human subcutaneous AT, the expression of SCD1 gene remained unchanged after the chronic exercise (Sjögren et al., 2012). Published data about the exercise-induced changes in the activity of other lipogenic enzymes are inconclusive. According to May et al. (2017), 3-week exercise contributed to an increase in acetyl-CoA carboxylase (ACC) mRNA level in mice subcutaneous WAT, but not in BAT whereby mRNA level for this enzyme was reduced. Similarly, a 6-week exercise resulted in an increase in ACC protein level in visceral WAT of rats (Holland et al., 2016). In contrast, 
TABLE 1 | The summary of effects of exercise on adipose tissue metabolism and adipokine secretion.

\begin{tabular}{|c|c|c|c|c|c|}
\hline \multicolumn{2}{|c|}{ The effect of exercise on: } & \multirow{2}{*}{$\begin{array}{l}\text { Subcutaneous WAT } \\
\uparrow^{h}\end{array}$} & \multirow{2}{*}{$\begin{array}{l}\text { Visceral WAT } \\
\text { DNF }\end{array}$} & \multirow{2}{*}{$\begin{array}{l}\text { BAT } \\
\text { DNF }\end{array}$} & \multirow{2}{*}{$\begin{array}{l}\text { Reference } \\
\text { Danner et al., } 1984\end{array}$} \\
\hline Fatty acid profile & $18: 0$ & & & & \\
\hline & $16: 1$ & $\downarrow^{h}$ & $\downarrow^{r}$ & DNF & $\begin{array}{l}\text { Danner et al., 1984; Petridou et al., 2005; } \\
\text { Rocha-Rodrigues et al., 2017b }\end{array}$ \\
\hline & $18: 1$ & $\downarrow^{h}$ & $\mathrm{NC}^{r}$ & DNF & $\begin{array}{l}\text { Allard et al., 1973; Sutherland et al., 1981; Petridou } \\
\text { et al., 2005; Rocha-Rodrigues et al., 2017b }\end{array}$ \\
\hline & MUFA & $\downarrow^{h}, N C$ or $\downarrow^{r}$ & $\downarrow^{r}$ & DNF & $\begin{array}{l}\text { Bailey et al., 1993; Nikolaidis and Mougios, 2004; } \\
\text { Petridou et al., 2005; Rocha-Rodrigues et al., 2017b }\end{array}$ \\
\hline & MUFA in TG & $\mathrm{NC}^{\mathrm{m}}$ & DNF & $\downarrow^{m}$ & May et al., 2017 \\
\hline & MUFA in PL & $\uparrow^{m}$ & DNF & $\downarrow^{m}$ & May et al., 2017 \\
\hline & $18: 2 n-6$ & $\uparrow^{h}, \uparrow^{r}$ & $N C^{r}$ & DNF & $\begin{array}{l}\text { Sutherland et al., 1981; Bailey et al., 1993; Sjögren } \\
\text { et al., } 2012\end{array}$ \\
\hline & $n-6$ PUFA & $\uparrow^{h}, \uparrow^{r}$ & DNF & DNF & Nikolaidis and Mougios, 2004 \\
\hline & PUFA in TG & $\downarrow^{m}$ & DNF & $\downarrow^{m}$ & May et al., 2017 \\
\hline & PUFA in PL & $\downarrow^{m}$ & DNF & $\uparrow^{m}$ & May et al., 2017 \\
\hline \multirow{6}{*}{$\begin{array}{l}\text { Expression/activity } \\
\text { of enzymes of lipid } \\
\text { metabolism }\end{array}$} & $\mathrm{HSL}$ & $\mathrm{NC}^{\mathrm{m}}$ & $\uparrow^{m}, \downarrow^{r}$ & DNF & $\begin{array}{l}\text { Chen et al., 2015; Holland et al., 2016; Woo and Kang, } \\
2016\end{array}$ \\
\hline & ATGL & $\mathrm{NC}^{\mathrm{m}}$ & $\uparrow m$ & DNF & Chen et al., 2015; Woo and Kang, 2016 \\
\hline & SCD1 & $N C^{h}, N C^{m}$ & DNF & $\downarrow^{m}$ & Sjögren et al., 2012; May et al., 2017 \\
\hline & ACC & $\uparrow^{m}$ & $\uparrow^{m}, \downarrow^{r}$ & $\downarrow^{m}$ & $\begin{array}{l}\text { May et al., 2017; Holland et al., 2016; } \\
\text { Rocha-Rodrigues et al., 2017a }\end{array}$ \\
\hline & FADS1 & DNF & $\uparrow^{r}$ & DNF & Rocha-Rodrigues et al., 2017a \\
\hline & ELOVL5 & DNF & $\uparrow^{r}$ & DNF & Rocha-Rodrigues et al., 2017a \\
\hline \multirow{4}{*}{$\begin{array}{l}\text { Expression/ } \\
\text { secretion of } \\
\text { adipokines }\end{array}$} & Adiponectin & $\mathrm{NC}^{r}, \uparrow$ or $\mathrm{NC}^{\mathrm{h}}$ & $\uparrow^{r}, \uparrow^{h}$ & DNF & Görgens et al., 2015; Kato et al., 2018 \\
\hline & Leptin & $\mathrm{NC}^{\mathrm{h}}$ & DNF & DNF & Görgens et al., 2015 \\
\hline & IL-6 & $\downarrow$ or $\mathrm{NC}^{h}$ & DNF & DNF & Bruun et al., 2006; Klimcakova et al., 2006 \\
\hline & Apelin & DNF & $\uparrow^{r}$ & DNF & Kazemi and Zahediasl, 2018 \\
\hline \multicolumn{2}{|c|}{ Adipose tissue beiging } & $\uparrow^{r}, \uparrow^{m}$ & DNF & - & Lehnig and Stanford, 2018 \\
\hline
\end{tabular}

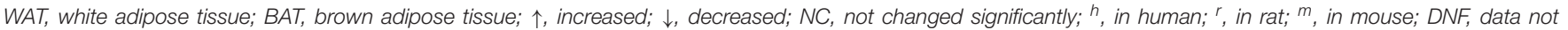

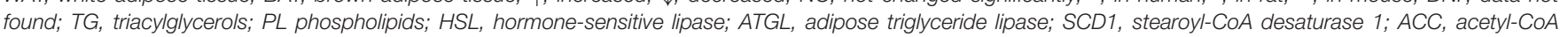
carboxylase; FADS1, fatty acid desaturase 1; ELOVL5, fatty acid elongase 1; I/6, interleukin 6.

Rocha-Rodrigues et al. (2017a) found reduced activity of ACC in visceral AT of rats subjected to an 8 -week endurance training. The same study demonstrated a post-exercise increase in the expressions of enzymes involved in PUFA metabolism, FA desaturase 1 and elongase 5 (Rocha-Rodrigues et al., 2017a); these findings are consistent with the results published by other authors who observed a chronic exercise-induced increase in PUFA content and elongase indices (Nikolaidis and Mougios, 2004). In line with those findings, May et al. (2017) found elevated levels of mRNA for elongase 3 and 4 in AT from chronically exercised mice. Taken altogether, the abovementioned findings suggest that the effect of exercise on the expression of enzymes involved in lipid metabolism may vary depending on FA group and AT depot.

\section{IMPACT OF EXERCISE ON ADIPOKINE SECRETION IN AT}

Muscle work during the exercise may activate a signaling cascade; specifically, myokines released from the muscle cells may trigger a release of adipokines, signaling molecules synthesized in the AT. Aside from the production of adipokines, AT can also synthesize many myokines, among others IL-6, MCP1, TNF $\alpha$, visfatin and myostatin, which are collectively referred to as adipomyokines (Görgens et al., 2015). Thus, plasma level of adipomyokines does not necessarily reflect solely the pool which is synthesized in the AT and acts on the muscles, and the origin of each molecule should be identified at a cellular level. Adiponectin is an insulin-sensitizing hormone that enhances FA oxidation in the muscles and downregulates the synthesis of lipids and glucose in the liver (Swierczynski and Sledzinski, 2012). The evidence from both human and animal studies analyzing the effects of exercise on serum adiponectin level is inconclusive; chronic exercise was either shown to increase the serum concentration or expression in AT of this adipokine or did not affect it at all (Kato et al., 2018; Lehnig and Stanford, 2018). Available data imply that the release of adiponectin from human AT may depend on exercise intensity (Görgens et al., 2015). Another adipokine, leptin, is synthesized primarily in the AT, regulates appetite and boosts peripheral metabolism (Swierczynski and Sledzinski, 2012). Chronic exercise was shown to contribute to 
a decrease in serum leptin concentration, but this effect was associated with the reduction of AT mass (Lehnig and Stanford, 2018). However, previous studies demonstrated that excess body weight was associated with leptin resistance (Swierczynski and Sledzinski, 2012), and chronic exercise might improve leptin sensitivity (Kang et al., 2013). Thus, the simultaneous reduction of serum leptin level and AT mass does not necessarily correspond to a decrease in the activity of this adipokine. Serum concentration of IL-6, acting as an anti-inflammatory myokine, was shown to increase substantially after acute exercise, and this effect was demonstrated to result from local synthesis of IL-6 by the working muscles. However, the level of IL-6 after chronic exercise was decreased or remained unchanged (Görgens et al., 2015). Also, the expression of IL-6 in the AT did not change or was reduced, depending on the type of chronic exercise (Bruun et al., 2006; Klimcakova et al., 2006). Generally, moderate chronic exercise seems to be associated with a decrease in the release of pro-inflammatory cytokines, such as TNF- $\alpha$, leptin and MCP-1, from the AT and working muscles; this may contribute to attenuation of systemic inflammation (Görgens et al., 2015). A recent study demonstrated that chronically exercised rats showed enhanced expression of apelin, an adipomyokine that decreases insulin resistance (Kazemi and Zahediasl, 2018). Apelin induced glucose uptake by AT, but at the same time decreases triglyceride amounts in mouse AT and lipid storage in 3T3-L1 preadipocytes (Indrakusuma et al., 2015). Also, serum concentration of resistin, an adipokine promoting insulin resistance, was shown to decrease in rats subjected to an chronic exercise (Shirvani and Arabzadeh, 2018). Altogether, these findings suggest that chronic exercise may improve the profile of adipokines released from the AT, and thus, may be beneficial for health.

\section{EXERCISE LEADS TO AT "BEIGING" - A PROCESS MEDIATED BY MYOKINES}

Aside from the metabolic processes discussed above (lipolysis, FA uptake, FA synthesis), FAs are also oxidized in mitochondria, in a process referred to as $\beta$-oxidation. A number of previous studies demonstrated that chronic exercise enhanced mitochondrial activity in visceral and subcutaneous AT, in both rodents (Stallknecht et al., 1991; Sutherland et al., 2009; Vernochet et al., 2012; Wu et al., 2012; Stanford et al., 2015a,b) and humans (Ruschke et al., 2010; Rönn et al., 2014). The process of mitochondrial $\beta$-oxidation in the AT is not as intensive as in the muscles but still can provide an extra pool of energy for adipocytes after the exercise. Furthermore, there is one AT depot that shows greater mitochondrial activity than visceral and subcutaneous WAT; this is BAT which contains numerous mitochondria whereby FAs undergo oxidization, becoming a source of energy for thermogenesis. The main protein involved in thermogenesis is uncoupling protein 1 (UCP1), mediating proton leakage across the inner mitochondrial membrane into the mitochondrial matrix, and thus, playing a role in heat production (Lehnig and Stanford, 2018). Recent research showed that chronic exercise may contribute to "beiging" of subcutaneous
WAT, a process which is also referred to as the "browning" of AT. During the process of "beiging," a phenotype and metabolism of white adipocytes in the AT change and resemble the respective characteristics of brown adipocytes in the BAT (Lehnig and Stanford, 2018). This phenotypic and functional switch includes an increase in mitochondrial respiration and enhancement of UCP1 protein expression, as well as the upregulation of other genes characteristic for BAT (Wu et al., 2012). In one study, ablation of beige adipocytes resulted in the development of obesity and insulin resistance in mice; this implies that these cells may play a role in the regulation of systemic energy metabolism (Stanford and Goodyear, 2016). While the exercise-induced adipocyte "beiging" has been well documented in rodents, still little is known about this phenomenon in humans (Lehnig and Stanford, 2018). However, it needs to be stressed that BAT in adult humans resembles murine beige AT, rather than the BAT (Wu et al., 2012). A number of potential mechanisms responsible for AT "beiging" have been proposed thus far. According to one hypothesis, the process may be mediated by myokines and small molecules released from working muscles (Lehnig and Stanford, 2018), specifically by irisin (Boström et al., 2012), myostatin (Feldman et al., 2006), meteorin-like 1 (Metrnl) (Rao et al., 2014), lactate (Carriere et al., 2014) and/or $\beta$-aminoisobutyric acid (BAIBA) (Roberts et al., 2014).

\section{CONCLUSION}

Physical exercise stimulates lipolysis, decreases FA uptake by the adipocytes, exerts an effect on FA composition within the AT and modulates the expression of enzymes involved in FA synthesis, elongation and desaturation. Moreover, exercise promotes "beiging" of AT and contributes to an increase in mitochondrial activity, which leads to enhanced FA oxidation in the AT. As a result of all those metabolic processes, physically active persons can maintain adequate volume of AT. Chronic exercise influences the release of adipokines, which may attenuate systemic inflammation and prevent insulin resistance. Moreover, transplantation of AT from trained to untrained mice was shown to improve glucose tolerance (Stanford et al., 2015b). Taken altogether, these findings imply that the exercise-induced changes in AT metabolism may exert a beneficial effect on global metabolic health.

\section{AUTHOR CONTRIBUTIONS}

$\mathrm{AM}, \mathrm{FM}, \mathrm{RB}, \mathrm{VD}$, and TS studied the literature and wrote the manuscript. All authors accepted the final version of manuscript.

\section{FUNDING}

This study was supported by the Medical University of Gdańsk (grant no. ST40), National Science Centre of Poland (grant no. NCN 2016/21/D/NZ5/00219), and by the Italian Ministry of University and Research (grant prot. 2012N8YJC3-Caporossi Daniela PRIN2012). 


\section{REFERENCES}

Allard, C., Alteresco, M., Ferguson, R. J., Chaniotis, L., Choquette, G., and Skinner, J. (1973). Changes in adipose tissue and increased serum cholesterol of coronary patients following training. Can. Med. Assoc. J. 109, 194-197.

Bailey, J. W., Walker, E., and Beauchene, R. E. (1993). Fatty acid composition of adipose tissue in aged rats: effects of dietary restriction and exercise. Exp. Gerontol. 28, 233-247. doi: 10.1016/0531-5565(93)90031-8

Boström, P., Wu, J., Jedrychowski, M. P., Korde, A., Ye, L., Lo, J. C., et al. (2012). A PGC1- $\alpha$-dependent myokine that drives brown-fat-like development of white fat and thermogenesis. Nature 481, 463-468. doi: 10.1038/nature10777

Bruun, J. M., Helge, J. W., Richelsen, B., and Stallknecht, B. (2006). Diet and exercise reduce low-grade inflammation and macrophage infiltration in adipose tissue but not in skeletal muscle in severely obese subjects. Am. J. Physiol. Metab. 290, E961-E967. doi: 10.1152/ajpendo.00506.2005

Carriere, A., Jeanson, Y., Berger-Muller, S., Andre, M., Chenouard, V., Arnaud, E., et al. (2014). Browning of white adipose cells by intermediate metabolites: an adaptive mechanism to alleviate redox pressure. Diabetes 63, 3253-3265. doi: $10.2337 / \mathrm{db} 13-1885$

Chen, N., Cheng, J., Zhou, L., Lei, T., Chen, L., Shen, Q., et al. (2015). Effects of treadmill running and rutin on lipolytic signaling pathways and TRPV4 protein expression in the adipose tissue of diet-induced obese mice. J. Physiol. Biochem. 71, 733-742. doi: 10.1007/s13105-015-0437-5

Danner, S. A., Wieling, W., Havekes, L., Leuven, J. G., Smit, E. M., and Dunning, A. J. (1984). Effect of physical exercise on blood lipids and adipose tissue composition in young healthy men. Atherosclerosis 53, 83-90. doi: 10.1016/ 0021-9150(84)90108-4

Feldman, B. J., Streeper, R. S., Farese, R. V., and Yamamoto, K. R. (2006). Myostatin modulates adipogenesis to generate adipocytes with favorable metabolic effects. Proc. Natl. Acad. Sci. U.S.A. 103, 15675-15680. doi: 10.1073/pnas.0607501103

Gao, S., Li, F., Li, H., Huang, Y., Liu, Y., and Chen, Y. (2016). Effects and molecular mechanism of GST-Irisin on lipolysis and autocrine function in 3T3-L1 adipocytes. PLoS One 11:e0147480. doi: 10.1371/journal.pone.0147480

Garaulet, M., Hernandez-Morante, J. J., Lujan, J., Tebar, F. J., and Zamora, S. (2006). Relationship between fat cell size and number and fatty acid composition in adipose tissue from different fat depots in overweight/obese humans. Int. J. Obes. 30, 899-905. doi: 10.1038/sj.ijo.0803219

Görgens, S. W., Eckardt, K., Jensen, J., Drevon, C. A., and Eckel, J. (2015). Exercise and regulation of adipokine and myokine production. Prog. Mol. Biol. Transl. Sci. 135, 313-336. doi: 10.1016/bs.pmbts.2015.07.002

Halliwell, K. J., Fielding, B. A., Samra, J. S., Humphreys, S. M., and Frayn, K. N. (1996). Release of individual fatty acids from human adipose tissue in vivo after an overnight fast. J. Lipid Res. 37, 1842-1848.

Holland, A. M., Kephart, W. C., Mumford, P. W., Mobley, C. B., Lowery, R. P., Shake, J. J., et al. (2016). Effects of a ketogenic diet on adipose tissue, liver, and serum biomarkers in sedentary rats and rats that exercised via resisted voluntary wheel running. Am. J. Physiol. Regul. Integr. Comp. Physiol. 311, R337-R351. doi: 10.1152/ajpregu.00156.2016

Indrakusuma, I., Sell, H., and Eckel, J. (2015). Novel mediators of adipose tissue and muscle crosstalk. Curr. Obes. Rep. 4, 411-417. doi: 10.1007/s13679-015-0174-7

Jakicic, J. M., and Otto, A. D. (2005). Physical activity considerations for the treatment and prevention of obesity. Am. J. Clin. Nutr. 82, 226S-229S. doi: 10.1093/ajcn/82.1.226S

Kang, S., Kim, K. B., and Shin, K. O. (2013). Exercise training improve leptin sensitivity in peripheral tissue of obese rats. Biochem. Biophys. Res. Commun. 435, 454-459. doi: 10.1016/j.bbrc.2013.05.007

Kato, H., Shibahara, T., Rahman, N., Takakura, H., Ohira, Y., and Izawa, T. (2018). Effect of a 9-week exercise training regimen on expression of developmental genes related to growth-dependent fat expansion in juvenile rats. Physiol. Rep. 6:e13880. doi: 10.14814/phy2.13880

Kazemi, F., and Zahediasl, S. (2018). Effects of exercise training on adipose tissue apelin expression in streptozotocin-nicotinamide induced diabetic rats. Gene 662, 97-102. doi: 10.1016/j.gene.2018.04.003

Klimcakova, E., Polak, J., Moro, C., Hejnova, J., Majercik, M., Viguerie, N., et al. (2006). Dynamic strength training improves insulin sensitivity without altering plasma levels and gene expression of adipokines in subcutaneous adipose tissue in obese men. J. Clin. Endocrinol. Metab. 91, 5107-5112. doi: 10.1210/jc.20060382
Lehnig, A. C., and Stanford, K. I. (2018). Exercise-induced adaptations to white and brown adipose tissue. J. Exp. Biol. 221(Pt Suppl. 1):jeb161570. doi: 10.1242/jeb. 161570

Liu, X., Strable, M. S., and Ntambi, J. M. (2011). Stearoyl CoA desaturase 1: role in cellular inflammation and stress. Adv. Nutr. 2, 15-22. doi: 10.3945/an.110. 000125

May, F. J., Baer, L. A., Lehnig, A. C., So, K., Chen, E. Y., Gao, F., et al. (2017). Lipidomic adaptations in white and brown adipose tissue in response to exercise demonstrate molecular species-specific remodeling. Cell Rep. 18, 1558-1572. doi: 10.1016/j.celrep.2017.01.038

Mika, A., Kaska, L., Korczynska, J., Mirowska, A., Stepnowski, P., Proczko, M., et al. (2015). Visceral and subcutaneous adipose tissue stearoyl-CoA desaturase1 mRNA levels and fatty acid desaturation index positively correlate with BMI in morbidly obese women. Eur. J. Lipid Sci. Technol. 117, 926-932. doi: 10.1002/ ejlt.201400372

Mika, A., and Sledzinski, T. (2017). Alterations of specific lipid groups in serum of obese humans: a review. Obes. Rev. 18, 247-272. doi: 10.1111/obr.12475

Motiani, P., Virtanen, K. A., Motiani, K. K., Eskelinen, J. J., Middelbeek, R. J., and Goodyear, L. J. (2017). Decreased insulin-stimulated brown adipose tissue glucose uptake after short-term exercise training in healthy middle-aged men. Diabetes Obes. Metab. 19, 1379-1388. doi: 10.1111/dom.12947

Nikolaidis, M. G., and Mougios, V. (2004). Effects of exercise on the fatty-acid composition of blood and tissue lipids. Sports Med. 34, 1051-1076. doi: 10.2165/ 00007256-200434150-00004

Ntambi, J. M., and Miyazaki, M. (2003). Recent insights into stearoylCoA desaturase-1. Curr. Opin. Lipidol. 14, 255-261. doi: 10.1097/01.mol. 0000073502.41685.c7

Petridou, A., Chatzinikolaou, A., Avloniti, A., Jamurtas, A., Loules, G., Papassotiriou, I., et al. (2017). Increased triacylglycerol lipase activity in adipose tissue of lean and obese men during endurance exercise. J. Clin. Endocrinol. Metab. 102, 3945-3952. doi: 10.1210/jc.2017-00168

Petridou, A., Nikolaidis, M. G., Matsakas, A., Schulz, T., Michna, H., and Mougios, V. (2005). Effect of exercise training on the fatty acid composition of lipid classes in rat liver, skeletal muscle, and adipose tissue. Eur. J. Appl. Physiol. 94, 84-92. doi: 10.1007/s00421-004-1294-z

Rao, R. R., Long, J. Z., White, J. P., Svensson, K. J., Lou, J., Lokurkar, I., et al. (2014). Meteorin-like is a hormone that regulates immune-adipose interactions to increase beige fat thermogenesis. Cell 157, 1279-1291. doi: 10.1016/j.cell. 2014.03.065

Roberts, L. D., Boström, P., O’Sullivan, J. F., Schinzel, R. T., Lewis, G. D., Dejam, A., et al. (2014). $\beta$-Aminoisobutyric acid induces browning of white fat and hepatic $\beta$-oxidation and is inversely correlated with cardiometabolic risk factors. Cell Metab. 19, 96-108. doi: 10.1016/j.cmet.2013.12.003

Rocha-Rodrigues, S., Rodríguez, A., Becerril, S., Ramírez, B., Gonçalves, I. O., Beleza, J., et al. (2017a). Physical exercise remodels visceral adipose tissue and mitochondrial lipid metabolism in rats fed a high-fat diet. Clin. Exp. Pharmacol. Physiol. 44, 386-394. doi: 10.1111/1440-1681.12706

Rocha-Rodrigues, S., Rodríguez, A., Gonçalves, I. O., Moreira, A., Maciel, E., Santos, S., et al. (2017b). Impact of physical exercise on visceral adipose tissue fatty acid profile and inflammation in response to a high-fat diet regimen. Int. J. Biochem. Cell Biol. 87, 114-124. doi: 10.1016/j.biocel.2017.04.008

Romain, A. J., Carayol, M., Desplan, M., Fedou, C., Ninot, G., Mercier, J., et al. (2012). Physical activity targeted at maximal lipid oxidation: a meta-analysis. J. Nutr. Metab. 2012:285395. doi: 10.1155/2012/285395

Rönn, T., Volkov, P., Tornberg, Å., Elgzyri, T., Hansson, O., Eriksson, K.F., et al. (2014). Extensive changes in the transcriptional profile of human adipose tissue including genes involved in oxidative phosphorylation after a 6-month exercise intervention. Acta Physiol. 211, 188-200.doi:10.1111/apha. 12247

Ruschke, K., Fishbein, L., Dietrich, A., Klöting, N., Tönjes, A., Oberbach, A., et al. (2010). Gene expression of PPARgamma and PGC-1alpha in human omental and subcutaneous adipose tissues is related to insulin resistance markers and mediates beneficial effects of physical training. Eur. J. Endocrinol. 162, 515-523. doi: 10.1530/EJE-09-0767

Shirvani, H., and Arabzadeh, E. (2018). Metabolic cross-talk between skeletal muscle and adipose tissue in high-intensity interval training vs. moderateintensity continuous training by regulation of PGC-1 $\alpha$. Eat. Weight Disord. doi: 10.1007/s40519-018-0491-4 [Epub ahead of print]. 
Sjögren, P., Sierra-Johnson, J., Kallings, L. V., Cederholm, T., Kolak, M., Halldin, M., et al. (2012). Functional changes in adipose tissue in a randomised controlled trial of physical activity. Lipids Health Dis. 11:80. doi: 10.1186/1476511X-11-80

Stallknecht, B., Vinten, J., Ploug, T., and Galbo, H. (1991). Increased activities of mitochondrial enzymes in white adipose tissue in trained rats. Am. J. Physiol. 261, E410-E414. doi: 10.1152/ajpendo.1991.261.3.E410

Stanford, K. I., and Goodyear, L. J. (2016). Exercise regulation of adipose tissue. Adipocyte 5, 153-162. doi: 10.1080/21623945.2016.1191307

Stanford, K. I., Middelbeek, R. J., and Goodyear, L. J. (2015a). Exercise effects on white adipose tissue: beiging and metabolic adaptations. Diabetes 64, $2361-$ 2368. doi: $10.2337 / \mathrm{db} 15-0227$

Stanford, K. I., Middelbeek, R. J., Townsend, K. L., Lee, M.-Y., Takahashi, H., So, K., et al. (2015b). A novel role for subcutaneous adipose tissue in exerciseinduced improvements in glucose homeostasis. Diabetes 64, 2002-2014. doi: 10.2337/db14-0704

Steinberg, G. R. (2009). Role of the AMP-activated protein kinase in regulating fatty acid metabolism during exercise. Appl. Physiol. Nutr. Metab. 34, 315-322. doi: 10.1139/H09-009

Sutherland, L. N., Bomhof, M. R., Capozzi, L. C., Basaraba, S. A. U., and Wright, D. C. (2009). Exercise and adrenaline increase PGC-1 1 alpha $\}$ mRNA expression in rat adipose tissue. J. Physiol. 587, 1607-1617. doi: 10.1113/jphysiol.2008. 165464

Sutherland, W. H., Woodhouse, S. P., and Heyworth, M. R. (1981). Physical training and adipose tissue fatty acid composition in men. Metabolism 30, 839-844. doi: 10.1016/0026-0495(81)90061-5

Swierczynski, J., and Sledzinski, T. (2012). "The role of adipokines and gastrointestinal tract hormones in obesity," in Principles of Metabolic Surgery, eds W. K. Karcz and O. Thomusch (Berlin: Springer), 53-79.

Vernochet, C., Mourier, A., Bezy, O., Macotela, Y., Boucher, J., Rardin, M. J., et al. (2012). Adipose-specific deletion of TFAM increases mitochondrial oxidation and protects mice against obesity and insulin resistance. Cell Metab. 16, 765776. doi: 10.1016/j.cmet.2012.10.016

Vosselman, M. J., Hoeks, J., Brans, B., Pallubinsky, H., Nascimento, E. B., van der Lans, A. A., et al. (2015). Low brown adipose tissue activity in endurancetrained compared with lean sedentary men. Int. J. Obes. 39, 1696-1702. doi: 10.1038/ijo.2015.130

Woo, J., and Kang, S. (2016). Diet change and exercise enhance protein expression of CREB, CRTC 2 and lipolitic enzymes in adipocytes of obese mice. Lipids Health Dis. 15:147. doi: 10.1186/s12944-016-0316-2

Wu, J., Boström, P., Sparks, L. M., Ye, L., Choi, J. H., Giang, A.-H., et al. (2012). Beige adipocytes are a distinct type of thermogenic fat cell in mouse and human. Cell 150, 366-376. doi: 10.1016/j.cell.2012.05.016

Xu, X., Ying, Z., Cai, M., Xu, Z., Li, Y., Jiang, S. Y., et al. (2011). Exercise ameliorates high-fat diet-induced metabolic and vascular dysfunction, and increases adipocyte progenitor cell population in brown adipose tissue. Am. J. Physiol. Integr. Comp. Physiol. 300, R1115-R1125. doi: 10.1152/ajpregu.00806. 2010

Conflict of Interest Statement: The authors declare that the research was conducted in the absence of any commercial or financial relationships that could be construed as a potential conflict of interest.

The handling Editor is currently co-organizing a Research Topic with one of the authors VD, and confirms the absence of any other collaboration.

Copyright (c) 2019 Mika, Macaluso, Barone, Di Felice and Sledzinski. This is an open-access article distributed under the terms of the Creative Commons Attribution License (CC BY). The use, distribution or reproduction in other forums is permitted, provided the original author(s) and the copyright owner(s) are credited and that the original publication in this journal is cited, in accordance with accepted academic practice. No use, distribution or reproduction is permitted which does not comply with these terms 


\section{OPEN ACCESS}

Edited by: Marilia Seelaender,

University of São Paulo, Brazil

Reviewed by:

Kunihiro Sakuma,

Tokyo Institute of Technology, Japan Nicolas J. Pillon,

Karolinska Institute (KI), Sweden

*Correspondence: Jong Han Lee jhleecw@gachon.ac.kr Hee-Sook Jun hsjun@gachon.ac.kr

Specialty section: This article was submitted to

Striated Muscle Physiology, a section of the journal Frontiers in Physiology

Received: 05 November 2018 Accepted: 14 January 2019 Published: 30 January 2019

Citation: Lee JH and Jun H-S (2019) Role of Myokines in Regulating Skeletal

Muscle Mass and Function. Front. Physiol. 10:42.

doi: 10.3389/fphys.2019.00042

\section{Role of Myokines in Regulating Skeletal Muscle Mass and Function}

\author{
Jong Han Lee ${ }^{1,2 *}$ and Hee-Sook Jun ${ }^{1,2,3 *}$ \\ ${ }^{1}$ College of Pharmacy, Gachon University, Incheon, South Korea, ${ }^{2}$ Lee Gil Ya Cancer and Diabetes Institute, Gachon \\ University, Incheon, South Korea, ${ }^{3}$ Gachon University Gil Medical Center, Gachon Medical and Convergence Institute, \\ Incheon, South Korea
}

Loss of skeletal muscle mass and strength has recently become a hot research topic with the extension of life span and an increasingly sedentary lifestyle in modern society. Maintenance of skeletal muscle mass is considered an essential determinant of muscle strength and function. Myokines are cytokines synthesized and released by myocytes during muscular contractions. They are implicated in autocrine regulation of metabolism in the muscle as well as in the paracrine/endocrine regulation of other tissues and organs including adipose tissue, the liver, and the brain through their receptors. Till date, secretome analysis of human myocyte culture medium has revealed over 600 myokines. In this review article, we summarize our current knowledge of major identified and characterized myokines focusing on their biological activity and function, particularly in muscle mass and function.

Keywords: muscular contraction, myocytes, myokines, skeletal muscle mass, muscle strength

\section{INTRODUCTION}

The muscle is a tissue composed of cells or fibers that produce force and movement of the body. They are primarily responsible for maintaining and changing body position, locomotion as well as the movement of internal organs. Different types of muscles perform different functions according to their location and type. Skeletal muscles are one of the most dynamic tissues involved in voluntary contraction according to command (Frontera and Ochala, 2015; Noto and Edens, 2018). They comprise approximately $40 \%$ of the total body weight (Frontera and Ochala, 2015; Noto and Edens, 2018). In contrast, cardiac and smooth muscles are associated with involuntary contraction without awareness (Frontera and Ochala, 2015; Hafen and Burns, 2018; Noto and Edens, 2018). Smooth muscles are found throughout the body and tightly regulate many of the body subsystems implicated in maintaining survival (Hafen and Burns, 2018).

Myokines are cytokines or peptides synthesized and released by myocytes in muscle tissue in response to muscular contractions (Pedersen et al., 2007). The term "myokine" was first introduced by a Swedish scientist, Bengt Saltin, in 2003 (Pedersen et al., 2003). Myokines are implicated in the autocrine regulation of metabolism in muscles as well as in the para/endocrine regulation of other tissues and organs including the adipose tissue, liver, and brain (Carson, 2017) through their receptors. Since myostatin was first identified as a myokine in 1997, secretome-based analysis of human myocyte culture medium has revealed over 600 myokines till date (Gorgens et al., 2015). However, the majority of these myokines are still not sufficiently characterized. Only few of them have been studied for their biological activity and function and have provided some clear evidence as being released directly from muscle contraction. Moreover, studies potentially associated with muscle atrophy barely exist. Understanding the biological and physiological roles 
of these myokines in skeletal muscle atrophy or weakness is important and valuable to find novel targets for therapeutic intervention.

In this review, we summarize our current knowledge focusing on myokines released directly by muscle contraction and their potential roles associated with skeletal muscle mass and function.

\section{MYOSTATIN}

Myostatin, growth differentiation factor 8, was the first identified myokine in 1997 by Se-Jin Lee and his colleagues (McPherron et al., 1997). It is encoded by the myostatin gene and is known as a highly conserved member of the TGF beta protein family (McPherron et al., 1997). It is abundantly expressed in skeletal muscles, but is also expressed to a lesser extent in cardiac muscles and fat tissues (McPherron et al., 1997; Sharma et al., 1999). Myostatin levels in the plasma of healthy young men has been shown to significantly decrease within $24 \mathrm{~h}$ post-exercise when compared to pre-exercise and has also been shown to positively correlate with plasma IL-6 (Kazemi, 2016). In contrast, serum myostatin has been shown to increase in patients with spinal cord injury after aerobic exercise (Han et al., 2016). Although heavily contradicting reports exist on both sides, circulating myostatin shows an obvious increase in females rather than males during sarcopenia (Bergen et al., 2015), and a decrease in cancer-cachexia (Loumaye and Thissen, 2017) and generic neuromuscular disease patients (Awano et al., 2008; Anaya-Segura et al., 2015; Burch et al., 2017).

The effects of myostatin are mediated through the activin type IIB receptor (ActRIIB), which is expressed ubiquitously (Pistilli et al., 2011; Amthor and Hoogaars, 2012). The downstream mediators of myostatin, Smad2 and Smad3, are phosphorylated and form a complex with Smad4. This complex in turn stimulates FoxO-dependent transcription and regulates the transcription of genes associated with the proliferation and differentiation in skeletal muscle precursor cells as well as protein degradation pathways (such as the ubiquitin-proteasome processes, and autophagy) in mature myofibers (Burks and Cohn, 2011; Han et al., 2013). In addition, myostatin-mediated Smad signaling activation inhibits protein synthesis in muscle tissues by suppressing the Akt-mediated mTOR signaling pathway (Han et al., 2013). Functionally, myostatin is a negative regulator of muscle growth thereby leading to inhibition of myogenesis through muscle cell differentiation and growth (McPherron et al., 1997). Animals blocking myostatin activity with substance show significantly increased muscle mass (myofiber hypertrophy rather than hyperplasia) (Morvan et al., 2017). In myostatinknockout mice, the muscle mass is approximately twice increased compared to that in normal mice (McPherron et al., 1997). In humans, individuals with mutations in both copies of the myostatin gene showed significantly increased muscle mass and muscle strength compared to that observed in normal individuals (Schuelke et al., 2004). Growing evidence indicates that increasing myostatin and its analog activin A contribute to the incidence of muscle atrophy (Morvan et al., 2017). Thus, myostatin is considered a promising target molecule for the treatment of muscle wasting. In the past two decades, several agents, such as follistatin (myostatin antagonist), and selective antibody-based approaches targeting ActR-IIB, myostatin, and activin A were developed to antagonize/suppress myostatin signaling. These molecules were evaluated under various pathological conditions such as muscular wasting or atrophy. For example, a myostatin antibody, MYO-029/stamulumab, was tested in broad muscle dystrophic models, including Becker's muscular dystrophy (BMD) and facioscapulohumeral dystrophy, but failed to show clinical efficacy in elevating muscle strength (Leung et al., 2015). Overexpression of the follistatin isoform, FS344, using an AVV vector showed improved ambulation in patients with BMD and inclusion body myositis (Al-Zaidy et al., 2015; Mendell et al., 2015; Mendell et al., 2017). However, so far none of these treatments have proven to be clinically sufficient as shown in Table 1 (Cohen et al., 2015; Mariot et al., 2017). There are still obstacles (such as lack of target specificity and potential clinical toxicities) to overcome for their use in human patients. In addition, a recent study showed that activin A prominently regulates muscle mass in primates than does myostatin in rodents (Busquets et al., 2012; Cohen et al., 2015), suggesting that targeting myostatin alone may not be sufficient to treat muscle atrophy in humans.

\section{IRISIN}

Irisin is a cleaved form of Fibronectin type III domain-containing protein 5 (FNDC5), which was simultaneously discovered by two independent groups in 2002 (Teufel et al., 2002; Colaianni et al., 2014) It was first reported as a potential mediator of the beneficial effect of exercise (Raschke et al., 2013b). Initially, the expression of PGC1 $\alpha$ in muscle stimulates FNDC5 expression, which drives brown fat-like development of white fat cells named beige cells and increases thermogenesis (Bostrom et al., 2012). Although exercise-induced increase in the level of irisin in the blood is heavily debated (Pekkala et al., 2013), many reports have continuously shown an increase in FNDC5 mRNA expression upon exercise in rodent models (Dun et al., 2013; Roberts et al., 2013) and humans (Huh et al., 2012; Lecker et al., 2012), thus triggering renewed interest in exercise-induced myokines. In line with these observations, expression of mitochondrialspecific transcription factors, such as PGC- $1 \alpha$ and mitochondrial transcription factor $\mathrm{A}$, increases in $\mathrm{C} 2 \mathrm{C} 12$ myotubes exposure to recombinant irisin for $24 \mathrm{~h}$. They are all involved in elevated mitochondrial content and oxygen consumption (Vaughan et al., 2015). Moreover, irisin and myostatin are inversely secreted from skeletal muscles after physical exercise (MacKenzie et al., 2013), thereby suggesting its potential myogenic role. Reza et al. reported that irisin induced skeletal muscle hypertrophy and attenuated denervation-induced atrophy by activating IL-6 signaling in rodents (Reza et al., 2017). The effects of irisin on hypertrophy were shown to be established by the activation of muscle satellite cells and elevation of protein synthesis (Reza et al., 2017). This study substantially opened up potential research avenues on irisin with respect to muscle atrophy. Moreover, a latest study showed that circulating irisin levels were lower 
TABLE 1 | Summary of current trials of myostatin-activin pathway inhibitors.

\begin{tabular}{|c|c|c|c|}
\hline Mechanism of action & Drug/compound & Test pathological models & Current states \\
\hline Myostatin antibody & $\begin{array}{l}\text { MYO-029/Stamulumab } \\
\text { PF-06252616/Domagrozumab } \\
\text { Ly-2495655/Landogrozumab } \\
\text { REGN-1033/Trevogrumab }\end{array}$ & $\begin{array}{l}\text { Muscle dystrophy (BMD, FSHD, and LGMD) } \\
\text { Duchenne muscular dystrophy } \\
\text { Cancer cachexia } \\
\text { Sarcopenia }\end{array}$ & $\begin{array}{l}\text { Dropped } \\
\text { Dropped } \\
\text { Ongoing } \\
\text { Ongoing }\end{array}$ \\
\hline Myostatin peptide & AMG-745/PINTA-745 & Chronic kidney disease & Dropped \\
\hline Activin A antibody & REGN-2477 & Healthy subjects & Ongoing \\
\hline $\begin{array}{l}\text { ActRllb-FC (myostatin decoy } \\
\text { receptor) }\end{array}$ & ACE-031 & Duchenne muscular dystrophy & Dropped \\
\hline Anti-myostatin adnectin & BMS-986089 & Ambulatory boys with DMD & Ongoing \\
\hline ActRIIB antibody & BYM-338/Bimagrumab & $\begin{array}{l}\text { Cancer cachexia Sarcopenia } \\
\text { Type } 2 \text { diabetes }\end{array}$ & Ongoing \\
\hline Myostatin antagonist & Follistatin isoform FS344 & Becker muscular dystrophy & Ongoing \\
\hline
\end{tabular}

ACE-031, Ramatercept; REGN, Trevogrumab; BMD, Becker's muscular dystrophy; FSHD, Facioscapulohumeral dystrophy; LGMD, limb-girdle muscular dystrophy.

in women with postmenopausal sarcopenia when compared to those with pre-sarcopenia and that they negatively correlated with the quadricep cross-sectional area (Park et al., 2018), suggesting that irisin may also function as a potential promyogenic factor in human pathological conditions. Further studies are needed to reveal the biological effects of human irisin and the underlying mechanism in human skeletal muscles.

\section{IL-6}

Interleukin 6 (IL-6) was identified in 2000 and is the most studied myokine (Steensberg et al., 2000; Pedersen and Febbraio, 2008). It is secreted from muscles into the blood vessel in response to muscle contractions (Pedersen and Febbraio, 2008), by which skeletal muscles communicate with central and peripheral organs (Pedersen et al., 2003). The circulatory level of IL-6 is affected by both the duration and intensity of muscle contraction in humans (Steensberg et al., 2000; Helge et al., 2003). Interestingly, IL-6 is highly produced and released after post-exercise while insulin action is enhanced. However, IL-6 is also associated with obesity and insulin resistance (Pedersen and Febbraio, 2008). IL-6 has an insulin-like effect on glucose metabolism. IL-6 increases insulinstimulated glucose disposal in humans as well as glucose uptake and fatty acid oxidation in vitro through AMP-activated protein kinase and PI3K-Akt signaling pathways (Al-Khalili et al., 2006; Carey et al., 2006). Individuals with spinal cord injury (SCI) are prone to develop metabolic diseases due to the lack of exerciserelated IL- 6 response, suggesting that IL- 6 plays a pivotal role in regulating glucose homeostasis (Kouda et al., 2012).

On the other hand, the role of IL-6 on muscle atrophy seems to be a negative effect rather than a beneficial effect. Increased circulating angiotensin II (AngII) reduces lean body mass in chronic kidney disease. In mice, AngII infusion resulted in increased circulating IL- 6 and its hepatic production, suggesting that AngII-induced inflammation might be a trigger for muscle loss (Zhang et al., 2009). In contrast, AngII-induced muscle atrophy was suppressed in IL-6-deficient mice (Zhang et al., 2009). IL-6 is overproduced in patients with Duchenne muscular dystrophy and in muscles of the mdx animal model. Inhibition of IL-6 activity with an interleukin-6 receptor (Il-6r) neutralizing antibody attenuates the dystrophic phenotype, severe muscle degeneration, inflammation, as well as accumulation of nonfunctional fat and fibrotic tissues (Wada et al., 2017). In addition, pharmacological inhibition of IL- 6 activity in mdx male mice inhibits anti-inflammatory responses and improvement in muscle repair (Pelosi et al., 2015). Therefore, inhibition of IL-6 might be beneficial for preventing muscle loss.

\section{BRAIN-DERIVED NEUROTROPHIC FACTOR}

Brain-derived neurotrophic factor (BDNF) is the second member of the neurotrophin family of growth factors, which regulates neuronal survival, plasticity, growth, and death through tropomyosin-related kinase receptor B (TrkB). It was for the first time purified from pig brain in 1982 (Barde et al., 1982). After 11 years, the BDNF gene was identified by two independent groups (Metsis et al., 1993; Binder and Scharfman, 2004). Initially, BDNF has been studied mostly in relation with nervous system development and function (Clow and Jasmin, 2010). However, the expression of several neurotrophin receptors is identified in skeletal muscles, thus implicating the certain role of BDNF. Indeed, Chevrel et al. (2006) reported that BDNF is differentially expressed in skeletal muscles according to physiological or pathological conditions. In adult skeletal muscles, BDNF is also expressed in muscle satellite cells (Mousavi et al., 2004) and is upregulated in muscle injury followed by the activation and proliferation of satellite cells, suggesting that BDNF might play an important role in mediating the satellite cell response to muscle injury (Omura et al., 2005). Jasmin et al. showed that BDNF substantially regulates satellite cell differentiation and skeletal muscle regeneration, by using BDNF null and muscle-specific BDNF KO mice (Clow and Jasmin, 2010). These results indicate that BDNF might be involved in the regulation of damaged muscles. Although there are many studies associated with the role of BDNF in muscle development and function, there is no clear evidence indicating that it is a myokine. In fact, the effect of muscle contraction on circulating BDNF 


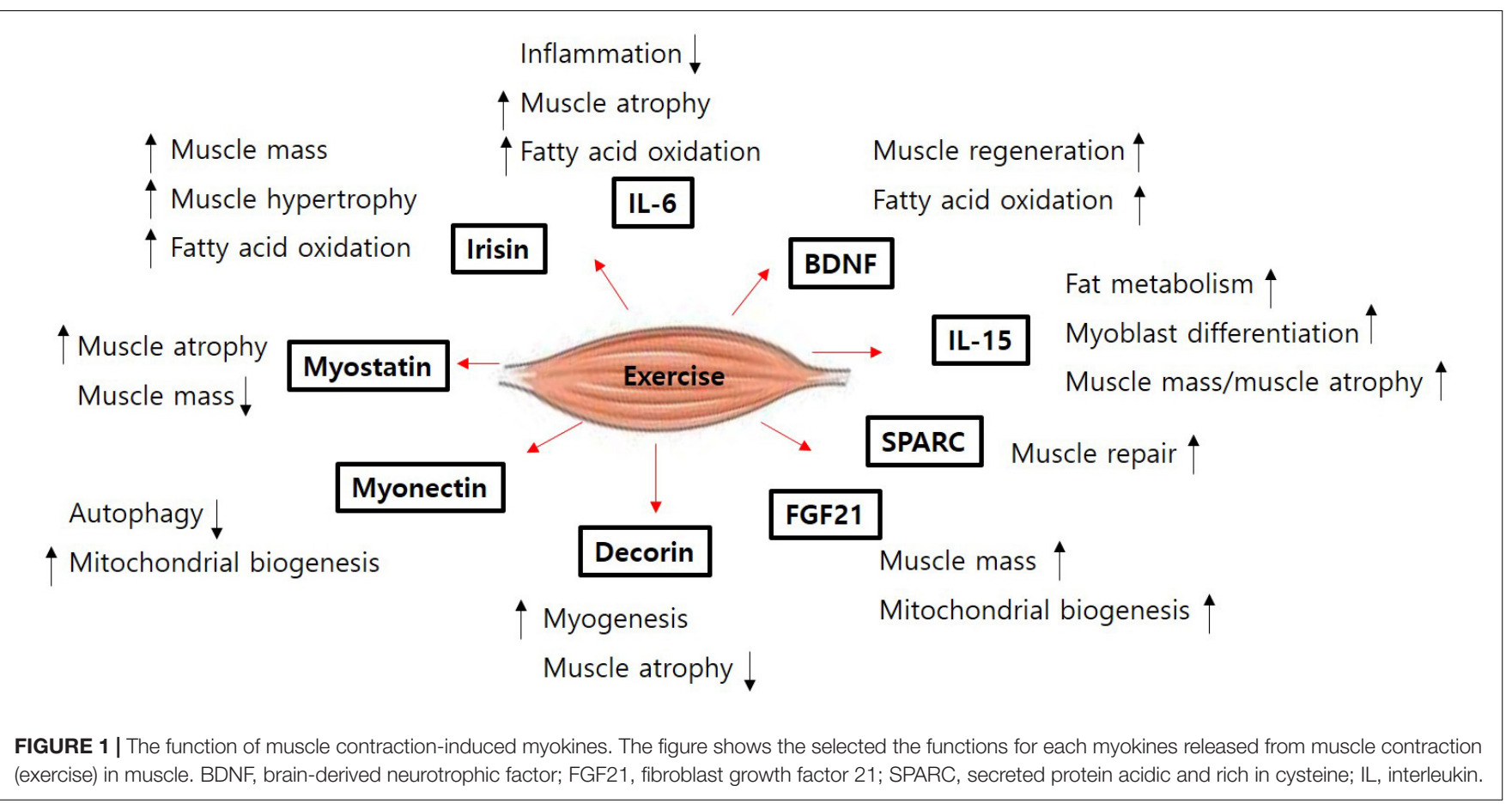

levels is controversial. Some studies have reported no change in serum BDNF right after either acute or chronic exercise. On the other hand, several studies have shown that circulating BDNF increases with physical exercise (Ferris et al., 2007; Yarrow et al., 2010; Pereira et al., 2018). In skeletal muscle cells, BDNF mRNA expression is increased by contraction and increased fat oxidation through activation of AMP-activated protein kinase (Matthews et al., 2009). Overall, these studies suggest that musclederived BDNF is important for regulating muscle regeneration right after muscle injury. However, many key questions on the biological functions of BDNF in skeletal muscles remain unresolved. A major issue would be to elucidate the mechanism by which BDNF regulates satellite cell differentiation and skeletal muscle regeneration, and in which BDNF substantially recover muscle strength and function. Manipulating BDNF may thus represent an important therapeutic tool for alleviating dystrophic muscle atrophy.

\section{IL-15}

Interleukin-15 (IL-15) is a cytokine with a structure similar to interleukin-2 (IL-2). It was discovered by two different research groups in 1994 and was characterized as a T cell growth factor (Steel et al., 2012). Later on, several studies showed that IL15 is accumulated in the muscles as a result of regular exercise training, indicating that it is a myokine (Pedersen, 2011; Tamura et al., 2011; Brunelli et al., 2015). Moreover, IL-15 mRNA expression is upregulated along with myoblast differentiation (Pedersen and Febbraio, 2008). Supportively, several studies showed that exogenously treated IL-15 or IL-15 overexpression promotes myoblast differentiation and increases muscle mass in the mouse C2 skeletal myogenic cell line (Quinn et al., 1995, 2002). In rats with cancer cachexia, IL-15 treatment attenuates skeletal muscle wasting by suppressing protein degradation through inhibition of the ATP-dependent ubiquitin proteolytic pathway (Carbo et al., 2000). IL-15 administration was found to improve diaphragm strength with increased muscle fiber cross-sectional area and decreased collagen accumulation in dystrophic mdx mice (Harcourt et al., 2005). In contrast, systemic infusion of IL-15 induces muscle atrophy in skeletal muscles of rodents (Pistilli and Alway, 2008). IL-15 treatment increased the glucose uptake in skeletal muscle cells via activation of the Jak3/STAT3 signaling pathway (Krolopp et al., 2016) or the AMPK signaling pathway (Gray and Kamolrat, 2011). In addition, Quinn L et al. and coworkers reported that IL15 transgenic mice exhibited increased fat oxidation, energy expenditure and running endurance even with lower muscle mass compared to that in wild type mice. Interestingly, these mice also expressed troponin I and myosin heavy chain mRNA isoform indicating the conversion of muscles to a more oxidative phenotype (Quinn et al., 2013; Chalkiadaki et al., 2014). Collectively, the above controversial reports indicate that IL-15 acts differently according to the normal and pathological conditions. Thus, further studies should be focused on clarifying the different factors influencing the varying roles of IL-15 between different physiological conditions.

\section{MYONECTIN (CTRP15)}

Myonectin is a myokine belonging to the C1q/TNF-related protein (CTRP) family, and was discovered by Seldin et al. (2012). It is a novel nutrient-responsive myokine secreted from skeletal 


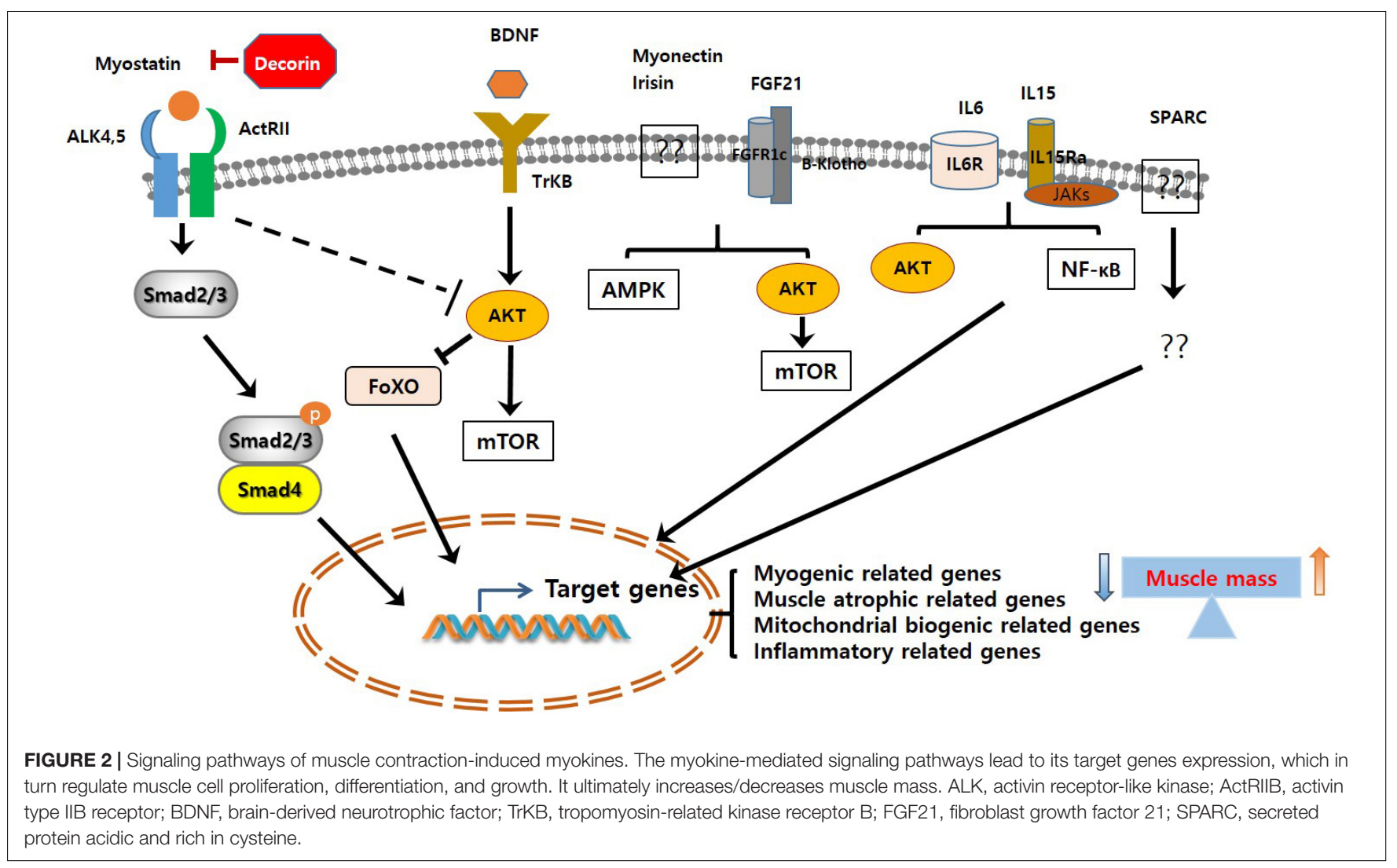

muscles (Seldin et al., 2012; Peterson et al., 2014). Myonectin is released into the blood stream by muscular contraction, and is functionally similar to insulin as it promotes fatty acid uptake into cells by increased expression of fatty acid transport genes (CD36, FATP1, Fabp1, and Fabp4) (Seldin and Wong, 2012; Seldin et al., 2012). In the mouse liver and cultured hepatocytes, recombinant myonectin treatment suppresses starvation-induced autophagy by inhibiting LC3-dependent autophagosome formation, p62 degradation, and other autophagy-related genes expression. Furthermore, the ability of myonectin to suppress autophagy is abolished by inhibition of the PI3K/Akt/mTOR signaling pathway (Seldin et al., 2013). Autophagy is considered to be a mechanism that induces muscle atrophy (Bonaldo and Sandri, 2013). In addition, the PI3K/Akt signaling pathway is involved in anabolic responses in the body. Therefore, these observations indicate that myonectin may play an important role in increasing muscle mass by elevating protein synthesis and inhibiting protein degradation. On a related note, mitochondrial content in muscles is an important determinant for muscle type and function. Myonectin is remarkably increased following depletion of mtDNA and increases glucose uptake and fatty acid oxidation through activation of the AMPK signaling pathway in rat skeletal myocytes (Park et al., 2009; Lim et al., 2012). Interestingly, oxidative, slow-twitch muscle fiber types express a higher level of myonectin than does glycolytic, fast-twitch muscle fiber types, suggesting that it may be involved in mitochondrial biogenesis and sensing cellular energy state (Seldin and Wong, 2012). However, there is no study associated with its biological function and mechanism on muscle mass and muscle mitochondrial biogenesis in normal physiology and in the diseased state.

\section{DECORIN}

Decorin is small leucine-rich proteoglycan identified as a myokine by Kanzleiter et al. (2014). It is secreted in skeletal muscles during muscular contraction and plays an important role in muscle growth. Decorin directly binds to and inactivates myostatin (a potent inhibitor of muscle growth) in a zincdependent manner, and inhibits its anti-myogenic effects (El Shafey et al., 2016). In vivo over-expression of Decorin in murine skeletal muscles promotes expression of the pro-myogenic factor Mighty (Marshall et al., 2008). Mighty is ubiquitously expressed but appears to be negatively regulated by myostatin in skeletal muscles. Decorin overexpression increases the expression of Myod1 and follistatin, whereas it reduces the muscle-specific ubiquitin ligases atrogin1 and MuRF1 (Marshall et al., 2008). Thus, Decorin might act as a myogenic factor and might be a possible therapeutic target for the treatment of muscle wasting.

\section{FIBROBLAST GROWTH FACTOR (FGF) 21}

Fibroblast growth factors (FGFs) are signaling proteins with diverse biological functions in development and metabolism. 
FGFs are classified as para, intra, and endocrine according to their action manners. Paracrine FGFs mostly function as local signaling molecules in developmental processes whereas intracrine FGFs mainly serve as intracellular molecules in neuronal processes (Itoh and Ornitz, 2011). FGF21 functions as endocrinal hormone-like or local signaling molecules in metabolism. FGF21 does not have proliferative activity as other paracrine and endocrine FGFs family and is only associated with metabolism (Itoh, 2014). FGFs activate several intracellular signaling pathways including phosphatidylinositol 3-kinase $(\mathrm{PI} 3 \mathrm{~K}) /$ serine-threonine protein kinase AKT, signaling transducer and activator of transcription (STAT), mitogen activation protein kinase (MAPK), and phosphoinositide phospholipase C (PLC) $\gamma$ (Itoh, 2014). Specifically, FGF21 acts through FGF receptor $1 c$ with $\beta$-Klotho as a cofactor. Skeletal muscle-specific Akt1 transgenic mice showed skeletal muscle fiber hypertrophy with increasing Fgf21 expression in the muscle and in serum indicating that FGF21 plays an important role in regulating muscle mass (Izumiya et al., 2008). In addition, FGF21 expression is coupled to mitochondrial dysfunction and insults of various stresses in skeletal muscles. Autophagy deficiency and subsequent mitochondrial dysfunction elevates the level of FGF21 as a myokine, thereby protecting against diet-induced obesity and insulin resistance (Kim et al., 2013; Keipert et al., 2014). In cultured myoblasts, mitochondrial complex inhibitor treatment increased expression by promoting the binding of activating transcription factor 2 (ATF2) for the promoter region of the Fgf21 gene (Ribas et al., 2014). Moreover, in human brain vascular smooth muscle cells, FGF21 protects against angiotensin II-induced cerebrovascular aging by elevating mitochondrial biogenesis (Wang et al., 2016). All the above studies suggest that FGF21 may be potentially involved in switching muscle type and regulating mitophagy, thereby regulating muscle mass and function. Thus, targeting FGF21 might be an attractive approach to treat mitochondrial-based myopathy and muscle dysfunction.

\section{SECRETED PROTEIN ACIDIC AND RICH IN CYSTEINE (SPARC)}

Aoi et al. (2013) reported SPARC/osteonectin as a novel myokine, which is released from the skeletal muscles of both humans and mice after exercise, even though it was identified earlier (Aoi et al., 2013). Exercise-stimulated SPARC secretion was shown to inhibit colon tumorigenesis by enhancing apoptosis in colon cancer cells (Aoi et al., 2013). SPARC was also shown to be upregulated in inherited and idiopathic muscle wasting diseases such as Duchenne muscular dystrophy and congenital muscular dystrophy (Jorgensen et al., 2009). SPARC overexpression almost completely abolished myogenic differentiation in the muscle

\section{REFERENCES}

Al-Khalili, L., Bouzakri, K., Glund, S., Lonnqvist, F., Koistinen, H. A., and Krook, A. (2006). Signaling specificity of interleukin-6 action on glucose and lipid metabolism in skeletal muscle. Mol. Endocrinol. 20, 3364-3375. doi: 10.1210/me.2005-0490 progenitor cell line, C2C12 (Petersson et al., 2013). Thus, SPARC may play a certain functional role in the repair of muscle damage in muscle satellite cells. However, very limited studies associated with the role of myokines are currently available. Further studies need to first determine and address the expression profile and role of SPARC in muscle development and regeneration. The underlying signaling pathways also need to be studied in detail.

\section{CONCLUSION}

Skeletal muscle atrophy is an emerging medical problem worldwide owing to increasing elderly populations and various classical reasons including genetic mutation, disease-derived cachexia, and accidents. However, although our understanding of molecular mechanisms regulating muscle atrophy/muscle weakness has substantially progressed, there is no specific treatment for muscle atrophy. Recently, a number of myokines have been identified through secretome analysis and some have proven to be very informative while searching for novel myokines (Raschke et al., 2013a; Hartwig et al., 2014; Grube et al., 2018). However, the majority of myokines are not still sufficiently characterized with regard to their biological activity and function. Only few myokines have been restrictively characterized (Figure 1) and their potential signaling pathways, implicated in muscle cell proliferation, differentiation, and growth in order to maintain muscle mass, muscle strength, and function, been identified (Figure 2). Therefore, it is important to better understand their precise role and function on skeletal muscles under normal physiological and pathophysiological conditions. Targeting novel myokines for either increasing or suppressing their functional activity in certain pathological states could be an attractive novel therapeutic tool for combating skeletal muscle atrophy.

\section{AUTHOR CONTRIBUTIONS}

Both authors conceived and wrote the manuscript and approved it for publication.

\section{FUNDING}

This work was supported by a Basic Science Research Program grant of the National Research Foundation of Korea, the Ministry of Education of South Korea (2017R1D1A1B03036210 to JL) and the Korea Health Technology R\&D Project through the Korea Health Industry Development Institute (KHIDI), funded by the Ministry of Health and Welfare, South Korea (HI14C1135 to H-SJ).

Al-Zaidy, S. A., Sahenk, Z., Rodino-Klapac, L. R., Kaspar, B., and Mendell, J. R. (2015). Follistatin gene therapy improves ambulation in becker muscular dystrophy. J. Neuromuscul. Dis. 2, 185-192. doi: 10.3233/JND-150083

Amthor, H., and Hoogaars, W. M. (2012). Interference with myostatin/ActRIIB signaling as a therapeutic strategy for Duchenne muscular dystrophy. Curr. Gene Ther. 12, 245-259. doi: 10.2174/156652312800840577 
Anaya-Segura, M. A., Garcia-Martinez, F. A., Montes-Almanza, L. A., Diaz, B. G., Avila-Ramirez, G., Alvarez-Maya, I., et al. (2015). Non-invasive biomarkers for duchenne muscular dystrophy and carrier detection. Molecules 20, 1115411172. doi: 10.3390/molecules200611154

Aoi, W., Naito, Y., Takagi, T., Tanimura, Y., Takanami, Y., Kawai, Y., et al. (2013). A novel myokine, secreted protein acidic and rich in cysteine (SPARC), suppresses colon tumorigenesis via regular exercise. Gut 62, 882-889. doi: 10. 1136/gutjnl-2011-300776

Awano, H., Takeshima, Y., Okizuka, Y., Saiki, K., Yagi, M., and Matsuo, M. (2008). Wide ranges of serum myostatin concentrations in Duchenne muscular dystrophy patients. Clin. Chim. Acta 391, 115-117. doi: 10.1016/j.cca.2008.01. 024

Barde, Y. A., Edgar, D., and Thoenen, H. (1982). Purification of a new neurotrophic factor from mammalian brain. EMBO J. 1, 549-553. doi: 10.1002/j.1460-2075. 1982.tb01207.x

Bergen, H. R. III, Farr, J. N., Vanderboom, P. M., Atkinson, E. J., White, T. A., Singh, R. J., et al. (2015). Myostatin as a mediator of sarcopenia versus homeostatic regulator of muscle mass: insights using a new mass spectrometry-based assay. Skeletal Muscle 5:21. doi: 10.1186/s13395-015-0047-5

Binder, D. K., and Scharfman, H. E. (2004). Brain-derived neurotrophic factor. Growth Factors 22, 123-131. doi: 10.1080/08977190410001723308

Bonaldo, P., and Sandri, M. (2013). Cellular and molecular mechanisms of muscle atrophy. Dis. Models Mech. 6, 25-39. doi: 10.1242/dmm.010389

Bostrom, P., Wu, J., Jedrychowski, M. P., Korde, A., Ye, L., Lo, J. C., et al. (2012). A PGC1-alpha-dependent myokine that drives brown-fat-like development of white fat and thermogenesis. Nature 481, 463-468. doi: 10.1038/nature 10777

Brunelli, D. T., Chacon-Mikahil, M. P., Gaspari, A. F., Lopes, W. A., Bonganha, V., Bonfante, I. L., et al. (2015). Combined training reduces subclinical inflammation in obese middle-age men. Med. Sci. Sports Exerc. 47, 2207-2215. doi: 10.1249/MSS.0000000000000658

Burch, P. M., Pogoryelova, O., Palandra, J., Goldstein, R., Bennett, D., Fitz, L., et al. (2017). Reduced serum myostatin concentrations associated with genetic muscle disease progression. J. Neurol. 264, 541-553. doi: 10.1007/s00415-0168379-6

Burks, T. N., and Cohn, R. D. (2011). Role of TGF-beta signaling in inherited and acquired myopathies. Skeletal Muscle 1:19. doi: 10.1186/2044-5040-1-19

Busquets, S., Toledo, M., Orpi, M., Massa, D., Porta, M., Capdevila, E., et al. (2012). Myostatin blockage using actRIIB antagonism in mice bearing the Lewis lung carcinoma results in the improvement of muscle wasting and physical performance. J. Cachexia Sarcopenia Muscle 3, 37-43. doi: 10.1007/s13539-0110049-z

Carbo, N., Lopez-Soriano, J., Costelli, P., Busquets, S., Alvarez, B., Baccino, F. M., et al. (2000). Interleukin-15 antagonizes muscle protein waste in tumourbearing rats. Br. J. Cancer 83, 526-531. doi: 10.1054/bjoc.2000.1299

Carey, A. L., Steinberg, G. R., Macaulay, S. L., Thomas, W. G., Holmes, A. G., Ramm, G., et al. (2006). Interleukin-6 increases insulin-stimulated glucose disposal in humans and glucose uptake and fatty acid oxidation in vitro via AMP-activated protein kinase. Diabetes 55, 2688-2697. doi: 10.2337/db05-1404

Carson, B. P. (2017). The potential role of contraction-induced myokines in the regulation of metabolic function for the prevention and treatment of type 2 diabetes. Front. Endocrinol. 8:97. doi: 10.3389/fendo.2017. 00097

Chalkiadaki, A., Igarashi, M., Nasamu, A. S., Knezevic, J., and Guarente, L. (2014). Muscle-specific SIRT1 gain-of-function increases slow-twitch fibers and ameliorates pathophysiology in a mouse model of duchenne muscular dystrophy. PLoS Genet. 10:e1004490. doi: 10.1371/journal.pgen.10 04490

Chevrel, G., Hohlfeld, R., and Sendtner, M. (2006). The role of neurotrophins in muscle under physiological and pathological conditions. Muscle Nerve 33, 462-476. doi: 10.1002/mus.20444

Clow, C., and Jasmin, B. J. (2010). Brain-derived neurotrophic factor regulates satellite cell differentiation and skeltal muscle regeneration. Mol. Biol. Cell 21, 2182-2190. doi: 10.1091/mbc.E10-02-0154

Cohen, S., Nathan, J. A., and Goldberg, A. L. (2015). Muscle wasting in disease: molecular mechanisms and promising therapies. Nat. Rev. Drug Discov. 14, 58-74. doi: 10.1038/nrd4467
Colaianni, G., Cuscito, C., Mongelli, T., Oranger, A., Mori, G., Brunetti, G., et al. (2014). Irisin enhances osteoblast differentiation in vitro. Int. J. Endocrinol. 2014:902186. doi: 10.1155/2014/902186

Dun, S. L., Lyu, R. M., Chen, Y. H., Chang, J. K., Luo, J. J., and Dun, N. J. (2013). Irisin-immunoreactivity in neural and non-neural cells of the rodent. Neuroscience 240, 155-162. doi: 10.1016/j.neuroscience.2013.02.050

El Shafey, N., Guesnon, M., Simon, F., Deprez, E., Cosette, J., Stockholm, D., et al. (2016). Inhibition of the myostatin/Smad signaling pathway by short decorinderived peptides. Exp. Cell Res. 341, 187-195. doi: 10.1016/j.yexcr.2016.01.019

Ferris, L. T., Williams, J. S., and Shen, C. L. (2007). The effect of acute exercise on serum brain-derived neurotrophic factor levels and cognitive function. Med. Sci. Sports Exerc. 39, 728-734. doi: 10.1249/mss.0b013e31802f04c7

Frontera, W. R., and Ochala, J. (2015). Skeletal muscle: a brief review of structure and function. Calcified Tissue Int. 96, 183-195. doi: 10.1007/s00223-014-9915-y

Gorgens, S. W., Eckardt, K., Jensen, J., Drevon, C. A, and Eckel, J. (2015). Exercise and regulation of adipokine and myokine production. Prog. Mol. Biol. Transl. Sci. 135, 313-336. doi: 10.1016/bs.pmbts.2015.07.002

Gray, S. R, and Kamolrat, T. (2011). The effect of exercise induced cytokines on insulin stimulated glucose transport in C2C12 cells. Cytokine 55, 221-228. doi: 10.1016/j.cyto.2011.04.019

Grube, L., Dellen, R., Kruse, F., Schwender, H., Stuhler, K., and Poschmann, G. (2018). Mining the secretome of C2C12 muscle cells: data dependent experimental approach to analyze protein secretion using label-free quantification and peptide based analysis. J. Proteome Res. 17, 879-890. doi: 10.1021/acs.jproteome.7b00684

Hafen, B. B., and Burns, B. (2018). StatPearls. Treasure Island, FL. StatPearls Publishing.

Han, D. S., Hsiao, M. Y., Wang, T. G., Chen, S. Y., and Yang, W. S. (2016). Association of serum myokines and aerobic exercise training in patients with spinal cord injury: an observational study. BMC Neurol. 16:142. doi: 10.1186/ s12883-016-0661-9

Han, H. Q., Zhou, X., Mitch, W. E., and Goldberg. A. L. (2013). Myostatin/activin pathway antagonism: molecular basis and therapeutic potential. Int. J. Biochem. Cell Biol. 45, 2333-2347. doi: 10.1016/j.biocel.2013.05.019

Harcourt, L. J., Holmes, A. G., Gregorevic, P., Schertzer, J. D., Stupka, N., Plant. D. R., and Lynch, G. S. (2005). Interleukin-15 administration improves diaphragm muscle pathology and function in dystrophic $\mathrm{mdx}$ mice. Am. J. Pathol. 166, 1131-1141. doi: 10.1016/S0002-9440(10)62 333-4

Hartwig, S., Raschke, S., Knebel, B., Scheler, M., Irmler, M., Passlack, W., et al. (2014). Secretome profiling of primary human skeletal muscle cells. Biochim. Biophys. Acta 1844, 1011-1017. doi: 10.1016/j.bbapap.2013.08.004

Helge, J. W., Stallknecht, B., Pedersen, B. K., Galbo, H., Kiens, B., and Richter, E. A. (2003). The effect of graded exercise on IL-6 release and glucose uptake in human skeletal muscle. J. Physiol. 546(Pt 1), 299-305. doi: 10.1113/jphysiol. 2002.030437

Huh, J. Y., Panagiotou, G., Mougios, V., Brinkoetter, M., Vamvini, M. T., Schneider, B. E., et al. (2012). FNDC5 and irisin in humans: I. Predictors of circulating concentrations in serum and plasma and II. mRNA expression and circulating concentrations in response to weight loss and exercise. Metabolism 61, 17251738. doi: 10.1016/j.metabol.2012.09.002

Itoh, N. (2014). FGF21 as a hepatokine, adipokine, and myokine in metabolism and diseases. Front. Endocrinol. 5:107. doi: 10.3389/fendo.2014.00107

Itoh, N., and Ornitz, D. M. (2011). Fibroblast growth factors: from molecular evolution to roles in development, metabolism and disease. J. Biochem. 149, 121-130. doi: 10.1093/jb/mvq121

Izumiya, Y., Bina, H. A., Ouchi, N., Akasaki, Y., Kharitonenkov, A., and Walsh, K. (2008). FGF21 is an Akt-regulated myokine. FEBS Lett. 582, 3805-3810. doi: 10.1016/j.febslet.2008.10.021

Jorgensen, L. H., Petersson, S. J., Sellathurai, J., Andersen, D. C., Thayssen, S., Sant, D. J., et al. (2009). Secreted protein acidic and rich in cysteine (SPARC) in human skeletal muscle. J. Histochem. Cytochem. 57, 29-39. doi: 10.1369/jhc. 2008.951954

Kanzleiter, T., Rath, M., Gorgens, S. W., Jensen, J., Tangen, D. S., Kolnes, A. J., et al. (2014). The myokine decorin is regulated by contraction and involved in muscle hypertrophy. Biochem. Biophys. Res. Commun. 450, 1089-1094. doi: 10.1016/j.bbrc.2014.06.123 
Kazemi, F. (2016). The correlation of resistance exercise-induced myostatin with insulin resistance and plasma cytokines in healthy young men. J. Endocrinol. Investigat. 39, 383-388. doi: 10.1007/s40618-015-0373-9

Keipert, S., Ost, M., Johann, K., Imber, F., Jastroch, M., van Schothorst, E. M., et al. (2014). Skeletal muscle mitochondrial uncoupling drives endocrine crosstalk through the induction of FGF21 as a myokine. Am. J. Physiol. Endocrinol. Metab. 306, E469-E482. doi: 10.1152/ajpendo.00330.2013

Kim, K. H., Jeong, Y. T., Oh, H., Kim, S. H., Cho, J. M., Kim, Y. N., et al. (2013). Autophagy deficiency leads to protection from obesity and insulin resistance by inducing Fgf21 as a mitokine. Nat. Med. 19, 83-92. doi: 10.1038/ nm.3014

Kouda, K., Furusawa, K., Sugiyama, H., Sumiya, T., Ito, T., Tajima, F., et al. (2012). Does 20-min arm crank ergometer exercise increase plasma interleukin-6 in individuals with cervical spinal cord injury? Eur. J. Appl. Physiol. 112, 597-604. doi: 10.1007/s00421-011-2004-2

Krolopp, J. E., Thornton, S. M., and Abbott, M. J. (2016). IL-15 activates the Jak3/STAT3 signaling pathway to mediate glucose uptake in skeletal muscle cells. Front. Physiol. 7:626. doi: 10.3389/fphys.2016.00626

Lecker, S. H., Zavin, A., Cao, P., Arena, R., Allsup, K., Daniels, K. M., et al. (2012). Expression of the irisin precursor FNDC5 in skeletal muscle correlates with aerobic exercise performance in patients with heart failure. Circ. Heart Failure 5, 812-818. doi: 10.1161/CIRCHEARTFAILURE.112.969543

Leung, D. G., Carrino, J. A., Wagner, K. R., and Jacobs, M. A. (2015). Wholebody magnetic resonance imaging evaluation of facioscapulohumeral muscular dystrophy. Muscle Nerve 52, 512-520. doi: 10.1002/mus.24569

Lim, S., Choi, S. H., Koo, B. K., Kang, S. M., Yoon, J. W., Jang, H. C., et al. (2012). Effects of aerobic exercise training on C1q tumor necrosis factor alpharelated protein isoform 5 (myonectin): association with insulin resistance and mitochondrial DNA density in women. J. Clin. Endocrinol. Metab. 97, E88-E93. doi: 10.1210/jc.2011-1743

Loumaye, A., and Thissen, J. P. (2017). Biomarkers of cancer cachexia. Clin. Biochem. 50, 1281-1288. doi: 10.1016/j.clinbiochem.2017.07.011

MacKenzie, M. G., Hamilton, D. L., Pepin, M., Patton, A., and Baar, K. (2013). Inhibition of myostatin signaling through Notch activation following acute resistance exercise. PLoS One 8:e68743. doi: 10.1371/journal.pone.0068743

Mariot, V., Joubert. R., Hourde, C., Feasson, L., Hanna, M., Muntoni, F., et al. (2017). Downregulation of myostatin pathway in neuromuscular diseases may explain challenges of anti-myostatin therapeutic approaches. Nat. Commun. 8:1859. doi: 10.1038/s41467-017-01486-4

Marshall, A., Salerno, M. S., Thomas, M., Davies, T., Berry, C., Dyer, K., et al. (2008). Mighty is a novel promyogenic factor in skeletal myogenesis. Exp. Cell Res. 314, 1013-1029. doi: 10.1016/j.yexcr.2008.01.004

Matthews, V. B., Astrom, M. B., Chan, M. H., Bruce, C. R., Krabbe, K. S., Prelovsek, O., et al. (2009). Brain-derived neurotrophic factor is produced by skeletal muscle cells in response to contraction and enhances fat oxidation via activation of AMP-activated protein kinase. Diabetologia 52, 1409-1418. doi: 10.1007/s00125-009-1364-1

McPherron, A. C., Lawler, A. M., and Lee, S. J. (1997). Regulation of skeletal muscle mass in mice by a new TGF-beta superfamily member. Nature $387,83-90$. doi: $10.1038 / 387083 \mathrm{a} 0$

Mendell, J. R., Sahenk. Z., Al-Zaidy, S., Rodino-Klapac, L. R., Lowes, L. P., Alfano, L. N., et al. (2017). Follistatin gene therapy for sporadic inclusion body myositis improves functional outcomes. Mol. Ther. 25, 870-879. doi: 10.1016/j.ymthe. 2017.02.015

Mendell, J. R., Sahenk, Z., Malik, V., Gomez, A. M., Flanigan, K. M., Lowes, L. P., et al. (2015). A phase $1 / 2$ a follistatin gene therapy trial for becker muscular dystrophy. Mol. Ther. 23, 192-201. doi: 10.1038/mt.2014.200

Metsis, M., Timmusk, T., Arenas, E., and Persson, H. (1993). Differential usage of multiple brain-derived neurotrophic factor promoters in the rat brain following neuronal activation. Proc. Natl. Acad. Sci. U.S.A. 90, 8802-8806. doi: 10.1073/ pnas. 90.19 .8802

Morvan, F., Rondeau, J. M., Zou, C., Minetti, G., Scheufler, C., Scharenberg, M., et al. (2017). Blockade of activin type II receptors with a dual anti-ActRIIA/IIB antibody is critical to promote maximal skeletal muscle hypertrophy. Proc. Natl. Acad. Sci. U.S.A. 114, 12448-12453. doi: 10.1073/pnas.1707925114

Mousavi, K., Parry, D. J., and Jasmin, B. J. (2004). BDNF rescues myosin heavy chain IIB muscle fibers after neonatal nerve injury. Am. J. Physiol. Cell Physiol. 287, C22-C29. doi: 10.1152/ajpcell.00583.2003
Noto, R. E., and Edens, M. A. (2018). StatPearls. Treasure Island, FL. StatPearls Publishing.

Omura, T., Sano, M., Omura, K., Hasegawa, T., Doi, M., Sawada, T., and Nagano, A. (2005). Different expressions of BDNF, NT3, and NT4 in muscle and nerve after various types of peripheral nerve injuries. J. Peripheral Nerv. Syst. 10, 293-300. doi: 10.1111/j.1085-9489.2005.10307.x

Park, H. S., Kim, H. C., Zhang, D., Yeom, H., and Lim, S. K. (2018). The novel myokine irisin: clinical implications and potential role as a biomarker for sarcopenia in postmenopausal women. Endocrine doi: 10.1007/s12020-0181814-y [Epub ahead of print].

Park, S. Y., Choi, J. H., Ryu, H. S., Pak, Y. K., Park, K. S., Lee, H. K., and Lee. W. (2009). C1q tumor necrosis factor alpha-related protein isoform 5 is increased in mitochondrial DNA-depleted myocytes and activates AMP-activated protein kinase. J. Biol. Chem. 284, 27780-27789. doi: 10.1074/jbc.M109.005611

Pedersen, B. K. (2011). Muscles and their myokines. J. Exp. Biol. 214(Pt 2), 337-346. doi: $10.1242 /$ jeb. 048074

Pedersen, B. K., and Febbraio, M. A. (2008). Muscle as an endocrine organ: focus on muscle-derived interleukin-6. Physiol. Rev. 88, 1379-1406. doi: 10.1152/ physrev.90100.2007

Pedersen, B. K., Akerstrom, T. C., Nielsen, A. R., and Fischer, C. P. (2007). Role of myokines in exercise and metabolism. J. Appl. Physiol. 103, 1093-1098. doi: 10.1152/japplphysiol.00080.2007

Pedersen, B. K., Steensberg, A., Fischer, C., Keller, C., Keller, P., Plomgaard, P., et al. (2003). Searching for the exercise factor: is IL-6 a candidate? J. Muscle Res. Cell Motil. 24, 113-119. doi: 10.1023/A:1026070911202

Pekkala, S., Wiklund, P. K., Hulmi, J. J., Ahtiainen, J. P., Horttanainen, M., Pollanen, E., et al. (2013). Are skeletal muscle FNDC5 gene expression and irisin release regulated by exercise and related to health? J. Physiol. 591, 5393-5400. doi: 10.1113/jphysiol.2013.263707

Pelosi, L., Berardinelli, M. G., De Pasquale, L., Nicoletti, C., D’Amico, A., Carvello, F., et al. (2015). Functional and morphological improvement of dystrophic muscle by interleukin 6 receptor blockade. eBioMedicine 2, 285-293. doi: 10.1016/j.ebiom.2015.02.014

Pereira, E. S., Krause Neto, W., Calefi, A. S., Georgetti, M., Guerreiro, L., Zocoler, C. A. S., et al. (2018). Significant acute response of brain-derived neurotrophic factor following a session of extreme conditioning program is correlated with volume of specific exercise training in trained men. Front. Physiol. 9:823. doi: 10.3389/fphys.2018.00823.

Peterson, J. M., Mart, R., and Bond, C. E. (2014). Effect of obesity and exercise on the expression of the novel myokines, Myonectin and Fibronectin type III domain containing 5. PeerJ 2:e605. doi: 10.7717/peerj.605

Petersson, S. J., Jorgensen, L. H., Andersen, D. C., Norgaard, R. C., Jensen, C. H., and Schroder, H. D. (2013). SPARC is up-regulated during skeletal muscle regeneration and inhibits myoblast differentiation. Histol. Histopathol. 28, 1451-1460. doi: 10.14670/HH-28.1451

Pistilli, E. E., and Alway, S. E. (2008). Systemic elevation of interleukin-15 in vivo promotes apoptosis in skeletal muscles of young adult and aged rats. Biochem. Biophys. Res. Commun. 373, 20-24. doi: 10.1016/j.bbrc.2008. 05.188

Pistilli, E. E., Bogdanovich, S., Goncalves, M. D., Ahima, R. S., Lachey, J., Seehra, J., et al. (2011). Targeting the activin type IIB receptor to improve muscle mass and function in the mdx mouse model of Duchenne muscular dystrophy. Am. J. Pathol. 178, 1287-1297. doi: 10.1016/j.ajpath.2010.11.071

Quinn, L. S., Anderson, B. G., Conner, J. D., and Wolden-Hanson, T. (2013). IL-15 overexpression promotes endurance, oxidative energy metabolism, and muscle PPARdelta, SIRT1, PGC-1alpha, and PGC-1beta expression in male mice. Endocrinology 154, 232-245. doi: 10.1210/en.2012-1773

Quinn, L. S., Anderson, B. G., Drivdahl, R. H., Alvarez, B., and Argiles, J. M. (2002). Overexpression of interleukin-15 induces skeletal muscle hypertrophy in vitro: implications for treatment of muscle wasting disorders. Exp. Cell Res. 280, 55-63. doi: 10.1006/excr.2002.5624

Quinn, L. S.,Haugk, K. L., and Grabstein, K. H. (1995). Interleukin-15: a novel anabolic cytokine for skeletal muscle. Endocrinology 136, 3669-3672. doi: 10.1210/endo.136.8.7628408

Raschke, S., Eckardt, K., Bjorklund Holven, K., Jensen, J., and Eckel. J. (2013a). Identification and validation of novel contraction-regulated myokines released from primary human skeletal muscle cells. PLoS One 8:e62008. doi: 10.1371/ journal.pone.0062008 
Raschke, S., Elsen, M., Gassenhuber, H., Sommerfeld, M., Schwahn, U., Brockmann, B., et al. (2013b). Evidence against a beneficial effect of irisin in humans. PLoS One 8:e73680. doi: 10.1371/journal.pone.0073680

Reza, M. M., Subramaniyam, N., Sim, C. M., Ge, X., Sathiakumar, D., McFarlane, C., et al. (2017). Irisin is a pro-myogenic factor that induces skeletal muscle hypertrophy and rescues denervation-induced atrophy. Nat. Commun. 8:1104. doi: 10.1038/s41467-017-01131-0

Ribas, F., Villarroya, J., Hondares, E., Giralt, M., and Villarroya, F. (2014). FGF21 expression and release in muscle cells: involvement of MyoD and regulation by mitochondria-driven signalling. Biochem. J. 463, 191-199. doi: 10.1042/ BJ20140403

Roberts, M. D., Bayless, D. S., Company, J. M., Jenkins, N. T., Padilla. J., Childs, T. E., et al. (2013). Elevated skeletal muscle irisin precursor FNDC5 mRNA in obese OLETF rats. Metabolism 62, 1052-1056. doi: 10.1016/j.metabol.2013.02.002

Schuelke, M., Wagner, K. R., Stolz, L. E., Hubner, C., Riebel, T., Komen, W., et al. (2004). Myostatin mutation associated with gross muscle hypertrophy in a child. N. Engl. J. Med. 350, 2682-2688. doi: 10.1056/NEJMoa040933

Seldin, M. M., and Wong, G. W. (2012). Regulation of tissue crosstalk by skeletal muscle-derived myonectin and other myokines. Adipocyte 1, 200-202. doi: 10.4161/adip. 20877

Seldin, M. M., Lei, X., Tan, S. Y., Stanson, K. P., Wei, Z., and Wong, G. W. (2013). Skeletal muscle-derived myonectin activates the mammalian target of rapamycin (mTOR) pathway to suppress autophagy in liver. J. Biol. Chem. 288, 36073-36082. doi: 10.1074/jbc.M113.500736

Seldin, M. M., Peterson, J. M., Byerly, M. S., Wei, Z., and Wong, G. W. (2012). Myonectin (CTRP15), a novel myokine that links skeletal muscle to systemic lipid homeostasis. J. Biol. Chem. 287, 11968-11980. doi: 10.1074/jbc.M111. 336834

Sharma, M., Kambadur, R., Matthews, K. G., Somers, W. G., Devlin, G. P., Conaglen, J. V., et al. (1999). Myostatin, a transforming growth factor-beta superfamily member, is expressed in heart muscle and is upregulated in cardiomyocytes after infarct. J. Physiol. 180, 1-9.

Steel, J. C., Waldmann, T. A., and Morris, J. C. (2012). Interleukin-15 biology and its therapeutic implications in cancer. Trends Pharmacol. Sci. 33, 35-41. doi: 10.1016/j.tips.2011.09.004

Steensberg, A., van Hall, G., Osada, T., Sacchetti, M., Saltin, B., and Klarlund Pedersen, B. (2000). Production of interleukin-6 in contracting human skeletal muscles can account for the exercise-induced increase in plasma interleukin-6. J. Physiol. 529(Pt 1), 237-242. doi: 10.1111/j.1469-7793.2000. 00237.x

Tamura, Y., Watanabe, K., Kantani, T., Hayashi, J., Ishida, N., and Kaneki, M. (2011). Upregulation of circulating IL-15 by treadmill running in healthy individuals: is IL-15 an endocrine mediator of the beneficial effects of endurance exercise? Endocrine J. 58, 211-215. doi: 10.1507/endocri.K10E-400

Teufel, A., Malik, N., Mukhopadhyay, M., and Westphal, H. (2002). Frcp1 and Frcp2, two novel fibronectin type III repeat containing genes. Gene 297, 79-83. doi: 10.1016/S0378-1119(02)00828-4

Vaughan, R. A., Gannon, N. P., Mermier, C. M., and Conn, C. A. (2015). Irisin, a unique non-inflammatory myokine in stimulating skeletal muscle metabolism. J. Physiol. Biochem. 71, 679-689. doi: 10.1007/s13105-015-0433-9

Wada, E., Tanihata, J., Iwamura, A., Takeda, S., Hayashi, Y. K., and Matsuda, R. (2017). Treatment with the anti-IL-6 receptor antibody attenuates muscular dystrophy via promoting skeletal muscle regeneration in dystrophin-/utrophindeficient mice. Skeletal Muscle 7:23. doi: 10.1186/s13395-017-0140-z

Wang, X. M., Xiao, H., Liu, L. L., Cheng, D., Li, X. J., and Si. L. Y. (2016). FGF21 represses cerebrovascular aging via improving mitochondrial biogenesis and inhibiting p53 signaling pathway in an AMPK-dependent manner. Exp. Cell Res. 346, 147-156. doi: 10.1016/j.yexcr.2016.06.020

Yarrow, J. F., White, L. J., McCoy, S. C., and Borst, S. E. (2010). Training augments resistance exercise induced elevation of circulating brain derived neurotrophic factor (BDNF). Neurosci. Lett. 479, 161-165. doi: 10.1016/j.neulet.2010.05.058

Zhang, L., Du, J., Hu, Z., Han, G., Delafontaine, P., Garcia, G., et al. (2009). IL-6 and serum amyloid A synergy mediates angiotensin II-induced muscle wasting. J. Am. Soc. Nephrol. 20, 604-612. doi: 10.1681/ASN.2008060628

Conflict of Interest Statement: The authors declare that the research was conducted in the absence of any commercial or financial relationships that could be construed as a potential conflict of interest.

Copyright $\odot 2019$ Lee and Jun. This is an open-access article distributed under the terms of the Creative Commons Attribution License (CC BY). The use, distribution or reproduction in other forums is permitted, provided the original author(s) and the copyright owner(s) are credited and that the original publication in this journal is cited, in accordance with accepted academic practice. No use, distribution or reproduction is permitted which does not comply with these terms. 


\section{OPEN ACCESS}

Edited by:

Marilia Seelaender,

University of São Paulo, Brazil

Reviewed by:

Parco M. Siu,

The University of Hong Kong,

Hong Kong

David C. Hughes,

The University of lowa, United States

*Correspondence:

Kathryn H. Myburgh

khm@sun.ac.za

${ }^{\dagger}$ These authors have contributed equally to this work and joint first

authors

Specialty section

This article was submitted to

Striated Muscle Physiology, a section of the journal

Frontiers in Physiology

Received: 14 November 2018

Accepted: 24 January 2019

Published: 11 February 2019

Citation:

Isaacs AW, Macaluso F, Smith C and Myburgh KH (2019) C-Reactive

Protein Is Elevated Only in High

Creatine Kinase Responders

to Muscle Damaging Exercise.

Front. Physiol. 10:86.

doi: 10.3389/fphys.2019.00086

\section{C-Reactive Protein Is Elevated Only in High Creatine Kinase Responders to Muscle Damaging Exercise}

\author{
Ashwin W. Isaacs ${ }^{1 \dagger}$, Filippo Macaluso ${ }^{1,2,3,4+}$, Carine Smith ${ }^{1}$ and Kathryn H. Myburgh ${ }^{1,3 *}$ \\ ${ }^{1}$ Department of Physiological Sciences, Stellenbosch University, Stellenbosch, South Africa, ${ }^{2}$ Department of Experimental \\ Biomedicine and Clinical Neurosciences, University of Palermo, Palermo, Italy, ${ }^{3}$ Euro-Mediterranean Institute of Science \\ and Technology, Palermo, Italy, ${ }^{4}$ SMART Engineering Solutions \& Technologies Research Center, eCampus University, \\ Novedrate, Italy
}

The purpose of this study was to investigate if exertional rhabdomyolysis induced by an acute bout of plyometric exercise in untrained individuals was associated with histological characteristics of skeletal muscle, creatine kinase $(\mathrm{CK})$ polymorphism or secondary damage. Twenty-six healthy male untrained individuals completed a bout of plyometric exercise (10 sets of 10 maximal squat jumps, with each standardized to achieve at least $95 \%$ of individual maximal jump height). Blood samples were taken, and perceived pain was scored immediately before the exercise intervention and 6 h, 1, 2, and 3 days post-intervention. Muscle biopsies were collected 9 or 4 days before (baseline) and 3 days after plyometric jumps. Subjects were divided into two groups, high $(n=10)$ and low responders $(n=16)$, based on a cut-off limit for exertional rhabdomyolysis of peak CK activity $\geq 1000 \mathrm{U} / \mathrm{L}$ in any post-exercise blood sample. Perceived pain was more severe assessed in squat than standing position. Low responders perceived more pain at $6 \mathrm{~h}$ and 1 day, while high responders perceived more pain than low responders on days three and four after exercise; structural (dystrophin staining) and ultra-structural (transmission electron microscopy) analysis of muscle fibers revealed no baseline pathology; damage was evident in all individuals in both groups, with no difference between high and low responders in either damage or fiber type proportion. High responders had significantly higher total white blood cell and neutrophil counts $6 \mathrm{~h}$ and significantly higher C-reactive protein (CRP) $6 \mathrm{~h}$ and days one and two after exercise compared to low responders. High responders had significantly greater muscle myeloperoxidase (MPO) levels in baseline and 3 day post-exercise biopsies compared to baseline of low responders. MLCK C49T single polymorphism was present in $26 \%$ of volunteers, whose CK responses were not higher than those with MLCK CC or CT genotype. In conclusion, perceived pain is more effectively assessed with potentially affected muscle under eccentric strain, even if static. High CK responders also have pronounced CRP responses to unaccustomed plyometric exercise intervention. Exertional rhabdomyolysis after unaccustomed eccentric exercise may be related to underlying inability to resolve intramuscular MPO.

Keywords: eccentric exercise and muscle damage, creatine kinase, myoglobin, single nucleotide polymorphism, myeloperoxidase 


\section{INTRODUCTION}

Elevated circulating creatine kinase (CK) is a hallmark of muscle damage caused by intense and unaccustomed exercise including muscle-group targeted eccentric resistance exercise, downhill running and plyometric jumping (Nosaka et al., 2001; Macaluso et al., 2012b, 2013; van de Vyver and Myburgh, 2012). Literature reports that certain individuals, despite apparently similar characteristics to other study participants, may experience greater increases in CK activity following unaccustomed eccentric exercise (Clarkson et al., 2005; Devaney et al., 2007; Yamin et al., 2008). This biological phenomenon remains unexplained (Sayers and Clarkson, 2002).

Individuals who experience severe muscle damage may present with exertional rhabdomyolysis which is characterized by the continued release of myoglobin $(\mathrm{Mb})$ into the circulation (Sayers and Clarkson, 2002; Scalco et al., 2016). Typically, elevations in $\mathrm{CK}$ and $\mathrm{Mb}$ go hand and in hand after extreme bouts of unaccustomed eccentric exercise (Clarkson et al., 2005), but CK activity is most commonly measured (Damas et al., 2016). The laboratory diagnostic cut-off limit for exertional rhabdomyolysis varies across studies and study populations (Sayers and Clarkson, 2002; Warren et al., 2002; Lauritzen et al., 2009), however, a lower limit for exertional rhabdomyolysis has been suggested to be $\mathrm{CK} \geq 1000 \mathrm{U} / \mathrm{L}$ (Lee and Clarkson, 2003; Thoenes, 2010). Although this phenomenon is well described, including the potential for clinical sequelae (Hill et al., 2017), the potential mechanisms pre-disposing certain individuals remains to be elucidated fully.

Potential role players could include background genetic polymorphisms, the pre-exercise condition of skeletal muscle itself or pre-exercise systemic profile - such as relative leukocyte distribution or inflammatory processes. Several genetic polymorphisms of skeletal muscle proteins have been associated with exertional muscle damage variability. A few studies have indicated that genes involved in muscle structure (ACTN3) (Clarkson et al., 2005), growth (IGF2) (Devaney et al., 2007) or regulation of force production (MYLK) (Clarkson et al., 2005), can present polymorphisms that affect baseline CK activity and exacerbate the muscle damage response to eccentric exercise. Similarly, inflammation-related polymorphisms (TNFA and IL6) have been associated with elevated CK activity following unaccustomed eccentric exercise (Yamin et al., 2008). For a recent review, see Baumert et al. (2016).

Muscle damage results in the activation and mobilization of circulating leukocytes to the damaged area, where they release reactive oxygen species (ROS) and proteolytic enzymes (Smith et al., 2008). Oxidative stress has been shown to play an important role in muscle damage (Lee and Clarkson, 2003; Yamin et al., 2008) and redox individuality has been implicated in damage variability observed after eccentric exercise (Margaritelis et al., 2014). More specifically, neutrophils are early inflammatory responders and known to be a source of oxidative stress (Vollaard et al., 2005; Smith et al., 2008). In addition, myeloperoxidase (MPO), which is also highly expressed in neutrophils and released during the respiratory burst (Childs et al., 2001), is not an oxygen radical per se, but at elevated concentrations is also known to cause tissue damage. Schneider and Tiidus (2007) suggested inclusion of intramuscular MPO assessment in investigations aimed at elucidating the extent of neutrophil activity in muscle (Schneider and Tiidus, 2007). Chronologically, in response to mechanical damage and neutrophil-induced secondary damage, C-reactive protein (CRP) dependent complement activation facilitates removal of cellular debris (Pereira Panza et al., 2015). CRP elevation after an acute bout of eccentric biceps exercise has been shown to be sensitive to a nutritional intervention in the form of a supplement containing antioxidants and docohexanoic acid (Phillips et al., 2003). The supplement had no effect on CK levels. This suggests that CRP should be investigated in more detail as a biomarker of secondary damage following unaccustomed exercise that may induce muscle damage.

Unaccustomed eccentric exercise damages type II muscle fibers in greater proportion than type I fibers (Macaluso et al., 2012b). Therefore, sedentary individuals with a larger percentage of type II muscle fibers may present with higher values of the indirect markers used to describe exertional muscle damage. However, whether participants with higher proportions of Type II fibers also present with higher CK activity responses, within the range of exertional rhabdomyolysis, is currently not known.

The aim of this study was to probe in untrained individuals different mechanisms which may predispose to exertional rhabdomyolysis, induced by an acute bout of plyometric exercise that results in varying levels of post-exercise circulating CK. Variables assessed were selected from different categories of potential contributors namely genetic background (MYLCK genotype), skeletal muscle phenotype (fiber type; MPO content) and inflammatory profile (circulating leukocytes; CRP).

\section{MATERIALS AND METHODS}

\section{Study Design}

The study was granted ethical clearance (reference no. N09/05/164) by Sub-committee C of the Research Committee of University of Stellenbosch. On the day of the plyometric exercise intervention, blood samples and perceived pain scores were collected both immediately before (day 0) and $6 \mathrm{~h} \mathrm{1,2,3}$ and 4 days after exercise intervention; whilst muscle biopsies were obtained 4 or 9 days before and 3 days after the acute bout of plyometric exercise.

\section{Subjects}

Twenty-six healthy male untrained individuals (age $=21.5 \pm 1.6$ years; height $=175.9 \pm 8.9 \mathrm{~cm}$; weight $=73.0 \pm 14.1 \mathrm{~kg}$ ) were recruited. Participants were informed of the criteria for inclusion and exclusion and all the experimental procedures and associated risk before providing written informed consent. All participants completed a habitual and recent physical activity and health history questionnaire, and they were healthy and had no medical contraindications or any muscle or lower limb injury in the previous 6 months. No participants were currently or chronically treated by any corticosteroid-containing medication (including inhaled forms). Exercise criteria for inclusion were: habitually physically active 
0-2 times per week without any systematic training of the lower body; no plyometric exercise of the lower body other than typical daily activities. All participants refrained from unaccustomed exercise or vigorous physical activity during the study. Volunteers were informed to maintain their normal dietary habits, and not to take any anti-inflammatory drugs (e.g., non-steroidal anti-inflammatory drugs) or nutritional supplements (e.g., vitamins, protein/amino acids) during the experimental period. The habitual exercise and healthy history questionnaire confirmed that none of the participants practiced plyometric exercise before the study.

\section{Plyometric Exercise Intervention}

Volunteer subjects first completed $5 \mathrm{~min}$ of backward and forward running together with light stretching as a warm-up before performing three maximal squat jumps. The maximal height reached by the head was recorded and 95\% of this height served as a target height which subjects had to maintain during the exercise intervention. The plyometric exercise intervention consisted of 10 sets of 10 maximal squat jumps, separated by $1 \mathrm{~min}$ recovery time between sets. This protocol has previously been used to induce transient muscle damage in the knee extensor muscles (Vissing et al., 2008; Macaluso et al., 2012b). Subjects were allowed to swing their arms which assisted with balance and generating momentum with each jump. However, by not adopting a $90^{\circ}$ knee joint angle on landing and not maintaining an upright trunk position and the minimum jump height all constituted incorrect jumps. The jump technique was observed by the researcher and when incorrect jumps were observed, the subjects were instructed to stop and given a 1 min rest period before completing that set, thereby completing 100 proper squat jumps (Macaluso et al., 2012b). Static stretching for less than $60 \mathrm{~s}$ was permitted since data in literature reported that this would not affect maximal muscle performance (Kay and Blazevich, 2012).

\section{Blood Sampling and Analysis}

After an equilibration time of $5 \mathrm{~min}$ in a supine position, blood samples were drawn from the antecubital vein and collected in SST and EDTA vacutainer tubes (Becton Dickinson and Company). Tubes were inverted 5-6 times, and then centrifuged at $3500 \mathrm{rpm}$ for $10 \mathrm{~min}$ at $4^{\circ} \mathrm{C}$. Blood samples were then analyzed by commercial laboratory PathCare pathology laboratory (Stellenbosch Medi Clinic, South Africa) for CK activity (Access one-step sandwich assay CK-MB assay), $\mathrm{Mb}$ (Mbchemiluminescence), CRP (CRP-immunoturbidimetric method Beckman Coulter, Inc.), total and differential white blood cell count (WBC) using a CellDyne 3700CS Hematology Analyzer (Abbott Diagnostics, Fullerton, CA, United States).

\section{High and Low Responders Groups}

Subjects were categorized as high $(n=10)$ or low $(n=16)$ responders based on $\mathrm{CK}$ activity: individuals for whom $\mathrm{CK}$ activity at all time points assessed was lower than $1000 \mathrm{U} / \mathrm{L}$, were considered low responders, whereas individuals exhibiting CK activity in access of $1000 \mathrm{U} / \mathrm{L}$ at any time point, were categorized as high responders. In literature $\mathrm{CK} \geq 1000 \mathrm{U} / \mathrm{L}$ has been suggested to be lower limit for exertional rhabdomyolysis (Lee and Clarkson, 2003; Thoenes, 2010).

From this point forth the groups will be defined as high (height $=180 \pm 0.05 \mathrm{~cm}$; weight $=71.1 \pm 10.05 \mathrm{~kg}$; $95 \%$ jump height $211 \pm 0.06 \mathrm{~cm}$ ) and low responders (height $=180 \pm 0.11 \mathrm{~cm}$; weight $=74.2 \pm 16.37 \mathrm{~kg}$; $95 \%$ jump height $211 \pm 0.14 \mathrm{~cm})$.

\section{Perceived Pain}

Perceived pain was measured using a visual pain scale. Participants indicated soreness of the knee-extensors ranging from 0, None; 2, Discomfort; 4, Annoying; 6, Horrible; 8, Dreadful; 10, Agonizing. This was done in two different positions (standing and squatting position) as described by Macaluso et al. (2012b).

\section{Muscle Sampling}

Muscle biopsies of vastus lateralis muscle were obtained 9 or 4 days prior and 3 days after the plyometric exercise intervention using the suction-assisted technique (Macaluso et al., 2012a). Biopsies were taken by a medical doctor experienced in the technique and experienced in obtaining follow-up biopsies from the same depth. The second biopsy was taken from the opposite leg. Each biopsy was split into three parts: one was snap frozen in liquid nitrogen, the second was embedded in tissue freezing medium and frozen in isopentane (cooled in liquid nitrogen) for subsequent cryosectioning and immunofluorescent microscopy, and the third $(1 \times 3 \mathrm{~mm})$ was fixed in $2.5 \%$ glutaraldehyde.

\section{Immunofluorescence Staining}

Cross sections of the muscle tissue were cut using a cryostat microtome (Leica CM1100, Leica Microsystem Nussloch GmbH, Germany) at $-22^{\circ} \mathrm{C}$, mounted on slides and stored at $-20^{\circ} \mathrm{C}$. The next day the slides were brought to room temperature, rinsed in $0.01 \mathrm{M}$ phosphate buffered saline (PBS) containing $0.25 \%$ Triton X-100 (15 min) and washed with PBS $(3 \times 5 \mathrm{~min})$. The following primary antibodies were used: MHC II (1:250; A4.74, mouse monoclonal antibody, Developmental Studies Hybridoma Bank, Iowa City, IA, United States) to identify fast twitch muscle fibers and dystrophin (1:250, rabbit polyclonal, Santa Cruz Biotechnology, Santa Cruz, CA, United States) to identify the sarcolemma. The first primary antibody was left to incubate for $1 \mathrm{~h}$ at room temperature, after which sections were revealed with Alexa fluor 488 conjugated secondary antibody (1:250, goat anti-mouse, Invitrogen, Eugene, OR, United States) and Alexa fluor 594 (1:250, goat anti-rabbit, Invitrogen, Eugene) conjugated secondary antibody for $1 \mathrm{~h}$ at room temperature. bisBenzimide $\mathrm{H}$ 33342 trihydrochloride (Hoechst, 1:200; Sigma Aldrich, B2261) was added to all sections to visualize nuclei. Samples were then washed in PBS $(3 \times 5 \mathrm{~min})$, mounted with fluorescent mounting medium (DAKO; GLostrup, Denmark) and analyzed with a direct fluorescent microscope (model DM 5000 CTR; Leica Microsystem Nussloch $\mathrm{GmbH}$ ) with $10 \times$ and $20 \times$ objectives. Images obtained were assessed for fiber positivity to MHC II antibody for fiber type determination (MHC-IIa fibers are highly positive; MHC IIx fibers are positive with lower intensity; MHC-I 
fibers are negative) and loss of dystrophin staining to determine which were damaged (Macaluso et al., 2012b, 2014).

\section{Transmission Electron Microscopy Analysis}

Muscle specimens from each volunteer for both time points were cut in a longitudinal orientation, fixed in $2.5 \%$ glutaraldehyde and post-fixed in $1 \%$ osmium tetroxide for $1 \mathrm{~h}$. Next the samples were dehydrated with graded ethanol 30, 50, 70, 95, and $100 \% \mathrm{vlv}$ and tissue pieces were placed in propylene oxide for $30 \mathrm{~min}$ and infiltrated with resin (EPON 812, Electron Microscopy Sciences, Hatfield, PA, United States) at passages of 1:3, 2:2 and 3:1 resin to propylene oxide) 1 day before polymerization at $50^{\circ} \mathrm{C}$ for $48 \mathrm{~h}$. Ultrathin $(50-70 \mathrm{~nm})$ longitudinal skeletal muscle sections were cut with an ultramicrotome (model RM2125 RT; Leica Microsytem Nussloch GmbH, Germany). Images of ultrathin sections of resin-embedded muscle were obtained using a transmission electron microscope (Jeol-Jem 1011 TEM, Leica Microsystem Nussloch GmbH, Germany). The longitudinal sections were assessed for the presence of damaged sarcomeres (Z-disk streaming) or necrotic and hypercontracted areas as described by Lauritzen et al. (2009).

\section{MPO Enzyme-Linked Immunosorbent Assay}

The concentration of secreted MPO in muscle tissue was determined using the Abcam MPO Human ELISA kit (ab119605), as recommended by the manufacturer. Briefly, $2 \mathrm{~mm}$ frozen muscle tissue was washed $2 x$ in Phosphate-buffered saline (PBS) and homogenized in RIPA ( $\mathrm{pH}$ 7.4, Tris-HCL $2.5 \mathrm{mM}$, EDTA $1 \mathrm{mM}$, NaF $50 \mathrm{mM}$, dithiothreitol $1 \mathrm{mM}$, phenylmethylsulfonyl fluoride (PMSF) $0.1 \mathrm{mM}$, benzamidine $1 \mathrm{mM}, 4 \mathrm{mg} / \mathrm{ml} \mathrm{SBTI}, 10 \mathrm{mg} / \mathrm{ml}$ leupeptin, $1 \%$ NP40, $0.1 \%$ SDS and $0.5 \% \mathrm{Na}$ deoxycholate) using a tissue homogenizer (Ultra-Turrax, Germany). The homogenate was then centrifuged at $5000 \times g$ for $5 \mathrm{~min}$ and immediately assayed. The sample readings were performed using the ELx800 universal microplate reader (Biotek Instruments, Inc., VT, United States).

\section{Genotyping Analysis}

Snap frozen muscle tissue (baseline muscle biopsy) was sent to the Central Analytical Facility (CAF, Stellenbosch University, South Africa) to test possible single nucleotide polymorphism (SNP) MYLCK C49T. DNA extraction was performed as follows: Tissue was placed in $1.7 \mathrm{ml}$ tube and homogenized with the TissueLyzer (Qiagen). Next $400 \mu \mathrm{l}$ of Buffer PL2 (NucleoSpin Plant II Kit, Separations) with $2 \mu \mathrm{l}$ of proteinase K $(10 \mathrm{mg} / \mathrm{ml}$; Sigma-Aldrich) was added. The sample was incubated overnight in a waterbath at $60^{\circ} \mathrm{C}$, following the protocol of the manufacturer. Extraction was performed on the Tecan TMP 2000 Liquid Handling Platform. The DNA extract was then analyzed for selected SNPs within the genes of selected muscle structure proteins. TaqMan primer sets for SNPs were: TTC AGA GCA ACT TCA GGA GCTT (forward primer); GCC AGT GGG ACA GGA AAGG (Reverse Primer). The PCR was performed in a cycler (Verity, Applied Biosystems, Life Technologies, CA,
United States) with the following cycling conditions: $95^{\circ} \mathrm{C}$ for $10 \mathrm{~min}$ followed by 44 cycles at $92^{\circ} \mathrm{C}$ for $15 \mathrm{~s}$ and a final extension of $60^{\circ} \mathrm{C}$ for $1 \mathrm{~min}$. Post-PCR purification was done using the NucleoFast purification system (NucleoFast, Separations, Germany). Sequencing was performed with BigDye Terminator (V1.3, Applied Biosystems, Life Technologies, CA, United States) followed by electrophoresis on the DNA Analyser (3730xl, Applied Biosystems, Life Technologies, CA, United States). Sequences were analyzed and trimmed using Sequencing Analysis (V5.3.1, Applied Biosystems, Life Technologies, CA, United States). Alignments were done using the ClustalW module, BioEdit version 7.0.4.1 with the downloaded SND-ID as reference. Possible polymorphism variants included MYLCK C49T genotype CC, CT, and TT (Clarkson et al., 2005).

\section{Statistical Analysis}

Data were assessed for normality by inspecting normal probability plots. A logarithmic transformation ( $\log 10)$ was applied to non-normally distributed data $(\mathrm{CK}, \mathrm{Mb}, \mathrm{CRP}$, and perceived pain) before analysis. Changes in perceived pain (in squat and in standing position), blood parameters (CK, $\mathrm{Mb}$, and (CRP), total and differential WBC in high responders vs. low responders over time were analyzed by two-way mixed model repeated-measures analysis of variance. If a significant difference was detected, this was further evaluated by the post hoc Fisher's LSD test. Statistical analyses were performed using PASW (version 18; SPSS Inc., Chicago, IL, United States). Significance was accepted at $P \leq 0.05$.

\section{RESULTS}

\section{Muscle Damage}

In the standing position, perceived pain scores for both low and high CK responders were fairly low and followed a similar time course, with significant elevations reported at $6 \mathrm{~h}$ and days 1 and 2 following plyometric exercise (Table 1). In contrast, in the squat position, both groups reported moderate to severe soreness, with highest scores on days 1 and 2 post-exercise, although on days 3 and 4, soreness scores were still significantly elevated from baseline in both groups. Time-dependent group differences were evident: while perceived soreness scores were higher in low responders at $6 \mathrm{~h}$ post-plyometric exercise, high responders rated their soreness at days 3 and 4 higher than low responders, but only in the squat position. At these later time points, the high responders' perceived pain scores were still at least 4 -fold higher than the $6 \mathrm{~h}$ time point, whilst that of low responders was only $\approx 1.5$-fold higher than $6 \mathrm{~h}$.

Electron micrographs of muscle biopsies performed on the 3rd day after the acute bout of plyometric exercise revealed muscle damage in the form of $Z$-disk streaming in all subjects (refer to Figures 1A,B for representative images of pre- and post-exercise sections in the longitudinal plane of vastus lateralis muscle). Necrotic and hyper-contracted areas were not evident in any of the muscle samples. Qualitative assessment of ultrastructural muscle damage did not reveal any overt differences between high and low responders. 
TABLE 1 | Perceived muscle soreness over time before and after a single bout of plyometric exercise.

\begin{tabular}{|c|c|c|c|c|}
\hline \multirow[b]{2}{*}{ Days } & \multicolumn{2}{|c|}{ Low responders } & \multicolumn{2}{|c|}{ High responders } \\
\hline & $\begin{array}{l}\text { Standing } \\
\text { position }\end{array}$ & $\begin{array}{c}\text { Squat } \\
\text { position }\end{array}$ & $\begin{array}{l}\text { Standing } \\
\text { position }\end{array}$ & $\begin{array}{c}\text { Squat } \\
\text { position }\end{array}$ \\
\hline-4 day & $0.0 \pm 0.0$ & $1.4 \pm 1,6$ & $0.0 \pm 0.0$ & $0.9 \pm 1.1$ \\
\hline 0 day & $0.0 \pm 0.0$ & $1.0 \pm 1.2$ & $0.0 \pm 0.0$ & $1.1 \pm 1.2$ \\
\hline $6 \mathrm{~h}$ & $1.3 \pm 1.4^{*}$ & $2.0 \pm 1.0^{*}$ & $0.9 \pm 1.0 * \psi$ & $1.0 \pm 1.0 \psi$ \\
\hline 1 day & $1.5 \pm 1.6^{*}$ & $5.8 \pm 1.7^{*}$ & $1.4 \pm 1.0^{*} \ddagger$ & $6.6 \pm 1.2^{*} \#$ \\
\hline 2 days & $1.5 \pm 2.4^{*}$ & $6.3 \pm 2.5^{*}$ & $0.9 \pm 0.9 *$ & $7.5 \pm 1.7^{*} \#$ \\
\hline 3 days & $0.60 \pm 1.0$ & $3.8 \pm 1.7^{*}$ & $0.6 \pm 1.0$ & $5.0 \pm 1.0 * \psi$ \\
\hline 4 days & $0.5 \pm 1.2$ & $2.8 \pm 1.5^{*}$ & $0.2 \pm 0.4$ & $4.4 \pm 1.2^{*} \psi$ \\
\hline
\end{tabular}

Soreness in the squat position and in the standing position for the low ( $n=16$ ) and high $(n=10)$ responders, respectively. *Significantly different from time points -4 and 0 day, $(P<0.0001)$, \#significantly different from time point $6 \mathrm{~h}$ $(P<0.001)$, łsignificantly different from time point 3 and 4 days $(P<0.05)$, ४significantly different response between high and low responders $(P<0.05)$. Data are expressed as mean $\pm S D$.
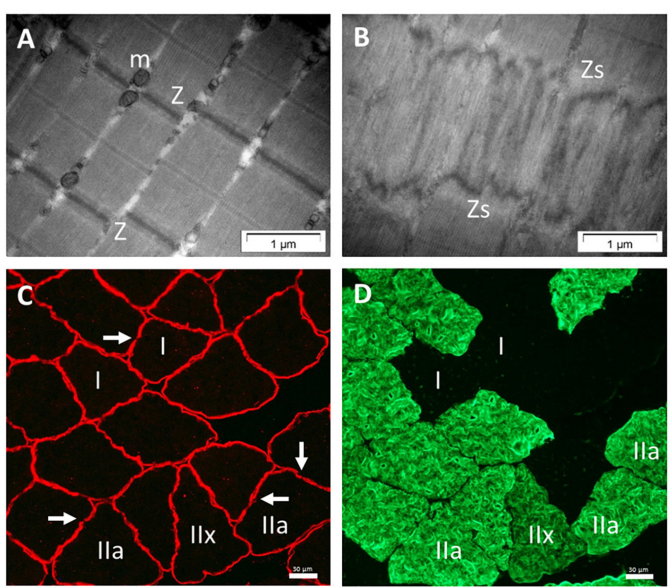

FIGURE 1 | Analysis of skeletal muscle sections. Electron micrographs of longitudinal sections. Illustration of undamaged sarcomeres (A). Arrangement of one sarcomere: Z, Z-line; and m, mitochondria. Damaged sarcomeres (B) on day 3 following eccentric exercise in human skeletal muscle: Zs, z-line streaming. No difference in ultrastructural damage was observed between low and high responder groups. Immunofluorescence of muscle cross-sections also on day 3 after the plyometric exercise intervention, using double immunostaining with anti-dystrophin (C) and anti-myosin heavy chain II (D). Arrows show examples of the loss in dystrophin staining; Ila and IIx indicate examples of MHC-II positive fibers; I, MHC-I fibers.

In line with the qualitative assessments of EM images, qualitative assessment of sarcolemmal damage, as assessed by loss of dystrophin continuity in membranes (Figure 1C) in the red channel of the double-stained sections, revealed breaks in dystrophin in both groups. Fiber type distribution did not differ between low and high responders (Table 2). Quantitative data of sarcolemmal damage for specific fiber types generated by direct comparisons of the red and green channels (Figures 1C,D), revealed no differences between high and low responders in terms of the proportion of each fiber type exhibiting sarcolemmal damage (Table 2).
In terms of indirect indicators of muscle damage, serum CK activity was significantly elevated from baseline and pre-exercise values at both $6 \mathrm{~h}(P<0.00001)$ and day $1(P<0.00001)$ after plyometric exercise intervention in the low responders, after which it returned toward baseline. In the high responders, a greater magnitude of increase (ANOVA effect of group, $P<0.0001)$ was observed for CK activity in response to the exercise intervention. In this group, $\mathrm{CK}$ activity remained at significantly higher levels when compared to pre-exercise values for all time points assessed (Figure 2A). A similar pattern to CK was observed for serum $\mathrm{Mb}$, with greater and more sustained response in the high responders (Figure $\mathbf{2 B}$ ). The only exception was that for $\mathrm{Mb}$, the values in low responders returned to baseline levels already at day 1 .

\section{Inflammation}

Total WBC and neutrophil counts for both high and low responders were significantly but transiently increased $6 \mathrm{~h}$ after exercise, following which it returned to baseline values (Table 2). Both WBC and neutrophil counts peaked at significantly higher levels in high responders than low responders at $6 \mathrm{~h}(P<0.5)$.

Serum CRP levels increased $(P<0.05)$ transiently on day 1 after exercise in the low responders (Figure 3A). High responders had a more robust response, with significantly higher CRP levels, which were sustained at levels significantly higher than preexercise levels for all post-exercise time points. A significant group difference was evident from the $6 \mathrm{~h}$ time point to day 2 . Pre-exercise intramuscular MPO levels were significantly higher $(P<0.05)$ in high responders when compared to low responders at baseline (Figure 3B). Neither group showed a significant MPO response to plyometric exercise at 3 days after plyometric exercise, which was the only time point after the intervention that a biopsy was taken.

(A) Serum C-reactive-protein (CRP) and (B) Myeloperoxidase (MPO) levels in skeletal muscle before and on day 3 after exercise intervention. The triangle and circle dot in the lines indicate high $(n=10)$ and low $(n=16)$ responders, respectively.

\section{Profile of MLCK Single Nucleotide Polymorphisms}

All three alleles for MLCK C49T were observed among our participants: Twelve participants presented with the homozygous wild type CC allele, 6 with the CT allele and 7 with the TT allele. Participants with the CC allele had circulating $\mathrm{CK}$ and $\mathrm{Mb}$ activities of $7806 \pm 5827 \mathrm{U} / \mathrm{L}$ and $299 \pm 132 \mathrm{ng} / \mathrm{ml}$, respectively, 3 days after plyometric jumping, versus somewhat lower values for those with the heterozygote allele (CT: $4485 \pm 2912 \mathrm{U} / \mathrm{L}$; $\mathrm{Mb} 227 \pm 58 \mathrm{ng} / \mathrm{ml}$ ) or the mutant homozygous allele (TT: CK $2442 \pm 1779 \mathrm{U} / \mathrm{L} ; \mathrm{Mb} 146 \pm 37 \mathrm{ng} / \mathrm{ml})$.

\section{DISCUSSION}

To date, it remains unclear why, within a group of individuals exercising in a similar environment and at similar intensity, the development of exertional rhabdomyolysis is only observed 
TABLE 2 | Percentage fiber type and the proportion within each fiber type category that were damaged fibers (loss of dystrophin continuity) comparing low responders and high responders.

\begin{tabular}{|c|c|c|c|c|c|c|c|c|}
\hline \multirow[b]{2}{*}{ Responders } & \multicolumn{2}{|c|}{ Type I muscle fiber } & \multicolumn{2}{|c|}{ Type II muscle fiber } & \multicolumn{2}{|c|}{ Type Ila muscle fiber } & \multicolumn{2}{|c|}{ Type IIx muscle fiber } \\
\hline & $(\%)$ & Damaged (\%) & $(\%)$ & Damaged (\%) & $(\%)$ & Damaged (\%) & $(\%)$ & Damaged (\%) \\
\hline Low & $35.2 \pm 10.5$ & $9.9 \pm 8.3$ & $64.8 \pm 10.5$ & $12.9 \pm 5.7$ & $59.8 \pm 16.6$ & $12.2 \pm 5.4$ & $8.8 \pm 7.2$ & $22.5 \pm 18.2$ \\
\hline High & $37.6 \pm 9.5$ & $10.4 \pm 6.1$ & $62.4 \pm 9.5$ & $13.2 \pm 7$ & $52.2 \pm 12.4$ & $13.6 \pm 8.2$ & $10.2 \pm 10$ & $16.2 \pm 10.7$ \\
\hline
\end{tabular}

Data are presented as mean $\pm S D$. No significant differences were observed between high and low responders in the percentage of muscle fiber type and in percentage of damaged muscle fibers at day 3 after the plyometric exercise intervention. The muscle biopsy was performed in the vastus lateralis.

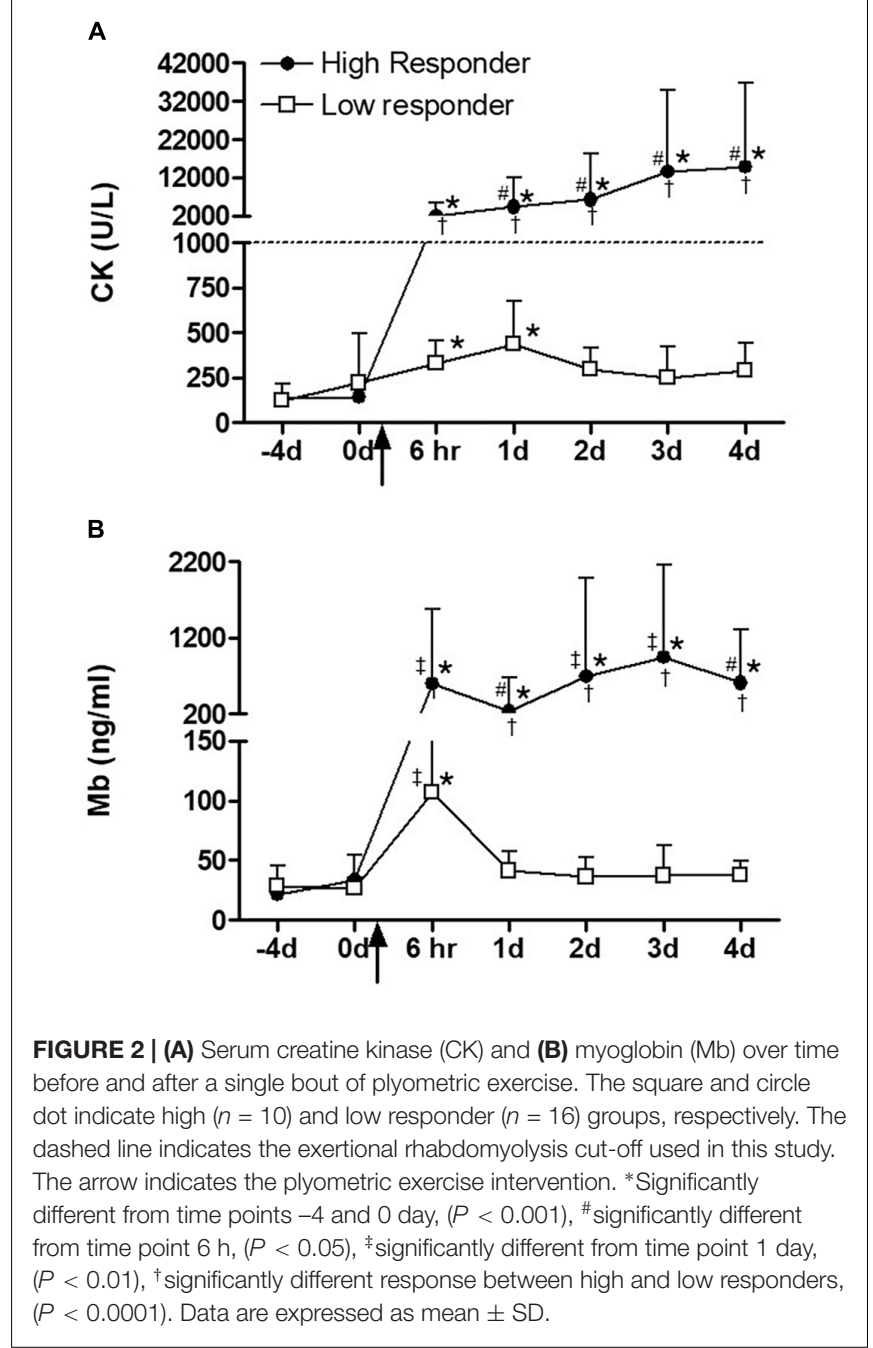

within a small percentage of these individuals (Clarkson et al., 2005; Devaney et al., 2007; Yamin et al., 2008). The strength of the current study is that the unexplained dilemma of low and high responders to eccentric exercise was assessed from multiple points of view with the same volunteers within the same study. These viewpoints ranged from perceived pain, which was not similar at all time points between the two groups, to blood biomarkers of damage and inflammation, to genetic polymorphism testing (variants of MLCK gene) and muscular

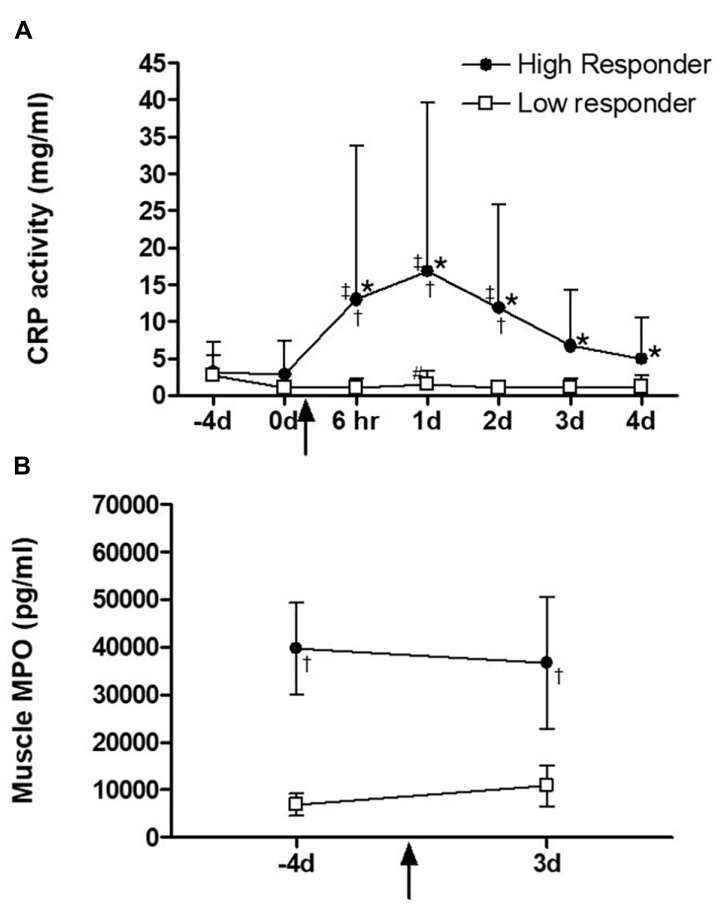

FIGURE 3 | (A) Serum C-reactive-protein (CRP) and (B) skeletal muscle myeloperoxidase (MPO) levels before and on day 3 after exercise intervention. The square and circle dot in the lines indicate high $(n=10)$ and low $(n=16)$ responders, respectively. *Significantly different from time points -4 and 0 day, $(P<0.001)$, " significantly different from time point $6 \mathrm{~h},(P<0.05)$, ${ }^{*}$ significantly different from time point 1 day, $(P<0.01),{ }^{\dagger}$ significantly different response between high and low responders, $(P<0.0001)$. Data are expressed as mean $\pm \mathrm{SD}$.

parameters. The results provide evidence suggesting that preexisting elevated skeletal muscle MPO pre-disposes to higher $\mathrm{CK}$ responsiveness following unaccustomed plyometric exercise and that the inflammatory response, assessed here by subsequent CRP elevations in the days after muscle damaging exercise, is exacerbated in high $\mathrm{CK}$ responders. While other factors, such as muscle and genetic characteristics, are suggested to be contributing factors to muscle damage, these are not clearly evident unless a larger number of individuals are assessed and are therefore unlikely to be predictive in the individual setting.

In the literature, reports have found a poor correlation between perceived pain reporting and the degree of change in other indirect markers of muscle damage. For example, pain 
was found to be elevated 1 day post-marathon and while pain resolved, CK levels remained elevated 2 days after (Clifford et al., 2017). Furthermore, CK activity does not correlate well with the amount of structural damage or the reduction of muscle function and thus is an unreliable marker (Sorichter et al., 1997; Chimera et al., 2004). Nosaka et al. (2002) who used an eccentric elbow flexor model of muscle damage, reported that delayed onset muscle soreness (DOMS) correlated poorly not only with eccentric exercise-induced muscle damage and loss of maximal isometric force, but also smaller elbow joint angles, larger upper arm circumference and plasma CK activity. In the current study, despite all participants completing the required number of jumps between 95 and 100\% of maximal jump height, the low $\mathrm{CK}$ responders reported significantly more perceived pain at the early time point of $6 \mathrm{~h}$ after plyometric jumping than the high responder group. The high responder group reported significantly higher delayed perceived soreness scores compared to the low responder group on days 3 and 4 following the exercise intervention, in particular when soreness was assessed in the squat position. This suggests that soreness should be assessed when the affected muscle groups are under eccentric strain, even if this is static. Coaches and athletic trainers should be aware that delayed soreness scores should be assessed for at least 4 days or more following intensive plyometric training in order to distinguish individuals possibly at risk of exertional rhabdomyolysis.

Muscle ultrastructural (Z-disk streaming) and structural damage (loss of dystrophin stain) was evident using electron and fluorescent microscopy, respectively, 3 days after the plyometric intervention. Nosaka et al. (2001) have reported that the repeated bout effect may last up to 6 months following an initial heavy eccentric exercise bout. However, some volunteers with a moderate to extreme response to unaccustomed eccentric exercise, who performed a second bout of eccentric exercise 3 weeks after the first bout, exhibited deformed fibers after the second bout (Paulsen et al., 2013). Given the qualitative evidence of muscle damage after the acute eccentric exercise intervention in all participants in the current study, it is unlikely that any of the participants did not fulfill the inclusion criterion of no habitual or recent training or plyometric exercise of the lower body. In previous studies, we observed acute bouts of plyometric jumping resulted in preferential fast twitch fiber damage (Macaluso et al., 2012b, 2014). McEwen and Hulland (1986) also observed preferential degeneration, but not exclusively to type II fibers, in horses affected by exertional rhabdomyolysis. Together, these findings could suggest that individuals with a greater percentage of fast twitch fibers may present more fiber damage, resulting in higher CK levels. However, no significant difference was observed between high and low responders in the proportions of fast and slow muscle fibers in the current study. The fiber type distribution was variable in both groups, which may explain this finding. A limitation is that with the size of the current study, there were no subjects with a very high proportion of fast twitch fibers. Therefore, it cannot be excluded that an even higher proportion of fast twitch fibers than that observed in the participants of the current study may be predictive of exertional rhabdomyolysis risk in other individuals.
Exertional rhabdomyolysis is common amongst military recruits (Hill et al., 2017), especially in the early phases after entry into service and if unaccustomed, excessive training is undertaken. The volume of such training is typically higher than that in laboratory research studies and the cut-off point for rhabdomyolysis may be as high as 5 times the upper limit of the normal, typically in the range of $1300 \mathrm{U} / \mathrm{L}$ (Warren et al., 2002). However, since signs such as dark colored urine are part of the diagnostic criteria for definitive clinical diagnosis of rhabdomyolysis (Hill et al., 2017), it has been found that exertional rhabdomyolysis may be underreported in some settings (Sauers et al., 2016). Therefore, in the current study a slightly lower cut-off limit of $1000 \mathrm{U} / \mathrm{L}$ was used, which is similar to other laboratory studies (Lee and Clarkson, 2003; Thoenes, 2010).

Recent reports suggest that SNPs may be responsible for inter-subject damage variability and CK response after eccentric exercise (Scalco et al., 2016). These studies have already established the genotypes of the different SNPs using larger study populations. Clarkson et al. (2005) demonstrated that individuals homozygous for the rare MLCK $49 \mathrm{~T}$ allele (4\% of 152 participants) had significantly greater increases in CK and $\mathrm{Mb}$ compared to the heterozygotes. The current data was not in line with this previous publication. Five of the seven individuals with this genotype were low responders for CK release and all of them were low responders for $\mathrm{Mb}$. A major difference between the current cohort and that of Clarkson et al. (2005) is that $28 \%$ of the small cohort actually presented with the TThomozygous allele. Therefore, this allele cannot be considered rare in our population. Although it might be considered a limitation for genotype assessment that the number of subjects in the current study was relatively low, taking the previous results and the current results together, they indicate the necessity of conducting genotype-phenotype studies in multiple populations around the world.

The two most interesting results of this study were the differences between the groups in the CRP profile and the baseline intramuscular MPO activity. High responders presented with significantly higher CRP levels following eccentric exercise when compared to their low responder counterparts, despite similar levels at baseline. Indeed, CRP was higher in high CK responders already at $6 \mathrm{~h}$ post-exercise and continued to be higher on days 1 and 2 when compared to the low responders, indicating prolonged cross-talk between damaged muscle and the liver. No group differences were evident at days 3 and 4 , although high responders were still elevated from their own baseline at these two later time points. Although circulating neutrophil levels were significantly greater in the high responder group at $6 \mathrm{~h}$ postexercise compared to the low responder group post-exercise, they did not remain elevated in the days following the intervention. Within $2 \mathrm{~h}$ after mechanical damage, neutrophils accumulate in the injured area (Hertel, 1997) and phagocytose cellular debris via the production of oxygen free radicals (Hertel, 1997; Toumi and Best, 2003; Pizza et al., 2005). Neutrophil activation may cause damage to cell membranes and surrounding non-injured tissue by rapidly releasing high concentrations of ROS and oxygen free radicals through a respiratory burst (Hertel, 1997; 
Brickson et al., 2001). In the context of unaccustomed eccentric exercise, this would be considered as secondary damage. By using a single stretch injury model in rabbit tibialis anterior muscle, Brickson et al. (2003) demonstrated that muscle fiber damage could be reduced by inhibiting neutrophil respiratory burst with a monoclonal antibody (M1/70). Furthermore, this model also showed the preservation of muscle structural proteins desmin and dystrophin (Brickson et al., 2003).

Myeloperoxidase is primarily expressed by neutrophils and plays an important role in microbicidal activity and cell debris destruction within the phagosome via oxidizing reactions (Hurst, 2012). Circulating monocytes also elevate intracellular MPO in response to eccentrically biased exercise such as downhill running (van de Vyver et al., 2016). Previous reports indicate that muscle loading during exhaustive exercise results in increased MPO activity per neutrophil (Suzuki et al., 1996) and downhill running increases intramuscular MPO (van de Vyver and Myburgh, 2014). Notably, the current data demonstrated higher MPO at baseline within the tissue targeted by the exercise in the high responders even before the plyometric intervention, which remained high at $3 \mathrm{~d}$ after. This suggests that an inability to resolve MPO in muscle may contribute to a relatively exacerbated CRP response independent of the magnitude of the acute neutrophil response. It is unclear if the intramuscular MPO observed was of neutrophilic or monocytic origin. Given that there was no evidence of overt muscle pathology in the baseline biopsy samples, it is possible that pro-inflammatory macrophages may have been basally active in the high responder group's muscle. However, MPO has also been shown to delay neutrophil apoptosis in an ex vivo model and to delay resolution of acute lung injury in mice (El Kebir et al., 2008). Neutrophil quantification in the muscle samples for future studies will be important. Not only in muscle fibers, but in the interstitial spaces between fibers; in the perivascular areas and beneath the epimysium in order to explain the difference in baseline MPO levels between high and low responders (Malm et al., 2004). In the current age

\section{REFERENCES}

Baumert, P., Lake, M. J., Stewart, C. E., Drust, B., and Erskine, R. M. (2016). Genetic variation and exercise-induced muscle damage: implications for athletic performance, injury and ageing. Eur. J. Appl. Physiol. 116, 1595-1625. doi: 10.1007/s00421-016-3411-1

Brickson, S., Hollander, J., Corr, D. T., Ji, L. L., and Best, T. M. (2001). Oxidant production and immune response after stretch injury in skeletal muscle. Med. Sci. Sports Exerc. 33, 2010-2015. doi: 10.1097/00005768-200112000-00006

Brickson, S., Ji, L. L., Schell, K., Olabisi, R., St Pierre Schneider, B., and Best, T. M. (2003). M1/70 attenuates blood-borne neutrophil oxidants, activation, and myofiber damage following stretch injury. J. Appl. Physiol. 95, 969-976. doi: 10.1152/japplphysiol.00005.2003

Childs, A., Jacobs, C., Kaminski, T., Halliwell, B., and Leeuwenburgh, C. (2001). Supplementation with vitamin $\mathrm{C}$ and $\mathrm{N}$-acetyl-cysteine increases oxidative stress in humans after an acute muscle injury induced by eccentric exercise. Free Radic. Biol. Med. 31, 745-753. doi: 10.1016/S0891-5849(01)00640-2

Chimera, N. J., Swanik, K. A., Swanik, C. B., and Straub, S. J. (2004). Effects of plyometric training on muscle-activation strategies and performance in female athletes. J. Athl. Train. 39, 24-31. of exercise prescription for rehabilitation and in groups with underlying disease, knowledge of baseline intramuscular MPO status may be valuable for decisions regarding the type of training prescribed. Merritt et al. (2013) showed that aged populations are particularly susceptible to muscle inflammation. This study also provided evidence that the skeletal muscle regenerative response is impaired with age following unaccustomed eccentric exercise, due to either high pro-inflammatory signaling at rest or a greater inflammatory response to damage and subsequent myoblast impairment.

In conclusion, in relatively healthy individuals, CRP responses to training are recommended for monitoring purposes and may indicate that a more conservative approach to training prescription should be followed where exercise interventions may be used as therapy for an individual's recovery and in aged populations. In aged individuals and other individuals with conditions that may already put them at risk for compromised intramuscular inflammation, response to eccentric contraction and perceived pain should be assessed under static eccentric conditions.

\section{AUTHOR CONTRIBUTIONS}

$\mathrm{AI}, \mathrm{FM}$, and KM performed the experiments. AI, FM, and CS conceived the project and analyzed the data. All authors contributed to critical analysis. AI, FM, CS, and KM wrote the manuscript and all authors approved the final manuscript for publication.

\section{FUNDING}

This research was supported by the Medical Research Council of South Africa and the National Research Foundation (NRF) to KM. FM was supported by a Claude Leon Trust Postdoctoral Fellowship and AI by scholarship awarded by the NRF.

Clarkson, P. M., Hoffman, E. P., Zambraski, E., Gordish-Dressman, H., Kearns, A. Hubal, M., et al. (2005). ACTN3 and MLCK genotype associations with exertional muscle damage. J. Appl. Physiol. 99, 564-569. doi: 10.1152/ japplphysiol.00130.2005

Clifford, T., Allerton, D. M., Brown, M. A., Harper, L., Horsburgh, S., Keane, K. M., et al. (2017). Minimal muscle damage after a marathon and no influence of beetroot juice on inflammation and recovery. Appl. Physiol. Nutr. Metab. 42, 263-270. doi: 10.1139/apnm-2016-0525

Damas, F., Nosaka, K., Libardi, C. A., Chen, T. C., and Ugrinowitsch, C. (2016). Susceptibility to exercise-induced muscle damage: a cluster analysis with a large sample. Int. J. Sports Med. 37, 633-640. doi: 10.1055/s-0042100281

Devaney, J. M., Hoffman, E. P., Gordish-Dressman, H., Kearns, A., Zambraski, E., and Clarkson, P. M. (2007). IGF-II gene region polymorphisms related to exertional muscle damage. J. Appl. Physiol. 102, 1815-1823. doi: 10.1152/ japplphysiol.01165.2006

El Kebir, D., József, L., Pan, W., and Filep, J. G. (2008). Myeloperoxidase delays neutrophil apoptosis through CD11b/CD18 integrins and prolongs inflammation. Circ. Res. 103, 352-359. doi: 10.1161/01.RES.0000326772. $76822.7 \mathrm{a}$ 
Hertel, J. (1997). The role of nonsteroidal anti-inflammatory drugs in the treatment of acute soft tissue injuries. J. Athl. Train 32, 350-358.

Hill, O. T., Scofield, D. E., Usedom, J., Bulathsinhala, L., McKinnon, C., Kwon, P., et al. (2017). Risk factors for rhabdomyolysis in the U.S. Army Mil. Med. 182, e1836-e1841. doi: 10.7205/MILMED-D-16-00076

Hurst, J. K. (2012). What really happens in the neutrophil phagosome? Free Radic. Biol. Med. 53, 508-520. doi: 10.1016/j.freeradbiomed.2012.05.008

Kay, A. D., and Blazevich, A. J. (2012). Effect of acute static stretch on maximal muscle performance: a systematic review. Med. Sci. Sports Exerc. 44, 154-164. doi: 10.1249/MSS.0b013e318225cb27

Lauritzen, F., Paulsen, G., Raastad, T., Bergersen, L. H., and Owe, S. G. (2009). Gross ultrastructural changes and necrotic fiber segments in elbow flexor muscles after maximal voluntary eccentric action in humans. J. Appl. Physiol. 107, 1923-1934. doi: 10.1152/japplphysiol.00148.2009

Lee, J., and Clarkson, P. M. (2003). Plasma creatine kinase activity and glutathione after eccentric exercise. Med. Sci. Sports Exerc. 35, 930-936. doi: 10.1249/01. MSS.0000069553.47739.36

Macaluso, F., Brooks, N. E., Niesler, C. U., and Myburgh, K. H. (2013). Satellite cell pool expansion is affected by skeletal muscle characteristics. Muscle Nerve 48, 109-116. doi: 10.1002/mus.23721

Macaluso, F., Brooks, N. E., van de Vyver, M., Van Tubbergh, K., Niesler, C. U., and Myburgh, K. H. (2012a). Satellite cell count, VO(2max), and p38 MAPK in inactive to moderately active young men. Scand. J. Med. Sci. Sports 22, e38-e44. doi: $10.1111 / j .1600-0838.2011 .01389 . x$

Macaluso, F., Isaacs, A. W., and Myburgh, K. H. (2012b). Preferential type II muscle fiber damage from plyometric exercise. J. Athl. Train 47, 414-420. doi: 10.4085/1062-6050-47.4.13

Macaluso, F., Isaacs, A. W., Di Felice, V., and Myburgh, K. H. (2014). Acute change of titin at mid-sarcomere remains despite $8 \mathrm{wk}$ of plyometric training. J. Appl. Physiol. 116, 1512-1519. doi: 10.1152/japplphysiol.00420.2013

Malm, C., Sjödin, T. L., Sjöberg, B., Lenkei, R., Renström, P., Lundberg, I. E., et al. (2004). Leukocytes, cytokines, growth factors and hormones in human skeletal muscle and blood after uphill or downhill running. J. Physiol. 556( $\mathrm{Pt} 3$ ), 983-1000. doi: 10.1113/jphysiol.2003.056598

Margaritelis, N. V., Kyparos, A., Paschalis, V., Theodorou, A. A., Panayiotou, G., Zafeiridis, A., et al. (2014). Reductive stress after exercise: the issue of redox individuality. Redox Biol. 2, 520-528. doi: 10.1016/j.redox.2014.02.003

McEwen, S. A., and Hulland, T. J. (1986). Histochemical and morphometric evaluation of skeletal muscle from horses with exertional rhabdomyolysis (tying-up). Vet. Pathol. 23, 400-410. doi: 10.1177/030098588602300409

Merritt, E. K., Stec, M. J., Thalacker-Mercer, A., Windham, S. T., Cross, J. M., Shelley, D. P., et al. (2013). Heightened muscle inflammation susceptibility may impair regenerative capacity in aging humans. J. Appl. Physiol. 115, 937-948. doi: 10.1152/japplphysiol.00019.2013

Nosaka, K., Newton, M., and Sacco, P. (2002). Delayed-onset muscle soreness does not reflect the magnitude of eccentric exercise-induced muscle damage. Scand. J. Med. Sci. Sports 12, 337-346. doi: 10.1034/j.1600-0838.2002.10178.x

Nosaka, K., Sakamoto, K., Newton, M., and Sacco, P. (2001). How long does the protective effect on eccentric exercise-induced muscle damage last? Med. Sci. Sports Exerc. 33, 1490-1495. doi: 10.1097/00005768-200109000-00011

Paulsen, G., Egner, I., Raastad, T., Reinholt, F., Owe, S., Lauritzen, F., et al. (2013). Inflammatory markers CD11b, CD16, CD66b, CD68, myeloperoxidase and neutrophil elastase in eccentric exercised human skeletal muscles. Histochem. Cell Biol. 139, 691-715. doi: 10.1007/s00418-012-1061-x

Pereira Panza, V. S., Diefenthaeler, F., and da Silva, E. L. (2015). Benefits of dietary phytochemical supplementation on eccentric exercise-induced muscle damage: is including antioxidants enough? Nutrition 31, 1072-1082. doi: 10.1016/j.nut. 2015.02.014

Phillips, T., Childs, A. C., Dreon, D. M., Phinney, S., and Leeuwenburgh, C. (2003). A dietary supplement attenuates IL-6 and CRP after eccentric exercise in untrained males. Med. Sci. Sports Exerc. 35, 2032-2037. doi: 10.1249/01.MSS. 0000099112.32342 .10

Pizza, F. X., Peterson, J. M., Baas, J. H., and Koh, T. J. (2005). Neutrophils contribute to muscle injury and impair its resolution after lengthening contractions in mice. J. Physiol. 562(Pt 3), 899-913. doi: 10.1113/jphysiol.2004. 073965
Sauers, S. E., Smith, L. B., Scofield, D. E., Cooper, A., and Warr, B. J. (2016). Selfmanagement of unreported musculoskeletal injuries in a U.S. Army brigade. Mil. Med. 181, 1075-1080. doi: 10.7205/MILMED-D-15-00233

Sayers, S. P., and Clarkson, P. M. (2002). Exercise-induced rhabdomyolysis. Curr. Sports Med. Rep. 1, 59-60. doi: 10.1249/00149619-20020400000001

Scalco, R. S., Snoeck, M., Quinlivan, R., Treves, S., Laforet, P., Jungbluth, H., et al. (2016). Exertional rhabdomyolysis: physiological response or manifestation of an underlying myopathy? BMJ Open Sport Exerc. Med. 2:e000151. doi: 10.1136/ bmjsem-2016-000151

Schneider, B. S., and Tiidus, P. M. (2007). Neutrophil infiltration in exerciseinjured skeletal muscle: how do we resolve the controversy? Sports Med. 37, 837-856. doi: 10.2165/00007256-200737100-00002

Smith, C., Kruger, M. J., Smith, R. M., and Myburgh, K. H. (2008). The inflammatory response to skeletal muscle injury: illuminating complexities. Sports Med. 38, 947-969. doi: 10.2165/00007256-20083811000005

Sorichter, S., Mair, J., Koller, A., Secnik, P., Parrak, V., Haid, C., et al. (1997). Muscular adaptation and strength during the early phase of eccentric training: influence of the training frequency. Med. Sci. Sports Exerc. 29, 1646-1652.

Suzuki, K., Sato, H., Kikuchi, T., Abe, T., Nakaji, S., Sugawara, K., et al. (1996). Capacity of circulating neutrophils to produce reactive oxygen species after exhaustive exercise. J. Appl. Physiol. 81, 1213-1222. doi: 10.1152/jappl.1996.81. 3.1213

Thoenes, M. (2010). Rhabdomyolysis: when exercising becomes a risk. J. Pediatr. Health Care 24, 189-193. doi: 10.1016/j.pedhc.2009.08.009

Toumi, H., and Best, T. M. (2003). The inflammatory response: friend or enemy for muscle injury? Br. J. Sports Med. 37, 284-286. doi: 10.1136/bjsm.37. 4.284

van de Vyver, M., Engelbrecht, L., Smith, C., and Myburgh, K. H. (2016). Neutrophil and monocyte responses to downhill running: intracellular contents of MPO, IL-6, IL-10, pstat3, and SOCS3. Scand. J. Med. Sci. Sports 26, 638-647. doi: $10.1111 / \mathrm{sms} .12497$

van de Vyver, M., and Myburgh, K. H. (2012). Cytokine and satellite cell responses to muscle damage: interpretation and possible confounding factors in human studies. J. Muscle Res. Cell Motil. 33, 177-185. doi: 10.1007/s10974-0129303-z

van de Vyver, M., and Myburgh, K. H. (2014). Variable inflammation and intramuscular STAT3 phosphorylation and myeloperoxidase levels after downhill running. Scand. J. Med. Sci. Sports 24, e360-e371. doi: 10.1111/sms. 12164

Vissing, K., Brink, M., Lonbro, S., Sorensen, H., Overgaard, K., Danborg, K., et al. (2008). Muscle adaptations to plyometric vs. resistance training in untrained young men. J. Strength Cond. Res. 22, 1799-1810. doi: 10.1519/JSC. ob013e318185f673

Vollaard, N. B., Shearman, J. P., and Cooper, C. E. (2005). Exercise-induced oxidative stress:myths, realities and physiological relevance. Sports Med. 35, 1045-1062. doi: 10.2165/00007256-200535120-00004

Warren, J. D., Blumbergs, P. C., and Thompson, P. D. (2002). Rhabdomyolysis: a review. Muscle Nerve 25, 332-347. doi: 10.1002/mus.10053

Yamin, C., Duarte, J. A., Oliveira, J. M., Amir, O., Sagiv, M., Eynon, N., et al. (2008). IL6 (-174) and TNFA (-308) promoter polymorphisms are associated with systemic creatine kinase response to eccentric exercise. Eur. J. Appl. Physiol. 104, 579-586. doi: 10.1007/s00421-008-0728-4

Conflict of Interest Statement: The authors declare that the research was conducted in the absence of any commercial or financial relationships that could be construed as a potential conflict of interest.

Copyright (C) 2019 Isaacs, Macaluso, Smith and Myburgh. This is an open-access article distributed under the terms of the Creative Commons Attribution License (CC BY). The use, distribution or reproduction in other forums is permitted, provided the original author(s) and the copyright owner(s) are credited and that the original publication in this journal is cited, in accordance with accepted academic practice. No use, distribution or reproduction is permitted which does not comply with these terms. 


\section{OPEN ACCESS}

Edited by:

Dario Coletti,

Sapienza University of Rome, Italy

Reviewed by:

Davide Cervia,

Università degli Studi della Tuscia, Italy Ashok Kumar,

University of Louisville, United States

${ }^{*}$ Correspondence:

Paola Costelli

paola.costelli@unito.it

Specialty section:

This article was submitted to

Striated Muscle Physiology,

a section of the journal

Frontiers in Physiology

Received: 05 November 2018

Accepted: 14 January 2019

Published: 18 February 2019

Citation:

Penna F, Ballarò R, Beltrà M,

De Lucia S, García Castillo L and Costelli P (2019) The Skeletal Muscle

as an Active Player Against Cancer

Cachexia. Front. Physiol. 10:41

doi: 10.3389/fphys.2019.00041

\section{The Skeletal Muscle as an Active Player Against Cancer Cachexia}

\author{
Fabio Penna, Riccardo Ballarò, Marc Beltrà, Serena De Lucia, Lorena García Castillo \\ and Paola Costelli*
}

Department of Clinical and Biological Sciences, Interuniversity Institute of Myology, University of Turin, Turin, Italy

The management of cancer patients is frequently complicated by the occurrence of cachexia. This is a complex syndrome that markedly impacts on quality of life as well as on tolerance and response to anticancer treatments. Loss of body weight, wasting of both adipose tissue and skeletal muscle and reduced survival rates are among the main features of cachexia. Skeletal muscle wasting has been shown to depend, mainly at least, on the induction of protein degradation rates above physiological levels. Such hypercatabolic pattern is driven by overactivation of different intracellular proteolytic systems, among which those dependent on ubiquitin-proteasome and autophagy. Selective rather than bulk degradation of altered proteins and organelles was also proposed to occur. Within the picture described above, the muscle is frequently considered a sort of by-stander tissue where external stimuli, directly or indirectly, can poise protein metabolism toward a catabolic setting. By contrast, several observations suggest that the muscle reacts to the wasting drive imposed by cancer growth by activating different compensatory strategies that include anabolic capacity, the activation of autophagy and myogenesis. Even if muscle response is eventually ill-fated, its occurrence supports the idea that in the presence of appropriate treatments the development of cancer-induced wasting might not be an ineluctable event in tumor hosts.

Keywords: muscle wasting, protein turnover, energy metabolism, myogenesis, adaptive response, oxidative stress

\section{INTRODUCTION}

Cachexia is a complex multiorgan syndrome that affects $50-80 \%$ of cancer patients and accounts for about $20 \%$ of cancer deaths (Argilés et al., 2014). Characteristic features of this syndrome are body weight loss, muscle wasting, adipose tissue depletion and metabolic abnormalities. The main symptoms include anorexia, anemia, asthenia and fatigue, that eventually result in severely impaired patient quality of life.

The pathogenesis of cancer cachexia is a complex phenomenon that includes nutritional changes, hypoanabolism, the onset of an overall hypercatabolic response that mainly affects proteins and lipids, chronic inflammation and altered energy metabolism. Last, but not least, the standard of care of neoplastic disease generally includes chemotherapy, further complicating the scenario. Indeed, cachectic patients very frequently cannot cope with anti-cancer treatment schedule and often require dosage limitation and/or therapy interruption, reducing both drug effectiveness and patient survival (Pin et al., 2018).

Several potential anti-cachexia drugs are currently being tested in clinical trials, but none of them has proved effective enough to be routinely applied in the clinical practice (Morley et al., 2014). In this regard, the small number of clinical trials and the poor knowledge about the pathogenesis of cancer cachexia likely account for the lack of effective treatments (Penna et al., 2016b). 
The present review will discuss the mechanisms underlying cancer-induced wasting and will focus on the adaptive response set up by the skeletal muscle to the challenge posed by alterations at the systemic level. In this regard, most of the existent studies and reviews consider the skeletal muscle as a passive target of stimuli that eventually lead to reduced anabolism and/or increased catabolism. By contrast, muscle tissue attempts to strike back by modulating metabolism in order to set up a sort of defensive strategy against a hostile external environment. This is particularly relevant since understanding and potentiating such reactive behavior could improve the effectiveness of anticachexia treatments.

\section{MALNUTRITION}

Cancer-associated malnutrition reflects into involuntary body weight loss that results from the combination of several factors such as reduced food intake, malabsorption, altered substrate utilization and increased substrate demand. The occurrence of malnutrition is highly unfavorable, since both recovery and tolerance to antineoplastic treatments are definitely improved in well-nourished than in malnourished subjects (e.g., with unintentional body weight loss $>5 \%$ ). Being malnutrition a potent predictor of bad outcome, the achievement of a good nutritional state in cancer patients should become a goal to be pursued in the clinical practice (Aversa et al., 2017). In this regard, helpful guidelines for the management of malnutrition in cancer patients have been set up by both the European Society of Parenteral and Enteral Nutrition (ESPEN; Arends et al., 2017a) and the Academy of Nutrition and Dietetics (AND; Thompson et al., 2017). These documents include the need of an early nutritional assessment, the relevance of nutritional counseling during the course of the disease, the definition of an appropriate and personalized nutritional support, the identification of the right moment for the adoption of such support.

Nutritional habits in cancer patients can be affected very early, resulting in latent malnutrition that becomes frankly evident while the disease progresses, accompanying the appearance of cachexia. Multiple mechanisms contribute to malnutrition, such as tumor localization at the gastrointestinal tract, pain, anxiety, malabsorption, and anorexia. While this latter is very often recognized in cancer patients, in the early phases of disease it happens to be underestimated and for this reason it might not be promptly addressed. To circumvent this possibility, nutritional behavior should be accurately assessed in all cancer patients at first diagnosis (Arends et al., 2017b), in order to pick up and evaluate even small changes from usual habits.

Humoral factors produced by the host as well as by the tumor have been proposed to work in concert to modulate patient nutritional behavior, affecting, directly or indirectly, the central regulation of appetite. In this regard, several pro-inflammatory cytokines have been shown to contribute to anorexia in cancer, per se, but also enhancing the availability of neuropeptides acting at the central nervous system level such as melanocortin, neuropeptide Y, or leptin (Ezeoke and Morley, 2015). Particularly relevant in this regard is ghrelin, an orexigenic hormone mainly produced in the stomach, whose levels are usually increased in cancer patients. Such increase could result from both an attempt to counteract anorexia and the onset of ghrelin resistance (Argilés et al., 2017). The resulting picture is the induction of signaling pathways involved in causing anorexia at the expense of those able to stimulate food intake, eventually leading to loss of appetite and/or increased satiety (Argilés et al., 2014).

Despite reduced food intake is an important component of body weight loss, nutrient availability being lower than normal, cancer-associated malnutrition is quite different from that occurring in subjects exposed to fasting or to caloric restriction. Indeed, cancer patients very often are no more able to appropriately modulate their metabolism to meet the lack of nutrients. As an example, caloric restriction in healthy subjects results in increased glucose and lipid mobilization, sparing proteins as much as possible. By contrast, reduced food intake in cancer patients is associated with random mobilization of substrates, setting protein metabolism toward a persistently negative nitrogen balance.

\section{MUSCLE PROTEIN TURNOVER}

The loss of muscle mass and function, one of the main features of cancer cachexia, markedly impairs patient quality of life and survival. In addition, muscle mass depletion has been associated with reduced tolerance to anticancer treatments (Figure 1). In addition, recent observations show that immunotherapy by checkpoint inhibitors lose effectiveness in cachectic patients, likely due to the establishment of primary resistance (Coss et al., 2018). For these reasons, an accurate estimate of muscle mass and quality should be pursued during the management of cancer patients. By contrast, still nowadays the first evaluation of a patient mainly takes into account parameters such as body weight or body mass index (BMI), that do not provide any information about body composition. Indeed, normal body weight and/or BMI could result from increased adiposity or tissue water content, de facto masking the occurrence of muscle mass depletion.

Protein content, the most relevant component of muscle mass, depends on the balance between rates of protein synthesis and breakdown. Physiologically speaking, disruptions of such equilibrium activate an adaptive response aimed at reaching a new homeostasis that can alternatively result in muscle hypertrophy or hypotrophy, respectively depending on the prevalence of protein synthesis or degradation (Argilés et al., 2014).

\section{Protein Breakdown}

Intracellular protein degradation in the skeletal muscle relies on the activity of four main proteolytic pathways that depend on calpains, caspases, lysosomes, and proteasome. Results obtained in both experimental and clinical studies have clearly demonstrated that muscle wasting in cancer hosts is associated with supra-physiological activation of these proteolytic pathways (Penna et al., 2014), with particular reference to those depending on proteasome and lysosomes. These systems are involved in 


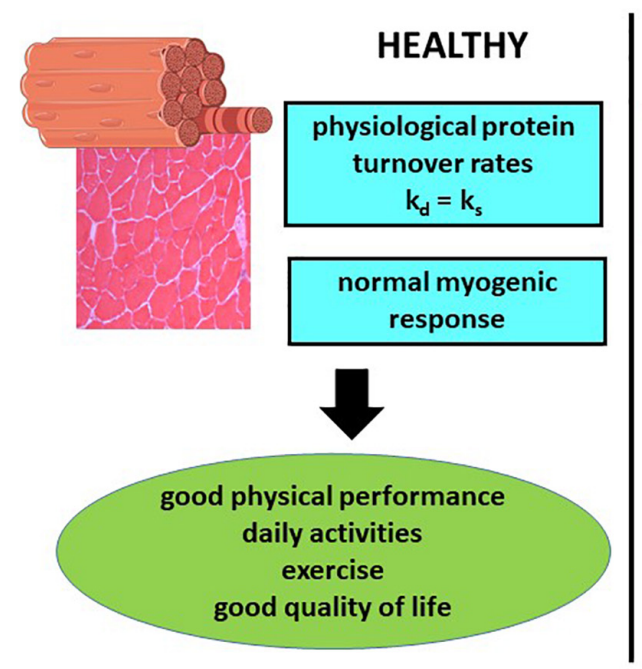

\section{CANCER}

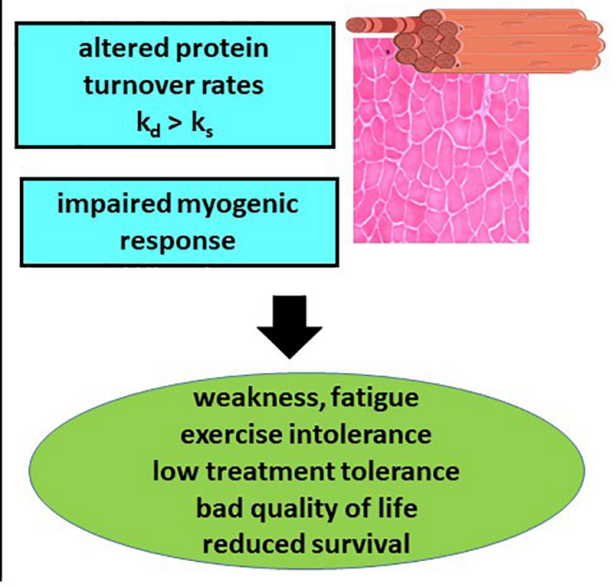

FIGURE 1 | Relevance of muscle wasting to cancer patient management. The occurrence of metabolic changes that result in muscle protein hypercatabolism and impaired regeneration capacity negatively impinges on both patient quality of life and survival. $\mathrm{k}_{\mathrm{s}}=$ fractional rate of protein synthesis; $\mathrm{k}_{\mathrm{d}}=$ fractional rate of protein degradation.

different aspects of intracellular protein degradation, the former breaking down short-lived and regulatory proteins, the latter being in charge of the disposal of altered organelles and structural proteins (Penna et al., 2014).

The activity of the proteasome-dependent proteolytic system depends on the availability of both ubiquitin and enzymes involved in protein substrate ubiquitylation, namely E1 (ubiquitin activating enzymes), E2 (ubiquitin conjugating enzymes) and E3 (ubiquitin ligases). As for the E3 family, some members are defined as muscle-specific. The most widely studied are MAFbx/atrogin-1 and MuRF1/TRIM63. The former is in charge of targeting proteins involved in cell cycle control, cell differentiation and cell death, while the latter mainly marks for degradation structural proteins (Argilés et al., 2014). The most recently discovered member of the muscle-specific E3 family is SMART (Milan et al., 2015). The expression levels of these muscle-specific ubiquitin ligases have been accepted as molecular markers of proteasome-dependent proteolysis and have been demonstrated to increase in different experimental models of cancer cachexia (Argilés et al., 2014). As for human studies, several reports show that in cancer patients this proteolytic system is activated above physiological levels. Of particular relevance, such enhanced activity has been observed also in non-weight losing gastric cancer patients (Bossola et al., 2003), recalling the need of early assessment of cachexia. On the other side, studies reporting unchanged levels of molecular and biochemical markers pertaining to the ubiquitin-proteasome proteolytic system in cancer patients do exist (Op den Kamp et al., 2012; Tardif et al., 2013).

The involvement of lysosomal proteolysis in muscle wasting is mainly referred to the overactivation of autophagy. This is a physiological process in charge of degrading cellular components, whose rate is increased by lack of nutrients or by the presence of damaged organelles, such as mitochondria or peroxisomes.
Some years ago the discovery of autophagy-related (ATG) genes has refreshed the field, providing useful tools to investigate the process. Indeed, at least some of the proteins encoded by these genes, such as beclin 1 and LC3B are now accepted markers of autophagy. The physiological protein homeostasis in the muscle is maintained by basal autophagy, in view of its role in the routine clearance of wasting products such as altered proteins and organelles. Disruption of autophagy has been shown to be associated with progressive muscle derangements, such as those occurring in mice lacking the Atg7 or the OPA1 genes (Masiero et al., 2009; Tezze et al., 2017) or carrying the BCL2 AAA mutation (He et al., 2012). On the other side, markers of autophagy are overexpressed in several muscle wastingassociated states such as denervation and fasting, suggesting that stress-induced autophagy is activated above physiological levels in these diseases. Consistently, the induction of autophagy in the skeletal muscle of both tumor-bearing animals and cancer patients is demonstrated by several reports (Penna et al., 2013; Tardif et al., 2013; Aversa et al., 2016; Pigna et al., 2016). However, despite autophagic flux is increased, the process does not reach its final step with complete cargo degradation, as indicated by the observation that autophagosomes accumulate in the muscle of cancer hosts, likely due to lysosomal engulfment (Penna et al., 2013; Aversa et al., 2016; Pigna et al., 2016). On the whole, these observations demonstrate that the relevance of autophagy, enhanced or inhibited, to muscle wasting can be significantly different, according to the specific situation. In other words, both excessive and defective autophagy are unwanted scenarios, and treatments impinging on these modality of protein degradation should be aimed at maintaining/restoring a physiologic autophagic flux.

Myofibrillar protein degradation depends on the preliminary disruption of myofilaments. Such preventive cleavage cannot be performed either by proteasome or lysosomes and has been 
proposed to involve other proteases, such as caspases and calpains (Penna et al., 2014). Consistently, these latter are upregulated and overactivated in the skeletal muscle of tumor-bearing animals (Llovera et al., 1995; Costelli et al., 2002; Pin et al., 2017) and of cancer patients (Smith et al., 2011).

Unfolded protein response (UPR) is important for the maintenance of skeletal muscle mass in adults. Because PERK regulates protein folding and calcium homeostasis, it is important to investigate how these functions of PERK are affected in skeletal muscle of smPERK-KO mice. How the activation of PERK is regulated in conditions of muscle growth and atrophy and whether modulation of its activity can improve skeletal muscle mass in various catabolic states is also an important area of future research.

\section{Protein Synthesis}

While the enhancement of muscle protein breakdown rates in cancer cachexia is clearly demonstrated, the same does not apply to protein synthesis, reduced, normal, or increased rates being reported (Argilés et al., 2014). As for experimental cachexia, the generally short time frame from tumor implantation and animal death frequently results in impaired anabolic capacity, at least in the skeletal muscle, although contrasting observations have been reported. As an example, results obtained in mice bearing the C26 or the LLC tumors show a reduction of muscle protein synthesis rates, that cannot be restored by treatments able to improve muscle mass (Toledo et al., 2016a; Nissinen et al., 2018). On the other side, protein synthesis rates close to those of controls have been observed in rats implanted with the Yoshida AH-130 hepatoma (Costelli et al., 1993). Further variability can be observed when tissues different from muscle are taken into consideration. As an example, enhanced pro-synthetic capacity has been measured in the liver of tumor-bearing animals, likely due to the increased demand of acute phase proteins. These observations put in evidence the limitations of the experimental models, where cachexia frequently develops markedly faster than in cancer patients.

The modulation of protein synthesis rates in cancer patients is less clear. Some old studies, but also a recent one, have reported that muscle protein synthesis is reduced in human pathology (Emery et al., 1984; Hanson et al., 2017). By contrast, another research group shows that pancreatic cancer patients are able to improve baseline protein synthesis rates, that result poised at values higher than those measured in healthy subjects (van Dijk et al., 2015). Between these opposites, several studies report muscle protein synthesis rates comparable to those of controls or of non-weight losing cancer patients (Engelen et al., 2016).

Stimulation of muscle protein synthesis has been proposed as a mean to improve muscle phenotype in cancer cachexia. Most of the attempts, mainly unsuccessful, have involved nutritional interventions or molecular tools aimed at improving the muscle anabolic capacity. Indeed, advanced cancer patients treated with both parenteral and enteral nutrition, including or not the supplementation with amino acids, do not show any improvement in muscle protein synthesis (Engelen et al., 2016). Similar results have been obtained in studies attempting to activate muscle protein anabolism by means of both drugs or genetic tools (Costelli et al., 2006; Penna et al., 2010a). Recent observations, however, show that there is the possibility to exploit a sort of anabolic window in cancer patients (see below).

\section{ENERGY METABOLISM}

The vast majority of cancer patients are hypermetabolic, presenting with resting energy expenditure (REE) higher than normal (Vazeille et al., 2017). The underlying tumor-driven mechanisms are still unclear, however released mediators and/or pro-inflammatory stimuli rather than tumor burden, that is usually quite low with respect to patient body weight (Purcell et al., 2016), are likely involved. A recent study describes the occurrence of hypermetabolism associated with body weight loss, inflammation, altered energy balance and low performance status in about $50 \%$ out of 390 cancer patients at first diagnosis, e.g., in the absence of any anticancer treatment (Vazeille et al., 2017). As stated above, metabolic alterations are an early feature in cancer patients. Indeed, the study by Vazeille et al. (2017) shows that about half of the hypermetabolic patients are non weight-losing and present with good performance status. Consistently, normal metabolism is rapidly restored in bladder cancer patients after surgery, while body weight recovery is markedly delayed (Tobert et al., 2017). These observations, coupled to those reported above on skeletal muscle protein depletion, stress the need of tools allowing an early detection of metabolic modulations in cancer hosts.

Additional mechanisms leading to increased REE in cancer patients may depend on alterations in thermogenesis. The tissues mainly in charge of this process are the brown adipose tissue (BAT) and the skeletal muscle. Both of them express high amounts of uncoupling proteins (UCPs), whose levels have been shown to further increase in tumor-bearing animals and cancer patients (Collins et al., 2002; Bing, 2011). In the last few years, white adipocytes have been proposed to convert into brown adipocyte-like (beige) cells. This process, known as 'browning,' relies on increased UCP1 expression, resulting in a shift of mitochondrial activity from ATP to heat production, increasing both lipolysis and energy expenditure (Argilés et al., 2014).

In addition to increased expenditure, also reduced energy production, due to undernutrition, decreased fat free mass and low physical activity, participates to generate the negative energy balance that frequently occurs in cancer patients. In this regard, in the last few years particular emphasis has been given to mitochondrial alterations occurring in the skeletal muscle, these organelles being the main source of energy production.

Muscle mitochondria in tumor-bearing animals show ultrastructural alterations (Shum et al., 2012; Fontes-Oliveira et al., 2013; Pin et al., 2015; Shum et al., 2018) associated with uncoupling, leading to reduced oxidative capacity (Julienne et al., 2012; Tzika et al., 2013). Such impairment can be associated with the systemic inflammatory response occurring in cachexia, as suggested by observations showing that activation of the transcription factor NF- $\kappa \mathrm{B}$ decreases muscle oxidative capacity and down-regulates mitochondrial biogenesis 
(Julienne et al., 2012). In addition, altered mitochondrial dynamics (fusion and fission) and biogenesis have been reported in the ApcMin/+ mice, characterized by high circulating levels of IL-6 (White et al., 2012). Moreover, mitochondria alterations, in addition to impinge on energy production, also favor the onset of oxidative stress, both working as catabolism-inducing stimuli.

\section{OXIDATIVE STRESS}

The intracellular sources of oxidative species such as reactive oxygen and nitrogen species (ROS/RNS) are organelles such as mitochondria and sarcoplasmic reticulum, and enzymes, in particular the nicotinamide adenine dinucleotide phosphate oxidase and the xanthine oxidase. The occurrence of oxidative stress depends on an altered balance between the production of ROS/RNS and the activity of the intracellular antioxidant systems. An altered redox homeostasis may exert important effects on the integrity of biological macromolecules, with potential consequences on both cell survival and metabolism (Gomez-Cabrera et al., 2009). In this regard, muscle wasting in cancer hosts has been associated with increased ROS/RNS levels and with enhanced oxidative damage to lipids and proteins (Mastrocola et al., 2008; Sullivan-Gunn et al., 2011; SalazarDegracia et al., 2018). At the muscle level, these feature have also been correlated with increased protein breakdown rates as well as with the activation of the inflammatory response (Gomes-Marcondes and Tisdale, 2002; Mastrocola et al., 2008; Puig-Vilanova et al., 2015).

The increase of oxidative species in cachectic subjects may rely on several mechanisms among which pro-inflammatory cytokines, antineoplastic treatments and the loss of coupling between phosphorylation and oxidation in mitochondria. As reported above, several intracellular signaling pathways are regulated, partially at least, by ROS/RNS. Particularly relevant are those leading to activation of the redox-sensitive transcription factors NF- $\kappa$ B and AP-1. The former, in particular, has been shown to contribute to impaired myogenesis (see below) and to enhance the expression of molecules pertaining to the intracellular proteolytic machinery. In this regard, oxidative stress has been proposed to impinge on the activation of $\mathrm{Ca}^{2+}$-dependent proteolysis in the presence of $\mathrm{Ca}^{2+}$ overload, on caspase- 3 dependent induction of proteasome activity and on up-regulation of muscle-specific ubiquitin ligases (Ábrigo et al., 2018). Moreover, a lot of evidence suggests that also autophagic degradation can be regulated by oxidative species, mainly acting on signaling pathways dependent on p38 or PI3K/Akt (Ábrigo et al., 2018).

Finally, redox homeostasis in the cell largely depends on mitochondria 'well-being.' Indeed, altered or damaged mitochondria fail to produce energy and may become both an uncontrolled source of ROS and a target for such oxidant species. The presence of altered mitochondria is an additional procatabolic stimulus, resulting in activation of mitophagy. While the goal of such activation is to get rid of useless organelles, the result is a reduction of muscle mitochondria abundance, that frequently in cachectic subjects is not associated with enhanced mitochondrial biogenesis, also in view of the reduced energy availability (Ábrigo et al., 2018).

\section{CYTOKINES AND HORMONES}

More than 150 years ago, Rudolf Virchow proposed that carcinogenesis requires a pro-inflammatory milieu, mainly on the basis that morphological analysis repeatedly reports infiltration of the tumor stroma by inflammatory cells (Liu et al., 2017). About one century later, the discovery of cytokines and of the role that these mediators play in tumor progression has provided an additional support to the relevance of inflammation to carcinogenesis. In addition, the same mediators have been shown to significantly contribute to the pathogenesis of cancer cachexia (Argilés et al., 2014).

In the last two decades, cancer cachexia has been mainly explained as the result of systemic inflammation (acute phase response) due to the host response to tumor growth (Figure 2). Several observations support such hypothesis. Indeed, in cancer patients plasma levels of acute phase reactants, $\mathrm{C}$ reactive protein in particular, are positively correlated with increased REE and predict reduced survival rates. Along this line, circulating $\mathrm{C}$ reactive protein levels contribute to build up the Glasgow prognostic score (Fearon et al., 2006). To obtain the amino acids necessary to synthesize the acute phase proteins, the liver markedly impinges on muscle metabolism, leading to increased protein degradation and, depending on the tumor, to reduced protein synthesis rates. Finally, also the skeletal muscle of tumorbearing mice has been shown to express mRNAs coding for several acute phase reactants (Bonetto et al., 2011), although the real meaning of this expression pattern is still unknown.

The hypercatabolic setting that characterizes cancer cachexia results, partially at least, from the complex interplay among humoral mediators, including proinflammatory cytokines, hormones and growth factors. Reciprocal regulations occur in cancer hosts, cytokines being able to impinge on the hormonal homeostasis and vice versa. High plasma levels of IL-6, TNF $\alpha$, and $\gamma$-INF have been observed in both tumor-bearing animals and cachectic cancer patients (Tisdale, 2010). Several cytokines, among which $\mathrm{TNF} \alpha$, have been shown to modulate peripheral insulin sensitivity, with a mechanism that affects the activation of the insulin receptor and of down-stream signaling molecules. In addition, $\mathrm{TNF} \alpha$ also down-regulates the signaling pathways dependent on the insulin-like growth factor (IGF)-1, reducing muscle anabolic capacity and enhancing the pro-catabolic stimuli (Tisdale, 2010). Few years ago, TGF $\beta$ deriving from bone resorption due to metastatic disease has been shown to significantly contribute to cancer-induced muscle wasting (Waning et al., 2015). Quite recently, TGF- $\beta$ and TNF $\alpha$ have been shown to mediate the expression of the zinc transporter ZIP14, that is overexpressed in the skeletal muscle of both cachectic tumor-bearing animals and patients affected by metastatic cancer and that plays a causative role in muscle wasting (Wang et al., 2018).

The relevance of cytokines to cachexia has been demonstrated by studies showing that their administration to healthy animals 


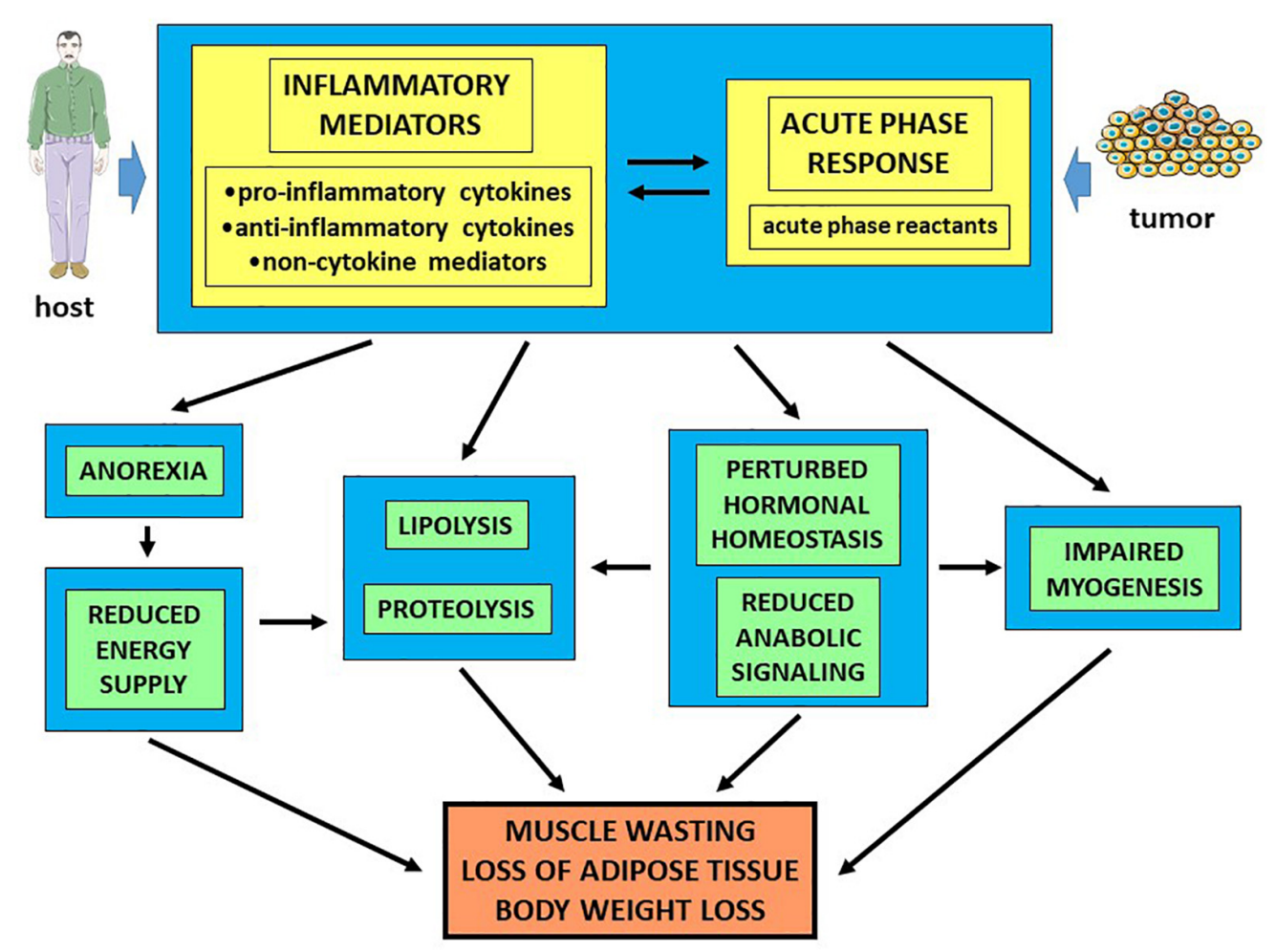

FIGURE 2 | Humoral mediators of cancer cachexia. Humoral mediators differentially involved in the inflammatory response play a crucial, but probably not exclusive, role in the pathogenesis of cancer cachexia.

results in skeletal muscle atrophy (Tisdale, 2010) as well as in alterations of lipid metabolism associated with down-regulation of lipoprotein lipase activity, induction of the hormone-sensitive lipase and stimulation of hepatic lipogenesis (Tisdale, 2009). Most of these effects also occur in tumor-bearing animals, and can be prevented treating the animals with specific anti-cytokine antibodies (Tisdale, 2009). Despite these observations, however, there is still no clear-cut evidence that anti-cytokine strategies can be useful to manage cachexia in cancer patients.

In addition to acute phase reactants, hormones and cytokines, other mediators have been proposed to contribute to the onset and progression of cancer cachexia. As an example, fat-derived leptin has been shown to inhibit both food intake and adipose tissue mass in healthy animals, although its circulating levels do not increase in both cancer patients and tumor-bearing animals, suggesting that its role in cachexia is not a causative one (Argilés et al., 2014). Among the mediators possibly involved in causing adipose tissue depletion in cachexia, the lipid mobilizing factor, increased in the circulation of cachectic cancer patients (Bing, 2011), has been shown to induce triglyceride disassembly by stimulating the activity of both the adipose triglyceride lipase and the hormone sensitive lipase, and to upregulate UCP2 expression in liver and muscle (Bing, 2011). Several reports have shown that the expression of myostatin, a molecule belonging to the TGF $\beta$ family and endowed with inhibitory activity on muscle enlargement, is enhanced in the muscle of tumor-bearing animals, although its relevance to muscle wasting in cancer hosts has not been completely defined. In this regard, recent observations show that myostatin can be secreted by the BAT, leading to impaired mTOR signaling in fast myofibers. As a result, the expression levels of several proteins, among which components of the mitochondrial electron transport chain, are reduced, affecting the energy balance (Kong et al., 2018). Several observations report the causal involvement of other TGF $\beta$ family members such as activins, and on the basis of these findings, drugs able to interfere with the activin receptor, which is also shared by myostatin, are currently under investigation as potential tools to manage cancer cachexia (Hatakeyama et al., 2016; Nissinen et al., 2018).

Recent reports suggest that receptors of the Toll-like family (TLRs) can be involved in mediating muscle wasting in cancer cachexia. In particular, TLR4 appears to directly activate muscle protein breakdown in mice hosting the Lewis lung carcinoma. Consistently, TLR4 inhibition exerts a protective effect against muscle wasting, likely by reducing the production of pro-inflammatory cytokines such as TNF $\alpha$ and IL-6 (Zhang et al., 2017a). In addition, the activation of TLR4 has also been shown to depend on Hsp70 and Hsp90 that reach the muscle through exosomes released by the tumor (Zhang et al., 2017b). Similarly, the activation of TLR7 by miR-21 contained into tumor-derived extracellular vesicles has been proposed to trigger apoptosis in cultured myoblasts, potentially contributing to the defective myogenesis reported in cancer cachexia (He et al., 2014). 


\section{MYOGENESIS}

Myogenesis is a physiological process induced during embryonal development and susceptible of activation in the adult after skeletal muscle injury, where it is more properly defined as regeneration. Irrespective of the trigger, muscle fiber degeneration following damage implies the recruitment and activation of satellite cells, stem cells resident in the muscle underneath myofiber basal lamina, that proliferate and fuse with existing fibers to restore the original muscle mass. When regeneration is triggered by chronic pathologies such as muscle dystrophies, satellite cell pool can be exhausted, leading to substitution of muscle with connective tissue.

The amount of satellite cells is not the same in every muscle type. As an example, it is higher in oxidative than in glycolytic muscles, respectively characterized by slow and fast contraction (Yin et al., 2013). In addition, satellite cell population is heterogeneous in terms of both myogenic potential and ability to perform the asymmetric division, the hallmark of stemness. In addition to satellite cells, several other cell types have been recognized as endowed with myogenic potential, such as mesoangioblasts and $\mathrm{PW}^{+}$cells. Finally, an important role during myogenesis/regeneration is played by fibroadipogenic progenitors (FAPs), cells that are unable to differentiate to muscle, but that are crucial to sustain myogenic precursors during proliferation and differentiation (Costamagna et al., 2015).

The activation of myogenesis in the adult strictly relies on modulations of the muscle microenvironment, that are different depending on the regenerative stimulus, e.g., acute damage or chronic diseases. Particularly relevant, in this regard, are the interactions occurring among immune cells, humoral mediators and myogenic precursors. Indeed, the recruitment of pro-inflammatory (M1) macrophages in the injured muscle is concomitant with satellite cell activation. However, the subsequent step of myogenic differentiation (satellite cell fusion with existing myofibers) strictly requires the induction of M1 cell apoptosis and their replacement with anti-inflammatory (M2) macrophages (St Pierre and Tidball, 1994). This transition must take place with an accurate timing (M1: 1-2 days postinjury; M2: 4 days post-injury) in order to ensure damage repair and the down-regulation of injury-induced inflammatory response. A crucial role in such process is played by cytokines, produced by macrophages but also deriving from other cellular sources. The production of TNF $\alpha$ and IL-10 allows the shift from pro-inflammatory to anti-inflammatory milieu, killing FAPs and M1 macrophages and allowing the recruitment of M2 cells (Perdiguero et al., 2011; Fiore et al., 2016). In addition, IL10 also promotes the differentiation of myogenic precursors different from satellite cells (Bosurgi et al., 2012). If cytokine production does not conform to the correct regeneration schedule, however, the process can be markedly impaired. As an example, if TNF $\alpha$ is produced earlier than days 3-4 post-injury, regeneration is impaired due to persistently cycling satellite cells (Guttridge et al., 2000; Bakkar et al., 2008). The other way round, if TNF $\alpha$ is not produced at the right moment, FAPs will not die and, taking advantage of anti-inflammatory cytokines such as IL-10 and TGF $\beta$, will result in the overproduction of extracellular matrix, eventually leading to muscle fibrosis (Fiore et al., 2016).

Alterations in the regenerative process have been proposed to contribute to muscle wasting occurring in several chronic diseases such as dystrophies, myopathies, autoimmune diseases, and cancer. Focusing on this latter, experimental studies performed in mice hosting the C26 tumor have shown in the muscle increased expression of Pax7, a marker of satellite cell activation, and reduced levels of myogenin, an indicator of ongoing differentiation. Similar observations have been reported in cancer patients (Ramamoorthy et al., 2009; Penna et al., 2010b; He et al., 2013). Consistently, in vivo regeneration is delayed in the muscle of tumor-bearing mice, with a mechanism that has been proposed to involve a persistent activation of the transcription factor NF-kB (He et al., 2013), and the increased phosphorylation of the stress kinase ERK (Penna et al., 2010b).

Recent observations have shown that overexpression of Twist1, a transcription factor associated with the malignant progression of several tumors, in myogenic precursors leads to skeletal muscle hypotrophy. Such a pattern is associated with increased myostatin expression in Twist $1^{+}$satellite cells. The authors hypothesize that in satellite cells Twist1 drives both myostatin synthesis and secretion. The released myostatin targets myofibers that respond by increasing Twist1 expression, resulting in muscle wasting (Parajuli et al., 2018). Of interest, Twistl levels higher than in healthy mice have been observed in the skeletal muscle of animals bearing different experimental tumors. Not only, if Twist1 expression is abrogated in satellite cells of tumor hosts, cancer-induced muscle wasting appears prevented (Parajuli et al., 2018). Finally, the zinc transporter ZIP14, recently shown to contribute to muscle wasting in cancer hosts, has also been proposed to interfere with regeneration, since it is induced in satellite cells isolated from cachectic muscles. Consistently, differentiation is impaired in ZIP14 overexpressing C2C12 myoblasts in the presence of zinc (Wang et al., 2018).

\section{THE SKELETAL MUSCLE STRIKES BACK: ADAPTIVE MECHANISMS TO FACE THE WASTING DRIVE}

Looking at the history of skeletal muscle biology, it is quite evident that while the relevance of this tissue to the organism life has been understood very early, for quite a long time the muscle has been considered as a sort of protein-containing black box, that receives nutrients, oxygen and signals from outside and returns the contractile activity necessary for breathing, moving, heart beating, etc. Only in the last few decades the idea that the skeletal muscle is indeed a metabolically active tissue able to release mediators and to influence, directly or indirectly, other body compartments has been gaining a growing consensus. However, still nowadays, when pathological muscle wasting is addressed, the general approach is to take into consideration, mainly at least, only alterations that take place in the extra-muscle body compartments and that target the muscle. This occurs, for example, when cancer-induced systemic inflammation and/or malnutrition are evoked to explain muscle wasting in cachexia. 
For sure, the role played by these features is overall recognized; what is lacking to this picture, however, is the response set up by the muscle to the systemic alterations. The general view, in this regard, is that the muscle passively suffers this sort of 'aggression' coming from the extra-muscle environment, being unable to face the external catabolic stimuli that eventually lead to the loss of muscle mass and function. However, several evidence suggest that the skeletal muscle is indeed able to set up compensatory, although eventually unsuccessful, strategies attempting to counteract cancer-induced hypercatabolism and hypoanabolism. Along this line, understanding these strategies will be highly valuable in order to design protocols aimed to support the muscle in the struggle against the wasting drive.

\section{Muscle Anabolic Response}

The above reported concept that slimming in cachexia is different from that due to caloric restriction is based on the observation that the latter is adopted in a physiological metabolic environment, while the former occurs in the presence of marked abnormalities, such as inflammation and hypermetabolism. In particular, cancer hosts might not properly activate protein synthesis following an increased nutrient loading, a condition that has been defined as 'anabolic resistance.' Its occurrence seriously questions the usefulness of anabolism-promoting strategies: if the patient cannot activate protein synthesis, no nutritional or pro-anabolic intervention will ever be successful. In the last few years, however, the idea that cancer hosts still maintain the ability to activate anabolism, at least until cachexia does not reach the refractory stage, has gained a growing consensus (Prado et al., 2013; Engelen et al., 2016; Antoun and Raynard, 2018).

Several observations derived from both experimental and clinical studies support this hypothesis. Muscle wasting in tumorbearing animals is unexpectedly associated with unchanged or increased levels of molecules involved in the protein synthetic machinery. As an example, the PI3K-Akt-mTOR signaling is poised toward activation in mice implanted with the C26 tumor (Penna et al., 2010a), while protein synthesis rates are unchanged with respect to controls in rats hosting the Yoshida AH-130 hepatoma or the MCA sarcoma (Tessitore et al., 1987; Stallion et al., 1995). As for human pathology, habitual myofibrillar protein synthesis rates in gastric cancer patients, either weight stable or weight losing, have been reported to be comparable to control subjects (MacDonald et al., 2015). Consistently, in the muscle of cancer patients, the pAkt/Akt ratio and the levels of pGSK $3 \beta$, both molecular markers of an intracellular pro-anabolic setting, are comparable to control levels or even increased (Aversa et al., 2012; Stephens et al., 2015). A study performed on patients affected by non-small cell lung cancer has shown the activation of a normal anabolic response after an euglycemic, hyperinsulinemic clamp associated with amino acid supplementation (Winter et al., 2012). Similarly, a significantly high anabolic response independent from body weight loss, muscle mass depletion and the occurrence of inflammation, has been obtained feeding lung cancer patients with an essential amino acid integration (Engelen et al., 2015). Finally, the association of conventional nutritional support with high leucine, fish oil and carbohydrate supplementation has enhanced the muscle anabolic response in advanced cancer patients (Deutz et al., 2011). On the whole, these observations support the idea that, if adequately supported, cancer patients might benefit from timely adopted nutritional, better if protein enriched, interventions.

In addition to the supply of amino acids, mainly mere 'bricks' to build up new proteins, nutritional supplements can also be useful in order to modulate both muscle and extramuscle environments. This applies, for example, when molecules able to reduce inflammation are included in the nutritional formula. Polyunsaturated fatty acids (PUFAs) belonging to the $\omega-3$ series have revealed promising in this regard: a recent meta-analysis based on the screening of a huge number of clinical trials involving chemotherapy-treated cancer patients has shown that oral nutritional supplementation exerts beneficial effects only when $\omega-3$ PUFAs are included in the formulation (de van der Schueren et al., 2018). It is still debated if $\omega$ 3 PUFA supplementation also results in improved patient outcome. While several studies support such possibility (reviewed in Laviano et al., 2018), contrasting evidence do exist. In this regard, no improvement of overall survival has been reported in a study investigating the effects of $\omega-3$ PUFA supplementation in cachectic gastrointestinal cancer patients treated with chemotherapy (Shirai et al., 2017).

Strategies aimed at counteracting members of the TGF $\beta$ family such as myostatin and activin have been tested. Particularly interesting appear the studies involving soluble activin receptor type IIB and antibodies directed against the activin II receptor (Bimargumab). In tumor-bearing mice the former has been reported to increase survival and muscle wasting as well as to improve the anabolic and anti-catabolic effect of formoterol (Toledo et al., 2016b; Nissinen et al., 2018). Similarly, Bimagrumab has proved effective in preventing muscle atrophy induced by glucocorticoids or cancer (Lach-Trifilieff et al., 2014; Hatakeyama et al., 2016). Clinical studies have been performed or are currently ongoing in order to test humanized antibodies targeting the activin IIB receptor. At present, the results available demonstrate that lean mass and muscle strength are increased in antibody-receiving volunteers (Becker et al., 2015).

Another exploitable strategy to improve anabolism is the administration of ghrelin. In this regard, increased circulating ghrelin markedly modulates protein and energy metabolism (Ezquerro et al., 2017). Consistently, tumor-bearing animals treated with ghrelin show increased food intake, improved body composition and increased tolerance to anti-cancer drugs (Graf and Garcia, 2017). Since ghrelin administration might also exert undesirable effects, ghrelin analogs have been produced and are currently studied in clinical trials. Among the most promising, anamorelin has been shown to improve body composition and muscle function in non-small cell lung cancer patients (Takayama et al., 2016). By contrast, other studies have clearly shown that anamorelin fails to improve motor function in cancer patients (Temel et al., 2016), likely suggesting that muscle function cannot be rescued by adopting single agent pharmacological strategies. Subsequent investigations have shown that, in selected groups of patients, anamorelin also leads to improved performance 
status (Katakami et al., 2018). Other appetite stimulants such as macimorelin and a synthetic human ghrelin are actually under investigation (Argilés et al., 2017).

\section{Enhanced Intracellular Protein Breakdown as a Salvage Pathway}

An adequate protein homeostasis, e.g., a balance between protein synthesis and degradation rates, is required to properly maintain cell functions and to prevent the onset and progression of diseases. In this regard, the observation that protein catabolism is generally increased in several conditions associated with muscle mass depletion, including cancer cachexia, has characterized this arm of protein metabolism with a negative connotation. However, normal cell function cannot disregard the presence of an adequate rate of protein turnover, meaning that the production of new proteins must be paralleled by the disassembly of pre-existing damaged or misfolded proteins. This is true in the whole organism and in the muscle in particular, where several myopathies arise due to the absence of physiological levels of protein catabolism (Bell et al., 2016). Indeed, the physiological rate of protein degradation in the skeletal muscle is considerably high, provided that this tissue is constantly exposed to damaging events such as mechanical stretch, force generation, and oxidative stress.

The observation that muscle intracellular proteolytic systems are activated above physiological levels in cancer cachexia (see above) has provided the basis for different experimental approaches aimed at contrasting the onset and progression of muscle mass depletion. As an example, genetic inhibition of muscle-specific ubiquitin ligases has been shown to effectively counteract the loss of muscle proteins (Rom and Reznick, 2016). Such an approach, however, has not yet been validated for the clinical use. Another tool has come from the discovery of rather specific proteasome inhibitors such as bortezomib. While this drug is widely used to treat hematologic malignancies, studies performed on experimental models of cancer cachexia have demonstrated that specific proteasome inhibition does not improve muscle phenotype (Penna et al., 2016a). The lack of effectiveness of the pharmacological approaches aimed at counteracting proteasome-dependent proteolysis likely depend on the compensatory activity set up by the other intracellular proteolytic systems, further supporting the idea that if muscle protein breakdown is overactivated in cancer cachexia, this does not happen by chance. In other words, such activation above physiological levels is likely part of an adaptive response due to the steadily increased abundance of damaged/unwanted proteins, ultimately aimed at maintaining vital functions. In this regard, targeting the mechanisms leading to protein alteration rather than protein degradation systems should be pursued.

Particularly relevant in terms of protein homeostasis maintenance is the proteolytic system that relies on autophagy. Enhancement of the autophagic-lysosomal protein degradation has been demonstrated in several conditions characterized by muscle wasting, including myopathies and cancer cachexia (Penna et al., 2014), suggesting that limiting the activation of this proteolytic system could be the goal to achieve in order to prevent or at least delay the loss of muscle mass and function. Such interpretation, however, does not take into account the physiological relevance of autophagy. In this regard, observations performed on experimental animals genetically manipulated in order to obtain a muscle-specific autophagy-defective phenotype have clearly shown that autophagy is required to maintain a correct muscle morphology, a healthy mitochondrial compartment and a proper force-generating capacity (Masiero et al., 2009). Consistently with these observations, gene strategies aimed at silencing Beclin-1, a key player of autophagosome formation, have proved ineffective in preventing the reduction of myofiber atrophy in mice hosting the C26 tumor (Penna et al., unpublished). In addition, frankly cachectic C26-bearing mice treated with pharmacological inhibitors of the autophagic flux do not survive (Penna et al., 2013). This observation suggests that autophagy in the muscle of the C26 hosts is activated above physiological levels also in order to provide substrates that are no more available from the usual sources. In this regard, at a certain point at least, such overactivation becomes an adaptive response that is crucial to maintain the body homeostasis or to support a new homeostatic level in stressful conditions. Partially consistent with these observations are the results reported by a study showing that stimulation of stress-induced autophagy obtained through mTOR inhibition improves muscle phenotype in tumor hosts (Pigna et al., 2016). However, long term treatment with mTOR inhibitors in patients has been shown to result in muscle wasting (Gyawali et al., 2016). Finally, overactivation of autophagy obtained through TP53INP2/Dor overexpression was shown to exacerbate the loss of muscle mass observed in experimental diabetes, confirming that both direct inhibition and stimulation of autophagy are detrimental in wasting conditions (Sala et al., 2014).

On the whole, these observations support the idea that modulations of autophagy can be good treatment options to manage muscle wasting in cancer cachexia only if they succeed in maintaining the physiological flux. In other words, too less autophagy is as detrimental as too much autophagy. Finally, the observation reported above clearly define that the prevention or delay of the onset and progression of muscle wasting in cancer cachexia cannot be obtained by targeting one specific proteolytic system. By contrast, acting on the mechanisms that activate the hypercatabolic drive appears a more promising strategy. Just as an example, $\beta_{2}$-adrenergic agonists have been demonstrated to effectively improve muscle phenotype in tumorbearing animals as well as in cancer patients (Busquets et al., 2004); such protection is associated with down-regulation of both proteasome activity and autophagy (Penna et al., unpublished).

The regulation of protein hypercatabolism in muscle wasting has also been associated with the activation of the UPR due to endoplasmic reticulum (ER) stress (Afroze and Kumar, 2017; Ma et al., 2017). Indeed, increased expression of ER stress markers has been reported in denervated muscles (Yu et al., 2011) as well as in the muscle of tumor-bearing animals (Bohnert et al., 2016). Such a response has been proposed to reflect a compensatory mechanism, since inhibition of both ER stress and UPR results in the induction of muscle wasting, the more so when the muscle is already depleted (Afroze and Kumar, 2017). More recently, 


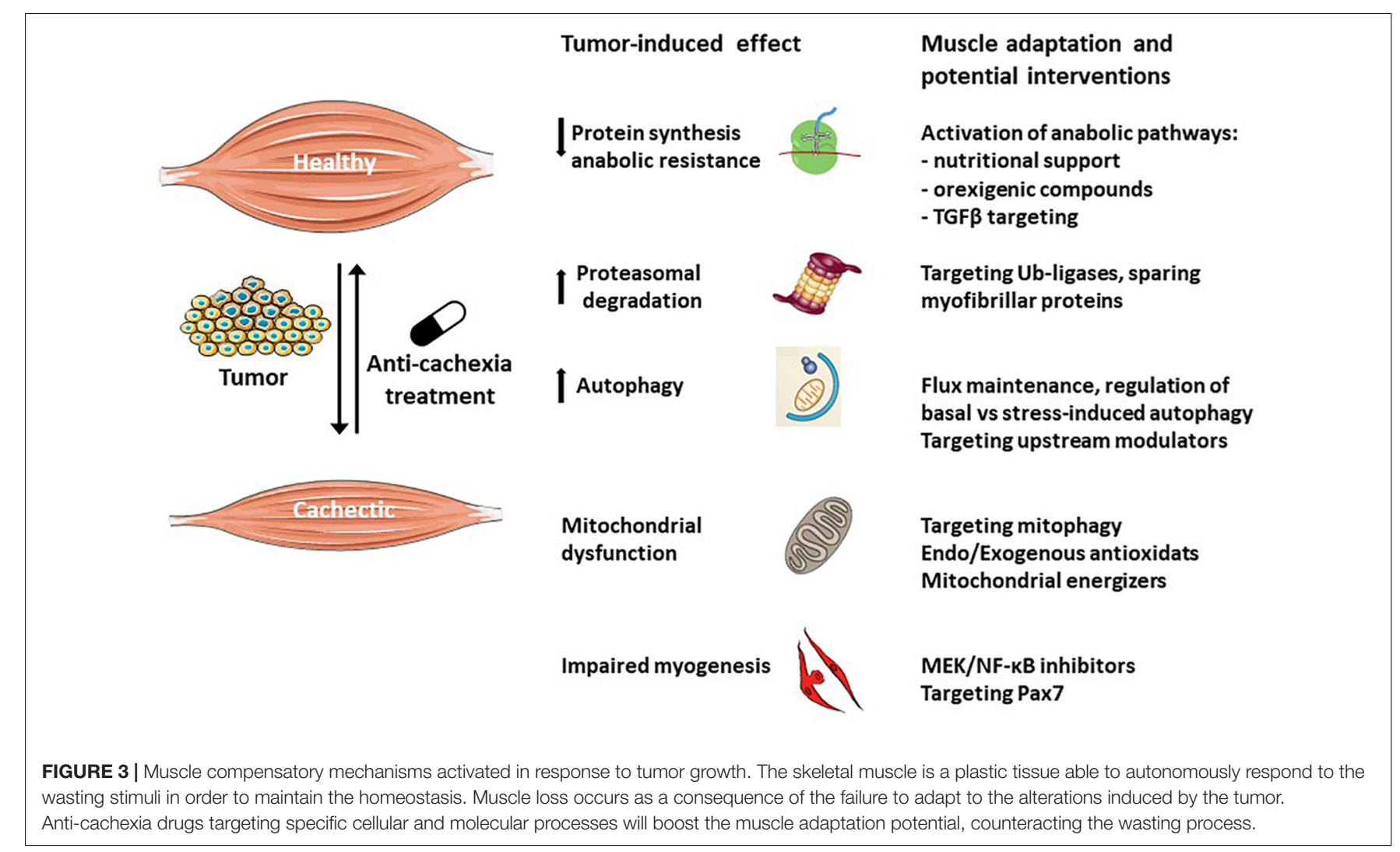

evidence suggesting the relevance of the PERK arm of the unfolded protein response to the maintenance of muscle mass and function has been reported (Gallot et al., 2018).

\section{Oxidative Stress Management}

Oxidative species such as ROS and RNS have been involved in the pathogenesis of cancer-induced muscle wasting (see above). Along this line, antioxidant compounds such as vitamins $\mathrm{C}$ and E, $\alpha$-lipoic acid, $N$-acetylcysteine, and polyphenols have been proposed as useful therapeutic tools. Polyphenols, in particular, have been shown to protect against the onset of cachexia in different types of tumors (Oelkrug et al., 2014; Gil da Costa et al., 2017), likely due to their inhibitory effect on NF-кB (Gil da Costa et al., 2017). On the other side, several pre-clinical and clinical studies have demonstrated that the anti-cachectic effects exerted by anti-oxidant drugs are poor, if not frankly detrimental (Busquets et al., 2007; Assi et al., 2016). In this regard, depending on the body compartment, tumor included, a prooxidant environment can be either detrimental or beneficial and anti-oxidant treatments can be helpful and exert unwanted effects at the same time.

Both systemic mediators and intramyofiber events, such as proinflammatory cytokines and mitochondrial alterations, respectively, can generate a pro-oxidant environment in the skeletal muscle of cancer hosts. However, even when muscle mass and function are significantly reduced with respect to control values, the tissue is able to activate an effective antioxidant response. Indeed, increased mRNA expression of both $\mathrm{Cu} / \mathrm{Zn}$
SOD and catalase, associated with unchanged levels of protein carbonylation and malondialdehyde, can be observed in the muscle of C26-bearing mice (Assi et al., 2016; Ballarò et al., unpublished). Such antioxidant response is further enhanced when tumor-bearing animals are exposed to a moderate exercise protocol, that also results in improved muscle mass and function (Ballarò et al., unpublished).

These results suggest that, despite profound metabolic alterations occur in the muscle of cancer hosts, this tissue is still able to activate and maintain an efficient anti-oxidant response, suggesting that tissue-specific interventions able to improve such adaptation would be beneficial to prevent or delay the progression of cachexia toward the refractory phase.

\section{Activation of the Myogenic Response}

Physiologically, the skeletal muscle activates the regeneration program to face myofiber injury. Previous observations have reported that this process is activated in the skeletal muscle of cancer hosts (see above), however, what is actually triggering regeneration in this situation is still debated. In this regard, few studies suggest that alterations of the dystrophin glycoprotein complex leading to sarcolemma leakage play a crucial role (Acharyya et al., 2005; He et al., 2013). However, the observation that an inflammatory infiltrate is lacking in the muscle of tumorbearing animals (Berardi et al., 2008) does not support this hypothesis. Another possibility is that the activation of the regenerative response is another face of the residual anabolic capacity reported in the muscle of cancer hosts. According to 
this possibility, but with unknown mechanisms, the muscle could react to the hypercatabolic state by recruiting and activating myogenic precursors, attempting to counteract the loss of muscle proteins. Such a strategy is however ineffective, since for unclear reasons committed $\mathrm{Pax}^{+}$myogenic precursors do not reach the complete differentiation and accumulate in the muscle without proceeding to fuse with existing myofibers. Consistently with this hypothesis, treatments able to release the impairment of myogenesis, such as MEK inhibitors or Pax7 silencing, also result in improved muscle mass and function (Penna et al., 2010b; Prado et al., 2012; He et al., 2013; Talbert et al., 2017).

Very little is known about the mechanisms that impair complete myogenic differentiation in the muscle of cancer hosts. The first possibility is that something has changed in muscle stem cells, leading to altered differentiation capacity. However, myogenic precursors isolated from the muscle of C26-bearing mice have been shown to perfectly differentiate in vitro (He et al., 2013; Inaba et al., 2018; Costamagna et al., unpublished data), demonstrating that the impaired regeneration does not depend on a cell autonomous defect. Taking into consideration these results, a possible alternative is that the presence of the tumor results in the generation of a muscle microenvironment that is not permissive for complete regeneration. Along this line, the persistently increased activation of both ERK and NF- $\mathrm{BB}$ (see above) are likely part of the mechanism that modulates muscle microenvironment. An additional mechanism can rely on the reduced recruitment to the muscle of neutrophils, macrophages, and mesenchymal progenitors occurring in tumor-bearing animals (Inaba et al., 2018).

Basal autophagy is also required to ensure both the maintenance of satellite cell homeostasis and a proper muscle regenerative response. Indeed, autophagy is steadily activated in quiescent satellite cells in order to get rid of potentially dangerous wasting products, preserving cell 'wellbeing.' Defective autophagy results in satellite cell senescence, mainly due to the accumulation of altered mitochondria that leads to oxidative stress, eventually reducing both the stem cell pool and function. Senescent muscle stem cells are unable to be recruited and activated in response to damaging stimuli (Sousa-Victor et al., 2018). Such pattern can be reversed by restoring basal flux of autophagic degradation or by pharmacological inhibition of ROS release above physiological levels (García-Prat et al., 2016). In addition, in the regenerating muscle autophagy has been proposed to impinge on myoblast differentiation (Fiacco et al., 2016; Fortini et al., 2016). Last but not least, autophagy is responsible for providing activated satellite cells with nutrients required to sustain the increased demand to exit quiescence and entering the cell cycle (Tang and Rando, 2014). Consistently with this hypothesis, the reduced expression of TP53INP2/DOR in the skeletal muscle of tumor-bearing mice and cancer hosts (Penna et al., unpublished) suggest that indeed basal autophagy is reduced in these conditions, potentially impinging also on Pax $7^{+}$cells.

The activation of satellite cells is characterized also by modulations in the oxidative pattern. Quite recent studies show that genetic manipulation of Pitx 2 and Pitx 3 transcription factors, known to regulate the redox homeostasis during embryonal myogenesis also affects the differentiation of adult satellite cells. In particular, muscle regeneration fails in animals depleted of both factors, that are characterized by markedly increased ROS levels (L'honoré et al., 2018). The same study reports that, while ROS increase is required by satellite cell to exit from quiescence and to proceed toward differentiation, excess ROS is detrimental (L'honoré et al., 2018). Along this line, reduced oxidative potential has been reported in satellite cells isolated from muscle biopsies of cancer patients, that are also endowed with reduced ability to differentiate (Brzeszczyńska et al., 2016). There is the possibility that the same alteration of redox state applies to myogenic precursors isolated from tumor-bearing animals, partially explaining the impaired regenerative response (Penna et al., 2010b; He et al., 2013). On the other side, the oxidative stress reported in the muscle of cancer hosts could also reflect the attempt to generate an environment permissive for satellite cell activation and differentiation.

\section{CONCLUSION}

Cancer cachexia arises from a complex milieu in which several factors such as metabolic alterations, malnutrition and systemic inflammation play a crucial role. Since this multiorgan syndrome is an important challenge in patient management, the definition of markers suitable to allow its early identification is actively pursued for therapeutic purposes.

Several reviews analyzed the therapeutic strategies actually available to counteract cancer cachexia (Advani et al., 2018; Di Girolamo et al., 2018; Solheim et al., 2018); while quite different approaches are adopted, all the studies agree in stating that they are far from being effective, likely because the mechanisms underlying this syndrome are only partially elucidated. Particularly relevant in this regard, is the observation that target tissues such as the skeletal muscle are able to react to the wasting stimuli activating compensatory responses (Figure 3). These counteractive strategies, however, are not potent enough and do not succeed in preventing the progression of cachexia. Along this line, the multimodal therapeutic protocol to treat cachexia could take advantage also of new approaches that, relying on understanding tissue-specific compensatory responses, contribute to support the organism effort in defeating the wasting drive.

\section{AUTHOR CONTRIBUTIONS}

FP prepared the first draft of the review. RB, MB, SD, and LGC contributed sections of the review. PC supervised the final version. All authors read and approved the submitted version.

\section{FUNDING}

This study was supported by University of Turin, Italy. 


\section{REFERENCES}

Ábrigo, J., Elorza, A. A., Riedel, C. A., Vilos, C., Simon, F., Cabrera, D., et al. (2018). Role of oxidative stress as key regulator of muscle wasting during Cachexia. Oxid. Med. Cell. Longev. 2018:2063179. doi: 10.1155/2018/2063179

Acharyya, S., Butchbach, M. E. R., Sahenk, Z., Wang, H., Saji, M., Carathers, M., et al. (2005). Dystrophin glycoprotein complex dysfunction: a regulatory link between muscular dystrophy and cancer cachexia. Cancer Cell 8, 421-432. doi: 10.1016/j.ccr.2005.10.004

Advani, S. M., Advani, P. G., VonVille, H. M., and Jafri, S. H. (2018). Pharmacological management of cachexia in adult cancer patients: a systematic review of clinical trials. BMC Cancer 18:1174. doi: 10.1186/s12885-018-5080-4

Afroze, D., and Kumar, A. (2017). ER stress in skeletal muscle remodeling and myopathies. FEBS J. doi: 10.1111/febs.14358 [Epub ahead of print].

Antoun, S., and Raynard, B. (2018). Muscle protein anabolism in advanced cancer patients: response to protein and amino acids support, and to physical activity. Ann. Oncol. 29(Suppl. 2), ii10-ii17. doi: 10.1093/annonc/mdx809

Arends, J., Bachmann, P., Baracos, V., Barthelemy, N., Bertz, H., Bozzetti, F., et al. (2017a). ESPEN guidelines on nutrition in cancer patients. Clin. Nutr. 36, 11-48. doi: 10.1016/j.clnu.2016.07.015

Arends, J., Baracos, V., Bertz, H., Bozzetti, F., Calder, P. C., Deutz, N. E. P., et al. (2017b). ESPEN expert group recommendations for action against cancerrelated malnutrition. Clin. Nutr. 36, 1187-1196. doi: 10.1016/j.clnu.2017.06. 017

Argilés, J. M., Busquets, S., Stemmler, B., and López-Soriano, F. J. (2014). Cancer cachexia: understanding the molecular basis. Nat. Rev. Cancer 14, 754-762. doi: $10.1038 / \mathrm{nrc} 3829$

Argilés, J. M., López-Soriano, F. J., Stemmler, B., and Busquets, S. (2017). Novel targeted therapies for cancer cachexia. Biochem. J. 474, 2663-2678. doi: 10.1042/ BCJ20170032

Assi, M., Derbré, F., Lefeuvre-Orfila, L., and Rébillard, A. (2016). Antioxidant supplementation accelerates cachexia development by promoting tumor growth in C26 tumor-bearing mice. Free Radic. Biol. Med. 91, 204-214. doi: 10.1016/j.freeradbiomed.2015.12.019

Aversa, Z., Bonetto, A., Penna, F., Costelli, P., Di Rienzo, G., Lacitignola, A., et al. (2012). Changes in myostatin signaling in non-weight-losing cancer patients. Ann. Surg. Oncol. 19, 1350-1356. doi: 10.1245/s10434-011-1720-5

Aversa, Z., Costelli, P., and Muscaritoli, M. (2017). Cancer-induced muscle wasting: latest findings in prevention and treatment. Ther. Adv. Med. Oncol. 9, 369-382. doi: $10.1177 / 1758834017698643$

Aversa, Z., Pin, F., Lucia, S., Penna, F., Verzaro, R., Fazi, M., et al. (2016). Autophagy is induced in the skeletal muscle of cachectic cancer patients. Sci. Rep. 6:30340. doi: 10.1038/srep30340

Bakkar, N., Wang, J., Ladner, K. J., Wang, H., Dahlman, J. M., Carathers, M., et al. (2008). IKK/NF- $\kappa B$ regulates skeletal myogenesis via a signaling switch to inhibit differentiation and promote mitochondrial biogenesis. J. Cell Biol. 180, 787-802. doi: 10.1083/jcb.200707179

Becker, C., Lord, S. R., Studenski, S. A., Warden, S. J., Fielding, R. A., Recknor, C. P., et al. (2015). Myostatin antibody (LY2495655) in older weak fallers: a proofof-concept, randomised, phase 2 trial. Lancet Diabetes Endocrinol. 3, 948-957. doi: 10.1016/S2213-8587(15)00298-3

Bell, R. A. V., Al-Khalaf, M., and Megeney, L. A. (2016). The beneficial role of proteolysis in skeletal muscle growth and stress adaptation. Skelet. Muscle 6:16. doi: 10.1186/s13395-016-0086-6

Berardi, E., Aulino, P., Murfuni, I., Toschi, A., Padula, F., Scicchitano, B. M., et al. (2008). Skeletal muscle is enriched in hematopoietic stem cells and not inflammatory cells in cachectic mice. Neurol. Res. 30, 160-169. doi: 10.1179/ 174313208 X281046

Bing, C. (2011). Lipid mobilization in cachexia. Curr. Opin. Support. Palliat. Care 5, 356-360. doi: 10.1097/SPC.0b013e32834bde0e

Bohnert, K. R., Gallot, Y. S., Sato, S., Xiong, G., Hindi, S. M., and Kumar, A. (2016). Inhibition of ER stress and unfolding protein response pathways causes skeletal muscle wasting during cancer cachexia. FASEB J. 30, 3053-3068. doi: 10.1096/fj.201600250RR

Bonetto, A., Aydogdu, T., Kunzevitzky, N., Guttridge, D. C., Khuri, S., Koniaris, L. G., et al. (2011). STAT3 activation in skeletal muscle links muscle wasting and the acute phase response in cancer cachexia. PLoS One 6:e22538. doi: 10.1371/journal.pone.0022538
Bossola, M., Muscaritoli, M., Costelli, P., Grieco, G., Bonelli, G., Pacelli, F., et al. (2003). Increased muscle proteasome activity correlates with disease severity in gastric cancer patients. Ann. Surg. 237, 384-389. doi: 10.1097/01.SLA. 0000055225.96357 .71

Bosurgi, L., Corna, G., Vezzoli, M., Touvier, T., Cossu, G., Manfredi, A. A., et al. (2012). Transplanted mesoangioblasts require macrophage IL-10 for survival in a mouse model of muscle injury. J. Immunol. 188, 6267-6277. doi: 10.4049/ jimmunol.1102680

Brzeszczyńska, J., Johns, N., Schilb, A., Degen, S., Degen, M., Langen, R., et al. (2016). Loss of oxidative defense and potential blockade of satellite cell maturation in the skeletal muscle of patients with cancer but not in the healthy elderly. Aging 8, 1690-1702. doi: 10.18632/aging.101006

Busquets, S., Figueras, M. T., Fuster, G., Almendro, V., Moore-Carrasco, R., Ametller, E., et al. (2004). Anticachectic effects of formoterol. Cancer Res. 64, 6725-6731. doi: 10.1158/0008-5472.CAN-04-0425

Busquets, S., Fuster, G., Ametller, E., Olivan, M., Figueras, M., Costelli, P., et al. (2007). Resveratrol does not ameliorate muscle wasting in different types of cancer cachexia models. Clin. Nutr. 26, 239-244. doi: 10.1016/j.clnu.2006.12. 001

Collins, P., Bing, C., McCulloch, P., and Williams, G. (2002). Muscle UCP3 mRNA levels are elevated in weight loss associated with gastrointestinal adenocarcinoma in humans. Br. J. Cancer 86, 372-375. doi: 10.1038/sj.bjc. 6600074

Coss, C. C., Clinton, S. K., and Phelps, M. A. (2018). Cachectic cancer patients: immune to checkpoint inhibitor therapy? Clin. Cancer Res. 24, 5787-5789. doi: 10.1158/1078-0432.CCR-18-1847

Costamagna, D., Costelli, P., Sampaolesi, M., and Penna, F. (2015). Role of inflammation in muscle homeostasis and myogenesis. Mediat. Inflamm. 2015:805172. doi: 10.1155/2015/805172

Costelli, P., Bossola, M., Muscaritoli, M., Grieco, G., Bonelli, G., Bellantone, R., et al. (2002). Anticytokine treatment prevents the increase in the activity of ATP-ubiquitin- and $\mathrm{Ca}(2+)$-dependent proteolytic systems in the muscle of tumour-bearing rats. Cytokine 19, 1-5. doi: 10.1006/cyto.2002.1036

Costelli, P., Carbó, N., Tessitore, L., Bagby, G. J., Lopez-Soriano, F. J., Argilés, J. M., et al. (1993). Tumor necrosis factor-alpha mediates changes in tissue protein turnover in a rat cancer cachexia model. J. Clin. Invest. 92, 2783-2789. doi: 10.1172/JCI116897

Costelli, P., Muscaritoli, M., Bossola, M., Penna, F., Reffo, P., Bonetto, A., et al. (2006). IGF-1 is downregulated in experimental cancer cachexia. AJP Regul. Integr. Comp. Physiol. 291, R674-R683. doi: 10.1152/ajpregu.00104.2006

de van der Schueren, M. A. E., Laviano, A., Blanchard, H., Jourdan, M., Arends, J., and Baracos, V. E. (2018). Systematic review and meta-analysis of the evidence for oral nutritional intervention on nutritional and clinical outcomes during chemo(radio)therapy: current evidence and guidance for design of future trials. Ann. Oncol. 29, 1141-1153. doi: 10.1093/annonc/mdy114

Deutz, N. E. P., Safar, A., Schutzler, S., Memelink, R., Ferrando, A., Spencer, H., et al. (2011). Muscle protein synthesis in cancer patients can be stimulated with a specially formulated medical food. Clin. Nutr. 30, 759-768. doi: 10.1016/j. clnu.2011.05.008

Di Girolamo, F. G., Guadagni, M., Fiotti, N., Situlin, R., and Biolo, G. (2018). Contraction and nutrition interaction promotes anabolism in cachectic muscle. Curr. Opin. Clin. Nutr. Metab. Care 22:1. doi: 10.1097/MCO.0000000000000527

Emery, P. W., Edwards, R. H., Rennie, M. J., Souhami, R. L., and Halliday, D. (1984). Protein synthesis in muscle measured in vivo in cachectic patients with cancer. Br. Med. J. 289, 584-586. doi: 10.1136/bmj.289.6445.584

Engelen, M. P. K. J., Safar, A. M., Bartter, T., Koeman, F., and Deutz, N. E. P. (2015). High anabolic potential of essential amino acid mixtures in advanced nonsmall cell lung cancer. Ann. Oncol. Off. J. Eur. Soc. Med. Oncol. 26, 1960-1966. doi: 10.1093/annonc/mdv271

Engelen, M. P. K. J., van der Meij, B. S., and Deutz, N. E. P. (2016). Protein anabolic resistance in cancer. Curr. Opin. Clin. Nutr. Metab. Care 19, 39-47. doi: 10.1097/MCO.0000000000000236

Ezeoke, C. C., and Morley, J. E. (2015). Pathophysiology of anorexia in the cancer cachexia syndrome. J. Cachexia. Sarcopenia Muscle 6, 287-302. doi: 10.1002/ jcsm. 12059

Ezquerro, S., Frühbeck, G., and Rodríguez, A. (2017). Ghrelin and autophagy. Curr. Opin. Clin. Nutr. Metab. Care 20, 402-408. doi: 10.1097/MCO. 0000000000000390 
Fearon, K. C., Voss, A. C., Hustead, D. S., and Cancer Cachexia Study Group (2006). Definition of cancer cachexia: effect of weight loss, reduced food intake, and systemic inflammation on functional status and prognosis. Am. J. Clin. Nutr. 83, 1345-1350. doi: 10.1093/ajcn/83.6.1345

Fiacco, E., Castagnetti, F., Bianconi, V., Madaro, L., De Bardi, M., Nazio, F., et al. (2016). Autophagy regulates satellite cell ability to regenerate normal and dystrophic muscles. Cell Death. Differ. 23, 1839-1849. doi: 10.1038/cdd.2016.70

Fiore, D., Judson, R. N., Low, M., Lee, S., Zhang, E., Hopkins, C., et al. (2016). Pharmacological blockage of fibro/adipogenic progenitor expansion and suppression of regenerative fibrogenesis is associated with impaired skeletal muscle regeneration. Stem Cell Res. 17, 161-169. doi: 10.1016/j.scr.2016.06.007

Fontes-Oliveira, C. C., Busquets, S., Toledo, M., Penna, F., Paz Aylwin, M., Sirisi, S., et al. (2013). Mitochondrial and sarcoplasmic reticulum abnormalities in cancer cachexia: altered energetic efficiency? Biochim. Biophys. Acta 1830, 2770-2778. doi: 10.1016/j.bbagen.2012.11.009

Fortini, P., Ferretti, C., Iorio, E., Cagnin, M., Garribba, L., Pietraforte, D., et al. (2016). The fine tuning of metabolism, autophagy and differentiation during in vitro myogenesis. Cell Death Dis. 7:e2168. doi: 10.1038/cddis.2016.50

Gallot, Y. S., Bohnert, K. R., Straughn, A. R., Xiong, G., Hindi, S. M., and Kumar, A. (2018). PERK regulates skeletal muscle mass and contractile function in adult mice. FASEB J. doi: 10.1096/fj.201800683RR [Epub ahead of print].

García-Prat, L., Martínez-Vicente, M., Perdiguero, E., Ortet, L., RodríguezUbreva, J., Rebollo, E., et al. (2016). Autophagy maintains stemness by preventing senescence. Nature 529, 37-42. doi: 10.1038/nature16187

Gil da Costa, R. M., Aragão, S., Moutinho, M., Alvarado, A., Carmo, D., Casaca, F., et al. (2017). HPV16 induces a wasting syndrome in transgenic mice: amelioration by dietary polyphenols via NF-кB inhibition. Life Sci. 169, 11-19. doi: $10.1016 /$ j.lfs.2016.10.031

Gomes-Marcondes, M. C. C., and Tisdale, M. J. (2002). Induction of protein catabolism and the ubiquitin-proteasome pathway by mild oxidative stress. Cancer Lett. 180, 69-74. doi: 10.1016/S0304-3835(02)00006-X

Gomez-Cabrera, M.-C., Viña, J., and Ji, L. L. (2009). Interplay of oxidants and antioxidants during exercise: implications for muscle health. Phys. Sportsmed. 37, 116-123. doi: 10.3810/psm.2009.12.1749

Graf, S., and Garcia, J. (2017). Anamorelin hydrochloride in the treatment of cancer anorexia-cachexia syndrome: design, development, and potential place in therapy. Drug Des. Devel. Ther. 11, 2325-2331. doi: 10.2147/DDDT.S110131

Guttridge, D. C., Mayo, M. W., Madrid, L. V., Wang, C. Y., and Baldwin, A. S. (2000). NF-kappaB-induced loss of MyoD messenger RNA: possible role in muscle decay and cachexia. Science 289, 2363-2366. doi: 10.1126/science.289. 5488.2363

Gyawali, B., Shimokata, T., Honda, K., Kondoh, C., Hayashi, N., Yoshino, Y., et al. (2016). Muscle wasting associated with the long-term use of mTOR inhibitors. Mol. Clin. Oncol. 5, 641-646. doi: 10.3892/mco.2016.1015

Hanson, E. D., Nelson, A. R., West, D. W. D., Violet, J. A., O'Keefe, L., Phillips, S. M., et al. (2017). Attenuation of resting but not load-mediated protein synthesis in prostate cancer patients on androgen deprivation. J. Clin. Endocrinol. Metab. 102, 1076-1083. doi: 10.1210/jc.2016-3383

Hatakeyama, S., Summermatter, S., Jourdain, M., Melly, S., Minetti, G. C., and Lach-Trifilieff, E. (2016). ActRII blockade protects mice from cancer cachexia and prolongs survival in the presence of anti-cancer treatments. Skelet. Muscle 6:26. doi: 10.1186/s13395-016-0098-2

He, C., Bassik, M. C., Moresi, V., Sun, K., Wei, Y., Zou, Z., et al. (2012). Exerciseinduced BCL2-regulated autophagy is required for muscle glucose homeostasis. Nature 481, 511-515. doi: 10.1038/nature10758

He, W. A., Berardi, E., Cardillo, V. M., Acharyya, S., Aulino, P., ThomasAhner, J., et al. (2013). NF-кB-mediated Pax7 dysregulation in the muscle microenvironment promotes cancer cachexia. J. Clin. Invest. 123, 4821-4835. doi: 10.1172/JCI68523

He, W. A., Calore, F., Londhe, P., Canella, A., Guttridge, D. C., and Croce, C. M. (2014). Microvesicles containing miRNAs promote muscle cell death in cancer cachexia via TLR7. Proc. Natl. Acad. Sci. U.S.A. 111, 4525-4529. doi: 10.1073/ pnas. 1402714111

Inaba, S., Hinohara, A., Tachibana, M., Tsujikawa, K., and Fukada, S. (2018). Muscle regeneration is disrupted by cancer cachexia without loss of muscle stem cell potential. PLoS One 13:e0205467. doi: 10.1371/journal.pone.0205467

Julienne, C. M., Dumas, J.-F., Goupille, C., Pinault, M., Berri, C., Collin, A., et al. (2012). Cancer cachexia is associated with a decrease in skeletal muscle mitochondrial oxidative capacities without alteration of ATP production efficiency. J. Cachexia Sarcopenia Muscle 3, 265-275. doi: 10.1007/s13539-0120071-9

Katakami, N., Uchino, J., Yokoyama, T., Naito, T., Kondo, M., Yamada, K., et al. (2018). Anamorelin (ONO-7643) for the treatment of patients with nonsmall cell lung cancer and cachexia: results from a randomized, double-blind, placebo-controlled, multicenter study of Japanese patients (ONO-7643-04). Cancer 124, 606-616. doi: 10.1002/cncr.31128

Kong, X., Yao, T., Zhou, P., Kazak, L., Tenen, D., Lyubetskaya, A., et al. (2018). Brown adipose tissue controls skeletal muscle function via the secretion of myostatin. Cell Metab. 28, 631.e-643.e. doi: 10.1016/j.cmet.2018.07.004

Lach-Trifilieff, E., Minetti, G. C., Sheppard, K., Ibebunjo, C., Feige, J. N., Hartmann, S., et al. (2014). An antibody blocking activin type ii receptors induces strong skeletal muscle hypertrophy and protects from atrophy. Mol. Cell. Biol. 34, 606-618. doi: 10.1128/MCB.01307-13

Laviano, A., Di Lazzaro, L., and Koverech, A. (2018). Nutrition support and clinical outcome in advanced cancer patients. Proc. Nutr. Soc. 77, 388-393. doi: $10.1017 /$ S0029665118000459

L'honoré, A., Commère, P.-H., Negroni, E., Pallafacchina, G., Friguet, B., Drouin, J., et al. (2018). The role of Pitx2 and Pitx 3 in muscle stem cells gives new insights into P38 $\alpha$ MAP kinase and redox regulation of muscle regeneration. eLife 7:e32991. doi: 10.7554/eLife.32991

Liu, M., Kalbasi, A., and Beatty, G. L. (2017). Functio laesa: cancer inflammation and therapeutic resistance. J. Oncol. Pract. 13, 173-180. doi: 10.1200/JOP.2016. 020347

Llovera, M., Garcia-Martinez, C., Agell, N., Lopez-Soriano, F. J., and Argiles, J. M. (1995). Muscle wasting associated with cancer cachexia is linked to an important activation of the ATP-dependent ubiquitin-mediated proteolysis. Int. J. Cancer 61, 138-141. doi: 10.1002/ijc.2910610123

Ma, L., Chu, W., Chai, J., Shen, C., Li, D., and Wang, X. (2017). ER stress and subsequent activated calpain play a pivotal role in skeletal muscle wasting after severe burn injury. PLoS One 12:e0186128. doi: 10.1371/journal.pone.0186128

MacDonald, A. J., Johns, N., Stephens, N., Greig, C., Ross, J. A., Small, A. C., et al. (2015). Habitual myofibrillar protein synthesis is normal in patients with upper GI cancer Cachexia. Clin. Cancer Res. 21, 1734-1740. doi: 10.1158/1078-0432. CCR-14-2004

Masiero, E., Agatea, L., Mammucari, C., Blaauw, B., Loro, E., Komatsu, M., et al. (2009). Autophagy is required to maintain muscle mass. Cell Metab. 10, 507-515. doi: 10.1016/j.cmet.2009.10.008

Mastrocola, R., Reffo, P., Penna, F., Tomasinelli, C. E., Boccuzzi, G., Baccino, F. M., et al. (2008). Muscle wasting in diabetic and in tumor-bearing rats: role of oxidative stress. Free Radic. Biol. Med. 44, 584-593. doi: 10.1016/j. freeradbiomed.2007.10.047

Milan, G., Romanello, V., Pescatore, F., Armani, A., Paik, J.-H., Frasson, L., et al. (2015). Regulation of autophagy and the ubiquitin-proteasome system by the FoxO transcriptional network during muscle atrophy. Nat. Commun. 6:6670. doi: $10.1038 /$ ncomms 7670

Morley, J. E., von Haehling, S., and Anker, S. D. (2014). Are we closer to having drugs to treat muscle wasting disease? J. Cachexia. Sarcopenia Muscle 5, 83-87. doi: 10.1007/s13539-014-0149-7

Nissinen, T. A., Hentilä, J., Penna, F., Lampinen, A., Lautaoja, J. H., Fachada, V., et al. (2018). Treating cachexia using soluble ACVR2B improves survival, alters mTOR localization, and attenuates liver and spleen responses. J. Cachexia. Sarcopenia Muscle 9, 514-529. doi: 10.1002/jcsm.12310

Oelkrug, C., Lange, C. M., Wenzel, E., Fricke, S., Hartke, M., Simasi, J., et al. (2014). Analysis of the tumoricidal and anti-cachectic potential of curcumin. Anticancer Res. 34, 4781-4788.

Op den Kamp, C. M., Langen, R. C., Minnaard, R., Kelders, M. C., Snepvangers, F. J., Hesselink, M. K., et al. (2012). Pre-cachexia in patients with stages I-III non-small cell lung cancer: systemic inflammation and functional impairment without activation of skeletal muscle ubiquitin proteasome system. Lung Cancer 76, 112-117. doi: 10.1016/j.lungcan.2011.09.012

Parajuli, P., Kumar, S., Loumaye, A., Singh, P., Eragamreddy, S., Nguyen, T. L., et al. (2018). Twist1 activation in muscle progenitor cells causes muscle loss akin to cancer cachexia. Dev. Cell 45, 712.e6-725.e6. doi: 10.1016/j.devcel.2018.05.026

Penna, F., Baccino, F. M., and Costelli, P. (2014). Coming back: autophagy in cachexia. Curr. Opin. Clin. Nutr. Metab. Care 17, 241-246. doi: 10.1097/MCO. 0000000000000048 
Penna, F., Bonetto, A., Aversa, Z., Minero, V. G., Rossi Fanelli, F., Costelli, P., et al. (2016a). Effect of the specific proteasome inhibitor bortezomib on cancerrelated muscle wasting. J. Cachexia Sarcopenia Muscle 7, 345-354. doi: 10.1002/ jcsm. 12050

Penna, F., Busquets, S., and Argilés, J. M. (2016b). Experimental cancer cachexia: evolving strategies for getting closer to the human scenario. Semin. Cell Dev. Biol. 54, 20-27. doi: 10.1016/j.semcdb.2015.09.002

Penna, F., Bonetto, A., Muscaritoli, M., Costamagna, D., Minero, V. G., Bonelli, G., et al. (2010a). Muscle atrophy in experimental cancer cachexia: is the IGF-1 signaling pathway involved? Int. J. Cancer 127, 1706-1717. doi: 10.1002/ijc. 25146

Penna, F., Costamagna, D., Fanzani, A., Bonelli, G., Baccino, F. M., and Costelli, P. (2010b). Muscle wasting and impaired Myogenesis in tumor bearing mice are prevented by ERK inhibition. PLoS One 5:e13604. doi: 10.1371/journal.pone. 0013604

Penna, F., Costamagna, D., Pin, F., Camperi, A., Fanzani, A., Chiarpotto, E. M., et al. (2013). Autophagic degradation contributes to muscle wasting in cancer cachexia. Am. J. Pathol. 182, 1367-1378. doi: 10.1016/j.ajpath.2012.12.023

Perdiguero, E., Sousa-Victor, P., Ruiz-Bonilla, V., Jardí, M., Caelles, C., Serrano, A. L., et al. (2011). p38/MKP-1-regulated AKT coordinates macrophage transitions and resolution of inflammation during tissue repair. J. Cell Biol. 195, 307-322. doi: 10.1083/jcb.201104053

Pigna, E., Berardi, E., Aulino, P., Rizzuto, E., Zampieri, S., Carraro, U., et al. (2016). Aerobic exercise and pharmacological treatments counteract cachexia by modulating autophagy in colon cancer. Sci. Rep. 6:26991. doi: 10.1038/ srep26991

Pin, F., Busquets, S., Toledo, M., Camperi, A., Lopez-Soriano, F. J., Costelli, P., et al. (2015). Combination of exercise training and erythropoietin prevents cancer-induced muscle alterations. Oncotarget 6, 43202-43215. doi: 10.18632/ oncotarget.6439

Pin, F., Couch, M. E., and Bonetto, A. (2018). Preservation of muscle mass as a strategy to reduce the toxic effects of cancer chemotherapy on body composition. Curr. Opin. Support. Palliat. Care 12, 420-426. doi: 10.1097/SPC. 0000000000000382

Pin, F., Minero, V. G., Penna, F., Muscaritoli, M., De Tullio, R., Baccino, F. M., et al. (2017). Interference with Ca2+-dependent proteolysis does not alter the course of muscle wasting in experimental cancer cachexia. Front. Physiol. 8:213. doi: 10.3389/fphys.2017.00213

Prado, C. M., Sawyer, M. B., Ghosh, S., Lieffers, J. R., Esfandiari, N., Antoun, S., et al. (2013). Central tenet of cancer cachexia therapy: do patients with advanced cancer have exploitable anabolic potential? Am. J. Clin. Nutr. 98, 1012-1019. doi: 10.3945/ajcn.113.060228

Prado, C. M. M., Bekaii-Saab, T., Doyle, L. A., Shrestha, S., Ghosh, S., Baracos, V. E., et al. (2012). Skeletal muscle anabolism is a side effect of therapy with the MEK inhibitor: selumetinib in patients with cholangiocarcinoma. Br. J. Cancer 106, 1583-1586. doi: 10.1038/bjc.2012.144

Puig-Vilanova, E., Rodriguez, D. A., Lloreta, J., Ausin, P., Pascual-Guardia, S., Broquetas, J., et al. (2015). Oxidative stress, redox signaling pathways, and autophagy in cachectic muscles of male patients with advanced COPD and lung cancer. Free Radic. Biol. Med. 79, 91-108. doi: 10.1016/j.freeradbiomed.2014.11. 006

Purcell, S. A., Elliott, S. A., Baracos, V. E., Chu, Q. S. C., and Prado, C. M. (2016) Key determinants of energy expenditure in cancer and implications for clinical practice. Eur. J. Clin. Nutr. 70, 1230-1238. doi: 10.1038/ejcn.2016.96

Ramamoorthy, S., Donohue, M., and Buck, M. (2009). Decreased Jun-D and myogenin expression in muscle wasting of human cachexia. Am. J. Physiol. Endocrinol. Metab. 297, E392-E401. doi: 10.1152/ajpendo.90529.2008

Rom, O., and Reznick, A. Z. (2016). The role of E3 ubiquitin-ligases MuRF-1 and MAFbx in loss of skeletal muscle mass. Free Radic. Biol. Med. 98, 218-230. doi: 10.1016/j.freeradbiomed.2015.12.031

Sala, D., Ivanova, S., Plana, N., Ribas, V., Duran, J., Bach, D., et al. (2014). Autophagy-regulating TP53INP2 mediates muscle wasting and is repressed in diabetes. J. Clin. Invest. 124, 1914-1927. doi: 10.1172/JCI72327

Salazar-Degracia, A., Busquets, S., Argilés, J. M., Bargalló-Gispert, N., LópezSoriano, F. J., and Barreiro, E. (2018). Effects of the beta 2 agonist formoterol on atrophy signaling, autophagy, and muscle phenotype in respiratory and limb muscles of rats with cancer-induced cachexia. Biochimie 149, 79-91. doi: 10.1016/j.biochi.2018.04.009
Shirai, Y., Okugawa, Y., Hishida, A., Ogawa, A., Okamoto, K., Shintani, M., et al. (2017). Fish oil-enriched nutrition combined with systemic chemotherapy for gastrointestinal cancer patients with cancer cachexia. Sci. Rep. 7:4826. doi: 10 . 1038/s41598-017-05278-0

Shum, A. M. Y., Mahendradatta, T., Taylor, R. J., Painter, A. B., Moore, M. M., Tsoli, M., et al. (2012). Disruption of MEF2C signaling and loss of sarcomeric and mitochondrial integrity in cancer-induced skeletal muscle wasting. Aging 4, 133-143. doi: 10.18632/aging.100436

Shum, A. M. Y., Poljak, A., Bentley, N. L., Turner, N., Tan, T. C., and Polly, P. (2018). Proteomic profiling of skeletal and cardiac muscle in cancer cachexia: alterations in sarcomeric and mitochondrial protein expression. Oncotarget 9, 22001-22022. doi: 10.18632/oncotarget.25146

Smith, I. J., Aversa, Z., Hasselgren, P.-O., Pacelli, F., Rosa, F., Doglietto, G. B., et al. (2011). CALPAIN activity is increased in skeletal muscle from gastric cancer patients with no or minimal weight loss. Muscle Nerve 43, 410-414. doi: $10.1002 /$ mus. 21893

Solheim, T. S., Laird, B. J. A., Balstad, T. R., Bye, A., Stene, G., Baracos, V., et al. (2018). Cancer cachexia: rationale for the MENAC (Multimodal-Exercise, Nutrition and Anti-inflammatory medication for Cachexia) trial. BMJ Support. Palliat. Care 8, 258-265. doi: 10.1136/bmjspcare-2017-001440

Sousa-Victor, P., García-Prat, L., and Muñoz-Cánoves, P. (2018). New mechanisms driving muscle stem cell regenerative decline with aging. Int. J. Dev. Biol. 62, 583-590. doi: 10.1387/ijdb.180041pm

St Pierre, B. A., and Tidball, J. G. (1994). Differential response of macrophage subpopulations to soleus muscle reloading after rat hindlimb suspension. J. Appl. Physiol. 77, 290-297. doi: 10.1152/jappl.1994.77.1.290

Stallion, A., Foley-Nelson, T., Chance, W. T., James, J. H., and Fischer, J. E. (1995). Anticatabolic effect of the beta 2-agonist cimaterol in vivo in tumor-bearing animals. J. Surg. Res. 59, 387-392. doi: 10.1006/jsre.1995.1180

Stephens, N. A., Skipworth, R. J. E., Gallagher, I. J., Greig, C. A., Guttridge, D. C., Ross, J. A., et al. (2015). Evaluating potential biomarkers of cachexia and survival in skeletal muscle of upper gastrointestinal cancer patients. J. Cachexia Sarcopenia Muscle 6, 53-61. doi: 10.1002/jcsm.12005

Sullivan-Gunn, M. J., Campbell-O’Sullivan, S. P., Tisdale, M. J., and Lewandowski, P. A. (2011). Decreased NADPH oxidase expression and antioxidant activity in cachectic skeletal muscle. J. Cachexia Sarcopenia Muscle 2, 181-188. doi: 10.1007/s13539-011-0037-3

Takayama, K., Katakami, N., Yokoyama, T., Atagi, S., Yoshimori, K., Kagamu, H., et al. (2016). Anamorelin (ONO-7643) in Japanese patients with non-small cell lung cancer and cachexia: results of a randomized phase 2 trial. Support. Care Cancer 24, 3495-3505. doi: 10.1007/s00520-016-3144-z

Talbert, E. E., Yang, J., Mace, T. A., Farren, M. R., Farris, A. B., Young, G. S., et al. (2017). Dual inhibition of MEK and PI3K/Akt rescues cancer cachexia through both tumor-extrinsic and -intrinsic activities. Mol. Cancer Ther. 16, 344-356. doi: 10.1158/1535-7163.MCT-16-0337

Tang, A. H., and Rando, T. A. (2014). Induction of autophagy supports the bioenergetic demands of quiescent muscle stem cell activation. EMBO J. 33, 2782-2797. doi: 10.15252/embj.201488278

Tardif, N., Klaude, M., Lundell, L., Thorell, A., and Rooyackers, O. (2013). Autophagic-lysosomal pathway is the main proteolytic system modified in the skeletal muscle of esophageal cancer patients. Am. J. Clin. Nutr. 98, 1485-1492. doi: 10.3945/ajen.113.063859

Temel, J. S., Abernethy, A. P., Currow, D. C., Friend, J., Duus, E. M., Yan, Y., et al. (2016). Anamorelin in patients with non-small-cell lung cancer and cachexia (ROMANA 1 and ROMANA 2): results from two randomised, doubleblind, phase 3 trials. Lancet Oncol. 17, 519-531.doi: 10.1016/S1470-2045(15) 00558-6

Tessitore, L., Bonelli, G., and Baccino, F. M. (1987). Early development of protein metabolic perturbations in the liver and skeletal muscle of tumour-bearing rats. A model system for cancer cachexia. Biochem. J. 241, 153-159. doi: 10.1042/ bj2410153

Tezze, C., Romanello, V., Desbats, M. A., Fadini, G. P., Albiero, M., Favaro, G., et al. (2017). Age-associated loss of OPA1 in muscle impacts muscle mass, metabolic homeostasis, systemic inflammation, and epithelial senescence. Cell Metab. 25, 1374.e6-1389.e6. doi: 10.1016/j.cmet.2017.04.021

Thompson, K. L., Elliott, L., Fuchs-Tarlovsky, V., Levin, R. M., Voss, A. C., and Piemonte, T. (2017). Oncology evidence-based nutrition practice guideline for adults. J. Acad. Nutr. Diet 117, 297.e47-310.e47. doi: 10.1016/j.jand.2016.05.010 
Tisdale, M. J. (2009). Mechanisms of cancer cachexia. Physiol. Rev. 89, 381-410. doi: $10.1152 /$ physrev.00016.2008

Tisdale, M. J. (2010). Cancer cachexia. Curr. Opin. Gastroenterol. 26, 146-151. doi: 10.1097/MOG.0b013e3283347e77

Tobert, C. M., Hamilton-Reeves, J. M., Norian, L. A., Hung, C., Brooks, N. A., Holzbeierlein, J. M., et al. (2017). Emerging impact of malnutrition on surgical patients: literature review and potential implications for cystectomy in bladder cancer. J. Urol. 198, 511-519. doi: 10.1016/j.juro.2017.01.087

Toledo, M., Busquets, S., Penna, F., Zhou, X., Marmonti, E., Betancourt, A., et al. (2016a). Complete reversal of muscle wasting in experimental cancer cachexia: additive effects of activin type II receptor inhibition and $\beta-2$ agonist. Int. J. Cancer 138, 2021-2029. doi: 10.1002/ijc.29930

Toledo, M., Penna, F., Oliva, F., Luque, M., Betancourt, A., Marmonti, E., et al. (2016b). A multifactorial anti-cachectic approach for cancer cachexia in a rat model undergoing chemotherapy. J. Cachexia Sarcopenia Muscle 7, 48-59. doi: 10.1002/jcsm. 12035

Tzika, A. A., Fontes-Oliveira, C. C., Shestov, A. A., Constantinou, C., Psychogios, N., Righi, V., et al. (2013). Skeletal muscle mitochondrial uncoupling in a murine cancer cachexia model. Int. J. Oncol. 43, 886-894. doi: 10.3892/ijo.2013.1998

van Dijk, D. P. J., van de Poll, M. C. G., Moses, A. G. W., Preston, T., Olde Damink, S. W. M., Rensen, S. S., et al. (2015). Effects of oral meal feeding on whole body protein breakdown and protein synthesis in cachectic pancreatic cancer patients. J. Cachexia Sarcopenia Muscle 6, 212-221. doi: 10.1002/jcsm.12029

Vazeille, C., Jouinot, A., Durand, J.-P., Neveux, N., Boudou-Rouquette, P., Huillard, O., et al. (2017). Relation between hypermetabolism, cachexia, and survival in cancer patients: a prospective study in 390 cancer patients before initiation of anticancer therapy. Am. J. Clin. Nutr. 105, 1139-1147. doi: 10.3945/ ajcn.116.140434

Wang, G., Biswas, A. K., Ma, W., Kandpal, M., Coker, C., Grandgenett, P. M., et al. (2018). Metastatic cancers promote cachexia through ZIP14 upregulation in skeletal muscle. Nat. Med. 24, 770-781. doi: 10.1038/s41591-018-0054-2

Waning, D. L., Mohammad, K. S., Reiken, S., Xie, W., Andersson, D. C., John, S., et al. (2015). Excess TGF- $\beta$ mediates muscle weakness associated with bone metastases in mice. Nat. Med. 21, 1262-1271.doi: 10.1038/nm. 3961

White, J. P., Puppa, M. J., Sato, S., Gao, S., Price, R. L., Baynes, J. W., et al. (2012). IL-6 regulation on skeletal muscle mitochondrial remodeling during cancer cachexia in the ApcMin/+ mouse. Skelet. Muscle 2:14. doi: 10.1186/2044-50402-14

Winter, A., MacAdams, J., and Chevalier, S. (2012). Normal protein anabolic response to hyperaminoacidemia in insulin-resistant patients with lung cancer cachexia. Clin. Nutr. 31, 765-773. doi: 10.1016/j.clnu.2012.05.003

Yin, H., Price, F., and Rudnicki, M. A. (2013). Satellite cells and the muscle stem cell niche. Physiol. Rev. 93, 23-67. doi: 10.1152/physrev.00043.2011

Yu, Z., Wang, A. M., Adachi, H., Katsuno, M., Sobue, G., Yue, Z., et al. (2011). Macroautophagy is regulated by the UPR-mediator CHOP and accentuates the phenotype of SBMA mice. PLoS Genet. 7:e1002321. doi: 10.1371/journal.pgen. 1002321

Zhang, G., Liu, Z., Ding, H., Miao, H., Garcia, J. M., and Li, Y.-P. (2017a). Tolllike receptor 4 mediates Lewis lung carcinoma-induced muscle wasting via coordinate activation of protein degradation pathways. Sci. Rep. 7:2273. doi: 10.1038/s41598-017-02347-2

Zhang, G., Liu, Z., Ding, H., Zhou, Y., Doan, H. A., Sin, K. W. T., et al. (2017b). Tumor induces muscle wasting in mice through releasing extracellular Hsp70 and Hsp90. Nat. Commun. 8:589. doi: 10.1038/s41467-017-00726-x

Conflict of Interest Statement: The authors declare that the research was conducted in the absence of any commercial or financial relationships that could be construed as a potential conflict of interest.

Copyright (c) 2019 Penna, Ballarò, Beltrà, De Lucia, García Castillo and Costelli. This is an open-access article distributed under the terms of the Creative Commons Attribution License (CC BY). The use, distribution or reproduction in other forums is permitted, provided the original author(s) and the copyright owner(s) are credited and that the original publication in this journal is cited, in accordance with accepted academic practice. No use, distribution or reproduction is permitted which does not comply with these terms. 


\section{OPEN ACCESS}

Edited by:

Dario Coletti,

Sapienza University of Rome, Italy

Reviewed by:

Antonio De Luca,

Università degli Studi della Campania Luigi Vanvitelli Caserta, Italy Giovanni Li Volti,

Università degli Studi di Catania, Italy

*Correspondence:

Marianna Bellafiore marianna.bellafiore@unipa.it

Specialty section: This article was submitted to Striated Muscle Physiology, a section of the journal

Frontiers in Physiology

Received: 02 January 2019 Accepted: 22 February 2019

Published: 28 March 2019

Citation:

Bellafiore M, Battaglia G,

Bianco A and Palma A (2019)

Expression Pattern of Angiogenic

Factors in Healthy Heart in Response to Physical Exercise Intensity.

Front. Physiol. 10:238

doi: 10.3389/fphys.2019.00238

\section{Expression Pattern of Angiogenic Factors in Healthy Heart in Response to Physical Exercise Intensity}

\author{
Marianna Bellafiore*, Giuseppe Battaglia, Antonino Bianco and Antonio Palma \\ Department of Psychology, Educational Science and Human Movement, University of Palermo, Palermo, Italy
}

Recently, many studies showing the regeneration potential of both cardiac and hematopoietic stem cells in adult heart following injury were definitively retracted by the literature. Therefore, stimulating myocardial angiogenesis becomes to be important for preventing cardiovascular diseases. Regular endurance exercise has been reported to induce capillary growth in healthy and diseased myocardium resulting in cardioprotective phenotype. Previously, we demonstrated a significantly increased capillary proliferation in mouse hearts following 30 and 45 days of endurance training. In the present study, we examined the localization and expression pattern of vascular endothelial growth factor receptors (VEGFR-1/Flt-1 and VEGFR-2/FIk-1), hypoxia-inducible factor-1 $\alpha(\mathrm{HIF-1} \alpha)$, and inducible nitric oxide synthase (iNOS) in heart neocapillarization in response to a mild, moderate, and high intensity of endurance training. Sixty-three Swiss male mice were divided into four untrained control groups and three groups trained for 15 (T15), 30 (T30), and 45 (T45) days with a gradually increasing intensity on a treadmill. We observed the localization of studied proteins with immunostaining and their expression level with Western blot analyses. We found that VEGFR-2/FIk-1 expression progressively increased in trained groups compared with controls, while VEGFR-1/FIt-1 and HIF-1 $\alpha$ were higher in T15 than in controls, T30, and T45 animals. Differently, iNOS levels enhanced after 15 and 30 days of exercise. The localization of these factors was not altered by exercise. The results showed that the expression of VEGFR-1/FIt-1, VEGFR-2/FIk-1, HIF-1 $\alpha$, and iNOS is differently regulated in cardiac angiogenesis according to the exercise intensity. VEGFR-1/FIt-1 and HIF- $1 \alpha$ are upregulated by a mild intensity exercise, while VEGFR-2/Flk-1 progressively enhances with increasing workload. Differently, iNOS protein is modulated by a moderate intensity exercise. VEGF pathway appears to be involved in exercise-related angiogenesis in heart and VEGF might act in a paracrine and endocrine manner. Understanding this relationship is important for developing exercise strategies to protect the heart by insults.

Keywords: heart, skeletal muscle, endurance training, angiogenesis, physical exercise, capillary growth, hypoxia, exercise intensity 


\section{INTRODUCTION}

Regular physical exercise is well known to reduce cardiovascular diseases, the main cause of death worldwide, increasing cardiac function and protecting against myocardial damage (Schuler et al., 2013; Tao et al., 2015). Heart adaptations can vary according to type, intensity, and duration of exercise and are commonly defined as the athlete's heart (Pluim et al., 2000; Fulghum and Hill, 2018). For instance, endurance exercises such as running or swimming performed for prolonged periods are a significant physiological stimulus for an enhanced demand of oxygen and nutrient leading to the formation of new capillary in myocardium (Brown, 2003; Bellafiore et al., 2007; Fulghum and Hill, 2018). In detail, modifications in the blood flow, muscle contraction, and oxygen levels associated with hemodynamic mechanical events have been shown to be key signals for triggering vessel wall remodeling and activation of growth factors involved in the proliferation, migration, and tube formation of endothelial cells in both heart and skeletal muscle (Brown, 2003; Prior et al., 2004). This implies a cross talk between skeletal and cardiac muscle in order to respond synergistically to various stimuli derived by physical exercise. Indeed, several studies recently reported that exercise stimulates the release of circulating cytokines and growth factors, called myokines, by skeletal muscle that acts with endocrine functions mediating exercise-induced cardiovascular adaptations (Giudice and Taylor, 2017; Hoffmann and Weigert, 2017).

Among the molecules involved in the angiogenesis induced by physical exercise, vascular endothelial growth factor (VEGF) plays a crucial role in the induction of endothelial cell mitosis and promotion of capillary sprouting (Hudlicka et al., 1995; Shibuya, 2006). The angiogenic action of VEGF is mediated by two primary receptors, such as VEGFR-1/Flt-1 and VEGFR-2/ Flk-1, both predominantly expressed on the endothelial cells but with different roles (Hudlicka et al., 1995; Iemitsu et al., 2006; Shibuya, 2006). VEGFR-1/Flt-1 has higher affinity for VEGF than VEGFR-2/Flk-1, and it was found to mediate chemotaxis, mitogenesis, and cytoskeletal reorganizations. In contrast, VEGFR-2/Flk-1 has a ligand-induced autophosphorylation activity much stronger than VEGFR-1/Flt-1 and is the major regulator of vasculogenesis and angiogenesis (Shibuya, 2006). Iemitsu et al. (2006) found an increase in mRNA and protein expression of VEGFR-1/Flt-1 and VEGFR-2/Flk-1 associated with an enhanced capillary density in aged rat hearts after 8 weeks of swim training. Furthermore, Milkiewicz et al. (2003) showed that these receptors were regulated by chronic ischemia/hypoxia and intermittent electrical stimulation in rat skeletal muscle. The role of hypoxia as a candidate for the initiation of angiogenesis in exercising muscle was reinforced by data indicating that hypoxia-inducible factor-1 a (HIF-1 $\alpha$ ) expression induced by hypoxia determined the upregulation of VEGF in trained rat muscles (Shweiki et al., 1992). Moreover, HIF-1a has been found to be significantly overexpressed in rat left ventricle $48 \mathrm{~h}$ after the last training session of a 10-week mild intensity aerobic exercise protocol and to participate in cardioprotection (Giusti et al., 2009). This effect might be due to "ischemic preconditioning" induced by physical exercise and detected in both humans and animals (Marongiu and Crisafulli, 2014). This phenomenon consists of ischemia short episodes that render the myocardium more resistant to subsequent more prolonged ischemic events. In addition, HIF- $1 \alpha$ has been discovered to activate the downstream genes of VEGF such as inducible nitric oxide synthase (iNOS) in cardiomyocytes and endothelial cells, resulting in an increase in angiogenesis and cardioprotection (Jung et al., 2000; Tekin et al., 2010).

In the past two decades, Anversa et al. discovered that c-kit-positive cardiac stem cells and hematopoietic stem cells derived from the bone marrow can regenerate the heart muscle following a myocardial infarction (Beltrami et al., 2001; Anversa et al., 2013). According to these studies, by injecting these stem cells into the heart, they could differentiate into cardiomyocytes and coronary vessels, leading to tissue repair. This result, whether it had been true, would have had a decisive relief for a very large number of patients all over the world. However, several of these studies were recently retracted from prestigious journals, and Harvard Medical School and Brigham and Women's Hospital have recommended the retraction of other 31 papers of Anversa's group (Drazen, 2018; Dyer, 2018). Therefore, the research of physical exercise-related stimuli inducing a cardioprotective phenotype results to be really important for preventing cardiovascular diseases and improving heart function following injury. To this regard, little is known about the signaling molecules that regulate capillary growth in healthy myocardium according to the exercise intensity. This limitation might be due to controversial evidence about the stimulation of new capillaries in healthy hearts by physical exercise and might depend on the intensity and length of exercise training selected for analyzing cardiac microvasculature (Brown, 2003). It has been indeed reported that exercise-induced formation of new capillaries is transient as capillaries transform into arterioles (Brown, 2003). In previous studies, we showed a significantly increased capillary proliferation in mouse hearts following 30 and 45 days of an endurance training protocol (Bellafiore et al., 2007, 2013). Therefore, the aim of the present study was to investigate the localization and expression pattern of VEGFR-1/Flt-1, VEGFR-2/Flk-1, HIF-1 $\alpha$, and iNOS in the angiogenesis of mouse hearts in response to mild, moderate, and high intensity of endurance training. We speculated response patterns that differ among examined molecules and depend on the training workload.

\section{MATERIALS AND METHODS}

\section{Experimental Design}

Sixty-three male 10-week-old Swiss mice were randomly divided into seven groups. Four groups were selected as sedentary controls (C) $(0,15,30$, and 45 days) and three groups were trained through an endurance protocol for 15 (T15), 30 (T30), and 45 days (T45). Mice were trained for 5 days/week on a rotating treadmill progressively increasing both workload intensity and training time (Di Felice et al., 2007). The exercise training intensity performed by T15, T30, and T45 mice corresponded to a mild, moderate, and high intensity, respectively. The study 
conforms to the Guide for the Care and Use of Laboratory Animals (NIH Publication No. 85-23, revised 1996) and was approved by the local Ethical Committee of Palermo University (Comitato Etico Palermo 1). We carried out this study using the same samples of heart prepared in our previous studies, in which we detected left ventricle hypertrophy associated with a significant increase in the capillary proliferation after 30 and 45 days of endurance training (Bellafiore et al., 2007, 2013).

\section{Immunostaining Analyses}

In order to examine the localization and expression of VEGFR-1/ Flt-1, VEGFR-2/Flk-1, HIF-1 $\alpha$, and iNOS, immunohistochemical analyses were performed. Hearts were fixed with formalin, embedded with paraffin, and cut to obtain $5 \mu \mathrm{m}$ sections. After incubation of sections for 10 min with $0.3 \% \mathrm{H}_{2} \mathrm{O}_{2}$, a serum-free protein block (DAKO, Carpinteria, USA) was added for $10 \mathrm{~min}$. Sections were then incubated with the primary antibodies against VEGFR-1/Flt-1 (1:200; Chemicon ${ }^{\circledR}$ International, Serological Company, EU), VEGFR-2/Flk-1 (1:100; Chemicon ${ }^{\circledR}$ International, Serological Company, EU), HIF-1a (1:100; Chemicon ${ }^{\circledR}$ International, Serological Company, EU), and iNOS (1:50; Calbiochem ${ }^{\circledR}$, San Diego, CA, USA) for $1 \mathrm{~h}$ at room temperature (RT). Non-immune mouse serum was substituted for negative controls. Before adding the primary antibodies for HIF- $1 \alpha$, the slides were treated with monohydrated citrate buffer $(\mathrm{pH} 6.0,0.01 \mathrm{M})$ in a water bath for 40 and $10 \mathrm{~min}$, respectively, at $100^{\circ} \mathrm{C}$ for the antigen retrieval. After incubation for 10 min with a biotinylated secondary antibody, AEC chromogen (DAKO, Carpinteria, CA, USA) was used to develop the horseradish peroxidase (HRP)-streptavidin complex.

\section{Western Blotting}

The expression levels of VEGFR-1/FLT-1, VEGFR-2/FLK-1, HIF-1a, and iNOS were measured in the left ventricle (LV) from each single mouse of the control and trained groups by immunoblot analyses. Frozen LV fragments were homogenized in a lysis buffer, containing protease inhibitors, and centrifuged at $13,000 \mathrm{rpm}$ for $10 \mathrm{~min}$ at $4^{\circ} \mathrm{C}$. Total cellular lysate fraction was collected, and protein concentration was determined using a colorimetric assay (Bio-4 Rad, Philadelphia, USA). About $20 \mu \mathrm{g}$ of protein samples for each lane and a protein marker (Bio-Rad, Philadelphia, USA) were separated by $8 \%$ SDS-PAGE and transferred to a nitrocellulose membrane. After $1 \mathrm{~h}$ at RT with a blocking buffer, each membrane was incubated with primary antibodies against VEGFR-1/Flt-1 (1:1000; Chemicon ${ }^{\circledR}$ International, Serological Company, EU), VEGFR-2/Flk-1 (1:500; Chemicon $^{\circledR}$ International, Serological Company, EU), HIF-1 $\alpha$ (1:500; Chemicon ${ }^{\circledR}$ International, Serological Company, EU), and iNOS (1:500; Calbiochem ${ }^{\circledR}$, San Diego, CA, USA) overnight at $4^{\circ} \mathrm{C}$. After washing, each membrane was incubated with HRP-conjugated secondary antibody for $1 \mathrm{~h}$ at RT, and signals were detected using an enhanced chemiluminescence (ECL, Amersham Bioscience, UK) for autoradiography. After stripping, the same membranes were incubated with $\beta$-actin antibody (1:1000; Sigma-Aldrich, USA), and the ratio of proteins of interest and $\beta$-actin was determined. The intensity of bands was quantified by computer-assisted image analysis (ImageJ, Media Cybernetics, Silver Spring, MD, USA) calculating pixel number per $\mathrm{cm}^{2}$.

\section{Statistical Analysis}

Data are expressed as means \pm standard deviations. One-way ANOVA test with Bonferroni's multiple comparison test was used to analyze significant differences. Values were considered significantly different at $p<0.05$.

\section{RESULTS}

\section{Cardiac Expression of VEGFR-1/FIt-1 and VEGFR-2/FIk-1 in Response to Physical Exercise}

Both receptors, VEGFR-1/Flt-1 and VEGFR-2/Flk-1, were expressed in subepicardial and subendocardial regions and specifically localized in the vascular endothelial cells as shown in Figure 1. We observed the same localization in the hearts from both control and trained mice (data not shown). From immunoblotting analyses, we obtained a band with molecular weight of about $180 \mathrm{kDa}$ corresponding to VEGFR-1/Flt-1 protein (Figure 2A) as reported in the literature. Because samples from C, T15, T30, and T45 groups did not significantly differ within the same group, a representative sample from each group has shown (Figures 2A,B). The expression of VEGFR-1/Flt-1 was higher in T15 mice than in C, T30, and T45 groups (T15: $1.17 \pm 0.005$ vs. C: $1.02 \pm 0.01$, T30: $1.03 \pm 0.01$, and T45: $0.94 \pm 0.01 ; p<0.05$; Figure 2B). T30 animals did not show any significant difference compared with $\mathrm{C}$ groups. Moreover, in T45 animals, VEGFR-1/Flt-1 expression was lower than C and T30 mice (T45: $0.94 \pm 0.01$ vs. C: $1.02 \pm 0.01$ and T30: $1.03 \pm 0.01 ; p<0.05$; Figure 2B).

As regards to VEGFR-2/Flk-1 expression, we found a band with molecular weight of about $200 \mathrm{kDa}$ that gradually increased in trained mice (T15, T30, T45) compared to controls (C) (T15: $0.97 \pm 0.01, \mathrm{~T} 30: 1.07 \pm 0.007$, and T45: $1.21 \pm 0.01$ vs. C: $0.93 \pm 0.01 ; p<0.05)$. In particular, VEGFR-2/Flk-1 expression was higher in groups T30 and T45 than in T15 (T30: $1.07 \pm 0.007$ and T45: $1.21 \pm 0.01$ vs. T15: $0.97 \pm 0.01$; $p<0.05)$ and in T45 rather than T30 mice (T45: $1.21 \pm 0.01$ vs. T30: $1.07 \pm 0.007 ; p<0.05$; Figure 2).

\section{Regulation of HIF-1 $\alpha$ Expression by the Endurance Training in the Myocardium}

Immunostaining analyses showed that HIF- $1 \alpha$ protein was specifically localized in the nucleus of vascular endothelial and myocardial cells of control and trained animals (Figure 1). The expression of HIF-1 $\alpha$ was confirmed by immunoblotting analyses (Figure 3A), which showed a band with molecular weight of about $120 \mathrm{kDa}$. As samples from C, T15, T30, and T45 did not significantly differ within the same group, a representative sample from each group is shown (Figure 3). As shown in Figure 3B, it is evident that HIF-1 $\alpha$ expression was significantly higher in T15 animals than in T30 and T45 

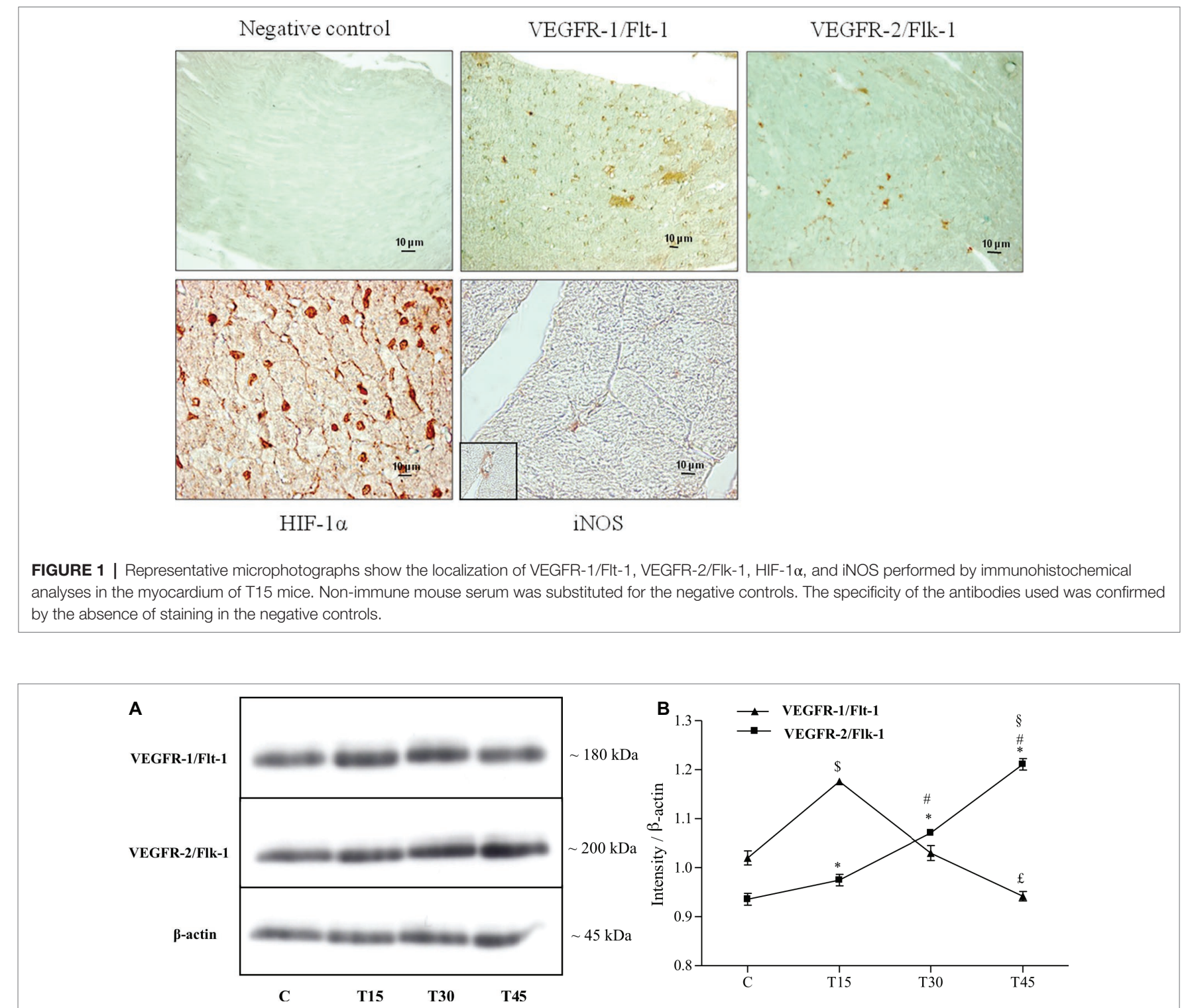

FIGURE 2 | Western blotting (A) and quantitative analysis (B) to measure VEGFR-1/FIt-1 and VEGFR-2/Flk-1 expression in hearts from trained and control mice. (B) VEGFR-1/FIt-1 protein levels resulted significantly higher in T15 than in groups C, T30, and T45. Conversely, T45 animals showed a lower VEGFR-1/FIt-1 expression than $\mathrm{C}$ and T30 mice ( $\$ p<0.05$ T15 vs. T30 and T45; $£ p<0.05$ T45 vs. C and T30). VEGFR-2/Flk-1 expression gradually increased in trained mice (T15, T30, and T45) than control ones. Moreover, VEGFR-2/FIk-1 expression was higher in groups T30 and T45 than T15 ones and in T45 mice than T30 ones ( ${ }^{*} p<0.05, T 15, T 30$ and T45 vs. C; $\# p<0.05$, T30 and T45 vs. T15; $\$ p<0.05, T 45$ vs. T30).

control mice (T15: $1.40 \pm 0.11$ vs. C: $1.24 \pm 0.05$, T30: $1.24 \pm 0.12$, and T45: $1.26 \pm 0.10 ; p<0.05)$. Moreover, control groups, T30, and T45 showed the same expression (Figure 3B).

\section{Analysis of iNOS Cardiac Expression Related to Physical Exercise}

In Figure 1, we observe that iNOS protein was expressed in the cells of interstitial connective tissue and smooth muscle of the hearts from T30 mice. The same localization was found in hearts from T15, T45, and control animals (data not shown). Evaluation of iNOS expression (Figures 4A,B) showed a significantly higher level in T15 and T30 groups than in C and T45 animals (T15:
$0.48 \pm 0.01$ and T30: $0.92 \pm 0.02$ vs. C: $0.23 \pm 0.009$ and T45: $0.36 \pm 0.01)$. Furthermore, no significant difference was detected between T45 and C animals. As samples from C, T15, T30, and T45 did not significantly differ within the same group, a representative sample from each group is shown (Figure 4).

\section{DISCUSSION}

In this study, we examined the relationship between VEGFR-1/ Flt-1, VEGFR-2/Flk-1, HIF-1 $\alpha$, iNOS expression and angiogenesis in heart in response to low, moderate, and high exercise intensity. 


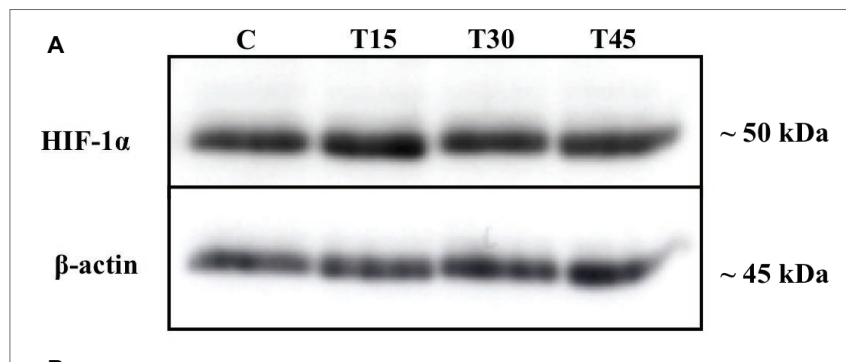

B

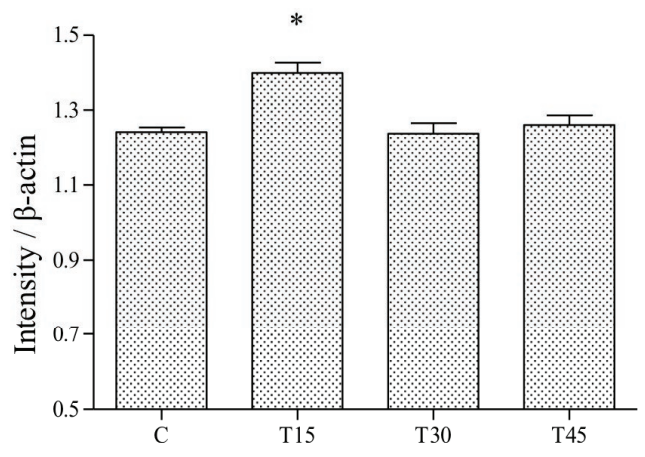

FIGURE 3 | HIF-1 $\alpha$ expression was evaluated by Western blotting analysis (A) in hearts from trained and control mice and its intensity measured with an image software (B). HIF-1 $\alpha$ levels resulted significantly higher in T15 than in C, T30, and T45 groups ( ${ }^{*} p<0.05, \mathrm{~T} 15$ vs. C, T30, and T45).

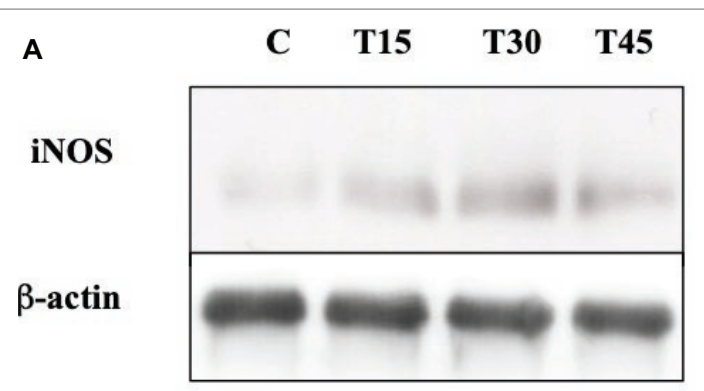

B

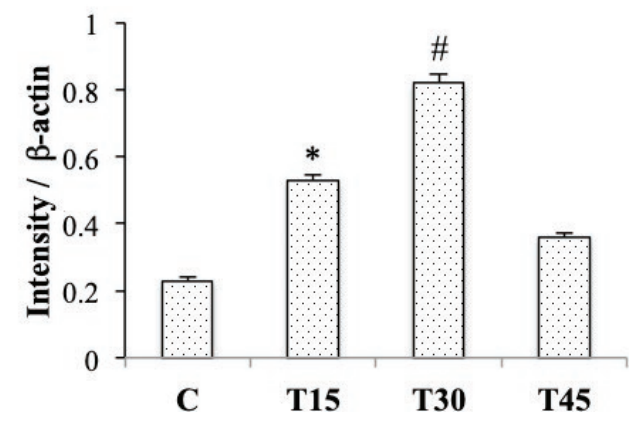

FIGURE 4 | Western blotting (A) and quantitative analyses (B) were performed to measure iNOS expression in hearts from trained and control mice. iNOS protein levels were significantly higher in T15 and in T30 than in control and T45 mice ( ${ }^{*} p<0.05, \mathrm{~T} 15$ vs. $C$ and T45; $\# p<0.05$, T30 vs. $C$ and T45).

To this end, we carried out the analyses in myocardial tissue samples in which we previously found a relevant increase in the capillary proliferation following 30 and 45 days of moderate and high intensity endurance training, respectively (Bellafiore et al., 2007, 2013). The main findings in the present study were that the expression levels of the examined molecules were dissimilar according to the neocapillarization extent and physical exercise intensity. Human epidemiological and animal studies have shown that a moderate to high intensity exercise is the best quantity that results in a reduction in the cardiovascular risk (Alleman et al., 2015). A recent paper reported that high intensity treadmill exercise training for up to 4 weeks produced greater increases in new capillary formation than low intensity exercise training in rat hearts (Waring et al., 2014). However, results concerning the regulation of VEGFR-1/Flt-1, VEGFR-2/Flk-1, HIF-1 $\alpha$, and iNOS expression by exercise different intensities in healthy heart angiogenesis are limited.

Several studies reported an increase in VEGFR-1/Flt-1 and VEGFR-2/Flk-1 expression after 8 weeks of swim training or 10 weeks of treadmill running in rat heart angiogenesis (Iemitsu et al., 2006; Marini et al., 2008). In our study, VEGFR-1/Flt-1 and VEGFR-2/Flk-1 proteins were specifically expressed in the vascular endothelial cells of myocardium and differently regulated by intensity and length of the endurance training. In particular, VEGFR-1/Flt-1 expression increased in T15 mice and reduced in T30 and T45 animals exhibiting the same level as the controls. On the other hand, VEGFR-2/Flk-1 expression gradually increased in mice trained for 15,30 , and 45 days. This dissimilar pattern of expression suggests different roles of these receptors in the angiogenesis process such as also reported by Shibuya (2006). According to this author, VEGFR-1/Flt-1 is a negative regulator for angiogenesis during embryogenesis, while it stimulates inflammation, tumor growth, and metastasis in adulthood. By contrast, VEGFR-2/Flk-1 transduces the major signals for angiogenesis. In effect, in old age myocardium, this receptor promotes an angiogenic cascade through phosphorylation of Akt and activation of endothelial nitric oxide (eNOS) pathway in response to exercise training (Iemitsu et al., 2006). Furthermore, Milkiewicz et al. (2003) showed a good spatial and temporal correlation between VEGFR2/Flk-1 protein levels and capillary growth induced by muscle activity. These findings are in agreement with our data that showed a similar pattern between VEGFR-2/Flk-1 expression and capillary proliferation in hearts of trained mice suggesting receptor's involvement in the proliferation of vascular endothelial cells in response to endurance exercise (Bellafiore et al., 2007, 2013).

The upregulation of VEGFR-1/Flt-1 after 15 days of exercise training might be explained by the request of this factor in the early steps of angiogenic sprouting when endothelial cell migration rather than cell proliferation is necessary. This hypothesis is supported by the same expression pattern between VEGFR-1/ Flt-1 and MMP-9 pro-enzyme in exercise-related cardiac angiogenesis (Bellafiore et al., 2013). Moreover, Waltenberger et al. (1994) showed that the activation of VEGFR-2/Flk-1 receptor by VEGF in cells devoid of VEGFR-1/Flt-1 resulted in a mitogenic response, while the activation of VEGFR-1/Flt-1 by VEGF did not induce cell proliferation in cells lacking VEGFR-2/Flk-1.

Hypoxia has been demonstrated to be a potent stimulus for the activation of HIF-1a protein generating capillary growth in heart tissue (Shweiki et al., 1992; Brown, 2003; Prior et al., 
2004) through the activation of VEGF pathway genes, including VEGFR-1/Flt-1 but not VEGFR-2/Flk-1 (Gerber et al., 1997). In our study, we detected that HIF-1 $\alpha$ was specifically expressed in the nucleus of vascular endothelial cells and myocardiocytes from both sedentary and trained mouse hearts. The expression of this protein under normoxic conditions was confirmed in the myocardium also by other authors (Stroka et al., 2001; Giusti et al., 2009). This finding might be due to a basal induction of genes that are necessary to providing cellular energy requirements. We found that HIF-1a expression significantly increased in mice trained for 15 days and reduced in animals trained for 30 and 45 days reaching the same level as the controls. The reduced expression in T30 and T45 animals might be explained by an increase in the supply of blood flow and $\mathrm{O}_{2}$ in the heart as a consequence of an increase in the capillary area, as shown in our previous study (Bellafiore et al., 2007). On the contrary, in the hearts of T15 mice that did not show a significantly increased capillary area, brief and transient episodes of hypoxia might occur during the cardiac cycle in response to exercise and determine the upregulation of HIF-1a as shown in other studies (Stroka et al., 2001; Brown, 2003; Marini et al., 2008; Giusti et al., 2009). The increased HIF-1a expression is indicative for an involvement of hypoxia signaling in the exercise-induced upregulation of VEGF receptors. Comparing the expression of HIF-1 $\alpha$ and VEGFR-1/Flt-1, we noted that their patterns are similar postulating an upregulation of VEGFR-1 by HIF-1 $\alpha$ after 15 days of endurance training in agreement with Gerber et al. (1997).

Nitric oxide (NO) as well as hypoxia has been reported to upregulate VEGF gene by enhancing HIF-1 $\alpha$ activity and significantly contributing to the prosurvival/proangiogenic program of capillary endothelium (Ziche and Morbidelli, 2000). Indeed, intermittent hypoxia has been described to induce protective effects against myocardial infarction in rodents via a signaling mechanism that depends upon iNOS (Tekin et al., 2010). Furthermore, Akita et al. (2007) showed that an increased expression of iNOS by eNOS upregulation was associated with late cardioprotection against ischemiareperfusion injury in mouse hearts after 7 days of treadmill exercise at a 60-70\% maximal oxygen uptake. In our study, iNOS cardiac expression was higher than corresponding controls in response to mild and moderate exercise intensity, and in our best knowledge, this is the first study that reports the involvement of iNOS in healthy heart neocapillarization according to the exercise intensity.

In the light of the increased expression of iNOS and HIF-1a in T15 hearts, we propose iNOS upregulation by HIF-1a protein in the angiogenic process in agreement with the study of Jung et al. (2000). In fact, these authors exhibited that HIF-1 $\alpha$-binding site was required for transcriptional activation of iNOS gene in rat cardiomyocytes under hypoxic conditions. In our study, the localization of HIF1a and iNOS in different cell types suggests a cross talk among myocardium, vasculature, and connective tissue to promote capillary growth related to exercise in a paracrine fashion.

$\mathrm{NO}$ also participates in the regulation of VEGFR-1/flt-1 gene in response to exercise as shown by NOS inhibition that reduced the exercise-induced increase in VEGFR-1/flt-1 mRNA in rat skeletal muscle (Gavin and Wagner, 2002). Therefore, in our experimental model, the upregulation of iNOS, HIF-1 $\alpha$, and VEGFR-1/flt-1 in T15 mouse hearts suggests the involvement of iNOS in the angiogenesis process related to endurance training.

A limitation of this study is the lack of data coming from the expression of circulating angiogenic factors produced by other organs that could be associated with the exercise-related adaptive effects of cardiac muscle. The release of circulating factors by the exercising skeletal muscle might have a role in supporting angiogenesis of healthy or pathological hearts for prevention and rehabilitation of heart failure (Di Raimondo et al., 2017). Recently, it has been reported that skeletal muscle cells communicate with heart cells in response to contraction or exercise training through the endocrine secretion of myokines (Giudice and Taylor, 2017). In particular, plasma levels of follistatin-like 1 (FSTL-1) glycoprotein increased after strength training (Norheim et al., 2011). Moreover, the administration of this myokine caused an increase in the number of dividing cardiomyocytes and angiogenesis in the peri-infarct region of mouse and swine heart (Wei et al., 2015). On the contrary, systemic levels of VEGF were frequently unaltered in response to exercise, pointing toward mainly local effects (Landers-Ramos et al., 2014).

Although the promising results of Anversa's studies on the application of stem cells for the repair of the infarcted heart region have been retracted, the activation of resident or circulating stem/precursor cells by physical exercise might induce a cardioprotective phenotype against insults (Bellafiore et al., 2006). Indeed, recently, an increase in number and activity state of these cells was discovered in exercised hearts, resulting in an amplified expression of transcription factors involved in the differentiation toward either the cardiomyocyte or capillary lineages. Moreover, these adaptations have been observed to be dependent on exercise duration and intensity (Waring et al., 2014). In skeletal muscle, the number of satellite cells is positively correlated to the capillarization of the myofiber, and angiogenesis results to be essential for muscle repair (Mounier et al., 2011). Therefore, increasing knowledge about molecular effectors of cardiac capillary growth related to exercise can be relevant in pre-clinical and clinical studies to generate native and synthetic biomaterials or three-dimensional structures able to induce stem cell differentiation and which might be used in pre-clinical and clinical studies (Di Felice et al., 2015).

In conclusion, the present study shows that VEGFR-1/ Flt-1, VEGFR-2/Flk-1, HIF-1 $\alpha$, and iNOS are involved in the cardiac angiogenesis, and their expression is regulated by the intensity of endurance training. In detail, the expression of VEGFR-1/Flt-1 and HIF-1 $\alpha$ is upregulated by an exercise mild intensity, while VEGFR-2/Flk-1 level progressively enhances with increasing workload. Differently, iNOS protein is modulated by a moderate intensity exercise. In the light of our results, we retain HIF-1a as a central regulator of exercise-induced angiogenesis pathway in the myocardium. HIF-1a might act as an upstream regulator of VEGFR-1 and iNOS in modulating cell proliferation and vascular 
relaxation. VEGF pathway appears to be involved in this regulation mechanism, and VEGF might act on heart endothelial cells in a paracrine and endocrine manner. Our endurance protocol was formulated for training human subjects and adapted to mice according to their body weight; therefore, understanding the molecular basis for exercise-induced angiogenesis is important in developing exercise strategies to protect the heart by insults.

\section{DATA AVAILABILITY}

All datasets generated for this study are included in the manuscript and/or the supplementary files.

\section{REFERENCES}

Akita, Y., Otani, H., Matsuhisa, S., Kyoi, S., Enoki, C., Hattori, R., et al. (2007). Exercise-induced activation of cardiac sympathetic nerve triggers cardioprotection via redox-sensitive activation of eNOS and upregulation of iNOS. Am. J. Physiol. Heart Circ. Physiol. 292, H2051-H2059. doi: 10.1152/ajpheart.01102.2006

Alleman, R. J., Stewart, L. M., Tsang, A. M., and Brown, D. A. (2015). Why does exercise "Trigger" adaptive protective responses in the heart? DoseResponse 13, 1-19. doi: 10.2203/dose-response.14-023.Alleman

Anversa, P., Kajstura, J., Rota, M., and Leri, A. (2013). Regenerating new heart with stem cells. J. Clin. Invest. 123, 62-70. doi: 10.1172/JCI63068

Bellafiore, M., Battaglia, G., Bianco, A., Farina, F., Palma, A., and Paoli, A. (2013). The involvement of MMP-2 and MMP-9 in heart exercise-related angiogenesis. J. Transl. Med. 11:283. doi: 10.1186/1479-5876-11-283

Bellafiore, M., Sivverini, G., Cappello, F., David, S., Palma, A., Farina, F., et al. (2006). Research of cardiomyocyte precursors in adult rat heart. Tissue Cell 38, 345-351. doi: 10.1016/j.tice.2006.08.003

Bellafiore, M., Sivverini, G., Palumbo, D., Macaluso, F., Bianco, A., Palma, A., et al. (2007). Increased $\mathrm{cx} 43$ and angiogenesis in exercised mouse hearts. Int. J. Sports Med. 28, 749-755. doi: 10.1055/s-2007-964899

Beltrami, A. P., Urbanek, K., Kajstura, J., Yan, S. M., Finato, N., Bussani, R., et al. (2001). Evidence that human cardiac myocytes divide after myocardial infarction. N. Engl. J. Med. 344, 1750-1757. doi: 10.1056/NEJM200106073442303

Brown, M. D. (2003). Exercise and coronary vascular remodelling in the healthy heart. Exp. Physiol. 88, 645-658. doi: 10.1113/eph8802618

Di Felice, V., Forte, G., and Coletti, D. (2015). Biomaterials and bioactive molecules to drive differentiation in striated muscle tissue engineering. Front. Physiol. 6:52. doi: 10.3389/fphys.2015.00052

Di Felice, V., Macaluso, F., Montalbano, A., Gammazza, A. M., Palumbo, D., Angelone, T., et al. (2007). Effects of conjugated linoleic acid and endurance training on peripheral blood and bone marrow of trained mice. J. Strength Cond. Res. 21, 193-198. doi: 10.1519/00124278-200702000-00035

Di Raimondo, D., Miceli, G., Musiari, G., Tuttolomondo, A., and Pinto, A. (2017). New insights about the putative role of myokines in the context of cardiac rehabilitation and secondary cardiovascular prevention. Ann. Transl. Med. 5:300. doi: 10.21037/atm.2017.07.30

Drazen, J. M. (2018). Evidence that human cardiac myocytes divide after myocardial infarction. N. Engl. J. Med. 379:1870. doi: 10.1056/NEJMe1813801

Dyer, O. (2018). NEJM retracts article from former researcher once hailed as heart stem cell pioneer. BMJ 363:k4432. doi: 10.1136/bmj.k4432

Fulghum, K., and Hill, B. G. (2018). Metabolic mechanisms of exercise-induced cardiac remodeling. Front. Cardiovasc. Med. 5:127. doi: 10.3389/fcvm.2018.00127

Gavin, T. P., and Wagner, P. D. (2002). Attenuation of the exercise-induced increase in skeletal muscle Flt-1 mRNA by nitric oxide synthase inhibition. Acta Physiol. Scand. 175, 201-209. doi: 10.1046/j.1365-201X.2002.00987.x

Gerber, H. P., Condorelli, F., Park, J., and Ferrara, N. (1997). Differential transcriptional regulation of the two vascular endothelial growth factor receptor genes. Flt-1, but not Flk-1/KDR, is upregulated by hypoxia. J. Biol. Chem. 272, 23659-23667. doi: 10.1074/jbc.272.38.23659

\section{AUTHOR CONTRIBUTIONS}

MB was responsible for study design, interpretation of data, and draft of manuscript. GB carried out data acquisition and participated in conceiving of the study and drafting manuscript. $\mathrm{AB}$ participated in exercise training sessions. AP participated in conceiving of the study and drafting manuscript. All authors read and approved the final manuscript.

\section{FUNDING}

This work was supported by a grant of Prof. A. Palma, PJ_CT_D12_Muovitem_0001.

Giudice, J., and Taylor, J. M. (2017). Muscle as a paracrine and endocrine organ. Curr. Opin. Pharmacol. 34, 49-55. doi: 10.1016/j.coph.2017.05.005

Giusti, B., Marini, M., Rossi, L., Lapini, I., Magi, A., Capalbo, A., et al. (2009). Gene expression profile of rat left ventricles reveals persisting changes following chronic mild exercise protocol: implications for cardioprotection. BMC Genomics 10:342. doi: 10.1186/1471-2164-10-342

Hoffmann, C., and Weigert, C. (2017). Skeletal muscle as an endocrine organ: the role of myokines in exercise adaptations. Cold Spring Harb. Perspect. Med. 7:a029793. doi: 10.1101/cshperspect.a029793

Hudlicka, O., Brown, M. D., Walter, H., Weiss, J. B., and Bate, A. (1995). Factors involved in capillary growth in the heart. Mol. Cell. Biochem. 147, 57-68. doi: 10.1007/BF00944784

Iemitsu, M., Maeda, S., Jesmin, S., Otsuki, T., and Miyauchi, T. (2006). Exercise training improves aging-induced downregulation of VEGF angiogenic signaling cascade in hearts. Am. J. Physiol. Heart Circ. Physiol. 291, H1290-H1298. doi: 10.1152/ajpheart.00820.2005

Jung, F., Palmer, L. A., Zhou, N., and Johns, R. A. (2000). Hypoxic regulation of inducible nitric oxide synthase via hypoxia inducible factor-1 in cardiac myocytes. Circ. Res. 86, 319-325. doi: 10.1161/01.RES.86.3.319

Landers-Ramos, R. Q., Jenkins, N. T., Spangenburg, E. E., Hagberg, J. M., and Prior, S. J. (2014). Circulating angiogenic and inflammatory cytokine responses to acute aerobic exercise in trained and sedentary young men. Eur. J. Appl. Physiol. 114, 1377-1384. doi: 10.1007/s00421-014-2861-6

Marini, M., Falcieri, E., Margonato, V., Treré, D., Lapalombella, R., Di Tullio, S., et al. (2008). Partial persistence of exercise-induced myocardial angiogenesis following 4-week detraining in the rat. Histochem. Cell Biol. 129, 479-487. doi: 10.1007/s00418-007-0373-8

Marongiu, E., and Crisafulli, A. (2014). Cardioprotection acquired through exercise: the role of ischemic preconditioning. Curr. Cardiol. Rev. 10, 336-348. doi: 10.2174/1573403X10666140404110229

Milkiewicz, M., Hudlicka, O., Verhaeg, J., Egginton, S., and Brown, M. D. (2003). Differential expression of Flk-1 and Flt-1 in rat skeletal muscle in response to chronic ischaemia: favourable effect of muscle activity. Clin. Sci. 105, 473-482. doi: 10.1042/CS20030035

Mounier, R., Chrétien, F., and Chazaud, B. (2011). Blood vessels and the satellite cell niche. Curr. Top. Dev. Biol. 96, 121-138. doi: 10.1016/ B978-0-12-385940-2.00005-X

Norheim, F., Raastad, T., Thiede, B., Rustan, A. C., Drevon, C. A., and Haugen, F. (2011). Proteomic identification of secreted proteins from human skeletal muscle cells and expression in response to strength training. Am. J. Physiol. Endocrinol. Metab. 301, E1013-E1021. doi: 10.1152/ajpendo.00326.2011

Pluim, B. M., Zwinderman, A. H., van der Laarse, A., and van der Wall, E. E. (2000). The athlete's heart. A meta-analysis of cardiac structure and function. Circulation 101, 336-344. doi: 10.1161/01.CIR.101.3.336

Prior, B. M., Yang, H. T., and Terjung, R. L. (2004). What makes vessels grow with exercise training? J. Appl. Physiol. 97, 1119-1128. doi: 10.1152/ japplphysiol.00035.2004

Shibuya, M. (2006). Differential roles of vascular endothelial growth factor receptor-1 and receptor-2 in angiogenesis. J. Biochem. Mol. Biol. 39, 469-478. 
Schuler, G., Adams, V., and Goto, Y. (2013). Role of exercise in the prevention of cardiovascular disease: results, mechanisms, and new perspectives. Eur. Heart J. 3, 1790-1799. doi: 10.1093/eurheartj/eht111

Shweiki, D., Itin, A., Soffer, D., and Keshet, E. (1992). Vascular endothelial growth factor induced by hypoxia may mediate hypoxia-initiated angiogenesis. Nature 359, 843-845. doi: 10.1038/359843a0

Stroka, D. M., Burkhardt, T., Desbaillets, I., Wenger, R. H., Neil, D. A., Bauer, C., et al. (2001). HIF-1 is expressed in normoxic tissue and displays an organ-specific regulation under systemic hypoxia. FASEB J. 15, 2445-2453. doi: 10.1096/fi.01-0125com

Tao, L., Bei, Y., Zhang, H., Xiao, J., and Li, X. (2015). Exercise for the heart: signaling pathways. Oncotarget 6, 20773-20784. doi: 10.18632/ oncotarget. 4770

Tekin, D., Dursun, A. D., and Xi, L. (2010). Hypoxia inducible factor 1 (HIF-1) and cardioprotection. Acta Pharmacol. Sin. 31, 1085-1094. doi: 10.1038/ aps. 2010.132

Waltenberger, J., Claessonwelsh, L., Siegbahn, A., Shibuya, M., and Heldin, C. H. (1994). Different signal transduction properties of KDR and Flt1, two receptors for vascular endothelial growth factor. J. Biol. Chem. 269, 26988-26995.
Waring, C. D., Vicinanza, C., Papalamprou, A., Smith, A. J., Purushothaman, S., Goldspink, D. F., et al. (2014). The adult heart responds to increased workload with physiologic hypertrophy, cardiac stem cell activation, and new myocyte formation. Eur. Heart J. 35, 2722-2731. doi: 10.1093/eurheartj/ehs338

Wei, K., Serpooshan, V., Hurtado, C., Diez-Cunado, M., Zhao, M., Maruyama, S., et al. (2015). Epicardial FSTL1 reconstitution regenerates the adult mammalian heart. Nature 525, 479-485. doi: 10.1038/nature15372

Ziche, M., and Morbidelli, L. (2000). Nitric oxide and angiogenesis. J. NeuroOncol. 50, 139-148.

Conflict of Interest Statement: The authors declare that the research was conducted in the absence of any commercial or financial relationships that could be construed as a potential conflict of interest.

Copyright (c) 2019 Bellafiore, Battaglia, Bianco and Palma. This is an open-access article distributed under the terms of the Creative Commons Attribution License (CC BY). The use, distribution or reproduction in other forums is permitted, provided the original author(s) and the copyright owner(s) are credited and that the original publication in this journal is cited, in accordance with accepted academic practice. No use, distribution or reproduction is permitted which does not comply with these terms. 
OPEN ACCESS

Edited by:

Dario Coletti,

Sapienza University of Rome, Italy

Reviewed by:

Jennifer Stevenson Moylan, University of Kentucky, United States Emanuele Berardi,

VIB-KU Leuven Center for Cancer Biology, Belgium

*Correspondence:

Rosanna Piccirillo rosanna.piccirillo@marionegri.it

Specialty section: This article was submitted to

Striated Muscle Physiology, a section of the journal Frontiers in Physiology

Received: 21 December 2018 Accepted: 04 March 2019

Published: 29 March 2019

Citation:

Piccirillo R (2019) Exercise-Induced Myokines With Therapeutic Potential for Muscle Wasting. Front. Physiol. 10:287. doi: 10.3389/fphys.2019.00287

\section{Exercise-Induced Myokines With Therapeutic Potential for Muscle Wasting}

\author{
Rosanna Piccirillo* \\ Department of Neurosciences, Mario Negri Institute for Pharmacological Research IRCCS, Milan, Italy
}

Skeletal muscle is a highly vascularized tissue that can secrete proteins called myokines. These muscle-secreted factors exert biological functions in muscle itself (autocrine effect) or on short- or long-distant organs (paracrine/endocrine effects) and control processes such as metabolism, angiogenesis, or inflammation. Widely differing diseases ranging from genetic myopathies to cancers are emerging as causing dysregulated secretion of myokines from skeletal muscles. Myokines are also involved in the control of muscle size and may be important to be restored to normal levels to alleviate muscle wasting in various conditions, such as cancer, untreated diabetes, chronic obstructive pulmonary disease, aging, or heart failure. Interestingly, many myokines are induced by exercise (muscle-derived exerkines) and some even by specific types of physical activity, but more studies are needed on this issue. Most exercise-induced myokines travel throughout the body by means of extracellular vesicles. Restoring myokines by physical activity may be added to the list of mechanisms by which exercise exerts preventative or curative effects against a large number of diseases, including the deleterious muscle wasting they may cause. Extending our understanding about which myokines could be usefully restored in certain diseases might help in prescribing more tailored exercise or myokine-based drugs.

Keywords: myokines, exercise, exerkines, skeletal muscles, atrophy

\section{INTRODUCTION}

Skeletal muscle is the largest tissue in the human body, accounting for about $30 \%$ of body mass in women and $40 \%$ in men. Although, it can suffer in many incurable diseases, it is unexpectedly the most "undrugged" tissue. Skeletal muscle is very plastic because it can be enlarged with adaptation to specific exercise and/or high-protein content diet or reduced in catabolic conditions like fasting, cancer, untreated diabetes, heart failure, AIDS, chronic obstructive pulmonary disease (COPD), or aging (Piccirillo et al., 2014; Furrer and Handschin, 2018). This tissue is the main body's protein reservoir, able to break down its own proteins and release aminoacids into the bloodstream during stress or fasting or disease. This process goes under the name of muscle wasting or cachexia, and ultimately aggravates the disease state, even leading individuals to death (Kalantar-Zadeh et al., 2013). Losing more than about $40 \%$ of normal body mass was incompatible with life in starved, lager-confined Jews (Winick, 1979) and in HIV-infected patients before the successful advent of antiretroviral therapy (Kotler et al., 1989; Roubenoff and Kehayias, 1991). 
Reduction in muscle size mainly consists of a decrease in cell size caused by loss of organelles, cytosol and proteins. This deleterious process is mainly driven by increased protein breakdown through enhanced proteasomal and lysosomal activities (coordinatively activated by FoxO3-dependent transcription) (Sandri et al., 2004; Zhao et al., 2007) and reduced protein synthesis (mainly regulated by the IGF-1/PI3K/AKT pathway). During muscle atrophy, the removal of ATP-producing organelles, such as mitochondria, through enhanced autophagy (i.e., mitophagy) explains the increased propensity to fatigue of muscle-losing patients (VanderVeen et al., 2017).

Physical exercise is among the ways by which muscles may be protected against disease-induced muscle wasting. Physical activity can be grossly divided into two types: aerobic (or endurance) and anaerobic (or strength) exercise. The former shifts muscle fiber types toward those with increased capacity for aerobic metabolism and better ability to resist fatigue, due to a larger number of mitochondria and vessels (types I and IIA fibers), this is typical in runners, long-distance cross-country skiers, bicyclists, swimmers. These fibers contract slowly with a low peak force and generate ATP through oxidative phosphorylation of glucose and non-esterified fatty acids. The second type of exercise causes hypertrophy, especially of myofibers IIX (and IIB in rodents), due to enhanced synthesis of contractile proteins, resulting in increased strength with no change in the number of mitochondria (typically occurring in weight-lifters and body-builders). These fibers generate fast contractions with a high peak force, and metabolize phosphocreatine and glucose anaerobically to make ATP (Flück and Hoppeler, 2003; Hawley et al., 2014).

Aerobic exercise induces in humans (Pilegaard et al., 2003) and in rodents (Terada et al., 2002) the peroxisome proliferatoractivated receptor $\gamma$ coactivator $1-\alpha(P G C 1-\alpha)$. PGC1- $\alpha$ not only promotes mitochondrial biogenesis, contrasting the propensity to fatigue of cachectic muscles, but also directly antagonizes protein catabolism by blocking FoxO3 (Sandri et al., 2006), the master transcription factor coordinating both proteasomal and lysosomal protein degradation (Sandri et al., 2004; Mammucari et al., 2007; Zhao et al., 2007). As a result, oxidative fibers are more resistant to cancer-induced atrophy than glycolytic ones, typically enlarged upon muscle adaptation to anaerobic exercise (Ciciliot et al., 2013). Conversely, anaerobic exercise, by stimulating mainly myofibrillar protein synthesis through activation of the PI3K/AKT pathway and overproduction of IGF1 (McCall et al., 2003), may obviate muscle wasting by enhancing protein synthesis and inactivating FoxO3 through AKT-mediated phosphorylation (Sandri et al., 2004).

It is becoming clear that physical activity mediates the release from muscles of factors with anti-atrophic effects in an autocrine fashion but, surprisingly, these muscle-derived molecules can even contrast the primary disease, as is the case of the recently identified anti-tumoral molecules of muscle origin (Aoi et al., 2013; Gannon et al., 2015). Some examples are oncostatin M, able to restrain mammary cancer cell growth in vitro (Hojman et al., 2011), fatigue substance (F-Substance) isolated from muscles of trained rats and displaying inhibitory effect on the breast cancer cell line MCF-7 (Munoz et al., 2013) and secreted protein acidic and rich in cysteine (SPARC). The latter is induced in plasma of trained individuals or mice and it is able to suppress colon tumorigenesis via regular exercise in mice (Aoi et al., 2013).

Skeletal muscle is a highly vascularized tissue and has secretory abilities (Pedersen and Febbraio, 2008). In fact, muscles release not only aminoacids in response to increased energy demand and fuel the liver to undergo gluconeogenesis, but also proteins to mediate inter-tissue crosstalk. These molecules have been named myokines to underline their muscle origin. The current definition of myokines is "cytokines or peptides which are secreted by skeletal muscle cells and subsequently released into the circulation to exert endocrine or paracrine effects in other cells, tissues or organs" (Pedersen and Febbraio, 2012). Not all myokines are exclusively originating by skeletal muscles. Some myokines are mainly muscle-restricted proteins, like myostatin, while others can also be secreted by other tissues, as is the case of the adipomyokines (for example, IL-8 and MCP-1) (Trayhurn et al., 2011). However, skeletal muscle is probably the main source of most myokines secreted also by other tissues in the circulation because it is amply vascularized and makes up $30-40 \%$ of the human body mass.

More than 3000 myokines have been reported (Whitham and Febbraio, 2016) and, among them, it is worth mentioning those identified in humans: angiopoietin-like 4 (ANGPTL4), apelin, brain-derived neurotrophic factor (BDNF), CCL2 or MCP-1, CX3CL1 of fractalkine (FKN), fibroblast growth factor 21 (FGF21), interleukin-6 (IL-6), IL-7, IL-8, IL-15, irisin, leukemia inhibitory factor (LIF), meteorin-like protein (Metrnl), myostatin and SPARC (for a review see Catoire and Kersten, 2015). Myokine secretion is a process conserved among species: Drosophila melanogaster (Zhao and Karpac, 2017), adult Danio rerio (i.e., Zebrafish) (Rovira et al., 2017), and mammals (Demontis et al., 2013b), among others, all have muscles able to release such factors. The first reported myokine, Interleukin-6 (IL-6), is conserved in Drosophila melanogaster where three orthologs exist, Unpaired (Upd) also called Outstretched, Upd2 and Upd3, increases with exercise (Ostrowski et al., 2000) and has antiinflammatory properties, at least in mammals (Pedersen and Febbraio, 2008). Another example is myostatin that is secreted by skeletal muscles and can stimulate activin type II receptors (ActRII), ultimately leading to muscle atrophy; it is conserved in Drosophila melanogaster (myoglianin) and in Zebrafish (myostatin b). Notably, myostatin inactivation by spontaneous missense mutations or intentional genetic ablation results in hypermuscularity in a large number of species (McPherron and Lee, 1997; McPherron et al., 1997; Prontera et al., 2009).

Some myokines are preferentially secreted by glycolytic fibers [e.g., musclin (Banzet et al., 2007; Subbotina et al., 2015), osteoprotegerin and angiogenin (Rutti et al., 2018)], and others by oxidative fibers [e.g., myonectin (Seldin et al., 2012)] but the fiber-type preference, if it exists, is unknown for most of the other myokines. Along the same line, FGF21 that is an hormonelike molecule involved in metabolism has been described as an AKT-regulated myokine (Izumiya et al., 2008a; He et al., 2018), hence, more typically secreted by glycolytic fibers, while irisin expression is induced by PGC1 $\alpha$, thus, more typically secreted by oxidative ones (Boström et al., 2012). Some myokines have been renamed muscle-derived exerkines (Safdar and Tarnopolsky, 2018) because they are induced by physical activity and - most 
interestingly - some of them show a preference for muscle induction through specific types of physical exercise (Figure 1A) (Kanzleiter et al., 2014; Bazgir et al., 2015; Shin et al., 2015; Laker et al., 2017; Ishiuchi et al., 2018).
Lecker et al. (2004) found a subset of genes that were differentially expressed in muscles undergoing wasting in unrelated conditions (fasting, uremia, tumor, and diabetes) in rodent models and named them "atrogenes." Among them,

A

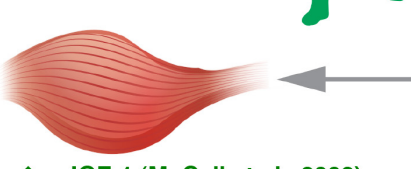

$\uparrow$ IGF-1 (McCall et al., 2003)

Myostatin (Walker et al., 2004)

$\uparrow$ Decorin (Kanzleiter et al., 2014)

$\uparrow$ IL-15 (Bazgir et al., 2015)

$\uparrow$ Irisin (Kim et al., 2015)

^ BMP7 (Kim et al., 2016)

$\uparrow$ Angiopoietin-like 4 or ANGPL4 (Laker et al., 2017)

FGF21 (He et al., 2018)
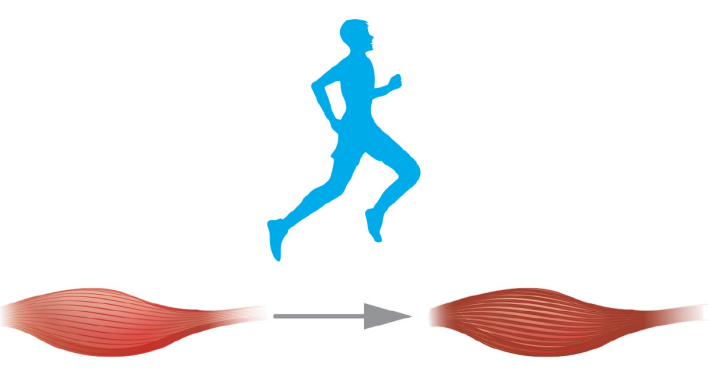

$\uparrow \quad$ IL-6 (Ostrowski et al., 1998)

$\uparrow \quad$ LIF (Broholm et al., 2008)

$\uparrow \operatorname{VEGF}$ (Arany et al., 2008)

$\downarrow$ Myostatin (Hittel et al., 2010)

$\uparrow \quad$ Irisin (Boström et al., 2012)

$\uparrow$ Myonectin (Seldin et al., 2012)

$\uparrow$ SPARC (Aoi et al., 2013)

$\uparrow$ Apelin (Besse-Patin et al., 2014)

$\uparrow$ CCL2 or MCP-1 (Catoire et al., 2014)

$\uparrow$ CX3CL1 or fractalkine or FKN

(Catoire et al., 2014)

$\uparrow \quad$ IL-15 (Shin et al., 2015)

$\uparrow$ Musclin (Subbotina et al., 2015)

$\uparrow$ SDF1 (Puchert et al., 2016)

$\uparrow$ FGF21 (Tanimura et al., 2016)

$\Downarrow \quad$ CCL5/RANTES (Ishiuchi et al., 2018)

B

$\uparrow$ IL=8 (Amir Levy et at., 2015)

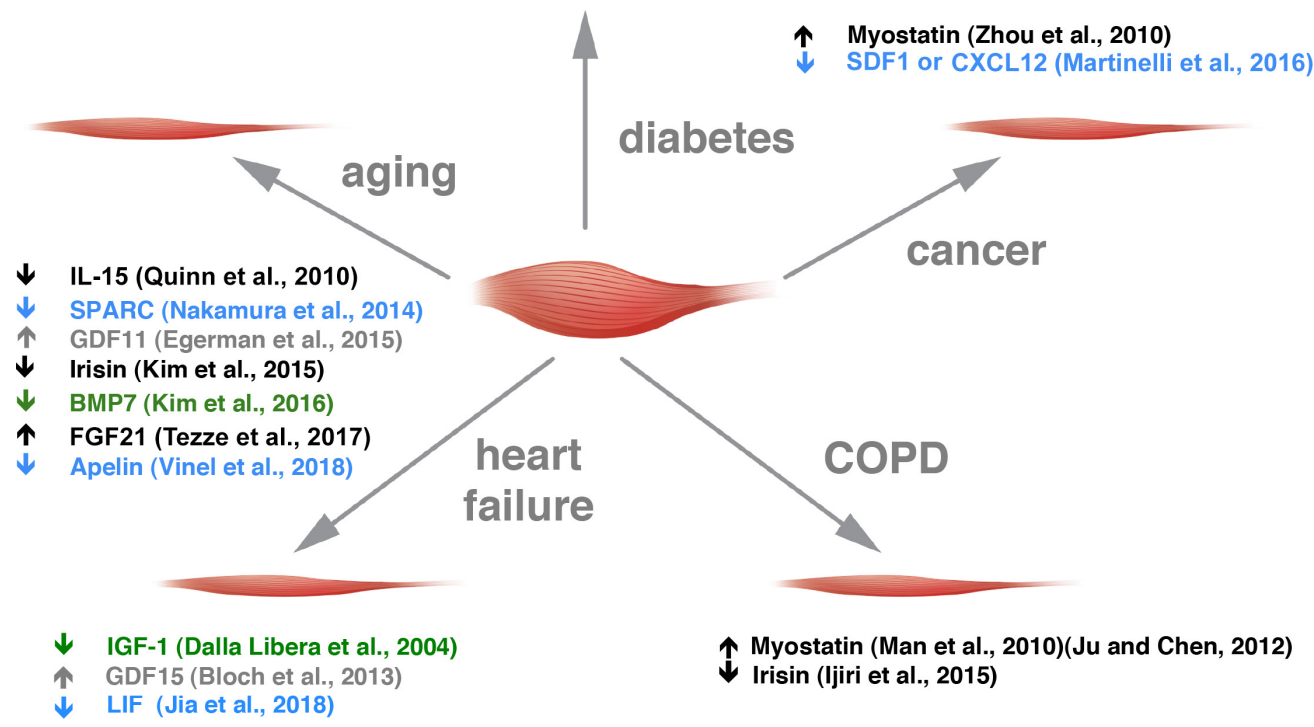

FIGURE 1 | Exercise-sensitive myokines with a preference for aerobic or anaerobic exercise are altered in different muscle wasting disorders. (A) Muscle adaptation to anaerobic and aerobic exercise is grossly depicted. In green, myokines altered in anaerobic exercise are listed with the appropriate references. In blue, those altered by endurance training are reported. (B) Myokine alterations in the five diseases indicated are listed. Myokines sensitive to anaerobic or aerobic exercise are depicted in green and blue, respectively, and those that change in both kinds of exercise in black. Myokines whose alteration by exercise is not clear are indicated in gray. 
a subset of potentially secreted proteins, called "extracellular matrix proteins," were all downregulated in all conditions of muscle atrophy tested and included fibrillin 1, fibronectin 1, galectin 1 , osteoblast specific factor 2 , secreted modular calciumbinding protein 2, and insulin-like growth factor binding protein 5. It would be worth clarifying whether these include some that could be useful to reverse to basal levels to contrast atrophy, and whether they can be classified as myokines or not (i.e., "atromyokines").

Most exercise-induced myokines travel through the body by means of exosomes measuring between 30 and $100 \mathrm{~nm}$ in diameter, or micro- or nano-sized extracellular vesicles (EVs), through analyses in Vesciclepedia and ExoCarta (Safdar and Tarnopolsky, 2018). These muscle-derived EVs increase in number in the bloodstream upon exercise, perhaps through calcium-dependent secretion coupled with muscle contractions (Whitham et al., 2018). In a very elegant work, Whitham et al. (2018) showed that when EV liberated in the blood of healthy individuals after $1 \mathrm{~h}$ bout of cycling exercise were labeled and transferred to donor mice, they were able to target organs like the liver, but not all organs have been scrutinized. The search for exercise factors of muscle origin mediated by these structures has just started and could help explain why myokines are not rapidly degraded by the numerous proteases in the extracellular environment. Their protective lipid bilayers could facilitate them to target muscle-unrelated tissues, affecting overall body homeostasis. This would further help explain how genetic modifications restricted to muscles impact on other tissues (Rai and Demontis, 2016) as the reduced adiposity reported in animals with muscle deficiency of myostatin (McPherron and Lee, 2002) or the increased resistance to diet-induced obesity and steatosis of mice with muscles overexpressing AKT1 (Izumiya et al., 2008b).

The secretion of myokines is altered in a growing number of diseases, including congenital myotonic dystrophy (Nakamori et al., 2017), and modulable through physical activities (Benatti and Pedersen, 2015). This minireview gives an overview of such factors in health and muscle atrophy-causing diseases (Figure 1B) and how physical exercise could correct some of them toward healthier levels, to spare muscle mass.

\section{MYOKINES ALTERED IN DIABETES}

Tissue cross-talk is emerging as vital to coordinate the different organs implicated in glucose homeostasis. Among the crosstalking factors, muscle-secreted myokines can influence the function and survival of pancreatic $\beta$-cells as is the case of angiogenin (previously known as an angiogenesis-promoting factor) (Gao and $\mathrm{Xu}, 2008$ ) and osteoprotegerin (involved also in bone density's regulation) (Simonet et al., 1997) that protect $\beta$ cells from the deleterious effects of proinflammatory cytokines (Rutti et al., 2018).

Diabetes accounts for about $15 \%$ of total diseases related to selected myokines (apelin, BDNF, IL-15, irisin, SPARC) (Son et al., 2018). In adult humans, an insulin-resistant state preceding diabetes onset, already alters myokine secretion, as is the case of increased release of myonectin, but not FGF21 or myostatin
(Toloza et al., 2018), but the biological effects of this increase are not clear yet. In another study, FGF21 was found increased in the plasma of diabetic individuals, but if this is a compensatory mechanism to increase glucose uptake from muscles remains to be explored (Mashili et al., 2011). This myokine was found increased also in human and murine plasma after a single bout of endurance exercise (Tanimura et al., 2016). In streptozotocininduced diabetic mice, myostatin is increased and causes muscle wasting (Hulmi et al., 2012). Molecules able to neutralize this myokine are under tests for the treatment of diabetic myopathy in animal models, but with contradictory results so far (Wang et al., 2015; Dong et al., 2016).

Puzzingly, both CX3CL1 (Shah et al., 2011) and Metrln (Chung et al., 2018) were elevated in the blood of patients afflicted by type II diabetes and also in plasma and/or muscles of individuals after acute endurance exercise (Catoire et al., 2014) or short-term interval training (Eaton et al., 2018), but so far their possible contribution to muscle size control has not been explored. Finally, increased plasma apelin has been found to predict type II diabetes but only in men, for reasons we still do not understand (Ma et al., 2014). While the role of apelin in improving insulin resistance by enhancing glucose utilization by muscle and fat is clear (Dray et al., 2008), its direct ability to protect muscles from diabetes-induced atrophy deserves further studies, but its elevated expression upon aerobic exercise is of note (Besse-Patin et al., 2014). IL-8, a chemokine whose muscle secretion is altered by exercise in humans, though mostly after extreme conditions in response to an ultraendurance exercise bout in experienced athletes (Marklund et al., 2013), was found to be overproduced by myotubes from type II diabetes patients and to disfavor muscle capillarization, reducing muscle exposure to glucose, so possibly further impinging on muscle wasting and the primary disease itself (Amir Levy et al., 2015).

Further studies are needed to see whether and which other myokine(s) is/are altered in muscles undergoing diabetic atrophy (for a further summary on this topic see Carson, 2017), and whether it might be useful to target for the cure of this kind of atrophy.

\section{MYOKINES ALTERED IN CANCER CACHEXIA}

Muscle wasting occurs in $80 \%$ of patients with advanced cancer and causes death in 30-40\% of cases (Fearon et al., 2011). Various myokines may be involved in this kind of atrophy (for a review see Manole et al., 2018). Cancer accounts for only 3.3\% of total diseases related to selected myokines (apelin, BDNF, IL-15, irisin, SPARC) (Son et al., 2018), but additional myokines have been shown to be more clearly involved in cancer cachexia.

We found stromal derived factor 1 (SDF1), also referred to as CXCL12, as specifically and strongly downregulated only in skeletal muscles of tumor-bearing mice and not in those undergoing wasting for other reasons (i.e., uremia, diabetes, fasting, or disuse) (Martinelli et al., 2016). Overexpression of SDF1 in muscle through in vivo electroporation of plasmid spared not only the cross-sectional area of transfected fibers but also that of adjacent untransfected ones, indicating that 
SDF1 also exerts its anti-atrophic effects in a paracrine fashion (Martinelli et al., 2016). Interestingly, Puchert and coworkers found it to be induced in muscles of mice trained by running for 4 weeks (Puchert et al., 2016). This muscle-secreted factor has a role in myogenesis, muscle regeneration (Bobadilla et al., 2014) and angiogenesis, similarly to VEGF (Arany et al., 2008) and could be involved in increased muscle capillarization upon adaptation to aerobic exercise. Overall, elevated muscle secretion of SDF1 could be ascribed to the mechanisms by which aerobic exercise or some drugs (e.g., Sunitinib) alleviate cancer-associated muscle wasting (Pretto et al., 2015).

Myostatin-based signaling was found increased in muscles of Yoshida hepatoma-bearing rats (Costelli et al., 2008), in C26-bearing mice (Zhou et al., 2010), and in patients with various malignancies (for a review see Han et al., 2013), while in pre-cachectic cancer patients circulating levels of myostatin increased only with certain types of tumors (Aversa et al., 2012). Importantly, while myostatin is the main factor limiting muscle size in mice, it seems that in humans activin A and growth differentiation factor 11 (GDF11) also play major roles and could be targeted to spare muscles during human diseases (LachTrifilieff et al., 2014). Anti-ActRII drugs have in fact been tested in cancer patients but unfortunately, there were no improvements in functional parameters (for a review see Furrer and Handschin, 2018). Myostatin is one of the few myokines whose secretion is reduced with both anaerobic (Walker et al., 2004) and aerobic exercise (Hittel et al., 2010), in concordance with its role as a negative modulator of muscle size.

The type of exercise that seems most effective at sparing muscles during cancer seems the aerobic and not the anaerobic one. In fact, the $\mathrm{AKT} / \mathrm{mTOR}$ signaling appears even hyperactivated in muscles of tumor-bearing rodents (Penna et al., 2010). So, future research should focus on understanding which aerobic exercise-induced myokine(s), besides SDF1, have anticatabolic or proanabolic action in muscles during cancer cachexia.

\section{MYOKINES ALTERED IN COPD}

Chronic obstructive pulmonary disease is an inexorable lung disease caused by excessive inflammation and subsequent damage to the airways and lung tissue, mainly occurring after long-term exposure to cigarette smoking (Passey et al., 2016).

Very few myokines have been studied in relation to COPD-induced muscle wasting, even though COPD is the most prevalent disease worldwide causing excessive myopenia and death (Lozano et al., 2012). COPD accounts for about $5 \%$ of total diseases related to selected myokines (apelin, BDNF, IL-15, irisin, SPARC) (Son et al., 2018). Consistent with its role in reducing muscle mass, myostatin expression is increased in the vastus lateralis (Plant et al., 2010) and quadriceps (Man et al., 2010) from COPD patients. Circulating myostatin protein is also elevated in serum from COPD patients and correlates with reduced muscle mass in males (Ju and Chen, 2012). Clinical trials to test bimagrumab (anti-ActRII-based drug) in COPD patients have recently showed increases in lean body mass and muscle volume in treated patients but unfortunately, no improvements in functional parameters (for a review see, Furrer and Handschin, 2018).

Irisin, a prominent PGC1 $\alpha$-induced myokine, is the cleaved product of the transmembrane protein fibronectin type III domain-containing protein 5 (FNDC5) (Boström et al., 2012) and is induced by resistance exercise in mice and humans (Kim et al., 2015). Most importantly, circulating irisin was low in COPD patients and its levels correlate with exercise capacity (Ijiri et al., 2015). More studies are still needed to dissect the mechanisms behind COPD-related muscle atrophy, because efficacious therapies are lacking.

\section{MYOKINES ALTERED IN AGING}

Many alterations occur in skeletal muscle with aging (Demontis et al., 2013a), including enhanced activity of the proteasomal pathway causing muscle atrophy (i.e., sarcopenia) (Altun et al., 2010), but the question whether muscle's ability to secrete myokines changes with aging has only recently started to attract attention. The increased sarcolemmal permeability of aged muscles may favor increased release of myokines during aging. Nonetheless, the expression of bone morphogenetic factor 7 (BMP7) is low in muscles of aged rats but can be restored through either uphill or gradual downhill running (Kim et al., 2016). This myokine may act by stabilizing the neuromuscular junction (NMJ) that is reduced in number and function with aging (for a review see, Demontis et al., 2013b; Larsson et al., 2019). Moreover, BMP7 has been found to cause muscle hypertrophy in mice through Smad1/5-mediated activation of mTOR signaling (Winbanks et al., 2013). Similar results have been obtained for irisin, whose secretion decreases in aging mice and is restored with resistance exercise training (Kim et al., 2015). While myostatin was unchanged in the elderly (Welle et al., 2002), another member of the same transforming growth factor $\beta$ (TGF $\beta$ ) family, GDF11, increases in blood (and muscles) during aging in rats and humans and, even more importantly, inhibits muscle regeneration (Egerman et al., 2015). The secretion of FGF21 also seems dysregulated in aged mice (Tezze et al., 2017).

Apelin is an exerkine that has been recently found to have a major role in counteracting age-associated muscle wasting (Vinel et al., 2018), also by promoting mitochondriogenesis, alleviating muscle-related inflammation and stimulating its regenerative capacity. Its possible role in disease-associated muscle atrophy is still unclear. SPARC is induced in plasma of humans by a single bout of steady-state cycling exercise at $70 \%$ of $\mathrm{VO}_{2}$ max for $30 \mathrm{~min}$, or after 4 weeks of cycling, three times a week for $30 \mathrm{~min}$ at $70 \%$ of $\mathrm{VO}_{2} \mathrm{max}$, and similarly in exercising mice (Aoi et al., 2013). This protein was low in aged muscle because its internalization in skeletal muscle progenitors becomes defective with age, so its anti-adipogenic effect could be limited and may contribute to fat infiltration in muscle upon aging (Nakamura et al., 2014).

Finally, IL-15 and the soluble form of its receptor also decrease in serum of aged mice (Quinn et al., 2010). These findings are in concordance with the single nucleotide polymorphisms (SNP) found in the human gene for such 
receptor that affects the muscularity and exercise capacity of individuals (Pistilli et al., 2008) and may influence interindividual propensity to sarcopenia.

\section{MYOKINES ALTERED IN HEART FAILURE}

Muscle wasting in patients with chronic heart failure (HF) identifies those at high risk of death (Anker et al., 1997). IGF1 decreases in the skeletal muscle of pre-cachectic HF patients (Hambrecht et al., 2002) and in animal models. Treatment with IGF-1 helped contrast the HF-induced muscle atrophy, improving exercise capacity (Dalla Libera et al., 2004).

GDF15 (also known as macrophage-inhibiting factor-1 and involved in appetite regulation) was found elevated in the acute muscle wasting following cardiac surgery and, interestingly, GDF15-treated myotubes undergo wasting, supporting a procachectic role of this factor (Bloch et al., 2013). A recent study showed the effect on muscle expression of an IL-6-like cytokine, LIF, in rats undergoing myocardial infarction. In this model the myocardial infarction-associated muscle wasting was recovered upon interval exercise and LIF was upregulated as well in gastrocnemii (Jia et al., 2018). These results contrast with other reports of a pro-cachectic role of (tumor-derived) LIF in cancerassociated muscle wasting (Seto et al., 2015), so further studies are needed to dissect the signaling pathway activated by LIF in muscle fibers and verify whether endogenous expression of LIF is altered in muscles atrophying because of various conditions. The upregulated expression of LIF by exercise is confirmed by other studies (Broholm et al., 2008) and further proven by the existence in the human LIF promoter of two nuclear factor of activated $\mathrm{T}$ cells (NFAT) binding sites (Bamberger et al., 2004), a transcription factor well-known to be activated by muscle contractions through the $\mathrm{Ca}^{2+}$ calmodulin-calcineurin axis (Schiaffino and Serrano, 2002).

Searching for similar binding sites in the gene regulatory regions of other putatively exercise-induced myokines should be

\section{REFERENCES}

Altun, M., Besche, H. C., Overkleeft, H. S., Piccirillo, R., Edelmann, M. J., Kessler, B. M., et al. (2010). Muscle wasting in aged, sarcopenic rats is associated with enhanced activity of the ubiquitin proteasome pathway. J. Biol. Chem. 285, 39597-39608. doi: 10.1074/jbc.M110.129718

Amir Levy, Y., Ciaraldi, T. P., Mudaliar, S. R., Phillips, S. A., and Henry, R. R. (2015). Excessive secretion of IL-8 by skeletal muscle in type 2 diabetes impairs tube growth: potential role of PI3K and the Tie2 receptor. Am. J. Physiol. Endocrinol. Metab. 309, E22-E34. doi: 10.1152/ajpendo.00 513.2014

Anker, S. D., Ponikowski, P., Varney, S., Chua, T. P., Clark, A. L., Webb-Peploe, K. M., et al. (1997). Wasting as independent risk factor for mortality in chronic heart failure. Lancet 349, 1050-1053. doi: 10.1016/S0140-6736(96)07015-8

Aoi, W., Naito, Y., Takagi, T., Tanimura, Y., Takanami, Y., Kawai, Y., et al. (2013). A novel myokine, secreted protein acidic and rich in cysteine (SPARC), suppresses colon tumorigenesis via regular exercise. Gut 62, 882-889. doi: 10. 1136/gutjnl-2011-300776

Arany, Z., Foo, S.-Y., Ma, Y., Ruas, J. L., Bommi-Reddy, A., Girnun, G., et al. (2008). HIF-independent regulation of VEGF and angiogenesis by the transcriptional a topic for future research to expand our knowledge of myokine regulation at the transcriptional level.

\section{CONCLUSION}

An "exercise pill" to reproduce the multiple beneficial effects of exercise on various organs seems to be unpractical because exercise affects too many interconnected pathways and its holistic effects also depend on the host genetics. Nonetheless, expanding our knowledge about which types of activity in humans control beneficial myokine secretion could give valuable, more personalized suggestions to patients about which kind of exercise to practice. Selected myokine-based drugs could be given to act on specific pathways and help patients who for various reasons cannot exercise.

\section{AUTHOR CONTRIBUTIONS}

The author confirms being the sole contributor of this work and has approved it for publication. RP conceptualized the manuscript, reviewed the literature, and wrote the manuscript in its entirety.

\section{FUNDING}

This work was supported by Italian Association for Cancer Research (AIRC-IG 19927).

\section{ACKNOWLEDGMENTS}

I am grateful to the Italian Association for Cancer Research (AIRC-IG 19927) for financial support and I thank J. D. Baggott for help with the editing.

coactivator PGC-1alpha. Nature 451, 1008-1012. doi: 10.1038/nature 06613

Aversa, Z., Bonetto, A., Penna, F., Costelli, P., Di Rienzo, G., Lacitignola, A., et al. (2012). Changes in myostatin signaling in non-weight-losing cancer patients. Ann. Surg. Oncol. 19, 1350-1356. doi: 10.1245/s10434-011-1720-5

Bamberger, A.-M., Jenatschke, S., Schulte, H. M., Ellebrecht, I., Beil, F. U., and Bamberger, C. M. (2004). Regulation of the human leukemia inhibitory factor gene by ETS transcription factors. Neuroimmunomodulation 11, 10-19. doi: $10.1159 / 000072964$

Banzet, S., Koulmann, N., Sanchez, H., Serrurier, B., Peinnequin, A., and Bigard, A. X. (2007). Musclin gene expression is strongly related to fast-glycolytic phenotype. Biochem. Biophys. Res. Commun. 353, 713-718. doi: 10.1016/j.bbrc. 2006.12.074

Bazgir, B., Salesi, M., Koushki, M., and Amirghofran, Z. (2015). Effects of eccentric and concentric emphasized resistance exercise on IL-15 serum levels and its relation to inflammatory markers in athletes and non-athletes. Asian J. Sports Med. 6:e27980. doi: 10.5812/asjsm.27980

Benatti, F. B., and Pedersen, B. K. (2015). Exercise as an anti-inflammatory therapy for rheumatic diseases-myokine regulation. Nat. Rev. Rheumatol. 11, 86-97. doi: 10.1038/nrrheum.2014.193 
Besse-Patin, A., Montastier, E., Vinel, C., Castan-Laurell, I., Louche, K., Dray, C., et al. (2014). Effect of endurance training on skeletal muscle myokine expression in obese men: identification of apelin as a novel myokine. Int. J. Obes. 38, 707-713. doi: 10.1038/ijo.2013.158

Bloch, S. A. A., Lee, J. Y., Wort, S. J., Polkey, M. I., Kemp, P. R., and Griffiths, M. J. D. (2013). Sustained elevation of circulating growth and differentiation factor-15 and a dynamic imbalance in mediators of muscle homeostasis are associated with the development of acute muscle wasting following cardiac surgery. Crit. Care Med. 41, 982-989. doi: 10.1097/CCM.0b013e318 $274671 \mathrm{~b}$

Bobadilla, M., Sainz, N., Abizanda, G., Orbe, J., Rodriguez, J. A., Páramo, J. A., et al. (2014). The CXCR4/SDF1 axis improves muscle regeneration through MMP-10 activity. Stem Cells Dev. 23, 1417-1427. doi: 10.1089/scd.2013.0491

Boström, P., Wu, J., Jedrychowski, M. P., Korde, A., Ye, L., Lo, J. C., et al. (2012). A PGC1-alpha-dependent myokine that drives brown-fat-like development of white fat and thermogenesis. Nature 481, 463-468. doi: 10.1038/nature 10777

Broholm, C., Mortensen, O. H., Nielsen, S., Akerstrom, T., Zankari, A., Dahl, B., et al. (2008). Exercise induces expression of leukaemia inhibitory factor in human skeletal muscle. J. Physiol. 586, 2195-2201. doi: 10.1113/jphysiol.2007. 149781

Catoire, M., and Kersten, S. (2015). The search for exercise factors in humans. FASEB J. 29, 1615-1628. doi: 10.1096/fj.14-263699

Catoire, M., Mensink, M., Kalkhoven, E., Schrauwen, P., and Kersten, S. (2014). Identification of human exercise-induced myokines using secretome analysis. Physiol. Genomics 46, 256-267. doi: 10.1152/physiolgenomics. 00174.2013

Carson, B. P. (2017). The potential role of contraction-induced myokines in the regulation of metabolic function for the prevention and treatment of Type 2 diabetes. Front. Endocrinol. 8:97. doi: 10.3389/fendo.2017.00097

Chung, H. S., Hwang, S. Y., Choi, J. H., Lee, H. J., Kim, N. H., Yoo, H. J., et al. (2018). Implications of circulating Meteorin-like (Metrnl) level in human subjects with type 2 diabetes. Diabetes Res. Clin. Pract. 136, 100-107. doi: 10.1016/j.diabres.2017.11.031

Ciciliot, S., Rossi, A. C., Dyar, K. A., Blaauw, B., and Schiaffino, S. (2013). Muscle type and fiber type specificity in muscle wasting. Int. J. Biochem. Cell Biol. 45, 2191-2199. doi: 10.1016/j.biocel.2013.05.016

Costelli, P., Muscaritoli, M., Bonetto, A., Penna, F., Reffo, P., Bossola, M., et al. (2008). Muscle myostatin signalling is enhanced in experimental cancer cachexia. Eur. J. Clin. Invest. 38, 531-538. doi: 10.1111/j.1365-2362.2008.01970.x

Dalla Libera, L., Ravara, B., Volterrani, M., Gobbo, V., Della Barbera, M., Angelini, A., et al. (2004). Beneficial effects of GH/IGF-1 on skeletal muscle atrophy and function in experimental heart failure. Am. J. Physiol. Cell Physiol. 286, C138-C144.

Demontis, F., Piccirillo, R., Goldberg, A. L., and Perrimon, N. (2013a). Mechanisms of skeletal muscle aging: insights from Drosophila and mammalian models. Dis. Model. Mech. 6, 1339-1352. doi: 10.1242/dmm.012559

Demontis, F., Piccirillo, R., Goldberg, A. L., and Perrimon, N. (2013b). The influence of skeletal muscle on systemic aging and lifespan. Aging Cell 12, 943-949. doi: 10.1111/acel.12126

Dong, J., Dong, Y., Dong, Y., Chen, F., Mitch, W. E., and Zhang, L. (2016). Inhibition of myostatin in mice improves insulin sensitivity via irisin-mediated cross talk between muscle and adipose tissues. Int. J. Obes. 40, 434-442. doi: 10.1038/ijo.2015.200

Dray, C., Knauf, C., Daviaud, D., Waget, A., Boucher, J., Buleon, M., et al. (2008). Apelin stimulates glucose utilization in normal and obese insulin-resistant mice. Cell Metab. 8, 437-445. doi: 10.1016/j.cmet.2008.10.003

Eaton, M., Granata, C., Barry, J., Safdar, A., Bishop, D., and Little, J. P. (2018). Impact of a single bout of high-intensity interval exercise and short-term interval training on interleukin-6, FNDC5, and METRNL mRNA expression in human skeletal muscle. J. Sport Health Sci. 7, 191-196. doi: 10.1016/j.jshs. 2017.01.003

Egerman, M. A., Cadena, S. M., Gilbert, J. A., Meyer, A., Nelson, H. N., Swalley, S. E., et al. (2015). GDF11 increases with age and inhibits skeletal muscle regeneration. Cell Metab. 22, 164-174. doi: 10.1016/j.cmet. 2015.05.010
Fearon, K., Strasser, F., Anker, S. D., Bosaeus, I., Bruera, E., Fainsinger, R. L., et al. (2011). Definition and classification of cancer cachexia: an international consensus. Lancet Oncol. 12, 489-495. doi: 10.1016/S1470-2045(10)70218-7

Flück, M., and Hoppeler, H. (2003). Molecular basis of skeletal muscle plasticityfrom gene to form and function. Rev. Physiol. Biochem. Pharmacol. 146, 159-216. doi: 10.1007/s10254-002-0004-7

Furrer, R., and Handschin, C. (2018). Muscle wasting diseases: novel targets and treatments. Annu. Rev. Pharmacol. Toxicol. 59, 315-339. doi: 10.1146/annurevpharmtox-010818-021041

Gannon, N. P., Vaughan, R. A., Garcia-Smith, R., Bisoffi, M., and Trujillo, K. A. (2015). Effects of the exercise-inducible myokine irisin on malignant and nonmalignant breast epithelial cell behavior in vitro. Int. J. Cancer 136, E197-E202. doi: 10.1002/ijc.29142

Gao, X., and Xu, Z. (2008). Mechanisms of action of angiogenin. Acta Biochim. Biophys. Sin. 40, 619-624. doi: 10.1111/j.1745-7270.2008.00442.x

Hambrecht, R., Schulze, P. C., Gielen, S., Linke, A., Möbius-Winkler, S., Yu, J., et al. (2002). Reduction of insulin-like growth factor-I expression in the skeletal muscle of noncachectic patients with chronic heart failure. J. Am. Coll. Cardiol. 39, 1175-1181. doi: 10.1016/S0735-1097(02) 01736-9

Han, H. Q., Zhou, X., Mitch, W. E., and Goldberg, A. L. (2013). Myostatin/activin pathway antagonism: molecular basis and therapeutic potential. Int. J. Biochem. Cell Biol. 45, 2333-2347. doi: 10.1016/j.biocel.2013.05.019

Hawley, J. A., Hargreaves, M., Joyner, M. J., and Zierath, J. R. (2014). Integrative biology of exercise. Cell 159, 738-749. doi: 10.1016/j.cell.2014.10.029

He, Z., Tian, Y., Valenzuela, P. L., Huang, C., Zhao, J., Hong, P., et al. (2018). Myokine response to high-intensity interval vs. resistance exercise: an individual approach. Front. Physiol. 9:1735. doi: 10.3389/fphys.2018.01735

Hittel, D. S., Axelson, M., Sarna, N., Shearer, J., Huffman, K. M., and Kraus, W. E. (2010). Myostatin decreases with aerobic exercise and associates with insulin resistance. Med. Sci. Sports Exerc. 42, 2023-2029. doi: 10.1249/MSS. 0b013e3181e0b9a8

Hojman, P., Dethlefsen, C., Brandt, C., Hansen, J., Pedersen, L., and Pedersen, B. K. (2011). Exercise-induced muscle-derived cytokines inhibit mammary cancer cell growth. Am. J. Physiol. Endocrinol. Metab. 301, E504-E510. doi: 10.1152/ ajpendo.00520.2010

Hulmi, J. J., Silvennoinen, M., Lehti, M., Kivelä, R., and Kainulainen, H. (2012). Altered REDD1, myostatin, and Akt/mTOR/FoxO/MAPK signaling in streptozotocin-induced diabetic muscle atrophy. Am. J. Physiol. Endocrinol. Metab. 302, E307-E315. doi: 10.1152/ajpendo.00398.2011

Ijiri, N., Kanazawa, H., Asai, K., Watanabe, T., Hirata, K., and Irisin, A. (2015). newly discovered myokine, is a novel biomarker associated with physical activity in patients with chronic obstructive pulmonary disease. Respirology 20, 612-617. doi: 10.1111/resp.12513

Ishiuchi, Y., Sato, H., Komatsu, N., Kawaguchi, H., Matsuwaki, T., Yamanouchi, K., et al. (2018). Identification of CCL5/RANTES as a novel contraction-reducible myokine in mouse skeletal muscle. Cytokine 108, 17-23. doi: 10.1016/j.cyto. 2018.03.012

Izumiya, Y., Bina, H. A., Ouchi, N., Akasaki, Y., Kharitonenkov, A., and Walsh, K. (2008a). FGF21 is an Akt-regulated myokine. FEBS Lett. 582, 3805-3810. doi: 10.1016/j.febslet.2008.10.021

Izumiya, Y., Hopkins, T., Morris, C., Sato, K., Zeng, L., Viereck, J., et al. (2008b). Fast/Glycolytic muscle fiber growth reduces fat mass and improves metabolic parameters in obese mice. Cell Metab. 7, 159-172. doi: 10.1016/j.cmet.2007.11.003

Jia, D., Cai, M., Xi, Y., Du, S., and Zhenjun, T. (2018). Interval exercise training increases LIF expression and prevents myocardial infarction-induced skeletal muscle atrophy in rats. Life Sci. 193, 77-86. doi: 10.1016/j.lfs.2017.12.009

Ju, C.-R., and Chen, R.-C. (2012). Serum myostatin levels and skeletal muscle wasting in chronic obstructive pulmonary disease. Respir. Med. 106, 102-108. doi: 10.1016/j.rmed.2011.07.016

Kalantar-Zadeh, K., Rhee, C., Sim, J. J., Stenvinkel, P., Anker, S. D., and Kovesdy, C. P. (2013). Why cachexia kills: examining the causality of poor outcomes in wasting conditions. J. Cachexia Sarcopenia Muscle 4, 89-94. doi: 10.1007/ s13539-013-0111-0

Kanzleiter, T., Rath, M., Gorgens, S. W., Jensen, J., Tangen, D. S., Kolnes, A. J., et al. (2014). The myokine decorin is regulated by contraction and involved 
in muscle hypertrophy. Biochem. Biophys. Res. Commun. 450, 1089-1094. doi: 10.1016/j.bbrc.2014.06.123

Kim, H.-J., So, B., Choi, M., Kang, D., and Song, W. (2015). Resistance exercise training increases the expression of irisin concomitant with improvement of muscle function in aging mice and humans. Exp. Gerontol. 70, 11-17. doi: 10.1016/j.exger.2015.07.006

Kim, J.-S., Lee, Y.-H., and Yi, H.-K. (2016). Gradual downhill running improves age-related skeletal muscle and bone weakness: implication of autophagy and bone morphogenetic proteins. Exp. Physiol. 101, 1528-1540. doi: 10.1113/ EP085852

Kotler, D. P., Tierney, A. R., Wang, J., and Pierson, R. N. (1989). Magnitude of body-cell-mass depletion and the timing of death from wasting in AIDS. Am. J. Clin. Nutr. 50, 444-447. doi: 10.1093/ajcn/50.3.444

Lach-Trifilieff, E., Minetti, G. C., Sheppard, K., Ibebunjo, C., Feige, J. N., Hartmann, S., et al. (2014). An antibody blocking activin type II receptors induces strong skeletal muscle hypertrophy and protects from atrophy. Mol. Cell Biol. 34, 606-618. doi: 10.1128/MCB.01307-13

Laker, R. C., Garde, C., Camera, D. M., Smiles, W. J., Zierath, J. R., Hawley, J. A., et al. (2017). Transcriptomic and epigenetic responses to short-term nutrient-exercise stress in humans. Sci. Rep. 7:15134. doi: 10.1038/s41598-017-15420-7

Larsson, L., Degens, H., Li, M., Salviati, L., Lee, Y. I., Thompson, W., et al. (2019). Sarcopenia: aging-related loss of muscle mass and function. Physiol. Rev. 99, 427-511. doi: 10.1152/physrev.00061.2017

Lecker, S. H., Jagoe, R. T., Gilbert, A., Gomes, M., Baracos, V., Bailey, J., et al. (2004). Multiple types of skeletal muscle atrophy involve a common program of changes in gene expression. FASEB J. 18, 39-51. doi: 10.1096/fj.030610com

Lozano, R., Naghavi, M., Foreman, K., Lim, S., Shibuya, K., Aboyans, V., et al. (2012). Global and regional mortality from 235 causes of death for 20 age groups in 1990 and 2010: a systematic analysis for the Global Burden of Disease Study 2010. Lancet 380, 2095-2128. doi: 10.1016/S0140-6736(12)61728-0

Ma, W. Y., Yu, T. Y., Wei, J. N., Hung, C. S., Lin, M. S., Liao, Y. J., et al. (2014). Plasma apelin: a novel biomarker for predicting diabetes. Clin. Chim. Acta 435, 18-23. doi: $10.1016 /$ j.cca.2014.03.030

Mammucari, C., Milan, G., Romanello, V., Masiero, E., Rudolf, R., Del Piccolo, P., et al. (2007). FoxO3 controls autophagy in skeletal muscle in vivo. Cell Metab. 6, 458-471. doi: 10.1016/j.cmet.2007.11.001

Man, W. D.-C., Natanek, S. A., Riddoch-Contreras, J., Lewis, A., Marsh, G. S., Kemp, P. R., et al. (2010). Quadriceps myostatin expression in COPD. Eur. Respir. J. 36, 686-688. doi: 10.1183/09031936.00032510

Manole, E., Ceafalan, L. C., Popescu, B. O., Dumitru, C., and Bastian, A. E. (2018). Myokines as possible therapeutic targets in cancer cachexia. J. Immunol. Res. 2018:8260742. doi: 10.1155/2018/8260742

Marklund, P., Mattsson, C. M., Wåhlin-Larsson, B., Ponsot, E., Lindvall, B., Lindvall, L., et al. (2013). Extensive inflammatory cell infiltration in human skeletal muscle in response to an ultraendurance exercise bout in experienced athletes. J. Appl. Physiol. 114, 66-72. doi: 10.1152/japplphysiol.01538.2011

Martinelli, G. B., Olivari, D., Re Cecconi, A. D., Talamini, L., Ottoboni, L., Lecker, S. H., et al. (2016). Activation of the SDF1/CXCR4 pathway retards muscle atrophy during cancer cachexia. Oncogene 35, 6212-6222. doi: 10.1038/onc. 2016.153

Mashili, F. L., Austin, R. L., Deshmukh, A. S., Fritz, T., Caidahl, K., Bergdahl, K., et al. (2011). Direct effects of FGF21 on glucose uptake in human skeletal muscle: implications for type 2 diabetes and obesity. Diabetes. Metab. Res. Rev. 27, 286-297. doi: 10.1002/dmrr.1177

McCall, G. E., Allen, D. L., Haddad, F., and Baldwin, K. M. (2003). Transcriptional regulation of IGF-I expression in skeletal muscle. Am. J. Physiol. Cell Physiol. 285, C831-C839. doi: 10.1152/ajpcell.00047.2003

McPherron, A. C., Lawler, A. M., and Lee, S. J. (1997). Regulation of skeletal muscle mass in mice by a new TGF-beta superfamily member. Nature $387,83-90$. doi: $10.1038 / 387083 \mathrm{a} 0$

McPherron, A. C., and Lee, S. J. (1997). Double muscling in cattle due to mutations in the myostatin gene. Proc. Natl. Acad. Sci. U.S.A. 94, 12457-12461. doi: 10. 1073/pnas.94.23.12457

McPherron, A. C., and Lee, S.-J. (2002). Suppression of body fat accumulation in myostatin-deficient mice. J. Clin. Invest. 109, 595-601. doi: 10.1172/JCI0213562
Munoz, R. M., Han, H., Tegeler, T., Petritis, K., Von Hoff, D. D., and Hoffman, S. A. (2013). Isolation and characterization of muscle fatigue substance with anti-tumor activities. J. Cancer 4, 343-349. doi: 10.7150/jca.5418

Nakamori, M., Hamanaka, K., Thomas, J. D., Wang, E. T., Hayashi, Y. K., Takahashi, M. P., et al. (2017). Aberrant myokine signaling in congenital myotonic dystrophy. Cell Rep. 21, 1240-1252. doi: 10.1016/j.celrep.2017.10.018

Nakamura, K., Yamanouchi, K., and Nishihara, M. (2014). Secreted protein acidic and rich in cysteine internalization and its age-related alterations in skeletal muscle progenitor cells. Aging Cell 13, 175-184. doi: 10.1111/acel.12168

Ostrowski, K., Schjerling, P., and Pedersen, B. K. (2000). Physical activity and plasma interleukin-6 in humans-effect of intensity of exercise. Eur. J. Appl. Physiol. 83, 512-515. doi: 10.1007/s004210000312

Passey, S. L., Hansen, M. J., Bozinovski, S., McDonald, C. F., Holland, A. E., and Vlahos, R. (2016). Emerging therapies for the treatment of skeletal muscle wasting in chronic obstructive pulmonary disease. Pharmacol. Ther. 166, 56-70. doi: 10.1016/j.pharmthera.2016.06.013

Pedersen, B. K., and Febbraio, M. A. (2008). Muscle as an endocrine organ: focus on muscle-derived interleukin-6. Physiol. Rev. 88, 1379-1406. doi: 10.1152/ physrev. 90100.2007

Pedersen, B. K., and Febbraio, M. A. (2012). Muscles, exercise and obesity: skeletal muscle as a secretory organ. Nat. Rev. Endocrinol. 8, 457-465. doi: 10.1038/ nrendo.2012.49

Penna, F., Bonetto, A., Muscaritoli, M., Costamagna, D., Minero, V. G., Bonelli, G., et al. (2010). Muscle atrophy in experimental cancer cachexia: is the IGF-1 signaling pathway involved? Int. J. Cancer 127, 1706-1717. doi: 10.1002/ijc. 25146

Piccirillo, R., Demontis, F., Perrimon, N., and Goldberg, A. L. (2014). Mechanisms of muscle growth and atrophy in mammals and Drosophila. Dev. Dyn. 243, 201-215. doi: 10.1002/dvdy.24036

Pilegaard, H., Saltin, B., and Neufer, P. D. (2003). Exercise induces transient transcriptional activation of the PGC-1alpha gene in human skeletal muscle. J. Physiol. 546, 851-858. doi: 10.1113/jphysiol.2002.034850

Pistilli, E. E., Devaney, J. M., Gordish-Dressman, H., Bradbury, M. K., Seip, R. L., Thompson, P. D., et al. (2008). Interleukin-15 and interleukin-15R alpha SNPs and associations with muscle, bone, and predictors of the metabolic syndrome. Cytokine 43, 45-53. doi: 10.1016/j.cyto.2008.04.008

Plant, P. J., Brooks, D., Faughnan, M., Bayley, T., Bain, J., Singer, L., et al. (2010). Cellular markers of muscle atrophy in chronic obstructive pulmonary disease. Am. J. Respir. Cell Mol. Biol. 42, 461-471. doi: 10.1165/rcmb.2008-0 $382 \mathrm{OC}$

Pretto, F., Ghilardi, C., Moschetta, M., Bassi, A., Rovida, A., Scarlato, V., et al. (2015). Sunitinib prevents cachexia and prolongs survival of mice bearing renal cancer by restraining STAT3 and MuRF-1 activation in muscle. Oncotarget 6, 3043-3054. doi: 10.18632/oncotarget.2812

Prontera, P., Bernardini, L., Stangoni, G., Capalbo, A., Rogaia, D., Ardisia, C., et al. (2009). 2q31.2q32.3 deletion syndrome: report of an adult patient. Am. J. Med. Genet. A 149A, 706-712. doi: 10.1002/ajmg.a.32688

Puchert, M., Adams, V., Linke, A., and Engele, J. (2016). Evidence for the involvement of the CXCL12 system in the adaptation of skeletal muscles to physical exercise. Cell. Signal. 28, 1205-1215. doi: 10.1016/j.cellsig. 2016.05.019

Quinn, L. S., Anderson, B. G., Strait-Bodey, L., and Wolden-Hanson, T. (2010). Serum and muscle interleukin-15 levels decrease in aging mice: correlation with declines in soluble interleukin-15 receptor alpha expression. Exp. Gerontol. 45, 106-112. doi: 10.1016/j.exger.2009.10.012

Rai, M., and Demontis, F. (2016). Systemic nutrient and stress signaling via myokines and myometabolites. Annu. Rev. Physiol. 78, 85-107. doi: 10.1146/ annurev-physiol-021115-105305

Roubenoff, R., and Kehayias, J. J. (1991). The meaning and measurement of lean body mass. Nutr. Rev. 49, 163-175. doi: 10.1111/j.1753-4887.1991.tb03013.x

Rovira, M., Arrey, G., and Planas, J. V. (2017). Exercise-induced hypertrophic and oxidative signaling pathways and myokine expression in fast muscle of adult zebrafish. Front. Physiol. 8:1063. doi: 10.3389/fphys.2017.01063

Rutti, S., Dusaulcy, R., Hansen, J. S., Howald, C., Dermitzakis, E. T., Pedersen, B. K., et al. (2018). Angiogenin and Osteoprotegerin are type II muscle specific myokines protecting pancreatic beta-cells against proinflammatory cytokines. Sci. Rep. 8:10072. doi: 10.1038/s41598-018-28117-2 
Safdar, A., and Tarnopolsky, M. A. (2018). Exosomes as mediators of the systemic adaptations to endurance exercise. Cold Spring Harb. Perspect. Med. 8:a029827. doi: 10.1101/cshperspect.a029827

Sandri, M., Lin, J., Handschin, C., Yang, W., Arany, Z. P., Lecker, S. H., et al. (2006). PGC-1alpha protects skeletal muscle from atrophy by suppressing FoxO3 action and atrophy-specific gene transcription. Proc. Natl. Acad. Sci. U.S.A. 103, 16260-16265. doi: 10.1073/pnas.0607795103

Sandri, M., Sandri, C., Gilbert, A., Skurk, C., Calabria, E., Picard, A., et al. (2004). Foxo transcription factors induce the atrophy-related ubiquitin ligase atrogin1 and cause skeletal muscle atrophy. Cell 117, 399-412. doi: 10.1016/S00928674(04)00400-3

Schiaffino, S., and Serrano, A. (2002). Calcineurin signaling and neural control of skeletal muscle fiber type and size. Trends Pharmacol. Sci. 23, 569-575. doi: 10.1016/S0165-6147(02)02111-9

Seldin, M. M., Peterson, J. M., Byerly, M. S., Wei, Z., and Wong, G. W. (2012). Myonectin (CTRP15), a novel myokine that links skeletal muscle to systemic lipid homeostasis. J. Biol. Chem. 287, 11968-11980. doi: 10.1074/jbc.M111. 336834

Seto, D. N., Kandarian, S. C., and Jackman, R. W. (2015). A key role for leukemia inhibitory factor in C26 cancer cachexia. J. Biol. Chem. 290, 19976-19986. doi: 10.1074/jbc.M115.638411

Shah, R., Hinkle, C. C., Ferguson, J. F., Mehta, N. N., Li, M., Qu, L., et al. (2011). Fractalkine is a novel human adipochemokine associated with type 2 diabetes. Diabetes 60, 1512-1518. doi: 10.2337/db10-0956

Shin, K. O., Bae, J. Y., Woo, J., Jang, K. S., Kim, K. S., Park, J. S., et al. (2015). The effect of exercise on expression of myokine and angiogenesis mRNA in skeletal muscle of high fat diet induced obese rat. J. Exerc. Nutr. Biochem. 19, 91-98. doi: 10.5717/jenb.2015.15061006

Simonet, W. S., Lacey, D. L., Dunstan, C. R., Kelley, M., Chang, M. S., Lüthy, R., et al. (1997). Osteoprotegerin: a novel secreted protein involved in the regulation of bone density. Cell 89, 309-319. doi: 10.1016/S0092-8674(00) 80209-3

Son, J. S., Chae, S. A., Testroet, E. D., Du, M., and Jun, H.-P. (2018). Exerciseinduced myokines: a brief review of controversial issues of this decade. Expert Rev. Endocrinol. Metab. 13, 51-58. doi: 10.1080/17446651.2018.1416290

Subbotina, E., Sierra, A., Zhu, Z., Gao, Z., Koganti, S. R., Reyes, S., et al. (2015). Musclin is an activity-stimulated myokine that enhances physical endurance. Proc. Natl. Acad. Sci. U.S.A. 112, 16042-16047. doi: 10.1073/pnas.1514250112

Tanimura, Y., Aoi, W., Takanami, Y., Kawai, Y., Mizushima, K., Naito, Y., et al. (2016). Acute exercise increases fibroblast growth factor 21 in metabolic organs and circulation. Physiol. Rep. 4:e12828. doi: 10.14814/phy2.12828

Terada, S., Goto, M., Kato, M., Kawanaka, K., Shimokawa, T., and Tabata, I. (2002). Effects of low-intensity prolonged exercise on PGC-1 mRNA expression in rat epitrochlearis muscle. Biochem. Biophys. Res. Commun. 296, 350-354. doi: 10.1016/S0006-291X(02)00881-1

Tezze, C., Romanello, V., Desbats, M. A., Fadini, G. P., Albiero, M., Favaro, G., et al. (2017). Age-associated loss of OPA1 in muscle impacts muscle mass, metabolic homeostasis, systemic inflammation, and epithelial senescence. Cell Metab. 25, 1374-1389.e6. doi: 10.1016/j.cmet.2017.04.021

Toloza, F. J. K., Mantilla-Rivas, J. O., Pérez-Matos, M. C., Ricardo-Silgado, M. L., Morales-Alvarez, M. C., Pinzón-Cortés, J. A., et al. (2018). Plasma levels of myonectin but not myostatin or fibroblast-derived growth factor 21 are associated with insulin resistance in adult humans without diabetes mellitus. Front. Endocrinol. 9:5. doi: 10.3389/fendo.2018.00005
Trayhurn, P., Drevon, C. A., and Eckel, J. (2011). Secreted proteins from adipose tissue and skeletal muscle - adipokines, myokines and adipose/muscle cross-talk. Arch. Physiol. Biochem. 117, 47-56. doi: 10.3109/13813455.2010. 535835

VanderVeen, B. N., Fix, D. K., and Carson, J. A. (2017). Disrupted skeletal muscle mitochondrial dynamics, mitophagy, and biogenesis during cancer cachexia: a role for inflammation. Oxid. Med. Cell. Longev. 2017:3292087. doi: 10.1155/ 2017/3292087

Vinel, C., Lukjanenko, L., Batut, A., Deleruyelle, S., Pradère, J.-P., Le Gonidec, S., et al. (2018). The exerkine apelin reverses age-associated sarcopenia. Nat. Med. 24, 1360-1371. doi: 10.1038/s41591-018-0131-6

Walker, K. S., Kambadur, R., Sharma, M., and Smith, H. K. (2004). Resistance training alters plasma myostatin but not IGF-1 in healthy men. Med. Sci. Sports Exerc. 36, 787-793. doi: 10.1249/01.MSS.0000126384.04 778.29

Wang, Q., Guo, T., Portas, J., and McPherron, A. C. (2015). A soluble activin receptor type IIB does not improve blood glucose in streptozotocin-treated mice. Int. J. Biol. Sci. 11, 199-208. doi: 10.7150/ijbs.10430

Welle, S., Bhatt, K., Shah, B., and Thornton, C. (2002). Insulin-like growth factor-1 and myostatin mRNA expression in muscle: comparison between 62-77 and 21-31 yr old men. Exp. Gerontol. 37, 833-839. doi: 10.1016/S0531-5565(02)00025-6

Whitham, M., and Febbraio, M. A. (2016). The ever-expanding myokinome: discovery challenges and therapeutic implications. Nat. Rev. Drug Discov. 15, 719-729. doi: 10.1038/nrd.2016.153

Whitham, M., Parker, B. L., Friedrichsen, M., Hingst, J. R., Hjorth, M., Hughes, W. E., et al. (2018). Extracellular vesicles provide a means for tissue crosstalk during exercise. Cell Metab. 27, 237-251.e4. doi: 10.1016/j.cmet.2017.12.001

Winbanks, C. E., Chen, J. L., Qian, H., Liu, Y., Bernardo, B. C., Beyer, C., et al. (2013). The bone morphogenetic protein axis is a positive regulator of skeletal muscle mass. J. Cell Biol. 203, 345-357. doi: 10.1083/jcb.201211134

Winick, M. (1979). Hunger Disease-Studies by Jewish Physicians in the Warsaw Ghetto. New York, NY: John Wiley \& Sons.

Zhao, J., Brault, J. J., Schild, A., Cao, P., Sandri, M., Schiaffino, S., et al. (2007). FoxO3 coordinately activates protein degradation by the autophagic/lysosomal and proteasomal pathways in atrophying muscle cells. Cell Metab. 6, 472-483. doi: 10.1016/j.cmet.2007.11.004

Zhao, X., and Karpac, J. (2017). Muscle directs diurnal energy homeostasis through a myokine-dependent hormone module in Drosophila. Curr. Biol. 27, 1941-1955.e6. doi: 10.1016/j.cub.2017.06.004

Zhou, X., Wang, J. L., Lu, J., Song, Y., Kwak, K. S., Jiao, Q., et al. (2010). Reversal of cancer cachexia and muscle wasting by ActRIIB antagonism leads to prolonged survival. Cell 142, 531-543. doi: 10.1016/j.cell.2010.07.011

Conflict of Interest Statement: The author declares that the research was conducted in the absence of any commercial or financial relationships that could be construed as a potential conflict of interest.

Copyright (c) 2019 Piccirillo. This is an open-access article distributed under the terms of the Creative Commons Attribution License (CC BY). The use, distribution or reproduction in other forums is permitted, provided the original author(s) and the copyright owner(s) are credited and that the original publication in this journal is cited, in accordance with accepted academic practice. No use, distribution or reproduction is permitted which does not comply with these terms. 


\section{OPEN ACCESS}

Edited by:

Marilia Seelaender,

University of São Paulo, Brazil

Reviewed by:

Martina Krüger,

Heinrich Heine Universität Düsseldorf,

Germany

Bruno Bastide,

Lille University of Science and

Technology, France

*Correspondence:

David H. St-Pierre

st-pierre.david_h@uqam.ca

Specialty section:

This article was submitted to

Striated Muscle Physiology,

a section of the journal

Frontiers in Physiology

Received: 01 November 2018 Accepted: 18 March 2019

Published: 10 April 2019

Citation:

Andrich DE, Melbouci L, OU Y, Auclair N, Mercier J, Grenier J-C, Lira FS, Barreiro LB, Danialou G,

Comtois A-S, Lavoie J-C and

St-Pierre DH (2019) A Short-Term High-Fat Diet Alters Glutathione Levels and IL-6 Gene Expression in Oxidative Skeletal Muscles of Young Rats. Front. Physiol. 10:372. doi: 10.3389/fphys.2019.00372

\section{A Short-Term High-Fat Diet Alters Glutathione Levels and IL-6 Gene Expression in Oxidative Skeletal Muscles of Young Rats}

David E. Andrich ${ }^{1,2,3}$, Lilya Melbouci ${ }^{1,2,4}$, Ya Ou ${ }^{1,2,4}$, Nickolas Auclair $^{1,2,4}$, Jocelyne Mercier ${ }^{1,2,4}$, Jean-Christophe Grenier ${ }^{4}$, Fábio Santos Lira ${ }^{1,2,5}$, Luis B. Barreiro ${ }^{4,6}$, Gawiyou Danialou ${ }^{1,7}$, Alain-Steve Comtois ${ }^{1,2}$, Jean-Claude Lavoie ${ }^{4,8}$ and David H. St-Pierre ${ }^{1,2,4 *}$

'Département des Sciences de l'Activité Physique, Université du Québec à Montréal (UQAM), Montréal, QC, Canada, ${ }^{2}$ Groupe de Recherche en Activité Physique Adaptée (GRAPA), Université du Québec à Montréal (UQAM), Montréal, QC, Canada, ${ }^{3}$ Département des Sciences Biologiques, Université du Québec à Montréal (UQAM), Montréal, QC, Canada, ${ }^{4}$ Centre de Recherche du CHU Sainte-Justine, Montréal, QC, Canada, ${ }^{5}$ Department of Physical Education, São Paulo State University, São Paulo, Brazil, ${ }^{6}$ Département de Pédiatrie, Faculté de Médecine, Université de Montréal, Montréal, QC, Canada, ${ }^{7}$ Royal Military College Saint-Jean, Saint-Jean-sur-Richelieu, QC, Canada, ${ }^{8}$ Département de Nutrition, Faculté de Médecine, Université de Montréal, Montréal, QC, Canada

Obesity and ensuing disorders are increasingly prevalent worldwide. High-fat diets (HFD) and diet-induced obesity have been shown to induce oxidative stress and inflammation while altering metabolic homeostasis in many organs, including the skeletal muscle. We previously observed that 14 days of HFD impairs contractile functions of the soleus (SOL) oxidative skeletal muscle. However, the mechanisms underlying these effects are not clarified. In order to determine the effects of a short-term HFD on skeletal muscle glutathione metabolism, young male Wistar rats (100-125 g) were fed HFD or a regular chow diet (RCD) for 14 days. Reduced (GSH) and disulfide (GSSG) glutathione levels were measured in the SOL. The expression of genes involved in the regulation of glutathione metabolism, oxidative stress, antioxidant defense and inflammation were measured by RNA-Seq. We observed a significant 25\% decrease of GSH levels in the SOL muscle. Levels of GSSG and the GSH:GSSG ratio were similar in both groups. Further, we observed a 4.5 fold increase in the expression of pro-inflammatory cytokine interleukin 6 (IL-6) but not of other cytokines or markers of inflammation and oxidative stress. We hereby demonstrate that a short-term HFD significantly lowers SOL muscle GSH levels. This effect could be mediated through the increased expression of IL-6. Further, the skeletal muscle antioxidant defense could be impaired under cellular stress. We surmise that these early alterations could contribute to HFD-induced insulin resistance observed in longer protocols.

Keywords: high-fat diet, young rats, muscle glutathione, oxidative stress, inflammation, gene expression 


\section{INTRODUCTION}

Obesity has become a major health, social, and economic burden worldwide (Hruby and $\mathrm{Hu}$, 2015). This is particularly concerning among children, since the prevalence of obesity and overweightness in this age group has risen by nearly $10 \%$ in the last 4 decades (Rao et al., 2016). Indeed, the risk of carrying excess weight into adulthood and of developing morbid obesity is much greater among obese children and adolescents (The et al., 2010). Overweight children are also more at risk of developing obesity related diseases like type 2 diabetes and the metabolic syndrome in later stages of life (Biro and Wien, 2010).

A sedentary lifestyle and poor diet quality are known as two main contributors to obesity, as calorie-rich diets promote a positive energy balance leading to weight gain. Obesity increases the risk of developing a large number of metabolic disorders (Head, 2015). For instance, we have recently reported that only 2 weeks of high fat diet (HFD) significantly altered contractile functions of the oxidative skeletal muscle soleus in young rats, although the same result could not be observed in the glycolytic extensor digitorum longus (EDL) muscle (Andrich et al., 2018b). Hence, long known to cause excess lipid accumulation (Peckham et al., 1962), HFDs have also been shown to stimulate the production of reactive oxygen species (ROS), thus leading to oxidative stress (Auberval et al., 2014). Strong evidence shows that sub-clinical inflammation and oxidative stress are two of the main contributors for the pathogenesis of metabolic dysfunctions in the obese state (Galassetti, 2012). It appears as though HFD-stimulated excess ROS production (Vial et al., 2011) can precede observable weight gain and insulin resistance (Matsuzawa-Nagata et al., 2008), indicating that oxidative stress might be a result of the diet itself and not a consequence of excess lipid accumulation. Further, HFD induces inflammation and oxidative stress in the skeletal muscle of rodents (Yokota et al., 2009; Gortan Cappellari et al., 2016). Beyond its role in locomotion and posture maintenance, skeletal muscle is a key player in the regulation of metabolic homeostasis (Frontera and Ochala, 2015). In fact, skeletal muscle insulin resistance is considered as the primary cause of type 2 diabetes (DeFronzo and Tripathy, 2009). Skeletal muscle dysfunctions can be induced by oxidative stress in type 2 diabetes patients (Tsutsui et al., 2011; DiazMorales et al., 2016; Wang et al., 2016), highlighting its role in the pathogenesis of the disease (Giacco and Brownlee, 2010). Further, 6 weeks of HFD has been shown to decrease reduced glutathione (GSH) levels, an important antioxidant, in the gastrocnemius of 8-week-old Sprague-Dawley rats (Anderson et al., 2009). After a similar exposure to HFD, higher glutathione disulfide (GSSG) levels and a lower GSH:GSSG ratio were also observed in the gastrocnemius muscle of 18 -week old rats (Fisher-Wellman et al., 2013). However, the early mechanisms underlying such alterations remain to be elucidated. Further, it was previously shown that glutathione levels are more prone to undergo HFD-induced alterations in oxidative muscle (Pinho et al., 2017). Therefore, this study aimed to investigate the effects of a short-term (14 days) HFD on glutathione metabolism in the soleus muscle of young rats. To do so, we measured glutathione levels as well as gene expression of known factors regulating glutathione metabolism, oxidative stress, and inflammation. Thus, we hypothesized that a short-term exposure to an obesogenic diet alters glutathione production and redox potential while inducing oxidative stress and inflammation in the soleus muscle.

\section{MATERIALS AND METHODS}

\section{Animal Procedures}

This study was carried out in strict accordance with recommendations of the National Institutes of Health guide for the care and use of Laboratory animals. Before undergoing the experimental work, the protocol was approved by the Comite Institutionnel de Protection des Animaux (CIPA) of UQAM (Permit Number: 0515-R3-759-0516). After a 3-day acclimatization period at UQAM's animal facility, young (100-125 g; approximately 4 weeks old) male Wistar rats (Charles River, St-Constant, QC, Canada) were randomly fed with a regular chow diet (RCD; $n=13)$ or HFD $(n=12)$ for 14 days and submitted to a 12-h light/dark cycle starting at 06:00. Animals were given ad libitum access to the diets and water throughout the experimental protocol. Sacrifice was achieved under anesthesia (3\% isoflurane at $0.5 \mathrm{~L} / \mathrm{min}$ of $\mathrm{O}_{2}$ ) after a $4 \mathrm{~h}$ fast to standardize the feeding status of each animal. The soleus (SOL) muscle of both legs was collected for glutathione determination and RNA extraction.

\section{Diets}

Physiological fuel values were calculated from modified Atwater factors $(3.5 \mathrm{kcal} / \mathrm{g}$ carbohydrate; $3.5 \mathrm{kcal} / \mathrm{g}$ protein; $8.5 \mathrm{kcal} / \mathrm{g}$ fat). The high fat diet was prepared from purified food-grade reagents according to a commercial formulation (D12492 diet, Research Diets Inc., New Brunswick, NJ, USA). It had a macronutrient weight content of $26.3 \%$ carbohydrate (19.2\% kcal), $26.2 \%$ protein (19\% kcal), and $34.9 \%$ fat $(61.8 \% \mathrm{kcal})$ and a physiological fuel value of $4.80 \mathrm{kcal} / \mathrm{g}$. Carbohydrate sources were maltodextrin and sucrose (64.5 and 35.5\%, respectively), protein sources were casein and L-cystine (98.5 and $1.5 \%$, respectively), while lipid sources were lard and soybean oil ( 90.7 and $9.3 \%$, respectively). The diet also contained cellulose $(64.6 \mathrm{~g} / \mathrm{kg})$, calcium carbonate $(7.1 \mathrm{~g} / \mathrm{kg})$, dicalcium phosphate $(16.8 \mathrm{~g} / \mathrm{kg})$, potassium citrate $(21.3 \mathrm{~g} / \mathrm{kg})$, and choline bitartrate $(2.6 \mathrm{~g} / \mathrm{kg})$, as well as mineral $(12.9 \mathrm{~g} / \mathrm{kg})$ and vitamin $(12.9 \mathrm{~g} / \mathrm{kg})$ mixes. The regular chow diet (Charles River Rodent Diet \# 5075, Cargill Animal Nutrition, MN, USA) had a macronutrient weight content of $55.2 \%$ carbohydrate $(65.6 \% \mathrm{kcal}), 18 \%$ protein $(21.4 \% \mathrm{kcal})$, and $4.5 \%$ fat $(13 \% \mathrm{kcal})$ and a physiological fuel value of $2.89 \mathrm{kcal} / \mathrm{g}$.

\section{Glutathione Measurements}

Immediately after collection, $0.25 \mathrm{~g}$ of SOL muscle was homogenized $(2 \times 10 \mathrm{~s}$ with Polytron Teador; Biospec Products Inc, DremelRacine, WI) in $1.25 \mathrm{ml}$ of iced and freshly prepared 5\% (w/v) metaphosphoric acid (Fisher A280-100) and centrifuged for $3 \mathrm{~min}$ at $7200 \mathrm{~g}$. Pellets and supernatants were kept at $-80^{\circ} \mathrm{C}$ 
until protein and glutathione determinations, respectively. Reduced glutathione (GSH) and glutathione disulfide (GSSG) were quantified by capillary $(75-\mu \mathrm{m} \times 50$-cm silica) electrophoresis $(75 \mathrm{mM}$ boric acid and $25 \mathrm{mM}$ Bis-Tris, $\mathrm{pH} 8.4,28^{\circ} \mathrm{C}, 18 \mathrm{kV}$ ) as described previously (Lavoie et al., 2008). The redox potential was defined as the half-cell reduction potential of the GSSG $\left(2 \mathrm{H}^{+} / 2 \mathrm{GSH}\right.$ couple) and calculated by using the Nernst equation $\left(25^{\circ} \mathrm{C}\right.$, pH 7.0) (Turcot et al., 2009).

\section{RNA Extraction}

Collected tissue samples were stored in RNAlater stabilization solution (Ambion) and stored at $-20^{\circ} \mathrm{C}$ for later use. About 15 to $60 \mathrm{mg}$ of tissue per sample was homogenized in $1 \mathrm{ml}$ of TRIzol Reagent (Ambion) with a TissueLyserII homogenizer (Qiagen) and extracted according to the manufacturer's instructions. Samples were further processed using the PureLink RNA Mini Kit (Ambion), and contaminating DNA was removed via DNase on-column digestion. A BioDrop spectrophotometer was used to determine RNA concentrations, and the ratio of absorbance at 260 and $280 \mathrm{~nm}$ is used to assess purity. RNA integrity was evaluated by visualization of intact $18 \mathrm{~S}$ and $28 \mathrm{~S}$ RNA bands following agarose gel electrophoresis. SuperScript VILO Master Mix (Invitrogen) was used to synthesize cDNA with $1 \mu \mathrm{g}$ of RNA per $20 \mu \mathrm{l}$ reaction.

\section{RNA Sequencing}

RNA sequencing methodology was adapted from Pai et al. (2016). Briefly, libraries were prepared using the Illumina TruSeq protocol. Once prepared, indexed cDNA libraries were pooled (six libraries per pool) in equimolar amounts, and the majority was sequenced with single-end $101 \mathrm{bp}$ reads on the Illumina HiSeq4000. Low quality score bases and adaptor sequences were first trimmed using Trim Galore (version 0.2.7). The resulting reads were then mapped to a genome reference sequence (Ensembl Rnor_6.0 release 81) with STAR (version 2.4.2) using the 1-pass protocol. The number of mismatches allowed for the pairs was of 5, and a soft-clipping step that optimizes alignment scores was automatically applied by the STAR software. Read counting on each gene was done with HTseq (version 0.6.1p1), which was launched separately on each alignment file with the intersection-nonempty option, supported by SAMtools (version 0.1.19) using the same gene reference file as for the alignments.

\section{Statistical Analyses}

Sample sizes were calculated as recommended (Charan and Kantharia, 2013) using data from previously published studies, as well as our own pilot studies using power set at $0.8(80 \%)$ and significance set at $p<0.05$. All values are presented as means $\pm \mathrm{SD}$, except where noted. Normality was assessed using the Shapiro-Wilk test. Unpaired Student's $t$ tests were used to compare values between the two groups. Statistical analyses were performed using the SPSS 16.0 (IBM Corporation, Armonk, NY) software. For RNA-Seq analyses, the DESeq2 (version 1.18.1) software was used to identify genes with a significantly different expression in the HF group. A FDR-adjusted $p<0.10$, corresponding to the treatment variable, and an absolute fold change of mean expression level greater than 1.5 was required to qualify a gene as significantly differently expressed (Love et al., 2014). Significance for all other statistical analyses was set at $p<0.05$.

\section{RESULTS}

As previously reported (Andrich et al., 2018a,b), we found no significant difference in body weight between both groups (data not shown). We observed significantly lower total glutathione levels in the soleus muscle of the HFD group $(p=0.046$; Figure 1A), which was largely due to the significant $25 \%$ decrease of GSH levels ( $p=0.042$; Figure 1B). However, we did not find any difference in GSSG levels ( $p=0.722$; Figure 1C), GSH:GSSG ratio ( $p=0.693$; Figure $2 \mathrm{~A}$ ), or glutathione redox potential ( $p=0.534$; Figure 2B).

When looking at gene expression levels, we did not find any significant differences in glutathione metabolism (Pizzorno, 2014) enzymes glutamate cysteine ligase catalytic subunit (GCLC; adjusted $p=0.865$ ), glutamate cysteine ligase modifier subunit (GCLM; adjusted $p=0.800$ ), glutathione synthase (GSS; adjusted $p=0.984$ ), methionine synthase (MTR; adjusted $p=0.917$ ), glutathione reductase (GSR; adjusted $p=0.978$ ), $\gamma$-glutamyltransferase-7 (GGT7; adjusted $p=0.248$ ), or the Nrf2 transcription factor (NFE2L2; adjusted $p=0.990$; Figure 3A). Further, we did not find any differences in major antioxidant enzymes glutathione peroxidase (GPX; adjusted $p=0.912$ ), catalase (CAT; adjusted $p=0.399$ ), or mitochondrial superoxide dismutase (SOD2; adjusted $p=0.600$; Figure 3B).
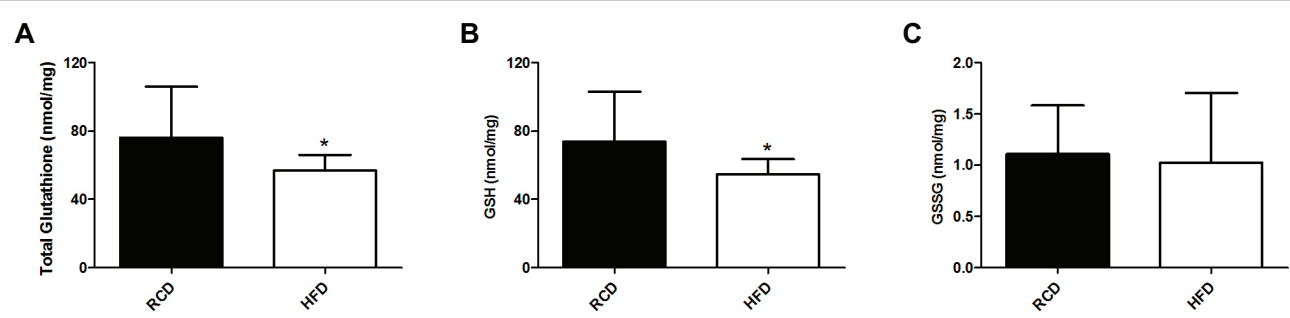

FIGURE 1 | Soleus muscle levels of total glutathione (A), GSH (B) and GSSG (C) in young rats submitted to 14 days of HFD or RCD. Results are presented as means \pm SD for $n=12-13$; * indicates significant difference between the two groups $(p<0.05)$. 
A

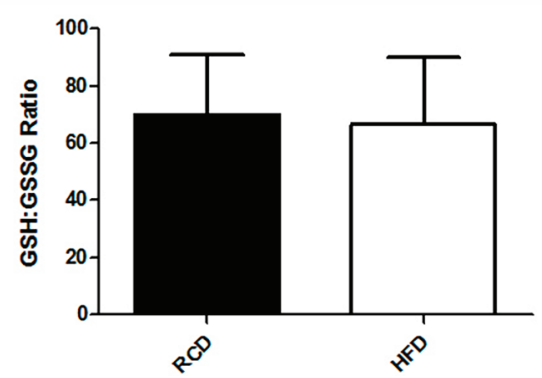

B

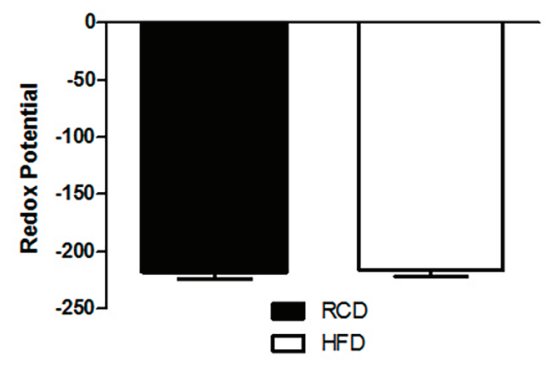

FIGURE 2 | Soleus muscle GSH:GSSG ratio (A) and glutathione redox potential (B) in young rats submitted to 14 days of HFD or RCD. Results are presented as means \pm SD for $n=12-13$.

A

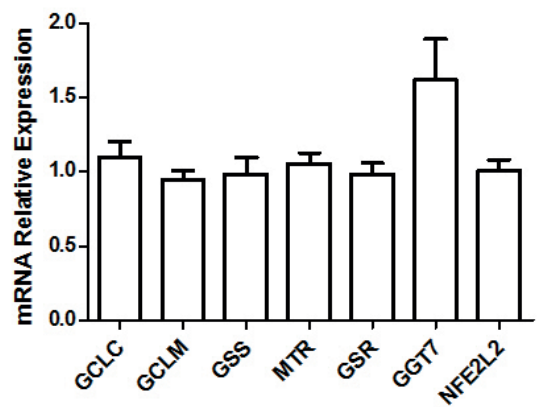

B

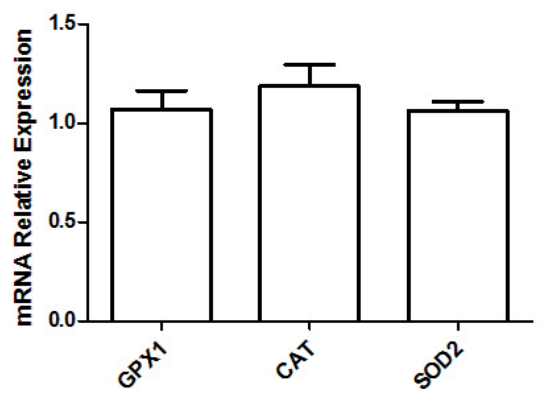

FIGURE 3 | Relative gene expression levels of various enzymes and transcription factors implicated in the glutathione metabolism (A) and relative gene expression levels of major antioxidant enzymes (B) in the soleus muscle of young rats submitted to 14 days of HFD. Results are presented as mean fold change, compared to the RCD group, \pm SEM for 5-6 replicates per condition.
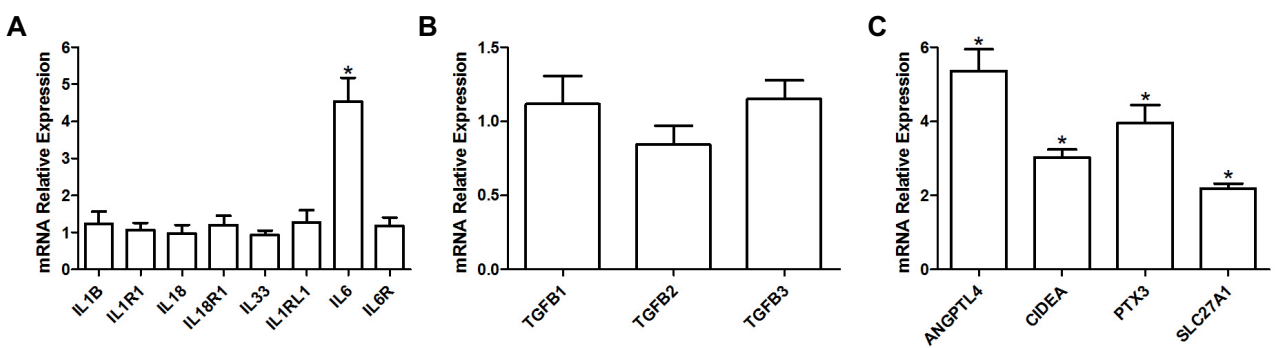

FIGURE 4 | Relative gene expression levels of various interleukins and their respective receptors (A), relative gene expression levels of TGF- $\beta$ cytokines (B) as well as relative gene expression of various pro-inflammatory proteins $\mathbf{( C )}$ in the soleus muscle of young rats submitted to 14 days of HFD. Results are presented as mean fold change, compared to the RCD group, \pm SEM for 5-6 replicates per condition; * indicates significant difference between the two groups (adjusted $p<0.10$ ).

We observed a significant 4.5 fold increase in the expression of interleukin 6 (IL6; adjusted $p=0.05$ ) in the HFD group, but not of its receptor (IL6R; adjusted $p=0.913$ ) or of any other interleukins or their respective receptor (adjusted $p \geq 0.902$; Figure 4A). Expression levels were also similar for the cytokine transforming growth factor $\beta$ (TGFB; adjusted $p \geq 0.579$; Figure 4B) superfamily genes. However, we observed a significant increase in the expression of other proteins implicated in pro-inflammatory pathways, such as a 5.4 fold increase in angiopoietin-like 4 (ANGPTL4; adjusted $p=0.009$ ), a 3 fold increase in cell death activator CIDE-A (CIDEA; adjusted $p<0.001$ ), a 4 fold increase in pentraxin-related protein PTX3 (PTX3; adjusted $p=0.006$ ), and a 2.2 fold increase in long-chain fatty acid transport protein 1 (SLC27A1/FATP1; adjusted $p<0.001$; Figure 4C).

Finally, we did not observe any difference in the gene expression levels of NF- $\kappa B$ (NFKB; adjusted $p \geq 0.801$; Figure 5A) 

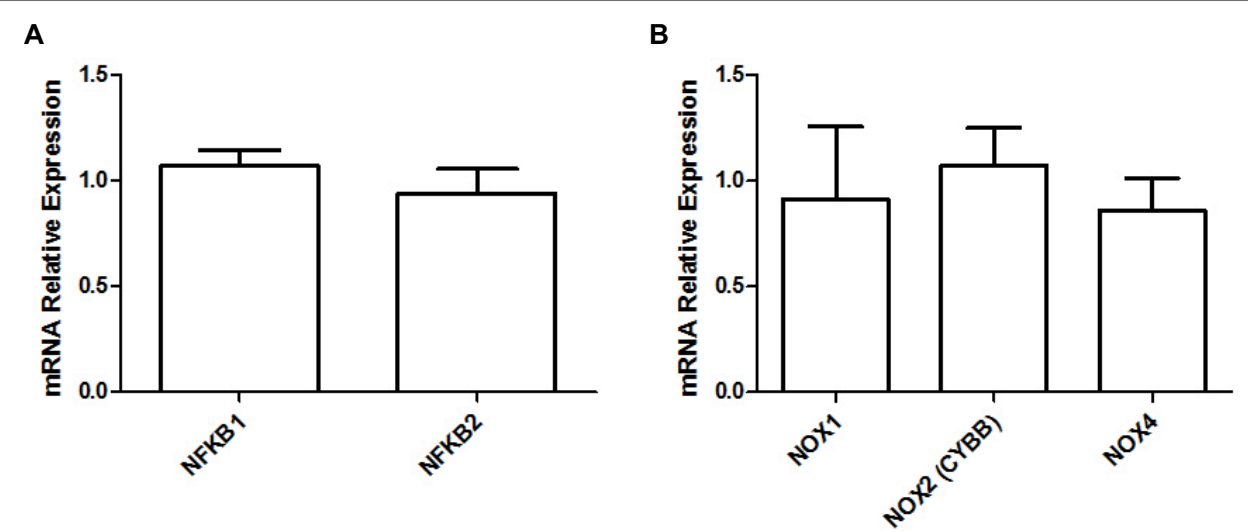

FIGURE 5 | Relative gene expression levels of NF-kB (A) and relative gene expression levels of various NADPH oxidase isoforms (B) in the soleus muscle of young rats submitted to 14 days of HFD. Results are presented as mean fold change, compared to the RCD group, \pm SEM for 5-6 replicates per condition.

protein complex members or NADPH oxidase isoforms (NOX; adjusted $p \geq 0.801$; Figure 5B) between both groups.

\section{DISCUSSION}

The present study intended to clarify the effects of a shortterm HFD on the mechanisms regulating glutathione metabolism, the development of oxidative stress, and inflammation in the soleus (SOL) muscle of young rats. To our knowledge, the present results are first to demonstrate reduced glutathione levels after such a short exposure to HFD. After measuring the expression levels of a host of enzymes involved in the regulation of glutathione metabolism, results suggest that this decrease in GSH is not due to an alteration in de novo synthesis or GSSG recycling via GSR. Further, a significant increase in the expression of the pro-inflammatory cytokine interleukin 6 (IL-6) in the SOL muscle suggests an involvement in early metabolic alterations that can disrupt lipid and glucose metabolisms. These alterations precede any observable weight gain but could contribute to the mechanisms of impaired insulin signaling (Matsuzawa-Nagata et al., 2008), which could ultimately lead to the development of type 2 diabetes (Wang et al., 2003), as well as other metabolic disorders (Lumeng and Saltiel, 2011).

Multiple studies have previously reported HFD-induced altered GSH or GSSG levels or ratio in rodent skeletal muscle (Anderson et al., 2009; Ritchie and Dyck, 2012; Espinosa et al., 2013; Yuzefovych et al., 2013; Gortan Cappellari et al., 2016; Pinho et al., 2017). However, the expression of both GCL subunits (the rate-limiting enzymes in the de novo synthesis of GSH), or of GSR (catalyzing the reduction of GSSG into GSH), was not altered, as previously reported in mice liver (Zhou et al., 2018). The latter study hypothesized that HFD could alter GSH levels via glutathione synthesis-related gene promoters hypermethylation. In the same study, a diet supplemented with serine, a cysteine precursor, was shown to counteract the alterations in GSH production and the development of oxidative stress induced by a HFD in hepatic tissues. This is of great interest, since cysteine availability is the rate-limiting factor of cellular GSH synthesis $(\mathrm{Lu}, 2013)$. As shown in previous work (Andrich et al., 2018a), our HFD formulation is supplemented with L-cystine, the oxidized dimer form of cysteine. Therefore, we conclude that reduced glutathione levels observed in this study were not a consequence of decreased cysteine availability caused by a lack of nutritional intake, as L-cystine supplementation was previously shown to stimulate GSH production (Yin et al., 2016). In that same study, using the same diet and protocol, we observed significantly lighter livers in HFD rats (Andrich et al., 2018a). Glutathione levels are at their highest in liver (where it is primarily synthesized), which also plays an important role in glutathione inter-organ homeostasis (Ookhtens and Kaplowitz, 1998). However, it appears that hepatic GSH needs to reach extreme depletion before it can affect skeletal muscle GSH concentrations (Burk and Hill, 1995). Further, the hepatic cysteine concentration is not considered to be a limiting step of GSH synthesis, as methionine is converted to cysteine. Nevertheless, the first enzyme in this metabolic cascade, methionine adenosyltransferase, can be inhibited by oxidative molecules (Elremaly et al., 2012, 2016). On the other hand, a more recent study hypothesized that skeletal muscle glutamine levels could influence hepatic GSH production in the presence of oxidative stress (Bilinsky et al., 2015). Thus, HFD-modulated interactions between liver and skeletal muscle glutathione metabolism need to be clarified.

As GSH reduces hydrogen peroxide $\left(\mathrm{H}_{2} \mathrm{O}_{2}\right)$ through GPX, the levels of GSSG, a product of that reaction, rise. Under cellular stress, GSH levels drop as GSSG accumulates in the cell, although it can also react with the free sulfhydryl group of a protein to form a mixed disulfide or be transported out of the cell $(\mathrm{Lu}, 2013)$. Hence, the GSH:GSSG ratio is a good indicator of cellular oxidative stress (Schafer and Buettner, 2001). We did not observe any significant HFD-induced changes in GSSG levels or in the GSH:GSSG ratio. This, combined with the lack of difference in the expression of 
major antioxidant enzymes GPX, CAT, and MnSOD or in various isoforms of NADPH oxidase, a superoxide precursor, would suggest that the soleus muscle is not under cellular stress, yet. This would confirm earlier findings from our group that showed no increase in $\operatorname{ROS}\left(\mathrm{H}_{2} \mathrm{O}_{2}\right)$ production from permeabilized soleus muscle fibers in rats submitted to the same 14-day HFD (Leduc-Gaudet et al., 2018). Nonetheless, in vivo measurements of $\mathrm{H}_{2} \mathrm{O}_{2}$ and malondialdehyde (MDA), a product of lipid peroxidation and widely used marker of oxidative stress (Nielsen et al., 1997), could provide further confirmation of these observations. The present results do not show any difference in the glutathione redox potential as calculated by the Nernst equation. However, this equation's validity as an indicator of cellular redox potential is currently debated in the literature, as it appears that the redox potential is highly dependent of GSH but not GSSG levels (Flohe, 2013). Moreover, further evidence points toward the redox potential, depending predominantly on kinetics per se rather than thermodynamic constraints (Deponte, 2017). A major consequence of the observed 25\% drop in GSH levels is a decreased capacity to detoxify endogenous peroxides via GPX. Thus, it is appealing to postulate that, under exercise-induced physical stress and accelerated ROS production (Steinbacher and Eckl, 2015), the SOL antioxidant defense system will be compromised in HFD rats due to significantly lower GSH levels. Measurements of skeletal muscle glutathione levels following an exercise bout could confirm this hypothesis.
The other major finding of this study is the 4.5 fold increase in the gene expression of pro-inflammatory cytokine IL-6, which has previously been shown to be increased in the obese state (Eder et al., 2009) and diminished after weight loss (Bougoulia et al., 2006). Further, elevated IL-6 levels are a good indicator of an inflamed state, which can play a key role in the development of insulin resistance and other associated diseases (Yamashita et al., 2018). Its production can be regulated by various factors like C/EBP $\beta$ (Hungness et al., 2002) and PPAR $\gamma$-activated proteins ANGPTL4 (Phua et al., 2017), CIDEA (Chatterjee et al., 2015), and FATP1 (Nishiyama et al., 2018). Interestingly, PPAR $\gamma$ has often been associated to Il-6 inhibition through STAT3 inactivation (Wang et al., 2004). However, other evidence suggests that PPAR $\gamma$ could trigger IL- 6 production in skeletal muscle (Assi et al., 2017) and other cell types and tissues (Wanichkul et al., 2003; Zhang et al., 2014). Here, our data suggest that PPAR $\gamma$, whose activity has been shown to be modulated by lipid ingestion (den Besten et al., 2015), could stimulate IL- 6 expression through the activation of other proteins. In turn, IL- 6 can induce the production of other pro-inflammatory proteins like PTX3 (Figure 6; Atar et al., 2017). Co-incidence of elevated IL-6 and lower GSH levels was previously reported (Valles et al., 2013), while obesogenic diets were shown to induce both of these effects in mice (Han et al., 2017) and rats (Govindaraj and Sorimuthu Pillai, 2015). In individuals with type 2 diabetes, increased IL-6 levels have been suspected as a cause of lowered GSH levels (Lagman et al., 2015). In mice, IL-6 was associated with

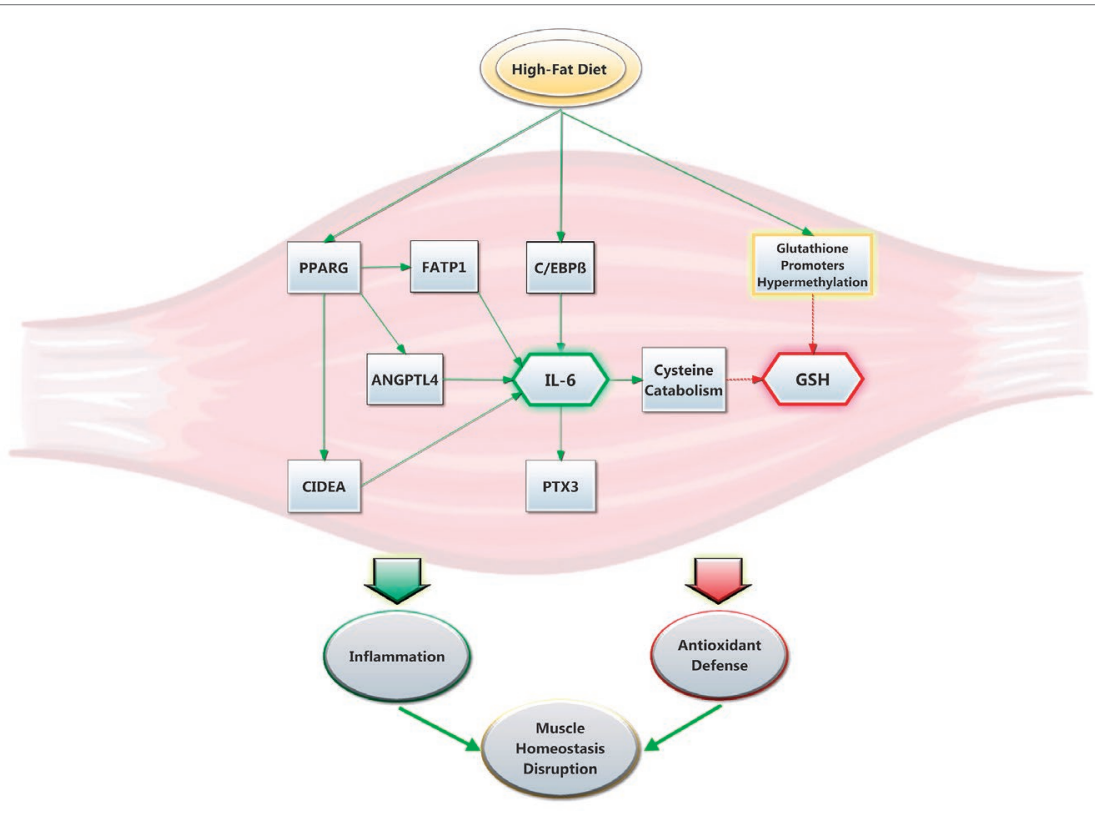

FIGURE 6 | Suggested interplay between HFD, GSH levels and IL-6 expression in rat soleus muscle. The high-fat diet promptly promotes the expression of IL-6. This is stimulated by an increase in C/EBP $\beta$ (Hungness et al., 2002) and PPAR $\gamma$ activity (den Besten et al., 2015), the latter which yields the upregulation of pro-inflammatory proteins ANGPTL4, CIDEA and FATP1. In turn, IL-6 increases the expression of PTX3 and promotes cysteine catabolism (Hack et al., 1996), which lowers GSH levels. The latter are also decreased via HFD through a mechanism that was previously proposed to involve glutathione synthesis-related gene promoters hypermethylation (Zhou et al., 2018). Ultimately, the present results show that HFD promptly alters the antioxidant defense system while promoting inflammation and disruption in skeletal muscle homeostasis. 
glutathione depletion in the skeletal muscle, a mechanism possibly involving increased cysteine catabolism (Hack et al., 1996). Further, IL-6 has been shown to promote GSH release, but not production, from the liver into blood (Obrador et al., 2011). It remains to be seen if such a phenomenon could occur in the skeletal muscle. Furthermore, GSH has been shown to inhibit IL-6 production in patients with liver cirrhosis (Pena et al., 1999). Induced GSH depletion has also been demonstrated to inhibit $\mathrm{T}$ helper cell $\mathrm{T}_{\mathrm{h}} 1$ response in favor of $\mathrm{T}_{\mathrm{h}} 2$ response, which is responsible for IL-6 production (Peterson et al., 1998; Brundu et al., 2016). Thus, elevated IL-6 expression could also be consequential to low GSH levels. On the other hand, we did not observe a different expression of the IL-33 gene in the HFD group, which stimulates the production of $\mathrm{T}_{\mathrm{h}} 2$-associated cytokines (Schmitz et al., 2005). In both groups, we also found similar gene expression of other pro-inflammatory cytokines of the interleukin-1 superfamily, including IL-1 $\beta$, whose expression has been shown to be stimulated by HFD in the vastus lateralis muscle of rats (Collins et al., 2016). In light of those results, it seems appropriate to recommend that the underlying mechanisms of glutathione and interleukin interactions should be further investigated in future studies.

In order to better assess the inflammatory status of the SOL muscle, the expression of TGF- $\beta$ cytokine superfamily isoforms was also considered, as it was reported to decrease GSH levels in multiple cell types, in vitro (Liu and Gaston Pravia, 2010), possibly via the suppression of GCLC expression (Arsalane et al., 1997). Further, HFD was shown to induce a rise in TGF- $\beta$ levels in both rats and mice (Yadav et al., 2011; Sousa-Pinto et al., 2016). We could not, however, observe similar results after submitting young rats to a 2-week HFD. Therefore, we cannot postulate that TGF- $\beta$ influences glutathione metabolism at this early stage. As discussed above, a decrease in the GSH concentration, as a glutathione peroxidase substrate, will result in a lower detoxification of endogenous peroxide, allowing an increase in the intracellular concentration of $\mathrm{H}_{2} \mathrm{O}_{2}$. Because it activates NF- $\kappa B, \mathrm{H}_{2} \mathrm{O}_{2}$ could therefore stimulate the increase of IL-6. Indeed, NF- $\kappa \mathrm{B}$ is a major mediator of the inflammation cascade and its activation can be either stimulated or inhibited by GHS, depending on the tissue of interest and the experimental model (Hammond et al., 2001). Increased NF- $\kappa \mathrm{B}$ phosphorylation was also observed in the gastrocnemius muscle of rats after 16 weeks of HFD (Sishi et al., 2011). Chronic NF- $\kappa$ B activation may be involved in the development of several diseases, including obesity and type 2 diabetes (Lira et al., 2012). We could not, however, detect any difference in the expression of NF- $\kappa \mathrm{B}$ between HFD and RCD rats after 2 weeks, underlining that, in our model, HFD could impact endogenous metabolism rather than gene expression.

Results presented in this study demonstrate that a shortterm high fat diet induces lower GSH levels in the SOL muscle of young rats. This effect can neither be attributed to a decrease in the expression of glutathione synthesisimplicated enzymes nor to observable oxidative stress. However, decreased GSH levels suggest a potentially altered antioxidant defense system. Moreover, 2 weeks of HFD induced a significant increase in IL-6 gene expression, which suggests its interaction with skeletal muscle glutathione metabolism. It was previously reported that IL- 6 mRNA expression is increased in response to contractions or to glycogen depletion in the skeletal muscle (Munoz-Canoves et al., 2013). The present data, coinciding with our previous results, raise the hypothesis that disruptions in the antioxidant defense system, coupled to inflammation activation, could play a role in the impairment of contractile functions in the soleus muscle of young rats submitted to only 14 days of HFD. It was previously shown that IL-6 plays a pivotal role in muscle wasting mechanisms (Belizario et al., 2016), although we could not observe evidence of atrophy in neither SOL nor EDL in our previous results (Andrich et al., 2018b). On the other hand, GSH has been shown to improve $\mathrm{Ca}^{2+}$ sensitivity in rat skeletal muscle, although this was only observed in fast twitch fibers (Murphy et al., 2008) in which fast skeletal muscle troponin isoforms are highly expressed. Further, it appears as though diets rich in saturated fatty acids could alter fast skeletal muscle troponin T (TNNT3 gene) expression through alternative splicing of pre-mRNA in rat skeletal muscle (Black et al., 2017), although it remains to be seen if HFD could also alter the expression of other proteins of the troponin complex (troponin C and troponin I). Thus, further studies are needed to elucidate what role glutathione and inflammation could play in impaired oxidative muscle contractile functions and to better clarify the mechanisms (including the role of the liver and IL-6) underlying the reduction of GSH levels observed in the SOL muscle of young rats submitted to a short-term HFD.

\section{ETHICS STATEMENT}

This study was carried out in strict accordance with recommendations of the National Institutes of Health guide for the care and use of Laboratory animals. Before undergoing the experimental work, the protocol was approved by the Comité Institutionnel de Protection des Animaux (CIPA) of UQAM (Permit Number: 0515-R3-759-0516).

\section{AUTHOR CONTRIBUTIONS}

DA and DS-P wrote the manuscript. DA, LM, YO, NA, JM, J-CG, LB, J-CL, and DS-P performed the experiments. DA contributed to data treatment, statistical analyses, tables and figures. FL, GD, AC, J-CL, and DS-P designed the study. All authors contributed to manuscript revision, read and approved the submitted version.

\section{FUNDING}

DS-P is funded by a NSERC Discovery Grant \#418509-2012 and his salary support is provided by a FQRS Chercheur Boursier Junior 1 Award. 


\section{REFERENCES}

Anderson, E. J., Lustig, M. E., Boyle, K. E., Woodlief, T. L., Kane, D. A., Lin, C. T., et al. (2009). Mitochondrial $\mathrm{H}_{2} \mathrm{O}_{2}$ emission and cellular redox state link excess fat intake to insulin resistance in both rodents and humans. J. Clin. Invest. 119, 573-581. doi: 10.1172/JCI37048

Andrich, D. E., Melbouci, L., Ou, Y., Leduc-Gaudet, J. P., Chabot, F., Lalonde, F, et al. (2018a). Altered feeding behaviors and adiposity precede observable weight gain in young rats submitted to a short-term high-fat diet. J. Nutr. Metab. 2018. doi: 10.1155/2018/1498150

Andrich, D. E., Ou, Y., Melbouci, L., Leduc-Gaudet, J. P., Auclair, N., Mercier, J., et al. (2018b). Altered lipid metabolism impairs skeletal muscle force in young rats submitted to a short-term high-fat diet. Front. Physiol. 9:1327. doi: 10.3389/ fphys.2018.01327

Arsalane, K., Dubois, C. M., Muanza, T., Begin, R., Boudreau, F., Asselin, C., et al. (1997). Transforming growth factor-betal is a potent inhibitor of glutathione synthesis in the lung epithelial cell line A549: transcriptional effect on the GSH rate-limiting enzyme gamma-glutamylcysteine synthetase. Am. J. Respir. Cell Mol. Biol. 17, 599-607. doi: 10.1165/ajrcmb.17.5.2833

Assi, M., Kenawi, M., Ropars, M., and Rebillard, A. (2017). Interleukin-6, C/EBP-beta and PPAR-gamma expression correlates with intramuscular liposarcoma growth in mice: the impact of voluntary physical activity levels. Biochem. Biophys. Res. Commun. 490, 1026-1032. doi: 10.1016/j.bbrc.2017.06.158

Atar, A., Kural, A., Yenice, G., Comez, I., and Tugcu, V. (2017). Role of interleukin-6 and pentraxin 3 as an early marker in Peyronie's disease. Kaohsiung J. Med. Sci. 33, 195-200. doi: 10.1016/j.kjms.2017.01.007

Auberval, N., Dal, S., Bietiger, W., Pinget, M., Jeandidier, N., Maillard-Pedracini, E., et al. (2014). Metabolic and oxidative stress markers in Wistar rats after 2 months on a high-fat diet. Diabetol. Metab. Syndr. 6:130. doi: 10.1186/1758-5996-6-130

Belizario, J. E., Fontes-Oliveira, C. C., Borges, J. P., Kashiabara, J. A., and Vannier, E. (2016). Skeletal muscle wasting and renewal: a pivotal role of myokine IL-6. Springerplus 5:619. doi: 10.1186/s40064-016-2197-2

Bilinsky, L. M., Reed, M. C., and Nijhout, H. F. (2015). The role of skeletal muscle in liver glutathione metabolism during acetaminophen overdose. J. Theor. Biol. 376, 118-133. doi: 10.1016/j.jtbi.2015.04.006

Biro, F. M., and Wien, M. (2010). Childhood obesity and adult morbidities. Am. J. Clin. Nutr. 91, 1499S-1505S. doi: 10.3945/ajcn.2010.28701B

Black, A. J., Ravi, S., Jefferson, L. S., Kimball, S. R., and Schilder, R. J. (2017). Dietary fat quantity and type induce transcriptome-wide effects on alternative splicing of pre-mRNA in rat skeletal muscle. J. Nutr. 147, 1648-1657. doi: 10.3945/jn.117.254482

Bougoulia, M., Triantos, A., and Koliakos, G. (2006). Plasma interleukin-6 levels, glutathione peroxidase and isoprostane in obese women before and after weight loss. Association with cardiovascular risk factors. Hormones 5, 192-199. doi: 10.14310/horm.2002.11182

Brundu, S., Palma, L., Picceri, G. G., Ligi, D., Orlandi, C., Galluzzi, L., et al. (2016). Glutathione depletion is linked with Th2 polarization in mice with a retrovirus-induced immunodeficiency syndrome, murine AIDS: role of proglutathione molecules as immunotherapeutics. J. Virol. 90, 7118-7130. doi: 10.1128/JVI.00603-16

Burk, R. F., and Hill, K. E. (1995). Reduced glutathione release into rat plasma by extrahepatic tissues. Am. J. Phys. 269, G396-G399. doi: 10.1152/ ajpgi.1995.269.3.G396

Charan, J., and Kantharia, N. D. (2013). How to calculate sample size in animal studies? J. Pharmacol. Pharmacother. 4, 303-306. doi: 10.4103/0976-500X.119726

Chatterjee, A., Mondal, P., Ghosh, S., Mehta, V. S., and Sen, E. (2015). PPARgamma regulated CIDEA affects pro-apoptotic responses in glioblastoma. Cell Death Dis. 1:15038. doi: 10.1038/cddiscovery.2015.38

Collins, K. H., Hart, D. A., Reimer, R. A., Seerattan, R. A., Waters-Banker, C., Sibole, S. C., et al. (2016). High-fat high-sucrose diet leads to dynamic structural and inflammatory alterations in the rat vastus lateralis muscle. $J$. Orthop. Res. 34, 2069-2078. doi: 10.1002/jor.23230

DeFronzo, R. A., and Tripathy, D. (2009). Skeletal muscle insulin resistance is the primary defect in type 2 diabetes. Diabetes Care 32(Suppl. 2), S157-S163. doi: $10.2337 / \mathrm{dc} 09-\mathrm{S} 302$

den Besten, G., Bleeker, A., Gerding, A., van Eunen, K., Havinga, R., van Dijk, T. H., et al. (2015). Short-chain fatty acids protect against high-fat diet-induced obesity via a PPARgamma-dependent switch from lipogenesis to fat oxidation. Diabetes 64, 2398-2408. doi: 10.2337/db14-1213

Deponte, M. (2017). The incomplete glutathione puzzle: just guessing at numbers and figures? Antioxid. Redox Signal. 27, 1130-1161. doi: 10.1089/ars.2017.7123

Diaz-Morales, N., Rovira-Llopis, S., Escribano-Lopez, I., Banuls, C., Lopez-Domenech, S., Falcon, R., et al. (2016). Role of oxidative stress and mitochondrial dysfunction in skeletal muscle in type 2 diabetic patients. Curr. Pharm. Des. 22, 2650-2656. doi: 10.217 4/1381612822666160217142949

Eder, K., Baffy, N., Falus, A., and Fulop, A. K. (2009). The major inflammatory mediator interleukin-6 and obesity. Inflamm. Res. 58, 727-736. doi: 10.1007/ s00011-009-0060-4

Elremaly, W., Mohamed, I., Rouleau, T., and Lavoie, J. C. (2016). Impact of glutathione supplementation of parenteral nutrition on hepatic methionine adenosyltransferase activity. Redox Biol. 8, 18-23. doi: 10.1016/j. redox.2015.12.003

Elremaly, W., Rouleau, T., and Lavoie, J. C. (2012). Inhibition of hepatic methionine adenosyltransferase by peroxides contaminating parenteral nutrition leads to a lower level of glutathione in newborn Guinea pigs. Free Radic. Biol. Med. 53, 2250-2255. doi: 10.1016/j.freeradbiomed.2012.10.541

Espinosa, A., Campos, C., Diaz-Vegas, A., Galgani, J. E., Juretic, N., Osorio-Fuentealba, C., et al. (2013). Insulin-dependent $\mathrm{H}_{2} \mathrm{O}_{2}$ production is higher in muscle fibers of mice fed with a high-fat diet. Int. J. Mol. Sci. 14, 15740-15754. doi: 10.3390/ijms140815740

Fisher-Wellman, K. H., Gilliam, L. A., Lin, C. T., Cathey, B. L., Lark, D. S., and Neufer, P. D. (2013). Mitochondrial glutathione depletion reveals a novel role for the pyruvate dehydrogenase complex as a key $\mathrm{H}_{2} \mathrm{O}_{2}$-emitting source under conditions of nutrient overload. Free Radic. Biol. Med. 65, 1201-1208. doi: 10.1016/j.freeradbiomed.2013.09.008

Flohe, L. (2013). The fairytale of the GSSG/GSH redox potential. Biochim. Biophys. Acta 1830, 3139-3142. doi: 10.1016/j.bbagen.2012.10.020

Frontera, W. R., and Ochala, J. (2015). Skeletal muscle: a brief review of structure and function. Calcif. Tissue Int. 96, 183-195. doi: 10.1007/s00223-014-9915-y

Galassetti, P. (2012). Inflammation and oxidative stress in obesity, metabolic syndrome, and diabetes. Exp. Diabetes Res. 2012:943706. doi: $10.1155 / 2012 / 943706$

Giacco, F., and Brownlee, M. (2010). Oxidative stress and diabetic complications. Circ. Res. 107, 1058-1070. doi: 10.1161/CIRCRESAHA.110.223545

Gortan Cappellari, G., Zanetti, M., Semolic, A., Vinci, P., Ruozi, G., Falcione, A., et al. (2016). Unacylated ghrelin reduces skeletal muscle reactive oxygen species generation and inflammation and prevents high-fat diet-induced hyperglycemia and whole-body insulin resistance in rodents. Diabetes 65, 874-886. doi: 10.2337/ db15-1019

Govindaraj, J., and Sorimuthu Pillai, S. (2015). Rosmarinic acid modulates the antioxidant status and protects pancreatic tissues from glucolipotoxicity mediated oxidative stress in high-fat diet: streptozotocin-induced diabetic rats. Mol. Cell. Biochem. 404, 143-159. doi: 10.1007/s11010-015-2374-6

Hack, V., Gross, A., Kinscherf, R., Bockstette, M., Fiers, W., Berke, G., et al. (1996). Abnormal glutathione and sulfate levels after interleukin 6 treatment and in tumor-induced cachexia. FASEB J. 10, 1219-1226.

Hammond, C. L., Lee, T. K., and Ballatori, N. (2001). Novel roles for glutathione in gene expression, cell death, and membrane transport of organic solutes. J. Hepatol. 34, 946-954. doi: 10.1016/S0168-8278(01)00037-X

Han, H., Qiu, F., Zhao, H., Tang, H., Li, X., and Shi, D. (2017). Dietary flaxseed oil prevents western-type diet-induced nonalcoholic fatty liver disease in apolipoprotein-e knockout mice. Oxidative Med. Cell. Longev. 2017:3256241. doi: $10.1155 / 2017 / 3256241$

Head, G. A. (2015). Cardiovascular and metabolic consequences of obesity. Front. Physiol. 6:32. doi: 10.3389/fphys.2015.00032

Hruby, A., and $\mathrm{Hu}, \mathrm{F}$. B. (2015). The epidemiology of obesity: a big picture. PharmacoEconomics 33, 673-689. doi: 10.1007/s40273-014-0243-X

Hungness, E. S., Luo, G. J., Pritts, T. A., Sun, X., Robb, B. W., Hershko, D., et al. (2002). Transcription factors C/EBP-beta and -delta regulate IL-6 production in IL-1beta-stimulated human enterocytes. J. Cell. Physiol. 192, 64-70. doi: $10.1002 /$ jcp.10116

Lagman, M., Ly, J., Saing, T., Kaur Singh, M., Vera Tudela, E., Morris, D., et al. (2015). Investigating the causes for decreased levels of glutathione in individuals with type II diabetes. PLoS One 10:e0118436. doi: 10.1371/journal. pone.0118436 
Lavoie, J. C., Rouleau, T., Tsopmo, A., Friel, J., and Chessex, P. (2008). Influence of lung oxidant and antioxidant status on alveolarization: role of lightexposed total parenteral nutrition. Free Radic. Biol. Med. 45, 572-577. doi: 10.1016/j.freeradbiomed.2008.04.018

Leduc-Gaudet, J. P., Reynaud, O., Chabot, F., Mercier, J., Andrich, D. E., St-Pierre, D. H., et al. (2018). The impact of a short-term high-fat diet on mitochondrial respiration, reactive oxygen species production, and dynamics in oxidative and glycolytic skeletal muscles of young rats. Physiol. Rep. 6. doi: $10.14814 /$ phy2.13548

Lira, F. S., Rosa, J. C., Pimentel, G. D., Seelaender, M., Damaso, A. R., Oyama, L. M., et al. (2012). Both adiponectin and interleukin-10 inhibit LPS-induced activation of the NF- $\hat{I}^{\circ} \mathrm{B}$ pathway in $3 \mathrm{~T} 3-\mathrm{L} 1$ adipocytes. Cytokine $57,98-106$. doi: $10.1016 /$ j.cyto.2011.10.001

Liu, R. M., and Gaston Pravia, K. A. (2010). Oxidative stress and glutathione in TGF-beta-mediated fibrogenesis. Free Radic. Biol. Med. 48, 1-15. doi: 10.1016/j.freeradbiomed.2009.09.026

Love, M. I., Huber, W., and Anders, S. (2014). Moderated estimation of fold change and dispersion for RNA-seq data with DESeq2. Genome Biol. 15:550. doi: 10.1186/s13059-014-0550-8

Lu, S. C. (2013). Glutathione synthesis. Biochim. Biophys. Acta 1830, 3143-3153. doi: $10.1016 /$ j.bbagen.2012.09.008

Lumeng, C. N., and Saltiel, A. R. (2011). Inflammatory links between obesity and metabolic disease. J. Clin. Invest. 121, 2111-2117. doi: 10.1172/JCI57132

Matsuzawa-Nagata, N., Takamura, T., Ando, H., Nakamura, S., Kurita, S., Misu, H., et al. (2008). Increased oxidative stress precedes the onset of high-fat diet-induced insulin resistance and obesity. Metabolism 57, 1071-1077. doi: 10.1016/j.metabol.2008.03.010

Munoz-Canoves, P., Scheele, C., Pedersen, B. K., and Serrano, A. L. (2013). Interleukin-6 myokine signaling in skeletal muscle: a double-edged sword? FEBS J. 280, 4131-4148. doi: 10.1111/febs.12338

Murphy, R. M., Dutka, T. L., and Lamb, G. D. (2008). Hydroxyl radical and glutathione interactions alter calcium sensitivity and maximum force of the contractile apparatus in rat skeletal muscle fibres. J. Physiol. 586, 2203-2216. doi: 10.1113/jphysiol.2007.150516

Nielsen, F., Mikkelsen, B. B., Nielsen, J. B., Andersen, H. R., and Grandjean, P. (1997). Plasma malondialdehyde as biomarker for oxidative stress: reference interval and effects of life-style factors. Clin. Chem. 43, 1209-1214.

Nishiyama, K., Fujita, T., Fujimoto, Y., Nakajima, H., Takeuchi, T., and Azuma, Y. T. (2018). Fatty acid transport protein 1 enhances the macrophage inflammatory response by coupling with ceramide and c-Jun N-terminal kinase signaling. Int. Immunopharmacol. 55, 205-215. doi: 10.1016/j. intimp.2017.12.003

Obrador, E., Benlloch, M., Pellicer, J. A., Asensi, M., and Estrela, J. M. (2011). Intertissue flow of glutathione (GSH) as a tumor growth-promoting mechanism: interleukin 6 induces GSH release from hepatocytes in metastatic B16 melanoma-bearing mice. J. Biol. Chem. 286, 15716-15727. doi: 10.1074/jbc. M110.196261

Ookhtens, M., and Kaplowitz, N. (1998). Role of the liver in interorgan homeostasis of glutathione and cyst(e)ine. Semin. Liver Dis. 18, 313-329. doi: 10.1055/s-2007-1007167

Pai, A. A., Baharian, G., Page Sabourin, A., Brinkworth, J. F., Nedelec, Y., Foley, J. W., et al. (2016). Widespread shortening of 3' untranslated regions and increased exon inclusion are evolutionarily conserved features of innate immune responses to infection. PLoS Genet. 12:e1006338. doi: 10.1371/ journal.pgen.1006338

Peckham, S. C., Entenman, C., and Carroll, H. W. (1962). The influence of a hypercalric diet on gross body and adipose tissue composition in the rat. J. Nutr. 77, 187-197. doi: 10.1093/jn/77.2.187

Pena, L. R., Hill, D. B., and McClain, C. J. (1999). Treatment with glutathione precursor decreases cytokine activity. JPEN J. Parenter. Enteral Nutr. 23, 1-6. doi: 10.1177/014860719902300101

Peterson, J. D., Herzenberg, L. A., Vasquez, K., and Waltenbaugh, C. (1998). Glutathione levels in antigen-presenting cells modulate Th1 versus Th2 response patterns. Proc. Natl. Acad. Sci. U. S. A. 95, 3071-3076.

Phua, T., Sng, M. K., Tan, E. H., Chee, D. S., Li, Y., Wee, J. W., et al. (2017). Angiopoietin-like 4 mediates colonic inflammation by regulating chemokine transcript stability via tristetraprolin. Sci. Rep. 7:44351. doi: 10.1038/srep44351

Pinho, R. A., Sepa-Kishi, D. M., Bikopoulos, G., Wu, M. V., Uthayakumar, A., Mohasses, A., et al. (2017). High-fat diet induces skeletal muscle oxidative stress in a fiber type-dependent manner in rats. Free Radic. Biol. Med. 110, 381-389. doi: 10.1016/j.freeradbiomed.2017.07.005

Pizzorno, J. (2014). Glutathione! Integr. Med. 13, 8-12.

Rao, D. P., Kropac, E., Do, M. T., Roberts, K. C., and Jayaraman, G. C. (2016). Childhood overweight and obesity trends in Canada. Health Promot. Chronic Dis. Prev. Can. 36, 194-198. doi: 10.24095/hpcdp.36.9.03

Ritchie, I. R., and Dyck, D. J. (2012). Rapid loss of adiponectin-stimulated fatty acid oxidation in skeletal muscle of rats fed a high fat diet is not due to altered muscle redox state. PLoS One 7:e52193. doi: 10.1371/journal. pone.0052193

Schafer, F. Q., and Buettner, G. R. (2001). Redox environment of the cell as viewed through the redox state of the glutathione disulfide/glutathione couple. Free Radic. Biol. Med. 30, 1191-1212. doi: 10.1016/S0891-5849(01)00480-4

Schmitz, J., Owyang, A., Oldham, E., Song, Y., Murphy, E., McClanahan, T. K., et al. (2005). IL-33, an interleukin-1-like cytokine that signals via the IL-1 receptor-related protein ST2 and induces T helper type 2-associated cytokines. Immunity 23, 479-490. doi: 10.1016/j.immuni.2005.09.015

Sishi, B., Loos, B., Ellis, B., Smith, W., du Toit, E. F., and Engelbrecht, A. M. (2011). Diet-induced obesity alters signalling pathways and induces atrophy and apoptosis in skeletal muscle in a prediabetic rat model. Exp. Physiol. 96, 179-193. doi: 10.1113/expphysiol.2010.054189

Sousa-Pinto, B., Goncalves, L., Rodrigues, A. R., Tomada, I., Almeida, H., Neves, D., et al. (2016). Characterization of TGF-beta expression and signaling profile in the adipose tissue of rats fed with high-fat and energy-restricted diets. J. Nutr. Biochem. 38, 107-115. doi: 10.1016/j.jnutbio.2016.07.017

Steinbacher, P., and Eckl, P. (2015). Impact of oxidative stress on exercising skeletal muscle. Biomolecules 5, 356-377. doi: 10.3390/biom5020356

The, N. S., Suchindran, C., North, K. E., Popkin, B. M., and Gordon-Larsen, P. (2010). Association of adolescent obesity with risk of severe obesity in adulthood. JAMA 304, 2042-2047. doi: 10.1001/jama.2010.1635

Tsutsui, H., Kinugawa, S., Matsushima, S., and Yokota, T. (2011). Oxidative stress in cardiac and skeletal muscle dysfunction associated with diabetes mellitus. J. Clin. Biochem. Nutr. 48, 68-71. doi: 10.3164/jcbn.11-012FR

Turcot, V., Rouleau, T., Tsopmo, A., Germain, N., Potvin, L., Nuyt, A. M., et al. (2009). Long-term impact of an antioxidant-deficient neonatal diet on lipid and glucose metabolism. Free Radic. Biol. Med. 47, 275-282. doi: 10.1016/j.freeradbiomed.2009.04.026

Valles, S. L., Benlloch, M., Rodriguez, M. L., Mena, S., Pellicer, J. A., Asensi, M., et al. (2013). Stress hormones promote growth of B16-F10 melanoma metastases: an interleukin 6- and glutathione-dependent mechanism. J. Transl. Med. 11:72. doi: $10.1186 / 1479-5876-11-72$

Vial, G., Dubouchaud, H., Couturier, K., Cottet-Rousselle, C., Taleux, N., Athias, A., et al. (2011). Effects of a high-fat diet on energy metabolism and ROS production in rat liver. J. Hepatol. 54, 348-356. doi: 10.1016/j. jhep.2010.06.044

Wang, X., Feng, Z., Yang, L., Han, S., Cao, K., Xu, J., et al. (2016). O-GlcNAcase deficiency suppresses skeletal myogenesis and insulin sensitivity in mice through the modulation of mitochondrial homeostasis. Diabetologia 59, 1287-1296. doi: 10.1007/s00125-016-3919-2

Wang, Y., Wang, P. Y., Qin, L. Q., Davaasambuu, G., Kaneko, T., Xu, J., et al. (2003). The development of diabetes mellitus in Wistar rats kept on a high-fat/low-carbohydrate diet for long periods. Endocrine 22, 85-92. doi: 10.1385/ENDO:22:2:85

Wang, L. H., Yang, X. Y., Zhang, X., Huang, J., Hou, J., Li, J., et al. (2004). Transcriptional inactivation of STAT3 by PPARgamma suppresses IL-6responsive multiple myeloma cells. Immunity 20, 205-218. doi: 10.1016/ S1074-7613(04)00030-5

Wanichkul, T., Han, S., Huang, R. P., and Sidell, N. (2003). Cytokine regulation by peroxisome proliferator-activated receptor gamma in human endometrial cells. Fertil. Steril. 79(Suppl. 1), 763-769. doi: 10.1016/s0015-0282(02)04835-5

Yadav, H., Quijano, C., Kamaraju, A. K., Gavrilova, O., Malek, R., Chen, W., et al. (2011). Protection from obesity and diabetes by blockade of TGF-beta/ Smad3 signaling. Cell Metab. 14, 67-79. doi: 10.1016/j.cmet.2011.04.013

Yamashita, A. S., Belchior, T., Lira, F., Bishop, N. C., Wessner, B., Rosa, J. C., et al. (2018). Regulation of metabolic disease-associated inflammation by nutrient sensors. Mediat. Inflamm. 2018:18. doi: 10.1155/2018/8261432

Yin, J., Ren, W., Yang, G., Duan, J., Huang, X., Fang, R., et al. (2016). L-Cysteine metabolism and its nutritional implications. Mol. Nutr. Food Res. 60, 134-146. doi: $10.1002 / \mathrm{mnfr} .201500031$ 
Yokota, T., Kinugawa, S., Hirabayashi, K., Matsushima, S., Inoue, N., Ohta, Y., et al. (2009). Oxidative stress in skeletal muscle impairs mitochondrial respiration and limits exercise capacity in type 2 diabetic mice. Am. J. Physiol. Heart Circ. Physiol. 297, H1069-H1077. doi: 10.1152/ ajpheart.00267.2009

Yuzefovych, L. V., Musiyenko, S. I., Wilson, G. L., and Rachek, L. I. (2013). Mitochondrial DNA damage and dysfunction, and oxidative stress are associated with endoplasmic reticulum stress, protein degradation and apoptosis in high fat diet-induced insulin resistance mice. PLoS One 8:e54059. doi: 10.1371/journal.pone.0054059

Zhang, Y., Hu, L., Cui, Y., Qi, Z., Huang, X., Cai, L., et al. (2014). Roles of PPARgamma/NF-kappaB signaling pathway in the pathogenesis of intrahepatic cholestasis of pregnancy. PLoS One 9:e87343. doi: 10.1371/journal.pone.0087343

Zhou, X., He, L., Zuo, S., Zhang, Y., Wan, D., Long, C., et al. (2018). Serine prevented high-fat diet-induced oxidative stress by activating AMPK and epigenetically modulating the expression of glutathione synthesis-related genes. Biochim. Biophys. Acta 1864, 488-498. doi: 10.1016/j.bbadis.2017.11.009

Conflict of Interest Statement: The authors declare that the research was conducted in the absence of any commercial or financial relationships that could be construed as a potential conflict of interest.

Copyright $\odot 2019$ Andrich, Melbouci, Ou, Auclair, Mercier, Grenier, Lira, Barreiro, Danialou, Comtois, Lavoie and St-Pierre. This is an open-access article distributed under the terms of the Creative Commons Attribution License (CC BY). The use, distribution or reproduction in other forums is permitted, provided the original author(s) and the copyright owner(s) are credited and that the original publication in this journal is cited, in accordance with accepted academic practice. No use, distribution or reproduction is permitted which does not comply with these terms. 


\title{
FGF21 as Modulator of Metabolism in Health and Disease
}

\author{
Caterina Tezze ${ }^{1,2 *}$, Vanina Romanello ${ }^{1,2}$ and Marco Sandri ${ }^{1,2,3,4 *}$ \\ ${ }^{1}$ Veneto Institute of Molecular Medicine, Padua, Italy, ${ }^{2}$ Department of Biomedical Science, University of Padua, Padua, Italy, \\ ${ }^{3}$ Department of Medicine, McGill University, Montreal, QC, Canada, ${ }^{4}$ Department of Biomedical Science, Myology Center, \\ University of Padua, Padua, Italy
}

Fibroblast growth factor 21 (FGF21) is a hormone that regulates important metabolic pathways. FGF21 is expressed in several metabolically active organs and interacts with different tissues. The FGF21 function is complicated and well debated due to its different sites of production and actions. Striated muscles are plastic tissues that undergo adaptive changes within their structural and functional properties in order to meet their different stresses, recently, they have been found to be an important source of FGF21. The FGF21 expression and secretion from skeletal muscles happen in both mouse and in humans during their different physiological and pathological conditions, including

\section{OPEN ACCESS}

Edited by:

Valentina Di Felice,

University of Palermo, Italy

Reviewed by:

Ashok Kumar,

University of Louisville, United States

Fabio Penna,

University of Turin, Italy

*Correspondence:

Caterina Tezze

caterina.tezze@gmail.com

Marco Sandri

marco.sandri@unipd.it

Specialty section:

This article was submitted to

Striated Muscle Physiology,

a section of the journal

Frontiers in Physiology

Received: 30 November 2018

Accepted: 27 March 2019

Published: 17 April 2019

Citation:

Tezze C, Romanello $\mathrm{V}$ and Sandri M (2019) FGF21 as Modulator of Metabolism in Health and Disease.

Front. Physiol. 10:419.

doi: 10.3389/fphys.2019.00419 exercise and mitochondrial dysfunction. In this review, we will discuss the recent findings that identify FG21 as beneficial and/or detrimental cytokine interacting as an autocrine or endocrine in order to modulate cellular function, metabolism, and senescence.

Keywords: FGF21, skeletal muscle, metabolism, regulation, myokine, cytokine, mitochondria

\section{INTRODUCTION}

Nearly $40-50 \%$ of total body mass in non-obese mammals is composed of skeletal muscle. Striated muscles are plastic tissues that undergo adaptive changes in their structural or functional properties in order to meet new challenges. Skeletal muscle tissues have generally been considered to be pure locomotor organs, but in the 2000s it was recognized a secretory function for these muscles. In 2003 it was proposed that IL- 6 and other cytokines were induced and secreted by skeletal muscles into their circulation during physical activity. In consideration of these findings, the muscle-secreted molecules were named "myokines." Increasing evidence underlines that skeletal muscle secretes a wide variety of molecules like cytokines, miRNA, exosomes, mtDNA during exercise, but also in different acquired and inherited diseases (Inagaki et al., 2007; Alipoor et al., 2016; Hoffmann and Weigert, 2017; Delezie and Handschin, 2018; Safdar and Tarnopolsky, 2018). Thus, skeletal muscles are understood to be a source of myokines, metabolites, and muscle-derived molecules. They mediate communication between distant organs to adapt whole body metabolism to nutritional and environmental pressures (Rai and Demontis, 2016; Whitham and Febbraio, 2016). This systemic regulation helps to explain why physical activity, and thereby muscle contraction, elicits several beneficial effects in a variety of diseases. However, the nature and the function of most of the myokines and muscle-derived molecules are still unclear. In this review, we will focus on fibroblast growth factor 21 (FGF21) and its role as a "myokine." Several studies have proven that FGF21 stimulates the oxidation of fatty acids, the production of ketone bodies, and the inhibition of lipogenesis (Fisher and Maratos-Flier, 2016; Staiger et al., 2017). Therefore, the finding that FGF21 
regulates glucose-lipid metabolism has made it a promising therapeutic target for metabolic disease. However, some studies have shown that administrating FGF21 prevents diet-induced obesity and insulin resistance in mice and humans (Fisher and Maratos-Flier, 2016; Staiger et al., 2017). There is a paradoxically positive correlation with elevated serum FGF21 levels and metabolic disorders like obesity, diabetes, mitochondrial diseases, and aging (Staiger et al., 2017; Tezze et al., 2017). Interestingly, all these conditions have muscle loss as a common factor. In addition, several reports have indicated that a pathophysiological role for FGF21 includes:

(a) the promotion of muscle atrophy (Oost et al., 2019), bone loss and reduced bone mineral density (Wei et al., 2012; Fazeli et al., 2015).

(b) that it acts as a stress-induced myokine, which is released during starvation, ER stress, and mitochondrial dysfunction (Izumiya et al., 2008; Keipert et al., 2013; Pereira et al., 2017; Tezze et al., 2017; Rodríguez-Nuevo et al., 2018).

Thus, FGF21 is a powerful and debatable hormone that belongs to the list of factors that controls energy homeostasis and metabolism. As described below, the beneficial and/or detrimental actions of FGF21 are affected by multiple variables including tissue source, serum concentration, animal age, and the presence of synergizing factors.

\section{FGF21 AND METABOLISM}

The fibroblast growth factor (FGF) family is a group of multifunctional signaling molecules that have a wide variety of functions. The family comprises of 22 related proteins, each grouped into subfamilies which are based on genetic and functional similarity. In Nishimura et al. (2000) the Fgf21 gene was identified in mouse embryos by RT-PCR. Making it the 21st discovered Fgf gene, which was later to be included into the FGF19 subfamily (also called endocrine FGF), together with Fgf15 (mouse ortholog of human FGF19) and Fgf23. FGF21 function remained completely unknown until 2005 when it was proposed to be a novel metabolic regulator and a potential anti-diabetic drug (Kharitonenkov et al., 2005). Kharitonenkov et al. (2005) demonstrated that the administration of FGF21 reduced plasma glucose and triglycerides to an almost normal level in both ob/ob and $\mathrm{db} / \mathrm{db}$ mice. These effects persisted for up to $24 \mathrm{~h}$ after which the last FGF21 was administrated. Over the following years, several groups investigated the metabolic role of FGF21 in mice. Their results showed that FGF21 mediates the adaptive starvation response to induce ketogenesis, gluconeogenesis, lipolysis, and lipid $\beta$-oxidation (Inagaki et al., 2007; Izumiya et al., 2008). In line with Kharitonenkov results, FGF21 treatment improved metabolic parameters in obese and diabetic animal models (Coskun et al., 2008; Xu et al., 2009). In humans, FGF21 is a starvation-induced protein that is elevated after 7 days of fasting and regulates the utilization of fuel to adapt metabolism in the late phase of the absence of nutrients
(Fazeli et al., 2015). FGF21 is expressed in several tissues such as liver (Nishimura et al., 2000), adipocytes (Zhang et al., 2008), pancreas (Johnson et al., 2009), and brain where it passes the blood-brain barrier (Hsuchou et al., 2007). Other studies reported the expression of FGF21 mRNA in testes, gastrointestinal tract, brain, skeletal muscle, brown adipose tissue (BAT), and heart (Patel et al., 2015). Based on its role in glucose and lipid metabolism and its wide tissue expression, it was hypothesized that FGF21 is a fasting-adaptation hormone in rodents (Fazeli et al., 2015). However, it was recently demonstrated that FGF21-knockout mice do not exhibit an impaired response to fasting (Antonellis et al., 2016) suggesting that FGF21 is not required for the physiological response to low nutrients.

\section{FGF21 EXPRESSION AND REGULATION}

The FGF21 regulation is complicated because of its different tissue production and action. The liver is considered to be the main site of FGF21 production (Badman et al., 2007; Inagaki et al., 2007). Badman and Inagaki independently showed a cross-talk between FGF21 and PPAR $\alpha$ signaling in the liver during fasting. FGF21 expression was increased in mice that were either fed with a ketogenic diet or through fasting and in these conditions, $\operatorname{PPAR} \alpha$ was recruited in the different regions of the $F g f 21$ promoter (Badman et al., 2007; Inagaki et al., 2007). However, this finding was not confirmed in other metabolic conditions in which FGF21 was induced, suggesting that other transcription factors might also regulate hepatic FGF21 expression. Indeed, Fgf21 was found to be under the regulation of the Unfolding Protein Response (UPR) in hepatocytes (Schaap et al., 2013; Kim et al., 2015). Kim et al. (2015) started from the observation that FGF21 increased in the liver of patients who were affected by steatosis, and mouse models of obesity or non-alcoholic fatty liver disease (NAFLD) while at the same time, observing that an ER stress became triggered. They found that FGF21 expression was dependent from PKR-like ER kinase (PERK)-eukaryotic initiation factor $2 \alpha$ (eIF $2 \alpha$ )-activating transcription factor 4 (ATF4) pathways both, in vitro and in vivo (Kim et al., 2015). Moreover, FGF21-null mice displayed induction of ER stress markers in genes and observed more lipid accumulation in the liver after tunicamycin treatment, an inhibitor of glycoprotein biosynthesis that promotes ER stress (Kim et al., 2015). Several studies have suggested that other transcription factors are involved in the regulation of hepatic FGF21 expression. Adams et al. (2010) reported that thyroid hormone receptor $\beta$, which mediates the action of tri-iodothyronine in the liver, stimulates lipolysis, and hepatic fatty acid oxidation via FGF21 induction. Moreover, several researchers have speculated that FGF21 expression is regulated by RAR $\beta$ (Wang et al., 2010, p. 21; Berry et al., 2012; Tsuchiya et al., 2012; Li et al., 2013). This is because the metabolic effect of the RARb ligand, retinoic acid, on body weight loss and glucose/lipid metabolism are similar to FGF21, this has been recently demonstrated in vitro in $\mathrm{C} 2 \mathrm{C} 12$ (Hirai et al., 2019) but has not yet been confirmed in vivo. 
Retinoic acid receptor-related orphan receptor (ROR) $\alpha$ has been implicated in various physiological functions, including the immune system, inflammation, and circadian rhythms. In the present study, the synthetic $\operatorname{ROR} \alpha / \gamma$ agonist SR1078 stimulated the production and gene expression of FGF21 in C2C12 myotubes. FGF21, a member of the FGF family, plays an important role in the regulation of peripheral glucose tolerance and lipid metabolism while improving metabolic health. The mRNA expression and secretion of FGF21 were significantly weaker in $\operatorname{ROR} \alpha$-silenced cells than in cells transfected with non-targeting control siRNA. SR1078 significantly up-regulated $\mathrm{C} / \mathrm{EBP}$ homologous protein (CHOP), an established marker of ER stress, in a dose-dependent manner in $\mathrm{C} 2 \mathrm{C} 12$ myotubes, while CHOP expression was decreased in $\mathrm{ROR} \alpha$-silenced $\mathrm{C} 2 \mathrm{C} 12$ cells, suggesting that $\mathrm{ROR} \alpha$ is involved in the regulation of FGF21 expression and stimulates ER stress in C2C12 myotubes. The naturally occurring compound baicalein upregulated FGF21 expression and secretion in C2C12 myotubes. Additionally, the up-regulation of CHOP mRNA and protein expression was observed in $\mathrm{C} 2 \mathrm{C} 12$ myotubes after the baicalein treatment. Furthermore, the knockdown of ROR $\alpha$ prevented the augmentation of FGF21 and up-regulation of CHOP in response to baicalein in $\mathrm{C} 2 \mathrm{C} 12$ cells. Collectively, these results suggest that baicalein stimulates the ER stress response and FGF21 expression through a $\mathrm{ROR} \alpha$-dependent mechanism in $\mathrm{C} 2 \mathrm{C} 12$ myotubes, and indicates the potential of baicalein as an effective anti-obesity therapy via its ability to enhance FGF21 production (Hirai et al., 2019). Other reports have shown that the hepatic expression of FGF21 is either regulated positively or negatively by glucocorticoid receptor (GR) (Patel et al., 2015), cAMP-responsive element-binding protein $\mathrm{H}$ (CREBH) (Lee et al., 2017), carbohydrate response element-binding protein (ChREBP) (Iizuka et al., 2009), PPAR $\gamma$ (Moyers et al., 2007; Wang et al., 2008), farnesoid X receptor (FXR) (Cyphert et al., 2012), and liver X receptor (LXR) (Archer et al., 2012; Uebanso et al., 2012) under various conditions. Extrahepatic tissues, such as skeletal muscle, white adipose, and brown adipose tissue regulate FGF21 via different transcription factors. Indeed, PPAR $\gamma$ activation increases FGF21 production in white adipose tissue where it acts as an autocrine or endocrine factor to improve insulin action (Dutchak et al., 2012). Brown adipose tissue (BAT), FGF21 is regulated by ATF2 (Hondares et al., 2011) while the skeletal muscle is controlled by ATF4 (Kim et al., 2013) and by the PI3K-AKT signaling pathway (Izumiya et al., 2008).

\section{FGF21 DOWNSTREAM SIGNALING}

The FGF21-dependent signaling downstream FGF receptors (FGFr) are extremely complicated and well debated. Its complexity is due to the fact that FGF21 is activated and released in different conditions such as energy stress, ER stress, mitochondrial dysfunction, and cold-stress. In vitro studies have failed to identify a direct interaction of FGF19 subfamily with their FGF receptor. This negative finding, and the very weak heparin binding affinity of these family members, implies that the FGF19 subfamily requires additional cofactors to stably bind the FGFRs in the target tissue (Gälman et al., 2008). The aging-suppressor gene Klotho and Beta-Klotho are indeed the essential co-receptors for the interaction and activation of FGFRs (Gälman et al., 2008). The complex FGF21-Klotho binds multiple FGFRs, including FGFR1c, $-2 c,-3 c$, and -4 both, in vitro and in vivo (Agrawal et al., 2018). However, the signaling downstream FGF receptors are tissue specific. In cultured adipocytes, FGF21 treatment activates Ras/Raf MAPK and the downstream effector ERK1 and ERK2 (Moyers et al., 2007; Ge et al., 2011). Transcriptomic analyses on adipocytes identified that ERK/MAPK signaling pathways are enriched after FGF21 administration in vivo, a second study showed that existing ERK1/2- independent pathways in mTOR act on FGF21 activating on adiponectin in adipose tissue in vivo (Minard et al., 2016). In liver, FGF21 positively controls the PI3K/AKT, insulin-like growth factor 1 (IGF-1) and mTOR pathways as well as triglyceride homeostasis, glucose uptake, amino acid transport, and energy expenditure (Muise et al., 2013). Moreover, FGF21 inhibits Growth Hormone (GH) action in hepatocytes resulting in STAT5 inhibition, decreased expression, secretion of IGF-I, and the reduction of body size (Inagaki et al., 2008). Finally, FGF21 also induces the expression of IGFBP1, an inhibitor of IGF1 and suppressor of cytokine signaling 2, which is a negative modulator of $\mathrm{GH}$ signaling, furthering blocking the GH-IGF1 axis in the liver. It has also been reported that FGF21 works on the central nervous system and dorsal vagal complex of the hindbrain to regulate circadian rhythm, which is important for the adaptive starvation response (Bookout et al., 2013).

\section{SERUM LEVELS OF FGF21 IN ANIMAL MODELS}

In published studies, the serum FGF21 concentrations measured in chow-fed mice ranging from 0 to $3000 \mathrm{ng} / \mathrm{mL}$ (Badman et al., 2007; Fisher et al., 2010; Dutchak et al., 2012; Murata et al., 2013; Tezze et al., 2017; Jimenez et al., 2018). The difference in the reported FGF21 serum levels may be dependent on the mice strain, animal age, time of the day in which serum was collected, as well as the methodology used to quantify the serum levels. This complicates the interpretation of the data, since basal FGF21 concentration, as well as the increasing degree, may trigger different metabolic actions. As shown in Table 1, the fold increase of FGF21 serum in knockout/transgenic versus controls mice and in different experimental conditions are witness to a range from 2 to 100. These observations are particularly important considering that FGF21 is a molecule with a very short half-life that, in absence of specific stimuli, has been calculated from 1 to $2 \mathrm{~h}$ (Kharitonenkov et al., 2007; Xu et al., 2009; Hecht et al., 2012). This short half-life in the serum is due to enzymatic degradation and/or renal clearance. In fact, fibroblast activation protein $\alpha$ (FAP) was recently identified as the serine protease that cleaves and inactivates FGF21 (Zhen et al., 2016). Interestingly, FAP is also secreted in human muscle during exercise (Parmar et al., 2018). Therefore, because FGF21 is secreted from the kidney it 
TABLE 1 | Serum level, absolute and normalize, of FGF21 regarding the transgenic mice, reported into the literature.

\begin{tabular}{|c|c|c|c|c|}
\hline Transgenic mouse & Paper & $\begin{array}{l}\text { Basal condition/control } \\
\text { animals serum level (pg/mL) }\end{array}$ & $\begin{array}{l}\text { Transgenic/virus serum level } \\
\text { (pg/mL) }\end{array}$ & $\begin{array}{l}\text { Fold increase compare to } \\
\text { basal condition/control } \\
\text { mouse }\end{array}$ \\
\hline $\begin{array}{l}\text { Virus-mediated FGF21 } \\
\text { knock-down - liver specific }\end{array}$ & Badman et al., 2007 & & Not shown & \\
\hline FGF21 total ko & Antonellis et al., 2016 & & Not shown & \\
\hline FGF21 tg -liver specific & Inagaki et al., 2007 & & Not shown & \\
\hline $\begin{array}{l}\text { Virus-mediated FGF21 - liver } \\
\text { specific }\end{array}$ & Jimenez et al., 2018 & $\begin{array}{l}1000 \text { (null-chow) } 5000 \\
\text { (null-HFD) }\end{array}$ & $\begin{array}{l}\text { Not shown (chow) } 6000 \text { - } \\
25000 \text { (HFD) }\end{array}$ & 1-5 (HFD) (HFD) \\
\hline $\begin{array}{l}\text { Virus-mediated Fgf21 - muscle } \\
\text { specific }\end{array}$ & Jimenez et al., 2018 & 3000 (fed) & 45000(fed) & 15 (fed) \\
\hline atg7 ko - muscle specific & Kim et al., 2013 & Detectable (fed) - 2000(fasted) & $500($ fed $)-4000$ (fasted) & Not clear (fed) - 2 (fasted) \\
\hline Ucp1 tg - muscle specific & Keipert et al., 2013 & $1000($ fed $)-2000$ (fasted) & 4000(fed) - 5000(fasted) & 4 (fed) -2 (fasted) \\
\hline 4ebp1 tg - muscle specific & Tsai et al., 2015 & 1000 (fasted) & 4000 (fasted) & 4 (fasted) \\
\hline Tsc1 ko - muscle specific & Guridi et al., 2015 & 500 (fed) & $1500(\mathrm{fed})$ & 3 (fed) \\
\hline $\begin{array}{l}\text { Opa1 ko - inducible muscle } \\
\text { specific }\end{array}$ & Tezze et al., 2017 & n.d. ( < $50 \mathrm{pg} / \mathrm{mL})$ & $4000(\mathrm{fed})$ & $100(\mathrm{fed})$ \\
\hline $\begin{array}{l}\text { Opa1 ko - constitutive muscle } \\
\text { specific }\end{array}$ & $\begin{array}{l}\text { Rodríguez-Nuevo et al., 2018, } \\
\text { p. } 1\end{array}$ & 222 (fed) & 544 (fed) & 2,4 \\
\hline $\begin{array}{l}\text { Opa1 ko - inducible muscle } \\
\text { specific heterozygous }\end{array}$ & Pereira et al., 2017 & $1069(\mathrm{fed})$ & 11500 (fed) & 10 \\
\hline Fundc1 ko - muscle specific & Fu et al., 2018 & 400 & 900 (HFD) & 2,24 \\
\hline
\end{tabular}

has a short half-life and its activity is modulated by the presence of Klotho and FAP, simply monitoring serum levels may not be enough to claim a direct FGF21 action on targeted tissues without checking the activation of the pathway downstream FGFr.

\section{FGF21 IN CARDIAC AND SKELETAL MUSCLE}

Originally, the heart was not considered to be an FGF21 source or target, primarily because $\beta$-Klotho mRNA is required to be co-receptors for cellular responsiveness to FGF21, and this was only found to be modestly expressed (Fon Tacer et al., 2010). However, recent studies have demonstrated that FGF21 plays a key role in cardiac remodeling. In fact, the heart expresses FGFR1, fibroblast growth factor receptor $1, \beta$-klotho, as well as FGF21. Moreover, in rodents, it has been reported that FGF21 expression protects against pathologic cardiac hypertrophy, oxidative stress, and myocardial infarction (Planavila et al., 2013, 2015; Joki et al., 2015). In the heart, FGF21 acts in the manner of an autocrine and controls autophagy in obesityinduced cardiomyopathy (Rupérez et al., 2018). In skeletal muscle, FGF21 expression was found undetectable or to be limitedly expressed in basal conditions (Izumiya et al., 2008; Hojman et al., 2009; Pereira et al., 2017; Tezze et al., 2017; Rodríguez-Nuevo et al., 2018). However, several physiological and/or pathological conditions triggered FGF21 expression in muscle as well as showing a secretion into the blood. This was first demonstrated in muscle-specific AKT1 transgenic mice, where the activation of AKT1 brought a reduction of adipose tissue via FGF21 secretion (Izumiya et al., 2008). In Kim et al. (2013), it was found that autophagy inhibition, specifically in skeletal muscle, was protected from obesity and HFD because FGF21 was dramatically induced in muscles. As a consequence of the autophagic impairment muscles accumulate dysfunctional mitochondria, resulting in ER stress, UPR activation, and FGF21 induction via the transcription factor of ATF4. Similarly, muscle-specific deletion of Tuberous sclerosis 1 (TSC1) resulted in the activation of mTOR. At the same time FGF21 upregulation, via UPR, improved the insulin sensitivity, leading to an association with a leaner phenotype when compared with controls (Guridi et al., 2015). Consistent expression of constitutively active 4EBP1 in skeletal muscles caused FGF21 secretion into the circulation which then ameliorates lipid metabolism in WAT via PPARs (Tsai et al., 2015). Mild mitochondrial dysfunction is a consequent of UCP1, Uncoupling Protein 1, expression in skeletal muscle resulting in FGF21 expression and the diminution of the size of myofibers, but at the same time also improved the metabolic profile due to the browning of WAT (Keipert et al., 2013). We have now shown that mitochondrial dysfunction is due to OPA1 ablation in skeletal muscle and triggers a dramatic increase of muscle FGF21 transcript and serum levels (Tezze et al., 2017; Pereira et al., 2017; Rodríguez-Nuevo et al., 2018). This important FGF21 induction is mediated by mitochondrialdependent oxidative stress that had caused UPR activation (Tezze et al., 2017; Rodríguez-Nuevo et al., 2018). ER stress, muscle atrophy, and FGF21 upregulation were found in several muscle-specific OPA1-deficient mice, but the phenotypes of these knockout mice were different. These discrepancies may be a consequence to the age of the animal in which OPA1 was deleted, and/or the degree of mitochondrial dysfunction, and/or the blood levels of FGF21. In Tezze et al. (2017) the 100-fold (Table 1) showed an increase of FGF21 blood 


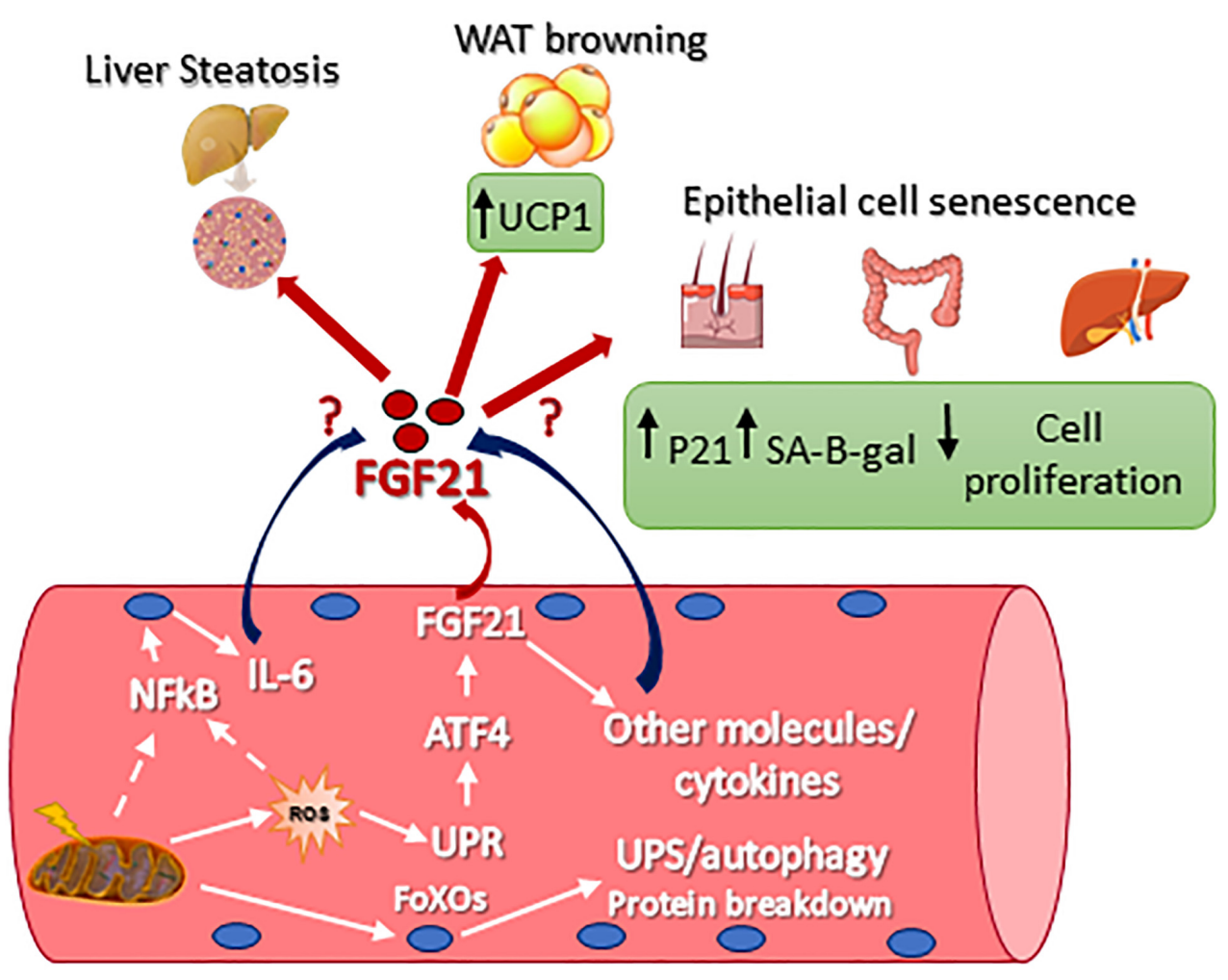

FIGURE 1 | Signaling pathways that control FGF21 expression in skeletal muscle and FGF21-mediated metabolic effect on different tissues. UCP1, uncoupling protein 1; p21, Cdkn1a cyclin-dependent kinase inhibitor 1A; SA-B-gal, senescence-associated beta-galactosidase; Nf-kb, nuclear factor kappa-light-chain-enhancer of activated B cells; IL-6, interleukin 6; ROS, reactive oxygen species; ATF4, activating transcription factor 4; UPR, unfolded protein response; FoXO, Forkhead box O.

levels after acute deletion of OPA1 mice at 5 months of age, causing several detrimental systemic effects. OPA1 inhibition induces important metabolic changes and a precocious aging phenotype of epithelial tissues, a systemic inflammatory response and a premature animal death (Figure 1). Importantly, FGF21 deletion in OPA1-null mice reverted almost all the effects linked to aging, while muscle and white adipose tissue loss were attenuated, but still present, in the double FGF21/OPA1 knockout mice. Interestingly, $\beta$ klotho and FGFRs were upregulated in OPA1 deficient skeletal muscles when compared to the controls supporting the role of this pathway in muscle mass regulation (Tezze et al., 2017). Similar to Tezze et al. (2017) also Rodríguez-Nuevo et al. (2018) showed precocious mouse death and a systemic inflammatory response when OPA1 was deleted. In this work, the group of Zorzano used a myogenindriven Cre transgenic mouse line to delete OPA1 in developing muscle. The finding that the phenotype of these knockout mice was not rescued by FGF21 inhibition is probably due to its reliance on unknown bioenergetics issues. In fact, the presence of mitochondrial dysfunction in growing muscles is too bioenergetically detrimental to be counteracted by concomitant FGF21 ablation. Alternatively, the secretory pattern induced by a mitochondrial defect in new-born mice is neither the same of adult muscle nor adequate to promote tissue senescence. Dale group has also generated an inducible muscle-specific knockout mouse that shows mild muscle loss and, surprisingly, several beneficial metabolic changes in terms of resistance to obesity when the animals were challenged with a high-fat diet. However, OPA1 deletion was induced in very young mice (4 weeks of age), and not in adult animals [12 weeks in Tezze et al. (2017)] and the reduction of OPA1 was partial (around $50-70 \%)$. This is probably due to the fact that stem cells muscles are still fused to myofibers, bringing the OPA1 gene inside the knockout fibers and causing the partial inhibition of OPA1 mildly affecting the mitochondrial function. In fact, mitochondrial respiratory complexes and supercomplexes, as well as mitochondrial DNA content and citrate synthase, were unaffected in these knockout mice. The partial OPA1 inhibition induced morphological changes (swelling) and minor alterations of respiration. This mild mitochondrial phenotype results in a minor level of FGF21 serum fold increase (Table 1). This phenotype differs from our animal model in which OPA1 deletion occurred in adult mice (3-5 months of age) and resulted in the decrease of mtDNA, respiratory complexes and supercomplexes content, complex activity, respiration, mitochondrial membrane potential and in an increase of ROS production. Altogether these mitochondrial changes resulted in a dramatic increase of FGF21 in muscle and serum (100 fold, Table 1), its range has been reported in patients with mitochondrial diseases (Morovat et al., 2017) and unhealthy aging (Conte et al., 2018). Therefore, the 
beneficial or detrimental effect of FGF21 is age, dose, time, and context/tissue-dependent.

\section{FGF21 IN HUMANS, A BENEFICIAL OR DETRIMENTAL MOLECULE?}

In healthy humans, a large variation level of serum FGF21 is shown between individuals, ranging from $5 \mathrm{pg} / \mathrm{mL}$ to $5 \mathrm{ng} / \mathrm{mL}$ (Gälman et al., 2008; Zhang et al., 2008; Li et al., 2009; Dushay et al., 2010; Fazeli et al., 2015). This finding is similar to the rodents' data and further underlines a problem in the method of FGF21 quantification and/or in the variability in terms (Pedersen et al., 2003). For years the search for the stimulus that initiates and maintains the change of excitability or sensibility of the regulating centers in exercise has been progressing. For lack of more precise knowledge, it has been called the "work stimulus," "the work factor," or "the exercise factor." In other terms, one big challenge for muscle and exercise physiologists has been to determine how muscles signal to central and peripheral organs. Here, we discuss the possibility that interleukin-6 (IL-6) could mediate some of the health beneficial effects of exercise. In resting muscle, the IL-6 gene is silent, but it is rapidly activated by contractions. The transcription rate is very fast and the fold changes of IL-6 mRNA is marked. IL-6 is released from working muscles into the circulation in high amounts. The IL6 production is modulated by the glycogen content in muscles, and IL- 6 thus works as an energy sensor. IL- 6 exerts its effect on adipose tissue, inducing lipolysis and gene transcription in abdominal subcutaneous fat and increases whole body lipid oxidation. Furthermore, IL-6 inhibits low-grade TNF-alphaproduction and may thereby inhibit TNF-alpha-induced insulin resistance and atherosclerosis development. We propose that IL- 6 and other cytokines, which are produced and released by skeletal muscles, exerting their effects in other organs of the body, should be named "myokines" (Pedersen et al., 2003). Of the patient's age, time of the day when the blood is collected, and nutritional state of the patient (fed or fasted) that may affect FGF21 secretion or turnover. The Fgf21 gene under basal conditions is considered to be mainly expressed in the liver, and to a much lesser extent, also in the brain (Kharitonenkov and Adams, 2014) and in the pancreas (Fon Tacer et al., 2010). Similar to rodents, the PPAR $\alpha$ response element was identified in the human FGF21 promoters meaning that they may regulate FGF21 expression (Lundåsen et al., 2007). However, there are other tissues contributing to the FGF21 blood levels in physio-pathological conditions. In skeletal muscle, FGF21 is expressed in response to insulin stimulation, suggesting that FGF21 is an insulin-regulated myokine (Hojman et al., 2009) and an association between chronic hyperinsulinemia and levels of FGF21 were found in humans (Hojman et al., 2009). Fazeli et al. (2015) showed that FGF21 works as a fasting-induced hormone for the adaptive response to starvation and for the utilization of fuel derived from tissues breakdown. Others claimed that serum levels of FGF21 are closely related to adiposity, lipid metabolism, and are used as a biomarker of liver injury but not of insulin secretion and sensitivity (Li et al., 2009). Despite the beneficial action of FGF21 in rodents, the literature in humans is not homogeneous and is supportive of some detrimental effects. For instance, FGF21 serum levels are increased in patients with mutations of mitochondrial DNA in skeletal muscles but not when similar mutations accumulate in other organs. Due to this specificity to mitochondrial myopathies, FGF21 was proposed as a biomarker of mitochondrial dysfunction in skeletal muscles and used as a diagnostic test for these inherited disorders (Suomalainen et al., 2011, p. 21). In other conditions FG21 serum levels have been used as a predictor of disease progression. For instance, higher circulating FGF21 levels were associated with a high mortality rate in end-stage renal disease patients (Kohara et al., 2017). It was also reported in patients with diastolic dysfunction of heart failure who have preserved ejection fraction (Chou et al., 2016), and in those who are serum FGF21 level had been found to be an independent predictor of coronary heart disease (Lee et al., 2017). Moreover, circulating FGF21 levels are elevated in various metabolic disease states, such as obesity, insulin resistance, and type 2 diabetes mellitus (Zhang et al., 2008; Chavez et al., 2009). Interestingly, patients with type 2 diabetes also have elevated circulating levels of FAP, and this is associated with a diminished ratio of bioactive to total FGF21 in response to an oral glucose tolerance test (Samms et al., 2017). The increase of plasma FGF21 levels correlated with the severity of whole-body mass (primarily muscle) and hepatic insulin resistance (Chavez et al., 2009). Unexpectedly, in obese humans, the levels of FGF21 did not change after chronic exercise (Besse-Patin et al., 2014) despite the well-known beneficial effect of physical activity. This could be related to the level of the myokine FAP, which is known to increase during exercise (Parmar et al., 2018). Increased FAP could weaken the ratio of bioactive FGF21 in obesity as well as in TDM2 patients. Moreover, we found that FGF21 serum levels positively and significantly correlated with age (Tezze et al., 2017; Conte et al., 2018). Importantly, the presence of high FGF21 serum levels in association with GDF15 and humanin, which are two other markers of mitochondrial function, had been found to be a predictor of morbidity and mortality (Conte et al., 2018).

\section{FINAL CONSIDERATIONS}

The understanding that the control of whole-body metabolism has been greatly advanced in the last years. Impacting these factors are the different molecules which are secreted by skeletal muscles in different conditions. In particularly, FGF21 emerged as an important myokine with several metabolic effects but whether its action is beneficial or detrimental in physiological or pathological conditions are still unclear, especially in humans. There are several issues that should be addressed and clarified soon. The most problematic and urgent one is related to the great variability in the measurements of blood levels between different studies, both in mice and humans. Therefore, a standardization of the procedure for FGF21 measurement is required to understand whether a threshold of serum concentrations is a determinant for its different actions. Nevertheless, we have a clear picture of the different tissues source and the targets that are involved in FGF21 effects. We also know the conditions that trigger FGF21 
expression in skeletal muscles (e.g., mitochondrial dysfunction and ER stress). What is still unclear is the relationship between FGF21-mediated metabolic changes and the contribution of these alterations to disease progression/onset. Moreover, the molecules that synergize, enhance or counteract FGF21-action are still unknown. Our recent findings are that high versus low serum levels correlate with mortality in elderly people, underline the concept that FGF21 actions are dose-dependent. Therefore, first, it is necessary to better address which concentration, or fold increase in plasma, is the most relevant for obtaining a beneficial or detrimental effect. Second, it is important to dissect the other factors (e.g., cytokines, metabolites) that may modulate FGF21 action in target tissues and consequently elicit a positive (healthy) or negative (unhealthy) effect. Finally, the downstream signaling pathway should be better dissected in beneficial or detrimental action. In conclusion, FGF21 belongs to a promising class of cytokines that are induced in response to stress and that can be used as a drug, drug target, or through a biomarker, depending on the physio-pathological context. All these findings

\section{REFERENCES}

Adams, A. C., Astapova, I., Fisher, M., Badman, M. K., Kurgansky, K. E., Flier, J. S., et al. (2010). Thyroid hormone regulates hepatic expression of fibroblast growth factor 21 in a PPAR $\alpha$-dependent manner. J. Biol. Chem. 285, 14078-14082. doi: $10.1074 /$ jbc.C110.107375

Archer, A., Venteclef, N., Mode, A., Pedrelli, M., Gabbi, C., Clément, K., et al. (2012). Fasting-induced FGF21 is repressed by LXR activation via recruitment of an HDAC3 corepressor complex in mice. Mol. Endocrinol. 26, 1980-1990. doi: 10.1210/me.2012-1151

Agrawal, A., Parlee, S., Perez-Tilve, D., Li, P., Pan, J., Mroz, P. A., et al. (2018). Molecular elements in FGF19 and FGF21 defining KLB/FGFR activity and specificity. Mol. Metab. 13, 45-55. doi: 10.1016/j.molmet.2018.05.003

Alipoor, S. D., Mortaz, E., Garssen, J., Movassaghi, M., Mirsaeidi, M., and Adcock, I. M. (2016). Exosomes and exosomal miRNA in respiratory diseases. Med. Inflamm. 2016:5628404. doi: 10.1155/2016/5628404

Antonellis, P. J., Hayes, M. P., and Adams, A. C. (2016). fibroblast growth factor 21-null mice do not exhibit an impaired response to fasting. Front. Endocrinol. 7:77. doi: 10.3389/fendo.2016.00077

Badman, M. K., Pissios, P., Kennedy, A. R., Koukos, G., Flier, J. S., and MaratosFlier, E. (2007). Hepatic fibroblast growth factor 21 is regulated by PPARalpha and is a key mediator of hepatic lipid metabolism in ketotic states. Cell Metab. 5, 426-437. doi: 10.1016/j.cmet.2007.05.002

Berry, D. C., DeSantis, D., Soltanian, H., Croniger, C. M., and Noy, N. (2012). Retinoic acid upregulates preadipocyte genes to block adipogenesis and suppress diet-induced obesity. Diabetes Metab. Res. Rev. 61, 1112-1121. doi: $10.2337 / \mathrm{db} 11-1620$

Besse-Patin, A., Montastier, E., Vinel, C., Castan-Laurell, I., Louche, K., Dray, C., et al. (2014). Effect of endurance training on skeletal muscle myokine expression in obese men: identification of apelin as a novel myokine. Int. J. Obes. 38, 707-713. doi: 10.1038/ijo.2013.158

Bookout, A. L., de Groot, M. H. M., Owen, B. M., Lee, S., Gautron, L., Lawrence, H. L., et al. (2013). FGF21 regulates metabolism and circadian behavior by acting on the nervous system. Nat. Med. 19, 1147-1152. doi: 10.1038/nm.3249

Chavez, A. O., Molina-Carrion, M., Abdul-Ghani, M. A., Folli, F., DeFronzo, R. A., and Tripathy, D. (2009). Circulating fibroblast growth factor-21 is elevated in impaired glucose tolerance and type 2 diabetes and correlates with muscle and hepatic insulin resistance. Diabetes Care 32, 1542-1546. doi: 10.2337/dc090684

Chou, R.-H., Huang, P.-H., Hsu, C.-Y., Chang, C.-C., Leu, H.-B., Huang, C.-C., et al. (2016). Circulating fibroblast growth factor 21 is associated with diastolic dysfunction in heart failure patients with preserved ejection fraction. Sci. Rep. 6:33953. doi: 10.1038/srep33953 will become clear when FGF21 will be used as a therapeutic molecule, exploiting the beneficial effects of FGF21 for treating metabolic disease or when it will be blocked to ameliorate disease progression and the onset of disease.

\section{AUTHOR CONTRIBUTIONS}

CT and MS wrote the manuscript. VR contributed to the discussion.

\section{FUNDING}

This work was supported from the AFM-Telethon (19524), Italian Ministry of Education (MiUR) (PRIN 2010/2011), AIRC (17388), H2020-MSCA-RISE-2014 project no 645648 "Muscle Stress Relief", and CARIPARO to MS. Starting Grants CARIPARO to VR and MS.

Conte, M., Ostan, R., Fabbri, C., Santoro, A., Guidarelli, G., Vitale, G., et al. (2018). Human aging and longevity are characterized by high levels of mitokines. J. Gerontol. A Biol. Sci. Med. Sci. doi: 10.1093/gerona/gly153 [Epub ahead of print].

Coskun, T., Bina, H. A., Schneider, M. A., Dunbar, J. D., Hu, C. C., Chen, Y., et al. (2008). Fibroblast growth factor 21 corrects obesity in mice. Endocrinology 149, 6018-6027. doi: 10.1210/en.2008-0816

Cyphert, H. A., Ge, X., Kohan, A. B., Salati, L. M., Zhang, Y., and Hillgartner, F. B. (2012). Activation of the farnesoid X receptor induces hepatic expression and secretion of fibroblast growth factor 21. J. Biol. Chem. 287, 25123-25138. doi: 10.1074/jbc.M112.375907

Delezie, J., and Handschin, C. (2018). Endocrine crosstalk between skeletal muscle and the brain. Front. Neurol. 9:698. doi: 10.3389/fneur.2018.00698

Dushay, J., Chui, P. C., Gopalakrishnan, G. S., Varela-Rey, M., Crawley, M., Fisher, F. M., et al. (2010). Increased fibroblast growth factor 21 in obesity and nonalcoholic fatty liver disease. Gastroenterology 139, 456-463. doi: 10.1053/j. gastro.2010.04.054

Dutchak, P. A., Katafuchi, T., Bookout, A. L., Choi, J. H., Yu, R. T., Mangelsdorf, D. J., et al. (2012). Fibroblast growth factor-21 regulates PPAR $\gamma$ activity and the antidiabetic actions of thiazolidinediones. Cell 148, 556-567. doi: 10.1016/j.cell. 2011.11.062

Fazeli, P. K., Lun, M., Kim, S. M., Bredella, M. A., Wright, S., Zhang, Y., et al. (2015). FGF21 and the late adaptive response to starvation in humans. J. Clin. Invest. 125, 4601-4611. doi: 10.1172/JCI83349

Fisher, F. M., Chui, P. C., Antonellis, P. J., Bina, H. A., Kharitonenkov, A., Flier, J. S., et al. (2010). Obesity is a fibroblast growth factor 21 (FGF21)-resistant state. Diabetes Metab. Res. Rev 59, 2781-2789. doi: 10.2337/db10-0193

Fisher, F. M., and Maratos-Flier, E. (2016). Understanding the physiology of FGF21. Annu. Rev. Physiol. 78, 223-241. doi: 10.1146/annurev-physiol-021115105339

Fon Tacer, K., Bookout, A. L., Ding, X., Kurosu, H., John, G. B., Wang, L., et al. (2010). Research resource: comprehensive expression atlas of the fibroblast growth factor system in adult mouse. Mol. Endocrinol. 24, 2050-2064. doi: 10.1210/me.2010-0142

Fu, T., Xu, Z., Liu, L., Guo, Q., Wu, H., Liang, X. et al. (2018). Mitophagy directs muscle-adipose crosstalk to alleviate dietary obesity. Cell Rep. 23, 1357-1372. doi: 10.1016/j.celrep.2018.03.127

Gälman, C., Lundåsen, T., Kharitonenkov, A., Bina, H. A., Eriksson, M., Hafström, I., et al. (2008). The circulating metabolic regulator FGF21 is induced by prolonged fasting and PPARalpha activation in man. Cell Metab. 8, 169-174. doi: 10.1016/j.cmet.2008.06.014

Ge, X., Chen, C., Hui, X., Wang, Y., Lam, K. S. L., and Xu, A. (2011). Fibroblast growth factor 21 induces glucose transporter-1 expression 
through activation of the serum response factor/Ets-like protein-1 in adipocytes. J. Biol. Chem. 286, 34533-34541. doi: 10.1074/jbc.M111.24 8591

Guridi, M., Tintignac, L. A., Lin, S., Kupr, B., Castets, P., and Rüegg, M. A. (2015). Activation of mTORC1 in skeletal muscle regulates whole-body metabolism through FGF21. Sci. Signal. 8:ra113. doi: 10.1126/scisignal.aab3715

Hecht, R., Li, Y.-S., Sun, J., Belouski, E., Hall, M., Hager, T., et al. (2012). Rationalebased engineering of a potent long-acting FGF21 analog for the treatment of type 2 diabetes. PLoS One 7:e49345. doi: 10.1371/journal.pone.0049345

Hirai, T., Nomura, K., Ikai, R., Nakashima, K.-I., and Inoue, M. (2019). Baicalein stimulates fibroblast growth factor 21 expression by up-regulating retinoic acid receptor-related orphan receptor $\alpha$ in $\mathrm{C} 2 \mathrm{C} 12$ myotubes. Biomed. Pharmacother. 109, 503-510. doi: 10.1016/j.biopha.2018.10.154

Hoffmann, C., and Weigert, C. (2017). Skeletal muscle as an endocrine organ: the role of myokines in exercise adaptations. Cold Spring Harb. Perspect. Med. 7:a029793. doi: 10.1101/cshperspect.a029793

Hojman, P., Pedersen, M., Nielsen, A. R., Krogh-Madsen, R., Yfanti, C., Åkerstrom, T., et al. (2009). Fibroblast growth factor-21 is induced in human skeletal muscles by hyperinsulinemia. Diabetes Metab. Res. Rev. 58, 2797-2801. doi: $10.2337 / \mathrm{db} 09-0713$

Hondares, E., Iglesias, R., Giralt, A., Gonzalez, F. J., Giralt, M., Mampel, T., et al. (2011). Thermogenic activation induces FGF21 expression and release in brown adipose tissue. J. Biol. Chem. 286, 12983-12990. doi: 10.1074/jbc.M110. 215889

Hsuchou, H., Pan, W., and Kastin, A. J. (2007). The fasting polypeptide FGF21 can enter brain from blood. Peptides 28, 2382-2386. doi: 10.1016/j.peptides.2007. 10.007

Iizuka, K., Takeda, J., and Horikawa, Y. (2009). Glucose induces FGF21 mRNA expression through ChREBP activation in rat hepatocytes. FEBS Lett. 583, 2882-2886. doi: 10.1016/j.febslet.2009.07.053

Inagaki, T., Dutchak, P., Zhao, G., Ding, X., Gautron, L., Parameswara, V., et al. (2007). Endocrine regulation of the fasting response by PPAR $\alpha$-mediated induction of fibroblast growth factor 21. Cell Metab. 5, 415-425. doi: 10.1016/j. cmet.2007.05.003

Inagaki, T., Lin, V. Y., Goetz, R., Mohammadi, M., Mangelsdorf, D. J., and Kliewer, S. A. (2008). Inhibition of growth hormone signaling by the fasting-induced hormone FGF21. Cell Metab. 8, 77-83. doi: 10.1016/j.cmet.2008.05.006

Izumiya, Y., Bina, H. A., Ouchi, N., Akasaki, Y., Kharitonenkov, A., and Walsh, K. (2008). FGF21 is an Akt-regulated myokine. FEBS Lett. 582, 3805-3810. doi: 10.1016/j.febslet.2008.10.021

Jimenez, V., Jambrina, C., Casana, E., Sacristan, V., Muñoz, S., Darriba, S., et al. (2018). FGF21 gene therapy as treatment for obesity and insulin resistance. EMBO Mol. Med. 10:e8791. doi: 10.15252/emmm.201708791

Johnson, C. L., Weston, J. Y., Chadi, S. A., Fazio, E. N., Huff, M. W., Kharitonenkov, A., et al. (2009). Fibroblast growth factor 21 reduces the severity of cerulein-induced pancreatitis in mice. Gastroenterology 137, 1795-1804. doi: 10.1053/j.gastro.2009.07.064

Joki, Y., Ohashi, K., Yuasa, D., Shibata, R., Ito, M., Matsuo, K., et al. (2015). FGF21 attenuates pathological myocardial remodeling following myocardial infarction through the adiponectin-dependent mechanism. Biochem. Biophys. Res. Commun. 459, 124-130. doi: 10.1016/j.bbrc.2015.02.081

Keipert, S., Ost, M., Johann, K., Imber, F., Jastroch, M., van Schothorst, E. M., et al. (2013). Skeletal muscle mitochondrial uncoupling drives endocrine crosstalk through the induction of FGF21 as a myokine. Am. J. Physiol. Endocrinol. Metab. 306, E469-E482. doi: 10.1152/ajpendo.00330.2013

Kharitonenkov, A., and Adams, A. C. (2014). Inventing new medicines: the FGF21 story. Mol. Metab. 3, 221-229. doi: 10.1016/j.molmet.2013.12.003

Kharitonenkov, A., Shiyanova, T. L., Koester, A., Ford, A. M., Micanovic, R., Galbreath, E. J., et al. (2005). FGF-21 as a novel metabolic regulator. J. Clin. Invest. 115, 1627-1635. doi: 10.1172/JCI23606

Kharitonenkov, A., Wroblewski, V. J., Koester, A., Chen, Y.-F., Clutinger, C. K., Tigno, X. T., et al. (2007). The metabolic state of diabetic monkeys is regulated by fibroblast growth factor-21. Endocrinology 148, 774-781. doi: 10.1210/en. 2006-1168

Kim, K. H., Jeong, Y. T., Oh, H., Kim, S. H., Cho, J. M., Kim, Y.-N., et al. (2013). Autophagy deficiency leads to protection from obesity and insulin resistance by inducing Fgf21 as a mitokine. Nat. Med. 19, 83-92. doi: 10.1038/nm. 3014
Kim, S. H., Kim, K. H., Kim, H.-K., Kim, M.-J., Back, S. H., Konishi, M., et al. (2015). Fibroblast growth factor 21 participates in adaptation to endoplasmic reticulum stress and attenuates obesity-induced hepatic metabolic stress. Diabetologia 58, 809-818. doi: 10.1007/s00125-014-3475-6

Kohara, M., Masuda, T., Shiizaki, K., Akimoto, T., Watanabe, Y., Honma, S., et al. (2017). Association between circulating fibroblast growth factor 21 and mortality in end-stage renal disease. PLoS One 12:e0178971. doi: 10.1371/ journal.pone.0178971

Lee, C. H., Woo, Y. C., Chow, W. S., Cheung, C. Y. Y., Fong, C. H. Y., Yuen, M. M. A., et al. (2017). Role of circulating fibroblast growth factor 21 measurement in primary prevention of coronary heart disease among chinese patients with type 2 diabetes mellitus. J. Am. Heart Assoc. 6:e005344. doi: 10.1161/JAHA.116.005344

Li, H., Bao, Y., Xu, A., Pan, X., Lu, J., Wu, H., et al. (2009). Serum fibroblast growth factor 21 is associated with adverse lipid profiles and gammaglutamyltransferase but not insulin sensitivity in Chinese subjects. J. Clin. Endocrinol. Metab. 94, 2151-2156. doi: 10.1210/jc.2008-2331

Li, Y., Wong, K., Walsh, K., Gao, B., and Zang, M. (2013). Retinoic acid receptor $\beta$ stimulates hepatic induction of fibroblast growth factor 21 to promote fatty acid oxidation and control whole-body energy homeostasis in mice. J. Biol. Chem. 288, 10490-10504. doi: 10.1074/jbc.M112.42 9852

Lundåsen, T., Hunt, M. C., Nilsson, L.-M., Sanyal, S., Angelin, B., Alexson, S. E. H., et al. (2007). PPARalpha is a key regulator of hepatic FGF21. Biochem. Biophys. Res. Commun. 360, 437-440. doi: 10.1016/j.bbrc.2007. 06.068

Minard, A. Y., Tan, S.-X., Yang, P., Fazakerley, D. J., Domanova, W., Parker, B. L., et al. (2016). mTORC1 is a major regulatory node in the FGF21 signaling network in adipocytes. Cell Rep. 17, 29-36. doi: 10.1016/j.celrep.2016.08.086

Morovat, A., Weerasinghe, G., Nesbitt, V., Hofer, M., Agnew, T., Quaghebeur, G., et al. (2017). Use of FGF-21 as a biomarker of mitochondrial disease in clinical practice. J. Clin. Med. 6:E80. doi: 10.3390/jcm6080080

Moyers, J. S., Shiyanova, T. L., Mehrbod, F., Dunbar, J. D., Noblitt, T. W., Otto, K. A., et al. (2007). Molecular determinants of FGF-21 activity-synergy and cross-talk with PPARgamma signaling. J. Cell. Physiol. 210, 1-6. doi: 10.1002/ jcp. 20847

Muise, E. S., Souza, S., Chi, A., Tan, Y., Zhao, X., Liu, F., et al. (2013). Downstream signaling pathways in mouse adipose tissues following acute in vivo administration of fibroblast growth factor 21. PLoS One 8:e73011. doi: 10.1371/journal.pone.0073011

Murata, Y., Nishio, K., Mochiyama, T., Konishi, M., Shimada, M., Ohta, H., et al. (2013). Fgf21 impairs adipocyte insulin sensitivity in mice fed a lowcarbohydrate, high-fat ketogenic diet. PLoS One 8:e69330. doi: 10.1371/journal. pone.0069330

Nishimura, T., Nakatake, Y., Konishi, M., and Itoh, N. (2000). Identification of a novel FGF, FGF-21, preferentially expressed in the liver. Biochim. Biophys. Acta 1492, 203-206.

Oost, L. J., Kustermann, M., Armani, A., Blaauw, B., and Romanello, V. (2019). Fibroblast growth factor 21 controls mitophagy and muscle mass. J. Cachexia Sarcopenia Muscle. doi: 10.1002/jcsm.12409

Parmar, B., Lewis, J. E., Samms, R. J., Ebling, F. J. P., Cheng, C. C., Adams, A. C., et al. (2018). Eccentric exercise increases circulating fibroblast activation protein $\alpha$ but not bioactive fibroblast growth factor 21 in healthy humans. Exp. Physiol. 103, 876-883. doi: 10.1113/EP086669

Patel, R., Bookout, A. L., Magomedova, L., Owen, B. M., Consiglio, G. P., Shimizu, M., et al. (2015). Glucocorticoids regulate the metabolic hormone FGF21 in a feed-forward loop. Mol. Endocrinol. 29, 213-223. doi: 10.1210/me. 2014- 1259

Pedersen, B. K., Steensberg, A., Fischer, C., Keller, C., Keller, P., Plomgaard, P., et al. (2003). Searching for the exercise factor: is IL-6 a candidate? J. Muscle Res. Cell. Motil. 24, 113-119.

Pereira, R. O., Tadinada, S. M., Zasadny, F. M., Oliveira, K. J., Pires, K. M. P., Olvera, A., et al. (2017). OPA1 deficiency promotes secretion of FGF21 from muscle that prevents obesity and insulin resistance. EMBO J. 36, 2126-2145. doi: $10.15252 /$ embj.201696179

Planavila, A., Redondo, I., Hondares, E., Vinciguerra, M., Munts, C., Iglesias, R., et al. (2013). Fibroblast growth factor 21 protects against cardiac hypertrophy in mice. Nat. Commun. 4:2019. doi: 10.1038/ncomms3019 
Planavila, A., Redondo-Angulo, I., Ribas, F., Garrabou, G., Casademont, J., Giralt, M., et al. (2015). Fibroblast growth factor 21 protects the heart from oxidative stress. Cardiovasc. Res. 106, 19-31. doi: 10.1093/cvr/cvu263

Rai, M., and Demontis, F. (2016). Systemic nutrient and stress signaling via myokines and myometabolites. Annu. Rev. Physiol. 78, 85-107. doi: 10.1146/ annurev-physiol-021115-105305

Rodríguez-Nuevo, A., Díaz-Ramos, A., Noguera, E., Díaz-Sáez, F., Duran, X., Muñoz, J. P., et al. (2018). Mitochondrial DNA and TLR9 drive muscle inflammation upon opal deficiency. EMBO J. 37:e96553. doi: 10.15252/embj. 201796553

Rupérez, C., Lerin, C., Ferrer-Curriu, G., Cairo, M., Mas-Stachurska, A., Sitges, M., et al. (2018). Autophagic control of cardiac steatosis through FGF21 in obesityassociated cardiomyopathy. Int. J. Cardiol. 260, 163-170. doi: 10.1016/j.ijcard. 2018.02.109

Safdar, A., and Tarnopolsky, M. A. (2018). Exosomes as mediators of the systemic adaptations to endurance exercise. Cold Spring Harb. Perspect Med. 8:a029827. doi: $10.1101 /$ cshperspect.a029827

Samms, R. J., Lewis, J. E., Norton, L., Stephens, F. B., Gaffney, C. J., Butterfield, T., et al. (2017). FGF21 is an insulin-dependent postprandial hormone in adult humans. J. Clin. Endocrinol. Metab. 102, 3806-3813. doi: 10.1210/jc.201701257

Schaap, F. G., Kremer, A. E., Lamers, W. H., Jansen, P. L. M., and Gaemers, I. C. (2013). Fibroblast growth factor 21 is induced by endoplasmic reticulum stress. Biochimie 95, 692-699. doi: 10.1016/j.biochi.2012.10.019

Staiger, H., Keuper, M., Berti, L., Hrabe de Angelis, M., and Häring, H.-U. (2017). Fibroblast growth factor 21-metabolic role in mice and men. Endocr. Rev. 38, 468-488. doi: 10.1210/er.2017-00016

Suomalainen, A., Elo, J. M., Pietiläinen, K. H., Hakonen, A. H., Sevastianova, K., Korpela, M., et al. (2011). FGF-21 as a biomarker for muscle-manifesting mitochondrial respiratory chain deficiencies: a diagnostic study. Lancet Neurol. 10, 806-818. doi: 10.1016/S1474-4422(11)70155-7

Tezze, C., Romanello, V., Desbats, M. A., Fadini, G. P., Albiero, M., Favaro, G., et al. (2017). Age-associated loss of opal in muscle impacts muscle mass, metabolic homeostasis, systemic inflammation, and epithelial senescence. Cell Metab. 25, 1374.e6-1389.e6. doi: 10.1016/j.cmet.2017.04.021

Tsai, S., Sitzmann, J. M., Dastidar, S. G., Rodriguez, A. A., Vu, S. L., McDonald, C. E., et al. (2015). Muscle-specific 4E-BP1 signaling activation improves metabolic parameters during aging and obesity. J. Clin. Invest. 125, 2952-2964. doi: 10.1172/JCI77361

Tsuchiya, H., Ikeda, Y., Ebata, Y., Kojima, C., Katsuma, R., Tsuruyama, T., et al. (2012). Retinoids ameliorate insulin resistance in a leptin-dependent manner in mice. Hepatology 56, 1319-1330. doi: 10.1002/hep.25798
Uebanso, T., Taketani, Y., Yamamoto, H., Amo, K., Tanaka, S., Arai, H., et al. (2012). Liver X receptor negatively regulates fibroblast growth factor 21 in the fatty liver induced by cholesterol-enriched diet. J. Nutr. Biochem. 23, 785-790. doi: 10.1016/j.jnutbio.2011.03.023

Wang, H., Qiang, L., and Farmer, S. R. (2008). Identification of a domain within peroxisome proliferator-activated receptor gamma regulating expression of a group of genes containing fibroblast growth factor 21 that are selectively repressed by SIRT1 in adipocytes. Mol. Cell. Biol. 28, 188-200. doi: 10.1128/ MCB.00992-07

Wang, Y., Solt, L. A., and Burris, T. P. (2010). Regulation of FGF21 expression and secretion by retinoic acid receptor-related orphan receptor $\alpha$. J. Biol. Chem. 285, 15668-15673. doi: 10.1074/jbc.M110.102160

Wei, W., Dutchak, P. A., Wang, X., Ding, X., Wang, X., Bookout, A. L., et al. (2012). Fibroblast growth factor 21 promotes bone loss by potentiating the effects of peroxisome proliferator-activated receptor $\gamma$. Proc. Natl. Acad. Sci. U.S.A. 109, 3143-3148. doi: 10.1073/pnas.1200797109

Whitham, M., and Febbraio, M. A. (2016). The ever-expanding myokinome: discovery challenges and therapeutic implications. Nat. Rev. Drug Discov. 15, 719-729. doi: 10.1038/nrd.2016.153

Xu, J., Stanislaus, S., Chinookoswong, N., Lau, Y. Y., Hager, T., Patel, J., et al. (2009). Acute glucose-lowering and insulin-sensitizing action of FGF21 in insulin-resistant mouse models-association with liver and adipose tissue effects. Am. J. Physiol. Endocrinol. Metab. 297, E1105-E1114. doi: 10.1152/ajpendo.00348.2009

Zhang, X., Yeung, D. C. Y., Karpisek, M., Stejskal, D., Zhou, Z.-G., Liu, F., et al. (2008). Serum FGF21 levels are increased in obesity and are independently associated with the metabolic syndrome in humans. Diabetes Metab. Res. Rev 57, 1246-1253. doi: 10.2337/db07-1476

Zhen, E. Y., Jin, Z., Ackermann, B. L., Thomas, M. K., and Gutierrez, J. A. (2016). Circulating FGF21 proteolytic processing mediated by fibroblast activation protein. Biochem. J. 473, 605-614. doi: 10.1042/BJ20151085

Conflict of Interest Statement: The authors declare that the research was conducted in the absence of any commercial or financial relationships that could be construed as a potential conflict of interest.

Copyright (c) 2019 Tezze, Romanello and Sandri. This is an open-access article distributed under the terms of the Creative Commons Attribution License (CC BY). The use, distribution or reproduction in other forums is permitted, provided the original author(s) and the copyright owner(s) are credited and that the original publication in this journal is cited, in accordance with accepted academic practice. No use, distribution or reproduction is permitted which does not comply with these terms. 


\section{OPEN ACCESS}

Edited by:

J.-P. Jin,

Wayne State University, United States

Reviewed by:

Jennifer Stevenson Moylan, University of Kentucky, United States

David C. Hughes,

The University of lowa, United States

*Correspondence:

Zhigang Xue

zhigang.xue@upmc.fr

Specialty section: This article was submitted to

Striated Muscle Physiology, a section of the journal

Frontiers in Physiology

Received: 09 November 2018

Accepted: 22 March 2019

Published: 24 April 2019

Citation:

Baccam A, Benoni-Sviercovich A, Rocchi M, Moresi V, Seelaender $M$, Li Z, Adamo S, Xue Z and Coletti D (2019) The Mechanical Stimulation of Myotubes Counteracts the Effects

of Tumor-Derived Factors Through the Modulation of the Activin/Follistatin Ratio. Front. Physiol. 10:401. doi: 10.3389/fphys.2019.00401
The Mechanical Stimulation of Myotubes Counteracts the Effects of Tumor-Derived Factors Through the Modulation of the Activin/Follistatin Ratio

\author{
Alexandra Baccam ${ }^{1,2,3}$, Alexandra Benoni-Sviercovich ${ }^{1,2,3}$, Marco Rocchi $^{4}$, \\ Viviana Moresi ${ }^{2,3}$, Marilia Seelaender ${ }^{5}$, Zhenlin Li $^{2}$, Sergio Adamo ${ }^{1,3}$, Zhigang Xue ${ }^{2 \star}$ and \\ Dario Coletti 1,2,3 \\ ${ }^{1}$ Biology of Adaptation and Aging (B2A), Sorbonne Université, UMR8256 - INSERM ERL U1164, Paris, France, ${ }^{2}$ Section \\ of Histology, Department of Anatomical, Histological, Forensic and Orthopedic Sciences, Sapienza University of Rome, \\ Rome, Italy, ${ }^{3}$ Interuniversity Institute of Myology, Rome, Italy, ${ }^{4}$ Department of Biomolecular Sciences, University of Urbino, \\ Urbino, Italy, ${ }^{5}$ Institute of Biomedical Sciences, Faculdade de Medicina, University of São Paulo, São Paulo, Brazil
}

Activin negatively affects muscle fibers and progenitor cells in aging (sarcopenia) and in chronic diseases characterized by severe muscle wasting (cachexia). High circulating activin levels predict poor survival in cancer patients. However, the relative impact of activin in mediating muscle atrophy and hampered homeostasis is still unknown. To directly assess the involvement of activin, and its physiological inhibitor follistatin, in cancer-induced muscle atrophy, we cultured $\mathrm{C} 2 \mathrm{C} 12$ myotubes in the absence or in the presence of a mechanical stretching stimulus and in the absence or presence of C26 tumor-derived factors (CM), so as to mimic the mechanical stimulation of exercise and cancer cachexia, respectively. We found that $\mathrm{CM}$ induces activin release by myotubes, further exacerbating the negative effects of tumor-derived factors. In addition, mechanical stimulation is sufficient to counteract the adverse tumor-induced effects on muscle cells, in association with an increased follistatin/activin ratio in the cell culture medium, indicating that myotubes actively release follistatin upon stretching. Recombinant follistatin counteracts tumor effects on myotubes exclusively by rescuing fusion index, suggesting that it is only partially responsible for the stretch-mediated rescue. Therefore, besides activin, other tumor-derived factors may play a significant role in mediating muscle atrophy. In addition to increasing follistatin secretion mechanical stimulation induces additional beneficial responses in myotubes. We propose that in animal models of cancer cachexia and in cancer patients purely mechanical stimuli play an important role in mediating the rescue of the muscle homeostasis reported upon exercise.

Keywords: skeletal muscle atrophy, myokines, mechanotransduction, exercise, C26 colon carcinoma, FlexCell apparatus 


\section{INTRODUCTION}

Cancer cachexia is multifactorial and characterized by tumorand host-derived factors leading to muscle wasting (Fearon et al., 2012). TGF- $\beta$ family members, including activin and myostatin, are key regulators of muscle development and homeostasis (Chen et al., 2016) and have been reported to mediate cachexia (Costelli et al., 2008; Chen et al., 2014). Since they bind to activin type IIB receptor (ActRIIB), the latter has been targeted to counteract muscle wasting (Barreto et al., 2016; Hatakeyama et al., 2016; Toledo et al., 2016) or to promote muscle hypertrophy (Morvan et al., 2017) and the regenerative capacity of muscle (Formicola et al., 2018). Activin induces muscle catabolism via p38 $\beta$ (Ding et al., 2017) and SMAD2/3 activation (Winbanks et al., 2016). Chemotherapy activates this pathway too, further worsening muscle atrophy (Barreto et al., 2016; Coletti, 2018). Follistatin (or activin-binding protein) is a potent, physiological inhibitor of activin and myostatin (Zheng et al., 2017). Several organs such as the gonads (Tilbrook et al., 1996) and skeletal muscle (Ciaraldi et al., 2016) are sources of follistatin.

Exercise modulates follistatin and other myokines (Yeo et al., 2012) and the plasma profile of cytokines (Lira et al., 2009; Donatto et al., 2013), producing marked beneficial effects on muscle homeostasis (Barone et al., 2016; Coletti et al., 2016; Pigna et al., 2016). This is the reason why exercise is recommended to treat cachexia (Lira et al., 2012, 2014).

Even though a high level of circulating activin is an adverse prognostic factor in cancer patients (Loumaye et al., 2017), it is yet unknown if activin is a direct player or only a mere marker of cachexia. Besides, it is not known if beneficial exercise effects are mediated by purely mechanical stimuli in the muscle, nor whether exercise itself modulates the follistatin/activin ratio and its paracrine consequences. To address these questions, we cultured $\mathrm{C} 2 \mathrm{C} 12$ myotubes in the absence or in the presence of mechanical stretching, and in the absence or in the presence of tumor-derived factors, so as to mimic the mechanical stimulation of exercise and cancer cachexia, respectively.

\section{MATERIALS AND METHODS}

\section{Cell Cultures}

C2C12 were seeded at a density of 20000 cells $/ \mathrm{cm}^{2}$ in the multiwell plate of the Flexcell ${ }^{\circledast}$ FX- $6000^{\mathrm{TM}}$ Tension System designed for unidirectional stretching (collagen coated silicon membrane, $3.89 \mathrm{~cm}^{2}$ ). A vacuum was constantly applied to stretch the membrane by $6 \%$, in order to increase membrane stiffness, a condition that preliminary experiments showed to be necessary to allow a proper differentiation of the $\mathrm{C} 2 \mathrm{C} 12$ cells into myotubes (data not shown). The following day, at $80 \%$ confluence, GM (DMEM with $15 \%$ FBS, $4.5 \mathrm{~g} / \mathrm{L}$ of glucose, $2 \mathrm{mM}$ of L-glutamine, $100 \mu \mathrm{g} / \mathrm{mL}$ of penicillinstreptomycin; Sigma-Aldrich, St. Louis, MO, United States) was replaced with DM containing 2\% Horse Serum (HS, Carotenuto et al., 2016). A mixed culture of myoblasts and myotubes was kept for 4 days in $\mathrm{DM}$ at $37^{\circ} \mathrm{C}, 5 \%$ of $\mathrm{CO} 2$ in the Flexcell apparatus, always under constant stretch. For the experiments, the initial myotubes cultures were further cultured for additional $2 \mathrm{~d}$ under continuous stretching (SC, Static Condition) or were subjected to 2 daily series of $2 \mathrm{~h}$ cyclic stretching $(0,5 \mathrm{~Hz}, 6 \%$ stretch), with a $3 \mathrm{~h}$-pause between them (DC, Dynamic Condition). As to media composition during the $2 \mathrm{~d}$ experimental treatments see figure legends (Sigma reagents).

In order to obtain a tumor cell conditioned medium (CM), C26 carcinoma cells (Cell Lines Service) were cultured for $2 \mathrm{~d}$ in serum-free DMEM and the supernatant being diluted to $20 \%$ in HS medium. To obtain the control medium, DMEM was incubated for $2 \mathrm{~d}$ at $37^{\circ} \mathrm{C}$ in the absence of $\mathrm{C} 26$ cells.

\section{Immunofluorescence, ELISA}

Samples underwent standard procedures (De Arcangelis et al., 2005; Aulino et al., 2015). Antibodies: MF20 anti-MHC and F5D anti-myogenin (DSHB); Alexafluor 555 or 488 secondary Abs (Molecular probes). Regarding the ELISA quantification of some specific factors secreted in $\mathrm{C} 26 \mathrm{CM}$ or in the myotube culture media DAC00B Human/Mouse Activin A and DFN00 Human Follistatin (R\&D) systems were used following manufacturer's instructions.

\section{Western Blot}

Protein extraction and electrophoresis were performed as previously described (Coletti et al., 2016). In brief: samples were treated with lysis buffer RIPA containing Tris-Cl $50 \mathrm{Mm}$ $\mathrm{pH}=7.5,150 \mathrm{Mm} \mathrm{NaCl}, 1 \% \mathrm{NP} 40,0.5 \%$ desoxyclorate de sodium, EGTA $20 \mathrm{mM}$, DTT $1 \mathrm{mM}$, and a protease inhibitor cocktail. Proteins were denatured with a Bolt kit (Molecular probes, Invitrogen). Total protein content was measured by Bradford and GAPDH was used as a loading control. Membranes of nitrocellulose were incubated with blocking buffer TBS-Tween with 5\% not fat milk. Antibodies: anti-MyoD, anti Phospho-SMAD2/3, anti-SMAD2/3 (Cell Signalling), antiGAPDH (Sigma). The sample size was 9, however, triplicate independent samples were pooled to obtain a sufficient amount of proteins to be loaded in each lane. Secondary antibody fluorescence was detected by using the Odyssey system; for each band, quantification of the signal was obtained by ImageJ, following background subtraction, and each value was normalized by the corresponding GAPDH band intensity; for SMADs, the ratio between the normalized P-SMAD and SMAD values was calculated.

\section{Quantitative PCR}

Total RNA was isolated with Trizol $^{\circledR}$ reagent (Invitrogen) following the manufacturer's recommendations and homogenized. RNA concentration was determined by measuring the absorbance in $260 \mathrm{~nm} / 280 \mathrm{~nm}$ in a NanoDrop spectrophotometer. cDNA synthesis was carried out using the High capacity applied Reverse Transcription Kit (Biosystem). Lightcycler 480 was used to detect SYBR Green signal in Q-PCR. The mRNA levels were determined by the comparative $\mathrm{Ct}$ method; the average $\Delta \mathrm{Ct}$ value of the control group was 
Aa

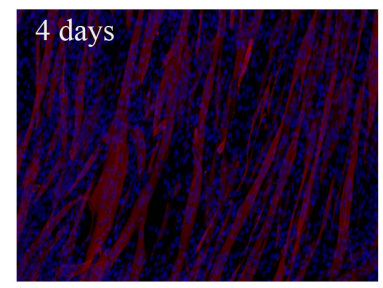

Ab
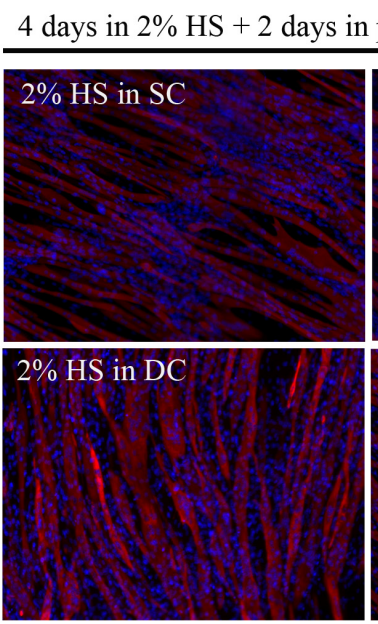

$\mathrm{Ca}$

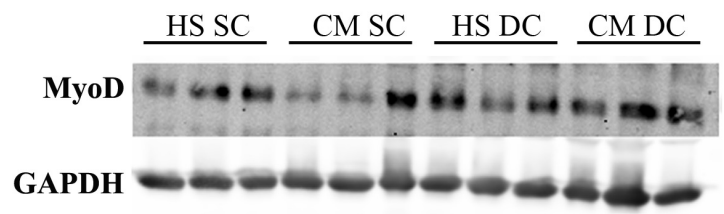

$\mathrm{Da}$

4 days in $2 \% \mathrm{HS}+2$ days in presence of treatment below
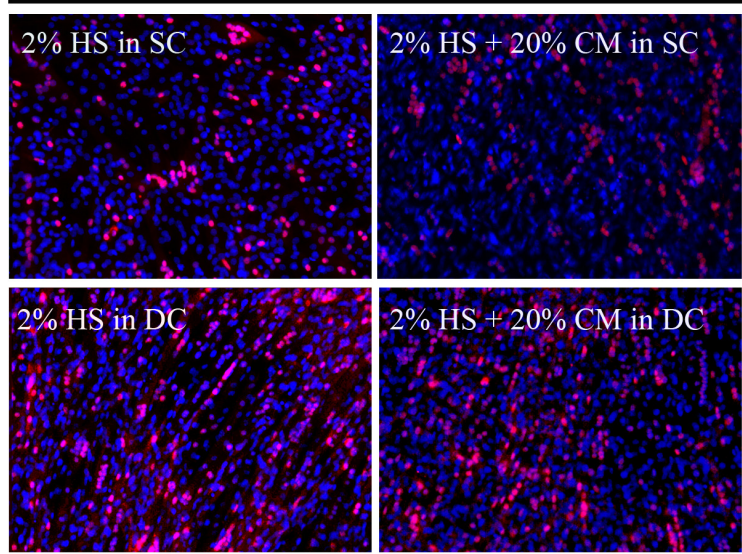

$2 \% \mathrm{HS}+20 \% \mathrm{CM}$ in $\mathrm{DC}$

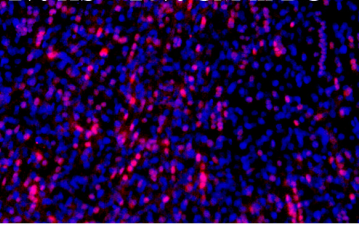

$\mathrm{Ba}$

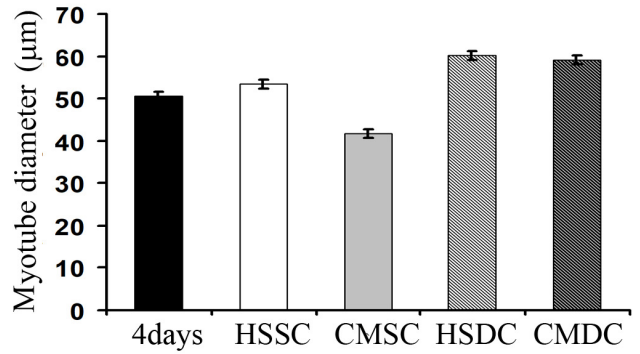

$\mathrm{Bb}$

$\mathrm{Bc}$
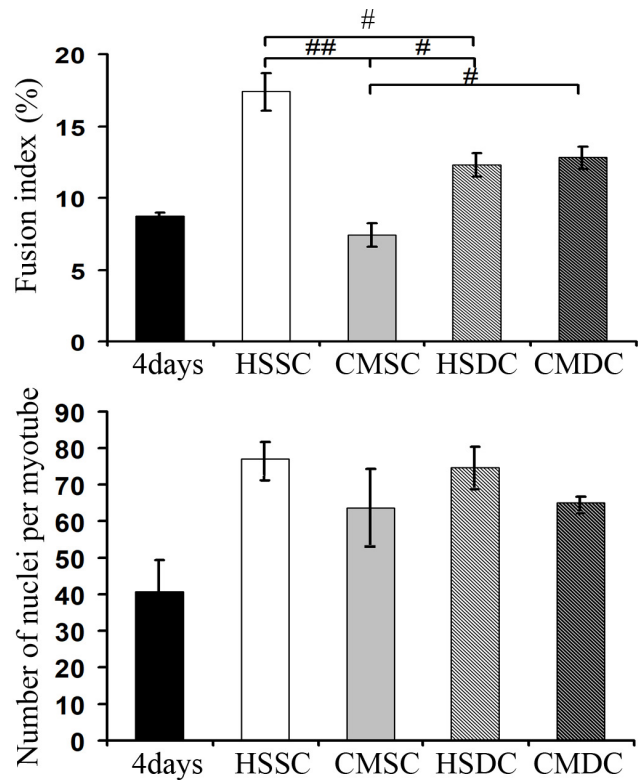

$\mathrm{Cb}$

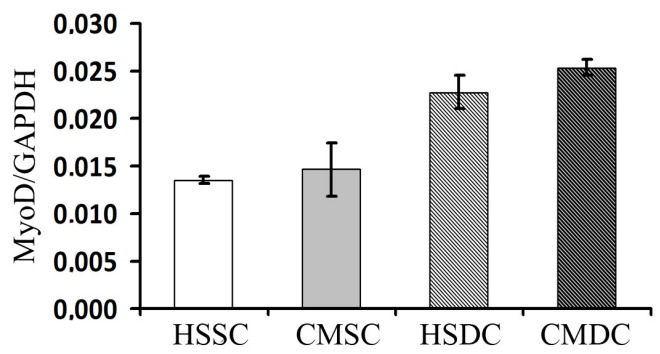

Db

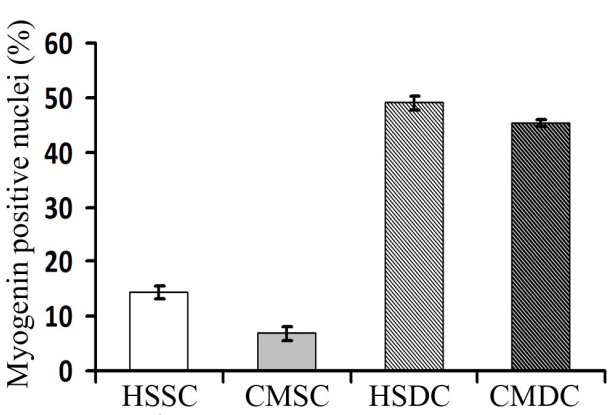


FIGURE 1 | Mechanical stimulation counteracts the negative effect of tumor-derived factors. (A) Myosin (red) localization and nuclei (blue) by immunofluorescence in $\mathrm{C} 2 \mathrm{C} 12$ myotubes at $4 \mathrm{~d}(\mathbf{A a})$ and $6 \mathrm{~d}(\mathbf{A b})$ of culture in a differentiation medium in the absence (HS) or presence (CM) of C26-conditioned medium, in combination with the absence (SC) or presence (DC) of cyclic stretching. (B) Morphometric analyses were performed on replicate samples ( $n=6)$. One-way ANOVA performed on data from $4 \mathrm{~d}$ and $6 \mathrm{~d}$ (five groups) followed by Dunnet's test indicated a significant increase in the fusion index (FI) and in the number of nuclei/myotube (NpM) between 4d and 6d in any condition except CM. Two-way ANOVA performed on $6 \mathrm{~d}$ values showed a significant effect for: (Ba) DC on myotube diameter ( $F=12.66$; $d f=1 ; p<0.05$ ); (Bb) CM and interaction with DC (for CM: $F=24.73 ; d f=1 ; p<0.001$; for interaction: $F=30.2 ; d f=1 ; p<0.001$ ) on fusion index; ${ }^{\prime} p<0.05$, $\#$ \# $p .01$ by Tukey HSD test); (Bc) CM on number of nuclei/myotube ( $F=5.64 ; d f=1 ; p<0.05)$. (C) WB analysis for MyoD (Ca) and relative average density (Cb) following normalization over the GAPDH signal in the same conditions as above. Two-way ANOVA showed a significant effect for DC $(F=19.47 ; d f=1 ; p<0.001)$. (D) Myogenin (red) and nuclei (blue) by IF (Da) in C2C12 myotubes at 6d of culture in the same conditions as above, and quantification of the percentage of myogenin+ nuclei (Db). Two-way ANOVA showed the significant effect of both $\mathrm{CM}(F=13.55 ; d f=1 ; p<0.01)$ and DC $(F=451.7 ; d f=1 ; p<0.0001)$, indicating that the DC rescues myogenin expression. Data are shown as mean $\pm \mathrm{SEM}$.

subtracted from the test value to derive a $-\Delta \Delta \mathrm{Ct}$ value. The expression of each gene was evaluated by $2^{-\Delta \Delta C t}$, according to Livak and Schmittgen (2001). List of primers used:

\section{ActRIIBL: CTG-TGC-GGA-CTC-CTT-TAA-GC ActRIIBR: TCT-TCA-CAG-CCA-CAA-AGT-CG Activin-AL: CAG-TGG-GGA-GGT-CCT-AGA-CA Activin-AR: CAA-AAG-GAG-CAG-CAG-AGA-CC FollistatinL: CCT-CCT-GCT-GCT-GCT-ACT-CT FollistatinR: TGC-TGC-AAC-ACT-CTT-CCT-TG Wnt4L: CTG-GAG-AAG-TGT-GGC-TGT-GA Wnt4R: GGA-CGT-CCA-CAA-AGG-ACT-GT MyoDL: GAG-ATG-CGC-TCC-ACT-ATG-CT MyoDR: TGG-CAT-GAT-GGA-TTA-CAG-CG MyogeninL: GCA-CTG-GAG-TTC-GGT-CCC-AA MyogeninR: TAT-CCT-CCA-CCG-TGA-TGC-TG}

\section{Imaging and Morphometry}

Images were acquired by a Zeiss EM S3/SyCoP3 Macroapotome equipped with Zen software in the imaging facility of the Institute of Biology Paris-Seine. The ImageJ software was used for the morphometric analysis. Fusion index (FI) was defined as the number of nuclei in myotubes on total nuclei in 5 fields/sample; myotube diameter (DIA) was measured in 50 myotubes/sample; nuclei per myotube (NpM) were counted in 50 randomly chosen myotubes. Myotubes diameter was measured as the average from three independent measurements per myotube according to previously published methods (Trendelenburg et al., 2009; Deane et al., 2013). At least triplicate samples from two independent experiments were analyzed for each condition; thus, $6<n<10$ for each data group.

\section{Statistical Analysis}

Comparisons of quantitative variables were performed through 2-way ANOVA, after verifying parametric assumptions. In case these assumptions were violated, some transformations (square root or arcsin, as appropriate) were used. Post hoc comparisons were performed through Tukey's significant difference method. When a comparison of each treatment group with a single control group was necessary, a Dunnett post hoc test was employed. The significance level was set at 0.05 . Statistical analyses were performed by SPSS 25.0.

\section{RESULTS}

\section{Mechanical Stimulation Counteracts the Negative Effect of Tumor-Derived Factors on Muscle Cells}

C2C12 cultures, following $4 \mathrm{~d}$ in DM, contained both multinucleated myotubes and undifferentiated myoblasts (Figure 1Aa). We further cultured these cells for $2 \mathrm{~d}$ in control conditions (i.e., in HS) in the absence (static condition, SC) or presence (dynamic condition, DC) of mechanical stimulation, represented by cyclical stretching of the substratum; furthermore, we treated the cells with C26 tumor-conditioned medium (CM), in a SC or a DC, and we analyzed $6 \mathrm{~d}$ cultures undergoing four combinatorial treatments (Figure 1Ab). The morphometric analysis focused on myotube diameter (DIA), as a marker of fiber size, on fusion index (FI), as a marker of the extent of myogenic differentiation, and on the number of nuclei per myotube (NpM), as an indication of myotube growth because of the addition of nuclei deriving from the myoblasts. On day 6 myotube cultures showed a significant increase in FI and NpM as compared to $4 \mathrm{~d}$ cultures, indicating that the myotubes continuously grew in size by incorporating the nuclei from myoblasts, or, possibly, that additional newborn myotubes formed (Figure 1B). Two-way ANOVA on 6d-culture morphological features showed that: CM decreased, while DC significantly increased, myotube DIA even in the presence of CM (Figure 1Ba); CM diminished FI, while DC interfered with CM and rescued FI. Given the significance of the negative interaction between CM and DC we could perform post hoc tests, which showed not only that the FI in the presence of CM is lower compared to all the other treatments, but also that the DC does not promote fusion per se (Figure 1Bb); indeed CM had a negative effect on the number of $\mathrm{NpM}$, with no interaction with the DC, while the latter did not significantly affect the number of NpM (Figure 1Bc).

Consistently with the previous results, the positive effects of mechanical stimulation on myogenesis were confirmed by the significant upregulation of the MyoD level (Figure 1Ca), likely mirroring a sustained activation of differentiating myoblasts; we also observed a decreased, albeit not significant, amount of MyoD protein following CM treatment, with no interaction between the two variables as shown by ANOVA (Figure 1Cb). The immunofluorescence analysis of myogenin (Figure 1Da), which is a later differentiation marker and is required for terminal myogenic differentiation, revealed statistically 


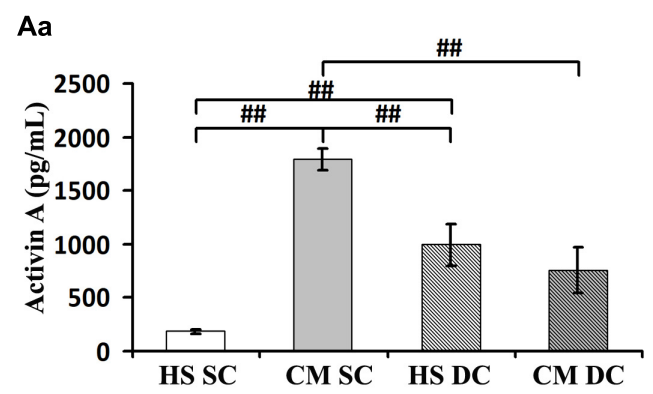

Ab

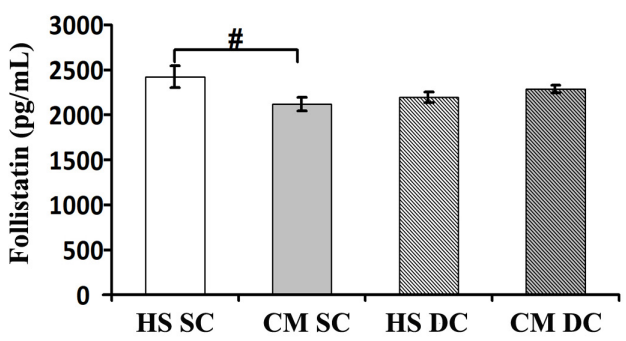

Ac

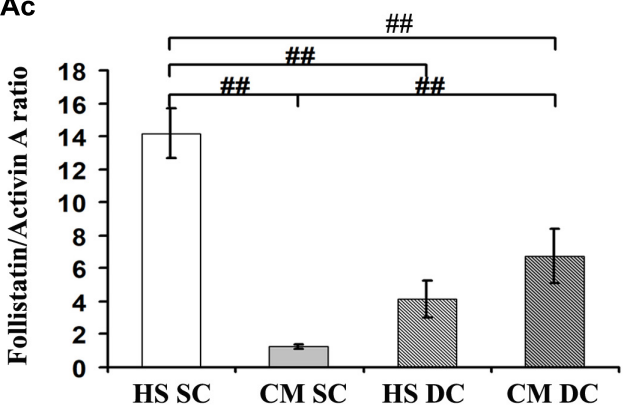

Ca
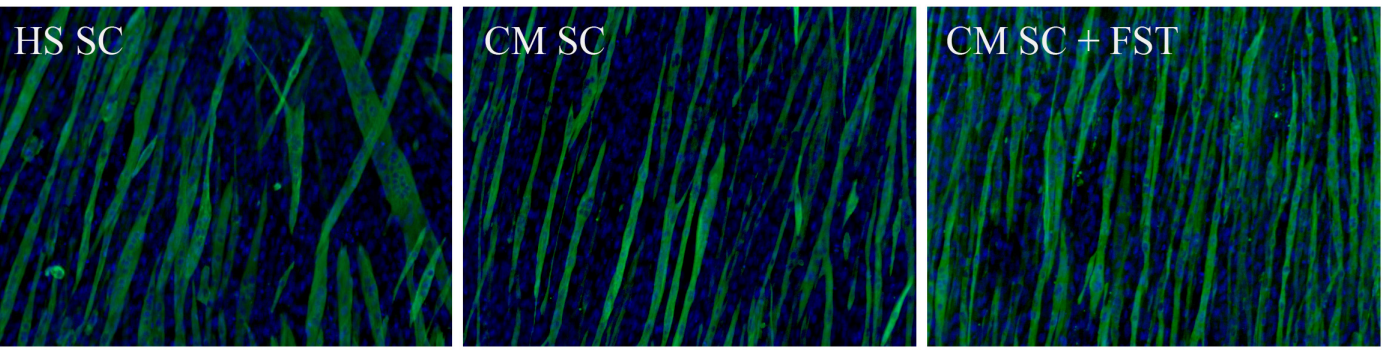

$\mathrm{Cb}$
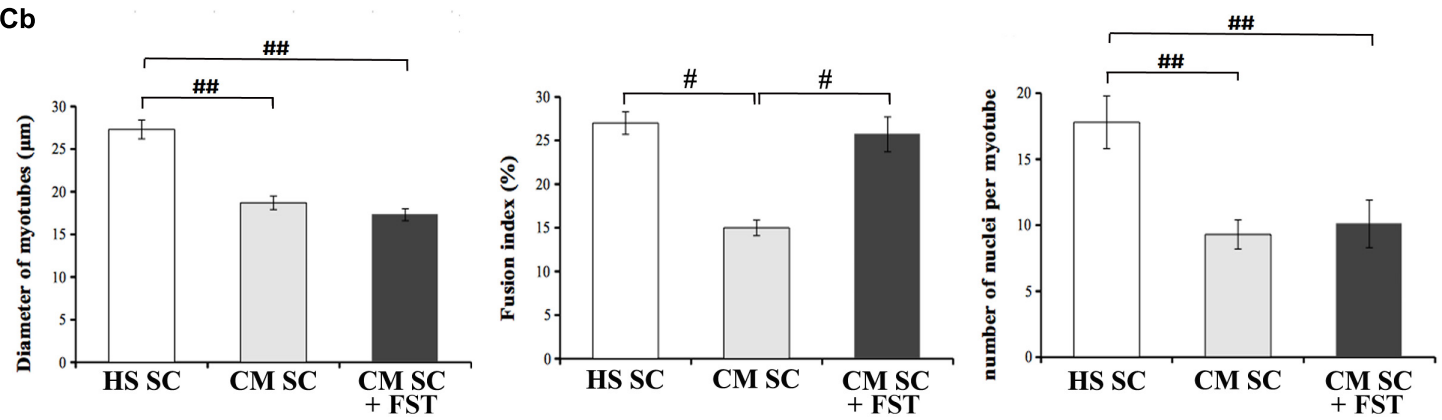

FIGURE 2 | Continued 
FIGURE 2 | Follistatin is not sufficient to rescue myotube size in the presence of tumor-derived factors. (A) Quantification of activin (Aa) and follistatin (Ab) by ELISA in $6 \mathrm{~d}$ culture supernatant, in differentiation medium in the absence (HS) or presence (CM) of C26-conditioned medium, in combination with the absence (SC) or presence (DC) of cyclic stretch. The follistatin/activin ratio was calculated (Ac). Two-way ANOVA showed: CM effect and a interaction with DC on activin levels; interaction between CM and DC on follistatin levels; CM effect and interaction with DC on follistatin/activin ratio. ${ }^{\#} p<0.05$, \#\# $p<0.01$ by Tukey HSD test. (B) WB analysis for P-SMAD2/3 and SMAD 2/3 (Ba) and relative average density (Bb) following normalization over the GAPDH. Two-way ANOVA showed a significant effect for DC $(F=6.25 ; d f=1 ; p<0.05)$ and interaction of Dc with CM ( $F=12.36$; $d f=1 ; p<0.01)$. ${ }^{*} p<0.05$, \#\# $p<0.01$ by Tukey HSD test. (C) Immunofluorescence for Myosin (green) and nuclei (blue) in $\mathrm{C} 2 \mathrm{C} 12$ myotubes at $6 \mathrm{~d}$ of culture (Ca) in differentiation medium in the absence (HS) or presence (CM) of C26-conditioned medium and in CM supplemented with $100 \mathrm{ng} / \mathrm{ml}$ recombinant follistatin, in the absence of cyclic stretch (SC). The treatments were performed starting on $4 \mathrm{~d}$ culture and changing the medium daily. Morphometric analyses $\mathbf{( C b )}$ were performed on replicate samples $(n=6)$. One-way ANOVA indicated a significant effect of treatments on myotube diameter and on the number of nuclei/myotube. ${ }^{* *} p<0.01$ by Tukey HSD test.

significant, opposite effects of both CM and DC (Figure 1Db) on the percentage of the myogenin positive nuclei.

\section{Follistatin Is Sufficient to Rescue Myogenic Differentiation but Not Myotube Size in the Presence of Tumor-Derived Factors}

To assess the contribution of myokines and putative tumorderived factors on myotube size and myoblast recruitment, we measured both activin and follistatin levels in the $6 \mathrm{~d}$ culture media. Worth noting, the C26 CM used throughout this work contained $2800 \pm 380 \mathrm{pg} / \mathrm{mL}$ activin (data not shown), implying that, following a dilution to $20 \%$ in the culture medium, the latter contained $590 \pm 76 \mathrm{pg} / \mathrm{mL}$ activin of tumor origin at the beginning of the treatments, i.e., on day 4 (data not shown). Following 2 days in culture, activin concentration decreased to $190 \pm 18 \mathrm{pg} / \mathrm{mL}$ in control conditions (HS SC) indicating a non-specific or myotube-mediated degradation or internalization/absorption (Figure 2Aa); on the contrary, in the presence of CM activin increased about 3 times, which suggests an activin release from muscle cells (Figure 2Aa). In the DC activin levels were significantly reduced, both in the absence or presence of CM, even though they remained higher than in unstimulated, control cultures (Figure 2Aa).

In order to better understand the novel finding of the release of activin from muscle cells, we measured activin A expression in the four culture conditions failing to see any statistically significant difference, even though we noticed that activin expression doubled in CM SC (Table 1); this suggests that activin release from the myotubes could be partially dependent on activin expression but mostly depends on post-translational events.

We also measured follistatin concentration in the four conditions above and noticed that it significantly decreased in the CM SC (Figure 2Ab), consistently with the physiological role of follistatin as an activin-binding protein, and that its level was rescued in the presence of mechanical stimulation, i.e., DC. Worth noting, the CM and the DC have opposite, significant effects on follistatin expression (Table 1), indicating that the exposure to tumor-derived factors, including activin, downregulates follistatin production in muscle cells, while the mechanical stimulation rescues follistatin expression and release.

Since free activin binds to the activin receptor type-2B (actRIIB) we could not exclude that mechanical stimulation counteracted CM effects by affecting the actRIIB expression as well. Therefore, we measured actRIIB receptor expression in muscle cell cultures and we found a quasi-significant effect of both $\mathrm{CM}$ and $\mathrm{DC}$ on its expression. In addition, we found that mechanical stimulation interferes with the CMinduced actRIIB expression increase, further contributing to the myotube desensitization to activin (Table 1). Altogether, these data suggest an adverse effect on myotubes of tumor celland muscle-derived activin, which could be counteracted by the mechanically stimulated secretion of follistatin by myotubes (Figure 2A). Activin effects are further exacerbated by the differential modulation of actRIIB by CM and DC (Table 1).

Given the pivotal role of actRIIB in mediating cachexia in vivo (Zhou et al., 2010) and the negative effects of tumor-derived

TABLE 1 | Mechanical stimulation counteracts the negative effect of tumor-derived factors on P-SMAD transcriptional targets (MRF) and on Follistatin expression.

\begin{tabular}{lccccc}
\hline Gene & HS SC & CM SC & HS DC & CM DC & ANOVA \\
\hline ActRIII & $1.00 \pm 0.36$ & $5.35 \pm 1.52$ & $1.56 \pm 0.51$ & $0.99 \pm 0.14$ & DC, CM QS interaction \\
Activin A & $1.00 \pm 0.30$ & $2.02 \pm 0.53$ & $1.21 \pm 0.38$ & $1.18 \pm 0.29$ & NS \\
Follistatin & $1.00 \pm 0.17$ & $0.50 \pm 0.09$ & $1.77 \pm 0.43$ & $1.02 \pm 0.25$ & DC and CM effects \\
Wnt4 & $1.00 \pm 0.27$ & $2.70 \pm 0.74$ & $1.58 \pm 0.36$ & $1.23 \pm 0.27$ & interaction \\
MyoD & $1.00 \pm 0.09$ & $0.44 \pm 0.05$ & $0.70 \pm 0.15$ & $0.66 \pm 0.15$ & DC and CM effects interaction \\
Myogenin & $1.00 \pm 0.16$ & $0.59 \pm 0.08$ & $0.97 \pm 0.08$ & $0.92 \pm 0.11$ & DC and CM effects
\end{tabular}

Gene expression was assessed by Q-PCR in myotubes following $2 d$ culture as indicated in the absence (HS) or presence (CM) of C26-conditioned medium, in combination with the absence (SC) or presence (DC) of cyclic stretch. Gene expression is shown as fold induction in respect to control (HS SC), following normalization over GAPDH. Data are shown as mean \pm SEM of replicate samples $(8<n<16)$. Two-way ANOVA significance is reported in the last column (ANOVA) and ANOVA results are reported in parentheses for each gene, as follows: ActRIIB, activin Receptor IIB (for DC and for CM: Q.S.; interaction F = 8.67; $d f=1 ; p<0.01$ ); activin A (NS); Follistatin (for DC: $F=6.71 ; d f=1 ; p<0.05 ;$ for $C M: F=6.09 ; d f=1 ; p<0.05)$; Wingless-related integration site 4, Wnt4 $(F=4.74 ; d f=1 ; p<0.05) ; M y o D(F o r D C: F=12.58 ; d f=1 ;$ $p<0.01$; for $C M: F=6.85 ; d f=1 ; p<0.05$; interaction: $F=6.43$; $d f=1 ; p<0.05$ ); myogenin (for $D C: F=3.97 ; d f=1 ; p<0.05 ;$ for $C M: F=4.42 ; d f=1 ; p<0.05$; interaction: QS). NS and QS = not and quasi $(p=0.05)$ significant, respectively. 
factors on myotubes reported above, we aimed to confirm that the ActRIIB signaling pathway was differentially activated by CM and DC. Therefore, we measured SMAD2/3 activation (expressed as P-SMAD2/3 over total SMADs, following normalization by the housekeeping gene GAPDH, Figure 2Ba) and found consistent results, i.e., SMAD2/3 activation by the $\mathrm{CM}$ and inhibition by the DC (Figure 2Bb). To further confirm these results, we analyzed SMAD2/3 transcriptional targets, including Wnt4 and, noticeably, the MRF MyoD and myogenin (Table 1). As expected, a negative interaction was found between DC and CM on Wnt4 expression: the latter was increased by the CM, albeit not significantly (possibly a false negative result in this case); in addition, a return to control levels was observed in the DC (Table 1). As is known, SMAD2/3 transcriptional effects on MRF are the opposite than those on Wnt4, since their expression is inhibited by P-SMAD2/3: so, as expected and in line with the protein levels shown in Figure 1, the CM significantly decreased both MyoD and myogenin expression, while the DC restored MyoD and myogenin expression to control levels (Table 1).

The correlation between a high follistatin/activin ratio and the improvement of myogenesis prompted us to investigate whether follistatin was sufficient to counteract CM effects upon myotubes. To this purpose, we incubated myotube cultures in SC with CM in the absence or presence of recombinant follistatin (Figure 2Ca). While CM decreased myotube DIA and hampered FI and NpM increase, follistatin rescued FI but failed to counteract CM effects on DIA and NpM (Figure 2Cb). Worth noting, in a control experiment recombinant follistatin alone was able to counteract the adverse effects of recombinant activin upon myotube DIA and FI, as a proof of concept of its inhibitory activity on activin (Supplementary Figure S1).

\section{DISCUSSION}

Act receptor ligands are becoming increasingly important as triggers of muscle wasting and as pharmacological targets to treat cachexia. The myostatin-activin-SMAD cascade has been shown to activate FOXO3a, a crucial activator of muscle-atrophyrelated gene expression (Mathew, 2011); ActRIIB antagonism suffices to revert muscle wasting and prolong survival in animals affected by cancer cachexia (Zhou et al., 2010). Additional studies highlighted activin relevance to humans, since increased circulating concentrations of activin may contribute to the development of cachexia in cancer patients (Loumaye et al., 2015). Here we showed that activin is present in the tumorconditioned medium, inducing myotube atrophy and inhibiting the incorporation of myoblasts into nascent myotubes. We found that a mechanical stimulation-dependent rescue of the myotube size is indeed associated to an increase in the follistatin/activin ratio, showing that this is an effective in vitro model to identify beneficial muscle derived factors. In addition, CM seems to promote the release of activin from myotubes inducing a vicious circle ultimately leading to myotube atrophy and hampered myotube growth - while mechanical stretching appears to diminish activin levels and increase the levels of the activin inhibitor follistatin. However, follistatin per se is not sufficient to fully revert CM negative effects, since recombinant follistatin only rescues FI without affecting myotube size (both in terms of diameter and recruitment of additional nuclei). As a consequence, additional factors in the tumor CM control

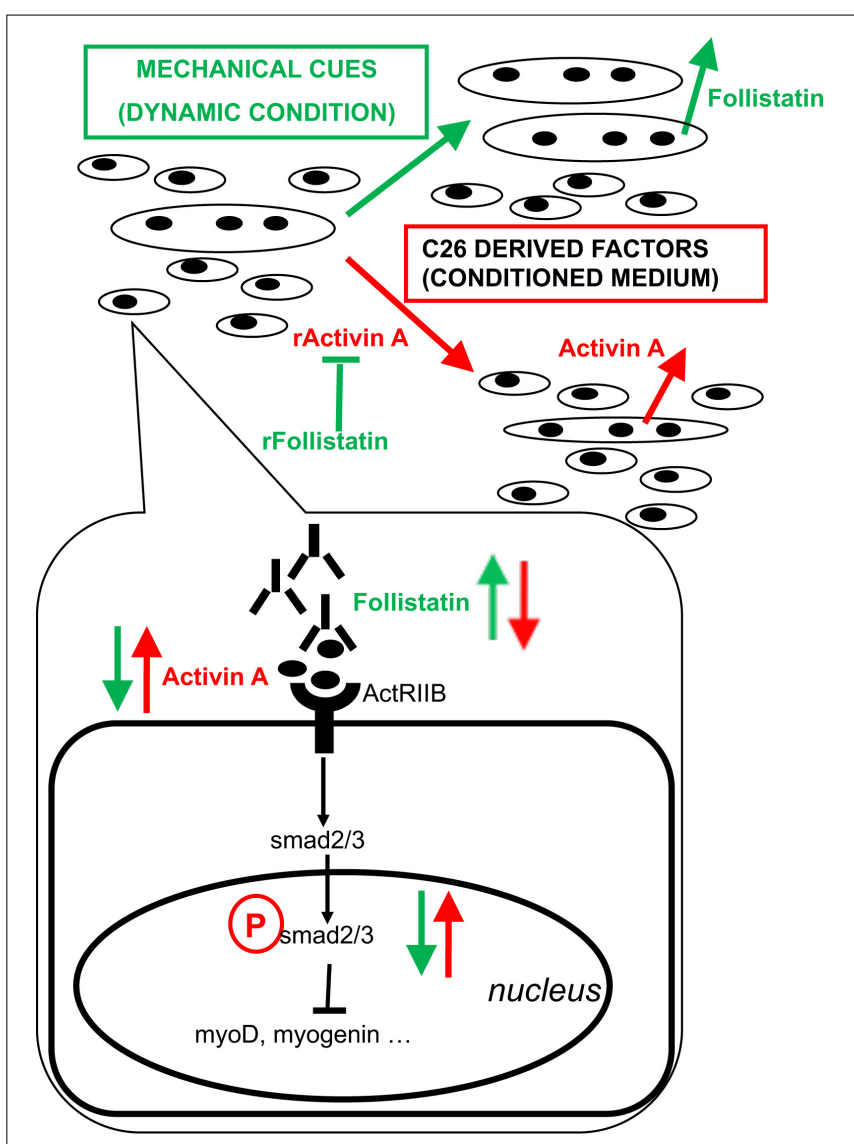

FIGURE 3 | Proposed model of action of tumor-derived factors and mechanical stimulation on myotubes and myoblasts. Mixed cultures of myotubes and myoblasts mature in culture by increasing the diameter of myotubes, the fusion index (i.e., myogenic differentiation tout court, including the formation of novel myotubes) and the number of nuclei per myotube (i.e., myotube accretion by incorporation of myoblasts). C26 tumor-derived factors include activin and induce further expression and release of activin as well as a decrease of follistatin expression and its release by muscle cells, ultimately leading to myotube atrophy, a block of myogenic differentiation and hampered incorporation of myoblasts into myotubes. On the other hand, mechanical stimulation counteracts the negative effects exerted by tumor-derived factors on muscle cells by diminishing the levels of activin available to bind actRIIB: this is obtained by reducing activin concentration in the medium and by rescuing follistatin release by muscle cells. Recombinant activin (rActivin A) mimics tumor $\mathrm{CM}$ and its effects are counteracted by recombinant follistatin (rFollistatin). However, rFollistatin only partially counteracts CM: since, in the presence of $\mathrm{CM}$, follistatin rescues the fusion index but not myotube diameter nor the number of NpM, while mechanical stimulation also reverts

CM-mediated effects on myotube size, follistatin is mostly responsible for the regulation of myogenic differentiation, while mechanical stimulation preserves myotube size through additional mechanisms. The signaling pathways downstream of actRIIB involve the activation of SMAD2/3 transcriptional activity, which is increased by tumor-derived factors and decreased by mechanical stimulation, resulting in the regulation of MRF expression leading to myoblast differentiation and fusion. 
myotube size and their negative effects are hampered by mechanical stimulation independently of follistatin release from myotubes. A model of the action of mechanical stimulation combined with tumor-derived factors on the release of activin and follistatin from myotubes is shown in Figure 3. In this context, actRIIB plays a major role, since its expression does not significantly change, through the activin-mediated SMAD2/3 transcriptional activity. The ratio between available activin and follistatin is likely the major player in these responses, even though the mechanisms underlying activin availability and the inhibition of activin by follistatin remain to be elucidated.

In conclusion: (a) the development of novel activin-targeted therapeutic approaches should consider the existence of further significant tumor-secreted factors mediating cachexia, even though activin plays a major role; (b) upon mechanical stimulation myotubes activate other pathways in addition to follistatin, which effectively counteract the adverse effect of tumor-derived factors; (c) in particular, in the presence of tumor-derived factors follistatin alone is not sufficient to recruit additional cells (nuclei) toward the myotubes, even though it increases the fusion index, representing the formation of new myotubes. In vivo muscle acts as a secretory organ (Pedersen, 2013); our results suggest that the pleiotropic effects of exercise are not limited to contraction-dependent endocrinological effects and that pure mechanical stimuli have a direct and relevant effect on muscle homeostasis.

\section{AUTHOR CONTRIBUTIONS}

$\mathrm{AB}, \mathrm{AB}-\mathrm{S}$, and $\mathrm{ZX}$ performed the experiments and collaborated to the writing of the manuscript. MR performed the statistical analysis and contributed to the interpretation of the data and discussion. VM, MS, ZL, and SA supervised the experimental

\section{REFERENCES}

Aulino, P., Costa, A., Chiaravalloti, E., Perniconi, B., Adamo, S., Coletti, D., et al. (2015). Muscle extracellular matrix scaffold is a multipotent environment. Int. J. Med. Sci. 12, 336-340. doi: 10.7150/ijms. 10761

Barone, R., Macaluso, F., Sangiorgi, C., Campanella, C., Marino Gammazza, A., Moresi, V., et al. (2016). Skeletal muscle Heat shock protein 60 increases after endurance training and induces peroxisome proliferator-activated receptor gamma coactivator 1 alphal expression. Sci. Rep. 6:19781. doi: 10.1038/ srep19781

Barreto, R., Mandili, G., Witzmann, F. A., Novelli, F., Zimmers, T. A., and Bonetto, A. (2016). Cancer and chemotherapy contribute to muscle loss by activating common signaling Pathways. Front. Physiol. 7:472.

Carotenuto, F., Coletti, D., Di Nardo, P., and Teodori, L. (2016). alpha-linolenic acid reduces TNF-induced apoptosis in $\mathrm{C} 2 \mathrm{C} 12$ myoblasts by regulating expression of apoptotic proteins. Eur. J. Transl. Myol. 26:6033. doi: 10.4081/ ejtm.2016.6033

Chen, J. L., Colgan, T. D., Walton, K. L., Gregorevic, P., and Harrison, C. A. (2016). The TGF-beta signalling network in muscle development, adaptation and disease. Adv. Exp. Med. Biol. 900, 97-131. doi: 10.1007/978-3-31927511-6_5

Chen, J. L., Walton, K. L., Winbanks, C. E., Murphy, K. T., Thomson, R. E., Makanji, Y., et al. (2014). Elevated expression of activins promotes muscle wasting and cachexia. FASEB J. 28, 1711-1723. doi: 10.1096/fj.13-245894 work, contributed to discussion, and revised the manuscript. DC supervised and coordinated the experimental work, contributed to data analysis, and wrote the first draft of the manuscript.

\section{FUNDING}

DC was funded by AFM (2017-20603), EFEM 2016, IBPS Action Incitative (2014), and ANR (2013-J13R191). SA was funded by 2016 Sapienza Research projects RM116154ECE34AF4 (2016) and RM11715C78539BD8 (2017).

\section{ACKNOWLEDGMENTS}

The authors are grateful to Anna Luisa Mazzotti for the editing of the manuscript.

\section{SUPPLEMENTARY MATERIAL}

The Supplementary Material for this article can be found online at: https://www.frontiersin.org/articles/10.3389/fphys. 2019.00401/full\#supplementary-material

FIGURE S1 | (A) Immunofluorescence for Myosin (red) in C2C12 myotubes at 6d of culture, following $2 \mathrm{~d}$ of treatment with $10 \mathrm{ng} / \mathrm{ml}$ recombinant activin (rActivin), $25 \mathrm{ng} / \mathrm{ml}$ recombinant follistatin (rFollistantin) or both, with daily changes of medium. (B) Morphometric analysis was performed on replicate samples $(n=7)$ indicating a significant effect of both treatments and a negative interaction between them. Two-way ANOVA showed: significant effects on myotube diameter for activin $(F=7.72 ; d f=1 ; p<0.05)$, follistatin $(F=4.35 ; d f=1 ; p<0.05)$ and an interaction of follistatin with activin $(F=7.91 ; d f=1 ; p<0.01)$; significant effects on fusion index for activin ( $F=4.68$; $d f=1 ; p<0.05)$, follistatin $(F=9.1$; $d f=1 ; p<0.01) . \# p<0.05{ }^{\# \#} p<0.01$ by Tukey HSD test. $\# p<0.05$ by Tukey HSD test.

Ciaraldi, T. P., Ryan, A. J., Mudaliar, S. R., and Henry, R. R. (2016). Altered myokine secretion is an intrinsic property of skeletal muscle in type 2 diabetes. PLoS One 11:e0158209. doi: 10.1371/journal.pone.0158209

Coletti, D. (2018). Chemotherapy-induced muscle wasting: an update. Eur. J. Transl. Myol. 28:7587. doi: 10.4081/ejtm.2018.7587

Coletti, D., Aulino, P., Pigna, E., Barteri, F., Moresi, V., Annibali, D., et al. (2016). Spontaneous physical activity downregulates Pax7 in cancer cachexia. Stem Cells Int. 2016:6729268. doi: 10.1155/2016/6729268

Costelli, P., Muscaritoli, M., Bonetto, A., Penna, F., Reffo, P., Bossola, M., et al. (2008). Muscle myostatin signalling is enhanced in experimental cancer cachexia. Eur. J. Clin. Invest. 38, 531-538. doi: 10.1111/j.13652362.2008.01970.x

De Arcangelis, V., Coletti, D., Canato, M., Molinaro, M., Adamo, S., Reggiani, C., et al. (2005). Hypertrophy and transcriptional regulation induced in myogenic cell line L6-C5 by an increase of extracellular calcium. J. Cell Physiol. 202, 787-795. doi: 10.1002/jcp.20174

Deane, C. S., Hughes, D. C., Sculthorpe, N., Lewis, M. P., Stewart, C. E., and Sharples, A. P. (2013). Impaired hypertrophy in myoblasts is improved with testosterone administration. J. Steroid Biochem. Mol. Biol. 138, 152-161. doi: 10.1016/j.jsbmb.2013.05.005

Ding, H., Zhang, G., Sin, K. W., Liu, Z., Lin, R. K., Li, M., et al. (2017). Activin A induces skeletal muscle catabolism via p38beta mitogen-activated protein kinase. J. Cachexia Sarcopenia Muscle 8, 202-212. doi: 10.1002/jcsm.12145

Donatto, F. F., Neves, R. X., Rosa, F. O., Camargo, R. G., Ribeiro, H., MatosNeto, E. M., et al. (2013). Resistance exercise modulates lipid plasma profile 
and cytokine content in the adipose tissue of tumour-bearing rats. Cytokine 61, 426-432. doi: 10.1016/j.cyto.2012.10.021

Fearon, K. C., Glass, D. J., and Guttridge, D. C. (2012). Cancer cachexia: mediators, signaling, and metabolic pathways. Cell Metab. 16, 153-166. doi: 10.1016/j. cmet.2012.06.011

Formicola, L., Pannerec, A., Correra, R. M., Gayraud-Morel, B., Ollitrault, D., Besson, V., et al. (2018). Inhibition of the activin receptor type-2B pathway restores regenerative capacity in satellite cell-depleted skeletal muscle. Front. Physiol. 9:515. doi: 10.3389/fphys.2018.00515

Hatakeyama, S., Summermatter, S., Jourdain, M., Melly, S., Minetti, G. C., and Lach-Trifilieff, E. (2016). ActRII blockade protects mice from cancer cachexia and prolongs survival in the presence of anti-cancer treatments. Skeletal Muscle 6:26. doi: 10.1186/s13395-016-0098-2

Lira, F. S., Neto, J. C., and Seelaender, M. (2014). Exercise training as treatment in cancer cachexia. Appl. Physiol. Nutr. Metab. 39, 679-686. doi: 10.1139/apnm2013-0554

Lira, F. S., Rosa, J. C., Zanchi, N. E., Yamashita, A. S., Lopes, R. D., Lopes, A. C., et al. (2009). Regulation of inflammation in the adipose tissue in cancer cachexia: effect of exercise. Cell Biochem. Funct. 27, 71-75. doi: 10.1002/cbf.1540

Lira, F. S., Yamashita, A. S., Rosa, J. C., Koyama, C. H., Caperuto, E. C., Batista, M. L. Jr., et al. (2012). Exercise training decreases adipose tissue inflammation in cachectic rats. Horm. Metab. Res. 44, 91-98. doi: 10.1055/s-0031-1299694

Livak, K. J., and Schmittgen, T. D. (2001). Analysis of relative gene expression data using real-time quantitative PCR and the 2(-Delta Delta C(T)) method. Methods 25, 402-408. doi: 10.1006/meth.2001.1262

Loumaye, A., de Barsy, M., Nachit, M., Lause, P., Frateur, L., van Maanen, A., et al. (2015). Role of Activin A and myostatin in human cancer cachexia. J. Clin. Endocrinol. Metab. 100, 2030-2038. doi: 10.1210/jc.2014-4318

Loumaye, A., de Barsy, M., Nachit, M., Lause, P., van Maanen, A., Trefois, P., et al. (2017). Circulating Activin A predicts survival in cancer patients. J. Cachexia Sarcopenia Muscle 8, 768-777. doi: 10.1002/jcsm.12209

Mathew, S. J. (2011). InACTIVatINg cancer cachexia. Dis. Model. Mech. 4, 283-285. doi: $10.1242 / \mathrm{dmm} .007658$

Morvan, F., Rondeau, J. M., Zou, C., Minetti, G., Scheufler, C., Scharenberg, M., et al. (2017). Blockade of activin type II receptors with a dual anti-ActRIIA/IIB antibody is critical to promote maximal skeletal muscle hypertrophy. Proc. Natl. Acad. Sci. U.S.A. 114, 12448-12453. doi: 10.1073/pnas.17079 25114

Pedersen, B. K. (2013). Muscle as a secretory organ. Compr. Physiol. 3, 1337-1362. doi: $10.1002 /$ cphy.c120033
Pigna, E., Berardi, E., Aulino, P., Rizzuto, E., Zampieri, S., Carraro, U., et al. (2016). Aerobic exercise and pharmacological treatments counteract cachexia by modulating autophagy in colon cancer. Sci. Rep. 6:26991. doi: 10.1038/ srep26991

Tilbrook, A. J., de Kretser, D. M., Dunshea, F. R., Klein, R., Robertson, D. M., Clarke, I. J., et al. (1996). The testis is not the major source of circulating follistatin in the ram. J. Endocrinol. 149, 55-63. doi: 10.1677/joe.0.1490055

Toledo, M., Busquets, S., Penna, F., Zhou, X., Marmonti, E., Betancourt, A., et al. (2016). Complete reversal of muscle wasting in experimental cancer cachexia: additive effects of activin type II receptor inhibition and beta-2 agonist. Int. J. Cancer 138, 2021-2029. doi: 10.1002/ijc.29930

Trendelenburg, A. U., Meyer, A., Rohner, D., Boyle, J., Hatakeyama, S., and Glass, D. J. (2009). Myostatin reduces Akt/TORC1/p70S6K signaling, inhibiting myoblast differentiation and myotube size. Am. J. Physiol. Cell Physiol. 296, C1258-C1270. doi: 10.1152/ajpcell.00105.2009

Winbanks, C. E., Murphy, K. T., Bernardo, B. C., Qian, H., Liu, Y., Sepulveda, P. V., et al. (2016). Smad7 gene delivery prevents muscle wasting associated with cancer cachexia in mice. Sci. Transl. Med. 8:348ra398. doi: 10.1126/ scitranslmed.aac 4976

Yeo, N. H., Woo, J., Shin, K. O., Park, J. Y., and Kang, S. (2012). The effects of different exercise intensity on myokine and angiogenesis factors. J. Sports Med. Phys. Fitness 52, 448-454.

Zheng, H., Qiao, C., Tang, R., Li, J., Bulaklak, K., Huang, Z., et al. (2017). Follistatin $\mathrm{N}$ terminus differentially regulates muscle size and fat in vivo. Exp. Mol. Med. 49:e377. doi: 10.1038/emm.2017.135

Zhou, X., Wang, J. L., Lu, J., Song, Y., Kwak, K. S., Jiao, Q., et al. (2010). Reversal of cancer cachexia and muscle wasting by ActRIIB antagonism leads to prolonged survival. Cell 142, 531-543. doi: 10.1016/j.cell.2010.07.011

Conflict of Interest Statement: The authors declare that the research was conducted in the absence of any commercial or financial relationships that could be construed as a potential conflict of interest.

Copyright (ㄷ 2019 Baccam, Benoni-Sviercovich, Rocchi, Moresi, Seelaender, Li, Adamo, Xue and Coletti. This is an open-access article distributed under the terms of the Creative Commons Attribution License (CC BY). The use, distribution or reproduction in other forums is permitted, provided the original author(s) and the copyright owner(s) are credited and that the original publication in this journal is cited, in accordance with accepted academic practice. No use, distribution or reproduction is permitted which does not comply with these terms. 


\section{OPEN ACCESS}

Edited by:

Marilia Seelaender,

University of São Paulo, Brazil

Reviewed by:

Marcin Wysoczynski,

University of Louisville, United States

Paul D. Allen,

University of Leeds, United Kingdom Laszlo Csernoch,

University of Debrecen, Hungary

${ }^{*}$ Correspondence:

Libera Berghella

libera@caltech.edu

Specialty section:

This article was submitted to Striated Muscle Physiology, a section of the journal Frontiers in Physiology

Received: 25 November 2018 Accepted: 08 April 2019

Published: 30 April 2019

Citation:

Moresi V, Adamo S and Berghella L (2019) The JAK/STAT Pathway in Skeletal Muscle Pathophysiology.

Front. Physiol. 10:500

doi: 10.3389/fphys.2019.00500

\section{The JAK/STAT Pathway in Skeletal Muscle Pathophysiology}

\author{
Viviana Moresi ${ }^{1,2}$, Sergio Adamo ${ }^{1,2}$ and Libera Berghella ${ }^{1,3 *}$ \\ 'Unit of Histology and Medical Embryology, DAHFMO, University La Sapienza, Rome, Italy, ${ }^{2}$ Interuniversity Institute of \\ Myology, Rome, Italy, ${ }^{3}$ Division of Biology and Biological Engineering, California Institute of Technology, Pasadena, CA, \\ United States
}

The Janus kinase (JAK)/signal transducer and activator of transcription (STAT) pathway is a key intracellular mediator of a variety of metabolically relevant hormones and cytokines, including the interleukin-6 (IL-6) family of cytokines. The JAK/STAT pathway transmits extracellular signals to the nucleus, leading to the transcription of genes involved in multiple biological activities. The JAK/STAT pathway has been reported to be required for the homeostasis of different tissues and organs. Indeed, when deregulated, it promotes the initiation and progression of pathological conditions, including cancer, obesity, diabetes, and other metabolic diseases. In skeletal muscle, activation of the JAK/STAT pathway by the IL-6 cytokines accounts for opposite effects: on the one hand, it promotes muscle hypertrophy, by increasing the proliferation of satellite cells; on the other hand, it contributes to muscle wasting. The expression of IL-6 and of key members of the JAK/STAT pathway is regulated at the epigenetic level through histone methylation and histone acetylation mechanisms. Thus, manipulation of the JAK/STAT signaling pathway by specific inhibitors and/or drugs that modulate epigenetics is a promising therapeutic intervention for the treatment of numerous diseases. We focus this review on the JAK/STAT pathway functions in striated muscle pathophysiology and the potential role of IL-6 as an effector of the cross talk between skeletal muscle and other organs.

Keywords: IL-6 cytokine, JAK/STAT pathway, skeletal muscle, organ cross talk, epigenetics

\section{INTRODUCTION}

The Janus kinase (JAK)/signal transducer and activator of transcription (STAT) pathway is a potent signaling cascade, evolutionarily conserved from flies to humans. It is upstream of multiple cellular activities such as proliferation, differentiation, migration, apoptosis, and cell communication or complex biological processes including inflammation, immunesystem development, immune response, and cancer (Darnell et al., 1994; O'Shea et al., 2002, 2015; Bousoik and Montazeri Aliabadi, 2018). The JAK/STAT pathway was initially identified as responsive to interferon-gamma, although a variety of extracellular polypeptide signals and their transmembrane receptors were later found to activate it (Schindler et al., 1992; Heinrich et al., 1998; Aaronson and Horvath, 2002; O'Shea and Plenge, 2012).

In mammals, four members of the JAK proteins (JAK1, JAK2, JAK3, and TYK2) and seven members of the STAT family (STAT 1-4, STAT 5A/B, and STAT 6) were identified. They all share structurally and functionally conserved domains. JAK/STAT proteins are 
ubiquitously expressed, and different combinations of them respond to specific cytokines or growth factor signals, assuring a high degree of specificity with distinct in vivo roles (Aaronson and Horvath, 2002; Kisseleva et al., 2002; Rawlings et al., 2004). The mechanism of IL-6/JAK/STAT signaling cascade allows a direct communication between transmembrane receptors and the nucleus and can be summarized by the following steps: IL-6, the ligand, binds the IL-6r-Gp130 receptor complex and activates the JAK tyrosine kinases recruited to the intracellular domains of their receptors. Once activated, JAK proteins change their conformation, dimerize, phosphorylate, and activate their primary substrates, the STAT proteins. Tyrosinephosphorylated STAT proteins homo- or hetero-dimerize and translocate to the nucleus, where they interact with coactivators and bind to specific regulatory elements in the promoter regions of thousands of different target protein-coding genes, along with microRNAs and long non-coding RNAs. STAT activity is regulated by phosphorylation, acetylation, and methylation, promoting STAT dimer stabilization, DNA binding, interaction with transcriptional coactivators, and target gene expression (Zuang, 2013; Yu et al., 2014; Zimmers et al., 2016). A further level of control is provided by negative regulators of JAK/STAT signaling that guarantee a cytokineinducible feedback inhibition of signals from specific cytokine receptors (Greenhalgh and Hilton, 2001; Aaronson and Horvath, 2002; Linossi et al., 2013). JAK/STAT signaling operates also in response to IL-6 trans-signaling. Indeed, a soluble form of IL-6 receptor (sIL-6R), comprising the extracellular portion of the receptor, binds to IL-6, and the IL-6-sIL-6R complex is able to bind to and activate gp130 homodimers in cells which lack the membrane bound IL-6R (Kallen, 2002; Scheller et al., 2006). Thus, the JAK/STAT signaling cascade provides a remarkable direct and tuned translation of extracellular signals into a transcriptional response in a vast range of cells.

Primarily identified as functioning in hematopoietic cells, the JAK/STAT signaling cascade has been found to play a critical role in different cell types and tissues, including skeletal muscle. As skeletal muscle contracts, it secretes several cytokines into the circulation and the JAK/STAT pathway mediates the signaling of many of the myokines secreted by skeletal muscle (Pedersen and Febbraio, 2008; Hoffmann and Weigert, 2017).

Here, we will review the IL-6/JAK/STAT signaling cascade in myogenesis and skeletal muscle pathophysiology, focusing on its dichotomic role in myogenic cell proliferation and differentiation, as well as in muscle growth and muscle wasting. We will also discuss some examples of cross talk between muscle and other tissues. Finally, we will examine IL-6/JAK/STAT activity regulation, emphasizing the epigenetic mechanisms.

\footnotetext{
Abbreviations: AT, Adipose tissue; C/EBP $\delta$, CCAAT/enhancer binding protein; DMD, Duchenne muscular dystrophy; DNMT, DNA methyltransferase; FAP, Fibro-adipogenic progenitors; FoxO, Forkhead box; IL-6, Interleukin 6; JAK, Janus kinase; MAFbx, Muscle atrophy F-box; MuRF, Muscle RING finger; MuSC, Muscle satellite cell; sIL-6R, Soluble interleukin 6 receptor; STAT, Signal transducer and activator of transcription; UPS, Ubiquitin proteasome system.
}

\section{IL-6/JAK/STAT SIGNALING CASCADE IN SKELETAL MUSCLE}

It is now widely accepted that through IL-6 family signals, the JAK/STAT pathway is required for efficient muscle fiber adaptation during development and regeneration. It was proposed that different combinations of the JAK/STAT pathway members have opposite effects on muscle differentiation and myogenesis. Indeed, the JAK1/STAT1/STAT3 axis promotes myoblast proliferation, preventing the premature differentiation into myotubes. Conversely, JAK2/STAT2/STAT3 induces myogenic differentiation, suggesting that other intracellular ligands act on JAK/STAT factors, to obtain distinct cellular responses at each step during development and myogenesis (Sun et al., 2007; Wang et al., 2008; Jang and Baik, 2013; Muñoz-Cánoves et al., 2013). Several studies demonstrated a role of the JAK/ STAT pathway in regulating the myogenic progression of adult satellite cells (MuSCs), a population of cells that play a fundamental role in skeletal muscle postnatal growth and repair upon injury. MuSCs from IL-6 KO mice showed decreased proliferative capacity, both in vivo and in vitro. This impairment was caused by a lack of IL-6-mediated activation of STAT3 signaling. STAT3 induces the transcription of downstream genes involved in several biological functions, including myoblast proliferation, differentiation, and survival (Serrano et al., 2008; Toth et al., 2011). More recently, it has been shown that STAT3 knock-down (elicited by transient pharmacological or siRNA inhibition) in MuSCs, induced their expansion upon regeneration, but inhibited their differentiation, thus impairing muscle regeneration. Moreover, repeated intermittent administration of a STAT3 inhibitor in mdx mice, determined a sustained expansion of MuSC, contributing to an overall improvement in skeletal muscle repair (Tierney et al., 2014). Elsewhere, it was described that JAK2 or STAT3 KO in isolated MuSCs and pharmacological inhibition in vivo promoted symmetric satellite cell division and markedly improved their homing and repairing ability when transplanted into regenerating muscle (Price et al., 2014). However, different evidences were described when STAT3 depletion was investigated specifically by genetic deletion in MuSCs of mdx mice. By this approach, a progressive reduction of MuSC accompanied with aggravated fibrosis and muscle inflammation was observed. Then, a permanent knockout of STAT3 and a direct and long-term treatment with STAT3 inhibitors, which causes a gradual depletion of MuSCs, might have adverse effects on MuSCs and regeneration in DMD patients (Zhu et al., 2016), in contrast with other approaches such as transient inhibition by chemical inhibitors or siRNA, shown elsewhere (Price et al., 2014; Tierney et al., 2014). It may be speculated that transient and periodic reduction of STAT3 in cellular component of the MuSC niche, such as macrophages or fibro/adipogenic progenitors, known for playing an essential role in muscle regeneration (Bentzinger et al., 2013), is responsible for the beneficial effects observed in dystrophic muscle. Alternatively, IL-6 downstream effectors other than STAT3 could be active in MuSC in promoting muscle regeneration. Nevertheless, studies demonstrated that IL-6-mediated immunological responses may promote additional 
muscle fiber damage under conditions of dystrophin deficiency in mdx mice (Pelosi et al., 2015). Accordingly, IL-6 receptor blockade with the anti-IL-6 receptor antibody attenuated muscular dystrophy via promoting skeletal muscle regeneration in $\mathrm{mdx}$ and dystrophin-/utrophin-deficient mice (Pelosi et al., 2015; Wada et al., 2017).

IL-6/JAK/STAT pathway mediates increased proliferation of MuSC in other conditions such as acute exercise. Indeed, in a model of increasing mechanical loading, muscle hypertrophy resulted attenuated in IL-6 KO mice, due to an impaired MuSC proliferation and migration (Serrano et al., 2008). Moreover, mRNA expression for STAT3 target genes that regulate MuSC proliferation, migration, and differentiation was reduced (Serrano et al., 2008). Acute resistance exercise and resistance training activate the IL-6/STAT1/STAT3 signaling pathway in rat skeletal muscle (Begue et al., 2013) and in human (Trenerry et al., 2007, 2011), suggesting a potential role for STAT3 in the adaptive growth of skeletal muscle mediated by MuSCs. Nevertheless, more recent studies in human muscle biopsies and in STAT3 KO murine model concluded that STAT3 localized to the MuSCs is not required in load-induced skeletal muscle hypertrophy (Amorese and Spangenburg, 2017; Perez-Schindler et al., 2017). The cause of this contradictory evidence may be related to the methods for inducing hypertrophy and to the cell types where STAT3 activation occurs. Indeed, STAT3 activation in immune cells or other cells resident in skeletal muscle may also play significant roles in regulating muscle responses to exercise training (van de Vyver et al., 2016).

While sudden and acute induction of the IL- 6 cascade promotes muscle growth, IL- 6 sustained and elevated release and STAT3 activation have been associated with muscle atrophy occurrence in several catabolic conditions, such as obesity, diabetes, and age-induced sarcopenia or cancer (Zimmers et al., 2016). IL-6 overexpression in transgenic mice caused muscular atrophy, though entirely reversed by treatment with the membrane IL-6 receptor antibody (Tsujinaka et al., 1996). Interestingly, the negative role of IL- 6 in the control of muscle mass was initially demonstrated using animal models of inflammation and cancer-associated cachexia. Cachexia is a muscle wasting syndrome accompanying many acute and chronic diseases, including cancer (Fearon et al., 2012; He et al., 2013; Argiles et al., 2014; Pigna et al., 2016). In cachexia experimental models, STAT3 expression is induced and correlates with increased expression of skeletal muscle ubiquitin E3 ligases. STAT3 dominant negative activity blocked the skeletal muscle loss downstream of IL-6, partly by inhibiting the activity of the ubiquitin proteasome system (UPS), in vitro and in vivo (Baltgalvis et al., 2009; Bonetto et al., 2011, 2012). Coherently, treatment with neutralizing antibodies prevented the increase of IL-6 concentration, exerting a protective effect on body weight loss in cachectic mice and blocking STAT3 activation reduced muscle wasting (Strassmann et al., 1992; Oldenburg et al., 1993; Haddad et al., 2005; Zimmers et al., 2016). Moreover, treatment of cachectic mice with pharmacological inhibitors of the JAK/STAT pathway components, partially prevented muscle mass loss (Gilabert et al., 2014; Pretto et al., 2015; Silva et al., 2015). JAK/STAT pathway activation is responsible for muscle atrophy by several potential mechanisms. In cachexia and chronic kidney disease models, both of which exhibit muscle mass loss, STAT3 initiated muscle wasting by stimulating CCAAT/enhancer binding protein (C/EBP $\delta$ ) expression and activity, which in turn increased myostatin, MAFbx/Atrogin-1, and MuRF-1 expression in myofibers (Zhang et al., 2013). Direct UPS activation can be mediated by STAT3 or indirectly via caspase-3 activation (Silva et al., 2015) or dependent on FoxO transcription factors (Hutchins et al., 2013; Judge et al., 2014). Cachexia has been also associated with posttranslational modifications of JAK/STAT3 components, such as increased muscle phospho-Y705-STAT3 and increased STAT3 localization in myonuclei (Bonetto et al., 2011). Muscle catabolic profile may also be caused by the reduction in ribosomal protein kinase S6K1 phosphorylation and the increase of SOCS3 transcription, an inhibitor of the JAK/STAT pathway (Haddad et al., 2005). Nevertheless, others found that IL-6 does not stimulate muscle loss per se (Baltgalvis et al., 2008), thus supposing that other cytokines activate JAK/STAT, which triggers skeletal muscle proteolysis (Zhang et al., 2013). More recently, it was also shown that IL-6 trans-signaling works as a novel potent inducer of autophagy in myotubes inducing pathway that may be important in cancer cachexia development (Pettersen et al., 2017). Furthermore, IL-6 trans-signaling/STAT3 axis was identified as a therapeutic target in advanced cancer patients presenting cachexia (Miller et al., 2017).

In the muscle microenvironment, a JAK/STAT pathway contribution in catabolic conditions can be considered in relation to its role in promoting the expansion of the satellite cell pool in vitro and in vivo, impairing differentiation and muscle repair (He et al., 2013; Muñoz-Cánoves et al., 2013; Price et al., 2014; Tierney et al., 2014; Sala and Sacco, 2016; Zhu et al., 2016). Other than activation of STAT3 in MuSCs, secreted and elevated IL-6 levels and persistent STAT3 activation were observed in atrophic conditions in the fibro/adipogenic progenitors (FAPs), a population of cells resident in skeletal muscle, fundamental for muscular regeneration and inducible source of IL-6 (Joe et al., 2010; Madaro et al., 2018). IL-6/ STAT3 signaling inactivation in FAPs counteracted muscle atrophy and fibrosis in mouse models of acute denervation and amyotrophic lateral sclerosis (ALS) (Gurney et al., 1994). This suggests an alternative IL-6/JAK/STAT-mediated mechanism, which induces muscle mass loss and represents a possible therapeutic target for neurogenic atrophy diseases (Madaro et al., 2018; Marazzi and Sassoon, 2018).

Altogether, the autocrine and paracrine action of the IL-6/ JAK/STAT pathway on skeletal muscle has opposite effects on satellite cells differentiation and proliferation, hence on muscle homeostasis. Moreover, it causes both deleterious (pro-atrophy) and beneficial (pro-repair and pro-growth) effects on muscle fiber size (Figure 1). The balance between these opposite outcomes may depend on the fine tuning of the JAK/STAT pathway. This effect can be mediated by the interaction of the JAK/STAT molecular effectors with the myofibers or with the multiple cell types of the muscle niche. Further studies will provide new insights to elucidate the molecular mechanism underlying this complex regulation. 


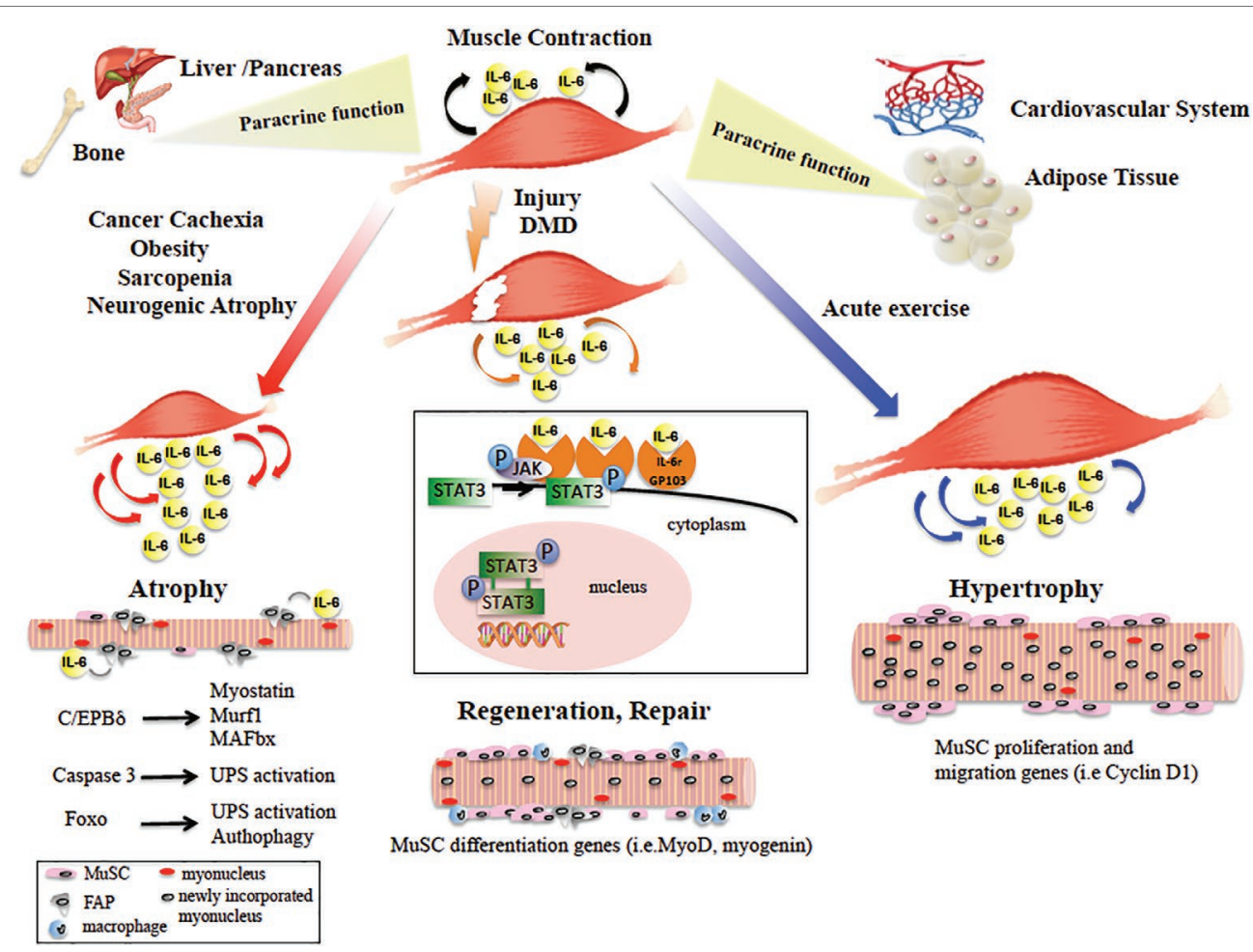

FIGURE 1 | Diagram showing the main paracrine and dichotomic autocrine functions of the IL-6/JAK/STAT3 pathway in the pathophysiology of skeletal muscle. Skeletal muscle physiological contraction induces IL-6 release (black arrows), with paracrine effects on other organ metabolism. Upon injury or in DMD, IL-6 is released (orange arrows) following the inflammatory response and IL-6/JAK/STAT pathway promotes muscle repair by activating pro-myogenic genes (such as MyoD) that allow MuSC differentiation and fusion into new or existing myofibers. In catabolic conditions, IL-6 levels are elevated (red arrows) and induce muscle size loss, by activation of different pro-atrophic pathways in myofibers. In neurogenic atrophy, FAPs activate the IL-6/JAK/STAT pathway. In response to acute exercise, IL-6 is highly produced (blue arrows) and IL-6/JAK/STAT pathway is activated, inducing pro-proliferation and pro-fusion genes that control contribution of MuSC to myofiber growth. In the box, the IL-6/JAK/STAT3 signaling model is shown. IL-6 binds the IL-6r-Gp130 receptor complex and activates the JAK tyrosine kinases. Once activated, JAK proteins dimerize, phosphorylate, and activate their primary substrates, the STAT proteins. Phosphorylated STAT proteins dimerize and translocate to the nucleus, where they activate different target protein-coding genes.

\section{IL-6 AS A MEDIATOR OF THE CROSS TALK BETWEEN SKELETAL MUSCLE AND OTHER ORGANS}

Progressive discovery of new myokines by application of new technologies contributed to the definition of the muscle secretome and to provide new insights regarding their therapeutic potential in the treatment of obesity, metabolic disease, and cancer (Whitham and Febbraio, 2016).

IL-6 was the first cytokine to be proposed as a myokine (Pedersen et al., 2003), and the first myokine found to be secreted during exercise, playing important roles in regulating the metabolism of other organs (Goldstein, 1961). One of the main paracrine functions of IL-6 is to lead to an increase in hepatic glucose production, which works as an energy source for contracting muscles (Febbraio et al., 2004). Furthermore, during exercise, skeletal muscle performs also an "energy sensing" role, affecting some metabolic processes and, through IL-6, mediates the cross talk with insulin-sensitive tissues. By activation of AMP-activated protein kinase (AMPK) and/or PI3-kinase, IL-6 leads to enhanced glucose uptake, lipolysis and fatty acid oxidation, which provide energy from skeletal muscle (Keller et al., 2001;
Al-Khalili et al., 2006). Moreover, IL-6/JAK/STAT plays also a major role in mediating communication between skeletal muscle and pancreas, enhancing glucose tolerance by activating glucagonlike peptide 1 (GLP1) in pancreatic islets. This allows adaption to changes in insulin demand, reduction of food intake and body weight, though having a role in improving metabolic homeostasis in obesity and type 2 diabetes (Bouzakri et al., 2011; Plomgaard et al., 2012; Ellingsgaard et al., 2015).

A direct cross talk between muscle tissue and adipose tissue (AT) occurs in obese mice. In this condition, subcutaneous adipose tissue does not contribute to IL-6 secretion during exercise, so the increased IL-6 produced following prolonged exercise probably derives from skeletal muscle (Eder et al., 2009). In fact, obese mice exposed to acute exercise showed an IL-6 induction, accompanied by increase in STAT3 phosphorylation, reduction in M1 macrophages, and inflammation in infiltrates in AT (Macpherson et al., 2015).

Skeletal muscle-derived growth factors and cytokines have a critical role in maintaining the cardiovascular system. The trophic cascade initiated by skeletal muscle JAK/STAT3 signaling increases growth factor levels in multiple tissues, leading to elevated circulating HGF and VEGF. Their synergistic actions 
further activate the myocardial repair mechanisms orchestrated by PI3K-AKT, ERK (Shabbir et al., 2010). The cardioprotective events of the IL-6/JAK/STAT3 apparently contradict its activity in promoting skeletal muscle wasting, underlying the multiple role of JAK-STAT3 signaling in different tissues.

It is known that the myokines mediate direct communication from skeletal muscle to bone. Elevated IL-6 induces bone loss in IL-6 KO mice (De Benedetti et al., 2006) and is a systemic mediator of the bone loss in dystrophy. In this context, elevated levels of IL-6 produced by inflamed skeletal muscle induce osteoclast increase, which can be reduced by treatment with an anti-IL-6 antibody (Rufo et al., 2011). Interestingly, IL-6, by affecting the functions of liver, fat, and intestine, induces secretion of hepatokines and adipokines to regulate bone formation and bone resorption (Rufo et al., 2011; Guo et al., 2017).

Collectively, these data show that IL- 6 produced by contracting skeletal muscle plays important roles in regulating metabolism in other organs (Figure 1). Hence, lack of physical activity appears to affect a whole network of organs such as liver, pancreas, fat, and bone. From this perspective, the IL-6/JAK/ STAT pathway is nodal in novel therapeutic approaches for the preventive treatment of diseases including cardiovascular diseases, type 2 diabetes, cancer, and osteoporosis.

\section{EPIGENETIC CONTROL OF IL-6/JAK/STAT PATHWAY}

Considering that IL-6 mediates cellular response to stress or metabolic changes, it is not surprising that the IL-6 pathway is also modulated at the epigenetic level, at least by two main mechanisms, i.e., DNA methylation and histone modifications (Figure 2A). IL-6 gene transcription itself is directly modulated by histone acetylation and methylation in macrophages and in cancer cell lines (Lee et al., 2011; Zhang et al., 2015; Hu et al., 2016; Serresi et al., 2016; Chen et al., 2018). Moreover, an association between IL6/JAK/STAT DNA altered methylation and depression has been recently described (Ryan et al., 2017). JAK and STAT gene hypomethylation might also exert influences on erythroid lineage choice by specifically upregulating erythropoiesis transcription factors (Liu et al., 2017). In B cells activating pathway, the lysine-specific histone methyltransferase KMT2D affected H3K4 methylation and expression of a specific set of JAK-STAT genes (Figure 2B; Ortega-Molina et al., 2015).

Interestingly, IL-6 signaling modulates or cooperates with epigenetic mechanisms in regulating chromatin accessibility in tumorigenesis and development (Figure 2C). For instance, IL-6 promotes hypermethylation of the miR142-3p promoter in glioblastoma cells and of certain tumor suppressor genes in oral squamous cell carcinoma (Gasche et al., 2011; Chiou et al., 2013). IL-6-induced hypermethylation and gene silencing are mediated by DNA methyltransferases (DNMTs). IL-6 contributes to tumor growth by increasing DNMT expression and epigenetically repressing tumor suppressor genes or several microRNA in cancer cell lines (Hodge et al., 2005; Zhang et al., 2005, 2006; Braconi et al., 2010; Takeuchi et al., 2015). IL-6 also promotes DNA methylation of the promoter-bound
STAT3, leading to a decrease in STAT3 DNA binding in human colon cancer cells (Yang et al., 2010), or of the Foxp3 gene, thus influencing regulatory $\mathrm{T}$ cell development (Lal et al., 2009). $\mathrm{T}$ cells differentiation is also regulated by STAT3-dependent histone trimethylation at target gene loci (Durant et al., 2010).

STAT proteins have also been implicated in epigenetic switches involving somatic cell and metabolic reprogramming, inflammation, and transformation. JAK/STAT3 activity plays a fundamental role in facilitating DNA demethylation/de novo methylation to complete reprogramming of pre-iPSC (Tang et al., 2012). Both STAT3 and STAT5 mediate trans-activation and epigenetic remodeling of IL-10 through their interaction with the histone acetyltransferase p300 in lupus T cells (Hedrich et al., 2014). Furthermore, STAT proteins can recruit and form a repressor complex with either the histone methyltransferases, or with $\mathrm{NCoR}$ associated with histone deacetylases, repressing the transcription of genes or microRNA promoters (Nakajima et al., 2001; Litterst et al., 2003; Wang et al., 2004; Mandal et al., 2011; Chang et al., 2015).

To date, no evidence about the epigenetic control of IL-6 pathway has been reported in skeletal muscle. Identification of the epigenetic mechanisms regulating IL-6 gene expression, or the expression of the IL-6 pathway downstream effectors, as well as STAT protein interaction studies in specific muscle cell types or during muscle differentiation and their effects on muscle cell biology remain puzzling.

\section{CONCLUSION AND PERSPECTIVES}

The IL-6/JAK/STAT signaling cascade plays a dominant role in skeletal muscle pathophysiology. IL-6 autocrine, paracrine, and endocrine functions assign to its downstream effectors pivotal importance in skeletal muscle-wasting-associated diseases and other multiple system diseases where muscle acts in communication with other organs. Targeting the components of the JAK/STAT pathway recently emerged as a strategic approach for the treatment of inflammatory diseases and human cancer.

This review highlights the opposite outcomes on muscle biology caused by the amount of local and systemic release of IL-6. Transient release and short-term acute action have positive effects, by increasing the source of progenitors for regeneration and growth in skeletal muscle. This also affects metabolic processes in other organs, since it stimulates glucose production. In different circumstances, chronically elevated levels of IL-6 have negative consequences, promoting muscle atrophy through different mechanisms not completely yet elucidated. These antithetical effects can also be a key to the several discrepancies observed with different experimental approaches aimed to decipher the IL-6/JAK/STAT role in skeletal muscle functions. Moreover, the different cell and tissue compartments where IL-6 is produced and acts can account for the conflicting effects observed on muscle repair, growth, and wasting. Additionally, a role in these dichotomous outcomes can also be carried out by the combined action of the IL- 6 trans-signaling, which is pro-inflammatory and the classic IL-6 signaling via 

A Histone octamer
- Methyated cytosines
Acetyl group
RNA polymerase
$\boldsymbol{\Delta}$ Methyl group (H3K79, H3K4)
$\triangle$ Methyl group (H3K9, H3K27)
$i^{\text {Acetylated Histone tail }}$
P Transcription

Transcriptionally Active Chromatin
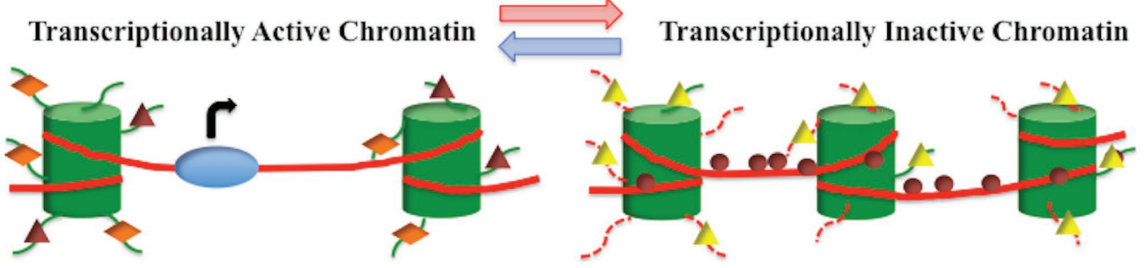

B

\begin{tabular}{c|l|l|l} 
Target gene & \multicolumn{1}{|c|}{ Epigenetic modification } & \multicolumn{1}{c|}{ Cell, Tissue } & \multicolumn{1}{c}{ Ref } \\
\hline IL-6 & Histone Deacetylation (HDAC2) & myeloid cells & Zhang et al. 2015 \\
\hline IL-6 & Histone Deacetylation & macrophages & Hu et al. 2016 \\
\hline IL-6 & DNA Methylation & $\begin{array}{l}\text { human buccal } \\
\text { swab cells }\end{array}$ & Ryan et al. 2017 \\
\hline IL-6 & Histone Methylation (EZH2) & $\begin{array}{l}\text { MCF-7 breast cancer cell } \\
\text { line }\end{array}$ & Lee et al. 2011 \\
\hline IL-6 & Histone Methylation (EZH2) & $\begin{array}{l}\text { human non- small cell } \\
\text { lung } \\
\text { cancer (NSCLC) }\end{array}$ & Serresi et al. 2016 \\
\hline JAK-STAT & DNA Methylation & hESCs & Liu et al. 2017 \\
\hline SOCS3 & Histone Methylation (KMT2D) & human B cells & $\begin{array}{l}\text { Ortega-Molina et al. } \\
\text { 2015 }\end{array}$ \\
\hline IL-6 & Histone Acetylation & macrophages & Hu et al. 2016 \\
\hline IL-6 & Histone Methylation (Dot11) & macrophages & Chen et al. 2018 \\
\hline
\end{tabular}

C

\begin{tabular}{c|l|l|l} 
Target gene & \multicolumn{1}{|c|}{ Epigenetic modification } & \multicolumn{1}{|c|}{ Cell, Tissue } & \multicolumn{1}{c}{ Ref } \\
\hline miR142-3p & DNA Methylation (DNMT1) & glyoblastoma cells & Chiou et al. 2013 \\
\hline p53 & DNA Methylation (DNMT1) & $\begin{array}{l}\text { human myeloma cell line } \\
\text { (KAS) }\end{array}$ & Gasche et al. 2011 \\
\hline Nanog, Oct4 & DNA Mehylation (DNMT1) & $\begin{array}{l}\text { induced pluripotent stem } \\
\text { cells (iPSCs) }\end{array}$ & Tang et al. 2012 \\
\hline miR-200c & Histone methylation (G9a) & $\begin{array}{l}\text { cancer stem-like cells } \\
\text { (CSC) }\end{array}$ & Chang et al. 2015 \\
\hline Stat3 & $\begin{array}{l}\text { Histone deacetylation (repressor } \\
\text { complex NCoR/SMRT) }\end{array}$ & $\begin{array}{l}\text { human myeloma cells } \\
\text { (MM) }\end{array}$ & Wang et al. 2004 \\
\hline igK & Histone methylation (Ezh2) & pro-B cells & Mandal et al. 2011 \\
\hline II17a, II17f, II21, Il6r & Histone methylation & T cells & Durant et al. 2010 \\
\hline b-casein & Histone Acetylation (p300/CPB) & $\begin{array}{l}\text { Systemic lupus } \\
\text { erythematosus (SLE) } \\
\text { T cells }\end{array}$ & Hedrich et al. 2014 \\
\hline H300) & Hela cells & Litterst et al. 2003 \\
\hline
\end{tabular}

FIGURE 2 | (A) DNA methylation and histone modifications are involved in epigenetic modulations of IL-6/JAK/STAT pathway members. They induce chromatin conformational transitions, altering accessibility of the transcriptional machinery (transcriptional active chromatin - blue arrow; transcriptional inactive chromatin - red arrow). DNA methylation is a process by which methyl groups are added to the cytosine of the DNA molecule and acts to repress gene transcription. Histone acetylation transfers acetyl groups to the histones and increases gene expression. Histone deacetylation removes acetyl groups from histones, allowing the histone to wrap more tightly the DNA and preventing transcription. Histone methylation adds methyl groups to the amino acids of the histones. Methylation of histones can either increase (i.e., H3K79, H3K4) or decrease (i.e. H3K9, H3K27) gene transcription. (B) Epigenetic modifications of IL-6/JAK/STAT pathway member genes that lead to gene repression (red) or gene activation (blue). (C) Epigenetic switches involving IL-6/JAK/STAT pathway members that lead to gene repression (red) or gene activation (blue) in tumorigenesis and development. 
the membrane bound IL-6R, which instead is needed for regenerative or anti-inflammatory activities of the cytokine (Scheller et al., 2011; Rose-John, 2012; Belizário et al., 2016).

Development of specific inhibitors or neutralizing antibodies against IL-6/JAK/STAT pathway factors may be proposed for diseases that cause muscle wasting, including DMD, cancer cachexia, and diabetes. Indeed, many studies demonstrated that they could ameliorate muscle wasting in mice (Zhang et al., 2013; Pretto et al., 2015; Silva et al., 2015). Nevertheless, they can act by nonspecific mechanisms and on cells and tissues other than myofibers.

In the light of this evidence, any therapeutic approach for skeletal muscle-wasting diseases targeting IL-6/JAK/STAT pathway should ideally consider the rate and the site of IL-6 production, in order to promote the benefits and avoid the detrimental effects.

Future studies on the mechanisms of action underlying the IL-6/JAK/STAT signaling cascade will provide new insights to tailor therapeutic strategies for each physiopathological condition. Further investigation of epigenetic mechanisms regulating and

\section{REFERENCES}

Aaronson, D. S., and Horvath, C. M. (2002). A road map for those who know JAK-STAT. Science 296, 1653-1655. doi: 10.1126/science.1071545

Al-Khalili, L., Bouzakri, K., Glund, S., Lönnqvist, F., Koistinen, H. A., and Krook, A. (2006). Signaling specificity of interleukin-6 action on glucose and lipid metabolism in skeletal muscle. Mol. Endocrinol. 20, 3364-3375. doi: 10.1210/me.2005-0490

Amorese, A. J., and Spangenburg, E. E. (2017). Defining the status quo in muscle hypertrophy. Focus on "overload-mediated skeletal muscle hypertrophy is not impaired by loss of myofiber STAT3". Am. J. Physiol. Cell Physiol. 313, C255-C256. doi: 10.1152/ajpcell.00165.2017

Argiles, J. M., Busquets, S., Stemmler, B., and López-Soriano, F. J. (2014). Cancer cachexia: understanding the molecular basis. Nat. Rev. Cancer 14, 754-762. doi: 10.1038/nrc3829

Baltgalvis, K. A., Berger, F. G., Pena, M. M., Davis, J. M., Muga, S. J., and Carson, J. A. (2008). Interleukin-6 and cachexia in ApcMin/+ mice. Am. J. Physiol. Regul. Integr. Comp. Physiol. 294, R393-R401. doi: 10.1152/ ajpregu.00716.2007

Baltgalvis, K. A., Berger, F. G., Pena, M. M., Davis, J. M., White, J. P., and Carson, J. A. (2009). Muscle wasting and interleukin-6-induced atrogin-I expression in the cachectic Apc Min/+ mouse. Pflugers Arch. 457, 989-1001. doi: 10.1007/s00424-008-0574-6

Begue, G., Douillard, A., Galbes, O., Rossano, B., Vernus, B., Candau, R., et al. (2013). Early activation of rat skeletal muscle IL-6/STAT1/STAT3 dependent gene expression in resistance exercise linked to hypertrophy. PLoS One 8:e57141. doi: 10.1371/journal.pone.0057141

Belizário, J. E., Fontes-Oliveira, C. C., Borges, J. P., Kashiabara, J. A., and Vannier, E. (2016). Skeletal muscle wasting and renewal: a pivotal role of myokine IL-6. Springerplus 5:619. doi: 10.1186/s40064-016-2197-2

Bentzinger, C. F., Wang, Y. X., Dumont, N. A., and Rudnicki, M. A. (2013). Cellular dynamics in the muscle satellite cell niche. EMBO Rep. 14, 1062-1072. doi: 10.1038/embor.2013.182

Bonetto, A., Aydogdu, T., Jin, X., Zhang, Z., Zhan, R., Puzis, L., et al. (2012). Jak/STAT3 pathway inhibition blocks skeletal muscle wasting downstream of IL-6 and in experimental cancer cachexia. Am. J. Physiol. Endocrinol. Metab. 303, E410-E421. doi: 10.1152/ajpendo.00039.2012

Bonetto, A., Aydogdu, T., Kunzevitzky, N., Guttridge, D. C., Khuri, S., Koniaris, L. G., et al. (2011). STAT3 activation in skeletal muscle links muscle wasting and the acute phase response in cancer cachexia. PLoS One 6:e22538. doi: 10.1371/journal.pone.0022538 involving IL-6/JAK/STAT signaling cascade may identify epigenetics modification of IL- 6 and its effectors as biomarkers of several diseases. Moreover, the IL-6/JAK/STAT molecular factors may represent new targets of the evolving epigenetics therapies directed to systemic pathologies and neuromuscular diseases, where combinations of epigenetic modulators may provide a tool to discriminate among alternative therapeutic effects.

\section{AUTHOR CONTRIBUTIONS}

All authors listed have made a substantial, direct and intellectual contribution to the work, and approved it for publication.

\section{FUNDING}

VM and SA are supported by Sapienza University research grants. LB is supported by the French Muscular Dystrophy Association (AFM-Telethon).

Bousoik, E., and Montazeri Aliabadi, H. (2018). "Do we know Jack" About JAK? A closer look at JAK/STAT signaling pathway. Front. Oncol. 8:287. doi: $10.3389 /$ fonc. 2018.00287

Bouzakri, K., Plomgaard, P., Berney, T., Donath, M. Y., Pedersen, B. K., and Halban, P. A. (2011). Bimodal effect on pancreatic beta-cells of secretory products from normal or insulin-resistant human skeletal muscle. Diabetes 60, 1111-1121. doi: 10.2337/db10-1178

Braconi, C., Huang, N., and Patel, T. (2010). MicroRNA dependent regulation of DNMT-1 and tumor suppressor gene expression by interleukin-6 in human malignant cholangiocytes. Hepatology 51, 881-890. doi: 10.1002/ hep. 23381

Chang, C. C., Wu, M. J., Yang, J. Y., Camarillo, I. G., and Chang, C. J. (2015). Leptin-STAT3-G9a signaling promotes obesity-mediated breast cancer progression. Cancer Res. 75, 2375-2386. doi: 10.1158/0008-5472. CAN-14-3076

Chen, X., Liu, X., Zhang, Y., Huai, W., Zhou, Q., Xu, S., et al. (2018). Methyltransferase Dot1l preferentially promotes innate IL-6 and IFN- $\beta$ production by mediating $\mathrm{H} 3 \mathrm{~K} 79 \mathrm{me} 2 / 3$ methylation in macrophages. Cell. Mol. Immunol. doi: 10.1038/s41423-018-0170-4

Chiou, G. Y., Chien, C. S., Wang, M. L., Chen, M. T., Yang, Y. P., Yu, Y. L., et al. (2013). Epigenetic regulation of the miR142-3p/interleukin-6 circuit in glioblastoma. Mol. Cell 52, 693-706. doi: 10.1016/j. molcel.2013.11.009

Darnell, J. E. Jr., Kerr, I. M., and Stark, G. R. (1994). Jak-STAT pathways and transcriptional activation in response to IFNs and other extracellular signaling proteins. Science 264, 1415-1421. doi: 10.1126/science.8197455

De Benedetti, F., Rucci, N., Del Fattore, A., Peruzzi, B., Paro, R., Longo, M., et al. (2006). Impaired skeletal development in interleukin-6-transgenic mice: a model for the impact of chronic inflammation on the growing skeletal system. Arthritis Rheum. 54, 3551-3563. doi: 10.1002/art.22175

Durant, L., Watford, W. T., Ramos, H. L., Laurence, A., Vahedi, G., Wei, L., et al. (2010). Diverse targets of the transcription factor STAT3 contribute to $\mathrm{T}$ cell pathogenicity and homeostasis. Immunity $32,605-615$. doi: 10.1016/j. immuni.2010.05.003

Eder, K., Baffy, N., Falus, A., and Fulop, A. K. (2009). The major inflammatory mediator interleukin-6 and obesity. Inflamm. Res. 58, 727-736. doi: 10.1007/ s00011-009-0060-4

Ellingsgaard, H., Hauselmann, I., Schuler, B., Habib, A. M., Baggio, L. L., Meier, D., et al. (2015). Interleukin-6 enhances insulin secretion by increasing glucagon-like peptide-1 secretion from L cells and alpha cells. Nat. Med. 17, 1481-1489. doi: 10.1038/nm.2513 
Fearon, K. C. H., Glass, D. J., and Guttridge, D. C. (2012). Cancer cachexia: mediators, signaling, and metabolic pathways. Cell Metab. 16, 153-166. doi: 10.1016/j.cmet.2012.06.011

Febbraio, M. A., Hiscock, N., Sacchetti, M., Fischer, C. P., and Pedersen, B. K. (2004). Interleukin 6 is a novel factor mediating glucose homeostasis during skeletal muscle contraction. Diabetes 53, 1643-1648. doi: 10.2337/diabetes.53.7.1643

Gasche, J. A., Hoffmann, J., Boland, C. R., and Goel, A. (2011). Interleukin-6 promotes tumorigenesis by altering DNA methylation in oral cancer cells. Int. J. Cancer 129, 1053-1063. doi: 10.1002/ijc.25764

Gilabert, M., Calvo, E., Airoldi, A., Hamidi, T., Moutardier, V., Turrini, O., et al. (2014). Pancreatic cancer-induced cachexia is Jak2-dependent in mice. J. Cell. Physiol. 229, 1437-1443. doi: 10.1002/jcp.24580

Goldstein, M. S. (1961). Humoral nature of the hypoglycemic factor of muscular work. Diabetes 10, 232-234. doi: 10.2337/diab.10.3.232

Greenhalgh, C. J., and Hilton, D. J. (2001). Negative regulation of cytokine signaling. J. Leukoc. Biol. 70, 348-356.

Guo, B., Zhang, Z. K., Liang, C., Li, J., Liu, J., Lu, A., et al. (2017). Molecular communication from skeletal muscle to bone: a review for muscle-derived myokines regulating bone metabolism. Calcif. Tissue Int. 100, 184-192. doi: 10.1007/s00223-016-0209-4

Gurney, M. E., Pu, H., Chiu, A. Y., Dal Canto, M. C., Polchow, C. Y., Alexander, D. D., et al. (1994). Motor neuron degeneration in mice that express a human $\mathrm{Cu}, \mathrm{Zn}$ superoxide dismutase mutation. Science 264, 1772-1775. doi: 10.1126/science.8209258

Haddad, F., Zaldivar, F., Cooper, D. M., and Adams, G. R. (2005). IL-6-induced skeletal muscle atrophy. J. Appl. Physiol. 98, 911-917. doi: 10.1152/ japplphysiol.01026.2004

He, W. A., Berardi, E., Cardillo, V. M., Acharyya, S., Aulino, P., Thomas-Ahner, J., et al. (2013). Nf-Kappab-mediated Pax7 dysregulation in the muscle microenvironment promotes cancer cachexia. J. Clin. Investig. 123, 4821-4835. doi: 10.1172/JCI68523

Hedrich, C. M., Rauen, T., Apostolidis, S. A., Grammatikos, A. P., Rodriguez Rodriguez, N., Ioannidis, C., et al. (2014). Stat3 promotes IL-10 expression in lupus $\mathrm{T}$ cells through trans-activation and chromatin remodeling. Proc. Natl. Acad. Sci. USA 111, 13457-13462. doi: 10.1073/pnas.1408023111

Heinrich, P. C., Behrmann, I., Müller-Newen, G., Schaper, F., and Graeve, L. (1998). Interleukin-6-type cytokine signalling through the gp130/Jak/STAT pathway. Biochem. J. 334, 297-314. doi: 10.1042/bj3340297

Hodge, D. R., Peng, B., Cherry, J. C., Hurt, E. M., Fox, S. D., Kelley, J. A., et al. (2005). Interleukin 6 supports the maintenance of p53 tumor suppressor gene promoter methylation. Cancer Res. 65, 4673-4682. doi: 10.1158/0008-5472. CAN-04-3589

Hoffmann, C., and Weigert, C. (2017). Skeletal muscle as an endocrine organ: the role of myokines in exercise adaptations. Cold Spring Harb. Perspect. Med. 7, pii:a029793. doi: 10.1101/cshperspect.a029793

Hu, L., Yu, Y., Huang, H., Fan, H., Hu, L., Yin, C., et al. (2016). Epigenetic regulation of interleukin 6 by histone acetylation in macrophages and its role in paraquat-induced pulmonary fibrosis. Front. Immunol. 7:696. doi: 10.3389/fimmu.2016.00696

Hutchins, A. P., Diez, D., Takahashi, Y., Ahmad, S., Jauch, R., Tremblay, M. L., et al. (2013). Distinct transcriptional regulatory modules underlie STAT3's cell type-independent and cell type-specific functions. Nucleic Acids Res. 41, 2155-2170. doi: 10.1093/nar/gks1300

Jang, Y. -N., and Baik, E. J. (2013). JAK-STAT pathway and myogenic differentiation. JAK-STAT 2:e23282. doi: 10.4161/jkst.23282

Joe, A. W. B., Yi, L., Natarajan, A., Le Grand, F., So, L., Wang, J., et al. (2010). Muscle injury activates resident fibro/adipogenic progenitors that facilitate myogenesis. Nat. Cell Biol. 12, 153-163. doi: 10.1038/ncb2015

Judge, S. M., Wu, C. L., Beharry, A. W., Roberts, B. M., Ferreira, L. F., Kandarian, S. C., et al. (2014). Genome-wide identification of FoxO-dependent gene networks in skeletal muscle during C26 cancer cachexia. BMC Cancer 14:997. doi: 10.1186/1471-2407-14-997

Kallen, K. J. (2002). The role of transsignalling via the agonistic soluble IL-6 receptor in human diseases. Biochim. Biophys. Acta 1592, 323-343.

Keller, C., Steensberg, A., Pilegaard, H., Osada, T., Saltin, B., Pedersen, B. K., et al. (2001). Transcriptional activation of the IL-6 gene in human contracting skeletal muscle: influence of muscle glycogen content. FASEB J. 15, 2748-2750. doi: 10.1096/fj.01-0507fje
Kisseleva, T., Bhattacharya, S., Braunstein, J., and Schindler, C. W. (2002). Signaling through the JAK/STAT pathway, recent advances and future challenges. Gene 285, 1-24. doi: 10.1016/S0378-1119(02)00398-0

Lal, G., Zhang, N., van der Touw, W., Ding, Y., Ju, W., Bottinger, E. P., et al (2009). Epigenetic regulation of Foxp3 expression in regulatory $\mathrm{T}$ cells by DNA methylation. J. Immunol. 182, 259-273.

Lee, S. T., Li, Z., Wu, Z., Aau, M., Guan, P., Karuturi, R. K., et al. (2011). Context-specific regulation of NF- $\mathrm{KB}$ target gene expression by EZH2 in breast cancers. Mol. Cell 43, 798-810. doi: 10.1016/j.molcel.2011.08.011

Linossi, E. M., Babon, J. J., Hilton, D. J., and Nicholson, S. E. (2013). Suppression of cytokine signaling: the SOCS perspective. Cytokine Growth Factor Rev. 24, 241-248. doi: 10.1016/j.cytogfr.2013.03.005

Litterst, C. M., Kliem, S., Marilley, D., and Pfitzner, E. (2003). NCoA-1/SRC-1 is an essential coactivator of STAT5 that binds to the FDL motif in the alpha-helical region of the STAT5 transactivation domain. J. Biol. Chem. 278, 45340-45351. doi: 10.1074/jbc.M303644200

Liu, Z., Feng, Q., Sun, P., Lu, Y., Yang, M., Zhang, X., et al. (2017). Genomewide DNA methylation drives human embryonic stem cell erythropoiesis by remodeling gene expression dynamics. Epigenomics 9, 1543-1558. doi: 10.2217/epi-2017-0039

Macpherson, R. E., Huber, J. S., Frendo-Cumbo, S., Simpson, J. A., and Wright, D. C. (2015). Adipose tissue insulin action and IL-6 signaling after exercise in obese mice. Med. Sci. Sports Exerc. 47, 2034-2042. doi: 10.1249/ MSS.0000000000000660

Madaro, L., Passafaro, M., Sala, D., Etxaniz, U., Lugarini, F., Proietti, D., et al. (2018). Denervation-activated STAT3-IL-6 signalling in fibro-adipogenic progenitors promotes myofibres atrophy and fibrosis. Nat. Cell Biol. 20, 917-927. doi: 10.1038/s41556-018-0151-y

Mandal, M., Powers, S. E., Maienschein-Cline, M., Bartom, E. T., Hamel, K. M., Kee, B. L., et al. (2011). Epigenetic repression of the Igk locus by STAT5mediated Ezh2 recruitment. Nat. Immunol. 12, 1212-1220. doi: 10.1038/ ni.2136

Marazzi, G., and Sassoon, D. (2018). FAPs are sensors for skeletal myofibre atrophy. Nat. Cell Biol. 20, 864-865. doi: 10.1038/s41556-018-0149-5

Miller, A., McLeod, L., Alhayyani, S., Szczepny, A., Watkins, D. N., Chen, W., et al. (2017). Blockade of the IL-6 trans-signalling/STAT3 axis suppresses cachexia in Kras-induced lung adenocarcinoma. Oncogene 36, 3059-3066. doi: 10.1038/onc.2016.437

Muñoz-Cánoves, P., Scheele, C., Pedersen, B. K., and Serrano, A. L. (2013). Interleukin-6 myokine signaling in skeletal muscle: a double-edged sword? FEBS J. 280, 4131-4148. doi: 10.1111/febs.12338

Nakajima, H., Brindle, P. K., Handa, M., and Ihle, J. N. (2001). Functional interaction of STAT5 and nuclear receptor co-repressor SMRT: implications in negative regulation of STAT5-dependent transcription. EMBO J. 20, 6836-6844. doi: $10.1093 / \mathrm{emboj} / 20.23 .6836$

O'Shea, J. J., Gadina, M., and Schreiber, R. D. (2002). Cytokine signaling in 2002: new surprises in the Jak/Stat pathway. Cell 109 (Suppl.), S121-S131.

O'Shea, J. J., and Plenge, R. (2012). JAK and STAT signaling molecules in immunoregulation and immune-mediated disease. Immunity 36, 542-550. doi: 10.1016/j.immuni.2012.03.014

O'Shea, J. J., Schwartz, D. M., Villarino, A. V., Gadina, M., McInnes, I. B., and Laurence, A. (2015). The JAK-STAT pathway: impact on human disease and therapeutic intervention. Annu. Rev. Med. 66, 311-328. doi: 10.1146/ annurev-med-051113-024537

Oldenburg, H. S., Rogy, M. A., Lazarus, D. D., Van Zee, K. J., Keeler, B. P., Chizzonite, R. A., et al. (1993). Cachexia and the acute-phase protein response in inflammation are regulated by interleukin-6. Eur. J. Immunol. 23, 1889-1894. doi: 10.1002/eji.1830230824

Ortega-Molina, A., Boss, I. W., Canela, A., Pan, H., Jiang, Y., Zhao, C., et al. (2015). The histone lysine methyltransferase KMT2D sustains a gene expression program that represses B cell lymphoma development. Nat. Med. 21, 1199-1208. doi: $10.1038 / \mathrm{nm} .3943$

Pedersen, B. K., and Febbraio, M. A. (2008). Muscle as an endocrine organ: focus on muscle-derived interleukin-6. Physiol. Rev. 88, 1379-1406. doi: 10.1152/physrev.90100.2007

Pedersen, B. K., Steensberg, A., Fischer, C., Keller, C., Keller, P., Plomgaard, P., et al. (2003). Searching for the exercise factor: is IL-6 a candidate? J. Muscle Res. Cell Motil. 23, 113-119. 
Pelosi, L., Berardinelli, M. G., Forcina, L., Spelta, E., Rizzuto, E., Nicoletti, C., et al. (2015). Increased levels of interleukin-6 exacerbate the dystrophic phenotype in mdx mice. Hum. Mol. Genet. 24, 6041-6053. doi: 10.1093/ hmg/ddv323

Perez-Schindler, J., Esparza, M. C., McKendry, J., Breen, L., Philp, A., and Schenk, S. (2017). Overload-mediated skeletal muscle hypertrophy is not impaired by loss of myofiber STAT3. Am. J. Physiol. Cell Physiol. 313, C257-C261. doi: 10.1152/ajpcell.00100.2017

Pettersen, K., Andersen, S., Degen, S., Tadini, V., Grosjean, J., Hatakeyama, S., et al. (2017). Cancer cachexia associates with a systemic autophagy-inducing activity mimicked by cancer cell-derived IL-6 trans-signaling. Sci. Rep. 7:2046. doi: 10.1038/s41598-017-02088-2

Pigna, E., Berardi, E., Aulino, P., Rizzuto, E., Zampieri, S., Carraro, U., et al. (2016). Aerobic exercise and pharmacological treatments counteract cachexia by modulating autophagy in colon cancer. Sci. Rep. 6:26991. doi: 10.1038/ srep26991

Plomgaard, P., Halban, P. A., and Bouzakri, K. (2012). Bimodal impact of skeletal muscle on pancreatic $\beta$-cell function in health and disease. Diabetes Obes. Metab. 14, 78-84. doi: 10.1111/j.1463-1326.2012.01641.x

Pretto, F., Ghilardi, C., Moschetta, M., Bassi, A., Rovida, A., Scarlato, V., et al. (2015). Sunitinib prevents cachexia and prolongs survival of mice bearing renal cancer by restraining STAT3 and MuRF-1 activation in muscle. Oncotarget 6, 3043-3054. doi: 10.18632/oncotarget.2812

Price, F. D., von Maltzahn, J., Bentzinger, C. F., Dumont, N. A., Yin, H., Chang, N. C., et al. (2014). Inhibition of JAK-STAT signaling stimulates adult satellite cell function. Nat. Med. 20, 1174-1181. doi: 10.1038/nm.3655

Rawlings, J. S., Rosler, K. M., and Harrison, D. A. (2004). The JAK/STAT signaling pathway. J. Cell Sci. 117, 1281-1283. doi: 10.1242/jcs.00963

Rose-John, S. (2012). IL-6 trans-signaling via the soluble IL-6 receptor: importance for the pro-inflammatory activities of IL-6. Int. J. Biol. Sci. 8, 1237-1247. doi: $10.7150 /$ ijbs. 4989

Rufo, A., Del Fattore, A., Capulli, M., Carvello, F., De Pasquale, L., Ferrari, S., et al. (2011). Mechanisms inducing low bone density in Duchenne muscular dystrophy in mice and humans. J. Bone Miner. Res. 26, 1891-1903. doi: $10.1002 / \mathrm{jbmr} .410$

Ryan, J., Pilkington, L., Neuhaus, K., Ritchie, K., Ancelin, M. L., and Saffery, R. (2017). Investigating the epigenetic profile of the inflammatory gene IL-6 in late-life depression. BMC Psychiatry 17:354. doi: 10.1186/s12888-017-1515-8

Sala, D., and Sacco, A. (2016). STAT3 signaling as a potential target to treat muscle-wasting diseases. Curr. Opin. Clin. Nutr. Metab. Care 19, 171-176. doi: 10.1097/MCO.0000000000000273

Scheller, J., Chalaris, A., Schmidt-Arras, D., and Rose-John, S. (2011). The pro- and anti-inflammatory properties of the cytokine interleukin-6. Biochim. Biophys. Acta, Mol. Cell Res. 1813, 878-888. doi: 10.1016/j.bbamcr.2011.01.034

Scheller, J., Ohnesorge, N., and Rose-John, S. (2006). Interleukin-6 trans-signalling in chronic inflammation and cancer. Scand. J. Immunol. 63, 321-329. doi: 10.1111/j.1365-3083.2006.01750.x

Schindler, C., Shuai, K., Prezioso, V. R., and Darnell, J. E. Jr. (1992). Interferondependent tyrosine phosphorylation of a latent cytoplasmic transcription factor. Science 257, 809-813. doi: 10.1126/science.1496401

Serrano, A. L., Baeza-Raja, B., Perdiguero, E., Jardí, M., and Muñoz-Cánoves, P. (2008). Interleukin-6 is an essential regulator of satellite cell-mediated skeletal muscle hypertrophy. Cell Metab. 7, 33-44. doi: 10.1016/j.cmet.2007.11.011

Serresi, M., Gargiulo, G., Proost, N., Siteur, B., Cesaroni, M., Koppens, M., et al. (2016). Polycomb repressive complex 2 is a barrier to KRAS-driven inflammation and epithelial-mesenchymal transition in non-small-cell lung cancer. Cancer Cell 29, 17-31. doi: 10.1016/j.ccell.2015.12.006

Shabbir, A., Zisa, D., Lin, H., Mastri, M., Roloff, G., Suzuki, G., et al. (2010). Activation of host tissue trophic factors through JAK-STAT3 signaling: a mechanism of mesenchymal stem cell-mediated cardiac repair. Am. J. Physiol. Heart Circ. Physiol. 299, H1428-H1438. doi: 10.1152/ajpheart.00488.2010

Silva, K. A., Dong, J., Dong, Y., Dong, Y., Schor, N., Tweardy, D. J., et al. (2015). Inhibition of STAT3 activation suppresses caspase-3 and the ubiquitinproteasome system, leading to preservation of muscle mass in cancer cachexia. J. Biol. Chem. 290, 11177-11187. doi: 10.1074/jbc.M115.641514

Strassmann, G., Fong, M., Kenney, J. S., and Jacob, C. O. (1992). Evidence for the involvement of interleukin 6 in experimental cancer cachexia. J. Clin. Invest. 89, 1681-1684. doi: 10.1172/JCI115767
Sun, L., Ma, K., Wang, H., Xiao, F., Gao, Y., Zhang, W., et al. (2007). JAK1STAT1-STAT3, a key pathway promoting proliferation and preventing premature differentiation of myoblasts. J. Cell Biol. 179, 129-138. doi: 10.1083/ jcb.200703184

Takeuchi, A., Nishioka, C., Ikezoe, T., Yang, J., and Yokoyama, A. (2015). STAT5A regulates DNMT3A in CD34(+)/CD38(-) AML cells. Leuk. Res. 39, 897-905. doi: 10.1016/j.leukres.2015.05.006

Tang, Y., Luo, Y., Jiang, Z., Ma, Y., Lin, C. J., Kim, C., et al. (2012). Jak/Stat3 signaling promotes somatic cell reprogramming by epigenetic regulation. Stem Cells 30, 2645-2656. doi: 10.1002/stem.1225

Tierney, M. T., Aydogdu, T., Sala, D., Malecova, B., Gatto, S., Puri, P. L., et al. (2014). STAT3 signaling controls satellite cell expansion and skeletal muscle repair. Nat. Med. 20, 1182-1186. doi: 10.1038/nm.3656

Toth, K. G., McKay, B. R., De Lisio, M., Little, J. P., Tarnopolsky, M. A., and Parise, G. (2011). IL-6 induced STAT3 signalling is associated with the proliferation of human muscle satellite cells following acute muscle damage. PLoS One 6:e17392. doi: 10.1371/journal.pone.0017392

Trenerry, M. K., Carey, K. A., Ward, A. C., and Cameron-Smith, D. (2007). STAT3 signaling is activated in human skeletal muscle following acute resistance exercise. J. Appl. Physiol. 102, 1483-1489. doi: 10.1152/ japplphysiol.01147.2006

Trenerry, M. K., Della Gatta, P. A., Larsen, A. E., Garnham, A. P., and Cameron-Smith, D. (2011). Impact of resistance exercise training on interleukin-6 and JAK/STAT in young men. Muscle Nerve 43, 385-392. doi: 10.1002/mus. 21875

Tsujinaka, T., Fujita, J., Ebisui, C., Yano, M., Kominami, E., Suzuki, K., et al. (1996). Interleukin 6 receptor antibody inhibits muscle atrophy and modulates proteolytic systems in interleukin 6 transgenic mice. J. Clin. Invest. 97, 244-249. doi: 10.1172/JCI118398

van de Vyver, M., Engelbrecht, L., Smith, C., and Myburgh, K. H. (2016). Neutrophil and monocyte responses to downhill running: intracellular contents of MPO, IL-6, IL-10, pstat3, and SOCS3. Scand. J. Med. Sci. Sports 26, 638-647. doi: 10.1111/sms.12497

Wada, E., Tanihata, J., Iwamura, A., Takeda, S., Hayashi, Y. K., and Matsuda, R. (2017). Treatment with the anti-IL-6 receptor antibody attenuates muscular dystrophy via promoting skeletal muscle regeneration in dystrophin-/utrophin-deficient mice. Skelet. Muscle 7:23. doi: 10.1186/ s13395-017-0140-z

Wang, K., Wang, C., Xiao, F., Wang, H., and Wu, Z. (2008). JAK2/STAT2/ STAT3 are required for myogenic differentiation. J. Biol. Chem. 283, 34029-34036. doi: 10.1074/jbc.M803012200

Wang, L. H., Yang, X. Y., Zhang, X., Huang, J., Hou, J., Li, J., et al. (2004). Transcriptional inactivation of STAT3 by PPARgamma suppresses IL-6responsive multiple myeloma cells. Immunity 20, 205-218. doi: 10.1016/ S1074-7613(04)00030-5

Whitham, M., and Febbraio, M. A. (2016). The ever-expanding myokinome: discovery challenges and therapeutic implications. Nat. Rev. Drug Discov. 15, 719-729. doi: 10.1038/nrd.2016.153

Yang, J., Huang, J., Dasgupta, M., Sears, N., Miyagi, M., Wang, B., et al. (2010). Reversible methylation of promoter-bound STAT3 by histone-modifying enzymes. Proc. Natl. Acad. Sci. USA 107, 21499-21504. doi: 10.1073/ pnas. 1016147107

Yu, H., Lee, H., Herrmann, A., Buettner, R., and Jove, R. (2014). Revisiting STAT3 signalling in cancer: new and unexpected biological functions. Nat. Rev. Cancer 14, 736-746. doi: 10.1038/nrc3818

Zhang, L., Pan, J., Dong, Y., Tweardy, D. J., Dong, Y., Garibotto, G., et al. (2013). Stat 3 activation links a C/EBP $\delta$ to myostatin pathway to stimulate loss of muscle mass. Cell Metab. 18, 368-379. doi: 10.1016/j. cmet.2013.07.012

Zhang, Q., Wang, H. Y., Marzec, M., Raghunath, P. N., Nagasawa, T., and Wasik, M. A. (2005). STAT3- and DNA methyltransferase 1-mediated epigenetic silencing of SHP-1 tyrosine phosphatase tumor suppressor gene in malignant T lymphocytes. Proc. Natl. Acad. Sci. USA 102, 6948-6953. doi: 10.1073/ pnas.0501959102

Zhang, Q., Wang, H. Y., Woetmann, A., Raghunath, P. N., Odum, N., and Wasik, M. A. (2006). STAT3 induces transcription of the DNA methyltransferase 1 gene (DNMT1) in malignant T lymphocytes. Blood 108, 1058-1064. doi: 10.1182/blood-2005-08-007377 
Zhang, Q., Zhao, K., Shen, Q., Han, Y., Gu, Y., Li, X., et al. (2015). Tet2 is required to resolve inflammation by recruiting Hdac2 to specifically repress IL-6. Nature 525, 389-393. doi: 10.1038/nature15252

Zhu, H., Xiao, F., Wang, G., Wei, X., Jiang, L., Chen, Y., et al. (2016). STAT3 regulates self-renewal of adult muscle satellite cells during injury-induced muscle regeneration. Cell Rep. 16, 2102-2115. doi: 10.1016/j.celrep.2016.07.041

Zimmers, T. A., Fishel, M. L., and Bonetto, A. (2016). STAT3 in the systemic inflammation of cancer cachexia. Semin. Cell Dev. Biol. 54, 28-41. doi: 10.1016/j.semcdb.2016.02.009

Zuang, S. (2013). Regulation of STAT signaling by acetylation. Cell. Signal. 25, 1924-1931. doi: 10.1016/j.cellsig.2013.05.007
Conflict of Interest Statement: The authors declare that the research was conducted in the absence of any commercial or financial relationships that could be construed as a potential conflict of interest.

Copyright (c) 2019 Moresi, Adamo and Berghella. This is an open-access article distributed under the terms of the Creative Commons Attribution License (CC BY). The use, distribution or reproduction in other forums is permitted, provided the original author(s) and the copyright owner(s) are credited and that the original publication in this journal is cited, in accordance with accepted academic practice. No use, distribution or reproduction is permitted which does not comply with these terms. 


\section{OPEN ACCESS}

Edited by:

Dario Coletti,

Sapienza University of Rome, Italy

Reviewed by:

Damien Vitiello,

Université Paris Descartes, France Nissrine Daou,

Pierre and Marie Curie University,

France

*Correspondence: Stéphanie Hody

shody@uliege.be

Specialty section: This article was submitted to

Striated Muscle Physiology, a section of the journal Frontiers in Physiology

Received: 10 December 2018 Accepted: 15 April 2019

Published: 03 May 2019

Citation:

Hody S, Croisier J-L, Bury T, Rogister B and Leprince P (2019) Eccentric Muscle Contractions: Risks and Benefits. Front. Physiol. 10:536.

doi: 10.3389/fphys.2019.00536

\section{Eccentric Muscle Contractions: Risks and Benefits}

\author{
Stéphanie Hody ${ }^{1 *}$, Jean-Louis Croisier ${ }^{1}$, Thierry Bury ${ }^{1}$, Bernard Rogister ${ }^{2,3,4}$ and \\ Pierre Leprince ${ }^{2,4}$
}

${ }^{1}$ Department of Motricity Sciences, University of Liège, Liege, Belgium, ${ }^{2}$ GIGA-Neurosciences, University of Liège, Liege, Belgium, ${ }^{3}$ Department of Neurology, The University Hospital Center, University of Liège, Liege, Belgium, ${ }^{4}$ GIGA - Laboratory of Nervous System Disorders and Therapy, University of Liège, Liege, Belgium

Eccentric contractions, characterized by the lengthening of the muscle-tendon complex, present several unique features compared with other types of contractions, which may lead to unique adaptations. Due to its specific physiological and mechanical properties, there is an increasing interest in employing eccentric muscle work for rehabilitation and clinical purposes. However, unaccustomed eccentric exercise is known to cause muscle damage and delayed pain, commonly defined as "Delayed-Onset Muscular Soreness" (DOMS). To date, the most useful preventive strategy to avoid these adverse effects consists of repeating sessions involving submaximal eccentric contractions whose intensity is progressively increased over the training. Despite an increased number of investigations focusing on the eccentric contraction, a significant gap still remains in our understanding of the cellular and molecular mechanisms underlying the initial damage response and subsequent adaptations to eccentric exercise. Yet, unraveling the molecular basis of exercise-related muscle damage and soreness might help uncover the mechanistic basis of pathological conditions as myalgia or neuromuscular diseases. In addition, a better insight into the mechanisms governing eccentric training adaptations should provide invaluable information for designing therapeutic interventions and identifying potential therapeutic targets.

Keywords: skeletal muscle, eccentric contraction, exercise-induced muscle damage (EIMD), delayed-onset muscle soreness (DOMS), eccentric muscle training

\section{INTRODUCTION}

An eccentric (lengthening) muscle contraction occurs when a force applied to the muscle exceeds the momentary force produced by the muscle itself, resulting in the forced lengthening of the muscle-tendon system while contracting (Lindstedt et al., 2001). During this process, the muscle absorbs energy developed by an external load, explaining why eccentric action is also called "negative work" as opposed to concentric (shortening) contraction or "positive work" (Abbott et al., 1952). Although not always obvious, eccentric muscle contractions are an integral part of most movements during daily or sport activities. Skeletal muscles contract eccentrically to support the weight of the body against gravity and to absorb shock or to store elastic recoil energy in preparation for concentric (or accelerating) contractions (LaStayo et al., 2003b). The slowing-down role of such 
contractions is classically illustrated by downhill running or walking down the stairs during which the eccentric work of the knee extensor muscles is accentuated (Gault and Willems, 2013).

Compared to concentric or isometric (constant length) contractions, eccentric muscle actions possess several unique features that may be responsible for unique adaptations (Guilhem et al., 2010; Duchateau and Baudry, 2014). Greater forces are generated during eccentric contraction compared to other contraction types for a given angular velocity (Hortobagyi and Katch, 1990). In addition, eccentric contractions require less motor unit activation and consume less oxygen and energy for a given muscle force than concentric contractions (Abbott et al., 1952). Indeed, the metabolic cost required for eccentric exercise is approximately fourfold lower than for the same exercise performed concentrically. Reduced cardiorespiratory and hemodynamic responses have been reported following eccentric exercise when compared to concentric exercise at the same absolute workload (Overend et al., 2000; Meyer et al., 2003). While many questions remains unanswered, it is well accepted that neural strategies controlling eccentric contractions considerably differ from concentric or isometric contractions (Duchateau and Baudry, 2014). Differences are detected on the level of the contracting muscle as well as on the cortical level. Most studies indicate a reduced central activation (evidenced by a lower EMG amplitude) during maximal eccentric contractions than maximal concentric or isometric contractions. This has implications on eccentric coordination: fine motor control in eccentrically biased actions appears more difficult as fewer motor-units are required for the same work (Hoppeler, 2016). The twitch interpolation technique also revealed a greater voluntary deficit in eccentric compared to concentric contractions, such that untrained individuals are usually unable to fully activate their muscles during maximal eccentric muscle contractions. Further characteristics of eccentric contraction are a greater cortical excitability but a lower motor units discharge. Collectively, the mechanisms underpinning the unique features of eccentric contraction are not well understood (Hoppeler and Herzog, 2014).

Due to its specific physiological and mechanical properties, the eccentric contraction has gained a growing interest in several fields. Besides its interest in sport training or in physical medicine and rehabilitation (Croisier et al., 2002; Kjaer and Heinemeier, 2014; Vogt and Hoppeler, 2014), evidence is accumulating regarding the benefits of eccentric exercise in special populations of aged individuals or patients with chronic health diseases such as neuromuscular pathologies (Roig et al., 2008; Gault and Willems, 2013; Isner-Horobeti et al., 2013; Hyldahl and Hubal, 2014). Indeed, the two main defining properties of eccentric contraction "highest forces and lower energy requirement" makes this contraction regime a judicious alternative to conventional muscle training. To date, it is well accepted that the benefits of eccentric exercise transcend improved muscle function, as this mode of training has been shown to induce a number of favorable repercussions on neural drive or health-related factors (Paschalis et al., 2010, 2013). For many years, eccentric regime has been largely used in sport training to improve maximal muscular strength, power as well as coordination during eccentric tasks. Robust evidence support its wide prescription in the sport rehabilitation field, notably in the treatment of tendinopathies (Croisier et al., 2007; Kaux et al., 2013). In addition, implementing eccentric exercise in athletes showed its effectiveness to prevent sport injuries such as hamstring strain (Croisier et al., 2002). While research has mainly focused on the functional outcomes following eccentric resistance training using high-loads, the potential of low/moderate load regimes received much attention over the last decade (LaStayo et al., 2014; Hoppeler, 2016). With the increasing consideration of the physical activity in numerous medical fields over the last decades, a novel training modality based on low to moderate load ECC exercise has emerged. This modality, referred as RENEW (Resistance Exercise via Eccentric Work) by LaStayo et al. (2014), appears to result in similar gains in muscle strength and volume as traditional strength training. Since eccentric modality provides a strong mechanical stress at a lower metabolic cost (Lastayo et al., 1999), it appears particularly suitable for training individuals with medical conditions associated to muscle wasting and reduction in muscle strength, mobility and aerobic capacity (Hoppeler, 2016). Eccentric training is increasingly proposed to patients with cardiorespiratory problems, sarcopenia of old age, cachexia, diabetes type 2, neurological and musculoskeletal diseases (Julian et al., 2018). Along with the positive effects on the muscle function, aerobic eccentric exercise induces specific effects on muscle energetic metabolism, insulin resistance and blood lipid profile, reducing disease risks. It is thus recognized as a promising lifestyle factor to combat obesity and dyslipidemias (Paschalis et al., 2010; Julian et al., 2018, 2019).

However, despite the above-mentioned advantages, the use of eccentric exercise in clinical conditions has been frequently the object of contrasting opinions, because of its potential undesirable associated effects. Indeed, eccentric exercise induces greater muscle damage and negative functional consequences in an healthy naïve muscle than other types of exercise (Friden and Lieber, 1992). Indeed, the combination of high force and reduced recruitment of fiber number during eccentric contractions causes a high mechanical stress on the involved structures that may lead to focal microlesions of the muscle fibers (Lieber and Friden, 1999). Numerous histological studies described widespread Z-line streaming with myofibrillar disruption and necrosis following intense and/or unaccustomed eccentric exercise (Friden and Lieber, 1998; Crameri et al., 2007; Lauritzen et al., 2009). The sarcomeric disorganization has been associated with disruptions to the sarcolemma and the extracellular matrix, swelling of mitochondria, dilation of the transverse tubule system and fragmentation of the sarcoplasmic reticulum (Takekura et al., 2001; Crameri et al., 2004). Sarcolemmal disruption may be highlighted by the appearance of sarcoplasmic proteins into the blood (as creatine kinase, CK and myoglobin, $\mathrm{Mb}$ ) or by the cytoplasmic accumulation of proteins that are normally not present in muscle fibers (as albumin and immunoglobulins) (McNeil and Khakee, 1992; Clarkson and Hubal, 2002). Vital dye such as Evans blue is also used in rodents to demonstrate increased sarcolemmal permeability (Hamer et al., 2002). Damage to extracellular matrix and connective tissue components also occur following a novel 
eccentric exercise (Brown et al., 1997; Crameri et al., 2007). Morphological abnormalities observed immediately after exercise gradually extent to a larger number of muscle fibers and appear exacerbated 2-3 days post-exercise (Friden et al., 1983a). These observations have led authors to define primary and secondary damage phases (Morgan and Allen, 1999). Both human and animal studies supported that Type II (in particular IIb) muscle fibers are more damaged after eccentric exercise than Type I fibers (Friden et al., 1983b; Jones et al., 1986; Lieber and Friden, 1988). Several hypotheses could explain the higher susceptibility of Type II fibers to exercise-induced muscle damage (EIMD). Among these are differences in their structural composition (Z-line, fiber type specific protein isoforms such as titin), a reduced oxidative capacity, a lower ability to regulate calcium homeostasis or a selective recruitment of fast-twitch muscle fibers during eccentric contraction (Lieber and Friden, 1999; McHugh et al., 1999a; Byrne et al., 2004).

The EIMD manifests itself by a range of clinical symptoms including delayed-onset muscle soreness (DOMS), stiffness, swelling and various functional deficits such as a loss in force generating capacity or decreased proprioceptive function (Clarkson, 1992). To avoid the invasive nature of muscle biopsies, these clinical manifestations as well as the plasma CK activity are frequently used to indirectly assess the presence of muscle damage (Warren et al., 1999; Clarkson and Hubal, 2002). The magnitude of changes in EIMD indirect markers (in particular, the plasma CK activity) shows a marked interindividual variability even when subjects are submitted to standardized eccentric protocols (Clarkson et al., 1992; Nosaka and Clarkson, 1996; Hody et al., 2013c). Multiple factors as muscle architecture, muscle typology, individual fitness, age, sex, and genetic variability may contribute to the wide inter-subject variability in the response to eccentric exercise (Vincent and Vincent, 1997; Clarkson and Hubal, 2002; Yamin et al., 2007; Hody et al., 2009; Hyldahl and Hubal, 2014). Even if DOMS and associated clinical symptoms spontaneously disappear after few days, these negative consequences can delay or disturb rehabilitation and/or training programs. Exercise is necessary to maintain a good health and to prevent physical inactivity-related diseases, but unpleasant sensations resulting from unaccustomed exercise can discourage people to continue physical activity. Moreover, due to the mechanical fragility, the risk of further injuries (e.g., muscle tears or ligament rupture) increases if intense physical activities are performed during a DOMS episode or the following days (Nicol et al., 2006). It is worth noting that muscle soreness disappears before the full recovery of muscle function, further elevating the injury incidence (Strojnik et al., 2001). Although exceptional, extreme $\mathrm{CK}$ and $\mathrm{Mb}$ elevations associated with EIMD could be severe enough to provoke a kidney tubulopathy (Sayers et al., 1999).

Given the risks and drawbacks related to the occurrence of EIMD described above, the development of strategies to prevent or reduce the intensity of its clinical manifestations has become a primary goal of many studies. The most commonly used approaches include stretching, cryotherapy, electric or manual therapies, whole-body vibration or nutritional and pharmacological interventions (Cheung et al., 2003;
Barnett, 2006; Bloomer, 2007; Howatson and van Someren, 2008). Despite the large number of clinical trials, there are very few evidence-based guidelines for the application of these interventions. The inconsistencies in the dose and frequency of the investigated interventions may account for the lack of consensus regarding their efficacy. Conversely, there is unequivocal evidence that a first bout of eccentric exercise confers protection against EIMD following a subsequent bout of the similar exercise. This muscle adaptation process, commonly called "the repeated-bout effect" ( $\mathrm{RBE}$ ), is characterized by reduced increases in muscular proteins in the blood, attenuated DOMS, less muscle swelling, reduced abnormality in echo intensity of B-mode ultrasound and/or magnetic resonance images and faster recovery of muscle strength and range of motion following the repeated bout (McHugh, 2003; Nosaka and Aoki, 2011). Although a significant protective effect occurs after a single eccentric bout (Clarkson et al., 1992; Nosaka and Clarkson, 1995), the adaptive process appears more complete after several sessions (Croisier et al., 1999; Hody et al., 2011). The RBE seems to imply long-lasting adaptation since it persists for several weeks and even up to 6 months but the magnitude of the protection decreases over time (Nosaka et al., 2001, 2005). It is interesting to note that the magnitude of the protective effect is not necessarily dependent on the severity of the initial muscle damage. It has been demonstrated that repeating bouts of "non-damaging" eccentric exercise can provide strong protective adaptations against subsequent bouts of maximal eccentric exercise (Chen et al., 2013). Therefore, to date, performing repeated sessions with submaximal eccentric contractions appears to be the most efficient strategy to induce eccentric training-induced adaptations that would prevent further EIMD and DOMS. The demonstration that eccentric actions can be performed without damage and soreness allowed considering the potential of eccentric trainings in medical conditions. Studies conducted first with healthy subjects and then, with patient populations, have supported the application of eccentric trainings as a safe, feasible and efficient strategy for rehabilitation purposes (LaStayo et al., 2000; Hoppeler, 2016). Numerous studies have attempted to elucidate the mechanisms underlying the RBE, but this feature of the skeletal muscle is not fully understood (McHugh et al., 1999a; McHugh, 2003; Nosaka and Aoki, 2011).

Despite considerable amount of available data at the clinical and histological levels, a significant gap still remains in the understanding of the mechanisms that mediate morphological, cellular, and molecular responses to muscle damaging eccentric exercise (Hoppeler and Herzog, 2014). In addition, the molecular events underlying the specific eccentric training muscle adaptations are not fully understood (McHugh, 2003; Nosaka and Aoki, 2011). This review begins by describing the potential mechanisms leading to muscle damage and soreness following unaccustomed eccentric exercise. Then, are discussed the current knowledge of the eccentric training-induced adaptations including the main hypotheses of the protective effect against EIMD. Finally, the multiple applications of eccentric training, justifying the need of an improved understanding of its underlying molecular and cellular mechanisms, are exposed. 


\section{UNACCUSTOMED ECCENTRIC EXERCISE}

\section{Mechanisms of Exercise-Induced Muscle Damage}

It is generally accepted that the damage process is initiated due to a lack of homogeneity in sarcomeres stretching (asymmetric lengthening). This theory initially proposed by Morgan (1990) suggests that during eccentric contractions, the weakest sarcomeres or even half-sarcomeres will absorb most of the length change (Morgan, 1990). These may be stretched beyond the point of myofilament overlap resulting in disrupted or "popped" sarcomeres. In line with this proposal, several studies have clearly shown that the length of the muscle during eccentric contraction is a critical factor in determining the extent of damage (Talbot and Morgan, 1998). Eccentric contractions performed at longer muscle length results in greater symptoms of damage than similar contractions at shorter muscle length (Lieber and Friden, 1993).

The initial mechanical damage would trigger a cascade of events leading to more severe secondary damage (Figure 1). Loss of calcium homeostasis, possible inflammatory reaction and reactive oxygen species (ROS) production are thought to contribute to the secondary damage phase. The disturbances in $\mathrm{Ca}^{2+}$ homeostasis observed following unaccustomed eccentric exercise may be the consequence of membrane damage (Friden and Lieber, 2001) or opening of stretch-activated channels (Overgaard et al., 2002). Abnormal increase in calcium concentration inside muscle cells is responsible for the activation of muscle proteases, named calpains. Since these proteases cleave important structural proteins in charge of myofibril integrity (as desmin and alpha-actinin), they have been suggested to contribute to EIMD. The degradation of proteins released from myofibrillar structures by the calpains could be enhanced by other proteolytic pathways as the ubiquitinproteasome system (Raastad et al., 2010). Activation of calpains may also result in the destruction of membrane constituents, which in turn, will increase calcium entry. Elevated calcium concentrations in skeletal muscle mitochondria, which can alter mitochondrial respiratory function, also occur following unaccustomed eccentric exercise (Rattray et al., 2011, 2013). This calcium overload may be associated with the opening of the mitochondrial permeability transition pore (mPTP) leading to the activation of cell death signaling or with the increased calpain proteolytic activity which is capable of targeting proteins resulting in mitochondrial dysfunction. Furthermore, the increased calpains activity can promote neutrophils and macrophages activation, leading to ROS production (Powers and Jackson, 2008). Besides the clinical symptoms associated with EIMD (such as DOMS and decline in muscle strength), EIMD have been reported to induce metabolic consequences at the acute phase: decreased glucose uptake and insulin sensitivity, impaired glycogen synthesis, elevated metabolic rate and a shift toward non-oxidative metabolism (Tee et al., 2007).

\section{Inflammatory and Immune Responses to Eccentric Exercise}

While the development of an inflammatory reaction after eccentric exercise has been debated (Yu et al., 2002; Malm and Yu, 2012), many studies have now provided clear evidence of systemic and local inflammatory responses in both rodents and humans following various types of eccentric exercise (Peake J. et al., 2005; Paulsen et al., 2012). However, in contrast to extensive works describing the histological and clinical signs associated to EIMD, the mechanisms underlying the inflammation-immune responses and the subsequent regenerative events are less well understood (Peake J. et al., 2005; Paulsen et al., 2012). The inflammation processes following damaging exercise was initially considered as a detrimental event due to its association with muscle damage, soreness and delayed recovery but it is now well accepted that the inflammatory stages are crucial for functional recovery of the muscle to EIMD. The inflammation would ensure the removal of tissue debris from the injured area and promote muscle repair by activating muscle cells. Over the last decade, more studies

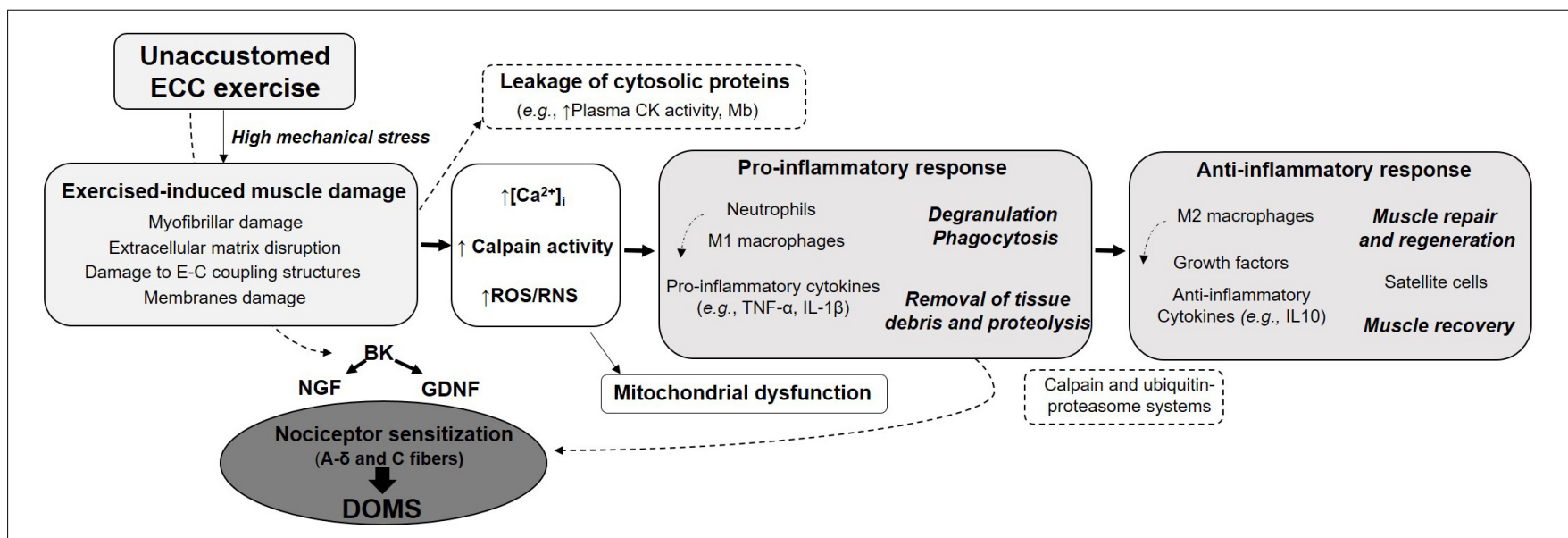

FIGURE 1 | Summary of the main specific features of eccentric contraction, its multi-target beneficial effects and potential risks associated with unaccustomed and/or maximal eccentric exercise. 
have focused on the implication of multiple immune cell types interacting with the muscle and emphasized the undeniable role of satellite cells for muscle regeneration following one bout of eccentric exercise (Crameri et al., 2004; Paulsen et al., 2012).

Early accumulation of leukocytes, primarily neutrophils, has been observed in micro-blood vessels of the damaged muscle, as well as in the perimysium, immediately after exercise. In case of moderate to severe EIMD, histological studies have consistently shown that neutrophils infiltrate into the muscle and accumulate in the damaged area from 1 and $24 \mathrm{~h}$ after eccentric exercise (Paulsen et al., 2010). It is likely that secretion and/or passive release of chemoattractant proteins due to modifications to membrane permeability are involved in the recruitment of circulating inflammatory cells. They initiate the pro-inflammatory stage through phagocytosis and by releasing proteolytic enzymes (such as elastase or myeloperoxidase) and reactive species. At later time points, when neutrophils are cleared from muscle, pro-inflammatory macrophages start to accumulate. This type of macrophages, referred as M1, contribute to the phagocytosis of the damaged tissue by secreting pro-inflammatory cytokines (e.g., TNF- $\alpha$, IL-6, and IL-1 $\beta$ ) and secretory leukocyte protease inhibitor. Tissue-resident monocytes may also become activated after exercise, in addition to the leukocytes originating from the blood circulation. Neutrophils and M1 macrophages interact with each other to regulate the proinflammatory response of muscle damage. Their influx inside injured myofibers appears to be dependent on the magnitude of EIMD and may lead to an exacerbation of the initial cellular alterations. Conversely, M2 macrophages that appear later generally produce anti-inflammatory cytokines and signaling molecules involved in the muscle recovery and regeneration. Large variations across healthy individuals are observed, some presenting substantial leukocyte accumulation whereas others displayed very little leukocytes invasion. Furthermore, the magnitude of the inflammation response appears to be dependent on the initial perturbations induced by the exercise. It is assumed that minor perturbations result in a cell-signaling-mediated adaptive response, whereas intense eccentric actions seem to generate a more severe response leading to secondary damage to myofibers and increased risk of necrosis. While significant necrosis is observed after electrically stimulated contractions, segmental myofiber necrosis may occur without affecting the whole myofiber, even in severe cases of EIMD. Interestingly, the degree of leucocyte accumulation seems to be related to the changes in force-generating capacity of the muscle (Paulsen et al., 2010). Therefore, measuring the decline of muscular strength following exercise, which is recognized as the best indirect marker of EIMD, may inform on the status of the muscle. In contrast, the level of leukocyte invasion into injured myofibers is not necessarily related to DOMS.

The muscle inflammatory response appears to intimately coregulate with muscle regeneration. Indeed, additionally to their immune functions, macrophages also participate to myogenesis and contribute to the extracellular matrix remodeling. M1 macrophages stimulate satellite cells proliferation whereas M2 macrophages interact with differentiating satellite cells
(Paulsen et al., 2012). The latter can also promote general protein synthesis within muscle fibers. The replacement of macrophages M1 to anti-inflammatory M2 macrophages is a key stage for the transition from proinflammatory to anti-inflammatory stages. This process is regulated by different signals including the phagocytosis of cell debris, IL10 and AMP-activated protein kinase (Chazaud, 2016). While a large body of research has primarily focused on neutrophils and macrophages, other cell types interact with the muscle and are important in the inflammation and muscle regeneration processes. These include notably mast cells, T lymphocytes, eosinophils, fibro-adipogenic progenitors, and pericytes (Paulsen et al., 2012).

The inflammation and immune responses are mediated by various growth factors and the actions of exercise-responsive cytokines (i.e., IL-6, CCL2, and interferon- $\gamma$ ), pro-inflammatory cytokines (TNF- $\alpha$ and IL-1 $\beta$ ) and the anti-inflammatory cytokine IL-10. Collectively, all these cytokines appear to activate myoblast proliferation and some of them are involved in myoblast differentiation (Peake J. et al., 2005). Interestingly, the satellite cells activity is differentially affected by the contraction mode in human muscle following exercise of the same work load. Resistance eccentric, but not concentric, exercise has been shown to elicit the proliferation of satellite cells immediately after exercise, suggesting that EIMD is the main stimulus for activating the satellite cells pool (Hyldahl and Hubal, 2014; Hyldahl et al., 2014).

The precise source of production for cytokines found in the circulation during and after exercise is not well established. Indeed, the cytokines can be produced not only by leucocytes, but also by myofibers and peri-tendinous tissue (Paulsen et al., 2010). The term "myokines" has been introduced to refer to muscle derived-cytokines and chemokines. Myokines are secreted by the skeletal muscle in order to communicate with non-muscle tissues and act as auto-, para- and endocrine mediators. These might be molecular mediators which link muscle exercise and the whole body physiology (Schnyder and Handschin, 2015). While research to date has focused primarily on the biological functions of the myokines in regulating metabolism, much less attention has been made regarding their role in inflammatory and adaptation to EIMD (Paulsen et al., 2010). Nevertheless, studies investigating the cytokine responses to eccentric exercise demonstrated increased activity of some cytokines such as MCP-1 and IL-10 after eccentric but not concentric exercise (Hyldahl and Hubal, 2014). The anti-inflammatory cytokine IL-10 may attract T lymphocytes, which activate muscle cell proliferation and muscle regeneration. Systemic increase of IL-8 and upregulation in intramuscular IL-8 mRNA expression and plasma levels after downhill running and eccentric actions of the quadriceps has also been reported (Hubal et al., 2008; Buford et al., 2009). IL-8 plasma levels are also increased after eccentric muscle contractions, but unchanged following concentric exercise. IL-8 is known to attract primary neutrophils but this chemokine may also promote neovascularization of muscle tissue through its association with CXCR2 (Schnyder and Handschin, 2015). Some studies also showed an increase in plasma concentration of IL-1ra and G-CSF (granulocyte-colony stimulating factor) in the hours after eccentric exercise (Peake J. et al., 2005; Peake J.M. et al., 2005). 
A large body of science has focused on IL-6. This myokine, considered as one "exercise factor," is regulated by exercise and acts both locally within the muscle and on distal organs in an endocrine-like fashion (Catoire and Kersten, 2015). IL-6 has initially been characterized as a prototypical pro-inflammatory cytokine by contributing to neutrophil mobilization and activation and promoting impaired peripheral insulin resistance. In contrast, anti-inflammatory properties of IL- 6 have been proposed later. Indeed, its exercise-induced systemic increase generates the elevation of plasma level of several antiinflammatory cytokines (IL-1ra and IL-10) and inhibits the production of the pro-inflammatory cytokine TNF- $\alpha$ (Pedersen and Febbraio, 2008; Pedersen, 2012). Various cell types secrete IL-6, including the skeletal muscle fibers during and after exercise. Alongside the systemic increase, IL- 6 mRNA levels are augmented in contracting muscle fibers. IL- 6 is considered as an energy sensor of the muscle (Pedersen, 2012) since its secretion from the exercising muscles increases glucose uptake and fatty acid oxidation locally and improves insulin secretion, which further increases glucose uptake into muscle fibers. Hepatic glucose delivery and fatty acid release from adipose tissue are also stimulated supporting the maintenance of metabolic homeostasis during exercise (Febbraio and Pedersen, 2002). Muscle derived IL-6 was first thought to be related to injury but "non-damaging exercise" has been shown to lead to substantial IL-6 increase (Croisier et al., 1999). Nevertheless, IL-6 contributes with TNF- $\alpha$ and MCP-1 to muscle regeneration after EIMD by stimulating the proliferation and differentiation of myoblasts (Schnyder and Handschin, 2015). Moreover, the transforming growth factorbeta is another cytokine involved in muscle recovery and repair after muscle damage that regulates extracellular matrix remodeling and promotes fibrosis (Kim and Lee, 2017).

\section{Delayed-Onset Muscle Soreness}

Delayed-onset muscle soreness (DOMS) refers to unpleasant, dull, aching pain, usually felt during palpation, contraction or stretching of the affected muscle. Such muscle soreness typically appears 12-24 h after unaccustomed eccentric exercise, peaks at between 24 and $72 \mathrm{~h}$ before progressively subsiding and disappearing within 5-7 days post-exercise. Interestingly, DOMS intensity is poorly correlated with other EIMD indirect markers and seems thus not to reflect the magnitude of muscle damage (Nosaka et al., 2002). Although DOMS is an extremely common symptom, why DOMS occurs with a delay, and why eccentric contraction but not shortening contraction induces DOMS is not clearly understood. Several hypotheses have been put forward to explain the mechanism of DOMS. These include lactic acid release, spasm, connective tissue damage, muscle damage, inflammation and oxidative stress (Hyldahl and Hubal, 2014). For many years, the most widely supported hypothesis was that the biochemical, thermal and mechanical changes associated with the inflammatory response sensitize small diameter muscles afferents (types III and IV) that may then be at the origin of the sensation of muscle soreness (Friden and Lieber, 1992). It was only in 2010 that Murase and coworkers provided new insights into the molecular mechanisms of DOMS generation. They highlighted bradykinin and nerve growth factor (NGF) as important players in the development of DOMS following eccentric contractions (Figure 1). Using a rodent model, they demonstrated that a bradykinin-like substance released from the muscle during eccentric exercise triggers the process of muscular mechanical hyperalgesia by upregulating NGF through B2 receptors in exercised muscle of rats. In humans, NGF has been shown to be involved in the generation and potentiation of pain following eccentric exercise (Nie et al., 2009). Another pathway proposed to be involved in the development of DOMS is the activation of the COX-2-glial cell line-derived neurotrophic factor (GDNF) (Murase et al., 2013; Mizumura and Taguchi, 2016). Similarly to NGF pathway, this agent likely generates muscle mechanical hyperalgesia directly by stimulating muscle nociceptors, or by binding to extracellular receptors. While myofibers micro-damage were believed to be necessary to initiate inflammation and DOMS, some studies reported mechanical hyperalgesia after eccentric exercises without any signs of muscle damage. This supports the crucial roles of NGF and GDNF in DOMS and suggests that the mechanical hyperalgesia development may be associated with inflammation in the extracellular matrix (Peake J. M. et al., 2017). NGF and GDNF are also known to play a role in pathological pain conditions and are increasingly recognized as active players in the whole pain process and upregulated in ischemic skeletal muscle (Turrini et al., 2002). NGF is increasingly regarded as an active player in the whole pain process (McKelvey et al., 2013). Thus, advances in the understanding of the mechanisms and cellular origins of muscle soreness could lead to development of effective interventions for not only exercise-related muscle soreness, but also common myalgia.

\section{ECCENTRIC TRAINING-INDUCED ADAPTATIONS}

\section{Molecular Aspects}

The distinct features of the eccentric contraction compared to other contraction modes are the source of specific training adaptations. A significant body of evidence suggests that compared to concentric contractions, chronically performed eccentric contractions promote greater gains in strength, muscle mass and neural adaptations (Reeves et al., 2009; Roig et al., 2009). The mechanisms responsible for these adaptations are underlined by modifications in gene expression. Indeed the process of exercise-induced adaptations in skeletal muscle involves multiple signaling mechanisms initiating transcription of specific genes that enable subsequent translation into a series of new proteins (Coffey and Hawley, 2007). Several studies have reported that eccentric and concentric actions activate distinct muscular molecular pathways in humans (Kostek et al., 2007) and in rats (Chen et al., 2002). It has been shown that eccentric exercise triggers a progressive activation of genes responsible for cellular growth and development, involved in muscular cell hypertrophy processes. The expression levels of these genes are more stimulated by eccentric actions than by isometric or concentric actions (Chen et al., 2002; Barash et al., 2004; Kostek et al., 2007), presumably due to the unique mechanical 


\section{FEATURES OF ECCENTRIC CONTRACTION}

- High muscle forces

- Distinct molecular response

- Higher satellite cell activity

- Greater anabolism signaling
- Low metabolic demand

- Low fatigue

- Low perceived exercise

- Low cardiorespiratory demand
- Unique neural strategies

- Reduced central nervous activity

- Fewer motor unit recruitement

- Lower motor unit discharge

- Greater cortical excitability

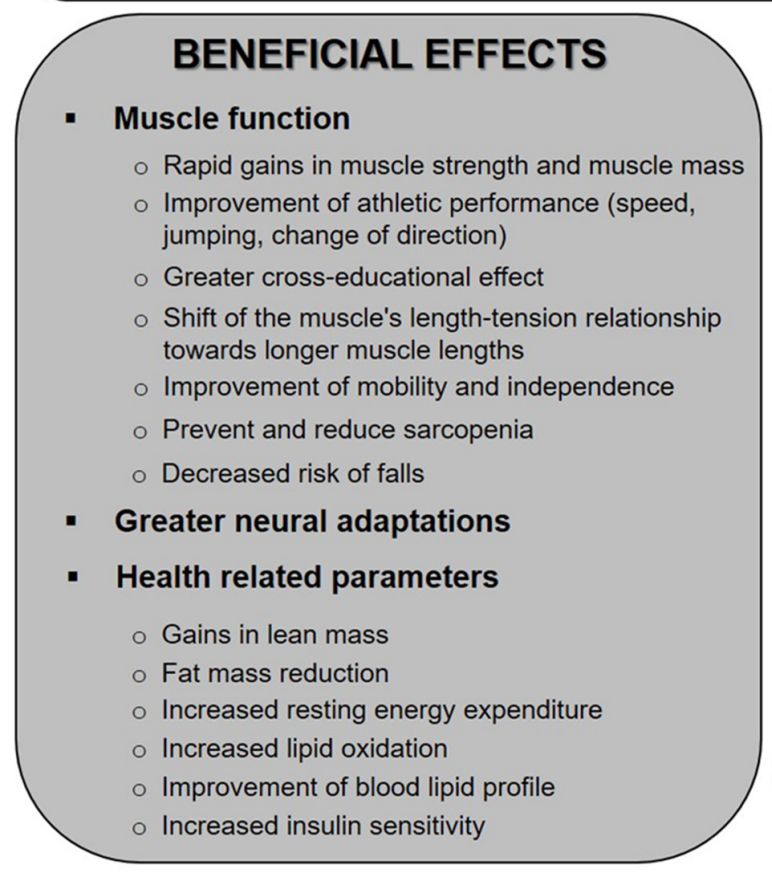

\section{RISKS}

- Exercise-induced muscle damage

- Ultrastructural damage

- Impaired sarcolemma permeability

- Damage of extracellular matrix

- Delayed-onset muscle soreness

- Mechanical hyperalgesia

- Pain, tenderness, swelling, stiffness

- Impaired muscle function

- Loss in force generating capacity

- Decreased range of motion

- Impaired proprioceptive function

- Modified locomotion of biomechanics (gait, running, sport actions)

- Decreased athletic performance

- Increased risks of muscle, tendon and joint structures injuries

- Exertional rhabdomyolysis and associated kidney tubulopathy

FIGURE 2 | Schematic representation of the potential mechanisms associated with eccentric exercise-induced muscle damage and involved in the development of DOMS. BD, bradykinin; CK, creatine kinase; DOMS, delayed-onset muscle soreness; E-C, excitation-contraction; GDNF, glial cell line-derived neurotrophic factor; IL, interleukin; NGF, nerve growth factor; ROS/RNS, reactive oxygen and nitrogen species; TNF, tumor necrosis factor.

stress placed on the eccentrically contracted muscles. For example, in skeletal muscle, the effect of eccentric training was greater than concentric training for liver-type insulin-like growth factor I and mechano-growth factor (positive regulators of muscle growth) (Barash et al., 2004). Such modifications in gene expression profiles are thought to be regulated by mechanical signaling pathways involving proteins that are sensitive to the mechanical status of muscle cell (i.e., Microtubules-Associated Proteins or MAP proteins) (Hentzen et al., 2006). Transcriptome analyses in eccentric-exercised muscles also revealed substantial transcriptional activity related to the presence of leukocytes, immune-related signaling and adaptive remodeling of the intramuscular extracellular matrix until $96 \mathrm{~h}$ after exercise (Neubauer et al., 2014). In comparison to concentric or isometric contractions, eccentric contractions appear to upregulate muscle cell activity and anabolic signaling pathway to a greater extent (Douglas et al., 2017).

\section{Specific Muscle Adaptations to Chronic Eccentric Exercise}

Because the eccentric contraction differs from other contraction types notably in terms of force generation, maximum force produced and energy cost, it could provide different stimuli leading to distinct muscular and functional adaptations (Figure 2) (Franchi et al., 2017a). A significant body of evidence have suggested the superiority of eccentric resistance training in terms of muscular hypertrophy over concentric or conventional strength trainings (Julian et al., 2018). Some studies also reported earlier increments in muscle mass with eccentric-based resistance training when compared with concentric training. However, the findings appear extremely variable to clearly confirm greater gains in muscle mass following eccentric modalities (Julian et al., 2018). Indeed, in their review, Franchi et al. (2014) draw the conclusion that the changes in muscle size are similar between eccentric and concentric training when matched for load or work. A systematic review and meta-analysis about the contribution of the different muscle actions to muscle growth showed a greater muscle mass gain with eccentric contractions but the results did not reach significance (Schoenfeld et al., 2017). Nevertheless, taking into account the energy demand to produce similar force or work, eccentric exercise may be considered as more efficient (Julian et al., 2018). Interestingly, contraction type tends to induce a region-specific hypertrophy. Greater increase in distal muscle size has been observed following eccentric 
training, while concentric training favors median-muscle hypertrophy (Franchi et al., 2014). In addition, the hypertrophic responses to eccentric versus concentric contractions might be obtained by different structural adaptations mediated by distinct myogenic and molecular responses. While both training regimes appeared to increase muscle fascicle length and pennation angle (Blazevich et al., 2007), conventional strength training would increase pennation angle more than eccentric training. In contrast, eccentric-only resistance training seems to favor fascicle length increase (Reeves et al., 2009), with the implication that eccentric training is able to shift the optimum of the length-tension relationship to longer muscle length (Hoppeler, 2016). This muscle architectural change appears thus particularly interesting for injury prevention and athletic performance (Brughelli and Cronin, 2007). Regarding muscle thickness, similar increases have been observed with both training modes. There are evidence that eccentric training promotes significantly greater increase in muscle strength, whereas the differences in isometric and concentric measures seems less significant (Roig et al., 2009). Findings also showed that the increase in eccentric strength after eccentric training is greater than the gain in concentric strength after concentric training (Vikne et al., 2006). The systematic review of Douglas et al. (2017) also reported mode-specific strength increase and revealed that greater overall strength increases can be achieved after eccentric training than concentric or traditional training. Furthermore, in comparison with concentric exercise, eccentric actions have been reported to induce a greater cross-education effect. Only few studies examined changes in muscle power. Performance in actions involving muscle power or stretch-shortening cycle (such as vertical jump) appeared to be improved to a greater extent with eccentric training compared with concentric or traditional resistance training (Liu et al., 2013; Douglas et al., 2017).

\section{The Repeated-Bout Effect (RBE)}

Skeletal muscle exhibits an intriguing plasticity to repeated bouts of eccentric exercises. Among the adaptations specifically triggered by the eccentric contraction, some contribute to the $\mathrm{RBE}$, aiming thus to protect muscle against EIMD. A large number of theories have been proposed to explain the RBE, suggesting a multifactorial origin of this adaptive process. Potential adaptations have been categorized as (Lindstedt et al., 2001) neural, (Abbott et al., 1952) mechanical and (LaStayo et al., 2003b) cellular theories (McHugh et al., 1999a; McHugh, 2003). However, although many studies have attempted to elucidate the mechanisms behind the RBE, a unified theory is not yet available.

According to the neural theory, the EIMD results from the high mechanical stress imposed on a small number of active muscle fibers during intense eccentric contractions. Although not commonly accepted, this theory also supports a preferential recruitment of fast-twitch motor units during eccentric contractions to explain the higher susceptibility to disruption of the fast muscle fibers. Therefore, it has been postulated that changes in neural activation may contribute to reduce subsequent myofibrillar damage (McHugh, 2003). Suggested neural adaptations involve improved motor units (MUs) synchronization and activation of a large pool of MUs, mainly by recruiting a greater number of slow-twitch fibers (Warren et al., 2000; Chen, 2003; Starbuck and Eston, 2012). Such mechanisms would allow a better distribution of the workload over a greater number of active muscle fibers in repeated bouts (Nosaka and Clarkson, 1995). The fast-setting adaptations but also the existence of contralateral protective effect (Howatson and van Someren, 2007; Starbuck and Eston, 2012; Hody et al., 2013b) support the contribution of neurophysiologic processes in the RBE. Indeed, some studies have reported that an initial bout of eccentric exercise in one limb provides protection from the symptoms of EIMD during a second eccentric bout in the contralateral limb. Nevertheless, the magnitude of protection in the contralateral limb is lower than that observed in the ipsilateral limb, indicating that neural adaptations cannot entirely explain the RBE (Howatson and van Someren, 2007). Moreover, the demonstration of RBE with electrically stimulated eccentric contractions (Black and McCully, 2008) suggests that the RBE can occur independently of neural adaptations and involves thus a peripheral and/or muscular adaptation.

The mechanical origin of initial muscle damage has led authors to suggest that changes in mechanical properties of the musculoskeletal system could render the muscle more resilient to EIMD. With respect to this hypothesis, both the passive and dynamic stiffness of the muscle-tendon complex has been shown to increase after eccentric training (Howell et al., 1993; Reich et al., 2000). These modifications have been, respectively, attributed to an increase in intramuscular connective tissue improving the ability to withstand myofibrillar stress and to a reinforcement of intermediate filament system, in charge of maintaining the alignment and structure of the sarcomeres (i.e., titin, desmin) (McHugh, 2003). In agreement with the reorganization of cytoskeletal proteins, the level of certain structural proteins, such as desmin, was found to increase in the days following eccentric exercise (Feasson et al., 2002; Lehti et al., 2007). This suggests that muscle-specific cytoskeletal remodeling could play a role to protect from future sarcomere disruption. Desmin, the major protein of the muscle intermediate filament, would act as mechanical integrator for the repair of the filaments (Yu et al., 2002). Its reinforcement secondary to transcriptional upregulation may provide mechanical protection from future sarcomere disruption (Peters et al., 2003). However, some studies showing that stiffer muscles are more prone to damage questioned the mechanical theory ( $\mathrm{McHugh}$ et al., 1999b). Moreover, desmin knockout (KO) mice have been found to exhibit less exercise-induced than wild-type mice (Sam et al., 2000). This finding was, however, imputed to more compliant muscles of $\mathrm{KO}$ mice. Recent works have suggested that in addition to their function of structural support to the cell, the intermediate filaments may play an active role in biological processes such as signaling, mechanotransduction and gene regulation. The mechanisms behind these processes are not well understood. Desmin, which is responsible for transmission of stress among myofibrils appears to be required for the maintenance of myofiber alignment, nuclear deformation, stress production and JNK-mediated stress sensing (Palmisano et al., 2015). Growing evidence supports the role of the skeletal muscle intermediate filaments as a stress-transmitting 
and stress-signaling network. Notably, cytoskeletal proteins help mitochondria not only in their movement and proper cellular positioning, but also to maintain their biogenesis, morphology, function, and regulation of energy fluxes. The functionality of these cytoskeletal proteins may thus influence the mitochondria functions, including the regulation of $\mathrm{Ca}^{2+}$ signals and apoptosis (Mado et al., 2019). Remodeling of the intermediate filaments network may also impact cell migratory behaviors important to development (Sanghvi-Shah and Weber, 2017).

Another group of theories explaining the RBE relies on cellular adaptation. Given the focal feature of muscle damage, speculation has been made that the muscle becomes more resistant to EIMD thanks to the removal of stress-susceptible fibers or sarcomeres resulting from the initial eccentric bout (Armstrong, 1984; Newham et al., 1987). However, this is inconsistent with the fact that the initial bout does not have to cause appreciable damage to confer a protective effect. Several works provided evidence that eccentric exercise promotes an increase in series sarcomeres (Lynn and Morgan, 1994; Yu et al., 2004). Such longitudinal addition of sarcomeres is thought to contribute to the protective effect as it would avoid the sarcomere stretching beyond their overlap and thus, their disruption. The sarcolemma and sarcoplasmic reticulum would also become stronger following the initial bout of eccentric exercise ( $\mathrm{McHugh}$, 2003). This may limit perturbations of calcium homeostasis and thus, may prevent the calpain activation and the degradation of cytoskeletal proteins. The reduced calpain activity could then explain the attenuation of mitochondrial dysfunction following chronic exposure of eccentric exercise. Other potential adaptations such a decreased susceptibility to calcium-induced mPTP (mitochondrial permeability transition pore) opening or upregulation of heat shock proteins, in particular Hsp70, may also be contribute to protect mitochondrial function (Rattray et al., 2013). In addition, changes in the inflammatory response, such as a reduced activation of the monocytes and neutrophils, have been described after repeated eccentric bouts and may also be related to the RBE (Pizza et al., 1996). Nevertheless, whether adaptation in the inflammatory process is the cause or a consequence of reduced muscle damage is not elucidated. Adaptation may also rely on the monocyte chemoattractant protein 1 (MCP-1), a chemokine involved in activation and attraction of inflammatory cells. Indeed, MCP-1 is dramatically overexpressed at the transcript level after a single bout of eccentric exercise and it appeared even more upregulated after a second bout (Hubal et al., 2008). Authors have thus suggested that MCP-1 enhances muscle recovery after a repeated bout of eccentric exercise via improved signaling between macrophages and satellite cells. Other chemokines may contribute to the protective adaptation to exercise-induced muscle damage. Upregulation of CCL2 and a decreased of NF-kB DNA-binding activity occur following repeated bouts of eccentric exercise. These observations supports the hypothesis that the immune response becomes more efficient to promote the regeneration of muscle tissue after an initial bout of eccentric exercises, notably through enhancement in inflammatory cell infiltration into the muscle and myoblast proliferation (Peake J. et al., 2005). Furthermore, a remodeling of the surrounding extracellular matrix might also occur during the RBE. A strengthening of the extracellular matrix such as an improved integrin support may help to recover faster after eccentric contractions (Hyldahl et al., 2015).

Other potential cellular adaptations include increased protein synthesis, adaptation in the excitation-contraction coupling and increased stress proteins (i.e., heat shock proteins) (McHugh, 2003). In particular, the role of heat shock proteins (HSPs) in protection against muscle damage constitutes an exciting new area of research. The small HSPs (sHSPs) named HSPB1 (Hsp27) and alphaB-crystallin, seem to play important roles in cellular adaptation as they have been implicated in the chaperoning of unfolded proteins, the stabilization of the cytoskeleton as well as in the regulation of the cellular redox state and inhibition of apoptosis (Orejuela et al., 2007). Following one bout of eccentric exercise, the sHSPs translocate from the cytosol to the cytoskeletal/myofibrillar compartment, presumably to stabilize and protect the myofibrillar filament organization (Paulsen et al., 2007, 2009; Frankenberg et al., 2014). Such an observation was not found after concentric exercise (Frankenberg et al., 2014). AlphaB-crystallin interacts with desmin intermediate filaments and, Hsp27, together with alphaB-crystallin, has been suggested to interact with various microfilaments (Orejuela et al., 2007). These data strongly support the idea that alphaB-crystallin and Hsp27 are crucial for the maintenance and the remodeling of myofibrillar structures. Therefore, in line with the reinforcement of the cytoskeletal/myofibrillar structures, appropriate adaptation in the protection systems of HSPs might be important as well (Paulsen et al., 2007). Moreover, because the HSPs are involved in the development of stress tolerance against several stressful insults, it is likely that the HSPs response elicited by an initial damaging bout bestows resistance to a second potentially damaging exercise. Only few studies investigated the HSPs response to repeated bouts of eccentric exercise. Paulsen et al. (2009) revealed that two bouts of maximal eccentric exercise separated by 3 weeks resulted in comparable increased levels of HSPs in the cytoskeletal fraction, despite less damage inflicted during the second bout. The large amount of Hsp27, alphaBcrystallin, and Hsp70 in the cytoskeletal compartment after the repeated bout suggests that a more efficient translocation of these HSPs is plausibly a mechanism behind the RBE. Similarly, Thompson et al. (2002) reported a similar relative increase of Hsp27 and Hsp70 2 days after the first and second eccentric bouts, but, intriguingly, the basal levels of these HSPs appeared to be lower before the second bout. This finding casts doubt on the HSPs as important players in the RBE. Contrary to these findings, results from a study by Vissing et al. (2009) did not point out a role for the HSPs in reducing EIMD, as they observed a blunted translocation of HSPs after the second bout. In this latter study, the low degree of muscle damage inflicted during the exercise and/or the long duration between bouts ( 8 weeks) could explain the lack of HSPs movement following the second bout. Future studies appear thus to be necessary to delineate the HSPs response after repeated eccentric bouts.

Finally, using a proteomic approach, a short isokinetic eccentric training in human quadriceps was found to induce proteome modifications that suggest an isoform shift in fiber type components (Hody et al., 2011). Indeed, a decreased 
expression of several glycolytic enzymes coupled with a lower expression of the fast isoforms of some contractile and structural proteins was observed after five sessions of submaximal eccentric contractions. Adaptation in the muscle fiber typology following eccentric training was further supported by a study in mice. This highlighted significant changes in the size and number of muscle fiber types following eccentrically biased trained in comparison with untrained or concentrically biased trained mice: the eccentric training specifically resulted in an increased proportion of slow and fast oxidative muscle fibers (Hody et al., 2013a). Nevertheless, whether a shift to a more oxidative muscle phenotype is really involved in protection against EIMD is still a question to resolve.

\section{MULTIPLE APPLICATIONS OF ECCENTRIC TRAINING}

Eccentric training has sparked a growing interest over the last decade, particularly in light of the emerging healthrelated benefits of improved muscle mass. Moreover, because a greater volume of exercise can be done at less metabolic and cardiorespiratory cost, eccentric muscle work constitutes a promising training strategy, not only to improve athletes' performances, but also to help maintain or restore the exercise capacity and quality of life in individuals with reduced tolerance for physical activity (i.e., the elderly or patients with chronic disabilities) (Gault and Willems, 2013; Hyldahl and Hubal, 2014; LaStayo et al., 2014). The lower perceived exertion to perform eccentric exercises helps to increase the adherence of patients to exercise programs. As eccentric contractions have traditionally been associated with muscle damage, the prescription of eccentric training programs in clinical practice has been discouraged for a long time. Nowadays, it is well accepted that when the duration, frequency and intensity of the eccentric training sessions are progressively increased, symptoms of damage can be minimized and even avoided (Croisier et al., 1999; Chen et al., 2013). Additionally, it is now accepted that neither muscle damage nor inflammation are prerequisites for stimulating positive muscle adaptations as protection against EIMD or increased muscle mass (LaStayo et al., 2007). Eccentric training interventions are thus considered as a safe and suitable alternative to traditional resistance exercise. Before discussing the numerous applications of eccentric training, it should be mentioned that the identification of its specific effects in comparison to the other training modalities remain difficult due to methodological reasons. First, the training programs used in several studies involved usual daily movements that do not isolate eccentric and concentric contractions. Secondly, a limited number of studies employed an appropriate calibration of the eccentric and concentric exercises, making conclusive comparison between the contraction modes impossible. Since they imply different metabolic cost for the same mechanical work, eccentric and concentric exercises must be matched for similar mechanical output, metabolic rate or oxygen consumption level, or similar total training load. Additionally, the techniques and equipment to perform eccentric exercises is often sophisticated, require specific experience and may represent financial constraints. These reasons may contribute to the few number of studies comparing eccentric training with other modalities and the difficulty to draw definitive conclusions (Julian et al., 2018).

\section{Competitive Sports}

While few studies have been devoted to the effects of eccentric training in elite athletes compared to untrained subjects, the systematic inclusion of eccentric-based protocols into training programs is recommended for most competitive sports for performance enhancement or injury prevention purposes (IsnerHorobeti et al., 2013; Vogt and Hoppeler, 2014). Indeed, because of its distinct characteristics, eccentric training modalities can further enhance maximal muscular strength and optimize improvements to power, optimal muscle length for strength development, as well as coordination during eccentric tasks (LaStayo et al., 2003a). Eccentric training may also be especially efficient in enhancing speed performance or in rebound activities such as jump (Franchi et al., 2017b; Chaabene et al., 2018). This has notably been demonstrated in basketball players. Those subjected to eccentric training for 6 weeks exhibited a significant improvement in jumping height of $8 \%$ while the performance of the players that performed traditional weight-lifting was unchanged (Lindstedt et al., 2002). A change in titin protein isoform has been proposed to explain the increased stiffness of the muscle-tendon unit and enhanced recovery of elastic strain energy (Hoppeler, 2016). These functional adaptations in skeletal muscles are based on increases in muscle mass, fascicle length, number of sarcomeres, and cross-sectional area of type II fibers. In terms of injury prevention, the isokinetic assessment of muscle function, in particular through the eccentric mode, appears to be of great importance for detecting athletes at high risk of injuries before the start of the season (Croisier et al., 2002; Forthomme et al., 2013). Moreover, preventive interventions with controlled eccentric exercises have been shown to decrease the risk of hamstring injury in professional soccer players (Croisier et al., 2008) or of shoulder pain in volleyball players (Forthomme et al., 2013).

\section{Rehabilitation}

Over the last 20 years, eccentric muscle actions have been frequently integrated in the treatment of several pathologies of the locomotor system (Croisier et al., 2009). In particular, chronic eccentric exercise has become a mainstay in the treatment of tendinopathies mainly of the Achilles, patellar and lateral epicondylar tendonitis (Croisier et al., 2007; Hoppeler and Herzog, 2014; Kjaer and Heinemeier, 2014). To justify the relevance of eccentric exercise for strengthening tendon tissues, a stimulating impact of such exercise on collagen synthesis and an increase in blood flow around tendon cells after eccentric actions have been proposed (Guilhem et al., 2010). Eccentric intervention has also been shown to be safe and effective after anterior cruciate ligament reconstruction (ACLR). The studies of Gerber et al. (2007, 2009) reported superior short and long-term results in strength, performance and activity level after surgery when eccentric exercise is part of the rehabilitation after ACL-R in comparison to standard rehabilitation programs. Otherwise, 
ipsilateral eccentric training has been demonstrated to increase muscles' strength in the contralateral homologous muscle group, and this in a greater extent than concentric training (Higbie et al., 1996; Hortobagyi et al., 1997). Thus, implementing unilateral eccentric contractions in rehabilitation programs could improve the muscle function of the opposite injured limb without it being solicited.

\section{Sarcopenia}

Given the ever-increasing aging population, the development of strategies to improve the quality of life of the elderly has become a major concern. One of the most evident and disabling consequences of aging is sarcopenia, a process characterized by a progressive and steady loss of lean skeletal muscle mass. Muscle loss is also associated with an increase in intramuscular fat and connective tissue, a reduction in muscle strength, in addition to cardiovascular dysfunction reducing aerobic capacity (Gault and Willems, 2013). Such changes contribute to a decline in functional independence and severely compromise the function, quality of life, and life expectancy in older individuals. Multiple lines of evidence suggest that exercise training can prevent or reverse muscle aging. Indeed, studies comparing muscle characteristics of highly trained young and senior athletes demonstrated that trained subjects can maintain and improve muscle function regardless of their age (Roig et al., 2008; Dickinson et al., 2013). However, the implementation of conventional resistance training programs in the elderly may be hampered by the difficulty of such programs as reduced initial levels of force and cardiovascular dysfunction are frequent in old adults. Conversely, eccentric training programs can massively overload the muscular system with a low cardiopulmonary stress. Interestingly, numerous studies reported that older individuals exhibit a relatively preserved capacity of producing eccentric strength. Indeed, when compared to concentric or isometric strength, the magnitude of the age-related decline in eccentric strength is less pronounced. This provides an additional advantage for eccentric exercises to initiate resistance training and rehabilitation programs (LaStayo et al., 2003b; Roig et al., 2010). In addition to the suitability of eccentric training in old individuals, it is important to emphasize that resistance training with eccentric contractions induces greater beneficial effects than concentric training to improve mobility and independence of the elderly. As in young individuals, high-intensity eccentric resistance training has been shown to be more effective than concentric training in increasing muscle strength and mass in older adults (LaStayo et al., 2003a; Reeves et al., 2009). Other appreciable benefits resulting from eccentric training in old individuals are the improved ability to complete functional tasks and the decreased risk of fall (Gault and Willems, 2013). LaStayo et al. (2003a) demonstrated that using eccentric modality in very frail elderly (mean age, 80 years) was more efficient to reverse sarcopenia and its related functional limitations than traditional weight training. Indeed, the elderly who performed 10-20 min of eccentric resistance exercise 3 times per week over 11 weeks showed significant improvements in strength (60\%), balance (7\%), stair descent (21\%) abilities and a reduced risk of fall. These positive outcomes were not found in the elderly subjects submitted to traditional resistance exercises. Additionally, the subjects of the eccentric group reported the training to be relatively effortless. Besides resistance training, eccentric endurance exercise involving large muscle groups (ECC cycling, downhill treadmill walking, and stepping) seems to be particularly convenient for the elderly (in particular for frail elderly). This training modality minimizes the substantial mechanical stress on single joints occurring during resistance training and provides benefits for strength, muscle mass and potentially aerobic adaptations (Gault and Willems, 2013; LaStayo et al., 2014). The study of Mueller et al. (2009) compared the effects of a moderate load eccentric exercise on an eccentric ergometer to a conventional resistance exercise training. Both trainings were carried out for 12 weeks with 2 sessions per week. A significant increase in maximal isometric strength (8.4\%) was observed only for the eccentric group (Mueller et al., 2009). Improvements in body composition characterized by a decrease in intramyocellular lipid content concomitantly with total body fat have also been observed in the elderly after 12 weeks of eccentric ergometer training (Mueller et al., 2011). In contrast, tight lean mass increased similarly after both training modalities. Interestingly, the gain in muscle mass in the elderly following eccentric training was not paralleled by an increase in muscle fiber cross-sectional area (hypertrophy) as observed with traditional exercise training (Mueller et al., 2011). Muscle growth after eccentric training thus seem to occur by the addition of sarcomeres in series or by hyperplasia. While available evidence suggest that eccentric training protocols are well tolerated in elderly individuals, it should kept in mind that old adults show an increased vulnerability to exercise-related muscle damage. Indeed, biopsies from the human $m$. vastus lateralis immediately after a bout of eccentric cycling showed disorganization of sarcomeres, with a higher percentage of disorganization in older (59-63-years) compared to younger adults (20-30-years) (Manfredi et al., 1991). Therefore, careful and safe progression of the intensity of eccentric training is thus strongly advised when initiating eccentric programs in the elderly.

\section{Chronic Diseases}

Musculoskeletal dysfunction is relatively common in patients with chronic conditions such as chronic obstructive pulmonary disease, chronic heart failure or stroke (Hyldahl and Hubal, 2014). Although the exact etiology of the muscle function decline in these patients is not yet clear, it is believed that the lack of physical activity contributes at least to some of the deleterious changes in muscle function (Roig et al., 2008). Moreover, the ability of exercise to maintain mobility and minimize muscle wasting in most people with chronic conditions is commonly accepted. Until now, only few studies explored the use of eccentric-biased programs in persons with chronic health conditions. Nevertheless, current evidence exists regarding the effectiveness and safety of eccentric exercise in restoring musculoskeletal function in patients with different chronic conditions. For instance, compared to conventional training programs, judicious eccentric-based protocols result in greater strength gains and enhancement of functional capacity in cancer survivors, Parkinson disease patients or total knee 
replacement patients (Hyldahl and Hubal, 2014). However, such favorable effects were not observed in individuals with multiple sclerosis (Hayes et al., 2011). Studies exploring the use of resistance training in individuals recovering from a stroke revealed that eccentric contractions were more effective for improving neuromuscular activation, strength, and walking speed than concentric contractions (Engardt et al., 1995; Clark and Patten, 2013). Since eccentric training seems to provide greater central neural adaptation than concentric modes of exercise, the use of eccentric exercise may be particularly effective for patients with central nervous system diseases. The physiologic characteristics of eccentric contraction (attenuated cardiopulmonary stress, low metabolic cost) seem to be well suited for their incorporation into the revalidation of patients intolerant to intense cardiac and respiratory efforts (i.e., patients with heart disorders or lung pathologies) (Meyer et al., 2003; Roig et al., 2008). Eccentric training has been suggested to attenuate reductions in arterial compliance, thus potentially limiting the risks commonly associated with resistance training in patients with coronary disease (Okamoto et al., 2006). Steiner et al. (2004) compared concentric and eccentric training at similar heart rate $(85 \%$ of $\mathrm{HR})$ in patients suffering from cardiac problems. Training was carried out 3 times per week during 8 weeks, with a progressive increase of the exercise intensity the first 5 weeks. The authors showed a significant gain in muscle torque following the eccentric training. Both training modalities induce a small 3\% increase in leg muscle mass but leg and whole body fat mass appeared to decrease only in patients trained eccentrically. Interestingly, despite working at fourfold higher mechanical loads, the eccentric group did not show different changes in cardiovascular variables (such as heart rate, mean arterial pressure, or vascular resistance) than the concentrically trained subjects (Meyer et al., 2003). Collectively, all studies reported eccentric exercise to be a safe training modality for patients with various cardiac conditions.

Eccentric exercise may also be useful in the prevention or treatment of metabolic diseases given its rapid and favorable effects on health related parameters (Roig et al., 2008; Paschalis et al., 2010; Isner-Horobeti et al., 2013). For instance, eccentric training is more effective to improve glucose tolerance than concentric training. Additionally, Paschalis et al. (2010) demonstrated that a weekly bout of intense eccentric exercise and not concentric exercise - is sufficient to improve health risk factors. They found that only $30 \mathrm{~min}$ of eccentric exercise per week for 8 weeks markedly increased resting energy expenditure and lipid oxidation as well as decreased insulin resistance and blood lipid profile. The study of Marcus et al. (2008) compared the effects of a 16-week aerobic exercise training alone to aerobic exercise combined with moderate load eccentric exercise in diabetes type 2 patients. While glycemic control and physical performance were similarly improved in all patients, the improvements in tight lean mass and body mass index were larger when eccentric exercise was performed.

In regard with muscular dystrophy pathology, no human study investigated the potential effects of eccentric training in this disease. It is likely that eccentric contractions may accelerate the degenerative process given that the degenerative nature of dystrophic muscle can partially be accounted for by exhaustive regenerative cycles (Hyldahl and Hubal, 2014). Nevertheless, some recent animal studies indicate a favorable adaptation to moderate exercise in dystrophic animals (Lovering and Brooks, 2014). In fact, despite the increased vulnerability of dystrophic $(m d x)$ muscles to eccentric contractions, young $m d x$ mice were found to recover from and adapt more quickly to EIMD than wild-type mice (Ridgley et al., 2009; Call et al., 2011). However, such increased regenerative capacity was lost in older animals (Carter et al., 2002) and it is still unclear whether an eccentricbased training program would be helpful or detrimental to the long-term health of the muscle.

Notwithstanding recent evidence demonstrate the benefits of eccentric training interventions in several fields, there is a real need to further study the physiology of eccentric contraction. Indeed, it is still unclear whether this high specificity of eccentric training adaptations compromises the transferability of strength gains to more functional movements (Roig et al., 2010). Moreover, long-term implications of eccentric training in old individuals or in patients with chronic diseases should be explored in more details. Likewise, further investigations are required to optimize parameters as intensity, duration, and modes of eccentric training leading to the favorable effects on muscle performance, health and quality of life.

\section{PRACTICAL CONSIDERATIONS}

Eccentric actions can be integrated in different types of muscle training. Plyometric exercises, such as drop jump, is frequently used to improve speed and jumping ability in athletes. The literature recommends specific habituation training and knowledgeable supervision due to the inherent risk of injuries in such exercises (Hoppeler, 2016). Eccentric based resistance training, characterized by high muscle loads at low metabolic cost, has been increasingly prescribed for individuals with a centrally limited exercise tolerance (LaStayo et al., 2014). However, in most patient populations, the use of high mechanical loads may constrain their adherence to resistance muscle training. Therefore, the new modality "moderate load eccentric exercise" represents an attractive choice in various medical conditions (Hoppeler, 2016). Over the last decades, various motorized ergometers or similar devices allowing safe and controlled application of eccentric loads, have been developed for rehabilitation and performance purposes.

The prescription of eccentric muscle training require specific experience. Practitioners must respect fundamental precepts and consider important safety considerations concerning the applications of eccentric muscle training, especially during the initial implementation phase. Exercise professionals should be aware of the potential detrimental effects of eccentric contractions as well as the ways to prevent their occurrence. Nowadays, it is well accepted that repeated exposure to eccentric exercises confers protective adaptations against potential further damage (McHugh, 2003; Nosaka and Aoki, 2011). Even if the magnitude of this "repeated-bout effect" appeared larger if the initial eccentric bout involved high workloads, this 
strategy could be problematic, especially for those undergoing rehabilitation or elite athletes. Indeed, DOMS and the functional consequences associated with EIMD may frequently disturb the progress of rehabilitation and/or training programs. Moreover, the uncomfortable sensations may discourage people to continue exercise training. Therefore, an initial phase consisting of submaximal eccentric muscle actions with incremental loading over multiple sessions should be used to introduce individuals to eccentric muscle training (LaStayo et al., 2003b). Flann et al. (2011) demonstrated that a 3-week gradual "ramp up" eccentric protocol was effective at promoting muscle hypertrophy in the absence of demonstrable markers of muscle damage. In clinical interventions, the progressive ramping eccentric protocol typically starts with load of 50-75 W to reach the target training load of 400-500 W. Higher loads, over 1,200 W, can be achieved in competitive athletes (Hoppeler, 2016). A period of 2-4 days between the exposure stimulus and progressively higher levels of loading has been suggested as optimal (Hoppeler, 2016). Guidelines to design ramping protocols in rehabilitation conditions are described in more details by LaStayo et al. (2014). The exercise duration generally increases from 5-10 $\mathrm{min}$ to 20-30 min over the sessions. When using higher load trainings, four bouts of $5 \mathrm{~min}$ seems to be equally effective and less tiring for subjects (Steiner et al., 2004; Vogt and Hoppeler, 2014). A training frequency of two sessions per week seems to be the lower limit to induce measurable gains (Mueller et al., 2009).

When conceiving eccentric interventions, practitioners should also take into account the parameters affecting the extent and duration of EIMD and/or slower recovery. Exercises performed at high vs. low eccentric torque, at long vs. short muscle length and increasing numbers of eccentric contractions appear to result in more severe EIMD (Nosaka and Newton, 2002). Skeletal muscles do not display the same vulnerability to EIMD. The upper limb muscles appear mostly more affected than lower limb muscles and the knee flexors more than the knee extensors (Chen et al., 2011). The untrained status, genetic variations, aging and chronic diseases are other factors increasing the severity of potential EIMD (Tidball, 2011; Gault and Willems, 2013; Baumert et al., 2016). Special attention should thus be given to these populations when establishing the initial eccentric exercise prescription in rehabilitation settings.

Even if trained athletes are generally less affected by EIMD than untrained people when submitting to the same eccentric protocol, they might not escape to the detrimental effects of eccentric contractions in some circumstances. They might be particularly vulnerable to EIMD at the start of the season, or when they return to competition after injury or following an unaccustomed eccentric session (Cheung et al., 2003). The occurrence of EIMD associated with unaccustomed eccentric exercise could be problematic in athletes because of the associated negative consequences on locomotor biomechanics and sport performance within the short term (Cheung et al., 2003; Assumpcao Cde et al., 2013). Moreover, when athletes suffered from DOMS, they are frequently unable to train at their maximal intensity which can compromise the quality of the training programs. Even if the negative functional consequences of EIMD are transitory, it seems important to avoid their onset even in healthy athletes. If EIMD have not been avoided, it is recommended not to perform high intensity exercises, particularly explosive efforts. Indeed, the risk of injuries such as muscle tears or ligament rupture has been shown to increase due to the disturbed muscle function and mechanical fragility. Accordingly, it should be kept in mind that even when muscle hyperalgesia is resolved, a decrease in muscle function may persist. Care must thus be exerted in the days following an episode of DOMS (Damas et al., 2016). When experiencing EIMD, stretching should also be avoided since it could interfere with recovery. Since EIMD triggers inflammation response, some practitioners have used non-steroid anti-inflammatory drugs (NSAIDs) in an attempt to attenuate the clinical symptoms (Paulsen et al., 2012). Nevertheless, studies demonstrated that reducing or blocking potential inflammation response may negatively perturb the muscle cell activity and hinder the hypertrophy and regenerative processes (Mackey et al., 2007). Therefore, NSAIDs should be avoided in healthy subjects (Paulsen et al., 2012). Contrary to this, evidence suggests that NSAIDs may be beneficial for subjects characterized by a low-grade systemic inflammation contributing to sarcopenia (Bautmans et al., 2005; Rieu et al., 2009). In the elderly or in individuals with chronic disease, the use of NSAIDs may help to maintain muscle mass (Rieu et al., 2009).

\section{CONCLUSION}

The study of eccentric contraction is no longer confined to muscle physiology and sport sciences but is becoming central in clinical medicine and is likely to expand in the near future. Indeed, due to its unique neural, mechanical and metabolic properties, the eccentric mode has gained a growing interest in several fields. In addition to its efficiency in sports performance and rehabilitation, the eccentric training interventions constitute an attractive strategy to prevent muscle wasting in sarcopenia or in many chronic diseases. Increasing evidence also support the beneficial effects of eccentric exercises on body composition and other health-related parameters, making this contraction mode a promising tool for various patient populations. However, unaccustomed eccentric exercise is well known to induce muscle damage that manifests by a range of clinical symptoms including DOMS and decreased muscle function. Up to now, there is no equivocal therapeutic approach allowing a significant attenuation in the symptoms of damage. Conversely, it has been clearly demonstrated that repeated exposures to eccentric actions with progressively increasing loads can prevent the occurrence of muscle damage or DOMS.

\section{FUTURE PERSPECTIVES}

Although the eccentric contraction has received more attention over the last decade, many questions remain unanswered with regard to both the initial damaging response to unaccustomed eccentric contraction and the subsequent adaptations. Furthermore, the mechanisms behind the protective effect conferred 
by a repeated eccentric bout are still in great part speculative. Yet, the numerous practical applications of eccentric exercise in sports, rehabilitation and pathological conditions justify the need to elucidate the mechanisms underlying the acute and chronic effect of eccentric exercise on the skeletal muscle. In addition, a better knowledge of the transient eccentric induced damage and subsequent adaptations on a mechanistic level may help to further understand the degeneration/regeneration cycles in healthy skeletal muscle and to identify abnormalities in these processes in pathological conditions as in neuromuscular diseases. Given some similarities in the histopathological alterations that follow unaccustomed eccentric actions with those observed in muscular dystrophy pathology, further investigations on the eccentric exercise may unravel crucial issues in molecular mechanisms frequently involved in neuromuscular diseases. Investigations employing rigorous standardization of the experimental conditions in the eccentric and other training groups are necessary to determine the specific multi-target and to

\section{REFERENCES}

Abbott, B. C., Bigland, B., and Ritchie, J. M. (1952). The physiological cost of negative work. J. Physiol. 117, 380-390.

Armstrong, R. B. (1984). Mechanisms of exercise-induced delayed onset muscular soreness: a brief review. Med. Sci. Sports Exerc. 16, 529-538.

Assumpcao Cde, O., Lima, L. C., Oliveira, F. B., Greco, C. C., and Denadai, B. S. (2013). Exercise-induced muscle damage and running economy in humans. ScientificWorldJournal 2013:189149. doi: 10.1155/2013/189149

Barash, I. A., Mathew, L., Ryan, A. F., Chen, J., and Lieber, R. L. (2004). Rapid muscle-specific gene expression changes after a single bout of eccentric contractions in the mouse. Am. J. Physiol. Cell Physiol. 286, C355-C364.

Barnett, A. (2006). Using recovery modalities between training sessions in elite athletes: does it help? Sports Med. 36, 781-796.

Baumert, P., Lake, M. J., Stewart, C. E., Drust, B., and Erskine, R. M. (2016). Interindividual variability in the response to maximal eccentric exercise. Eur. J. Appl. Physiol. 116, 2055-2056.

Bautmans, I., Njemini, R., Lambert, M., Demanet, C., and Mets, T. (2005). Circulating acute phase mediators and skeletal muscle performance in hospitalized geriatric patients. J. Gerontol. A Biol. Sci. Med. Sci. 60, 361-367.

Black, C. D., and McCully, K. K. (2008). Muscle injury after repeated bouts of voluntary and electrically stimulated exercise. Med. Sci. Sports Exerc. 40, 1605-1615. doi: 10.1249/MSS.0b013e3181788dbe

Blazevich, A. J., Cannavan, D., Coleman, D. R., and Horne, S. (2007). Influence of concentric and eccentric resistance training on architectural adaptation in human quadriceps muscles. J. Appl. Physiol. 103, 1565-1575.

Bloomer, R. J. (2007). The role of nutritional supplements in the prevention and treatment of resistance exercise-induced skeletal muscle injury. Sports Med. 37, 519-532.

Brown, S. J., Child, R. B., Day, S. H., and Donnelly, A. E. (1997). Exercise-induced skeletal muscle damage and adaptation following repeated bouts of eccentric muscle contractions. J. Sports Sci. 15, 215-222.

Brughelli, M., and Cronin, J. (2007). Altering the length-tension relationship with eccentric exercise: implications for performance and injury. Sports Med. 37, $7-26$.

Buford, T. W., Cooke, M. B., Shelmadine, B. D., Hudson, G. M., Redd, L., and Willoughby, D. S. (2009). Effects of eccentric treadmill exercise on inflammatory gene expression in human skeletal muscle. Appl. Physiol. Nutr. Metab. 34, 745-753. doi: 10.1139/H09-067

Byrne, C., Twist, C., and Eston, R. (2004). Neuromuscular function after exerciseinduced muscle damage: theoretical and applied implications. Sports Med. 34, 49-69.

Call, J. A., Eckhoff, M. D., Baltgalvis, K. A., Warren, G. L., and Lowe, D. A. (2011). Adaptive strength gains in dystrophic muscle exposed to repeated draw guidelines for eccentric activity prescriptions. In particular, more efforts should be devoted to develop intensity, duration and modes of eccentric training optimizing efficiency of this method.

\section{AUTHOR CONTRIBUTIONS}

$\mathrm{SH}$ wrote the manuscript. J-LC, TB, BR, and PL revised the manuscript according to their respective field of expertise.

\section{FUNDING}

This work was supported by "Fonds de la Recherche Scientifique Médicale" grant (FRSM 3.4559.11) from the Belgian "Fonds de la Recherche Scientifique-Fonds National de la Recherche Scientifique" (F.R.S.-FNRS). PL is a Senior Research Associate of F.R.S.-FNRS.

bouts of eccentric contraction. J. Appl. Physiol. 111, 1768-1777. doi: 10.1152/ japplphysiol.00942.2011

Carter, G. T., Abresch, R. T., and Fowler, W. M. Jr. (2002). Adaptations to exercise training and contraction-induced muscle injury in animal models of muscular dystrophy. Am. J. Phys. Med. Rehabil. 81(Suppl. 11), S151-S161.

Catoire, M., and Kersten, S. (2015). The search for exercise factors in humans. FASEB J. 29, 1615-1628. doi: 10.1096/fj.14-263699

Chaabene, H., Prieske, O., Negra, Y., and Granacher, U. (2018). Change of direction speed: toward a strength training approach with accentuated eccentric muscle actions. Sports Med. 48, 773-779. doi: 10.1007/s40279-018-0907-3

Chazaud, B. (2016). Inflammation during skeletal muscle regeneration and tissue remodeling: application to exercise-induced muscle damage management. Immunol. Cell Biol. 94, 140-145. doi: 10.1038/icb.2015.97

Chen, T. C. (2003). Effects of a second bout of maximal eccentric exercise on muscle damage and electromyographic activity. Eur. J. Appl. Physiol. Occup. Physiol. 89, 115-121.

Chen, T. C., Lin, K. Y., Chen, H. L., Lin, M. J., and Nosaka, K. (2011). Comparison in eccentric exercise-induced muscle damage among four limb muscles. Eur. J. Appl. Physiol. 111, 211-223. doi: 10.1007/s00421-010-1648-7

Chen, T. C., Tseng, W. C., Huang, G. L., Chen, H. L., Tseng, K. W., and Nosaka, K. (2013). Low-intensity eccentric contractions attenuate muscle damage induced by subsequent maximal eccentric exercise of the knee extensors in the elderly. Eur. J. Appl. Physiol. Occup. Physiol. 113, 1005-1015. doi: 10.1007/s00421-0122517-3

Chen, Y. W., Nader, G. A., Baar, K. R., Fedele, M. J., Hoffman, E. P., and Esser, K. A. (2002). Response of rat muscle to acute resistance exercise defined by transcriptional and translational profiling. J. Physiol. 545(Pt 1), 7-41.

Cheung, K., Hume, P., and Maxwell, L. (2003). Delayed onset muscle soreness: treatment strategies and performance factors. Sports Med. 33, 145-164.

Clark, D. J., and Patten, C. (2013). Eccentric versus concentric resistance training to enhance neuromuscular activation and walking speed following stroke. Neurorehabil. Neural Repair 27, 335-344. doi: 10.1177/154596831246 9833

Clarkson, P. M. (1992). Exercise-induced muscle damage-animal and human models. Med. Sci. Sports Exerc. 24, 510-511.

Clarkson, P. M., and Hubal, M. J. (2002). Exercise-induced muscle damage in humans. Am. J. Phys. Med. Rehabil. 81(Suppl. 11), S52-S69.

Clarkson, P. M., Nosaka, K., and Braun, B. (1992). Muscle function after exerciseinduced muscle damage and rapid adaptation. Med. Sci. Sports Exerc. 24, 512-520.

Coffey, V. G., and Hawley, J. A. (2007). The molecular bases of training adaptation. Sports Med. 37, 737-763.

Crameri, R. M., Aagaard, P., Qvortrup, K., Langberg, H., Olesen, J., and Kjaer, M. (2007). Myofibre damage in human skeletal muscle: effects of 
electrical stimulation versus voluntary contraction. J. Physiol. 583(Pt 1), 365-380.

Crameri, R. M., Langberg, H., Magnusson, P., Jensen, C. H., Schroder, H. D., Olesen, J. L., et al. (2004). Changes in satellite cells in human skeletal muscle after a single bout of high intensity exercise. J. Physiol. 558(Pt 1), 333-340.

Croisier, J. L., Camus, G., Venneman, I., Deby-Dupont, G., Juchmes-Ferir, A., Lamy, M., et al. (1999). Effects of training on exercise-induced muscle damage and interleukin 6 production. Muscle Nerve 22, 208-212.

Croisier, J. L., Foidart-Dessalle, M., Tinant, F., Crielaard, J. M., and Forthomme, B. (2007). An isokinetic eccentric programme for the management of chronic lateral epicondylar tendinopathy. Br. J. Sports Med. 41, 69-75.

Croisier, J. L., Forthomme, B., Namurois, M. H., Vanderthommen, M., and Crielaard, J. M. (2002). Hamstring muscle strain recurrence and strength performance disorders. Am. J. Sports Med. 30, 199-203.

Croisier, J. L., Ganteaume, S., Binet, J., Genty, M., and Ferret, J. M. (2008). Strength imbalances and prevention of hamstring injury in professional soccer players: a prospective study. Am. J. Sports Med. 36, 469-475. doi: 10.1177/ 0363546508316764

Croisier, J. L., Maquet, D., Crielaard, J. M., and Forthomme, B. (2009). Quelles applications du travail excentrique en reeducation? Kinésithér. Rev. 9, 85-86.

Damas, F., Nosaka, K., Libardi, C. A., Chen, T. C., and Ugrinowitsch, C. (2016). Susceptibility to exercise-induced muscle damage: a cluster analysis with a large sample. Int. J. Sports Med. 37, 33-40. doi: 10.1055/s-0042-100281

Dickinson, J. M., Volpi, E., and Rasmussen, B. B. (2013). Exercise and nutrition to target protein synthesis impairments in aging skeletal muscle. Exerc. Sport Sci. Rev. 41, 216-223. doi: 10.1097/JES.0b013e3182a4e699

Douglas, J., Pearson, S., Ross, A., and McGuigan, M. (2017). Chronic adaptations to eccentric training: a systematic review. Sports Med. 47, 17-41.

Duchateau, J., and Baudry, S. (2014). Insights into the neural control of eccentric contractions. J. Appl. Physiol. 116, 1418-1425. doi: 10.1152/japplphysiol.00002. 2013

Engardt, M., Knutsson, E., Jonsson, M., and Sternhag, M. (1995). Dynamic muscle strength training in stroke patients: effects on knee extension torque, electromyographic activity, and motor function. Arch. Phys. Med. Rehabil. 76, 419-425.

Feasson, L., Stockholm, D., Freyssenet, D., Richard, I., Duguez, S., Beckmann, J. S., et al. (2002). Molecular adaptations of neuromuscular disease-associated proteins in response to eccentric exercise in human skeletal muscle. J. Physiol. 543(Pt 1), 297-306.

Febbraio, M. A., and Pedersen, B. K. (2002). Muscle-derived interleukin6: mechanisms for activation and possible biological roles. FASEB J. 16, 1335-1347.

Flann, K. L., LaStayo, P. C., McClain, D. A., Hazel, M., and Lindstedt, S. L. (2011). Muscle damage and muscle remodeling: no pain, no gain? J. Exp. Biol. 214(Pt 4), 674-679. doi: 10.1242/jeb.050112

Forthomme, B., Wieczorek, V., Frisch, A., Crielaard, J. M., and Croisier, J. L. (2013). Shoulder pain among high-level volleyball players and preseason features. Med. Sci. Sports Exerc. 45, 1852-1860. doi: 10.1249/MSS.0b013e318296128d

Franchi, M. V., Atherton, P. J., Reeves, N. D., Fluck, M., Williams, J., Mitchell, W. K., et al. (2014). Architectural, functional and molecular responses to concentric and eccentric loading in human skeletal muscle. Acta Physiol. 210, 642-654. doi: 10.1111/apha.12225

Franchi, M. V., Mitchell, K. W., Hoppeler, H., and Narici, M. V. (2017a). Editorial: physiology and clinical potential of eccentric exercise. Front. Physiol. 8:891. doi: 10.3389/fphys.2017.00891

Franchi, M. V., Reeves, N. D., and Narici, M. V. (2017b). Skeletal muscle remodeling in response to eccentric vs. concentric loading: morphological, molecular, and metabolic adaptations. Front. Physiol. 8:447. doi: 10.3389/fphys. 2017.00447

Frankenberg, N. T., Lamb, G. D., Overgaard, K., Murphy, R. M., and Vissing, K. (2014). Small heat shock proteins translocate to the cytoskeleton in human skeletal muscle following eccentric exercise independently of phosphorylation. J. Appl. Physiol. 116, 1463-1472. doi: 10.1152/japplphysiol.01026.2013

Friden, J., and Lieber, R. L. (1992). Structural and mechanical basis of exerciseinduced muscle injury. Med. Sci. Sports Exerc. 24, 521-530.

Friden, J., and Lieber, R. L. (1998). Segmental muscle fiber lesions after repetitive eccentric contractions. Cell Tissue Res. 293, 165-171.
Friden, J., and Lieber, R. L. (2001). Eccentric exercise-induced injuries to contractile and cytoskeletal muscle fibre components. Acta Physiol. Scand. 171, 321-326.

Friden, J., Seger, J., Sjostrom, M., and Ekblom, B. (1983a). Adaptive response in human skeletal muscle subjected to prolonged eccentric training. Int. J. Sports Med. 4, 177-183.

Friden, J., Sjostrom, M., and Ekblom, B. (1983b). Myofibrillar damage following intense eccentric exercise in man. Int. J. Sports Med. 4, 170-176.

Gault, M. L., and Willems, M. E. (2013). Aging, functional capacity and eccentric exercise training. Aging Dis. 4, 351-363. doi: 10.14336/AD.2013.0400351

Gerber, J. P., Marcus, R. L., Dibble, L. E., Greis, P. E., Burks, R. T., and Lastayo, P. C. (2007). Safety, feasibility, and efficacy of negative work exercise via eccentric muscle activity following anterior cruciate ligament reconstruction. J. Orthop. Sports Phys. Ther. 37, 10-18.

Gerber, J. P., Marcus, R. L., Dibble, L. E., Greis, P. E., Burks, R. T., and LaStayo, P. C. (2009). Effects of early progressive eccentric exercise on muscle size and function after anterior cruciate ligament reconstruction: a 1-year follow-up study of a randomized clinical trial. Phys. Ther. 89, 51-59. doi: 10.2522/ptj. 20070189

Guilhem, G., Cornu, C., and Guevel, A. (2010). Neuromuscular and muscle-tendon system adaptations to isotonic and isokinetic eccentric exercise. Ann. Phys. Rehabil. Med. 53, 319-341. doi: 10.1016/j.rehab.2010.04.003

Hamer, P. W., McGeachie, J. M., Davies, M. J., and Grounds, M. D. (2002). Evans Blue Dye as an in vivo marker of myofibre damage: optimising parameters for detecting initial myofibre membrane permeability. J. Anat. 200(Pt 1), 9-79.

Hayes, H. A., Gappmaier, E., and LaStayo, P. C. (2011). Effects of high-intensity resistance training on strength, mobility, balance, and fatigue in individuals with multiple sclerosis: a randomized controlled trial. J. Neurol. Phys. Ther. 35, 2-10. doi: 10.1097/NPT.0b013e31820b5a9d

Hentzen, E. R., Lahey, M., Peters, D., Mathew, L., Barash, I. A., Friden, J., et al. (2006). Stress-dependent and -independent expression of the myogenic regulatory factors and the MARP genes after eccentric contractions in rats. J. Physiol. 570(Pt 1), 157-167.

Higbie, E. J., Cureton, K. J., Warren, G. L. III, and Prior, B. M. (1996). Effects of concentric and eccentric training on muscle strength, cross-sectional area, and neural activation. J. Appl. Physiol. 81, 2173-2181.

Hody, S., Lacrosse, Z., Leprince, P., Collodoro, M., Croisier, J. L., and Rogister, B. (2013a). Effects of eccentrically and concentrically biased training on mouse muscle phenotype. Med. Sci. Sports Exerc. 45, 1460-1468. doi: 10.1249/MSS. ob013e3182894a33

Hody, S., Rogister, B., Leprince, P., Laglaine, T., and Croisier, J. L. (2013b). The susceptibility of the knee extensors to eccentric exercise-induced muscle damage is not affected by leg dominance but by exercise order. Clin. Physiol. Funct. Imaging 33, 373-380. doi: 10.1111/cpf.12040

Hody, S., Rogister, B., Leprince, P., Wang, F., and Croisier, J. L. (2013c). Muscle fatigue experienced during maximal eccentric exercise is predictive of the plasma creatine kinase (CK) response. Scand. J. Med. Sci. Sports 23, 501-507. doi: 10.1111/j.1600-0838.2011.01413.x

Hody, S., Leprince, P., Sergeant, K., Renaut, J., Croisier, J. L., Wang, F., et al. (2011). Human muscle proteome modifications after acute or repeated eccentric exercises. Med. Sci. Sports Exerc. 43, 2281-2296. doi: 10.1249/MSS. ob013e318222edf3

Hody, S., Rogister, B., Croisier, J. L., Wang, F., and Leprince, P. (2009). "Courbatures après exercice excentrique: high et Low responders?," in Exercice Musculaire Excentrique, Vol. 65, eds J. L. Croisier and P. Codine (Paris: Masson), 183-191.

Hoppeler, H. (2016). Moderate load eccentric exercise; a distinct novel training modality. Front. Physiol. 7:483. doi: 10.3389/fphys.2016.00483

Hoppeler, H., and Herzog, W. (2014). Eccentric exercise: many questions unanswered. J. Appl. Physiol. 116, 405-406.

Hortobagyi, T., and Katch, F. I. (1990). Eccentric and concentric torque-velocity relationships during arm flexion and extension. Influence of strength level. Eur. J. Appl. Physiol. Occup. Physiol. 60, 395-401.

Hortobagyi, T., Lambert, N. J., and Hill, J. P. (1997). Greater cross education following training with muscle lengthening than shortening. Med. Sci. Sports Exerc. 29, 107-112. 
Howatson, G., and van Someren, K. A. (2007). Evidence of a contralateral repeated bout effect after maximal eccentric contractions. Eur. J. Appl. Physiol. 101, 207-214.

Howatson, G., and van Someren, K. A. (2008). The prevention and treatment of exercise-induced muscle damage. Sports Med. 38, 483-503.

Howell, J. N., Chleboun, G., and Conatser, R. (1993). Muscle stiffness, strength loss, swelling and soreness following exercise-induced injury in humans. J. Physiol. 464, 183-196.

Hubal, M. J., Chen, T. C., Thompson, P. D., and Clarkson, P. M. (2008). Inflammatory gene changes associated with the repeated-bout effect. Am. J. Physiol. Regul. Integr. Comp. Physiol. 294, R1628-R1637. doi: 10.1152/ajpregu. 00853.2007

Hyldahl, R. D., and Hubal, M. J. (2014). Lengthening our perspective: morphological, cellular, and molecular responses to eccentric exercise. Muscle Nerve 49, 155-170. doi: 10.1002/mus.24077

Hyldahl, R. D., Nelson, B., Xin, L., Welling, T., Groscost, L., Hubal, M. J., et al. (2015). Extracellular matrix remodeling and its contribution to protective adaptation following lengthening contractions in human muscle. FASEB J. 29, 2894-2904. doi: 10.1096/fj.14-266668

Hyldahl, R. D., Olson, T., Welling, T., Groscost, L., and Parcell, A. C. (2014). Satellite cell activity is differentially affected by contraction mode in human muscle following a work-matched bout of exercise. Front. Physiol. 5:485. doi: $10.3389 /$ fphys.2014.00485

Isner-Horobeti, M. E., Dufour, S. P., Vautravers, P., Geny, B., Coudeyre, E., and Richard, R. (2013). Eccentric exercise training: modalities, applications and perspectives. Sports Med. 43, 483-512. doi: 10.1007/s40279-0130052-y

Jones, D. A., Newham, D. J., Round, J. M., and Tolfree, S. E. (1986). Experimental human muscle damage: morphological changes in relation to other indices of damage. J. Physiol. 375, 435-448.

Julian, V., Thivel, D., Costes, F., Touron, J., Boirie, Y., Pereira, B., et al. (2018). Eccentric training improves body composition by inducing mechanical and metabolic adaptations: a promising approach for overweight and obese individuals. Front. Physiol. 9:1013. doi: 10.3389/fphys.2018.01013

Julian, V., Thivel, D., Miguet, M., Pereira, B., Costes, F., Coudeyre, E., et al. (2019). Eccentric cycling is more efficient in reducing fat mass than concentric cycling in adolescents with obesity. Scand. J. Med. Sci. Sports 29, 4-15. doi: 10.1111/sms. 13301

Kaux, J. F., Drion, P., Libertiaux, V., Colige, A., Hoffmann, A., Nusgens, B., et al. (2013). Eccentric training improves tendon biomechanical properties: a rat model. J. Orthop. Res. 31, 119-124. doi: 10.1002/jor.22202

Kim, J., and Lee, J. (2017). Role of transforming growth factor-beta in muscle damage and regeneration: focused on eccentric muscle contraction. J. Exerc. Rehabil. 13, 621-626. doi: 10.12965/jer.1735072.536

Kjaer, M., and Heinemeier, K. M. (2014). Eccentric exercise: acute and chronic effects on healthy and diseased tendons. J. Appl. Physiol. 116, 1435-1438. doi: 10.1152/japplphysiol.01044.2013

Kostek, M. C., Chen, Y. W., Cuthbertson, D. J., Shi, R., Fedele, M. J., Esser, K. A., et al. (2007). Gene expression responses over $24 \mathrm{~h}$ to lengthening and shortening contractions in human muscle: major changes in CSRP3, MUSTN1, SIX1, and FBXO32. Physiol. Genomics 31, 42-52.

LaStayo, P., Marcus, R., Dibble, L., Frajacomo, F., and Lindstedt, S. (2014). Eccentric exercise in rehabilitation: safety, feasibility, and application. J. Appl. Physiol. 116, 1426-1434. doi: 10.1152/japplphysiol.00008.2013

LaStayo, P., McDonagh, P., Lipovic, D., Napoles, P., Bartholomew, A., Esser, K., et al. (2007). Elderly patients and high force resistance exercise-a descriptive report: can an anabolic, muscle growth response occur without muscle damage or inflammation? J. Geriatr. Phys. Ther. 30, 128-134.

LaStayo, P. C., Ewy, G. A., Pierotti, D. D., Johns, R. K., and Lindstedt, S. (2003a). The positive effects of negative work: increased muscle strength and decreased fall risk in a frail elderly population. J. Gerontol. Ser. A Biol. Sci. Med. Sci. 58, M419-M424.

LaStayo, P. C., Woolf, J. M., Lewek, M. D., Snyder-Mackler, L., Reich, T., and Lindstedt, S. L. (2003b). Eccentric muscle contractions: their contribution to injury, prevention, rehabilitation, and sport. J. Orthop. Sports Phys. Ther. 33, 557-571.

LaStayo, P. C., Pierotti, D. J., Pifer, J., Hoppeler, H., and Lindstedt, S. L. (2000). Eccentric ergometry: increases in locomotor muscle size and strength at low training intensities. Am. J. Physiol. Regul. Integr. Comp. Physiol. 278, R1282R1288.

Lastayo, P. C., Reich, T. E., Urquhart, M., Hoppeler, H., and Lindstedt, S. L. (1999). Chronic eccentric exercise: improvements in muscle strength can occur with little demand for oxygen. Am. J. Physiol. 276, R611-R615. doi: 10.1152/ajpregu. 1999.276.2.R611

Lauritzen, F., Paulsen, G., Raastad, T., Bergersen, L. H., and Owe, S. G. (2009). Gross ultrastructural changes and necrotic fiber segments in elbow flexor muscles after maximal voluntary eccentric action in humans. J. Appl. Physiol. 107, 1923-1934. doi: 10.1152/japplphysiol.00148.2009

Lehti, T. M., Kalliokoski, R., and Komulainen, J. (2007). Repeated bout effect on the cytoskeletal proteins titin, desmin, and dystrophin in rat skeletal muscle. J. Muscle Res. Cell Motil. 28, 39-47.

Lieber, R. L., and Friden, J. (1988). Selective damage of fast glycolytic muscle fibres with eccentric contraction of the rabbit tibialis anterior. Acta Physiol. Scand. $133,587-588$.

Lieber, R. L., and Friden, J. (1993). Muscle damage is not a function of muscle force but active muscle strain. J. Appl. Physiol. 74, 520-526.

Lieber, R. L., and Friden, J. (1999). Mechanisms of muscle injury after eccentric contraction. J. Sci. Med. Sport 2, 253-265.

Lindstedt, S. L., LaStayo, P. C., and Reich, T. E. (2001). When active muscles lengthen: properties and consequences of eccentric contractions. News Physiol. Sci. 16, 256-261.

Lindstedt, S. L., Reich, T. E., Keim, P., and LaStayo, P. C. (2002). Do muscles function as adaptable locomotor springs? J. Exp. Biol. 205(Pt 15), 2211-2216.

Liu, C., Chen, C. S., Ho, W. H., Fule, R. J., Chung, P. H., and Shiang, T. Y. (2013). The effects of passive leg press training on jumping performance, speed, and muscle power. J. Strength. Cond. Res. 27, 1479-1486. doi: 10.1519/JSC. 0b013e31826bde9f

Lovering, R. M., and Brooks, S. V. (2014). Eccentric exercise in aging and diseased skeletal muscle: good or bad? J. Appl. Physiol. 116, 1439-1445. doi: 10.1152/ japplphysiol.00174.2013

Lynn, R., and Morgan, D. L. (1994). Decline running produces more sarcomeres in rat vastus intermedius muscle fibers than does incline running. J. Appl. Physiol. 77, 1439-1444.

Mackey, A. L., Kjaer, M., Dandanell, S., Mikkelsen, K. H., Holm, L., Dossing, S., et al. (2007). The influence of anti-inflammatory medication on exerciseinduced myogenic precursor cell responses in humans. J. Appl. Physiol. 103, 425-431.

Mado, K., Chekulayev, V., Shevchuk, I., Puurand, M., Tepp, K., and Kaambre, T. (2019). On the role of tubulin, plectin, desmin and vimentin in the regulation of mitochondrial energy fluxes in muscle cells. Am. J. Physiol. Cell Physiol. doi: 10.1152/ajpcell.00303.2018

Malm, C., and Yu, J. G. (2012). Exercise-induced muscle damage and inflammation: re-evaluation by proteomics. Histochem. Cell Biol. 138, 89-99. doi: $10.1007 / \mathrm{s} 00418-012-0946-\mathrm{z}$

Manfredi, T. G., Fielding, R. A., O’Reilly, K. P., Meredith, C. N., Lee, H. Y., and Evans, W. J. (1991). Plasma creatine kinase activity and exercise-induced muscle damage in older men. Med. Sci. Sports Exerc. 23, 1028-1034.

Marcus, R. L., Smith, S., Morrell, G., Addison, O., Dibble, L. E., Wahoff-Stice, D., et al. (2008). Comparison of combined aerobic and high-force eccentric resistance exercise with aerobic exercise only for people with type 2 diabetes mellitus. Phys. Ther. 88, 1345-1354. doi: 10.2522/ptj.20080124

McHugh, M. P. (2003). Recent advances in the understanding of the repeated bout effect: the protective effect against muscle damage from a single bout of eccentric exercise. Scand. J. Med. Sci. Sports 13, 88-97.

McHugh, M. P., Connolly, D. A., Eston, R. G., and Gleim, G. W. (1999a). Exerciseinduced muscle damage and potential mechanisms for the repeated bout effect. Sports Med. 27, 157-170.

McHugh, M. P., Connolly, D. A., Eston, R. G., Kremenic, I. J., Nicholas, S. J., and Gleim, G. W. (1999b). The role of passive muscle stiffness in symptoms of exercise-induced muscle damage. Am. J. Sports Med. 27, 594-599.

McKelvey, L., Shorten, G. D., and O’Keeffe, G. W. (2013). Nerve growth factormediated regulation of pain signalling and proposed new intervention strategies in clinical pain management. J. Neurochem. 124, 276-289. doi: 10.1111/jnc. 12093

McNeil, P. L., and Khakee, R. (1992). Disruptions of muscle fiber plasma membranes. Role in exercise-induced damage. Am. J. Pathol. 140, 1097-1109. 
Meyer, K., Steiner, R., Lastayo, P., Lippuner, K., Allemann, Y., Eberli, F., et al. (2003). Eccentric exercise in coronary patients: central hemodynamic and metabolic responses. Med. Sci. Sports Exerc. 35, 1076-1082.

Mizumura, K., and Taguchi, T. (2016). Delayed onset muscle soreness: involvement of neurotrophic factors. J. Physiol. Sci. 66, 43-52.

Morgan, D. L. (1990). New insights into the behavior of muscle during active lengthening. Biophys. J. 57, 209-221.

Morgan, D. L., and Allen, D. G. (1999). Early events in stretch-induced muscle damage. J. Appl. Physiol. 87, 2007-2015.

Mueller, M., Breil, F. A., Lurman, G., Klossner, S., Fluck, M., Billeter, R., et al. (2011). Different molecular and structural adaptations with eccentric and conventional strength training in elderly men and women. Gerontology 57, 528-538. doi: 10.1159/000323267

Mueller, M., Breil, F. A., Vogt, M., Steiner, R., Lippuner, K., Popp, A., et al. (2009). Different response to eccentric and concentric training in older men and women. Eur. J. Appl. Physiol. 107, 145-153. doi: 10.1007/s00421-009-1108-4

Murase, S., Terazawa, E., Hirate, K., Yamanaka, H., Kanda, H., Noguchi, K., et al. (2013). Upregulated glial cell line-derived neurotrophic factor through cyclooxygenase- 2 activation in the muscle is required for mechanical hyperalgesia after exercise in rats. J. Physiol. 591, 3035-3048. doi: 10.1113/ jphysiol.2012.249235

Neubauer, O., Sabapathy, S., Ashton, K. J., Desbrow, B., Peake, J. M., Lazarus, R., et al. (2014). Time course-dependent changes in the transcriptome of human skeletal muscle during recovery from endurance exercise: from inflammation to adaptive remodeling. J. Appl. Physiol. 116, 274-287. doi: 10.1152/japplphysiol. 00909.2013

Newham, D. J., Jones, D. A., and Clarkson, P. M. (1987). Repeated high-force eccentric exercise: effects on muscle pain and damage. J. Appl. Physiol. 63, 1381-1386.

Nicol, C., Avela, J., and Komi, P. V. (2006). The stretch-shortening cycle: a model to study naturally occurring neuromuscular fatigue. Sports Med. 36, 977-999.

Nie, H., Madeleine, P., Arendt-Nielsen, L., and Graven-Nielsen, T. (2009). Temporal summation of pressure pain during muscle hyperalgesia evoked by nerve growth factor and eccentric contractions. Eur. J. Pain 13, 704-710. doi: 10.1016/j.ejpain.2008.06.015

Nosaka, K., and Aoki, M. S. (2011). Repeated bout effect: research update and future perspective. Braz. J. Biomotricity 5, 5-15.

Nosaka, K., and Clarkson, P. M. (1995). Muscle damage following repeated bouts of high force eccentric exercise. Med. Sci. Sports Exerc. 27, 1263-1269.

Nosaka, K., and Clarkson, P. M. (1996). Variability in serum creatine kinase response after eccentric exercise of the elbow flexors. Int. J. Sports Med. 17, 120-127.

Nosaka, K., and Newton, M. (2002). Difference in the magnitude of muscle damage between maximal and submaximal eccentric loading. J. Strength. Cond. Res. 16, 202-208.

Nosaka, K., Newton, M., and Sacco, P. (2002). Delayed-onset muscle soreness does not reflect the magnitude of eccentric exercise-induced muscle damage. Scand. J. Med. Sci. Sports 12, 337-346.

Nosaka, K., Newton, M. J., and Sacco, P. (2005). Attenuation of protective effect against eccentric exercise-induced muscle damage. Can. J. Appl. Physiol. 30, 529-542.

Nosaka, K., Sakamoto, K., Newton, M., and Sacco, P. (2001). How long does the protective effect on eccentric exercise-induced muscle damage last? Med. Sci. Sports Exerc. 33, 1490-1495.

Okamoto, T., Masuhara, M., and Ikuta, K. (2006). Effects of eccentric and concentric resistance training on arterial stiffness. J. Hum. Hypertens. 20, 348-354.

Orejuela, D., Bergeron, A., Morrow, G., and Tanguay, R. (2007). "Small heat shock proteins in physiological and stress-related processes," in Cell Stress Proteins. Protein Reviews, Vol. 7, ed. S. K. Calderwood (New York, NY: Springer), $143-177$.

Overend, T. J., Versteegh, T. H., Thompson, E., Birmingham, T. B., and Vandervoort, A. A. (2000). Cardiovascular stress associated with concentric and eccentric isokinetic exercise in young and older adults. J. Gerontol. Ser. A Biol. Sci. Med. Sci. 55, B177-B182.

Overgaard, K., Lindstrom, T., Ingemann-Hansen, T., and Clausen, T. (2002). Membrane leakage and increased content of $\mathrm{Na}^{+}-\mathrm{K}^{+}$pumps and $\mathrm{Ca}^{2+}$ in human muscle after a 100-km run. J. Appl. Physiol. 92, 1891-1898.
Palmisano, M. G., Bremner, S. N., Hornberger, T. A., Meyer, G. A., Domenighetti, A. A., Shah, S. B., et al. (2015). Skeletal muscle intermediate filaments form a stress-transmitting and stress-signaling network. J. Cell Sci. 128, 219-224. doi: $10.1242 /$ jcs. 142463

Paschalis, V., Nikolaidis, M. G., Giakas, G., Theodorou, A. A., Sakellariou, G. K., Fatouros, I. G., et al. (2010). Beneficial changes in energy expenditure and lipid profile after eccentric exercise in overweight and lean women. Scand. J. Med. Sci. Sports 20, e103-e111. doi: 10.1111/j.1600-0838.2009.00920.x

Paschalis, V., Nikolaidis, M. G., Theodorou, A. A., Deli, C. K., Raso, V., Jamurtas, A. Z., et al. (2013). The effects of eccentric exercise on muscle function and proprioception of individuals being overweight and underweight. J. Strength. Cond. Res. 27, 2542-2551. doi: 10.1519/JSC.0b013e31827fc9a6

Paulsen, G., Crameri, R., Benestad, H. B., Fjeld, J. G., Morkrid, L., Hallen, J., et al. (2010). Time course of leukocyte accumulation in human muscle after eccentric exercise. Med. Sci. Sports Exerc. 42, 75-85. doi: 10.1249/MSS. 0b013e3181ac7adb

Paulsen, G., Lauritzen, F., Bayer, M. L., Kalhovde, J. M., Ugelstad, I., Owe, S. G., et al. (2009). Subcellular movement and expression of HSP27, alphaB-crystallin, and HSP70 after two bouts of eccentric exercise in humans. J. Appl. Physiol. 107, 570-582. doi: 10.1152/japplphysiol.00209.2009

Paulsen, G., Mikkelsen, U. R., Raastad, T., and Peake, J. M. (2012). Leucocytes, cytokines and satellite cells: what role do they play in muscle damage and regeneration following eccentric exercise? Exerc. Immunol. Rev. 18, 42-97.

Paulsen, G., Vissing, K., Kalhovde, J. M., Ugelstad, I., Bayer, M. L., Kadi, F., et al. (2007). Maximal eccentric exercise induces a rapid accumulation of small heat shock proteins on myofibrils and a delayed HSP70 response in humans. Am. J. Physiol. Regul. Integr. Comp. Physiol. 293, R844-R853.

Peake, J., Nosaka, K., and Suzuki, K. (2005). Characterization of inflammatory responses to eccentric exercise in humans. Exerc. Immunol. Rev. 11, 64-85.

Peake, J. M., Neubauer, O., Della Gatta, P. A., and Nosaka, K. (2017). Muscle damage and inflammation during recovery from exercise. J. Appl. Physiol. 122, 559-570.

Peake, J. M., Suzuki, K., Hordern, M., Wilson, G., Nosaka, K., and Coombes, J. S. (2005). Plasma cytokine changes in relation to exercise intensity and muscle damage. Eur. J. Appl. Physiol. 95, 514-521.

Pedersen, B. K. (2012). Muscular interleukin-6 and its role as an energy sensor. Med. Sci. Sports Exerc. 44, 392-396. doi: 10.1249/MSS.0b013e31822f94ac

Pedersen, B. K., and Febbraio, M. A. (2008). Muscle as an endocrine organ: focus on muscle-derived interleukin-6. Physiol. Rev. 88, 1379-1406. doi: 10.1152/ physrev.90100.2007

Peters, D., Barash, I. A., Burdi, M., Yuan, P. S., Mathew, L., Friden, J., et al. (2003). Asynchronous functional, cellular and transcriptional changes after a bout of eccentric exercise in the rat. J. Physiol. 553(Pt 3), 947-957.

Pizza, F. X., Davis, B. H., Henrickson, S. D., Mitchell, J. B., Pace, J. F., Bigelow, N., et al. (1996). Adaptation to eccentric exercise: effect on CD64 and CD11b/CD18 expression. J. Appl. Physiol. 80, 47-55.

Powers, S. K., and Jackson, M. J. (2008). Exercise-induced oxidative stress: cellular mechanisms and impact on muscle force production. Physiol. Rev. 88, 12431276. doi: 10.1152/physrev.00031.2007

Raastad, T., Owe, S. G., Paulsen, G., Enns, D., Overgaard, K., Crameri, R., et al. (2010). Changes in calpain activity, muscle structure, and function after eccentric exercise. Med. Sci. Sports Exerc. 42, 86-95. doi: 10.1249/MSS. 0b013e3181ac7afa

Rattray, B., Caillaud, C., Ruell, P. A., and Thompson, M. W. (2011). Heat exposure does not alter eccentric exercise-induced increases in mitochondrial calcium and respiratory dysfunction. Eur. J. Appl. Physiol. 111, 2813-2821.

Rattray, B., Thompson, M., Ruell, P., and Caillaud, C. (2013). Specific training improves skeletal muscle mitochondrial calcium homeostasis after eccentric exercise. Eur. J. Appl. Physiol. 113, 427-436. doi: 10.1007/s00421-0122446-1

Reeves, N. D., Maganaris, C. N., Longo, S., and Narici, M. V. (2009). Differential adaptations to eccentric versus conventional resistance training in older humans. Exp. Physiol. 94, 825-833. doi: 10.1113/expphysiol.2009.046599

Reich, T. E., Lindstedt, S. L., LaStayo, P. C., and Pierotti, D. J. (2000). Is the spring quality of muscle plastic? Am. J. Physiol. Regul. Integr. Comp. Physiol. 278, R1661-R1666.

Ridgley, J. A., Pinniger, G. J., Hamer, P. W., and Grounds, M. D. (2009). The physiological effects of IGF-1 (class 1:Ea transgene) over-expression on 
exercise-induced damage and adaptation in dystrophic muscles of mdx mice. Pflugers Arch. 457, 1121-1132. doi: 10.1007/s00424-008-0568-4

Rieu, I., Magne, H., Savary-Auzeloux, I., Averous, J., Bos, C., Peyron, M. A., et al. (2009). Reduction of low grade inflammation restores blunting of postprandial muscle anabolism and limits sarcopenia in old rats. J. Physiol. 587(Pt 22), 5483-5492. doi: 10.1113/jphysiol.2009.178319

Roig, M., Macintyre, D. L., Eng, J. J., Narici, M. V., Maganaris, C. N., and Reid, W. D. (2010). Preservation of eccentric strength in older adults: evidence, mechanisms and implications for training and rehabilitation. Exp. Gerontol. 45, 400-409. doi: 10.1016/j.exger.2010.03.008

Roig, M., O’Brien, K., Kirk, G., Murray, R., McKinnon, P., Shadgan, B., et al. (2009). The effects of eccentric versus concentric resistance training on muscle strength and mass in healthy adults: a systematic review with meta-analysis. Br. J. Sports Med. 43, 556-568. doi: 10.1136/bjsm.2008.051417

Roig, M., Shadgan, B., and Reid, W. D. (2008). Eccentric exercise in patients with chronic health conditions: a systematic review. Physiother. Can. 60, 146-160. doi: 10.3138/physio.60.2.146

Sam, M., Shah, S., Friden, J., Milner, D. J., Capetanaki, Y., and Lieber, R. L. (2000). Desmin knockout muscles generate lower stress and are less vulnerable to injury compared with wild-type muscles. Am. J. Physiol. Cell Physiol. 279, C1116-C1122.

Sanghvi-Shah, R., and Weber, G. F. (2017). Intermediate filaments at the junction of mechanotransduction, migration, and development. Front. Cell. Dev. Biol. 5:81. doi: 10.3389/fcell.2017.00081

Sayers, S. P., Clarkson, P. M., Rouzier, P. A., and Kamen, G. (1999). Adverse events associated with eccentric exercise protocols: six case studies. Med. Sci. Sports Exerc. 31, 1697-1702.

Schnyder, S., and Handschin, C. (2015). Skeletal muscle as an endocrine organ: PGC-1alpha, myokines and exercise. Bone 80, 115-125. doi: 10.1016/j.bone. 2015.02.008

Schoenfeld, B. J., Ogborn, D. I., Vigotsky, A. D., Franchi, M. V., and Krieger, J. W. (2017). Hypertrophic effects of concentric vs. eccentric muscle actions: a systematic review and meta-analysis. J. Strength. Cond. Res. 31, 2599-2608. doi: 10.1519/JSC.0000000000001983

Starbuck, C., and Eston, R. G. (2012). Exercise-induced muscle damage and the repeated bout effect: evidence for cross transfer. Eur. J. Appl. Physiol. Occup. Physiol. 112, 1005-1013. doi: 10.1007/s00421-011-2053-6

Steiner, R., Meyer, K., Lippuner, K., Schmid, J. P., Saner, H., and Hoppeler, H. (2004). Eccentric endurance training in subjects with coronary artery disease: a novel exercise paradigm in cardiac rehabilitation? Eur. J. Appl. Physiol. 91, $572-578$.

Strojnik, V., Komi, P. V., and Nicol, C. (2001). "Fatigue during one-week tourist alpine skiing," in Science and Skiing II, ed. E. Müller (Hamburg: Kovac), 599-607.

Takekura, H., Fujinami, N., Nishizawa, T., Ogasawara, H., and Kasuga, N. (2001). Eccentric exercise-induced morphological changes in the membrane systems involved in excitation-contraction coupling in rat skeletal muscle. J. Physiol. 533(Pt 2), 571-583.

Talbot, J. A., and Morgan, D. L. (1998). The effects of stretch parameters on eccentric exercise-induced damage to toad skeletal muscle. J. Muscle Res. Cell Motil. 19, 237-245.
Tee, J. C., Bosch, A. N., and Lambert, M. I. (2007). Metabolic consequences of exercise-induced muscle damage. Sports Med. 37, 27-36.

Thompson, H. S., Clarkson, P. M., and Scordilis, S. P. (2002). The repeated bout effect and heat shock proteins: intramuscular HSP27 and HSP70 expression following two bouts of eccentric exercise in humans. Acta Physiol. Scand. 174, 47-56.

Tidball, J. G. (2011). Mechanisms of muscle injury, repair, and regeneration. Compr. Physiol. 1, 2029-2062. doi: 10.1002/cphy.c100092

Turrini, P., Gaetano, C., Antonelli, A., Capogrossi, M. C., and Aloe, L. (2002). Nerve growth factor induces angiogenic activity in a mouse model of hindlimb ischemia. Neurosci. Lett. 323, 109-112.

Vikne, H., Refsnes, P. E., Ekmark, M., Medbo, J. I., Gundersen, V., and Gundersen, K. (2006). Muscular performance after concentric and eccentric exercise in trained men. Med. Sci. Sports Exerc. 38, 1770-1781.

Vincent, H. K., and Vincent, K. R. (1997). The effect of training status on the serum creatine kinase response, soreness and muscle function following resistance exercise. Int. J. Sports Med. 18, 431-437.

Vissing, K., Bayer, M. L., Overgaard, K., Schjerling, P., and Raastad, T. (2009). Heat shock protein translocation and expression response is attenuated in response to repeated eccentric exercise. Acta Physiol. 196, 283-293. doi: 10.1111/j.17481716.2008.01940.x

Vogt, M., and Hoppeler, H. H. (2014). Eccentric exercise: mechanisms and effects when used as training regime or training adjunct. J. Appl. Physiol. 116, 14461454. doi: 10.1152/japplphysiol.00146.2013

Warren, G. L., Hermann, K. M., Ingalls, C. P., Masselli, M. R., and Armstrong, R. B. (2000). Decreased EMG median frequency during a second bout of eccentric contractions. Med. Sci. Sports Exerc. 32, 820-829.

Warren, G. L., Lowe, D. A., and Armstrong, R. B. (1999). Measurement tools used in the study of eccentric contraction-induced injury. Sports Med. 27, 43-59.

Yamin, C., Amir, O., Sagiv, M., Attias, E., Meckel, Y., Eynon, N., et al. (2007). ACE ID genotype affects blood creatine kinase response to eccentric exercise. J. Appl. Physiol. 103, 2057-2061.

Yu, J. G., Carlsson, L., and Thornell, L. E. (2004). Evidence for myofibril remodeling as opposed to myofibril damage in human muscles with DOMS: an ultrastructural and immunoelectron microscopic study. Histochem. Cell Biol. 121, 219-227.

Yu, J. G., Malm, C., and Thornell, L. E. (2002). Eccentric contractions leading to DOMS do not cause loss of desmin nor fibre necrosis in human muscle. Histochem. Cell Biol. 118, 29-34.

Conflict of Interest Statement: The authors declare that the research was conducted in the absence of any commercial or financial relationships that could be construed as a potential conflict of interest.

Copyright (C) 2019 Hody, Croisier, Bury, Rogister and Leprince. This is an open-access article distributed under the terms of the Creative Commons Attribution License (CC BY). The use, distribution or reproduction in other forums is permitted, provided the original author(s) and the copyright owner(s) are credited and that the original publication in this journal is cited, in accordance with accepted academic practice. No use, distribution or reproduction is permitted which does not comply with these terms. 


\section{OPEN ACCESS}

Edited by:

Lars Larsson,

Karolinska Institute (KI), Sweden

Reviewed by:

Kunihiro Sakuma,

Tokyo Institute of Technology, Japan Jorge Lira Ruas,

Karolinska Institute (KI), Sweden

*Correspondence:

Valentina Di Felice

valentina.difelice@unipa.it; vdfelice@inwind.it

Specialty section: This article was submitted to

Striated Muscle Physiology, a section of the journal Frontiers in Physiology

Received: 24 November 2018 Accepted: 11 April 2019

Published: 07 May 2019

Citation:

Trovato E, Di Felice V and Barone R (2019) Extracellular

Vesicles: Delivery Vehicles

of Myokines. Front. Physiol. 10:522. doi: 10.3389/fphys.2019.00522

\section{Extracellular Vesicles: Delivery Vehicles of Myokines}

\author{
Eleonora Trovato ${ }^{1}$, Valentina Di Felice ${ }^{1,2 *}$ and Rosario Barone ${ }^{1,3}$ \\ ${ }^{1}$ Department of Biomedicine, Neurosciences and Advanced Diagnostic (BIND), Human Anatomy and Histology Institute, \\ University of Palermo, Palermo, Italy, ${ }^{2}$ Innovation and Biotechnology for Health and Exercise (iBioTHEx), Palermo, Italy, \\ ${ }^{3}$ Euro-Mediterranean Institute of Science and Technology (IEMEST), Palermo, Italy
}

Movement and regular physical activity are two important factors that help the human body prevent, reduce and treat different chronic diseases such as obesity, type 2 diabetes, heart diseases, hypertension, sarcopenia, cachexia and cancer. During exercise, several tissues release molecules into the blood stream, and are able to mediate beneficial effects throughout the whole body. In particular, contracting skeletal muscle cells have the capacity to communicate with other organs through the release of humoral factors that play an important role in the mechanisms of adaptation to physical exercise. These muscle-derived factors, today recognized as myokines, act as endocrine and paracrine hormones. Moreover, exercise may stimulate the release of small membranous vesicles into circulation, whose composition is influenced by the same exercise. Combining the two hypotheses, these molecules related to exercise, named exer-kines, might be secreted from muscle cells inside small vesicles (nanovesicles). These could act as messengers in tissue cross talk during physical exercise. Thanks to their ability to deliver useful molecules (such as proteins and miRNA) in both physiological and pathological conditions, extracellular vesicles can be thought of as promising candidates for potential therapeutic and diagnostic applications for several diseases.

Keywords: exercise, muscle cells, exocytosis, extracellular bodies, exosomes

\section{INTRODUCTION}

Skeletal muscle is the largest organ of our body, responsible for our posture and our movement. It is equivalent to $2 / 5$ of the whole body weight and it is responsible for more than $3 / 4$ of the total human metabolism. It is mainly composed of proteins and a fine balance between protein synthesis and protein degradation regulates its mass. An unbalance in one of these two processes can lead to the establishment and progression of pathological conditions (Bowen et al., 2015) such as sarcopenia (slow and normal loss of muscle due to aging, in absence of other diseases) and cachexia (multifactorial syndrome, characterized by a severe and involuntary loss of muscle mass, with or without loss of fat mass) (Fearon et al., 2011).

A sedentary lifestyle, physical inactivity and malnutrition (reduction or hyper-caloric intake), are among the causes that emphasize the accumulation of visceral fat. Therefore, a lifestyle based on a greater physical activity and a lower energy intake, helps to decrease visceral fat mass content (Miyatake et al., 2002; Shojaee-Moradie et al., 2007), inflammation (Petersen and Pedersen, 2005; Mathur and Pedersen, 2008; Nilsson et al., 2019) and the risk of several chronic diseases such as obesity (Roh and So, 2017), type 2 diabetes (Hu et al., 1999) and cancer (Hojman et al., 2011; Barone et al., 2016). 
Muscle fiber is the principal unit of skeletal muscle and it is able to shorten its length because of nervous stimulation. Its development requires the involvement of several proteins and it is promoted by the differentiation and fusion of muscle cell progenitors into myotubes (Mauro, 1961). During physical activity, these cells are subjected to energetic (metabolic) and mechanical (contractile) stimuli that improve metabolic health of skeletal muscle and promote the release of specific molecules (called myokines), that can alter the function of other tissues (Stanford and Goodyear, 2018).

Recently, physical activity has also been associated with the release of extracellular vesicles (EVs) into the circulation (Fruhbeis et al., 2015). These are nano-sized vesicles that appear to be involved into cell-to-cell communication and may probably bring cytokines to distal organs, such as the heart (Bei et al., 2017). Communication is an essential process in multicellular organisms, both in physiological and pathological conditions and it is actuated by the exchange of information through different mechanisms: direct contact between cells (Cartwright and Arnold, 1980; Kalimi and Lo, 1988; Franke, 2009), secretion of soluble factors (Sicard, 1986; Lukacs et al., 1995) or interaction ligand-receptor (Qi et al., 2001). They are spherical organelles (originating from intracellular lipid compartments and released into the extracellular space and the systemic circulation) that have been discovered as new protagonists of intercellular communication (Pap et al., 2009; Zomer et al., 2010; Desrochers et al., 2016; Verweij et al., 2019).

Extracellular vesicles were first considered as cell waste products, but several studies revealed that they can transfer signaling molecules among cells in an autocrine, paracrine or endocrine manner (Lobb et al., 2015). They play a crucial role in regulating physiological processes (Schweitzer, 1973; Ratajczak et al., 2006; Kurachi et al., 2016; Niu et al., 2016; Bidarimath et al., 2017), inducing local and systemic changes that can develop, in some cases, into the progression of some diseases like cancer (Becker et al., 2016; Ohyashiki et al., 2018), neurodegenerative diseases (Vella et al., 2007; Danzer et al., 2012; Guix et al., 2018) and viral infections (Jaworski et al., 2014; Kalamvoki et al., 2014).

Interest in EVs has grown exponentially in the last 30 years, in part because most of the cells are able to secrete them. Skeletal muscle, the organ most represented in our organism, with its mechanical and secretory activities (Pedersen, 2013) may be responsible for the release of most of the circulating EVs during exercise. In fact, our research group has recently demonstrated that a single bout of exercise induces an increase in the release of EVs in the blood of healthy mice (Barone et al., 2016). While another paper described how an event of injury in response to exercise alters the composition of circulating EVs such as the content of some micro-RNAs (mi-Rs) (Guescini et al., 2015; Lovett et al., 2018).

\section{MYOKINES AND THE CONTRACTING MUSCLE}

Physical activity is known to exert beneficial effects on the prevention of chronic diseases (Zheng et al., 2006;
Mikus et al., 2010; Pedersen and Saltin, 2015), this may be due to the release of contraction-regulated molecules (cytokines and myokines) that play a crucial role in the communication between muscle and other tissues (such as adipose tissue, liver, and pancreatic cells) (Ahima and Park, 2015). Despite the term "myokine" is generally referred to any secreted protein synthesized by the skeletal muscle tissue, it should be effectively used to describe only those proteins secreted by muscle cells. In fact, the skeletal muscle also comprise fibrous connective tissue and endothelial and nerve cells.

Several myokines are synthesized and secreted during contraction (Pourteymour et al., 2017) like Fibroblast Growth Factor 21 (FGF-21) (Kim and Song, 2017), Interleukin-6 (IL6) (Brown et al., 2018), Interleukin-8 (IL-8) (Covington et al., 2016), Interleukin-15 (IL-15) (Hingorjo et al., 2018), Leukemia Inhibitory Factor (LIF) (Broholm et al., 2011), irisin (Lu et al., 2016), Myostatin (Hjorth et al., 2016), Angiopoietin-like 4 (ANGPTL4) (Norheim et al., 2014), Brain-Derived Neurotrophic Factor (BDNF) (Fortunato et al., 2018), Follistatin-like 1 (FSTL1) (Xi et al., 2016) and Vascular Endothelial Growth Factor (VEGF) (Gomes et al., 2017). Their expression in skeletal muscle is generally very low, but the levels of some of these myokines increase considerably during muscle contraction (FGF-21, IL-6, IL-15, irisin and BDNF among others) (Table 1). Moreover, the use of different exercise-based protocols (aerobic or resistance exercise training) can affect their secretion (Guescini et al., 2015; Schild et al., 2016; Abd El-Kader and Al-Shreef, 2018; Brown et al., 2018).

FGF-21 is an endocrine hormone belonging to the family of fibroblast growth factors (FGFs), which plays an important role in response to liver starvation, in lipolysis and glucose uptake in adipose tissues and skeletal muscle. It enhances the utilization of energy substrates (fatty acids, ketones and glucose) and meddles with energy consuming processes (lipogenesis and growth). Moreover, under certain conditions (such as cold), it can stimulate the activation of brown adipocytes and promote adaptive thermogenesis (Wu et al., 2013). It is also involved in mechanisms related to physical activity; in fact, its levels increase in acute exercise (in both human and mice), enhancing the phosphorylation of protein kinase B (Akt) and the translocation of Glucose Transporter Type 4 (GLUT4) to the muscle cell membrane, with consequent glucose uptake (Tanimura et al., 2016). Some results are controversial. For example, Cuevas-Ramos et al. (2012) proved that its serum levels increase only after 2 weeks of daily physical activity and no change was observed after a single bout of exercise. Chronic exercise and FGF-21 also induces the expression of Peroxisome proliferator-activated receptor Gamma Coactivator -1 $\alpha$ (PGC$1 \alpha$ ), a transcriptional protein factor that interacts with various DNA-binding proteins resulting in increased gluco-neogenesis, fatty acid oxidation, ketogenesis and mitochondrial biogenesis (Barone et al., 2016, 2017).

Interleukins are another important class of molecules released during exercise, especially IL-6, IL-8 and IL-15 (Ostrowski et al., 1999; Peake et al., 2015). They are small molecules belonging to the class of cytokines and constitute one of the most 
TABLE 1 | Principal myokines and exerkines released and regulated during physical activity.

\begin{tabular}{|c|c|c|}
\hline Contracting Exerkines & Levels of myokine & Reference \\
\hline FGF-21 & Increase & Cuevas-Ramos et al., 2012; Kim and Song, 2017 \\
\hline IL-6 & Increase & Papanicolaou et al., 1996 \\
\hline IL-8 & Increase & Mucci et al., 2000; Covington et al., 2016 \\
\hline IL-15 & Increase & Nielsen et al., 2007; Hingorjo et al., 2018 \\
\hline Irisin & Increase & Kraemer et al., 2014 \\
\hline BDNF & Increase & Gomez-Pinilla et al., 2002; Fortunato et al., 2018 \\
\hline ANGPTL4 & Increase & Norheim et al., 2014 \\
\hline LIF & Increase & Broholm et al., 2011 \\
\hline FSTL-1 & Increase & Xi et al., 2016 \\
\hline VEGF & Increase & Gustafsson et al., 2001; Gomes et al., 2017 \\
\hline Myostatin & Decrease & Hittel et al., 2010 \\
\hline
\end{tabular}

important communication systems among cells, defending the body (Nelson and Summer, 1998).

IL-6 is a pleiotropic cytokine, able to work as a pro- and anti-inflammatory molecule (Petersen and Pedersen, 2006; Yao et al., 2014), cardiovascular risk factor and regulator of lipid metabolism (Bao et al., 2015). It also acts as a myokine and its concentration increases up to 100 -fold in response to muscle contraction (Agarwal et al., 2017), mediating anti-inflammatory responses and metabolic adaptations. It may reduce the incidence of cardiovascular diseases through lipid and glucose metabolism and the suppression of pro-inflammatory cytokines. During exercise, it acts both locally (inside the muscle) and peripherally (in several organs, such as white adipose tissue WAT) acting as a hormone. In WAT, it affects adipose tissue metabolism and lipolysis, with the release in the circulation and oxidation of fatty acids (Knudsen et al., 2017) while in skeletal muscle, it activates 5 '-Adenosine Monophosphate-activated Protein (AMP) and/or Phosphatidylinositol 3-kinase (PI3 kinase) to increase glucose uptake and fat oxidation (Pedersen, 2009).

IL-15 is one of the most abundantly expressed cytokines in human muscle, involved in the regulation of adiposity, muscle mass, exercise capacity and mitochondrial activity in muscle cells (Thornton et al., 2016). Historically studied as an activator of Natural Killer (NK) cells (with anti-tumorigenic and anti-inflammatory properties) (Gosselin et al., 1999), its levels strongly increase after resistance exercise, promoting muscle growth (Nielsen et al., 2007). Similar to IL-6, its activity is strongly correlated to the AMP-protein kinase (AMPK), a central regulator of metabolism; indeed, it was shown that lacking muscle AMPK reduces serum IL-15, causing the acceleration of skin aging (Crane et al., 2015). IL-15 has also anti-tumorigenic effects; in fact, Carbo et al. (2000) showed that, in tumorigenic rats treated with IL-15, it partly inhibits skeletal muscle wasting protein rates (eight-fold) to values even lower than those observed in non-tumor-bearing animals.

Irisin is a secreted myokine that originates from the cleavage of its precursor fibronectin type III domain-containing protein 5 (FNDC5) (Huh et al., 2012). Firstly known as a molecule responsible for the browning of WAT (Giralt and Villarroya, 2013), it was also shown that its levels strongly increase after resistance exercise (Miyamoto-Mikami et al., 2015;
Zhao et al., 2017), but not in aerobic training (Kim et al., 2016). Moreover, it was also recently proposed as a novel marker for patients with cardiac cachexia (Kalkan et al., 2018). Even if it was shown that it can mediate those beneficial effects that follow exercise (inducing the expression of pro-myogenic genes in myotubes), its role in physical activity still remain controversial. In fact, on one hand, Blizzard Leblanc et al. (2017) shown a significant increase in irisin plasma levels after an acute bout of aerobic exercise, also associated with the improvement in insulin sensitivity. These data were also confirmed by Nygaard et al. (2015), which further showed that single sessions of intense endurance exercise and heavy strength training led to transient increase of plasma concentration, without an increase in FNDC5 expression (Pang et al., 2018). However, on the other hand, a pivot study in hemo-dyalised patients proved that there was no correlation between intense intra-dialytic strength exercise and the increase of circulating irisin (Esgalhado et al., 2018). Also, the research group of Biniaminov et al. (2018) investigated the association among resting irisin concentrations, regular physical activity and physical fitness in serum of healthy humans; they found that nor physical activity level, neither fitness status were related to resting irisin concentrations in healthy humans.

Considering its potential beneficial effects during training, Reza et al. (2017) investigated its capability to act as an exercise mimetic. They injected wild-type mice with irisin and observed an increase in body weight, skeletal muscle mass and muscle strength, suggesting that irisin induces fiber hypertrophy and enhances regeneration of injured muscle cells, through the activation of satellite cells and protein synthesis.

Overall, the way irisin works is still largely unknown. However, it may be that it exerts its activity in a way similar to that of interleukins, through AMP kinase.

Another important myokine released during physical exercise is the BDNF (Gomez-Pinilla et al., 2002), a type of neurotrophic molecule mainly involved in memory and cognitive development (Heldt et al., 2007). This molecule is the product of the proteolytic cleavage of its precursor protein (proBDNF) (Koshimizu et al., 2009). BDNF and proBDNF often have opposing actions; in fact, while BDNF promotes synaptic long-term potentiation and stress resistance, proBDNF enhances longterm depression (Teng et al., 2005). 
Brain-derived neurotrophic factor has neurobiological and metabolic effects, regulating the survival (Hofer and Barde, 1988), growth (Kalcheim and Gendreau, 1988) and maintenance of neurons (Griesbeck et al., 1995). Its association with physical activity is already known (Almeida et al., 2016; Church et al., 2016); in fact, its expression not only increases after exercise (Park and Kwak, 2017) but also depends on the intensity of exercise (Jeon and Ha, 2017). Previous studies demonstrated that its mRNA and protein expression levels increase in human skeletal muscle after exercise without any release into the circulation (Matthews et al., 2009). Indeed, it acts as a contraction-inducible protein that enhances fatty acid oxidation through the activation of AMPK in the skeletal muscle. Thus, it does not act in a hormone like manner, but as an autocrine and/or paracrine molecule within the skeletal muscle tissue (Matthews et al., 2015).

\section{EXTRACELLULAR VESICLES: ORIGINS AND FUNCTIONS}

First characterized in 1967 in hematopoietic cells (Wolf, 1967), EVs are small particles composed of a lipid bi-layer containing multiple molecules derived from the cytosol and from the cellular membrane of the donor cell (Akers et al., 2013). They constitute a heterogeneous population that differs in cellular origin, size, morphology, antigenic composition and functional properties (Table 2). Isolated from different body fluids (including blood, urine, saliva, breast milk, amniotic fluid, ascites, cerebrospinal fluid, bile and seminal fluid) (Colombo et al., 2014), these vesicles are involved in the communication of both prokaryotes and eukaryotes. Despite the fact that their name originally referred to their size (apoptotic bodies $>800 \mathrm{~nm}$, microparticles in a range of $0.1-1 \mu \mathrm{m}$ and exosomes with diameter of 40-150 nm), their tissue of origin (prostasomes, oncosomes) and their function or their presence outside the cells (exosomes), one of the last classifications of EVs, focused on their biogenesis pathways (Kalra et al., 2016). However, there is still no consensus on specific EV subtypes markers, such as endosome-origin "exosomes" and plasma membrane-derived "ectosomes" (microparticles/microvesicles); therefore, assigning an EV to a particular biogenesis pathway remains difficult unless the $\mathrm{EV}$ is caught during its release in live imaging techniques. Hence, in order to classify a particular vesicle to a specific EV subtype, MISEV 2018 suggested considering several parameters such as: size ["small EVs" (sEVs) and "medium/large EVs" (m/lEVs)], density (low, middle, high), biochemical composition (CD63+, CD81+, Annexin A5), description of their tissue of origin (prostasomes, oncosomes), their function or their presence outside the cells, or their biogenesis pathway (Théry et al., 2018).

Extracellular vesicles obtained from differential ultracentrifugation have been classified into: large EVs, pelleted at low speed; medium-sized EVs, pelleted at intermediate speed and small EVs (sEVs), pelleted at high speed. The latter were further subdivided into four sub-categories: (1) sEVs rich in tetraspanines CD63, CD9 and CD81 and endosomal markers (better known as exosomes); (2) sEVs without CD63 and CD81, but rich in CD9; (3) sEVs without CD63/CD9/CD81; (4) sEVs rich in extracellular matrix or serum factors. The last two-listed sEV are not associated with exosomes (Kowal et al., 2016).

Their molecular composition reflects the specific functions of the cells from which they originate and, for this reason, their cargo is defined as cell-type specific. They generally carry several molecules, such as proteins, nucleic acids (largely represented by mRNA and miRNA) and lipids, and their protein composition is similar to that of the plasma membrane and of the endocytotic and subcellular compartments of the budding cell (Gutierrez-Vazquez et al., 2013). In particular, exosome protein content is mainly divided in four categories: transmembrane or lipid bound extracellular proteins (tetraspaninins like CD9, CD81, CD63), cytosolic proteins normally involved in their biogenesis (RAB proteins, Hsp70, Hsp90), intracellular proteins unique of cellular organelles and typically absent in exosomes (calnexin, Golgi and ER proteins) and extracellular proteins, such as acetylcholinesterase (ACHE). When characterizing these particles, at least one protein of each category should be identified (Lotvall et al., 2014). Therefore, membrane and cytoskeletal proteins, lysosomal markers enzymes, death receptors (FasL, TRAIL), cytokines, HLA class I and II proteins, and some HSPs can be part of these vesicles. As well as their molecular content, even their functions are closely related to their cellular origin, being involved in several mechanisms such as immune response and inflammation (Zhang et al., 2014).

Despite the fact that the lipid composition of these nanovesicles is still not well known, we know that EVs are mainly composed of sphyngolipids, phosphatidyl serine, cholesterol (possibly involved in exosomes release), saturated fatty acids and ceramide (Pfrieger and Vitale, 2018).

The mechanisms underlying the biogenesis of EVs are different among various types of vesicles. Apoptotic bodies $(>800 \mathrm{~nm})$ are particles that cells produce during the apoptotic process. During programmed cell death, membrane protrusion known as apoptopodia (Atkin-Smith et al., 2015) release (in the extracellular space) vesicles (apoptotic bodies) resulting from the fragmentation of the apoptotic cell (Coleman et al., 2001). Ectosomes (0.1-1 $\mu \mathrm{m})$, also known as shedding vesicles or microparticles, originate from plasma membrane through an outward budding of the membrane; in this process, the increase of intracellular $\mathrm{Ca}^{2+}$, induced by an external signal, causes changes in lipid distribution and membrane blebbing, through the alteration of the enzymatic activity of flippases, translocases and scramblases (Willms et al., 2018). This increment of internal $\mathrm{Ca}^{2+}$ enhances the activation of cytosolic proteases (such as calpain and gelsolin), which re-organize the cytoskeleton (through the deconstruction of the actin cytoskeletal protein network) and causing plasma membrane protrusion with consequent detachment of these vesicles (Turturici et al., 2014; Cocucci and Meldolesi, 2015).

Exosomes $(<150 \mathrm{~nm})$ are the most studied small vesicles, especially because of their small size and internal content that reflects that of the cell of origin. They are mostly defined by their size and their protein content, despite the fact that in literature the term "exosome" is improperly used to refer to small EVs (Wang et al., 2017). Their biogenesis is a well-organized process, mainly characterized by exocytosis through an active 
TABLE 2 | Types of extracellular vesicles and most common markers.

\begin{tabular}{|c|c|c|c|}
\hline & Apoptotic Bodies & Microvesicles-Ectosomes & $\begin{array}{l}\text { Exosomes } \\
\text { (small-medium-large) }\end{array}$ \\
\hline Size & $>800 \mathrm{~nm}$ & $0.1 \mathrm{~nm}-1 \mu \mathrm{m}$ & $30-150 \mathrm{~nm}$ \\
\hline Biogenesis & Apoptosis & Outward budding of plasma membrane & Endocytosis \\
\hline Common Markers & $\begin{array}{l}\text { Chemokine (C-X3-C motif) ligand } 1 \text { X3CL1 } \\
\text { Intercellular adhesion molecule } 3 \text { (ICAM-3) }\end{array}$ & $\begin{array}{l}\beta 1 \text { integrins; Selectins } \\
\text { CD40 } \\
\text { Matrix metalloproteinase (MMP) }\end{array}$ & $\begin{array}{l}\text { Tetraspanins (CD63; CD9; } \\
\text { CD81) } \\
\text { Hsps (Hsp70; Hsp90) } \\
\text { Tumor susceptibility gene } 101 \\
\text { (TSG101) } \\
\text { Annexin V-VI } \\
\text { Metallopeptidase domain } 10 \\
\text { (ADAM10) } \\
\text { Alix }\end{array}$ \\
\hline Reference & $\begin{array}{l}\text { Groves and Ellwood, 1985; Catchpoole and } \\
\text { Stewart, } 1995\end{array}$ & Surman et al., 2017 & Kowal et al., 2016 \\
\hline
\end{tabular}

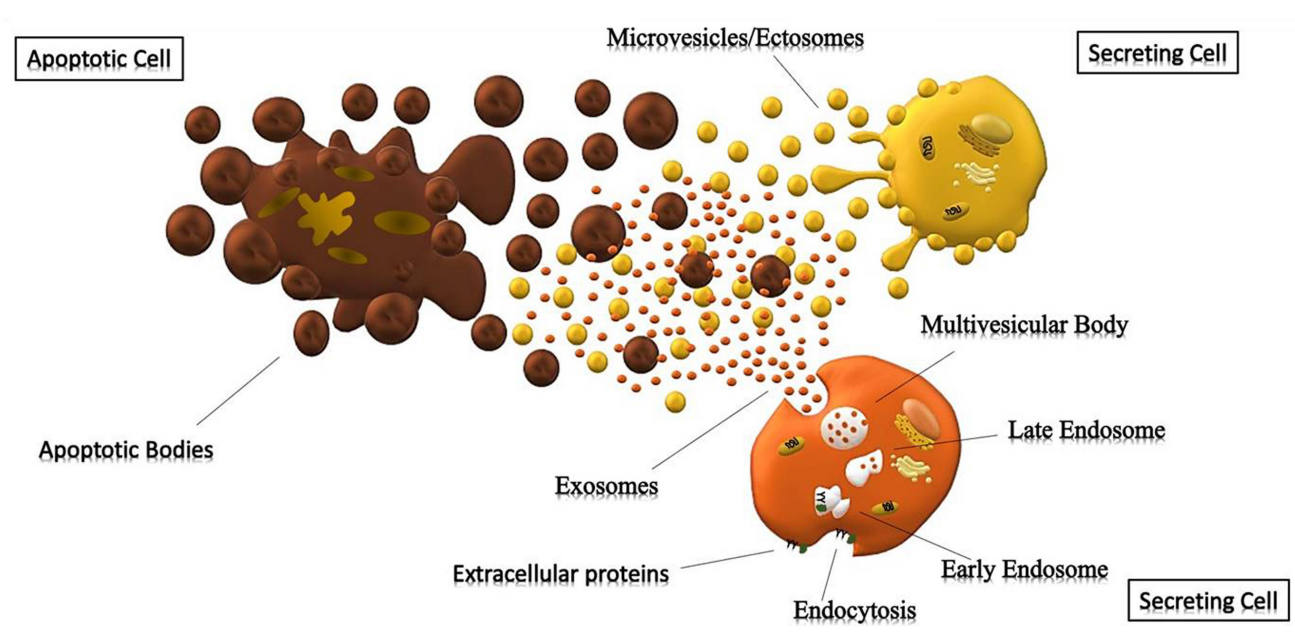

FIGURE 1 | Cells releasing extracellular vesicles. Representation of different extracellular vesicles. Brown particles represent Apoptotic Bodies (>500 nm), forming during the apoptotic process; while the yellow and orange vesicles represent Microparticles (>150 nm) and Exosomes (30-150 nm) generated respectively by outward budding of the membrane and endocytosis.

involvement of the membrane. The process starts with the invagination of the plasma membrane and the development of the early endosome (EE), a membrane bounded compartment within the cell. Subsequently, the inner budding of the membrane of the early endosome replaces the already existing endosomal luminal space with small intraluminal vesicles (ILVs) and forms a body called Multi-Vescicular Body (MVB) or late endosome (LE). The latter is filled with proteins, lipids, and cytoplasm specifically sorted (Gutierrez-Vazquez et al., 2013; Hessvik and Llorente, 2018). At this point vesicles within the MVB can undergo three different fates: merge with the lysosomes and be degraded in their protein content; constitute a momentary deposit compartment or merge with the plasma membrane, releasing its intraluminar vesicles in the extracellular space as exosomes (Figure 1) (Caruso Bavisotto et al., 2017; Sutaria et al., 2017). The development of ILVs and MVBs is a process that requires the participation of the Endosomal Sorting Complex Required for Transport (ESCRT), a complex composed by almost 30 proteins assembled into 4 components: ESCRT 0, ESCRT I, ESCRT II and ESCRT III.
ESCRT 0 is involved in the recognition and the sequestration of ubiquitinated transmembrane proteins into the endosomal membrane; ESCRT II and I are responsible for the membrane deformation into buds with specific cargo; the last complex is implicated into the detachment of the formed vesicle. Even though the mechanism involved in exosomes secretion is still not well understood, it is likely that the increase of internal $\mathrm{Ca}^{2+}$, followed by a cytoskeleton remodeling, is involved in their release. Once in the extracellular space, these vesicles can be either internalized by the receiving cell through endocytosis processes, or they act as transmembrane signals by binding receptors on the plasma membrane and activate specific cellular pathways (Feng et al., 2010; Mulcahy et al., 2014). It was also thought that their uptake can be due by a "passive endocytosis" which occurs during the natural recycling of the plasma membrane and could passively take up exosomes attached to the surface of a cell.

Currently, the characterization of these vesicles is a combination of several methods that include microscopy (TEM, SEM, CrioTEM), Western Blot, Flow Cytometry, 
Nanoparticle Tracking Analysis (NTA), Tunable Resistive Pulse Sensing (TRPS), Dynamic Light Scattering (DLS) and immunohistochemical analysis of specific EVs markers, used to describe their morphology, biochemical composition and the receptors localized on these vesicles (Szatanek et al., 2015) (Table 3).

However, one of the main issue when purifying these particles is that, currently, there is no consensus for a unique standard isolation protocol. Ideally, the method used for their isolation should be simple, fast and inexpensive.

Overall, there are three main methodologies used for their isolation: differential centrifugation/ultracentrifugation with/without a sucrose gradient/cushion, adsorption to magnetic/non-magnetic micro-beads and size exclusion chromatography. Each has its own advantages and the choice of one method rather than another can result in different EV subpopulations with different properties (Konoshenko et al., 2018). Hence, one important challenge is the absence of a unique method able to minimize co-isolating protein aggregates and other membranous particles from a pure sample of EVs. Currently, the gold standard for pure exosome preparation is differential ultracentrifugation coupled with sucrose/iodixanol density gradient. Van Deun et al. (2014) isolated EVs with 4 commonly used methods (ultracentrifugation, density gradient, exo-kit and total exosome isolation) for the evaluation of yield, size, morphology, protein and RNA content of exosome. They demonstrated that density gradient ultracentrifugation gave the purest exosome preparations, while the other three techniques also co-isolate contaminating factors. Similarly, Skottvoll et al. (2019) evaluated the performance of different isolation methods based on differential ultracentrifugation and a commercial isolation kit (total exosome isolation reagent). These results shown that the two isolation methods had similar performance with only some differences based on the origin of the cell. In another study, the results published by Gamez-Valero et al. (2016) suggested that size-exclusion chromatography (SEC) is capable of eliminating most of the abundant proteins contained in a body fluid, also maintaining the EVs vesicular structure and conformation, thus making this procedure ideal for biomarker discovery as well as for therapeutic applications. Benedikter et al. (2017) also confirmed these data, demonstrating that ultrafiltration followed by size exclusion chromatography (UF-SEC), provides well-concentrated EVs for proteomic and functional analysis. Indeed, because of its efficient capability to separate EVs from contaminant proteins (especially from large initial volumes) UF-SEC gives a higher yield of pure vesicles if compared to those isolated by simple ultracentrifugation.

Interest on EVs is growing very fast over the years. Thanks to their characteristics (specifically their non-immunogenic nature due to the similar composition to the cell from which they originate) they were recently taken into consideration for their use as drug delivery vehicles (Batrakova and Kim, 2015).

Actually, liposomes are used as drug delivery vehicles, but their biocompatibility and their safety are still unknown. Unlike these synthetic systems, exosomes have long circulating half-life, promise to be biocompatible and stable, and have minimal or no inherent toxicity issues. Moreover, thanks to their small size, they are able to cross the blood-brain barrier ( $\mathrm{BBB})$, thus providing a useful carrier for the delivery of small drugs across this area.

Ninety-eight percent of drugs potentially important for the central nervous system cannot cross the BBB and their conceptual efficacy shown in labs has not a counterpart in clinical trials (Haney et al., 2015). Moreover, thanks to their capability to carry different molecules (protein and miRNA among others), they can also eliminate problems related to the instability of nucleic acid based drugs (Liang et al., 2018). Furthermore, the possibility to isolate them from all biological fluids, suggests their use for diagnostic applications, providing a non-invasive diagnostic method. For instance, they can be used for diagnosis since circulating exosomes can be correlated to specific diseases (Aryani and Denecke, 2016). For these reasons, if compared with their synthetic counterparts, they seem to be a better choice,

TABLE 3 | Most common extracellular vesicles isolation and characterization methods.

\begin{tabular}{|c|c|c|c|}
\hline Isolation & References & Characterization & References \\
\hline Ultracentrifugation & Thery et al., 2006 & $\begin{array}{l}\text { Electron Microscopy: TEM; SEM; } \\
\text { cryo-EM }\end{array}$ & $\begin{array}{l}\text { Escola et al., 1998; Poliakov } \\
\text { et al., 2009; Sokolova et al., } \\
2011\end{array}$ \\
\hline $\begin{array}{l}\text { Density gradient } \\
\text { Ultracentrifugation }\end{array}$ & Zhang et al., 2014 & $\begin{array}{l}\text { Dynamic Light Scattering (DLS); } \\
\text { Nanoparticle Tracking Analysis (NTA); } \\
\text { Tunable Resistive Pulse Sensing (TRPS) }\end{array}$ & $\begin{array}{l}\text { Kesimer and Gupta, } 2015 \\
\text { Gercel-Taylor et al., } 2012 \\
\text { Maas et al., } 2014\end{array}$ \\
\hline $\begin{array}{l}\text { Size Exclusion } \\
\text { Chromatography (SEC) }\end{array}$ & Boing et al., 2014 & Biochemical analysis (Western Blot) & Théry et al., 2018 \\
\hline Immunological separation: & Logozzi et al., 2009; Pedersen et al., 2017 & Flow cytometry & Pospichalova et al., 2015 \\
\hline
\end{tabular}

Magnetic beads; ELISA

Logozzi et al., 2009; Pedersen et al., 2017

Omics analysis: Proteomic; Lipidomic

Schey et al., 2015; Haraszti et al., 2016 
exceeding those synthetic nanoparticles limitations. That's why they have aroused a lot of interest as a drug delivery system for the treatment of several chronic and neurodegenerative diseases (Ha et al., 2016).

Nowadays their use as a drug delivery system for small molecules, proteins and nucleic acids is already a true reality; Alvarez-Erviti et al. (2011) showed that intravenously injected Rabies Virus Glycoprotein (RVG) targeted exosomes delivered GAPDH siRNA specifically in the brain, resulting in a specific gene knockdown. A similar result was also confirmed by Liu et al. (2015), showing that exogenous siRNA transfected into cells can be packaged by exosomes and delivered into recipient cells to regulate gene silencing, indicating that exosomes can serve as siRNA delivery vesicles in gene therapy for cancer and other diseases. In another study, large size plasmid DNA encapsulated into exosomes was successfully transferred to MSCs (Lin et al., 2018). Sun et al. (2010) treated mice with curcumin carrying exosomes, demonstrating that they were protected against lipopolysaccharide (LPS)-induced septic shock. Curcumin was more stable both in vitro and in vivo. In a similar study, Gomari et al. (2018) encapsulated doxorubicin (a drug currently used for breast and solid cancer) into exosomes to increase local dosage of the molecule and reduce its adverse effects on other organs. Considering their ability to deliver large molecules (such as proteins), Khatua et al. (2009) demonstrated that human cytidine deaminase APOBEC3G (A3G), a cellular defense system against human immunodeficiency virus type 1 (HIV-1) and other retroviruses, can be secreted in exosomes conferring an antiviral phenotype to target cells, also limiting replication of the virus in recipient cells.

However, despite several evidences of their potential as drug delivery system, one of the principal obstacles for the application of exosomes in clinic is their final yield from donor cells, which is often very limited and strongly related to the protocol of isolation (Van Deun et al., 2014). In addition, they may act as vehicles for the replication and propagation of transmissible pathogens, since exosomes derived from bacteria, or virus-infected cells, might contain pathogen-derived factors that activate a proinflammatory pathway. Plus exosomes have also a different effect on health and diseases; indeed, despite some of them can prevent tumor development (Naseri et al., 2018; Rosenberger et al., 2019), others provide a communication system between tumor cells and the surrounding tissues (Haga et al., 2015; Keklikoglou et al., 2019).

In the last few years, researchers have started to combine exosomes with synthetic nanoparticles, developing engineered particles more efficiently than their natural counterparts do. For instance, Sato et al. (2016), to control and modify the performance of exosomal nanocarriers, realized hybrid exosomes fusing them with polyethylene glycol (PEG) liposomes. This modification facilitated cellular uptake of the PEG modified exosomes, reducing also their circulation time in the blood. Other researchers created exosome-mimetic nanovesicles by serial extrusions through polycarbonate membranes with pore sizes of 10,5 , and $1 \mu \mathrm{m}$, for their utilization in tissue repair and regeneration. These exosome-mimetic nanovesicles (NVs) had a final yield almost 100 times higher than exosomes and promoted cell proliferation and liver regeneration similar to that induced by exosomes (Wu et al., 2018).

\section{EVs AND EXERCISE: EVs CHANGES INDUCED BY EXERCISE}

The capacity of myokines to positively influence the metabolism and homeostasis of the body, makes them promising targets for treatment of several diseases. However, little is known about the mechanisms that regulate the release of these factors, especially regarding the final steps in recruitment and exocytosis of specific secretory vesicles. In fact, despite muscle cells express several secretory vesicle transport proteins (Romancino et al., 2013), the mechanisms that target containing vesicles to particular regions in the plasma membrane to control myokine secretion are largely unknown. Some studies demonstrated that glucose receptor GLUT4 is translocated to the plasma membrane in Vesicle-Associated Membrane Protein 2 (VAMP2) labeled vesicles and, its translocation, requires an active remodeling of actin filament which can be induced by insulin (Giudice and Taylor, 2017).

Exercise triggers the release of exerkines (EXs) into the circulation, possibly through their encapsulation within EVs (Gorgens et al., 2015; Lombardi et al., 2016) that mediates the systemic benefits of physical exercise, in both physiological and pathological conditions (Safdar et al., 2016). In fact, it was shown that in patients with cardio-metabolic risk factors, acute exercise promoted a large release of plasma EVs (Bei et al., 2017). A similar mechanism of communication among cells was already seen during high energy demand related to exercise; in this case, cells released enzymes of the glycolytic pathway into the EVs, that probably influenced the glycolytic rate in the recipient cells (Garcia et al., 2016; Zhao et al., 2016).

Whitham et al. (2018) analyzed (by means of the NanoUHPLC followed by mass spectrometry) the proteome of the EVs of human plasma (before and after exercise), demonstrating how the EV trafficking was involved in tissue cross talk during physical activity. Indeed, exercise induces an increase of more than 300 proteins in the circulation, many of them associated with the biogenesis and function of "small vesicles" and exosomes. They identified 35 new myokine candidates, supporting the idea that the skeletal muscle is one of the major distributors of secreted molecules during exercise (Deshmukh et al., 2015). Some of these molecules were also found in EVs collected from myotubes conditioned medium (Forterre et al., 2014), but also from plasma and serum of participants who had walking speed decline (Suire et al., 2017). Another interesting data obtained by Petersen et al. (2011), showed the amino acid sequences of each protein transported inside these vesicles. Using the SignalP 4.0 server, they revealed that these proteins were deprived of the signal peptide sequence, typical of the classical secretion pathway. According to the obtained results, they postulated that the increase of EVs into the circulation (induced by exercise), can be related to the mechanism by which the skeletal muscle releases myokines, in a way independent of the classic secretory pathway. Moreover, exosome release is generally associated with 
an increase of intracellular calcium (Savina et al., 2003); since the motoneuron stimulates skeletal muscle fibers (causing an immediate release of $\mathrm{Ca}^{2+}$ from the sarcoplasmic reticulum) (Melzer et al., 1984), it may be plausible that the release of muscle small vesicles is faster than in other organs. However, uptake of the EVs in the recipient cells during exercise is a necessary step to talk about tissue cross talk and the reduction of the amount of proteins within the EVs ( $4 \mathrm{~h}$ after exercise), suggests that these proteins are partially removed from the circulation by tissue absorbing.

Therefore, exosomes and sEVs may act as communicator factors among cells, through the packaging of proteins inside their lumen.

\section{CONCLUSION}

Even though it has been widely proved that, during exercise, muscle cells release several myokines into the circulation with a potential role in whole-body homeostasis (Aswad et al., 2014), the mechanisms underlying this secretion process are still vaguely

\section{REFERENCES}

Abd El-Kader, S. M., and Al-Shreef, F. M. (2018). Inflammatory cytokines and immune system modulation by aerobic versus resisted exercise training for elderly. Afr. Health Sci. 18, 120-131.

Agarwal, M., Singh, S., Narayan, J., Pandey, S., Tiwari, S., and Sharma, P. (2017). Cardiovascular response and serum interleukin-6 level in concentric Vs. Eccentric exercise. J. Clin. Diagn. Res. 11, CC04-CC08.

Ahima, R. S., and Park, H. K. (2015). Connecting myokines and metabolism. Endocrinol. Metab. (Seoul) 30, 235-245.

Akers, J. C., Gonda, D., Kim, R., Carter, B. S., and Chen, C. C. (2013). Biogenesis of extracellular vesicles (EV): exosomes, microvesicles, retrovirus-like vesicles, and apoptotic bodies. J. Neurooncol. 113, 1-11. doi: 10.1007/s11060-013-1084-8

Almeida, M. F., Chaves, R. S., Silva, C. M., Chaves, J. C. S., Melo, K. P., and Ferrari, M. F. R. (2016). BDNF trafficking and signaling impairment during early neurodegeneration is prevented by moderate physical activity. IBRO Rep. 1, 19-31. doi: 10.1016/j.ibror.2016. 08.001

Alvarez-Erviti, L., Seow, Y., Yin, H., Betts, C., Lakhal, S., and Wood, M. J. (2011). Delivery of siRNA to the mouse brain by systemic injection of targeted exosomes. Nat. Biotechnol. 29, 341-345. doi: 10.1038/nbt.1807

Aryani, A., and Denecke, B. (2016). Exosomes as a nanodelivery system: a key to the future of neuromedicine? Mol. Neurobiol. 53, 818-834. doi: 10.1007/s12035014-9054-5

Aswad, H., Forterre, A., Wiklander, O. P., Vial, G., Danty-Berger, E., Jalabert, A., et al. (2014). Exosomes participate in the alteration of muscle homeostasis during lipid-induced insulin resistance in mice. Diabetologia 57, 2155-2164. doi: 10.1007/s00125-014-3337-2

Atkin-Smith, G. K., Tixeira, R., Paone, S., Mathivanan, S., Collins, C., Liem, M., et al. (2015). A novel mechanism of generating extracellular vesicles during apoptosis via a beads-on-a-string membrane structure. Nat. Commun. 6:7439.

Bao, P., Liu, G., and Wei, Y. (2015). Association between IL-6 and related risk factors of metabolic syndrome and cardiovascular disease in young rats. Int. J. Clin. Exp. Med. 8, 13491-13499.

Barone, R., Macaluso, F., Sangiorgi, C., Campanella, C., Marino Gammazza, A., Moresi, V., et al. (2016). Skeletal muscle Heat shock protein 60 increases after endurance training and induces peroxisome proliferator-activated receptor gamma coactivator 1 alphal expression. Sci. Rep. 6:19781.

Barone, R., Sangiorgi, C., Marino Gammazza, A., D'amico, D., Salerno, M., Cappello, F., et al. (2017). Effects of conjugated linoleic acid associated with endurance exercise on muscle fibres and peroxisome proliferator-activated known. Since calcium is an essential ion involved in both extracellular vesicles secretion and contraction of skeletal muscle fibers, it may be possible that, during exercise, the stimulation of muscle cells from motoneurons enhances the release of small vesicles potentially carrying myokines. However, there are almost no studies about that, hence, further analysis are needed in order to better understand the relationship between nanovesicles and myokines.

\section{AUTHOR CONTRIBUTIONS}

All authors listed have made a substantial, direct and intellectual contribution to the work, and approved it for publication.

\section{FUNDING}

This study was funded by "Ministero dell'Istruzione, dell'Università e della Ricerca” (PRIN2012-prot. 2012N8YJC3Prof. Daniela Caporossi, Prof. Felicia Farina, and VDF).

receptor gamma coactivator 1 alpha isoforms. J. Cell Physiol. 232, 1086-1094. doi: $10.1002 /$ jcp. 25511

Batrakova, E. V., and Kim, M. S. (2015). Using exosomes, naturally-equipped nanocarriers, for drug delivery. J. Control Release 219, 396-405. doi: 10.1016/ j.jconrel.2015.07.030

Becker, A., Thakur, B. K., Weiss, J. M., Kim, H. S., Peinado, H., and Lyden, D. (2016). Extracellular vesicles in cancer: cell-to-cell mediators of metastasis. Cancer Cell 30, 836-848. doi: 10.1016/j.ccell.2016.10.009

Bei, Y., Xu, T., Lv, D., Yu, P., Xu, J., Che, L., et al. (2017). Exercise-induced circulating extracellular vesicles protect against cardiac ischemia-reperfusion injury. Basic Res Cardiol. 112:38.

Benedikter, B. J., Bouwman, F. G., Vajen, T., Heinzmann, A. C. A., Grauls, G., Mariman, E. C., et al. (2017). Ultrafiltration combined with size exclusion chromatography efficiently isolates extracellular vesicles from cell culture media for compositional and functional studies. Sci. Rep. 7:15297.

Bidarimath, M., Khalaj, K., Kridli, R. T., Kan, F. W., Koti, M., and Tayade, C. (2017). Extracellular vesicle mediated intercellular communication at the porcine maternal-fetal interface: a new paradigm for conceptus-endometrial cross-talk. Sci. Rep. 7:40476.

Biniaminov, N., Bandt, S., Roth, A., Haertel, S., Neumann, R., and Bub, A. (2018). Irisin, physical activity and fitness status in healthy humans: no association under resting conditions in a cross-sectional study. PLoS One 13:e0189254. doi: 10.1371/journal.pone.0189254

Blizzard Leblanc, D. R., Rioux, B. V., Pelech, C., Moffatt, T. L., Kimber, D. E., Duhamel, T. A., et al. (2017). Exercise-induced irisin release as a determinant of the metabolic response to exercise training in obese youth: the EXIT trial. Physiol. Rep. 5:e13539. doi: 10.14814/phy2.13539

Boing, A. N., Van Der Pol, E., Grootemaat, A. E., Coumans, F. A., Sturk, A., and Nieuwland, R. (2014). Single-step isolation of extracellular vesicles by size-exclusion chromatography. J. Extracell. Vesicles 8:3.

Bowen, T. S., Schuler, G., and Adams, V. (2015). Skeletal muscle wasting in cachexia and sarcopenia: molecular pathophysiology and impact of exercise training. J. Cachexia Sarcop. Muscle 6, 197-207. doi: 10.1002/jcsm.12043

Broholm, C., Laye, M. J., Brandt, C., Vadalasetty, R., Pilegaard, H., Pedersen, B. K., et al. (2011). LIF is a contraction-induced myokine stimulating human myocyte proliferation. J. Appl. Physiol. (1985) 111, 251-259. doi: 10.1152/japplphysiol. 01399.2010

Brown, M., Mcclean, C. M., Davison, G. W., Brown, J. C. W., and Murphy, M. H. (2018). The acute effects of walking exercise intensity on systemic cytokines and oxidative stress. Eur. J. Appl. Physiol. 118, 2111-2120. doi: 10.1007/s00421-0183930-z 
Brown, P. N., and Yin, H. (2017). Polymer-based purification of extracellular vesicles. Methods Mol. Biol. 1660, 91-103. doi: 10.1007/978-1-4939-7253-1_8

Carbo, N., Lopez-Soriano, J., Costelli, P., Busquets, S., Alvarez, B., Baccino, F. M., et al. (2000). Interleukin-15 antagonizes muscle protein waste in tumourbearing rats. Br. J. Cancer 83, 526-531. doi: 10.1054/bjoc.2000.1299

Cartwright, J. Jr., and Arnold, J. M. (1980). Intercellular bridges in the embryo of the Atlantic squid, Loligo pealei. I. Cytoplasmic continuity and tissue differentiation. J. Embryol. Exp. Morphol. 57, 3-24.

Caruso Bavisotto, C., Cappello, F., Macario, A. J. L., Conway, De Macario, E., Logozzi, M., et al. (2017). Exosomal HSP60: a potentially useful biomarker for diagnosis, assessing prognosis, and monitoring response to treatment. Expert Rev. Mol. Diagn. 17, 815-822. doi: 10.1080/14737159.2017.1356230

Catchpoole, D. R., and Stewart, B. W. (1995). Formation of apoptotic bodies is associated with internucleosomal DNA fragmentation during drug-induced apoptosis. Exp. Cell Res. 216, 169-177. doi: 10.1006/excr.1995.1021

Church, D. D., Hoffman, J. R., Mangine, G. T., Jajtner, A. R., Townsend, J. R., Beyer, K. S., et al. (2016). Comparison of high-intensity vs. high-volume resistance training on the BDNF response to exercise. J. Appl. Physiol. (1985) 121, 123-128. doi: 10.1152/japplphysiol.00233.2016

Cocucci, E., and Meldolesi, J. (2015). Ectosomes and exosomes: shedding the confusion between extracellular vesicles. Trends Cell Biol. 25, 364-372. doi: 10.1016/j.tcb.2015.01.004

Coleman, M. L., Sahai, E. A., Yeo, M., Bosch, M., Dewar, A., and Olson, M. F. (2001). Membrane blebbing during apoptosis results from caspase-mediated activation of ROCK I. Nat. Cell Biol. 3, 339-345. doi: 10.1038/35070009

Colombo, M., Raposo, G., and Thery, C. (2014). Biogenesis, secretion, and intercellular interactions of exosomes and other extracellular vesicles. Annu. Rev. Cell Dev. Biol. 30, 255-289. doi: 10.1146/annurev-cellbio-101512- 122326

Covington, J. D., Tam, C. S., Bajpeyi, S., Galgani, J. E., Noland, R. C., Smith, S. R., et al. (2016). Myokine expression in muscle and myotubes in response to exercise stimulation. Med. Sci. Sports Exerc. 48, 384-390. doi: 10.1249/mss. 0000000000000787

Crane, J. D., Macneil, L. G., Lally, J. S., Ford, R. J., Bujak, A. L., Brar, I. K., et al. (2015). Exercise-stimulated interleukin-15 is controlled by AMPK and regulates skin metabolism and aging. Aging Cell 14, 625-634. doi: 10.1111/acel.12341

Cuevas-Ramos, D., Almeda-Valdes, P., Meza-Arana, C. E., Brito-Cordova, G., Gomez-Perez, F. J., Mehta, R., et al. (2012). Exercise increases serum fibroblast growth factor 21 (FGF21) levels. PLoS One 7:e38022. doi: 10.1371/journal.pone. 0038022

Danzer, K. M., Kranich, L. R., Ruf, W. P., Cagsal-Getkin, O., Winslow, A. R., Zhu, L., et al. (2012). Exosomal cell-to-cell transmission of alpha synuclein oligomers. Mol. Neurodegener. 7:42. doi: 10.1186/1750-1326-7-42

Deshmukh, A. S., Murgia, M., Nagaraj, N., Treebak, J. T., Cox, J., and Mann, M. (2015). Deep proteomics of mouse skeletal muscle enables quantitation of protein isoforms, metabolic pathways, and transcription factors. Mol. Cell Proteomics 14, 841-853. doi: 10.1074/mcp.m114.044222

Desrochers, L. M., Bordeleau, F., Reinhart-King, C. A., Cerione, R. A., and Antonyak, M. A. (2016). Microvesicles provide a mechanism for intercellular communication by embryonic stem cells during embryo implantation. Nat. Commun. 7:11958

Ding, M., Wang, C., Lu, X., Zhang, C., Zhou, Z., Chen, X., et al. (2018). Comparison of commercial exosome isolation kits for circulating exosomal microRNA profiling. Anal. Bioanal. Chem. 410, 3805-3814. doi: 10.1007/s00216-018-1052-4

Escola, J. M., Kleijmeer, M. J., Stoorvogel, W., Griffith, J. M., Yoshie, O., and Geuze, H. J. (1998). Selective enrichment of tetraspan proteins on the internal vesicles of multivesicular endosomes and on exosomes secreted by human B-lymphocytes. J. Biol. Chem. 273, 20121-20127. doi: 10.1074/jbc.273.32.20121

Esgalhado, M., Stockler-Pinto, M. B., Cardozo, L., Barboza, J. E., and Mafra, D. (2018). Does high intensity exercise affects irisin plasma levels in hemodialysis patients? A pilot study. J. Bras. Nefrol. 40, 53-58. doi: 10.1590/1678-4685-jbn3802

Fearon, K., Strasser, F., Anker, S. D., Bosaeus, I., Bruera, E., Fainsinger, R. L., et al. (2011). Definition and classification of cancer cachexia: an international consensus. Lancet Oncol. 12, 489-495. doi: 10.1016/s1470-2045(10)70218-7

Feng, D., Zhao, W. L., Ye, Y. Y., Bai, X. C., Liu, R. Q., Chang, L. F., et al. (2010). Cellular internalization of exosomes occurs through phagocytosis. Traffic 11, 675-687. doi: 10.1111/j.1600-0854.2010.01041.x
Forterre, A., Jalabert, A., Berger, E., Baudet, M., Chikh, K., Errazuriz, E., et al. (2014). Proteomic analysis of $\mathrm{C} 2 \mathrm{C} 12$ myoblast and myotube exosome-like vesicles: a new paradigm for myoblast-myotube cross talk? PLoS One 9:e84153. doi: 10.1371/journal.pone.0084153

Fortunato, A. K., Pontes, W. M., De Souza, D. M. S., Prazeres, J. S. F., MarcucciBarbosa, L. S., Santos, J. M. M., et al. (2018). Strength Training Session induces important changes on physiological, immunological, and inflammatory biomarkers. J. Immunol. Res. 2018:9675216.

Franke, W. W. (2009). Discovering the molecular components of intercellular junctions-a historical view. Cold Spring Harb. Perspect. Biol. 1:a003061. doi: 10.1101/cshperspect.a003061

Fruhbeis, C., Helmig, S., Tug, S., Simon, P., and Kramer-Albers, E. M. (2015). Physical exercise induces rapid release of small extracellular vesicles into the circulation. J. Extracell. Vesicles 4:28239. doi: 10.3402/jev.v4. 28239

Gamez-Valero, A., Monguio-Tortajada, M., Carreras-Planella, L., Franquesa, M., Beyer, K., and Borras, F. E. (2016). Size-exclusion chromatography-based isolation minimally alters extracellular vesicles' characteristics compared to precipitating agents. Sci. Rep. 6:33641.

Garcia, N. A., Moncayo-Arlandi, J., Sepulveda, P., and Diez-Juan, A. (2016). Cardiomyocyte exosomes regulate glycolytic flux in endothelium by direct transfer of GLUT transporters and glycolytic enzymes. Cardiovasc. Res. 109, 397-408. doi: 10.1093/cvr/cvv260

Gercel-Taylor, C., Atay, S., Tullis, R. H., Kesimer, M., and Taylor, D. D. (2012). Nanoparticle analysis of circulating cell-derived vesicles in ovarian cancer patients. Anal. Biochem. 428, 44-53. doi: 10.1016/j.ab.2012.06.004

Giralt, M., and Villarroya, F. (2013). White, brown, beige/brite: different adipose cells for different functions? Endocrinology 154, 2992-3000. doi: 10.1210/en. 2013-1403

Giudice, J., and Taylor, J. M. (2017). Muscle as a paracrine and endocrine organ. Curr. Opin. Pharmacol. 34, 49-55. doi: 10.1016/j.coph.2017.05.005

Gomari, H., Forouzandeh Moghadam, M., and Soleimani, M. (2018). Targeted cancer therapy using engineered exosome as a natural drug delivery vehicle. Onco Targets Ther. 11, 5753-5762. doi: 10.2147/ott.s173110

Gomes, J. L., Fernandes, T., Soci, U. P., Silveira, A. C., Barretti, D. L., Negrao, C. E., et al. (2017). Obesity downregulates MicroRNA-126 inducing capillary rarefaction in skeletal muscle: effects of aerobic exercise training. Oxid. Med. Cell. Longev. 2017:2415246.

Gomez-Pinilla, F., Ying, Z., Roy, R. R., Molteni, R., and Edgerton, V. R. (2002). Voluntary exercise induces a BDNF-mediated mechanism that promotes neuroplasticity. J. Neurophysiol. 88, 2187-2195. doi: 10.1152/jn.00152.2002

Gorgens, S. W., Eckardt, K., Jensen, J., Drevon, C. A., and Eckel, J. (2015). Exercise and regulation of adipokine and myokine production. Prog. Mol. Biol. Transl. Sci. 135, 313-336. doi: 10.1016/bs.pmbts.2015.07.002

Gosselin, J., Tomoiu, A., Gallo, R. C., and Flamand, L. (1999). Interleukin-15 as an activator of natural killer cell-mediated antiviral response. Blood 94, 4210-4219.

Griesbeck, O., Parsadanian, A. S., Sendtner, M., and Thoenen, H. (1995). Expression of neurotrophins in skeletal muscle: quantitative comparison and significance for motoneuron survival and maintenance of function. J. Neurosci. Res. 42, 21-33. doi: 10.1002/jnr.490420104

Groves, J. A., and Ellwood, P. A. (1985). A comparison of the analyses of respirable quartz by infra-red spectrophotometry at HSE field and headquarters laboratories. Ann. Occup. Hyg. 29, 429-433.

Guescini, M., Canonico, B., Lucertini, F., Maggio, S., Annibalini, G., Barbieri, E., et al. (2015). Muscle releases alpha-sarcoglycan positive extracellular vesicles carrying miRNAs in the bloodstream. PLoS One 10:e0125094. doi: 10.1371/ journal.pone.0125094

Guix, F. X., Corbett, G. T., Cha, D. J., Mustapic, M., Liu, W., Mengel, D., et al. (2018). Detection of aggregation-competent tau in neuron-derived extracellular vesicles. Int. J. Mol. Sci. 19:E663.

Gustafsson, T., Bodin, K., Sylven, C., Gordon, A., Tyni-Lenne, R., and Jansson, E. (2001). Increased expression of VEGF following exercise training in patients with heart failure. Eur. J. Clin. Invest. 31, 362-366. doi: 10.1046/j.1365-2362. 2001.00816.x

Gutierrez-Vazquez, C., Villarroya-Beltri, C., Mittelbrunn, M., and Sanchez-Madrid, F. (2013). Transfer of extracellular vesicles during immune cell-cell interactions. Immunol. Rev. 251, 125-142. doi: 10.1111/imr.12013 
Ha, D., Yang, N., and Nadithe, V. (2016). Exosomes as therapeutic drug carriers and delivery vehicles across biological membranes: current perspectives and future challenges. Acta Pharm. Sin. B 6, 287-296. doi: 10.1016/j.apsb.2016.02.001

Haga, H., Yan, I. K., Takahashi, K., Wood, J., Zubair, A., and Patel, T. (2015). Tumour cell-derived extracellular vesicles interact with mesenchymal stem cells to modulate the microenvironment and enhance cholangiocarcinoma growth. J. Extracell. Vesicles 4:24900. doi: 10.3402/jev.v4.24900

Haney, M. J., Klyachko, N. L., Zhao, Y., Gupta, R., Plotnikova, E. G., He, Z., et al. (2015). Exosomes as drug delivery vehicles for Parkinson's disease therapy. J. Control. Release 207, 18-30. doi: 10.1016/j.jconrel.2015.03.033

Haraszti, R. A., Didiot, M. C., Sapp, E., Leszyk, J., Shaffer, S. A., Rockwell, H. E., et al. (2016). High-resolution proteomic and lipidomic analysis of exosomes and microvesicles from different cell sources. J. Extracell. Vesicles 5:32570. doi: 10.3402/jev.v5.32570

Heldt, S. A., Stanek, L., Chhatwal, J. P., and Ressler, K. J. (2007). Hippocampusspecific deletion of BDNF in adult mice impairs spatial memory and extinction of aversive memories. Mol. Psychiatry 12, 656-670. doi: 10.1038/sj.mp.4001957

Hessvik, N. P., and Llorente, A. (2018). Current knowledge on exosome biogenesis and release. Cell. Mol. Life Sci. 75, 193-208. doi: 10.1007/s00018-017-2595-9

Hingorjo, M. R., Zehra, S., Saleem, S., and Qureshi, M. A. (2018). Serum Interleukin-15 and its relationship with adiposity Indices before and after short-term endurance exercise. Pak. J. Med. Sci. 34, 1125-1131.

Hittel, D. S., Axelson, M., Sarna, N., Shearer, J., Huffman, K. M., and Kraus, W. E. (2010). Myostatin decreases with aerobic exercise and associates with insulin resistance. Med. Sci. Sports Exerc. 42, 2023-2029. doi: 10.1249/mss. 0b013e3181e0b9a8

Hjorth, M., Pourteymour, S., Gorgens, S. W., Langleite, T. M., Lee, S., Holen, T., et al. (2016). Myostatin in relation to physical activity and dysglycaemia and its effect on energy metabolism in human skeletal muscle cells. Acta Physiol. (Oxf.) 217, 45-60. doi: 10.1111/apha.12631

Hofer, M. M., and Barde, Y. A. (1988). Brain-derived neurotrophic factor prevents neuronal death in vivo. Nature 331, 261-262. doi: 10.1038/331261a0

Hojman, P., Dethlefsen, C., Brandt, C., Hansen, J., Pedersen, L., and Pedersen, B. K. (2011). Exercise-induced muscle-derived cytokines inhibit mammary cancer cell growth. Am. J. Physiol. Endocrinol. Metab. 301, E504-E510.

Hu, F. B., Sigal, R. J., Rich-Edwards, J. W., Colditz, G. A., Solomon, C. G., Willett, W. C., et al. (1999). Walking compared with vigorous physical activity and risk of type 2 diabetes in women: a prospective study. JAMA 282, 1433-1439.

Huh, J. Y., Panagiotou, G., Mougios, V., Brinkoetter, M., Vamvini, M. T., Schneider, B. E., et al. (2012). FNDC5 and irisin in humans: I. Predictors of circulating concentrations in serum and plasma and II. mRNA expression and circulating concentrations in response to weight loss and exercise. Metabolism 61, 1725-1738. doi: 10.1016/j.metabol.2012. 09.002

Jaworski, E., Narayanan, A., Van Duyne, R., Shabbeer-Meyering, S., Iordanskiy, S., Saifuddin, M., et al. (2014). Human T-lymphotropic virus type 1-infected cells secrete exosomes that contain Tax protein. J. Biol. Chem. 289, 22284-22305. doi: 10.1074/jbc.m114.549659

Jeon, Y. K., and Ha, C. H. (2017). The effect of exercise intensity on brain derived neurotrophic factor and memory in adolescents. Environ. Health Prev. Med. 22:27.

Kalamvoki, M., Du, T., and Roizman, B. (2014). Cells infected with herpes simplex virus 1 export to uninfected cells exosomes containing STING, viral mRNAs, and microRNAs. Proc. Natl. Acad. Sci. U.S.A. 111, E4991-E4996.

Kalcheim, C., and Gendreau, M. (1988). Brain-derived neurotrophic factor stimulates survival and neuronal differentiation in cultured avian neural crest. Brain Res. 469, 79-86. doi: 10.1016/0165-3806(88)90171-x

Kalimi, G. H., and Lo, C. W. (1988). Communication compartments in the gastrulating mouse embryo. J. Cell. Biol. 107, 241-255. doi: $10.1083 /$ jcb.107.1.241

Kalkan, A. K., Cakmak, H. A., Erturk, M., Kalkan, K. E., Uzun, F., Tasbulak, O., et al. (2018). Adropin and Irisin in patients with cardiac cachexia. Arq. Bras Cardiol. 111, 39-47.

Kalra, H., Drummen, G. P., and Mathivanan, S. (2016). Focus on extracellular vesicles: introducing the next small big thing. Int. J. Mol. Sci. 17:170. doi: 10.3390/ijms17020170

Keklikoglou, I., Cianciaruso, C., Guc, E., Squadrito, M. L., Spring, L. M., Tazzyman, S., et al. (2019). Chemotherapy elicits pro-metastatic extracellular vesicles in breast cancer models. Nat. Cell Biol. 21, 190-202. doi: 10.1038/ s41556-018-0256-3

Kesimer, M., and Gupta, R. (2015). Physical characterization and profiling of airway epithelial derived exosomes using light scattering. Methods 87, 59-63. doi: 10.1016/j.ymeth.2015.03.013

Khatua, A. K., Taylor, H. E., Hildreth, J. E., and Popik, W. (2009). Exosomes packaging APOBEC3G confer human immunodeficiency virus resistance to recipient cells. J. Virol. 83, 512-521. doi: 10.1128/jvi.01658-08

Kim, H. J., Lee, H. J., So, B., Son, J. S., Yoon, D., and Song, W. (2016). Effect of aerobic training and resistance training on circulating irisin level and their association with change of body composition in overweight/obese adults: a pilot study. Physiol. Res. 65, 271-279.

Kim, H. J., and Song, W. (2017). Resistance training increases fibroblast growth factor-21 and irisin levels in the skeletal muscle of Zucker diabetic fatty rats. J. Exerc. Nutr. Biochem. 21, 50-54. doi: 10.20463/jenb.2017.0008

Knudsen, J. G., Gudiksen, A., Bertholdt, L., Overby, P., Villesen, I., Schwartz, C. L., et al. (2017). Skeletal muscle IL-6 regulates muscle substrate utilization and adipose tissue metabolism during recovery from an acute bout of exercise. PLoS One 12:e0189301. doi: 10.1371/journal.pone.0189301

Konoshenko, M. Y., Lekchnov, E. A., Vlassov, A. V., and Laktionov, P. P. (2018). Isolation of extracellular vesicles: general methodologies and latest trends. Biomed. Res. Int. 2018:8545347.

Koshimizu, H., Kiyosue, K., Hara, T., Hazama, S., Suzuki, S., Uegaki, K., et al. (2009). Multiple functions of precursor BDNF to CNS neurons: negative regulation of neurite growth, spine formation and cell survival. Mol. Brain 2:27. doi: 10.1186/1756-6606-2-27

Kowal, J., Arras, G., Colombo, M., Jouve, M., Morath, J. P., Primdal-Bengtson, B., et al. (2016). Proteomic comparison defines novel markers to characterize heterogeneous populations of extracellular vesicle subtypes. Proc. Natl. Acad. Sci. U.S. A 113, E968-E977.

Kraemer, R. R., Shockett, P., Webb, N. D., Shah, U., and Castracane, V. D. (2014). A transient elevated irisin blood concentration in response to prolonged, moderate aerobic exercise in young men and women. Horm. Metab. Res. 46, 150-154. doi: 10.1055/s-0033-1355381

Kurachi, M., Mikuni, M., and Ishizaki, Y. (2016). Extracellular vesicles from vascular endothelial cells promote survival, proliferation and motility of oligodendrocyte precursor cells. PLoS One 11:e0159158. doi: 10.1371/journal. pone. 0159158

Liang, G., Kan, S., Zhu, Y., Feng, S., Feng, W., and Gao, S. (2018). Engineered exosome-mediated delivery of functionally active miR-26a and its enhanced suppression effect in HepG2 cells. Int. J. Nanomed. 13, 585-599. doi: 10.2147/ ijn.s 154458

Lin, Y., Wu, J., Gu, W., Huang, Y., Tong, Z., Huang, L., et al. (2018). Exosomeliposome hybrid nanoparticles deliver CRISPR/Cas9 system in MSCs. Adv. Sci. (Weinh) 5:1700611. doi: 10.1002/advs.201700611

Liu, Y., Li, D., Liu, Z., Zhou, Y., Chu, D., Li, X., et al. (2015). Targeted exosomemediated delivery of opioid receptor $\mathrm{Mu}$ siRNA for the treatment of morphine relapse. Sci. Rep. 5:17543.

Lobb, R. J., Becker, M., Wen, S. W., Wong, C. S., Wiegmans, A. P., Leimgruber, A., et al. (2015). Optimized exosome isolation protocol for cell culture supernatant and human plasma. J. Extracell. Vesicles 4:27031. doi: 10.3402/jev.v4.27031

Logozzi, M., De Milito, A., Lugini, L., Borghi, M., Calabro, L., Spada, M., et al. (2009). High levels of exosomes expressing CD63 and caveolin-1 in plasma of melanoma patients. PLoS One 4:e5219. doi: 10.1371/journal.pone.0005219

Lombardi, G., Sanchis-Gomar, F., Perego, S., Sansoni, V., and Banfi, G. (2016). Implications of exercise-induced adipo-myokines in bone metabolism. Endocrine 54, 284-305. doi: 10.1007/s12020-015-0834-0

Lotvall, J., Hill, A. F., Hochberg, F., Buzas, E. I., Di Vizio, D., Gardiner, C., et al. (2014). Minimal experimental requirements for definition of extracellular vesicles and their functions: a position statement from the international society for extracellular vesicles. J. Extracell. Vesicles 3:26913. doi: 10.3402/jev.v3.26913

Lovett, J. A. C., Durcan, P. J., and Myburgh, K. H. (2018). Investigation of circulating extracellular vesicle MicroRNA following two consecutive bouts of muscle-damaging exercise. Front. Physiol. 9:1149. doi: 10.3389/fphys.2018. 01149

Lu, Y., Li, H., Shen, S. W., Shen, Z. H., Xu, M., Yang, C. J., et al. (2016). Swimming exercise increases serum irisin level and reduces body fat mass in high-fat-diet fed Wistar rats. Lipids Health Dis. 15:93. 
Lukacs, N. W., Strieter, R. M., Elner, V., Evanoff, H. L., Burdick, M. D., and Kunkel, S. L. (1995). Production of chemokines, interleukin- 8 and monocyte chemoattractant protein-1, during monocyte: endothelial cell interactions. Blood 86, 2767-2773.

Maas, S. L., De Vrij, J., and Broekman, M. L. (2014). Quantification and sizeprofiling of extracellular vesicles using tunable resistive pulse sensing. J. Vis. Exp. 19:e51623.

Mathur, N., and Pedersen, B. K. (2008). Exercise as a mean to control low-grade systemic inflammation. Mediat. Inflamm. 2008:109502.

Matthews, V. B., Astrom, M. B., Chan, M. H., Bruce, C. R., Krabbe, K. S., Prelovsek, O., et al. (2009). Brain-derived neurotrophic factor is produced by skeletal muscle cells in response to contraction and enhances fat oxidation via activation of AMP-activated protein kinase. Diabetologia 52, 1409-1418. doi: 10.1007/ s00125-009-1364-1

Matthews, V. B., Astrom, M. B., Chan, M. H., Bruce, C. R., Krabbe, K. S., Prelovsek, O., et al. (2015). Erratum to: brain-derived neurotrophic factor is produced by skeletal muscle cells in response to contraction and enhances fat oxidation via activation of AMP-activated protein kinase. Diabetologia 58, 854-855. doi: 10.1007/s00125-0153502-2

Mauro, A. (1961). Satellite cell of skeletal muscle fibers. J. Biophys. Biochem. Cytol. 9, 493-495. doi: 10.1083/jcb.9.2.493

Melzer, W., Rios, E., and Schneider, M. F. (1984). Time course of calcium release and removal in skeletal muscle fibers. Biophys. J. 45, 637-641. doi: 10.1016/ s0006-3495(84)84203-4

Mikus, C. R., Rector, R. S., Arce-Esquivel, A. A., Libla, J. L., Booth, F. W., Ibdah, J. A., et al. (2010). Daily physical activity enhances reactivity to insulin in skeletal muscle arterioles of hyperphagic Otsuka Long-Evans Tokushima Fatty rats. J. Appl. Physiol. (1985) 109, 1203-1210. doi: 10.1152/japplphysiol.00064. 2010

Miyamoto-Mikami, E., Sato, K., Kurihara, T., Hasegawa, N., Fujie, S., Fujita, S., et al. (2015). Endurance training-induced increase in circulating irisin levels is associated with reduction of abdominal visceral fat in middleaged and older adults. PLoS One 10:e0120354. doi: 10.1371/journal.pone.012 0354

Miyatake, N., Nishikawa, H., Morishita, A., Kunitomi, M., Wada, J., Suzuki, H., et al. (2002). Daily walking reduces visceral adipose tissue areas and improves insulin resistance in Japanese obese subjects. Diabetes Res. Clin. Pract. 58, 101-107. doi: 10.1016/s0168-8227(02)00129-8

Mucci, P., Durand, F., Lebel, B., Bousquet, J., and Prefaut, C. (2000). Interleukins 1-beta, -8, and histamine increases in highly trained, exercising athletes. Med. Sci. Sports Exerc. 32, 1094-1100. doi: 10.1097/00005768-20000600000009

Mulcahy, L. A., Pink, R. C., and Carter, D. R. (2014). Routes and mechanisms of extracellular vesicle uptake. J. Extracell. Vesicles 3.

Naseri, Z., Oskuee, R. K., Jaafari, M. R., and Forouzandeh Moghadam, M. (2018). Exosome-mediated delivery of functionally active miRNA-142-3p inhibitor reduces tumorigenicity of breast cancer in vitro and in vivo. Int. J. Nanomed. 13, 7727-7747. doi: 10.2147/ijn.s182384

Nelson, S., and Summer, W. R. (1998). Innate immunity, cytokines, and pulmonary host defense. Infect. Dis. Clin. N. Am. 12, 555-567. doi: 10.1016/s0891-5520(05) 70198-7

Nielsen, A. R., Mounier, R., Plomgaard, P., Mortensen, O. H., Penkowa, M., Speerschneider, T., et al. (2007). Expression of interleukin-15 in human skeletal muscle effect of exercise and muscle fibre type composition. J. Physiol. 584, 305-312. doi: 10.1113/jphysiol.2007.139618

Nilsson, M. I., Bourgeois, J. M., Nederveen, J. P., Leite, M. R., Hettinga, B. P., Bujak, A. L., et al. (2019). Lifelong aerobic exercise protects against inflammaging and cancer. PLoS One 14:e0210863. doi: 10.1371/journal.pone.021 0863

Niu, C., Wang, X., Zhao, M., Cai, T., Liu, P., Li, J., et al. (2016). Macrophage foam cell-derived extracellular vesicles promote vascular smooth muscle cell migration and adhesion. J. Am. Heart Assoc. 5:e004099.

Norheim, F., Hjorth, M., Langleite, T. M., Lee, S., Holen, T., Bindesboll, C., et al. (2014). Regulation of angiopoietin-like protein 4 production during and after exercise. Physiol. Rep. 2:e12109. doi: 10.14814/phy2. 12109
Nygaard, H., Slettalokken, G., Vegge, G., Hollan, I., Whist, J. E., Strand, T., et al. (2015). Irisin in blood increases transiently after single sessions of intense endurance exercise and heavy strength training. PLoS One 10:e0121367. doi: 10.1371/journal.pone.0121367

Ohyashiki, J. H., Umezu, T., and Ohyashiki, K. (2018). Extracellular vesiclemediated cell-cell communication in haematological neoplasms. Philos. Trans. R. Soc. Lond. B Biol. Sci. 373:20160484. doi: 10.1098/rstb.2016.0484

Ostrowski, K., Rohde, T., Asp, S., Schjerling, P., and Pedersen, B. K. (1999). Pro- and anti-inflammatory cytokine balance in strenuous exercise in humans. J. Physiol. 515( Pt 1), 287-291. doi: 10.1111/j.1469-7793.1999.287ad.x

Pang, M., Yang, J., Rao, J., Wang, H., Zhang, J., Wang, S., et al. (2018). Timedependent changes in increased levels of plasma irisin and muscle PGC1alpha and FNDC5 after exercise in mice. Tohoku J. Exp. Med. 244, 93-103. doi: $10.1620 /$ tjem. 244.93

Pap, E., Pallinger, E., Pasztoi, M., and Falus, A. (2009). Highlights of a new type of intercellular communication: microvesicle-based information transfer. Inflamm. Res. 58, 1-8. doi: 10.1007/s00011-008-8210-7

Papanicolaou, D. A., Petrides, J. S., Tsigos, C., Bina, S., Kalogeras, K. T., Wilder, R., et al. (1996). Exercise stimulates interleukin-6 secretion: inhibition by glucocorticoids and correlation with catecholamines. Am. J. Physiol. 271, E601E605.

Park, C. H., and Kwak, Y. S. (2017). Analysis of energy restriction and physical activity on brain function: the role of ketone body and brain-derived neurotrophic factor. J. Exerc. Rehabil. 13, 378-380. doi: 10.12965/jer.1735028. 514

Peake, J. M., Della Gatta, P., Suzuki, K., and Nieman, D. C. (2015). Cytokine expression and secretion by skeletal muscle cells: regulatory mechanisms and exercise effects. Exerc. Immunol. Rev. 21, 8-25.

Pedersen, B. K. (2009). The diseasome of physical inactivity-and the role of myokines in muscle-fat cross talk. J. Physiol. 587, 5559-5568. doi: 10.1113/ jphysiol.2009.179515

Pedersen, B. K. (2013). Muscle as a secretory organ. Compr. Physiol. 3, 1337-1362.

Pedersen, B. K., and Saltin, B. (2015). Exercise as medicine - Evidence for prescribing exercise as therapy in 26 different chronic diseases. Scand. J. Med. Sci. Sports 25(Suppl. 3), 1-72. doi: 10.1111/sms.12581

Pedersen, K. W., Kierulf, B., and Neurauter, A. (2017). Specific and generic isolation of extracellular vesicles with magnetic beads. Methods Mol. Biol. 1660, 65-87. doi: 10.1007/978-1-4939-7253-1_7

Petersen, A. M., and Pedersen, B. K. (2005). The anti-inflammatory effect of exercise. J. Appl. Physiol. (1985) 98, 1154-1162.

Petersen, A. M., and Pedersen, B. K. (2006). The role of IL-6 in mediating the anti-inflammatory effects of exercise. J. Physiol. Pharmacol. 57(Suppl. 10), 43-51.

Petersen, T. N., Brunak, S., Von Heijne, G., and Nielsen, H. (2011). SignalP 4.0: discriminating signal peptides from transmembrane regions. Nat. Methods 8, 785-786. doi: 10.1038/nmeth.1701

Pfrieger, F. W., and Vitale, N. (2018). Cholesterol and the journey of extracellular vesicles. J. Lipid Res. 59, 2255-2261. doi: 10.1194/jlr.r08 4210

Poliakov, A., Spilman, M., Dokland, T., Amling, C. L., and Mobley, J. A. (2009). Structural heterogeneity and protein composition of exosome-like vesicles (prostasomes) in human semen. Prostate 69, 159-167. doi: 10.1002/pros.20860

Pospichalova, V., Svoboda, J., Dave, Z., Kotrbova, A., Kaiser, K., Klemova, D., et al. (2015). Simplified protocol for flow cytometry analysis of fluorescently labeled exosomes and microvesicles using dedicated flow cytometer. J. Extracell. Vesicles 4:25530. doi: 10.3402/jev.v4. 25530

Pourteymour, S., Eckardt, K., Holen, T., Langleite, T., Lee, S., Jensen, J., et al. (2017). Global mRNA sequencing of human skeletal muscle: search for novel exerciseregulated myokines. Mol. Metab. 6, 352-365. doi: 10.1016/j.molmet.2017.01. 007

Qi, S. Y., Groves, J. T., and Chakraborty, A. K. (2001). Synaptic pattern formation during cellular recognition. Proc. Natl. Acad. Sci. U.S.A. 98, 6548-6553. doi: 10.1073/pnas. 111536798

Ratajczak, J., Miekus, K., Kucia, M., Zhang, J., Reca, R., Dvorak, P., et al. (2006). Embryonic stem cell-derived microvesicles reprogram hematopoietic progenitors: evidence for horizontal transfer of mRNA 
and protein delivery. Leukemia 20, 847-856. doi: 10.1038/sj.leu.240 4132

Reza, M. M., Subramaniyam, N., Sim, C. M., Ge, X., Sathiakumar, D., Mcfarlane, C., et al. (2017). Irisin is a pro-myogenic factor that induces skeletal muscle hypertrophy and rescues denervation-induced atrophy. Nat. Commun. 8:1104.

Roh, H. T., and So, W. Y. (2017). The effects of aerobic exercise training on oxidant-antioxidant balance, neurotrophic factor levels, and blood-brain barrier function in obese and non-obese men. J. Sport Health Sci. 6, 447-453. doi: 10.1016/j.jshs.2016.07.006

Romancino, D. P., Paterniti, G., Campos, Y., De Luca, A., Di Felice, V., D’azzo, A., et al. (2013). Identification and characterization of the nano-sized vesicles released by muscle cells. FEBS Lett. 587, 1379-1384. doi: 10.1016/j.febslet.2013. 03.012

Rosenberger, L., Ezquer, M., Lillo-Vera, F., Pedraza, P. L., Ortuzar, M. I., Gonzalez, P. L., et al. (2019). Stem cell exosomes inhibit angiogenesis and tumor growth of oral squamous cell carcinoma. Sci. Rep. 9:663.

Safdar, A., Saleem, A., and Tarnopolsky, M. A. (2016). The potential of endurance exercise-derived exosomes to treat metabolic diseases. Nat. Rev. Endocrinol. 12, 504-517. doi: 10.1038/nrendo.2016.76

Sato, Y. T., Umezaki, K., Sawada, S., Mukai, S. A., Sasaki, Y., Harada, N., et al. (2016). Engineering hybrid exosomes by membrane fusion with liposomes. Sci. Rep. 6:21933.

Savina, A., Furlan, M., Vidal, M., and Colombo, M. I. (2003). Exosome release is regulated by a calcium-dependent mechanism in K562 cells. J. Biol. Chem. 278, 20083-20090. doi: 10.1074/jbc.m301642200

Schey, K. L., Luther, J. M., and Rose, K. L. (2015). Proteomics characterization of exosome cargo. Methods 87, 75-82. doi: 10.1016/j.ymeth.2015.03.018

Schild, M., Eichner, G., Beiter, T., Zugel, M., Krumholz-Wagner, I., Hudemann, J., et al. (2016). Effects of acute endurance exercise on plasma protein profiles of endurance-trained and untrained individuals over time. Mediat. Inflamm. 2016:4851935.

Schweitzer, H. (1973). [Remarks from practice on liver and alcohol]. Z. Allgemeinmed. 49, 1170-1171.

Shojaee-Moradie, F., Baynes, K. C., Pentecost, C., Bell, J. D., Thomas, E. L., Jackson, N. C., et al. (2007). Exercise training reduces fatty acid availability and improves the insulin sensitivity of glucose metabolism. Diabetologia 50, 404-413. doi: 10.1007/s00125-006-0498-7

Sicard, R. E. (1986). Hormones, neurosecretions, and growth factors as signal molecules for intercellular communication. Dev. Compar. Immunol. 10, 269-272. doi: 10.1016/0145-305x(86)90011-x

Sitar, S., Kejzar, A., Pahovnik, D., Kogej, K., Tusek-Znidaric, M., Lenassi, M., et al. (2015). Size characterization and quantification of exosomes by asymmetricalflow field-flow fractionation. Anal. Chem. 87, 9225-9233. doi: 10.1021/acs. analchem.5b01636

Skottvoll, F. S., Berg, H. E., Bjorseth, K., Lund, K., Roos, N., Bekhradnia, S., et al. (2019). Ultracentrifugation versus kit exosome isolation: nanoLC-MS and other tools reveal similar performance biomarkers, but also contaminations. Future Sci. OA 5:FSO359.

Sokolova, V., Ludwig, A. K., Hornung, S., Rotan, O., Horn, P. A., Epple, M., et al. (2011). Characterisation of exosomes derived from human cells by nanoparticle tracking analysis and scanning electron microscopy. Colloids Surf. B Biointerfaces 87, 146-150. doi: 10.1016/j.colsurfb.2011.05.013

Stanford, K. I., and Goodyear, L. J. (2018). Muscle-adipose tissue cross talk. Cold Spring Harb. Perspect. Med. 8:a029801. doi: 10.1101/cshperspect.a029801

Suire, C. N., Eitan, E., Shaffer, N. C., Tian, Q., Studenski, S., Mattson, M. P., et al. (2017). Walking speed decline in older adults is associated with elevated pro-BDNF in plasma extracellular vesicles. Exp. Gerontol. 98, 209-216. doi: 10.1016/j.exger.2017.08.024

Sun, D., Zhuang, X., Xiang, X., Liu, Y., Zhang, S., Liu, C., et al. (2010). A novel nanoparticle drug delivery system: the anti-inflammatory activity of curcumin is enhanced when encapsulated in exosomes. Mol. Ther. 18, 1606-1614. doi: $10.1038 / \mathrm{mt} .2010 .105$

Surman, M., Stepien, E., Hoja-Lukowicz, D., and Przybylo, M. (2017). Deciphering the role of ectosomes in cancer development and progression: focus on the proteome. Clin. Exp. Metastasis 34, 273-289. doi: 10.1007/s10585-0179844-z

Sutaria, D. S., Badawi, M., Phelps, M. A., and Schmittgen, T. D. (2017). Achieving the promise of therapeutic extracellular vesicles: the devil is in details of therapeutic loading. Pharm. Res. 34, 1053-1066. doi: 10.1007/s11095-0172123-5

Szatanek, R., Baran, J., Siedlar, M., and Baj-Krzyworzeka, M. (2015). Isolation of extracellular vesicles: determining the correct approach (Review). Int. J. Mol. Med. 36, 11-17. doi: 10.3892/ijmm.2015.2194

Tanimura, Y., Aoi, W., Takanami, Y., Kawai, Y., Mizushima, K., Naito, Y., et al. (2016). Acute exercise increases fibroblast growth factor 21 in metabolic organs and circulation. Physiol. Rep. 4:e12828. doi: 10.14814/phy2.12828

Teng, H. K., Teng, K. K., Lee, R., Wright, S., Tevar, S., Almeida, R. D., et al. (2005). ProBDNF induces neuronal apoptosis via activation of a receptor complex of p75NTR and sortilin. J. Neurosci. 25, 5455-5463. doi: 10.1523/jneurosci.512304.2005

Thery, C., Amigorena, S., Raposo, G., and Clayton, A. (2006). Isolation and characterization of exosomes from cell culture supernatants and biological fluids. Curr. Protoc. Cell Biol. Chap. 3:Unit 3, 22.

Théry, C., Witwer, K. W., Aikawa, E., Alcaraz, M. J., Anderson, J. D., Andriantsitohaina, R., et al. (2018). Minimal information for studies of extracellular vesicles 2018 (MISEV2018): a position statement of the International Society for Extracellular Vesicles and update of the MISEV2014 guidelines. J. Extracell. Vesicles 7:1535750. doi: 10.1080/20013078.2018.1535750

Thornton, S. M., Krolopp, J. E., and Abbott, M. J. (2016). IL-15 mediates mitochondrial activity through a PPARdelta-dependentPPARalpha-independent mechanism in skeletal muscle cells. PPAR Res. 2016:5465804.

Turturici, G., Tinnirello, R., Sconzo, G., and Geraci, F. (2014). Extracellular membrane vesicles as a mechanism of cell-to-cell communication: advantages and disadvantages. Am. J. Physiol. Cell Physiol. 306, C621-C633.

Van Deun, J., Mestdagh, P., Sormunen, R., Cocquyt, V., Vermaelen, K., Vandesompele, J., et al. (2014). The impact of disparate isolation methods for extracellular vesicles on downstream RNA profiling. J. Extracell. Vesicles 18:3.

Vella, L. J., Sharples, R. A., Lawson, V. A., Masters, C. L., Cappai, R., and Hill, A. F. (2007). Packaging of prions into exosomes is associated with a novel pathway of PrP processing. J. Pathol. 211, 582-590. doi: 10.1002/path.2145

Verweij, F. J., Revenu, C., Arras, G., Dingli, F., Loew, D., Pegtel, D. M., et al. (2019). Live tracking of inter-organ communication by endogenous exosomes in vivo. Dev. Cell 48:e574.

Wang, Y., Wang, Q., Wei, X., Shao, J., Zhao, J., Zhang, Z., et al. (2017). Global scientific trends on exosome research during 2007-2016: a bibliometric analysis. Oncotarget 8, 48460-48470.

Whitham, M., Parker, B. L., Friedrichsen, M., Hingst, J. R., Hjorth, M., Hughes, W. E., et al. (2018). Extracellular vesicles provide a means for tissue crosstalk during exercise. Cell Metab. 27:e234.

Willms, E., Cabanas, C., Mager, I., Wood, M. J. A., and Vader, P. (2018). Extracellular vesicle heterogeneity: subpopulations, isolation techniques, and diverse functions in cancer progression. Front. Immunol. 9:738. doi: 10.3389/ fimmu.2018.00738

Wolf, P. (1967). The nature and significance of platelet products in human plasma. Br. J. Haematol. 13, 269-288. doi: 10.1111/j.1365-2141.1967.tb08741.x

Wu, J., Cohen, P., and Spiegelman, B. M. (2013). Adaptive thermogenesis in adipocytes: is beige the new brown? Genes Dev. 27, 234-250. doi: 10.1101/gad. 211649.112

Wu, J. Y., Ji, A. L., Wang, Z. X., Qiang, G. H., Qu, Z., Wu, J. H., et al. (2018). Exosome-mimetic nanovesicles from hepatocytes promote hepatocyte proliferation in vitro and liver regeneration in vivo. Sci. Rep. 8:2471.

Xi, Y., Gong, D. W., and Tian, Z. (2016). FSTL1 as a potential mediator of exercise-induced cardioprotection in post-myocardial infarction rats. Sci. Rep. $6: 32424$.

Yao, X., Huang, J., Zhong, H., Shen, N., Faggioni, R., Fung, M., et al. (2014). Targeting interleukin-6 in inflammatory autoimmune diseases and cancers. Pharmacol. Ther. 141, 125-139. doi: 10.1016/j.pharmthera.2013.09.004

Zhang, Z., Wang, C., Li, T., Liu, Z., and Li, L. (2014). Comparison of ultracentrifugation and density gradient separation methods for isolating Tca8113 human tongue cancer cell line-derived exosomes. Oncol. Lett. 8, 1701-1706. doi: 10.3892/ol.2014. 2373

Zhao, H., Yang, L., Baddour, J., Achreja, A., Bernard, V., Moss, T., et al. (2016). Tumor microenvironment derived exosomes pleiotropically modulate cancer cell metabolism. Elife 5:e10250. 
Zhao, J., Su, Z., Qu, C., and Dong, Y. (2017). Effects of 12 weeks resistance training on serum irisin in older male adults. Front. Physiol. 8:171. doi: 10.3389/fphys. 2017.00171

Zheng, H., Liu, Y., Li, W., Yang, B., Chen, D., Wang, X., et al. (2006). Beneficial effects of exercise and its molecular mechanisms on depression in rats. Behav. Brain Res. 168, 47-55. doi: 10.1016/j.bbr.2005. 10.007

Zomer, A., Vendrig, T., Hopmans, E. S., Van Eijndhoven, M., Middeldorp, J. M., and Pegtel, D. M. (2010). Exosomes: fit to deliver small RNA. Commun. Integr. Biol. 3, 447-450. doi: 10.4161/cib.3.5.12339
Conflict of Interest Statement: The authors declare that the research was conducted in the absence of any commercial or financial relationships that could be construed as a potential conflict of interest.

Copyright (c) 2019 Trovato, Di Felice and Barone. This is an open-access article distributed under the terms of the Creative Commons Attribution License (CC BY). The use, distribution or reproduction in other forums is permitted, provided the original author(s) and the copyright owner(s) are credited and that the original publication in this journal is cited, in accordance with accepted academic practice. No use, distribution or reproduction is permitted which does not comply with these terms. 


\section{OPEN ACCESS}

Edited by:

Wataru Aoi,

Kyoto Prefectural University, Japan

Reviewed by:

Kunihiro Sakuma,

Tokyo Institute of Technology, Japan

Daniel W. D. West,

University of Toronto, Canada

*Correspondence:

Tomasz Sledzinski tsledz@gumed.edu.pl

Specialty section: This article was submitted to

Striated Muscle Physiology, a section of the journal Frontiers in Physiology

Received: 02 August 2018 Accepted: 26 April 2019 Published: 15 May 2019

Citation: Mika A, Czumaj A, Stepnowski P, Macaluso F, Spinoso G, Barone R, Di Felice $V$ and Sledzinski T (2019) Exercise and Conjugated Linoleic Acid Supplementation Induce Changes in the Composition of Liver Fatty Acids. Front. Physiol. 10:602. doi: 10.3389/fphys.2019.00602

\section{Exercise and Conjugated Linoleic Acid Supplementation Induce Changes in the Composition of Liver Fatty Acids}

\author{
Adriana Mika ${ }^{1,2}$, Aleksandra Czumaj', Piotr Stepnowski'2, Filippo Macaluso ${ }^{3,4,5}$, \\ Giulio Spinoso ${ }^{3}$, Rosario Barone ${ }^{3,4}$, Valentina Di Felice ${ }^{3,4}$ and Tomasz Sledzinski ${ }^{\text {* }}$ \\ ${ }^{1}$ Department of Pharmaceutical Biochemistry, Faculty of Pharmacy, Medical University of Gdańsk, Gdańsk, Poland, \\ ${ }^{2}$ Department of Environmental Analysis, Faculty of Chemistry, University of Gdańsk, Gdańsk, Poland, ${ }^{3}$ Department \\ of Experimental Biomedicine and Clinical Neurosciences, University of Palermo, Palermo, Italy, ${ }^{4}$ Euro-Mediterranean Institute \\ of Science and Technology, Palermo, Italy, ${ }^{5}$ SMART Engineering Solutions \& Technologies Research Center, eCampus \\ University, Novedrate, Italy
}

Exercise and supplementation with conjugated linoleic acid (CLA) are used to reduce body weight and to improve health. Applied together, they may exert a synergistic effect. However, the effects of exercise and CLA supplementation on liver metabolism are poorly understood. The aim of this study was to examine the influence of exercise and CLA supplementation on fatty acids (FA) composition in mouse liver. We analyzed 44 of known FAs of this organ by gas chromatography-mass spectrometry. Our results demonstrated that exercise contributed to a decrease in odd-chain FA and an increase in n-6 polyunsaturated FA contents. In turn, CLA stimulated an increase in branchedchain FAs and n-3 polyunsaturated FAs. Exercise combined with CLA supplementation caused a substantial increase in branched-chain FA content and a slight increase in n-6 polyunsaturated FAs. The elevated liver content of branched-chain FAs after the exercise combined with CLA supplementation, as well as the increase in n-3 polyunsaturated FAs after CLA may be favorable since both these FA groups were previously shown to produce health benefits. However, the expression pattern of enzymes involved in fatty acid synthesis did not match the changes in FA composition. Thus, the mechanism of exercise- and CLA-induced changes in liver FA profile is yet to be established. Also, the consequences of CLA- and/or exercise-induced changes in the composition of liver FAs need to be elucidated.

Keywords: fatty acid, liver, exercise, myokine, conjugated linoleic acid

\section{INTRODUCTION}

The hepatic fat composition is determined by a number of processes, e.g., de novo lipogenesis, delivery of lipids to the liver from diet or adipose tissue, hepatic lipid oxidation and secretion of intrahepatic lipids to the circulation (Lira et al., 2012; Brouwers et al., 2016). Alterations of these processes are responsible for deposition of the intrahepatic lipids and development of nonalcoholic fatty liver (Brouwers et al., 2016), a condition associated with obesity, insulin resistance 
and metabolic syndrome (Mika et al., 2017). The hepatic fat composition may also be modified by extrinsic factors, including physical exercise and supplementation with various FAs. While the beneficial effects of exercise on skeletal muscle metabolism have been described in detail, little is known on the influence of physical training on liver metabolism (Brouwers et al., 2016). Previous studies demonstrated that moderate exercise might prevent accumulation of fat in the liver through diminished delivery of lipids to this organ, enhanced hepatic oxidation and increased incorporation of triacylglycerol (TG) into very low-density lipoprotein (VLDL) (Lira et al., 2012; Brouwers et al., 2016; Oh et al., 2017). Conjugated linoleic acid (CLA) has been advertised and promoted as a pro-health and weight loss promoting supplement since the mid-1990s. While supplementation with CLA, indeed, leads to a decrease in body adiposity (Barone et al., 2013; Macaluso et al., 2013), it was also shown to induce hepatic steatosis and to reduce the levels of n-3 and n-6 polyunsaturated FAs (PUFAs) in mouse liver, although these effects seem to be CLA isomer-specific (Vyas et al., 2012). Furthermore, the results of another study involving rats suggest that CLA may, in fact, attenuate liver steatosis (Purushotham et al., 2007).

The aim of the present study was to explore and discuss, using preliminary data, the effects of physical exercise and supplementation with CLA, applied either alone or in combination, on the composition of FAs in mouse liver. Various FAs exert different effects on cell metabolism; for example, n-3 PUFAs are anti-inflammatory fatty acids (FA), whereas saturated FAs (SFAs) induce inflammation (Mika and Sledzinski, 2017). Both physical exercise and supplementation with CLA are used to reduce body weight and to improve health. The effect of both treatments on FA composition in the liver may be vital for the functioning of this organ.

\section{MATERIALS AND METHODS}

\section{Animals and Treatment}

The study included 32 young (7-week-old) male mice (BALB/cAnNHsd) obtained from Harlan Laboratories S.r.l. (Italy). The mice were maintained under a constant 12:12-h light-dark cycle with free access to food and water. The protocols of all animal experiments were approved by the Committee on the Ethics of Animal Experiments at the University of Palermo (Italy), and compliant with the Guide for the Care and Use of Laboratory Animals of the National Institute of Health (NIH).

The study mice were randomized into four groups, eight animals each: placebo sedentary (PLA-SED), CLA sedentary (CLA-SED), placebo trained (PLA-TR), and CLA trained (CLATR). Through the 6-week experiment period, animals from both CLA groups (CLA-SED and CLA-TR) were gavaged with $35 \mu \mathrm{l}$ d-1 of Tonalin ${ }^{\circledR}$ FFA 80 food supplement (Cognis Nutrition and Health, Germany) containing CLA, whereas mice from the placebo groups (PLA-SED and PLA-TR) received $35 \mu \mathrm{d}-1$ of sunflower oil via the same route (Macaluso et al., 2012; Barone et al., 2013, 2017). The gavaged quantities correspond to the $0.5 \%$ of food ingested, approximately $4 \mathrm{~g}$ (Barone et al., 2013).
Tonalin ${ }^{\circledR}$ FFA 80 is derived from safflower oil and contains a 50:50 ratio of the active CLA isomers (C18:2 c9, t11 and C18:2 t10, c12).

Animals from both trained groups (PLA-TR and CLA-TR) were trained on a motorized Rota-Rod (Ugo Basile, Biological Research Apparatus, Italy) 5 days/week over a period of 6 weeks. The endurance training began at $3.2 \mathrm{~m} / \mathrm{min}$ for 5 days/week; during week 1 , the mice exercised for $15 \mathrm{~min}$, and the time and speed were systematically increased until week 6 , where they were training at $4.8 \mathrm{~m} / \mathrm{min}$ for $60 \mathrm{~min}$ (Di Felice et al., 2007; Barone et al., 2016a). Mice from the sedentary groups (PLA-SED and CLA-SED) did not perform any controlled physical activity throughout the period of the experiment. At $48 \mathrm{~h}$ from the end of the last exercise session, all mice were sacrificed by cervical dislocation, and their livers were harvested and weighed (Barone et al., 2016b). The liver was frozen in liquid nitrogen and stored at $-80^{\circ} \mathrm{C}$ for further analyses.

\section{GCMS Analysis of Fatty Acid Composition in the Liver}

The liver samples were extracted in a chloroform-methanol mixture (2:1, v/v) (Folch et al., 1957). Extracted lipids were hydrolyzed with $0.5 \mathrm{M} \mathrm{KOH}$, and the profile of FA methyl esters were determined by gcms using Zebron 5MSi capillary column and electron ionization source, as described previously (Sledzinski et al., 2013).

\section{Analysis of mRNA Levels}

Total mRNA was isolated from $25 \mathrm{mg}$ of mouse liver using GenElute Mammalian Total RNA Miniprep Kit (Sigma-Aldrich, MO, United States), in line with the manufacturer's instructions. The quality of the resulting RNA was assessed by automated gel electrophoresis (Experion, Bio-Rad Laboratories). Reverse transcription and quantitative real-time PCR were conducted as described previously (Czumaj et al., 2018). The primer sequences are presented in Supplementary Table 1.

\section{Immunofluorescence and Confocal Analysis}

For immunofluorescence, deparaffinised sections were incubated in the antigen unmasking solution $(10 \mathrm{mM}$ tri-sodium citrate, $0.05 \%$ Tween-20) for $8 \mathrm{~min}$ at $75^{\circ} \mathrm{C}$, and treated with a blocking solution (3\% BSA in PBS) for $30 \mathrm{~min}$. The primary antibody (anti-BCKDHA, diluted 1:50 in PBS, rabbit polyclonal ab90691, Abcam; Cambridge, United Kingdom; anti-BCKDHA (phospho S293), diluted 1:50 in PBS, rabbit polyclonal ab200577, Abcam; anti-ELOVL6, diluted 1:200 in PBS, rabbit polyclonal ab69857, Abcam) was applied and the sections were incubated in a humidified chamber overnight at $4^{\circ} \mathrm{C}$. Then, the sections were incubated for $1 \mathrm{~h}$ at room temperature with a conjugated secondary antibody (anti-rabbit IgG-FITC antibody produced in goat, diluted 1:100 in PBS, F0382, Sigma-Aldrich, St. Louis, MO, United States). Nuclei were stained with Hoechst Stain Solution (diluted 1:1000 in PBS, Hoechst 33258, Sigma-Aldrich). The slides were treated with PermaFluor Mountant (Thermo Fisher Scientific, Inc., Waltham, MA, United States) and cover slipped. The images were captured using a Leica Confocal Microscope 
TABLE 1 | Fatty acid composition of livers in the studied groups of mice.

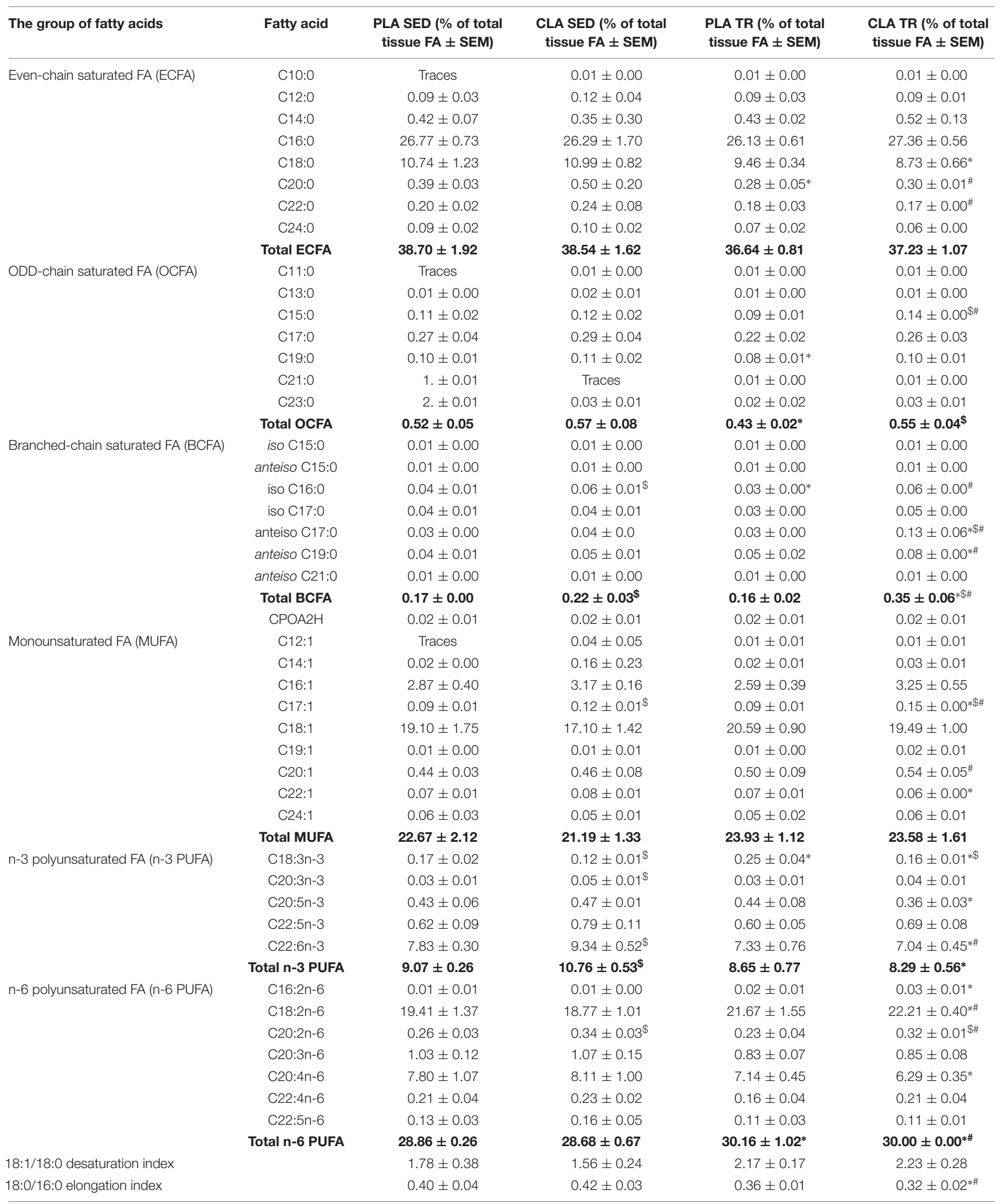

CPOA2H - Cyclopropaneoctanoic acid 2-hexyl; PLA - placebo, SED - sedentary, TR - trained, ${ }^{*} p<0.05$ trained compared to corresponding sedentary group; $\$ p<0.05$ $C L A$ treated compared to corresponding placebo group ${ }^{\#} p<0.05$ comparing PLA SED to CLA TR. 
TCS SP8 (Leica Microsystems). The staining intensity was expressed as the mean value pixel intensity (PI) using the software Leica application suite advanced fluorescences software.

\section{Statistical Analysis}

The results are presented as means \pm SEM for each study group, if not otherwise specified. The significance of intergroup differences was verified with one-way analysis of variance (ANOVA), or ANOVA on ranks for not normally distributed data, followed by an appropriate post hoc test. The threshold of statistical significance for all tests was set at $P<0.05$. All analyses were carried out with STATISTICA 12 package (StatSoft).

\section{RESULTS}

The results documenting the effects of CLA supplementation and endurance exercise on body weight, skeletal muscle strength, and endurance performance in the study groups have been published elsewhere (Barone et al., 2013). Endurance training or CLA supplementation did not exert a statistically significant effect on the liver weight [PLA-SED: $0.058 \pm 0.020 \mathrm{~g}$ (liver weight/body weight \pm Standard Deviation - SD), PLA-TR: $0.041 \pm 0.003 \mathrm{~g}$, CLA-SED: $0.055 \pm 0.022$ g, CLA-TR: $0.046 \pm 0.003$ g], however, we observed a trend to decrease of liver weight/body weight ratio after exercise.

\section{Fatty Acid Composition in Liver After Exercise and CLA Supplementation}

The analysis of FA composition in mouse livers demonstrated that physical exercise and supplementation with CLA applied either alone or in combination, induced significant changes in the contents of various FA (Table 1). The training contributed to a significant increase in n-6 PUFAs and a decrease in odd chain FAs (OCFAs), with the latter effect observed solely in PLATR mice. Moreover, a tendency for higher values of 18:1/18:0 desaturation index (DI) and lower values of 18:0/16:0 elongation index (EI) was observed in trained animals, but the results did not differ significantly from those for sedentary animals (Table 1). Supplementation with CLA contributed to a significant increase in branched-chain FA (BCFA) and n-3 PUFA contents in sedentary mice. The exposure to both physical exercise and CLA supplementation resulted in a significant increase in BCFA content and a slight increase in n-6 PUFAs. However, the trained

TABLE 2 | Relative mRNA levels of liver enzymes of fatty acid metabolism.

\begin{tabular}{lcccc}
\hline Enzyme & PLA SED & CLA SED & PLA TR & CLA TR \\
\hline BHKDHA & 1.00 & $6.63 \pm 1.45^{\$}$ & $2.32 \pm 0.15$ & $3.81 \pm 1.27^{\# *}$ \\
SCD1 & 1.00 & $2.97 \pm 0.23^{\$}$ & $1.83 \pm 0.18^{*}$ & $1.76 \pm 0.57^{\# *}$ \\
ELOVL6 & 1.00 & $7.68 \pm 2.2^{\$}$ & $3.67 \pm 1.2^{*}$ & $1.14 \pm 0.47^{*}$
\end{tabular}

PLA - placebo, SED - sedentary, TR - trained, BHKDHA - alpha subunit of branched-chain alpha-keto acid dehydrogenase enzyme complex, SCD1 - stearoilCOA desaturase, ELOVL6 - fatty acid elongase $6, * p<0.05$ trained compared to corresponding sedentary group; $\$ p<0.05$ CLA compared to corresponding placebo group, \# $p<0.05$ comparing PLA SED to CLA TR. and CLA-supplemented mice did not show an increase in n3 PUFA content, which was observed in PLA-TR animals. The combination of CLA supplementation and exercise contributed also to a non-significant increase in DI values and a significant decrease in EI values (Table 1).

\section{The Expression of the Enzymes of Fatty Acid Metabolism in Liver After Exercise and CLA Supplementation}

To verify if the training- and supplementation-induced differences in liver FA composition resulted from changes in the synthesis, elongation, and desaturation of FA in the liver, we measured the expressions of some enzymes known to be involved in these processes. As the combination of exercise and CLA supplementation exerted the most evident effect on BCFA content, we measured the expression of the alpha subunit of branched-chain alpha-keto acid dehydrogenase enzyme complex (BCKDHA), an enzyme responsible for BCFA synthesis. Moreover, we determined the expression of another two enzymes, stearoyl-CoA desaturase (SCD1), and FA elongase 6 (ELOVL6). Although the analysis demonstrated that both CLA supplementation and physical exercise exerted an effect on BHKDHA, SCD1, and ELOVL6 expressions (Table 2), the pattern of changes in mRNA levels in hepatocytes of trained and supplemented mice did not match with the changes in their BCFA content or 18:1/18:0 and 18:0/16:0 index values (Tables 1, 2).

Confocal microscopy analyses are showed in Figure 1, which indicated that BCKDHA is localized mainly in the mitochondrion matrix and minimally in the nucleus, while phospho-BCKDHA is localized only in the nucleus. The protein level of BCKDHA was significantly higher in CLA-TR group than in PLA-SED, while no differences were observed among the others groups. The endoplasmic reticulum membrane protein, ELOVL6, was significantly higher in PLA-TR group than in PLA-SED, while no differences were observed among the others groups. Thus, changes in protein levels were consistent with the mRNA levels.

\section{DISCUSSION}

This study revealed that physical exercise, supplementation with CLA and combination thereof might induce significant changes in FA composition of mouse liver. The most spectacular change was observed in the case of BCFA content in CLATR mice, which was twice as high as in the untreated controls. It should be emphasized that such a significant increase in BCFAs was not observed after either the exercise or the CLA supplementation alone. The effect of exercise and CLA supplementation on BCFA content should probably be considered a favorable finding since elevated levels of FA from this group were previously shown to be associated with some health benefits (Wongtangtintharn et al., 2004, 2005, Ran-Ressler et al., 2011), and their serum levels correlated inversely with hypertriglyceridemia, inflammation and insulin concentration (Mika et al., 2016). Moreover, Garcia Caraballo et al. (2014) 


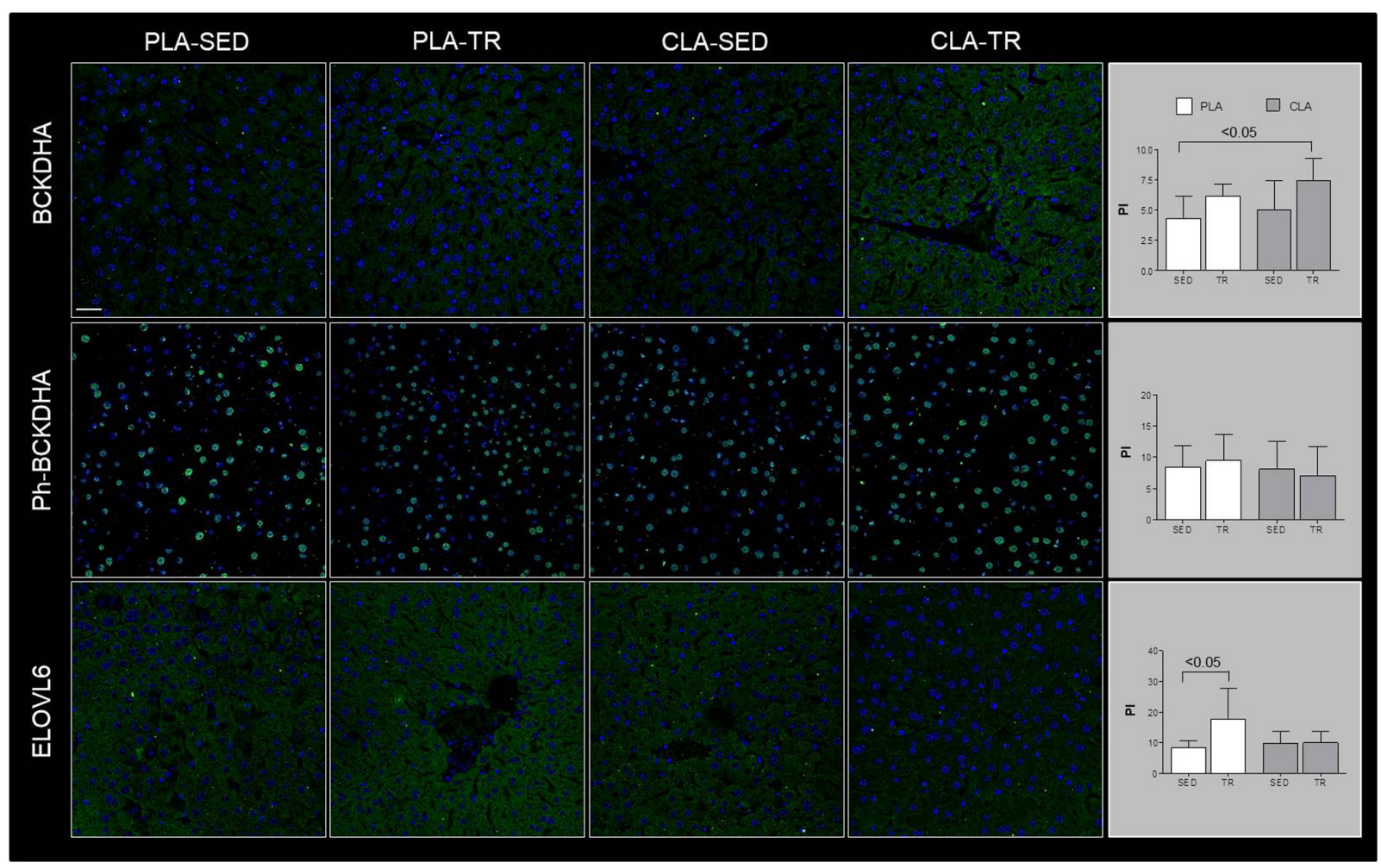

FIGURE 1 | Confocal microscopy analyses. Representative images of immunofluorescence stain for BCKDHA, phospho-BCKDHA and ELOVL6 in liver sections of placebo sedentary (PLA-SED), placebo trained (PLA-TR), CLA sedentary (CLA-SED), and CLA trained (CLA-TR). The staining intensity for BCKDHA,

phospho-BCKDHA, and ELOVL6 was expressed as the mean value pixel intensity (PI) using the software Leica application suite advanced fluorescences software.

Data are presented as the means $\pm \mathrm{SD}$. Bar $=25 \mu \mathrm{m}$ for all panels.

found an inverse correlation between liver contents of BCFAs and $\mathrm{TG}$ in mice maintained on a high-protein diet, and suggested that the elevated BCFA content may predispose to a decrease in hepatic TG level and lesser steatosis. In mammals, BCFA can be synthesized from branched-chain amino acids (Garcia Caraballo et al., 2014), and the rate-limiting step of these amino acids catabolism is catalyzed by branch chain keto-acid dehydrogenase (BCKDHA) enzyme complex (Crown et al., 2015). However, the patterns of post-treatment changes in BCKDHA mRNA and protein level and BCFA content in our trained and CLA-supplemented mice did not match. We also did not find any differences in phosphorylated (inactive) BCKDHA levels. This implies that the increase in BCFA content observed after exercise and CLA supplementation might have been mediated by other mechanisms (i.e., increased influx of BCFA into hepatocyte).

Another beneficial effect of CLA supplementation documented in our study was an increase in n-3 PUFA content. Previous studies demonstrated that n-3 PUFA might exert anti-inflammatory, insulin-sensitizing, and cardioprotective effects (Mika and Sledzinski, 2017). However, in our study, the beneficial effect of CLA supplementation on n-3 PUFA content was counterbalanced by physical exercise, since we did not find a statistically significant difference in the levels of these FA in supplemented and non-supplemented mice subjected to the Rota-Rod training. In turn, the liver content of n-6 PUFAs increased after training but remained unchanged after CLA supplementation. n- 6 PUFAs are precursors of many proinflammatory molecules (Mika and Sledzinski, 2017). Physical exercise is known to attenuate inflammation and oxidative stress in the liver (de Castro et al., 2017). However, the inflammatory response may be triggered by many various factors, and the elevated content of $n-6$ PUFAs might not be enough to induce inflammation in liver tissues.

We also found that physical exercise resulted in an increase of 18:1/18:0 DI values and in a decrease in 18:0/16:0 EI values. DI and EI are calculated based on the contents of some specific FAs and are considered the measures of SCD1 and ELOVLs activities, respectively (Murakami et al., 2008; Popeijus et al., 2008). However, when we analyzed the profiles of exercise- and supplementation-induced changes in both indices against the patterns of changes in SCD1 mRNA and ELOVL6 mRNA or protein levels, they seemed to be unrelated. This implies that DI and EI values in trained and CLA-supplemented mice did not depend on the expressions of SCD1 and ELOVL6 genes, but were rather influenced by other processes, such as FA oxidation, supply of dietary lipids or release thereof from adipose tissue, and secretion of hepatic lipids to the circulation in the form of lipoproteins. 
An important issue that needs to be addressed is the mechanism through which CLA and exercise modulate liver profiles of FAs. A proteomic study showed that some specific CLA isomers might influence the activities of enzymes involved in beta-oxidation and fructose metabolism (Della Casa et al., 2016). Furthermore, exercising muscles release various myokines, and many of them have already been shown to influence the liver metabolism (Carson, 2017). This problem should be elucidated during future research.

In conclusion, this study demonstrated that both supplementation with CLA and physical exercise might induce significant changes in FA profile of the liver. However, the mechanisms and the consequences of CLA- and/or exerciseinduced changes in the composition of liver FAs need to be elucidated.

\section{ETHICS STATEMENT}

The protocols of all animal experiments were approved by the Committee on the Ethics of Animal Experiments at the University of Palermo (Italy), and compliant with the Guide for the Care and Use of Laboratory Animals of the National Institute of Health (NIH).

\section{REFERENCES}

Barone, R., Macaluso, F., Catanese, P., Marino Gammazza, A., Rizzuto, L., Marozzi, P., et al. (2013). Endurance exercise and conjugated linoleic acid (CLA) supplementation up-regulate CYP17A1 and stimulate testosterone biosynthesis. PLoS One 8:e79686. doi: 10.1371/journal.pone.0079686

Barone, R., Macaluso, F., Sangiorgi, C., Campanella, C., Marino Gammazza, A., Moresi, V., et al. (2016a). Skeletal muscle Heat shock protein 60 increases after endurance training and induces peroxisome proliferator-activated receptor gamma coactivator $1 \alpha 1$ expression. Sci. Rep. 27:19781. doi: 10.1038/srep19781

Barone, R., Rappa, F., Macaluso, F., Caruso Bavisotto, C., Sangiorgi, C., Di Paola, G., et al. (2016b). Alcoholic liver disease: a mouse model reveals protection by Lactobacillus fermentum. Clin. Transl. Gastroenterol. 21:e138. doi: 10.1038/ctg. 2015.66

Barone, R., Sangiorgi, C., Marino Gammazza, A., D’Amico, D., Salerno, M., Cappello, F., et al. (2017). Effects of conjugated linoleic acid associated with endurance exercise on muscle fibres and peroxisome proliferatoractivated receptor $\gamma$ coactivator $1 \alpha$ Isoforms. J. Cell. Physiol. 232, 1086-1094. doi: $10.1002 /$ jcp. 25511

Brouwers, B., Hesselink, M. K. C., Schrauwen, P., and Schrauwen-Hinderling, V. B. (2016). Effects of exercise training on intrahepatic lipid content in humans. Diabetologia 59, 2068-2079. doi: 10.1007/s00125-016-4037-x

Carson, B. P. (2017). The potential role of contraction-induced myokines in the regulation of metabolic function for the prevention and treatment of type 2 diabetes. Front. Endocrinol. 8:97. doi: 10.3389/fendo.2017.00097

Crown, S. B., Marze, N., and Antoniewicz, M. R. (2015). Catabolism of branched chain amino acids contributes significantly to synthesis of odd-chain and evenchain fatty acids in 3T3-L1 adipocytes. PLoS One 10:e0145850. doi: 10.1371/ journal.pone. 0145850

Czumaj, A., Mika, A., Chmielewski, M., and Sledzinski, T. (2018). Cyclopropaneoctanoic acid 2-hexyl upregulates the expression of genes responsible for lipid synthesis and release in human hepatic HEPG2 Cells. Lipids 53, 345-351. doi: 10.1002/lipd.12034

de Castro, M. R. T., Ferreira, A. P. O., Busanello, G. L., da Silva, L. R. H., da Silveira, M. E. P. Jr., Fiorin, F. D. S., et al. (2017). Previous physical exercise alters the hepatic profile of oxidative-inflammatory status and limits the secondary

\section{AUTHOR CONTRIBUTIONS}

FM, RB, and VDF performed the animal experiments. AM and PS performed and interpreted GCMS analysis. AC and TS performed and interpreted Real-time PCR analysis. GS performed and interpreted immunofluorescence and confocal analysis. AM, VDF, and TS wrote the manuscript. All authors accepted the final version of manuscript.

\section{FUNDING}

This study was supported by the Medical University of Gdańsk (Grant No. ST40), National Science Centre of Poland (Grant No. NCN 2016/21/D/NZ5/00219), and by the Italian Ministry of University and Research (Grant Prot. 2012N8YJC3 - Caporossi Daniela PRIN2012).

\section{SUPPLEMENTARY MATERIAL}

The Supplementary Material for this article can be found online at: https://www.frontiersin.org/articles/10.3389/fphys. 2019.00602/full\#supplementary-material

brain damage induced by severe traumatic brain injury in rats. J. Physiol. 595, 6023-6044. doi: 10.1113/JP273933

Della Casa, L., Rossi, E., Romanelli, C., Gibellini, L., and Iannone, A. (2016). Effect of diets supplemented with different conjugated linoleic acid (CLA) isomers on protein expression in C57/BL6 mice. Genes Nutr. 11:26. doi: 10.1186/s12263016-0542-2

Di Felice, V., Macaluso, F., Montalbano, A., Gammazza, A. M., Palumbo, D., Angelone, T., et al. (2007). Effects of conjugated linoleic acid and endurance training on peripheral blood and bone marrow of trained mice. J. Strength Cond. Res. 21, 193-198. doi: 10.1519/R-18955.1

Folch, J., Lees, M., and Sloane Stanley, G. H. (1957). A simple method for the isolation and purification of total lipides from animal tissues. J. Biol. Chem. 226, 497-509.

Garcia Caraballo, S. C., Comhair, T. M., Houten, S. M., Dejong, C. H. C., Lamers, W. H., and Koehler, S. E. (2014). High-protein diets prevent steatosis and induce hepatic accumulation of monomethyl branched-chain fatty acids. J. Nutr. Biochem. 25, 1263-1274. doi: 10.1016/j.jnutbio.2014.07.005

Lira, F. S., Carnevali, L. C., Zanchi, N. E., Santos, R. V., Lavoie, J. M., and Seelaender, M. (2012). Exercise intensity modulation of hepatic lipid metabolism. J. Nutr. Metab. 2012, 1-8. doi: 10.1155/2012/809576

Macaluso, F., Barone, R., Catanese, P., Carini, F., Rizzuto, L., Farina, F., et al. (2013). Do fat supplements increase physical performance? Nutrients 7, 509-524. doi: 10.3390/nu5020509

Macaluso, F., Morici, G., Catanese, P., Ardizzone, N. M., Marino Gammazza, A., Bonsignore, G., et al. (2012). Effect of conjugated linoleic acid on testosterone levels in vitro and in vivo after an acute bout of resistance exercise. J. Strength Cond. Res. 26, 1667-1674. doi: 10.1519/JSC.0b013e318231ab78

Mika, A., and Sledzinski, T. (2017). Alterations of specific lipid groups in serum of obese humans: a review. Obes. Rev. 18, 247-271. doi: 10.1111/obr.12475

Mika, A., Sledzinski, T., and Stepnowski, P. (2017). Current progress of lipid analysis in metabolic diseases by mass spectrometry methods. Curr. Med. Chem. 24, 60-103. doi: 10.2174/0929867324666171003121127

Mika, A., Stepnowski, P., Kaska, L., Proczko, M., Wisniewski, P., Sledzinski, M., et al. (2016). Obesity a comprehensive study of serum odd- and branchedchain fatty acids in patients with excess weight. Obesity 24, 1669-1676. doi: $10.1002 /$ oby. 21560 
Murakami, K., Sasaki, S., Takahashi, Y., Uenishi, K., Watanabe, T., Kohri, T., et al. (2008). Lower estimates of delta-5 desaturase and elongase activity are related to adverse profiles for several metabolic risk factors in young Japanese women. Nutr. Res. 28, 816-824. doi: 10.1016/j.nutres.2008.08.009

Oh, S., So, R., Shida, T., Matsuo, T., Kim, B., Akiyama, K., et al. (2017). Highintensity aerobic exercise improves both hepatic fat content and stiffness in sedentary obese men with nonalcoholic fatty liver disease. Sci. Rep. 7:43029. doi: $10.1038 /$ srep43029

Popeijus, H. E., Saris, W. H. M., and Mensink, R. P. (2008). Role of stearoyl-CoA desaturases in obesity and the metabolic syndrome. Int. J. Obes. 32, 1076-1082. doi: 10.1038/ijo.2008.55

Purushotham, A., Shrode, G. E., Wendel, A. A., Liu, L.-F., and Belury, M. A. (2007). Conjugated linoleic acid does not reduce body fat but decreases hepatic steatosis in adult Wistar rats. J. Nutr. Biochem. 18, 676-684. doi: 10.1016/J.JNUTBIO. 2006.10.008

Ran-Ressler, R. R., Khailova, L., Arganbright, K. M., Adkins-Rieck, C. K., Jouni, Z. E., Koren, O., et al. (2011). Branched chain fatty acids reduce the incidence of necrotizing enterocolitis and alter gastrointestinal microbial ecology in a neonatal rat model. PLoS One 6:e29032. doi: 10.1371/journal.pone.0029032

Sledzinski, T., Mika, A., Stepnowski, P., Proczko-Markuszewska, M., Kaska, L., Stefaniak, T., et al. (2013). Identification of cyclopropaneoctanoic acid 2-hexyl in human adipose tissue and serum. Lipids 48, 839-848. doi: 10.1007/s11745013-3806-2
Vyas, D., Kadegowda, A. K. G., and Erdman, R. A. (2012). Dietary conjugated linoleic acid and hepatic steatosis: species-specific effects on liver and adipose lipid metabolism and gene expression. J. Nutr. Metab. 2012, 1-13. doi: 10.1155/ 2012/932928

Wongtangtintharn, S., Oku, H., Iwasaki, H., Inafuku, M., Toda, T., and Yanagita, T. (2005). Incorporation of branched-chain fatty acid into cellular lipids and caspase-independent apoptosis in human breast cancer cell line, SKBR-3. Lipids Health Dis. 4:29. doi: 10.1186/1476-511X-4-29

Wongtangtintharn, S., Oku, H., Iwasaki, H., and Toda, T. (2004). Effect of branched-chain fatty acids on fatty acid biosynthesis of human breast cancer cells. J. Nutr. Sci. Vitaminol. 50, 137-143.

Conflict of Interest Statement: The authors declare that the research was conducted in the absence of any commercial or financial relationships that could be construed as a potential conflict of interest.

Copyright (c) 2019 Mika, Czumaj, Stepnowski, Macaluso, Spinoso, Barone, Di Felice and Sledzinski. This is an open-access article distributed under the terms of the Creative Commons Attribution License (CC BY). The use, distribution or reproduction in other forums is permitted, provided the original author(s) and the copyright owner(s) are credited and that the original publication in this journal is cited, in accordance with accepted academic practice. No use, distribution or reproduction is permitted which does not comply with these terms. 


\section{OPEN ACCESS}

Edited by:

Dario Coletti

Sapienza University of Rome, Italy

Reviewed by: Emanuele Rinninella, Università Cattolica del Sacro Cuore,

Adamasco Cupisti, University of Pisa, Italy

Pablo Molina

Doctor Peset University Hospital,

Spain

*Correspondence:

Alessio Molfino

alessio.molfino@uniroma1.it

Specialty section This article was submitted to Striated Muscle Physiology, a section of the journal

Frontiers in Physiology

Received: 21 January 2019 Accepted: 06 June 2019

Published: 25 June 2019

Citation:

Molfino A, Amabile MI,

Ammann T, Lai S, Grosso A Lionetto L, Spagnoli A, Simmaco M, Monti M, Laviano A, Chiappini MG and Muscaritoli M (2019) Longitudinal Physical Activity Change During Hemodialysis and Its Association With Body Composition and Plasma BAIBA Levels. Front. Physiol. 10:805 doi: 10.3389/fphys.2019.00805
Longitudinal Physical Activity Change During Hemodialysis and Its Association With Body Composition and Plasma BAIBA Levels

\author{
Alessio Molfino ${ }^{1 *}$, Maria Ida Amabile ${ }^{1,2}$, Thomas Ammann ${ }^{3}$, Silvia Lai', \\ Alessandra Grosso ${ }^{3}$, Luana Lionetto ${ }^{4}$, Alessandra Spagnoli5, Maurizio Simmaco6, \\ Massimo Monti ${ }^{2}$, Alessandro Laviano ${ }^{1}$, Maria Grazia Chiappini ${ }^{3}$ and \\ Maurizio Muscaritoli ${ }^{1}$
}

${ }^{1}$ Department of Translational and Precision Medicine, Sapienza University of Rome, Rome, Italy, ${ }^{2}$ Department of Surgical Sciences, Sapienza University of Rome, Rome, Italy, ${ }^{3}$ Hemodialysis Unit, Fatebenefratelli Hospital, Rome, Italy, ${ }^{4}$ Advanced Molecular Diagnostic Unit, Istituto Dermopatico dell'Immacolata-IRCCS, Rome, Italy, ${ }^{5}$ Department of Public Health and Infectious Diseases, Sapienza University of Rome, Rome, Italy, ${ }^{6}$ Analytical Laboratory Unit, Sant'Andrea Hospital, Department NESMOS, Sapienza University of Rome, Rome, Italy

Rationale: Low physical activity is frequent in end stage renal disease. We evaluated the longitudinal change in physical activity and its barriers in hemodialysis (HD) patients and the association between the patterns of physical activity change, body composition, and beta-aminoisobutyric acid (BAIBA), as circulating myokine.

Methods: This is an observational study, where HD patients were considered in a 24-month follow-up. We assessed overtime the change of physical inactivity and its barriers by validated questionnaires, body composition by bioimpedance analysis, muscle strength by hand-dynamometer, and plasma BAIBA levels by liquid chromatography spectrometry. Parametric and non-parametric analyses were performed, as appropriate.

Results: Out of the 49 patients studied at baseline, 39 completed the first-year followup, and 29 completed the second year. At month 12, active patients had higher intracellular water (ICW) $(P=0.001)$ and cellular mass $(P<0.001)$, as well as at month $24(P=0.012, P=0.002$; respectively) with respect to inactive. A significant reduction in ICW was shown at month $12(P=0.011)$ and month $24(P=0.014)$ in all patients. The barrier "reduced walking ability" was more frequent in inactive patients with respect to active at month $12(P=0.003)$ and at month $24(P=0.05)$. At month 24 , plasma BAIBA levels were higher among active patients with respect to inactive $(P=0.043)$ and a correlation was seen between muscle strength and ICW $(r=0.51, P=0.005)$; normalizing BAIBA per body mass index, we found it lower with respect to baseline $(P=0.004)$, as well as after correcting per ICW $(P=0.001)$, as marker of muscle mass.

Conclusion: A high prevalence of physical inactivity persisted during a 24-month followup in this cohort. We found an association between physical activity and a decline in marker of muscularity and reduced plasma BAIBA levels.

Keywords: hemodialysis, physical activity, body composition, beta-aminoisobutyric acid, longitudinal study 


\section{INTRODUCTION}

The assessment of physical activity among hemodialysis (HD) patients is a crucial clinical aspect which has improved the understanding of the association between frailty and poor outcomes (Sy and Johansen, 2017). This assessment allows to recognize that inactive patients with end-stage renal disease (ESRD) undergoing maintenance HD are at higher risk for morbidity and mortality (Sy and Johansen, 2017; Ku et al., 2018). Physical inactivity is associated with the risk of falls, hospitalizations, cognitive impairment, vascular access failure, and poor quality of life among HD patients (Johansen et al., 2013a; Sy and Johansen, 2017) and exercise may be effective to reduce this risk (Johansen et al., 2013b). We recently investigated the presence of physical inactivity and its barriers among a cohort of HD patients and the association with the myokine beta-aminoisobutyric acid (BAIBA) (Molfino et al., 2017), which is formed by the catabolism of valine, determining several beneficial effects on muscle metabolism in an autocrine/paracrine manner (Molfino et al., 2017; Huh, 2018). In particular, BAIBA secretion increases with exercise and induces browning of white fat and hepatic beta-oxidation having potential implication in the physiological mechanisms of physical activity (Roberts et al., 2014).

Although low physical activity accompanies ESRD progression (Robinson-Cohen et al., 2014), few studies have documented the time course and the features of physical activity changes that occur with ESRD progression (Johansen et al., 2000).

The patterns of change in physical activity and barriers appear clinically relevant because they may provide insights into an appropriate timing of diagnosis and intervention to achieve the greatest impact on outcomes among HD patients. In addition, to date few data are available on the role of exercise-induced myokines, as possible biomarkers of physical activity and its variation in humans and in particular among HD patients who are known to be extremely sedentary (Johansen et al., 2000). In this light, research on possible novel biomarker(s), such as BAIBA, to easily assess and monitor muscularity associated or not to low physical activity, appears clinically important.

In this study, we first aimed to determine the longitudinal changes (up to 24 months) in physical activity levels among patients on HD, including barriers to physical activity. We next analyzed the association between these patterns of physical activity changes, BAIBA levels, body composition, and muscle strength.

\section{PATIENTS AND METHODS}

This longitudinal study was performed on patients from the Dialysis Unit of "Fatebenefratelli" Hospital, Isola Tiberina, in Rome, Italy. Women and men $\geq 18$ years chronically maintained on $\mathrm{HD}$, participating on a study investigating the role of nutrition and quality of life, whose baseline characteristics were previously described (Molfino et al., 2017), underwent to a follow-up of 24 months. In summary, 52 patients are chronically treated at the HD center and the inclusion criteria consisted in enrolling the entire HD cohort with the exception of patients with highly catabolic diseases, such as cancer, chronic infections, and AIDS, and the absence of informed consent, as previously shown (Molfino et al., 2017). The sample size we used was representative of the entire number of patients, respecting the inclusion criteria, treated at the single Dialysis Unit.

The study was approved by the local ethics committee. Written informed consent was obtained from all patients. Patients were maintained on a regular HD prescription, three times a week, for 4-h sessions during the 2-year follow-up.

\section{Patient Clinical Characteristics}

Participants' demographic and clinical characteristics, including age, gender, weight, height, body mass index (BMI), dialysis vintage, and $\mathrm{Kt} / \mathrm{V}$ were recorded, and comorbidities and current therapies were collected. Nutritional and inflammatory biomarkers were assessed at baseline (Molfino et al., 2017), at 12 and 24 months.

\section{Body Composition Analysis}

Body impedance analysis (BIA) was performed in all $\mathrm{HD}$ patients to assess body composition overtime (Model 101, singlefrequency BIA, Akern, Florence, Italy). Blinded research staff performed BIA $1 \mathrm{~h}$ after the end of the second HD session of the week. As previously shown, the patient maintained a supine position during this period (Jankowska et al., 2006; Molfino et al., 2017). The pairs of electrodes were placed on the nonaccess side of the body on the hand to the foot for injecting current and on the wrist to the ankle for measuring voltage. Total body water was estimated using the resistance extrapolated to frequency and parameters, including intracellular water (ICW) and cellular mass, as the metabolically active component of lean tissue mass, were calculated using a program provided by the producer (Jankowska et al., 2006; Molfino et al., 2017).

\section{Physical Activity Level}

As for baseline (Molfino et al., 2017), at month 12 and month 24, we asked enrolled patients to answer questions on the self-reported frequency of physical activity, including recreational walking, running, and so on, during leisure time. This questionnaire was shown to be reliable in large cohorts of dialysis patients (Tentori et al., 2010; Delgado and Johansen, 2012).

A second questionnaire was used to evaluate the barriers to physical activity in general or to lower physical activity levels than desired, investigating 23 barriers, designed on the basis of previously validated questionnaire in the $\mathrm{HD}$ population (Delgado and Johansen, 2012; Johansen et al., 2014b).

Patients who experienced exercise at least once a week were considered active, those who never exercised or reported physical exercise less than once a week were considered inactive (Delgado and Johansen, 2012; Johansen et al., 2014b).

\section{Muscle Strength}

The muscle function was assessed on the same day of the recruitment immediately before starting the HD session by 
handgrip strength measurement, using a handle dynamometer (JAMAR, Sammons Preston Rolyan, Bolingbrook, IL, United States). The test was performed conducting three attempts with each hand, and the mean of the strongest hand was used to determine muscle strength (Johansen et al., 2014a; Molfino et al., 2017).

\section{Plasma BAIBA Levels Assay}

Blood samples were obtained from the HD patients immediately before the second HD session of the week and kept at room temperature for $1 \mathrm{~h}$ before the plasma was isolated. Aliquots of plasma were stored at $-80^{\circ} \mathrm{C}$ until analysis. Taking into account the expected association between the myokine BAIBA and body size and body composition, in particular muscularity, we also used BAIBA/BMI and more specifically BAIBA/ICW (\%) ratio (ICW, as marker of muscularity) (Johansen et al., 2014a) in our analyses. Data presented here utilize the mean of two measurements.

Plasma BAIBA levels were assessed by a Liquid chromatography-tandem mass spectrometry (LC-MS/MS) method, as previously described (Molfino et al., 2017). Briefly, $40 \mu \mathrm{L}$ of plasma samples were added to $160 \mu \mathrm{L}$ of internal standard in acetonitrile. The samples were vortex-mixed, centrifuged and $100 \mu \mathrm{L}$ of clean upper layer were then diluted with $100 \mu \mathrm{L}$ of $0.1 \%$ formic acid and transferred in autosampler vials. Chromatographic separation was achieved using a Phenomenex Luna HILIC column $(100 \times 2.0 \mathrm{~mm}, 3 \mu \mathrm{m}$; Phenomenex, Torrance, CA, United States), with an elution gradient (Molfino et al., 2017). The HPLC-MS/MS analysis were performed using an Agilent Liquid Chromatography System series 1100 (Agilent Technologies, Santa Clara, CA, United States) coupled with a 3200 triple quadrupole system (Applied Biosystems, Foster City, CA, United States), with a Turbo Ion Spray source. Data were acquired and processed by Analyst 1.5.1 Software.

\section{Statistical Analyses}

Patient characteristics were described using mean ( \pm SD) for continuous normally distributed variables and percent for dichotomous variables. Variables that were not normally distributed were described using median with 25th and 75th percentiles. The proportion of patients self-reporting each barrier and the average number of barriers perceived per patient were calculated. Chi-square, Mann-Whitney, and Kruskall-Wallis tests were used to study the association between reported barriers to physical activity and patient characteristics, the association between barriers and reported levels of physical activity, between barriers and time (baseline, 12, 24 months) and between BAIBA, levels of physical activity and its barriers, body composition and muscle strength overtime. Spearman's correlation index was used to assess associations between continuous measurements. A linear mixed-effect model was used to evaluate the change of body composition overtime, as well as a model to evaluate changes of clinical parameters and biomarkers overtime. The model included physical activity, categorical time point, physical activity level per time point interaction as fixed effect and subject as a random effect.
A standard two-tailed a $(P<0.05)$ was considered statistically significant. Statistical analysis was performed in R v.3.4.3.

\section{RESULTS}

\section{Patient Characteristics}

Characteristics of the participants are reported in Table $\mathbf{1 .}$

In summary, 49 patients (13 women) were studied at baseline with a mean age of $66.6 \pm 15.5$ years. Median dialysis vintage was 37 months [interquartile range (IQR), 13-92] with a mean $\mathrm{Kt} / \mathrm{V}$ of $1.3 \pm 0.3$ (Molfino et al., 2017) and $20 \%$ of patients were on hemodiafiltration.

At 12-month follow-up, 7 patients died, 2 were transferred to another dialysis facility and 1 patient received renal transplantation. Therefore, 39 patients were considered, with a mean age of $66 \pm 15.1$ years and a median dialysis vintage of 40.5 months (IQR, 25-70.2) (Table 1).

At 24-month follow-up, 5 patients died, 2 were transferred to another dialysis facility and 3 patients received renal transplantation. A total of 29 patients were studied, with a mean age of $66.9 \pm 14.7$ years and a median dialysis vintage of 50 months (IQR, 37-77) (Table 1).

During the entire follow-up period, as for baseline (Molfino et al., 2017), more than $50 \%$ of the patients were treated with antihypertensive therapies, statins, vitamin supplements, erythropoietin, and potassium, and phosphate binders and supplemented with folic acid.

\section{Physical Activity Level and Its Change}

At baseline, inactive patients were 51 and $88 \%$ reported having barriers to physical activity (Molfino et al., 2017). Similarly, at 12 months $51 \%$ of the patients reported performing physical activity during leisure time less than once per week, almost never, or never, and therefore were considered inactive, and 90\%

TABLE 1 | Patient characteristics.

\begin{tabular}{|c|c|c|c|}
\hline & Month 12 & Month 24 & \\
\hline & Patients $N=39$ & Patients $N=29$ & $\mathbf{P}$ \\
\hline Sex (male) & 31 & 23 & \\
\hline Age, years & $66 \pm 15.2$ & $66.9 \pm 14.7$ & 0.85 \\
\hline BMl, weight $(\mathrm{kg}) /$ height $^{2}(\mathrm{~m})$ & $24.5 \pm 5.3$ & $25.0 \pm 5.82$ & 0.87 \\
\hline $\mathrm{Hb}, \mathrm{g} / \mathrm{dl}$ & $11.2 \pm 1.3$ & $11.4 \pm 1.22$ & 0.96 \\
\hline Albumin, g/l & $39.7 \pm 3.04$ & $37.4 \pm 4.08$ & 0.49 \\
\hline Urea ${ }^{\#}, \mathrm{mg} / \mathrm{dl}$ & $118.6 \pm 31.8$ & $132.6 \pm 36.1$ & 0.68 \\
\hline Creatinine, mg/dl & $9.1 \pm 2.5$ & $8.9 \pm 2.4$ & 0.41 \\
\hline CRP, mg/dl & $0.4(0.2 ; 1.3)$ & $0.37(0.19 ; 0.9)$ & 0.97 \\
\hline Dialysis vintage, months & $40.5(25 ; 70.2)$ & $50(37 ; 77)$ & 0.21 \\
\hline $\mathrm{nPCR}, \mathrm{g} / \mathrm{kg} / \mathrm{d}$ & $0.92 \pm 0.2$ & $0.94 \pm 0.17$ & 0.97 \\
\hline $\mathrm{Kt} / \mathrm{N}$ & $1.29 \pm 0.24$ & $1.34 \pm 0.26$ & 0.39 \\
\hline Physical inactivity (yes) & $20(51 \%)$ & 19 (63\%) & 0.27 \\
\hline
\end{tabular}

\#Urea levels are 2.14 times the corresponding BUN levels. BMI, body mass index; BUN, blood urea nitrogen; CRP, C-reactive protein; Hb, hemoglobin; $n P C R$, normalized protein catabolic rate. 
reported barriers to physical activity. Four patients, who were active at baseline, resulted inactive at month 12 months.

At month 24, 65\% of patients resulted inactive and $96 \%$ reported having barriers to physical activity. From baseline to month 24, according to the questionnaire, 7 active patients resulted inactive. In the entire cohort of the enrolled patients, the median number of barriers endorsed at baseline $(n=4)$, month $12(n=3)$, and month $24(n=3)$ did not significantly change overtime $(P=0.30)$. From baseline to 24 -month followup, the type of barriers did not significantly change in all HD patients and also when considering active and inactive patients separately. We did not observe differences between active and inactive patients in terms of type and numbers of comorbidities, and in nutritional and inflammatory biomarkers. In addition, we did not find a significant association between inactivity at baseline and mortality $(P=0.114)$.

During the entire follow-up, the most frequently endorsed barrier was "fatigue on dialysis days." The barrier "reduced walking ability" was confirmed to be more frequent in inactive patients with respect to active patients at month $12(P=0.003)$ and month $24(P=0.05)$.

\section{Body Composition and Physical Activity Change}

Differences in body composition parameters between active and inactive patients during the follow-up are reported in Table 2. As for baseline (Molfino et al., 2017), at month 12, we found that active patients had higher ICW $(\%)(P=0.001)$ and cellular mass $(\%)(P<0.001)$ with respect to inactive patients. Similarly, at month 24 active patients showed compared to inactive patients higher ICW (\%) $(P=0.012)$ and cellular mass (\%) $(P=0.002)$ (Table 2).

Independent of the presence of physical inactivity, our cohort of $\mathrm{HD}$ patients showed, compared to baseline, a significant reduction in ICW $(\%)$ at month $12(P=0.011)$ and at month $24(P=0.014)$.

This trend overtime was confirmed after adjusting for the presence of physical inactivity, although at the limit of significance (at month 12, $P=0.046$ and at month 24, $P=0.059$, respectively)

\section{BAIBA, Physical Activity Level and Its Barriers, Body Composition and Muscle Strength Overtime}

In all the participants, at baseline BAIBA $(\mu \mathrm{M})$ median value was 0.40 (IQR, 0.21-0.61) (Molfino et al., 2017) and at month 24 was 0.21 (IQR, 0.10-0.32). During the 24-month followup, BAIBA median level did not significantly change among all the participants; however, at month 24, median BAIBA level was higher among active patients with respect to inactive (Figure 1) $(P=0.043)$.

No significant association was found between plasma BAIBA levels and the number and type of barriers at month 24 .

Independent of the presence of physical inactivity, when considering BAIBA normalized per BMI (BAIBA/BMI), we found this ratio at month 24 significantly lower with respect to baseline $(P=0.004)$ (Figure 2A). Similarly, when BAIBA was normalized per ICW (BAIBA/ICW), we documented this ratio lower with respect to baseline $(P=0.001)$ (Figure $2 \mathrm{~B}$ ).

At month 24, median values of hand-grip measurement were not different between active $(21 \mathrm{mmHg})$ and inactive patients $(21 \mathrm{mmHg})$. Moreover, no association was documented overtime between muscle strength and BAIBA levels, physical inactivity and its barriers. However, at month 24, a significant correlation was observed between muscle strength and ICW $(r=0.51, P=0.005)$.

\section{DISCUSSION}

There have been robust data on physical inactivity and its association with frailty during $\mathrm{HD}$ and how this condition may affect patient's prognosis (Johansen et al., 2013b, 2016; Sy and Johansen, 2017). This suggests to physicians to evaluate physical activity level to prevent clinical deterioration and functional dependence during ESRD (Rodríguez-Mañas et al., 2013). The prevalence of HD patients physically inactive and sedentary is high (Johansen et al., 2007, 2014b), and its percentage depends on how physical activity is defined (Delgado and Johansen, 2012; Bossola et al., 2014; Johansen et al., 2016).

TABLE 2 | Biompendance analysis parameters.

\begin{tabular}{|c|c|c|c|c|c|c|c|c|c|}
\hline & \multicolumn{3}{|c|}{ Baseline } & \multicolumn{3}{|c|}{ 12-month follow-up } & \multicolumn{3}{|c|}{ 24-month follow-up } \\
\hline & $\begin{array}{c}\text { Active patients } \\
N=24(49 \%)\end{array}$ & $\begin{array}{l}\text { Inactive patients } \\
\qquad N=25 \text { (51\%) }\end{array}$ & $\mathbf{P}$ & $\begin{array}{l}\text { Active patient } \\
N=19(49 \%)\end{array}$ & $\begin{array}{l}\text { Inactive patients } \\
\qquad N=20 \text { (51\%) }\end{array}$ & $\mathbf{P}$ & $\begin{array}{l}\text { Active patient } \\
N=10(35 \%)\end{array}$ & $\begin{array}{l}\text { Inactive patients } \\
\qquad N=19(65 \%)\end{array}$ & $\mathbf{P}$ \\
\hline Body weight (Kg) & $69.9 \pm 18.4$ & $69.2 \pm 14.7$ & 0.88 & $66.6 \pm 18.0$ & $71.2 \pm 13.7$ & 0.38 & $62.25 \pm 13.8$ & $73.65 \pm 16.9$ & 0.07 \\
\hline \multicolumn{10}{|l|}{ Biompendance analysis } \\
\hline \multicolumn{10}{|l|}{ Fat mass } \\
\hline Intracellular water (\%) & $51.2 \pm 7.3$ & $44.5 \pm 9.5$ & 0.008 & $52.4 \pm 5.98$ & $43.1 \pm 9.4$ & 0.001 & $53.1 \pm 5.97$ & $45.4 \pm 7.7$ & 0.012 \\
\hline Cellular mass (\%) & $42.3 \pm 7.3$ & $35.9 \pm 7.8$ & 0.005 & $43.1 \pm 4.02$ & $33.04 \pm 9.4$ & $<0.001$ & $43.08 \pm 4.2$ & $35.9 \pm 6.3$ & 0.002 \\
\hline Body mass cell index $\left(\mathrm{kg} / \mathrm{m}^{2}\right)$ & $6.7 \pm 1.9$ & $5.6 \pm 1.8$ & 0.04 & $6.88 \pm 1.9$ & $5.65 \pm 1.9$ & 0.059 & $6.18 \pm 1.72$ & $6.95 \pm 4.2$ & 0.58 \\
\hline Phase angle $\left({ }^{\circ}\right)$ & $5.2 \pm 1.2$ & $4.1 \pm 1.1$ & $<0.001$ & $5.2 \pm 1.0$ & $4.0 \pm 1.1$ & $<0.001$ & $5.4 \pm 1.1$ & $4.3 \pm 1.2$ & 0.02 \\
\hline
\end{tabular}




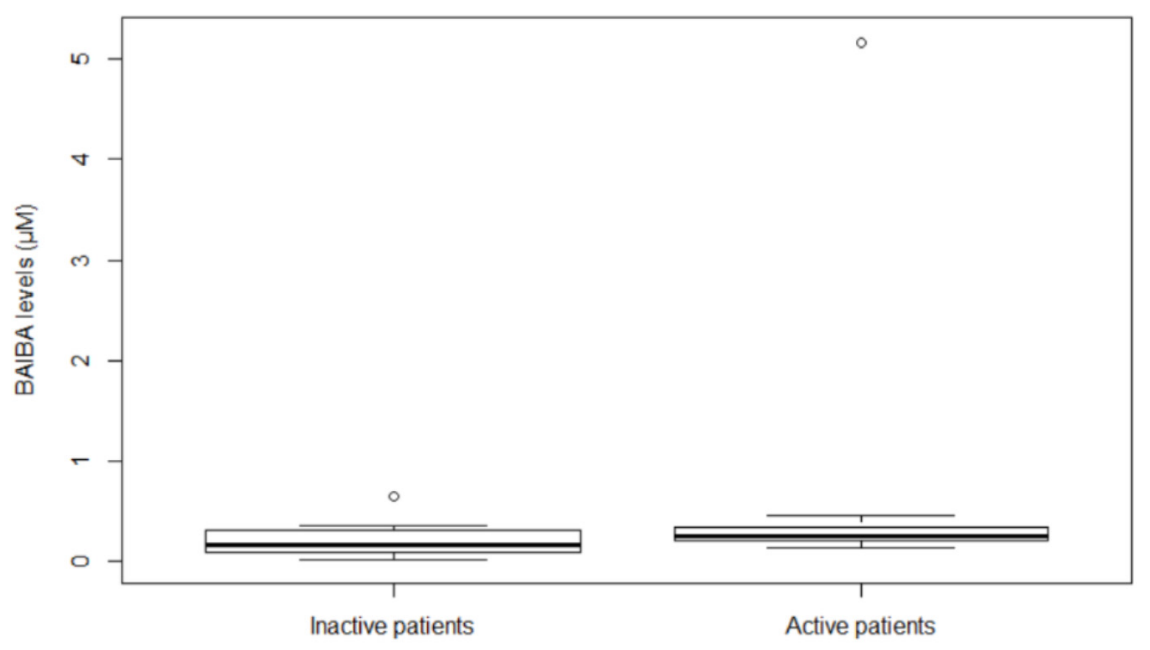

FIGURE 1 | Plasma BAIBA levels in hemodialysis inactive and active patients at month 24. Box plot of BAIBA levels ( $\mu$ M) stratified by inactive or active patients. BAIBA levels were significantly higher in active with respect to inactive patients $(P=0.043)$. Lines represent the median, 25th and 75 th percentiles, and the whiskers (error bars) below and above the box indicate the 10th and 90th percentiles. BAIBA, beta-aminoisobutyric acid.

We previously confirmed in a cohort of HD patients a high prevalence of physical inactivity, defined using self-reported physical function (Molfino et al., 2017). The majority of the data available in the literature on this topic have been obtained in cross-sectional studies (Johansen et al., 2014a, 2016). Novel information may be obtained by longitudinal studies by comparing the timing of changes in body composition with the development of several conditions, including low muscle mass and frailty (Johansen et al., 2014a). In this light, longitudinal data are important to ascertain whether modifications in body composition are strictly related with physical activity changes and whether possible biomarker(s) of muscularity may identify or predict changes in body composition and in physical activity level which are associated with adverse outcomes.

In the present study, we evaluated overtime (up to 24 months) in a single-center cohort of HD patients, being representative of a homogenous population, the physical activity level during leisure time. We administered, as for baseline (Molfino et al., 2017), a first questionnaire on the self-reported frequency of physical activity and a second questionnaire regarding barriers to physical activity in general or to lower physical activity levels than desired. The participation in physical activity, already low at baseline (Molfino et al., 2017), was confirmed to be reduced after the 24-month follow-up, as $63 \%$ of the patients reported participating in light activity never, almost never, or less than once per week, and $96 \%$ of patients reported endorsing barriers to physical activity. Interestingly, although not statistically different, the median number of barriers at month 24 was lower than baseline. Considering that low level of physical activity is associated with reduced survival, we believe that those patients in our cohort who survived at month 24 may be less inactive. The most frequent barriers observed at month 12 and 24 were "fatigue on dialysis day" and "reduced walking ability." The barrier "reduced walking ability" was more frequently reported by inactive patients with respect to active during the entire follow-up at month 12 and 24. This highlights the importance of walking difficulty in conditioning reduced mobility in HD patients. Moreover, results of a recent multicenter study documented that barriers to physical activity and non-proactive health-care staff's attitude reduced the implementation of physical activity in the HD population and that patients not reporting barriers were those who showed greater beneficial effects from a proactive staff's attitude (Regolisti et al., 2018).

Physical inactivity may be the cause and/or the effect of body composition derangements. In fact, low activity level may determine and/or worsen low muscle mass; on the other hand, lower muscularity may reduce physical performance. In this light, it appears advisable to evaluate body composition overtime in association with the level of physical activity.

During our follow-up, we found that lower ICW, a proxy of muscle mass (Johansen et al., 2014a), was associated with physical inactivity at month 12 and 24 . A significant decline in ICW was observed from baseline to month 24 in the entire cohort. This is in line with the results obtained in a larger cohort of HD patients where lower ICW was associated with frailty (Johansen et al., 2014a).

When performing a multivariable analysis accounting for physical activity, the trend of ICW reduction overtime was confirmed in the inactive group, suggesting the possibility that the time-altered body composition association might be explained, at least in part, by physical inactivity.

In addition, we focused on the possible relationship between changes overtime of body composition, physical activity, and BAIBA, as circulating biomarker of muscularity during HD. This aspect appears relevant considering that BAIBA seems to present different actions within skeletal muscle, including attenuation of insulin resistance, inflammation and inducing fatty acid oxidation (Jung et al., 2015). Not conclusive data are yet available on the physiology/pathophysiology of BAIBA and we do not have information on BAIBA removal from HD. 

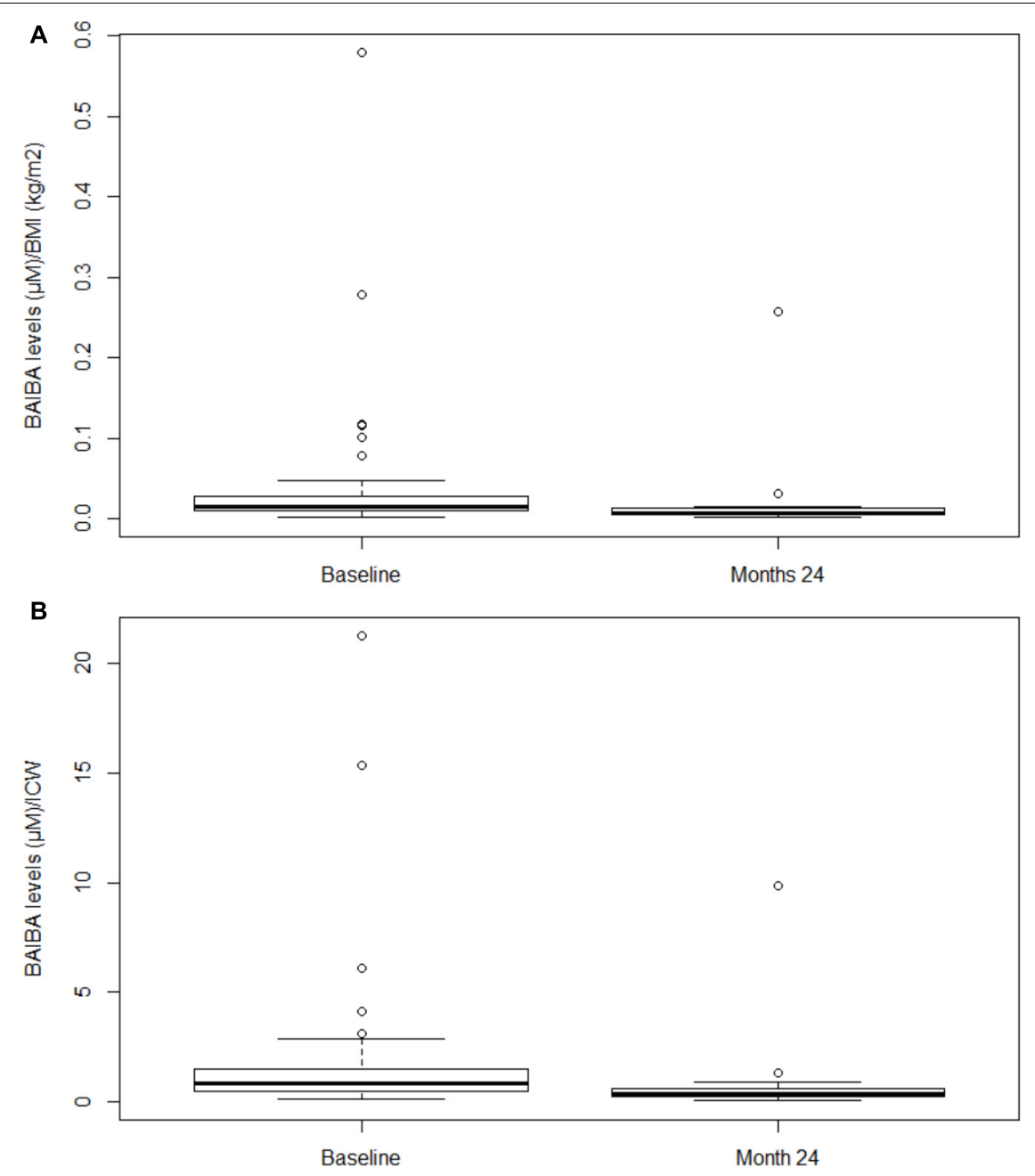

FIGURE 2 | Plasma BAIBA levels normalized per BMI and per ICW in hemodialysis patients at baseline and at month 24. In panel (A) Box plot of BAIBA/BMI ratio at baseline vs. month $24(P=0.004)$. Lines represent the median, 25th and 75th percentiles, and the whiskers (error bars) below and above the box indicate the 10th and 90th percentiles. BAIBA, betaaminoisobutyric acid; BMI, body mass index. In panel (B) Box plot of BAIBA/ICW ratio at baseline vs. month 24 ( $P$ = 0.001$)$. Lines represent the median, 25th and 75th percentiles, and the whiskers (error bars) below and above the box indicate the 10th and 90th percentiles. BAIBA,

betaaminoisobutyric acid; ICW, intracellular water.

However, Roberts et al. showed robust data in humans documenting that BAIBA levels increased with physical activity and were inversely related with metabolic risk factors (Roberts et al., 2014).

In the present study, at the end of the follow-up (month 24), inactive patients showed lower plasma BAIBA levels with respect to active patients and, independent of the level of physical activity, we found lower BAIBA/BMI ratio with respect to baseline. This trend was confirmed when BAIBA was corrected per marker of muscle mass. Although not at baseline, a correlation was present at month 24 between muscle mass and its function (muscle strength). However, as for baseline, no correlation was seen between BAIBA and muscle function indicating a possible different mechanism(s) regulating BAIBA expression and muscle mass and its function. In particular, our results did not allow us to ascertain a cause-effect relationship between plasma BAIBA levels and changes of physical activity and muscularity overtime. However, our data give us the perspective to possibly use BAIBA as a muscle-specific biomarker that might help physicians in monitoring muscularity in the HD population.

Our study has several limitations, including the patient sampling (single center study) and, in particular, the reduced sample size at month 12 and 24, possibly affecting the investigated associations, and the study group is highly variable including a wide range of dialysis vintage. As for baseline, physical activity is only subjectively assessed by the patients and not by more objective instruments such as accelerometers or pedometers, and the tool used to evaluate physical activity did not include questions on potential additional activities (Molfino et al., 2017). The association found between physical inactivity and body composition does not imply a clear causality in one or 
other direction, as previously suggested by others (Johansen et al., 2014a). We could not determine if low ICW caused physical inactivity or if being more sedentary determined body composition derangements. Using BIA, possible errors in estimating body composition and fluid distribution remain (Kyle et al., 2004). Moreover, the biomarker BAIBA has not yet been validated in HD population, with poor availability of information regarding its accumulation in HD patients and its removal with dialysis.

\section{CONCLUSION}

In conclusion, we observed in our cohort of $\mathrm{HD}$ patients a high prevalence of physical inactivity during a 24-month follow-up and a clinically relevant number of endorsed barriers. These conditions were associated with a decline in markers of muscularity overtime. The levels of the myokine BAIBA after 24-month follow-up were significantly lower among inactive patients and, independent of the level of physical activity, BAIBA levels significantly reduced overtime when corrected per index of muscularity. Larger clinical investigations are essential to clarify the role of BAIBA and body composition changes overtime in $\mathrm{HD}$, as well as to assess BAIBA levels evaluating its changes after interventional

\section{REFERENCES}

Bossola, M., Pellu, V., Di Stasio, E., Tazza, L., Giungi, S., and Nebiolo, P. E. (2014). Self-reported physical activity in patients on chronic hemodialysis: correlates and barriers. Blood Purif. 38, 24-29. doi: 10.1159/000363599

Delgado, C., and Johansen, K. L. (2012). Barriers to exercise participation among dialysis patients. Nephrol. Dial. Transplant. 27, 1152-1157. doi: 10.1093/ndt/ gfr404

Huh, J. Y. (2018). The role of exercise-induced myokines in regulating metabolism. Arch. Pharm. Res. 41, 14-29. doi: 10.1007/s12272-017-0994-y

Jankowska, M., Debska-Slizień, A., and Rutkowski, B. (2006). Bioelectrical impedance analysis before versus after a hemodialysis session in evaluation of nutritional status. J. Ren. Nutr. 6, 137-140. doi: 10.1053/j.jrn.2006. 01.005

Johansen, K. L., Chertow, G. M., Jin, C., and Kutner, N. G. (2007). Significance of frailty among dialysis patients. J. Am. Soc. Nephrol. 18, 2960-2967. doi: 10.1681/asn.2007020221

Johansen, K. L., Chertow, G. M., Ng, A. V., Mulligan, K., Carey, S., Schoenfeld, P. Y., et al. (2000). Physical activity levels in patients on hemodialysis and healthy sedentary controls. Kidney Int. 57, 2564-2570. doi: 10.1046/j.15231755.2000.00116.x

Johansen, K. L., Dalrymple, L. S., Delgado, C., Kaysen, G. A., Kornak, J., Grimes, B., et al. (2014a). Association between body composition and frailty among prevalent hemodialysis patients: a US Renal Data System special study. J. Am. Soc. Nephrol. 25, 381-389. doi: 10.1681/ASN.2013040431

Johansen, K. L., Dalrymple, L. S., Delgado, C., Kaysen, G. A., Kornak, J., Grimes, B., et al. (2014b). Comparison of self-report-based and physical performance-based frailty definitions among patients receiving maintenance hemodialysis. Am. J. Kidney Dis. 64, 600-607. doi: 10.1053/j.ajkd.2014. 03.016

Johansen, K. L., Dalrymple, L. S., Glidden, D., Delgado, C., Kaysen, G. A., Grimes, B., et al. (2016). Association of performance-based and self-reported function-based definitions of frailty with mortality among patients receiving hemodialysis. Clin. J. Am. Soc. Nephrol. 11, 626-632. doi: 10.2215/CJN. 03710415 studies by administering nutritional support and/or physical activity interventions.

\section{ETHICS STATEMENT}

Ethics Committee of Fatebenefratelli Hospital, Rome, Italy, approved the protocol. Written informed consent was obtained from each participant. All subjects gave written informed consent in accordance with the Declaration of Helsinki.

\section{AUTHOR CONTRIBUTIONS}

AM designed the research, conducted the research, analyzed the data, and wrote the manuscript. MIA conducted the research, collected the data, and wrote the manuscript. TA and AG collected the data. AS analyzed the data and performed the statistical analyses. LL performed the laboratory dosage. MS analyzed the laboratory data. SL conducted the research. MMo reviewed the manuscript. AL designed the research and reviewed the manuscript. MGC conducted the research, enrolled the patients, and reviewed the manuscript. $\mathrm{MMu}$ designed the research, reviewed the manuscript, and had primary responsibility for the final content.

Johansen, K. L., Delgado, C., Bao, Y., and Tamura, M. K. (2013a). Frailty and dialysis initiation. Semin. Dial. 26, 690-696. doi: 10.1111/sdi.12126

Johansen, K. L., Kaysen, G. A., Dalrymple, L. S., Grimes, B. A., Glidden, D. V., Anand, S., et al. (2013b). Association of physical activity with survival among ambulatory patients on dialysis: the comprehensive dialysis study. Clin. J. Am. Soc. Nephrol. 8, 248-253. doi: 10.2215/CJN. 08560812

Jung, T. W., Hwang, H. J., Hong, H. C., Yoo, H. J., Baik, S. H., and Choi, K. M. (2015). BAIBA attenuates insulin resistance and inflammation induced by palmitate or a high fat diet via an AMPK-PPAR $\delta$-dependent pathway in mice. Diabetologia 58, 2096-2105. doi: 10.1007/s00125-0153663-z

Ku, E., Kopple, J. D., Johansen, K. L., McCulloch, C. E., Go, A. S., Xie, D., et al. (2018). Longitudinal weight change during CKD progression and its association with subsequent mortality. Am. J. Kidney Dis. 71, 657-665. doi: 10.1053/j.ajkd. 2017.09.015

Kyle, U. G., Bosaeus, I., De Lorenzo, A. D., Deurenberg, P., Elia, M., Gómez, J. M., et al. (2004). Bioelectrical impedance analysis-part I: review of principles and methods. Clin. Nutr. 23, 1226-1243. doi: 10.1016/j.clnu.2004. 06.004

Molfino, A., Amabile, M. I., Ammann, T., Farcomeni, A., Lionetto, L., Simmaco, M., et al. (2017). The metabolite beta-aminoisobutyric acid and physical inactivity among hemodialysis patients. Nutrition 34, 101-107. doi: 10.1016/j. nut.2016.07.012

Regolisti, G., Maggiore, U., Sabatino, A., Gandolfini, I., Pioli, S., Torino, C., et al. (2018). Interaction of healthcare staff's attitude with barriers to physical activity in hemodialysis patients: a quantitative assessment. PLoS One 13:e0196313. doi: 10.1371/journal.pone.0196313

Roberts, L. D., Boström, P., O’Sullivan, J. F., Schinzel, R. T., Lewis, G. D., Dejam, A., et al. (2014). Beta-aminoisobutyric acid induces browning of white fat and hepatic betaoxidation and is inversely correlated with cardiometabolic risk factors. Cell Metab. 19, 96-108. doi: 10.1016/j.cmet.2013. 12.003

Robinson-Cohen, C., Littman, A. J., Duncan, G. E., Weiss, N. S., Sachs, M. C., Ruzinski, J., et al. (2014). Physical activity and change in estimated GFR 
among persons with CKD. J. Am. Soc. Nephrol. 25, 399-406. doi: 10.1681/ASN. 2013040392

Rodríguez-Mañas, L., Féart, C., Mann, G., Viña, J., Chatterji, S., Chodzko-Zajko, W., et al. (2013). Searching for an operational definition of frailty: a Delphi method based consensus statement: the frailty operative definition-consensus conference project. J. Gerontol. A. Biol. Sci. Med. Sci. 68, 62-67. doi: 10.1093/ gerona/gls119

Sy, J., and Johansen, K. L. (2017). The impact of frailty on outcomes in dialysis. Curr. Opin. Nephrol. Hypertens. 26, 537-542. doi: 10.1097/mnh. 0000000000000364

Tentori, F., Elder, S. J., Thumma, J., Pisoni, R. L., Bommer, J., Fissell, R. B., et al. (2010). Physical exercise among participants in the Dialysis Outcomes and Practice Patterns Study (DOPPS): correlates and associated outcomes. Nephrol. Dial. Transplant. 25, 3050-3062. doi: 10.1093/ndt/ gfq138
Conflict of Interest Statement: The authors declare that the research was conducted in the absence of any commercial or financial relationships that could be construed as a potential conflict of interest.

The handling Editor declared a shared affiliation, though no other collaboration, with several of the authors, AM, MIA, SL, AS, MS, MMo, AL, and MMu, at the time of review.

Copyright (c) 2019 Molfino, Amabile, Ammann, Lai, Grosso, Lionetto, Spagnoli, Simmaco, Monti, Laviano, Chiappini and Muscaritoli. This is an open-access article distributed under the terms of the Creative Commons Attribution License (CC BY).

The use, distribution or reproduction in other forums is permitted, provided the original author(s) and the copyright owner(s) are credited and that the original publication in this journal is cited, in accordance with accepted academic practice. No use, distribution or reproduction is permitted which does not comply with these terms. 
OPEN ACCESS

Edited by:

Marilia Seelaender,

University of São Paulo, Brazil

Reviewed by:

Nicolas J. Pillon,

Karolinska Institutet (KI), Sweden

Vera C. Mazurak,

University of Alberta, Canada

${ }^{*}$ Correspondence:

Sunyoung Kim

sunyoung@snu.ac.kr

Specialty section:

This article was submitted to Striated Muscle Physiology,

a section of the journal

Frontiers in Physiology

Received: 28 September 2018

Accepted: 04 July 2019

Published: 25 July 2019

Citation:

Choi W, Lee J, Lee J, Lee SH and Kim S (2019) Hepatocyte Growth

Factor Regulates Macrophage Transition to the M2 Phenotype and

Promotes Murine Skeletal Muscle Regeneration.

Front. Physiol. 10:914.

doi: 10.3389/fphys.2019.00914

\section{Hepatocyte Growth Factor Regulates Macrophage Transition to the M2 Phenotype and Promotes Murine Skeletal Muscle Regeneration}

\author{
Wooshik Choi', Jaeman Lee', Junghun Lee ${ }^{2}$, Sang Hwan Lee ${ }^{1}$ and Sunyoung Kim ${ }^{1,2 \star}$
}

${ }^{1}$ Department of Biological Sciences, College of Natural Sciences, Seoul National University, Seoul, South Korea, ${ }^{2} R \& D$ Center for Innovative Medicines, ViroMed Co., Ltd, Seoul, South Korea

Hepatocyte growth factor (HGF) is well known for its role in the migration of embryonic muscle progenitors and the activation of adult muscle stem cells, yet its functions during the adult muscle regeneration process remain to be elucidated. In this study, we showed that HGF/c-met signaling was activated during muscle regeneration, and that among various infiltrated cells, the macrophage is the major cell type affected by HGF. Pharmacological inhibition of the c-met receptor by PHA-665752 increased the expression levels of pro-inflammatory (M1) macrophage markers such as IL-1 $\beta$ and iNOS while lowering those of pro-regenerative (M2) macrophage markers like IL-10 and TGF- $\beta$, resulting in compromised muscle repair. In Raw 264.7 cells, HGF decreased the RNA level of LPS-induced TNF- $\alpha$, IL-1 $\beta$, and iNOS while enhancing that of IL-10. HGF was also shown to increase the phosphorylation of AMPK $\alpha$ through CaMKK $\beta$, thereby overcoming the effects of the LPS-induced deactivation of AMPK $\alpha$. Transfection with specific siRNA to AMPK $\alpha$ diminished the effects of HGF on the LPS-induced gene expressions of M1 and M2 markers. Exogenous delivery of HGF through intramuscular injection of the HGF-expressing plasmid vector promoted the transition to M2 macrophage and facilitated muscle regeneration. Taken together, our findings suggested that HGF/c-met might play an important role in the transition of the macrophage during muscle repair, indicating the potential use of HGF as a basis for developing therapeutics for muscle degenerative diseases.

Keywords: hepatocyte growth factor, muscle regeneration, macrophage transition, AMPK, CaMKK $\beta$

\section{INTRODUCTION}

Resolution of tissue damage requires tight interaction between the immune system and the target tissue undergoing repair. Immune cells detect the injury, remove damaged tissues, and then promote repair mechanisms to restore tissue integrity. In addition, they strongly influence the growth and differentiation of stem cells and progenitors to repair the inflicted damage (Wynn and Vannella, 2016). Upon muscle injury, macrophages have been reported to play critical roles in this process as they occupy major cell population infiltrated in injured muscle. They are responsible for the removal of damaged myofibers and also contribute to subsequent regrowth and differentiation of 
muscle progenitors (Tidball, 2017). These procedures are tightly regulated by the coordinated transition of macrophage between pro-inflammatory (M1) and pro-regenerative (M2) phenotypes in the immune environment (Wang et al., 2014).

Hepatocyte growth factor (HGF) is a multifunctional protein which contains mitogenic, morphogenic, motogenic, and angiogenic activities by interacting with its cellular receptor, c-met (Nakamura and Mizuno, 2010). This interaction subsequently turns on a variety of signaling pathways depending on cell types. HGF has been shown to play important roles in the regeneration process of various tissues by stimulating the proliferation and migration of respective progenitor cells (Kawaida et al., 1994; Huh et al., 2004; Wu et al., 2004; Watanabe et al., 2005). For example, HGF has been implicated in both skeletal muscle development and its regeneration after injury (Sisson et al., 2009).

The interaction between HGF and c-met is required for migration of myogenic progenitors into the limb buds during embryogenesis (Dietrich et al., 1999). Upon muscle injury, HGF activates muscle stem cells that reside in muscle fiber, rendering them to make a myogenic commitment (Jennische et al., 1993; Allen et al., 1995; Tatsumi et al., 1998; Sheehan et al., 2000). Exogenously added recombinant HGF protein was reported to increase myoblast proliferation while inhibiting differentiation, resulting in delayed regeneration of damaged muscle (Miller et al., 2000). However, recent studies have shown that HGF activated by urokinase plasminogen activator (uPA) promotes muscle regeneration (Sisson et al., 2009), and its receptor, c-met, is responsible for the transition of quiescent muscle stem cells into GAlert, a cellular state in which they have an increased ability to participate in tissue repair (Rodgers et al., 2014). Thus, the role of HGF in muscle regeneration remains to be clarified. In particular, although the immune system plays an instrumental role in the muscle regeneration process, the effect of HGF on immune cells infiltrated in injured muscle tissue is not yet clearly understood.

Here, we report the role of HGF in the transition of infiltrated macrophages during muscle regeneration. HGF expression was upregulated following muscle injury. When mice were treated with PHA-665752, an inhibitor of the c-met receptor, muscle regeneration was delayed. Consistently, the population of M1 and M2 macrophages during muscle regeneration was deregulated. HGF overexpression by intramuscular (i.m.) injection of plasmid expression vector facilitated muscle regeneration. Data from experiments involving Raw 264.7 cells indicated that HGF might regulate the transition of macrophage to the M2 phenotype through CaMKK $\beta$-AMPK signaling. Taken together, our data suggested that HGF might be used as a platform for developing therapeutic agents to treat diseases associated with defects in muscle regeneration.

\section{MATERIALS AND METHODS}

\section{Animal Cares}

Ten-week-old male C57BL/6 mice were purchased from Orient Bio Inc. (Seongnam, Korea) for animal studies. Mice were housed at $24^{\circ} \mathrm{C}$ with a $12 \mathrm{~h}$ light-dark cycle. All experiments were performed in compliance with the guideline set by the International Animal Care and Use Committee at Seoul National University.

\section{Surgical Procedures}

All surgical protocols were approved by the International Animal Care and Use Committee at Seoul National University. For muscle injury, ten-week-old male C57BL/6 mice were anesthetized with isoflurane, and the TAs were injected with $50 \mu \mathrm{l}$ CTX (Latoxan, Valence, France), diluted to $10 \mu \mathrm{M}$ in phosphate buffered saline (PBS). Sham treatment was performed by following the same procedure except injecting TAs with PBS. PHA-665752 (Tocris Bioscience, MO), a c-met inhibitor, was dissolved in DMSO (Sigma Aldrich, MO) and i.p. administered in each mouse on a daily basis with a dose of $20 \mathrm{mg} / \mathrm{kg}$. For i.m. injection, $0.3 \mathrm{~mm}$ needle size, $0.5 \mathrm{ml}$ insulin syringe $(\mathrm{BD}$, $\mathrm{NJ}$ ) was used. $\mathrm{pCK}$ or pCK-HGF-X7 plasmid expression vector was dissolved in $50 \mu \mathrm{l}$ PBS $(2 \mu \mathrm{g} / \mu \mathrm{l})$. The injection procedure was performed by injecting the needle parallel to the tibia and then delivering plasmid into the middle of the TA.

\section{Immunohistochemistry}

Immunohistochemical analyses were performed as previously described (Ahn et al., 2014). Briefly, TAs were isolated and fixed in $4 \%$ paraformaldehyde in PBS and cryo-sectioned to $6 \mu \mathrm{m}$ thickness. Sections were washed in 0.1 M PBS ( $\mathrm{pH}$ 7.4) twice, then blocked for $1 \mathrm{~h}$ with PBS containing 5\% fetal bovine serum (Corning, NY), 5\% donkey serum (Jackson ImmunoResearch Laboratories, PA), 2\% BSA (Sigma Aldrich, MA) and $0.1 \%$ Triton X-100 (Sigma Aldrich, MA). Samples were incubated with primary antibodies diluted in blocking buffer overnight at $4^{\circ} \mathrm{C}$. Sections were washed 4 times in PBS and incubated for $1 \mathrm{~h}$ at room temperature with secondary antibodies (Invitrogen, CA) diluted in PBS. Immunostained samples were further washed 6 times and counterstained with DAPI (Sigma Aldrich, MA) for nuclear staining. The fluorescence images were obtained using a Zeiss LSM 700 confocal microscope (Zeiss, Oberkochen, Germany).

\section{Hematoxylin and Eosin Staining and Morphometric Analysis}

TAs were fixed in 10\% normalized buffered formalin (Sigma Aldrich, MA) and dehydrated with a gradient series of ethanol from 70 to $100 \%$. Samples were embedded in the paraffin block and sectioned to $6 \mu \mathrm{m}$ thickness. A paraffin section of the TA was stained by hematoxylin and eosin, and the morphology of each cross-section was analyzed by Image J software (National Institutes of Health, MD). More than 300 myofibers were assessed from four individual mice in each group.

\section{RNA Isolation and RT-qPCR}

TAs were prepared and mechanistically homogenized using Bullet Blender Storm (Next Advance, NY), and total RNA was extracted from homogenized TA or cultured cells with RNAiso (Takara, Kusatsu, Japan) following the manufacturer's instructions. 
One microgram of RNA was converted to cDNA using oligo dT primers (Qiagen, Hilden, Germany) and Reverse Transcriptase XL (AMV) (Takara, Kusatsu, Japan). Gene expression was assessed using quantitative real-time PCR with Thermal Cycler Dice Real Time System TP800 (Takara, Kusatsu, Japan) and SYBR Premix Ex Taq (Takara, Kusatsu, Japan). Primers used in this study are listed in Supplementary Table S1A.

\section{ELISA}

TAs were prepared and mechanistically homogenized using Bullet Blender Storm (Next Advance, NY), and total proteins were extracted in RIPA lysis buffer (Sigma Aldrich, MO) containing a protease inhibitor (Roche, Basel, Switzerland), phosphatase inhibitor (Roche, Basel, Switzerland), and PMSF (Sigma Aldrich, MO). Samples were centrifuged at 12,000 rpm for $15 \mathrm{~min}$ at $4^{\circ} \mathrm{C}$, and the supernatants containing total protein were subjected to mHGF ELISA ( $R \& D$ systems, $\mathrm{MN}$ ) following the manufacturer's protocol.

\section{Western Blot}

For immunoblotting, TAs or cultured cells were prepared and homogenized in RIPA lysis buffer (Sigma Aldrich, MO) containing a protease inhibitor (Roche, Basel, Switzerland) and phosphatase inhibitor (Roche, Basel, Switzerland) using Bullet Blender Storm (Next Advance, NY). Equal amounts of protein were then separated by $10 \%$ SDS-polyacrylamide gel and electrophoretically transferred to polyvinylidene fluoride membranes (Millipore, MA). The membranes were blocked with 5\% BSA (Gibco, MA) in TBST ( $1 \mathrm{M}$ Tris- $\mathrm{HCl}, \mathrm{pH} 7.4,0.9 \% \mathrm{NaCl}$ and $0.1 \%$ Tween-20) for $1 \mathrm{~h}$ and probed with antibodies diluted in $3 \%$ BSA blocking solution overnight at $4^{\circ} \mathrm{C}$. Membranes were then incubated with HRP-conjugated anti-mouse or anti-rabbit IgG (1:100,000; Sigma Aldrich, MO) for $1 \mathrm{~h}$, and the protein bands were visualized with the enhanced chemiluminescence system (Millipore, MA). Antibodies used in this study are listed in Supplementary Table S1B.

\section{Cell Culture and Reagents}

Raw 264.7 cells (American Type Culture Collection, VA) were grown in DMEM (Welgene, Gyeongsan, Korea) supplemented with $10 \%$ FBS (Corning, NY) and antibiotics [100 U/ml penicillin and $100 \mu \mathrm{g} / \mathrm{ml}$ streptomycin (Sigma Aldrich, MO)]. Recombinant human HGF protein (R\&D systems, MN) and LPS (Sigma Aldrich, MO) were used at appropriate concentrations. STO-609 (CaMKK $\beta$ inhibitor, Tocris Bioscience, MO) was used at 5 or $20 \mu \mathrm{M}$ for experiments.

\section{siRNA Transfection}

Raw 264.7 cells were transfected with siRNA specific to AMPKa or scramble siRNA (Santa Cruz Biotechnology, TX) using RNAiMAX (ThermoFisher Scientific, MA) according to the manufacturer's protocol. Briefly, Raw 264.7 cells were plated at $1 \times 10^{6}$ cells per well in 6 -well culture plates. Twenty-four hours later, $25 \mathrm{pmol}(2.5 \mu \mathrm{l}, 10 \mu \mathrm{M})$ of AMPKa or scramble siRNA was diluted into $125 \mu \mathrm{l}$ of Opti-MEM (Gibco, MA), and $5 \mu \mathrm{l}$ of RNAiMAX was diluted in $125 \mu \mathrm{l}$ of Opti-MEM. Diluted siRNA and RNAiMAX were then combined and incubated at room temperature for $5 \mathrm{~min}$. Subsequently, $250 \mu \mathrm{l}$ of the siRNARNAiMAX mixtures were added to each well of a 6-well plate. Twenty-four hours after transfection, cells were subjected to the analysis. Knock-down efficiency was evaluated by western blot using antibodies against AMPKa (Cell Signaling Technology, MA).

\section{Statistical Analysis}

All values are represented as mean \pm SEM from two or more independent experiments. Statistical significance was determined using unpaired student's $t$ test or one way ANOVA followed by Bonferroni's multiple comparison tests, provided by the GraphPad Prism 7 (GraphPad, CA) software.

\section{RESULTS}

\section{HGF/c-Met Signaling Was Upregulated During Muscle Regeneration}

To investigate the possible involvement of HGF during muscle regeneration, cardiotoxin (CTX)-induced muscle injury model was used. Injection of CTX provides homogenous damage to the whole muscle and induces the infiltration of various immune cells including monocytes and macrophages into the regenerating muscle until the repair is completed (Arnold et al., 2007). Tibialis anterior (TA) muscle of a 10-week-old C57BL/6 mouse was injected with CTX or PBS (sham), and total proteins were prepared from the TA at appropriate time points followed by ELISA. The basal level of the HGF protein in the sham (PBS-injected) muscle was maintained at $120-160 \mathrm{pg} / \mathrm{mg}$ of total cellular protein in the TA (Figure 1A). After muscle injury, the level of the HGF protein in the injured side was gradually increased, reaching a peak at approximately $1.1 \mathrm{ng} \mathrm{mg}$ of total cellular protein at day 4 , and then steadily decreased before returning to the sham level at day 12. A similar magnitude of HGF RNA induction was observed during muscle regeneration as measured by RT-qPCR (Figure 1B). These data suggested that HGF expression was highly induced after muscle injury at both RNA and protein levels.

c-met is the only known receptor for HGF. When HGF is expressed, the c-met protein becomes phosphorylated to be activated. Therefore, the level and content of the c-met protein was analyzed after muscle injury. Total proteins were prepared from the TA followed by western blot using antibodies to total c-met or phosphorylated form (Figure 1C). During muscle regeneration, the level of total c-met protein rapidly increased and the phosphorylated form of the c-met protein was also upregulated in the damaged muscle.

To identify the cell types that express c-met, cells known to be infiltrated in the injured muscle were analyzed by IHC using antibodies to phosphorylated c-met, CD11b for macrophages, CD31 for endothelial cells, and Ly6G for neutrophils, 3 days after muscle injury. As shown in Figure 1D, the major cell type containing activated c-met was macrophages. In sham-treated muscle, there were no cells expressing phosphorylated c-met (Supplementary Figure S1A). Since macrophages are known to be a primary 

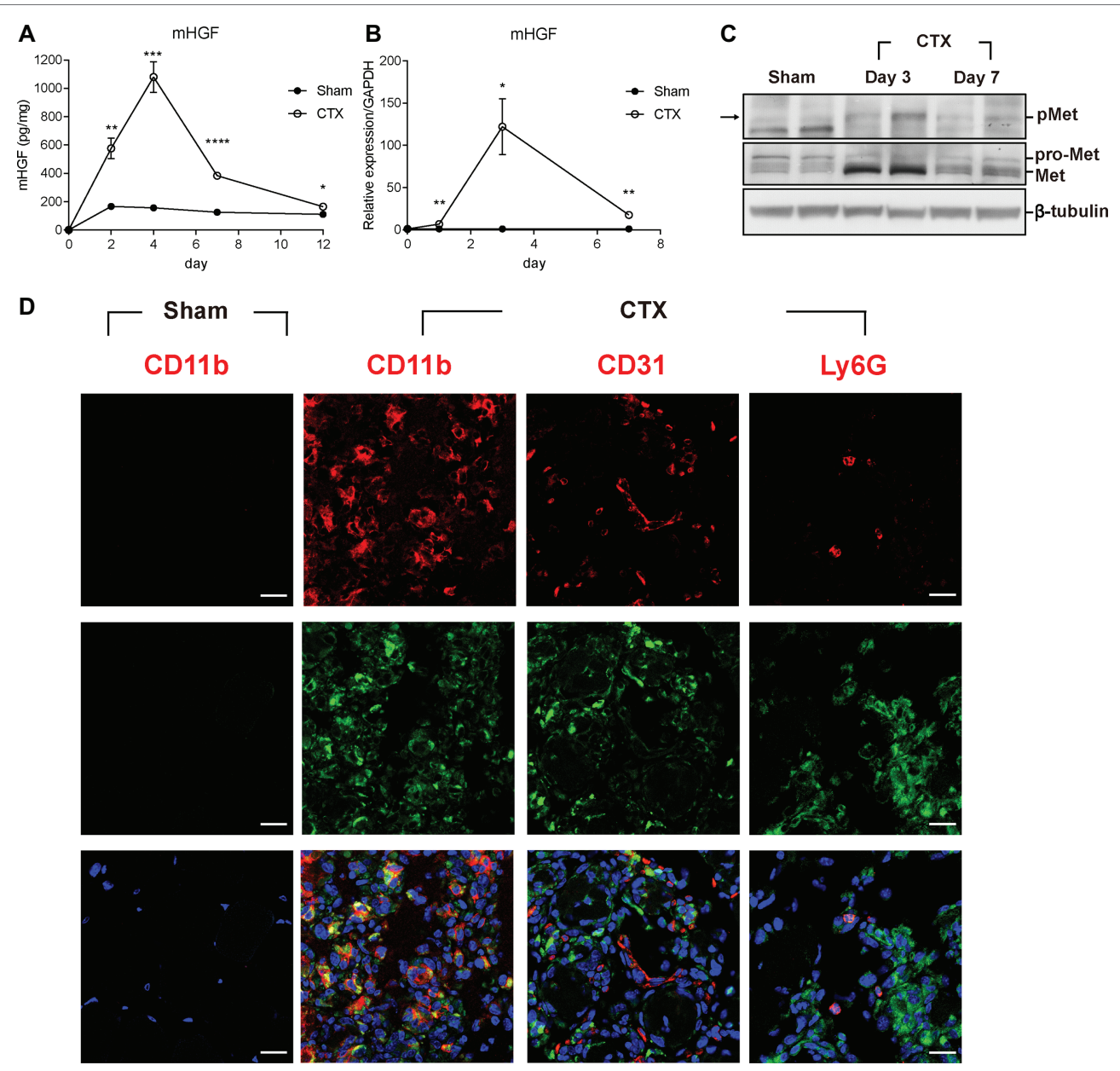

pMet DAPI

FIGURE 1 | HGF/c-met signaling is activated during muscle regeneration. (A) Expression kinetics of HGF protein during muscle injury by CTX and regeneration. The muscle was isolated at $2,4,7$, and 12 days after CTX injection, and total proteins were analyzed by ELISA to measure the protein levels of HGF. ${ }^{*} p<0.05$, ${ }^{* \star} p<0.01,{ }^{* \star *} p<0.001,{ }^{* \star \star *} p<0.0001$ versus sham-treated muscles (unpaired student's $t$ test), $n=4$ per group. (B) Expression kinetics of the RNA levels of HGF during muscle injury and regeneration. RNA was prepared from TAs 1,3 , and 7 days after CTX injection followed by RT-qPCR, ${ }^{\star} p<0.05,{ }^{* \star} p<0.01$ versus shamtreated muscles (unpaired student's $t$ test), $n=4$ per group. The values were normalized to glyceraldehyde-3-phosphate dehydrogenase (GAPDH). (C) Expression kinetics of total and phosphorylated c-met proteins in CTX-injured TAs. Muscles were isolated at 3 and 7 days postinjury, and total proteins were prepared followed by western blot using specific antibodies to total or phosphorylated c-met. $\beta$-tubulin was used as a loading control. Each lane represents a sample from an individual mouse. Two representative mice are shown here. Two independent experiments were performed (with a total of four mice), and similar results were obtained. Arrow indicates the protein of interest in blots. (D) Identification of cell types expressing c-met. CTX-injured TA was isolated 3 days postinjury and subjected to immunofluorescence assay using antibodies to CD11b for macrophages, CD31 for endothelial cells, Ly6G for neutrophils (all red), and phosphorylated c-met (green). Nuclei were counterstained with DAPI (blue). $n=4$ per group. Scale bars, $20 \mu \mathrm{m}$. All data are presented as mean \pm SEM. See also Supplementary Figure S1.

source of HGF after injury (Sisson et al., 2009), these data indicated that in an injured muscle, HGF might act on macrophage in an autocrine manner.

\section{Inhibition of c-Met Signaling Delayed Muscle Regeneration}

It was tested whether increased expression of HGF would contribute to or inhibit muscle regeneration using an inhibitor specific to the c-met receptor, PHA-665752. After CTX injection, mice were intraperitoneally (i.p.) injected with PHA-665752 on a daily basis. We have previously reported that i.p. injection of PHA-665752 could effectively inhibit the phosphorylation of c-met in vivo including in the muscle tissue (Choi et al., 2018; Ko et al., 2018). Treatment with PHA-665752 also suppressed c-met phosphorylation in macrophages infiltrating the injured muscle (Supplementary Figure S2A). Seven days after CTX injection, TA mass from vehicle (DMSO)-treated animals was found to be reduced by $12 \%$ from $38.7 \pm 0.7$ to $33.9 \pm 0.9 \mathrm{mg}$, compared to that of the sham-operated group, while PHA-665752 treated mice showed a larger reduction, by $19 \%$ (Figure 2A). The skeletal muscle cross-section was analyzed by hematoxylin and eosin (H\&E) staining of the TA (Figure 2B). In vehicle-treated mice, muscle fiber size was decreased by $40 \pm 3 \%$ from $1,690 \pm 134$ to $1,014 \pm 55 \mu \mathrm{m}^{2}$ 
A

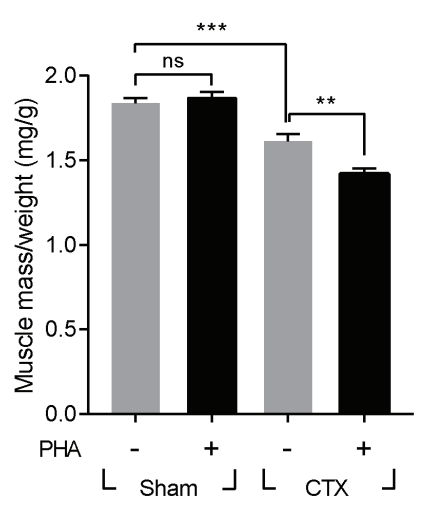

C

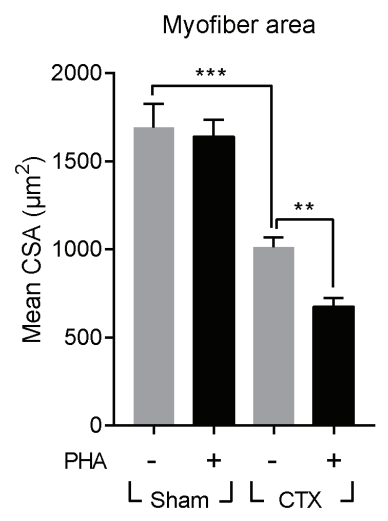

B

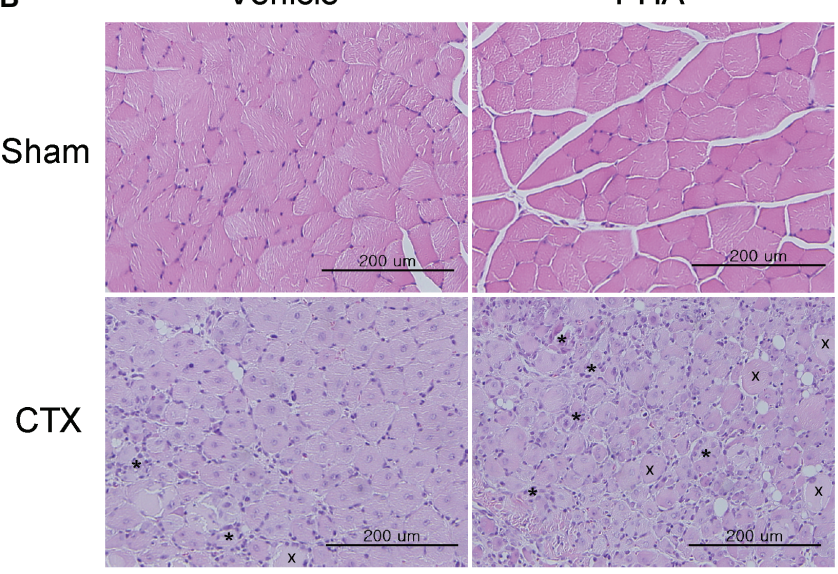

D

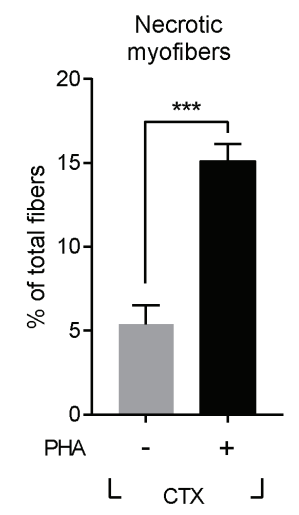

PHA

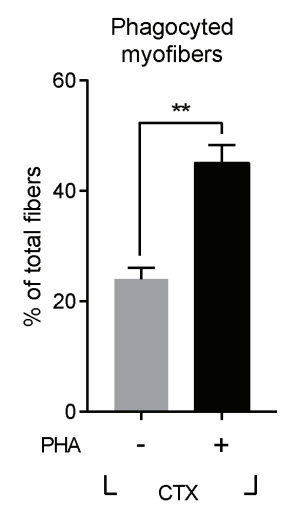

FIGURE 2 | Effects of c-met inhibitor PHA-665752 on muscle regeneration. After CTX injury, mice were i.p. injected with 20 mg/kg of PHA-665752 on a daily basis until sacrificed. CTX injured TAs were analyzed 7 days postinjury. (A) Effect on muscle weight. Muscle mass was normalized with the weight of mice. PHA, PHA-665752. ns, not significant, ${ }^{* *} p<0.01,{ }^{* * *} p<0.001$ (one-way ANOVA), $n=4$ per group. (B) H\&E staining of regenerating muscle. Crosses (x) and asterisks $\left({ }^{*}\right.$ ) indicate necrotic and phagocyted myofibers, respectively. Scale bars, $200 \mu \mathrm{m}$. (C) Effect on cross-sectional areas of muscle fibers. Mean value of area sizes is indicated in the graph. ${ }^{\star \star} p<0.01,{ }^{\star \star \star} p<0.001$ (one-way ANOVA), $n=4$ per group. (D) Quantification of necrotic or phagocyted fibers expressed as a percentage of total myofibers. At least 300 muscle fiber areas were counted per sample. ${ }^{* \star} p<0.01,{ }^{\star \star *} p<0.001$ (unpaired student's $t$ test), $n=4$ per group. All data are presented as mean \pm SEM. See also Supplementary Figure $\mathbf{S 2}$.

compared with that of the sham-operated animals. In PHA-665752 treated mice, it was further reduced, by $60 \pm 3 \%$ compared to the sham-operated group (Figure 2C). In addition, the number of necrotic or damaged phagocytic fibers in PHA-treated animal was significantly higher in comparison to the vehicletreated group (Figure 2D). These data indicated that the inhibition of c-met signaling could delay the restoration of muscle mass and muscle fiber regeneration, suggesting that HGF might play a positive role(s) in muscle repair.

It was reported that defects in the process of removing damaged muscle fibers are associated with the improper transition of macrophage from M1 toward M2 phenotype (Mounier et al., 2013). Therefore, the effect of PHA-665752 on the expression of M1 and M2 macrophage markers were tested. Three days after muscle injury, RNAs were isolated from TAs of mice when the RNA level of M1 and M2 macrophage markers were greatly induced. In animals treated with PHA-665752, the level of IL-1 $\beta$, iNOS, and CCL2 (M1 markers) further increased
(Figure 3A), while that of IL-10, TGF- $\beta$, arginase 1 (Arg1), CD163, and resistin-like alpha (Retnla) (M2 markers) was reduced, in comparison to the vehicle-treated group (Figure 3B).

To identify whether the regulation of M1 and M2 marker genes was caused by the changes in the population of macrophage, injured muscle was analyzed by immunofluorescence assay using antibodies to iNOS for M1 macrophages, and CD206 for M2 macrophages. Three days after muscle injury, the number of iNOS-positive macrophages was highly increased and was further enhanced in animals treated with PHA-665752 (Figure 3C). The number of CD206-positive macrophages was also increased upon injury, but PHA-665752 treatment significantly reduced it (Figure 3D). These results indicated that the HGF/c-met signaling pathway might be involved in the transition of macrophages to an appropriate type during muscle regeneration.

Muscle regeneration is controlled by spatiotemporal regulation of various myogenic regulatory factors (MRFs) during which 

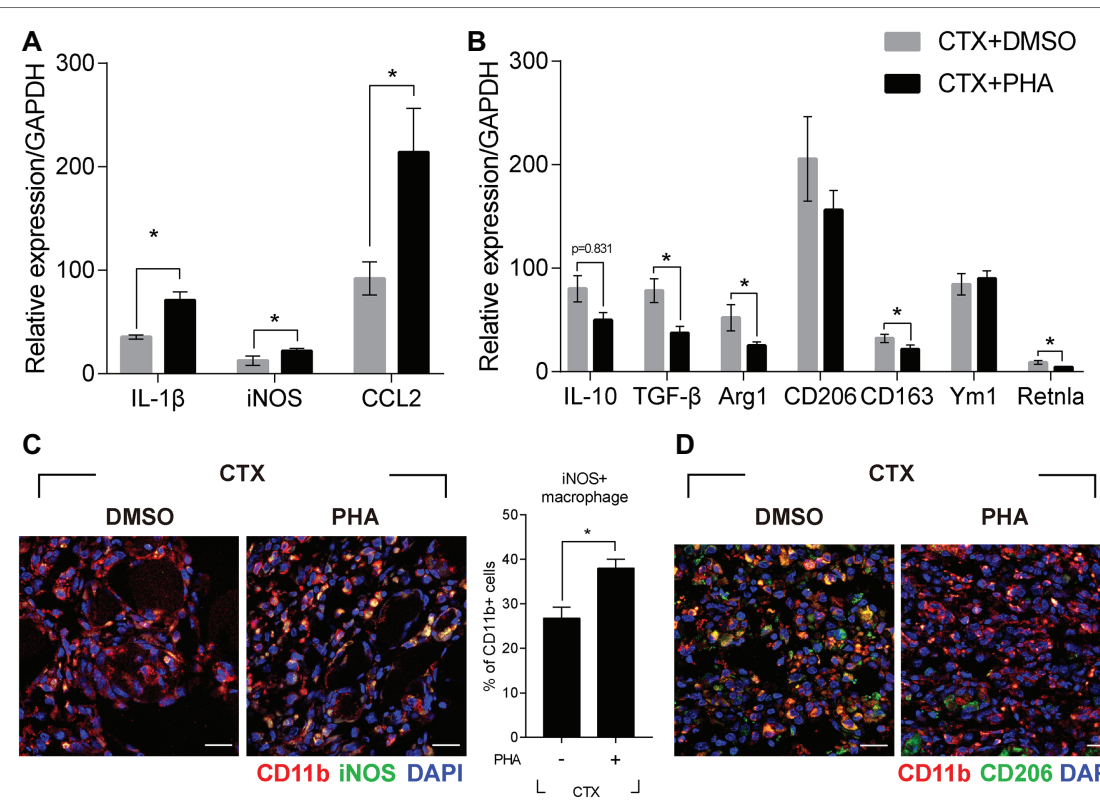

D CTX
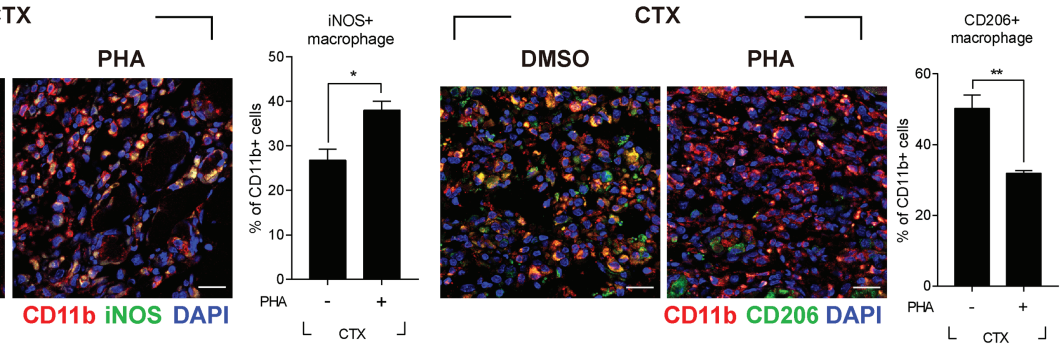

E

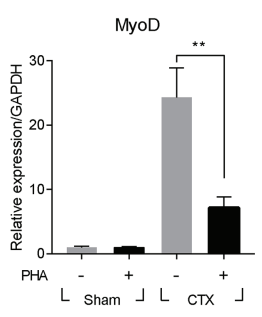

$\mathbf{F}$

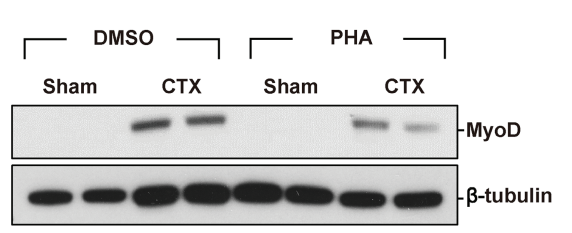

FIGURE 3 | Effects of c-met inhibitor PHA-665752 on macrophage population infiltrated in the muscle. After CTX injury, mice were i.p. injected with 20 mg/kg of PHA-665752 on a daily basis until sacrificed. (A) Effects on the RNA levels of M1 markers (iNOS, IL-1 $\beta$, and CCL2). (B) Effects on the RNA levels of M2 markers (IL-10, TGF- $\beta$, Arg1, CD206, CD163, Ym1, and Retnla). The RNA level of these genes isolated from TAs 3 days after injury was determined by RT-qPCR. The relative expression level of sham-operated, vehicle-treated mice is presented as 1. (C) Effect on iNOS-positive macrophages and (D) CD206-positive macrophages. CTX-injured TA was isolated 3 days postinjury and subjected to immunofluorescence assay using antibodies to CD11b (red) and iNOS or CD206 (green). Nuclei were counterstained with DAPI (blue). Percentage of iNOS+ or CD206+ macrophages was indicated in the graph. ${ }^{\star} p<0.05$, ${ }^{\star *} p<0.01$ (unpaired student's $t$ test), $n=4$ per group. Scale bars, 20 m. (E) Effects on MyoD RNA. * $p<0.05$ (unpaired student's $t$ test), $n=4$ per group. (F) Effects on MyoD protein. Two representative results are shown here, $n=4$ per group. All data are presented as mean \pm SEM.

infiltrated immune cells play key roles (Di Marco et al., 2005; Tidball and Villalta, 2010). Therefore, the expression of myoD, the key player in the regulation of myogenic commitment, was tested. Three days after muscle injury, the RNA level of myoD was markedly increased in CTX-injured TA, but treatment with PHA-665752 inhibited injury-induced myoD expression (Figure 3E). The protein level of myoD showed a similar pattern (Figure 3F). Taken together, our results implied that $\mathrm{HGF} / \mathrm{c}$-met signaling would act to regulate macrophage M1-M2 transition, thereby contributing to regeneration of injured muscle.

\section{HGF Promotes M2 Macrophage Transition via CaMKK $\beta$-AMPK Signaling}

To understand the mechanism(s) underlying the effect of HGF on macrophage transition at the molecular and cellular levels, Raw 264.7 cells, a murine macrophage line, was used. Lipopolysaccharide (LPS) was used to activate these cells to the pro-inflammatory phenotype $(\mathrm{M} 1)$ in order to mimic in vivo muscle injury. Cells were treated with various concentrations of the recombinant human HGF (hHGF) protein in the presence of $100 \mathrm{ng} / \mathrm{ml}$ of LPS for $24 \mathrm{~h}$. When Raw 264.7 cells were treated with LPS only, the expression level of M1 markers (IL-1 $\beta$, iNOS, and TNFa) was greatly induced compared to the untreated control. Co-treatment with HGF inhibited an LPS-mediated increase in the level of M1 maker genes in a dose-dependent manner resulting in an approximate $40 \%$ decrease compared to the LPS only group (Figure 4A). On the other hand, LPS stimulation increased the expression level of IL-10 and Arg1, representative M2 marker genes, and HGF treatment further increased it in a dose-dependent manner (Figure 4B), suggesting that HGF might indeed control macrophage M2 transition.

The transition of macrophages from M1 to M2 during muscle regeneration is known to be controlled by two different pathways; CREB-C/EBP $\beta$ pathway (Ruffell et al., 2009) or AMPK signaling (Mounier et al., 2013). HGF had little or no effect on the former, since treatment with HGF did not affect the phosphorylation of $\mathrm{CREB}$ and the expression of $\mathrm{C} / \mathrm{EBP} \beta$ 

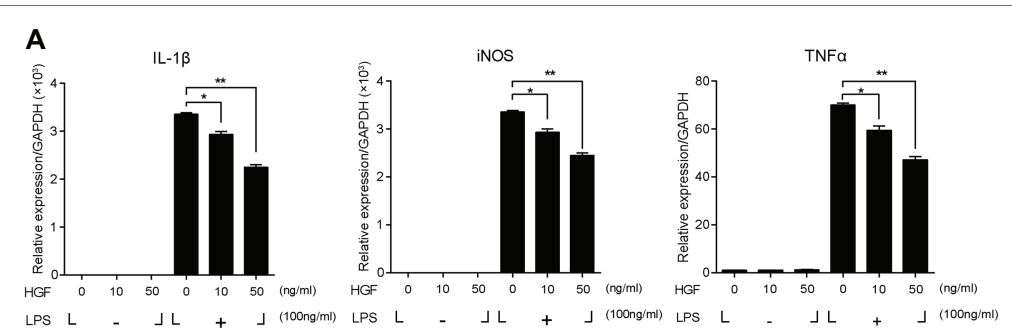

B
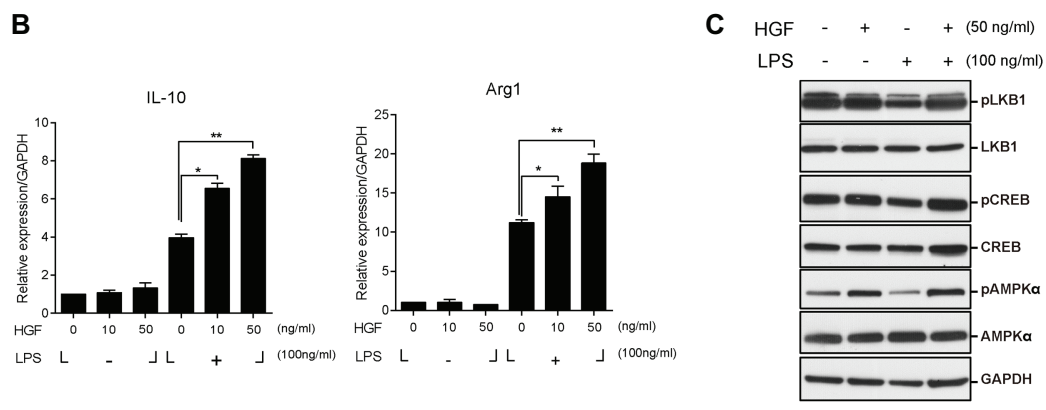

D
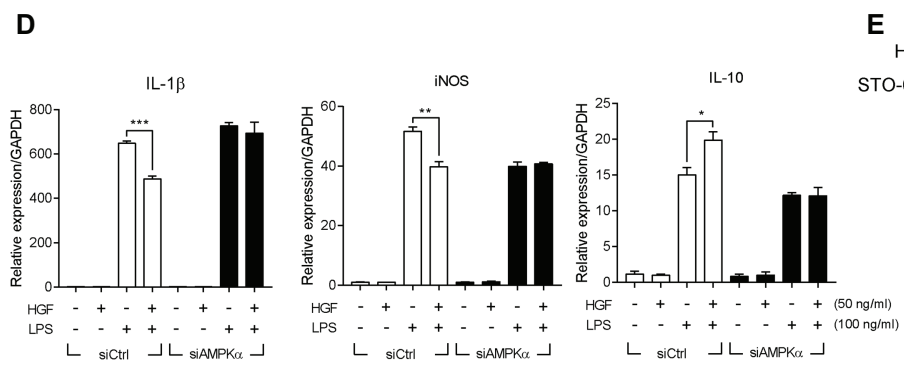

E

HGF - + - + + $+(50 \mathrm{ng} / \mathrm{ml})$

$\begin{array}{lllllll} & -6 & 5 & 20 & 20 & (\mathrm{ng} / \mathrm{ml})\end{array}$

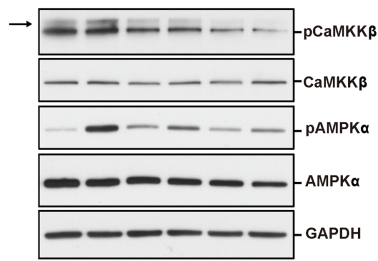

FIGURE 4 | Roles of CaMKKK-AMPK $\alpha$ on HGF-mediated control of the expression of M1 and M2 markers in Raw 264.7 cells. Raw 264.7 cells were cultured in the presence or absence of LPS and recombinant HGF proteins. Total RNA and proteins were prepared and analyzed by RT-qPCR and western blot, respectively. (A) Effects of HGF on the RNA levels of M1 markers (IL-1 $\beta$, iNOS, and TNF $\alpha$ ). ${ }^{*} p<0.05,{ }^{* *} p<0.01$ (unpaired student's $t$ test), $n=3$ per group. (B) Effects of HGF on RNA levels of M2 markers (IL-10 and Arg1). Values were normalized to GAPDH. ${ }^{*} p<0.05,{ }^{* *} p<0.01$ (unpaired student's $t$ test), $n=3$ per group. (C) Effects of HGF on signaling pathways related to macrophage polarization. (D) Effects of AMPK $\alpha$ knockdown on HGF-mediated regulation of the RNA level of IL-1 $\beta$, iNOS, and IL-10, marker genes of M1 and M2. Raw 264.7 cells were transfected with AMPK $\alpha$ or control siRNAs, and then treated with LPS and HGF. Values were normalized to GAPDH. ${ }^{*} p<0.05,{ }^{* *} p<0.01,{ }^{* * *} p<0.001$ (unpaired student's $t$ test), $n=3$ per group. (E) Effects of CaMKK $\beta$ inhibitor, STO-609, on the HGF-mediated phosphorylation of AMPK $\alpha$. Arrow indicates the protein of interest in blots. In western blot hybridization, GAPDH was used as a loading control. All data are presented as mean \pm SEM. See also Supplementary Figure $\mathbf{S} 2$ and $\mathbf{S 3}$.

(Figure 4C, Supplementary Figure S3B). To test whether HGF regulates the macrophage transition through AMPK, Raw 264.7 cells were treated with $50 \mathrm{ng} / \mathrm{ml}$ of the hHGF protein and $100 \mathrm{ng} / \mathrm{ml}$ LPS for $30 \mathrm{~min}$, followed by western blot, using antibodies to total or phosphorylated AMPKa. LPS stimulation decreased the level of phosphorylated AMPKa as previously reported (Sag et al., 2008), but the presence of HGF restored the phosphorylation of AMPKa to the normal level. The level of total AMPKa remained unchanged in all conditions (Figure 4C).

To test if AMPK is involved in the effects of HGF on inflammatory cytokines production, Raw 264.7 cells were transfected with siRNA against AMPKa followed by treatment with LPS and HGF. The protein level of AMPKa was highly reduced by siRNA transfection (Supplementary Figure S3A). Raw 264.7 cells transfected with siAMPKa had no effect on the HGF-mediated control of M1 and M2 marker gene expressions (Figure 4D). These results indicated that HGF controlled the expression of M1 markers (IL-1 $\beta$ and iNOS) and IL-10 by upregulating AMPK phosphorylation.

AMPK phosphorylation is known to be regulated by upstream effectors such as LKB1 and CaMKK $\beta$ (Carling et al., 2008). It was tested as to which upstream effectors would be involved in the phosphorylation of AMPK mediated by HGF. LKB1 did not seem to play a role as HGF did not induce the phosphorylation of LKB1 in Raw 264.7 cells (Figure 4C). However, treatment with HGF induced the phosphorylation of CaMKK $\beta$ and treatment with STO-609, CaMKK $\beta$ inhibitor, lowered the level of HGF-mediated phosphorylation of AMPKa (Figure 4E). Therefore, in Raw 264.7 cells, CaMKK $\beta$, but not LKB1, appeared to act as an upstream effector of HGF-mediated control of AMPK.

Finally, it was investigated whether the phosphorylation of AMPK was regulated by HGF during skeletal muscle regeneration. At day 3 postinjury, TAs were isolated and subjected to western blot and immunofluorescence. The level of phosphorylated 


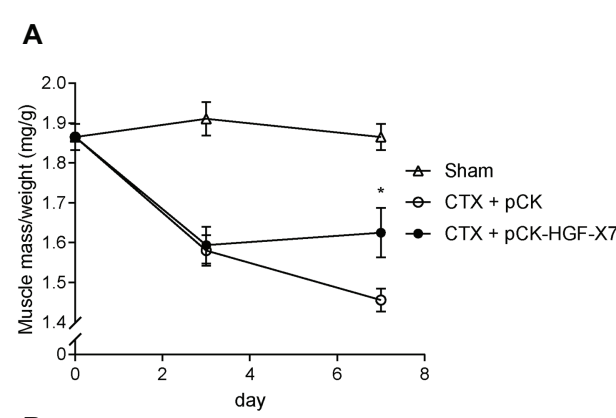

B

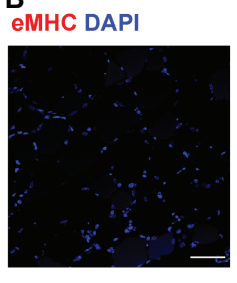

Sham

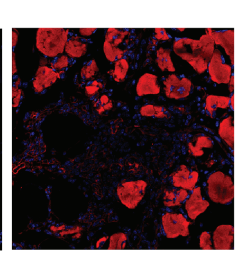

pCK

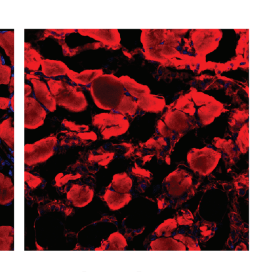

pCK-HGF-X7

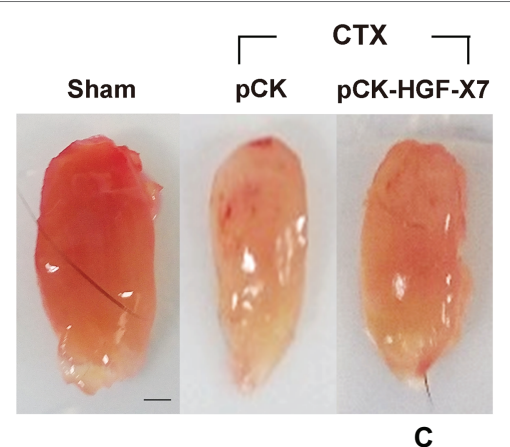

C
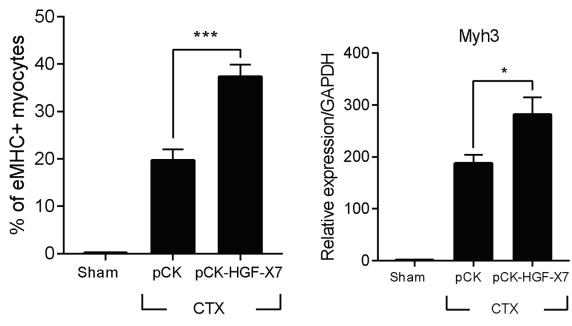

CTX

D

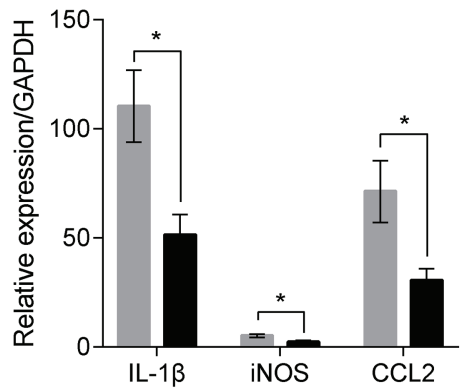

E

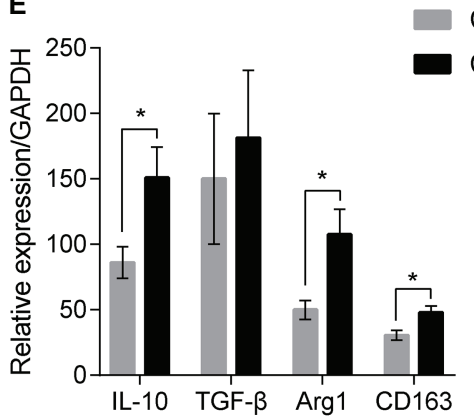

FIGURE 5 | Effects of HGF overexpression by intramuscular injection of HGF expressing plasmid on muscle regeneration. pCK or pCK-HGF-X7 was i.m. injected 3 days prior to the CTX injection. TAs were prepared at appropriate times after injury. (A) Effect on TA weight. Representative TAs from 7 days postinjury are shown in the photos. ${ }^{*} p<0.05$ versus CTX + pCK group (one-way ANOVA), $n=4$ per group. Scale bar, $1 \mathrm{~mm}$. (B) Effects on cross-sectional areas of regenerating fibers. CTX-injured TA was isolated 3 days postinjury and subjected to immunofluorescence assay using antibodies to eMHC (red) for regenerating myofibers. Percentage of eMHC+ myocytes was indicated in the graph. ${ }^{\star \star \star} p<0.001$ (one-way ANOVA), $n=4$ per group. Scale bar, $50 \mu \mathrm{m}$. (C-E) Three days after injury, the TA was isolated, and total RNAs were analyzed by RT-qPCR. (C) Effects on the expression of Myh3. * $p<0.05$ (one-way ANOVA), $n=4$ per group. (D) Effects on the RNA levels of M1 markers (IL-1 $\beta$, iNOS, and CCL2). * $p<0.05$ (one-way ANOVA), $n=4$ per group. (E) Effects on RNA levels of M2 markers (IL-10, TGF- $\beta$, Arg1, and CD163). ${ }^{\star} p<0.05$ (one-way ANOVA), $n=4$ per group. Values were normalized to GAPDH. All data are presented as mean \pm SEM. See also Supplementary Figure S4.

AMPKa was highly increased in CTX-injured TA, and treatment with PHA-665752 had no significant effect (Supplementary Figure S2B). When the phosphorylation of AMPK was measured in situ specifically in macrophages, however, a completely different picture emerged; PHA-665752 treatment significantly reduced the injury-mediated phosphorylation of AMPK in macrophages (Supplementary Figure S2C). Taken together, these data suggested that $\mathrm{HGF} / \mathrm{c}$-met pathway regulate M2 macrophage transition by regulating CAMKK $\beta$ and AMPK.

\section{Exogenous Delivery of HGF Facilitated Muscle Regeneration}

Based on the above results indicating a positive role(s) of HGF in macrophage transition during muscle regeneration, we tested the effects of the exogenous addition of HGF in the same CTX muscle injury model. Since HGF has a very short half-life, less than $5 \mathrm{~min}$ in serum (Kawaida et al., 1994), we delivered HGF in the form of a plasmid DNA expression vector. pCK-HGF-X7 (or VM202) is a plasmid designed to produce two isoforms of human HGF, HGF723 (or dHGF), and HGF728 (or cHGF), at high levels in vivo (Cho et al., 2008; Pyun et al., 2010; Hahn et al., 2011), and it has been known to work in a variety of clinical studies and animal models (Cho et al., 2008; Pyun et al., 2010; Hahn et al., 2011; Kessler et al., 2015; Kibbe et al., 2016). The in vivo protein expression kinetics of this plasmid in the muscle have been well established previously (Supplementary Figure S4A) (Cho et al., 2008; Pyun et al., 2010; Hahn et al., 2011; Nho et al., 2018).

Three days prior to the muscle injury, $100 \mu \mathrm{g}$ of pCK-HGF-X7 or pCK control vector lacking the HGF sequence was i.m. administered into the TA, and muscle injury was introduced 
by injecting CTX to the TA of a 10-week-old C57BL/6 mouse. The TA was isolated, and muscle mass was quantitated at different time points after muscle injury. As shown in Figure 5A, in CTX-injured mice injected with pCK control vector, muscle mass was decreased by 18 and $22 \%$ at days 3 and 7, respectively. When mice were injected with pCK-HGF-X7, the restoration of muscle weight was facilitated, to 17 and $13 \%$ decrease, compared to the control at days 3 and 7 , respectively.

The regenerating muscle fibers were analyzed by immunostaining embryonic myosin heavy chain (eMHC), 3 days after muscle injury. In pCK-treated animals, the percentage of eMHC+ myocytes was about $20 \%$, while it increased to $37 \%$ when treated with pCK-HGF-X7 (Figure 5B). Similar patterns were observed when the RNA level of eMHC (Myh3) was measured by qRT-PCR (Figure 5C). Overall, our data showed that the exogenous addition of HGF, delivered in the form of plasmid expression vector, could facilitate muscle regeneration.

The effects of i.m. injection of pCK-HGF-X7 on M1 and M2 markers were also measured. Muscle injury was induced, and pCK or pCK-HGF-X7 was i.m. injected into the TA. Three days after muscle injury, TA was isolated, and the RNA level of M1 and M2 markers were measured using RT-qPCR. The level of M1 markers, IL1 $\beta$, iNOS, and CCL2 was highly increased after muscle injury, but pCK-HGF-X7 injection reduced the expression of these genes (Figure 5D). The level of M2 markers, IL-10, Arg1, and CD163, also increased upon muscle injury and further increased in animals treated with PCK-HGF-X7 (Figure 5E). These data strongly indicated that HGF overexpression by gene transfer technology could trigger transition of macrophages to the M2 phenotype to promote muscle regeneration.

\section{DISCUSSION}

In this report, we demonstrated that HGF/c-met signaling plays a key role in the regulation of macrophage transition during muscle regeneration after necrotic injury. The $\mathrm{HGF} / \mathrm{c}$-met signaling was highly activated after muscle damage, and the macrophage was the major cell type affected by HGF among cells that infiltrated the muscle. Treating CTX-injured mice with PHA-665752, a specific inhibitor for c-met, deregulated the population of macrophages and delayed muscle regeneration. Exogenous supply of the HGF protein to the affected region through i.m. injection of a highly efficient plasmid expression vector promoted the transition of macrophage to the M2 phenotype and facilitated muscle regeneration. Data from Raw 264.7 cells showed that HGF controls macrophage transition via CaMKK $\beta$-AMPK signaling. Taken together, HGF/c-met signaling plays a key role in the transition of the macrophage infiltrated during muscle regeneration.

HGF is secreted by a variety of cell types in cases of muscle injury. Relevant to our work is that fact that, macrophages are known to be the primary source of HGF during muscle regeneration (Sisson et al., 2009). Consistent with previous reports, we found that the kinetics of the RNA and protein levels of HGF paralleled the curve of macrophagic appearance in regenerating muscle (Mounier et al., 2013). However, the underlying mechanism by which macrophages produce HGF has not yet been understood. One possibility is that HGF may be secreted when these cells are exposed to apoptotic neutrophils. It was previously reported that when macrophages encounter apoptotic cells, HGF is produced via a RhoAdependent signal in vitro (Park et al., 2011). Interestingly, our study showed that the expression level of HGF peaked at 3-4 days postinjury, and this is the time when neutrophils induce an early immune response and became apoptotic and cleared by macrophages (Arnold et al., 2007; Nguyen et al., 2011). It remains to be elucidated whether HGF expression in macrophages would indeed be regulated by their exposure to apoptotic neutrophils in vivo.

We found that HGF regulated phosphorylation of AMPK through the CaMKK $\beta$ upstream regulator. Since HGF/c-met signaling promotes the influx of calcium ions into a variety of cells, it is possible that calcium-dependent kinases are activated (Baffy et al., 1992; Tyndall et al., 2007; Gomes et al., 2008). In our study involving Raw 264.7 cells, another wellknown upstream regulator, LKB1, was not phosphorylated by HGF. This is different from the previous study showing that HGF regulates the phosphorylation of AMPK through LKB1 in primary hepatocytes (Vázquez Chantada et al., 2009). In cases of muscle injury, however, it was previously reported that mice lacking LKB1 in their myeloid cells did not show any significant defects during muscle regeneration (Mounier et al., 2013). Therefore, LKB1 in macrophages may play a negligible role in the HGF/AMPK-mediated regulation of macrophage transition during muscle repair. These data imply that HGF might regulate the phosphorylation of AMPK through a different pathway depending on cell type.

In this study, we focused on the role of HGF/c-met signaling on macrophages during muscle regeneration, as they are the major cell type infiltrated after muscle injury. We found that HGF regulated the transition of the macrophage phenotype to promote muscle repair; while others reported that when c-met is knocked out specifically in muscle stem cells, mice showed delayed stem cell activation, decreased myoblast motility, abnormalities in myocyte fusion, and impaired muscle regeneration (Webster and Fan, 2013). These results suggest that $\mathrm{HGF} / \mathrm{c}-\mathrm{met}$ signaling could act on various cell types, including muscle progenitors and invading immune cells, to coordinate muscle repair during muscle regeneration. Also, HGF may be able to produce multiple positive effects in various cells during muscle regeneration.

In summary, $\mathrm{HGF} / \mathrm{c}-$ met signaling appears to play a role(s) in muscle repair by promoting macrophage transition to the M2 phenotype. Various muscle diseases, including muscular dystrophy or myositis, are accompanied by a high inflammatory burden, leading to tissue lysis and compromising of the regeneration process. Currently, no drug can discriminate between the different phenotypes of the macrophage. Without such specificity, there is a chance of indiscriminate macrophage depletion, which can lead to undesirable systemic side effects 
(Balaban et al., 2005). Given the safety and efficacy records of pCK-HGF-X7 (VM202) observed in several clinical studies for other indications, further studies are warranted to investigate the possibility of using HGF, and in particular the plasmid DNA vector expressing HGF, for various muscle degenerative diseases.

\section{AUTHOR CONTRIBUTIONS}

WC designed the study, performed the experiments, analyzed the data, and wrote the manuscript. JaL, JuL, and SL conducted the experiments. SK designed the study and wrote the manuscript.

\section{REFERENCES}

Ahn, J., Jang, J., Choi, J., Lee, J., Oh, S.-H., Lee, J., et al. (2014). GSK3 $\beta$, but not GSK3 $\alpha$, inhibits the neuronal differentiation of neural progenitor cells as a downstream target of mammalian target of rapamycin complex1. Stem Cells Dev. 23, 1121-1133. doi: 10.1089/scd.2013.0397

Allen, R. E., Sheehan, S. M., Taylor, R. G., Kendall, T. L., and Rice, G. M. (1995). Hepatocyte growth factor activates quiescent skeletal muscle satellite cells in vitro. J. Cell. Physiol. 165, 307-312. doi: 10.1002/jcp.1041650211

Arnold, L., Henry, A., Poron, F., Baba-Amer, Y., Van Rooijen, N., Plonquet, A., et al. (2007). Inflammatory monocytes recruited after skeletal muscle injury switch into antiinflammatory macrophages to support myogenesis. J. Exp. Med. 204, 1057-1069. doi: 10.1084/jem.20070075

Baffy, G., Yang, L., Michalopoulos, G. K., and Williamson, J. R. (1992). Hepatocyte growth factor induces calcium mobilization and inositol phosphate production in rat hepatocytes. J. Cell. Physiol. 153, 332-339. doi: 10.1002/jcp.1041530213

Balaban, B., Matthews, D. J., Clayton, G. H., and Carry, T. (2005). Corticosteroid treatment and functional improvement in Duchenne muscular dystrophy: long-term effect. Am. J. Phys. Med. Rehabil. 84, 843-850. doi: 10.1097/01. phm.0000184156.98671.d0

Carling, D., Sanders, M., and Woods, A. (2008). The regulation of AMPactivated protein kinase by upstream kinases. Int. J. Obes. 32, S55-S59. doi: 10.1038/ijo.2008.124

Cho, K. R., Choi, J.-S., Hahn, W., Kim, D. S., Park, J. S., Lee, D. S., et al. (2008). Therapeutic angiogenesis using naked DNA expressing two isoforms of the hepatocyte growth factor in a porcine acute myocardial infarction model. Eur. J. Cardiothorac. Surg. 34, 857-863. doi: 10.1016/j. ejcts.2008.05.045

Choi, W., Lee, J., Lee, J., Ko, K. R., and Kim, S. (2018). Hepatocyte growth factor regulates miR-206-HDAC4 cascade to control the neurogenic muscle atrophy following surgical denervation in mice. Mol. Ther. Nucleic Acids 12, 568-577. doi: 10.1016/j.omtn.2018.06.013

Di Marco, S., Mazroui, R., Dallaire, P., Chittur, S., Tenenbaum, S. A., Radzioch, D., et al. (2005). NF- $\kappa B-$ mediated MyoD decay during muscle wasting requires nitric oxide synthase mRNA stabilization, HuR protein, and nitric oxide release. Mol. Cell. Biol. 25, 6533-6545. doi: 10.1128/MCB.25.15.6533-6545.2005

Dietrich, S., Abou-Rebyeh, F., Brohmann, H., Bladt, F., Sonnenberg-Riethmacher, E., Yamaai, T., et al. (1999). The role of SF/HGF and c-met in the development of skeletal muscle. Development 126, 1621-1629.

Gomes, D. A., Rodrigues, M. A., Leite, M. F., Gomez, M. V., Varnai, P., Balla, T., et al. (2008). c-Met must translocate to the nucleus to initiate calcium signals. J. Biol. Chem. 283, 4344-4351. doi: 10.1074/jbc.M706550200

Hahn, W., Pyun, W. B., Kim, D. S., Yoo, W. S., Lee, S. D., Won, J. H., et al. (2011). Enhanced cardioprotective effects by coexpression of two isoforms of hepatocyte growth factor from naked plasmid DNA in a rat ischemic heart disease model. J. Gene Med. 13, 549-555. doi: 10.1002/jgm.1603

Huh, C.-G., Factor, V. M., Sánchez, A., Uchida, K., Conner, E. A., and Thorgeirsson, S. S. (2004). Hepatocyte growth factor/c-met signaling pathway is required for efficient liver regeneration and repair. Proc. Natl. Acad. Sci. 101, 4477-4482. doi: 10.1073/pnas.0306068101

\section{FUNDING}

This work was supported by research grants from the basic Science Research Programs through the National Research Foundations of Korea (NRF) (2012R1A1A2008018y) funded by the Ministry of Education, and ViroMed Co., Ltd. (SK).

\section{SUPPLEMENTARY MATERIAL}

The Supplementary Material for this article can be found online at: https://www.frontiersin.org/articles/10.3389/fphys.2019.00914/ full\#supplementary-material

Jennische, E., Ekberg, S., and Matejka, G. L. (1993). Expression of hepatocyte growth factor in growing and regenerating rat skeletal muscle. Am. J. Phys. Cell Phys. 265, C122-C128. doi: 10.1152/ajpcell.1993.265.1.C122

Kawaida, K., Matsumoto, K., Shimazu, H., and Nakamura, T. (1994). Hepatocyte growth factor prevents acute renal failure and accelerates renal regeneration in mice. Proc. Natl. Acad. Sci. 91, 4357-4361. doi: 10.1073/pnas.91.10.4357

Kessler, J. A., Smith, A. G., Cha, B. S., Choi, S. H., Wymer, J., Shaibani, A., et al. (2015). Double blind, placebo controlled study of HGF gene therapy in diabetic neuropathy. Ann. Clin. Transl. Neurol. 2, 465-478. doi: 10.1002/ acn3.186

Kibbe, M., Hirsch, A., Mendelsohn, F., Davies, M., Pham, H., Saucedo, J., et al. (2016). Safety and efficacy of plasmid DNA expressing two isoforms of hepatocyte growth factor in patients with critical limb ischemia. Gene Ther. 23, 306-312. doi: 10.1038/gt.2015.110

Ko, K. R., Lee, J., Lee, D., Nho, B., and Kim, S. (2018). Hepatocyte growth factor (HGF) promotes peripheral nerve regeneration by activating repair Schwann cells. Sci. Rep. 8:8316. doi: 10.1038/s41598-018-26704-x

Miller, K. J., Thaloor, D., Matteson, S., and Pavlath, G. K. (2000). Hepatocyte growth factor affects satellite cell activation and differentiation in regenerating skeletal muscle. Am. J. Phys. Cell Phys. 278, C174-C181. doi: 10.1152/ ajpcell.2000.278.1.C174

Mounier, R., Théret, M., Arnold, L., Cuvellier, S., Bultot, L., Göransson, O., et al. (2013). AMPKal regulates macrophage skewing at the time of resolution of inflammation during skeletal muscle regeneration. Cell Metab. 18, 251-264. doi: 10.1016/j.cmet.2013.06.017

Nakamura, T., and Mizuno, S. (2010). The discovery of hepatocyte growth factor (HGF) and its significance for cell biology, life sciences and clinical medicine. Proc. Jpn. Acad. Ser. B 86, 588-610. doi: 10.2183/pjab.86.588

Nguyen, M.-H., Cheng, M., and Koh, T. J. (2011). Impaired muscle regeneration in ob/ob and $\mathrm{db} / \mathrm{db}$ mice. Sci. World J. 11, 1525-1535. doi: 10.1100/ tsw.2011.137

Nho, B., Lee, J., Lee, J., Ko, K. R., Lee, S. J., and Kim, S. (2018). Effective control of neuropathic pain by transient expression of hepatocyte growth factor in a mouse chronic constriction injury model. FASEB J. 32, 5119-5131. doi: 10.1096/fj.201800476R

Park, H. J., Choi, Y. H., Cho, Y. J., Henson, P. M., and Kang, J. L. (2011). RhoA-mediated signaling up-regulates hepatocyte growth factor gene and protein expression in response to apoptotic cells. J. Leukoc. Biol. 89, 399-411. doi: $10.1189 /$ jlb.0710414

Pyun, W., Hahn, W., Kim, D., Yoo, W., Lee, S., Won, J., et al. (2010). Naked DNA expressing two isoforms of hepatocyte growth factor induces collateral artery augmentation in a rabbit model of limb ischemia. Gene Ther. 17, 1442-1452. doi: 10.1038/gt.2010.101

Rodgers, J. T., King, K. Y., Brett, J. O., Cromie, M. J., Charville, G. W., Maguire, K. K., et al. (2014). mTORC1 controls the adaptive transition of quiescent stem cells from G 0 to G alert. Nature 510, 393-396. doi: 10.1038/nature13255

Ruffell, D., Mourkioti, F., Gambardella, A., Kirstetter, P., Lopez, R. G., Rosenthal, N., et al. (2009). A CREB-C/EBP $\beta$ cascade induces M2 macrophage-specific gene expression and promotes muscle injury repair. Proc. Natl. Acad. Sci. 106, 17475-17480. doi: 10.1073/pnas.0908641106 
Sag, D., Carling, D., Stout, R. D., and Suttles, J. (2008). Adenosine $5^{\prime}$-monophosphate-activated protein kinase promotes macrophage polarization to an anti-inflammatory functional phenotype. J. Immunol. 181, 8633-8641. doi: 10.4049 /jimmunol.181.12.8633

Sheehan, S. M., Tatsumi, R., Temm-Grove, C. J., and Allen, R. E. (2000). HGF is an autocrine growth factor for skeletal muscle satellite cells in vitro. Muscle Nerve 23, 239-245. doi: 10.1002/(SICI)1097-4598(200002)23:2<239:: AID-MUS15>3.0.CO;2-U

Sisson, T. H., Nguyen, M.-H., Yu, B., Novak, M. L., Simon, R. H., and Koh, T. J. (2009). Urokinase-type plasminogen activator increases hepatocyte growth factor activity required for skeletal muscle regeneration. Blood 114, 5052-5061. doi: 10.1182/blood-2008-12-196212

Tatsumi, R., Anderson, J. E., Nevoret, C. J., Halevy, O., and Allen, R. E. (1998). HGF/SF is present in normal adult skeletal muscle and is capable of activating satellite cells. Dev. Biol. 194, 114-128. doi: 10.1006/ dbio. 1997.8803

Tidball, J. G. (2017). Regulation of muscle growth and regeneration by the immune system. Nat. Rev. Immunol. 17, 165-178. doi: 10.1038/nri. 2016.150

Tidball, J. G., and Villalta, S. A. (2010). Regulatory interactions between muscle and the immune system during muscle regeneration. Am. J. Phys. Regul. Integr. Comp. Phys. 298, R1173-R1187. doi: 10.1152/ajpregu.00735.2009

Tyndall, S. J., Patel, S. J., and Walikonis, R. S. (2007). Hepatocyte growth factor-induced enhancement of dendritic branching is blocked by inhibitors of N-methyl-D-aspartate receptors and calcium/calmodulin-dependent kinases. J. Neurosci. Res. 85, 2343-2351. doi: 10.1002/jnr.21390

Vázquez Chantada, M., Ariz, U., Varela Rey, M., Embade, N., Martínez Lopez, N., Fernández Ramos, D., et al. (2009). Evidence for LKB1/AMP activated protein kinase/endothelial nitric oxide synthase cascade regulated by hepatocyte growth factor, S adenosylmethionine, and nitric oxide in hepatocyte proliferation. Hepatology 49, 608-617. doi: 10.1002/hep.22660
Wang, H., Melton, D. W., Porter, L., Sarwar, Z. U., McManus, L. M., and Shireman, P. K. (2014). Altered macrophage phenotype transition impairs skeletal muscle regeneration. Am. J. Pathol. 184, 1167-1184. doi: 10.1016/j. ajpath.2013.12.020

Watanabe, M., Ebina, M., Orson, F. M., Nakamura, A., Kubota, K., Koinuma, D., et al. (2005). Hepatocyte growth factor gene transfer to alveolar septa for effective suppression of lung fibrosis. Mol. Ther. 12, 58-67. doi: 10.1016/j. ymthe.2005.02.019

Webster, M. T., and Fan, C.-M. (2013). c-MET regulates myoblast motility and myocyte fusion during adult skeletal muscle regeneration. PLoS One 8:e81757. doi: 10.1371/journal.pone.0081757

Wu, M., Yokozeki, H., Takagawa, S., Yamamoto, T., Satoh, T., Kaneda, Y., et al. (2004). Hepatocyte growth factor both prevents and ameliorates the symptoms of dermal sclerosis in a mouse model of scleroderma. Gene Ther. 11, 170-180. doi: 10.1038/sj.gt.3302165

Wynn, T. A., and Vannella, K. M. (2016). Macrophages in tissue repair, regeneration, and fibrosis. Immunity 44, 450-462. doi: 10.1016/j.immuni.2016.02.015

Conflict of Interest Statement: JuL and SK are employees or shareholders of ViroMed Co., Ltd., whose plasmid DNA (pCK-HGF-X7) was used in this work.

The remaining authors declare that the research was conducted in the absence of any commercial or financial relationships that could be construed as a potential conflict of interest.

Copyright (c) 2019 Choi, Lee, Lee, Lee and Kim. This is an open-access article distributed under the terms of the Creative Commons Attribution License (CC BY). The use, distribution or reproduction in other forums is permitted, provided the original author(s) and the copyright owner(s) are credited and that the original publication in this journal is cited, in accordance with accepted academic practice. No use, distribution or reproduction is permitted which does not comply with these terms. 
OPEN ACCESS

Edited by:

Dario Coletti,

Sapienza University of Rome, Italy

Reviewed by:

John Joseph McCarthy, University of Kentucky, United States

Bianca Maria Scicchitano,

Catholic University of the Sacred

Heart, Italy

*Correspondence:

Chiara Mozzetta

chiara.mozzetta@uniroma1.it

Luca Madaro

I.madaro@hsantalucia.it

Specialty section:

This article was submitted to

Striated Muscle Physiology,

a section of the journal

Frontiers in Physiology

Received: 01 April 2019

Accepted: 05 August 2019

Published: 21 August 2019

Citation:

Biferali B, Proietti D, Mozzetta C and Madaro L (2019)

Fibro-Adipogenic Progenitors Cross-Talk in Skeletal Muscle:

The Social Network.

Front. Physiol. 10:1074.

doi: 10.3389/fphys.2019.01074

\section{Fibro-Adipogenic Progenitors Cross-Talk in Skeletal Muscle: The Social Network}

\author{
Beatrice Biferali1,2, Daisy Proietti3,4, Chiara Mozzetta ${ }^{2 *}$ and Luca Madaro ${ }^{3 *}$ \\ ${ }^{1}$ Department of Biology and Biotechnology "C. Darwin," Sapienza University of Rome, Rome, Italy, ${ }^{2}$ Institute of Molecular \\ Biology and Pathology (IBPM), CNR National Research Council of Italy, c/o Department of Biology and Biotechnology "C. \\ Darwin," Sapienza University of Rome, Rome, Italy, ${ }^{3}$ IRCCS Santa Lucia Foundation, Rome, Italy, ${ }^{4}$ DAHFMO-Unit of \\ Histology and Medical Embryology, Sapienza University of Rome, Rome, Italy
}

Skeletal muscle is composed of a large and heterogeneous assortment of cell populations that interact with each other to maintain muscle homeostasis and orchestrate regeneration. Although satellite cells (SCs) - which are muscle stem cells - are the protagonists of functional muscle repair following damage, several other cells such as inflammatory, vascular, and mesenchymal cells coordinate muscle regeneration in a finely tuned process. Fibro-adipogenic progenitors (FAPs) are a muscle interstitial mesenchymal cell population, which supports SCs differentiation during tissue regeneration. During the first days following muscle injury FAPs undergo massive expansion, which is followed by their macrophage-mediated clearance and the reestablishment of their steady-state pool. It is during this critical time window that FAPs, together with the other cellular components of the muscle stem cell niche, establish a dynamic network of interactions that culminate in muscle repair. A number of different molecules have been recently identified as important mediators of this cross-talk, and its alteration has been associated with different muscle pathologies. In this review, we will focus on the soluble factors that regulate FAPs activity, highlighting their roles in orchestrating the inter-cellular interactions between FAPs and the other cell populations that participate in muscle regeneration.

Keywords: FAPs, fibrosis, cytokine - immunological terms, muscle regeneration, stem cell

\section{INTRODUCTION}

Skeletal muscle is the most abundant tissue in healthy humans, accounting for $40 \%$ of body weight. It is composed of multinucleated contractile cells called myofibers, which are formed during development by fusion of differentiated mononuclear muscle cells, and their number remains constant during post-natal growth. The regenerative potential of skeletal muscle relies primarily on satellite cells (SCs), the prototypical muscle stem cells. Upon muscle injury SCs enter the cell cycle, proliferate, and differentiate to repair damaged myofibers, while self-renewing to repopulate the reserve pool (Feige et al., 2018).

Recently, several studies have indicated that the establishment of functional cross-talk between SCs and other cell types within the muscle niche, including motor neurons, endothelial cells, immune cells, fibrogenic cells, and adipogenic precursors, is crucial for muscle repair and homeostasis (Tatsumi et al., 2009; Joe et al., 2010; Uezumi et al., 2010; Heredia et al., 2013; 
Saccone et al., 2014; Kuswanto et al., 2016; Verma et al., 2018; Giordani et al., 2019; Lukjanenko et al., 2019). Indeed, different stem/progenitor cell types are recruited to the regenerative niche and influence muscle regeneration either by directly differentiating into muscle cells or by releasing paracrine factors (i.e., growth factors, cytokines) that control the regenerative response of SCs (Pannérec et al., 2012). Among the noncellular components of the SCs niche the extra-cellular matrix (ECM) plays a crucial role by undergoing a transient remodeling during acute injury, followed by a prompt termination to avoid pathological fibrosis and tissue degeneration. Although recent findings have shown that myogenic cells can produce ECM components (Fry et al., 2017b; González et al., 2017; Baghdadi et al., 2018), and a recently identified population of interstitial tenocytes has been implicated in ECM deposition in vivo (Giordani et al., 2019), the main cellular sources of ECM proteins are fibroblasts, myo-fibroblast, and fibro-adipogenic progenitors (FAPs) (Serrano and Muñoz-Cánoves, 2010; Lemos et al., 2015; Contreras et al., 2016; Mueller et al., 2016).

Since their discovery FAPs have attracted a considerable attention (Joe et al., 2010; Uezumi et al., 2010), in particular, their phenotypical plasticity, which appears critical for efficient muscle repair. FAPs have been defined as multi-potent progenitors, having the ability to differentiate into fibroblasts, adipocytes, and possibly into osteoblasts and chondrocytes, although not into myoblasts (Joe et al., 2010; Uezumi et al., 2010). They share the expression of cell surface markers, such as Sca-1 and PDGFR $\alpha$ with mesenchymal stem cells and can therefore be broadly defined as mesenchymal precursors (Joe et al., 2010; Mueller et al., 2016; Judson et al., 2017; Malecova et al., 2018; Giordani et al., 2019).

Under quiescent conditions FAPs frequently localize close to blood vessels but unlike pericytes FAPs reside outside the capillary basement membrane and do not express NG2 (Joe et al., 2010).

However, upon muscle injury, FAPs become activated, proliferate and expand, and provide a transient favorable environment to promote SCs-mediated regeneration (Joe et al., 2010; Heredia et al., 2013; Mozzetta et al., 2013). FAPs expansion is critical during regeneration in order to sustain SCs differentiation in a paracrine manner and to maintain the SCs pool (Wosczyna et al., 2019). Indeed, in vivo depletion of FAPs clearly established their absolute requirement for regeneration and long-term maintenance of skeletal muscle (Wosczyna et al., 2019). However, as regeneration proceeds, FAPs are cleared from the regenerative niche by apoptosis (Lemos et al., 2015) and failure in doing so has been associated with their pathological accumulation and with a number of muscle dysfunctions. In fact, beyond their supportive role in muscle regeneration, FAPs have been identified as the major source of infiltrating fibroblasts and adipocytes in degenerating dystrophic muscles (Uezumi et al., 2010, 2011; Mozzetta et al., 2013; Kopinke et al., 2017). Similarly, in chronic atrophic conditions, caused by moto-neurons deficits, increased fibrosis is associated with accumulation of FAPs in the interstitium of denervated muscles (Contreras et al., 2016; Fry et al., 2017a; Madaro et al., 2018; Rebolledo et al., 2019). Likewise, intra-muscular fatty infiltration and obesity-associated muscle dysfunctions have been also linked to FAPs accumulation and fibro-adipogenic differentiation (Dammone et al., 2018; Gorski et al., 2018; Kang et al., 2018; Pagano et al., 2018; Buras et al., 2019).

These findings emphasize that the FAPs lineage decisions are dramatically influenced by signals released in their microenvironment, whose pathological alteration might culminate in excessive ECM accumulation (Lemos et al., 2015; Contreras et al., 2016; Dammone et al., 2018; Madaro et al., 2018), acquisition of altered cell fates, as in the case of heterotypic ossification (Lees-Shepard et al., 2018), and impaired myogenesis. In physiological conditions, FAPs' crosstalk with other cell populations is emerging as an important and finely orchestrated process crucial for a successful muscle regeneration. While it is now well established that a cross-talk between SCs and fibrogenic cells is necessary for efficient SCs expansion in response to injury, and to prevent interstitial fibrosis accumulation (Murphy et al., 2011; Fry et al., 2017b; Lukjanenko et al., 2019), increasing evidence indicates that FAPs also actively interact with immune cells in a finely tuned manner (Heredia et al., 2013; Lemos et al., 2015; Malecova et al., 2018; Moratal et al., 2018).

Taken together, these observations demonstrate that FAPs orchestrate a plethora of processes involved in regenerative myogenesis, which have been recently reviewed elsewhere (Wosczyna and Rando, 2018). In this mini-review, we will instead specifically focus on the secreted signals, cytokines, and paracrine factors controlling FAPs function and those released by FAPs monitoring the different cell types involved in muscle repair. We will first describe the signals secreted by the various cell populations present in the regenerative niche known to directly influence FAPs activity and then discuss the signals released by FAPs themselves, highlighting their cellular targets and functions (Table 1).

\section{THE SECRETOME THAT REGULATES FAPS ACTIVITIES}

\section{IL-4 and IL-13 Family}

Interleukin-4 (IL-4) and IL-13 are Th2 cytokines, which have been implicated as mediators in the cross-talk between the immune system and FAPs (Heredia et al., 2013). The innate immune system is activated rapidly upon muscle injury and triggers the recruitment of Th2 lymphocytes, macrophages, mast cells, and eosinophils to the injured sites (Tidball and Villalta, 2010; Heredia et al., 2013).

Interleukin-4/IL-13 signaling is crucial for skeletal muscle repair, as demonstrated by studies showing a complete absence of regenerated myofibers, persistence of cellular debris, and an inflammatory infiltrate, in the muscles of $I L-4 / I L-13^{-/-}$ mice following cardiotoxin-induced injury (Heredia et al., 2013). Although activation of type 2 immune responses has been classically associated with alternatively activated (M2) macrophages (Allen and Wynn, 2011; Palm et al., 2012), eosinophils have been recently identified as the dominant cell source of IL-4 and IL-13 (Heredia et al., 2013) during 
TABLE 1 | Schematic table illustrating the principal molecules, the producing and target cells, and the corresponding biological effects, that have been shown to act on, or be released by, FAPs.

FAPs social network

\begin{tabular}{|c|c|c|c|c|c|}
\hline \multicolumn{6}{|c|}{ FAPs social network } \\
\hline Molecule & $\begin{array}{l}\text { Production } \\
\text { cells }\end{array}$ & Target & Effects & References & $\begin{array}{l}\text { Experimental } \\
\text { conditions }\end{array}$ \\
\hline TGF- $\beta$ & Macrophages & FAPs & $\begin{array}{l}\text { TGF- } \beta \text { induces FAPs fibrogenic differentiation and blocks } \\
\text { TNF-induced FAPs apoptosis. }\end{array}$ & $\begin{array}{l}\text { Uezumi et al., 2011; Lemos } \\
\text { et al., 2015; Davies et al., } \\
2016\end{array}$ & In vitro and in vivo \\
\hline IL-15 & $\begin{array}{l}\text { Muscle } \\
\text { fibers }\end{array}$ & FAPs & $\begin{array}{l}\text { IL-15 stimulates FAPs proliferation and prevents adipogenic } \\
\text { differentiation in vitro and in vivo and promotes } \\
\text { FAPs-induced SC differentiation. }\end{array}$ & Kang et al., 2018 & In vitro and in vivo \\
\hline IL-4 & Eosinophils & FAPs & $\begin{array}{l}\text { IL-4 inhibits adipogenic differentiation of FAPs and } \\
\text { increases FAPs ability to remove cellular debris enhancing } \\
\text { regeneration. }\end{array}$ & $\begin{array}{l}\text { Heredia et al., 2013; Dong } \\
\text { et al., } 2014\end{array}$ & In vitro and in vivo \\
\hline IL-13 & & FAPs & $\begin{array}{l}\text { IL-13 promotes FAPs proliferation that supports } \\
\text { myogenesis, while inhibits FAPs differentiation into } \\
\text { adipocytes. }\end{array}$ & Heredia et al., 2013 & In vitro and in vivo \\
\hline TNF- $\alpha$ & Macrophages & FAPs & $\begin{array}{l}\text { TNF- } \alpha \text { induces FAPs apoptosis preventing excessive } \\
\text { deposition of extracellular matrix during regeneration. }\end{array}$ & Lemos et al., 2015 & In vitro and in vivo \\
\hline \multirow[t]{2}{*}{ IL-6 } & FAPs & Myotubes & IL-6 promotes myogenic differentiation. & Joe et al., 2010 & In vitro \\
\hline & & FAPs & $\begin{array}{l}\text { IL-6 promotes pro-atrophic FAPs phenotype during } \\
\text { denervation. }\end{array}$ & Madaro et al., 2018 & In vitro and in vivo \\
\hline IL-33 & FAPs & $\begin{array}{l}\text { Regulatory } \\
\text { T cells }\end{array}$ & $\begin{array}{l}\text { IL-33 increases Treg cells proliferation promoting muscle } \\
\text { repair. }\end{array}$ & Kuswanto et al., 2016 & In vitro and in vivo \\
\hline Follistatin & FAPs & $\begin{array}{l}\text { Satellite } \\
\text { cells }\end{array}$ & $\begin{array}{l}\text { FAPs-secreted follistatin promotes multinucleated } \\
\text { myotubes formation. }\end{array}$ & Mozzetta et al., 2013 & In vitro \\
\hline $\mathrm{IL}-10$ & FAPs & Myotubes & $\begin{array}{l}\mathrm{IL}-10 \text { is upregulated in FAPs during muscle regeneration. Its } \\
\text { role is still unknown but the hypothesis is that the secretion } \\
\text { of IL-10 facilitates myoblast differentiation by preventing the } \\
\text { antimyogenic activity of TNF and IL-1 } 1 \beta \text {. }\end{array}$ & Lemos et al., 2012 & $\mathrm{n} / \mathrm{a}$ \\
\hline $\begin{array}{l}\text { BMP1- } \\
\text { MMP14 }\end{array}$ & FAPs & Macrophages & $\begin{array}{l}\text { FAPs-secreted BMP1 and MMP14 activate TGF- } \beta \\
\text { produced by macrophages in fibrotic DMD muscle. }\end{array}$ & Juban et al., 2018 & In vitro \\
\hline WISP1 & FAPs & $\begin{array}{l}\text { Satellite } \\
\text { cells }\end{array}$ & $\begin{array}{l}\text { FAPS-secreted WISP1 regulates satellite cell expansion and } \\
\text { asymmetric differentiation. FAPs-derived WISP1 is lost } \\
\text { during aging impairing muscle regeneration. }\end{array}$ & Lukjanenko et al., 2019 & In vitro and in vivo \\
\hline
\end{tabular}

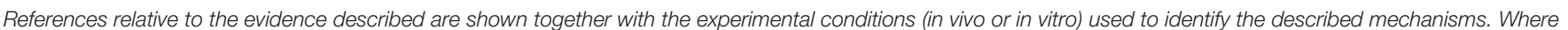
experimental evidence is lacking we indicated the experimental procedure with n/a.

skeletal muscle regeneration. Specifically, it has been shown that eosinophils secrete IL-4 to activate the regenerative actions of FAPs. Indeed, Heredia et al. (2013) identified FAPs as the cells specifically expressing the IL- $4 \mathrm{R} \alpha$, demonstrating, both in vitro and in vivo, that FAPs are the cellular targets of IL-4/IL-13 signaling during muscle regeneration. Intriguingly, they also unveiled a previously unrecognized function of FAPs: their capacity to phagocytoze necrotic debris, a crucial process for successful completion of muscle repair (Heredia et al., 2013). In addition, IL-4/IL-13 signaling, via activation of STAT6, promotes FAPs proliferation to support myogenesis, while inhibiting their differentiation into adipocytes (Heredia et al., 2013). Accordingly, in the injured muscles of IL-4-knockout mice, the levels of adipocytes are increased, while in vitro and in vivo administration of IL-4 inhibits FAPs adipogenesis (Heredia et al., 2013; Dong et al., 2014). In agreement with these observations, glucocorticoids (GCs)-induced repression of IL-4 leads to intramuscular adipogenic accumulation by promoting FAPs proliferation and differentiation into adipocytes (Dong et al., 2014). Since GCs are known to suppress eosinophils, it is likely that they inhibit IL-4 signaling by reducing the number of infiltrating eosinophils upon muscle injury (Dong et al., 2014). Yet, IL-4-polarized, anti-inflammatory macrophages have been shown to induce adipogenesis of human FAPs isolated from dystrophic muscles (Moratal et al., 2018), suggesting that IL-4 signaling might govern more complex cellular interactions than previously expected.

\section{IL-15}

Interleukin-15 is expressed in human skeletal muscle and it has been identified as an anabolic factor involved in muscle growth (Quinn et al., 2002; Furmanczyk and Quinn, 2003). Indeed, IL-15 can decrease protein degradation in muscle (Busquets et al., 2005) and modulate muscle-adipose tissues interactions (Quinn et al., 2005). A recent work identified IL-15 as a myokine able to prevent intramuscular fatty infiltration, likely affecting FAPs differentiation capacities (Kang et al., 2018). In this work, the authors showed that IL-15 stimulates FAPs proliferation and it directly inhibits their adipogenic differentiation, both in vitro and in vivo, ultimately facilitating myofibers regeneration (Kang et al., 2018). Moreover, intramuscular administration of a recombinant IL-15 prevented fat accumulation in the murine 
model of glycerol-induced fatty degeneration (Kang et al., 2018). Accordingly, in vitro treatment of FAPs with IL-15 impaired their capacity to differentiate into adipocytes (Kang et al., 2018), likely through the induction of desert Hedgehog (DHH) signaling, a known repressor of FAPs adipogenesis (Kopinke et al., 2017). Although these results suggest a positive role for IL-15 in muscle regeneration, the evidence that IL-15 administration, and expression, correlates with increased collagen deposition in vivo after muscle damage (Kang et al., 2018), poses several unresolved issues that warrant further investigation. Indeed, whether IL15 directly promotes FAPs differentiation into fibroblasts has not been tested yet. Furthermore, even though FAPs expansion and regenerative fibrogenesis have a positive impact on acute muscle regeneration (Heredia et al., 2013; Fiore et al., 2016), the evidence that IL-15 expression is positively correlated with the number of FAPs and collagen deposition in subjects with rotator cuff tear indicates that IL-15 might serve, instead, as a signal to sustain FAPs pathogenic fibrogenesis in chronically fibrotic muscles.

\section{TNF- $\alpha$}

Tumor necrosis factor-alpha (TNF- $\alpha$ ) is a pleiotropic cytokine secreted rapidly upon muscle damage by infiltrating inflammatory cells and its impact on muscle regeneration is still under debate. Indeed, while pharmacological blockade of TNF- $\alpha$ has been associated with reduced muscle necrosis and amelioration of the histological profile of dystrophic muscles (Hodgetts et al., 2006; Huang et al., 2009; Piers et al., 2011; Ermolova et al., 2014). More recently, TNF- $\alpha$ has been implicated in preventing FAPs aberrant accumulation (Lemos et al., 2015; Fiore et al., 2016), suggesting that anti-TNF therapies might instead exert a pro-fibrotic effect.

During acute injury, TNF- $\alpha$ has been reported to promote muscle repair by activating promyogenic $\mathrm{p} 38$ signaling (Chen et al., 2007), thus inducing SCs differentiation (Palacios et al., 2010). Recently, it has been suggested that TNF- $\alpha$ regulates matrix production during acute damage, thus unveiling a crucial function for TNF- $\alpha$ in mediating FAPs apoptosis and clearance (Lemos et al., 2015; Fiore et al., 2016). Specifically, TNF$\alpha$ was found to be predominantly expressed and produced by infiltrating monocytes that rapidly differentiate into proinflammatory macrophages (M1) that accumulate in close proximity to expanding FAPs. By using a mouse model unable to recruit circulating monocytes to damaged muscles [the CC chemokine receptor type $2(\mathrm{Ccr} 2)^{-/-}$mouse strain (Warren et al., 2005)], Lemos et al. (2015) elegantly demonstrated that in the absence of infiltrating TNF-a-producing macrophages, FAPs accumulate in the sites of damage and aberrantly differentiate into fibrogenic cells. Inflammatory cell-derived TNF- $\alpha$ production is therefore required for FAPs clearance to prevent pathological ECM accumulation. Of note, this physiological role is altered in chronically damaged muscles, such as those of dystrophic mice, where the abundance of transforming growth factor beta 1 (TGF- $\beta 1$ ) signaling impairs the pro-apoptotic effects of TNF- $\alpha$ on FAPs (Lemos et al., 2015). These data might offer a possible explanation for the apparent controversial results reporting a positive effect of anti-TNF- $\alpha$ therapies on dystrophic mice (Hodgetts et al., 2006; Huang et al., 2009; Piers et al., 2011; Ermolova et al., 2014). Indeed, when TGF- $\beta 1$ is abundant, as in chronic degenerating muscles, the anti-fibrotic role of TNF- $\alpha$ is irrelevant and pharmacological approaches aimed at inhibiting its activity most likely exert their beneficial effects through targeting of the pro-myogenic, SC-mediated function of TNF- $\alpha$.

\section{TGF- $\beta$}

The transforming growth factor beta (TGF- $\beta$ ) superfamily comprises pleiotropic and multifunctional secreted peptides implicated in a wide range of cell functions, including tissue homeostasis and repair, immune and inflammatory responses, ECM deposition, cell differentiation, and growth (Biernacka et al., 2011; Meng et al., 2016). Studies in a wide range of experimental models have firmly established TGF- $\beta 1$ as a crucial mediator of fibrinogenesis and inhibition of its activity has consistently been associated with reduced fibrosis (Biernacka et al., 2011; Meng et al., 2016).

In the context of skeletal muscle, inhibition of TGF- $\beta 1$ has been linked to improvement in muscle regeneration and decreased fibrosis (Davies et al., 2016; Song et al., 2017; Zhang et al., 2019), consistent with the importance of the TGF- $\beta 1$ signaling in regulating both regeneration and matrix production. Several works have elucidated the detrimental, cell-autonomous, impact of TGF- $\beta$ signaling on muscle stem cells by inhibiting their activation (Carlson et al., 2008; Wang et al., 2016) and terminal differentiation (Carlson et al., 2008) while promoting a fibrogenic switch in chronically degenerating muscles (Biressi et al., 2014).

Nonetheless, recent findings point toward a prominent role for inflammatory cell-derived TGF- $\beta$ signaling in the survival and fibrotic differentiation of FAPs. Specifically, during chronic muscle damage, macrophages express and secrete high levels of TGF- $\beta 1$, antagonizing the TNF-mediated apoptosis of FAPs, and instead induce their fibrogenic differentiation and consequent ECM deposition (Lemos et al., 2015; Davies et al., 2016; Fiore et al., 2016; Juban et al., 2018). Thus, in conditions of chronic muscle damage, TGF- $\beta 1$ acts as a dominant, prosurvival signal that overrides the beneficial effect of the proinflammatory cell-derived, and anti-fibrotic cytokine, TNF- $\alpha$. Thus, treatment with nilotinib, via specific inhibition of TGF$\beta 1$-induced p38 signaling, restores FAPs apoptosis and prevents fibrotic accumulation in dystrophic mice (Lemos et al., 2015).

Of note, FAPs from chronic fibrotic dystrophic muscles have been identified as the main source of TGF- $\beta$-activating enzymes (Juban et al., 2018). Indeed, once released, latent TGF- $\beta 1$ must be activated, either via enzymatic or mechanical mechanisms, to exert its properties and to bind to its receptors (Travis and Sheppard, 2014). FAPs exhibit high expression of a series of latent TGF- $\beta 1$ activators, among which matrix metallo proteinase 14 (MMP14) and bone morphogenetic protein 1 (BMP1) are able to activate the latent TGF- $\beta 1$ released by pro-inflammatory macrophages (Juban et al., 2018). Notably, pharmacological inhibition of BMP1 or MMP14 reduced muscle fibrosis in dystrophic mice resulting in increased muscle fiber size and reduced necrosis (Juban et al., 2018). 
In summary, these data support a model through which chronic inflammation and fibrosis reciprocally sustain themselves in degenerating dystrophic muscles, by reinforcing a regulatory cross-talk between inflammatory cells and FAPs.

\section{MOLECULES SECRETED BY FAPS}

\section{IL-6}

In skeletal muscle, IL-6 is classified as a myokine produced and released by muscle fibers in response to contraction (Pedersen and Febbraio, 2008). It promotes lipolytic and anti-inflammatory beneficial effects during exercise (Pedersen et al., 2003), working as an energy sensor and exerting both local and endocrine metabolic effects.

Interleukin-6 regulates both muscle hypertrophy and regeneration (Muñoz-Cánoves et al., 2013). Indeed, IL-6 knockout mice show a reduced hypertrophic response to overloading, ascribed to impaired myonuclei incorporation as a consequence of the defective proliferation and migration of SCs. Treatment with IL-6 promotes murine SCs proliferation, via regulation of cell-cycle associated genes, Cyclin D1 and c-Myc (Serrano et al., 2008), while during regeneration the IL-6/STAT3 axis controls SCs fate (Tierney et al., 2014). Interestingly, FAPs were identified as one of the main source of IL- 6 during muscle regeneration. Upon muscle injury, IL-6 expression remains constant in myogenic progenitors but increases nearly 10-fold in FAPs and in vitro co-culture experiments have shown that IL-6 mediates the pro-myogenic activity of FAPs (Joe et al., 2010).

The positive effect of IL-6, and others myokines, is normally associated with transient production and short-term action. By contrast, persistent inflammatory conditions, denervation, and some types of cancer and other chronic diseases have been associated with long-lasting elevated IL-6 levels. In agreement with this notion, IL-6 has been shown to promote skeletal muscle atrophy (Haddad et al., 2005). Accordingly, in denervated muscles, FAPs show a persistent activation of IL-6, thus promoting muscle atrophy without other systemic effects (Madaro et al., 2018). Notably, in vivo pharmacological inhibition of IL-6 effectively counteracts denervation-mediated muscle atrophy (Madaro et al., 2018) and accumulation of FAPs with hyper-activation of IL-6 signaling has also been found in mouse models of amyotrophic lateral sclerosis (ALS) (Contreras et al., 2016; Madaro et al., 2018).

Taken together, these observations suggest two apparently opposing effects of FAPs-derived IL-6 during muscle regeneration or in denervation-induced muscle wasting, and further studies are needed to shed light on the molecular mechanisms behind these apparently contradictory roles.

\section{IL-33}

Interleukin-33 is a nuclear chromatin-associated cytokine, belonging to the IL-1 family, and constitutively expressed in the nucleus of a wide variety of cell types, including fibroblasts, epithelial cells, and endothelial cells (Carriere et al., 2007). IL-33 appears to function as an alarmin (alarm signal) that is rapidly released upon cellular damage and stress (Liew et al., 2016) and mediates a potent effect on the activation of regulatory $\mathrm{T}$ cell lymphocytes (Treg) (Matta et al., 2014; Alvarez et al., 2019).

In skeletal muscle, the major IL-33-producing cell type has been identified within the FAP cell population (Kuswanto et al., 2016). FAPs start to express IL-33 within $6-12 \mathrm{~h}$ after acute injury, inducing proliferation of muscle resident Treg (Kuswanto et al., 2016). As previously demonstrated, Treg cells promote muscle repair, accumulating in both acutely and chronically injured skeletal muscles (Burzyn et al., 2013; Castiglioni et al., 2015; Panduro et al., 2018). Interestingly, a severe decline in Treg accumulation, caused by an impairment in IL-33-producing FAPs, has been linked to regeneration defects in aged muscles (Kuswanto et al., 2016). On the other hand, in vivo treatment with IL-33 restored the Treg population in injured muscles of old mice, enhancing tissue regeneration.

Intriguingly, IL-33-expressing FAPs have been found in close association with muscle spindles (Kuswanto et al., 2016), which are stretch-sensitive mechanoreceptors that lie within the skeletal muscle and comprise both sensory and motor neurons. This finding raises the possibility that FAPs might function as mechano-cellular sensors that modulate the crosstalk between neural and immune cells to facilitate proper homeostatic reorganization of skeletal muscle and neural circuits upon injury. In agreement with this possibility, IL-33 expression is increased in fibroblasts upon mechanical stress (Kakkar et al., 2012) and PDGFR $\alpha^{+}$mesenchymal precursors, found within the endoneurium of peripheral nerves, have been recently implicated in tissue repair and regeneration (Carr et al., 2019).

\section{WISP1}

Wnt family member 1 (WNT1) inducible signaling pathway protein 1 (WISP1) is encoded by the cellular communication network factor 4 (CCN4) gene, a member of the CCN family of matricellular proteins that are involved in diverse biological processes, such as ECM remodeling, tissue repair, and tumor growth. CCN4/WISP1 is important in the musculoskeletal system, where it regulates osteogenesis and chondrogenesis, as well as skin repair (Ono et al., 2011, 2018; Maeda et al., 2015).

A recent study showed that in young mice CCN4/WISP1 is upregulated in FAPs following muscle injury, but this induction is lost in FAPs of old muscles (Lukjanenko et al., 2019). The FAP-secreted WISP1 plays an important role in SCs expansion and asymmetric commitment to myogenic differentiation. Indeed, similar to aging, the loss of WISP1 in knockout mice affects SCs function and impairs myogenesis. In agreement with this, the transplantation of young, but not aged or WISP $^{-/-}$FAPs, rescues the myogenic dysfunction of aged SCs and their regeneration ability (Lukjanenko et al., 2019). Even better, systemic treatment with recombinant WISP1 mimics rejuvenation beneficial effects, opening new prospects in the use of this approach as a strategy to counteract aging and associated muscular diseases.

Interestingly, these findings together with the reported impaired interplay between FAPs and Treg during aging (Kuswanto et al., 2016), the sensitivity of FAPs to muscle 

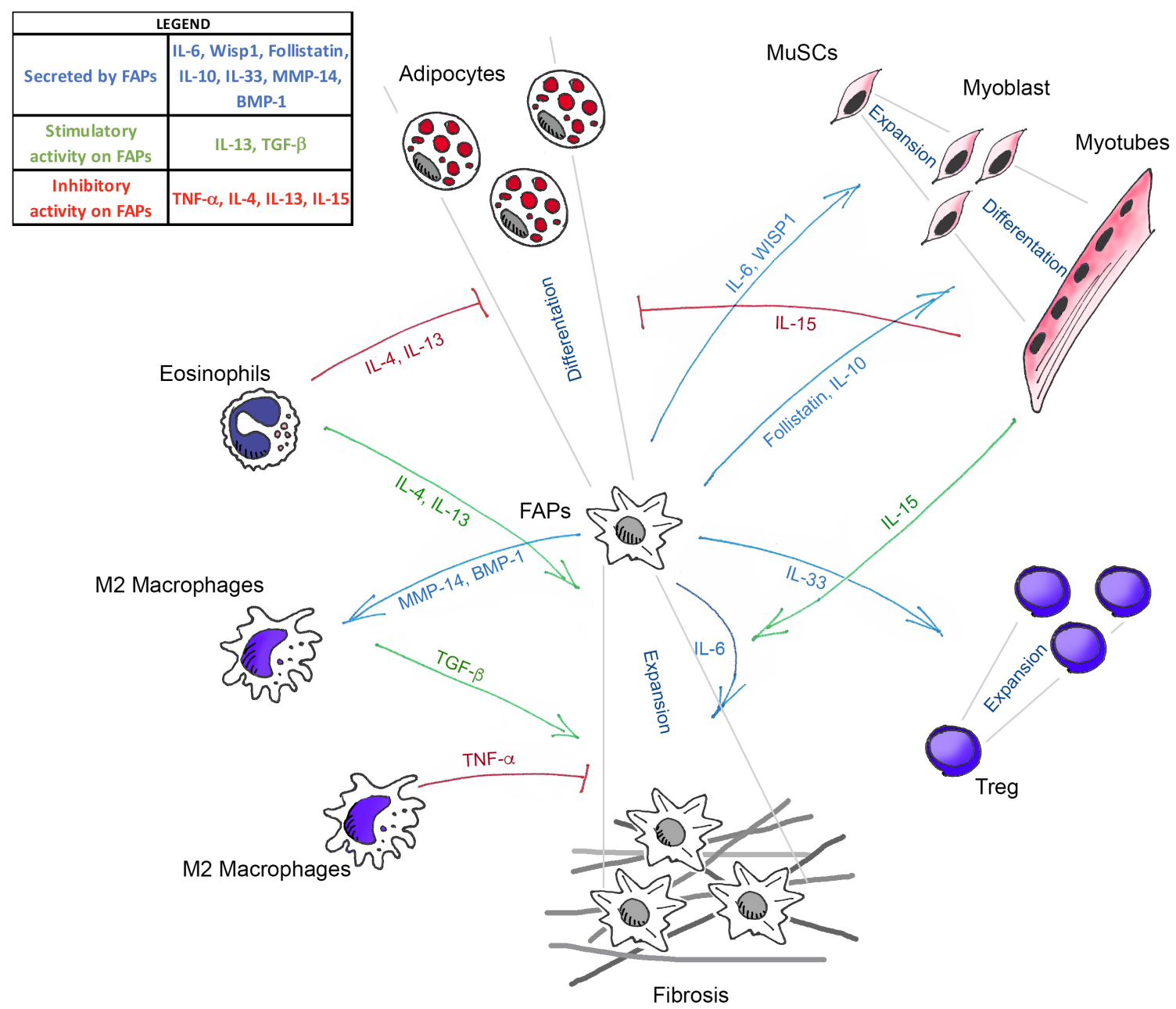

FIGURE 1 | Schematic illustration showing the known mediators that govern the interaction between FAPs, muscle stem cells (MuSCs), and the different immune cells in skeletal muscle homeostasis. Green arrows (TGF- $\beta$, IL-13, IL-4, and IL-15) indicate the molecules that positively regulate FAPs expansion. Blue arrows (IL-33, IL-6, Follistatin, IL-10, WISP1, MMP-14, and BMP-1) represent the molecules secreted by FAPs that act on the different cell targets. Red lines (TNF- $\alpha$, IL-4, IL-13, and IL-15) show the factors that inhibit the fibro-adipogenic differentiation of FAPs.

denervation (Madaro et al., 2018), and the recently reported atrophic phenotype of FAPs-depleted skeletal muscles (Wosczyna et al., 2019) clearly point toward FAPs as promising new cellular targets for the treatment of muscle defects associated with sarcopenia.

\section{Follistatin}

Follistatin is a potent natural antagonist of myostatin and activin A, two TGF- $\beta$ superfamily cytokines implicated in muscle growth inhibition, and it is therefore a potent pro-myogenic factor (Lee, 2007; Nakatani et al., 2008; Guo et al., 2009; Kota et al., 2009; Rodino-Klapac et al., 2009; Winbanks et al., 2012).

In the context of skeletal muscle regeneration, follistatin expression is induced $12 \mathrm{~h}$ after muscle injury (Iezzi et al., 2004) and remains elevated for 5 days, concurrent with SCs activation. Of note, FAPs have been described as the major source of follistatin, displaying 10-fold higher expression levels than SCs (Mozzetta et al., 2013; Formicola et al.,
2019). Follistatin is considered the central mediator of the fusogenic effects exerted by histone deacetylase inhibitors (HDACi) on skeletal muscles (Iezzi et al., 2004; Minetti et al., 2006; Mozzetta et al., 2013). Indeed, HDACi treatment in dystrophic mice induces the upregulation of follistatin in muscle progenitor cells, promoting the formation of multinucleated myotubes. In agreement with its promyogenic activity, follistatin knock-down in FAPs reduced the ability of HDACi to stimulate SCs-mediated formation of myotubes, suggesting a crucial role of FAP-derived follistatin as a mediator of the pro-differentiative activity of FAPs (Mozzetta et al., 2013).

A proper balance between follistatin and its antagonists is crucial to preserve reciprocal functional interactions between FAPs and SCs and to preserve muscle homeostasis (Baccam et al., 2019; Formicola et al., 2019). Indeed, pharmacological inhibition of the activin receptor type-2B pathway (AcvR2B), which blocks both myostatin and activin A activity, reverses muscle 
atrophy in SC-depleted skeletal muscles, while also restoring stem cells regenerative potential (Formicola et al., 2019). Of note, beneficial effects of AcvR2B targeting in SCs-depleted muscles are accompanied by an increased number of FAPs (Formicola et al., 2019), an observation that further supports the notion that restoration of a proper balance of regulatory factors between the different cells within the regenerative niche is key for muscle repair.

Once again, a role of FAPs emerges not only in regulating muscle regeneration but also in mediating signaling pathways associated with maintaining muscle mass. Future experiments should elucidate the possible use of FAPs as a source of trophic factors.

\section{IL-10}

Interleukin is a broadly expressed anti-inflammatory cytokine that inhibits the activation of the innate immune system and Th1 activation, preventing inflammatory and autoimmune pathologies (Saraiva and O'Garra, 2010; Ouyang et al., 2011). Like IL-6, IL-10 is also considered a myokine expressed in skeletal muscle in a wide range of conditions. It influences different aspects of muscle biology, such as regeneration, exercise, metabolism, and aging (Furmanczyk and Quinn, 2003; Nunes et al., 2008; Villalta et al., 2011; Deng et al., 2012; Dagdeviren et al., 2016, 2017). Its anti-inflammatory activity has been investigated in different muscle-related disorders (Hong et al., 2009; NitaharaKasahara et al., 2014; Villalta et al., 2014; Dagdeviren et al., 2016, 2017; Zhang et al., 2018). The main source of IL-10 in regenerating skeletal muscle is macrophages and Tregs (Villalta et al., 2011, 2014; Deng et al., 2012) although it has also been demonstrated that FAPs increase IL-10 expression upon muscle damage (Lemos et al., 2012). This evidence is in line with the crucial pro-myogenic activity of FAPs, which likely contribute to muscle repair also through the secretion of an anti-inflammatory cytokine, such as IL-10, to counteract the anti-myogenic activity of TNF- $\alpha$. Although the function of IL-10 released by FAPs has not yet been demonstrated in vivo, this work underscores the complexity of the interplay between inflammatory cells and the

\section{REFERENCES}

Allen, J. E., and Wynn, T. A. (2011). Evolution of Th2 immunity: a rapid repair response to tissue destructive pathogens. PLoS Pathog. 7:e1002003. doi: 10.1371/ journal.ppat.1002003

Alvarez, F., Istomine, R., Shourian, M., Pavey, N., Al-Aubodah, T. A.-F., Qureshi, S., et al. (2019). The alarmins IL-1 and IL-33 differentially regulate the functional specialisation of Foxp3+ regulatory $\mathrm{T}$ cells during mucosal inflammation. Mucosal Immunol. 12, 746-760. doi: 10.1038/s41385-0190153-5

Baccam, A., Benoni-Sviercovich, A., Rocchi, M., Moresi, V., Seelaender, M., Li, Z., et al. (2019). The mechanical stimulation of myotubes counteracts the effects of tumor-derived factors through the modulation of the activin/follistatin ratio. Front. Physiol. 24:401. doi: 10.3389/fphys.2019.00401

Baghdadi, M. B., Castel, D., Machado, L., Fukada, S., Birk, D. E., Relaix, F., et al. (2018). Reciprocal signalling by notch-collagen V-CALCR retains muscle stem cells in their niche. Nature 557, 714-718. doi: 10.1038/s41586-018-0144-149

Biernacka, A., Dobaczewski, M., and Frangogiannis, N. G. (2011). TGF- $\beta$ signaling in fibrosis. Growth Factors 29, 196-202. doi: 10.3109/08977194.2011.595714 other players in muscle regeneration. Future studies are needed to better understand this mechanism.

\section{CONCLUDING REMARKS}

In conclusion, the available evidence reviewed above clearly indicates that FAPs act as crucial regulators of skeletal muscle homeostasis (Figure 1). However, several critical issues need to be addressed before defining them as the co-star of skeletal muscle repair. First, their molecular heterogeneity makes it difficult to target them genetically, to uniquely assess their requirement, and to define the function of FAP-specific expression of the different factors described above. Future single-cell transcriptomic approaches will help identify subpopulations differently altered during the diverse stages of muscle regeneration and, more importantly, in pathological situations.

Finally, the evidence of the association of FAPs with nerve structures (Kuswanto et al., 2016), and the ability of FAPs to respond to nerve lesions (Contreras et al., 2016; Madaro et al., 2018), suggest a mechano-sensitivity of FAPs and emphasize the urgency to improve our understanding of the molecular regulation governing FAPs activity during muscle adaptation.

\section{AUTHOR CONTRIBUTIONS}

LM and CM wrote and edited the manuscript. BB and DP wrote the manuscript.

\section{FUNDING}

This work was supported by the Italian Ministry of Health (Grant No. GR-2013-02356592) to LM. CM was supported by a grant from the Italian Ministry of University and Research (SIR, Scientific Independence of Young Researcher no. RBSI14QMG0) and by a My First grant from the Italian Association for Cancer Research (AIRC), no. 18993.

Biressi, S., Miyabara, E. H., Gopinath, S. D., Carlig, P. M. M., and Rando, T. A. (2014). A Wnt-TGF $\beta 2$ axis induces a fibrogenic program in muscle stem cells from dystrophic mice. Sci. Transl. Med. 6:267ra176. doi: 10.1126/scitranslmed. 3008411

Buras, E. D., Converso-Baran, K., Davis, C. S., Akama, T., Hikage, F., Michele, D. E., et al. (2019). Fibro-adipogenic remodeling of the diaphragm in obesityassociated respiratory dysfunction. Diabetes 68, 45-56. doi: 10.2337/db180209

Burzyn, D., Kuswanto, W., Kolodin, D., Shadrach, J. L., Cerletti, M., Jang, Y., et al. (2013). A special population of regulatory T cells potentiates muscle repair. Cell 155, 1282-1295. doi: 10.1016/j.cell.2013.10.054

Busquets, S., Figueras, M. T., Meijsing, S., Carbó, N., Quinn, L. S., Almendro, V., et al. (2005). Interleukin-15 decreases proteolysis in skeletal muscle: a direct effect. Int. J. Mol. Med. 16, 471-476.

Carlson, M. E., Hsu, M., and Conboy, I. M. (2008). Imbalance between pSmad3 and Notch induces CDK inhibitors in old muscle stem cells. Nature 454, 528-532. doi: $10.1038 /$ nature 07034

Carr, M. J., Toma, J. S., Johnston, A. P. W., Steadman, P. E., Yuzwa, S. A., Mahmud, N., et al. (2019). Mesenchymal precursor cells in adult nerves contribute to 
mammalian tissue repair and regeneration. Cell Stem Cell 24, 240-256.e9. doi: 10.1016/j.stem.2018.10.024

Carriere, V., Roussel, L., Ortega, N., Lacorre, D.-A., Americh, L., Aguilar, L., et al. (2007). IL-33, the IL-1-like cytokine ligand for ST2 receptor, is a chromatinassociated nuclear factor in vivo. Proc. Natl. Acad. Sci. U.S.A. 104, 282-287. doi: 10.1073/pnas.0606854104

Castiglioni, A., Corna, G., Rigamonti, E., Basso, V., Vezzoli, M., Monno, A., et al. (2015). FOXP3 + T cells recruited to sites of sterile skeletal muscle injury regulate the fate of satellite cells and guide effective tissue regeneration. PLoS One 10:e0128094. doi: 10.1371/journal.pone.0128094

Chen, S.-E., Jin, B., and Li, Y.-P. (2007). TNF- $\alpha$ regulates myogenesis and muscle regeneration by activating p38 MAPK. Am. J. Physiol. Physiol. 292, C1660-C1671. doi: 10.1152/ajpcell.00486.2006

Contreras, O., Rebolledo, D. L., Oyarzún, J. E., Olguín, H. C., and Brandan, E. (2016). Connective tissue cells expressing fibro/adipogenic progenitor markers increase under chronic damage: relevance in fibroblast-myofibroblast differentiation and skeletal muscle fibrosis. Cell Tissue Res. 364, 647-660. doi: 10.1007/s00441-015-2343-0

Dagdeviren, S., Jung, D. Y., Friedline, R. H., Noh, H. L., Kim, J. H., Patel, P. R., et al. (2017). IL-10 prevents aging-associated inflammation and insulin resistance in skeletal muscle. FASEB J. 31, 701-710. doi: 10.1096/fj.201600832R

Dagdeviren, S., Jung, D. Y., Lee, E., Friedline, R. H., Noh, H. L., Kim, J. H., et al. (2016). Altered interleukin-10 signaling in skeletal muscle regulates obesitymediated inflammation and insulin resistance. Mol. Cell. Biol. 36, 2956-2966. doi: 10.1128/MCB.00181-116

Dammone, G., Karaz, S., Lukjanenko, L., Winkler, C., Sizzano, F., Jacot, G., et al. (2018). PPAR $\gamma$ controls ectopic adipogenesis and cross-talks with myogenesis during skeletal muscle regeneration. Int. J. Mol. Sci. 19:E2044. doi: 10.3390/ ijms 19072044

Davies, M. R., Liu, X., Lee, L., Laron, D., Ning, A. Y., Kim, H. T., et al. (2016). TGF- $\beta$ small molecule inhibitor SB431542 reduces rotator cuff muscle fibrosis and fatty infiltration by promoting fibro/adipogenic progenitor apoptosis. PLoS One 11:e0155486. doi: 10.1371/journal.pone.0155486

Deng, B., Wehling-Henricks, M., Villalta, S. A., Wang, Y., and Tidball, J. G. (2012). IL-10 triggers changes in macrophage phenotype that promote muscle growth and regeneration. J. Immunol. 189, 3669-3680. doi: 10.4049/jimmunol.110 3180

Dong, Y., Silva, K. A. S., Dong, Y., and Zhang, L. (2014). Glucocorticoids increase adipocytes in muscle by affecting IL-4 regulated FAP activity. FASEB J. 28, 4123-4132. doi: 10.1096/fj.14-254011

Ermolova, N. V., Martinez, L., Vetrone, S. A., Jordan, M. C., Roos, K. P., Sweeney, H. L., et al. (2014). Long-term administration of the TNF blocking drug remicade $(\mathrm{cVlq})$ to $\mathrm{mdx}$ mice reduces skeletal and cardiac muscle fibrosis, but negatively impacts cardiac function. Neuromuscul. Disord. 24, 583-595. doi: 10.1016/j.nmd.2014.04.006

Feige, P., Brun, C. E., Ritso, M., and Rudnicki, M. A. (2018). Orienting muscle stem cells for regeneration in homeostasis. Aging Dis. Cell Stem Cell 23, 653-664. doi: $10.1016 /$ j.stem.2018.10.006

Fiore, D., Judson, R. N., Low, M., Lee, S., Zhang, E., Hopkins, C., et al. (2016). Pharmacological blockage of fibro/adipogenic progenitor expansion and suppression of regenerative fibrogenesis is associated with impaired skeletal muscle regeneration. Stem Cell Res. 17, 161-169. doi: 10.1016/j.scr.2016. 06.007

Formicola, L., Pannérec, A., Correra, R. M., Gayraud-Morel, B., Ollitrault, D., Besson, V., et al. (2019). Inhibition of the activin receptor type-2B pathway restores regenerative capacity in satellite cell-depleted skeletal muscle. Front. Physiol. 9:515. doi: 10.3389/fphys.2018.00515

Fry, C. S., Johnson, D. L., Ireland, M. L., and Noehren, B. (2017a). ACL injury reduces satellite cell abundance and promotes fibrogenic cell expansion within skeletal muscle. J. Orthop. Res. 35, 1876-1885. doi: 10.1002/jor.23502

Fry, C. S., Kirby, T. J., Kosmac, K., McCarthy, J. J., and Peterson, C. A. (2017b). Myogenic progenitor cells control extracellular matrix production by fibroblasts during skeletal muscle hypertrophy. Cell Stem Cell 20, 56-69. doi: 10.1016/j. stem.2016.09.010

Furmanczyk, P. S., and Quinn, L. S. (2003). Interleukin-15 increases myosin accretion in human skeletal myogenic cultures. Cell Biol. Int. 27, 845-851. doi: 10.1016/s1065-6995(03)00172-0
Giordani, L., He, G. J., Negroni, E., Sakai, H., Law, J. Y. C., Siu, M. M., et al. (2019). High-dimensional single-cell cartography reveals novel skeletal muscleresident cell populations. Mol. Cell. 74, 609-621.e6. doi: 10.1016/j.molcel.2019. 02.026

González, M. N., de Mello, W., Butler-Browne, G. S., Silva-Barbosa, S. D., Mouly, V., Savino, W., et al. (2017). HGF potentiates extracellular matrix-driven migration of human myoblasts: involvement of matrix metalloproteinases and MAPK/ERK pathway. Skelet. Muscle 7:20. doi: 10.1186/s13395-017-0138-6

Gorski, T., Mathes, S., and Krützfeldt, J. (2018). Uncoupling protein 1 expression in adipocytes derived from skeletal muscle fibro/adipogenic progenitors is under genetic and hormonal control. J. Cachexia. Sarcopenia Muscle 9, 384-399. doi: 10.1002/jcsm. 12277

Guo, T., Jou, W., Chanturiya, T., Portas, J., Gavrilova, O., and McPherron, A. C. (2009). Myostatin inhibition in muscle, but not adipose tissue, decreases fat mass and improves insulin sensitivity. PLoS One 4:e4937. doi: 10.1371/journal. pone.0004937

Haddad, F., Zaldivar, F., Cooper, D. M., and Adams, G. R. (2005). IL-6-induced skeletal muscle atrophy. J. Appl. Physiol. 98, 911-917. doi: 10.1152/japplphysiol. 01026.2004

Heredia, J. E., Mukundan, L., Chen, F. M., Mueller, A. A., Deo, R. C., Locksley, R. M., et al. (2013). Type 2 innate signals stimulate fibro/adipogenic progenitors to facilitate muscle regeneration. Cell 153, 376-388. doi: 10.1016/j.cell.2013.02. 053

Hodgetts, S., Radley, H., Davies, M., and Grounds, M. D. (2006). Reduced necrosis of dystrophic muscle by depletion of host neutrophils, or blocking TNF $\alpha$ function with etanercept in mdx mice. Neuromuscul. Disord. 16, 591-602. doi: 10.1016/j.nmd.2006.06.011

Hong, E.-G., Ko, H. J., Cho, Y.-R., Kim, H.-J., Ma, Z., Yu, T. Y., et al. (2009). Interleukin-10 prevents diet-induced insulin resistance by attenuating macrophage and cytokine response in skeletal muscle. Diabetes 58, 2525-2535. doi: $10.2337 / \mathrm{db} 08-1261$

Huang, P., Zhao, X. S., Fields, M., Ransohoff, R. M., and Zhou, L. (2009). Imatinib attenuates skeletal muscle dystrophy in mdx mice. FASEB J. 23, 2539-2548. doi: 10.1096/fj.09-129833

Iezzi, S., Di Padova, M., Serra, C., Caretti, G., Simone, C., Maklan, E., et al. (2004). Deacetylase inhibitors increase muscle cell size by promoting myoblast recruitment and fusion through induction of follistatin. Dev. Cell 6, 673-684. doi: 10.1016/s1534-5807(04)00107-8

Joe, A. W. B., Yi, L., Natarajan, A., Le Grand, F., So, L., Wang, J., et al. (2010). Muscle injury activates resident fibro/adipogenic progenitors that facilitate myogenesis. Nat. Cell Biol. 12, 153-163. doi: 10.1038/ncb2015

Juban, G., Saclier, M., Yacoub-Youssef, H., Kernou, A., Arnold, L., Boisson, C., et al. (2018). AMPK activation regulates LTBP4-dependent TGF- $\beta 1$ secretion by pro-inflammatory macrophages and controls fibrosis in duchenne muscular dystrophy. Cell Rep. 25, 2163-2176.e6. doi: 10.1016/j.celrep.2018.10.077

Judson, R. N., Low, M., Eisner, C., and Rossi, F. M. (2017). Isolation, culture, and differentiation of fibro/adipogenic progenitors (FAPs) from skeletal muscle. Methods Mol. Biol. 1668, 93-103. doi: 10.1007/978-1-4939-72 83-8-7

Kakkar, R., Hei, H., Dobner, S., and Lee, R. T. (2012). Interleukin 33 as a mechanically responsive cytokine secreted by living cells. J. Biol. Chem. 287, 6941-6948. doi: 10.1074/jbc.M111.298703

Kang, X., Yang, M., Shi, Y., Xie, M., Zhu, M., Zheng, X., et al. (2018). Interleukin15 facilitates muscle regeneration through modulation of fibro/adipogenic progenitors. Cell Commun. Signal. 16:42. doi: 10.1186/s12964-0180251-0

Kopinke, D., Roberson, E. C., and Reiter, J. F. (2017). Ciliary hedgehog signaling restricts injury-induced adipogenesis. Cell 170, 340-351.e12. doi: 10.1016/j.cell. 2017.06.035

Kota, J., Handy, C. R., Haidet, A. M., Montgomery, C. L., Eagle, A., RodinoKlapac, L. R., et al. (2009). Follistatin Gene Delivery enhances muscle growth and strength in nonhuman primates. Sci. Transl. Med. 1:6ra15. doi: 10.1126/ scitranslmed.3000112

Kuswanto, W., Burzyn, D., Panduro, M., Wang, K. K., Jang, Y. C., Wagers, A. J., et al. (2016). Poor repair of skeletal muscle in aging mice reflects a defect in local, interleukin-33-dependent accumulation of regulatory T cells. Immunity 44, 355-367. doi: 10.1016/j.immuni.2016.01.009 
Lee, S.-J. (2007). Quadrupling muscle mass in mice by targeting TGF- $\beta$ signaling pathways. PLoS One 2:e789. doi: 10.1371/journal.pone.0000789

Lees-Shepard, J. B., Yamamoto, M., Biswas, A. A., Stoessel, S. J., Nicholas, S.-A. E., Cogswell, C. A., et al. (2018). Activin-dependent signaling in fibro/adipogenic progenitors causes fibrodysplasia ossificans progressiva. Nat. Commun. 9:471. doi: 10.1038/s41467-018-02872-2

Lemos, D. R., Babaeijandaghi, F., Low, M., Chang, C.-K., Lee, S. T., Fiore, D., et al. (2015). Nilotinib reduces muscle fibrosis in chronic muscle injury by promoting TNF-mediated apoptosis of fibro/adipogenic progenitors. Nat. Med. 21, 786-794. doi: 10.1038/nm.3869

Lemos, D. R., Paylor, B., Chang, C., Sampaio, A., Underhill, T. M., and Rossi, F. M. V. (2012). Functionally convergent white adipogenic progenitors of different lineages participate in a diffused system supporting tissue regeneration. Stem Cells 30, 1152-1162. doi: 10.1002/stem.1082

Liew, F. Y., Girard, J.-P., and Turnquist, H. R. (2016). Interleukin-33 in health and disease. Nat. Rev. Immunol. 16, 676-689. doi: 10.1038/nri.2016.95

Lukjanenko, L., Karaz, S., Stuelsatz, P., Gurriaran-Rodriguez, U., Michaud, J., Dammone, G., et al. (2019). Aging disrupts muscle stem cell function by impairing matricellular WISP1 secretion from fibro-adipogenic progenitors. Cell Stem Cell 24, 433-446.e7. doi: 10.1016/j.stem.2018.12.014

Madaro, L., Passafaro, M., Sala, D., Etxaniz, U., Lugarini, F., Proietti, D., et al. (2018). Denervation-activated STAT3-IL-6 signalling in fibro-adipogenic progenitors promotes myofibres atrophy and fibrosis. Nat. Cell Biol. 20, 917927. doi: 10.1038/s41556-018-0151-y

Maeda, A., Ono, M., Holmbeck, K., Li, L., Kilts, T. M., Kram, V., et al. (2015). WNT1-induced secreted protein-1 (WISP1), a novel regulator of bone turnover and wnt signaling. J. Biol. Chem. 290, 14004-14018. doi: 10.1074/jbc.M114. 628818

Malecova, B., Gatto, S., Etxaniz, U., Passafaro, M., Cortez, A., Nicoletti, C., et al. (2018). Dynamics of cellular states of fibro-adipogenic progenitors during myogenesis and muscular dystrophy. Nat. Commun. 9:3670. doi: 10.1038/ s41467-018-06068-6

Matta, B. M., Lott, J. M., Mathews, L. R., Liu, Q., Rosborough, B. R., Blazar, B. R., et al. (2014). IL-33 is an unconventional alarmin that stimulates IL-2 secretion by dendritic cells to selectively expand IL-33R/ST2 + regulatory T cells. J. Immunol. 193, 4010-4020. doi: 10.4049/jimmunol.1400481

Meng, X., Nikolic-Paterson, D. J., and Lan, H. Y. (2016). TGF- $\beta$ : the master regulator of fibrosis. Nat. Rev. Nephrol. 12, 325-338. doi: 10.1038/nrneph. 2016.48

Minetti, G. C., Colussi, C., Adami, R., Serra, C., Mozzetta, C., Parente, V., et al. (2006). Functional and morphological recovery of dystrophic muscles in mice treated with deacetylase inhibitors. Nat. Med. 12, 1147-1150. doi: 10.1038/ nm1479

Moratal, C., Raffort, J., Arrighi, N., Rekima, S., Schaub, S., Dechesne, C. A., et al. (2018). IL-1 $\beta$ - and IL-4-polarized macrophages have opposite effects on adipogenesis of intramuscular fibro-adipogenic progenitors in humans. Sci. Rep. 8:17005. doi: 10.1038/s41598-018-35429-w

Mozzetta, C., Consalvi, S., Saccone, V., Tierney, M., Diamantini, A., Mitchell, K. J., et al. (2013). Fibroadipogenic progenitors mediate the ability of HDAC inhibitors to promote regeneration in dystrophic muscles of young, but not old Mdx mice. EMBO Mol. Med. 5, 626-639. doi: 10.1002/emmm.20120 2096

Mueller, A. A., van Velthoven, C. T., Fukumoto, K. D., Cheung, T. H., and Rando, T. A. (2016). Intronic polyadenylation of PDGFR $\alpha$ in resident stem cells attenuates muscle fibrosis. Nature 540, 276-279. doi: 10.1038/nature20160

Muñoz-Cánoves, P., Scheele, C., Pedersen, B. K., and Serrano, A. L. (2013). Interleukin-6 myokine signaling in skeletal muscle: a double-edged sword. FEBS J. 280, 4131-4148. doi: 10.1111/febs.12338

Murphy, M. M., Lawson, J. A., Mathew, S. J., Hutcheson, D. A., and Kardon, G. (2011). Satellite cells, connective tissue fibroblasts and their interactions are crucial for muscle regeneration. Development 138, 3625-3637. doi: 10.1242/dev. 064162

Nakatani, M., Takehara, Y., Sugino, H., Matsumoto, M., Hashimoto, O., Hasegawa, Y., et al. (2008). Transgenic expression of a myostatin inhibitor derived from follistatin increases skeletal muscle mass and ameliorates dystrophic pathology in mdx mice. FASEB J. 22, 477-487. doi: 10.1096/fj.07-8673com

Nitahara-Kasahara, Y., Hayashita-Kinoh, H., Chiyo, T., Nishiyama, A., Okada, H., Takeda, S., et al. (2014). Dystrophic mdx mice develop severe cardiac and respiratory dysfunction following genetic ablation of the anti-inflammatory cytokine IL-10. Hum. Mol. Genet. 23, 3990-4000. doi: 10.1093/hmg/ ddu113

Nunes, R. B., Tonetto, M., Machado, N., Chazan, M., Heck, T. G., Veiga, A. B. G., et al. (2008). Physical exercise improves plasmatic levels of IL-10, left ventricular end-diastolic pressure, and muscle lipid peroxidation in chronic heart failure rats. J. Appl. Physiol. 104, 1641-1647. doi: 10.1152/japplphysiol.00062. 2008

Ono, M., Inkson, C. A., Kilts, T. M., and Young, M. F. (2011). WISP-1/CCN4 regulates osteogenesis by enhancing BMP-2 activity. J. Bone Miner. Res. 26, 193-208. doi: 10.1002/jbmr.205

Ono, M., Masaki, A., Maeda, A., Kilts, T. M., Hara, E. S., Komori, T., et al. (2018). CCN4/WISP1 controls cutaneous wound healing by modulating proliferation, migration and ECM expression in dermal fibroblasts via $\alpha 5 \beta 1$ and TNF $\alpha$. Matrix Biol. 6, 533-546. doi: 10.1016/j.matbio.2018.01.004

Ouyang, W., Rutz, S., Crellin, N. K., Valdez, P. A., and Hymowitz, S. G. (2011). Regulation and functions of the IL-10 family of cytokines in inflammation and disease. Annu. Rev. Immunol. 29, 71-109. doi: 10.1146/annurev-immunol031210- 101312

Pagano, A. F., Brioche, T., Arc-Chagnaud, C., Demangel, R., Chopard, A., and Py, G. (2018). Short-term disuse promotes fatty acid infiltration into skeletal muscle. J. Cachexia. Sarcopenia Muscle 9, 335-347. doi: 10.1002/JCSM.12259

Palacios, D., Mozzetta, C., Consalvi, S., Caretti, G., Saccone, V., Proserpio, V., et al. (2010). TNF/p38 $\alpha$ /Polycomb signaling to Pax7 locus in satellite cells links inflammation to the epigenetic control of muscle regeneration. Cell Stem Cell 7, 455-469. doi: 10.1016/j.stem.2010.08.013

Palm, N. W., Rosenstein, R. K., and Medzhitov, R. (2012). Allergic host defences. Nature 484, 465-472. doi: 10.1038/nature11047

Panduro, M., Benoist, C., and Mathis, D. (2018). T reg cells limit IFN- $\gamma$ production to control macrophage accrual and phenotype during skeletal muscle regeneration. Proc. Natl. Acad. Sci. U.S.A. 115, E2585-E2593. doi: 10. 1073/pnas. 1800618115

Pannérec, A., Marazzi, G., and Sassoon, D. (2012). Stem cells in the hood: the skeletal muscle niche. Trends Mol. Med. 18, 599-606. doi: 10.1016/j.molmed. 2012.07.004

Pedersen, B. K., and Febbraio, M. A. (2008). Muscle as an endocrine organ: focus on muscle-derived interleukin-6. Physiol. Rev. 88, 1379-1406. doi: 10.1152/ physrev.90100.2007

Pedersen, B. K., Steensberg, A., Keller, P., Keller, C., Fischer, C., Hiscock, N., et al. (2003). Muscle-derived interleukin-6: lipolytic, anti-inflammatory and immune regulatory effects. Pflügers Arch. Eur. J. Physiol. 446, 9-16. doi: 10.1007/s00424002-0981-z

Piers, A. T., Lavin, T., Radley-Crabb, H. G., Bakker, A. J., Grounds, M. D., and Pinniger, G. J. (2011). Blockade of TNF in vivo using cV1q antibody reduces contractile dysfunction of skeletal muscle in response to eccentric exercise in dystrophic mdx and normal mice. Neuromuscul. Disord. 21, 132-141. doi: 10.1016/j.nmd.2010.09.013

Quinn, L., Straitbodey, L., Anderson, B., Argiles, J., and Havel, P. (2005). Interleukin-15 stimulates adiponectin secretion by 3T3-L1 adipocytes: evidence for a skeletal muscle-to-fat signaling pathway. Cell Biol. Int. 29, 449-457. doi: 10.1016/j.cellbi.2005.02.005

Quinn, L. S., Anderson, B. G., Drivdahl, R. H., Alvarez, B., and Argilés, J. M. (2002). Overexpression of interleukin-15 induces skeletal muscle hypertrophy in vitro: implications for treatment of muscle wasting disorders. Exp. Cell Res. 280, 55-63. doi: 10.1006/excr.2002.5624

Rebolledo, D. L., González, D., Faundez-Contreras, J., Contreras, O., Vio, C. P., Murphy-Ullrich, J. E., et al. (2019). Denervation-induced skeletal muscle fibrosis is mediated by CTGF/CCN2 independently of TGF- $\beta$. Matrix Biol. doi: 10.1016/j.matbio.2019.01.002 [Epub ahead of print].

Rodino-Klapac, L. R., Haidet, A. M., Kota, J., Handy, C., Kaspar, B. K., and Mendell, J. R. (2009). Inhibition of myostatin with emphasis on follistatin as a therapy for muscle disease. Muscle Nerve 39, 283-296. doi: 10.1002/mus.21244

Saccone, V., Consalvi, S., Giordani, L., Mozzetta, C., Barozzi, I., Sandona, M., et al. (2014). HDAC-regulated myomiRs control BAF60 variant exchange and direct the functional phenotype of fibro-adipogenic progenitors in dystrophic muscles. Genes Dev. 28, 841-857. doi: 10.1101/gad.234468.113

Saraiva, M., and O'Garra, A. (2010). The regulation of IL-10 production by immune cells. Nat. Rev. Immunol. 10, 170-181. doi: 10.1038/nri2711 
Serrano, A. L., Baeza-Raja, B., Perdiguero, E., Jardí, M., and Muñoz-Cánoves, P. (2008). Interleukin-6 is an essential regulator of satellite cell-mediated skeletal muscle hypertrophy. Cell Metab. 7, 33-44. doi: 10.1016/j.cmet.2007. 11.011

Serrano, A. L., and Muñoz-Cánoves, P. (2010). Regulation and dysregulation of fibrosis in skeletal muscle. Exp. Cell Res. 316, 3050-3058. doi: 10.1016/J.YEXCR. 2010.05.035

Song, Y., Yao, S., Liu, Y., Long, L., Yang, H., Li, Q., et al. (2017). Expression levels of TGF- $\beta 1$ and CTGF are associated with the severity of duchenne muscular dystrophy. Exp. Ther. Med. 13, 1209-1214. doi: 10.3892/etm.2017. 4105

Tatsumi, R., Sankoda, Y., Anderson, J. E., Sato, Y., Mizunoya, W., Shimizu, N., et al. (2009). Possible implication of satellite cells in regenerative motoneuritogenesis: HGF upregulates neural chemorepellent Sema3A during myogenic differentiation. Am. J. Physiol. Cell Physiol. 297, C238-C252. doi: 10.1152/ajpcell.00161.2009

Tidball, J. G., and Villalta, S. A. (2010). Regulatory interactions between muscle and the immune system during muscle regeneration. Am. J. Physiol. Integr. Comp. Physiol. 298, R1173-R1187. doi: 10.1152/ajpregu.00735.2009

Tierney, M. T., Aydogdu, T., Sala, D., Malecova, B., Gatto, S., Puri, P. L., et al. (2014). STAT3 signaling controls satellite cell expansion and skeletal muscle repair. Nat. Med. 20, 1182-1186. doi: 10.1038/nm.3656

Travis, M. A., and Sheppard, D. (2014). TGF- $\beta$ activation and function in immunity. Annu. Rev. Immunol. 32, 51-82. doi: 10.1146/annurev-immunol032713-120257

Uezumi, A., Fukada, S., Yamamoto, N., Takeda, S., and Tsuchida, K. (2010). Mesenchymal progenitors distinct from satellite cells contribute to ectopic fat cell formation in skeletal muscle. Nat. Cell Biol. 12, 143-152. doi: 10.1038/ ncb2014

Uezumi, A., Ito, T., Morikawa, D., Shimizu, N., Yoneda, T., Segawa, M., et al. (2011). Fibrosis and adipogenesis originate from a common mesenchymal progenitor in skeletal muscle. J. Cell Sci. 124, 3654-3664. doi: 10.1242/jcs. 086629

Verma, M., Asakura, Y., Murakonda, B. S. R., Pengo, T., Latroche, C., Chazaud, B., et al. (2018). Muscle satellite cell cross-talk with a vascular niche maintains quiescence via VEGF and notch signaling. Cell Stem Cell 23, 530-543.e9. doi: 10.1016/j.stem.2018.09.007

Villalta, S. A., Rinaldi, C., Deng, B., Liu, G., Fedor, B., and Tidball, J. G. (2011). Interleukin-10 reduces the pathology of $\mathrm{mdx}$ muscular dystrophy by deactivating M1 macrophages and modulating macrophage phenotype. Hum. Mol. Genet. 20, 790-805. doi: 10.1093/hmg/ddq523
Villalta, S. A., Rosenthal, W., Martinez, L., Kaur, A., Sparwasser, T., Tidball, J. G., et al. (2014). Regulatory T cells suppress muscle inflammation and injury in muscular dystrophy. Sci. Transl. Med. 6:258ra142. doi: 10.1126/scitranslmed. 3009925

Wang, X., Shen, Q. W., Wang, J., Zhang, Z., Feng, F., Chen, T., et al. (2016). KLF7 regulates satellite cell quiescence in response to extracellular signaling. Stem Cells 34, 1310-1320. doi: 10.1002/stem.2346

Warren, G. L., Hulderman, T., Mishra, D., Gao, X., Millecchia, L., O'Farrell, L., et al. (2005). Chemokine receptor CCR2 involvement in skeletal muscle regeneration. FASEB J. 19, 413-415. doi: 10.1096/fj.04-2421fje

Winbanks, C. E., Weeks, K. L., Thomson, R. E., Sepulveda, P. V., Beyer, C., Qian, H., et al. (2012). Follistatin-mediated skeletal muscle hypertrophy is regulated by Smad3 and mTOR independently of myostatin. J. Cell Biol. 197, 997-1008. doi: $10.1083 /$ jcb.201109091

Wosczyna, M. N., Konishi, C. T., Perez Carbajal, E. E., Wang, T. T., Walsh, R. A., Gan, Q., et al. (2019). Mesenchymal stromal cells are required for regeneration and homeostatic maintenance of skeletal muscle. Cell Rep. 27, 2029-2035.e5. doi: 10.1016/j.celrep.2019.04.074

Wosczyna, M. N., and Rando, T. A. (2018). A muscle stem cell support group: coordinated cellular responses in muscle regeneration. Dev. Cell. 46, 135-143. doi: 10.1016/j.devcel.2018.06.018

Zhang, C., Qiao, Y., Huang, L., Li, F., Zhang, Z., Ping, Y., et al. (2018). Regulatory T cells were recruited by CCL3 to promote cryo-injured muscle repair. Immunol. Lett. 204, 29-37. doi: 10.1016/j.imlet.2018.10.004

Zhang, P., He, J., Wang, F., Gong, J., Wang, L., Wu, Q., et al. (2019). Hemojuvelin is a novel suppressor for duchenne muscular dystrophy and age-related muscle wasting. J. Cachexia. Sarcopenia Muscle 10, 557-573. doi: 10.1002/jcsm.12414

Conflict of Interest Statement: The authors declare that the research was conducted in the absence of any commercial or financial relationships that could be construed as a potential conflict of interest.

The handling Editor declared a shared affiliation, though no other collaboration, with several of the authors, $\mathrm{BB}, \mathrm{CM}$, and DP, at the time of review.

Copyright (c) 2019 Biferali, Proietti, Mozzetta and Madaro. This is an open-access article distributed under the terms of the Creative Commons Attribution License (CC BY). The use, distribution or reproduction in other forums is permitted, provided the original author(s) and the copyright owner(s) are credited and that the original publication in this journal is cited, in accordance with accepted academic practice. No use, distribution or reproduction is permitted which does not comply with these terms. 


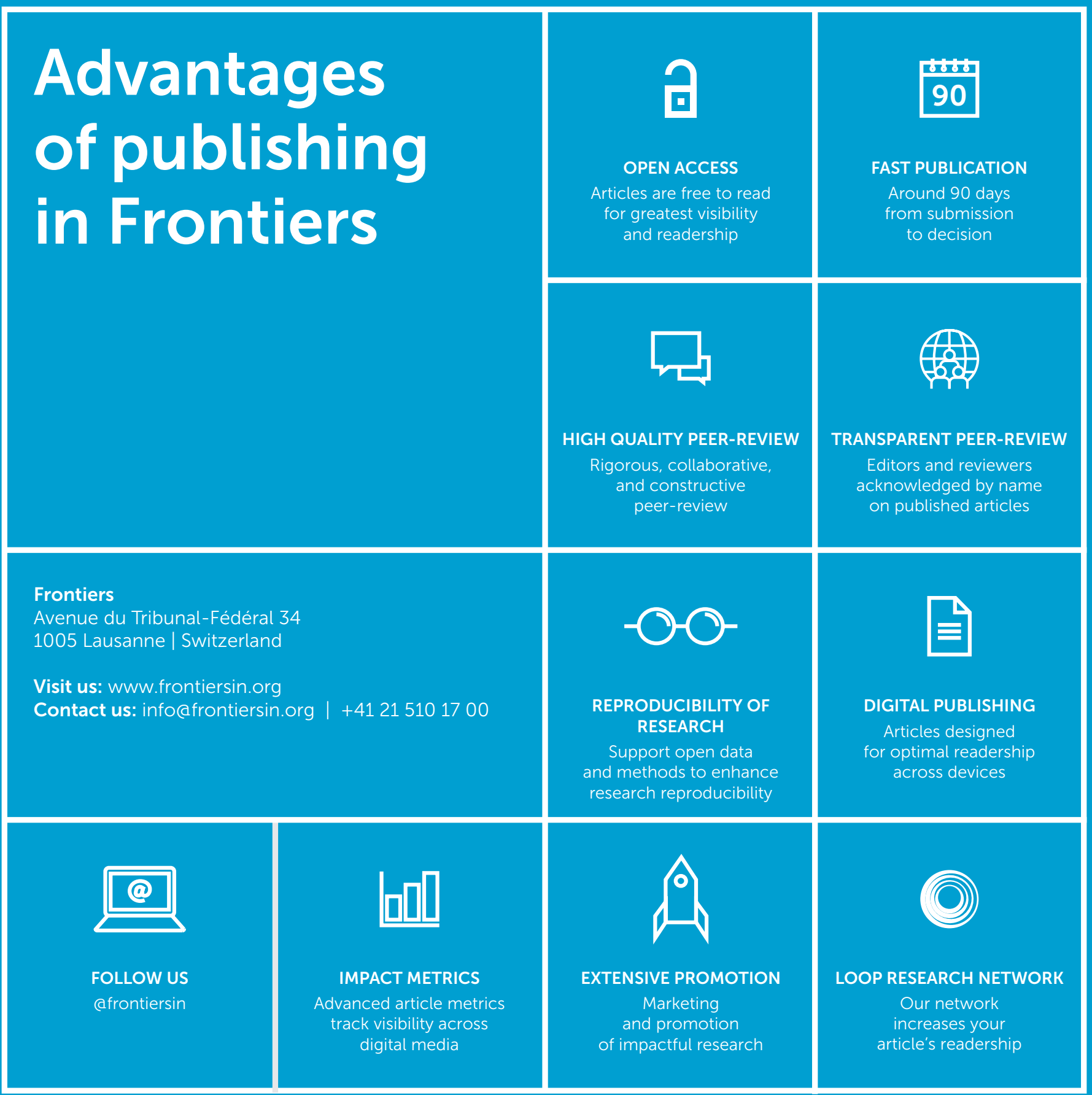

